\title{
All100 988850
}

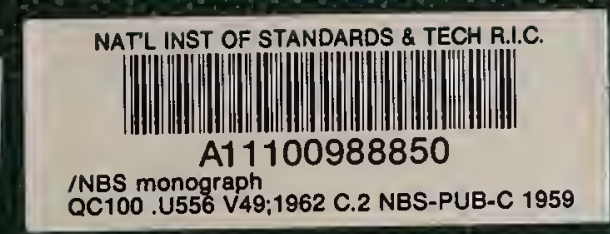

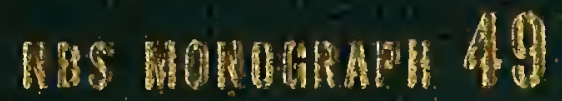

\section{Tables of Finstein Functions}

Wibrationall contributions to

The Thermodynanic leunations

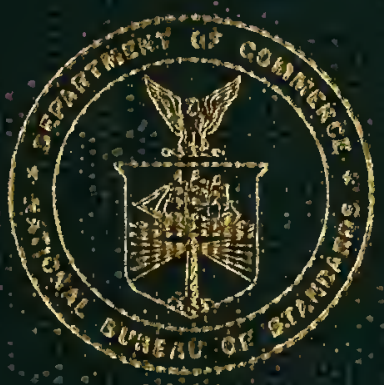



UNITED STATES DEPARTMENT OF COMMERCE - Luther H. Hodges, Secretary NATIONAL BUREAU OF STANDARDS - A. V. Astin, Director

\section{Tables of Einstein Functions}

\section{Vibrational Contributions to}

\section{the Thermodynamic Functions}

Joseph Hilsenrath and Guy G. Ziegler

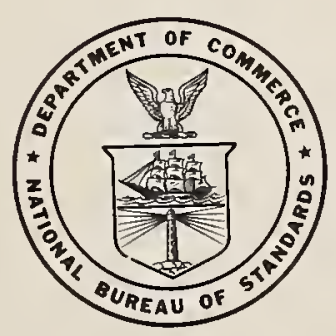

National Bureau of Standards, Monograph 49

Issued July 12, 1962 
atonal Bureau of Standards

AUG 241962

109,387 


\section{Foreword}

This Monograph is one in a series of publications presenting definitive thermodynamic data of interest in pure and applied science and in technology. The tables of Einstein functions should be useful in estimating the thermodynamic properties of polyatomic gases at high and low temperature, as well as the heat conductivity and electrical conductivity of solids.

A. V. Astin, Director. 


\section{Contents}

Foreword

1. Introduction $\ldots \ldots \ldots$

2. Harmonic oscillator contributions to the thermodynamic properties.-

3. The tables and their use

III

4. Computation of the tables.

5. References

Table I. Harmonic oscillator contributions (in dimensionless form) to the thermodynamic functions for values of $X=h c v / k T \ldots \ldots$

Table II. Harmonic oscillator contributions to the thermodynamic

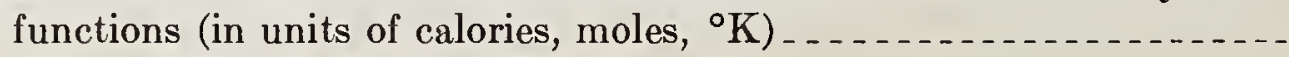




\title{
Tables of Einstein Functions
}

\section{Vibrational Contributions to the Thermodynamic Functions}

\author{
Joseph Hilsenrath and Guy G. Ziegler
}

\begin{abstract}
Tables are presented for the contribution of a harmonic oscillator to the free energy function, enthalpy function, entropy, and heat capacity of gases. Dimensionless values of the Planck-Einstein functions are given as a function of $x=h c \nu / k T$ for $x=0.0010(.0001)$ $0.1500(.001) 4.000(.01) 10.00(.2) 16.0$. A second table which gives the contributions in cal $/ \mathrm{mole}$ - $\mathrm{K}$ directly as a function of frequency $\nu$, and temperature $T$, was computed using the values 1.43880 for the second radiation constant $h c / k$, and 1.98717 for the universal gas constant $R$. The arguments for the latter table are spaced at 10 wave number intervals from 100 $\mathrm{cm}^{-1}$ to $4000 \mathrm{~cm}^{-1}$.
\end{abstract}

\section{Introduction}

Since the publication in 1940 [1] ${ }^{1}$ of a short table of harmonic oscillator contributions obtained by interpolation from unpublished calculations of $\mathrm{H}$. L. Johnston, the interest and need for such tables has increased steadily. In 1942 the tables were modified as a result of changes in the physical constants, and published as an appendix to a treatise on physical chemistry [2]. In the same year independent calculations were published by Sherman and Ewell [3].

The advent of punched-card computing equipment stimulated the computation in 1948 of more extensive tables by Krieger [4] and by Johnston, Savedoff, and Belzer [5]. These tables incorporated the then most recent physical constants. The Krieger tables were issued as a Rand report. The extensive tables of Johnston et al.-perhaps the most convenient tabulation for thermodynamic calculations - were published as an ONR

1 Figures in brackets indicate the literature references on p. vii. report and have long since been out of print. Other works on this subject include shorter but useful tables by Miller, West, and Bernstein [6], Torkington [7], and Overton and Hancock [8].

The recent acceptance by physicists and chemists of new values for the fundamental constants and the continued demand for expanded and convenient tables prompted the recomputation and preparation of this volume. The authors have tried to incorporate in this work a number of the more desirable features of the above-cited works, particularly with respect to their use in calculations involving: Thermodynamic properties of gases; specific heats, electrical and thermal conductivity of solids; and molecular structure and spectroscopy of diatomic and polyatomic molecules. It is the hope of the authors that the formal character of this publication will insure a more adequate notice and dissemination than was accorded the earlier works, thereby reducing the probability of still further duplication of effort.

\section{Harmonic Oscillator Contributions to the Thermodynamic Properties}

The application of statistical mechanical methods to the computation of thermodynamic properties of gases from the energy states of atoms or molecuses requires, in part, the evaıuation of the vibrational and electronic contributions to the partition function. These can be written in terms of sums of exponential functions with suitable weighting factors, provided the partition function is assumed separable. In computing the vibrational contributions to the thermodynamic properties, it is usually the practice to assume a rigid-rotator-harmonic oscillator as a first approximation and then to add corrections for anharmonicities, centrifugal stretching, etc., when suitable experimental data are available. The harmonic oscillator contributions can be written in terms of exponentials of the variable $x=h c \nu / k T$ as follows:

$$
\begin{gathered}
-\left(F^{\circ}-E_{0}^{\circ}\right) / R T=-\ln \left(1-e^{-x}\right) \\
\left(H^{\circ}-E_{0}^{\circ}\right) / R T=x e^{-x}\left(1-e^{-x}\right)^{-1} \\
S^{\circ} / R=x e^{-x}\left(1-e^{-x}\right)^{-1}-\ln \left(1-e^{-x}\right) \\
C_{p}^{\circ} / R=x^{2} e^{-x}\left(1-e^{-x}\right)^{-2}
\end{gathered}
$$

where: $\nu$ is the oscillator or vibrational frequency in wave numbers, $T$ is the absolute temperature in $^{\circ} \mathrm{K}, h c / k=1.43880 \mathrm{~cm} /{ }^{\circ} \mathrm{K}$, and $R=1.98717 \mathrm{cal} /$ mole ${ }^{\circ} \mathrm{K}$. The value of the universal gas constant, $R$, is consistent with $T_{0}=273.15^{\circ} \mathrm{K}$ for the ice point, $[9,10]$ and with the new scale of atomic weights based on $\mathrm{C}^{12}=12$ [11]. The value used for $h c / k$ in this calculation is very close to the value $1.43879 \mathrm{~cm} /{ }^{\circ} \mathrm{K}$ of the new least squares adjusted physical constants [12]. 


\section{The Tables and Their Use}

Two tables have been generated from the present calculations. The first constitutes a basic mathematical table, independent of any physical constants, tabulated at closely spaced intervals of the argument. The second table is presented primarily for the convenience of those engaged in thermodynamic investigations to facilitate calculations at specific temperatures physically attainable.

Table I gives the contributions to the four thermodynamic functions in dimensionless form for closely spaced values of $x=h i \nu / k T$. The values can easily be converted to any desired set of units by multiplying by the gas constant $R$, in appropriate units. For properties on a molar basis $R=1.98717 \mathrm{cal} / \mathrm{mole}{ }^{\circ} \mathrm{K}$. The use of this table requires the evaluation of a single argument $x=1.43879 \nu / T$ for each frequency at aach temperature.

Interpolation, if required, is facilitated by the listing of first differences for each of the four tabulated functions. The spacing of the argument $x$ is as follows: $0.0010(.0001) 0.1500(.001) 4.000(.01)$ $10.00(.2) 16.0$. The spacing of table $I$ is such that, except for values on the first two pages, linear interpolation will give values accurate to one unit in the fifth decimal place. For values of $x$ below 0.01 (on the first two pages) the error resulting from linear interpolation is at most one-eighth of the second difference. For such applications where this error is excessive, use may be made of a 3 or 4 point nonlinear interpolation scheme using Lagrangian interpolation coefficients or an equivalent method employing higher order differences.

Table II lists the vibrational contributions to the free energy function, entropy and heat capacity (in cal $/ \mathrm{mole}^{\circ} \mathrm{K}$ ) explicitly as a function of fre- quency $\nu$ in $\mathrm{cm}^{-1}$ and temperature $T$ in ${ }^{\circ} \mathrm{K}$. The tabulated temperatures are $273.15{ }^{\circ} \mathrm{K}, 298.15^{\circ} \mathrm{K}$, $400{ }^{\circ} \mathrm{K}$ and from there on in $100^{\circ}$ intervals to $5000^{\circ} \mathrm{K}$. For each temperature the tables start uniformly at $\nu=100 \mathrm{~cm}^{-1}$ and extend to $4000 \mathrm{~cm}^{-1}$.

The usefulness of table II can be easily extended to temperatures below $400^{\circ} \mathrm{K}$ or to values other than those listed by simple inspection. The tabulated contributions to the thermal function depend only on the ratio $\nu / T$. Thus the values listed at $1000^{\circ} \mathrm{K}$ are equally applicable to 10 and $100{ }^{\circ} \mathrm{K}$. In the former case the corresponding frequency would be divided by 100 giving a range of 1 to $40 \mathrm{~cm}^{-1}$ at easily interpolatable intervals of $0.1 \mathrm{~cm}^{-1}$. In the latter case the frequency would be divided by 10 giving a frequency range of 10 to $400 \mathrm{~cm}^{-1}$ at intervals of $1 \mathrm{~cm}^{-1}$. This reduction of the frequency range which accompanies any calculations at lower temperatures is not restrictive in practice. In the examples cited, frequencies greater than $40 \mathrm{~cm}^{-1}$ at $10^{\circ} \mathrm{K}$, and $400 \mathrm{~cm}^{-1}$ at $100{ }^{\circ} \mathrm{K}$ do not contribute appreciably to the thermal functions. In a similar fashion, the present construction of table II permits simple conversion of the tabulated values to a range of intermediate temperatures within the tabulated range. For example, between 500 and $600^{\circ} \mathrm{K}$, one can obtain contributions to the thermal function at $550^{\circ} \mathrm{K}$ by doubling the frequencies and reading the values from the $1100^{\circ} \mathrm{K}$ table.

Table II is intended for use at the tabulated arguments or at equivalent arguments easily derivable from them. Since it is not recommended for situations involving interpolations (except as outlined above), no differences are given. Where interpolation cannot be avoided, use should be made of table $I$.

\section{Computation of the Tables}

The tables were computed on the IBM 704 Computer from a program written in the Fortran language and employing mathematical subroutines obtained from the Fortran Library. These were Bell Laboratory modifications of Fortran versions of Los Alamos programs distributed by SHARE under numbers LA S816 for $e^{x}$ and LA S820 for $\ln x$. These subroutines, which are now fairly standard, have an error of at most 3 in the 8 th significant decimal digit.

The calculations were carried out in single precision to machine accuracy and rounded to the figures tabulated. Accordingly, the tables should be correct to within $1 / 2$ unit in the last place tabulated. The tables were printed directly from the machine-printed copy, except for the headings, which were hand typed. Such comparisons as were made between our table I and the corresponding table of Johnston, Savedoff, and Belzer indicate exact agreement over all but the first few entries where the difference is only one in the 5th decimal. The differences between our table II and the earlier works are small and result from the use of different fundamental constants.

The authors acknowledge the assistance rendered by Miss Carla Harms in checking the tables, and by Mrs. Eleanor F. Rozsics in preparing the manuscript. Thanks are also due Dr. David White for stimulating discussions and helpful editorial suggestions. 


\section{References}

[1] Wilson, E. B. Jr., The present status of the statistical method of calculating thermodynamic functions, Chem. Revs. 27, 17-38 (1940).

[2] Taylor and Glasstone, Treatise on physical chemistry, Van Nostrand Co., Appendix I, Harmonic Oscillator Contributions (1942).

[3] Sherman, J., and Ewell, R. B., Six place table of the Einstein functions, J. Chem. Phys. 46, 641-62 (1942).

[4] Krieger, F. J., A table of vibrational contributions of a harmonic oscillator to thermodynamic functions, Rand Report RA 15088, Douglas Aircraft Co., Inc. (July 1, 1948).

[5] Johnston, H. L., Savedoff, L., and Belzer, J., Contributions to the thermodynamic functions by a Planck-Einstein oscillator in one degree of freedom, Tech. Rept. No. 3, The Ohio State University Research Foundation, Columbus, Ohio, May 8, 1949. Prepared under ONR contract. Later published by the Superintendent of Documents, Government Printing Office, Washington 25, D.C. (1949).

[6] Miller, E., West, K., and Bernstein, H. J., Tables of functions for the vibrational contributions to ther- modynamic quantities, NRC Bull. No. 1, National Research Council, Ottawa, Canada (1951).

[7] Torkington, P., Harmonic oscillator contributions to the thermodynamic functions, J. Chem. Phys. 18, 1373-79, (Oct. 1950).

[8] Overton, W. C. Jr., and Hancock, J. H., Tables of Einstein energy, specific heat, and derivative specific heat functions, NRL Report 5502, Naval Research Laboratory,. Washington, D.C. (July 6, 1960).

[9] Comptes Rendus de la Onzieme Conference Generale des Poids et Measures (1960).

[10] Stimson, H. F., International Practical Temperature Scale of 1948: Text Revision of 1960, J. Research NBS 65A (Phys. and Chem.) 139-145 (1961); also issued as NBS Mono. 37 (Sept. 8, 1961).

[11] Batuecas, T., and Gueron, J., Preliminary report of the Commission on Atomic Weights. Reprint from Information Bulletin No. 14b, International Union of Pure and Applied Chemistry (1961).

[12] Cohen, E. R., Dumond, J. IV. M., McNish, A. G. (to be published). 



\section{Table I.}

\section{Harmonic Oscillator Contributions}

(in dimensionless form)

to the Thermodynamic Functions

for Values of $X=h c v / k T$ 

Table I Harmonic Oscillator Contributions (in dimensionless form) to the Thermodynamic Functions for Values of $\mathrm{X}=\mathrm{hcv} / \mathrm{kT}$

\begin{tabular}{|c|c|c|c|c|c|c|c|}
\hline $\mathbf{X}$ & $-\left(F^{\circ}-E_{O} O\right) / R T$ & $\Delta$ & $\left(H^{\circ}-E_{O}^{\circ}\right) / R T$ & $\Delta$ & $S \circ / R$ & $\Delta$ & $\stackrel{\mathrm{p}}{\mathrm{p}} / \mathrm{R}$ \\
\hline .0010 & 6.90827 & 9526 & 0.99951 & 4 & 7.90778 & 9530 & 1.00000 \\
\hline $\begin{array}{l}0.0011 \\
0.0012 \\
0.0013 \\
0.0014 \\
0.0015\end{array}$ & $\begin{array}{l}6.81301 \\
6.72604 \\
6.64605 \\
6.57198 \\
6.50304\end{array}$ & $\begin{array}{l}8697 \\
7999 \\
7407 \\
6894 \\
6449\end{array}$ & $\begin{array}{l}0.99947 \\
0.99940 \\
0.99936 \\
0.99930 \\
0.99925\end{array}$ & $\begin{array}{l}7 \\
4 \\
6 \\
5 \\
5\end{array}$ & $\begin{array}{l}7.81248 \\
7.72544 \\
7.64541 \\
7.57129 \\
7.50230\end{array}$ & $\begin{array}{l}8704 \\
8003 \\
7412 \\
6899 \\
6455\end{array}$ & $\begin{array}{l}1.00000 \\
1.00000 \\
1.00000 \\
1.00000 \\
1.00000\end{array}$ \\
\hline $\begin{array}{l}0.0016 \\
0.0017 \\
0.0018 \\
0.0019 \\
0.0020\end{array}$ & $\begin{array}{l}6 \cdot 43855 \\
6 \cdot 37798 \\
6 \cdot 32088 \\
6 \cdot 26686 \\
6.21562\end{array}$ & $\begin{array}{l}6057 \\
5710 \\
5402 \\
5124 \\
4875\end{array}$ & $\begin{array}{l}0.99920 \\
0.99915 \\
0.99911 \\
0.99906 \\
0.99901\end{array}$ & $\begin{array}{l}5 \\
4 \\
5 \\
5 \\
6\end{array}$ & $\begin{array}{l}7.43775 \\
7.37713 \\
7.31999 \\
7.26591 \\
7.214 ; 2\end{array}$ & $\begin{array}{l}6062 \\
5714 \\
5408 \\
5129 \\
4880\end{array}$ & $\begin{array}{l}1.00000 \\
1.00000 \\
1.00000 \\
1.00000 \\
1.00000\end{array}$ \\
\hline $\begin{array}{l}0.0021 \\
0.0022 \\
0.0023 \\
0.0024 \\
0.0025\end{array}$ & $\begin{array}{l}6.16687 \\
6.12040 \\
6.07600 \\
6.03349 \\
5.99271\end{array}$ & $\begin{array}{l}4647 \\
4440 \\
4251 \\
4078 \\
3916\end{array}$ & $\begin{array}{l}0.99895 \\
0.99891 \\
0.99885 \\
0.99880 \\
0.99875\end{array}$ & $\begin{array}{l}4 \\
6 \\
5 \\
5 \\
5\end{array}$ & $\begin{array}{l}7.16582 \\
7.11931 \\
7.07485 \\
7.03229 \\
6.99147\end{array}$ & $\begin{array}{l}4651 \\
4446 \\
4256 \\
4082 \\
3922\end{array}$ & $\begin{array}{l}1.00000 \\
1.00000 \\
1.00000 \\
1.00000 \\
1.00000\end{array}$ \\
\hline $\begin{array}{l}0.0026 \\
0.0027 \\
0.0028 \\
0.0029 \\
0.0030\end{array}$ & $\begin{array}{l}5.95355 \\
5.91586 \\
5.87954 \\
5.84450 \\
5.81064\end{array}$ & $\begin{array}{l}3769 \\
3632 \\
3504 \\
3386 \\
3273\end{array}$ & $\begin{array}{l}0.99870 \\
0.99866 \\
0.99860 \\
0.99855 \\
0.99850\end{array}$ & $\begin{array}{l}4 \\
6 \\
5 \\
5 \\
5\end{array}$ & $\begin{array}{l}6.95225 \\
6.91452 \\
6.87814 \\
6.84305 \\
6.80915\end{array}$ & $\begin{array}{l}3773 \\
3638 \\
3509 \\
3390 \\
3279\end{array}$ & $\begin{array}{l}1.00000 \\
1.00000 \\
1.00000 \\
1.00000 \\
1.00000\end{array}$ \\
\hline $\begin{array}{l}0.0031 \\
0.0032 \\
0.0333 \\
0.0034 \\
0.0035\end{array}$ & $\begin{array}{l}5.77791 \\
5.74620 \\
5.71548 \\
5.68568 \\
5.65674\end{array}$ & $\begin{array}{l}3171 \\
3072 \\
2980 \\
2894 \\
2811\end{array}$ & $\begin{array}{l}0.99845 \\
0.99840 \\
0.99835 \\
0.99830 \\
0.99825\end{array}$ & $\begin{array}{l}5 \\
5 \\
5 \\
5 \\
4\end{array}$ & $\begin{array}{l}6.77636 \\
6.74461 \\
6.71384 \\
6.68398 \\
6.65500\end{array}$ & $\begin{array}{l}3175 \\
3077 \\
2986 \\
2898 \\
2817\end{array}$ & $\begin{array}{l}1.00000 \\
1.00000 \\
1.00000 \\
1.00000 \\
1.00000\end{array}$ \\
\hline $\begin{array}{l}0.0036 \\
0.0037 \\
0.0038 \\
0.0039 \\
0.0040\end{array}$ & $\begin{array}{l}5.62863 \\
5.60127 \\
5.57466 \\
5.54873 \\
5.52346\end{array}$ & $\begin{array}{l}2736 \\
2661 \\
2593 \\
2527 \\
2464\end{array}$ & $\begin{array}{l}0.99821 \\
0.99815 \\
0.99810 \\
0.99805 \\
0.99800\end{array}$ & $\begin{array}{l}6 \\
5 \\
5 \\
5 \\
5\end{array}$ & $\begin{array}{l}6.62683 \\
6.59943 \\
6.57276 \\
6.54678 \\
6.52147\end{array}$ & $\begin{array}{l}2740 \\
2667 \\
2598 \\
2531 \\
2470\end{array}$ & $\begin{array}{l}1.00000 \\
1.00000 \\
1.00000 \\
1.00000 \\
1.00000\end{array}$ \\
\hline $\begin{array}{l}0.0041 \\
0.0042 \\
0.0043 \\
0.0044 \\
0.0045\end{array}$ & $\begin{array}{l}5.49882 \\
5.47477 \\
5.45129 \\
5.42835 \\
5.40593\end{array}$ & $\begin{array}{l}2405 \\
2348 \\
2294 \\
2242 \\
2193\end{array}$ & $\begin{array}{l}0.99795 \\
0.99790 \\
0.99785 \\
0.99780 \\
0.99775\end{array}$ & $\begin{array}{l}5 \\
5 \\
5 \\
5 \\
5\end{array}$ & $\begin{array}{l}6.49677 \\
6.47268 \\
6.44914 \\
6.42615 \\
6.40368\end{array}$ & $\begin{array}{l}2409 \\
2354 \\
2299 \\
2247 \\
2198\end{array}$ & $\begin{array}{l}1.00000 \\
1.00000 \\
1.00000 \\
1.00000 \\
1.00000\end{array}$ \\
\hline $\begin{array}{l}0.0046 \\
0.0047 \\
0.0048 \\
0.0049 \\
0.0050\end{array}$ & $\begin{array}{l}5.38400 \\
5.36255 \\
5.34154 \\
5 \cdot 32097 \\
5.30082\end{array}$ & $\begin{array}{l}2145 \\
2101 \\
2057 \\
2015\end{array}$ & $\begin{array}{l}0.99770 \\
0.99766 \\
0.99760 \\
0.99755 \\
0.99750\end{array}$ & $\begin{array}{l}4 \\
6 \\
5 \\
5\end{array}$ & $\begin{array}{l}6.38170 \\
6.36020 \\
6.33914 \\
6.31852 \\
6.29832\end{array}$ & $\begin{array}{l}2150 \\
2106 \\
2062 \\
2020\end{array}$ & $\begin{array}{l}1.00000 \\
1.00000 \\
1.00000 \\
1.00000 \\
1.00000\end{array}$ \\
\hline
\end{tabular}


Table I Harmonic Oscillator Contributions (in dimensionless form) to the Thermodynamic Functions for Values of $\mathrm{X}=\mathrm{hc} / \mathrm{kT}$

\begin{tabular}{|c|c|c|c|c|c|c|c|}
\hline $\mathbf{I}$ & $-\left(F^{\circ}-E_{O} O\right) / R T$ & $\Delta$ & $\left(H^{\circ}-E_{O}^{O}\right) / R T$ & $\Delta$ & $\mathrm{S} / \mathrm{R}$ & $\Delta$ & $\mathrm{Co} / \mathrm{R}$ \\
\hline 0.0050 & $5 \cdot 30082$ & 1976 & 0.99750 & 5 & 6.29832 & 1980 & 1.00000 \\
\hline $\begin{array}{l}0.0051 \\
0.0052 \\
0.0053 \\
0.0054 \\
0.0055\end{array}$ & $\begin{array}{l}5.28106 \\
5.26170 \\
5.24270 \\
5.22406 \\
5.20576\end{array}$ & $\begin{array}{l}1937 \\
1899 \\
1864 \\
1830 \\
1797\end{array}$ & $\begin{array}{l}0.99745 \\
0.99740 \\
0.99735 \\
0.99731 \\
0.99725\end{array}$ & $\begin{array}{l}5 \\
5 \\
4 \\
6 \\
5\end{array}$ & $\begin{array}{l}6.27852 \\
6.25910 \\
6.24005 \\
6.22136 \\
6.20301\end{array}$ & $\begin{array}{l}1942 \\
1905 \\
1869 \\
1835 \\
1802\end{array}$ & $\begin{array}{l}1.00000 \\
1.00000 \\
1.00000 \\
1.00000 \\
1.00000\end{array}$ \\
\hline $\begin{array}{l}0.0056 \\
0.0057 \\
0.0058 \\
0.0259 \\
0.0060\end{array}$ & $\begin{array}{l}5.18779 \\
5.17014 \\
5.15280 \\
5.13575 \\
5.11900\end{array}$ & $\begin{array}{l}1765 \\
1734 \\
1705 \\
1675 \\
1649\end{array}$ & $\begin{array}{l}0.99720 \\
0.99715 \\
0.99710 \\
0.99705 \\
0.99700\end{array}$ & $\begin{array}{l}5 \\
5 \\
5 \\
5 \\
5\end{array}$ & $\begin{array}{l}6.18499 \\
6.16729 \\
6.14990 \\
6.13280 \\
6.11600\end{array}$ & $\begin{array}{l}1770 \\
1739 \\
1710 \\
1680 \\
1653\end{array}$ & $\begin{array}{l}1.00000 \\
1.00000 \\
1.00000 \\
1.00000 \\
1.00000\end{array}$ \\
\hline $\begin{array}{l}0.0061 \\
0.0062 \\
0.0063 \\
0.0064 \\
0.0065\end{array}$ & $\begin{array}{l}5.10251 \\
5.08631 \\
5.07036 \\
5.05466 \\
5.03920\end{array}$ & $\begin{array}{l}1620 \\
1595 \\
1570 \\
1546 \\
1522\end{array}$ & $\begin{array}{l}0.99695 \\
0.99691 \\
0.99686 \\
0.99680 \\
0.99676\end{array}$ & $\begin{array}{l}4 \\
5 \\
6 \\
4 \\
6\end{array}$ & $\begin{array}{l}6.09947 \\
6.08321 \\
6.06721 \\
6.05146 \\
6.03596\end{array}$ & $\begin{array}{l}1626 \\
1600 \\
1575 \\
1550 \\
1527\end{array}$ & $\begin{array}{l}1.00000 \\
1.00000 \\
1.00000 \\
1.00000 \\
1.00000\end{array}$ \\
\hline $\begin{array}{l}0.0066 \\
0.0067 \\
0.0068 \\
0.0069 \\
0.0070\end{array}$ & $\begin{array}{l}5.02398 \\
5.00900 \\
4.99423 \\
4.97968 \\
4.96534\end{array}$ & $\begin{array}{l}1498 \\
1477 \\
1455 \\
1434 \\
1413\end{array}$ & $\begin{array}{l}0.99670 \\
0.99665 \\
0.99660 \\
0.99655 \\
0.99650\end{array}$ & $\begin{array}{l}5 \\
5 \\
5 \\
5 \\
4\end{array}$ & $\begin{array}{l}6.02069 \\
6.00565 \\
5.99084 \\
5.97624 \\
5.96185\end{array}$ & $\begin{array}{l}1504 \\
1481 \\
1460 \\
1439 \\
1419\end{array}$ & $\begin{array}{l}1.00000 \\
1.00000 \\
1.00000 \\
1.00000 \\
1.00000\end{array}$ \\
\hline $\begin{array}{l}0.0071 \\
0.0072 \\
0.0073 \\
0.0074 \\
0.0075\end{array}$ & $\begin{array}{l}4.95121 \\
4.93727 \\
4.92353 \\
4.90997 \\
4.89660\end{array}$ & $\begin{array}{l}1394 \\
1374 \\
1356 \\
1337 \\
1319\end{array}$ & $\begin{array}{l}0.99646 \\
0.99641 \\
0.99636 \\
0.99631 \\
0.99626\end{array}$ & $\begin{array}{l}5 \\
5 \\
5 \\
5 \\
5\end{array}$ & $\begin{array}{l}5.94766^{\circ} \\
5.93368 \\
5.91988 \\
5.90628 \\
5.89286\end{array}$ & $\begin{array}{l}1398 \\
1380 \\
1360 \\
1342 \\
1325\end{array}$ & $\begin{array}{l}1.00000 \\
1.00000 \\
1.00000 \\
1.00000 \\
1.00000\end{array}$ \\
\hline $\begin{array}{l}0.0076 \\
0.0077 \\
0.0078 \\
0.0079 \\
0.0080\end{array}$ & $\begin{array}{l}4.88341 \\
4.87038 \\
4.85753 \\
4.84484 \\
4.83231\end{array}$ & $\begin{array}{l}1303 \\
1285 \\
1269 \\
1253 \\
1237\end{array}$ & $\begin{array}{l}0.99621 \\
0.99615 \\
0.99611 \\
0.99605 \\
0.99601\end{array}$ & $\begin{array}{l}6 \\
4 \\
6 \\
4 \\
5\end{array}$ & $\begin{array}{l}5.37961 \\
5.86654 \\
5.85354 \\
5.84089 \\
5.82832\end{array}$ & $\begin{array}{l}1307 \\
1290 \\
1275 \\
1257 \\
1242\end{array}$ & $\begin{array}{l}1.00000 \\
0.99999 \\
1.00000 \\
0.99999 \\
1.00000\end{array}$ \\
\hline $\begin{array}{l}0.0081 \\
0.0082 \\
0.0083 \\
0.0084 \\
0.0085\end{array}$ & $\begin{array}{l}4.81994 \\
4.80772 \\
4.79565 \\
4.78372 \\
4.77194\end{array}$ & $\begin{array}{l}1222 \\
1207 \\
1193 \\
1178 \\
1165\end{array}$ & $\begin{array}{l}0.99596 \\
0.99591 \\
0.99586 \\
0.99581 \\
0.99576\end{array}$ & $\begin{array}{l}5 \\
5 \\
5 \\
5 \\
5\end{array}$ & $\begin{array}{l}5.81590 \\
5.80363 \\
5.79151 \\
5.77953 \\
5.76769\end{array}$ & $\begin{array}{l}1227 \\
1212 \\
1198 \\
1184 \\
1169\end{array}$ & $\begin{array}{l}1.00000 \\
1.00000 \\
1.00000 \\
0.99999 \\
1.00000\end{array}$ \\
\hline $\begin{array}{l}0.0086 \\
0.0087 \\
0.0088 \\
0.0089 \\
0.0090\end{array}$ & $\begin{array}{l}4.76029 \\
4.74878 \\
4.73740 \\
4.72615 \\
4.71503\end{array}$ & $\begin{array}{l}1151 \\
1138 \\
1125 \\
11112 \\
1100\end{array}$ & $\begin{array}{l}0.99571 \\
0.99566 \\
0.99561 \\
0.99556 \\
0.99551\end{array}$ & $\begin{array}{l}5 \\
5 \\
5 \\
5 \\
5\end{array}$ & $\begin{array}{l}5.75600 \\
5.74444 \\
5.73301 \\
5.72171 \\
5.71054\end{array}$ & $\begin{array}{l}1156 \\
1143 \\
1130 \\
1117 \\
1105\end{array}$ & $\begin{array}{l}0.99999 \\
0.99999 \\
0.99999 \\
0.99999 \\
1.00000\end{array}$ \\
\hline $\begin{array}{l}0.0091 \\
0.0092 \\
0.0093 \\
0.0094 \\
0.0095\end{array}$ & $\begin{array}{l}4.70403 \\
4.69315 \\
4.68239 \\
4.67174 \\
4.66121\end{array}$ & $\begin{array}{l}1088 \\
1076 \\
1065 \\
1053 \\
1042\end{array}$ & $\begin{array}{l}0.99546 \\
0.99541 \\
0.99536 \\
0.99531 \\
0.99526\end{array}$ & $\begin{array}{l}5 \\
5 \\
5 \\
5 \\
5\end{array}$ & $\begin{array}{l}5.69949 \\
5.68856 \\
5.67775 \\
5.66705 \\
5.65647\end{array}$ & $\begin{array}{l}1093 \\
1081 \\
1070 \\
1058 \\
1047\end{array}$ & $\begin{array}{l}0.99999 \\
1.00000 \\
0.99999 \\
0.99999 \\
0.99999\end{array}$ \\
\hline $\begin{array}{l}0.0096 \\
0.0097 \\
0.0098 \\
0.0099 \\
0.0100\end{array}$ & $\begin{array}{l}4.65079 \\
4.64048 \\
4.63027 \\
4.62017 \\
4.61017\end{array}$ & $\begin{array}{l}1031 \\
1021 \\
1010 \\
1000\end{array}$ & $\begin{array}{l}0.99521 \\
0.99516 \\
0.99511 \\
0.99506 \\
0.99501\end{array}$ & $\begin{array}{l}5 \\
5 \\
5 \\
5\end{array}$ & $\begin{array}{l}5.64600 \\
5.63564 \\
5.62538 \\
5.61523 \\
5.60518\end{array}$ & $\begin{array}{l}1036 \\
1026 \\
1015 \\
1005\end{array}$ & $\begin{array}{l}0.99999 \\
1.00000 \\
0.99999 \\
0.99999 \\
0.99999\end{array}$ \\
\hline
\end{tabular}


Table 1 Harmonic Oscillator Contributions (in dimensionless form) to the Thermodynamic Functions for Values of $\mathrm{X}=\mathrm{hc} / \mathrm{kT}$

\begin{tabular}{|c|c|c|c|c|c|c|c|}
\hline 8 & $-\left(F^{\circ}-E_{O} O\right) / R T$ & $\Delta$ & $\left(H^{\circ}-E_{O}^{\circ}\right) / R T$ & $\Delta$ & $\mathrm{S} \% / \mathrm{R}$ & $\Delta$ & $\mathrm{C}_{\mathrm{p}}^{\circ} / \mathrm{R}$ \\
\hline 0.0100 & 4.61017 & 990 & 0.99501 & 5 & 5.60518 & 995 & 0.99999 \\
\hline $\begin{array}{l}0.0101 \\
0.0102 \\
0.0103 \\
0.0104 \\
0.0105\end{array}$ & $\begin{array}{l}4.60027 \\
4.59046 \\
4.58076 \\
4.57114 \\
4.56163\end{array}$ & $\begin{array}{l}981 \\
970 \\
962 \\
951 \\
943\end{array}$ & $\begin{array}{l}0.99496 \\
0.99491 \\
0.99486 \\
0.99481 \\
0.99476\end{array}$ & $\begin{array}{l}5 \\
5 \\
5 \\
5 \\
5\end{array}$ & $\begin{array}{l}5.59523 \\
5.58537 \\
5.57562 \\
5.56595 \\
5.55638\end{array}$ & $\begin{array}{l}986 \\
975 \\
967 \\
957 \\
947\end{array}$ & $\begin{array}{l}0.99999 \\
0.99999 \\
0.99999 \\
0.99999 \\
0.99999\end{array}$ \\
\hline $\begin{array}{l}0.0106 \\
0.0107 \\
0.0108 \\
0.0109 \\
0.0110\end{array}$ & $\begin{array}{l}4.55220 \\
4.54286 \\
4.53361 \\
4.52444 \\
4.51536\end{array}$ & $\begin{array}{l}934 \\
925 \\
917 \\
908 \\
901\end{array}$ & $\begin{array}{l}0.99471 \\
0.99466 \\
0.99461 \\
0.99456 \\
0.99451\end{array}$ & $\begin{array}{l}5 \\
5 \\
5 \\
5 \\
5\end{array}$ & $\begin{array}{l}5.54691 \\
5.53752 \\
5.52822 \\
5.51900 \\
5.50987\end{array}$ & $\begin{array}{l}939 \\
930 \\
922 \\
913 \\
906\end{array}$ & $\begin{array}{l}0.99999 \\
0.99999 \\
0.99999 \\
0.99999 \\
0.99999\end{array}$ \\
\hline $\begin{array}{l}0.0111 \\
0.0112 \\
0.0113 \\
0.0114 \\
0.0115\end{array}$ & $\begin{array}{l}4.50635 \\
4.49744 \\
4.48860 \\
4.47984 \\
4.47115\end{array}$ & $\begin{array}{l}891 \\
884 \\
876 \\
869 \\
860\end{array}$ & $\begin{array}{l}0.99446 \\
0.99441 \\
0.99436 \\
0.99431 \\
0.99426\end{array}$ & $\begin{array}{l}5 \\
5 \\
5 \\
5 \\
5\end{array}$ & $\begin{array}{l}5.50081 \\
5.49185 \\
5.48296 \\
5.47415 \\
5.46542\end{array}$ & $\begin{array}{l}896 \\
889 \\
881 \\
873 \\
866\end{array}$ & $\begin{array}{l}0.99999 \\
0.99999 \\
0.99999 \\
0.99999 \\
0.99999\end{array}$ \\
\hline $\begin{array}{l}0.0116 \\
0.0117 \\
0.0118 \\
0.0119 \\
0.0120\end{array}$ & $\begin{array}{l}4.46255 \\
4.45401 \\
4.44555 \\
4.43716 \\
4.42884\end{array}$ & $\begin{array}{l}854 \\
846 \\
839 \\
832 \\
825\end{array}$ & $\begin{array}{l}0.99421 \\
0.99416 \\
0.99411 \\
0.99406 \\
0.99401\end{array}$ & $\begin{array}{l}5 \\
5 \\
5 \\
5 \\
5\end{array}$ & $\begin{array}{l}5.45676 \\
5.44817 \\
5.43966 \\
5.43122 \\
5.42286\end{array}$ & $\begin{array}{l}859 \\
851 \\
844 \\
836 \\
830\end{array}$ & $\begin{array}{l}0.99999 \\
0.99999 \\
0.99999 \\
0.99999 \\
0.99999\end{array}$ \\
\hline $\begin{array}{l}0.0121 \\
0.0122 \\
0.0123 \\
0.0124 \\
0.0125\end{array}$ & $\begin{array}{l}4.42059 \\
4.41241 \\
4.40430 \\
4.39625 \\
4.38827\end{array}$ & $\begin{array}{l}818 \\
811 \\
805 \\
798 \\
792\end{array}$ & $\begin{array}{l}0.99396 \\
0.99391 \\
0.99386 \\
0.99381 \\
0.99376\end{array}$ & $\begin{array}{l}5 \\
5 \\
5 \\
5 \\
5\end{array}$ & $\begin{array}{l}5.41456 \\
5.40632 \\
5.39816 \\
5.39007 \\
5.38203\end{array}$ & $\begin{array}{l}824 \\
816 \\
809 \\
804 \\
796\end{array}$ & $\begin{array}{l}0.99999 \\
0.99999 \\
0.99999 \\
0.99999 \\
0.99999\end{array}$ \\
\hline $\begin{array}{l}0.0126 \\
0.0127 \\
0.0128 \\
0.0129 \\
0.0130\end{array}$ & $\begin{array}{l}4.38035 \\
4 \cdot 37250 \\
4 \cdot 36470 \\
4.35697 \\
4.34930\end{array}$ & $\begin{array}{l}785 \\
780 \\
773 \\
767 \\
761\end{array}$ & $\begin{array}{l}0.99371 \\
0.99366 \\
0.99361 \\
0.99356 \\
0.99351\end{array}$ & $\begin{array}{l}5 \\
5 \\
5 \\
5 \\
5\end{array}$ & $\begin{array}{l}5.37407 \\
5.36616 \\
5.35832 \\
5.35053 \\
5.34281\end{array}$ & $\begin{array}{l}791 \\
784 \\
779 \\
772 \\
766\end{array}$ & $\begin{array}{l}0.99999 \\
0.99999 \\
0.99999 \\
0.99999 \\
0.99999\end{array}$ \\
\hline $\begin{array}{l}0.0131 \\
0.0132 \\
0.0133 \\
0.0134 \\
0.0135\end{array}$ & $\begin{array}{l}4.34169 \\
4.33413 \\
4.32664 \\
4.31919 \\
4.31181\end{array}$ & $\begin{array}{l}756 \\
749 \\
745 \\
738 \\
733\end{array}$ & $\begin{array}{l}0.99346 \\
0.99341 \\
0.99337 \\
0.99332 \\
0.99327\end{array}$ & $\begin{array}{l}5 \\
4 \\
5 \\
5 \\
5\end{array}$ & $\begin{array}{l}5.33515 \\
5.32755 \\
5.32000 \\
5.31251 \\
5.30507\end{array}$ & $\begin{array}{l}760 \\
755 \\
749 \\
744 \\
738\end{array}$ & $\begin{array}{l}0.99999 \\
0.99999 \\
0.99999 \\
0.99999 \\
0.99999\end{array}$ \\
\hline $\begin{array}{l}0.0136 \\
0.0137 \\
0.0138 \\
0.0139 \\
0.0140\end{array}$ & $\begin{array}{l}4.30448 \\
4.29720 \\
4.28998 \\
4.28281 \\
4.27569\end{array}$ & $\begin{array}{l}728 \\
722 \\
717 \\
712 \\
707\end{array}$ & $\begin{array}{l}0.99322 \\
0.99317 \\
0.99312 \\
0.99307 \\
0.99302\end{array}$ & $\begin{array}{l}5 \\
5 \\
5 \\
5 \\
5\end{array}$ & $\begin{array}{l}5.29759 \\
5.29037 \\
5.28379 \\
5.27588 \\
5.26871\end{array}$ & $\begin{array}{l}732 \\
728 \\
721 \\
717 \\
712\end{array}$ & $\begin{array}{l}0.99999 \\
0.99999 \\
0.99998 \\
0.99999 \\
0.99998\end{array}$ \\
\hline $\begin{array}{l}0.0141 \\
0.0142 \\
0.0143 \\
0.0144 \\
0.0145\end{array}$ & $\begin{array}{l}4.26862 \\
4.26161 \\
4.25464 \\
4.24772 \\
4.24085\end{array}$ & $\begin{array}{l}701 \\
697 \\
692 \\
687 \\
682\end{array}$ & $\begin{array}{l}0.99297 \\
0.99292 \\
0.99287 \\
0.99282 \\
0.99277\end{array}$ & $\begin{array}{l}5 \\
5 \\
5 \\
5 \\
5\end{array}$ & $\begin{array}{l}5.26159 \\
5.25452 \\
5.24750 \\
5.24054 \\
5.23362\end{array}$ & $\begin{array}{l}707 \\
702 \\
696 \\
692 \\
688\end{array}$ & $\begin{array}{l}0.99999 \\
0.99999 \\
0.99998 \\
0.99998 \\
0.99998\end{array}$ \\
\hline $\begin{array}{l}0.0146 \\
0.0147 \\
0.0148 \\
0.0149 \\
0.0150\end{array}$ & $\begin{array}{l}4.23403 \\
4.22725 \\
4 \cdot 22052 \\
4.21383 \\
4.20720\end{array}$ & $\begin{array}{l}678 \\
673 \\
669 \\
663\end{array}$ & $\begin{array}{l}0.99272 \\
0.99267 \\
0.99262 \\
0.99257 \\
0.99252\end{array}$ & $\begin{array}{l}5 \\
5 \\
5 \\
5\end{array}$ & $\begin{array}{l}5.22674 \\
5.21992 \\
5.21314 \\
5.20640 \\
5.19972 .\end{array}$ & $\begin{array}{l}682 \\
678 \\
674 \\
668\end{array}$ & $\begin{array}{l}0.99998 \\
0.99998 \\
0.99998 \\
0.99998 \\
0.99998\end{array}$ \\
\hline
\end{tabular}


Table I Harmonic Oscillator Contributions (in dimensionless form) to the Thermodynamic Functions for Values of $\mathrm{X}=\mathrm{hc} / \mathrm{kT}$

\begin{tabular}{|c|c|c|c|c|c|c|c|}
\hline$x$ & $-\left(F^{\circ}-E_{O}^{\circ}\right) / R T$ & $\Delta$ & $\left(H^{\circ}-E_{0}^{O}\right) / R T$ & $\Delta$ & So/R & $\Delta$ & $\mathrm{Co} / \mathrm{R}$ \\
\hline 0.0150 & 4.20720 & 660 & 0.99252 & 5 & 5.19972 & 665 & 0.99998 \\
\hline $\begin{array}{l}0.0151 \\
0.0152 \\
0.0153 \\
0.0154 \\
0.0155\end{array}$ & $\begin{array}{l}4.20060 \\
4.19405 \\
4.18754 \\
4.18108 \\
4.17466\end{array}$ & $\begin{array}{l}655 \\
651 \\
646 \\
642 \\
639\end{array}$ & $\begin{array}{l}0.99247 \\
0.99242 \\
0.99237 \\
0.99232 \\
0.99227\end{array}$ & $\begin{array}{l}5 \\
5 \\
5 \\
5 \\
5\end{array}$ & $\begin{array}{l}5.19307 \\
5.18647 \\
5.17991 \\
5.17340 \\
5.16693\end{array}$ & $\begin{array}{l}660 \\
656 \\
651 \\
647 \\
644\end{array}$ & $\begin{array}{l}0.99998 \\
0.99998 \\
0.99998 \\
0.99998 \\
0.99998\end{array}$ \\
\hline $\begin{array}{l}0.0156 \\
0.0157 \\
0.0158 \\
0.0159 \\
0.0160\end{array}$ & $\begin{array}{l}4.16827 \\
4.16193 \\
4.15563 \\
4.14938 \\
4.14316\end{array}$ & $\begin{array}{l}634 \\
630 \\
625 \\
622 \\
618\end{array}$ & $\begin{array}{l}0.99222 \\
0.99217 \\
0.99212 \\
0.99207 \\
0.99202\end{array}$ & $\begin{array}{l}5 \\
5 \\
5 \\
5 \\
5\end{array}$ & $\begin{array}{l}5.16049 \\
5.15411 \\
5.14776 \\
5.14145 \\
5.13518\end{array}$ & $\begin{array}{l}638 \\
635 \\
631 \\
627 \\
623\end{array}$ & $\begin{array}{l}0.99998 \\
0.99998 \\
0.99998 \\
0.99998 \\
0.99998\end{array}$ \\
\hline $\begin{array}{l}0.0161 \\
0.0162 \\
0.0163 \\
0.0164 \\
0.0165\end{array}$ & $\begin{array}{l}4.13698 \\
4.13083 \\
4.12473 \\
4.11866 \\
4.11263\end{array}$ & $\begin{array}{l}615 \\
610 \\
607 \\
603 \\
599\end{array}$ & $\begin{array}{l}0.99197 \\
0.99192 \\
0.99187 \\
0.99182 \\
0.99177\end{array}$ & $\begin{array}{l}5 \\
5 \\
5 \\
5 \\
5\end{array}$ & $\begin{array}{l}5.12895 \\
5.12276 \\
5.11660 \\
5.11049 \\
5.10441\end{array}$ & $\begin{array}{l}619 \\
616 \\
611 \\
608 \\
605\end{array}$ & $\begin{array}{l}0.99998 \\
0.99998 \\
0.99998 \\
0.99998 \\
0.99998\end{array}$ \\
\hline $\begin{array}{l}0.0166 \\
0.0167 \\
0.0168 \\
0.0169 \\
0.0170\end{array}$ & $\begin{array}{l}4.10664 \\
4.10068 \\
4.09477 \\
4.08888 \\
4.08303\end{array}$ & $\begin{array}{l}596 \\
591 \\
589 \\
585 \\
581\end{array}$ & $\begin{array}{l}0.99172 \\
0.99167 \\
0.99162 \\
0.99157 \\
0.99152\end{array}$ & $\begin{array}{l}5 \\
5 \\
5 \\
5 \\
5\end{array}$ & $\begin{array}{l}5.09836 \\
5.09236 \\
5.08639 \\
5.08046 \\
5.07455\end{array}$ & $\begin{array}{l}600 \\
597 \\
593 \\
591 \\
586\end{array}$ & $\begin{array}{l}0.99998 \\
0.99998 \\
0.99998 \\
0.99998 \\
0.99998\end{array}$ \\
\hline $\begin{array}{l}0.0171 \\
0.0172 \\
0.0173 \\
0.0174 \\
0.0175\end{array}$ & $\begin{array}{l}4.07722 \\
4.07143 \\
4.06569 \\
4.05997 \\
4.05429\end{array}$ & $\begin{array}{l}579 \\
574 \\
572 \\
568 \\
565\end{array}$ & $\begin{array}{l}0.99147 \\
0.99142 \\
0.99138 \\
0.99133 \\
0.99128\end{array}$ & $\begin{array}{l}5 \\
4 \\
5 \\
5 \\
5\end{array}$ & $\begin{array}{l}5.06869 \\
5.06286 \\
5.05706 \\
5.05130 \\
5.04557\end{array}$ & $\begin{array}{l}583 \\
580 \\
576 \\
573 \\
570\end{array}$ & $\begin{array}{l}0.99998 \\
0.99998 \\
0.99998 \\
0.99997 \\
0.99997\end{array}$ \\
\hline $\begin{array}{l}0.0176 \\
0.0177 \\
0.0178 \\
0.0179 \\
0.0180\end{array}$ & $\begin{array}{l}4.04864 \\
4.04303 \\
4.03744 \\
4.03189 \\
4.02637\end{array}$ & $\begin{array}{l}561 \\
559 \\
555 \\
552 \\
549\end{array}$ & $\begin{array}{l}0.99123 \\
0.99118 \\
0.99113 \\
0.99108 \\
0.99103\end{array}$ & $\begin{array}{l}5 \\
5 \\
5 \\
5 \\
5\end{array}$ & $\begin{array}{l}5.03987 \\
5.03421 \\
5.02857 \\
5.02297 \\
5.01740\end{array}$ & $\begin{array}{l}566 \\
564 \\
560 \\
557 \\
554\end{array}$ & $\begin{array}{l}0.99997 \\
0.99998 \\
0.99998 \\
0.99997 \\
0.99997\end{array}$ \\
\hline $\begin{array}{l}0.0181 \\
0.0182 \\
0.0183 \\
0.0184 \\
0.0185\end{array}$ & $\begin{array}{l}4.02088 \\
4.01542 \\
4.00999 \\
4.00459 \\
3.99922\end{array}$ & $\begin{array}{l}546 \\
543 \\
540 \\
537 \\
534\end{array}$ & $\begin{array}{l}0.99098 \\
0.99093 \\
0.99088 \\
0.99083 \\
0.99078\end{array}$ & $\begin{array}{l}5 \\
5 \\
5 \\
5 \\
5\end{array}$ & $\begin{array}{l}5.01186 \\
5.00635 \\
5.00087 \\
4.99542 \\
4.99000\end{array}$ & $\begin{array}{l}551 \\
548 \\
545 \\
542 \\
539\end{array}$ & $\begin{array}{l}0.99997 \\
0.99997 \\
0.99997 \\
0.99997 \\
0.99997\end{array}$ \\
\hline $\begin{array}{l}0.0186 \\
0.0187 \\
0.0188 \\
0.0189 \\
0.0190\end{array}$ & $\begin{array}{l}3.99388 \\
3.98857 \\
3.98328 \\
3.97803 \\
3.97280\end{array}$ & $\begin{array}{l}531 \\
529 \\
525 \\
523 \\
520\end{array}$ & $\begin{array}{l}0.99073 \\
0.99068 \\
0.99063 \\
0.99058 \\
0.99053\end{array}$ & $\begin{array}{l}5 \\
5 \\
5 \\
5 \\
5\end{array}$ & $\begin{array}{l}4.98461 \\
4.97925 \\
4.97391 \\
4.96861 \\
4.96333\end{array}$ & $\begin{array}{l}536 \\
534 \\
530 \\
528 \\
525\end{array}$ & $\begin{array}{l}0.99997 \\
0.99997 \\
0.99997 \\
0.99997 \\
0.99997\end{array}$ \\
\hline $\begin{array}{l}0.0191 \\
0.0192 \\
0.0193 \\
0.0194 \\
0.0195\end{array}$ & $\begin{array}{l}3.96760 \\
3.96243 \\
3.95728 \\
3.95217 \\
3.94708\end{array}$ & $\begin{array}{l}517 \\
515 \\
511 \\
509 \\
507\end{array}$ & $\begin{array}{l}0.99048 \\
0.99043 \\
0.99038 \\
0.99033 \\
0.99028\end{array}$ & $\begin{array}{l}5 \\
5 \\
5 \\
5 \\
5\end{array}$ & $\begin{array}{l}4.95808 \\
4.95286 \\
4.94767 \\
4.94250 \\
4.93736\end{array}$ & $\begin{array}{l}522 \\
519 \\
517 \\
514 \\
512\end{array}$ & $\begin{array}{l}0.99997 \\
0.99997 \\
0.99997 \\
0.99997 \\
0.99997\end{array}$ \\
\hline $\begin{array}{l}0.0196 \\
0.0197 \\
0.0198 \\
0.0199 \\
0.0200\end{array}$ & $\begin{array}{l}3.94201 \\
3.93697 \\
3.93196 \\
3.92697 \\
3.92201\end{array}$ & $\begin{array}{l}504 \\
501 \\
499 \\
496\end{array}$ & $\begin{array}{l}0.99023 \\
0.99018 \\
0.99013 \\
0.99008 \\
0.99003\end{array}$ & $\begin{array}{l}5 \\
5 \\
5 \\
5\end{array}$ & $\begin{array}{l}4.93224 \\
4.92715 \\
4.92239 \\
4.91705 \\
4.91204\end{array}$ & $\begin{array}{l}509 \\
506 \\
504 \\
501\end{array}$ & $\begin{array}{l}0.99997 \\
0.99997 \\
0.99997 \\
0.99997 \\
0.99997\end{array}$ \\
\hline
\end{tabular}


Table I Harmonic Oscillator Contributions (in dimensionless form) to the Thermodynamic Functions for Values of $\mathrm{X}=\mathrm{hc} / \mathrm{kT}$

\begin{tabular}{|c|c|c|c|c|c|c|c|}
\hline $\boldsymbol{Z}$ & $-\left(F^{\circ}-E_{O}^{O}\right) / R T$ & $\Delta$ & $\left(\mathrm{H}^{\circ}-\mathrm{E}_{\mathrm{O}}^{\mathrm{O}}\right) / \mathrm{RT}$ & $\Delta$ & So/R & $\Delta$ & $\mathrm{Co} / \mathrm{R}$ \\
\hline 0.0200 & 3.92201 & 494 & 0.99003 & 5 & $4 \cdot 91204$ & 499 & 0.99997 \\
\hline $\begin{array}{l}0.0201 \\
0.0202 \\
0.0203\end{array}$ & $\begin{array}{l}3.91707 \\
3.91216 \\
3.90727\end{array}$ & $\begin{array}{l}491 \\
489 \\
487\end{array}$ & $\begin{array}{l}0.98998 \\
0.98993 \\
0.98989\end{array}$ & $\begin{array}{l}5 \\
4 \\
5\end{array}$ & $\begin{array}{l}4.90705 \\
4.90209 \\
4.89715\end{array}$ & $\begin{array}{l}496 \\
494 \\
491\end{array}$ & $\begin{array}{l}0.99997 \\
0.99997 \\
0.99997\end{array}$ \\
\hline $\begin{array}{l}0.0204 \\
0.0205\end{array}$ & $\begin{array}{l}3.90240 \\
3.89756\end{array}$ & $\begin{array}{l}484 \\
481\end{array}$ & $\begin{array}{l}0.98984 \\
0.98979\end{array}$ & $\begin{array}{l}5 \\
5\end{array}$ & $\begin{array}{l}4.89224 \\
4.88735\end{array}$ & $\begin{array}{l}489 \\
487\end{array}$ & $\begin{array}{l}0.999 .97 \\
0.99997\end{array}$ \\
\hline $\begin{array}{l}0.0206 \\
0.0207 \\
0.0208 \\
0.0209 \\
0.0210\end{array}$ & $\begin{array}{l}3.89275 \\
3.88795 \\
3.88318 \\
3.87844 \\
3.87371\end{array}$ & $\begin{array}{l}480 \\
477 \\
474 \\
473 \\
470\end{array}$ & $\begin{array}{l}0.98974 \\
0.98969 \\
0.98964 \\
0.98959 \\
0.98954\end{array}$ & $\begin{array}{l}5 \\
5 \\
5 \\
5 \\
5\end{array}$ & $\begin{array}{l}4.88248 \\
4.87764 \\
4.87282 \\
4.86802 \\
4.86325\end{array}$ & $\begin{array}{l}484 \\
482 \\
480 \\
477 \\
475\end{array}$ & $\begin{array}{l}0.99996 \\
0.99997 \\
0.99996 \\
0.99996 \\
0.99996\end{array}$ \\
\hline $\begin{array}{l}0.0211 \\
0.0212 \\
0.0213 \\
0.0214 \\
0.0215\end{array}$ & $\begin{array}{l}3.86901 \\
3.86434 \\
3.85968 \\
3.85505 \\
3.85043\end{array}$ & $\begin{array}{l}467 \\
466 \\
463 \\
462 \\
459\end{array}$ & $\begin{array}{l}0.98949 \\
0.98944 \\
0.98939 \\
0.98934 \\
0.98929\end{array}$ & $\begin{array}{l}5 \\
5 \\
5 \\
5 \\
5\end{array}$ & $\begin{array}{l}4.85850 \\
4.85377 \\
4.84907 \\
4.84438 \\
4.83972\end{array}$ & $\begin{array}{l}473 \\
470 \\
469 \\
466 \\
464\end{array}$ & $\begin{array}{l}0.99996 \\
0.99996 \\
0.99996 \\
0.99996 \\
0.99996\end{array}$ \\
\hline $\begin{array}{l}0.0216 \\
0.0217 \\
0.0218 \\
0.0219 \\
0.0220\end{array}$ & $\begin{array}{l}3.84584 \\
3.84127 \\
3.83673 \\
3.83220 \\
3.82769\end{array}$ & $\begin{array}{l}457 \\
454 \\
453 \\
451 \\
448\end{array}$ & $\begin{array}{l}0.98924 \\
0.98919 \\
0.98914 \\
0.98909 \\
0.98904\end{array}$ & $\begin{array}{l}5 \\
5 \\
5 \\
5 \\
5\end{array}$ & $\begin{array}{l}4.83508 \\
4.83046 \\
4.82587 \\
4.82129 \\
4.81673\end{array}$ & $\begin{array}{l}462 \\
459 \\
458 \\
456 \\
453\end{array}$ & $\begin{array}{l}0.99996 \\
0.99996 \\
0.99996 \\
0.99996 \\
0.99996\end{array}$ \\
\hline $\begin{array}{l}0.0221 \\
0.0222 \\
0.0223 \\
0.0224 \\
0.0225\end{array}$ & $\begin{array}{l}3.82321 \\
3.81874 \\
3.81430 \\
3.80987 \\
3.80547\end{array}$ & $\begin{array}{l}447 \\
444 \\
443 \\
440 \\
439\end{array}$ & $\begin{array}{l}0.98899 \\
0.98894 \\
0.98889 \\
0.98884 \\
0.98879\end{array}$ & $\begin{array}{l}5 \\
5 \\
5 \\
5 \\
5\end{array}$ & $\begin{array}{l}4.81220 \\
4.80768 \\
4.80319 \\
4.79872 \\
4.79426\end{array}$ & $\begin{array}{l}452 \\
449 \\
447 \\
446 \\
443\end{array}$ & $\begin{array}{l}0.99996 \\
0.99996 \\
0.99996 \\
0.99996 \\
0.99996\end{array}$ \\
\hline $\begin{array}{l}0.0226 \\
0.0227 \\
0.0228 \\
0.0229 \\
0.0230\end{array}$ & $\begin{array}{l}3.80108 \\
3.79672 \\
3.79237 \\
3.78805 \\
3.78374\end{array}$ & $\begin{array}{l}436 \\
435 \\
432 \\
431 \\
429\end{array}$ & $\begin{array}{l}0.98874 \\
0.98869 \\
0.98864 \\
0.98859 \\
0.98854\end{array}$ & $\begin{array}{l}5 \\
5 \\
5 \\
5 \\
5\end{array}$ & $\begin{array}{l}4.78983 \\
4.78541 \\
4.78102 \\
4.77664 \\
4.77228\end{array}$ & $\begin{array}{l}442 \\
439 \\
438 \\
436 \\
433\end{array}$ & $\begin{array}{l}0.99996 \\
0.99996 \\
0.99996 \\
0.99996 \\
0.99996\end{array}$ \\
\hline $\begin{array}{l}C .0231 \\
C .0232 \\
C .0233 \\
C .0234 \\
C .0235\end{array}$ & $\begin{array}{l}3.77945 \\
3.77518 \\
3.77093 \\
3.76670 \\
3.76248\end{array}$ & $\begin{array}{l}427 \\
425 \\
423 \\
422 \\
419\end{array}$ & $\begin{array}{l}0.98849 \\
0.98845 \\
0.98840 \\
0.98835 \\
0.98830\end{array}$ & $\begin{array}{l}4 \\
5 \\
5 \\
5 \\
5\end{array}$ & $\begin{array}{l}4.76795 \\
4.76363 \\
4.75932 \\
4.75504 \\
4.75078\end{array}$ & $\begin{array}{l}432 \\
431 \\
428 \\
426 \\
425\end{array}$ & $\begin{array}{l}0.99996 \\
0.99996 \\
0.99996 \\
0.99996 \\
0.99995\end{array}$ \\
\hline $\begin{array}{l}0.0236 \\
0.0237 \\
0.0238 \\
0.0239 \\
0.0240\end{array}$ & $\begin{array}{l}3.75829 \\
3.75411 \\
3.74995 \\
3.74580 \\
3.74168\end{array}$ & $\begin{array}{l}418 \\
416 \\
415 \\
412 \\
411\end{array}$ & $\begin{array}{l}0.98825 \\
0.98820 \\
0.98815 \\
0.98810 \\
0.98805\end{array}$ & $\begin{array}{l}5 \\
5 \\
5 \\
5 \\
5\end{array}$ & $\begin{array}{l}4.74653 \\
4.74230 \\
4.73809 \\
4.73390 \\
4.72973\end{array}$ & $\begin{array}{l}423 \\
421 \\
419 \\
417 \\
416\end{array}$ & $\begin{array}{l}0.99995 \\
0.99995 \\
0.99995 \\
0.99995 \\
0.999 .95\end{array}$ \\
\hline $\begin{array}{l}0.0241 \\
0.0242 \\
0.0243 \\
0.0244 \\
0.0245\end{array}$ & $\begin{array}{l}3.73757 \\
3.73348 \\
3.72940 \\
3.72535 \\
3.72131\end{array}$ & $\begin{array}{l}409 \\
408 \\
405 \\
404 \\
403\end{array}$ & $\begin{array}{l}0.98800 \\
0.98795 \\
0.98790 \\
0.98785 \\
0.98780\end{array}$ & $\begin{array}{l}5 \\
5 \\
5 \\
5 \\
5\end{array}$ & $\begin{array}{l}4.72557 \\
4.72143 \\
4.71730 \\
4.71320 \\
4.70911\end{array}$ & $\begin{array}{l}414 \\
413 \\
410 \\
409 \\
408\end{array}$ & $\begin{array}{l}0.99995 \\
0.99995 \\
0.99995 \\
0.99995 \\
0.99995\end{array}$ \\
\hline $\begin{array}{l}0.0246 \\
0.0247 \\
0.0248 \\
0.0249 \\
0.0250\end{array}$ & $\begin{array}{l}3.71728 \\
3.71328 \\
3.70929 \\
3.70531 \\
3.70135\end{array}$ & $\begin{array}{l}400 \\
399 \\
398 \\
396\end{array}$ & $\begin{array}{l}0.98775 \\
0.98770 \\
0.98765 \\
0.98760 \\
0.98755\end{array}$ & $\begin{array}{l}5 \\
5 \\
5 \\
5\end{array}$ & $\begin{array}{l}4.70503 \\
4.70098 \\
4.69694 \\
4.69291 \\
4.68891\end{array}$ & $\begin{array}{l}405 \\
404 \\
403 \\
400\end{array}$ & $\begin{array}{l}0.99995 \\
0.99995 \\
0.99995 \\
0.99995 \\
0.99995\end{array}$ \\
\hline
\end{tabular}


Table I Harmonic Oscillator Contributions (in dimensionless form) to the Thermodynamic Functions for Values of $X=h c v / k T$

\begin{tabular}{|c|c|c|c|c|c|c|c|}
\hline $\bar{I}$ & $-\left(F^{\circ}-E_{O}^{\circ}\right) / R T$ & $\Delta$ & $\left(\mathrm{H}^{\circ}-\mathrm{E}_{\mathrm{O}}^{\circ}\right) / \mathrm{RT}$ & $\Delta$ & $\mathrm{S} \% / \mathrm{R}$ & $\Delta$ & $\mathrm{CO}_{\mathrm{p}} / \mathrm{R}$ \\
\hline 0.0250 & 3.70135 & 394 & 0.98755 & 5 & 4.68891 & 400 & 0.99995 \\
\hline $\begin{array}{l}0.0251 \\
0.0252\end{array}$ & $\begin{array}{l}3.69741 \\
3.69349\end{array}$ & $\begin{array}{l}392 \\
392\end{array}$ & $\begin{array}{l}0.98750 \\
0.98745\end{array}$ & $\begin{array}{l}5 \\
5\end{array}$ & $\begin{array}{l}4.58491 \\
4.68094\end{array}$ & $\begin{array}{l}397 \\
396\end{array}$ & $\begin{array}{l}0.99995 \\
0.99995\end{array}$ \\
\hline $\begin{array}{l}0.0253 \\
0.0254 \\
0.0255\end{array}$ & $\begin{array}{l}3.68957 \\
3.68568 \\
3.68180\end{array}$ & $\begin{array}{l}389 \\
388 \\
386\end{array}$ & $\begin{array}{l}0.98740 \\
0.98735 \\
0.98730\end{array}$ & $\begin{array}{l}5 \\
5 \\
5\end{array}$ & $\begin{array}{l}4.67678 \\
4.67303 \\
4.66910\end{array}$ & $\begin{array}{l}395 \\
393 \\
391\end{array}$ & $\begin{array}{l}0.99995 \\
0.99995 \\
0.99995\end{array}$ \\
\hline $\begin{array}{l}0.0256 \\
0.0257 \\
0.0258 \\
0.0259 \\
0.0260\end{array}$ & $\begin{array}{l}3.67794 \\
3.67409 \\
3.67025 \\
3.66643 \\
3.66263\end{array}$ & $\begin{array}{l}385 \\
384 \\
382 \\
380 \\
379\end{array}$ & $\begin{array}{l}0.98725 \\
0.98721 \\
0.98716 \\
0.98711 \\
0.98706\end{array}$ & $\begin{array}{l}4 \\
5 \\
5 \\
5 \\
5\end{array}$ & $\begin{array}{l}4.66519 \\
4.66129 \\
4.65741 \\
4.65354 \\
4.64969\end{array}$ & $\begin{array}{l}390 \\
388 \\
387 \\
385 \\
384\end{array}$ & $\begin{array}{l}0.99995 \\
0.99995 \\
0.99994 \\
0.99995 \\
0.99994\end{array}$ \\
\hline $\begin{array}{l}0.0261 \\
0.0262 \\
0.0263 \\
0.0264 \\
0.0265\end{array}$ & $\begin{array}{l}3.65884 \\
3.65507 \\
3.65131 \\
3.64756 \\
3.64383\end{array}$ & $\begin{array}{l}377 \\
376 \\
375 \\
373 \\
371\end{array}$ & $\begin{array}{l}0.98701 \\
0.98696 \\
0.98691 \\
0.98686 \\
0.98681\end{array}$ & $\begin{array}{l}5 \\
5 \\
5 \\
5 \\
5\end{array}$ & $\begin{array}{l}4.64585 \\
4.64202 \\
4.63822 \\
4.63442 \\
4.63064\end{array}$ & $\begin{array}{l}383 \\
380 \\
380 \\
378 \\
377\end{array}$ & $\begin{array}{l}0.99994 \\
0.99994 \\
0.99994 \\
0.99994 \\
0.99994\end{array}$ \\
\hline $\begin{array}{l}0.0 .266 \\
0.0267 \\
0.0268 \\
0.0269 \\
0.0270\end{array}$ & $\begin{array}{l}3.64012 \\
3.63641 \\
3.63272 \\
3.62905 \\
3.62539\end{array}$ & $\begin{array}{l}371 \\
369 \\
367 \\
366 \\
365\end{array}$ & $\begin{array}{l}0.98676 \\
0.98671 \\
0.98666 \\
0.98661 \\
0.98656\end{array}$ & $\begin{array}{l}5 \\
5 \\
5 \\
5 \\
5\end{array}$ & $\begin{array}{l}4.62687 \\
4.62312 \\
4.61938 \\
4.61566 \\
4.6119 .5\end{array}$ & $\begin{array}{l}375 \\
374 \\
372 \\
371 \\
370\end{array}$ & $\begin{array}{l}0.99994 \\
0.99994 \\
0.99994 \\
0.99994 \\
0.99994\end{array}$ \\
\hline $\begin{array}{l}0.0271 \\
0.0272\end{array}$ & $\begin{array}{l}3.62174 \\
3.61811\end{array}$ & $\begin{array}{l}363 \\
362\end{array}$ & $\begin{array}{l}0.98651 \\
0.98646\end{array}$ & $\begin{array}{l}5 \\
5\end{array}$ & $\begin{array}{l}4.60825 \\
4.60457\end{array}$ & $\begin{array}{l}368 \\
367\end{array}$ & $\begin{array}{l}0.99994 \\
0.99994\end{array}$ \\
\hline $\begin{array}{l}0.0273 \\
0.0274 \\
0.0275\end{array}$ & $\begin{array}{l}3.61449 \\
3.61088 \\
3.60729\end{array}$ & $\begin{array}{l}361 \\
359 \\
358\end{array}$ & $\begin{array}{l}0.98641 \\
0.98636 \\
0.98631\end{array}$ & $\begin{array}{l}5 \\
5 \\
5\end{array}$ & $\begin{array}{l}4.60090 \\
4.59724 \\
4.59360\end{array}$ & $\begin{array}{l}366 \\
364 \\
363\end{array}$ & $\begin{array}{l}0.99994 . \\
0.99994 \\
0.99994\end{array}$ \\
\hline $\begin{array}{l}0.0276 \\
0.0277 \\
0.0278 \\
0.0279 \\
0.0280\end{array}$ & $\begin{array}{l}3.60371 \\
3.60014 \\
3.59659 \\
3.59305 \\
3.58952\end{array}$ & $\begin{array}{l}357 \\
355 \\
354 \\
353 \\
352\end{array}$ & $\begin{array}{l}0.98626 \\
0.98621 \\
0.98616 \\
0.98612 \\
0.98607\end{array}$ & $\begin{array}{l}5 \\
5 \\
4 \\
5 \\
5\end{array}$ & $\begin{array}{l}4 \cdot 58997 \\
4 \cdot 58636 \\
4.58275 \\
4.57916 \\
4.57558\end{array}$ & $\begin{array}{l}361 \\
361 \\
359 \\
358 \\
356\end{array}$ & $\begin{array}{l}0.99994 \\
0.99994 \\
0.99994 \\
0.99994 \\
0.99993\end{array}$ \\
\hline $\begin{array}{l}0.0281 \\
0.0282 \\
0.0283 \\
0.0284 \\
0.0285\end{array}$ & $\begin{array}{l}3.58600 \\
3.58250 \\
3.57901 \\
3.57553 \\
3.57207\end{array}$ & $\begin{array}{l}350 \\
349 \\
348 \\
346 \\
346\end{array}$ & $\begin{array}{l}0.98602 \\
0.98597 \\
0.98592 \\
0.98587 \\
0.98582\end{array}$ & $\begin{array}{l}5 \\
5 \\
5 \\
5 \\
5\end{array}$ & $\begin{array}{l}4.57202 \\
4.56847 \\
4.56493 \\
4.56140 \\
4.55739\end{array}$ & $\begin{array}{l}355 \\
354 \\
353 \\
351 \\
351\end{array}$ & $\begin{array}{l}0.99993 \\
0.99994 \\
0.99993 \\
0.99993 \\
0.99993\end{array}$ \\
\hline $\begin{array}{l}0.0286 \\
0.0287 \\
0.0288 \\
0.0289 \\
0.0290\end{array}$ & $\begin{array}{l}3.56861 \\
3.56517 \\
3.56175 \\
3.55833 \\
3.55492\end{array}$ & $\begin{array}{l}344 \\
342 \\
342 \\
341 \\
339\end{array}$ & $\begin{array}{l}0.98577 \\
0.98572 \\
0.98567 \\
0.98562 \\
0.98557\end{array}$ & $\begin{array}{l}5 \\
5 \\
5 \\
5 \\
5\end{array}$ & $\begin{array}{l}4.55438 \\
4.55 C 39 \\
4.54742 \\
4.54395 \\
4.54049\end{array}$ & $\begin{array}{l}349 \\
347 \\
347 \\
346 \\
344\end{array}$ & $\begin{array}{l}0.99993 \\
0.99993 \\
0.99993 \\
0.99993 \\
0.99993\end{array}$ \\
\hline $\begin{array}{l}0.0291 \\
0.0292 \\
0.0293 \\
0.0294 \\
0.0295\end{array}$ & $\begin{array}{l}3.55153 \\
3.54815 \\
3.54478 \\
3.54142 \\
3.53808\end{array}$ & $\begin{array}{l}338 \\
337 \\
336 \\
334 \\
334\end{array}$ & $\begin{array}{l}0.98552 \\
0.98547 \\
0.98542 \\
0.98537 \\
0.98532\end{array}$ & $\begin{array}{l}5 \\
5 \\
5 \\
5 \\
5\end{array}$ & $\begin{array}{l}4.53705 \\
4.53362 \\
4.53020 \\
4.52680 \\
4.52340\end{array}$ & $\begin{array}{l}343 \\
342 \\
340 \\
340 \\
338\end{array}$ & $\begin{array}{l}0.99993 \\
0.99993 \\
0.99993 \\
0.99993 \\
0.99993\end{array}$ \\
\hline $\begin{array}{l}0.0296 \\
0.0297 \\
0.0298 \\
0.0299 \\
0.0300\end{array}$ & $\begin{array}{l}3.53474 \\
3.53142 \\
3.52811 \\
3.52481 \\
3.52152\end{array}$ & $\begin{array}{l}332 \\
331 \\
330 \\
329\end{array}$ & $\begin{array}{l}0.98527 \\
0.98522 \\
0.98517 \\
0.98512 \\
0.98508\end{array}$ & $\begin{array}{l}5 \\
5 \\
5 \\
4\end{array}$ & $\begin{array}{l}4.52002 \\
4.51665 \\
4.51328 \\
4.50993 \\
4.50660\end{array}$ & $\begin{array}{l}337 \\
337 \\
335 \\
333\end{array}$ & $\begin{array}{l}0.99993 \\
0.99993 \\
0.99993 \\
0.99993 \\
0.99993\end{array}$ \\
\hline
\end{tabular}


Table I Harmonic Oscillator Contributions (in dimensionless form) to the Thermodynamic Functions for Values of $\mathrm{X}=\mathrm{hcv} / \mathrm{kT}$

\begin{tabular}{|c|c|c|c|c|c|c|c|}
\hline 8 & $-\left(\mathrm{F}^{\circ}-\mathrm{E}_{\mathrm{O}} \mathrm{O}\right) / \mathrm{RT}$ & $\Delta$ & $\left(\mathrm{H}^{\circ}-\mathrm{E}_{\mathrm{O}}^{\circ}\right) / \mathrm{RT}$ & $\Delta$ & $\mathrm{SO} / \mathrm{R}$ & $\Delta$ & $\mathrm{co} / \mathrm{R}$ \\
\hline 0.0300 & 3.52152 & 328 & 0.98508 & 5 & 4.50660 & 333 & 0.99993 \\
\hline $\begin{array}{l}0.0301 \\
0.0302 \\
0.0303\end{array}$ & $\begin{array}{l}3.51824 \\
3.514 .98 \\
3.51172\end{array}$ & $\begin{array}{l}326 \\
326 \\
325\end{array}$ & $\begin{array}{l}0.98503 \\
0.98498 \\
0.98493\end{array}$ & $\begin{array}{l}5 \\
5 \\
5\end{array}$ & $\begin{array}{l}4.50327 \\
4.49995 \\
4.49665\end{array}$ & $\begin{array}{l}332 \\
330 \\
330\end{array}$ & $\begin{array}{l}0.99992 \\
0.99992 \\
0.99992\end{array}$ \\
\hline $\begin{array}{l}0.0304 \\
0.0305\end{array}$ & $\begin{array}{l}3.50847 \\
3.50524\end{array}$ & $\begin{array}{l}323 \\
322\end{array}$ & $\begin{array}{l}0.98488 \\
0.98483\end{array}$ & $\begin{array}{l}5 \\
5\end{array}$ & $\begin{array}{l}4.49335 \\
4.49007\end{array}$ & $\begin{array}{l}328 \\
328\end{array}$ & $\begin{array}{l}0.99992 \\
0.99992\end{array}$ \\
\hline $\begin{array}{l}0.0306 \\
0.0307 \\
0.0308 \\
0.0309 \\
0.0310\end{array}$ & $\begin{array}{l}3.50202 \\
3.49880 \\
3.49560 \\
3.49241 \\
3.48923\end{array}$ & $\begin{array}{l}322 \\
320 \\
319 \\
318 \\
317\end{array}$ & $\begin{array}{l}0.98478 \\
0.98473 \\
0.98468 \\
0.98463 \\
0.98458\end{array}$ & $\begin{array}{l}5 \\
5 \\
5 \\
5 \\
5\end{array}$ & $\begin{array}{l}4.48679 \\
4.48353 \\
4.48028 \\
4.47704 \\
4.47381\end{array}$ & $\begin{array}{l}326 \\
325 \\
324 \\
323 \\
322\end{array}$ & $\begin{array}{l}0.99992 \\
0.99992 \\
0.99992 \\
0.99992 \\
0.99992\end{array}$ \\
\hline $\begin{array}{l}0.0311 \\
0.0312 \\
0.0313 \\
0.0314 \\
0.0315\end{array}$ & $\begin{array}{l}3.48606 \\
3.48290 \\
3.47975 \\
3.47661 \\
3.47348\end{array}$ & $\begin{array}{l}316 \\
315 \\
314 \\
313 \\
312\end{array}$ & $\begin{array}{l}0.98453 \\
0.98448 \\
0.98443 \\
0.98438 \\
0.98433\end{array}$ & $\begin{array}{l}5 \\
5 \\
5 \\
5 \\
5\end{array}$ & $\begin{array}{l}4.47059 \\
4.46738 \\
4.46418 \\
4.46099 \\
4.45781\end{array}$ & $\begin{array}{l}321 \\
320 \\
319 \\
318 \\
317\end{array}$ & $\begin{array}{l}0.99992 \\
0.99992 \\
0.99992 \\
0.99992 \\
0.99992\end{array}$ \\
\hline $\begin{array}{l}0.0316 \\
0.0317 \\
0.0318 \\
0.0319 \\
0.0320\end{array}$ & $\begin{array}{l}3.47036 \\
3.46725 \\
3.46415 \\
3.46106 \\
3.45798\end{array}$ & $\begin{array}{l}311 \\
310 \\
309 \\
308 \\
307\end{array}$ & $\begin{array}{l}0.98428 \\
0.98423 \\
0.98418 \\
0.98414 \\
0.98409\end{array}$ & $\begin{array}{l}5 \\
5 \\
4 \\
5 \\
5\end{array}$ & $\begin{array}{l}4.45464 \\
4.45148 \\
4.44833 \\
4.44519 \\
4.44206\end{array}$ & $\begin{array}{l}316 \\
315 \\
314 \\
313 \\
312\end{array}$ & $\begin{array}{l}0.99992 \\
0.99992 \\
0.99992 \\
0.99992 \\
0.99992\end{array}$ \\
\hline $\begin{array}{l}0.0321 \\
0.0322 \\
0.0323 \\
0.0324 \\
0.0325\end{array}$ & $\begin{array}{l}3.45491 \\
3.45185 \\
3.44879 \\
3.44575 \\
3.44272\end{array}$ & $\begin{array}{l}306 \\
306 \\
304 \\
303 \\
302\end{array}$ & $\begin{array}{l}0.98404 \\
0.98399 \\
0.98394 \\
0.98389 \\
0.98384\end{array}$ & $\begin{array}{l}5 \\
5 \\
5 \\
5 \\
5\end{array}$ & $\begin{array}{l}4.43894 \\
4.43583 \\
4.43273 \\
4.42964 \\
4.42656\end{array}$ & $\begin{array}{l}311 \\
310 \\
309 \\
308 \\
307\end{array}$ & $\begin{array}{l}0.99991 \\
0.99991 \\
0.99991 \\
0.99991 \\
0.99991\end{array}$ \\
\hline $\begin{array}{l}0.0326 \\
0.0327 \\
0.0328 \\
0.0329 \\
0.0330\end{array}$ & $\begin{array}{l}3.43970 \\
3.43669 \\
3.43368 \\
3.43069 \\
3.42770\end{array}$ & $\begin{array}{l}301 \\
301 \\
299 \\
299 \\
297\end{array}$ & $\begin{array}{l}0.98379 \\
0.98374 \\
0.98369 \\
0.98364 \\
0.98359\end{array}$ & $\begin{array}{l}5 \\
5 \\
5 \\
5 \\
5\end{array}$ & $\begin{array}{l}4.42349 \\
4.42043 \\
4.41737 \\
4.41433 \\
4.41129\end{array}$ & $\begin{array}{l}306 \\
306 \\
304 \\
304 \\
302\end{array}$ & $\begin{array}{l}0.99991 \\
0.99991 \\
0.99991 \\
0.99991 \\
0.99991\end{array}$ \\
\hline $\begin{array}{l}0.0331 \\
0.0332 \\
0.0333 \\
0.0334 \\
0.0335\end{array}$ & $\begin{array}{l}3.42473 \\
3.42176 \\
3.41880 \\
3.41585 \\
3.41291\end{array}$ & $\begin{array}{l}297 \\
296 \\
295 \\
294 \\
293\end{array}$ & $\begin{array}{l}0.98354 \\
0.98349 \\
0.98344 \\
0.983339 \\
0.98334\end{array}$ & $\begin{array}{l}5 \\
5 \\
5 \\
5 \\
5\end{array}$ & $\begin{array}{l}4.40827 \\
4.40525 \\
4.40224 \\
4.39925 \\
4.39626\end{array}$ & $\begin{array}{l}302 \\
301 \\
299 \\
299 \\
298\end{array}$ & $\begin{array}{l}0.99991 \\
0.99991 \\
0.99991 \\
0.99991 \\
0.99991\end{array}$ \\
\hline $\begin{array}{l}0.0336 \\
0.0337 \\
0.0338 \\
0.0339 \\
0.0340\end{array}$ & $\begin{array}{l}3.40998 \\
3.40706 \\
3.40415 \\
3.40124 \\
3.39835\end{array}$ & $\begin{array}{l}292 \\
291 \\
291 \\
289 \\
289\end{array}$ & $\begin{array}{l}0.98329 \\
0.98324 \\
0.98320 \\
0.98315 \\
0.98310\end{array}$ & $\begin{array}{l}5 \\
4 \\
5 \\
5 \\
5\end{array}$ & $\begin{array}{l}4.39328 \\
4.39030 \\
4.38734 \\
4.38439 \\
4.38144\end{array}$ & $\begin{array}{l}298 \\
296 \\
295 \\
295 \\
293\end{array}$ & $\begin{array}{l}0.99991 \\
0.99991 \\
0.99991 \\
0.99990 \\
0.99990\end{array}$ \\
\hline $\begin{array}{l}0.0341 \\
0.0342 \\
0.0343 \\
0.0344 \\
0.0345\end{array}$ & $\begin{array}{l}3.39546 \\
3.39258 \\
3.38971 \\
3.38685 \\
3.38400\end{array}$ & $\begin{array}{l}288 \\
287 \\
286 \\
285 \\
285\end{array}$ & $\begin{array}{l}0.98305 \\
0.98300 \\
0.98295 \\
0.98290 \\
0.98285\end{array}$ & $\begin{array}{l}5 \\
5 \\
5 \\
5 \\
5\end{array}$ & $\begin{array}{l}4 \cdot 37851 \\
4 \cdot 37558 \\
4 \cdot 37266 \\
4 \cdot 36975 \\
4 \cdot 36635\end{array}$ & $\begin{array}{l}293 \\
292 \\
291 \\
290 \\
290\end{array}$ & $\begin{array}{l}0.99990 \\
0.99990 \\
0.99990 \\
0.99990 \\
0.99990\end{array}$ \\
\hline $\begin{array}{l}0.0346 \\
0.0347 \\
0.0348 \\
0.0349 \\
0.0350\end{array}$ & $\begin{array}{l}3.38115 \\
3.37832 \\
3.37549 \\
3.37267 \\
3.36986\end{array}$ & $\begin{array}{l}283 \\
283 \\
282 \\
281\end{array}$ & $\begin{array}{l}0.98280 \\
0.98275 \\
0.98270 \\
0.98265 \\
0.98260\end{array}$ & $\begin{array}{l}5 \\
5 \\
5 \\
5\end{array}$ & $\begin{array}{l}4.36395 \\
4.36107 \\
4.35819 \\
4.35532 \\
4.35246\end{array}$ & $\begin{array}{l}288 \\
288 \\
287 \\
286\end{array}$ & $\begin{array}{l}0.99990 \\
0.99990 \\
0.99990 \\
0.99990 \\
0.99990\end{array}$ \\
\hline
\end{tabular}


Table I Harmonic Oscillator Contributions (in dimensionless form) to the Thermodynamic Functions for Values of $\mathrm{X}=\mathrm{hc} / \mathrm{kT}$

\begin{tabular}{|c|c|c|c|c|c|c|c|}
\hline $\mathbb{Z}$ & $-\left(F^{\circ}-E_{O}^{\circ}\right) / R T$ & $\Delta$ & $\left(\mathrm{H}^{\circ}-\mathrm{E}_{\mathrm{O}}^{\circ}\right) / \mathrm{RT}$ & $\Delta$ & So/R & $\Delta$ & $\mathrm{C}_{\mathrm{p}}^{\circ} / \mathrm{R}$ \\
\hline 0.0350 & 3.36986 & 281 & 0.98260 & $5^{\circ}$ & 4.35246 & 285 & 0.99990 \\
\hline $\begin{array}{l}0.0351 \\
0.0352 \\
0.0353 \\
0.0354 \\
0.0355\end{array}$ & $\begin{array}{l}3.36705 \\
3.36426 \\
3.36147 \\
3.35869 \\
3.35592\end{array}$ & $\begin{array}{l}279 \\
279 \\
278 \\
277 \\
276\end{array}$ & $\begin{array}{l}0.98255 \\
0.98250 \\
0.98245 \\
0.98240 \\
0.98236\end{array}$ & $\begin{array}{l}5 \\
5 \\
5 \\
4 \\
5\end{array}$ & $\begin{array}{l}4.34961 \\
4.34676 \\
4.34392 \\
4.34110 \\
4.33828\end{array}$ & $\begin{array}{l}285 \\
284 \\
282 \\
282 \\
282\end{array}$ & $\begin{array}{l}0.99990 \\
0.99990 \\
0.99990 \\
0.99990 \\
0.99990\end{array}$ \\
\hline $\begin{array}{l}0.0356 \\
0.0357 \\
0.0358 \\
0.0359 \\
0.0360\end{array}$ & $\begin{array}{l}3.35316 \\
3.35040 \\
3.34765 \\
3.34491 \\
3.34218\end{array}$ & $\begin{array}{l}276 \\
275 \\
274 \\
273 \\
272\end{array}$ & $\begin{array}{l}0.98231 \\
0.98226 \\
0.98221 \\
0.98216 \\
0.98211\end{array}$ & $\begin{array}{l}5 \\
5 \\
5 \\
5 \\
5\end{array}$ & $\begin{array}{l}4.33546 \\
4.33266 \\
4 \cdot 32986 \\
4.32707 \\
4.32429\end{array}$ & $\begin{array}{l}280 \\
280 \\
279 \\
278 \\
277\end{array}$ & $\begin{array}{l}0.99989 \\
0.99989 \\
0.99989 \\
0.99989 \\
0.99989\end{array}$ \\
\hline $\begin{array}{l}0.0361 \\
0.0362 \\
0.0363 \\
0.0364 \\
0.0365\end{array}$ & $\begin{array}{l}3.33946 \\
3.33674 \\
3.33403 \\
3.33133 \\
3.32864\end{array}$ & $\begin{array}{l}272 \\
271 \\
270 \\
269 \\
269\end{array}$ & $\begin{array}{l}0.98206 \\
0.98201 \\
0.98196 \\
0.98191 \\
0.98186\end{array}$ & $\begin{array}{l}5 \\
5 \\
5 \\
5 \\
5\end{array}$ & $\begin{array}{l}4.32152 \\
4.31875 \\
4.31599 \\
4.31324 \\
4.31050\end{array}$ & $\begin{array}{l}277 \\
276 \\
275 \\
274 \\
274\end{array}$ & $\begin{array}{l}0.99989 \\
0.99989 \\
0.99989 \\
0.99989 \\
0.99989\end{array}$ \\
\hline $\begin{array}{l}0.0366 \\
0.0367 \\
0.0368 \\
0.0369 \\
0.0370\end{array}$ & $\begin{array}{l}3.32595 \\
3.32327 \\
3.32060 \\
3.31794 \\
3.31528\end{array}$ & $\begin{array}{l}268 \\
267 \\
266 \\
266 \\
265\end{array}$ & $\begin{array}{l}0.98181 \\
0.98176 \\
0.98171 \\
0.98166 \\
0.98161\end{array}$ & $\begin{array}{l}5 \\
5 \\
5 \\
5 \\
5\end{array}$ & $\begin{array}{l}4.30776 \\
4.30503 \\
4.30231 \\
4.29960 \\
4.29690\end{array}$ & $\begin{array}{l}273 \\
272 \\
271 \\
270 \\
270\end{array}$ & $\begin{array}{l}0.99989 \\
0.99989 \\
0.99989 \\
0.99989 \\
0.99989\end{array}$ \\
\hline $\begin{array}{l}0.0371 \\
0.0372 \\
0.0373 \\
0.0374 \\
0.0375\end{array}$ & $\begin{array}{l}3.31263 \\
3.30999 \\
3.30735 \\
3.30473 \\
3.30211\end{array}$ & $\begin{array}{l}264 \\
264 \\
262 \\
262 \\
262\end{array}$ & $\begin{array}{l}0.98156 \\
0.98152 \\
0.98147 \\
0.98142 \\
0.98137\end{array}$ & $\begin{array}{l}4 \\
5 \\
5 \\
5 \\
5\end{array}$ & $\begin{array}{l}4.29420 \\
4.29150 \\
4.28832 \\
4.28614 \\
4.28347\end{array}$ & $\begin{array}{l}270 \\
268 \\
268 \\
267 \\
266\end{array}$ & $\begin{array}{l}0.99989 \\
0.99989 \\
0.99988 \\
0.99988 \\
0.99988\end{array}$ \\
\hline $\begin{array}{l}0.0376 \\
0.0377 \\
0.0378 \\
0.0379 \\
0.0380\end{array}$ & $\begin{array}{l}3.29949 \\
3.29689 \\
3.29429 \\
3.29169 \\
3.28911\end{array}$ & $\begin{array}{l}260 \\
260 \\
260 \\
258 \\
258\end{array}$ & $\begin{array}{l}0.98132 \\
0.98127 \\
0.98122 \\
0.98117 \\
0.98112\end{array}$ & $\begin{array}{l}5 \\
5 \\
5 \\
5 \\
5\end{array}$ & $\begin{array}{l}4.28081 \\
4.27815 \\
4.27551 \\
4.27286 \\
4.27023\end{array}$ & $\begin{array}{l}266 \\
264 \\
265 \\
263 \\
263\end{array}$ & $\begin{array}{l}0.99988 \\
0.99988 \\
0.99988 \\
0.99988 \\
0.99988\end{array}$ \\
\hline $\begin{array}{l}0.0381 \\
0.0382 \\
0.0383 \\
0.0384 \\
0.0385\end{array}$ & $\begin{array}{l}3.28653 \\
3.28396 \\
3.28139 \\
3.27884 \\
3.27629\end{array}$ & $\begin{array}{l}257 \\
257 \\
255 \\
255 \\
255\end{array}$ & $\begin{array}{l}0.98107 \\
0.98102 \\
0.98097 \\
0.98092 \\
0.98087\end{array}$ & $\begin{array}{l}5 \\
5 \\
5 \\
5 \\
5\end{array}$ & $\begin{array}{l}4.26760 \\
4.26498 \\
4.26237 \\
4.25976 \\
4.25716\end{array}$ & $\begin{array}{l}262 \\
261 \\
261 \\
260 \\
260\end{array}$ & $\begin{array}{l}0.99988 \\
0.99988 \\
0.99988 \\
0.99988 \\
0.99988\end{array}$ \\
\hline $\begin{array}{l}0.0386 \\
0.0387 \\
0.0388 \\
0.0389 \\
0.0390\end{array}$ & $\begin{array}{l}3.27374 \\
3.27120 \\
3.26867 \\
3.26615 \\
3.26363\end{array}$ & $\begin{array}{l}254 \\
253 \\
252 \\
252 \\
251\end{array}$ & $\begin{array}{l}0.98082 \\
0.98077 \\
0.98073 \\
0.98068 \\
0.98063\end{array}$ & $\begin{array}{l}5 \\
4 \\
5 \\
5 \\
5\end{array}$ & $\begin{array}{l}4.25456 \\
4.25198 \\
4.24940 \\
4.24682 \\
4.24426\end{array}$ & $\begin{array}{l}258 \\
258 \\
258 \\
256 \\
256\end{array}$ & $\begin{array}{l}0.99988 \\
0.99988 \\
0.99988 \\
0.99987 \\
0.99987\end{array}$ \\
\hline $\begin{array}{l}0.0391 \\
0.0392 \\
0.0393 \\
0.0394 \\
0.0395\end{array}$ & $\begin{array}{l}3.26112 \\
3.25861 \\
3.25612 \\
3.25362 \\
3.25114\end{array}$ & $\begin{array}{l}251 \\
249 \\
250 \\
248 \\
248\end{array}$ & $\begin{array}{l}0.98058 \\
0.98053 \\
0.98048 \\
0.98043 \\
0.98038\end{array}$ & $\begin{array}{l}5 \\
5 \\
5 \\
5 \\
5\end{array}$ & $\begin{array}{l}4.24170 \\
4.23914 \\
4.23660 \\
4.23405 \\
4.23152\end{array}$ & $\begin{array}{l}256 \\
254 \\
255 \\
253 \\
253\end{array}$ & $\begin{array}{l}0.99987 \\
0.99987 \\
0.99987 \\
0.99987 \\
0.99987\end{array}$ \\
\hline $\begin{array}{l}0.0396 \\
0.0397 \\
0.0398 \\
0.0399 \\
0.0400\end{array}$ & $\begin{array}{l}3.24866 \\
3.24619 \\
3.24372 \\
3.24126 \\
3.23881\end{array}$ & $\begin{array}{l}247 \\
247 \\
246 \\
245\end{array}$ & $\begin{array}{l}0.98033 \\
0.98028 \\
0.98023 \\
0.98018 \\
0.98013\end{array}$ & $\begin{array}{l}5 \\
5 \\
5 \\
5\end{array}$ & $\begin{array}{l}4.22899 \\
4.22647 \\
4.22395 \\
4.22145 \\
4.21894\end{array}$ & $\begin{array}{l}252 \\
252 \\
250 \\
251\end{array}$ & $\begin{array}{l}0.99987 \\
0.99987 \\
0.99987 \\
0.99987 \\
0.99987\end{array}$ \\
\hline
\end{tabular}


Table I Harmonic Oscillator Contributions (in dimensionless form) to the Thermodynamic Functions for Values of $X=h c v / k T$

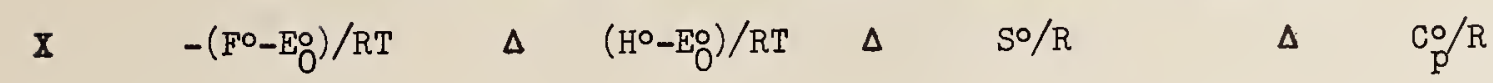

\begin{tabular}{|c|c|c|c|c|c|c|c|}
\hline 0.0400 & 3.23881 & 245 & 0.98013 & 5 & 4.21894 & 249 & 0.99987 \\
\hline $\begin{array}{l}0.0401 \\
0.0402 \\
0.0403\end{array}$ & $\begin{array}{l}3.23636 \\
3.23392 \\
3.23149\end{array}$ & $\begin{array}{l}244 \\
243 \\
243\end{array}$ & $\begin{array}{l}0.98008 \\
0.98003 \\
0.97999\end{array}$ & $\begin{array}{l}5 \\
4 \\
5\end{array}$ & $\begin{array}{l}4.21645 \\
4.21396 \\
4.21147\end{array}$ & $\begin{array}{l}249 \\
249 \\
248\end{array}$ & $\begin{array}{l}0.99987 \\
0.99987 \\
0.99987\end{array}$ \\
\hline $\begin{array}{l}0.0404 \\
0.0405\end{array}$ & $\begin{array}{l}3.22906 \\
3.22664\end{array}$ & $\begin{array}{l}242 \\
242\end{array}$ & $\begin{array}{l}0.97994 \\
0.97989\end{array}$ & $\begin{array}{l}5 \\
5\end{array}$ & $\begin{array}{l}4.20899 \\
4.20652\end{array}$ & $\begin{array}{l}247 \\
246\end{array}$ & $\begin{array}{l}0.99986 \\
0.99986\end{array}$ \\
\hline $\begin{array}{l}0.0406 \\
0.0407 \\
0.0408 \\
0.0409 \\
0.0410\end{array}$ & $\begin{array}{l}3.22422 \\
3.22181 \\
3.21940 \\
3.21701 \\
3.21461\end{array}$ & $\begin{array}{l}241 \\
241 \\
239 \\
240 \\
238\end{array}$ & $\begin{array}{l}0.97984 \\
0.97979 \\
0.97974 \\
0.97969 \\
0.97964\end{array}$ & $\begin{array}{l}5 \\
5 \\
5 \\
5 \\
5\end{array}$ & $\begin{array}{l}4.20406 \\
4.20160 \\
4.19914 \\
4.19670 \\
4.19425\end{array}$ & $\begin{array}{l}246 \\
246 \\
244 \\
245 \\
243\end{array}$ & $\begin{array}{l}0.99986 \\
0.99986 \\
0.99986 \\
0.99986 \\
0.99986\end{array}$ \\
\hline $\begin{array}{l}0.0411 \\
0.0412 \\
0.0413 \\
0.0 \$ 14 \\
0.0415\end{array}$ & $\begin{array}{l}3.21223 \\
3.20985 \\
3.20747 \\
3.20510 \\
3.20274\end{array}$ & $\begin{array}{l}238 \\
238 \\
237 \\
236 \\
236\end{array}$ & $\begin{array}{l}0.97959 \\
0.97954 \\
0.97949 \\
0.97944 \\
0.97939\end{array}$ & $\begin{array}{l}5 \\
5 \\
5 \\
5 \\
5\end{array}$ & $\begin{array}{l}4.19182 \\
4.18939 \\
4.18696 \\
4.18455 \\
4.18213\end{array}$ & $\begin{array}{l}243 \\
243 \\
241 \\
242 \\
240\end{array}$ & $\begin{array}{l}0.99986 \\
0.99986 \\
0.99986 \\
0.99986 \\
0.99986\end{array}$ \\
\hline $\begin{array}{l}0.0416 \\
0.0417 \\
0.0418 \\
0.0419 \\
0.0420\end{array}$ & $\begin{array}{l}3.20038 \\
3.19803 \\
3.19569 \\
3.19335 \\
3.19101\end{array}$ & $\begin{array}{l}235 \\
234 \\
234 \\
234 \\
233\end{array}$ & $\begin{array}{l}0.97934 \\
0.97930 \\
0.97925 \\
0.97920 \\
0.97915\end{array}$ & $\begin{array}{l}4 \\
5 \\
5 \\
5 \\
5\end{array}$ & $\begin{array}{l}4.17973 \\
4.17733 \\
4.17493 \\
4.17254 \\
4.17016\end{array}$ & $\begin{array}{l}240 \\
240 \\
239 \\
238 \\
238\end{array}$ & $\begin{array}{l}0.99986 \\
0.99986 \\
0.99985 \\
0.99985 \\
0.99985\end{array}$ \\
\hline $\begin{array}{l}0.0421 \\
0.0422\end{array}$ & $\begin{array}{l}3.18868 \\
3.18636\end{array}$ & $\begin{array}{l}232 \\
232\end{array}$ & $\begin{array}{l}0.97910 \\
0.97905\end{array}$ & $\begin{array}{l}5 \\
5\end{array}$ & $\begin{array}{l}4.16778 \\
4.16541\end{array}$ & $\begin{array}{l}237 \\
237\end{array}$ & $\begin{array}{l}0.99985 \\
0.99985\end{array}$ \\
\hline $\begin{array}{l}0.0423 \\
0.0424 \\
0.0425\end{array}$ & $\begin{array}{l}3.18404 \\
3.18173 \\
3.17943\end{array}$ & $\begin{array}{l}231 \\
230 \\
230\end{array}$ & $\begin{array}{l}0.97900 \\
0.97895 \\
0.97890\end{array}$ & $\begin{array}{l}5 \\
5 \\
5\end{array}$ & $\begin{array}{l}4.16304 \\
4.16068 \\
4.15833\end{array}$ & $\begin{array}{l}236 \\
235 \\
235\end{array}$ & $\begin{array}{l}0.99985 \\
0.99985 \\
0.99985\end{array}$ \\
\hline $\begin{array}{l}0.0426 \\
0.0427 \\
0.0428 \\
0.0429 \\
0.0430\end{array}$ & $\begin{array}{l}3.17713 \\
3.17483 \\
3.17254 \\
3.17026 \\
3.16798\end{array}$ & $\begin{array}{l}230 \\
229 \\
228 \\
228 \\
228\end{array}$ & $\begin{array}{l}0.97885 \\
0.97880 \\
0.97875 \\
0.97870 \\
0.97865\end{array}$ & $\begin{array}{l}5 \\
5 \\
5 \\
5 \\
5\end{array}$ & $\begin{array}{l}4.15598 \\
4.15363 \\
4.15129 \\
4.14896 \\
4.14663\end{array}$ & $\begin{array}{l}235 \\
234 \\
233 \\
233 \\
232\end{array}$ & $\begin{array}{l}0.99985 \\
0.99985 \\
0.99985 \\
0.99985 \\
0.99985\end{array}$ \\
\hline $\begin{array}{l}0.0431 \\
0.0432 \\
0.0433 \\
0.0434 \\
0.0435\end{array}$ & $\begin{array}{l}3.16570 \\
3.16344 \\
3.16117 \\
3.15892 \\
3.15667\end{array}$ & $\begin{array}{l}226 \\
227 \\
225 \\
225 \\
225\end{array}$ & $\begin{array}{l}0.97860 \\
0.97856 \\
0.97851 \\
0.97846 \\
0.97841\end{array}$ & $\begin{array}{l}4 \\
5 \\
5 \\
5 \\
5\end{array}$ & $\begin{array}{l}4.14431 \\
4.14139 \\
4 \cdot 13958 \\
4.13737 \\
4.13507\end{array}$ & $\begin{array}{l}232 \\
231 \\
231 \\
230 \\
229\end{array}$ & $\begin{array}{l}0.99985 \\
0.99984 \\
0.99984 \\
0.99984 \\
0.99984\end{array}$ \\
\hline $\begin{array}{l}0.0436 \\
0.043^{-} \\
0.0436 \\
0.043 ! \\
0.0440\end{array}$ & $\begin{array}{l}3.15442 \\
3.15218 \\
3.14994 \\
3.14771 \\
3.14548\end{array}$ & $\begin{array}{l}224 \\
224 \\
223 \\
223 \\
222\end{array}$ & $\begin{array}{l}0.97836 \\
0.97831 \\
0.97826 \\
0.97821 \\
0.97816\end{array}$ & $\begin{array}{l}5 \\
5 \\
5 \\
5 \\
5\end{array}$ & $\begin{array}{l}4 \cdot 13278 \\
4 \cdot 13049 \\
4 \cdot 12820 \\
4 \cdot 12592 \\
4 \cdot 12365\end{array}$ & $\begin{array}{l}229 \\
229 \\
228 \\
227 \\
227\end{array}$ & $\begin{array}{l}0.99984 \\
0.99984 \\
0.99984 \\
0.99984 \\
0.99984\end{array}$ \\
\hline $\begin{array}{l}0.0441 \\
0.0442 \\
0.0443 \\
0.0444 \\
0.0445\end{array}$ & $\begin{array}{l}3.14326 \\
3.14105 \\
3.13884 \\
3.13663 \\
3.13443\end{array}$ & $\begin{array}{l}221 \\
221 \\
221 \\
220 \\
219\end{array}$ & $\begin{array}{l}0.97811 \\
0.97806 \\
0.97801 \\
0.97796 \\
0.97792\end{array}$ & $\begin{array}{l}5 \\
5 \\
5 \\
4 \\
5\end{array}$ & $\begin{array}{l}4.12138 \\
4.11911 \\
4 \cdot 11685 \\
4.111460 \\
4.11235\end{array}$ & $\begin{array}{l}227 \\
226 \\
225 \\
225 \\
225\end{array}$ & $\begin{array}{l}0.99984 \\
0.99984 \\
0.99984 \\
0.99984 \\
0.99983\end{array}$ \\
\hline $\begin{array}{l}0.0446 \\
0.0447 \\
0.0448 \\
0.0449 \\
0.0450\end{array}$ & $\begin{array}{l}3.13224 \\
3.13005 \\
3.12786 \\
3.12568 \\
3.12351\end{array}$ & $\begin{array}{l}219 \\
219 \\
218 \\
217\end{array}$ & $\begin{array}{l}0.97787 \\
0.97782 \\
0.97777 \\
0.97772 \\
0.97767\end{array}$ & $\begin{array}{l}5 \\
5 \\
5 \\
5\end{array}$ & $\begin{array}{l}4.11010 \\
4.10786 \\
4.10563 \\
4.10340 \\
4.10118\end{array}$ & $\begin{array}{l}224 \\
223 \\
223 \\
222\end{array}$ & $\begin{array}{l}0.99983 \\
0.99983 \\
0.99983 \\
0.99983 \\
0.99983\end{array}$ \\
\hline
\end{tabular}


Table I Harmonic Oscillator Contributions (in dimensionless form) to the Thermodynamic Functions for Values of $X=\mathrm{hcv} / \mathrm{kT}$

\begin{tabular}{|c|c|c|c|c|c|c|c|}
\hline $\mathbf{X}$ & $-\left(F^{\circ}-E_{O}^{\circ}\right) / R T$ & $\Delta$ & $\left(H^{\circ}-E_{0}^{\circ}\right) / R T$ & $\Delta$ & $S \circ / \Omega$ & $\Delta$ & $\mathrm{C}_{\mathrm{p}}^{\circ} / \mathrm{R}$ \\
\hline 0.0450 & 3.12351 & 217 & 0.97767 & 5 & 4.10118 & 222 & 0.99983 \\
\hline $\begin{array}{l}0.0451 \\
0.0452\end{array}$ & $\begin{array}{l}3.12134 \\
3.11917\end{array}$ & $\begin{array}{l}217 \\
216\end{array}$ & $\begin{array}{l}0.97762 \\
0.97757\end{array}$ & $\begin{array}{l}5 \\
5\end{array}$ & $\begin{array}{l}4.09896 \\
4.09674\end{array}$ & $\begin{array}{l}222 \\
221\end{array}$ & $\begin{array}{l}0.99983 \\
0.99983\end{array}$ \\
\hline $\begin{array}{l}0.0453 \\
0.0454 \\
0.0455\end{array}$ & $\begin{array}{l}3.11701 \\
3.11486 \\
3.11271\end{array}$ & $\begin{array}{l}215 \\
215 \\
215\end{array}$ & $\begin{array}{l}0.97752 \\
0.97747 \\
0.97742\end{array}$ & $\begin{array}{l}5 \\
5 \\
5\end{array}$ & $\begin{array}{l}4.09453 \\
4.09233 \\
4.09013\end{array}$ & $\begin{array}{l}220 \\
220 \\
220\end{array}$ & $\begin{array}{l}0.99983 \\
0.99983 \\
0.99983\end{array}$ \\
\hline $\begin{array}{l}0.0456 \\
0.0457 \\
0.0458 \\
0.0459 \\
0.0460\end{array}$ & $\begin{array}{l}3.11056 \\
3.10842 \\
3.10628 \\
3.10415 \\
3.10203\end{array}$ & $\begin{array}{l}214 \\
214 \\
213 \\
212 \\
213\end{array}$ & $\begin{array}{l}0.97737 \\
0.97732 \\
0.97728 \\
0.97723 \\
0.97718\end{array}$ & $\begin{array}{l}5 \\
4 \\
5 \\
5 \\
5\end{array}$ & $\begin{array}{l}4.08793 \\
4.08574 \\
4.08356 \\
4.08138 \\
4.07920\end{array}$ & $\begin{array}{l}219 \\
218 \\
218 \\
218 \\
217\end{array}$ & $\begin{array}{l}0.99983 \\
0.99983 \\
0.99983 \\
0.99982 \\
0.99982\end{array}$ \\
\hline $\begin{array}{l}0.0461 \\
0.0462 \\
0.0463 \\
0.0464 \\
0.0465\end{array}$ & $\begin{array}{l}3.09990 \\
3.09779 \\
3.09567 \\
3.09357 \\
3.09146\end{array}$ & $\begin{array}{l}211 \\
212 \\
210 \\
211 \\
210\end{array}$ & $\begin{array}{l}0.97713 \\
0.97708 \\
0.97703 \\
0.97698 \\
0.97693\end{array}$ & $\begin{array}{l}5 \\
5 \\
5 \\
5 \\
5\end{array}$ & $\begin{array}{l}4.07703 \\
4.07486 \\
4.07270 \\
4.07055 \\
4.06839\end{array}$ & $\begin{array}{l}217 \\
216 \\
215 \\
216 \\
214\end{array}$ & $\begin{array}{l}0.99982 \\
0.99982 \\
0.99982 \\
0.99982 \\
0.99982\end{array}$ \\
\hline $\begin{array}{l}0.0466 \\
0.0467 \\
0.0468 \\
0.0469 \\
0.0470\end{array}$ & $\begin{array}{l}3.08936 \\
3.08727 \\
3.08518 \\
3.08310 \\
3.08102\end{array}$ & $\begin{array}{l}209 \\
209 \\
208 \\
208 \\
208\end{array}$ & $\begin{array}{l}0.97688 \\
0.97683 \\
0.97678 \\
0.97673 \\
0.97668\end{array}$ & $\begin{array}{l}5 \\
5 \\
5 \\
5 \\
4\end{array}$ & $\begin{array}{l}4.06625 \\
4.06410 \\
4.06196 \\
4.05983 \\
4.05770\end{array}$ & $\begin{array}{l}215 \\
214 \\
213 \\
213 \\
213\end{array}$ & $\begin{array}{l}0.99982 \\
0.99982 \\
0.99982 \\
0.99982 \\
0.99982\end{array}$ \\
\hline $\begin{array}{l}0.0471 \\
0.0472 \\
0.0473 \\
0.0474 \\
0.0475\end{array}$ & $\begin{array}{l}3.07894 \\
3.07687 \\
3.07480 \\
3.07274 \\
3.07068\end{array}$ & $\begin{array}{l}207 \\
207 \\
206 \\
206 \\
205\end{array}$ & $\begin{array}{l}0.97664 \\
0.97659 \\
0.97654 \\
0.97649 \\
0.97644\end{array}$ & $\begin{array}{l}5 \\
5 \\
5 \\
5 \\
5\end{array}$ & $\begin{array}{l}4.05558 \\
4.05345 \\
4.05134 \\
4.04923 \\
4.04712\end{array}$ & $\begin{array}{l}212 \\
211 \\
211 \\
211 \\
210\end{array}$ & $\begin{array}{l}0.99982 \\
0.99981 \\
0.99981 \\
0.99981 \\
0.99981\end{array}$ \\
\hline $\begin{array}{l}0.0476 \\
0.0477 \\
0.0478 \\
0.0479 \\
0.0480\end{array}$ & $\begin{array}{l}3.06863 \\
3.06658 \\
3.06453 \\
3.06249 \\
3.06046\end{array}$ & $\begin{array}{l}205 \\
205 \\
204 \\
203 \\
203\end{array}$ & $\begin{array}{l}0.97639 \\
0.97634 \\
0.97629 \\
0.97624 \\
0.97619\end{array}$ & $\begin{array}{l}5 \\
5 \\
5 \\
5 \\
5\end{array}$ & $\begin{array}{l}4.04502 \\
4.04292 \\
4.04083 \\
4.03874 \\
4.03665\end{array}$ & $\begin{array}{l}210 \\
209 \\
209 \\
209 \\
208\end{array}$ & $\begin{array}{l}0.99981 \\
0.99981 \\
0.99981 \\
0.99981 \\
0.99981\end{array}$ \\
\hline $\begin{array}{l}0.0481 \\
0.0482 \\
0.0483 \\
0.0484 \\
0.0485\end{array}$ & $\begin{array}{l}3.05843 \\
3.05640 \\
3.05438 \\
3.05236 \\
3.05034\end{array}$ & $\begin{array}{l}203 \\
202 \\
202 \\
202 \\
201\end{array}$ & $\begin{array}{l}0.97614 \\
0.97609 \\
0.97604 \\
0.97600 \\
0.97595\end{array}$ & $\begin{array}{l}5 \\
5 \\
4 \\
5 \\
5\end{array}$ & $\begin{array}{l}4.03457 \\
4.03249 \\
4.03042 \\
4.02835 \\
4.02629\end{array}$ & $\begin{array}{l}208 \\
207 \\
207 \\
206 \\
206\end{array}$ & $\begin{array}{l}0.99981 \\
0.99981 \\
0.99981 \\
0.99980 \\
0.99980\end{array}$ \\
\hline $\begin{array}{l}0.0486 \\
0.0487 \\
0.0488 \\
0.0489 \\
0.0490\end{array}$ & $\begin{array}{l}3.04833 \\
3.04633 \\
3.04433 \\
3.04233 \\
3.04033\end{array}$ & $\begin{array}{l}200 \\
200 \\
200 \\
200 \\
198\end{array}$ & $\begin{array}{l}0.97590 \\
0.97585 \\
0.97580 \\
0.97575 \\
0.97570\end{array}$ & $\begin{array}{l}5 \\
5 \\
5 \\
5 \\
5\end{array}$ & $\begin{array}{l}4.02423 \\
4.02218 \\
4.02012 \\
4.01808 \\
4.01603\end{array}$ & $\begin{array}{l}205 \\
206 \\
204 \\
205 \\
203\end{array}$ & $\begin{array}{l}0.99980 \\
0.99980 \\
0.99980 \\
0.99980 \\
0.99980\end{array}$ \\
\hline $\begin{array}{l}0.0491 \\
0.0492 \\
0.0493 \\
0.0494 \\
0.0495\end{array}$ & $\begin{array}{l}3.03835 \\
3.03636 \\
3.03438 \\
3.03240 \\
3.03043\end{array}$ & $\begin{array}{l}199 \\
198 \\
198 \\
197 \\
197\end{array}$ & $\begin{array}{l}0.97565 \\
0.97560 \\
0.97555 \\
0.97550 \\
0.97545\end{array}$ & $\begin{array}{l}5 \\
5 \\
5 \\
5 \\
4\end{array}$ & $\begin{array}{l}4.01400 \\
4.01196 \\
4.00933 \\
4.00791 \\
4.00588\end{array}$ & $\begin{array}{l}204 \\
203 \\
202 \\
203 \\
201\end{array}$ & $\begin{array}{l}0.99980 \\
0.99980 \\
0.99980 \\
0.99980 \\
0.99980\end{array}$ \\
\hline $\begin{array}{l}0.0496 \\
0.0497 \\
0.0498 \\
0.0499 \\
0.0500\end{array}$ & $\begin{array}{l}3.02846 \\
3.02650 \\
3.02454 \\
3.02258 \\
3.02063\end{array}$ & $\begin{array}{l}196 \\
196 \\
196 \\
195\end{array}$ & $\begin{array}{l}0.97541 \\
0.97536 \\
0.97531 \\
0.97526 \\
0.97521\end{array}$ & $\begin{array}{l}5 \\
5 \\
5 \\
5\end{array}$ & $\begin{array}{l}4.00387 \\
4.00185 \\
3.99984 \\
3.99784 \\
3.99584\end{array}$ & $\begin{array}{l}202 \\
201 \\
200 \\
200\end{array}$ & $\begin{array}{l}0.99980 \\
0.99979 \\
0.99979 \\
0.99979 \\
0.99979\end{array}$ \\
\hline
\end{tabular}


Table I Harmonic Oscillator Contributions (in dimensionless form) to the Thermodynamic Functions for Values of $X=h c v / k T$
$\mathbf{X}$
$-\left(\mathrm{FO}^{\circ}-\mathrm{EO}\right) / \mathrm{RT}$
$\Delta \quad\left(\mathrm{H}^{\circ}-\mathrm{EO}\right) / \mathrm{RT}$
$\Delta \quad \mathrm{S} \% / \mathrm{R}$
$\Delta \quad \mathrm{co} / \mathrm{R}$

0.0500

3.02063

195

0.97521

5

3.99584

200

0.99979

0.0501

3.01868

194

3.01674

0.97516

0.0502

3.01480

0.97511

3.01286

0.97506

0.0504

3.01093

193

193

0.97501

0.0505

193

0.97496

3.99384

199

0.99979

0.99979

0.99979

$3.98986 \quad 199$

$3.98787 \quad 198$

0.99979

0.0506

3.00900

193

0.97491

3.98589

198

0.99979

0.0507

3.00707

0.97486

3.00515

192

192

0.0509

3.00323

0.97482

3.98391

197

0.99979

3.98194

197

0.99979

3. 97997

197

191

0.97472

3.97800

196

0.99979

0.99978

0.0510

2.99941

190

0.0511

2.99941
2.99751

0.97467

0.0512

2.99560

0.97462

0.0513

191

189

2.99371

0.97457

0.97452

0.0514

2.99181

189

0.97447

3.97604

0.99978

0.0516

2. 98992

0.97442

188

0.0517

2. 98804

0.97437

2.98615

0.97432

0.0519

2. 98427

188

0.0520

2.98240

187

187

0.97427

0.97423

0.0521

2.98053

187

0.0522

2.97866

0.97418

186

2. 97680

0.97413

0.0524

2.97493

0.97408

185

0.0525

2.97308

0.97403

186

0.0526

2. 97122

185

2.96937

0.97398

3.97408

3.97213

196

0.99978

194

$3.96823 \quad 195$

0.99978

0.99978

0.99978

0.99978

0.0527

2.96753

2.96569

0.97393

184

184

0.0529

2.96385

184

0.97388

0.97383

0.0530

184

0.97378

3.96628

194

0.99978

0.99978

0.99978

0.99978

0.99978

0.0531

2. 96201

0.97373

$3.96434 \quad 193$

$3.96048 \quad 193$

$\begin{array}{ll}3.35855 & 193 \\ 3.95632 & 192\end{array}$

0.99977

$3.95470 \quad 191$

0.99977

0.99977

$3.95087 \quad 191$

$3.94896 \quad 190$

0.99977

0.99977

0.0532

183

2. 96018

0.97369

3.94706

191

0.99977

0.99977

0.99977

0.99977

$3.94136 \quad 189$

189

0.99977

0.0533

2.95835

183

182

$2.95653 \quad 183$

0.0535

2.95470

181

0.97364

0.97359

0.97354

0.97349

3.93758

188

0.99977

$3.93570 \quad 188$

0.99976

0.99976

$3.93194 \quad 188$

$3.93006 \quad 187$

0.99976

3.92819

186

0.99976

0.0536

2.95289

182

0.97344

181

$2.94926 \quad 181$

0.0538

0.97339

$3.92633 \quad 187$

0.99976

0.97334

0.97329

0.0539

2. 94745

180

2.94565

180

0.97324

0.0541

2.94385

180

2.94205

0.97319

179

0.0542

2.94026

2.93847

0.0544

2.93668

179

178

0.97314

0.97310

0.97305

0.97300

3.92446

186

0.99976

0.99976

$3.92260 \quad 185$

0.99976

$3.91889 \quad 185$

0.99976

0.0546

2.93490

178

2.93312

0.97295

178

0.0548

2.93134

0.97290

177

0.97285

0.0549

2. 92957

0.97280

0.97275

$3.91704 \quad 184$

0.99976

0.99976

0.99975

$3.91335 \quad 184$

0.99975

$3.91151 \quad 183$

0.99975

0.0550

2. 92780

3.90785

183

0.99975

$3.90602 \quad 183$

$3.90419 \quad 182$

0.99975

0.99975

0.99975

3.90055

0.99975 
Table I Harmonic Oscillator Contributions (in dimensionless form) to the Thermodynamic Functions for Values of $\mathrm{X}=\mathrm{hcv} / \mathrm{kT}$

\begin{tabular}{|c|c|c|c|c|c|c|c|}
\hline$X$ & $-\left(F^{\circ}-E_{O} O\right) / R T$ & $\Delta$ & $\left(\mathrm{H}^{\circ}-\mathrm{E} \mathrm{O}\right) / \mathrm{RT}$ & $\Delta$ & $\mathrm{S} \circ / \mathrm{R}$ & $\Delta$ & $\mathrm{C} \circ / \mathrm{R}$ \\
\hline 0.0550 & 2.92780 & 177 & 0.97275 & 5 & 3.90055 & 182 & 0.99975 \\
\hline $\begin{array}{l}0.0551 \\
0.0552 \\
0.0553 \\
0.0554 \\
0.0555\end{array}$ & $\begin{array}{l}2.92603 \\
2.92427 \\
2.92250 \\
2.92075 \\
2.91899\end{array}$ & $\begin{array}{l}176 \\
177 \\
175 \\
176 \\
175\end{array}$ & $\begin{array}{l}0.97270 \\
0.97265 \\
0.97260 \\
0.97256 \\
0.97251\end{array}$ & $\begin{array}{l}5 \\
5 \\
4 \\
5 \\
5\end{array}$ & $\begin{array}{l}3.89873 \\
3.89692 \\
3.85511 \\
3.89330 \\
3.89150\end{array}$ & $\begin{array}{l}181 \\
181 \\
181 \\
180 \\
180\end{array}$ & $\begin{array}{l}0.99975 \\
0.99975 \\
0.99975 \\
0.99974 \\
0.99974\end{array}$ \\
\hline $\begin{array}{l}0.0556 \\
0.0557 \\
0.0558 \\
0.0559 \\
0.0560\end{array}$ & $\begin{array}{l}2.91724 \\
2.91550 \\
2.91375 \\
2.91201 \\
2.91027\end{array}$ & $\begin{array}{l}174 \\
175 \\
174 \\
174 \\
173\end{array}$ & $\begin{array}{l}0.97246 \\
0.97241 \\
0.97236 \\
0.97231 \\
0.97226\end{array}$ & $\begin{array}{l}5 \\
5 \\
5 \\
5 \\
5\end{array}$ & $\begin{array}{l}3.88970 \\
3.88790 \\
3.88611 \\
3.88432 \\
3.88253\end{array}$ & $\begin{array}{l}180 \\
179 \\
179 \\
179 \\
178\end{array}$ & $\begin{array}{l}0.99974 \\
0.99974 \\
0.99974 \\
0.99974 \\
0.99974\end{array}$ \\
\hline $\begin{array}{l}0.0561 \\
0.0562 \\
0.0563 \\
0.0564 \\
0.0565\end{array}$ & $\begin{array}{l}2.90854 \\
2.90681 \\
2.90508 \\
2.90335 \\
2.90163\end{array}$ & $\begin{array}{l}173 \\
173 \\
173 \\
172 \\
172\end{array}$ & $\begin{array}{l}0.97221 \\
0.97216 \\
0.97211 \\
0.97207 \\
0.97202\end{array}$ & $\begin{array}{l}5 \\
5 \\
4 \\
5 \\
5\end{array}$ & $\begin{array}{l}3.88075 \\
3.87897 \\
3.87719 \\
3.87542 \\
3.87365\end{array}$ & $\begin{array}{l}178 \\
178 \\
177 \\
177 \\
177\end{array}$ & $\begin{array}{l}0.99974 \\
0.99974 \\
0.99974 \\
0.99974 \\
0.99973\end{array}$ \\
\hline $\begin{array}{l}0.0566 \\
0.0567 \\
0.0568 \\
0.0569 \\
0.0570\end{array}$ & $\begin{array}{l}2.89991 \\
2.89820 \\
2.89648 \\
2.89478 \\
2.89307\end{array}$ & $\begin{array}{l}171 \\
172 \\
170 \\
171 \\
170\end{array}$ & $\begin{array}{l}0.97197 \\
0.97192 \\
0.97187 \\
0.97182 \\
0.97177\end{array}$ & $\begin{array}{l}5 \\
5 \\
5 \\
5 \\
5\end{array}$ & $\begin{array}{l}3.87188 \\
3.87012 \\
3.86835 \\
3.86659 \\
3.86484\end{array}$ & $\begin{array}{l}176 \\
177 \\
176 \\
175 \\
175\end{array}$ & $\begin{array}{l}0.99973 \\
0.99973 \\
0.99973 \\
0.99973 \\
0.99973\end{array}$ \\
\hline $\begin{array}{l}0.0571 \\
0.0572 \\
0.0573 \\
0.0574 \\
0.0575\end{array}$ & $\begin{array}{l}2.89137 \\
2.88967 \\
2.88797 \\
2.88627 \\
2.88458\end{array}$ & $\begin{array}{l}170 \\
170 \\
170 \\
169 \\
169\end{array}$ & $\begin{array}{l}0.97172 \\
0.97167 \\
0.97162 \\
0.97157 \\
0.97153\end{array}$ & $\begin{array}{l}5 \\
5 \\
5 \\
4 \\
5\end{array}$ & $\begin{array}{l}3.86309 \\
3.86134 \\
3.85959 \\
3.85785 \\
3.85611\end{array}$ & $\begin{array}{l}175 \\
175 \\
174 \\
174 \\
174\end{array}$ & $\begin{array}{l}0.99973 \\
0.99973 \\
0.99973 \\
0.99973 \\
0.99972\end{array}$ \\
\hline $\begin{array}{l}0.0576 \\
0.0577 \\
0.0578 \\
0.0579 \\
0.0580\end{array}$ & $\begin{array}{l}2.88289 \\
2.88121 \\
2.87953 \\
2.87785 \\
2.87617\end{array}$ & $\begin{array}{l}168 \\
168 \\
168 \\
168 \\
167\end{array}$ & $\begin{array}{l}0.97148 \\
0.97143 \\
0.97138 \\
0.97133 \\
0.97128\end{array}$ & $\begin{array}{l}5 \\
5 \\
5 \\
5 \\
5\end{array}$ & $\begin{array}{l}3.85437 \\
3.85264 \\
3.85091 \\
3.34918 \\
3.84745\end{array}$ & $\begin{array}{l}173 \\
173 \\
173 \\
173 \\
172\end{array}$ & $\begin{array}{l}0.99972 \\
0.99972 \\
0.99972 \\
0.99972 \\
0.99972\end{array}$ \\
\hline $\begin{array}{l}0.0581 \\
0.0582 \\
0.0583 \\
0.0584 \\
0.0585\end{array}$ & $\begin{array}{l}2.87450 \\
2.87283 \\
2.87116 \\
2.86950 \\
2.86784\end{array}$ & $\begin{array}{l}167 \\
167 \\
166 \\
166 \\
166\end{array}$ & $\begin{array}{l}0.97123 \\
0.97118 \\
0.97113 \\
0.97108 \\
0.97104\end{array}$ & $\begin{array}{l}5 \\
5 \\
5 \\
4 \\
5\end{array}$ & $\begin{array}{l}3.84573 \\
3.84401 \\
3.84229 \\
3.84058 \\
3.83887\end{array}$ & $\begin{array}{l}172 \\
172 \\
171 \\
171 \\
171\end{array}$ & $\begin{array}{l}0.99972 \\
0.99972 \\
0.99972 \\
0.99972 \\
0.99971\end{array}$ \\
\hline $\begin{array}{l}0.0586 \\
0.0587 \\
0.0588 \\
0.0589 \\
0.0590\end{array}$ & $\begin{array}{l}2.86618 \\
2.86452 \\
2.86287 \\
2.86122 \\
2.85957\end{array}$ & $\begin{array}{l}166 \\
165 \\
165 \\
165 \\
164\end{array}$ & $\begin{array}{l}0.97099 \\
0.97094 \\
0.97089 \\
0.97084 \\
0.97079\end{array}$ & $\begin{array}{l}5 \\
5 \\
5 \\
5 \\
5\end{array}$ & $\begin{array}{l}3.83716 \\
3.83546 \\
3.83376 \\
3.83206 \\
3.83036\end{array}$ & $\begin{array}{l}170 \\
170 \\
170 \\
170 \\
169\end{array}$ & $\begin{array}{l}0.99971 \\
0.99971 \\
0.99971 \\
0.99971 \\
0.99971\end{array}$ \\
\hline $\begin{array}{l}0.0591 \\
0.0592 \\
0.0593 \\
0.0594 \\
0.0595\end{array}$ & $\begin{array}{l}2.85793 \\
2.85629 \\
2.85465 \\
2.85301 \\
2.85138\end{array}$ & $\begin{array}{l}164 \\
164 \\
164 \\
163 \\
163\end{array}$ & $\begin{array}{l}0.97074 \\
0.97069 \\
0.97064 \\
0.97059 \\
0.97055\end{array}$ & $\begin{array}{l}5 \\
5 \\
5 \\
4 \\
5\end{array}$ & $\begin{array}{l}3.82867 \\
3.82698 \\
3.82529 \\
3.82361 \\
3.82193\end{array}$ & $\begin{array}{l}169 \\
169 \\
168 \\
168 \\
168\end{array}$ & $\begin{array}{l}0.99971 \\
0.99971 \\
0.99971 \\
0.99971 \\
0.99971\end{array}$ \\
\hline $\begin{array}{l}0.0596 \\
0.0597 \\
0.0598 \\
0.0599 \\
0.0600\end{array}$ & $\begin{array}{l}2.84975 \\
2.84812 \\
2.84650 \\
2.84488 \\
2.84326\end{array}$ & $\begin{array}{l}163 \\
162 \\
162 \\
162\end{array}$ & $\begin{array}{l}0.97050 \\
0.97045 \\
0.97040 \\
0.97035 \\
0.97030\end{array}$ & $\begin{array}{l}5 \\
5 \\
5 \\
5\end{array}$ & $\begin{array}{l}3.82025 \\
3.81857 \\
3.81690 \\
3.81523 \\
3.81356\end{array}$ & $\begin{array}{l}168 \\
167 \\
167 \\
167\end{array}$ & $\begin{array}{l}0.99970 \\
0.99970 \\
0.99970 \\
0.99970 \\
0.99970\end{array}$ \\
\hline
\end{tabular}


Table I Harmonic Oscillator Contributions (in dimensionless form) to the Thermodynamic Functions for Values of $\mathrm{X}=\mathrm{hcv} / \mathrm{kT}$

\begin{tabular}{|c|c|c|c|c|c|c|c|}
\hline$x$ & $-\left(\mathrm{F}^{\circ}-\mathrm{E}_{\mathrm{O}}\right) / \mathrm{RT}$ & $\Delta$ & $\left(\mathrm{H}^{\circ}-\mathrm{E} \mathrm{O}\right) / \mathrm{RT}$ & $\Delta$ & So/R & $\Delta$ & $\mathrm{C}_{\mathrm{p}} / \mathrm{R}$ \\
\hline 0.0600 & 2.84326 & 162 & 0.97030 & 5 & 3.81356 & 166 & 0.99970 \\
\hline $\begin{array}{l}0.0601 \\
0.0602 \\
0.0603\end{array}$ & $\begin{array}{l}2.84165 \\
2.84003 \\
2.83842\end{array}$ & $\begin{array}{l}161 \\
161 \\
161\end{array}$ & $\begin{array}{l}0.97025 \\
0.97020 \\
0.97015\end{array}$ & $\begin{array}{l}5 \\
5 \\
5\end{array}$ & $\begin{array}{l}3.81190 \\
3.81023 \\
3.80857\end{array}$ & $\begin{array}{l}167 \\
166 \\
165\end{array}$ & $\begin{array}{l}0.99970 \\
0.99970 \\
0.99970\end{array}$ \\
\hline $\begin{array}{l}0.0604 \\
0.0605\end{array}$ & $\begin{array}{l}2.83681 \\
2.83521\end{array}$ & $\begin{array}{l}160 \\
160\end{array}$ & $\begin{array}{l}0.97010 \\
0.97005\end{array}$ & $\begin{array}{l}5 \\
4\end{array}$ & $\begin{array}{l}3.80692 \\
3.80526\end{array}$ & $\begin{array}{l}166 \\
165\end{array}$ & $\begin{array}{l}0.99970 \\
0.99969\end{array}$ \\
\hline $\begin{array}{l}0.0606 \\
0.0607 \\
0.0608 \\
0.0609 \\
0.0610\end{array}$ & $\begin{array}{l}2.83361 \\
2.83201 \\
2.83041 \\
2.82882 \\
2.82723\end{array}$ & $\begin{array}{l}160 \\
160 \\
159 \\
159 \\
159\end{array}$ & $\begin{array}{l}0.97001 \\
0.96996 \\
0.96991 \\
0.96986 \\
0.96981\end{array}$ & $\begin{array}{l}5 \\
5 \\
5 \\
5 \\
5\end{array}$ & $\begin{array}{l}3.80361 \\
3.80196 \\
3.80032 \\
3.79868 \\
3.79704\end{array}$ & $\begin{array}{l}165 \\
164 \\
164 \\
164 \\
164\end{array}$ & $\begin{array}{l}0.99969 \\
0.99969 \\
0.99969 \\
0.99969 \\
0.99969\end{array}$ \\
\hline $\begin{array}{l}0.0611 \\
0.0612 \\
0.0613 \\
0.0614 \\
0.06 .15\end{array}$ & $\begin{array}{l}2.82564 \\
2.82405 \\
2.82247 \\
2.82089 \\
2.81931\end{array}$ & $\begin{array}{l}159 \\
158 \\
158 \\
158 \\
157\end{array}$ & $\begin{array}{l}0.96976 \\
0.96971 \\
0.96966 \\
0.96961 \\
0.96957\end{array}$ & $\begin{array}{l}5 \\
5 \\
5 \\
4 \\
5\end{array}$ & $\begin{array}{l}3.79540 \\
3.79376 \\
3.79213 \\
3.79050 \\
3.78888\end{array}$ & $\begin{array}{l}164 \\
163 \\
163 \\
162 \\
163\end{array}$ & $\begin{array}{l}0.99969 \\
0.99969 \\
0.99969 \\
0.99969 \\
0.99969\end{array}$ \\
\hline $\begin{array}{l}0.0616 \\
0.0617 \\
0.0618 \\
0.0619 \\
0.062 \mathrm{C}\end{array}$ & $\begin{array}{l}2.81774 \\
2.81616 \\
2.81459 \\
2.81303 \\
2.81146\end{array}$ & $\begin{array}{l}158 \\
157 \\
156 \\
157 \\
156\end{array}$ & $\begin{array}{l}0.96952 \\
0.96947 \\
0.96942 \\
0.96937 \\
0.96932\end{array}$ & $\begin{array}{l}5 \\
5 \\
5 \\
5 \\
5\end{array}$ & $\begin{array}{l}3.78725 \\
3.78563 \\
3.78401 \\
3.78239 \\
3.78078\end{array}$ & $\begin{array}{l}162 \\
162 \\
162 \\
161 \\
161\end{array}$ & $\begin{array}{l}0.99968 \\
0.99968 \\
0.99968 \\
0.99968 \\
0.99968\end{array}$ \\
\hline $\begin{array}{l}0.0621 \\
0.0622 \\
0.0623 \\
0.0624 \\
0.0625\end{array}$ & $\begin{array}{l}2.80990 \\
2.80834 \\
2.80678 \\
2.80523 \\
2.80368\end{array}$ & $\begin{array}{l}156 \\
156 \\
155 \\
155 \\
155\end{array}$ & $\begin{array}{l}0.96927 \\
0.96922 \\
0.96917 \\
0.96912 \\
0.96908\end{array}$ & $\begin{array}{l}5 \\
5 \\
5 \\
4 \\
5\end{array}$ & $\begin{array}{l}3.77917 \\
3.77756 \\
3.77596 \\
3.77435 \\
3.77275\end{array}$ & $\begin{array}{l}161 \\
160 \\
161 \\
160 \\
160\end{array}$ & $\begin{array}{l}0.99968 \\
0.99968 \\
0.99968 \\
0.99968 \\
0.99967\end{array}$ \\
\hline $\begin{array}{l}0.0626 \\
0.0627 \\
0.0628 \\
0.0629 \\
0.0630\end{array}$ & $\begin{array}{l}2.80213 \\
2.80058 \\
2.79904 \\
2.79749 \\
2.79596\end{array}$ & $\begin{array}{l}155 \\
154 \\
155 \\
153 \\
154\end{array}$ & $\begin{array}{l}0.96903 \\
0.96898 \\
0.96893 \\
0.96888 \\
0.96883\end{array}$ & $\begin{array}{l}5 \\
5 \\
5 \\
5 \\
5\end{array}$ & $\begin{array}{l}3.77115 \\
3.76956 \\
3.76796 \\
3.76637 \\
3.76479\end{array}$ & $\begin{array}{l}159 \\
160 \\
159 \\
158 \\
159\end{array}$ & $\begin{array}{l}0.99967 \\
0.99967 \\
0.99967 \\
0.99967 \\
0.99967\end{array}$ \\
\hline $\begin{array}{l}0.0631 \\
0.0632 \\
0.0633 \\
0.0634 \\
0.0635\end{array}$ & $\begin{array}{l}2.79442 \\
2.79288 \\
2.79135 \\
2.78982 \\
2.78830\end{array}$ & $\begin{array}{l}154 \\
153 \\
153 \\
152 \\
153\end{array}$ & $\begin{array}{l}0.96878 \\
0.96873 \\
0.96868 \\
0.96864 \\
0.96859\end{array}$ & $\begin{array}{l}5 \\
5 \\
4 \\
5 \\
5\end{array}$ & $\begin{array}{l}3.76320 \\
3.76162 \\
3.76004 \\
3.75846 \\
3.75688\end{array}$ & $\begin{array}{l}158 \\
158 \\
158 \\
158 \\
157\end{array}$ & $\begin{array}{l}0.99967 \\
0.99967 \\
0.99967 \\
0.99967 \\
0.99966\end{array}$ \\
\hline $\begin{array}{l}0.0636 \\
0.0637 \\
0.0638 \\
0.0639 \\
0.0640\end{array}$ & $\begin{array}{l}2.78677 \\
2.78525 \\
2.78373 \\
2.78222 \\
2.78070\end{array}$ & $\begin{array}{l}152 \\
152 \\
151 \\
152 \\
151\end{array}$ & $\begin{array}{l}0.96854 \\
0.96849 \\
0.96844 \\
0.96839 \\
0.96834\end{array}$ & $\begin{array}{l}5 \\
5 \\
5 \\
5 \\
5\end{array}$ & $\begin{array}{l}3.75531 \\
3.75374 \\
3.75217 \\
3.75061 \\
3.74904\end{array}$ & $\begin{array}{l}157 \\
157 \\
156 \\
157 \\
156\end{array}$ & $\begin{array}{l}0.99966 \\
0.99966 \\
0.99966 \\
0.99966 \\
0.99966\end{array}$ \\
\hline $\begin{array}{l}0.0641 \\
0.0642 \\
0.0643 \\
0.0644 \\
0.0645\end{array}$ & $\begin{array}{l}2.77919 \\
2.77768 \\
2.77617 \\
2.77467 \\
2.77317\end{array}$ & $\begin{array}{l}151 \\
151 \\
150 \\
150 \\
150\end{array}$ & $\begin{array}{l}0.96829 \\
0.96824 \\
0.96819 \\
0.96815 \\
0.96810\end{array}$ & $\begin{array}{l}5 \\
5 \\
4 \\
5 \\
5\end{array}$ & $\begin{array}{l}3.74748 \\
3 \cdot 74592 \\
3.74437 \\
3.74281 \\
3.74126\end{array}$ & $\begin{array}{l}156 \\
155 \\
156 \\
155 \\
155\end{array}$ & $\begin{array}{l}0.99966 \\
0.99966 \\
0.99966 \\
0.99965 \\
0.99965\end{array}$ \\
\hline $\begin{array}{l}0.0646 \\
0.0647 \\
0.0648 \\
0.0649 \\
0.0650\end{array}$ & $\begin{array}{l}2.77167 \\
2.77017 \\
2.76867 \\
2.76718 \\
2.76569\end{array}$ & $\begin{array}{l}150 \\
150 \\
149 \\
149\end{array}$ & $\begin{array}{l}0.96805 \\
0.96800 \\
0.96795 \\
0.96790 \\
0.96785\end{array}$ & $\begin{array}{l}5 \\
5 \\
5 \\
5\end{array}$ & $\begin{array}{l}3.73971 \\
3.73817 \\
3.73662 \\
3.73508 \\
3.73354\end{array}$ & $\begin{array}{l}154 \\
155 \\
154 \\
154\end{array}$ & $\begin{array}{l}0.99965 \\
0.99965 \\
0.99965 \\
0.99965 \\
0.99965\end{array}$ \\
\hline
\end{tabular}


Table I Harmonic Oscillator Contributions (in dimensionless form) to the Thermodynamic Functions for Values of $\mathrm{X}=\mathrm{hcv} / \mathrm{kT}$

\begin{tabular}{|c|c|c|c|c|c|c|c|}
\hline $\mathrm{X}$ & $-\left(F^{\circ}-E_{O}^{\circ}\right) / R T$ & $\Delta$ & $\left(\mathrm{H}^{\circ}-\mathrm{E}_{\mathrm{O}}^{\circ}\right) / \mathrm{RT}$ & $\Delta$ & $S \circ / R$ & $\Delta$ & $\mathrm{C}_{\mathrm{p}} / \mathrm{R}$ \\
\hline 0.0650 & 2.76569 & 149 & 0.96785 & 5 & 3.73354 & 153 & 0.99965 \\
\hline $\begin{array}{l}0.0651 \\
0.0652 \\
0.0653 \\
0.0654 \\
0.0655\end{array}$ & $\begin{array}{l}2.76420 \\
2.76272 \\
2.76124 \\
2.75975 \\
2.75828\end{array}$ & $\begin{array}{l}148 \\
148 \\
149 \\
147 \\
148\end{array}$ & $\begin{array}{l}0.96780 \\
0.96775 \\
0.96771 \\
0.96766 \\
0.96761\end{array}$ & $\begin{array}{l}5 \\
4 \\
5 \\
5 \\
5\end{array}$ & $\begin{array}{l}3.73201 \\
3.73047 \\
3.72894 \\
3.72741 \\
3.72588\end{array}$ & $\begin{array}{l}154 \\
153 \\
153 \\
153 \\
152\end{array}$ & $\begin{array}{l}0.99965 \\
0.99965 \\
0.99964 \\
0.99964 \\
0.99964\end{array}$ \\
\hline $\begin{array}{l}0.0656 \\
0.0657 \\
0.0658 \\
0.0659 \\
0.0660\end{array}$ & $\begin{array}{l}2.15680 \\
2.75533 \\
2.75386 \\
2.75239 \\
2.75092\end{array}$ & $\begin{array}{l}147 \\
147 \\
147 \\
147 \\
147\end{array}$ & $\begin{array}{l}0.96756 \\
0.96751 \\
0.96746 \\
0.96741 \\
0.96736\end{array}$ & $\begin{array}{l}5 \\
5 \\
5 \\
5 \\
5\end{array}$ & $\begin{array}{l}3.72436 \\
3.72284 \\
3.72132 \\
3.71980 \\
3.71828\end{array}$ & $\begin{array}{l}152 \\
152 \\
152 \\
152 \\
151\end{array}$ & $\begin{array}{l}0.99964 \\
0.99964 \\
0.99964 \\
0.99964 \\
0.99964\end{array}$ \\
\hline $\begin{array}{l}0.0661 \\
0.0662 \\
0.0663 \\
0.0664 \\
0.0665\end{array}$ & $\begin{array}{l}2.74945 \\
2.74799 \\
2.74653 \\
2.74507 \\
2.74362\end{array}$ & $\begin{array}{l}146 \\
146 \\
146 \\
145 \\
145\end{array}$ & $\begin{array}{l}0.96731 \\
0.96727 \\
0.96722 \\
0.96717 \\
0.96711\end{array}$ & $\begin{array}{l}4 \\
5 \\
5 \\
5 \\
5\end{array}$ & $\begin{array}{l}3.71677 \\
3.71526 \\
3.71375 \\
3.71224 \\
3.71074\end{array}$ & $\begin{array}{l}151 \\
151 \\
151 \\
150 \\
150\end{array}$ & $\begin{array}{l}0.99964 \\
0.99964 \\
0.99963 \\
0.99963 \\
0.99963\end{array}$ \\
\hline $\begin{array}{l}0.0666 \\
0.0667 \\
0.0668 \\
0.0669 \\
0.0670\end{array}$ & $\begin{array}{l}2.74217 \\
2.74072 \\
2.73927 \\
2.73782 \\
2.73638\end{array}$ & $\begin{array}{l}145 \\
145 \\
145 \\
144 \\
145\end{array}$ & $\begin{array}{l}0.96707 \\
0.96702 \\
0.96697 \\
0.96692 \\
0.96687\end{array}$ & $\begin{array}{l}5 \\
5 \\
5 \\
5 \\
4\end{array}$ & $\begin{array}{l}3.70924 \\
3.70774 \\
3.70624 \\
3.70474 \\
3.70325\end{array}$ & $\begin{array}{l}150 \\
150 \\
150 \\
149 \\
149\end{array}$ & $\begin{array}{l}0.99963 \\
0.99963 \\
0.99963 \\
0.99963 \\
0.99963\end{array}$ \\
\hline $\begin{array}{l}0.0671 \\
0.0672 \\
0.0673 \\
0.0674 \\
0.0675\end{array}$ & $\begin{array}{l}2.73493 \\
2.73349 \\
2.73206 \\
2.73062 \\
2.72919\end{array}$ & $\begin{array}{l}144 \\
143 \\
144 \\
143 \\
143\end{array}$ & $\begin{array}{l}0.96683 \\
0.96678 \\
0.96673 \\
0.96668 \\
0.96663\end{array}$ & $\begin{array}{l}3 \\
5 \\
5 \\
5 \\
5\end{array}$ & $\begin{array}{l}3.70176 \\
3.70027 \\
3.69878 \\
3.69730 \\
3.69582\end{array}$ & $\begin{array}{l}149 \\
149 \\
148 \\
148 \\
148\end{array}$ & $\begin{array}{l}0.99963 \\
0.99962 \\
0.99962 \\
0.99962 \\
0.99962\end{array}$ \\
\hline $\begin{array}{l}0.0676 \\
0.0677 \\
0.0678 \\
0.0679 \\
0.0680\end{array}$ & $\begin{array}{l}2.72776 \\
2.72633 \\
2.72490 \\
2.72348 \\
2.72205\end{array}$ & $\begin{array}{l}143 \\
143 \\
142 \\
143 \\
142\end{array}$ & $\begin{array}{l}0.96658 \\
0.96653 \\
0.96648 \\
0.96643 \\
0.96639\end{array}$ & $\begin{array}{l}5 \\
5 \\
5 \\
4 \\
5\end{array}$ & $\begin{array}{l}3.69434 \\
3.69286 \\
3.69138 \\
3.68991 \\
3.68844\end{array}$ & $\begin{array}{l}148 \\
148 \\
147 \\
147 \\
147\end{array}$ & $\begin{array}{l}0.99962 \\
0.99962 \\
0.99962 \\
0.99962 \\
0.99961\end{array}$ \\
\hline $\begin{array}{l}0.0581 \\
0.0682 \\
0.0683 \\
0.0684 \\
0.0685\end{array}$ & $\begin{array}{l}2.72063 \\
2.71922 \\
2.71780 \\
2.71639 \\
2.71498\end{array}$ & $\begin{array}{l}141 \\
142 \\
141 \\
141 \\
141\end{array}$ & $\begin{array}{l}0.96634 \\
0.96629 \\
0.96624 \\
0.96619 \\
0.96614\end{array}$ & $\begin{array}{l}5 \\
5 \\
5 \\
5 \\
5\end{array}$ & $\begin{array}{l}3.68697 \\
3.68550 \\
3.68404 \\
3.68258 \\
3.68112\end{array}$ & $\begin{array}{l}147 \\
146 \\
146 \\
146 \\
146\end{array}$ & $\begin{array}{l}0.99961 \\
0.99961 \\
0.99961 \\
0.99961 \\
0.99961\end{array}$ \\
\hline $\begin{array}{l}0.0686 \\
0.0687 \\
0.0688 \\
0.0689 \\
0.0690\end{array}$ & $\begin{array}{l}2.71357 \\
2.71216 \\
2.71075 \\
2.70935 \\
2.70795\end{array}$ & $\begin{array}{l}141 \\
141 \\
140 \\
140 \\
140\end{array}$ & $\begin{array}{l}0.96609 \\
0.96604 \\
0.96599 \\
0.96595 \\
0.96590\end{array}$ & $\begin{array}{l}5 \\
5 \\
4 \\
5 \\
5\end{array}$ & $\begin{array}{l}3.67966 \\
3.67820 \\
3.67675 \\
3.67530 \\
3.67385\end{array}$ & $\begin{array}{l}146 \\
145 \\
145 \\
145 \\
145\end{array}$ & $\begin{array}{l}0.99961 \\
0.99961 \\
0.99961 \\
0.99960 \\
0.99960\end{array}$ \\
\hline $\begin{array}{l}0.0691 \\
0.0692 \\
0.0693 \\
0.0694 \\
0.0695\end{array}$ & $\begin{array}{l}2.70655 \\
2.70515 \\
2.70376 \\
2.70237 \\
2.70098\end{array}$ & $\begin{array}{l}140 \\
139 \\
139 \\
139 \\
139\end{array}$ & $\begin{array}{l}0.96585 \\
0.96580 \\
0.96575 \\
0.96570 \\
0.96565\end{array}$ & $\begin{array}{l}5 \\
5 \\
5 \\
5 \\
5\end{array}$ & $\begin{array}{l}3.67240 \\
3.67095 \\
3.66951 \\
3.66807 \\
3.66663\end{array}$ & $\begin{array}{l}145 \\
144 \\
144 \\
144 \\
144\end{array}$ & $\begin{array}{l}0.99960 \\
0.99960 \\
0.99960 \\
0.99960 \\
0.99960\end{array}$ \\
\hline $\begin{array}{l}0.0696 \\
0.0697 \\
0.0698 \\
0.0699 \\
0.0700\end{array}$ & $\begin{array}{l}2.69959 \\
2.69820 \\
2.69682 \\
2.69544 \\
2.69406\end{array}$ & $\begin{array}{l}139 \\
138 \\
138 \\
138\end{array}$ & $\begin{array}{l}0.96560 \\
0.96556 \\
0.96551 \\
0.96546 \\
0.96541\end{array}$ & $\begin{array}{l}4 \\
5 \\
5 \\
5\end{array}$ & $\begin{array}{l}3.66519 \\
3.66376 \\
3.66232 \\
3.66089 \\
3.65946\end{array}$ & $\begin{array}{l}143 \\
144 \\
143 \\
143\end{array}$ & $\begin{array}{l}0.99960 \\
0.99960 \\
0.99959 \\
0.99959 \\
0.99959\end{array}$ \\
\hline
\end{tabular}


Table I Harmonic Oscillator Contributions (in dimensionless form) to the Thermodynamic Functions for Values of $\mathrm{X}=\mathrm{hcv} / \mathrm{kT}$

\begin{tabular}{|c|c|c|c|c|c|c|c|}
\hline $\mathbf{x}$ & $-\left(F^{\circ}-E_{0}^{\circ}\right) / R T$ & $\Delta$ & $\left(\mathrm{H}^{\circ}-\mathrm{EO}\right) / \mathrm{RT}$ & $\Delta$ & $S^{\circ} / \mathrm{R}$ & $\Delta$ & $\mathrm{co} / \mathrm{R}$ \\
\hline 0.0700 & 2.69406 & 138 & 0.96541 & 5 & 3.65946 & 142 & 0.99959 \\
\hline $\begin{array}{l}0.0701 \\
0.0702 \\
0.0703 \\
0.0704 \\
0.0705\end{array}$ & $\begin{array}{l}2.69268 \\
2.69130 \\
2.68993 \\
2.68856 \\
2.68719\end{array}$ & $\begin{array}{l}138 \\
137 \\
137 \\
137 \\
137\end{array}$ & $\begin{array}{l}0.96536 \\
0.96531 \\
0.96526 \\
0.96521 \\
0.96516\end{array}$ & $\begin{array}{l}5 \\
5 \\
5 \\
5 \\
4\end{array}$ & $\begin{array}{l}3.65804 \\
3.65661 \\
3.65519 \\
3.65377 \\
3.65235\end{array}$ & $\begin{array}{l}143 \\
142 \\
142 \\
142 \\
142\end{array}$ & $\begin{array}{l}0.99959 \\
0.99959 \\
0.99959 \\
0.99959 \\
0.99959\end{array}$ \\
\hline $\begin{array}{l}0.0706 \\
0.0707 \\
0.0708 \\
0.0709 \\
0.0710\end{array}$ & $\begin{array}{l}2.68582 \\
2.68445 \\
2.68309 \\
2.68173 \\
2.68037\end{array}$ & $\begin{array}{l}137 \\
136 \\
136 \\
136 \\
136\end{array}$ & $\begin{array}{l}0.96512 \\
0.96507 \\
0.96502 \\
0.96497 \\
0.96492\end{array}$ & $\begin{array}{l}5 \\
5 \\
5 \\
5 \\
5\end{array}$ & $\begin{array}{l}3.65093 \\
3.64952 \\
3.64811 \\
3.64669 \\
3.64529\end{array}$ & $\begin{array}{l}141 \\
141 \\
142 \\
140 \\
141\end{array}$ & $\begin{array}{l}0.99959 \\
0.99958 \\
0.99958 \\
0.99958 \\
0.99958\end{array}$ \\
\hline $\begin{array}{l}0.0711 \\
0.0712 \\
0.0713 \\
0.0714 \\
0.0715\end{array}$ & $\begin{array}{l}2.67901 \\
2.67765 \\
2.67630 \\
2.67495 \\
2.67359\end{array}$ & $\begin{array}{l}136 \\
135 \\
135 \\
136 \\
134\end{array}$ & $\begin{array}{l}0.96487 \\
0.96482 \\
0.96477 \\
0.96472 \\
0.96468\end{array}$ & $\begin{array}{l}5 \\
5 \\
5 \\
4 \\
5\end{array}$ & $\begin{array}{l}3.64388 \\
3.64247 \\
3.64107 \\
3.63967 \\
3.63827\end{array}$ & $\begin{array}{l}141 \\
140 \\
140 \\
140 \\
140\end{array}$ & $\begin{array}{l}0.99958 \\
0.99958 \\
0.99958 \\
0.99958 \\
0.99957\end{array}$ \\
\hline $\begin{array}{l}0.0716 \\
0.0717 \\
0.0718 \\
0.0719 \\
0.0720\end{array}$ & $\begin{array}{l}2.67225 \\
2.67090 \\
2.66956 \\
2.66821 \\
2.66687\end{array}$ & $\begin{array}{l}135 \\
134 \\
135 \\
134 \\
134\end{array}$ & $\begin{array}{l}0.96463 \\
0.96458 \\
0.96453 \\
0.96448 \\
0.96443\end{array}$ & $\begin{array}{l}5 \\
5 \\
5 \\
5 \\
5\end{array}$ & $\begin{array}{l}3.63687 \\
3.63548 \\
3.63409 \\
3.63269 \\
3.63130\end{array}$ & $\begin{array}{l}139 \\
139 \\
140 \\
139 \\
138\end{array}$ & $\begin{array}{l}0.99957 \\
0.99957 \\
0.99957 \\
0.99957 \\
0.99957\end{array}$ \\
\hline $\begin{array}{l}0.0721 \\
0.0722 \\
0.0723 \\
0.0724 \\
0.0725\end{array}$ & $\begin{array}{l}2.66553 \\
2.66420 \\
2.66286 \\
2.66153 \\
2.66020\end{array}$ & $\begin{array}{l}133 \\
134 \\
133 \\
133 \\
133\end{array}$ & $\begin{array}{l}0.96438 \\
0.96433 \\
0.96429 \\
0.96424 \\
0.96419\end{array}$ & $\begin{array}{l}5 \\
4 \\
5 \\
5 \\
5\end{array}$ & $\begin{array}{l}3.62992 \\
3.62853 \\
3.62715 \\
3.62577 \\
3.62439\end{array}$ & $\begin{array}{l}139 \\
138 \\
138 \\
138 \\
138\end{array}$ & $\begin{array}{l}0.99957 \\
0.99957 \\
0.99956 \\
0.99956 \\
0.99956\end{array}$ \\
\hline $\begin{array}{l}0.0726 \\
0.0727 \\
0.0728 \\
0.0729 \\
0.0730\end{array}$ & $\begin{array}{l}2.65887 \\
2.65754 \\
2.65622 \\
2.65490 \\
2.65357\end{array}$ & $\begin{array}{l}133 \\
132 \\
132 \\
133 \\
132\end{array}$ & $\begin{array}{l}0.96414 \\
0.96409 \\
0.96404 \\
0.96399 \\
0.96394\end{array}$ & $\begin{array}{l}5 \\
5 \\
5 \\
5 \\
4\end{array}$ & $\begin{array}{l}3.52301 \\
3.62163 \\
3.62026 \\
3.61889 \\
3.61752\end{array}$ & $\begin{array}{l}138 \\
137 \\
137 \\
137 \\
137\end{array}$ & $\begin{array}{l}0.99956 \\
0.99956 \\
0.99956 \\
0.99956 \\
0.99956\end{array}$ \\
\hline $\begin{array}{l}0.0731 \\
0.0732 \\
0.0733 \\
0.0734 \\
0.0735\end{array}$ & $\begin{array}{l}2.65225 \\
2.65094 \\
2.64962 \\
2.64831 \\
2.64699\end{array}$ & $\begin{array}{l}131 \\
132 \\
131 \\
132 \\
131\end{array}$ & $\begin{array}{l}0.96390 \\
0.96385 \\
0.96380 \\
0.96375 \\
0.96370\end{array}$ & $\begin{array}{l}5 \\
5 \\
5 \\
5 \\
5\end{array}$ & $\begin{array}{l}3.61615 \\
3.61478 \\
3.61342 \\
3.61206 \\
3.61070\end{array}$ & $\begin{array}{l}137 \\
136 \\
136 \\
136 \\
136\end{array}$ & $\begin{array}{l}0.99956 \\
0.99955 \\
0.99955 \\
0.99955 \\
0.99955\end{array}$ \\
\hline $\begin{array}{l}0.0736 \\
0.0737 \\
0.0738 \\
0.0739 \\
0.0740\end{array}$ & $\begin{array}{l}2.64568 \\
2.64438 \\
2.64307 \\
2.64176 \\
2.64046\end{array}$ & $\begin{array}{l}130 \\
131 \\
131 \\
130 \\
130\end{array}$ & $\begin{array}{l}0.96365 \\
0.96360 \\
0.96355 \\
0.96351 \\
0.96346\end{array}$ & $\begin{array}{l}5 \\
5 \\
4 \\
5 \\
5\end{array}$ & $\begin{array}{l}3.60934 \\
3.60798 \\
3.60662 \\
3.60527 \\
3.60392\end{array}$ & $\begin{array}{l}136 \\
136 \\
135 \\
135 \\
135\end{array}$ & $\begin{array}{l}0.99955 \\
0.99955 \\
0.99955 \\
0.99955 \\
0.99954\end{array}$ \\
\hline $\begin{array}{l}0.0741 \\
0.0742 \\
0.0743 \\
0.0744 \\
0.0745\end{array}$ & $\begin{array}{l}2.63916 \\
2.63786 \\
2.63656 \\
2.63527 \\
2.63397\end{array}$ & $\begin{array}{l}130 \\
130 \\
129 \\
130 \\
129\end{array}$ & $\begin{array}{l}0.96341 \\
0.96336 \\
0.96331 \\
0.96326 \\
0.96321\end{array}$ & $\begin{array}{l}5 \\
5 \\
5 \\
5 \\
5\end{array}$ & $\begin{array}{l}3.60257 \\
3.60122 \\
3.59987 \\
3.59853 \\
3.59719\end{array}$ & $\begin{array}{l}135 \\
135 \\
134 \\
134 \\
134\end{array}$ & $\begin{array}{l}0.99954 \\
0.99954 \\
0.99954 \\
0.99954 \\
0.99954\end{array}$ \\
\hline $\begin{array}{l}0.0746 \\
0.0747 \\
0.0748 \\
0.0749 \\
0.0750\end{array}$ & $\begin{array}{l}2.63268 \\
2.63139 \\
2.63010 \\
2.62882 \\
2.62753\end{array}$ & $\begin{array}{l}129 \\
129 \\
128 \\
129\end{array}$ & $\begin{array}{l}0.96316 \\
0.96311 \\
0.96307 \\
0.96302 \\
0.96297\end{array}$ & $\begin{array}{l}5 \\
4 \\
5 \\
5\end{array}$ & $\begin{array}{l}3.59585 \\
3.59451 \\
3.59317 \\
3.59184 \\
3.59050\end{array}$ & $\begin{array}{l}134 \\
134 \\
133 \\
134\end{array}$ & $\begin{array}{l}0.99954 \\
0.99954 \\
0.99953 \\
0.99953 \\
0.99953\end{array}$ \\
\hline
\end{tabular}


Table I Harmonic Oscillator Contributions (in dimensionless form) to the Thermodynamic Functions for Values of $\mathrm{X}=\mathrm{hc} / \mathrm{kT}$

\begin{tabular}{|c|c|c|c|c|c|c|c|}
\hline $\mathbf{X}$ & $-\left(F^{\circ}-E_{0}^{\circ}\right) / R T$ & $\Delta$ & $\left(\mathrm{H}^{\circ}-\mathrm{E}_{\mathrm{O}}^{\circ}\right) / \mathrm{RT}$ & $\Delta$ & $S \% / R$ & $\Delta$ & $\mathrm{C} \circ / \mathrm{R}$ \\
\hline 0.0750 & 2.62753 & 128 & 0.96297 & 5 & 3.59050 & 133 & 0.99953 \\
\hline $\begin{array}{l}0.0751 \\
0.0752 \\
0.0753 \\
0.0754 \\
0.0755\end{array}$ & $\begin{array}{l}2.62625 \\
2.62497 \\
2.62369 \\
2.62241 \\
2.62114\end{array}$ & $\begin{array}{l}128 \\
128 \\
128 \\
127 \\
128\end{array}$ & $\begin{array}{l}0.96292 \\
0.96287 \\
0.96282 \\
0.96277 \\
0.96272\end{array}$ & $\begin{array}{l}5 \\
5 \\
5 \\
5 \\
4\end{array}$ & $\begin{array}{l}3.58917 \\
3.58784 \\
3.58651 \\
3.58518 \\
3.58386\end{array}$ & $\begin{array}{l}133 \\
133 \\
133 \\
132 \\
132\end{array}$ & $\begin{array}{l}0.99953 \\
0.99953 \\
0.99953 \\
0.99953 \\
0.99953\end{array}$ \\
\hline $\begin{array}{l}0.0756 \\
0.0757 \\
0.0758 \\
0.0759 \\
0.0760\end{array}$ & $\begin{array}{l}2.61986 \\
2.61859 \\
2.61732 \\
2.61605 \\
2.61478\end{array}$ & $\begin{array}{l}127 \\
127 \\
127 \\
127 \\
126\end{array}$ & $\begin{array}{l}0.96268 \\
0.96263 \\
0.96258 \\
0.96253 \\
0.96248\end{array}$ & $\begin{array}{l}5 \\
5 \\
5 \\
5 \\
5\end{array}$ & $\begin{array}{l}3.58254 \\
3.58122 \\
3.57990 \\
3.57858 \\
3.57726\end{array}$ & $\begin{array}{l}132 \\
132 \\
132 \\
132 \\
131\end{array}$ & $\begin{array}{l}0.99952 \\
0.99952 \\
0.99952 \\
0.99952 \\
0.99952\end{array}$ \\
\hline $\begin{array}{l}0.0761 \\
0.0762 \\
0.0763 \\
0.0764 \\
0.0765\end{array}$ & $\begin{array}{l}2.61352 \\
2.61225 \\
2.61099 \\
2.60973 \\
2.60847\end{array}$ & $\begin{array}{l}127 \\
126 \\
126 \\
126 \\
126\end{array}$ & $\begin{array}{l}0.96243 \\
0.96238 \\
0.96234 \\
0.96229 \\
0.96224\end{array}$ & $\begin{array}{l}5 \\
4 \\
5 \\
5 \\
5\end{array}$ & $\begin{array}{l}3.57595 \\
3.57464 \\
3.57332 \\
3.57202 \\
3.57071\end{array}$ & $\begin{array}{l}131 \\
132 \\
130 \\
131 \\
131\end{array}$ & $\begin{array}{l}0.99952 \\
0.99952 \\
0.99952 \\
0.99951 \\
0.99951\end{array}$ \\
\hline $\begin{array}{l}0.0766 \\
0.0767 \\
0.0768 \\
0.0769 \\
0.0770\end{array}$ & $\begin{array}{l}2.60721 \\
2.60596 \\
2.60470 \\
2.60345 \\
2.60220\end{array}$ & $\begin{array}{l}125 \\
126 \\
125 \\
125 \\
125\end{array}$ & $\begin{array}{l}0.96219 \\
0.96214 \\
0.96209 \\
0.96204 \\
0.96199\end{array}$ & $\begin{array}{l}5 \\
5 \\
5 \\
5 \\
4\end{array}$ & $\begin{array}{l}3.56940 \\
3.56810 \\
3.56680 \\
3.56550 \\
3.56420\end{array}$ & $\begin{array}{l}130 \\
130 \\
130 \\
130 \\
130\end{array}$ & $\begin{array}{l}0.99951 \\
0.99951 \\
0.99951 \\
0.99951 \\
0.99951\end{array}$ \\
\hline $\begin{array}{l}0.0771 \\
0.0772 \\
0.0773 \\
0.0774 \\
0.0775\end{array}$ & $\begin{array}{l}2.60095 \\
2.59971 \\
2.59846 \\
2.59722 \\
2.59598\end{array}$ & $\begin{array}{l}124 \\
125 \\
124 \\
124 \\
124\end{array}$ & $\begin{array}{l}0.96195 \\
0.96190 \\
0.96185 \\
0.96180 \\
0.96175\end{array}$ & $\begin{array}{l}5 \\
5 \\
5 \\
5 \\
5\end{array}$ & $\begin{array}{l}3.56290 \\
3.56160 \\
3.56031 \\
3.55902 \\
3.55773\end{array}$ & $\begin{array}{l}130 \\
129 \\
129 \\
129 \\
129\end{array}$ & $\begin{array}{l}0.99950 \\
0.99950 \\
0.99950 \\
0.99950 \\
0.99950\end{array}$ \\
\hline $\begin{array}{l}0.0776 \\
0.0777 \\
0.0778 \\
0.0779 \\
0.0780\end{array}$ & $\begin{array}{l}2.59474 \\
2.59350 \\
2.59226 \\
2.59103 \\
2.58979\end{array}$ & $\begin{array}{l}124 \\
124 \\
123 \\
124 \\
123\end{array}$ & $\begin{array}{l}0.96170 \\
0.96165 \\
0.96160 \\
0.96156 \\
0.96151\end{array}$ & $\begin{array}{l}5 \\
5 \\
4 \\
5 \\
5\end{array}$ & $\begin{array}{l}3.55644 \\
3.55515 \\
3.55387 \\
3.55258 \\
3.55130\end{array}$ & $\begin{array}{l}129 \\
128 \\
129 \\
128 \\
128\end{array}$ & $\begin{array}{l}0.99950 \\
0.99950 \\
0.99950 \\
0.99949 \\
0.99949\end{array}$ \\
\hline $\begin{array}{l}0.0781 \\
0.0782 \\
0.0783 \\
0.0784 \\
0.0785\end{array}$ & $\begin{array}{l}2.58856 \\
2.58733 \\
2.58610 \\
2.58488 \\
2.58365\end{array}$ & $\begin{array}{l}123 \\
123 \\
122 \\
123 \\
122\end{array}$ & $\begin{array}{l}0.96146 \\
0.96141 \\
0.96136 \\
0.96131 \\
0.96126\end{array}$ & $\begin{array}{l}5 \\
5 \\
5 \\
5 \\
5\end{array}$ & $\begin{array}{l}3.55002 \\
3.54874 \\
3.54746 \\
3.54619 \\
3.54491\end{array}$ & $\begin{array}{l}128 \\
128 \\
127 \\
128 \\
127\end{array}$ & $\begin{array}{l}0.99949 \\
0.99949 \\
0.99949 \\
0.99949 \\
0.99949\end{array}$ \\
\hline $\begin{array}{l}0.0786 \\
0.0787 \\
0.0788 \\
0.0789 \\
0.0790\end{array}$ & $\begin{array}{l}2.58243 \\
2.58120 \\
2.57998 \\
2.57876 \\
2.57755\end{array}$ & $\begin{array}{l}123 \\
122 \\
122 \\
121 \\
122\end{array}$ & $\begin{array}{l}0.96121 \\
0.96117 \\
0.96112 \\
0.96107 \\
0.96102\end{array}$ & $\begin{array}{l}4 \\
5 \\
5 \\
5 \\
5\end{array}$ & $\begin{array}{l}3.54364 \\
3.54237 \\
3.54110 \\
3.53983 \\
3.53857\end{array}$ & $\begin{array}{l}127 \\
127 \\
127 \\
126 \\
127\end{array}$ & $\begin{array}{l}0.99949 \\
0.99948 \\
0.99948 \\
0.99948 \\
0.99948\end{array}$ \\
\hline $\begin{array}{l}0.0791 \\
0.0792 \\
0.0793 \\
0.0794 \\
0.0795\end{array}$ & $\begin{array}{l}2.57633 \\
2.57512 \\
2.57391 \\
2.57269 \\
2.57148\end{array}$ & $\begin{array}{l}121 \\
121 \\
122 \\
121 \\
120\end{array}$ & $\begin{array}{l}0.96097 \\
0.96092 \\
0.96087 \\
0.96083 \\
0.96078\end{array}$ & $\begin{array}{l}5 \\
5 \\
4 \\
5 \\
5\end{array}$ & $\begin{array}{l}3.53730 \\
3.53604 \\
3.53478 \\
3.53352 \\
3.53226\end{array}$ & $\begin{array}{l}126 \\
126 \\
126 \\
126 \\
125\end{array}$ & $\begin{array}{l}0.99948 \\
0.99948 \\
0.99948 \\
0.99948 \\
0.99947\end{array}$ \\
\hline $\begin{array}{l}0.0796 \\
0.0797 \\
0.0798 \\
0.0799 \\
0.0800\end{array}$ & $\begin{array}{l}2.57028 \\
2.56907 \\
2.56787 \\
2.56666 \\
2.56546\end{array}$ & $\begin{array}{l}121 \\
120 \\
121 \\
120\end{array}$ & $\begin{array}{l}0.96073 \\
0.96068 \\
0.96063 \\
0.96058 \\
0.96053\end{array}$ & $\begin{array}{l}5 \\
5 \\
5 \\
5\end{array}$ & $\begin{array}{l}3.53101 \\
3.52975 \\
3.52850 \\
3.52725 \\
3.52600\end{array}$ & $\begin{array}{l}126 \\
125 \\
125 \\
125\end{array}$ & $\begin{array}{l}0.99947 \\
0.99947 \\
0.99947 \\
0.99947 \\
0.99947\end{array}$ \\
\hline
\end{tabular}


Table I Harmonic Oscillator Contributions (in dimensionless form) to the Thermodynamic Functions for Values of $X=h c v / k T$

\begin{tabular}{|c|c|c|c|c|c|c|c|}
\hline $\mathbf{X}$ & $-\left(F^{\circ}-E_{O}^{\circ}\right) / R T$ & $\Delta$ & $\left(\mathrm{H}^{\circ}-\mathrm{E}_{\mathrm{O}}^{\circ}\right) / \mathrm{RT}$ & $\Delta$ & $\mathrm{S} \% / \mathrm{R}$ & $\Delta$ & $\mathrm{C}_{\mathrm{p}}^{\circ} / \mathrm{R}$ \\
\hline 0.0800 & 2.56546 & 120 & 0.96053 & 5 & 3.52600 & 125 & 0.99947 \\
\hline $\begin{array}{l}0.0801 \\
0.0802 \\
0.0803\end{array}$ & $\begin{array}{l}2.56426 \\
2.56306 \\
2.56187\end{array}$ & $\begin{array}{l}120 \\
119 \\
120\end{array}$ & $\begin{array}{l}0.96048 \\
0.96044 \\
0.96039\end{array}$ & $\begin{array}{l}4 \\
5 \\
5\end{array}$ & $\begin{array}{l}3.52475 \\
3.52350 \\
3.52225\end{array}$ & $\begin{array}{l}125 \\
125 \\
124\end{array}$ & $\begin{array}{l}0.99947 \\
0.99946 \\
0.99946\end{array}$ \\
\hline $\begin{array}{l}0.0804 \\
0.0805\end{array}$ & $\begin{array}{l}2.56067 \\
2.55948\end{array}$ & $\begin{array}{l}119 \\
119\end{array}$ & $\begin{array}{l}0.96034 \\
0.96029\end{array}$ & $\begin{array}{l}5 \\
5\end{array}$ & $\begin{array}{l}3.52101 \\
3.51977\end{array}$ & $\begin{array}{l}124 \\
124\end{array}$ & $\begin{array}{l}0.99946 \\
0.99946\end{array}$ \\
\hline $\begin{array}{l}0.0806 \\
0.0807 \\
0.0808 \\
0.0809 \\
0.0810\end{array}$ & $\begin{array}{l}2.55829 \\
2.55710 \\
2.55591 \\
2.55472 \\
2.55353\end{array}$ & $\begin{array}{l}119 \\
119 \\
119 \\
119 \\
118\end{array}$ & $\begin{array}{l}0.96024 \\
0.96019 \\
0.96014 \\
0.96010 \\
0.96005\end{array}$ & $\begin{array}{l}5 \\
5 \\
4 \\
5 \\
5\end{array}$ & $\begin{array}{l}3.51853 \\
3.51729 \\
3.51605 \\
3.51481 \\
3.51358\end{array}$ & $\begin{array}{l}124 \\
124 \\
124 \\
123 \\
123\end{array}$ & $\begin{array}{l}0.99946 \\
0.99946 \\
0.99946 \\
0.99945 \\
0.99945\end{array}$ \\
\hline $\begin{array}{l}0.0811 \\
0.0812 \\
0.0813 \\
0.0814 \\
0.0815\end{array}$ & $\begin{array}{l}2.55235 \\
2.55117 \\
2.54998 \\
2.54880 \\
2.54763\end{array}$ & $\begin{array}{l}118 \\
119 \\
118 \\
117 \\
118\end{array}$ & $\begin{array}{l}0.96000 \\
0.95995 \\
0.95990 \\
0.95985 \\
0.95980\end{array}$ & $\begin{array}{l}5 \\
5 \\
5 \\
5 \\
5\end{array}$ & $\begin{array}{l}3.51235 \\
3.51111 \\
3.50988 \\
3.50806 \\
3.50743\end{array}$ & $\begin{array}{l}124 \\
123 \\
122 \\
123 \\
123\end{array}$ & $\begin{array}{l}0.99945 \\
0.99945 \\
0.99945 \\
0.99945 \\
0.99945\end{array}$ \\
\hline $\begin{array}{l}0.0816 \\
0.0817 \\
0.0818 \\
0.0819 \\
0.0820\end{array}$ & $\begin{array}{l}2.54645 \\
2.54527 \\
2.54410 \\
2.54293 \\
2.54176\end{array}$ & $\begin{array}{l}118 \\
117 \\
117 \\
117 \\
117\end{array}$ & $\begin{array}{l}0.95975 \\
0.95971 \\
0.95966 \\
0.95961 \\
0.95956\end{array}$ & $\begin{array}{l}4 \\
5 \\
5 \\
5 \\
5\end{array}$ & $\begin{array}{l}3.50620 \\
3.50498 \\
3.50376 \\
3.50254 \\
3.50132\end{array}$ & $\begin{array}{l}122 \\
122 \\
122 \\
122 \\
122\end{array}$ & $\begin{array}{l}0.99945 \\
0.99944 \\
0.99944 \\
0.99944 \\
0.99944\end{array}$ \\
\hline $\begin{array}{l}0.0821 \\
0.0822 \\
0.0823 \\
0.0824 \\
0.0825\end{array}$ & $\begin{array}{l}2.54059 \\
2.53942 \\
2.53825 \\
2.53709 \\
2.53592\end{array}$ & $\begin{array}{l}117 \\
117 \\
116 \\
117 \\
116\end{array}$ & $\begin{array}{l}0.95951 \\
0.95946 \\
0.95941 \\
0.95937 \\
0.95932\end{array}$ & $\begin{array}{l}5 \\
5 \\
4 \\
5 \\
5\end{array}$ & $\begin{array}{l}3.50010 \\
3.49888 \\
3.49767 \\
3.49645 \\
3.49524\end{array}$ & $\begin{array}{l}122 \\
121 \\
122 \\
121 \\
121\end{array}$ & $\begin{array}{l}0.99944 \\
0.99944 \\
0.99944 \\
0.99943 \\
0.99943\end{array}$ \\
\hline $\begin{array}{l}0.0826 \\
0.0827 \\
0.0828 \\
0.0329 \\
0.0830\end{array}$ & $\begin{array}{l}2.53476 \\
2.53360 \\
2.53244 \\
2.53128 \\
2.53013\end{array}$ & $\begin{array}{l}116 \\
116 \\
116 \\
115 \\
116\end{array}$ & $\begin{array}{l}0.95927 \\
0.95922 \\
0.95917 \\
0.95912 \\
0.95907\end{array}$ & $\begin{array}{l}5 \\
5 \\
5 \\
5 \\
4\end{array}$ & $\begin{array}{l}3.49403 \\
3.49282 \\
3.49161 \\
3.49041 \\
3.48920\end{array}$ & $\begin{array}{l}121 \\
121 \\
120 \\
121 \\
120\end{array}$ & $\begin{array}{l}0.99943 \\
0.99943 \\
0.9 .9943 \\
0.99943 \\
0.99943\end{array}$ \\
\hline $\begin{array}{l}0.0831 \\
0.0832 \\
0.0833 \\
0.0834 \\
0.0835\end{array}$ & $\begin{array}{l}2.52897 \\
2.52782 \\
2.52667 \\
2.52552 \\
2.52437\end{array}$ & $\begin{array}{l}115 \\
115 \\
115 \\
115 \\
115\end{array}$ & $\begin{array}{l}0.95903 \\
0.95898 \\
0.95893 \\
0.95888 \\
0.95883\end{array}$ & $\begin{array}{l}5 \\
5 \\
5 \\
5 \\
5\end{array}$ & $\begin{array}{l}3.48800 \\
3.48680 \\
3.48560 \\
3.48440 \\
3.48320\end{array}$ & $\begin{array}{l}120 \\
120 \\
120 \\
120 \\
120\end{array}$ & $\begin{array}{l}0.99942 \\
0.99942 \\
0.99942 \\
0.99942 \\
0.99942\end{array}$ \\
\hline $\begin{array}{l}0.0836 \\
0.0837 \\
0.0838 \\
0.0839 \\
0.0840\end{array}$ & $\begin{array}{l}2.52322 \\
2.52207 \\
2.52093 \\
2.51979 \\
2.51864\end{array}$ & $\begin{array}{l}115 \\
114 \\
114 \\
115 \\
114\end{array}$ & $\begin{array}{l}0.95878 \\
0.95873 \\
0.95869 \\
0.95864 \\
0.95859\end{array}$ & $\begin{array}{l}5 \\
4 \\
5 \\
5 \\
5\end{array}$ & $\begin{array}{l}3.48200 \\
3.48081 \\
3.47961 \\
3.47842 \\
3.47723\end{array}$ & $\begin{array}{l}119 \\
120 \\
119 \\
119 \\
119\end{array}$ & $\begin{array}{l}0.99942 \\
0.99942 \\
0.99942 \\
0.99941 \\
0.99941\end{array}$ \\
\hline $\begin{array}{l}0.0841 \\
0.0842 \\
0.0843 \\
0.0844 \\
0.0845\end{array}$ & $\begin{array}{l}2.51750 \\
2.51636 \\
2.51523 \\
2.51409 \\
2.51296\end{array}$ & $\begin{array}{l}114 \\
113 \\
114 \\
113 \\
114\end{array}$ & $\begin{array}{l}0.95854 \\
0.95849 \\
0.95844 \\
0.95839 \\
0.95834\end{array}$ & $\begin{array}{l}5 \\
5 \\
5 \\
5 \\
4\end{array}$ & $\begin{array}{l}3.47604 \\
3.47486 \\
3.47367 \\
3.47248 \\
3.47130\end{array}$ & $\begin{array}{l}118 \\
119 \\
119 \\
118 \\
118\end{array}$ & $\begin{array}{l}0.99941 \\
0.99941 \\
0.99941 \\
0.99941 \\
0.99941\end{array}$ \\
\hline $\begin{array}{l}0.0846 \\
0.0847 \\
0.0848 \\
0.0849 \\
0.0850\end{array}$ & $\begin{array}{l}2.51182 \\
2.51069 \\
2.50956 \\
2.50843 \\
2.50730\end{array}$ & $\begin{array}{l}113 \\
113 \\
113 \\
113\end{array}$ & $\begin{array}{l}0.95830 \\
0.95825 \\
0.95820 \\
0.95815 \\
0.95810\end{array}$ & $\begin{array}{l}5 \\
5 \\
5 \\
5\end{array}$ & $\begin{array}{l}3.47012 \\
3.46894 \\
3.46776 \\
3.46658 \\
3.46541\end{array}$ & $\begin{array}{l}118 \\
118 \\
118 \\
117\end{array}$ & $\begin{array}{l}0.99940 \\
0.99940 \\
0.99940 \\
0.99940 \\
0.99940\end{array}$ \\
\hline
\end{tabular}


Table I Harmonic Oscillator Contributions (in dimensionless form) to the Thermodynamic Functions for Values of $\mathrm{X}=\mathrm{hcv} / \mathrm{kT}$

\begin{tabular}{|c|c|c|c|c|c|c|c|}
\hline 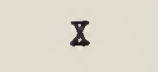 & $-\left(F^{\circ}-E_{O}^{\circ}\right) / R T$ & $\Delta$ & $\left(\mathrm{H}^{\circ}-\mathrm{E}_{\mathrm{O}}^{\circ}\right) / \mathrm{RT}$ & $\Delta$ & $\mathrm{S} / \mathrm{R}$ & $\Delta$ & $\mathrm{Co}_{\mathrm{p}} / \mathrm{R}$ \\
\hline 0.0850 & 2.50730 & 112 & 0.95810 & 5 & 3.46541 & 118 & 0.99940 \\
\hline $\begin{array}{l}0.0851 \\
0.0852 \\
0.0853 \\
0.0854 \\
0.0855\end{array}$ & $\begin{array}{l}2.50618 \\
2.50505 \\
2.50393 \\
2.50281 \\
2.50168\end{array}$ & $\begin{array}{l}1113 \\
1122 \\
1122 \\
1113 \\
112\end{array}$ & $\begin{array}{l}0.95805 \\
0.95800 \\
0.95796 \\
0.95791 \\
0.95786\end{array}$ & $\begin{array}{l}5 \\
4 \\
5 \\
5 \\
5\end{array}$ & $\begin{array}{l}3.46423 \\
3.46306 \\
3.46188 \\
3.46071 \\
3.45954\end{array}$ & $\begin{array}{l}117 \\
118 \\
117 \\
117 \\
116\end{array}$ & $\begin{array}{l}0.99940 \\
0.99940 \\
0.99939 \\
0.99939 \\
0.99939\end{array}$ \\
\hline $\begin{array}{l}0.0856 \\
0.0857 \\
0.0858 \\
0.0859 \\
0.0860\end{array}$ & $\begin{array}{l}2.50056 \\
2.49945 \\
2.49833 \\
2.49721 \\
2.49610\end{array}$ & $\begin{array}{lll}1 & 1 & 1 \\
1 & 1 & 2 \\
1 & 1 & 2 \\
1 & 1 & 1 \\
1 & 1 & 1\end{array}$ & $\begin{array}{l}0.95781 \\
0.95776 \\
0.95771 \\
0.95766 \\
0.95762\end{array}$ & $\begin{array}{l}5 \\
5 \\
5 \\
4 \\
5\end{array}$ & $\begin{array}{l}3.45838 \\
3.45721 \\
3.45604 \\
3.45488 \\
3.45372\end{array}$ & $\begin{array}{l}117 \\
117 \\
116 \\
116 \\
117\end{array}$ & $\begin{array}{l}0.99939 \\
0.99939 \\
0.99939 \\
0.99939 \\
0.99938\end{array}$ \\
\hline $\begin{array}{l}0.0861 \\
0.0862 \\
0.0863 \\
0.0864 \\
0.0865\end{array}$ & $\begin{array}{l}2.49499 \\
2.49388 \\
2.49277 \\
2.49166 \\
2.49055\end{array}$ & $\begin{array}{lll}1 & 1 & 1 \\
1 & 1 & 1 \\
1 & 1 & 1 \\
1 & 1 & 1 \\
1 & 1 & 1\end{array}$ & $\begin{array}{l}0.95757 \\
0.95752 \\
0.95747 \\
0.95742 \\
0.95737\end{array}$ & $\begin{array}{l}5 \\
5 \\
5 \\
5 \\
5\end{array}$ & $\begin{array}{l}3.45255 \\
3.45139 \\
3.45024 \\
3.44908 \\
3.44792\end{array}$ & $\begin{array}{l}116 \\
115 \\
116 \\
116 \\
115\end{array}$ & $\begin{array}{l}0.99938 \\
0.99938 \\
0.99938 \\
0.99938 \\
0.99938\end{array}$ \\
\hline $\begin{array}{l}0.0866 \\
0.0867 \\
0.0868 \\
0.0869 \\
0.0870\end{array}$ & $\begin{array}{l}2.48944 \\
2.48834 \\
2.48723 \\
2.48613 \\
2.48503\end{array}$ & $\begin{array}{l}110 \\
111 \\
110 \\
110 \\
110\end{array}$ & $\begin{array}{l}0.95732 \\
0.95728 \\
0.95723 \\
0.95718 \\
0.95713\end{array}$ & $\begin{array}{l}4 \\
5 \\
5 \\
5 \\
5\end{array}$ & $\begin{array}{l}3.44677 \\
3.44561 \\
3.44446 \\
3.44331 \\
3.44216\end{array}$ & $\begin{array}{l}116 \\
115 \\
115 \\
115 \\
115\end{array}$ & $\begin{array}{l}0.99938 \\
0.99937 \\
0.99937 \\
0.99937 \\
0.99937\end{array}$ \\
\hline $\begin{array}{l}0.0871 \\
0.0872 \\
0.0873 \\
0.0874 \\
0.0875\end{array}$ & $\begin{array}{l}2.48393 \\
2.48283 \\
2.48174 \\
2.48064 \\
2.47955\end{array}$ & $\begin{array}{l}110 \\
109 \\
110 \\
109 \\
110\end{array}$ & $\begin{array}{l}0.95708 \\
0.95703 \\
0.95699 \\
0.95694 \\
0.95689\end{array}$ & $\begin{array}{l}5 \\
4 \\
5 \\
5 \\
5\end{array}$ & $\begin{array}{l}3.44101 \\
3.43987 \\
3.43872 \\
3.43758 \\
3.43644\end{array}$ & $\begin{array}{l}114 \\
115 \\
114 \\
114 \\
115\end{array}$ & $\begin{array}{l}0.99937 \\
0.99937 \\
0.99937 \\
0.99936 \\
0.99936\end{array}$ \\
\hline $\begin{array}{l}0.0876 \\
0.0877 \\
0.0878 \\
0.0879 \\
0.0880\end{array}$ & $\begin{array}{l}2.47845 \\
2.47736 \\
2.47627 \\
2.47518 \\
2.47410\end{array}$ & $\begin{array}{l}109 \\
109 \\
109 \\
108 \\
109\end{array}$ & $\begin{array}{l}0.95684 \\
0.95679 \\
0.95674 \\
0.95669 \\
0.95665\end{array}$ & $\begin{array}{l}5 \\
5 \\
5 \\
4 \\
5\end{array}$ & $\begin{array}{l}3.43529 \\
3.43415 \\
3.43301 \\
3.43188 \\
3.43074\end{array}$ & $\begin{array}{l}114 \\
114 \\
113 \\
114 \\
113\end{array}$ & $\begin{array}{l}0.99936 \\
0.99936 \\
0.99936 \\
0.99936 \\
0.99935\end{array}$ \\
\hline $\begin{array}{l}0.0881 \\
0.0882 \\
0.0883 \\
0.0884 \\
0.0885\end{array}$ & $\begin{array}{l}2.47301 \\
2.47192 \\
2.47084 \\
2.46976 \\
2.46868\end{array}$ & $\begin{array}{l}109 \\
108 \\
108 \\
108 \\
108\end{array}$ & $\begin{array}{l}0.95660 \\
0.95655 \\
0.95650 \\
0.95645 \\
0.95640\end{array}$ & $\begin{array}{l}5 \\
5 \\
5 \\
5 \\
5\end{array}$ & $\begin{array}{l}3.42961 \\
3.42847 \\
3.42734 \\
3.42621 \\
3.42508\end{array}$ & $\begin{array}{l}114 \\
113 \\
113 \\
113 \\
113\end{array}$ & $\begin{array}{l}0.99935 \\
0.99935 \\
0.99935 \\
0.99935 \\
0.99935\end{array}$ \\
\hline $\begin{array}{l}0.0886 \\
0.0887 \\
0.0888 \\
0.0989 \\
0.0890\end{array}$ & $\begin{array}{l}2.46760 \\
2.46652 \\
2.46544 \\
2.46436 \\
2.46329\end{array}$ & $\begin{array}{l}108 \\
108 \\
108 \\
107 \\
107\end{array}$ & $\begin{array}{l}0.95635 \\
0.95631 \\
0.95626 \\
0.95621 \\
0.95616\end{array}$ & $\begin{array}{l}4 \\
5 \\
5 \\
5 \\
5\end{array}$ & $\begin{array}{l}3.42395 \\
3.42282 \\
3.42170 \\
3.42057 \\
3.41945\end{array}$ & $\begin{array}{l}113 \\
112 \\
113 \\
112 \\
112\end{array}$ & $\begin{array}{l}0.99935 \\
0.99934 \\
0.99934 \\
0.99934 \\
0.99934\end{array}$ \\
\hline $\begin{array}{l}0.0891 \\
0.0892\end{array}$ & $\begin{array}{l}2.46222 \\
2.46114\end{array}$ & $\begin{array}{l}108 \\
107\end{array}$ & $\begin{array}{l}0.95611 \\
0.95606\end{array}$ & $\begin{array}{l}5 \\
5\end{array}$ & $\begin{array}{l}3.41833 \\
3.41721\end{array}$ & $\begin{array}{l}112 \\
112\end{array}$ & $\begin{array}{l}0.99934 \\
0.99934\end{array}$ \\
\hline $\begin{array}{l}0.0893 \\
0.0894 \\
0.0895\end{array}$ & $\begin{array}{l}2.46007 \\
2.45900 \\
2.45793\end{array}$ & $\begin{array}{l}107 \\
107 \\
106\end{array}$ & $\begin{array}{l}0.95601 \\
0.95597 \\
0.95592\end{array}$ & $\begin{array}{l}4 \\
5 \\
5\end{array}$ & $\begin{array}{l}3.41609 \\
3.41497 \\
3.41385\end{array}$ & $\begin{array}{l}112 \\
112 \\
112\end{array}$ & $\begin{array}{l}0.99934 \\
0.99933 \\
0.99933\end{array}$ \\
\hline $\begin{array}{l}0.0896 \\
0.0897 \\
0.0898 \\
0.0899 \\
0.0900\end{array}$ & $\begin{array}{l}2.45687 \\
2.45580 \\
2.45473 \\
2.45367 \\
2.45261\end{array}$ & $\begin{array}{l}107 \\
107 \\
106 \\
106\end{array}$ & $\begin{array}{l}0.95587 \\
0.95582 \\
0.95577 \\
0.95572 \\
0.95568\end{array}$ & $\begin{array}{l}5 \\
5 \\
5 \\
4\end{array}$ & $\begin{array}{l}3.41273 \\
3.41162 \\
3.41051 \\
3.40939 \\
3.40828\end{array}$ & $\begin{array}{l}111 \\
111 \\
112 \\
111\end{array}$ & $\begin{array}{l}0.99933 \\
0.99933 \\
0.99933 \\
0.99933 \\
0.99933\end{array}$ \\
\hline
\end{tabular}


Table I Harmonic Oscillator Contributions (in dimensionless form) to the Thermodynamic Functions for Values of $X=h c v / k T$

\begin{tabular}{|c|c|c|c|c|c|c|c|}
\hline$X$ & $-\left(F^{\circ}-E_{O}^{\circ}\right) / R T$ & $\Delta$ & $\left(\mathrm{H}^{\circ}-\mathrm{E}_{\mathrm{O}}^{\circ}\right) / \mathrm{RT}$ & $\Delta$ & So/R & $\Delta$ & $\mathrm{CO}_{\mathrm{p}} / \mathrm{R}$ \\
\hline 0.0900 & 2.45261 & 106 & 0.95568 & 5 & 3.40828 & 111 & 0.99933 \\
\hline $\begin{array}{l}0.0901 \\
0.0902 \\
0.0903\end{array}$ & $\begin{array}{l}2.45155 \\
2.45049 \\
2.44943\end{array}$ & $\begin{array}{l}106 \\
106 \\
106\end{array}$ & $\begin{array}{l}0.95563 \\
0.95558 \\
0.95553\end{array}$ & $\begin{array}{l}5 \\
5 \\
5\end{array}$ & $\begin{array}{l}3.40717 \\
3.40606 \\
3.40436\end{array}$ & $\begin{array}{lll}1 & 1 & 1 \\
1 & 1 & 0 \\
1 & 1 & 1\end{array}$ & $\begin{array}{l}0.99932 \\
0.99932 \\
0.99932\end{array}$ \\
\hline $\begin{array}{l}0.0904 \\
0.0905\end{array}$ & $\begin{array}{l}2.44837 \\
2.44731\end{array}$ & $\begin{array}{l}106 \\
105\end{array}$ & $\begin{array}{l}0.95548 \\
0.95543\end{array}$ & $\begin{array}{l}5 \\
5\end{array}$ & $\begin{array}{l}3.40385 \\
3.40275\end{array}$ & $\begin{array}{l}110 \\
111\end{array}$ & $\begin{array}{l}0.99932 \\
0.99932\end{array}$ \\
\hline $\begin{array}{l}0.0906 \\
0.0907 \\
0.0908 \\
0.0909 \\
0.0910\end{array}$ & $\begin{array}{l}2.44626 \\
2.44521 \\
2.44415 \\
2.44310 \\
2.44205\end{array}$ & $\begin{array}{l}105 \\
106 \\
105 \\
105 \\
105\end{array}$ & $\begin{array}{l}0.95538 \\
0.95534 \\
0.95529 \\
0.95524 \\
0.95519\end{array}$ & $\begin{array}{l}4 \\
5 \\
5 \\
5 \\
5\end{array}$ & $\begin{array}{l}3.40164 \\
3.40054 \\
3.39944 \\
3.39834 \\
3.39724\end{array}$ & $\begin{array}{l}110 \\
110 \\
110 \\
110 \\
110\end{array}$ & $\begin{array}{l}0.99932 \\
0.99932 \\
0.99931 \\
0.99931 \\
0.99931\end{array}$ \\
\hline $\begin{array}{l}0.0911 \\
0.0912 \\
0.0913 \\
0.0914 \\
0.0915\end{array}$ & $\begin{array}{l}2.44100 \\
2.43995 \\
2.43891 \\
2.43786 \\
2.43682\end{array}$ & $\begin{array}{l}105 \\
104 \\
105 \\
104 \\
105\end{array}$ & $\begin{array}{l}0.95514 \\
0.95509 \\
0.95504 \\
0.95500 \\
0.95495\end{array}$ & $\begin{array}{l}5 \\
5 \\
4 \\
5 \\
5\end{array}$ & $\begin{array}{l}3.39614 \\
3.39505 \\
3.39395 \\
3.39286 \\
3.39177\end{array}$ & $\begin{array}{l}109 \\
110 \\
109 \\
109 \\
110\end{array}$ & $\begin{array}{l}0.99931 \\
0.99931 \\
0.99931 \\
0.99930 \\
0.99930\end{array}$ \\
\hline $\begin{array}{l}0.0916 \\
0.0917 \\
0.0918 \\
0.0919 \\
0.0920\end{array}$ & $\begin{array}{l}2.43577 \\
2.43473 \\
2.43369 \\
2.43265 \\
2.43161\end{array}$ & $\begin{array}{l}104 \\
104 \\
104 \\
104 \\
103\end{array}$ & $\begin{array}{l}0.95490 \\
0.95485 \\
0.95480 \\
0.95475 \\
0.95471\end{array}$ & $\begin{array}{l}5 \\
5 \\
5 \\
4 \\
5\end{array}$ & $\begin{array}{l}3.39067 \\
3.38958 \\
3.38849 \\
3.38741 \\
3.38632\end{array}$ & $\begin{array}{l}109 \\
109 \\
108 \\
109 \\
109\end{array}$ & $\begin{array}{l}0.99930 \\
0.99930 \\
0.99930 \\
0.99930 \\
0.99930\end{array}$ \\
\hline $\begin{array}{l}0.0921 \\
0.0922 \\
0.0923 \\
0.0924 \\
0.0925\end{array}$ & $\begin{array}{l}2.43058 \\
2.42954 \\
2.42851 \\
2.42747 \\
2.42644\end{array}$ & $\begin{array}{l}104 \\
103 \\
104 \\
103 \\
103\end{array}$ & $\begin{array}{l}0.95466 \\
0.95461 \\
0.95456 \\
0.95451 \\
0.95446\end{array}$ & $\begin{array}{l}5 \\
5 \\
5 \\
5 \\
5\end{array}$ & $\begin{array}{l}3.38523 \\
3.38415 \\
3.38307 \\
3.38198 \\
3.38090\end{array}$ & $\begin{array}{l}108 \\
108 \\
109 \\
108 \\
108\end{array}$ & $\begin{array}{l}0.99929 \\
0.99929 \\
0.99929 \\
0.99929 \\
0.99929\end{array}$ \\
\hline $\begin{array}{l}0.0926 \\
0.0927 \\
0.0928 \\
0.0929 \\
0.0930\end{array}$ & $\begin{array}{l}2.42541 \\
2.42438 \\
2.42335 \\
2.42232 \\
2.42130\end{array}$ & $\begin{array}{l}103 \\
103 \\
103 \\
102 \\
103\end{array}$ & $\begin{array}{l}0.95441 \\
0.95437 \\
0.95432 \\
0.95427 \\
0.95422\end{array}$ & $\begin{array}{l}4 \\
5 \\
5 \\
5 \\
5\end{array}$ & $\begin{array}{l}3.37982 \\
3.37875 \\
3.37767 \\
3.37659 \\
3.37552\end{array}$ & $\begin{array}{l}108 \\
107 \\
108 \\
107 \\
108\end{array}$ & $\begin{array}{l}0.99929 \\
0.99928 \\
0.99928 \\
0.99928 \\
0.99928\end{array}$ \\
\hline $\begin{array}{l}0.0931 \\
0.0932\end{array}$ & $\begin{array}{l}2.42027 \\
2.41925\end{array}$ & $\begin{array}{l}102 \\
103\end{array}$ & $\begin{array}{l}0.95417 \\
0.95412\end{array}$ & $\begin{array}{l}5 \\
4\end{array}$ & $\begin{array}{l}3.37444 \\
3.37337\end{array}$ & $\begin{array}{l}107 \\
107\end{array}$ & $\begin{array}{l}0.99928 \\
0.99928\end{array}$ \\
\hline $\begin{array}{l}0.0933 \\
0.0934 \\
0.0935\end{array}$ & $\begin{array}{l}2.41822 \\
2.41720 \\
2.41618\end{array}$ & $\begin{array}{l}102 \\
102 \\
102\end{array}$ & $\begin{array}{l}0.95408 \\
0.95403 \\
0.95398\end{array}$ & $\begin{array}{l}5 \\
5 \\
5\end{array}$ & $\begin{array}{l}3.37230 \\
3.37123 \\
3.37016\end{array}$ & $\begin{array}{l}107 \\
107 \\
107\end{array}$ & $\begin{array}{l}0.99927 \\
0.99927 \\
0.99927\end{array}$ \\
\hline $\begin{array}{l}0.0936 \\
0.0937 \\
0.0938 \\
0.0939 \\
0.0940\end{array}$ & $\begin{array}{l}2.41516 \\
2.41414 \\
2.41312 \\
2.41211 \\
2.41109\end{array}$ & $\begin{array}{l}102 \\
102 \\
101 \\
102 \\
101\end{array}$ & $\begin{array}{l}0.95393 \\
0.95388 \\
0.95383 \\
0.95378 \\
0.95374\end{array}$ & $\begin{array}{l}5 \\
5 \\
5 \\
4 \\
5\end{array}$ & $\begin{array}{l}3.36909 \\
3.36802 \\
3.36696 \\
3.36589 \\
3.36483\end{array}$ & $\begin{array}{l}107 \\
106 \\
107 \\
106 \\
106\end{array}$ & $\begin{array}{l}0.99927 \\
0.99927 \\
0.99927 \\
0.99927 \\
0.99926\end{array}$ \\
\hline $\begin{array}{l}0.0941 \\
0.0942 \\
0.0943 \\
0.0944 \\
0.0945\end{array}$ & $\begin{array}{l}2.41008 \\
2.40907 \\
2.40805 \\
2.40704 \\
2.40603\end{array}$ & $\begin{array}{l}101 \\
102 \\
101 \\
101 \\
101\end{array}$ & $\begin{array}{l}0.95369 \\
0.95364 \\
0.95359 \\
0.95354 \\
0.95349\end{array}$ & $\begin{array}{l}5 \\
5 \\
5 \\
5 \\
4\end{array}$ & $\begin{array}{l}3.36377 \\
3.36270 \\
3.36164 \\
3.36059 \\
3.35953\end{array}$ & $\begin{array}{l}107 \\
106 \\
105 \\
106 \\
106\end{array}$ & $\begin{array}{l}0.99926 \\
0.99926 \\
0.99926 \\
0.99926 \\
0.99926\end{array}$ \\
\hline $\begin{array}{l}0.0946 \\
0.0947 \\
0.0948 \\
0.0349 \\
0.0950\end{array}$ & $\begin{array}{l}2.40502 \\
2.40402 \\
2.40301 \\
2.40201 \\
2.40100\end{array}$ & $\begin{array}{l}100 \\
101 \\
100 \\
101\end{array}$ & $\begin{array}{l}0.95345 \\
0.95340 \\
0.95335 \\
0.95330 \\
0.95325\end{array}$ & $\begin{array}{l}5 \\
5 \\
5 \\
5\end{array}$ & $\begin{array}{l}3.35847 \\
3.35741 \\
3.35636 \\
3.35531 \\
3.35425\end{array}$ & $\begin{array}{l}106 \\
105 \\
105 \\
106\end{array}$ & $\begin{array}{l}0.99925 \\
0.99925 \\
0.99925 \\
0.99925 \\
0.99925\end{array}$ \\
\hline
\end{tabular}


Table I Harmonic Oscillator Contributions (in dimensionless form) to the Thermodynamic Functions for Values of $\mathrm{X}=\mathrm{hcv} / \mathrm{kT}$

\begin{tabular}{|c|c|c|c|c|c|c|c|}
\hline 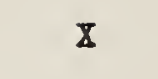 & $-\left(F^{\circ}-E_{0}^{O}\right) / R T$ & $\Delta$ & $\left(H^{\circ}-E_{O}^{\circ}\right) / R T$ & $\Delta$ & So/R & $\Delta$ & $\mathrm{C}_{\mathrm{p}}^{\circ} / \mathrm{R}$ \\
\hline 0.0950 & 2.40100 & 100 & 0.95325 & 5 & 3.35425 & 105 & 0.99925 \\
\hline $\begin{array}{l}0.0951 \\
0.0952\end{array}$ & $\begin{array}{l}2.40000 \\
2.39900\end{array}$ & $\begin{array}{l}100 \\
100\end{array}$ & $\begin{array}{l}0.95320 \\
0.95316\end{array}$ & $\begin{array}{l}4 \\
5\end{array}$ & $\begin{array}{l}3.35320 \\
3.35215\end{array}$ & $\begin{array}{l}105 \\
105\end{array}$ & $\begin{array}{l}0.99925 \\
0.99925\end{array}$ \\
\hline $\begin{array}{l}0.0953 \\
0.0954 \\
0.0955\end{array}$ & $\begin{array}{l}2.39800 \\
2.39700 \\
2.39600\end{array}$ & $\begin{array}{l}100 \\
100 \\
100\end{array}$ & $\begin{array}{l}0.95311 \\
0.95306 \\
0.95301\end{array}$ & $\begin{array}{l}5 \\
5 \\
5\end{array}$ & $\begin{array}{l}3.35110 \\
3.35006 \\
3.34901\end{array}$ & $\begin{array}{l}104 \\
105 \\
105\end{array}$ & $\begin{array}{l}0.99924 \\
0.99924 \\
0.99924\end{array}$ \\
\hline $\begin{array}{l}0.0956 \\
0.0957 \\
0.0958 \\
0.0959 \\
0.0960\end{array}$ & $\begin{array}{l}2.39500 \\
2.39401 \\
2.39301 \\
2.39202 \\
2.39102\end{array}$ & $\begin{array}{r}99 \\
100 \\
99 \\
100 \\
99\end{array}$ & $\begin{array}{l}0.95296 \\
0.95291 \\
0.95286 \\
0.95282 \\
0.95277\end{array}$ & $\begin{array}{l}5 \\
5 \\
4 \\
5 \\
5\end{array}$ & $\begin{array}{l}3.34796 \\
3.34692 \\
3.34587 \\
3.34483 \\
3.34379\end{array}$ & $\begin{array}{l}104 \\
105 \\
104 \\
104 \\
104\end{array}$ & $\begin{array}{l}0.99924 \\
0.99924 \\
0.99924 \\
0.99923 \\
0.99923\end{array}$ \\
\hline $\begin{array}{l}0.0961 \\
0.0962 \\
0.0963 \\
0.0964 \\
0.0965\end{array}$ & $\begin{array}{l}2.39003 \\
2.38904 \\
2.38805 \\
2.38706 \\
2.38607\end{array}$ & $\begin{array}{l}99 \\
99 \\
99 \\
99 \\
98\end{array}$ & $\begin{array}{l}0.95272 \\
0.95267 \\
0.95262 \\
0.95257 \\
0.95253\end{array}$ & $\begin{array}{l}5 \\
5 \\
5 \\
4 \\
5\end{array}$ & $\begin{array}{l}3.34275 \\
3.34171 \\
3.34057 \\
3.33964 \\
3.33860\end{array}$ & $\begin{array}{l}104 \\
104 \\
103 \\
104 \\
103\end{array}$ & $\begin{array}{l}0.99923 \\
0.99923 \\
0.99923 \\
0.99923 \\
0.99922\end{array}$ \\
\hline $\begin{array}{l}0.0966 \\
0.0967 \\
0.0968 \\
0.0969 \\
0.0970\end{array}$ & $\begin{array}{l}2.38509 \\
2.38410 \\
2.38312 \\
2.38213 \\
2.38115\end{array}$ & $\begin{array}{l}99 \\
98 \\
99 \\
98 \\
98\end{array}$ & $\begin{array}{l}0.95248 \\
0.95243 \\
0.95238 \\
0.95233 \\
0.95228\end{array}$ & $\begin{array}{l}5 \\
5 \\
5 \\
5 \\
4\end{array}$ & $\begin{array}{l}3.33757 \\
3.33653 \\
3.33550 \\
3.33447 \\
3.33344\end{array}$ & $\begin{array}{l}104 \\
103 \\
103 \\
103 \\
103\end{array}$ & $\begin{array}{l}0.99922 \\
0.99922 \\
0.99922 \\
0.99922 \\
0.99922\end{array}$ \\
\hline $\begin{array}{l}0.0971 \\
0.0972 \\
0.0973 \\
0.0974 \\
0.0975\end{array}$ & $\begin{array}{l}2 \cdot 38017 \\
2 \cdot 37919 \\
2 \cdot 37821 \\
2 \cdot 37723 \\
2 \cdot 37626\end{array}$ & $\begin{array}{l}98 \\
98 \\
98 \\
97 \\
98\end{array}$ & $\begin{array}{l}0.95224 \\
0.95219 \\
0.95214 \\
0.95209 \\
0.95204\end{array}$ & $\begin{array}{l}5 \\
5 \\
5 \\
5 \\
5\end{array}$ & $\begin{array}{l}3.33241 \\
3.33138 \\
3.33035 \\
3.32932 \\
3.32830\end{array}$ & $\begin{array}{l}103 \\
103 \\
103 \\
102 \\
103\end{array}$ & $\begin{array}{l}0.99921 \\
0.99921 \\
0.99921 \\
0.99921 \\
0.99921\end{array}$ \\
\hline $\begin{array}{l}0.0976 \\
0.0977 \\
0.0978 \\
0.0979 \\
0.0980\end{array}$ & $\begin{array}{l}2.37528 \\
2.37431 \\
2.37333 \\
2.37236 \\
2.37139\end{array}$ & $\begin{array}{l}97 \\
98 \\
97 \\
97 \\
97\end{array}$ & $\begin{array}{l}0.95199 \\
0.95195 \\
0.95190 \\
0.95185 \\
0.95180\end{array}$ & $\begin{array}{l}4 \\
5 \\
5 \\
5 \\
5\end{array}$ & $\begin{array}{l}3.32727 \\
3.32625 \\
3.32523 \\
3.32421 \\
3.32319\end{array}$ & $\begin{array}{l}102 \\
102 \\
102 \\
102 \\
102\end{array}$ & $\begin{array}{l}0.99921 \\
0.99920 \\
0.99920 \\
0.99920 \\
0.99920\end{array}$ \\
\hline $\begin{array}{l}0.0981 \\
0.0982 \\
0.0983 \\
0.0984 \\
0.0985\end{array}$ & $\begin{array}{l}2.37042 \\
2.36945 \\
2.36848 \\
2.36751 \\
2.36654\end{array}$ & $\begin{array}{l}97 \\
97 \\
97 \\
97 \\
96\end{array}$ & $\begin{array}{l}0.95175 \\
0.95170 \\
0.95166 \\
0.95161 \\
0.95156\end{array}$ & $\begin{array}{l}5 \\
4 \\
5 \\
5 \\
5\end{array}$ & $\begin{array}{l}3.32217 \\
3.32115 \\
3.32013 \\
3.31912 \\
3.31810\end{array}$ & $\begin{array}{l}102 \\
102 \\
101 \\
102 \\
101\end{array}$ & $\begin{array}{l}0.99920 \\
0.99920 \\
0.99920 \\
0.99919 \\
0.99919\end{array}$ \\
\hline $\begin{array}{l}0.0986 \\
0.0987 \\
0.0988 \\
0.0989 \\
0.0990\end{array}$ & $\begin{array}{l}2.36558 \\
2.36461 \\
2.36365 \\
2.36269 \\
2.36173\end{array}$ & $\begin{array}{l}97 \\
96 \\
96 \\
96 \\
96\end{array}$ & $\begin{array}{l}0.95151 \\
0.95146 \\
0.95141 \\
0.95136 \\
0.95132\end{array}$ & $\begin{array}{l}5 \\
5 \\
5 \\
4 \\
5\end{array}$ & $\begin{array}{l}3.31709 \\
3.31608 \\
3.31506 \\
3.31405 \\
3.31304\end{array}$ & $\begin{array}{l}101 \\
102 \\
101 \\
101 \\
101\end{array}$ & $\begin{array}{l}0.99919 \\
0.99919 \\
0.99919 \\
0.99919 \\
0.99918\end{array}$ \\
\hline $\begin{array}{l}0.0991 \\
0.0992 \\
0.0993 \\
0.0994 \\
0.0995\end{array}$ & $\begin{array}{l}2.36077 \\
2.35981 \\
2.35885 \\
2.35789 \\
2.35694\end{array}$ & $\begin{array}{l}96 \\
96 \\
96 \\
95 \\
96\end{array}$ & $\begin{array}{l}0.95127 \\
0.95122 \\
0.95117 \\
0.95112 \\
0.95108\end{array}$ & $\begin{array}{l}5 \\
5 \\
5 \\
4 \\
5\end{array}$ & $\begin{array}{l}3.31203 \\
3.31103 \\
3.31002 \\
3.30901 \\
3.30801\end{array}$ & $\begin{array}{l}100 \\
101 \\
101 \\
100 \\
100\end{array}$ & $\begin{array}{l}0.99918 \\
0.99918 \\
0.99918 \\
0.99918 \\
0.99918\end{array}$ \\
\hline $\begin{array}{l}0.0996 \\
0.0997 \\
0.0998 \\
0.0999 \\
0.1000\end{array}$ & $\begin{array}{l}2.35598 \\
2.35503 \\
2.35407 \\
2.35312 \\
2.35217\end{array}$ & $\begin{array}{l}95 \\
96 \\
95 \\
95\end{array}$ & $\begin{array}{l}0.95103 \\
0.95098 \\
0.95093 \\
0.95088 \\
0.95083\end{array}$ & $\begin{array}{l}5 \\
5 \\
5 \\
5\end{array}$ & $\begin{array}{l}3.30701 \\
3.30600 \\
3.30500 \\
3.30400 \\
3.30300\end{array}$ & $\begin{array}{l}101 \\
100 \\
100 \\
100\end{array}$ & $\begin{array}{l}0.99917 \\
0.99917 \\
0.99917 \\
0.99917 \\
0.99917\end{array}$ \\
\hline
\end{tabular}


Table I Harmonic Oscillator Contributions (in dimensionless form) to the Thermodynamic Functions for Values of $X=h c v / k T$

$\mathbb{1}$

$$
-\left(F^{\circ}-E_{0}^{\circ}\right) / R T
$$

$2 \cdot 35217$

2.35122

2.35027

$2 \cdot 34932$

2.34837

2.34743

2.34648

0.1006

0.1007

0.1008

0.1009

0.1010

0.1011

0.1012

0.1013

0.1014

0.1015

0.1016

0.1017

0.1018

0.1019

0.1020

0.1021

0.1022

0.1023

0.1024

0.1025

0.1026

0.1027

0.1028

0.1029

0.1030

0.1031

0.1032

0.1033

0.1034

0.1035

0.1236

0.1037

0.1038

0.1039

0.1040

0.1041

0.1042

0.1043

0.1044

0.1045

0.1046

0.1047

0.1048

0.1049

0.1050

2.34554

2.34459

2.34365

$2 \cdot 34271$

2.34177

2.34083

2.33989

2.33895

2.33802

2.33708

2.33615

2.33521

2.33428

2.33335

2.33242

2.33149

2.33056

2.32963

2.32870

2. 32778

2.32685

2.32593

2. 32501

$2 \cdot 32408$

2.32316

2.32224

2. 32132

2.32040

2. 31949

2.31857

2.31766

2.31674

2.31583

2.31491

2.31400

2.31309

2. 31218

2. 31127

2. 31036

2.30946

2.30855

2. 30764

2. 30674

2.30584
$\Delta \quad\left(\mathrm{H}^{\circ}-\mathrm{E}_{\mathrm{O}}^{\circ}\right) / \mathrm{RT}$
95

95

95

95

94

95

94

0.95083
0.95078
0.95074
0.95069
0.95064
0.95059

0.95054

0.95049

0.95045

$\begin{array}{ll}94 & 0.95040 \\ 94 & 0.95035\end{array}$

$94 \quad 0.95030$

940.95025

94

93

94

93

94

93

93

93

93

93

93

93

92

93

92

92

93

92

92

92

92

91

92

91

92

91

92

91

91

91

91

91

90

91

91

90

90
0.95021

0.95016

0.95011

0.95006

0.95001

0.94996

0.94992

0.94987

0.94982

0.94977

0.94972

0.94967

0.94963

0.94958

0.94953

0.94948

0.94943

0.94938

0.94934

0.94929

0.94924

0.94919

0.94914

0.94909

0.94905

0.94900

0.94895

0.94890

0.94885

0.94880

0.94871

0.94866

0.94861

0.94856

0.94852

0.94847

0.94842
0.94876
So/R

3.30300

3. 30200

3. 30101

3. 30001

3. 29901

3.29802

3. 29702

3. 29603

3. 29504

3. 29405

3. 29306

3.29207

3. 29108

3.29010

3.28911

3. 28813

3. 28714

3. 28616

3. 28518

3.28420

3.28322

3. 28224

3. 28126

3. $280 ? 8$

3. 27931

3.27833

3.27736

3. 27638

3.27541

3. 27444

3. 27347

3. 27250

3. 27153

3. 27056

3.26960

3.26863

3.26766

3. 26670

3. 26574

3. 26478

3.26382

3. 26285

3. 26190

3. 26094

3.25998

3.25902

3.25807

3. 25711

3. 25616

3. 25521

3.25425
$\Delta \quad \mathrm{Co}_{\mathrm{p}}^{\circ} / \mathrm{R}$

$\begin{array}{rl}100 & 0.99917 \\ 99 & 0.99917 \\ 100 & 0.99916 \\ 100 & 0.99916 \\ 99 & 0.99916 \\ 100 & 0.99916 \\ 99 & 0.99916 \\ 99 & 0.99916 \\ 99 & 0.99915 \\ 99 & 0.99915 \\ 99 & 0.99915 \\ 99 & 0.99915 \\ 98 & 0.99915 \\ 99 & 0.99915 \\ 98 & 0.99914 \\ 99 & 0.99914\end{array}$

98

98

98

98

98

98

98

97

98

97

98

97

97

97

97

97

97

96

97

97

96

96

96

96

97

95

96

96

96

95

96

95

95

96

0.99914

0.99914

0.99914

0.99914

0.99913

0.99913

0.99913 
Table I Harmonic Oscillator Contributions (in dimensionless form) to the Thermodynamic Functions for Values of $\mathrm{X}=\mathrm{hc} / \mathrm{kT}$

X

$-\left(F^{\circ}-E_{0}^{\circ}\right) / R T$

0.1050
0.1051
0.1052
0.1053
0.1054
0.1055
0.1056
0.1057
0.1058
0.1059

0.1060

0.1061

0.1062

0.1063

0.1364

0.1065

0.1066

0.1067

0.1068

0.1069

0.1070

0.1071

0.1072

0.1073

0.1074

0.1075

0.1076

0.1077

0.1078

0.1079

0.1080

0.1081

0.1082

0.1083

0.1084

0.1085

0.1086

0.1087

0.1088

0.1089

0.1090

0.1091

0.1092

0.1093

0.1094

0.1095

0.1096

0.1097

0.1098

0.1099

0.1100
2.30584

2.30493

2. 30403

2.30313

2.30223

2.30133

2.30043

2.29953

2. 29864

2. 29774

2. 29685

2.29595

2.29506

2. 29417

2.29328

2.29239

2.29150

2.29061

2.28972

2.28884

2.28795

2.28706

2.28618

2.28530

2. 28441

2.28353

2.28265

2.28177

2. 28089

2.28002

2. 27914

2.27826

2.27739

2. 27651

2. 27564

2.27476

2.27389

2.27302

2. 27215

2.27128

2.27041

2.26954

2. 26868

2.26781

2. 26695

2. 26608

2.26522

2. 26435

2.26349

2. 26263

2.26177
0.94692

0.94688

0.94683

0.94678

0.94673

0.94668

0.94663

0.94659

0.94654

0.94649

0.94644

0.94639

0.94635

0.94630

0.94625

0.94620

0.94615

0.94610

0.94606

0.94601
$\Delta \quad\left(\mathrm{H}^{\circ}-\mathrm{E}_{\mathrm{O}}^{\circ}\right) / \mathrm{RT}$

$\Delta$

So/R

$\begin{array}{ll}91 & 0.94842 \\ 90 & 0.94837 \\ 90 & 0.94832 \\ 90 & 0.94827 \\ 90 & 0.94823 \\ 90 & 0.94818 \\ & \\ 90 & 0.94813 \\ 89 & 0.94808 \\ 90 & 0.94803 \\ 89 & 0.94798 \\ 90 & 0.94794 \\ & \\ 89 & 0.94789 \\ 89 & 0.94784 \\ 89 & 0.94779 \\ 89 & 0.94774 \\ 89 & 0.94769 \\ & 0.94765 \\ 89 & 0.949 \\ 89 & 0.94760 \\ 88 & 0.94755 \\ 89 & 0.94750 \\ 89 & 0.94745\end{array}$

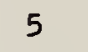

3.25425

95

$\Delta$

$\mathrm{Co}_{\mathrm{p}} \mathrm{R}$

88

88

89

88

88

0.94741

0.94736

0.94731

0.94726

0.94721

88

88

87

88

88

0.94716

0.94712

0.94707

0.94702

0.94697

87

88

87

88

87

87

87

87

87

87

86

87

86

87

86

87

86

86

86
3.25330

3.25235

3. 25140

3.25046

3.24951

3.24856

3.24762

3. 24667

3.24573

3. 24478

3.24384

3.24290

3.24196

3. 24102

3.24008

3.23915

3.23821

3. 23727

3. 23634

3.23540

3.23447

3. 23354

3. 23261

3. 23168

3.23075

3.22982

3.22889

3.22796

3. 22704

3.22611

3. 22519

3. 22426

3. 22334

3.22242

3.22150

3. 22058

3. 21966

3. 21874

3. 21782

3. 21690

3. 21599

3. 21507

3. 21416

3. 21324

3.21233

3. 21142

3. 21051

3.20960

3.20869

3. 20778
$95 \quad 0.99908$

95

94

95

95

94

95

$\begin{array}{ll}95 & 0.99907 \\ 94 & 0.99906\end{array}$

94

94

94

94

93

94

94

93

93

93

92

93

92

92

92

92

92

91

92

91

0.99908

0.99908

0.99908

0.99907

0.99907

0.99907

0.99907

0.99907

0.99906

0.99906

0.99906

0.99906

0.99906

0.99905

0.99905

0.99905

0.99905

0.99905

0.99904

0.99904

0.99904

0.99904

0.99904

0.99904

0.99903

0.99903

0.99903

0.99903

0.99903

0.99903

0.99902

0.99902

0.99902

0.99902

0.99902

0.99901

0.99901

0.99901

0.99901

0.99901

0.99901

0.99900

0.99900

0.99900 
Table I Harmonic Oscillator Contributions (in dimensionless form) to the Thermodynamic Functions for Values of $\mathrm{X}=\mathrm{hcv} / \mathrm{kT}$

$\begin{array}{lllllll}\mathbf{L} & -\left(\mathrm{F}^{\circ}-\mathrm{E}_{\mathrm{O}}\right) / \mathrm{RT} & \Delta & \left(\mathrm{H}^{\circ}-\mathrm{E}_{\mathrm{O}}^{\circ}\right) / \mathrm{RT} & \Delta & \mathrm{S} / \mathrm{R} & \Delta \quad \mathrm{CO}_{\mathrm{P}} / \mathrm{R}\end{array}$

$\begin{array}{llllllll}0.1100 & 2.26177 & 86 & 0.94601 & 5 & 3.20778 & 91 & 0.99899 \\ 0.11101 & 2.26091 & 86 & 0.94596 & 5 & 3.20687 & 91 & 0.99899 \\ 0.1102 & 2.26005 & 86 & 0.94591 & 5 & 3.20596 & 90 & 0.99899 \\ 0.1103 & 2.25919 & 85 & 0.94586 & 4 & 3.20506 & 91 & 0.99899 \\ 0.1104 & 2.25834 & 86 & 0.94582 & 5 & 3.20415 & 90 & 0.99899 \\ 0.1105 & 2.25748 & 85 & 0.94577 & 5 & 3.20325 & 91 & 0.99898 \\ 0.1106 & 2.25663 & 86 & 0.94572 & 5 & 3.20234 & 90 & 0.99898 \\ 0.11107 & 2.25577 & 85 & 0.94567 & 5 & 3.20144 & 90 & 0.99898 \\ 0.1108 & 2.2549 .2 & 86 & 0.94562 & 5 & 3.20054 & 90 & 0.99898 \\ 0.1109 & 2.25406 & 85 & 0.94557 & 4 & 3.19964 & 90 & 0.99898 \\ 0.1110 & 2.25321 & 85 & 0.94553 & 5 & 3.19874 & 90 & 0.99897 \\ 0.1111 & 2.25236 & 85 & 0.94548 & 5 & 3.19784 & 90 & 0.99897 \\ 0.1112 & 2.25151 & 85 & 0.94543 & 5 & 3.19694 & 90 & 0.99897 \\ 0.1113 & 2.25066 & 85 & 0.94538 & 5 & 3.19604 & 89 & 0.99897 \\ 0.1114 & 2.24981 & 85 & 0.94533 & 4 & 3.19515 & 90 & 0.99897 \\ 0.1115 & 2.24896 & 84 & 0.94529 & 5 & 3.19425 & 90 & 0.99896 \\ 0.1116 & 2.24812 & 85 & 0.94524 & 5 & 3.19335 & 89 & 0.99896 \\ 0.1117 & 2.24727 & 85 & 0.94519 & 5 & 3.19246 & 90 & 0.99896 \\ 0.1118 & 2.24642 & 84 & 0.94514 & 5 & 3.19156 & 89 & 0.99896 \\ 0.1119 & 2.24558 & 85 & 0.94509 & 4 & 3.19067 & 89 & 0.99896 \\ 0.1120 & 2.24473 & 84 & 0.94505 & 5 & 3.18978 & 89 & 0.99896\end{array}$

$\begin{array}{lll}0.1121 & 2.24389 & 84 \\ 0.1122 & 2.24305 & 8 \\ 0.1123 & 2.24221 & 8 \\ 0.1124 & 2.24137 & 8 \\ 0.1125 & 2.24052 & 8\end{array}$

$0.1126 \quad 2.23969 \quad 84$

$0.1127 \quad 2.23885$

$0.1128 \quad 2.23801$

$0.1129 \quad 2.23717$

$0.1130 \quad 2.23634$

0.1131

0.1132

0.1133

0.1134

0.1135

2.23550

2.23467

2.23383

2.23300

2. 23217

0.1136

2.23133

0.1137

0.1138

0.1139

0.1140

2.23050

2. 22967

2. 22884

2. 22802

0.1141

0.1142

0.1143

0.1144

0.1145

0.1146

0.1147

0.1148

0.1149

0.1150
2.22719

2. 22636

2. 22553

2. 22471

2. 22388

2. 22306

2. 22224

2. 22141

2. 22059

2. 21977
84

84

85

84

83

84

84

84

83

84

83

84

83

83

84

83

83

83

82

83

83

83

82

83

82

82

83

82

82
0.94500

0.94495

0.94490

0.94485

0.94480

0.94476

0.94471

0.94466

0.94461

0.94456

0.94452

0.94447

0.94442

0.94437

0.94432

0.94428

0.94423

0.94418

0.94413

0.94408

0.94403

0.94399

0.94394

0.94389

0.94384

0.94379

0.94375

0.94370

0.94365

0.94360
3.18889

3.18800

3.18711

3. 18622

3.18533

3. 18444

3. 18356

3. 18267

3. 18178

3.18090

3.18002

3. 17913

3.17825

3. 17737

3.17649

\section{9}

89

89

89

89

88

89

89

88

88

89

88

88

88

88

88

3.17561

3. 17473

3. 17385

3. 17297

3.17210

88

87

88

87

88

87

87

88

87

87

87

87

3.16685

3.16598

3.16511

3.16424
0.99895

0.99895

0.99895

0.99895

0.99895

0.99894

0.99894

0.99894

0.99894

0.99894

0.99893

0.99893

0.99893

0.99893

0.99893

0.99893

0.99892

0.99892

0.99892

0.99892

0.99892

0.99891

0.99891

0.99891

0.99891

0.99891

0.99890

0.99890

0.99890

3.16337

0.99890

$6389510-62-3$ 
Table I Harmonic Oscillator Contributions (in dimensionless form) to the Thermodynamic Functions for Values of $\mathrm{X}=\mathrm{hcv} / \mathrm{kT}$

\begin{tabular}{|c|c|c|c|c|c|c|c|}
\hline $\mathrm{X}$ & $-\left(F^{\circ}-E_{O}^{\circ}\right) / R T$ & $\Delta$ & $\left(\mathrm{H}^{\circ}-\mathrm{E}_{\mathrm{O}}^{\circ}\right) / \mathrm{RT}$ & $\Delta$ & $S \circ / R$ & $\Delta$ & $\mathrm{C}_{\mathrm{p}}^{\circ} / \mathrm{R}$ \\
\hline 0.1150 & 2.21977 & 82 & 0.94360 & 5 & 3.16337 & 86 & 0.99890 \\
\hline $\begin{array}{l}0.1151 \\
0.1152\end{array}$ & $\begin{array}{l}2.21895 \\
2.21813\end{array}$ & $\begin{array}{l}82 \\
82\end{array}$ & $\begin{array}{l}0.94355 \\
0.94351\end{array}$ & $\begin{array}{l}4 \\
5\end{array}$ & $\begin{array}{l}3.16251 \\
3.16164\end{array}$ & $\begin{array}{l}87 \\
87\end{array}$ & $\begin{array}{l}0.99890 \\
0.99889\end{array}$ \\
\hline 0.1153 & 2.21731 & 81 & 0.94346 & 5 & 3.16077 & 86 & 0.99889 \\
\hline 0.1154 & 2.21650 & 82 & 0.94341 & 5 & 3.15991 & 87 & 0.99889 \\
\hline 0.1155 & 2.21568 & 82 & 0.94336 & 5 & 3.15904 & 86 & 0.99889 \\
\hline 0.1156 & 2.21486 & 81 & 0.94331 & 4 & 3.15818 & 87 & 0.99889 \\
\hline 0.1157 & 2.21405 & 82 & 0.94327 & 5 & 3.15731 & 86 & 0.99889 \\
\hline 0.1158 & 2.21323 & 81 & 0.94322 & 5 & 3.15645 & 86 & 0.99888 \\
\hline 0.1159 & 2.21242 & 82 & 0.94317 & 5 & 3.15559 & 86 & 0.99888 \\
\hline 0.1160 & 2.21160 & 81 & 0.94312 & 5 & 3.15473 & 87 & 0.99888 \\
\hline 0.1161 & 2.21079 & 81 & 0.94307 & 4 & 3.15386 & 85 & 0.99888 \\
\hline 0.1162 & 2.20998 & 81 & 0.94303 & 5 & 3.15301 & 86 & 0.99888 \\
\hline 0.1163 & 2.20917 & 81 & 0.94298 & 5 & 3.15215 & 86 & 0.99887 \\
\hline 0.1164 & 2.20836 & 81 & 0.94293 & 5 & 3.15129 & 86 & 0.99887 \\
\hline 0.1165 & 2.20755 & 81 & 0.94288 & 5 & 3.15043 & 86 & 0.99887 \\
\hline 0.1166 & 2.20674 & 81 & 0.94283 & 5 & 3.14957 & 85 & 0.99887 \\
\hline 0.1167 & 2.20593 & 81 & 0.94278 & 4 & 3.14872 & 86 & 0.99887 \\
\hline 0.1168 & 2.20512 & 80 & 0.94274 & 5 & 3.14786 & 85 & 0.99886 \\
\hline 0.1169 & 2.20432 & 81 & 0.94269 & 5 & 3.14701 & 86 & 0.99886 \\
\hline 0.1170 & 2.20351 & 80 & 0.94264 & 5 & 3.14615 & 85 & 0.99886 \\
\hline 0.1171 & 2.20271 & 81 & 0.94259 & 5 & 3.14530 & 85 & 0.99886 \\
\hline 0.1172 & 2.20190 & 80 & 0.94254 & 4 & 3.14445 & 86 & 0.99886 \\
\hline 0.1173 & 2.20110 & 81 & 0.94250 & 5 & 3.14359 & 85 & 0.99885 \\
\hline 0.1174 & 2.20029 & 80 & 0.94245 & 5 & 3.14274 & 85 & 0.99885 \\
\hline 0.1175 & 2.19949 & 80 & 0.94240 & 5 & 3.14189 & 85 & 0.99885 \\
\hline 0.1176 & 2.19869 & 80 & 0.94235 & 5 & 3.14104 & 85 & 0.99885 \\
\hline 0.1177 & 2.19789 & 80 & 0.94230 & 4 & 3.14019 & 85 & 0.99885 \\
\hline 0.1178 & 2.19709 & 80 & 0.94226 & 5 & 3.13934 & 84 & 0.99884 \\
\hline 0.1179 & 2.19629 & 80 & 0.94221 & 5 & 3.13850 & 85 & 0.99884 \\
\hline 0.1180 & 2.19549 & 80 & 0.94216 & 5 & 3.13765 & 85 & 0.99884 \\
\hline 0.1181 & 2.19469 & 79 & 0.94211 & 5 & 3.13680 & 84 & 0.99884 \\
\hline 0.1182 & 2.19390 & 80 & 0.94206 & 4 & 3.13596 & 85 & 0.99884 \\
\hline 0.1183 & 2.19310 & 80 & 0.94202 & 5 & 3.13511 & 84 & 0.99883 \\
\hline 0.1184 & 2.19230 & 79 & 0.94197 & 5 & 3.13427 & 84 & 0.99883 \\
\hline 0.1185 & 2.19151 & 80 & 0.94192 & 5 & 3.13343 & 85 & 0.99883 \\
\hline 0.1186 & 2.19071 & 79 & 0.94187 & 5 & 3.13258 & 84 & 0.99883 \\
\hline 0.1187 & 2.18992 & 79 & 0.94182 & 4 & 3.13174 & 84 & 0.99883 \\
\hline 0.1188 & 2.18913 & 80 & 0.94178 & 5 & 3.13090 & 84 & 0.99882 \\
\hline 0.1189 & 2.18833 & 79 & 0.94173 & 5 & 3.13006 & 84 & 0.99882 \\
\hline 0.1190 & 2.18754 & 79 & 0.94168 & 5 & 3.12922 & 84 & 0.99882 \\
\hline 0.1191 & 2.18675 & 79 & 0.94163 & 5 & 3.12838 & 84 & 0.99882 \\
\hline 0.1192 & 2.18596 & 79 & 0.94158 & 4 & 3.12754 & 83 & 0.99882 \\
\hline 0.1193 & 2.18517 & 79 & 0.94154 & 5 & 3.12671 & 84 & 0.99881 \\
\hline 0.1194 & 2.18438 & 79 & 0.94149 & 5 & 3.12587 & 84 & 0.99881 \\
\hline 0.1195 & 2.18359 & 78 & 0.94144 & 5 & 3.12503 & 83 & 0.99881 \\
\hline 0.1196 & 2.18281 & 79 & 0.94139 & 5 & 3.12420 & 84 & 0.99881 \\
\hline 0.1197 & 2.18202 & 79 & 0.94134 & 4 & 3.12336 & 83 & 0.99881 \\
\hline 0.1198 & 2.18123 & 78 & 0.94130 & 5 & 3.12253 & 83 & 0.99881 \\
\hline 0.1199 & 2.18045 & 79 & 0.94125 & 5 & 3.12170 & 84 & 0.99880 \\
\hline 0.1200 & 2.17966 & & 0.94120 & ' & 3.12086 & & 0.99880 \\
\hline
\end{tabular}


Table I Harmonic Oscillator Contributions (in dimensionless form) to the Thermodynamic Functions for Values of $\mathrm{X}=\mathrm{hc} v / \mathrm{kT}$

\begin{tabular}{|c|c|c|c|c|c|c|c|}
\hline$X$ & $-\left(F^{\circ}-E_{O}^{O}\right) / R T$ & $\Delta$ & $\left(\mathrm{H}^{\circ}-\mathrm{E}_{\mathrm{O}}\right) / \mathrm{RT}$ & $\Delta$ & $S \% / R$ & $\Delta$ & $\mathrm{Co}_{\mathrm{p}}^{\circ} / \mathrm{R}$ \\
\hline 0.1200 & 2.17966 & 78 & 0.94120 & 5 & $3.12 C 86$ & 83 & 0.99880 \\
\hline $\begin{array}{l}0.1201 \\
0.1202 \\
0.1203\end{array}$ & $\begin{array}{l}2.17888 \\
2.17810 \\
2.17731\end{array}$ & $\begin{array}{l}78 \\
79 \\
78\end{array}$ & $\begin{array}{l}0.94115 \\
0.94110 \\
0.94106\end{array}$ & $\begin{array}{l}5 \\
4 \\
5\end{array}$ & $\begin{array}{l}3.12003 \\
3.11920 \\
3.11837\end{array}$ & $\begin{array}{l}83 \\
83 \\
83\end{array}$ & $\begin{array}{l}0.99880 \\
0.99880 \\
0.99879\end{array}$ \\
\hline $\begin{array}{l}0.1204 \\
0.1205\end{array}$ & $\begin{array}{l}2.17653 \\
2.17575\end{array}$ & $\begin{array}{l}78 \\
78\end{array}$ & $\begin{array}{l}0.94101 \\
0.94096\end{array}$ & $\begin{array}{l}5 \\
5\end{array}$ & $\begin{array}{l}3.11754 \\
3.11671\end{array}$ & $\begin{array}{l}83 \\
83\end{array}$ & $\begin{array}{l}0.99879 \\
0.99879\end{array}$ \\
\hline $\begin{array}{l}0.1206 \\
0.1207 \\
0.1208 \\
0.1209 \\
0.1210\end{array}$ & $\begin{array}{l}2.17497 \\
2.17419 \\
2.17341 \\
2.17263 \\
2.17185\end{array}$ & $\begin{array}{l}78 \\
78 \\
78 \\
78 \\
77\end{array}$ & $\begin{array}{l}0.94091 \\
0.94086 \\
0.94082 \\
0.94077 \\
0.94072\end{array}$ & $\begin{array}{l}5 \\
4 \\
5 \\
5 \\
5\end{array}$ & $\begin{array}{l}3.11588 \\
3.11505 \\
3.11423 \\
3.11340 \\
3.11257\end{array}$ & $\begin{array}{l}83 \\
82 \\
83 \\
83 \\
82\end{array}$ & $\begin{array}{l}0.99879 \\
0.99879 \\
0.99878 \\
0.99878 \\
0.99878\end{array}$ \\
\hline $\begin{array}{l}0.11111 \\
0.1212 \\
0.1213 \\
0.11214 \\
0.1215\end{array}$ & $\begin{array}{l}2.17108 \\
2.17030 \\
2.16953 \\
2.16875 \\
2.16798\end{array}$ & $\begin{array}{l}78 \\
77 \\
78 \\
77 \\
78\end{array}$ & $\begin{array}{l}0.94067 \\
0.94062 \\
0.94058 \\
0.94053 \\
0.94048\end{array}$ & $\begin{array}{l}5 \\
4 \\
5 \\
5 \\
5\end{array}$ & $\begin{array}{l}3.11175 \\
3.11092 \\
3.11010 \\
3.10928 \\
3.10846\end{array}$ & $\begin{array}{l}83 \\
82 \\
82 \\
82 \\
83\end{array}$ & $\begin{array}{l}0.99878 \\
0.99878 \\
0.99877 \\
0.99877 \\
0.99877\end{array}$ \\
\hline $\begin{array}{l}0.1216 \\
0.1217 \\
0.1218 \\
0.1219 \\
0.1220\end{array}$ & $\begin{array}{l}2.16720 \\
2.16643 \\
2.16566 \\
2.16489 \\
2.16411\end{array}$ & $\begin{array}{l}77 \\
77 \\
77 \\
78 \\
77\end{array}$ & $\begin{array}{l}0.94043 \\
0.94038 \\
0.94034 \\
0.94029 \\
0.94024\end{array}$ & $\begin{array}{l}5 \\
4 \\
5 \\
5 \\
5\end{array}$ & $\begin{array}{l}3.10763 \\
3.10681 \\
3.10599 \\
3.10517 \\
3.10435\end{array}$ & $\begin{array}{l}82 \\
82 \\
82 \\
82 \\
81\end{array}$ & $\begin{array}{l}0.99877 \\
0.99877 \\
0.99876 \\
0.99876 \\
0.99876\end{array}$ \\
\hline $\begin{array}{l}0.1221 \\
0.1222 \\
0.1223 \\
0.1224 \\
0.1225\end{array}$ & $\begin{array}{l}2.16334 \\
2.16257 \\
2.16181 \\
2.16104 \\
2.16027\end{array}$ & $\begin{array}{l}77 \\
76 \\
77 \\
77 \\
77\end{array}$ & $\begin{array}{l}0.94019 \\
0.94014 \\
0.94010 \\
0.94005 \\
0.94000\end{array}$ & $\begin{array}{l}5 \\
4 \\
5 \\
5 \\
5\end{array}$ & $\begin{array}{l}3.10354 \\
3.10272 \\
3.10190 \\
3.10109 \\
3.10027\end{array}$ & $\begin{array}{l}82 \\
82 \\
81 \\
82 \\
82\end{array}$ & $\begin{array}{l}0.99876 \\
0.99876 \\
0.99875 \\
0.99875 \\
0.99875\end{array}$ \\
\hline $\begin{array}{l}0.1226 \\
0.1227 \\
0.1228 \\
0.1229 \\
0.1230\end{array}$ & $\begin{array}{l}2.15950 \\
2.15874 \\
2.15797 \\
2.15721 \\
2.15644\end{array}$ & $\begin{array}{l}76 \\
77 \\
76 \\
77 \\
76\end{array}$ & $\begin{array}{l}0.93995 \\
0.93990 \\
0.93986 \\
0.93981 \\
0.93976\end{array}$ & $\begin{array}{l}5 \\
4 \\
5 \\
5 \\
5\end{array}$ & $\begin{array}{l}3.09945 \\
3.09864 \\
3.09783 \\
3.19701 \\
3.09620\end{array}$ & $\begin{array}{l}81 \\
81 \\
82 \\
81 \\
81\end{array}$ & $\begin{array}{l}0.99875 \\
0.99875 \\
0.99874 \\
0.99874 \\
0.99874\end{array}$ \\
\hline $\begin{array}{l}0.1231 \\
0.1232 \\
0.1233 \\
0.1234 \\
0.1235\end{array}$ & $\begin{array}{l}2.15568 \\
2.15491 \\
2.15415 \\
2.15339 \\
2.15263\end{array}$ & $\begin{array}{l}77 \\
76 \\
76 \\
76 \\
76\end{array}$ & $\begin{array}{l}0.93971 \\
0.93966 \\
0.93962 \\
0.93957 \\
0.93952\end{array}$ & $\begin{array}{l}5 \\
4 \\
5 \\
5 \\
5\end{array}$ & $\begin{array}{l}3.09539 \\
3.09438 \\
3.09377 \\
3.09296 \\
3.09215\end{array}$ & $\begin{array}{l}81 \\
81 \\
81 \\
81 \\
81\end{array}$ & $\begin{array}{l}0.99874 \\
0.99874 \\
0.99873 \\
0.99873 \\
0.99873\end{array}$ \\
\hline $\begin{array}{l}0.1236 \\
0.1237 \\
0.1238 \\
0.1239 \\
0.1240\end{array}$ & $\begin{array}{l}2.15187 \\
2.15111 \\
2.15035 \\
2.14959 \\
2.14883\end{array}$ & $\begin{array}{l}76 \\
76 \\
76 \\
76 \\
75\end{array}$ & $\begin{array}{l}0.93947 \\
0.93942 \\
0.93938 \\
0.93933 \\
0.93928\end{array}$ & $\begin{array}{l}5 \\
4 \\
5 \\
5 \\
5\end{array}$ & $\begin{array}{l}3.09134 \\
3.09053 \\
3.08973 \\
3.08892 \\
3.08811\end{array}$ & $\begin{array}{l}81 \\
80 \\
81 \\
81 \\
80\end{array}$ & $\begin{array}{l}0.99873 \\
0.99873 \\
0.99872 \\
0.99872 \\
0.99872\end{array}$ \\
\hline $\begin{array}{l}0.1241 \\
0.1242 \\
0.1243 \\
0.1244 \\
0.1245\end{array}$ & $\begin{array}{l}2.14808 \\
2.14732 \\
2.14656 \\
2.14581 \\
2.14505\end{array}$ & $\begin{array}{l}76 \\
76 \\
75 \\
76 \\
75\end{array}$ & $\begin{array}{l}0.93923 \\
0.93919 \\
0.93914 \\
0.93909 \\
0.93904\end{array}$ & $\begin{array}{l}4 \\
5 \\
5 \\
5 \\
5\end{array}$ & $\begin{array}{l}3.08731 \\
3.08650 \\
3.08570 \\
3.08490 \\
3.08410\end{array}$ & $\begin{array}{l}81 \\
80 \\
80 \\
80 \\
81\end{array}$ & $\begin{array}{l}0.99872 \\
0.99872 \\
0.99871 \\
0.99871 \\
0.99871\end{array}$ \\
\hline $\begin{array}{l}0.1246 \\
0.1247 \\
0.1248 \\
0.1249 \\
0.1250\end{array}$ & $\begin{array}{l}2.14430 \\
2.14355 \\
2.14279 \\
2.14204 \\
2.14129\end{array}$ & $\begin{array}{l}75 \\
76 \\
75 \\
75\end{array}$ & $\begin{array}{l}0.93899 \\
0.93895 \\
0.93890 \\
0.93885 \\
0.93880\end{array}$ & $\begin{array}{l}4 \\
5 \\
5 \\
5\end{array}$ & $\begin{array}{l}3.08329 \\
3.08249 \\
3.08169 \\
3.08089 \\
3.08009\end{array}$ & $\begin{array}{l}80 \\
80 \\
80 \\
80\end{array}$ & $\begin{array}{l}0.99871 \\
0.99871 \\
0.99870 \\
0.99870 \\
0.99870\end{array}$ \\
\hline
\end{tabular}


Table I Harmonic Oscillator Contributions (in dimensionless form) to the Thermodynamic Functions for Values of $X=h c v / k T$

$\mathbb{Z}$

\begin{tabular}{|c|c|}
\hline 0.1250 & 2.14129 \\
\hline $\begin{array}{l}0.1251 \\
0.1252\end{array}$ & $\begin{array}{l}2.14054 \\
2.13979\end{array}$ \\
\hline $\begin{array}{l}0.1253 \\
0.1254 \\
0.1255\end{array}$ & $\begin{array}{l}2.13904 \\
2.13829 \\
2.13754\end{array}$ \\
\hline $\begin{array}{l}0.1256 \\
0.1257 \\
0.1258 \\
0.1259 \\
0.1260\end{array}$ & $\begin{array}{l}2.13680 \\
2.13605 \\
2.13530 \\
2.13456 \\
2.13381\end{array}$ \\
\hline $\begin{array}{l}0.1261 \\
0.1262 \\
0.1263 \\
0.1264 \\
0.1265\end{array}$ & $\begin{array}{l}2.13307 \\
2.13232 \\
2.13158 \\
2.13084 \\
2.13010\end{array}$ \\
\hline $\begin{array}{l}0.1266 \\
0.1267 \\
0.1268 \\
0.1269 \\
0.1270\end{array}$ & $\begin{array}{l}2.12936 \\
2.12861 \\
2.12787 \\
2.12714 \\
2.12640\end{array}$ \\
\hline $\begin{array}{l}0.1271 \\
0.1272 \\
0.1273 \\
0.1274 \\
0.1275\end{array}$ & $\begin{array}{l}2.12566 \\
2.12492 \\
2.12418 \\
2.12345 \\
2.12271\end{array}$ \\
\hline $\begin{array}{l}0.1276 \\
0.1277 \\
0.1278 \\
0.1279 \\
0.1280\end{array}$ & $\begin{array}{l}2.12198 \\
2.12124 \\
2.12051 \\
2.11978 \\
2.11904\end{array}$ \\
\hline $\begin{array}{l}0.1281 \\
0.1282 \\
0.1283 \\
0.1284 \\
0.1285\end{array}$ & $\begin{array}{l}2.11831 \\
2.11758 \\
2.11685 \\
2.11612 \\
2.11539\end{array}$ \\
\hline $\begin{array}{l}0.1286 \\
0.1287 \\
0.1288 \\
0.1289 \\
0.1290\end{array}$ & $\begin{array}{l}2.11466 \\
2.11393 \\
2.11320 \\
2.111248 \\
2.11175\end{array}$ \\
\hline $\begin{array}{l}0.1291 \\
0.1292 \\
0.1293 \\
0.1294 \\
0.1295\end{array}$ & $\begin{array}{l}2.11102 \\
2.11030 \\
2.10957 \\
2.10885 \\
2.10813\end{array}$ \\
\hline $\begin{array}{l}0.1296 \\
0.1297 \\
0.1298 \\
0.1299 \\
0.1300\end{array}$ & $\begin{array}{l}2.10740 \\
2.10668 \\
2.10596 \\
2.10524 \\
2.10452\end{array}$ \\
\hline
\end{tabular}

$\Delta \quad\left(\mathrm{H}^{\circ}-\mathrm{E}_{\mathrm{O}}^{\circ}\right) / \mathrm{RT} \quad \Delta \quad \mathrm{S} / \mathrm{R}$

75

75

75

75

74

75

75

74

75

74

75

74

74

74

75

74

74

73

74

74

74

74

73

74

73

74

73

73

74

73

73

73

73

73

73

73

73

72

73

73

72

73

72

72

73

72

72

72

72

0.93880
0.93875
0.93871
0.93866
0.93861
0.93856
0.93851
0.93847
0.93842
0.93837
0.93832
0.93827
0.93823
0.93818
0.93813
0.93808
0.93804
0.93799
0.93794
0.93789
0.93784
0.93780
0.93775
0.93770
0.93765
0.93760

0.93756

0.93751

0.93746

0.93741

0.93732

0.93727

0.93722

0.93717

0.93713

0.93708

0.93703

0.93698

0.93693

0.93689

0.93684

0.93679

0.93674

0.93669

0.93665

0.93660

0.93655

0.93650

0.93646

0.93641
0.93736
$\Delta$

$\mathrm{Co}_{\mathrm{p}} / \mathrm{R}$

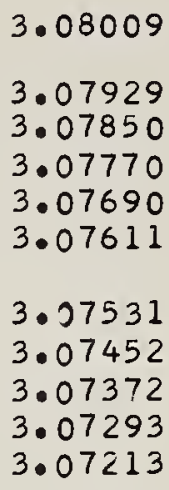

80

0.99870

79

0. 99870

80

80

79

80

0.99869

0.99869

0.99869

0.99869

79

0.99869

3.07134

3.07055

3. 06976

3.06897

3.06818

3. 06739

3. 06660

3.06581

3.06503

3.06424

3.06345

3. 06267

3.06188

3. 06110

3. 06032

3.05953

3. 05875

3. 05797

3. 05719

3. 05641

3.05563

3. 05485

3. 05407

3.05329

3. 05251

3. 05174

3. 05096

3. 05019

3. 24941

3.04854

3. 04786

3. 04709

3. 04632

3. 04554

3.04477

3. 04400

3. 04323

3. 04246

3. 04169

3. 04092
80

79

80

79

79

79

79

79

79

79

79

78

79

79

78

79

78

78

79

78

78

78

78

78

78

78

78

78

77

78

77

78

77

78

0.99868

0.99868

0.99868

0.99868

0.99868

0.99867

0.99867

0.99867

0.99867

0.99867

0.99866

0.99866

0.99866

0.99866

0. 99865

0.99865

0.99865

0.99865

0.99865

0.99864

0.99864

0.99864

0.99864

0.99864

0.99863

0.99863

0.99863

0.99863

0.99863

0.99862

0.99862

0.99862

0.99862

0.99861

0.99861

0.99861

0.99861

0.99861

0.99860

77

77

77

77
0.99860

0.99860

0.99860

0.99859
0.99860 
Table I Harmonic Oscillator Contributions (in dimensionless form) to the Thermodynamic Functions for Values of $X=h c v / k T$

\begin{tabular}{|c|c|c|c|c|c|c|c|}
\hline $\mathbf{X}$ & $-\left(F^{\circ}-E_{0}^{\circ}\right) / R T$ & $\Delta$ & $\left(\mathrm{H}^{\circ}-\mathrm{E}_{\mathrm{O}}^{\circ}\right) / \mathrm{RT}$ & $\Delta$ & So/R & $\Delta$ & $\mathrm{Co} / \Omega$ \\
\hline 0.1300 & 2.10452 & 72 & 0.93641 & 5 & 3.04092 & 76 & 0.99859 \\
\hline $\begin{array}{l}0.1301 \\
0.1302 \\
0.1303\end{array}$ & $\begin{array}{l}2.10380 \\
2.10308 \\
2.10236\end{array}$ & $\begin{array}{l}72 \\
72 \\
72\end{array}$ & $\begin{array}{l}0.93636 \\
0.93631 \\
0.93626\end{array}$ & $\begin{array}{l}5 \\
5 \\
4\end{array}$ & $\begin{array}{l}3.04016 \\
3.03939 \\
3.03862\end{array}$ & $\begin{array}{l}77 \\
77 \\
76\end{array}$ & $\begin{array}{l}0.99859 \\
0.99859 \\
0.99859\end{array}$ \\
\hline $\begin{array}{l}0.1304 \\
0.1305\end{array}$ & $\begin{array}{l}2.10164 \\
2.10092\end{array}$ & $\begin{array}{l}72 \\
71\end{array}$ & $\begin{array}{l}0.93622 \\
0.93617\end{array}$ & $\begin{array}{l}5 \\
5\end{array}$ & $\begin{array}{l}3.03786 \\
3.03709\end{array}$ & $\begin{array}{l}77 \\
76\end{array}$ & $\begin{array}{l}0.99858 \\
0.99858\end{array}$ \\
\hline $\begin{array}{l}0.1306 \\
0.1307 \\
0.1308 \\
0.11309 \\
0.1110\end{array}$ & $\begin{array}{l}2.10021 \\
2.09949 \\
2.09877 \\
2.09806 \\
2.09734\end{array}$ & $\begin{array}{l}72 \\
72 \\
71 \\
72 \\
71\end{array}$ & $\begin{array}{l}0.93612 \\
0.93607 \\
0.53603 \\
0.93598 \\
0.93593\end{array}$ & $\begin{array}{l}5 \\
4 \\
5 \\
5 \\
5\end{array}$ & $\begin{array}{l}3.03633 \\
3.03556 \\
3.03480 \\
3.03404 \\
3.03327\end{array}$ & $\begin{array}{l}77 \\
76 \\
76 \\
77 \\
76\end{array}$ & $\begin{array}{l}0.99858 \\
0.99858 \\
0.99858 \\
0.99857 \\
0.99857\end{array}$ \\
\hline $\begin{array}{l}0.1311 \\
0.1312 \\
0.1313 \\
0.1314 \\
0.1315\end{array}$ & $\begin{array}{l}2.09663 \\
2.09592 \\
2.09520 \\
2.09449 \\
2.09378\end{array}$ & $\begin{array}{l}71 \\
72 \\
71 \\
71 \\
71\end{array}$ & $\begin{array}{l}0.93588 \\
0.93583 \\
0.93579 \\
0.93574 \\
0.93569\end{array}$ & $\begin{array}{l}5 \\
4 \\
5 \\
5 \\
5\end{array}$ & $\begin{array}{l}3.03251 \\
3.03175 \\
3.03099 \\
3.03023 \\
3.02947\end{array}$ & $\begin{array}{l}76 \\
76 \\
76 \\
76 \\
76\end{array}$ & $\begin{array}{l}0.99857 \\
0.99857 \\
0.99856 \\
0.99856 \\
0.99856\end{array}$ \\
\hline $\begin{array}{l}0.1316 \\
0.1317 \\
0.1318 \\
0.1319 \\
0.1320\end{array}$ & $\begin{array}{l}2.09307 \\
2.09236 \\
2.09165 \\
2.09094 \\
2.09023\end{array}$ & $\begin{array}{l}71 \\
71 \\
71 \\
71 \\
71\end{array}$ & $\begin{array}{l}0.93564 \\
0.93560 \\
0.93555 \\
0.93550 \\
0.93545\end{array}$ & $\begin{array}{l}4 \\
5 \\
5 \\
5 \\
5\end{array}$ & $\begin{array}{l}3.12871 \\
3.02795 \\
3.02719 \\
3.02644 \\
3.02568\end{array}$ & $\begin{array}{l}76 \\
76 \\
75 \\
76 \\
76\end{array}$ & $\begin{array}{l}0.99856 \\
0.99856 \\
0.99855 \\
0.99855 \\
0.99855\end{array}$ \\
\hline $\begin{array}{l}0.1321 \\
0.1322 \\
0.1323 \\
0.1324 \\
0.1325\end{array}$ & $\begin{array}{l}2.08952 \\
2.08881 \\
2.08810 \\
2.08740 \\
2.08669\end{array}$ & $\begin{array}{l}71 \\
71 \\
70 \\
71 \\
70\end{array}$ & $\begin{array}{l}0.93540 \\
0.93536 \\
0.93531 \\
0.93526 \\
0.93521\end{array}$ & $\begin{array}{l}4 \\
5 \\
5 \\
b \\
5\end{array}$ & $\begin{array}{l}3.02492 \\
3.02417 \\
3.02341 \\
3.02266 \\
3.02190\end{array}$ & $\begin{array}{l}75 \\
76 \\
75 \\
76 \\
75\end{array}$ & $\begin{array}{l}0.99855 \\
0.99855 \\
0.99854 \\
0.99854 \\
0.99854\end{array}$ \\
\hline $\begin{array}{l}0.1326 \\
0.1327 \\
0.1328 \\
0.1329 \\
0.1330\end{array}$ & $\begin{array}{l}2.08599 \\
2.08528 \\
2.08458 \\
2.08387 \\
2.08317\end{array}$ & $\begin{array}{l}71 \\
70 \\
71 \\
70 \\
70\end{array}$ & $\begin{array}{l}0.93516 \\
0.93512 \\
0.93507 \\
0.93502 \\
0.93497\end{array}$ & $\begin{array}{l}4 \\
5 \\
5 \\
5 \\
4\end{array}$ & $\begin{array}{l}3.02115 \\
3.02040 \\
3.01963 \\
3.01889 \\
3.01814\end{array}$ & $\begin{array}{l}75 \\
75 \\
76 \\
75 \\
75\end{array}$ & $\begin{array}{l}0.99854 \\
0.99853 \\
0.99853 \\
0.99853 \\
0.99853\end{array}$ \\
\hline $\begin{array}{l}0.1331 \\
0.1332 \\
0.1333 \\
0.1334 \\
0.1335\end{array}$ & $\begin{array}{l}2.08247 \\
2.08176 \\
2.08106 \\
2.08036 \\
2.07966\end{array}$ & $\begin{array}{l}71 \\
70 \\
70 \\
70 \\
70\end{array}$ & $\begin{array}{l}0.93493 \\
0.93488 \\
0.93483 \\
0.93478 \\
0.93473\end{array}$ & $\begin{array}{l}5 \\
5 \\
5 \\
5 \\
4\end{array}$ & $\begin{array}{l}3.01739 \\
3.01664 \\
3.01589 \\
3.01514 \\
3.01440\end{array}$ & $\begin{array}{l}75 \\
75 \\
75 \\
74 \\
75\end{array}$ & $\begin{array}{l}0.99853 \\
0.99352 \\
0.99852 \\
0.99852 \\
0.99852\end{array}$ \\
\hline $\begin{array}{l}0.1336 \\
0.1337 \\
0.1338 \\
0.1339 \\
0.1340\end{array}$ & $\begin{array}{l}2.07896 \\
2.07826 \\
2.07756 \\
2.07687 \\
2.07617\end{array}$ & $\begin{array}{l}70 \\
70 \\
69 \\
70 \\
70\end{array}$ & $\begin{array}{l}0.93469 \\
0.93464 \\
0.93459 \\
0.93454 \\
0.93450\end{array}$ & $\begin{array}{l}5 \\
5 \\
5 \\
4 \\
5\end{array}$ & $\begin{array}{l}3.01365 \\
3.01290 \\
3.01215 \\
3.011141 \\
3.01066\end{array}$ & $\begin{array}{l}75 \\
75 \\
74 \\
75 \\
74\end{array}$ & $\begin{array}{l}0.99851 \\
0.99851 \\
0.99851 \\
0.99851 \\
0.99851\end{array}$ \\
\hline $\begin{array}{l}0.1341 \\
0.1342 \\
0.1343 \\
0.11344 \\
0.1345\end{array}$ & $\begin{array}{l}2.07547 \\
2.07477 \\
2.07408 \\
2.07338 \\
2.07269\end{array}$ & $\begin{array}{l}70 \\
69 \\
70 \\
69 \\
70\end{array}$ & $\begin{array}{l}0.93445 \\
0.93440 \\
0.93435 \\
0.93430 \\
0.93426\end{array}$ & $\begin{array}{l}5 \\
5 \\
5 \\
4 \\
5\end{array}$ & $\begin{array}{l}3.00992 \\
3.00917 \\
3.00843 \\
3.00769 \\
3.00694\end{array}$ & $\begin{array}{l}75 \\
74 \\
74 \\
75 \\
74\end{array}$ & $\begin{array}{l}0.99850 \\
0.99850 \\
0.99850 \\
0.99850 \\
0.99849\end{array}$ \\
\hline $\begin{array}{l}0.1346 \\
0.1347 \\
0.1348 \\
0.1349 \\
0.11350\end{array}$ & $\begin{array}{l}2.07199 \\
2.07130 \\
2.07061 \\
2.06991 \\
2.06922\end{array}$ & $\begin{array}{l}69 \\
69 \\
70 \\
69\end{array}$ & $\begin{array}{l}0.93421 \\
0.93416 \\
0.93411 \\
0.93407 \\
0.93402\end{array}$ & $\begin{array}{l}5 \\
5 \\
4 \\
5\end{array}$ & $\begin{array}{l}3.00620 \\
3.00546 \\
3.00472 \\
3.00398 \\
3.00324\end{array}$ & $\begin{array}{l}74 \\
74 \\
74 \\
74\end{array}$ & $\begin{array}{l}0.99849 \\
0.99849 \\
0.99849 \\
0.99848 \\
0.99848\end{array}$ \\
\hline
\end{tabular}


Table I Harmonic Oscillator Contributions (in dimensionless form) to the Thermodynamic Functions for Values of $\mathrm{X}=\mathrm{hc} / \mathrm{kT}$

\begin{tabular}{|c|c|c|c|c|c|c|c|}
\hline $\bar{Z}$ & $-\left(F^{\circ}-E_{O}\right) / R T$ & $\Delta$ & $\left(H^{\circ}-E_{O}^{\circ}\right) / R T$ & $\Delta$ & So/R & $\Delta$ & $\mathrm{C}_{\mathrm{p}}^{\circ} / \mathrm{R}$ \\
\hline 0.1350 & 2.06922 & 69 & 0.93402 & 5 & 3.00324 & 74 & 0.99848 \\
\hline $\begin{array}{l}0.1351 \\
0.1352\end{array}$ & $\begin{array}{l}2.06853 \\
2.06784\end{array}$ & $\begin{array}{l}69 \\
69\end{array}$ & $\begin{array}{l}0.93397 \\
0.93392\end{array}$ & $\begin{array}{l}5 \\
4\end{array}$ & $\begin{array}{l}3.00250 \\
3.00176\end{array}$ & $\begin{array}{l}74 \\
74\end{array}$ & $\begin{array}{l}0.99848 \\
0.99848\end{array}$ \\
\hline 0.1353 & 2.06715 & 69 & 0.93388 & 5 & 3.00102 & 73 & 0.99848 \\
\hline 0.1354 & 2.06646 & 69 & 0.93383 & 5 & 3.00029 & 74 & 0.99847 \\
\hline 0.1355 & 2.06577 & 69 & 0.93378 & 5 & 2.99955 & 74 & 0.99847 \\
\hline 0.1356 & 2.06508 & 69 & 0.93373 & 5 & 2.99881 & 73 & 0.99847 \\
\hline 0.1357 & 2.06439 & 69 & 0.93368 & 4 & 2.99808 & 74 & 0.99847 \\
\hline 0.1358 & 2.06370 & 68 & 0.93364 & 5 & 2.99734 & 73 & 0.99846 \\
\hline 0.1359 & 2.06302 & 69 & 0.93359 & 5 & 2.99661 & 74 & 0.99846 \\
\hline 0.1360 & 2.06233 & 69 & 0.93354 & 5 & 2.99587 & 73 & 0.99846 \\
\hline 0.1361 & 2.06164 & 68 & 0.93349 & 4 & 2.99514 & 74 & 0.99846 \\
\hline 0.1362 & 2.06096 & 69 & 0.93345 & 5 & 2.99440 & 73 & 0.99846 \\
\hline 0.1363 & 2.06027 & 68 & 0.93340 & 5 & 2.99367 & 73 & 0.99845 \\
\hline 0.1364 & 2.05959 & 69 & 0.93335 & 5 & 2.99294 & 73 & 0.99845 \\
\hline 0.1365 & 2.05890 & 68 & 0.93330 & 5 & 2.99221 & 73 & 0.99845 \\
\hline 0.1366 & 2.05822 & 68 & 0.93325 & 4 & 2.99148 & 74 & 0.99845 \\
\hline 0.1367 & 2.05754 & 68 & 0.93321 & 5 & 2.99074 & 73 & 0.99844 \\
\hline 0.1368 & 2.05686 & 69 & 0.93316 & 5 & 2.99001 & 72 & 0.99844 \\
\hline 0.1369 & 2.05617 & 68 & 0.93311 & 5 & 2.98929 & 73 & 0.99844 \\
\hline 0.1370 & 2.05549 & 68 & 0.93306 & 4 & 2.98856 & 73 & 0.99844 \\
\hline 0.1371 & 2.05481 & 68 & 0.93302 & 5 & 2.98783 & 73 & 0.99844 \\
\hline 0.1372 & 2.05413 & 68 & 0.93297 & 5 & 2.98710 & 73 & 0.99843 \\
\hline 0.1373 & 2.05345 & 68 & 0.93292 & 5 & 2.98637 & 72 & 0.99843 \\
\hline 0.1374 & 2.05277 & 68 & 0.93287 & 4 & 2.98565 & 73 & 0.99843 \\
\hline 0.1375 & 2.05209 & 67 & 0.93283 & 5 & 2.98492 & 73 & 0.99843 \\
\hline 0.1376 & 2.05142 & 68 & 0.93278 & 5 & $2.984 ! 9$ & 72 & 0.99842 \\
\hline 0.1377 & 2.05074 & 68 & 0.93273 & 5 & 2.98347 & 73 & 0.99842 \\
\hline 0.1378 & 2.05006 & 68 & 0.93268 & 3 & 2.98274 & 72 & 0.99842 \\
\hline 0.1379 & 2.04938 & 67 & 0.93263 & 4 & 2.98202 & 73 & 0.99842 \\
\hline 0.1380 & 2.04871 & 68 & 0.93259 & 5 & 2.98129 & 72 & 0.99841 \\
\hline 0.1381 & 2.04803 & 67 & 0.93254 & 5 & 2.98057 & 72 & 0.99841 \\
\hline 0.1382 & 2.04736 & 68 & 0.93249 & 5 & 2.97985 & 72 & 0.99841 \\
\hline 0.1383 & 2.04668 & 67 & 0.93244 & 4 & 2.97913 & 72 & 0.99841 \\
\hline 0.1384 & 2.04601 & 67 & 0.93240 & 5 & 2.97841 & 73 & 0.99841 \\
\hline 0.1385 & 2.04534 & 68 & 0.93235 & 5 & 2.97768 & 72 & 0.99840 \\
\hline $\begin{array}{l}0.1386 \\
0.1387\end{array}$ & $\begin{array}{l}2.04466 \\
2.04399\end{array}$ & $\begin{array}{l}67 \\
67\end{array}$ & $\begin{array}{l}0.93230 \\
0.93225\end{array}$ & $\begin{array}{l}5 \\
5\end{array}$ & $\begin{array}{l}2.97696 \\
2.97624\end{array}$ & $\begin{array}{l}72 \\
72\end{array}$ & $\begin{array}{l}0.99840 \\
0.99840\end{array}$ \\
\hline 0.1388 & 2.04332 & 67 & 0.93220 & 4 & 2.97552 & 72 & 0.99840 \\
\hline 0.1389 & 2.04265 & 67 & 0.93216 & 5 & 2.97480 & 71 & 0.99839 \\
\hline 0.1390 & $2 \cdot 04198$ & 67 & 0.93211 & 5 & 2.97409 & 72 & 0.99839 \\
\hline 0.1391 & 2.04131 & 67 & 0.93206 & 5 & 2.97337 & 72 & 0.99839 \\
\hline 0.1392 & 2.04064 & 67 & 0.93201 & 4 & 2.97265 & 72 & 0.99839 \\
\hline 0.1393 & 2.03997 & 67 & 0.93197 & 5 & 2.97193 & 71 & 0.99838 \\
\hline 0.1394 & 2.03930 & 67 & 0.93192 & 5 & 2.97122 & 72 & 0.99838 \\
\hline 0.1395 & 2.03863 & 67 & 0.93187 & 5 & 2.97050 & 71 & 0.99838 \\
\hline 0.1396 & 2.03796 & 67 & 0.93182 & 4 & 2.96979 & 72 & 0.99838 \\
\hline 0.1397 & 2.03729 & 66 & 0.93178 & 5 & 2.96907 & 71 & 0.99838 \\
\hline 0.1398 & 2.03663 & 67 & 0.93173 & 5 & 2.96836 & 72 & 0.99837 \\
\hline 0.1399 & 2.03596 & 66 & 0.93168 & 5 & 2.96764 & 71 & 0.99837 \\
\hline 0.1400 & 2.03530 & & 0.93163 & & 2.96693 & & 0.99837 \\
\hline
\end{tabular}


Table I Harmonic Oscillator Contributions (in dimensionless form) to the Thermodynamic Functions for Values of $X=h c v / k T$

\begin{tabular}{|c|c|c|c|c|c|c|c|}
\hline $\mathbf{X}$ & $-\left(F^{\circ}-E_{O} \circ\right) / R T$ & $\Delta$ & $\left(\mathrm{H}^{\circ}-\mathrm{E}_{\mathrm{O}}^{\mathrm{O}}\right) / \mathrm{RT}$ & $\Delta$ & $S \circ / R$ & $\Delta$ & $\mathrm{C}_{\mathrm{p}}^{\circ} / \mathrm{R}$ \\
\hline 0.1400 & 2.03530 & 67 & 0.93163 & 4 & 2.96693 & 71 & 0.99837 \\
\hline $\begin{array}{l}0.1401 \\
0.1402 \\
0.1403 \\
0.1404 \\
0.1405\end{array}$ & $\begin{array}{l}2.03463 \\
2.03397 \\
2.03330 \\
2.03264 \\
2.03198\end{array}$ & $\begin{array}{l}66 \\
67 \\
66 \\
66 \\
67\end{array}$ & $\begin{array}{l}0.93159 \\
0.93154 \\
0.93149 \\
0.93144 \\
0.93139\end{array}$ & $\begin{array}{l}5 \\
5 \\
5 \\
5 \\
4\end{array}$ & $\begin{array}{l}2.96622 \\
2.96550 \\
2.96479 \\
2.96408 \\
2.96337\end{array}$ & $\begin{array}{l}72 \\
71 \\
71 \\
71 \\
71\end{array}$ & $\begin{array}{l}0.99837 \\
0.99836 \\
0.99836 \\
0.99836 \\
0.99836\end{array}$ \\
\hline $\begin{array}{l}0.1406 \\
0.1407 \\
0.1408 \\
0.1409 \\
0.1410\end{array}$ & $\begin{array}{l}2.03131 \\
2.03065 \\
2.02999 \\
2.02933 \\
2.02867\end{array}$ & $\begin{array}{l}66 \\
66 \\
66 \\
66 \\
66\end{array}$ & $\begin{array}{l}0.93135 \\
0.93130 \\
0.93125 \\
0.93120 \\
0.93116\end{array}$ & $\begin{array}{l}5 \\
5 \\
5 \\
4 \\
5\end{array}$ & $\begin{array}{l}2.96266 \\
2.96195 \\
2.96124 \\
2.96053 \\
2.95982\end{array}$ & $\begin{array}{l}71 \\
71 \\
71 \\
71 \\
70\end{array}$ & $\begin{array}{l}0.99835 \\
0.99835 \\
0.99835 \\
0.99835 \\
0.99835\end{array}$ \\
\hline $\begin{array}{l}0.1411 \\
0.1412 \\
0.1413 \\
0.1414 \\
0.1415\end{array}$ & $\begin{array}{l}2.02801 \\
2.02735 \\
2.02669 \\
2.02603 \\
2.02537\end{array}$ & $\begin{array}{l}66 \\
66 \\
66 \\
66 \\
66\end{array}$ & $\begin{array}{l}0.93111 \\
0.93106 \\
0.93101 \\
0.93097 \\
0.93092\end{array}$ & $\begin{array}{l}5 \\
5 \\
4 \\
5 \\
5\end{array}$ & $\begin{array}{l}2.95912 \\
2.95841 \\
2.95770 \\
2.95700 \\
2.95629\end{array}$ & $\begin{array}{l}71 \\
71 \\
70 \\
71 \\
71\end{array}$ & $\begin{array}{l}0.99834 \\
0.99834 \\
0.99834 \\
0.99834 \\
0.99833\end{array}$ \\
\hline $\begin{array}{l}0.1416 \\
0.1417 \\
0.1418 \\
0.1119 \\
0.1420\end{array}$ & $\begin{array}{l}2.02471 \\
2.02406 \\
2.02340 \\
2.02274 \\
2.02209\end{array}$ & $\begin{array}{l}65 \\
66 \\
66 \\
65 \\
66\end{array}$ & $\begin{array}{l}0.93087 \\
0.93082 \\
0.93078 \\
0.93073 \\
0.93068\end{array}$ & $\begin{array}{l}5 \\
4 \\
5 \\
5 \\
5\end{array}$ & $\begin{array}{l}2.95558 \\
2.95488 \\
2.95418 \\
2.95347 \\
2.95277\end{array}$ & $\begin{array}{l}70 \\
70 \\
71 \\
70 \\
70\end{array}$ & $\begin{array}{l}0.99833 \\
0.99833 \\
0.99833 \\
0.99832 \\
0.99832\end{array}$ \\
\hline $\begin{array}{l}0.1421 \\
0.1422 \\
0.1423 \\
0.1424 \\
0.1425\end{array}$ & $\begin{array}{l}2.02143 \\
2.02078 \\
2.02012 \\
2.01947 \\
2.01882\end{array}$ & $\begin{array}{l}65 \\
66 \\
65 \\
65 \\
66\end{array}$ & $\begin{array}{l}0.93063 \\
0.93058 \\
0.93054 \\
0.93049 \\
0.93044\end{array}$ & $\begin{array}{l}5 \\
4 \\
5 \\
5 \\
5\end{array}$ & $\begin{array}{l}2.95207 \\
2.95136 \\
2.95066 \\
2.94996 \\
2.94926\end{array}$ & $\begin{array}{l}71 \\
70 \\
70 \\
70 \\
70\end{array}$ & $\begin{array}{l}0.99832 \\
0.99832 \\
0.99831 \\
0.99831 \\
0.99831\end{array}$ \\
\hline $\begin{array}{l}0.1426 \\
0.1427 \\
0.1428 \\
0.1429 \\
0.1430\end{array}$ & $\begin{array}{l}2.01816 \\
2.01751 \\
2.01686 \\
2.01621 \\
2.01556\end{array}$ & $\begin{array}{l}65 \\
65 \\
65 \\
65 \\
65\end{array}$ & $\begin{array}{l}0.93039 \\
0.93035 \\
0.93030 \\
0.93025 \\
0.93020\end{array}$ & $\begin{array}{l}4 \\
5 \\
5 \\
5 \\
4\end{array}$ & $\begin{array}{l}2.94856 \\
2.94786 \\
2.94716 \\
2.94646 \\
2.94576\end{array}$ & $\begin{array}{l}70 \\
70 \\
70 \\
70 \\
70\end{array}$ & $\begin{array}{l}0.99831 \\
0.99830 \\
0.99830 \\
0.99830 \\
0.99830\end{array}$ \\
\hline $\begin{array}{l}0.1431 \\
0.1432 \\
0.1433 \\
0.1434 \\
0.1435\end{array}$ & $\begin{array}{l}2.01491 \\
2.01426 \\
2.01361 \\
2.01296 \\
2.01231\end{array}$ & $\begin{array}{l}65 \\
65 \\
65 \\
65 \\
65\end{array}$ & $\begin{array}{l}0.93016 \\
0.93011 \\
0.93006 \\
0.93001 \\
0.92997\end{array}$ & $\begin{array}{l}5 \\
5 \\
5 \\
4 \\
5\end{array}$ & $\begin{array}{l}2.94506 \\
2.94437 \\
2.94367 \\
2.94297 \\
2.94228\end{array}$ & $\begin{array}{l}69 \\
70 \\
70 \\
69 \\
70\end{array}$ & $\begin{array}{l}0.99830 \\
0.99829 \\
0.99829 \\
0.99829 \\
0.99829\end{array}$ \\
\hline $\begin{array}{l}0.1436 \\
0.1437 \\
0.1438 \\
0.1439 \\
0.1440\end{array}$ & $\begin{array}{l}2.01166 \\
2.01102 \\
2.01037 \\
2.00972 \\
2.00908\end{array}$ & $\begin{array}{l}64 \\
65 \\
65 \\
64 \\
65\end{array}$ & $\begin{array}{l}0.92992 \\
0.92987 \\
0.92982 \\
0.92978 \\
0.92973\end{array}$ & $\begin{array}{l}5 \\
5 \\
4 \\
5 \\
5\end{array}$ & $\begin{array}{l}2.94158 \\
2.94039 \\
2.940119 \\
2.93950 \\
2.93881\end{array}$ & $\begin{array}{l}69 \\
70 \\
69 \\
69 \\
70\end{array}$ & $\begin{array}{l}0.99828 \\
0.99828 \\
0.99828 \\
0.99828 \\
0.99827\end{array}$ \\
\hline $\begin{array}{l}0.1441 \\
0.1442 \\
0.1443 \\
0.1444 \\
0.1445\end{array}$ & $\begin{array}{l}2.00843 \\
2.00779 \\
2.00714 \\
2.00650 \\
2.00586\end{array}$ & $\begin{array}{l}64 \\
65 \\
64 \\
64 \\
65\end{array}$ & $\begin{array}{l}0.92968 \\
0.92963 \\
0.92958 \\
0.92954 \\
0.92949\end{array}$ & $\begin{array}{l}5 \\
5 \\
4 \\
5 \\
5\end{array}$ & $\begin{array}{l}2.93811 \\
2.93742 \\
2.93673 \\
2.93604 \\
2.93535\end{array}$ & $\begin{array}{l}69 \\
69 \\
69 \\
69 \\
70\end{array}$ & $\begin{array}{l}0.99827 \\
0.99827 \\
0.99827 \\
0.99826 \\
0.99826\end{array}$ \\
\hline $\begin{array}{l}0.1446 \\
0.1447 \\
0.1448 \\
0.1449 \\
0.1450\end{array}$ & $\begin{array}{l}2.00521 \\
2.00457 \\
2.00393 \\
2.00329 \\
2.00265\end{array}$ & $\begin{array}{l}64 \\
64 \\
64 \\
64\end{array}$ & $\begin{array}{l}0.92944 \\
0.92939 \\
0.92935 \\
0.92930 \\
0.92925\end{array}$ & $\begin{array}{l}5 \\
4 \\
5 \\
5\end{array}$ & $\begin{array}{l}2.93465 \\
2.93396 \\
2.93328 \\
2.93259 \\
2.93190\end{array}$ & $\begin{array}{l}69 \\
68 \\
69 \\
69\end{array}$ & $\begin{array}{l}0.99826 \\
0.99826 \\
0.99825 \\
0.99825 \\
0.99825\end{array}$ \\
\hline
\end{tabular}


Table I Harmonic Oscillator Contributions (in dimensionless form) to the Thermodynamic Functions for Values of $\mathrm{X}=\mathrm{hcv} / \mathrm{kT}$

\begin{tabular}{|c|c|c|c|c|c|c|c|}
\hline $\mathbf{X}$ & $-\left(F^{\circ}-E_{O}^{\circ}\right) / R T$ & $\Delta$ & $\left(\mathrm{H}^{\circ}-\mathrm{E}_{\mathrm{O}}^{\circ}\right) / \mathrm{RT}$ & $\Delta$ & So/R & $\Delta$ & $C_{\mathrm{p}}^{\circ} / \mathrm{R}$ \\
\hline 0.1450 & 2.00265 & 65 & 0.92925 & 5 & 2.93190 & 69 & 0.99825 \\
\hline $\begin{array}{l}0.1451 \\
0.1452 \\
0.1453 \\
0.1454 \\
0.1455\end{array}$ & $\begin{array}{l}2.00200 \\
2.00136 \\
2.00073 \\
2.00009 \\
1.99945\end{array}$ & $\begin{array}{l}64 \\
63 \\
64 \\
64 \\
64\end{array}$ & $\begin{array}{l}0.92920 \\
0.92916 \\
0.92911 \\
0.92906 \\
0.92901\end{array}$ & $\begin{array}{l}4 \\
5 \\
5 \\
5 \\
4\end{array}$ & $\begin{array}{l}2.93121 \\
2.93052 \\
2.92983 \\
2.92915 \\
2.92846\end{array}$ & $\begin{array}{l}69 \\
69 \\
68 \\
69 \\
69\end{array}$ & $\begin{array}{l}0.99825 \\
0.99824 \\
0.99824 \\
0.99824 \\
0.99824\end{array}$ \\
\hline $\begin{array}{l}0.1456 \\
0.1457 \\
0.1458 \\
0.1459 \\
0.1460\end{array}$ & $\begin{array}{l}1.99881 \\
1.99817 \\
1.99753 \\
1.99690 \\
1.99626\end{array}$ & $\begin{array}{l}64 \\
64 \\
63 \\
64 \\
64\end{array}$ & $\begin{array}{l}0.92897 \\
0.92892 \\
0.92887 \\
0.92882 \\
0.92878\end{array}$ & $\begin{array}{l}5 \\
5 \\
5 \\
4 \\
5\end{array}$ & $\begin{array}{l}2.92777 \\
2.92709 \\
2.92640 \\
2.92572 \\
2.92504\end{array}$ & $\begin{array}{l}68 \\
69 \\
68 \\
68 \\
69\end{array}$ & $\begin{array}{l}0.99824 \\
0.99823 \\
0.99823 \\
0.99823 \\
0.99823\end{array}$ \\
\hline $\begin{array}{l}0.1461 \\
0.1462 \\
0.1463 \\
0.1464 \\
0.1465\end{array}$ & $\begin{array}{l}1.99562 \\
1.99499 \\
1.99435 \\
1.99372 \\
1.99309\end{array}$ & $\begin{array}{l}63 \\
64 \\
63 \\
63 \\
64\end{array}$ & $\begin{array}{l}0.92873 \\
0.92868 \\
0.92863 \\
0.92859 \\
0.92854\end{array}$ & $\begin{array}{l}5 \\
5 \\
4 \\
5 \\
5\end{array}$ & $\begin{array}{l}2.92435 \\
2.92367 \\
2.92299 \\
2.22231 \\
2.92152\end{array}$ & $\begin{array}{l}68 \\
68 \\
68 \\
69 \\
68\end{array}$ & $\begin{array}{l}0.99822 \\
0.99822 \\
0.99822 \\
0.99822 \\
0.99821\end{array}$ \\
\hline $\begin{array}{l}0.1466 \\
0.1467 \\
0.1468 \\
0.1469 \\
0.1470\end{array}$ & $\begin{array}{l}1.99245 \\
1.99182 \\
1.99119 \\
1.99055 \\
1.98992\end{array}$ & $\begin{array}{l}63 \\
63 \\
64 \\
63 \\
63\end{array}$ & $\begin{array}{l}0.92849 \\
0.92844 \\
0.92840 \\
0.92835 \\
0.92830\end{array}$ & $\begin{array}{l}5 \\
4 \\
5 \\
5 \\
5\end{array}$ & $\begin{array}{l}2.92094 \\
2.92026 \\
2.91958 \\
2.91890 \\
2.91822\end{array}$ & $\begin{array}{l}68 \\
68 \\
68 \\
68 \\
68\end{array}$ & $\begin{array}{l}0.99821 \\
0.99821 \\
0.99821 \\
0.99820 \\
0.99820\end{array}$ \\
\hline $\begin{array}{l}0.1471 \\
0.1472 \\
0.1473 \\
0.1474 \\
0.1475\end{array}$ & $\begin{array}{l}1.98929 \\
1.98866 \\
1.98803 \\
1.98740 \\
1.98677\end{array}$ & $\begin{array}{l}63 \\
63 \\
63 \\
63 \\
63\end{array}$ & $\begin{array}{l}0.92825 \\
0.92820 \\
0.92816 \\
0.92811 \\
0.92806\end{array}$ & $\begin{array}{l}5 \\
4 \\
5 \\
5 \\
5\end{array}$ & $\begin{array}{l}2.91754 \\
2.91687 \\
2.91619 \\
2.91551 \\
2.91483\end{array}$ & $\begin{array}{l}67 \\
68 \\
68 \\
68 \\
67\end{array}$ & $\begin{array}{l}0.99820 \\
0.99820 \\
0.99819 \\
0.99819 \\
0.99819\end{array}$ \\
\hline $\begin{array}{l}0.1476 \\
0.1477 \\
0.1478 \\
0.1+79 \\
0.1480\end{array}$ & $\begin{array}{l}1.98614 \\
1.98551 \\
1.98489 \\
1.98426 \\
1.98363\end{array}$ & $\begin{array}{l}63 \\
62 \\
63 \\
63 \\
63\end{array}$ & $\begin{array}{l}0.92801 \\
0.92797 \\
0.92792 \\
0.92787 \\
0.92782\end{array}$ & $\begin{array}{l}4 \\
5 \\
5 \\
5 \\
4\end{array}$ & $\begin{array}{l}2.91416 \\
2.91348 \\
2.91280 \\
2.91213 \\
2.91146\end{array}$ & $\begin{array}{l}68 \\
68 \\
67 \\
67 \\
68\end{array}$ & $\begin{array}{l}0.99819 \\
0.99818 \\
0.99818 \\
0.99818 \\
0.99818\end{array}$ \\
\hline $\begin{array}{l}0.1481 \\
0.1482 \\
0.1483 \\
0.1484 \\
0.1485\end{array}$ & $\begin{array}{l}1.98300 \\
1.98238 \\
1.98175 \\
1.98113 \\
1.98050\end{array}$ & $\begin{array}{l}62 \\
63 \\
62 \\
63 \\
62\end{array}$ & $\begin{array}{l}0.92778 \\
0.92773 \\
0.92768 \\
0.92763 \\
0.92759\end{array}$ & $\begin{array}{l}5 \\
5 \\
5 \\
4 \\
5\end{array}$ & $\begin{array}{l}2.91078 \\
2.91011 \\
2.90943 \\
2.90876 \\
2.90809\end{array}$ & $\begin{array}{l}67 \\
68 \\
67 \\
67 \\
67\end{array}$ & $\begin{array}{l}0.99817 \\
0.99817 \\
0.99817 \\
0.99817 \\
0.99816\end{array}$ \\
\hline $\begin{array}{l}0.1486 \\
0.1487 \\
0.1488 \\
0.1489 \\
0.1490\end{array}$ & $\begin{array}{l}1.97988 \\
1.97925 \\
1.97863 \\
1.97801 \\
1.97738\end{array}$ & $\begin{array}{l}63 \\
62 \\
62 \\
63 \\
62\end{array}$ & $\begin{array}{l}0.92754 \\
0.92749 \\
0.92744 \\
0.92740 \\
0.92735\end{array}$ & $\begin{array}{l}5 \\
5 \\
4 \\
5 \\
5\end{array}$ & $\begin{array}{l}2.90742 \\
2.90675 \\
2.90607 \\
2.90540 \\
2.90473\end{array}$ & $\begin{array}{l}67 \\
68 \\
67 \\
67 \\
67\end{array}$ & $\begin{array}{l}0.99816 \\
0.99816 \\
0.99816 \\
0.99815 \\
0.99815\end{array}$ \\
\hline $\begin{array}{l}0.1491 \\
0.1492 \\
0.1493 \\
0.1494 \\
0.1495\end{array}$ & $\begin{array}{l}1.97676 \\
1.97614 \\
1.97552 \\
1.97490 \\
1.97428\end{array}$ & $\begin{array}{l}62 \\
62 \\
62 \\
62 \\
62\end{array}$ & $\begin{array}{l}0.92730 \\
0.92725 \\
0.92721 \\
0.92716 \\
0.92711\end{array}$ & $\begin{array}{l}5 \\
4 \\
5 \\
5 \\
5\end{array}$ & $\begin{array}{l}2.90406 \\
2.90339 \\
2.90273 \\
2.90206 \\
2.90139\end{array}$ & $\begin{array}{l}67 \\
66 \\
67 \\
67 \\
67\end{array}$ & $\begin{array}{l}0.99815 \\
0.99815 \\
0.99814 \\
0.99814 \\
0.99814\end{array}$ \\
\hline $\begin{array}{l}0.1496 \\
0.1497 \\
0.1498 \\
0.1499 \\
0.1500\end{array}$ & $\begin{array}{l}1.97366 \\
1.97304 \\
1.97242 \\
1.97180 \\
1.97118\end{array}$ & $\begin{array}{l}62 \\
62 \\
62 \\
62\end{array}$ & $\begin{array}{l}0.92706 \\
0.92702 \\
0.92697 \\
0.92692 \\
0.92687\end{array}$ & $\begin{array}{l}4 \\
5 \\
5 \\
5\end{array}$ & $\begin{array}{l}2.90072 \\
2.90006 \\
2.89939 \\
2.89872 \\
2.89806\end{array}$ & $\begin{array}{l}66 \\
67 \\
67 \\
66\end{array}$ & $\begin{array}{l}0.99814 \\
0.99813 \\
0.99813 \\
0.99813 \\
0.99813\end{array}$ \\
\hline
\end{tabular}


Table I Harmonic Oscillator Contributions (in dimensionless form) to the Thermodynamic Functions for Values of $X=h c v / k T$

$\mathbf{X}$

0.150

0.151

0.152

0.153

0.154

0.155

0.156

0.157

0.158

0.159

0.160

0.161

0.162

0.163

0.164

0.165

0.166

0.167

0.168

0.169

0.170

0.171

0.172

0.173

0.174

0.175

0.176

0.177

0.178

0.179

0.180

0.181

0.182

0.183

0.184

0.185

0.186

0.187

0.188

0.189

0.190

0.191

0.192

0.193

0.194

0.195

0.196

0.197

0.198

0.199

0.200
$-\left(F^{\circ}-E_{0}^{\circ}\right) / R T$

$\Delta$

1.97118
1.96503
1.95891
1.95284
1.94681
1.94083
1.93489
1.92898
1.92312
1.91730
1.91151

615
612
607
603
598
594
591
586
582
579
574

1. 90577

1. 90007

1.89440

1.88877

1.88318

1.87762

1.87210

1.86662

1.86117

1.85575

1.85037

1.84503

1.83972

1.83444

1.82919

1.82398

1.81880

1.81365

1.80853

1. 80345

1.79839

1.79337

1.78837

1.78341

1. 77847

1.77357

1.76869

1.76384

1.75902

1.75423

1.74946

1.74472

1.74001

1.73533

1.73067

1.72604

1. 72144

1.71686

1.71230

1.70777
570

567

563

559

556

552

548

545

542

538

534

531

528

525

521

518

515

512

508

506

502

500

496

494

490

488

485

482

479

477

474

471

468

466

463

460

458

456

453
$\left(\mathrm{H}^{\circ}-\mathrm{E}_{\mathrm{O}}^{\circ}\right) / \mathrm{RT} \quad \Delta \quad \mathrm{S} \% / \mathrm{R}$

0.92687

0.92640
0.92592

0.92545

0.92498

0.92450

0.92403

0.92355

0.92308

0.92261

0.92213

0.92166

0.92119

0.92071

0.92024

0.91977

0.91930

0.91882

0.91835

0.91788

0.91741

0.91694

0.91646

0.91599

0.91552

0.91505

0.91458

0.91411

0.91364

0.91317

0.91270

0.91223

0.91176

0.91129

0.91082

0.91035

0.90988

0.90941

0.90894

0.90848

0.90801

0.90754

0.90707

0.90660

0.90613

0.90567

0.90520

0.90473

0.90426

0.90380

0.90333

$\begin{array}{ll}47 & 2.89806 \\ & \\ 48 & 2.89142 \\ 47 & 2.88484 \\ 47 & 2.87829 \\ 48 & 2.87179 \\ 47 & 2.86533 \\ 48 & 2.85891 \\ 47 & 2.85254 \\ 47 & 2.84620 \\ 48 & 2.83990 \\ 47 & 2.83365\end{array}$

$47 \quad 2.82743$

$48 \quad 2.82125$

$47 \quad 2.81511$

$47 \quad 2.80901$

$47 \quad 2.80294$

$48 \quad 2.79691$

$47 \quad 2.79092$

$47 \quad 2.78497$

$47 \quad 2.77905$

47

2.77316

$48 \quad 2.76731$

$47 \quad 2.76149$

$47 \quad 2.75571$

$47 \quad 2.74996$

$47 \quad 2.74424$

$47 \quad 2.73856$

$47 \quad 2.73291$

$47 \quad 2.72729$

$47 \quad 2.72170$

$47 \quad 2.71615$

$47 \quad 2.71062$

$47 \quad 2.70513$

$47 \quad 2.69966$

$47 \quad 2.69423$

$47 \quad 2.68882$

$47 \quad 2.68345$

$47 \quad 2.67810$

$46 \quad 2.67278$

$47 \quad 2.66750$

$47 \quad 2.66223$

$47 \quad 2.65700$

$47 \quad 2.65179$

$47 \quad 2.64662$

$46 \quad 2.64146$

$47 \quad 2.63634$

$47 \quad 2.63124$

$47 \quad 2.62617$

$46 \quad 2.62112$

$47 \quad 2.61610$

2.61110

$\Delta \quad \mathrm{co} / \mathrm{R}$

$\Delta$

$\begin{array}{lll}664 & 0.99813 & 3 \\ 658 & 0.99810 & 2 \\ 655 & 0.99808 & 3 \\ 650 & 0.99805 & 2 \\ 646 & 0.99803 & 3 \\ 642 & 0.99800 & 3 \\ 637 & 0.99797 & 2 \\ 634 & 0.99795 & 3 \\ 630 & 0.99792 & 2 \\ 625 & 0.99790 & 3 \\ 622 & 0.99787 & 3\end{array}$

618

614

610

607

603

599

595

592

589

585

582

578

575

572

568

565

562

559

555

553

549

547

543

541

537

535

532

528

527

523

521

517

516

512

510

$\begin{array}{ll}0.99784 & 2 \\ 0.99782 & 3 \\ 0.99779 & 3 \\ 0.99776 & 3 \\ 0.99773 & 2\end{array}$

0.99771

0.99768

0.99765

0.99762

0.99760

0.99757

0.99754

0.99751

0.99748

0.99745

0.99742

0.99739

0.99736

0.99733

0.99730

0.99727

0.99724

0.99721 
Table I Harmonic Oscillator Contributions (in dimensionless form) to the Thermodynamic Functions for Values of $\mathrm{X}=\mathrm{hcv} / \mathrm{kT}$
女
$-\left(\mathrm{F}^{\circ}-\mathrm{E} \mathrm{O}\right) / \mathrm{RT}$
$\Delta \quad\left(\mathrm{H}^{\circ}-\mathrm{EO}\right) / \mathrm{RT}$
$\Delta$
So/R
$\Delta \quad \mathrm{CO} / \mathrm{R}$

$\Delta$

\begin{tabular}{|c|c|c|c|c|c|c|c|}
\hline 0.200 & 1.70777 & 450 & 0.90333 & 47 & 2.61110 & 497 & 0.99667 \\
\hline $\begin{array}{l}0.201 \\
0.202 \\
0.203 \\
0.204 \\
0.205\end{array}$ & $\begin{array}{l}1.70327 \\
1.69879 \\
1.69433 \\
1.68990 \\
1.68549\end{array}$ & $\begin{array}{l}448 \\
446 \\
443 \\
441 \\
438\end{array}$ & $\begin{array}{l}0.90286 \\
0.90240 \\
0.90193 \\
0.90147 \\
0.90100\end{array}$ & $\begin{array}{l}46 \\
47 \\
46 \\
47 \\
47\end{array}$ & $\begin{array}{l}2.60613 \\
2.60119 \\
2.59626 \\
2.59137 \\
2.58649\end{array}$ & $\begin{array}{l}494 \\
493 \\
489 \\
488 \\
484\end{array}$ & $\begin{array}{l}0.99664 \\
0.99661 \\
0.99657 \\
0.99654 \\
0.99651\end{array}$ \\
\hline $\begin{array}{l}0.206 \\
0.207 \\
0.208 \\
0.209 \\
0.210\end{array}$ & $\begin{array}{l}1.68111 \\
1.67675 \\
1.67242 \\
1.66810 \\
1.66381\end{array}$ & $\begin{array}{l}436 \\
433 \\
432 \\
429 \\
427\end{array}$ & $\begin{array}{l}0.90053 \\
0.90007 \\
0.89960 \\
0.89914 \\
0.89867\end{array}$ & $\begin{array}{l}46 \\
47 \\
46 \\
47 \\
46\end{array}$ & $\begin{array}{l}2.58165 \\
2.57682 \\
2.57202 \\
2.56724 \\
2.56248\end{array}$ & $\begin{array}{l}483 \\
480 \\
478 \\
476 \\
473\end{array}$ & $\begin{array}{l}0.99647 \\
0.99644 \\
0.99640 \\
0.99637 \\
0.99633\end{array}$ \\
\hline $\begin{array}{l}0.211 \\
0.212 \\
0.213 \\
0.214 \\
0.215\end{array}$ & $\begin{array}{l}1.65954 \\
1.65530 \\
1.65107 \\
1.64687 \\
1.64269\end{array}$ & $\begin{array}{l}424 \\
423 \\
420 \\
418 \\
416\end{array}$ & $\begin{array}{l}0.89821 \\
0.89774 \\
0.89728 \\
0.89681 \\
0.89635\end{array}$ & $\begin{array}{l}47 \\
46 \\
47 \\
46 \\
47\end{array}$ & $\begin{array}{l}2.55775 \\
2.55304 \\
2.54835 \\
2.54369 \\
2.53904\end{array}$ & $\begin{array}{l}471 \\
469 \\
466 \\
465 \\
462\end{array}$ & $\begin{array}{l}0.99630 \\
0.99626 \\
0.99623 \\
0.99619 \\
0.99616\end{array}$ \\
\hline $\begin{array}{l}0.216 \\
0.217 \\
0.218 \\
0.219 \\
0.220\end{array}$ & $\begin{array}{l}1.63853 \\
1.63440 \\
1.63028 \\
1.62619 \\
1.62211\end{array}$ & $\begin{array}{l}413 \\
412 \\
409 \\
408 \\
405\end{array}$ & $\begin{array}{l}0.89588 \\
0.89542 \\
0.89496 \\
0.89449 \\
0.89403\end{array}$ & $\begin{array}{l}46 \\
46 \\
47 \\
46 \\
46\end{array}$ & $\begin{array}{l}2.53442 \\
2.52982 \\
2.52524 \\
2.52068 \\
2.51614\end{array}$ & $\begin{array}{l}460 \\
458 \\
456 \\
454 \\
451\end{array}$ & $\begin{array}{l}0.99612 \\
0.99609 \\
0.99605 \\
0.99601 \\
0.99598\end{array}$ \\
\hline $\begin{array}{l}0.221 \\
0.222 \\
0.223 \\
0.224 \\
0.225\end{array}$ & $\begin{array}{l}1.61806 \\
1.61403 \\
1.61001 \\
1.60602 \\
1.60205\end{array}$ & $\begin{array}{l}403 \\
402 \\
399 \\
397 \\
396\end{array}$ & $\begin{array}{l}0.89357 \\
0.89310 \\
0.89264 \\
0.89218 \\
0.89172\end{array}$ & $\begin{array}{l}47 \\
46 \\
46 \\
46 \\
47\end{array}$ & $\begin{array}{l}2.51163 \\
2.50713 \\
2.50265 \\
2.49820 \\
2.49376\end{array}$ & $\begin{array}{l}450 \\
448 \\
445 \\
444 \\
441\end{array}$ & $\begin{array}{l}0.99594 \\
0.99590 \\
0.99587 \\
0.99583 \\
0.99579\end{array}$ \\
\hline $\begin{array}{l}0.226 \\
0.227 \\
0.228 \\
0.229 \\
0.230\end{array}$ & $\begin{array}{l}1.59809 \\
1.59416 \\
1.59024 \\
1.58635 \\
1.58247\end{array}$ & $\begin{array}{l}393 \\
392 \\
389 \\
388 \\
385\end{array}$ & $\begin{array}{l}0.89125 \\
0.89079 \\
0.89033 \\
0.88987 \\
0.88940\end{array}$ & $\begin{array}{l}46 \\
46 \\
46 \\
47 \\
46\end{array}$ & $\begin{array}{l}2.48935 \\
2.48495 \\
2.48057 \\
2.47622 \\
2.47188\end{array}$ & $\begin{array}{l}440 \\
438 \\
435 \\
434 \\
432\end{array}$ & $\begin{array}{l}0.99575 \\
0.99572 \\
0.99568 \\
0.99564 \\
0.99560\end{array}$ \\
\hline $\begin{array}{l}0.231 \\
0.232 \\
0.233 \\
0.234 \\
0.235\end{array}$ & $\begin{array}{l}1.57862 \\
1.57478 \\
1.57096 \\
1.56715 \\
1.56337\end{array}$ & $\begin{array}{l}384 \\
382 \\
381 \\
378 \\
377\end{array}$ & $\begin{array}{l}0.88894 \\
0.88848 \\
0.88802 \\
0.88756 \\
0.88710\end{array}$ & $\begin{array}{l}46 \\
46 \\
46 \\
46 \\
46\end{array}$ & $\begin{array}{l}2.46756 \\
2.46326 \\
2.458 .98 \\
2.45471 \\
2.45047\end{array}$ & $\begin{array}{l}430 \\
428 \\
427 \\
424 \\
423\end{array}$ & $\begin{array}{l}0.99557 \\
0.99553 \\
0.99549 \\
0.99545 \\
0.99541\end{array}$ \\
\hline $\begin{array}{l}0.236 \\
0.237 \\
0.238 \\
0.239 \\
0.240\end{array}$ & $\begin{array}{l}1.55960 \\
1.55586 \\
1.55213 \\
1.54841 \\
1.54472\end{array}$ & $\begin{array}{l}374 \\
373 \\
372 \\
369 \\
368\end{array}$ & $\begin{array}{l}0.88664 \\
0.88618 \\
0.88572 \\
0.88526 \\
0.88480\end{array}$ & $\begin{array}{l}46 \\
46 \\
46 \\
46 \\
46\end{array}$ & $\begin{array}{l}2.44624 \\
2.44203 \\
2.43784 \\
2.43367 \\
2.42951\end{array}$ & $\begin{array}{l}421 \\
419 \\
417 \\
416 \\
414\end{array}$ & $\begin{array}{l}0.99537 \\
0.99533 \\
0.99529 \\
0.99525 \\
0.99521\end{array}$ \\
\hline $\begin{array}{l}0.241 \\
0.242 \\
0.243 \\
0.244 \\
0.245\end{array}$ & $\begin{array}{l}1.54104 \\
1.53738 \\
1.53373 \\
1.53011 \\
1.52650\end{array}$ & $\begin{array}{l}366 \\
365 \\
362 \\
361 \\
360\end{array}$ & $\begin{array}{l}0.88434 \\
0.88388 \\
0.88342 \\
0.88296 \\
0.88250\end{array}$ & $\begin{array}{l}46 \\
46 \\
46 \\
46 \\
46\end{array}$ & $\begin{array}{l}2.42537 \\
2.42125 \\
2.41715 \\
2.41306 \\
2.40899\end{array}$ & $\begin{array}{l}412 \\
410 \\
409 \\
407 \\
405\end{array}$ & $\begin{array}{l}0.99517 \\
0.99513 \\
0.99509 \\
0.99505 \\
0.99501\end{array}$ \\
\hline $\begin{array}{l}0.246 \\
0.247 \\
0.248 \\
0.249 \\
0.250\end{array}$ & $\begin{array}{l}1.52290 \\
1.51933 \\
1.51577 \\
1.51222 \\
1.50869\end{array}$ & $\begin{array}{l}357 \\
356 \\
355 \\
353\end{array}$ & $\begin{array}{l}0.88204 \\
0.88158 \\
0.88112 \\
0.88066 \\
0.88020\end{array}$ & $\begin{array}{l}46 \\
46 \\
46 \\
46\end{array}$ & $\begin{array}{l}2.40494 \\
2.40091 \\
2.39689 \\
2.39288 \\
2.38889\end{array}$ & $\begin{array}{l}403 \\
402 \\
401 \\
399\end{array}$ & $\begin{array}{l}0.99497 \\
0.99493 \\
0.99489 \\
0.99485 \\
0.99481\end{array}$ \\
\hline
\end{tabular}


Table I Harmonic Oscillator Contributions (in dimensionless form) to the Thermodynamic Functions for Values of $X=h c v / k T$

$\begin{array}{lllllllll}\mathbf{X} & -\left(\mathrm{F}^{\circ}-\mathrm{E}_{\mathrm{O}}^{\circ}\right) / \mathrm{RT} & \Delta & \left(\mathrm{H}^{\circ}-\mathrm{E}_{\mathrm{O}}\right) / \mathrm{RT} & \Delta & \mathrm{SO} / \mathrm{R} \quad \Delta \quad \mathrm{CO} / \mathrm{R}\end{array}$

$\Delta$

\begin{tabular}{|c|c|c|c|c|c|c|c|}
\hline 0.250 & 1.50869 & 351 & 0.88020 & 46 & 2.38889 & 397 & 0.99481 \\
\hline $\begin{array}{l}0.251 \\
0.252\end{array}$ & $\begin{array}{l}1.50518 \\
1.50168\end{array}$ & $\begin{array}{l}350 \\
348\end{array}$ & $\begin{array}{l}0.87974 \\
0.87929\end{array}$ & $\begin{array}{l}45 \\
46\end{array}$ & $\begin{array}{l}2.38492 \\
2.38097\end{array}$ & $\begin{array}{l}395 \\
394\end{array}$ & $\begin{array}{l}0.99477 \\
0.99472\end{array}$ \\
\hline $\begin{array}{l}0.253 \\
0.254 \\
0.255\end{array}$ & $\begin{array}{l}1.49820 \\
1.49473 \\
1.49128\end{array}$ & $\begin{array}{l}347 \\
345 \\
343\end{array}$ & $\begin{array}{l}0.87883 \\
0.87837 \\
0.87791\end{array}$ & $\begin{array}{l}46 \\
46 \\
45\end{array}$ & $\begin{array}{l}2.37703 \\
2.37310 \\
2.36920\end{array}$ & $\begin{array}{l}393 \\
390 \\
390\end{array}$ & $\begin{array}{l}0.99468 \\
0.99464 \\
0.99460\end{array}$ \\
\hline $\begin{array}{l}0.256 \\
0.257 \\
0.258 \\
0.259 \\
0.260\end{array}$ & $\begin{array}{l}1.48785 \\
1.48443 \\
1.48102 \\
1.47763 \\
1.47426\end{array}$ & $\begin{array}{l}342 \\
341 \\
339 \\
337 \\
336\end{array}$ & $\begin{array}{l}0.87746 \\
0.87700 \\
0.87654 \\
0.87608 \\
0.87563\end{array}$ & $\begin{array}{l}46 \\
46 \\
46 \\
45 \\
46\end{array}$ & $\begin{array}{l}2.36530 \\
2.36143 \\
2.35756 \\
2.35372 \\
2.34989\end{array}$ & $\begin{array}{l}387 \\
387 \\
384 \\
383 \\
382\end{array}$ & $\begin{array}{l}0.99456 \\
0.99451 \\
0.99447 \\
0.99443 \\
0.99439\end{array}$ \\
\hline $\begin{array}{l}0.261 \\
0.262 \\
0.263 \\
0.264 \\
0.265\end{array}$ & $\begin{array}{l}1.47090 \\
1.46755 \\
1.46422 \\
1.46090 \\
1.45760\end{array}$ & $\begin{array}{l}335 \\
333 \\
332 \\
330 \\
329\end{array}$ & $\begin{array}{l}0.87517 \\
0.87471 \\
0.87426 \\
0.87380 \\
0.87335\end{array}$ & $\begin{array}{l}46 \\
45 \\
46 \\
45 \\
46\end{array}$ & $\begin{array}{l}2.34607 \\
2.34227 \\
2.33848 \\
2.33471 \\
2.33095\end{array}$ & $\begin{array}{l}380 \\
379 \\
377 \\
376 \\
375\end{array}$ & $\begin{array}{l}0.99434 \\
0.99430 \\
0.99426 \\
0.99421 \\
0.99417\end{array}$ \\
\hline $\begin{array}{l}0.266 \\
0.267 \\
0.268 \\
0.269 \\
0.270\end{array}$ & $\begin{array}{l}1.45431 \\
1.45104 \\
1.44778 \\
1.44453 \\
1.44130\end{array}$ & $\begin{array}{l}327 \\
326 \\
325 \\
323 \\
322\end{array}$ & $\begin{array}{l}0.87289 \\
0.87243 \\
0.87198 \\
0.87152 \\
0.87107\end{array}$ & $\begin{array}{l}46 \\
45 \\
46 \\
45 \\
46\end{array}$ & $\begin{array}{l}2.32720 \\
2.32347 \\
2.31976 \\
2.31605 \\
2.31237\end{array}$ & $\begin{array}{l}373 \\
371 \\
371 \\
368 \\
368\end{array}$ & $\begin{array}{l}0.99412 \\
0.99408 \\
0.99404 \\
0.99399 \\
0.99395\end{array}$ \\
\hline $\begin{array}{l}0.271 \\
0.272 \\
0.273 \\
0.274 \\
0.275\end{array}$ & $\begin{array}{l}1.43808 \\
1.43487 \\
1.43168 \\
1.42850 \\
1.42534\end{array}$ & $\begin{array}{l}321 \\
319 \\
318 \\
316 \\
316\end{array}$ & $\begin{array}{l}0.87061 \\
0.87016 \\
0.86970 \\
0.86925 \\
0.86879\end{array}$ & $\begin{array}{l}45 \\
46 \\
45 \\
46 \\
45\end{array}$ & $\begin{array}{l}2.30869 \\
2.30503 \\
2.30138 \\
2.29775 \\
2.29413\end{array}$ & $\begin{array}{l}366 \\
365 \\
363 \\
362 \\
361\end{array}$ & $\begin{array}{l}0.99390 \\
0.99386 \\
0.99381 \\
0.99377 \\
0.99372\end{array}$ \\
\hline $\begin{array}{l}0.276 \\
0.277 \\
0.278 \\
0.279 \\
0.280\end{array}$ & $\begin{array}{l}1.42218 \\
1.41904 \\
1.41592 \\
1.41280 \\
1.40970\end{array}$ & $\begin{array}{l}314 \\
312 \\
312 \\
310 \\
309\end{array}$ & $\begin{array}{l}0.86834 \\
0.86789 \\
0.86743 \\
0.86698 \\
0.86652\end{array}$ & $\begin{array}{l}45 \\
46 \\
45 \\
46 \\
45\end{array}$ & $\begin{array}{l}2.29052 \\
2.28693 \\
2.28335 \\
2.27978 \\
2.27623\end{array}$ & $\begin{array}{l}359 \\
358 \\
357 \\
355 \\
355\end{array}$ & $\begin{array}{l}0.99368 \\
0.99363 \\
0.99358 \\
0.99354 \\
0.99349\end{array}$ \\
\hline $\begin{array}{l}0.281 \\
0.282 \\
0.283 \\
0.284 \\
0.285\end{array}$ & $\begin{array}{l}1.40661 \\
1.40354 \\
1.40047 \\
1.39742 \\
1.39438\end{array}$ & $\begin{array}{l}307 \\
307 \\
305 \\
304 \\
302\end{array}$ & $\begin{array}{l}0.86607 \\
0.86562 \\
0.86517 \\
0.86471 \\
0.86426\end{array}$ & $\begin{array}{l}45 \\
45 \\
46 \\
45 \\
45\end{array}$ & $\begin{array}{l}2.27268 \\
2.26916 \\
2.26564 \\
2.26213 \\
2.25864\end{array}$ & $\begin{array}{l}352 \\
352 \\
351 \\
349 \\
348\end{array}$ & $\begin{array}{l}0.99345 \\
0.99340 \\
0.99335 \\
0.99331 \\
0.99326\end{array}$ \\
\hline $\begin{array}{l}0.286 \\
0.287 \\
0.288 \\
0.289 \\
0.290\end{array}$ & $\begin{array}{l}1.39136 \\
1.38834 \\
1.38534 \\
1.38235 \\
1.37937\end{array}$ & $\begin{array}{l}302 \\
300 \\
299 \\
298 \\
296\end{array}$ & $\begin{array}{l}0.86381 \\
0.86335 \\
0.86290 \\
0.86245 \\
0.86200\end{array}$ & $\begin{array}{l}46 \\
45 \\
45 \\
45 \\
45\end{array}$ & $\begin{array}{l}2.25516 \\
2.25170 \\
2.24824 \\
2.24480 \\
2.24137\end{array}$ & $\begin{array}{l}346 \\
346 \\
344 \\
343 \\
342\end{array}$ & $\begin{array}{l}0.99321 \\
0.99316 \\
0.99312 \\
0.99307 \\
0.99302\end{array}$ \\
\hline $\begin{array}{l}0.291 \\
0.292 \\
0.293 \\
0.294 \\
0.295\end{array}$ & $\begin{array}{l}1.37641 \\
1.37345 \\
1.37051 \\
1.36758 \\
1.36466\end{array}$ & $\begin{array}{l}296 \\
294 \\
293 \\
292 \\
291\end{array}$ & $\begin{array}{l}0.86155 \\
0.86110 \\
0.86064 \\
0.86019 \\
0.85974\end{array}$ & $\begin{array}{l}45 \\
46 \\
45 \\
45 \\
45\end{array}$ & $\begin{array}{l}2.23795 \\
2.23455 \\
2.23115 \\
2.22777 \\
2.22440\end{array}$ & $\begin{array}{l}340 \\
.340 \\
338 \\
337 \\
336\end{array}$ & $\begin{array}{l}0.99297 \\
0.99292 \\
0.99288 \\
0.99283 \\
0.99278\end{array}$ \\
\hline $\begin{array}{l}0.296 \\
0.297 \\
0.298 \\
0.299 \\
0.300\end{array}$ & $\begin{array}{l}1.36175 \\
1.35885 \\
1.35596 \\
1.35309 \\
1.35023\end{array}$ & $\begin{array}{l}290 \\
289 \\
287 \\
286\end{array}$ & $\begin{array}{l}0.85929 \\
0.85884 \\
0.85839 \\
0.85794 \\
0.85749\end{array}$ & $\begin{array}{l}45 \\
45 \\
45 \\
45\end{array}$ & $\begin{array}{l}2.22104 \\
2.21769 \\
2.21435 \\
2.21103 \\
2.20771\end{array}$ & $\begin{array}{l}335 \\
334 \\
332 \\
332\end{array}$ & $\begin{array}{l}0.99273 \\
0.99268 \\
0.99263 \\
0.99258 \\
0.99253\end{array}$ \\
\hline
\end{tabular}


Table I Harmonic Oscillator Contributions (in dimensionless form) to the Thermodynamic Functions for Values of $X=h c v / k T$

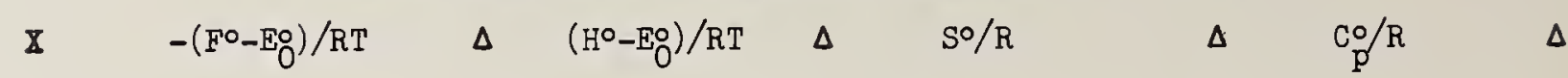

\begin{tabular}{|c|c|c|c|c|c|c|c|}
\hline 0.300 & 1.35023 & 286 & 0.85749 & 45 & 2.20771 & 330 & 0.99253 \\
\hline $\begin{array}{l}0.301 \\
0.302 \\
0.303 \\
0.304 \\
0.305\end{array}$ & $\begin{array}{l}1.34737 \\
1.34453 \\
1.34170 \\
1.33888 \\
1.33607\end{array}$ & $\begin{array}{l}284 \\
283 \\
282 \\
281 \\
280\end{array}$ & $\begin{array}{l}0.85704 \\
0.85659 \\
0.85614 \\
0.85569 \\
0.85524\end{array}$ & $\begin{array}{l}45 \\
45 \\
45 \\
45 \\
45\end{array}$ & $\begin{array}{l}2.20441 \\
2.20112 \\
2.19784 \\
2.19457 \\
2.19131\end{array}$ & $\begin{array}{l}329 \\
328 \\
327 \\
326 \\
325\end{array}$ & $\begin{array}{l}0.99248 \\
0.99243 \\
0.99238 \\
0.99233 \\
0.99228\end{array}$ \\
\hline $\begin{array}{l}0.306 \\
0.307 \\
0.308 \\
0.309 \\
0.310\end{array}$ & $\begin{array}{l}1.33327 \\
1.33048 \\
1.32771 \\
1.32494 \\
1.32218\end{array}$ & $\begin{array}{l}279 \\
277 \\
277 \\
276 \\
274\end{array}$ & $\begin{array}{l}0.85479 \\
0.85434 \\
0.85389 \\
0.85344 \\
0.85300\end{array}$ & $\begin{array}{l}45 \\
45 \\
45 \\
44 \\
45\end{array}$ & $\begin{array}{l}2.18806 \\
2.18483 \\
2.18160 \\
2.17838 \\
2.17518\end{array}$ & $\begin{array}{l}323 \\
323 \\
322 \\
320 \\
320\end{array}$ & $\begin{array}{l}0.99223 \\
0.99218 \\
0.99213 \\
0.99208 \\
0.99203\end{array}$ \\
\hline $\begin{array}{l}0.311 \\
0.312 \\
0.313 \\
0.314 \\
0.315\end{array}$ & $\begin{array}{l}1.31944 \\
1.31670 \\
1.31397 \\
1.31126 \\
1.30855\end{array}$ & $\begin{array}{l}274 \\
273 \\
271 \\
271 \\
269\end{array}$ & $\begin{array}{l}0.85255 \\
0.85210 \\
0.85165 \\
0.85120 \\
0.85076\end{array}$ & $\begin{array}{l}45 \\
45 \\
45 \\
44 \\
45\end{array}$ & $\begin{array}{l}2.17198 \\
2.16880 \\
2.16562 \\
2.16246 \\
2.15931\end{array}$ & $\begin{array}{l}318 \\
318 \\
316 \\
315 \\
315\end{array}$ & $\begin{array}{l}0.99198 \\
0.99193 \\
0.99188 \\
0.99182 \\
0.99177\end{array}$ \\
\hline $\begin{array}{l}0.316 \\
0.317 \\
0.318 \\
0.319 \\
0.320\end{array}$ & $\begin{array}{l}1.30586 \\
1.30317 \\
1.30049 \\
1.29783 \\
1.29517\end{array}$ & $\begin{array}{l}269 \\
268 \\
266 \\
266 \\
265\end{array}$ & $\begin{array}{l}0.85031 \\
0.84986 \\
0.84941 \\
0.84897 \\
0.84852\end{array}$ & $\begin{array}{l}45 \\
45 \\
44 \\
45 \\
45\end{array}$ & $\begin{array}{l}2.15616 \\
2.15303 \\
2.14991 \\
2.14679 \\
2.14369\end{array}$ & $\begin{array}{l}313 \\
312 \\
312 \\
310 \\
309\end{array}$ & $\begin{array}{l}0.99172 \\
0.99167 \\
0.99162 \\
0.99156 \\
0.99151\end{array}$ \\
\hline $\begin{array}{l}0.321 \\
0.322 \\
0.323 \\
0.324 \\
0.325\end{array}$ & $\begin{array}{l}1.29252 \\
1.28989 \\
1.28726 \\
1.28464 \\
1.28203\end{array}$ & $\begin{array}{l}263 \\
263 \\
262 \\
261 \\
260\end{array}$ & $\begin{array}{l}0.84807 \\
0.84763 \\
0.84718 \\
0.84673 \\
0.84629\end{array}$ & $\begin{array}{l}44 \\
45 \\
45 \\
44 \\
45\end{array}$ & $\begin{array}{l}2.14060 \\
2.13751 \\
2.13444 \\
2.13137 \\
2.12832\end{array}$ & $\begin{array}{l}309 \\
307 \\
307 \\
305 \\
305\end{array}$ & $\begin{array}{l}0.99146 \\
0.99140 \\
0.99135 \\
0.99130 \\
0.99124\end{array}$ \\
\hline $\begin{array}{l}0.326 \\
0.327 \\
0.328 \\
0.329 \\
0.330\end{array}$ & $\begin{array}{l}1.27943 \\
1.27684 \\
1.27426 \\
1.27169 \\
1.26913\end{array}$ & $\begin{array}{l}259 \\
258 \\
257 \\
256 \\
255\end{array}$ & $\begin{array}{l}0.84584 \\
0.84539 \\
0.84495 \\
0.84450 \\
0.84406\end{array}$ & $\begin{array}{l}45 \\
44 \\
45 \\
44 \\
45\end{array}$ & $\begin{array}{l}2.12527 \\
2.12224 \\
2.11921 \\
2.11620 \\
2.11319\end{array}$ & $\begin{array}{l}303 \\
303 \\
301 \\
301 \\
300\end{array}$ & $\begin{array}{l}0.99119 \\
0.99114 \\
0.99108 \\
0.99103 \\
0.99097\end{array}$ \\
\hline $\begin{array}{l}0.331 \\
0.332 \\
0.333 \\
0.334 \\
0.335\end{array}$ & $\begin{array}{l}1.26658 \\
1.26403 \\
1.26150 \\
1.25897 \\
1.25645\end{array}$ & $\begin{array}{l}255 \\
253 \\
253 \\
252 \\
251\end{array}$ & $\begin{array}{l}0.84361 \\
0.84317 \\
0.84272 \\
0.84228 \\
0.84183\end{array}$ & $\begin{array}{l}44 \\
45 \\
44 \\
45 \\
44\end{array}$ & $\begin{array}{l}2.11019 \\
2.10720 \\
2.10422 \\
2.10125 \\
2.09829\end{array}$ & $\begin{array}{l}299 \\
298 \\
297 \\
296 \\
296\end{array}$ & $\begin{array}{l}0.99092 \\
0.99087 \\
0.99081 \\
0.99076 \\
0.99070\end{array}$ \\
\hline $\begin{array}{l}0.336 \\
0.337 \\
0.338 \\
0.339 \\
0.340\end{array}$ & $\begin{array}{l}1.25394 \\
1.25144 \\
1.24895 \\
1.24647 \\
1.24400\end{array}$ & $\begin{array}{l}250 \\
249 \\
248 \\
247 \\
247\end{array}$ & $\begin{array}{l}0.84139 \\
0.84095 \\
0.84050 \\
0.84006 \\
0.83961\end{array}$ & $\begin{array}{l}44 \\
45 \\
44 \\
45 \\
44\end{array}$ & $\begin{array}{l}2.09533 \\
2.09239 \\
2.08946 \\
2.08653 \\
2.08361\end{array}$ & $\begin{array}{l}294 \\
293 \\
293 \\
292 \\
291\end{array}$ & $\begin{array}{l}0.99064 \\
0.99059 \\
0.99053 \\
0.99048 \\
0.99042\end{array}$ \\
\hline $\begin{array}{l}0.341 \\
0.342 \\
0.343 \\
0.344 \\
0.345\end{array}$ & $\begin{array}{l}1.24153 \\
1.23908 \\
1.23663 \\
1.23419 \\
1.23176\end{array}$ & $\begin{array}{l}245 \\
245 \\
244 \\
243 \\
243\end{array}$ & $\begin{array}{l}0.83917 \\
0.83873 \\
0.83828 \\
0.83784 \\
0.83740\end{array}$ & $\begin{array}{l}44 \\
45 \\
44 \\
44 \\
44\end{array}$ & $\begin{array}{l}2.08070 \\
2.07780 \\
2.07491 \\
2.07203 \\
2.06916\end{array}$ & $\begin{array}{l}290 \\
289 \\
288 \\
287 \\
287\end{array}$ & $\begin{array}{l}0.99037 \\
0.99031 \\
0.99025 \\
0.99020 \\
0.99014\end{array}$ \\
\hline $\begin{array}{l}0.346 \\
0.347 \\
0.348 \\
0.349 \\
0.350\end{array}$ & $\begin{array}{l}1.22933 \\
1.22692 \\
1.22451 \\
1.22211 \\
1.21972\end{array}$ & $\begin{array}{l}241 \\
241 \\
240 \\
239\end{array}$ & $\begin{array}{l}0.83696 \\
0.83651 \\
0.83607 \\
0.83563 \\
0.83519\end{array}$ & $\begin{array}{l}45 \\
44 \\
44 \\
44\end{array}$ & $\begin{array}{l}2.06629 \\
2.06343 \\
2.06058 \\
2.05774 \\
2.05491\end{array}$ & $\begin{array}{l}286 \\
285 \\
284 \\
283\end{array}$ & $\begin{array}{l}0.99008 \\
0.99003 \\
0.98997 \\
0.98991 \\
0.98985\end{array}$ \\
\hline
\end{tabular}


Table I Harmonic Oscillator Contributions (in dimensionless form) to the Thermodynamic Functions for Values of $\mathrm{X}=\mathrm{hc} / \mathrm{kT}$

$\mathbf{I}-\left(\mathrm{F}^{\circ}-\mathrm{E}_{\mathrm{O}}^{\circ}\right) / \mathrm{RT} \sim \Delta\left(\mathrm{H}^{\circ}-\mathrm{E}_{\mathrm{O}}\right) / \mathrm{RT} \quad \Delta \quad \mathrm{S} / \mathrm{R}$

\begin{tabular}{|c|c|c|c|}
\hline 0.350 & 1.21972 & 238 & 0.83519 \\
\hline $\begin{array}{l}0.351 \\
0.352 \\
0.353 \\
0.354 \\
0.355\end{array}$ & $\begin{array}{l}1.21734 \\
1.21497 \\
1.21260 \\
1.21024 \\
1.20789\end{array}$ & $\begin{array}{l}237 \\
237 \\
236 \\
235 \\
234\end{array}$ & $\begin{array}{l}0.83475 \\
0.83430 \\
0.83386 \\
0.83342 \\
0.83298\end{array}$ \\
\hline $\begin{array}{l}0.356 \\
0.357 \\
0.358 \\
0.359 \\
0.360\end{array}$ & $\begin{array}{l}1.20555 \\
1.20321 \\
1.20089 \\
1.19857 \\
1.19626\end{array}$ & $\begin{array}{l}234 \\
232 \\
232 \\
231 \\
231\end{array}$ & $\begin{array}{l}0.83254 \\
0.83210 \\
0.83166 \\
0.83122 \\
0.83078\end{array}$ \\
\hline $\begin{array}{l}0.361 \\
0.362 \\
0.363 \\
0.364 \\
0.365\end{array}$ & $\begin{array}{l}1.19395 \\
1.19166 \\
1.18937 \\
1.18709 \\
1.18481\end{array}$ & $\begin{array}{l}229 \\
229 \\
228 \\
228 \\
226\end{array}$ & $\begin{array}{l}0.83034 \\
0.82990 \\
0.82946 \\
0.82902 \\
0.82858\end{array}$ \\
\hline $\begin{array}{l}0.366 \\
0.367 \\
0.368 \\
0.369 \\
0.370\end{array}$ & $\begin{array}{l}1.18255 \\
1.18029 \\
1.17804 \\
1.17579 \\
1.17355\end{array}$ & $\begin{array}{l}226 \\
225 \\
225 \\
224 \\
223\end{array}$ & $\begin{array}{l}0.82814 \\
0.82770 \\
0.82726 \\
0.82682 \\
0.82638\end{array}$ \\
\hline $\begin{array}{l}0.371 \\
0.372 \\
0.373 \\
0.374 \\
0.375\end{array}$ & $\begin{array}{l}1.17132 \\
1.16910 \\
1.16689 \\
1.16468 \\
1.16248\end{array}$ & $\begin{array}{l}222 \\
221 \\
221 \\
220 \\
220\end{array}$ & $\begin{array}{l}0.82594 \\
0.82551 \\
0.82507 \\
0.82463 \\
0.82419\end{array}$ \\
\hline $\begin{array}{l}0.376 \\
0.377 \\
0.378 \\
0.379 \\
0.380\end{array}$ & $\begin{array}{l}1.16028 \\
1.15810 \\
1.15591 \\
1.15374 \\
1.15157\end{array}$ & $\begin{array}{l}218 \\
219 \\
217 \\
217 \\
216\end{array}$ & $\begin{array}{l}0.82375 \\
0.82332 \\
0.82288 \\
0.82244 \\
0.82200\end{array}$ \\
\hline $\begin{array}{l}0.381 \\
0.382 \\
0.383 \\
0.384 \\
0.385\end{array}$ & $\begin{array}{l}1.14941 \\
1.14726 \\
1.14512 \\
1.14298 \\
1.14084\end{array}$ & $\begin{array}{l}215 \\
214 \\
214 \\
214 \\
212\end{array}$ & $\begin{array}{l}0.82157 \\
0.82113 \\
0.82069 \\
0.82026 \\
0.81982\end{array}$ \\
\hline $\begin{array}{l}0.386 \\
0.387 \\
0.388 \\
0.389 \\
0.390\end{array}$ & $\begin{array}{l}1.13872 \\
1.13660 \\
1.13449 \\
1.13238 \\
1.13028\end{array}$ & 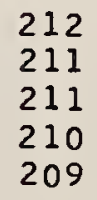 & $\begin{array}{l}0.81939 \\
0.81895 \\
0.81851 \\
0.81808 \\
0.81764\end{array}$ \\
\hline $\begin{array}{l}0.391 \\
0.392 \\
0.393 \\
0.394 \\
0.395\end{array}$ & $\begin{array}{l}1.12819 \\
1.12610 \\
1.12402 \\
1.12194 \\
1.11988\end{array}$ & $\begin{array}{l}209 \\
208 \\
208 \\
206 \\
206\end{array}$ & $\begin{array}{l}0.81721 \\
0.81677 \\
0.81634 \\
0.81590 \\
0.81547\end{array}$ \\
\hline $\begin{array}{l}0.396 \\
0.397 \\
0.398 \\
0.399 \\
0.400\end{array}$ & $\begin{array}{l}1.11782 \\
1.11576 \\
1.11371 \\
1.11167 \\
1.10963\end{array}$ & $\begin{array}{l}206 \\
205 \\
204 \\
204\end{array}$ & $\begin{array}{l}0.81503 \\
0.81460 \\
0.81117 \\
0.81373 \\
0.81330\end{array}$ \\
\hline
\end{tabular}

$\begin{array}{lllll}44 & 2.05491 & 282 & 0.98985 & 5 \\ 45 & 2.05209 & 282 & 0.98980 & 6 \\ 44 & 2.04927 & 281 & 0.98974 & 6 \\ 44 & 2.04646 & 280 & 0.98968 & 6 \\ 44 & 2.04366 & 279 & 0.98962 & 6 \\ 44 & 2.04087 & 278 & 0.98956 & 5\end{array}$

$0.98951 \quad 6$

$0.98945 \quad 6$

$0.98939 \quad 6$

0.989336

$0.98927 \quad 6$

$0.98921 \quad 6$

$0.98915 \quad 6$

$0.98909 \quad 6$

$0.98903 \quad 6$

$0.98897 \quad 6$

$0.98891 \quad 6$

$0.98885 \quad 6$

$0.98879 \quad 6$

$0.98873 \quad 6$

$0.98867 \quad 6$

$0.98861 \quad 6$

0.988556

0.988497

0.988426

$0.98836 \quad 6$

$0.98830 \quad 6$

$0.98824 \quad 6$

$0.98818 \quad 6$

0.988127

0.988056

$0.98799 \quad 6$

$0.98793 \quad 6$

$0.98787 \quad 7$

$0.98780 \quad 6$

0.987746

$1.96323 \quad 256$

257

255

$0.98768 \quad 7$

$0.98761 \quad 6$

$0.98755 \quad 7$

$0.98748 \quad 6$

0.987426

$0.98736 \quad 7$

1.94539

252

0.98729

0.98723

0.98716

0.98710

$0.98703 \quad 6$

1.93285

1.93036

1.92788

1.92540

1.92293
0.98697

0.98690

0.98684

0.98677 
Table I Harmonic Oscillator Contributions (in dimensionless form) to the Thermodynamic Functions for Values of $X=h c v / k T$

\begin{tabular}{|c|c|c|c|c|c|c|c|}
\hline $\mathrm{X}$ & $-\left(F^{\circ}-E_{O}^{\circ}\right) / R T$ & $\Delta$ & $\left(\mathrm{H}^{\circ}-\mathrm{E}_{\mathrm{O}}\right) / \mathrm{RT}$ & $\Delta$ & So/R & - & $\mathrm{Co} / \mathrm{R}$ \\
\hline 0.400 & 1.10963 & 203 & 0.81330 & 44 & 1.92293 & 246 & 0.98677 \\
\hline $\begin{array}{l}0.401 \\
0.402 \\
0.403 \\
0.404 \\
0.405\end{array}$ & $\begin{array}{l}1.10760 \\
1.10558 \\
1.10356 \\
1.10155 \\
1.09954\end{array}$ & $\begin{array}{l}202 \\
202 \\
201 \\
201 \\
200\end{array}$ & $\begin{array}{l}0.81286 \\
0.81243 \\
0.81200 \\
0.81156 \\
0.81113\end{array}$ & $\begin{array}{l}43 \\
43 \\
44 \\
43 \\
43\end{array}$ & $\begin{array}{l}1.92047 \\
1.91801 \\
1.91556 \\
1.91311 \\
1.91067\end{array}$ & $\begin{array}{l}246 \\
245 \\
245 \\
244 \\
243\end{array}$ & $\begin{array}{l}0.98671 \\
0.98664 \\
0.98658 \\
0.98651 \\
0.98644\end{array}$ \\
\hline $\begin{array}{l}0.406 \\
0.407 \\
0.408 \\
0.409 \\
0.410\end{array}$ & $\begin{array}{l}1.09754 \\
1.09555 \\
1.09356 \\
1.09158 \\
1.08960\end{array}$ & $\begin{array}{l}199 \\
199 \\
198 \\
198 \\
197\end{array}$ & $\begin{array}{l}0.81070 \\
0.81027 \\
0.80983 \\
0.80940 \\
0.80897\end{array}$ & $\begin{array}{l}43 \\
44 \\
43 \\
43 \\
43\end{array}$ & $\begin{array}{l}1.90824 \\
1.90582 \\
1.90340 \\
1.90098 \\
1.89857\end{array}$ & $\begin{array}{l}242 \\
242 \\
242 \\
241 \\
240\end{array}$ & $\begin{array}{l}0.98638 \\
0.98631 \\
0.98624 \\
0.98618 \\
0.98611\end{array}$ \\
\hline $\begin{array}{l}0.411 \\
0.412 \\
0.413 \\
0.414 \\
0.415\end{array}$ & $\begin{array}{l}1.08763 \\
1.08567 \\
1.08371 \\
1.08176 \\
1.07981\end{array}$ & $\begin{array}{l}196 \\
196 \\
195 \\
195 \\
194\end{array}$ & $\begin{array}{l}0.80854 \\
0.80811 \\
0.80767 \\
0.80724 \\
0.80681\end{array}$ & $\begin{array}{l}43 \\
44 \\
43 \\
43 \\
43\end{array}$ & $\begin{array}{l}1.89617 \\
1.89377 \\
1.89138 \\
1.88900 \\
1.88662\end{array}$ & $\begin{array}{l}240 \\
239 \\
238 \\
238 \\
237\end{array}$ & $\begin{array}{l}0.98604 \\
0.98597 \\
0.98591 \\
0.98584 \\
0.98577\end{array}$ \\
\hline $\begin{array}{l}0.416 \\
0.417 \\
0.418 \\
0.419 \\
0.420\end{array}$ & $\begin{array}{l}1.07787 \\
1.07593 \\
1.07400 \\
1.07208 \\
1.07016\end{array}$ & $\begin{array}{l}194 \\
193 \\
192 \\
192 \\
191\end{array}$ & $\begin{array}{l}0.80638 \\
0.80595 \\
0.80552 \\
0.80509 \\
0.80466\end{array}$ & $\begin{array}{l}43 \\
43 \\
43 \\
43 \\
43\end{array}$ & $\begin{array}{l}1.88425 \\
1.88188 \\
1.87952 \\
1.87717 \\
1.87482\end{array}$ & $\begin{array}{l}237 \\
236 \\
235 \\
235 \\
235\end{array}$ & $\begin{array}{l}0.98570 \\
0.98563 \\
0.98557 \\
0.98550 \\
0.98543\end{array}$ \\
\hline $\begin{array}{l}0.421 \\
0.422 \\
0.423 \\
0.424 \\
0.425\end{array}$ & $\begin{array}{l}1.06825 \\
1.06634 \\
1.06444 \\
1.06254 \\
1.06065\end{array}$ & $\begin{array}{l}191 \\
190 \\
190 \\
189 \\
188\end{array}$ & $\begin{array}{l}0.80423 \\
0.80380 \\
0.80337 \\
0.80294 \\
0.80251\end{array}$ & $\begin{array}{l}43 \\
43 \\
43 \\
43 \\
43\end{array}$ & $\begin{array}{l}1.87247 \\
1.87014 \\
1.86781 \\
1.86548 \\
1.86316\end{array}$ & $\begin{array}{l}233 \\
233 \\
233 \\
232 \\
232\end{array}$ & $\begin{array}{l}0.98536 \\
0.98529 \\
0.98522 \\
0.98515 \\
0.98508\end{array}$ \\
\hline $\begin{array}{l}0.426 \\
0.427 \\
0.428 \\
0.429 \\
0.430\end{array}$ & $\begin{array}{l}1.05877 \\
1.05689 \\
1.05501 \\
1.05314 \\
1.05128\end{array}$ & $\begin{array}{l}188 \\
188 \\
187 \\
186 \\
186\end{array}$ & $\begin{array}{l}0.80208 \\
0.80165 \\
0.80122 \\
0.80079 \\
0.80036\end{array}$ & $\begin{array}{l}43 \\
43 \\
43 \\
43 \\
43\end{array}$ & $\begin{array}{l}1.86084 \\
1.85853 \\
1.85623 \\
1.85393 \\
1.85164\end{array}$ & $\begin{array}{l}231 \\
230 \\
230 \\
229 \\
229\end{array}$ & $\begin{array}{l}0.98501 \\
0.98494 \\
0.98487 \\
0.98480 \\
0.98473\end{array}$ \\
\hline $\begin{array}{l}0.431 \\
0.432 \\
0.433 \\
0.434 \\
0.435\end{array}$ & $\begin{array}{l}1.04942 \\
1.04757 \\
1.04572 \\
1.04387 \\
1.04204\end{array}$ & $\begin{array}{l}185 \\
185 \\
185 \\
183 \\
184\end{array}$ & $\begin{array}{l}0.79993 \\
0.79950 \\
0.79908 \\
0.79865 \\
0.79822\end{array}$ & $\begin{array}{l}43 \\
42 \\
43 \\
43 \\
43\end{array}$ & $\begin{array}{l}1.84935 \\
1.84707 \\
1.84479 \\
1.84252 \\
1.84026\end{array}$ & $\begin{array}{l}228 \\
228 \\
227 \\
226 \\
226\end{array}$ & $\begin{array}{l}0.98466 \\
0.98459 \\
0.98452 \\
0.98445 \\
0.98438\end{array}$ \\
\hline $\begin{array}{l}0.436 \\
0.437 \\
0.438 \\
0.439 \\
0.440\end{array}$ & $\begin{array}{l}1.04020 \\
1.03838 \\
1.03656 \\
1.03474 \\
1.03293\end{array}$ & $\begin{array}{l}182 \\
182 \\
182 \\
181 \\
181\end{array}$ & $\begin{array}{l}0.79779 \\
0.79736 \\
0.79694 \\
0.79651 \\
0.79608\end{array}$ & $\begin{array}{l}43 \\
42 \\
43 \\
43 \\
43\end{array}$ & $\begin{array}{l}1.83800 \\
1.83574 \\
1.83349 \\
1.83125 \\
1.82901\end{array}$ & $\begin{array}{l}226 \\
225 \\
224 \\
224 \\
224\end{array}$ & $\begin{array}{l}0.98431 \\
0.98424 \\
0.98417 \\
0.98409 \\
0.98402\end{array}$ \\
\hline $\begin{array}{l}0.441 \\
0.442 \\
0.443 \\
0.444 \\
0.445\end{array}$ & $\begin{array}{l}1.03112 \\
1.02932 \\
1.02752 \\
1.02573 \\
1.02394\end{array}$ & $\begin{array}{l}180 \\
180 \\
179 \\
179 \\
178\end{array}$ & $\begin{array}{l}0.79565 \\
0.79523 \\
0.79480 \\
0.79437 \\
0.79395\end{array}$ & $\begin{array}{l}42 \\
43 \\
43 \\
42 \\
43\end{array}$ & $\begin{array}{l}1.82677 \\
1.82455 \\
1.82232 \\
1.82010 \\
1.81789\end{array}$ & $\begin{array}{l}222 \\
223 \\
222 \\
221 \\
221\end{array}$ & $\begin{array}{l}0.98395 \\
0.98388 \\
0.98381 \\
0.98373 \\
0.98366\end{array}$ \\
\hline $\begin{array}{l}0.446 \\
0.447 \\
0.448 \\
0.449 \\
0.450\end{array}$ & $\begin{array}{l}1.02216 \\
1.02039 \\
1.01861 \\
1.01685 \\
1.01508\end{array}$ & $\begin{array}{l}177 \\
178 \\
176 \\
177\end{array}$ & $\begin{array}{l}0.79352 \\
0.79310 \\
0.79267 \\
0.79224 \\
0.79182\end{array}$ & $\begin{array}{l}42 \\
43 \\
43 \\
42\end{array}$ & $\begin{array}{l}1.81568 \\
1.81348 \\
1.81128 \\
1.80909 \\
1.80690\end{array}$ & $\begin{array}{l}220 \\
220 \\
219 \\
219\end{array}$ & $\begin{array}{l}0.98359 \\
0.98351 \\
0.98344 \\
0.98337 \\
0.98329\end{array}$ \\
\hline
\end{tabular}


Table I Harmonic Oscillator Contributions (in dimensionless form) to the Thermodynamic Functions for Values of $\mathrm{X}=\mathrm{hc} v / \mathrm{kT}$
X $\quad-\left(\mathrm{F}^{\circ}-\mathrm{E}_{\mathrm{O}}^{\circ}\right) / \mathrm{RT}$
$\Delta \quad\left(\mathrm{H}^{\circ}-\mathrm{E}_{\mathrm{O}}\right) / \mathrm{RT}$
$\Delta \quad \mathrm{S} \% / \mathrm{R}$
$\Delta \quad \mathrm{C} \circ / \mathrm{R}$

$\Delta$

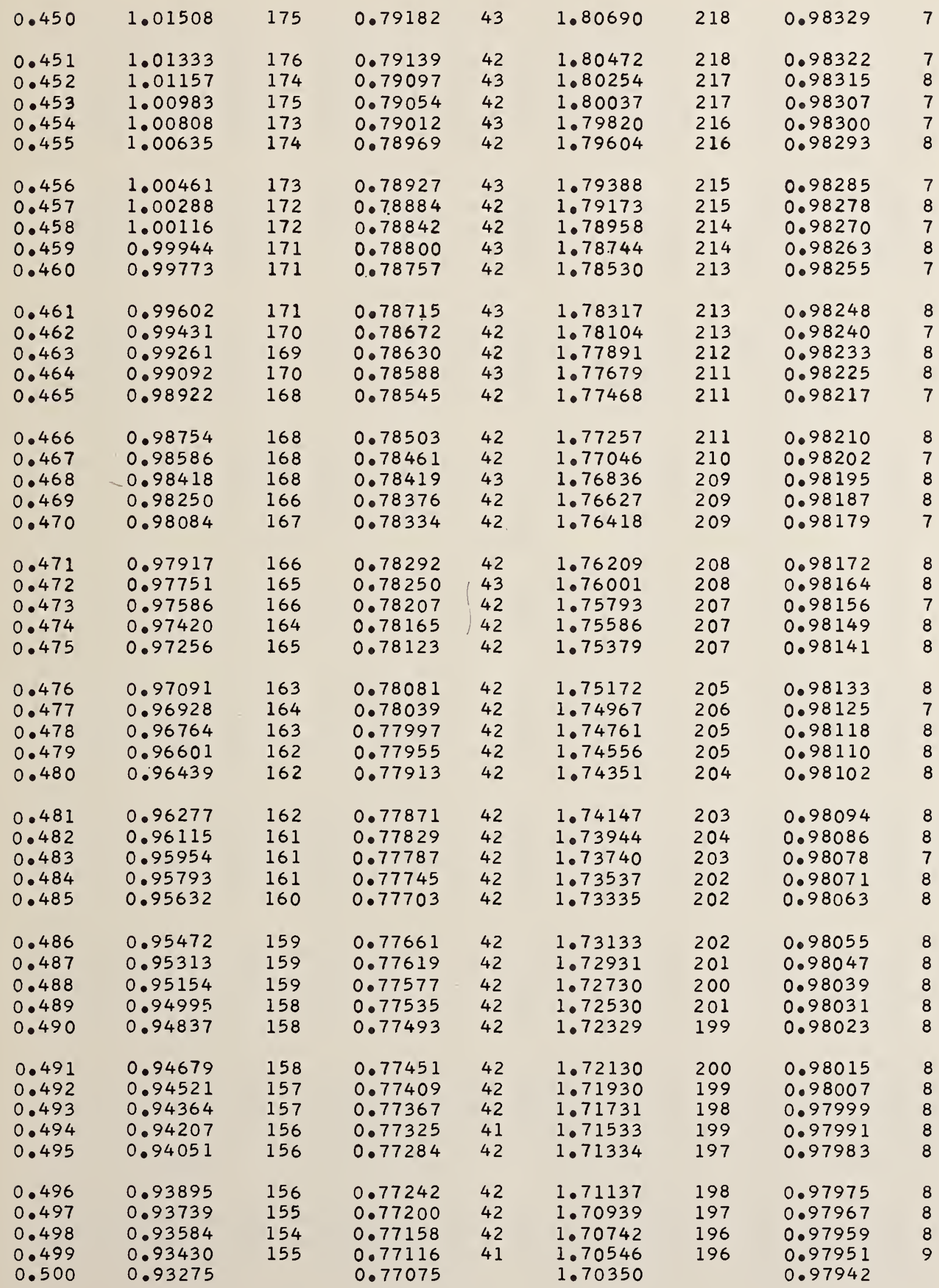


Table I Harmonic Oscillator Contributions (in dimensionless form) to the Thermodynamic Functions for Values of $X=h c v / k T$

$\begin{array}{lllllllll} & -\left(\mathrm{F}^{\circ}-\mathrm{E}_{\mathrm{O}}^{\circ}\right) / \mathrm{RT} & \Delta & \left(\mathrm{H}^{\circ}-\mathrm{E}_{\mathrm{O}}^{\circ}\right) / \mathrm{RT} & \Delta & \mathrm{S} / \mathrm{R} & \Delta \quad \mathrm{CO}_{\mathrm{p}}^{\circ} / \mathrm{R}\end{array}$

\begin{tabular}{|c|c|c|c|c|c|c|c|}
\hline 0.500 & 0.93275 & 154 & 0.77075 & 42 & 1.70350 & 196 & 0.97942 \\
\hline $\begin{array}{l}0.501 \\
0.502 \\
0.503 \\
0.504 \\
0.505\end{array}$ & $\begin{array}{l}0.93121 \\
0.92968 \\
0.92815 \\
0.92662 \\
0.92509\end{array}$ & $\begin{array}{l}153 \\
153 \\
153 \\
153 \\
152\end{array}$ & $\begin{array}{l}0.77033 \\
0.76991 \\
0.76950 \\
0.76908 \\
0.76866\end{array}$ & $\begin{array}{l}42 \\
41 \\
42 \\
42 \\
41\end{array}$ & $\begin{array}{l}1.70154 \\
1.69959 \\
1.69764 \\
1.69570 \\
1.69376\end{array}$ & $\begin{array}{l}195 \\
195 \\
194 \\
194 \\
194\end{array}$ & $\begin{array}{l}0.97934 \\
0.97926 \\
0.97918 \\
0.97910 \\
0.97902\end{array}$ \\
\hline $\begin{array}{l}0.506 \\
0.507 \\
0.508 \\
0.509 \\
0.510\end{array}$ & $\begin{array}{l}0.92357 \\
0.92206 \\
0.92054 \\
0.91904 \\
0.91753\end{array}$ & $\begin{array}{l}151 \\
152 \\
150 \\
151 \\
150\end{array}$ & $\begin{array}{l}0.76825 \\
0.76783 \\
0.76741 \\
0.76700 \\
0.76658\end{array}$ & $\begin{array}{l}42 \\
42 \\
41 \\
42 \\
41\end{array}$ & $\begin{array}{l}1.69182 \\
1.68989 \\
1.68796 \\
1.68603 \\
1.68411\end{array}$ & $\begin{array}{l}193 \\
193 \\
193 \\
192 \\
191\end{array}$ & $\begin{array}{l}0.97893 \\
0.97885 \\
0.97877 \\
0.97869 \\
0.97860\end{array}$ \\
\hline $\begin{array}{l}0.511 \\
0.512 \\
0.513 \\
0.514 \\
0.515\end{array}$ & $\begin{array}{l}0.91603 \\
0.91453 \\
0.91304 \\
0.91155 \\
0.91006\end{array}$ & $\begin{array}{l}150 \\
149 \\
149 \\
149 \\
148\end{array}$ & $\begin{array}{l}0.76617 \\
0.76575 \\
0.76534 \\
0.76492 \\
0.76450\end{array}$ & $\begin{array}{l}42 \\
41 \\
42 \\
42 \\
41\end{array}$ & $\begin{array}{l}1.68220 \\
1.68028 \\
1.67837 \\
1.67647 \\
1.67457\end{array}$ & $\begin{array}{l}192 \\
191 \\
190 \\
190 \\
190\end{array}$ & $\begin{array}{l}0.97852 \\
0.97844 \\
0.97835 \\
0.97827 \\
0.97819\end{array}$ \\
\hline $\begin{array}{l}0.516 \\
0.517 \\
0.518 \\
0.519 \\
0.520\end{array}$ & $\begin{array}{l}0.90858 \\
0.90710 \\
0.90562 \\
0.90415 \\
0.90269\end{array}$ & $\begin{array}{l}148 \\
148 \\
147 \\
146 \\
147\end{array}$ & $\begin{array}{l}0.76409 \\
0.76368 \\
0.76326 \\
0.76285 \\
0.76243\end{array}$ & $\begin{array}{l}41 \\
42 \\
41 \\
42 \\
41\end{array}$ & $\begin{array}{l}1.67267 \\
1.67078 \\
1.66889 \\
1.66700 \\
1.66512\end{array}$ & $\begin{array}{l}189 \\
189 \\
189 \\
188 \\
188\end{array}$ & $\begin{array}{l}0.97810 \\
0.97802 \\
0.97794 \\
0.97785 \\
0.97777\end{array}$ \\
\hline $\begin{array}{l}0.521 \\
0.522 \\
0.523 \\
0.524 \\
0.525\end{array}$ & $\begin{array}{l}0.90122 \\
0.89976 \\
0.89830 \\
0.89685 \\
0.89540\end{array}$ & $\begin{array}{l}146 \\
146 \\
145 \\
145 \\
145\end{array}$ & $\begin{array}{l}0.76202 \\
0.76160 \\
0.76119 \\
0.76078 \\
0.76036\end{array}$ & $\begin{array}{l}42 \\
41 \\
41 \\
42 \\
41\end{array}$ & $\begin{array}{l}1.66324 \\
1.66136 \\
1.65949 \\
1.65763 \\
1.65576\end{array}$ & $\begin{array}{l}188 \\
187 \\
186 \\
187 \\
186\end{array}$ & $\begin{array}{l}0.97768 \\
0.97760 \\
0.97751 \\
0.97743 \\
0.97734\end{array}$ \\
\hline $\begin{array}{l}0.526 \\
0.527 \\
0.528 \\
0.529 \\
0.530\end{array}$ & $\begin{array}{l}0.89395 \\
0.89251 \\
0.89107 \\
0.88963 \\
0.88820\end{array}$ & $\begin{array}{l}144 \\
144 \\
144 \\
143 \\
143\end{array}$ & $\begin{array}{l}0.75995 \\
0.75954 \\
0.75912 \\
0.75871 \\
0.75830\end{array}$ & $\begin{array}{l}41 \\
42 \\
41 \\
41 \\
41\end{array}$ & $\begin{array}{l}1.65390 \\
1.65205 \\
1.65019 \\
1.64835 \\
1.64650\end{array}$ & $\begin{array}{l}185 \\
186 \\
184 \\
185 \\
184\end{array}$ & $\begin{array}{l}0.97726 \\
0.97717 \\
0.97709 \\
0.97700 \\
0.97692\end{array}$ \\
\hline $\begin{array}{l}0.531 \\
0.532 \\
0.533 \\
0.534 \\
0.535\end{array}$ & $\begin{array}{l}0.88677 \\
0.88535 \\
0.88392 \\
0.88251 \\
0.88109\end{array}$ & $\begin{array}{l}142 \\
143 \\
141 \\
142 \\
141\end{array}$ & $\begin{array}{l}0.75789 \\
0.75747 \\
0.75706 \\
0.75665 \\
0.75624\end{array}$ & $\begin{array}{l}42 \\
41 \\
41 \\
41 \\
41\end{array}$ & $\begin{array}{l}1.64466 \\
1.64282 \\
1.6409 .9 \\
1.63916 \\
1.63733\end{array}$ & $\begin{array}{l}184 \\
183 \\
183 \\
183 \\
182\end{array}$ & $\begin{array}{l}0.97683 \\
0.97674 \\
0.97666 \\
0.97657 \\
0.97649\end{array}$ \\
\hline $\begin{array}{l}0.536 \\
0.537 \\
0.538 \\
0.539 \\
0.540\end{array}$ & $\begin{array}{l}0.87968 \\
0.87827 \\
0.87687 \\
0.87546 \\
0.87407\end{array}$ & $\begin{array}{l}141 \\
140 \\
141 \\
139 \\
140\end{array}$ & $\begin{array}{l}0.75583 \\
0.75542 \\
0.75500 \\
0.75459 \\
0.75418\end{array}$ & $\begin{array}{l}41 \\
42 \\
41 \\
41 \\
41\end{array}$ & $\begin{array}{l}1.63551 \\
1.63369 \\
1.63187 \\
1.63006 \\
1.62825\end{array}$ & $\begin{array}{l}182 \\
182 \\
181 \\
181 \\
181\end{array}$ & $\begin{array}{l}0.97640 \\
0.97631 \\
0.97622 \\
0.97614 \\
0.97605\end{array}$ \\
\hline $\begin{array}{l}0.541 \\
0.542 \\
0.543 \\
0.544 \\
0.545\end{array}$ & $\begin{array}{l}0.87267 \\
0.87128 \\
0.86989 \\
0.86851 \\
0.86712\end{array}$ & $\begin{array}{l}139 \\
139 \\
138 \\
139 \\
137\end{array}$ & $\begin{array}{l}0.75377 \\
0.75336 \\
0.75295 \\
0.75254 \\
0.75213\end{array}$ & $\begin{array}{l}41 \\
41 \\
41 \\
41 \\
41\end{array}$ & $\begin{array}{l}1.62644 \\
1.62464 \\
1.62284 \\
1.62105 \\
1.61925\end{array}$ & $\begin{array}{l}180 \\
180 \\
179 \\
180 \\
178\end{array}$ & $\begin{array}{l}0.97596 \\
0.97588 \\
0.97579 \\
0.97570 \\
0.97561\end{array}$ \\
\hline $\begin{array}{l}0.546 \\
0.547 \\
0.548 \\
0.549 \\
0.550\end{array}$ & $\begin{array}{l}0.86575 \\
0.86437 \\
0.86300 \\
0.86163 \\
0.86026\end{array}$ & $\begin{array}{l}138 \\
137 \\
137 \\
137\end{array}$ & $\begin{array}{l}0.75172 \\
0.75131 \\
0.75090 \\
0.75049 \\
0.75008\end{array}$ & $\begin{array}{l}41 \\
41 \\
41 \\
41\end{array}$ & $\begin{array}{l}1.61747 \\
1.61568 \\
1.61390 \\
1.61212 \\
1.61035\end{array}$ & $\begin{array}{l}179 \\
178 \\
178 \\
177\end{array}$ & $\begin{array}{l}0.97552 \\
0.97543 \\
0.97535 \\
0.97526 \\
0.97517\end{array}$ \\
\hline
\end{tabular}


Table I Harmonic Oscillator Contributions (in dimensionless form) to the Thermodynamic Functions for Values of $\mathrm{X}=\mathrm{hc} / \mathrm{kT}$
$\mathbf{Z}$
- (F०-EO)/RT
$\Delta \quad\left(\mathrm{H}^{\circ}-\mathrm{E}_{\mathrm{O}}^{\circ}\right) / \mathrm{RT}$
$\triangle \quad$ SO/R
$\triangle \quad \mathrm{co} / \mathrm{R}$

$\Delta$

\begin{tabular}{|c|c|c|c|c|c|c|c|}
\hline 0.550 & 0.86026 & 136 & 0.75008 & 41 & 1.61035 & 177 & 0.97517 \\
\hline $\begin{array}{l}0.551 \\
0.552 \\
0.553 \\
0.554 \\
0.555\end{array}$ & $\begin{array}{l}0.85890 \\
0.85754 \\
0.85619 \\
0.85483 \\
0.85349\end{array}$ & $\begin{array}{l}136 \\
135 \\
136 \\
134 \\
135\end{array}$ & $\begin{array}{l}0.74967 \\
0.74926 \\
0.74886 \\
0.74845 \\
0.74804\end{array}$ & $\begin{array}{l}41 \\
40 \\
41 \\
41 \\
41\end{array}$ & $\begin{array}{l}1.60858 \\
1.60681 \\
1.60504 \\
1.60328 \\
1.60152\end{array}$ & $\begin{array}{l}177 \\
177 \\
176 \\
176 \\
175\end{array}$ & $\begin{array}{l}0.97508 \\
0.97499 \\
0.97490 \\
0.97481 \\
0.97472\end{array}$ \\
\hline $\begin{array}{l}0.556 \\
0.557 \\
0.558 \\
0.559 \\
0.560\end{array}$ & $\begin{array}{l}0.85214 \\
0.85080 \\
0.84946 \\
0.84812 \\
0.84679\end{array}$ & $\begin{array}{l}134 \\
134 \\
134 \\
133 \\
133\end{array}$ & $\begin{array}{l}0.74763 \\
0.74722 \\
0.74681 \\
0.74641 \\
0.74600\end{array}$ & $\begin{array}{l}41 \\
41 \\
40 \\
41 \\
41\end{array}$ & $\begin{array}{l}1.59977 \\
1.59802 \\
1.59627 \\
1.59452 \\
1.59278\end{array}$ & $\begin{array}{l}175 \\
175 \\
175 \\
174 \\
173\end{array}$ & $\begin{array}{l}0.97463 \\
0.97454 \\
0.97445 \\
0.97436 \\
0.97427\end{array}$ \\
\hline $\begin{array}{l}0.561 \\
0.562 \\
0.563 \\
0.564 \\
0.565\end{array}$ & $\begin{array}{l}0.84546 \\
0.84413 \\
0.84280 \\
0.84148 \\
0.84016\end{array}$ & $\begin{array}{l}133 \\
133 \\
132 \\
132 \\
131\end{array}$ & $\begin{array}{l}0.74559 \\
0.74518 \\
0.74478 \\
0.74437 \\
0.74396\end{array}$ & $\begin{array}{l}41 \\
40 \\
41 \\
41 \\
41\end{array}$ & $\begin{array}{l}1.59105 \\
1.58931 \\
1.58758 \\
1.58585 \\
1.58413\end{array}$ & $\begin{array}{l}174 \\
173 \\
173 \\
172 \\
173\end{array}$ & $\begin{array}{l}0.97418 \\
0.97409 \\
0.97400 \\
0.97391 \\
0.97382\end{array}$ \\
\hline $\begin{array}{l}0.566 \\
0.567 \\
0.568 \\
0.569 \\
0.570\end{array}$ & $\begin{array}{l}0.83885 \\
0.83754 \\
0.83623 \\
0.83492 \\
0.83362\end{array}$ & $\begin{array}{l}131 \\
131 \\
131 \\
130 \\
130\end{array}$ & $\begin{array}{l}0.74355 \\
0.74315 \\
0.74274 \\
0.74234 \\
0.74193\end{array}$ & $\begin{array}{l}40 \\
41 \\
40 \\
41 \\
41\end{array}$ & $\begin{array}{l}1.58240 \\
1.58068 \\
1.57897 \\
1.57726 \\
1.57555\end{array}$ & $\begin{array}{l}172 \\
171 \\
171 \\
171 \\
171\end{array}$ & $\begin{array}{l}0.97373 \\
0.97363 \\
0.97354 \\
0.97345 \\
0.97336\end{array}$ \\
\hline $\begin{array}{l}0.571 \\
0.572 \\
0.573 \\
0.574 \\
0.575\end{array}$ & $\begin{array}{l}0.83232 \\
0.83102 \\
0.82973 \\
0.82844 \\
0.82715\end{array}$ & $\begin{array}{l}130 \\
129 \\
129 \\
129 \\
129\end{array}$ & $\begin{array}{l}0.74152 \\
0.74112 \\
0.74071 \\
0.74031 \\
0.73990\end{array}$ & $\begin{array}{l}40 \\
41 \\
40 \\
41 \\
40\end{array}$ & $\begin{array}{l}1.57384 \\
1.57214 \\
1.57044 \\
1.56874 \\
1.56705\end{array}$ & $\begin{array}{l}170 \\
170 \\
170 \\
169 \\
169\end{array}$ & $\begin{array}{l}0.97327 \\
0.97317 \\
0.97308 \\
0.97299 \\
0.97290\end{array}$ \\
\hline $\begin{array}{l}0.576 \\
0.577 \\
0.578 \\
0.579 \\
0.580\end{array}$ & $\begin{array}{l}0.82586 \\
0.82458 \\
0.82330 \\
0.82202 \\
0.82075\end{array}$ & $\begin{array}{l}128 \\
128 \\
128 \\
127 \\
127\end{array}$ & $\begin{array}{l}0.73950 \\
0.73909 \\
0.73869 \\
0.73828 \\
0.73788\end{array}$ & $\begin{array}{l}41 \\
40 \\
41 \\
40 \\
41\end{array}$ & $\begin{array}{l}1.56536 \\
1.56367 \\
1.56199 \\
1.56031 \\
1.55863\end{array}$ & $\begin{array}{l}169 \\
168 \\
168 \\
168 \\
168\end{array}$ & $\begin{array}{l}0.97280 \\
0.97271 \\
0.97262 \\
0.97253 \\
0.97243\end{array}$ \\
\hline $\begin{array}{l}0.581 \\
0.582 \\
0.583 \\
0.584 \\
0.585\end{array}$ & $\begin{array}{l}0.81948 \\
0.81821 \\
0.81695 \\
0.81568 \\
0.81442\end{array}$ & $\begin{array}{l}127 \\
126 \\
127 \\
126 \\
125\end{array}$ & $\begin{array}{l}0.73747 \\
0.73707 \\
0.73666 \\
0.73626 \\
0.73586\end{array}$ & $\begin{array}{l}40 \\
41 \\
40 \\
40 \\
41\end{array}$ & $\begin{array}{l}1.55695 \\
1.55528 \\
1.55361 \\
1.55194 \\
1.55028\end{array}$ & $\begin{array}{l}167 \\
167 \\
167 \\
166 \\
166\end{array}$ & $\begin{array}{l}0.97234 \\
0.97224 \\
0.97215 \\
0.97206 \\
0.97196\end{array}$ \\
\hline $\begin{array}{l}0.586 \\
0.587 \\
0.588 \\
0.589 \\
0.590\end{array}$ & $\begin{array}{l}0.81317 \\
0.81191 \\
0.81066 \\
0.80942 \\
0.80817\end{array}$ & $\begin{array}{l}126 \\
125 \\
124 \\
125 \\
124\end{array}$ & $\begin{array}{l}0.73545 \\
0.73505 \\
0.73465 \\
0.73424 \\
0.73384\end{array}$ & $\begin{array}{l}40 \\
40 \\
41 \\
40 \\
40\end{array}$ & $\begin{array}{l}1.54862 \\
1.54696 \\
1.54531 \\
1.54366 \\
1.54201\end{array}$ & $\begin{array}{l}166 \\
165 \\
165 \\
165 \\
164\end{array}$ & $\begin{array}{l}0.97187 \\
0.97177 \\
0.97168 \\
0.97158 \\
0.97149\end{array}$ \\
\hline $\begin{array}{l}0.591 \\
0.592 \\
0.593 \\
0.594 \\
0.595\end{array}$ & $\begin{array}{l}0.80693 \\
0.80569 \\
0.80445 \\
0.80322 \\
0.80199\end{array}$ & $\begin{array}{l}124 \\
124 \\
123 \\
123 \\
123\end{array}$ & $\begin{array}{l}0.73344 \\
0.73304 \\
0.73263 \\
0.73223 \\
0.73183\end{array}$ & $\begin{array}{l}40 \\
41 \\
40 \\
40 \\
40\end{array}$ & $\begin{array}{l}1.54037 \\
1.53872 \\
1.53709 \\
1.53545 \\
1.53382\end{array}$ & $\begin{array}{l}165 \\
163 \\
164 \\
163 \\
163\end{array}$ & $\begin{array}{l}0.97139 \\
0.97130 \\
0.97120 \\
0.97111 \\
0.97101\end{array}$ \\
\hline $\begin{array}{l}0.596 \\
0.597 \\
0.598 \\
0.599 \\
0.600\end{array}$ & $\begin{array}{l}0.80076 \\
0.79953 \\
0.79831 \\
0.79709 \\
0.79587\end{array}$ & $\begin{array}{l}123 \\
122 \\
122 \\
122\end{array}$ & $\begin{array}{l}0.73143 \\
0.73103 \\
0.73062 \\
0.73022 \\
0.72982\end{array}$ & $\begin{array}{l}40 \\
41 \\
40 \\
40\end{array}$ & $\begin{array}{l}1.53219 \\
1.53056 \\
1.52893 \\
1.52731 \\
1.52569\end{array}$ & $\begin{array}{l}163 \\
163 \\
162 \\
162\end{array}$ & $\begin{array}{l}0.97092 \\
0.97082 \\
0.97072 \\
0.97063 \\
0.97053\end{array}$ \\
\hline
\end{tabular}


Table I Harmonic Oscillator Contributions (in dimensionless form) to the

Thermodynamic Functions for Values of $X=h c v / k T$

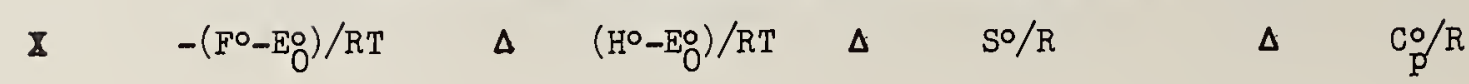

\begin{tabular}{|c|c|c|c|c|c|c|c|}
\hline 0.600 & 0.79587 & 121 & 0.72982 & 40 & 1.52569 & 161 & 0.97053 \\
\hline $\begin{array}{l}0.601 \\
0.602 \\
0.603 \\
0.604 \\
0.605\end{array}$ & $\begin{array}{l}0.79466 \\
0.79344 \\
0.79223 \\
0.79103 \\
0.78982\end{array}$ & $\begin{array}{l}122 \\
121 \\
120 \\
121 \\
120\end{array}$ & $\begin{array}{l}0.72942 \\
0.72902 \\
0.72862 \\
0.72822 \\
0.72782\end{array}$ & $\begin{array}{l}40 \\
40 \\
40 \\
40 \\
40\end{array}$ & $\begin{array}{l}1.52408 \\
1.52246 \\
1.52085 \\
1.51924 \\
1.51764\end{array}$ & $\begin{array}{l}162 \\
161 \\
161 \\
160 \\
160\end{array}$ & $\begin{array}{l}0.97044 \\
0.97034 \\
0.97024 \\
0.97015 \\
0.97005\end{array}$ \\
\hline $\begin{array}{l}0.606 \\
0.607 \\
0.608 \\
0.609 \\
0.610\end{array}$ & $\begin{array}{l}0.78862 \\
0.78742 \\
0.78622 \\
0.78503 \\
0.78384\end{array}$ & $\begin{array}{l}120 \\
120 \\
119 \\
119 \\
119\end{array}$ & $\begin{array}{l}0.72742 \\
0.72702 \\
0.72662 \\
0.72622 \\
0.72582\end{array}$ & $\begin{array}{l}40 \\
40 \\
40 \\
40 \\
40\end{array}$ & $\begin{array}{l}1.51604 \\
1.51444 \\
1.51284 \\
1.51125 \\
1.50966\end{array}$ & $\begin{array}{l}160 \\
160 \\
159 \\
159 \\
159\end{array}$ & $\begin{array}{l}0.96995 \\
0.96985 \\
0.96976 \\
0.96966 \\
0.96956\end{array}$ \\
\hline $\begin{array}{l}0.611 \\
0.612 \\
0.613 \\
0.614 \\
0.615\end{array}$ & $\begin{array}{l}0.78265 \\
0.78147 \\
0.78028 \\
0.77910 \\
0.77792\end{array}$ & $\begin{array}{l}118 \\
119 \\
118 \\
118 \\
117\end{array}$ & $\begin{array}{l}0.72542 \\
0.72502 \\
0.72462 \\
0.72422 \\
0.72382\end{array}$ & $\begin{array}{l}40 \\
40 \\
40 \\
40 \\
40\end{array}$ & $\begin{array}{l}1.50807 \\
1.50648 \\
1.50490 \\
1.50332 \\
1.50174\end{array}$ & $\begin{array}{l}159 \\
158 \\
158 \\
158 \\
157\end{array}$ & $\begin{array}{l}0.96946 \\
0.96936 \\
0.96927 \\
0.96917 \\
0.96907\end{array}$ \\
\hline $\begin{array}{l}0.616 \\
0.617 \\
0.618 \\
0.619 \\
0.620\end{array}$ & $\begin{array}{l}0.77675 \\
0.77557 \\
0.77440 \\
0.77324 \\
0.77207\end{array}$ & $\begin{array}{l}118 \\
117 \\
116 \\
117 \\
116\end{array}$ & $\begin{array}{l}0.72342 \\
0.72302 \\
0.72263 \\
0.72223 \\
0.72183\end{array}$ & $\begin{array}{l}40 \\
39 \\
40 \\
40 \\
40\end{array}$ & $\begin{array}{l}1.50017 \\
1.49860 \\
1.49703 \\
1.49546 \\
1.49390\end{array}$ & $\begin{array}{l}157 \\
157 \\
157 \\
156 \\
156\end{array}$ & $\begin{array}{l}0.96897 \\
0.96887 \\
0.96877 \\
0.96867 \\
0.96857\end{array}$ \\
\hline $\begin{array}{l}0.621 \\
0.622 \\
0.623 \\
0.624 \\
0.625\end{array}$ & $\begin{array}{l}0.77091 \\
0.76975 \\
0.76859 \\
0.76743 \\
0.76628\end{array}$ & $\begin{array}{l}116 \\
116 \\
116 \\
115 \\
115\end{array}$ & $\begin{array}{l}0.72143 \\
0.72103 \\
0.72064 \\
0.72024 \\
0.71984\end{array}$ & $\begin{array}{l}40 \\
39 \\
40 \\
40 \\
40\end{array}$ & $\begin{array}{l}1.49234 \\
1.49078 \\
1.48923 \\
1.48767 \\
1.48612\end{array}$ & $\begin{array}{l}156 \\
155 \\
156 \\
155 \\
155\end{array}$ & $\begin{array}{l}0.96847 \\
0.96837 \\
0.96827 \\
0.96817 \\
0.96807\end{array}$ \\
\hline $\begin{array}{l}0.626 \\
0.627 \\
0.628 \\
0.629 \\
0.630\end{array}$ & $\begin{array}{l}0.76513 \\
0.76398 \\
0.76284 \\
0.76169 \\
0.76055\end{array}$ & $\begin{array}{l}115 \\
114 \\
115 \\
114 \\
114\end{array}$ & $\begin{array}{l}0.71944 \\
0.71905 \\
0.71865 \\
0.71825 \\
0.71786\end{array}$ & $\begin{array}{l}39 \\
40 \\
40 \\
39 \\
40\end{array}$ & $\begin{array}{l}1.48457 \\
1.48303 \\
1.48149 \\
1.47995 \\
1.47841\end{array}$ & $\begin{array}{l}154 \\
154 \\
154 \\
154 \\
153\end{array}$ & $\begin{array}{l}0.96797 \\
0.96787 \\
0.96777 \\
0.96767 \\
0.96757\end{array}$ \\
\hline $\begin{array}{l}0.631 \\
0.632 \\
0.633 \\
0.634 \\
0.635\end{array}$ & $\begin{array}{l}0.75941 \\
0.75828 \\
0.75714 \\
0.75601 \\
0.75489\end{array}$ & $\begin{array}{l}113 \\
114 \\
113 \\
112 \\
113\end{array}$ & $\begin{array}{l}0.71746 \\
0.71707 \\
0.71667 \\
0.71627 \\
0.71588\end{array}$ & $\begin{array}{l}39 \\
40 \\
40 \\
39 \\
40\end{array}$ & $\begin{array}{l}1.47688 \\
1.47534 \\
1.47381 \\
1.47229 \\
1.47076\end{array}$ & $\begin{array}{l}154 \\
153 \\
152 \\
153 \\
152\end{array}$ & $\begin{array}{l}0.96747 \\
0.96737 \\
0.96727 \\
0.96717 \\
0.96706\end{array}$ \\
\hline $\begin{array}{l}0.636 \\
0.637 \\
0.638 \\
0.639 \\
0.640\end{array}$ & $\begin{array}{l}0.75376 \\
0.75264 \\
0.75151 \\
0.75039 \\
0.74928\end{array}$ & 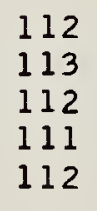 & $\begin{array}{l}0.71548 \\
0.71509 \\
0.71469 \\
0.71430 \\
0.71390\end{array}$ & $\begin{array}{l}39 \\
40 \\
39 \\
40 \\
39\end{array}$ & $\begin{array}{l}1.46924 \\
1.46772 \\
1.46621 \\
1.46469 \\
1.46318\end{array}$ & $\begin{array}{l}152 \\
151 \\
152 \\
151 \\
151\end{array}$ & $\begin{array}{l}0.96696 \\
0.96686 \\
0.96676 \\
0.96666 \\
0.96655\end{array}$ \\
\hline $\begin{array}{l}0.641 \\
0.642 \\
0.643 \\
0.644 \\
0.645\end{array}$ & $\begin{array}{l}0.74816 \\
0.74705 \\
0.74594 \\
0.74484 \\
0.74373\end{array}$ & $\begin{array}{lll}1 & 1 & 1 \\
1 & 1 & 1 \\
1 & 1 & 0 \\
1 & 1 & 1 \\
1 & 1 & 0\end{array}$ & $\begin{array}{l}0.71351 \\
0.71311 \\
0.711272 \\
0.71232 \\
0.71193\end{array}$ & $\begin{array}{l}40 \\
39 \\
40 \\
39 \\
39\end{array}$ & $\begin{array}{l}1.46167 \\
1.46017 \\
1.45866 \\
1.45716 \\
1.45566\end{array}$ & $\begin{array}{l}150 \\
151 \\
150 \\
150 \\
150\end{array}$ & $\begin{array}{l}0.96645 \\
0.96635 \\
0.96625 \\
0.96614 \\
0.96604\end{array}$ \\
\hline $\begin{array}{l}0.646 \\
0.647 \\
0.648 \\
0.649 \\
0.650\end{array}$ & $\begin{array}{l}0.74263 \\
0.74153 \\
0.74043 \\
0.73933 \\
0.73824\end{array}$ & 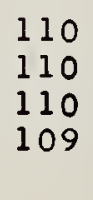 & $\begin{array}{l}0.71154 \\
0.71114 \\
0.711075 \\
0.71036 \\
0.70996\end{array}$ & $\begin{array}{l}40 \\
39 \\
39 \\
40\end{array}$ & $\begin{array}{l}1.45416 \\
1.45267 \\
1.45118 \\
1.44969 \\
1.44820\end{array}$ & $\begin{array}{l}149 \\
149 \\
149 \\
149\end{array}$ & $\begin{array}{l}0.96594 \\
0.96583 \\
0.96573 \\
0.96563 \\
0.96552\end{array}$ \\
\hline
\end{tabular}


Table I Harmonic Oscillator Contributions (in dimensionless form) to the

Thermodynamic Functions for Values of $\mathrm{X}=\mathrm{hcv} / \mathrm{kT}$
$\mathbf{X}$
$-\left(F^{\circ}-E_{0} \circ\right) / R T$
$\Delta \quad\left(\mathrm{H}^{\circ}-\mathrm{E}_{\mathrm{O}}^{\circ}\right) / \mathrm{RT}$
$\Delta \quad \mathrm{S} \% / \mathrm{R}$
$\Delta \quad \mathrm{C} \circ \mathrm{p}$

$\Delta$

\begin{tabular}{|c|c|c|c|c|c|c|c|}
\hline 0.650 & 0.73824 & 109 & 0.70996 & 39 & 1.44820 & 148 & 0.96552 \\
\hline $\begin{array}{l}0.651 \\
0.652\end{array}$ & $\begin{array}{l}0.73715 \\
0.73606\end{array}$ & $\begin{array}{l}109 \\
109\end{array}$ & $\begin{array}{l}0.70957 \\
0.70918\end{array}$ & $\begin{array}{l}39 \\
40\end{array}$ & $\begin{array}{l}1.44672 \\
1.44524\end{array}$ & $\begin{array}{l}148 \\
148\end{array}$ & $\begin{array}{l}0.96542 \\
0.96532\end{array}$ \\
\hline $\begin{array}{l}0.653 \\
0.654 \\
0.655\end{array}$ & $\begin{array}{l}0.73497 \\
0.73389 \\
0.73281\end{array}$ & $\begin{array}{l}108 \\
108 \\
108\end{array}$ & $\begin{array}{l}0.70878 \\
0.70839 \\
0.70800\end{array}$ & $\begin{array}{l}39 \\
39 \\
39\end{array}$ & $\begin{array}{l}1.44376 \\
1.44228 \\
1.44081\end{array}$ & $\begin{array}{l}148 \\
147 \\
148\end{array}$ & $\begin{array}{l}0.96521 \\
0.96511 \\
0.96500\end{array}$ \\
\hline $\begin{array}{l}0.656 \\
0.657 \\
0.658 \\
0.659 \\
0.660\end{array}$ & $\begin{array}{l}0.73173 \\
0.73065 \\
0.72957 \\
0.72850 \\
0.72743\end{array}$ & $\begin{array}{l}108 \\
108 \\
107 \\
107 \\
107\end{array}$ & $\begin{array}{l}0.70761 \\
0.70721 \\
0.70682 \\
0.70643 \\
0.70604\end{array}$ & $\begin{array}{l}40 \\
39 \\
39 \\
39 \\
39\end{array}$ & $\begin{array}{l}1.43933 \\
1.43786 \\
1.43640 \\
1.43493 \\
1.43347\end{array}$ & $\begin{array}{l}147 \\
146 \\
147 \\
146 \\
146\end{array}$ & $\begin{array}{l}0.96490 \\
0.96479 \\
0.96469 \\
0.96458 \\
0.96448\end{array}$ \\
\hline $\begin{array}{l}0.661 \\
0.662 \\
0.663 \\
0.664 \\
0.665\end{array}$ & $\begin{array}{l}0.72636 \\
0.72530 \\
0.72423 \\
0.72317 \\
0.72211\end{array}$ & $\begin{array}{l}106 \\
107 \\
106 \\
106 \\
106\end{array}$ & $\begin{array}{l}0.70565 \\
0.70526 \\
0.70487 \\
0.70447 \\
0.70408\end{array}$ & $\begin{array}{l}39 \\
39 \\
40 \\
39 \\
39\end{array}$ & $\begin{array}{l}1.43201 \\
1.43055 \\
1.42910 \\
1.42764 \\
1.42619\end{array}$ & $\begin{array}{l}146 \\
145 \\
146 \\
145 \\
145\end{array}$ & $\begin{array}{l}0.96437 \\
0.96427 \\
0.96416 \\
0.96405 \\
0.96395\end{array}$ \\
\hline $\begin{array}{l}0.666 \\
0.667 \\
0.668 \\
0.669 \\
0.670\end{array}$ & $\begin{array}{l}0.72105 \\
0.72000 \\
0.71894 \\
0.71789 \\
0.71684\end{array}$ & $\begin{array}{l}105 \\
106 \\
105 \\
105 \\
104\end{array}$ & $\begin{array}{l}0.70369 \\
0.70330 \\
0.70291 \\
0.70252 \\
0.70213\end{array}$ & $\begin{array}{l}39 \\
39 \\
39 \\
39 \\
39\end{array}$ & $\begin{array}{l}1.42474 \\
1.42330 \\
1.42185 \\
1.42041 \\
1.41897\end{array}$ & $\begin{array}{l}144 \\
145 \\
144 \\
144 \\
143\end{array}$ & $\begin{array}{l}0.96384 \\
0.96374 \\
0.96363 \\
0.96352 \\
0.96342\end{array}$ \\
\hline $\begin{array}{l}0.671 \\
0.672 \\
0.673 \\
0.674 \\
0.675\end{array}$ & $\begin{array}{l}0.71580 \\
0.71475 \\
0.71371 \\
0.71267 \\
0.71163\end{array}$ & $\begin{array}{l}105 \\
104 \\
104 \\
104 \\
104\end{array}$ & $\begin{array}{l}0.70174 \\
0.70135 \\
0.70096 \\
0.70057 \\
0.70018\end{array}$ & $\begin{array}{l}39 \\
39 \\
39 \\
39 \\
39\end{array}$ & $\begin{array}{l}1.41754 \\
1.41610 \\
1.41467 \\
1.41324 \\
1.41181\end{array}$ & $\begin{array}{l}144 \\
143 \\
143 \\
143 \\
142\end{array}$ & $\begin{array}{l}0.96331 \\
0.96320 \\
0.96310 \\
0.96299 \\
0.96288\end{array}$ \\
\hline $\begin{array}{l}0.676 \\
0.677 \\
0.678 \\
0.679 \\
0.680\end{array}$ & $\begin{array}{l}0.71059 \\
0.70956 \\
0.70853 \\
0.70750 \\
0.70647\end{array}$ & $\begin{array}{l}103 \\
103 \\
103 \\
103 \\
103\end{array}$ & $\begin{array}{l}0.69979 \\
0.69941 \\
0.69902 \\
0.69863 \\
0.69824\end{array}$ & $\begin{array}{l}38 \\
39 \\
39 \\
39 \\
39\end{array}$ & $\begin{array}{l}1.41039 \\
1.40896 \\
1.40754 \\
1.40613 \\
1.40471\end{array}$ & $\begin{array}{l}143 \\
142 \\
141 \\
142 \\
142\end{array}$ & $\begin{array}{l}0.96277 \\
0.96267 \\
0.96256 \\
0.96245 \\
0.96234\end{array}$ \\
\hline $\begin{array}{l}0.681 \\
0.682 \\
0.683 \\
0.684 \\
0.685\end{array}$ & $\begin{array}{l}0.70544 \\
0.70442 \\
0.70340 \\
0.70238 \\
0.70136\end{array}$ & $\begin{array}{l}102 \\
102 \\
102 \\
102 \\
101\end{array}$ & $\begin{array}{l}0.69785 \\
0.69746 \\
0.69708 \\
0.69669 \\
0.69630\end{array}$ & $\begin{array}{l}39 \\
38 \\
39 \\
39 \\
39\end{array}$ & $\begin{array}{l}1.40329 \\
1.40188 \\
1.40047 \\
1.39907 \\
1.39766\end{array}$ & $\begin{array}{l}141 \\
141 \\
140 \\
141 \\
140\end{array}$ & $\begin{array}{l}0.96223 \\
0.96212 \\
0.96202 \\
0.96191 \\
0.96180\end{array}$ \\
\hline $\begin{array}{l}0.686 \\
0.687 \\
0.688 \\
0.689 \\
0.690\end{array}$ & $\begin{array}{l}0.70035 \\
0.69933 \\
0.69832 \\
0.69731 \\
0.69630\end{array}$ & $\begin{array}{l}102 \\
101 \\
101 \\
101 \\
100\end{array}$ & $\begin{array}{l}0.69591 \\
0.69552 \\
0.69514 \\
0.69475 \\
0.69436\end{array}$ & $\begin{array}{l}39 \\
38 \\
39 \\
39 \\
38\end{array}$ & $\begin{array}{l}1.39626 \\
1.39486 \\
1.39346 \\
1.39206 \\
1.39067\end{array}$ & $\begin{array}{l}140 \\
140 \\
140 \\
139 \\
139\end{array}$ & $\begin{array}{l}0.96169 \\
0.96158 \\
0.96147 \\
0.96136 \\
0.96125\end{array}$ \\
\hline $\begin{array}{l}0.691 \\
0.692 \\
0.693 \\
0.694 \\
0.695\end{array}$ & $\begin{array}{l}0.69530 \\
0.69430 \\
0.69329 \\
0.69230 \\
0.69130\end{array}$ & $\begin{array}{r}100 \\
101 \\
99 \\
100 \\
100\end{array}$ & $\begin{array}{l}0.69398 \\
0.69359 \\
0.69320 \\
0.69282 \\
0.69243\end{array}$ & $\begin{array}{l}39 \\
39 \\
38 \\
39 \\
38\end{array}$ & $\begin{array}{l}1.38928 \\
1.38789 \\
1.38650 \\
1.38511 \\
1.38373\end{array}$ & $\begin{array}{l}139 \\
139 \\
139 \\
138 \\
138\end{array}$ & $\begin{array}{l}0.96114 \\
0.96103 \\
0.96092 \\
0.96081 \\
0.96070\end{array}$ \\
\hline $\begin{array}{l}0.696 \\
0.697 \\
0.698 \\
0.699 \\
0.700\end{array}$ & $\begin{array}{l}0.69030 \\
0.68931 \\
0.68832 \\
0.68733 \\
0.68634\end{array}$ & $\begin{array}{l}99 \\
99 \\
99 \\
99\end{array}$ & $\begin{array}{l}0.69205 \\
0.69166 \\
0.69127 \\
0.69089 \\
0.69050\end{array}$ & $\begin{array}{l}39 \\
39 \\
38 \\
39\end{array}$ & $\begin{array}{l}1.38235 \\
1.38097 \\
1.37959 \\
1.37822 \\
1.37684\end{array}$ & $\begin{array}{l}138 \\
138 \\
137 \\
138\end{array}$ & $\begin{array}{l}0.96059 \\
0.96048 \\
0.96037 \\
0.96026 \\
0.96015\end{array}$ \\
\hline
\end{tabular}


Table I Harmonic Oscillator Contributions (in dimensionless form) to the Thermodynamic Functions for Values of $\mathrm{X}=\mathrm{hcv} / \mathrm{kT}$

$\mathbf{x}$ $-\left(F^{\circ}-E O\right) / R T$

$\Delta \quad\left(H^{\circ}-E_{0}^{\circ}\right) / R T \quad \Delta \quad S \circ / R$

0.68634

0.700

0.701

0.702

0.703

0.704

0.705

0.706

0.707

0.708

0.709

0.710

0.711

0.712

0.713

0.714

0.715

0.716

0.717

0.718

0.719

0.720

0.721

0.722

0.723

0.724

0.725

0.726

0.727

0.728

0.729

0.730

0.731

0.732

0.733

0.734

0.735

0.736

0.737

0.738

0.739

0.740

0.741

0.742

0.743

0.744

0.745

0.746

0.747

0.748

0.749

0.750
0.68536

0.68437

0.68339

0.68241

0.68143

0.68046

0.67948

0.67851

0.67754

0.67657

0.67561

0.67464

0.67368

0.67272

0.67176

0.67080

0.66985

0.66890

0.66795

0.66700

0.66605

0.66510

0.66416

0.66322

0.66228

0.66134

0.66040

0.65947

0.65854

0.65760

0.65668

0.65575

0.65390

0.65298

0.65206

0.65114

0.65022

0.64930

0.64839

0.64748

0.64657

0.64566

0.64476

0.64385

0.64205

0.64115

0.64025

0.63935
0.65482

0.64295

$\begin{array}{ll}98 & 0.69050 \\ 99 & 0.69012 \\ 98 & 0.68973 \\ 98 & 0.68935 \\ 98 & 0.68896 \\ 97 & 0.68858 \\ 98 & 0.68820 \\ 97 & 0.68781 \\ 97 & 0.68743 \\ 97 & 0.68704 \\ 96 & 0.68666\end{array}$

38

$\begin{array}{ll}97 & 0.68628 \\ 96 & 0.68589 \\ 96 & 0.68551 \\ 96 & 0.68513 \\ 96 & 0.68474\end{array}$

0.68436

0.68398

0.68360

0.68321

38
38

39

38

0.68283

38

0.68245

0.68207

0.68169

0.68130

0.68092

38

38

39

38

94

94

93

93

94

92

0.68054

0.68016

0.67978

0.67940

0.67902

0.67864

0.67788

0.67750

0.67712

0.67674

0.67636

0.67598

0.67560

0.67522

0.67484

0.67446

0.67409

0.67371

0.67333

1.37684
1.37547
1.37411
1.37274
1.37137
1.37001
1.36865
1.36729
1.36594
1.36459
1.36323

137

$\Delta$

$\mathrm{Co} / \mathrm{R}$

$\Delta$

1.36188

1.36054

1.35919

1.35785

1.35651

1.35517

1.35383

1.35249

1.35116

1.34983

1.34850

1.34717

1.34585

1.34452

1.34320

1.34188

1.34056

1.33925

1.33793

1.33662

0.67826

1.33531

1.33401

1.33270

1.33140

1.33009

1.32879

1.32750

1.32620

1. 32491

1. 32361

1.32232

1.32103

1.31975

1.31846

1.31718

1.31590

1.31462

1.31334

1.31207

1.31079

0.96015

11

136

0.96004

11

137

137

136

136

0.95993

0.95981

0.95970

0.95959

11

11

11

136

$0.95948 \quad 11$

135

135

136

0.95937

0.95925

0.95914

0.95903

134

0.95892

0.95880

0.95869

0.95858

0.95847

12

11

11

11

134

134

134

134

134

133

133

0.95835

0.95824

0.95812

0.95801

0.95790

12

11

11

11

12

133

$0.95778 \quad 11$

133

132

133

132

0.95767

0.95755

0.95744

0.95733

11

12

11

11

12.

132

132

0.95721

0.95710

0.95698

0.95687

0.95675

12

11

11

12

132

131

131

130

131

130

131

130

$0.95663 \quad 11$

$0.95652 \quad 12$

$0.95640 \quad 11$

$0.95629 \quad 12$

$0.95617 \quad 11$

$0.95606 \quad 12$ 
Table I Harmonic Oscillator Contributions (in dimensionless form) to the Thermodynamic Functions for Values of $X=h c v / k T$

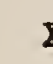

I $\quad-\left(\mathrm{F}^{\circ}-\mathrm{E}_{\mathrm{O}}\right) / \mathrm{RT}$

$\Delta$

$\left(\mathrm{H}^{\circ}-\mathrm{E}_{\mathrm{O}}\right) / \mathrm{RT}$

$\Delta$

So/R

0.67144

0.63846

0.751

0.752

0.753

0.754

0.755

0.756

0.757

0.758

0.759

0.760

0.761

0.762

0.763

0.764

0.765

0.766

0.767

0.768

0.769

0.770

0.771

0.772

0.773

0.774

0.775

0.776

0.777

0.778

0.779

0.780

0.781

0.782

0.783

0.784

0.785

0.786

0.787

0.788

0.789

0.790

0.791

0.792

0.793

0.794

0.795

0.796

0.797

0.798

0.799

0.800
0.63757

0.63668

0.63579

0.63490

0.63401

0.63313

0.63225

0.63136

0.63048

0.62961

0.62873

0.62786

0.62698

0.62611

0.62524

0.62438

0.62351

0.62264

0.62178

0.62092

0.62006

0.61920

0.61749

0.61664

0.61493

0.61409

0.61324

0.61239

0.61155

0.61071

0.60987

0.60903

0.60819

0.60735

0.60652

0.60568

0.60485

0.60402

0.60319

0.60237

0.60154

0.60072

0.59989

0.59907

0.59825

0.59743

0.59662
0.61835

0.61578

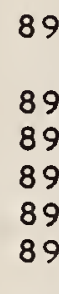

88

88

89

88

87

88

87

88

87

87

86

87

87

86

86

86

86

85

86

85

86

85

84

85

85

84

84

84

84

84

84

83

84

83

83

83

82

83

82

83

82

82

82

81

\subsection{6}

0.67031

0.66993

0.66956

0.66880

0.66843

0.66805

0.66730

0.66693

0.66655

0.66617

0.66580

0.66542

0.66505

0.66468

0.66430

0.66393

0.66355

0.66318

0.66281

0.66243

0.66206

0.66168

0.66131

0.66094

0.66057

0.66019

0.65982

0.65945

0.65908

0.65870

0.65833

0.65796

0.65759

0.65722

0.65685

0.65648

0.65610

0.65573

0.65536

0.65499

0.65462

0.65425

0.65388

0.65351

0.65314

0.65277
0.67069

0.66768
$\Delta \quad \mathrm{CO} / \mathrm{R}$

$\Delta$

$\begin{array}{lllll}38 & 1.31079 & 127 & 0.95441 & 11 \\ 37 & 1.30952 & 127 & 0.95430 & 12 \\ 38 & 1.30825 & 126 & 0.95418 & 12 \\ 38 & 1.30699 & 127 & 0.95406 & 12 \\ 37 & 1.30572 & 126 & 0.95394 & 12 \\ 38 & 1.30446 & 127 & 0.95382 & 12\end{array}$

$38 \quad 1.30319 \quad 126$

126

125

126

125

1.29942

1.29816

125

125

125

125

124

1.29316

1.29191

1.29067

1.28943

1.28818

1.28695

1.28571

124

125

123

124

124

1.28447

123

1.28324

1.28201

1.28078

1.27955

123

123

123

123

1.27832

122

123

122

122

122

1.27465

1.27343

121

122

121

121

121

121

121

120

120

1. 26373

1.26253

1.26133

120

120

120

120

119

119

120

1.25415

1.25295

1.25176

1.25058

1.24939

$0.95370 \quad 12$

$0.95358 \quad 12$

$0.95346 \quad 11$

$0.95335 \quad 12$

$0.95323 \quad 12$

$0.95311 \quad 12$

$0.95299 \quad 12$

$0.95287 \quad 12$

$0.95275 \quad 12$

$0.95263 \quad 12$ 
Table I Harmonic Oscillator Contributions (in dimensionless form) to the

Thermodynamic Functions for Values of $X=h c v / k T$

\begin{tabular}{|c|c|c|c|c|c|c|c|c|}
\hline $\mathbf{Z}$ & $-\left(F^{\circ}-E_{O}\right) / R T$ & $\Delta$ & $\left(\mathrm{H}^{\circ}-\mathrm{E}_{\mathrm{O}}\right) / \mathrm{RT}$ & $\Delta$ & $\mathrm{SO} / \mathrm{R}$ & $\Delta$ & $\mathrm{Co} / \mathrm{R}$ & $\Delta$ \\
\hline 0.800 & 0.59662 & 82 & 0.65277 & 37 & 1.24939 & 118 & 0.94833 & 12 \\
\hline $\begin{array}{l}0.801 \\
0.802 \\
0.803 \\
0.804 \\
0.805\end{array}$ & $\begin{array}{l}0.59580 \\
0.59499 \\
0.59418 \\
0.59337 \\
0.59256\end{array}$ & $\begin{array}{l}81 \\
81 \\
81 \\
81 \\
81\end{array}$ & $\begin{array}{l}0.65240 \\
0.65203 \\
0.65167 \\
0.65130 \\
0.65093\end{array}$ & $\begin{array}{l}37 \\
36 \\
37 \\
37 \\
37\end{array}$ & $\begin{array}{l}1.24821 \\
1.24702 \\
1.24584 \\
1.24466 \\
1.24348\end{array}$ & $\begin{array}{l}119 \\
118 \\
118 \\
118 \\
117\end{array}$ & $\begin{array}{l}0.94821 \\
0.94808 \\
0.94796 \\
0.94783 \\
0.94770\end{array}$ & $\begin{array}{l}13 \\
12 \\
13 \\
13 \\
12\end{array}$ \\
\hline $\begin{array}{l}0.806 \\
0.807 \\
0.808 \\
0.809 \\
0.810\end{array}$ & $\begin{array}{l}0.59175 \\
0.59094 \\
0.59014 \\
0.58933 \\
0.58853\end{array}$ & $\begin{array}{l}81 \\
80 \\
81 \\
80 \\
80\end{array}$ & $\begin{array}{l}0.65056 \\
0.65019 \\
0.64982 \\
0.64945 \\
0.64909\end{array}$ & $\begin{array}{l}37 \\
37 \\
37 \\
36 \\
37\end{array}$ & $\begin{array}{l}1.24231 \\
1.24113 \\
1.23996 \\
1.23879 \\
1.23762\end{array}$ & $\begin{array}{ll}1 & 18 \\
1 & 17 \\
1 & 17 \\
1 & 17 \\
1 & 17\end{array}$ & $\begin{array}{l}0.94758 \\
0.94745 \\
0.94733 \\
0.94720 \\
0.94707\end{array}$ & $\begin{array}{l}13 \\
12 \\
13 \\
13 \\
12\end{array}$ \\
\hline $\begin{array}{l}0.811 \\
0.812 \\
0.813 \\
0.814 \\
0.815\end{array}$ & $\begin{array}{l}0.58773 \\
0.58693 \\
0.58613 \\
0.58534 \\
0.58454\end{array}$ & $\begin{array}{l}80 \\
80 \\
79 \\
80 \\
79\end{array}$ & $\begin{array}{l}0.64872 \\
0.64835 \\
0.64798 \\
0.64762 \\
0.64725\end{array}$ & $\begin{array}{l}37 \\
37 \\
36 \\
37 \\
37\end{array}$ & $\begin{array}{l}1.23645 \\
1.23528 \\
1.23412 \\
1.23295 \\
1.23179\end{array}$ & $\begin{array}{ll}117 \\
116 \\
117 \\
116 \\
116\end{array}$ & $\begin{array}{l}0.94695 \\
0.94682 \\
0.94669 \\
0.94657 \\
0.94644\end{array}$ & $\begin{array}{l}13 \\
13 \\
12 \\
13 \\
13\end{array}$ \\
\hline $\begin{array}{l}0.816 \\
0.817 \\
0.818 \\
0.819 \\
0.820\end{array}$ & $\begin{array}{l}0.58375 \\
0.58296 \\
0.58217 \\
0.58138 \\
0.58059\end{array}$ & $\begin{array}{l}79 \\
79 \\
79 \\
79 \\
79\end{array}$ & $\begin{array}{l}0.64688 \\
0.64651 \\
0.64615 \\
0.64578 \\
0.64542\end{array}$ & $\begin{array}{l}37 \\
36 \\
37 \\
36 \\
37\end{array}$ & $\begin{array}{l}1.23063 \\
1.22947 \\
1.22831 \\
1.22716 \\
1.22600\end{array}$ & $\begin{array}{ll}1 & 16 \\
1 & 16 \\
1 & 15 \\
1 & 16 \\
1 & 15\end{array}$ & $\begin{array}{l}0.94631 \\
0.94618 \\
0.94606 \\
0.94593 \\
0.94580\end{array}$ & $\begin{array}{l}13 \\
12 \\
13 \\
13 \\
13\end{array}$ \\
\hline $\begin{array}{l}0.821 \\
0.822 \\
0.823 \\
0.824 \\
0.825\end{array}$ & $\begin{array}{l}0.57980 \\
0.57902 \\
0.57823 \\
0.57745 \\
0.57667\end{array}$ & $\begin{array}{l}78 \\
79 \\
78 \\
78 \\
78\end{array}$ & $\begin{array}{l}0.64505 \\
0.64468 \\
0.64432 \\
0.64395 \\
0.64359\end{array}$ & $\begin{array}{l}37 \\
36 \\
37 \\
36 \\
37\end{array}$ & $\begin{array}{l}1.22485 \\
1.22370 \\
1.22255 \\
1.22140 \\
1.22026\end{array}$ & $\begin{array}{l}115 \\
115 \\
115 \\
114 \\
115\end{array}$ & $\begin{array}{l}0.94567 \\
0.94555 \\
0.94542 \\
0.94529 \\
0.94516\end{array}$ & $\begin{array}{l}12 \\
13 \\
13 \\
13 \\
13\end{array}$ \\
\hline $\begin{array}{l}0.826 \\
0.827 \\
0.828 \\
0.829 \\
0.830\end{array}$ & $\begin{array}{l}0.57589 \\
0.57511 \\
0.57434 \\
0.57356 \\
0.57279\end{array}$ & $\begin{array}{l}78 \\
77 \\
78 \\
77 \\
77\end{array}$ & $\begin{array}{l}0.64322 \\
0.64285 \\
0.64249 \\
0.64212 \\
0.64176\end{array}$ & $\begin{array}{l}37 \\
36 \\
37 \\
36 \\
36\end{array}$ & $\begin{array}{l}1.21911 \\
1.21797 \\
1.21683 \\
1.21569 \\
1.21455\end{array}$ & $\begin{array}{l}114 \\
114 \\
114 \\
114 \\
114\end{array}$ & $\begin{array}{l}0.94503 \\
0.94490 \\
0.94477 \\
0.94465 \\
0.94452\end{array}$ & $\begin{array}{l}13 \\
13 \\
12 \\
13 \\
13\end{array}$ \\
\hline $\begin{array}{l}0.831 \\
0.832 \\
0.833 \\
0.834 \\
0.835\end{array}$ & $\begin{array}{l}0.57202 \\
0.57124 \\
0.57047 \\
0.56971 \\
0.56894\end{array}$ & $\begin{array}{l}78 \\
77 \\
76 \\
77 \\
77\end{array}$ & $\begin{array}{l}0.64140 \\
0.64103 \\
0.64067 \\
0.64030 \\
0.63994\end{array}$ & $\begin{array}{l}37 \\
36 \\
37 \\
36 \\
37\end{array}$ & $\begin{array}{l}1.21341 \\
1.21228 \\
1.21114 \\
1.21001 \\
1.20888\end{array}$ & $\begin{array}{ll}1 & 13 \\
1 & 14 \\
1 & 13 \\
1 & 13 \\
1 & 13\end{array}$ & $\begin{array}{l}0.94439 \\
0.94426 \\
0.94413 \\
0.94400 \\
0.94387\end{array}$ & $\begin{array}{l}13 \\
13 \\
13 \\
13 \\
13\end{array}$ \\
\hline $\begin{array}{l}0.836 \\
0.837 \\
0.838 \\
0.839 \\
0.840\end{array}$ & $\begin{array}{l}0.56817 \\
0.56741 \\
0.56665 \\
0.56588 \\
0.56512\end{array}$ & $\begin{array}{l}76 \\
76 \\
77 \\
76 \\
75\end{array}$ & $\begin{array}{l}0.63957 \\
0.63921 \\
0.63885 \\
0.63848 \\
0.63812\end{array}$ & $\begin{array}{l}36 \\
36 \\
37 \\
36 \\
36\end{array}$ & $\begin{array}{l}1.20775 \\
1.20662 \\
1.20549 \\
1.20437 \\
1.20324\end{array}$ & $\begin{array}{ll}1 & 13 \\
1 & 13 \\
1 & 12 \\
1 & 13 \\
1 & 12\end{array}$ & $\begin{array}{l}0.94374 \\
0.94361 \\
0.94348 \\
0.94335 \\
0.94322\end{array}$ & $\begin{array}{l}13 \\
13 \\
13 \\
13 \\
13\end{array}$ \\
\hline $\begin{array}{l}0.841 \\
0.842 \\
0.843 \\
0.844 \\
0.845\end{array}$ & $\begin{array}{l}0.56437 \\
0.56361 \\
0.56285 \\
0.56210 \\
0.56134\end{array}$ & $\begin{array}{l}76 \\
76 \\
75 \\
76 \\
75\end{array}$ & $\begin{array}{l}0.63776 \\
0.63739 \\
0.63703 \\
0.63667 \\
0.63631\end{array}$ & $\begin{array}{l}37 \\
36 \\
36 \\
36 \\
37\end{array}$ & $\begin{array}{l}1.20212 \\
1.20100 \\
1.19988 \\
1.19876 \\
1.19765\end{array}$ & $\begin{array}{ll}1 & 12 \\
1 & 12 \\
1 & 12 \\
1 & 1 \\
1 & 12\end{array}$ & $\begin{array}{l}0.94309 \\
0.94296 \\
0.94283 \\
0.94269 \\
0.94256\end{array}$ & $\begin{array}{l}13 \\
13 \\
14 \\
13 \\
13\end{array}$ \\
\hline $\begin{array}{l}0.846 \\
0.847 \\
0.848 \\
0.849 \\
0.850\end{array}$ & $\begin{array}{l}0.56059 \\
0.55984 \\
0.55909 \\
0.55834 \\
0.55759\end{array}$ & $\begin{array}{l}75 \\
75 \\
75 \\
75\end{array}$ & $\begin{array}{l}0.63594 \\
0.63558 \\
0.63522 \\
0.63486 \\
0.63450\end{array}$ & $\begin{array}{l}36 \\
36 \\
36 \\
36\end{array}$ & $\begin{array}{l}1.19653 \\
1.19542 \\
1.19431 \\
1.19320 \\
1.19209\end{array}$ & $\begin{array}{lll}1 & 1 & 1 \\
1 & 1 & 1 \\
1 & 1 & 1 \\
1 & 1 & 1\end{array}$ & $\begin{array}{l}0.94243 \\
0.94230 \\
0.94217 \\
0.94204 \\
0.94191\end{array}$ & $\begin{array}{l}13 \\
13 \\
13 \\
13\end{array}$ \\
\hline
\end{tabular}


Table I Harmonic Oscillator Contributions (in dimensionless form) to the Thermodynamic Functions for Values of $\mathrm{X}=\mathrm{hc} / \mathrm{kT}$

\begin{tabular}{|c|c|c|c|c|c|c|c|c|}
\hline $\mathbf{X}$ & $-\left(F^{\circ}-E_{O}^{O}\right) / R T$ & $\Delta$ & $\left(\mathrm{H}^{\circ}-\mathrm{E}_{\mathrm{O}}^{\mathrm{O}}\right) / \mathrm{RT}$ & $\Delta$ & $\mathrm{S} / \mathrm{R}$ & $\Delta$ & $\mathrm{C}_{\mathrm{p}}^{\circ} / \mathrm{R}$ & $\Delta$ \\
\hline 0.850 & 0.55759 & 74 & 0.63450 & 37 & 1.19209 & 111 & 0.94191 & 14 \\
\hline $\begin{array}{l}0.851 \\
0.852 \\
0.853 \\
0.854 \\
0.855\end{array}$ & $\begin{array}{l}0.55685 \\
0.55610 \\
0.55536 \\
0.55462 \\
0.55388\end{array}$ & $\begin{array}{l}75 \\
74 \\
74 \\
74 \\
74\end{array}$ & $\begin{array}{l}0.63413 \\
0.63377 \\
0.63341 \\
0.63305 \\
0.63269\end{array}$ & $\begin{array}{l}36 \\
36 \\
36 \\
36 \\
36\end{array}$ & $\begin{array}{l}1.19098 \\
1.18988 \\
1.18877 \\
1.18767 \\
1.18657\end{array}$ & $\begin{array}{lll}1 & 10 \\
1 & 1 & 1 \\
1 & 10 \\
1 & 1 & 0 \\
1 & 1 & 0\end{array}$ & $\begin{array}{l}0.94177 \\
0.94164 \\
0.94151 \\
0.94138 \\
0.94124\end{array}$ & $\begin{array}{l}13 \\
13 \\
13 \\
14 \\
13\end{array}$ \\
\hline $\begin{array}{l}0.856 \\
0.857 \\
0.858 \\
0.859 \\
0.860\end{array}$ & $\begin{array}{l}0.55314 \\
0.55240 \\
0.55166 \\
0.55093 \\
0.55019\end{array}$ & $\begin{array}{l}74 \\
74 \\
73 \\
74 \\
73\end{array}$ & $\begin{array}{l}0.63233 \\
0.63197 \\
0.63161 \\
0.63125 \\
0.63089\end{array}$ & $\begin{array}{l}36 \\
36 \\
36 \\
36 \\
36\end{array}$ & $\begin{array}{l}1.18547 \\
1.18437 \\
1.18327 \\
1.18218 \\
1.18108\end{array}$ & $\begin{array}{l}110 \\
110 \\
109 \\
110 \\
109\end{array}$ & $\begin{array}{l}0.94111 \\
0.94098 \\
0.94085 \\
0.94071 \\
0.94058\end{array}$ & $\begin{array}{l}13 \\
13 \\
14 \\
13 \\
13\end{array}$ \\
\hline $\begin{array}{l}0.861 \\
0.862 \\
0.863 \\
0.864 \\
0.865\end{array}$ & $\begin{array}{l}0.54946 \\
0.54873 \\
0.54800 \\
0.54727 \\
0.54654\end{array}$ & $\begin{array}{l}73 \\
73 \\
73 \\
73 \\
72\end{array}$ & $\begin{array}{l}0.63053 \\
0.63017 \\
0.62981 \\
0.62945 \\
0.62909\end{array}$ & $\begin{array}{l}36 \\
36 \\
36 \\
36 \\
36\end{array}$ & $\begin{array}{l}1.17999 \\
1.17890 \\
1.17781 \\
1.17672 \\
1.17563\end{array}$ & $\begin{array}{l}109 \\
109 \\
109 \\
109 \\
109\end{array}$ & $\begin{array}{l}0.94045 \\
0.94031 \\
0.94018 \\
0.94005 \\
0.93991\end{array}$ & $\begin{array}{l}14 \\
13 \\
13 \\
14 \\
13\end{array}$ \\
\hline $\begin{array}{l}0.866 \\
0.867 \\
0.868 \\
0.869 \\
0.870\end{array}$ & $\begin{array}{l}0.54582 \\
0.54509 \\
0.54437 \\
0.54364 \\
0.54292\end{array}$ & $\begin{array}{l}73 \\
72 \\
73 \\
72 \\
72\end{array}$ & $\begin{array}{l}0.62873 \\
0.62837 \\
0.62801 \\
0.62765 \\
0.62729\end{array}$ & $\begin{array}{l}36 \\
36 \\
36 \\
36 \\
36\end{array}$ & $\begin{array}{l}1.17454 \\
1.17346 \\
1.17238 \\
1.17129 \\
1.17021\end{array}$ & $\begin{array}{l}108 \\
108 \\
109 \\
108 \\
107\end{array}$ & $\begin{array}{l}0.93978 \\
0.93965 \\
0.93951 \\
0.93938 \\
0.93924\end{array}$ & $\begin{array}{l}13 \\
14 \\
13 \\
14 \\
13\end{array}$ \\
\hline $\begin{array}{l}0.871 \\
0.872 \\
0.873 \\
0.874 \\
0.875\end{array}$ & $\begin{array}{l}0.54220 \\
0.54148 \\
0.54076 \\
0.54005 \\
0.53933\end{array}$ & $\begin{array}{l}72 \\
72 \\
71 \\
72 \\
71\end{array}$ & $\begin{array}{l}0.62693 \\
0.62658 \\
0.62622 \\
0.62586 \\
0.62550\end{array}$ & $\begin{array}{l}35 \\
36 \\
36 \\
36 \\
36\end{array}$ & $\begin{array}{l}1.16914 \\
1.16806 \\
1.16698 \\
1.16591 \\
1.16483\end{array}$ & $\begin{array}{l}108 \\
108 \\
107 \\
108 \\
107\end{array}$ & $\begin{array}{l}0.93911 \\
0.93897 \\
0.93884 \\
0.93870 \\
0.93857\end{array}$ & $\begin{array}{l}14 \\
13 \\
14 \\
13 \\
14\end{array}$ \\
\hline $\begin{array}{l}0.876 \\
0.877 \\
0.878 \\
0.879 \\
0.880\end{array}$ & $\begin{array}{l}0.53862 \\
0.53790 \\
0.53719 \\
0.53648 \\
0.53577\end{array}$ & $\begin{array}{l}72 \\
71 \\
71 \\
71 \\
71\end{array}$ & $\begin{array}{l}0.62514 \\
0.62479 \\
0.62443 \\
0.62407 \\
0.62372\end{array}$ & $\begin{array}{l}35 \\
36 \\
36 \\
35 \\
36\end{array}$ & $\begin{array}{l}1.16376 \\
1.16269 \\
1.16162 \\
1.16055 \\
1.15949\end{array}$ & $\begin{array}{l}107 \\
107 \\
107 \\
106 \\
107\end{array}$ & $\begin{array}{l}0.93843 \\
0.93830 \\
0.93816 \\
0.93803 \\
0.93789\end{array}$ & $\begin{array}{l}13 \\
14 \\
13 \\
14 \\
14\end{array}$ \\
\hline $\begin{array}{l}0.881 \\
0.882 \\
0.883 \\
0.884 \\
0.885\end{array}$ & $\begin{array}{l}0.53506 \\
0.53436 \\
0.53365 \\
0.53295 \\
0.53224\end{array}$ & $\begin{array}{l}70 \\
71 \\
70 \\
71 \\
70\end{array}$ & $\begin{array}{l}0.62336 \\
0.62300 \\
0.62265 \\
0.62229 \\
0.62193\end{array}$ & $\begin{array}{l}36 \\
35 \\
36 \\
36 \\
35\end{array}$ & $\begin{array}{l}1.15842 \\
1.15736 \\
1.15630 \\
1.15524 \\
1.15418\end{array}$ & $\begin{array}{l}106 \\
106 \\
106 \\
106 \\
106\end{array}$ & $\begin{array}{l}0.93775 \\
0.93762 \\
0.93748 \\
0.93735 \\
0.93721\end{array}$ & $\begin{array}{l}13 \\
14 \\
13 \\
14 \\
14\end{array}$ \\
\hline $\begin{array}{l}0.886 \\
0.887 \\
0.888 \\
0.889 \\
0.890\end{array}$ & $\begin{array}{l}0.53154 \\
0.53084 \\
0.53014 \\
0.52944 \\
0.52874\end{array}$ & $\begin{array}{l}70 \\
70 \\
70 \\
70 \\
69\end{array}$ & $\begin{array}{l}0.62158 \\
0.62122 \\
0.62086 \\
0.62051 \\
0.62015\end{array}$ & $\begin{array}{l}36 \\
36 \\
35 \\
36 \\
35\end{array}$ & $\begin{array}{l}1.15312 \\
1.15206 \\
1.15101 \\
1.14995 \\
1.14890\end{array}$ & $\begin{array}{l}106 \\
105 \\
106 \\
105 \\
105\end{array}$ & $\begin{array}{l}0.93707 \\
0.93694 \\
0.93680 \\
0.93666 \\
0.93653\end{array}$ & $\begin{array}{l}13 \\
14 \\
14 \\
13 \\
14\end{array}$ \\
\hline $\begin{array}{l}0.891 \\
0.892 \\
0.893 \\
0.894 \\
0.895\end{array}$ & $\begin{array}{l}0.52805 \\
0.52735 \\
0.52666 \\
0.52597 \\
0.52528\end{array}$ & $\begin{array}{l}70 \\
69 \\
69 \\
69 \\
69\end{array}$ & $\begin{array}{l}0.61980 \\
0.61944 \\
0.61909 \\
0.61873 \\
0.61838\end{array}$ & $\begin{array}{l}36 \\
35 \\
36 \\
35 \\
36\end{array}$ & $\begin{array}{l}1.14785 \\
1.14680 \\
1.14575 \\
1.14470 \\
1.14365\end{array}$ & $\begin{array}{l}105 \\
105 \\
105 \\
105 \\
104\end{array}$ & $\begin{array}{l}0.93639 \\
0.93625 \\
0.93611 \\
0.93598 \\
0.93584\end{array}$ & $\begin{array}{l}14 \\
14 \\
13 \\
14 \\
14\end{array}$ \\
\hline $\begin{array}{l}0.896 \\
0.897 \\
0.898 \\
0.899 \\
0.900\end{array}$ & $\begin{array}{l}0.52459 \\
0.52390 \\
0.52321 \\
0.52252 \\
0.52184\end{array}$ & $\begin{array}{l}69 \\
69 \\
69 \\
68\end{array}$ & $\begin{array}{l}0.61802 \\
0.61767 \\
0.61731 \\
0.61696 \\
0.61661\end{array}$ & $\begin{array}{l}35 \\
36 \\
35 \\
35\end{array}$ & $\begin{array}{l}1.14261 \\
1.14156 \\
1.14052 \\
1.13948 \\
1.13844\end{array}$ & $\begin{array}{l}105 \\
104 \\
104 \\
104\end{array}$ & $\begin{array}{l}0.93570 \\
0.93556 \\
0.93542 \\
0.93529 \\
0.93515\end{array}$ & $\begin{array}{l}14 \\
14 \\
13 \\
14\end{array}$ \\
\hline
\end{tabular}


Table I Harmonic Oscillator Contributions (in dimensionless form) to the

Thermodynamic Functions for Values of $\mathrm{X}=\mathrm{hcv} / \mathrm{kT}$

$x$ $-\left(\mathrm{F}^{\circ}-\mathrm{E}_{\mathrm{O}}^{\circ}\right) / \mathrm{RT}$

\begin{tabular}{|c|c|}
\hline 0.900 & 0.52184 \\
\hline 0.901 & 0.52115 \\
\hline $\begin{array}{l}0.902 \\
0.903 \\
0.904 \\
0.905\end{array}$ & $\begin{array}{l}0.52047 \\
0.51979 \\
0.51910 \\
0.51842\end{array}$ \\
\hline $\begin{array}{l}0.906 \\
0.907 \\
0.908 \\
0.909 \\
0.910\end{array}$ & $\begin{array}{l}0.51775 \\
0.51707 \\
0.51639 \\
0.51572 \\
0.51504\end{array}$ \\
\hline $\begin{array}{l}0.911 \\
0.912 \\
0.913 \\
0.914 \\
0.915\end{array}$ & $\begin{array}{l}0.51437 \\
0.51370 \\
0.51303 \\
0.51236 \\
0.51169\end{array}$ \\
\hline $\begin{array}{l}0.916 \\
0.917 \\
0.918 \\
0.919 \\
0.920\end{array}$ & $\begin{array}{l}0.51102 \\
0.51035 \\
0.50969 \\
0.50902 \\
0.50836\end{array}$ \\
\hline $\begin{array}{l}0.921 \\
0.922 \\
0.923 \\
0.924 \\
0.925\end{array}$ & $\begin{array}{l}0.50770 \\
0.50704 \\
0.50638 \\
0.50572 \\
0.50506\end{array}$ \\
\hline $\begin{array}{l}0.926 \\
0.927 \\
0.928 \\
0.929 \\
0.930\end{array}$ & $\begin{array}{l}0.50440 \\
0.50375 \\
0.50309 \\
0.50244 \\
0.50179\end{array}$ \\
\hline $\begin{array}{l}0.931 \\
0.932 \\
0.933 \\
0.934 \\
0.935\end{array}$ & $\begin{array}{l}0.50114 \\
0.50049 \\
0.49984 \\
0.49919 \\
0.49854\end{array}$ \\
\hline $\begin{array}{l}0.936 \\
0.937 \\
0.938 \\
0.939 \\
0.940\end{array}$ & $\begin{array}{l}0.49790 \\
0.49725 \\
0.49661 \\
0.49597 \\
0.49533\end{array}$ \\
\hline $\begin{array}{l}0.941 \\
0.942 \\
0.943 \\
0.944 \\
0.945\end{array}$ & $\begin{array}{l}0.49469 \\
0.49405 \\
0.49341 \\
0.49277 \\
0.49213\end{array}$ \\
\hline $\begin{array}{l}0.946 \\
0.947 \\
0.948 \\
0.949 \\
0.950\end{array}$ & $\begin{array}{l}0.49150 \\
0.49086 \\
0.49023 \\
0.48960 \\
0.48897\end{array}$ \\
\hline
\end{tabular}

$\begin{array}{ll}69 & 0.61661 \\ 68 & 0.61625 \\ 68 & 0.61590 \\ 69 & 0.61554 \\ 68 & 0.61519 \\ 67 & 0.61484\end{array}$

36
35
36
35
35
35

68

68

67

68

67

67

67

67

67

67

67

66

67

66

66

66

66

66

66

66

65

66

65

65

65

65

65

65

65

64

65

64

64
64

64

0.61449

0.61413

0.61378

0.61343

0.61307

0.61272

0.61237

0.61202

0.61167

0.61131

0.61096

0.61061

0.61026

0.60991

0.60956

0.60921

0.60886

0.60851

0.60816

0.60781

0.60746

0.60711

0.60676

0.60641

0.60606

0.60571

0.60536

0.60501

0.60466

0.60431

0.60396

0.60362

0.60327

0.60292

0.60257

36

35

35

36

35

35

35

35

36

35

35

35

35

35

35

35

35

35.

35

35

35

35

35

35

35

35

35

35

35

35

34

35
35

35

35

0.60222

$64 \quad 0.60188$

$64 \quad 0.60153$

$64 \quad 0.60118$

$63 \quad 0.60083$

64

63

63

63

34

35

35

35

34

0.60049

0.60014

0.59979

0.59945
$\Delta$

So/R

1.13844
1.13740
1.13637
1.13533
1.13430
1.13326

1. 13223

1. 13120

1. 13017

1. 12914

1. 12812

\subsection{9}

1. 12607

1. 12504

1. 12402

1. 12300

\section{12198}

1. 12096

1. 11995

1. 11893

1. 11792

1. 11691

1. 11589

1. 11488

1. 11387

1. 11287

1.11186

1.11085

1.10985

1.10885

1.10785

1.10685

1. 10585

1. 10485

1. 10385

1.10286

1.10186

1.10087

1. 09988

1.09889

1.09790

0.59910

1.09691

1.09592

1.09494

1.09395

1.09297

1.09100

1.09002

1.08905

1.08807
$\Delta \quad \mathrm{co} / \mathrm{R}$

$\Delta$

$\begin{array}{lll}104 & 0.93515 & 14 \\ 103 & 0.93501 & 14 \\ 104 & 0.93487 & 14 \\ 103 & 0.93473 & 14 \\ 104 & 0.93459 & 14 \\ 103 & 0.93445 & 13 \\ 103 & 0.93432 & 14 \\ 103 & 0.93418 & 14 \\ 103 & 0.93404 & 14 \\ 102 & 0.93390 & 14 \\ 103 & 0.93376 & 14\end{array}$

$0.93362 \quad 14$

$0.93348 \quad 14$

$0.93334 \quad 14$

$0.93320 \quad 14$

$0.93306 \quad 14$

$0.93292 \quad 14$

$0.93278 \quad 14$

$0.93264 \quad 14$

$0.93250 \quad 15$

$0.93235 \quad 14$

$0.93221 \quad 14$

$0.93207 \quad 14$

$0.93193 \quad 14$

$0.93179 \quad 14$

$0.93165 \quad 14$

$0.93151 \quad 15$

$0.93136 \quad 14$

$0.93122 \quad 14$

$0.93108 \quad 14$

$0.93094 \quad 14$

100

$100 \quad 0.93080 \quad 15$

$100 \quad 0.93065 \quad 14$

$100 \quad 0.93051 \quad 14$

$\begin{array}{lll}99 & 0.93037 & 14\end{array}$

$\begin{array}{lll}100 & 0.93023 \quad 15\end{array}$

99

0.93008

0.92994

0.92980

0.92965

0.92951

0.92937

0.92922

0.92908

0.92893

0.92879

14

14

15

14

14

$35 \quad 1.09199$
0.92865

0.92850

0.92836

0.92821

0.92807
15

14

15

14

14

15

14

15

14 
Table I Harmonic Oscillator Contributions (in dimensionless form) to the Thermodynamic Functions for Values of $X=h c v / k T$

$\mathbf{X}$

$-\left(\mathrm{F}^{\circ}-\mathrm{E}_{\mathrm{O}}\right) / \mathrm{RT}$

$\Delta \quad\left(\mathrm{H}^{\circ}-\mathrm{E}_{\mathrm{O}}^{\circ}\right) / \mathrm{RT}$

$\Delta$

So/R

0.951

0.952

0.953

0.954

0.955

0.956

0.957

0.958

0.959

0.960

0.48897
0.48834
0.48771
0.48708
0.48645
0.48583

0.48520

0.48458

0.48396

0.48333

0.48271

0.961

0.962

0.963

0.964

0.965

0.48209

0.48147

0.48086

0.48024

0.47962

0.966

0.967

0.968

0.969

0.970

0.971

0.972

0.973

0.974

0.975

0.976

0.977

0.978

0.979

0.980

0.981

0.982

0.983

0.984

0.985

0.986

0.987

0.988

0.989

0.990

0.991

0.992

0.993

0.994

0.995

0.996

0.997

0.998

0.999

1.000
0.47901

0.47839

0.47778

0.47717

0.47656

0.47595

0.47534

0.47473

0.47412

0.47352

0.47291

0.47231

0.47171

0.47110

0.47050

0.46990

0.46930

0.46870

0.46811

0.46751

0.46691

0.46632

0.46573

0.46513

0.46454

0.46395

0.46336

0.46277

0.46218

0.46160

0.46101

0.46043

0.45984

0.45926

0.45868
$63 \quad 0.59910$

63

0.59875

0.59841

0.59806

63

62

63

0.59772

0.59737

35

34

35

34

35

34

0.59703

62

63

62

62

0.59668

0.59634

0.59599

0.59565

\section{5}

34

35

34

35

62

61

0.59530

34

0.59496

35

62

61

62

61

61

61

61

61

61

61

60

61

60

60

61

60

60

60

60

59

60

60

59

59

60

59

59

59

59

59

58

59

58

59

58

58

0.58673
0.59461

0.59427

0.59392

34

35

0.59358

0.59324

0.59289

0.59255

0.59221

0.59186

0.59152

0.59118

0.59083

0.59049

0.59015

0.58981

0.58946

0.58912

0.58878

0.58844

C. 58810

0.58776

0.58742

0.58707

0.58639

0.58605

0.58571

0.58537

0.58503

0.58469

0.58435

0.58401

0.58367

34

$$
\begin{aligned}
& 34 \\
& 35 \\
& 34
\end{aligned}
$$

34

35

34

34

35

34

34

34

35

34

34

34

34

34

34

35

34

0.58333

0.58299

0.58265

0.58232

0.58198
1.08807

1.08709

1. 08612

1.08514

1.08417

1.08320

1.08223

1. 08126

1.08029

1.07932

1.07836

1. 07739

1.07643

1. 07547

1. 07451

1.07355

1.07259

1.07163

1.07067

1. 06972

1.06876

1. 06781

1.06686

1. 06591

1. 06496

1.06401

1.06306

1.06211

1.06117

1.06023

1.05928

1.05834

1.05740

$1.0564 t$

1.05552

1.05458

1. 05365

1. 05271

1.05178

1. 05084

1. 04991

1.04898

1. 04805

1. 04712

1. 04620

1.04527

1.04434

1. 04342

1.04250

1.04157

1. 04065 
Table I Harmonic Oscillator Contributions (in dimensionless form) to the

Thermodynamic Functions for Values of $X=h c v / k T$

X $-\left(\mathrm{F}^{\circ}-\mathrm{E}_{\mathrm{O}}\right) / \mathrm{RT}$
$\Delta \quad\left(\mathrm{H}^{\circ}-\mathrm{E}_{\mathrm{O}}^{\circ}\right) / \mathrm{RT}$

$\Delta$

$\mathrm{S} / \mathrm{R}$

1.00

1.006

1.007

1.008

1.009

1.010

1.011

1.012

1.013

1.014

1.015

1.016

1.017

1.018

1.019

1.020

1.021

1.022

1.023

1.024

1.025

1.026

1.027

1.028

1.029

1.030

1.031

1.032

1.033

1.034

1.035

1.036

1.037

1.038

1.039

1.040

1.041

1.042

1.043

1.044

1.045

1.046

1.047

1.048

1.049

1.050
0.45868

0.45809

0.45751

0.45693

0.45635

0.45578

0.45520

0.45462

0.45405

0.45347

0.45290

0.45233

0.45176

0.45119

0.45062

0.45005

0.44948

0.44891

0.44835

0.44778

0.44722

0.44609

0.44497

0.44441

0.44385

0.44329

0.44273

0.44218

0.44162

0.44107

0.44051

0.43996

0.43941

0.43886

0.43831

0.43776

0.43721

0.43666

0.43611

0.43557

0.43502

0.43448

0.43393

0.43339

0.43285

0.43231

0.43177

0.43123

0.43069
0.44665

0.44553

58

57

58

57

57

\section{7}

57

57

57

57

57

56

57

56
57

0.57995

0.57961

34

0.57927

0.57893

$$
34
$$

0.57792

0.57759

0.57725

0.57691

33

34

34

33

$0.57658 \quad 34$

0.57624

0.57590

0.57557

34
34
33

33

34

0.57523

56

56

56

56

0.57490

0.57456

0.57423

0.57389

0.57356

$\begin{array}{lll}56 & 0.57322 \quad 33\end{array}$

56

55

56

55

0.57289

0.57222

$56 \quad 0.57155$

55

55

55

55

0.57122

0.57088

0.57055

0.57021

55

0.56988

0.56955

0.56921

0.56888

0.56822

34

54

55

54

54

0.56788

0.56755

0.56722

54

0.56656

0.56589

0.56556

$\begin{array}{llll}59 & 0.58198 & 34 & 1.04065 \\ 58 & 0.58164 & 34 & 1.03973 \\ 58 & 0.58130 & 34 & 1.03881 \\ 58 & 0.58096 & 34 & 1.03789 \\ 57 & 0.58062 & 33 & 1.03698 \\ 58 & 0.58029 & 34 & 1.03606\end{array}$

0.57860

1.03515

1.03423

1.03332

1.03241

1.03150

$\begin{array}{lll}0.57826 & 34 & 1.03059\end{array}$

\subsection{8}

1.02877

1.02787

1.02696

$\Delta \quad \mathrm{C}_{\mathrm{p}}^{\circ} / \mathrm{R}$

$\Delta$

1.02606

1.02515

1.02425

1.02335

1.02245

1.02155

1.02065

1.01976

1. 01886

1.01796

0.57255

1.01707

1.01618

1. 01529

1.01439

1.01350

1. 01262

1.01173

1.01084

1.00996

1.00907

1.00819

1.00730

1.00642

1. 00554

1. 00466

1.00378

1.00290

1.00203

1.00115

1.00028

0.99940

0.99853

0.99766

0.99679

0.99592

$\begin{array}{lll}92 & 0.92067 & 15 \\ 92 & 0.92052 & 15 \\ 92 & 0.92037 & 15 \\ 91 & 0.92022 & 15 \\ 92 & 0.92007 & 15 \\ 91 & 0.91992 & 15 \\ 92 & 0.91977 & 16 \\ 91 & 0.91961 & 15 \\ 91 & 0.91946 & 15 \\ 91 & 0.91931 & 15 \\ 91 & 0.91916 & 15\end{array}$

91

91

90

91

90

91

90

90

90

90

90

89

90

90

89

89

89

90

89

88

89

89

88

89

88

89

88

88

88

88

88

87

88

87

88

87

87

87

87

$0.91901 \quad 16$

$0.91885 \quad 15$

$0.91870 \quad 15$

$0.91855 \quad 15$

$0.91840 \quad 16$

$0.91824 \quad 15$

$0.91809 \quad 15$

$0.91794 \quad 16$

$0.91778 \quad 15$

$0.91763 \quad 15$

$0.91748 \quad 16$

$0.91732 \quad 15$

$0.91717 \quad 15$ 
Table I Harmonic Oscillator Contributions (in dimensionless form) to the Thermodynamic Functions for Values of $X=h c v / k T$

\begin{tabular}{|c|c|c|c|c|c|c|c|}
\hline $\mathbf{x}$ & $-\left(F^{\circ}-E_{O}^{\circ}\right) / R T$ & $\Delta$ & $\left(\mathrm{H}^{\circ}-\mathrm{E}_{0}^{\circ}\right) / \mathrm{RT}$ & $\Delta$ & $\mathrm{S} \% / \mathrm{R}$ & $\Delta$ & $\mathrm{Co}_{\mathrm{p}} / \mathrm{R}$ \\
\hline .050 & 0.43069 & 54 & 0.56523 & 33 & 0.99592 & 87 & 0.91298 \\
\hline $\begin{array}{l}1.051 \\
1.052\end{array}$ & $\begin{array}{l}0.43015 \\
0.42961\end{array}$ & $\begin{array}{l}54 \\
53\end{array}$ & $\begin{array}{l}0.56490 \\
0.56457\end{array}$ & $\begin{array}{l}33 \\
33\end{array}$ & $\begin{array}{l}0.99505 \\
0.99418\end{array}$ & $\begin{array}{l}87 \\
87\end{array}$ & $\begin{array}{l}0.91282 \\
0.91266\end{array}$ \\
\hline $\begin{array}{l}1.053 \\
1.054 \\
1.055\end{array}$ & $\begin{array}{l}0.42908 \\
0.42854 \\
0.42801\end{array}$ & $\begin{array}{l}54 \\
53 \\
54\end{array}$ & $\begin{array}{l}0.56424 \\
0.56391 \\
0.56358\end{array}$ & $\begin{array}{l}33 \\
33 \\
33\end{array}$ & $\begin{array}{l}0.99331 \\
0.99245 \\
0.99158\end{array}$ & $\begin{array}{l}86 \\
87 \\
86\end{array}$ & $\begin{array}{l}0.91251 \\
0.91235 \\
0.91219\end{array}$ \\
\hline $\begin{array}{l}1.056 \\
1.057 \\
1.058 \\
1.059 \\
1.060\end{array}$ & $\begin{array}{l}0.42747 \\
0.42694 \\
0.42641 \\
0.42588 \\
0.42535\end{array}$ & $\begin{array}{l}53 \\
53 \\
53 \\
53 \\
53\end{array}$ & $\begin{array}{l}0.56325 \\
0.56292 \\
0.56259 \\
0.56226 \\
0.56193\end{array}$ & $\begin{array}{l}33 \\
33 \\
33 \\
33 \\
33\end{array}$ & $\begin{array}{l}0.99072 \\
0.98985 \\
0.98899 \\
0.98813 \\
0.98727\end{array}$ & $\begin{array}{l}87 \\
86 \\
86 \\
86 \\
86\end{array}$ & $\begin{array}{l}0.91203 \\
0.91188 \\
0.91172 \\
0.91156 \\
0.91140\end{array}$ \\
\hline $\begin{array}{l}1.061 \\
1.062 \\
1.063 \\
1.064 \\
1.065\end{array}$ & $\begin{array}{l}0.42482 \\
0.42429 \\
0.42376 \\
0.42323 \\
0.42270\end{array}$ & $\begin{array}{l}53 \\
53 \\
53 \\
53 \\
52\end{array}$ & $\begin{array}{l}0.56160 \\
0.56127 \\
0.56094 \\
0.56061 \\
0.56028\end{array}$ & $\begin{array}{l}33 \\
33 \\
33 \\
33 \\
33\end{array}$ & $\begin{array}{l}0.98641 \\
0.98555 \\
0.98470 \\
0.98384 \\
0.98298\end{array}$ & $\begin{array}{l}86 \\
85 \\
86 \\
86 \\
85\end{array}$ & $\begin{array}{l}0.91124 \\
0.91108 \\
0.91093 \\
0.91077 \\
0.91061\end{array}$ \\
\hline $\begin{array}{l}1.066 \\
1.067 \\
1.068 \\
1.069 \\
1.070\end{array}$ & $\begin{array}{l}0.42218 \\
0.42165 \\
0.42113 \\
0.42061 \\
0.42008\end{array}$ & $\begin{array}{l}53 \\
52 \\
52 \\
53 \\
52\end{array}$ & $\begin{array}{l}0.55995 \\
0.55962 \\
0.55929 \\
0.55896 \\
0.55864\end{array}$ & $\begin{array}{l}33 \\
33 \\
33 \\
32 \\
33\end{array}$ & $\begin{array}{l}0.98213 \\
0.98128 \\
0.98042 \\
0.97957 \\
0.97872\end{array}$ & $\begin{array}{l}85 \\
86 \\
85 \\
85 \\
85\end{array}$ & $\begin{array}{l}0.91045 \\
0.91029 \\
0.91013 \\
0.90997 \\
0.90981\end{array}$ \\
\hline $\begin{array}{l}1.071 \\
1.072 \\
1.073 \\
1.074 \\
1.075\end{array}$ & $\begin{array}{l}0.41956 \\
0.41904 \\
0.41852 \\
0.41800 \\
0.41748\end{array}$ & $\begin{array}{l}52 \\
52 \\
52 \\
52 \\
51\end{array}$ & $\begin{array}{l}0.55831 \\
0.55798 \\
0.55765 \\
0.55732 \\
0.55700\end{array}$ & $\begin{array}{l}33 \\
33 \\
33 \\
32 \\
33\end{array}$ & $\begin{array}{l}0.97787 \\
0.97702 \\
0.97617 \\
0.97533 \\
0.97448\end{array}$ & $\begin{array}{l}85 \\
85 \\
84 \\
85 \\
84\end{array}$ & $\begin{array}{l}0.90966 \\
0.90950 \\
0.90934 \\
0.90918 \\
0.90902\end{array}$ \\
\hline $\begin{array}{l}1.076 \\
1.077 \\
1.078 \\
1.079 \\
1.080\end{array}$ & $\begin{array}{l}0.41697 \\
0.41645 \\
0.41593 \\
0.41542 \\
0.41490\end{array}$ & $\begin{array}{l}52 \\
52 \\
51 \\
52 \\
51\end{array}$ & $\begin{array}{l}0.55667 \\
0.55634 \\
0.55602 \\
0.55569 \\
0.55536\end{array}$ & $\begin{array}{l}33 \\
32 \\
33 \\
33 \\
33\end{array}$ & $\begin{array}{l}0.97364 \\
0.97279 \\
0.97195 \\
0.97111 \\
0.97026\end{array}$ & $\begin{array}{l}85 \\
84 \\
84 \\
85 \\
84\end{array}$ & $\begin{array}{l}0.90886 \\
0.90870 \\
0.90854 \\
0.90838 \\
0.90822\end{array}$ \\
\hline $\begin{array}{l}1.081 \\
1.082 \\
1.083 \\
1.084 \\
1.085\end{array}$ & $\begin{array}{l}0.41439 \\
0.41388 \\
0.41336 \\
0.41285 \\
0.41234\end{array}$ & $\begin{array}{l}51 \\
52 \\
51 \\
51 \\
51\end{array}$ & $\begin{array}{l}0.55503 \\
0.55471 \\
0.55438 \\
0.55406 \\
0.55373\end{array}$ & $\begin{array}{l}32 \\
33 \\
32 \\
33 \\
33\end{array}$ & $\begin{array}{l}0.96942 \\
0.96858 \\
0.96775 \\
0.96691 \\
0.96607\end{array}$ & $\begin{array}{l}84 \\
83 \\
84 \\
84 \\
83\end{array}$ & $\begin{array}{l}0.90806 \\
0.90790 \\
0.90773 \\
0.90757 \\
0.90741\end{array}$ \\
\hline $\begin{array}{l}1.086 \\
1.087 \\
1.088 \\
1.089 \\
1.090\end{array}$ & $\begin{array}{l}0.41183 \\
0.41132 \\
0.41081 \\
0.41031 \\
0.40980\end{array}$ & $\begin{array}{l}51 \\
51 \\
50 \\
51 \\
51\end{array}$ & $\begin{array}{l}0.55340 \\
0.55308 \\
0.55275 \\
0.55243 \\
0.55210\end{array}$ & $\begin{array}{l}32 \\
33 \\
32 \\
33 \\
32\end{array}$ & $\begin{array}{l}0.96524 \\
0.96440 \\
0.96357 \\
0.96273 \\
0.96190\end{array}$ & $\begin{array}{l}84 \\
83 \\
84 \\
83 \\
83\end{array}$ & $\begin{array}{l}0.90725 \\
0.90709 \\
0.90693 \\
0.90677 \\
0.90661\end{array}$ \\
\hline $\begin{array}{l}1.091 \\
1.092 \\
1.093 \\
1.094 \\
1.095\end{array}$ & $\begin{array}{l}0.40929 \\
0.40879 \\
0.40828 \\
0.40778 \\
0.40728\end{array}$ & $\begin{array}{l}50 \\
51 \\
50 \\
50 \\
51\end{array}$ & $\begin{array}{l}0.55178 \\
0.55145 \\
0.55113 \\
0.55080 \\
0.55048\end{array}$ & $\begin{array}{l}33 \\
32 \\
33 \\
32 \\
33\end{array}$ & $\begin{array}{l}0.96107 \\
0.96024 \\
0.95941 \\
0.95858 \\
0.95775\end{array}$ & $\begin{array}{l}83 \\
83 \\
83 \\
83 \\
82\end{array}$ & $\begin{array}{l}0.90645 \\
0.90628 \\
0.90612 \\
0.90596 \\
0.90580\end{array}$ \\
\hline $\begin{array}{l}1.096 \\
1.097 \\
1.098 \\
1.099 \\
1.100\end{array}$ & $\begin{array}{l}0.40677 \\
0.40627 \\
0.40577 \\
0.40527 \\
0.40477\end{array}$ & $\begin{array}{l}50 \\
50 \\
50 \\
50\end{array}$ & $\begin{array}{l}0.55015 \\
0.54983 \\
0.54950 \\
0.54918 \\
0.54886\end{array}$ & $\begin{array}{l}32 \\
33 \\
32 \\
32\end{array}$ & $\begin{array}{l}0.95693 \\
0.95610 \\
0.95528 \\
0.95445 \\
0.95363\end{array}$ & $\begin{array}{l}83 \\
82 \\
83 \\
82\end{array}$ & $\begin{array}{l}0.90564 \\
0.90547 \\
0.90531 \\
0.90515 \\
0.90499\end{array}$ \\
\hline
\end{tabular}


Table I Harmonic Oscillator Contributions (in dimensionless form) to the

Thermodynamic Functions for Values of $X=h c v / k T$

$\mathbf{z}$

$-\left(F^{\circ}-E_{0}^{\circ}\right) / R T$

$\Delta \quad\left(H^{\circ}-E_{O}^{\circ}\right) / R T$

$\Delta$

$S \circ / R$

1.100

1.102

1.103

1.104

1.105

1.106

1.107

1.108

1.109

1.110

1.111

1.112

1.113

1.114

1.115

1.116

1.117

1.118

1.119

1.120

1.121

1.122

1.123

1.124

1.125

1.126

1.127

1.128

1.129

1.130

1.131

1.132

1.133

1.134

1.135

1.136

1.137

1.138

1.139

1.140

1.141

1.142

1.143

1.144

1.145

1.146

1.147

1.148

1.149

1.150

\begin{abstract}
0.40477
0.40427

0.40378

0.40328

0.40278

0.40229

0.40179

0.40130

0.40080

0.40031

0.39982
\end{abstract}

0.39933

0.39884

0.39835

0.39786

0.39737

0.39688

0.39640

0.39591

0.39543

0.39494

0.39446

0.39397

0.39349

0.39301

0.39253

0.39205

0.39157

0.39109

0.39061

0.39013

0.38966

0.38918

0.38870

0.38823

0.38776

0.38681

0.38634

0.38587

0.38540

0.38493

0.38446

0.38399

0.38352

0.38305

0.38259

0.38212

0.38166

0.38119

0.38073
0.38728

\subsection{6}

0.54853

49

50

50

49

50

49

50

49

49

49

49

49

49

49

49

48

49

48

49

48

49

48

48

48

48

48

48

48

48

47

48

48

47

47

48

47

47

47

47

47

47

47

47

47

46

47

46

47

46

0.5478933

$0.54049 \quad 32$
32

$0.54756 \quad 32$

$0.54724 \quad 32$

$0.54692 \quad 33$

$0.54659 \quad 32$

$0.54627 \quad 32$

$0.54595 \quad 32$

$0.54563 \quad 33$

$0.54530 \quad 32$

$0.54498 \quad 32$

$0.54466 \quad 32$

$0.54434 \quad 32$

$0.54402 \quad 32$

$0.54370 \quad 33$

$0.54337 \quad 32$

$0.54305 \quad 32$

$0.54273 \quad 32$

0.5424132

$0.54209 \quad 32$

$0.54177 \quad 32$

$0.54145 \quad 32$

$0.54113 \quad 32$

0.54081

32

$0.54017 \quad 32$

$0.53985 \quad 32$

$0.53953 \quad 32$

$0.53921 \quad 32$

0.53889

0.53857

0.53825

0.53793

0.53762

32

32

32

31

32

0.53730

32

0.53698

0.53666

0.53634

0.53602

32

32

32

31

0.53571

32

0.53539

0.53507

0.53475

0.53444

32

32

31

32

0.53412

32

0.53380

31

0.53349

0.53317

0.53285

$\Delta \quad \mathrm{C} / \mathrm{R}$

$\Delta$

$\begin{array}{llll}0.95363 & 82 & 0.90499 & 17 \\ 0.95281 & 82 & 0.90482 & 16 \\ 0.95199 & 83 & 0.90466 & 16 \\ 0.95116 & 82 & 0.90450 & 17 \\ 0.95034 & 81 & 0.90433 & 16 \\ 0.94953 & 82 & 0.90417 & 16 \\ 0.94871 & 82 & 0.90401 & 17 \\ 0.94789 & 81 & 0.90384 & 16 \\ 0.94708 & 82 & 0.90368 & 16 \\ 0.94626 & 81 & 0.90352 & 17 \\ 0.94545 & 82 & 0.90335 & 16\end{array}$

0.94463

0.94382

0.94301

0.94220

0.94139

81

0.90319

0.90303

0.90286

0.90270

0.90253

0.94058

0.93977

0.93896

0.93816

0.93735

0.90237

0.90220

0.90204

0.90188

0.90171

0.93655

0.93574

0.93494

0.90155

0.90138

0.90122

0.90105

0.90088

0.93334

80

0.93254

0.93174

0.93094

0.93014

0.92934

0.92855

0.92775

0.92696

0.92616

0.92537

80

0.90072

0.90055

0.90039

0.90022

0.90006

0.92458

0.92379

0.92300

0.92221

0.92142

0.89989

0.89972

0.89956

0.89939

0.89922 
Table I Harmonic Oscillator Contributions (in dimensionless form) to the

Thermodynamic Functions for Values of $X=h c v / k T$

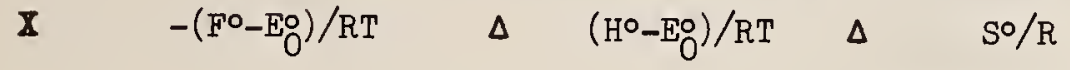

$\begin{array}{ll}1.150 & 0.38073 \\ 1.151 & 0.38027 \\ 1.152 & 0.37980 \\ 1.153 & 0.37934 \\ 1.154 & 0.37888 \\ 1.155 & 0.37842\end{array}$

1.156

1.157

1.158

1.159

1.160

1.161

1.162

1.163

1.164

1.165

1.166

1.167

1.168

1.169

1.170

1.171

1.172

1.173

2. 174

1.175

1.176

1.177

1.178

1.179

1.180

1.181

1.182

1.183

1.184

1.185

1.186

1.187

1.188

1.189

1.190

1.191

1.192

1.193

1.194

1.195

1.196

1.197

1.198

1.199

1.200
0.37796

0.37750

0.37704

0.37659

0.37613

0.37567

0.37522

0.37476

0.37431

0.37385

0.37340

0.37295

0.37250

0.37205

0.37160

0.37115

0.37070

0.37025

0.36980

0.36935

0.36891

0.36802

0.36757

0.36713

0.36668

0.36624

0.36580

0.36536

0.36492

0.36448

0.36404

0.36316

0.36272

0.36229

0.36185

0.36141

0.36098

0.36055

0.36011

0.35968

0.35925

0.35881

0.35838
0.36846

0.36360

$\begin{array}{lll}46 & 0.53285 & 31 \\ 47 & 0.53254 & 32 \\ 46 & 0.53222 & 32 \\ 46 & 0.53190 & 31 \\ 46 & 0.53159 & 32 \\ 46 & 0.53127 & 31\end{array}$

0.91358

0.91280

0.91202

0.91125

0.91047

0.90969

$46 \quad 0.53096 \quad 32$

$46 \quad 0.53064$

$45 \quad 0.53033$

46

46

0.53001

0.52970

45

46

45

46

45

45

45

45

45

45

45

45

45

45

44

45

44

45

44

45

0.52938

0.52907

0.52875

0.52844

0.52812

0.52781

0.52750

0.52718

0.52655

0.90892

0.90814

0.90737

0.90660

0.90583

0.90505

0.90428

0.90351

0.90275

0.90198

0.90121

0.90044

0.89968

0.89891

0.89815

0.52624

0.52593

0.52561

0.52530

0.52499

0.52468

0.52436

0.52405

0.52343

0.89739

0.89662

0.89586

0.89510

0.89434

0.89358

0.89282

0.89207

0.89131

0.89055

$44 \quad 0.52311 \quad 31$

44

44

44

44

44

44

44

44

43

44

44

43

43

44

43

43

44

43
0.88980

0.88904

0.88829

0.88754

0.88679

$0.52218 \quad 31$

$0.52187 \quad 31$

$0.52156 \quad 31$

$0.52125 \quad 31$

$0.52094 \quad 32$

$0.52062 \quad 31$

$0.52031 \quad 31$

0.52000

0.51969

0.51938

0.51907

0.51876

0.88603

0.88528

0.88453

0.88379

0.88304

0.51845

0.51814

0.51783

0.51752

0.51722

31

0.88229

0.88154
0.52687

0.52374
$\Delta$

$\mathrm{co} / \mathrm{R}$

$\Delta$

$\begin{array}{lll}78 & 0.89671 & 16 \\ 78 & 0.89655 & 17 \\ 77 & 0.89638 & 17 \\ 78 & 0.89621 & 17 \\ 78 & 0.89604 & 17 \\ 77 & 0.89587 & 17\end{array}$

78

77

77

77

78

77

\section{7}

76

77

77

7.7

76

77

76

76

77

76

76

76

76

76

75

76

76

75

$0.89570 \quad 17$

$0.89553 \quad 17$

$0.89536 \quad 16$

$0.89520 \quad 17$

$0.89503 \quad 17$

$0.89486 \quad 17$

$0.89469 \quad 17$

$0.89452 \quad 17$

$0.89435 \quad 17$

$0.89418 \quad 17$

$0.89401 \quad 17$

$0.89384 \quad 17$

$0.89367 \quad 17$

$0.89350 \quad 17$

$0.89333 \quad 17$

$0.89316 \quad 17$

$0.89299 \quad 17$

$0.89282 \quad 17$

$0.89265 \quad 17$

$0.89248 \quad 18$

$0.89230 \quad 17$

$0.89213 \quad 17$

$0.89196 \quad 17$

$0.89179 \quad 17$

$0.89162 \quad 17$

76

$0.89145 \quad 17$

$0.89128 \quad 18$

$0.89110 \quad 17$

$0.89093 \quad 17$

$0.89076 \quad 17$

$0.89059 \quad 17$

$0.89042 \quad 18$

$0.89024 \quad 17$

$0.89007 \quad 17$

$0.88990 \quad 17$ 
Table I Harmonic Oscillator Contributions (in dimensionless form) to the

Thermodynamic Functions for Values of $\mathrm{X}=\mathrm{hc} / \mathrm{kT}$

$\mathbf{x}$

$-\left(F^{\circ}-E O\right) / R T$

$\Delta \quad\left(H^{\circ}-E_{0}^{\circ}\right) / R T$

$\Delta$

$\mathrm{S} \% / \mathrm{R}$

1.200
1.201
1.202
1.203
1.204
1.205
1.206
1.207
1.208
1.20
1.210

1.211

1.212

1.213

1.214

1.215

1.216

1.217

1.218

1.219

1.220

1.221

1.222

1.223

1.224

1.225

1.226

1.227

1.228

1.229

1.230

1.231

1.232

1.233

1.234

1.235

1.236

1.237

1.238

1.239

1.240

1.241

1.242

1.243

1.244

1.245

1.246

1.247

1.248

1.249

1.250

0.35838
0.35795
0.35752
0.35709
0.35666
0.35624

0.35581

0.35538

0.35495

0.35453

0.35410

0.35368

0.35325

0.35283

0.35241

0.35199

0.35156

0.35114

0.35072

0.35030

0.34988

0.34947

0.34905

0.34863

0.34821

0.34780

0.34738

0.34697

0.34655

0.34614

0.34572

0.34531

0.34490

0.34449

0.34408

0.34367

0.34326

0.34285

0.34244

0.34203

0.34162

0.34122

0.34081

0.34040

0.34000

0.33959

0.33919

0.33879

0.33838

0.33798

0.33758

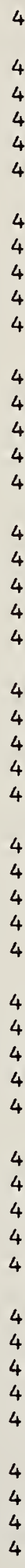

43

43

43

42

43

43

43

42

43

42

43

42

42

42

43

42

42

42

42

41

42

42

42

41

42

41

42

41

42

41

41

41

41

41

41

41

41

41

41

40

41

41

40

41

40

40

41

40

40
$0.51229 \quad 31$

$0.51198 \quad 31$

$0.51137 \quad 31$

$\begin{array}{lll}0.51076 & 31 & 0.86022\end{array}$

0.51045

0.51014

$0.50984 \quad 31 \quad 0.85805$

$0.50953 \quad 30$

0.50923

31

0.50892

0.50862

0.50831

0.50801

0.50770

30

0.50740

0.50709

$0.50648 \cdot 30 \quad 0.85015$

0.50618

0.50588

0.50557

$0.50527 \quad 30$

0.50436

0.50375

0.50254

0.50194
$\Delta$

$\mathrm{Co} / \mathrm{R}$

$\Delta$

$\begin{array}{llllll}0.51722 & 31 & 0.87560 & 74 & 0.88817 & 17 \\ 0.51691 & 31 & 0.87486 & 74 & 0.88800 & 18 \\ 0.51660 & 31 & 0.87412 & 74 & 0.88782 & 17 \\ 0.51629 & 31 & 0.87338 & 74 & 0.88765 & 17 \\ 0.51598 & 31 & 0.87264 & 73 & 0.88748 & 18 \\ 0.51567 & 31 & 0.87191 & 74 & 0.88730 & 17 \\ 0.51536 & 31 & 0.87117 & 73 & 0.88713 & 18 \\ 0.51505 & 30 & 0.87044 & 74 & 0.88695 & 17 \\ 0.51475 & 31 & 0.86970 & 73 & 0.88678 & 18 \\ 0.51444 & 31 & 0.86897 & 74 & 0.88660 & 17 \\ 0.51413 & 31 & 0.86823 & 73 & 0.88643 & 17\end{array}$

$\begin{array}{llllll}0.51382 & 30 & 0.86750 & 73 & 0.88626 & 18 \\ 0.51352 & 31 & 0.86677 & 73 & 0.88608 & 17 \\ 0.51321 & 31 & 0.86604 & 73 & 0.88591 & 18 \\ 0.51290 & 31 & 0.86531 & 73 & 0.88573 & 17 \\ 0.51259 & 30 & 0.86458 & 73 & 0.88556 & 18\end{array}$

0.86385

73

0.88538

17

0.86312

0.88521

0.86240

0.88503

18

0.86167

0.88485

18

0.86095

0.88468

18

0.85950

0.88450

17

0.88433

18

0.85877

0.88415

17

0.85733

0.88398

18

0.85661

0.85589

0.85517

0.85445

0.85373

0.88380

18

0.85301

0.85230

0.85158

0.85087

0.88362

17

0.88345

0.88327

18

0.88309

18

72

0.88292

17
18

71

0.88274

18

0.88256

0.88239

17

71

0.88221

18

72

0.88203

17

0.84944

72

0.88186

18

0.84872

0.88168

0.88150

18

0.84801

0.88132

18

0.84730

71

0.84659

0.88115

18

0.84588

0.88097

18

0.84517

71

0.88079

18

0.88061

0.88044

17

0.84446

0.84304

0.88026

18

0.84234

0.88008

18

0.84163

0.84093

0.87990

18

0.84022

0.83952

0.87972

18

0.87954

0.87937

17 
Table I Harmonic Oscillator Contributions (in dimensionless form) to the

Thermodynamic Functions for Values of $X=h c v / k T$
I $\quad-\left(F^{\circ}-E_{0}^{\circ}\right) / \mathrm{RT}$
$\Delta \quad\left(\mathrm{H}^{\circ}-\mathrm{E}_{0}^{\circ}\right) / \mathrm{RT}$
$\Delta$
So/R
$\Delta \quad \mathrm{C}_{\mathrm{p}}^{\circ} / \mathrm{R}$

$1 \cdot 25$

1.251

1.252

1.253

1.254

1.255

1. 256

1. 257

1.258

1.259

1.260

0.33758

40

0.33718

0.33678

0.33638

0.33598

0.33558

0.33518

0.33478

0.33439

0.33399

0.33359

1. 261

1.262

0.33320

0.33280

0.33241

0.33201

1.264

$1 \cdot 265$

0.33162

1.266

1.267

$1 \cdot 268$

1.269

1.270

1. 271

1.272

$1 \cdot 273$

1.274

1.275

1. 276

1.277

1.278

1.279

1.280

1. 281

1.282

1.283

$1 \cdot 284$

1.285

0.33123

0.33083

0.33044

0.33005

0.32966

0.32927

0.32888

0.32849

0.32810

0.32771

0.32733

0.32694

0.32655

0.32617

0.32578

0.32540

0.32501

0.32463

0.32425

0.32386

$1 \cdot 286$

1.287

$1 \cdot 288$

1. 289

1.290

0.32348

0.32310

0.32272

0.32234

0.32196

1. 291

1. 292

$1 \cdot 293$

1.294

1. 295

1.296

1.297

1. 298

1.299

1.300

0.32158

0.32120

0.32082

0.32044

0.32006

0.31969

0.31931

0.31894

0.31856

0.31818
$0.50194 \quad 30$

$40 \quad 0.50164 \quad 30$

$40 \quad 0.50134 \quad 31$

40

0.50103

0.50073

0.50043

30

30

40

40

39

40

40

39

40

39

40

39

39

40

39

39

39

39

39

39

39

39

38

39

39

38

39

38

39

38

38

39

38

38

38

38

38

38

38

38

38

38

37

38

37

38

38
0.50013

0.49983

0.49953

0.49923

0.49893

0.49863

0.49833

0.49803

0.49773

0.49743

0.49713

0.49683

0.49653

0.49623

0.49593

$0.49563 \quad 30$

0.49533

0.49503

0.49473

0.49443

0.49414

0.49384

0.49354

0.49324

0.49294

0.49265

0.49235

0.49205

0.49176

0.49146

0.49116

0.49086

0.49057

0.49027

0.48998

0.48968

0.48938

0.48909

0.48879

0.48850

0.48820

0.48790

0.48761

0.48731

0.48702
0.83811

0.83741

0.83671

0.83601

0.83531

0.83461

0.83391

0.83322

0.83252

0.83182

0.83113

0.83043

0.82974

0.82904

0.82835

0.82766

0.82697

0.82628

0.82490

0.82421

0.82352

0.82284

0.82215

0.82146

0.82078

0.82009

0.81941

0.81873

0.81804

0.81736

0.81668

0.81600

0.81532

0.81464

0.81396

0.81329

0.81261

0.81193

0.81126

0.81058

0.80991

0.80923

0.80789

0.80722

0.80654

0.80587

71

70

70

70

70

70

70

69

70

70

69

70

69

70

69

69

69

69

69

69

69

69

68

69

69

68

69
0.83882

0.82559

0.80856

0.80520 
Table I Harmonic Oscillator Contributions (in dimensionless form) to the

Thermodynamic Functions for Values of $\mathrm{X}=\mathrm{hc} v / \mathrm{kT}$

及 $-\left(F^{\circ}-E_{0}^{\circ}\right) / R T$

$\Delta \quad\left(H^{\circ}-E_{0}^{\circ}\right) / R T \quad \Delta \quad S \% / R$

$\Delta$

$\mathrm{Co}_{\mathrm{p}} / \mathrm{R}$

$\Delta$

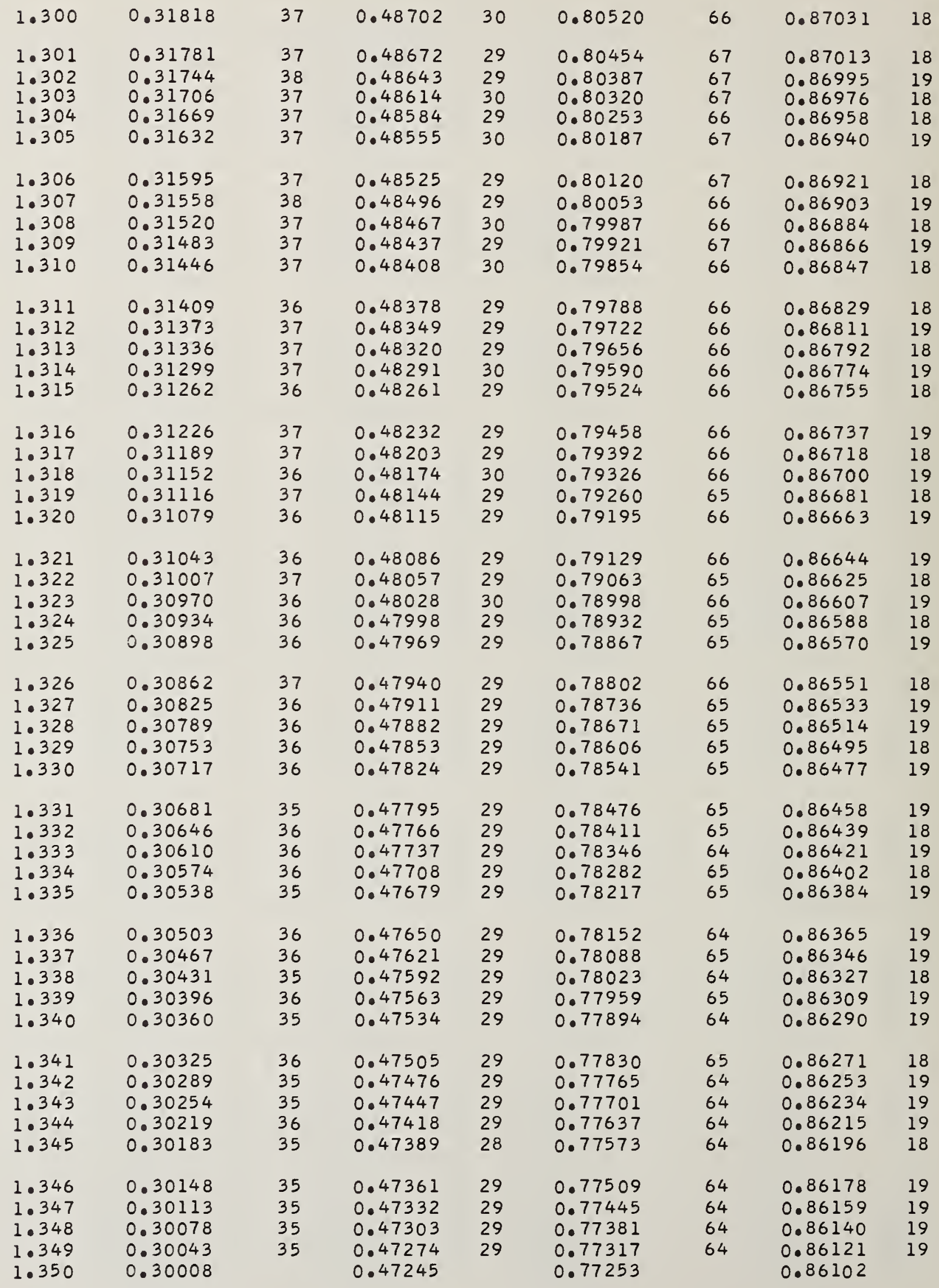


Table I Harmonic Oscillator Contributions (in dimensionless form) to the Thermodynamic Functions for Values of $\mathrm{X}=\mathrm{hcv} / \mathrm{kT}$

\begin{tabular}{|c|c|c|c|c|c|c|c|}
\hline $\bar{Z}$ & $-\left(F^{\circ}-E_{0}^{\circ}\right) / R T$ & $\Delta$ & $\left(\mathrm{H}^{\circ}-\mathrm{E}_{\mathrm{O}}^{\circ}\right) / \mathrm{RT}$ & $\Delta$ & So/R & $\Delta$ & $\mathrm{C}_{\mathrm{p}} / \mathrm{R}$ \\
\hline 1.350 & 0.30008 & 35 & 0.47245 & 28 & 0.77253 & 64 & 0.86102 \\
\hline $\begin{array}{l}1.351 \\
1.352 \\
1.353 \\
1.354 \\
1.355\end{array}$ & $\begin{array}{l}0.29973 \\
0.29938 \\
0.29903 \\
0.29868 \\
0.29833\end{array}$ & $\begin{array}{l}35 \\
35 \\
35 \\
35 \\
34\end{array}$ & $\begin{array}{l}0.47217 \\
0.47188 \\
0.47159 \\
0.47130 \\
0.47102\end{array}$ & $\begin{array}{l}29 \\
29 \\
29 \\
28 \\
29\end{array}$ & $\begin{array}{l}0.77189 \\
0.77126 \\
0.77062 \\
0.76999 \\
0.76935\end{array}$ & $\begin{array}{l}63 \\
64 \\
63 \\
64 \\
63\end{array}$ & $\begin{array}{l}0.86084 \\
0.86065 \\
0.86046 \\
0.86027 \\
0.86008\end{array}$ \\
\hline $\begin{array}{l}1.356 \\
1.357 \\
1.358 \\
1.359 \\
1.360\end{array}$ & $\begin{array}{l}0.29799 \\
0.29764 \\
0.29729 \\
0.29695 \\
0.29660\end{array}$ & $\begin{array}{l}35 \\
35 \\
34 \\
35 \\
34\end{array}$ & $\begin{array}{l}0.47073 \\
0.47044 \\
0.47016 \\
0.46987 \\
0.46958\end{array}$ & $\begin{array}{l}29 \\
28 \\
29 \\
29 \\
28\end{array}$ & $\begin{array}{l}0.76872 \\
0.76808 \\
0.76745 \\
0.76682 \\
0.76618\end{array}$ & $\begin{array}{l}64 \\
63 \\
63 \\
64 \\
63\end{array}$ & $\begin{array}{l}0.85989 \\
0.85971 \\
0.85952 \\
0.85933 \\
0.85914\end{array}$ \\
\hline $\begin{array}{l}1.361 \\
1.362 \\
1.363 \\
1.364 \\
1.365\end{array}$ & $\begin{array}{l}0.29626 \\
0.29591 \\
0.29557 \\
0.29523 \\
0.29488\end{array}$ & $\begin{array}{l}35 \\
34 \\
34 \\
35 \\
34\end{array}$ & $\begin{array}{l}0.46930 \\
0.46901 \\
0.46872 \\
0.46844 \\
0.46815\end{array}$ & $\begin{array}{l}29 \\
29 \\
28 \\
29 \\
28\end{array}$ & $\begin{array}{l}0.76555 \\
0.76492 \\
0.76429 \\
0.76366 \\
0.76303\end{array}$ & $\begin{array}{l}63 \\
63 \\
63 \\
63 \\
62\end{array}$ & $\begin{array}{l}0.85895 \\
0.85876 \\
0.85857 \\
0.85838 \\
0.85819\end{array}$ \\
\hline $\begin{array}{l}1.366 \\
1.367 \\
1.368 \\
1.369 \\
1.370\end{array}$ & $\begin{array}{l}0.29454 \\
0.29420 \\
0.29386 \\
0.29351 \\
0.29317\end{array}$ & $\begin{array}{l}34 \\
34 \\
35 \\
34 \\
34\end{array}$ & $\begin{array}{l}0.46787 \\
0.46758 \\
0.46729 \\
0.46701 \\
0.46672\end{array}$ & $\begin{array}{l}29 \\
29 \\
28 \\
29 \\
28\end{array}$ & $\begin{array}{l}0.76241 \\
0.76178 \\
0.76115 \\
0.76052 \\
0.75990\end{array}$ & $\begin{array}{l}63 \\
63 \\
63 \\
62 \\
63\end{array}$ & $\begin{array}{l}0.85800 \\
0.85781 \\
0.85762 \\
0.85743 \\
0.85724\end{array}$ \\
\hline $\begin{array}{l}1.371 \\
1 \cdot 372 \\
1.373 \\
1.374 \\
1.375\end{array}$ & $\begin{array}{l}0.29283 \\
0.29249 \\
0.29215 \\
0.29181 \\
0.29148\end{array}$ & $\begin{array}{l}34 \\
34 \\
34 \\
33 \\
34\end{array}$ & $\begin{array}{l}0.46644 \\
0.46615 \\
0.46587 \\
0.46559 \\
0.46530\end{array}$ & $\begin{array}{l}29 \\
28 \\
28 \\
29 \\
28\end{array}$ & $\begin{array}{l}0.75927 \\
0.75865 \\
0.75802 \\
0.75740 \\
0.75678\end{array}$ & $\begin{array}{l}62 \\
63 \\
62 \\
62 \\
63\end{array}$ & $\begin{array}{l}0.85705 \\
0.85686 \\
0.85667 \\
0.85648 \\
0.85629\end{array}$ \\
\hline $\begin{array}{r}.376 \\
.377 \\
.378 \\
.379 \\
.380\end{array}$ & $\begin{array}{l}0.29114 \\
0.29080 \\
0.29046 \\
0.29013 \\
0.28979\end{array}$ & $\begin{array}{l}34 \\
34 \\
33 \\
34 \\
34\end{array}$ & $\begin{array}{l}0.46502 \\
0.46473 \\
0.46445 \\
0.46416 \\
0.46388\end{array}$ & $\begin{array}{l}29 \\
28 \\
29 \\
28 \\
28\end{array}$ & $\begin{array}{l}0.75615 \\
0.75553 \\
0.75491 \\
0.75429 \\
0.75367\end{array}$ & $\begin{array}{l}62 \\
62 \\
62 \\
62 \\
62\end{array}$ & $\begin{array}{l}0.85610 \\
0.85591 \\
0.85572 \\
0.85553 \\
0.85534\end{array}$ \\
\hline $\begin{array}{l}1.381 \\
1.382 \\
1.383 \\
1.384 \\
1.385\end{array}$ & $\begin{array}{l}0.28945 \\
0.28912 \\
0.28878 \\
0.28845 \\
0.28811\end{array}$ & $\begin{array}{l}33 \\
34 \\
33 \\
34 \\
33\end{array}$ & $\begin{array}{l}0.46360 \\
0.46331 \\
0.46303 \\
0.46275 \\
0.46246\end{array}$ & $\begin{array}{l}29 \\
28 \\
28 \\
29 \\
28\end{array}$ & $\begin{array}{l}0.75305 \\
0.75243 \\
0.75181 \\
0.75120 \\
0.75058\end{array}$ & $\begin{array}{l}62 \\
62 \\
61 \\
62 \\
62\end{array}$ & $\begin{array}{l}0.85515 \\
0.85496 \\
0.85477 \\
0.85458 \\
0.85439\end{array}$ \\
\hline $\begin{array}{l}1.386 \\
1 \cdot 387 \\
1.388 \\
1.389 \\
1.390\end{array}$ & $\begin{array}{l}0.28778 \\
0.28745 \\
0.28711 \\
0.28678 \\
0.28645\end{array}$ & $\begin{array}{l}33 \\
34 \\
33 \\
33 \\
33\end{array}$ & $\begin{array}{l}0.46218 \\
0.46190 \\
0.46162 \\
0.46133 \\
0.46105\end{array}$ & $\begin{array}{l}28 \\
28 \\
29 \\
28 \\
28\end{array}$ & $\begin{array}{l}0.74996 \\
0.74935 \\
0.74873 \\
0.74812 \\
0.74750\end{array}$ & $\begin{array}{l}61 \\
62 \\
61 \\
62 \\
61\end{array}$ & $\begin{array}{l}0.85419 \\
0.85400 \\
0.85381 \\
0.85362 \\
0.85343\end{array}$ \\
\hline $\begin{array}{l}1.391 \\
1.392 \\
1.393 \\
1.394 \\
1.395\end{array}$ & $\begin{array}{l}0.28612 \\
0.28579 \\
0.28546 \\
0.28513 \\
0.28480\end{array}$ & $\begin{array}{l}33 \\
33 \\
33 \\
33 \\
33\end{array}$ & $\begin{array}{l}0.46077 \\
0.46049 \\
0.46020 \\
0.45992 \\
0.45964\end{array}$ & $\begin{array}{l}28 \\
29 \\
28 \\
28 \\
28\end{array}$ & $\begin{array}{l}0.74689 \\
0.74627 \\
0.74566 \\
0.74505 \\
0.74444\end{array}$ & $\begin{array}{l}62 \\
61 \\
61 \\
61 \\
61\end{array}$ & $\begin{array}{l}0.85324 \\
0.85305 \\
0.85285 \\
0.85266 \\
0.85247\end{array}$ \\
\hline $\begin{array}{l}1.396 \\
1.397 \\
1.398 \\
1.399 \\
1.400\end{array}$ & $\begin{array}{l}0.28447 \\
0.28414 \\
0.28381 \\
0.28348 \\
0.28315\end{array}$ & $\begin{array}{l}33 \\
33 \\
33 \\
33\end{array}$ & $\begin{array}{l}0.45936 \\
0.45908 \\
0.45880 \\
0.45852 \\
0.45824\end{array}$ & $\begin{array}{l}28 \\
28 \\
28 \\
28\end{array}$ & $\begin{array}{l}0.74383 \\
0.74322 \\
0.74261 \\
0.74200 \\
0.74139\end{array}$ & $\begin{array}{l}61 \\
61 \\
61 \\
61\end{array}$ & $\begin{array}{l}0.85228 \\
0.85209 \\
0.85189 \\
0.85170 \\
0.85151\end{array}$ \\
\hline
\end{tabular}


Table I Harmonic Oscillator Contributions (in dimensionless form) to the Thermodynamic Functions for Values of $\mathrm{X}=\mathrm{hc} / \mathrm{kT}$

$\mathbb{X}$ $-\left(F^{\circ}-E_{O}^{\circ}\right) / R T$

$\left(\mathrm{H}^{\circ}-\mathrm{E}_{\mathrm{O}}^{\circ}\right) / \mathrm{RT}$

0.28315

1.400

0.28283

1.401

1.402

1.403

1.404

1.405

0.28250

0.28217

0.28185

0.28152

1.406

1.407

1.408

1.409

1.410

1.411

1.412

1.413

1.414

1.415

1.416

1.417

1.418

1.419

1.420

1.421

1.422

1.423

1.424

1.425

1.426

1.427

1.428

1.429

1.430

1.431

1.432

1.433

1.434

1.435

1.436

1.437

1.438

1.439

1.440

1.441

1.442

1.443

1.444

1.445

1.446

1.447

1.448

1.449

1.450
0.28120

0.28087

0.28055

0.28023

0.27990

0.27958

0.27926

0.27894

0.27861

0.27829

0.27797

0.27765

0.27733

0.27701

0.27669

0.27638

0.27606

0.27574

0.27542

0.27511

0.27479

0.27447

0.27416

0.27384

0.27353

0.27321

0.27290

0.27259

0.27227

0.27196

0.27165

0.27134

0.27071

0.27040

0.27009

0.26978

0.26916

0.26886

0.26855

0.26824

0.26793

0.26762

0.26732
0.27102

0.26947

0.45292

0.45126

0.44849

0.44821

$\begin{array}{ll}32 & 0.45824 \\ 33 & 0.45795 \\ 33 & 0.45767 \\ 32 & 0.45739 \\ 33 & 0.45711 \\ 32 & 0.45683\end{array}$

$\Delta \quad \mathrm{S} \% / \mathrm{R}$

0.45655

33

32

32

33

32

0.45627

0.45599

0.45571

0.45543

0.45515

32

32

33

32

32

0.45487

0.45459

0.45432

0.45404

32

32

0.45376

0.45348

0.45320

0.45264

31

32

32

0.45237

0.45209

0.45181

0.45153

$0.45098 \quad 28$

0.45070

0.45042

0.45015

0.44987

$0.44959 \quad 27$

0.44932

27
28
28

0.44904

0.44876

0.44794

0.44766

0.44738

0.44711

0.44683

0.44656

0.44628

0.44601

0.44573

0.44546

0.44518

0.44491

0.44463

0.44436
29

28

28

28

28

28

28

28

28

28

28

28

28

27

28

28

28

28

28

28

27

28

28

28

27

28

28
28

27

28

28

27

28

27

28

28

27

28

27

28

27

28
27

0.74139

0.74078

0.74017

0.73957

0.73896

0.73836

0.73775

0.73715

0.73654

0.73594

0.73534

0.73473

0.73413

0.73353

0.73293

0.73233

0.73173

0.73113

0.73053

0.72994

0.72934

0.72874

0.72815

0.72755

0.72696

0.72636

0.72577

0.72517

0.72458

0.72399

0.72340

0.72281

0.72222

0.72163

0.72104

0.72045

0.71986

0.71927

0.71868

0.71810

0.71751

28

27

28

27

0.71693

0.71634

0.71576

0.71517

0.71459
$\Delta \quad \mathrm{Co} / \mathrm{R}$

$\Delta$

60
60

60

59
60

$61 \quad 0.85151$

19

$61 \quad 0.85132 \quad 20$

$\begin{array}{lll}60 & 0.85112 & 19\end{array}$

$\begin{array}{lll}61 & 0.85093 & 19\end{array}$

$\begin{array}{lll}60 & 0.8507 .4 & 19\end{array}$

$\begin{array}{lll}61 & 0.85055 & 20\end{array}$

$\begin{array}{lll}60 & 0.85035 & 19\end{array}$

$\begin{array}{lll}61 & 0.85016 & 19\end{array}$

$60 \quad 0.84997 \quad 20$

$\begin{array}{lll}60 & 0.84977 & 19\end{array}$

$\begin{array}{lll}61 & 0.84958 & 19\end{array}$

$\begin{array}{lll}60 & 0.84939 & 20\end{array}$

$\begin{array}{lll}60 & 0.84919 & 19\end{array}$

60

0.84900

0.84881

0.84861

19 
Table I Harmonic Oscillator Contributions (in dimensionless form) to the Thermodynamic Functions for Values of $\mathrm{X}=\mathrm{hcv} / \mathrm{kT}$

\begin{tabular}{|c|c|c|c|c|c|c|c|}
\hline$X$ & $-\left(\mathrm{F}^{\circ}-\mathrm{E}_{\mathrm{O}}^{\circ}\right) / \mathrm{RT}$ & $\Delta$ & $\left(\mathrm{H}^{\circ}-\mathrm{E}_{\mathrm{O}}^{\circ}\right) / \mathrm{RT}$ & $\Delta$ & $\mathrm{S} / \mathrm{R}$ & $\Delta$ & $\mathrm{C}_{\mathrm{p}} / \mathrm{R}$ \\
\hline 1.450 & 0.26732 & 31 & 0.44436 & 27 & 0.71168 & 58 & 0.84178 \\
\hline $\begin{array}{l}1.451 \\
1.452\end{array}$ & $\begin{array}{l}0.26701 \\
0.26671\end{array}$ & $\begin{array}{l}30 \\
31\end{array}$ & $\begin{array}{l}0.44409 \\
0.44381\end{array}$ & $\begin{array}{l}28 \\
27\end{array}$ & $\begin{array}{l}0.71110 \\
0.71052\end{array}$ & $\begin{array}{l}58 \\
58\end{array}$ & $\begin{array}{l}0.84158 \\
0.84139\end{array}$ \\
\hline $\begin{array}{l}1.453 \\
1.454\end{array}$ & $\begin{array}{l}0.26640 \\
0.26610\end{array}$ & $\begin{array}{l}30 \\
31\end{array}$ & $\begin{array}{l}0.44354 \\
0.44327\end{array}$ & $\begin{array}{l}27 \\
28\end{array}$ & $\begin{array}{l}0.70994 \\
0.70936\end{array}$ & $\begin{array}{l}58 \\
58\end{array}$ & $\begin{array}{l}0.84119 \\
0.84099\end{array}$ \\
\hline 1.455 & 0.26579 & 30 & 0.44299 & 27 & 0.70878 & 57 & 0.84080 \\
\hline $\begin{array}{l}1.456 \\
1.457 \\
1.458 \\
1.459 \\
1.460\end{array}$ & $\begin{array}{l}0.26549 \\
0.26518 \\
0.26488 \\
0.26458 \\
0.26427\end{array}$ & $\begin{array}{l}31 \\
30 \\
30 \\
31 \\
30\end{array}$ & $\begin{array}{l}0.44272 \\
0.44245 \\
0.44217 \\
0.44190 \\
0.44163\end{array}$ & $\begin{array}{l}27 \\
28 \\
27 \\
27 \\
28\end{array}$ & $\begin{array}{l}0.70821 \\
0.70763 \\
0.70705 \\
0.70648 \\
0.70590\end{array}$ & $\begin{array}{l}58 \\
58 \\
57 \\
58 \\
58\end{array}$ & $\begin{array}{l}0.84060 \\
0.84040 \\
0.84020 \\
0.84001 \\
0.83981\end{array}$ \\
\hline $\begin{array}{l}1.461 \\
1.462 \\
1.463 \\
1.464 \\
1.465\end{array}$ & $\begin{array}{l}0.26397 \\
0.26367 \\
0.26337 \\
0.26307 \\
0.26277\end{array}$ & $\begin{array}{l}30 \\
30 \\
30 \\
30 \\
30\end{array}$ & $\begin{array}{l}0.44135 \\
0.44108 \\
0.44081 \\
0.44054 \\
0.44026\end{array}$ & $\begin{array}{l}27 \\
27 \\
27 \\
28 \\
27\end{array}$ & $\begin{array}{l}0.70532 \\
0.70475 \\
0.70418 \\
0.70360 \\
0.70303\end{array}$ & $\begin{array}{l}57 \\
57 \\
58 \\
57 \\
57\end{array}$ & $\begin{array}{l}0.83961 \\
0.83941 \\
0.83922 \\
0.83902 \\
0.83882\end{array}$ \\
\hline $\begin{array}{l}1.466 \\
1.467 \\
1.468 \\
1.469 \\
1.470\end{array}$ & $\begin{array}{l}0.26247 \\
0.26217 \\
0.26187 \\
0.26157 \\
0.26127\end{array}$ & $\begin{array}{l}30 \\
30 \\
30 \\
30 \\
30\end{array}$ & $\begin{array}{l}0.43999 \\
0.43972 \\
0.43945 \\
0.43918 \\
0.43891\end{array}$ & $\begin{array}{l}27 \\
27 \\
27 \\
27 \\
28\end{array}$ & $\begin{array}{l}0.70246 \\
0.70189 \\
0.70132 \\
0.70074 \\
0.70017\end{array}$ & $\begin{array}{l}57 \\
57 \\
58 \\
57 \\
57\end{array}$ & $\begin{array}{l}0.83862 \\
0.83843 \\
0.83823 \\
0.83803 \\
0.83783\end{array}$ \\
\hline $\begin{array}{l}1.471 \\
1.472 \\
1.473 \\
1.474 \\
1.475\end{array}$ & $\begin{array}{l}0.26097 \\
0.26067 \\
0.26037 \\
0.26008 \\
0.25978\end{array}$ & $\begin{array}{l}30 \\
30 \\
29 \\
30 \\
30\end{array}$ & $\begin{array}{l}0.43863 \\
0.43836 \\
0.43809 \\
0.43782 \\
0.43755\end{array}$ & $\begin{array}{l}27 \\
27 \\
27 \\
27 \\
27\end{array}$ & $\begin{array}{l}0.69960 \\
0.69904 \\
0.69847 \\
0.69790 \\
0.69733\end{array}$ & $\begin{array}{l}56 \\
57 \\
57 \\
57 \\
57\end{array}$ & $\begin{array}{l}0.83763 \\
0.83743 \\
0.83724 \\
0.83704 \\
0.83684\end{array}$ \\
\hline $\begin{array}{l}.476 \\
.477 \\
.478 \\
.479 \\
.480\end{array}$ & $\begin{array}{l}0.25948 \\
0.25919 \\
0.25889 \\
0.25860 \\
0.25830\end{array}$ & $\begin{array}{l}29 \\
30 \\
29 \\
30 \\
29\end{array}$ & $\begin{array}{l}0.43728 \\
0.43701 \\
0.43674 \\
0.43647 \\
0.43620\end{array}$ & $\begin{array}{l}27 \\
27 \\
27 \\
27 \\
27\end{array}$ & $\begin{array}{l}0.69676 \\
0.69620 \\
0.69563 \\
0.69507 \\
0.69450\end{array}$ & $\begin{array}{l}56 \\
57 \\
56 \\
57 \\
56\end{array}$ & $\begin{array}{l}0.83664 \\
0.83644 \\
0.83624 \\
0.83604 \\
0.83584\end{array}$ \\
\hline $\begin{array}{r}.481 \\
.482 \\
.483 \\
.484 \\
4485\end{array}$ & $\begin{array}{l}0.25801 \\
0.25771 \\
0.25742 \\
0.25713 \\
0.25683\end{array}$ & $\begin{array}{l}30 \\
29 \\
29 \\
30 \\
29\end{array}$ & $\begin{array}{l}0.43593 \\
0.43566 \\
0.43539 \\
0.43512 \\
0.43485\end{array}$ & $\begin{array}{l}27 \\
27 \\
27 \\
27 \\
27\end{array}$ & $\begin{array}{l}0.69394 \\
0.69337 \\
0.69281 \\
0.69225 \\
0.69168\end{array}$ & $\begin{array}{l}57 \\
56 \\
56 \\
57 \\
56\end{array}$ & $\begin{array}{l}0.83565 \\
0.83545 \\
0.83525 \\
0.83505 \\
0.83485\end{array}$ \\
\hline $\begin{array}{l}1.486 \\
1.487 \\
1.488 \\
1.489 \\
1.490\end{array}$ & $\begin{array}{l}0.25654 \\
0.25625 \\
0.25596 \\
0.25566 \\
0.25537\end{array}$ & $\begin{array}{l}29 \\
29 \\
30 \\
29 \\
29\end{array}$ & $\begin{array}{l}0.43458 \\
0.43431 \\
0.43404 \\
0.43377 \\
0.43351\end{array}$ & $\begin{array}{l}27 \\
27 \\
27 \\
26 \\
27\end{array}$ & $\begin{array}{l}0.69112 \\
0.69056 \\
0.69000 \\
0.68944 \\
0.68888\end{array}$ & $\begin{array}{l}56 \\
56 \\
56 \\
56 \\
56\end{array}$ & $\begin{array}{l}0.83465 \\
0.83445 \\
0.83425 \\
0.83405 \\
0.83385\end{array}$ \\
\hline $\begin{array}{l}1.491 \\
1.492 \\
1.493 \\
1.494 \\
1.495\end{array}$ & $\begin{array}{l}0.25508 \\
0.25479 \\
0.25450 \\
0.25421 \\
0.25392\end{array}$ & $\begin{array}{l}29 \\
29 \\
29 \\
29 \\
29\end{array}$ & $\begin{array}{l}0.43324 \\
0.43297 \\
0.43270 \\
0.43243 \\
0.43216\end{array}$ & $\begin{array}{l}27 \\
27 \\
27 \\
27 \\
26\end{array}$ & $\begin{array}{l}0.68832 \\
0.68776 \\
0.68720 \\
0.68664 \\
0.68609\end{array}$ & $\begin{array}{l}56 \\
56 \\
56 \\
55 \\
56\end{array}$ & $\begin{array}{l}0.83365 \\
0.83345 \\
0.83325 \\
0.83305 \\
0.83285\end{array}$ \\
\hline $\begin{array}{l}1.496 \\
1.497 \\
1.498 \\
1.499 \\
1.500\end{array}$ & $\begin{array}{l}0.25363 \\
0.25335 \\
0.25306 \\
0.25277 \\
0.25248\end{array}$ & $\begin{array}{l}28 \\
29 \\
29 \\
29\end{array}$ & $\begin{array}{l}0.43190 \\
0.43163 \\
0.43136 \\
0.43109 \\
0.43083\end{array}$ & $\begin{array}{l}27 \\
27 \\
27 \\
26\end{array}$ & $\begin{array}{l}0.68553 \\
0.68497 \\
0.68442 \\
0.68386 \\
0.68331\end{array}$ & $\begin{array}{l}56 \\
55 \\
56 \\
55\end{array}$ & $\begin{array}{l}0.83265 \\
0.83245 \\
0.83225 \\
0.83205 \\
0.83185\end{array}$ \\
\hline
\end{tabular}


Table I Harmonic Oscillator Contributions (in dimensionless form) to the Thermodynamic Functions for Values of $\mathrm{X}=\mathrm{hcv} / \mathrm{kT}$

I $\quad-\left(F^{\circ}-E_{O}^{\circ}\right) / R T$

$\Delta \quad\left(\mathrm{H}^{\circ}-\mathrm{EO}\right) / \mathrm{RT}$

$\Delta$

So/R

1.501

1.502

1.503

1.504

1.505

1.506

1.507

1.508

1.509

1.510

1.511

1.512

1.513

1.514

1.515

1.516

1.517

1.518

1.519

1.520

1.521

1.522

1.523

1.524

1.525

1.526

1.527

1.528

1.529

1.530

1.531

1.532

1.533

1.534

1.535

1.536

1.537

1.538

1.539

1.540

1.541

1.542

1.543

1.544

1.545

1.546

1.547

1.548

1.549

1.550

0.25248
0.25220
0.25191
0.25162
0.25134
0.25105

0.25077

0.25048

0.25020

0.24991

0.24963

0.24935

0.24906

0.24878

0.24850

0.24822

\subsection{3}

0.24765

0.24737

0.24709

0.24681

\subsection{3}

0.24625

0.24597

0.24569

0.24542

\subsection{4}

0.24486

0.24458

0.24431

0.24403

0.24375

0.24348

0.24320

0.24293

0.24265

0.24238

0.24210

0.24183

0.24156

0.24128

0.24101

0.24074

0.24047

0.24019

0.23992

0.23965

0.23938

0.23911

0.23884

0.23857
0.43083

27

0.43056

$\begin{array}{lll}28 & 0.43083 & 27 \\ 29 & 0.43056 & 27 \\ 29 & 0.43029 & 27 \\ 28 & 0.43002 & 26 \\ 29 & 0.42976 & 27 \\ 28 & 0.42949 & 27 \\ 29 & 0.42922 & 26 \\ 28 & 0.42896 & 27 \\ 29 & 0.42869 & 27 \\ 28 & 0.42842 & 26 \\ 28 & 0.42816 & 27\end{array}$

0.42789

28

28

28

29

28

28

28

28

28

28

28

28

27

28

28

28

27

28

28

27

28

27

28

27

28

27

27

28

27

27

27

28

27

27

27

27

27

27

0.42313

0.42286

0.42260

0.42234

0.42208

0.42181

0.42155

0.42129

0.42102

0.42076

0.42050

0.42024

0.41998

0.41945

0.41919

0.41893

0.41867

0.41841

0.41815

0.41789
0.41971
$\Delta \quad \mathrm{CO} / \mathrm{R}$

$\Delta$ $\begin{array}{llll}0.67999 & 55 & 0.83064 & 20 \\ 0.67944 & 55 & 0.83044 & 20 \\ 0.67889 & 55 & 0.83024 & 20 \\ 0.67834 & 55 & 0.83004 & 20 \\ 0.67779 & 55 & 0.82984 & 20\end{array}$

0.41762

0.68331
0.68275
0.68220
0.68165
0.68109
0.68054

0.67724

0.67669

0.67614

0.67559

0.67505

0.67450

0.67395

0.67341

0.67286

0.67232

0.67177

0.67123

0.67068

0.67014

0.66960

0.66906

0.66852

0.66798

0.66743

0.66689

0.66636

0.66582

0.66528

0.66474

0.66420

0.66366

0.66313

0.66259

0.66206

0.66152

56

55

55

56

55

55

55

55

55

54

55

55

54

55

54

55

54

55

54

54

54

54

54

55

54

53

54

54

54

54

54

53

54

53

54

53

0.66099

0.66045

0.65992

0.65939

0.65885

0.65832

0.65779

0.65726

0.65673

0.65620

54

53

53

54

53
0.8296420

$0.82944 \quad 21$

$0.82923 \quad 20$

$0.82903 \quad 20$

$0.82883 \quad 20$

0.8286320

0.8284320

$0.82823 \quad 21$

$0.82802 \quad 20$

0.8278220

$0.82762 \quad 20$

$0.82742 \quad 20$

$0.82722 \quad 21$

0.8270120

0.8268120

0.8266120

$0.82641 \quad 21$

$0.82620 \quad 20$

$0.82600 \quad 20$

$0.82580 \quad 20$

$0.82560 \quad 21$

$0.82539 \quad 20$

0.8251920

0.8249921

0.8247820

$0.82458 \quad 20$

$0.82438 \quad 21$

$0.82417 \quad 20$

$0.82397 \quad 20$

$0.82377 \quad 21$

$0.82356 \quad 20$

$0.82336 \quad 20$ 
Table I Harmonic Oscillator Contributions (in dimensionless form) to the Thermodynamic Functions for Values of $X=\mathrm{hcv} / \mathrm{kT}$

\begin{tabular}{|c|c|c|c|c|c|c|c|}
\hline z & $-\left(F^{\circ}-E_{O} O\right) / R T$ & $\Delta$ & $\left(H^{\circ}-E_{O}^{\circ}\right) / R T$ & $\Delta$ & SO/R & $\Delta$ & $C O / R$ \\
\hline 550 & 0.23857 & 27 & 0.41762 & 26 & 0.65620 & 53 & 0.82173 \\
\hline $\begin{array}{l}.551 \\
.552 \\
.553 \\
.554 \\
.555\end{array}$ & $\begin{array}{l}0.23830 \\
0.23803 \\
0.23777 \\
0.23750 \\
0.23723\end{array}$ & $\begin{array}{l}27 \\
26 \\
27 \\
27 \\
27\end{array}$ & $\begin{array}{l}0.41736 \\
0.41710 \\
0.41684 \\
0.41658 \\
0.41632\end{array}$ & $\begin{array}{l}26 \\
26 \\
26 \\
26 \\
26\end{array}$ & $\begin{array}{l}0.65567 \\
0.65514 \\
0.65461 \\
0.65408 \\
0.65355\end{array}$ & $\begin{array}{l}53 \\
53 \\
53 \\
53 \\
53\end{array}$ & $\begin{array}{l}0.82152 \\
0.82132 \\
0.82111 \\
0.82091 \\
0.82071\end{array}$ \\
\hline $\begin{array}{l}.556 \\
.557 \\
.558 \\
559 \\
560\end{array}$ & $\begin{array}{l}0.23696 \\
0.23669 \\
0.23643 \\
0.23616 \\
0.23589\end{array}$ & $\begin{array}{l}27 \\
26 \\
27 \\
27 \\
26\end{array}$ & $\begin{array}{l}0.41606 \\
0.41580 \\
0.41554 \\
0.41528 \\
0.41502\end{array}$ & $\begin{array}{l}26 \\
26 \\
26 \\
26 \\
26\end{array}$ & $\begin{array}{l}0.65302 \\
0.65250 \\
0.65197 \\
0.65144 \\
0.65092\end{array}$ & $\begin{array}{l}52 \\
53 \\
53 \\
52 \\
53\end{array}$ & $\begin{array}{l}0.82050 \\
0.82030 \\
0.82009 \\
0.81989 \\
0.81968\end{array}$ \\
\hline $\begin{array}{l}561 \\
562 \\
563 \\
564 \\
565\end{array}$ & $\begin{array}{l}0.23563 \\
0.23536 \\
0.23510 \\
0.23483 \\
0.23457\end{array}$ & $\begin{array}{l}27 \\
26 \\
27 \\
26 \\
27\end{array}$ & $\begin{array}{l}0.41476 \\
0.41451 \\
0.41425 \\
0.41399 \\
0.41373\end{array}$ & $\begin{array}{l}25 \\
26 \\
26 \\
26 \\
26\end{array}$ & $\begin{array}{l}0.65039 \\
0.64987 \\
0.64934 \\
0.64882 \\
0.64830\end{array}$ & $\begin{array}{l}52 \\
53 \\
52 \\
52 \\
53\end{array}$ & $\begin{array}{l}0.81948 \\
0.81927 \\
0.81907 \\
0.81886 \\
0.81866\end{array}$ \\
\hline $\begin{array}{l}566 \\
567 \\
568 \\
569 \\
57.0\end{array}$ & $\begin{array}{l}0.23430 \\
0.23404 \\
0.23378 \\
0.23351 \\
0.23325\end{array}$ & $\begin{array}{l}26 \\
26 \\
27 \\
26 \\
26\end{array}$ & $\begin{array}{l}0.41347 \\
0.41321 \\
0.41295 \\
0.41269 \\
0.41244\end{array}$ & $\begin{array}{l}26 \\
26 \\
26 \\
25 \\
26\end{array}$ & $\begin{array}{l}0.64777 \\
0.64725 \\
0.64673 \\
0.64621 \\
0.64569\end{array}$ & $\begin{array}{l}52 \\
52 \\
52 \\
52 \\
52\end{array}$ & $\begin{array}{l}0.81845 \\
0.81825 \\
0.81804 \\
0.81783 \\
0.81763\end{array}$ \\
\hline $\begin{array}{l}571 \\
572 \\
573 \\
574 \\
575\end{array}$ & $\begin{array}{l}0.23299 \\
0.23273 \\
0.23246 \\
0.23220 \\
0.23194\end{array}$ & $\begin{array}{l}26 \\
27 \\
26 \\
26 \\
26\end{array}$ & $\begin{array}{l}0.41218 \\
0.41192 \\
0.41166 \\
0.41141 \\
0.41115\end{array}$ & $\begin{array}{l}26 \\
26 \\
25 \\
26 \\
26\end{array}$ & $\begin{array}{l}0.64517 \\
0.64465 \\
0.64413 \\
0.64361 \\
0.64309\end{array}$ & $\begin{array}{l}52 \\
52 \\
52 \\
52 \\
52\end{array}$ & $\begin{array}{l}0.81742 \\
0.81722 \\
0.81701 \\
0.81681 \\
0.81660\end{array}$ \\
\hline $\begin{array}{l}576 \\
577 \\
578 \\
579 \\
580\end{array}$ & $\begin{array}{l}0.23168 \\
0.23142 \\
0.23116 \\
0.23090 \\
0.23064\end{array}$ & $\begin{array}{l}26 \\
26 \\
26 \\
26 \\
26\end{array}$ & $\begin{array}{l}0.41089 \\
0.41063 \\
0.41038 \\
0.41012 \\
0.40986\end{array}$ & $\begin{array}{l}26 \\
25 \\
26 \\
26 \\
25\end{array}$ & $\begin{array}{l}0.64257 \\
0.64205 \\
0.64154 \\
0.64102 \\
0.64050\end{array}$ & $\begin{array}{l}52 \\
51 \\
52 \\
52 \\
51\end{array}$ & $\begin{array}{l}0.81639 \\
0.81619 \\
0.81598 \\
0.81578 \\
0.81557\end{array}$ \\
\hline $\begin{array}{l}581 \\
582 \\
583 \\
584 \\
585\end{array}$ & $\begin{array}{l}0.23038 \\
0.23012 \\
0.22986 \\
0.22961 \\
0.22935\end{array}$ & $\begin{array}{l}26 \\
26 \\
25 \\
26 \\
26\end{array}$ & $\begin{array}{l}0.40961 \\
0.40935 \\
0.40909 \\
0.40884 \\
0.40858\end{array}$ & $\begin{array}{l}26 \\
26 \\
25 \\
26 \\
26\end{array}$ & $\begin{array}{l}0.63999 \\
0.63947 \\
0.63896 \\
0.63844 \\
0.63793\end{array}$ & $\begin{array}{l}52 \\
51 \\
52 \\
51 \\
52\end{array}$ & $\begin{array}{l}0.81536 \\
0.81516 \\
0.81495 \\
0.81474 \\
0.81454\end{array}$ \\
\hline $\begin{array}{l}586 \\
587 \\
588 \\
589 \\
590\end{array}$ & $\begin{array}{l}0.22909 \\
0.22883 \\
0.22858 \\
0.22832 \\
0.22806\end{array}$ & $\begin{array}{l}26 \\
25 \\
26 \\
26 \\
25\end{array}$ & $\begin{array}{l}0.40832 \\
0.40807 \\
0.40781 \\
0.40756 \\
0.40730\end{array}$ & $\begin{array}{l}25 \\
26 \\
25 \\
26 \\
25\end{array}$ & $\begin{array}{l}0.63741 \\
0.63690 \\
0.63639 \\
0.63588 \\
0.63536\end{array}$ & $\begin{array}{l}51 \\
51 \\
51 \\
52 \\
51\end{array}$ & $\begin{array}{l}0.81433 \\
0.81412 \\
0.81392 \\
0.81371 \\
0.81350\end{array}$ \\
\hline $\begin{array}{l}591 \\
.592 \\
.593 \\
.594 \\
595\end{array}$ & $\begin{array}{l}0.22781 \\
0.22755 \\
0.22730 \\
0.22704 \\
0.22679\end{array}$ & $\begin{array}{l}26 \\
25 \\
26 \\
25 \\
26\end{array}$ & $\begin{array}{l}0.40705 \\
0.40679 \\
0.40653 \\
0.40628 \\
0.40603\end{array}$ & $\begin{array}{l}26 \\
26 \\
25 \\
25 \\
26\end{array}$ & $\begin{array}{l}0.63485 \\
0.63434 \\
0.63383 \\
0.63332 \\
0.63281\end{array}$ & $\begin{array}{l}51 \\
51 \\
51 \\
51 \\
51\end{array}$ & $\begin{array}{l}0.81330 \\
0.81309 \\
0.81288 \\
0.81267 \\
0.81247\end{array}$ \\
\hline $\begin{array}{l}1.596 \\
1.597 \\
1.598 \\
1.599 \\
1.600\end{array}$ & $\begin{array}{l}0.22653 \\
0.22628 \\
0.22602 \\
0.22577 \\
0.22552\end{array}$ & $\begin{array}{l}25 \\
26 \\
25 \\
25\end{array}$ & $\begin{array}{l}0.40577 \\
0.40552 \\
0.40526 \\
0.40501 \\
0.40475\end{array}$ & $\begin{array}{l}25 \\
26 \\
25 \\
26\end{array}$ & $\begin{array}{l}0.63230 \\
0.63179 \\
0.63128 \\
0.63078 \\
0.63027\end{array}$ & $\begin{array}{l}51 \\
51 \\
50 \\
51\end{array}$ & $\begin{array}{l}0.81226 \\
0.81205 \\
0.81184 \\
0.81164 \\
0.81143\end{array}$ \\
\hline
\end{tabular}


Table I Harmonic Oscillator Contributions (in dimensionless form) to the

Thermodynamic Functions for Values of $\mathrm{X}=\mathrm{hcv} / \mathrm{kT}$

$\mathbb{Z}$

$-\left(\mathrm{F}^{\circ}-\mathrm{E}_{\mathrm{O}}\right) / \mathrm{RT}$
$\Delta \quad\left(\mathrm{H}^{\circ}-\mathrm{E}_{\mathrm{O}}^{\circ}\right) / \mathrm{RT} \quad \Delta \quad \mathrm{S} / \mathrm{R}$

\subsection{1}

1.602

1.603

1.604

1.605

1.606

1.607

1.608

1.609

1.610

1.611

1.612

1.613

1.614

1.615

1.616

1.617

1.618

1.619

1.620

1.621

1.622

1.623

1.624

1.625

1.626

1.627

1.628

1.629

1.630

1.631

1.632

1.633

1.634

1.635

1.636

1.637

1.638

1.639

1.640

1.641

1.642

1.643

1.644

1.645

1.646

1.647

1.648

1.649

1.650

0.22552
0.22526
0.22501
0.22476
0.22451
0.22426

0.22400

0.22375

0.22350

0.22325

0.22300

0.22275

0.22250

0.22225

0.22201

0.22176

0.22151

0.22126

0.22101

0.22077

0.22052

0.22027

0.22003

0.21978

0.21954

0.21929

0.21905

0.21880

0.21856

0.21831

0.21807

0.21782

0.21758

0.21734

0.21710

0.21685

0.21661

0.21637

0.21613

0.21589

0.21565

0.21541

0.21517

0.21493

0.21469

0.21445

0.21421

0.21397

0.21373

0.21349

0.21325

$\begin{array}{ll}26 & 0.40475 \\ 25 & 0.40450 \\ 25 & 0.40424 \\ 25 & 0.40399 \\ 25 & 0.40374 \\ 26 & 0.40348\end{array}$

25

26

25

25

26

25

$0.40323 \quad 25$

25

25

25

25

25

25

25

24

25

25

25

25

24

25

25

24

25

24

25

24

25

24

25

24

25

24

24

25

24

0.40298

0.40272

0.40247

0.40222

0.40196

0.40171

0.40146

0.40121

0.40095

0.40070

0.40045

0.40020

0.39995

0.39970

0.39944

0.39919

0.39894

0.39869

0.39844

26

25

25

26

25

25

25

26

25

25

25

25

25

26

25

25

25

25

0.39819

0.39794

0.39769

0.39744

0.39719

0.39694

0.39669

0.39644

0.39619

0.39594

25

25

25

25

25

25

25

25

25

25

25

0.39569

24
24

24
24

24

24

0.39544

0.39519

0.39494

0.39469

25

25

25

25

25

0.39444

25

24

24

24

24

24

0.39419

0.39394

0.39369

0.39345

25

25

24

25

24

24

24

24

$\Delta \quad \mathrm{c} \% \mathrm{R}$

$\Delta$

$\begin{array}{llll}0.63027 & 51 & 0.81143 & 21 \\ 0.62976 & 50 & 0.81122 & 21 \\ 0.62926 & 51 & 0.81101 & 20 \\ 0.62875 & 51 & 0.81081 & 21 \\ 0.62824 & 50 & 0.81060 & 21 \\ 0.62774 & 51 & 0.81039 & 21\end{array}$

0.62723

0.62673

0.62623

0.62572

0.62522

50

50

51

50

50

0.62472

0.62422

0.62371

0.62321

0.62271

50

51

50

50

50

0.62221

0.62171

0.62121

0.62071

0.62022

50

50

50

49

50

0.61972

0.61922

0.61872

0.61823

0.61773

50

50

49

50

50

0.61723

0.61674

0.61624

0.61575

0.61525

49

50

49

50

49

0.61476

0.61427

0.61377

0.61328

0.61279 
Table I Harmonic Oscillator Contributions (in dimensionless form) to the

Thermodynamic Functions for Values of $X=h c v / k T$

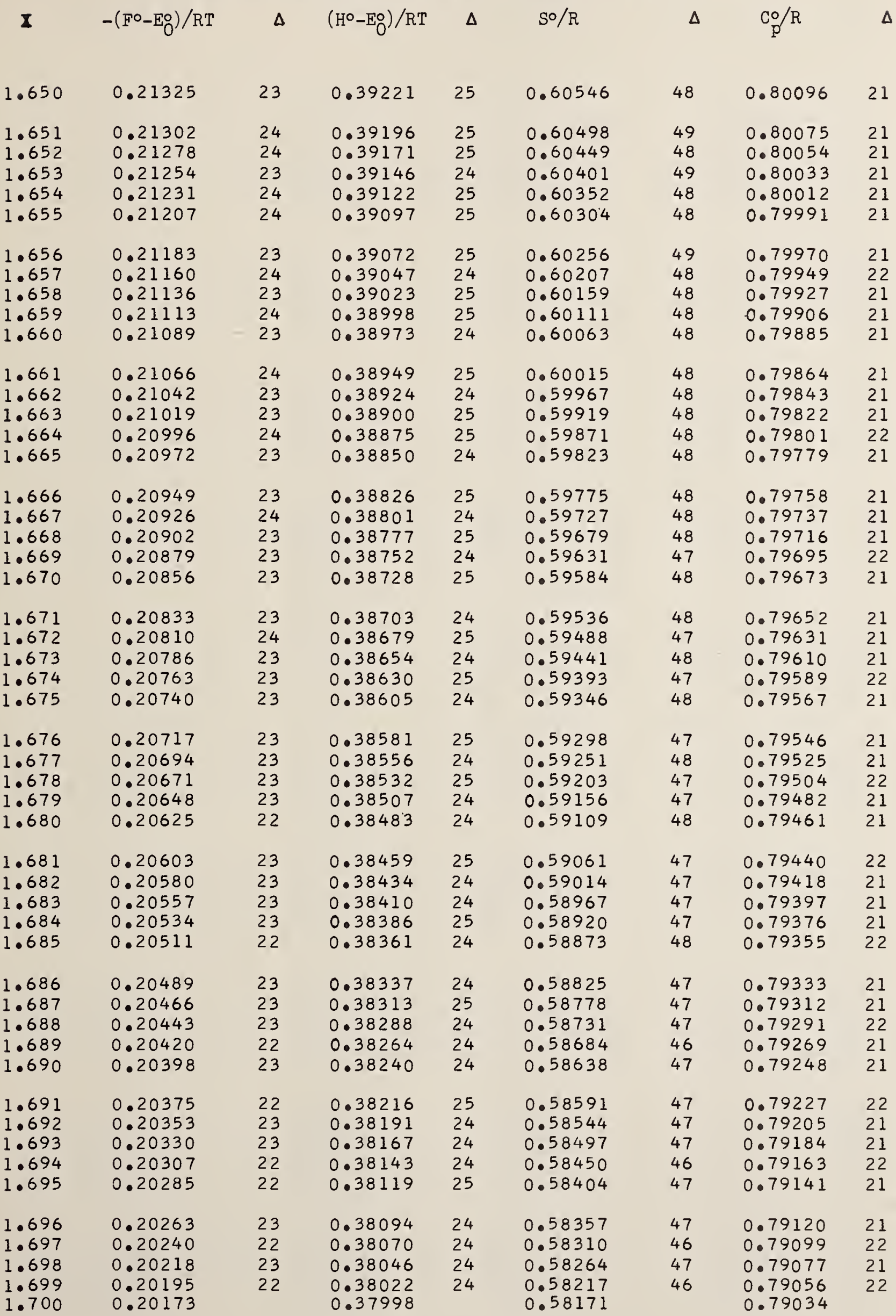


Table I Harmonic Oscillator Contributions (in dimensionless form) to the

Thermodynamic Functions for Values of $\mathrm{X}=\mathrm{hcv} / \mathrm{kT}$

I $-\left(F^{\circ}-E_{O}^{\circ}\right) / R T$
$\Delta \quad\left(H^{\circ}-E_{O}^{\circ}\right) / R T$

$\Delta$

So/R .

1.700
1.701
1.702
1.703
1.704
1.705
1.706
1.707
1.708
1.709
1.710
1.711
1.712
1.713
1.714
1.715
1.716
1.717
1.718
1.719
1.720
1.721
1.722
1.723
1.724
1.725
1.726
1.727
1.728
1.729
1.730

\subsection{3}

0.20151

0.20128

0.20106

0.20084

0.20061

0.20039

0.20017

0.19995

0.19973

0.19951

0.19929

0.19907

0.19885

0.19863

0.19841

0.19819

0.19797

0.19775

0.19753

0.19731

0.19709

0.19688

0.19666

0.19644

0.19623

0.19601

0.19579

0.19558

0.19536

0.19514

1.731

I. 732

I. 733

1.734

1.735

1.736

1.737

1.738

1.739

I. 740

1.741

1.742

I. 743

1.744

1.745

1.746

1.747

1.748

1.749

1.750
0.19493

0.19471

0.19450

0.19428

0.19407

0.19386

0.19364

0.19343

0.19322

0.19300

0.19279

0.19258

0.19237

0.19215

0.19194

0.19173

0.19152

0.19131

0.19110

0.19089

0.37998
0.37974
0.37950
0.37925
0.37901
0.37877

24

24

25

24

24

24

\section{2}

22

22

22

22

0.37853

0.37829

0.37805

0.37781

0.37757

24

24

24

24

22

22

22

22

22

0.37733

0.37709

0.37685

0.37661

0.37637

24

24

24

24

22

22

22

22

22

0.37613

0.37589

0.37565

0.37541

0.37517

21

0.37494

22

22

21

22

0.37470

0.37446

0.37422

0.37398

24

24

24

24

23

0.57294

22

0.37374

24

24

24

24

24

23

21

22

22

21

0.37351

0.37303

24
24

0.37279

22

0.37255

21

21

21

0.37232

0.37208

0.37184

0.37161

23

24

22

0.37137

21

21

22

21

0.37113

0.37090

0.37066

21

21

0.37019

0.36995

0.36971

0.36948

21

21

0.36924

21

21

0.36901

0.36877

0.36853

0.36830

0.36806

22
23
22
22
23
22

$\Delta \quad \mathrm{co} / \mathrm{R}$

$\Delta$

0.37327

0.37042

\subsection{1}

0.58124

0.58078

0.58031

0.57985

0.57939

0.57892

0.57846

0.57800

0.57754

0.57708

0.57662

0.57616

0.57570

0.57524

0.57478

0.57432

0.57386

0.57340

0.57249

0.57203

0.57157

0.57112

0.57066

0.57021

0.56975

0.56930

0.56884

0.56839

0.56794

0.56748

47

46

47

46

46
46

47

46

46

46

46

46

46

46

46

46

46

46

46

46

45

46

46

45

46

45

46

45

46

45

45

46

45

0.56703

0.56658

0.56613

0.56568

0.56523

0.56477

0.56432

0.56387

0.56343

0.56298

0.56253

0.56208

0.56163

0.56118

0.56074

0.56029

0.55984

0.55940

0.55895
$0.79034 \quad 21$

$0.79013 \quad 21$

$0.78992 \quad 22$

$0.78970 \quad 21$ 
Table I Harmonic Oscillator Contributions (in dimensionless form) to the

Thermodynamic Functions for Values of $\mathrm{X}=\mathrm{hcv} / \mathrm{kT}$

\begin{tabular}{|c|c|c|c|c|c|c|c|}
\hline $\bar{z}$ & $-\left(F^{\circ}-E_{O}^{\circ}\right) / R T$ & $\Delta$ & $\left(\mathrm{H}^{\circ}-\mathrm{E}_{\mathrm{O}}^{\circ}\right) / \mathrm{RT}$ & $\Delta$ & $\mathrm{S} / \mathrm{R}$ & $\Delta$ & $\mathrm{C}_{\mathrm{p}}^{\circ} / \mathrm{R}$ \\
\hline 1.750 & 0.19089 & 21 & 0.36806 & 23 & 0.55895 & 44 & 0.77958 \\
\hline $\begin{array}{l}1.751 \\
1.752 \\
1.753 \\
1.754 \\
1.755\end{array}$ & $\begin{array}{l}0.19068 \\
0.19047 \\
0.19026 \\
0.19005 \\
0.18984\end{array}$ & $\begin{array}{ll}2 & 1 \\
2 & 1 \\
2 & 1 \\
2 & 1 \\
2 & 1\end{array}$ & $\begin{array}{l}0.36783 \\
0.36759 \\
0.36736 \\
0.36712 \\
0.36689\end{array}$ & $\begin{array}{l}24 \\
23 \\
24 \\
23 \\
23\end{array}$ & $\begin{array}{l}0.55851 \\
0.55806 \\
0.55762 \\
0.55717 \\
0.55673\end{array}$ & $\begin{array}{l}45 \\
44 \\
45 \\
44 \\
44\end{array}$ & $\begin{array}{l}0.77937 \\
0.77915 \\
0.77893 \\
0.77872 \\
0.77850\end{array}$ \\
\hline $\begin{array}{l}1.756 \\
1.757 \\
1.758 \\
1.759 \\
1.760\end{array}$ & $\begin{array}{l}0.18963 \\
0.18942 \\
0.18921 \\
0.18900 \\
0.18880\end{array}$ & $\begin{array}{ll}2 & 1 \\
2 & 1 \\
2 & 1 \\
2 & 0 \\
2 & 1\end{array}$ & $\begin{array}{l}0.36666 \\
0.36642 \\
0.36619 \\
0.36595 \\
0.36572\end{array}$ & $\begin{array}{l}24 \\
23 \\
24 \\
23 \\
23\end{array}$ & $\begin{array}{l}0.55629 \\
0.55584 \\
0.55540 \\
0.55496 \\
0.55452\end{array}$ & $\begin{array}{l}45 \\
44 \\
44 \\
44 \\
45\end{array}$ & $\begin{array}{l}0.77828 \\
0.77807 \\
0.77785 \\
0.77763 \\
0.77742\end{array}$ \\
\hline $\begin{array}{l}1.761 \\
1.762 \\
1.763 \\
1.764 \\
1.765\end{array}$ & $\begin{array}{l}0.18859 \\
0.18838 \\
0.18817 \\
0.18797 \\
0.18776\end{array}$ & $\begin{array}{ll}2 & 1 \\
2 & 1 \\
2 & 0 \\
2 & 1 \\
2 & 1\end{array}$ & $\begin{array}{l}0.36549 \\
0.36525 \\
0.36502 \\
0.36478 \\
0.36455\end{array}$ & $\begin{array}{l}24 \\
23 \\
24 \\
23 \\
23\end{array}$ & $\begin{array}{l}0.55407 \\
0.55363 \\
0.55319 \\
0.55275 \\
0.55231\end{array}$ & $\begin{array}{l}44 \\
44 \\
44 \\
44 \\
44\end{array}$ & $\begin{array}{l}0.77720 \\
0.77698 \\
0.77676 \\
0.77655 \\
0.77633\end{array}$ \\
\hline $\begin{array}{l}1.766 \\
1.767 \\
1.768 \\
1.769 \\
1.770\end{array}$ & $\begin{array}{l}0.18755 \\
0.18735 \\
0.18714 \\
0.18694 \\
0.18673\end{array}$ & $\begin{array}{l}20 \\
21 \\
20 \\
21 \\
20\end{array}$ & $\begin{array}{l}0.36432 \\
0.36408 \\
0.36385 \\
0.36362 \\
0.36339\end{array}$ & $\begin{array}{l}24 \\
23 \\
23 \\
23 \\
24\end{array}$ & $\begin{array}{l}0.55187 \\
0.55143 \\
0.55099 \\
0.55056 \\
0.55012\end{array}$ & $\begin{array}{l}44 \\
44 \\
43 \\
44 \\
44\end{array}$ & $\begin{array}{l}0.77611 \\
0.77590 \\
0.77568 \\
0.77546 \\
0.77524\end{array}$ \\
\hline $\begin{array}{l}1.771 \\
1.772 \\
1.773 \\
1.774 \\
1.775\end{array}$ & $\begin{array}{l}0.18653 \\
0.18632 \\
0.18612 \\
0.18591 \\
0.18571\end{array}$ & $\begin{array}{ll}2 & 1 \\
2 & 0 \\
2 & 1 \\
2 & 0 \\
2 & 1\end{array}$ & $\begin{array}{l}0.36315 \\
0.36292 \\
0.36269 \\
0.36246 \\
0.36222\end{array}$ & $\begin{array}{l}23 \\
23 \\
23 \\
24 \\
23\end{array}$ & $\begin{array}{l}0.54968 \\
0.54924 \\
0.54880 \\
0.54837 \\
0.54793\end{array}$ & $\begin{array}{l}44 \\
44 \\
43 \\
44 \\
43\end{array}$ & $\begin{array}{l}0.77503 \\
0.77481 \\
0.77459 \\
0.77437 \\
0.77415\end{array}$ \\
\hline $\begin{array}{l}1.776 \\
1.777 \\
1.778 \\
1.779 \\
1.780\end{array}$ & $\begin{array}{l}0.18550 \\
0.18530 \\
0.18510 \\
0.18489 \\
0.18469\end{array}$ & $\begin{array}{l}20 \\
20 \\
21 \\
20 \\
20\end{array}$ & $\begin{array}{l}0.36199 \\
0.36176 \\
0.36153 \\
0.36130 \\
0.36107\end{array}$ & $\begin{array}{l}23 \\
23 \\
23 \\
23 \\
24\end{array}$ & $\begin{array}{l}0.54750 \\
0.54706 \\
0.54662 \\
0.54619 \\
0.54576\end{array}$ & $\begin{array}{l}44 \\
44 \\
43 \\
43 \\
44\end{array}$ & $\begin{array}{l}0.77394 \\
0.77372 \\
0.77350 \\
0.77328 \\
0.77306\end{array}$ \\
\hline $\begin{array}{l}1.781 \\
1.782 \\
1.783 \\
1.784 \\
1.785\end{array}$ & $\begin{array}{l}0.18449 \\
0.18428 \\
0.18408 \\
0.18388 \\
0.18368\end{array}$ & $\begin{array}{l}21 \\
20 \\
20 \\
20 \\
20\end{array}$ & $\begin{array}{l}0.36083 \\
0.36060 \\
0.36037 \\
0.36014 \\
0.35991\end{array}$ & $\begin{array}{l}23 \\
23 \\
23 \\
23 \\
23\end{array}$ & $\begin{array}{l}0.54532 \\
0.54489 \\
0.54445 \\
0.54402 \\
0.54359\end{array}$ & $\begin{array}{l}43 \\
44 \\
43 \\
43 \\
43\end{array}$ & $\begin{array}{l}0.77285 \\
0.77263 \\
0.77241 \\
0.77219 \\
0.77197\end{array}$ \\
\hline $\begin{array}{l}1.786 \\
1.787 \\
1.788 \\
1.789 \\
1.790\end{array}$ & $\begin{array}{l}0.18348 \\
0.18328 \\
0.18308 \\
0.18287 \\
0.18267\end{array}$ & $\begin{array}{l}20 \\
20 \\
21 \\
20 \\
20\end{array}$ & $\begin{array}{l}0.35968 \\
0.35945 \\
0.35922 \\
0.35899 \\
0.35876\end{array}$ & $\begin{array}{l}23 \\
23 \\
23 \\
23 \\
23\end{array}$ & $\begin{array}{l}0.54316 \\
0.54272 \\
0.54229 \\
0.54186 \\
0.54143\end{array}$ & $\begin{array}{l}44 \\
43 \\
43 \\
43 \\
43\end{array}$ & $\begin{array}{l}0.77175 \\
0.77154 \\
0.77132 \\
0.77110 \\
0.77088\end{array}$ \\
\hline $\begin{array}{l}1.791 \\
1.792 \\
1.793 \\
1.794 \\
1.795\end{array}$ & $\begin{array}{l}0.18247 \\
0.18227 \\
0.18207 \\
0.18187 \\
0.18167\end{array}$ & $\begin{array}{l}20 \\
20 \\
20 \\
20 \\
19\end{array}$ & $\begin{array}{l}0.35853 \\
0.35830 \\
0.35807 \\
0.35784 \\
0.35761\end{array}$ & $\begin{array}{l}23 \\
23 \\
23 \\
23 \\
23\end{array}$ & $\begin{array}{l}0.54100 \\
0.54057 \\
0.54014 \\
0.53971 \\
0.53928\end{array}$ & $\begin{array}{l}43 \\
43 \\
43 \\
43 \\
43\end{array}$ & $\begin{array}{l}0.77066 \\
0.77044 \\
0.77023 \\
0.77001 \\
0.76979\end{array}$ \\
\hline $\begin{array}{l}1.796 \\
1.797 \\
1.798 \\
1.799 \\
1.800\end{array}$ & $\begin{array}{l}0.18148 \\
0.18128 \\
0.18108 \\
0.18088 \\
0.18068\end{array}$ & $\begin{array}{l}20 \\
20 \\
20 \\
20\end{array}$ & $\begin{array}{l}0.35738 \\
0.35715 \\
0.35692 \\
0.35669 \\
0.35646\end{array}$ & $\begin{array}{l}23 \\
23 \\
23 \\
23\end{array}$ & $\begin{array}{l}0.53885 \\
0.53842 \\
0.53800 \\
0.53757 \\
0.53714\end{array}$ & $\begin{array}{l}43 \\
42 \\
43 \\
43\end{array}$ & $\begin{array}{l}0.76957 \\
0.76935 \\
0.76913 \\
0.76891 \\
0.76869\end{array}$ \\
\hline
\end{tabular}


Table I Harmonic Oscillator Contributions (in dimensionless form) to the

Thermodynamic Functions for Values of $\mathrm{X}=\mathrm{hc} v / \mathrm{kT}$

X

$-\left(\mathrm{F}^{\circ}-\mathrm{E}_{\mathrm{O}}^{\circ}\right) / \mathrm{RT}$
$\Delta \quad\left(\mathrm{H}^{\circ}-\mathrm{E}_{\mathrm{O}}^{\circ}\right) / \mathrm{RT}$

$\Delta$

1.800
1.801
1.802
1.803
1.804

1.805

1.806

1.807

1.808

1.809

1.810

1.811

1.812

1.813

1.814

1.815

1.816

1.817

1.818

1.819

1.820

1. 821

1.822

1.823

1.824

1.825

1.826

1.827

1.828

1.829

1.830

1.831

1.832

1. 833

1.834

1.835

1.836

1.837

1.838

1.839

1.840

1. 841

1. 842

1.843

1.844

1.845

1.846

1.847

1.848

1. 849

1.850

0.18068
0.18048
0.18029
0.18009
0.17989
0.17969

0.17950

0.17930

0.17910

0.17891

0.17871

0.17852

0.17832

0.17813

0.17793

0.17774

0.17754

0.17716

0.17696

0.17677

0.17657

0.17638

0.17619

0.17600

0.17580

0.17561

0.17542

0.17523

0.17504

0.17485

0.17465

0.17446

0.17427

0.17408

0.17389

0.17370

0.17351

0.17332

0.17314

0.17295

0.17276

0.17257

0.17238

0.17219

0.17201

0.17182

0.17163

0.17144

0.17126

0.17107
0.17735

20
19
20
20
20
19

0.35646
0.35623
0.35600
0.35577
0.35555
0.35532

23

23

23

22

23

\section{0}

20

19

20

19

$0.35509 \quad 23$

23

0.35486

0.35463

0.35440

0.35418

23

23

22

20

\section{9}

20

19

20

0.35395

0.35372

0.35349

0.35327

0.35304

0.35281

19

19

20

19

20

0.35258

0.35236

0.35213

0.35190

23

22

23

23

19

19

19

20

19

0.35168

0.35145

0.35123

0.35100

0.35077

19

19

19

19

20

0.35055

0.35032

0.35010

0.34987

0.34964

0.34942

19

\section{9}

\section{9}

$$
19
$$

19

0.34919

0.34897

0.34874

0.34852

0.34829

0.34807

0.34785

0.34762

0.34740

19

19

19

18

0.34717

0.34695

0.34672

0.34650

0.34628

19

19

19

0.34605

22

0.34583

0.34561

0.34538

$S^{\circ} / \mathrm{R}$

$\Delta$

$\mathrm{C}_{\mathrm{p}}^{\circ} / \mathrm{R}$

$\Delta$

$\begin{array}{llll}0.53714 & 42 & 0.76869 & 22 \\ 0.53672 & 43 & 0.76847 & 22 \\ 0.53629 & 43 & 0.76825 & 21 \\ 0.53586 & 42 & 0.76804 & 22 \\ 0.53544 & 43 & 0.76782 & 22 \\ 0.53501 & 42 & 0.76760 & 22\end{array}$

0.53459

0.53416

0.53374

0.53331

0.53289

43

42

43

42

42

0.53247

0.53204

0.53162

0.53120

0.53078

0.53035

0.52993

0.52951

0.52909

0.52867

43

42

42

42

43

42

42

42

42

42

0.52825

0.52783

0.52741

0.52700

0.52658

0.52616

0.52574

0.52532

0.52491

0.52449

0.52407

0.52366

0.52324

0.52283

0.52241

0.52200

0.52158

0.52117

0.52076

0.52034

0.51993

0.51952

0. 51911

0.51869

0.51828

42

42

41

42

42

42

42
42

41

42

42

41

42

41

42

41

42

41

41

42

41

41

41

42

41

41

0.51787 
Table I Harmonic Oscillator Contributions (in dimensionless form) to the

Thermodynamic Functions for Values of $\mathrm{X}=\mathrm{hcv} / \mathrm{kT}$

\begin{tabular}{|c|c|c|c|c|c|c|c|}
\hline $\mathbf{X}$ & $-\left(F^{\circ}-E_{O}^{\circ}\right) / R T$ & $\Delta$ & $\left(\mathrm{H}^{\circ}-\mathrm{E}_{\mathrm{O}}^{\circ}\right) / \mathrm{RT}$ & $\Delta$ & So/R & $\Delta$ & $\mathrm{Co} / \mathrm{R}$ \\
\hline .850 & 0.17107 & 19 & 0.34516 & 22 & 0.51623 & 41 & 0.75768 \\
\hline $\begin{array}{l}1.851 \\
1.852 \\
1.853 \\
1.854 \\
1.855\end{array}$ & $\begin{array}{l}0.17088 \\
0.17070 \\
0.17051 \\
0.17033 \\
0.17014\end{array}$ & $\begin{array}{l}18 \\
19 \\
18 \\
19 \\
19\end{array}$ & $\begin{array}{l}0.34494 \\
0.34472 \\
0.34449 \\
0.34427 \\
0.34405\end{array}$ & $\begin{array}{l}22 \\
23 \\
22 \\
22 \\
22\end{array}$ & $\begin{array}{l}0.51582 \\
0.51541 \\
0.51500 \\
0.51460 \\
0.51419\end{array}$ & $\begin{array}{l}41 \\
41 \\
40 \\
41 \\
41\end{array}$ & $\begin{array}{l}0.75746 \\
0.75724 \\
0.75702 \\
0.75680 \\
0.75658\end{array}$ \\
\hline $\begin{array}{l}1.856 \\
1.857 \\
1.858 \\
1.859 \\
1.860\end{array}$ & $\begin{array}{l}0.16995 \\
0.16977 \\
0.16958 \\
0.16940 \\
0.16921\end{array}$ & $\begin{array}{l}18 \\
19 \\
18 \\
19 \\
18\end{array}$ & $\begin{array}{l}0.34383 \\
0.34360 \\
0.34338 \\
0.34316 \\
0.34294\end{array}$ & $\begin{array}{l}23 \\
22 \\
22 \\
22 \\
22\end{array}$ & $\begin{array}{l}0.51378 \\
0.51337 \\
0.51296 \\
0.51256 \\
0.51215\end{array}$ & $\begin{array}{l}41 \\
41 \\
40 \\
41 \\
40\end{array}$ & $\begin{array}{l}0.75636 \\
0.75613 \\
0.75591 \\
0.75569 \\
0.75547\end{array}$ \\
\hline $\begin{array}{l}1.861 \\
1.862 \\
1.863 \\
1.864 \\
1.865\end{array}$ & $\begin{array}{l}0.16903 \\
0.16885 \\
0.16866 \\
0.16848 \\
0.16830\end{array}$ & $\begin{array}{l}18 \\
19 \\
18 \\
18 \\
19\end{array}$ & $\begin{array}{l}0.34272 \\
0.34249 \\
0.34227 \\
0.34205 \\
0.34183\end{array}$ & $\begin{array}{l}23 \\
22 \\
22 \\
22 \\
22\end{array}$ & $\begin{array}{l}0.51175 \\
0.51134 \\
0.51094 \\
0.51053 \\
0.51013\end{array}$ & $\begin{array}{l}41 \\
40 \\
41 \\
40 \\
41\end{array}$ & $\begin{array}{l}0.75525 \\
0.75503 \\
0.75480 \\
0.75458 \\
0.75436\end{array}$ \\
\hline $\begin{array}{l}1.866 \\
1.867 \\
1.868 \\
1.869 \\
1.870\end{array}$ & $\begin{array}{l}0.16811 \\
0.16793 \\
0.16775 \\
0.16756 \\
0.16738\end{array}$ & $\begin{array}{l}18 \\
18 \\
19 \\
18 \\
18\end{array}$ & $\begin{array}{l}0.34161 \\
0.34139 \\
0.34117 \\
0.34095 \\
0.34073\end{array}$ & $\begin{array}{l}22 \\
22 \\
22 \\
22 \\
23\end{array}$ & $\begin{array}{l}0.50972 \\
0.50932 \\
0.50891 \\
0.50851 \\
0.50811\end{array}$ & $\begin{array}{l}40 \\
41 \\
40 \\
40 \\
41\end{array}$ & $\begin{array}{l}0.75414 \\
0.75392 \\
0.75369 \\
0.75347 \\
0.75325\end{array}$ \\
\hline $\begin{array}{l}1.871 \\
1.872 \\
1.873 \\
1.874 \\
1.875\end{array}$ & $\begin{array}{l}0.16720 \\
0.16702 \\
0.16684 \\
0.16666 \\
0.16647\end{array}$ & $\begin{array}{l}18 \\
18 \\
18 \\
19 \\
18\end{array}$ & $\begin{array}{l}0.34050 \\
0.34028 \\
0.34006 \\
0.33984 \\
0.33962\end{array}$ & $\begin{array}{l}22 \\
22 \\
22 \\
22 \\
22\end{array}$ & $\begin{array}{l}0.50770 \\
0.50730 \\
0.50690 \\
0.50650 \\
0.50610\end{array}$ & $\begin{array}{l}40 \\
40 \\
40 \\
40 \\
40\end{array}$ & $\begin{array}{l}0.75303 \\
0.75281 \\
0.75258 \\
0.75236 \\
0.75214\end{array}$ \\
\hline $\begin{array}{l}.876 \\
.877 \\
.878 \\
.879 \\
.880\end{array}$ & $\begin{array}{l}0.16629 \\
0.16611 \\
0.16593 \\
0.16575 \\
0.16557\end{array}$ & $\begin{array}{l}18 \\
18 \\
18 \\
18 \\
18\end{array}$ & $\begin{array}{l}0.33940 \\
0.33918 \\
0.33896 \\
0.33874 \\
0.33852\end{array}$ & $\begin{array}{l}22 \\
22 \\
22 \\
22 \\
21\end{array}$ & $\begin{array}{l}0.50570 \\
0.50530 \\
0.50490 \\
0.50450 \\
0.50410\end{array}$ & $\begin{array}{l}40 \\
40 \\
40 \\
40 \\
40\end{array}$ & $\begin{array}{l}0.75192 \\
0.75169 \\
0.75147 \\
0.75125 \\
0.75103\end{array}$ \\
\hline $\begin{array}{l}.881 \\
.882 \\
.883 \\
.884 \\
.885\end{array}$ & $\begin{array}{l}0.16539 \\
0.16521 \\
0.16503 \\
0.16485 \\
0.16467\end{array}$ & $\begin{array}{l}18 \\
18 \\
18 \\
18 \\
18\end{array}$ & $\begin{array}{l}0.33831 \\
0.33809 \\
0.33787 \\
0.33765 \\
0.33743\end{array}$ & $\begin{array}{l}22 \\
22 \\
22 \\
22 \\
22\end{array}$ & $\begin{array}{l}0.50370 \\
0.50330 \\
0.50290 \\
0.50250 \\
0.50210\end{array}$ & $\begin{array}{l}40 \\
40 \\
40 \\
40 \\
40\end{array}$ & $\begin{array}{l}0.75080 \\
0.75058 \\
0.75036 \\
0.75014 \\
0.74991\end{array}$ \\
\hline $\begin{array}{l}.886 \\
.887 \\
.888 \\
.889 \\
.890\end{array}$ & $\begin{array}{l}0.16449 \\
0.16432 \\
0.16414 \\
0.16396 \\
0.16378\end{array}$ & $\begin{array}{l}17 \\
18 \\
18 \\
18 \\
18\end{array}$ & $\begin{array}{l}0.33721 \\
0.33699 \\
0.33677 \\
0.33655 \\
0.33634\end{array}$ & $\begin{array}{l}22 \\
22 \\
22 \\
21 \\
22\end{array}$ & $\begin{array}{l}0.50170 \\
0.50131 \\
0.50091 \\
0.50051 \\
0.50012\end{array}$ & $\begin{array}{l}39 \\
40 \\
40 \\
39 \\
40\end{array}$ & $\begin{array}{l}0.74969 \\
0.74947 \\
0.74924 \\
0.74902 \\
0.74880\end{array}$ \\
\hline $\begin{array}{l}.891 \\
.892 \\
.893 \\
.894 \\
.895\end{array}$ & $\begin{array}{l}0.16360 \\
0.16343 \\
0.16325 \\
0.16307 \\
0.16289\end{array}$ & $\begin{array}{l}17 \\
18 \\
18 \\
18 \\
17\end{array}$ & $\begin{array}{l}0.33612 \\
0.33590 \\
0.33568 \\
0.33546 \\
0.33525\end{array}$ & $\begin{array}{l}22 \\
22 \\
22 \\
21 \\
22\end{array}$ & $\begin{array}{l}0.49972 \\
0.49933 \\
0.49893 \\
0.49854 \\
0.49814\end{array}$ & $\begin{array}{l}39 \\
40 \\
39 \\
40 \\
39\end{array}$ & $\begin{array}{l}0.74858 \\
0.74835 \\
0.74813 \\
0.74791 \\
0.74768\end{array}$ \\
\hline $\begin{array}{l}1.896 \\
1.897 \\
1.898 \\
1.899 \\
1.900\end{array}$ & $\begin{array}{l}0.16272 \\
0.16254 \\
0.16236 \\
0.16219 \\
0.16201\end{array}$ & $\begin{array}{l}18 \\
18 \\
17 \\
18\end{array}$ & $\begin{array}{l}0.33503 \\
0.33481 \\
0.33459 \\
0.33438 \\
0.33416\end{array}$ & $\begin{array}{l}22 \\
22 \\
21 \\
22\end{array}$ & $\begin{array}{l}0.49775 \\
0.49735 \\
0.4965 t \\
0.4965 t \\
0.49617\end{array}$ & $\begin{array}{l}40 \\
39 \\
40 \\
39\end{array}$ & $\begin{array}{l}0.74746 \\
0.74724 \\
0.74701 \\
0.74679 \\
0.74657\end{array}$ \\
\hline
\end{tabular}


Table I Harmonic Oscillator Contributions (in dimensionless form) to the

Thermodynamic Functions for Values of $\mathrm{X}=\mathrm{hc} v / \mathrm{kT}$

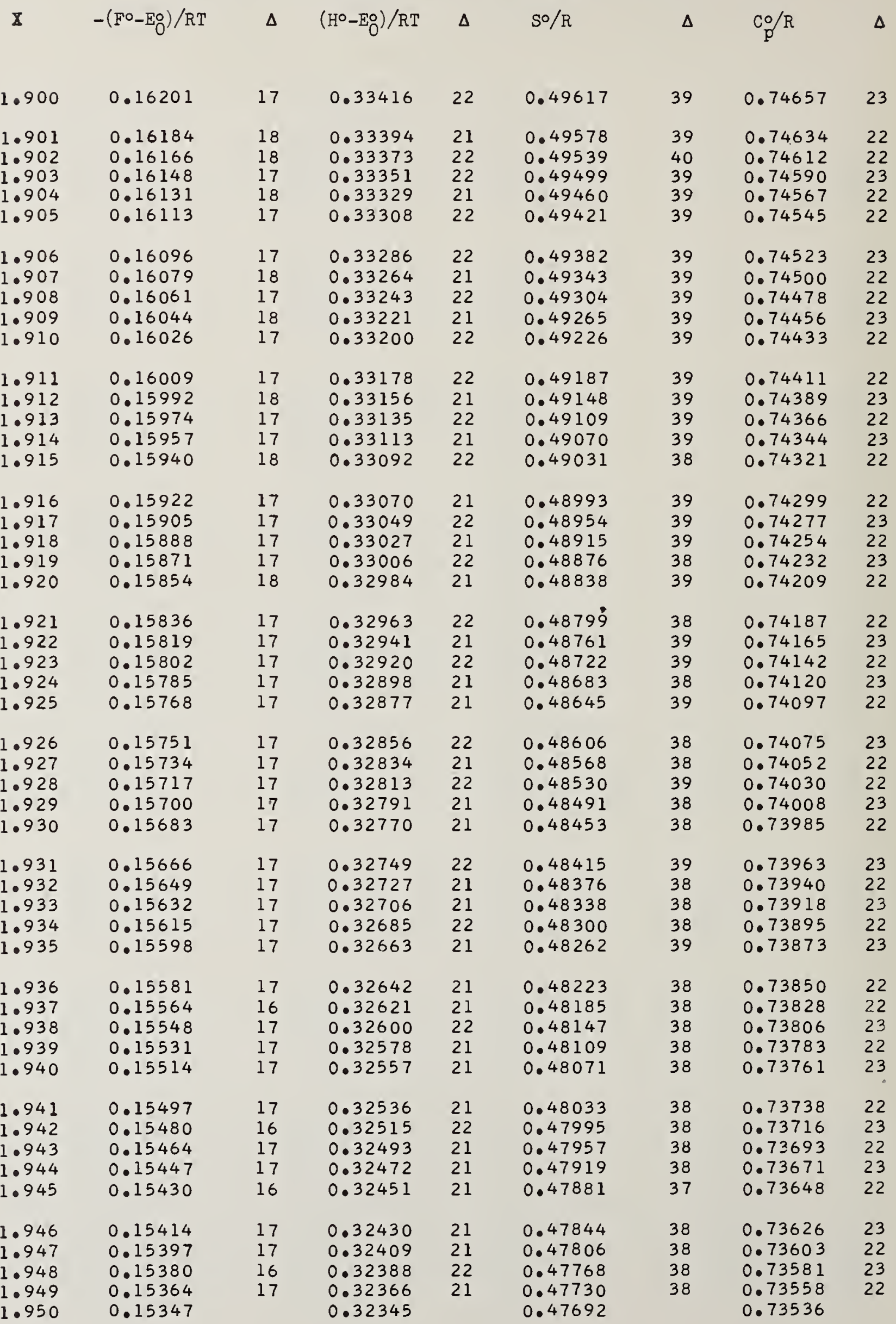


Table I Harmonic Oscillator Contributions (in dimensionless form) to the Thermodynamic Functions for Values of $\mathrm{X}=\mathrm{hcv} / \mathrm{kT}$

\begin{tabular}{|c|c|c|c|c|c|c|c|}
\hline $\mathbf{X}$ & $-\left(F^{\circ}-E_{O}^{\circ}\right) / R T$ & $\Delta$ & $\left(\mathrm{H}^{\circ}-\mathrm{E}_{\mathrm{O}}^{\circ}\right) / \mathrm{RT}$ & $\Delta$ & $\mathrm{S} / \mathrm{R}$ & $\Delta$ & $\mathrm{Co} / \mathrm{R}$ \\
\hline 950 & 0.15347 & 17 & .0 .32345 & 21 & 0.47692 & 37 & 0.73536 \\
\hline $\begin{array}{l}.951 \\
.952 \\
.953 \\
.954 \\
.955\end{array}$ & $\begin{array}{l}0.15330 \\
0.15314 \\
0.15297 \\
0.15281 \\
0.15264\end{array}$ & $\begin{array}{l}16 \\
17 \\
16 \\
17 \\
16\end{array}$ & $\begin{array}{l}0.32324 \\
0.32303 \\
0.32282 \\
0.32261 \\
0.32240\end{array}$ & $\begin{array}{ll}2 & 1 \\
2 & 1 \\
2 & 1 \\
2 & 1 \\
2 & 1\end{array}$ & $\begin{array}{l}0.47655 \\
0.47617 \\
0.47579 \\
0.47542 \\
0.47504\end{array}$ & $\begin{array}{l}38 \\
38 \\
37 \\
38 \\
37\end{array}$ & $\begin{array}{l}0.73513 \\
0.73491 \\
0.73468 \\
0.73446 \\
0.73423\end{array}$ \\
\hline $\begin{array}{r}.956 \\
.957 \\
.958 \\
.959 \\
.960\end{array}$ & $\begin{array}{l}0.15248 \\
0.15231 \\
0.15215 \\
0.15199 \\
0.15182\end{array}$ & $\begin{array}{l}17 \\
16 \\
16 \\
17 \\
16\end{array}$ & $\begin{array}{l}0.32219 \\
0.32198 \\
0.32177 \\
0.32156 \\
0.32135\end{array}$ & $\begin{array}{l}21 \\
21 \\
21 \\
21 \\
21\end{array}$ & $\begin{array}{l}0.47467 \\
0.47429 \\
0.47392 \\
0.47354 \\
0.47317\end{array}$ & $\begin{array}{l}38 \\
37 \\
38 \\
37 \\
38\end{array}$ & $\begin{array}{l}0.73401 \\
0.73378 \\
0.73355 \\
0.73333 \\
0.73310\end{array}$ \\
\hline $\begin{array}{l}.961 \\
.9662 \\
.963 \\
.964 \\
.965 \\
.965\end{array}$ & $\begin{array}{l}0.15166 \\
0.15149 \\
0.15133 \\
0.15117 \\
0.15100\end{array}$ & $\begin{array}{l}17 \\
16 \\
16 \\
17 \\
16\end{array}$ & $\begin{array}{l}0.32114 \\
0.32093 \\
0.32072 \\
0.32051 \\
0.32030\end{array}$ & $\begin{array}{l}21 \\
21 \\
21 \\
21 \\
21\end{array}$ & $\begin{array}{l}0.47279 \\
0.47242 \\
0.47205 \\
0.47167 \\
0.47130\end{array}$ & $\begin{array}{l}37 \\
37 \\
38 \\
37 \\
37\end{array}$ & $\begin{array}{l}0.73288 \\
0.73265 \\
0.73243 \\
0.73220 \\
0.73198\end{array}$ \\
\hline $\begin{array}{l}.966 \\
.967 \\
.968 \\
.969 \\
.9670\end{array}$ & $\begin{array}{l}0.15084 \\
0.15068 \\
0.15052 \\
0.15035 \\
0.15019\end{array}$ & $\begin{array}{l}16 \\
16 \\
17 \\
16 \\
16\end{array}$ & $\begin{array}{l}0.32009 \\
0.31988 \\
0.31967 \\
0.31946 \\
0.31925\end{array}$ & $\begin{array}{ll}21 \\
21 \\
21 \\
21 \\
21\end{array}$ & $\begin{array}{l}0.47093 \\
0.47056 \\
0.47019 \\
0.46981 \\
0.46944\end{array}$ & $\begin{array}{l}37 \\
37 \\
38 \\
37 \\
37\end{array}$ & $\begin{array}{l}0.73175 \\
0.73153 \\
0.73130 \\
0.73107 \\
0.73085\end{array}$ \\
\hline $\begin{array}{r}.971 \\
.972 \\
.973 \\
.974 \\
.975\end{array}$ & $\begin{array}{l}0.15003 \\
0.14987 \\
0.14971 \\
0.14954 \\
0.14938\end{array}$ & $\begin{array}{l}16 \\
16 \\
17 \\
16 \\
16\end{array}$ & $\begin{array}{l}0.31904 \\
0.31883 \\
0.31863 \\
0.31842 \\
0.31821\end{array}$ & $\begin{array}{l}21 \\
20 \\
21 \\
21 \\
21\end{array}$ & $\begin{array}{l}0.46907 \\
0.46870 \\
0.46833 \\
0.46796 \\
0.46759\end{array}$ & $\begin{array}{l}37 \\
37 \\
37 \\
37 \\
37\end{array}$ & $\begin{array}{l}0.73062 \\
0.73040 \\
0.73017 \\
0.72994 \\
0.72972\end{array}$ \\
\hline $\begin{array}{r}.976 \\
.977 \\
.978 \\
.979 \\
.979 \\
.980\end{array}$ & $\begin{array}{l}0.14922 \\
0.14906 \\
0.14890 \\
0.14874 \\
0.14858\end{array}$ & $\begin{array}{l}16 \\
16 \\
16 \\
16 \\
16\end{array}$ & $\begin{array}{l}0.31800 \\
0.31779 \\
0.31758 \\
0.31738 \\
0.31717\end{array}$ & $\begin{array}{l}21 \\
21 \\
20 \\
21 \\
21\end{array}$ & $\begin{array}{l}0.46722 \\
0.46685 \\
0.46649 \\
0.46612 \\
0.46575\end{array}$ & $\begin{array}{l}37 \\
36 \\
37 \\
37 \\
37\end{array}$ & $\begin{array}{l}0.72949 \\
0.72927 \\
0.72904 \\
0.72881 \\
0.72859\end{array}$ \\
\hline $\begin{array}{l}.981 \\
.982 \\
.983 \\
.984 \\
.985\end{array}$ & $\begin{array}{l}0.14842 \\
0.14826 \\
0.14810 \\
0.14794 \\
0.14778\end{array}$ & $\begin{array}{l}16 \\
16 \\
16 \\
16 \\
16\end{array}$ & $\begin{array}{l}0.31696 \\
0.31675 \\
0.31655 \\
0.31634 \\
0.31613\end{array}$ & $\begin{array}{l}21 \\
20 \\
21 \\
21 \\
21\end{array}$ & $\begin{array}{l}0.46538 \\
0.46501 \\
0.46465 \\
0.46428 \\
0.46391\end{array}$ & $\begin{array}{l}37 \\
36 \\
37 \\
37 \\
36\end{array}$ & $\begin{array}{l}0.72836 \\
0.72814 \\
0.72791 \\
0.72768 \\
0.72746\end{array}$ \\
\hline $\begin{array}{l}.986 \\
.987 \\
.988 \\
.989 \\
.990\end{array}$ & $\begin{array}{l}0.14762 \\
0.14746 \\
0.14730 \\
0.14715 \\
0.14699\end{array}$ & $\begin{array}{l}16 \\
16 \\
15 \\
16 \\
16\end{array}$ & $\begin{array}{l}0.31592 \\
0.31572 \\
0.31551 \\
0.31530 \\
0.31510\end{array}$ & $\begin{array}{l}20 \\
21 \\
21 \\
20 \\
21\end{array}$ & $\begin{array}{l}0.46355 \\
0.46318 \\
0.46281 \\
0.46245 \\
0.46208\end{array}$ & $\begin{array}{l}37 \\
37 \\
36 \\
37 \\
36\end{array}$ & $\begin{array}{l}0.72723 \\
0.72701 \\
0.72678 \\
0.72655 \\
0.72633\end{array}$ \\
\hline $\begin{array}{l}1.991 \\
1.992 \\
1.993 \\
1.994 \\
1.995\end{array}$ & $\begin{array}{l}0.14683 \\
0.14667 \\
0.14651 \\
0.14636 \\
0.14620\end{array}$ & $\begin{array}{l}16 \\
16 \\
15 \\
16 \\
16\end{array}$ & $\begin{array}{l}0.31489 \\
0.31468 \\
0.31448 \\
0.31427 \\
0.31406\end{array}$ & $\begin{array}{l}21 \\
20 \\
21 \\
21 \\
20\end{array}$ & $\begin{array}{l}0.46172 \\
0.46135 \\
0.46099 \\
0.46063 \\
0.46026\end{array}$ & $\begin{array}{l}37 \\
36 \\
36 \\
37 \\
36\end{array}$ & $\begin{array}{l}0.72610 \\
0.72587 \\
0.72565 \\
0.72542 \\
0.72519\end{array}$ \\
\hline $\begin{array}{l}1.996 \\
1.997 \\
1.998 \\
1.999 \\
2.000\end{array}$ & $\begin{array}{l}0.14604 \\
0.14588 \\
0.14573 \\
0.14557 \\
0.14541\end{array}$ & $\begin{array}{l}16 \\
15 \\
15 \\
16\end{array}$ & $\begin{array}{l}0.31386 \\
0.31365 \\
0.31345 \\
0.31324 \\
0.31304\end{array}$ & $\begin{array}{l}21 \\
20 \\
21 \\
20\end{array}$ & $\begin{array}{l}0.45990 \\
0.45954 \\
0.45917 \\
0.45881 \\
0.45845\end{array}$ & $\begin{array}{l}36 \\
37 \\
36 \\
36\end{array}$ & $\begin{array}{l}0.72497 \\
0.72474 \\
0.72451 \\
0.72429 \\
0.72406\end{array}$ \\
\hline
\end{tabular}


Table I Harmonic Oscillator Contributions (in dimensionless form) to the

Thermodynamic Functions for Values of $\mathrm{X}=\mathrm{hc} v / \mathrm{kT}$

I

$$
-\left(F^{\circ}-E_{O} \circ\right) / R T
$$

$\Delta \quad\left(\mathrm{H}^{\circ}-\mathrm{E}_{\mathrm{O}}^{\circ}\right) / \mathrm{RT}$

$\Delta$

So/R

0.31304

0.14541

2.000

2.002

2.003

2.004

2.005

2.006

2.007

2.008

2.009

2.010

2.011

2.012

2.013

2.014

2.015

2.016

2.017

2.018

2.019

2.020

2.021

2.022

2.023

2.024

2.025

2.026

2.027

2.028

2.029

2.030

2.031

2.032

2.033

2.034

2.035

2.036

2.037

2.038

2.039

2.040

2.041

2.042

2.043

2.044

2.045

2.046

2.047

2.048

2.049

2.050
0.14526

0.14510

0.14494

0.14479

0.14463

0.14448

0.14432

0.14417

0.14401

0.14386

0.14370

0.14355

0.14339

0.14324

0.14309

0.14293

0.14278

0.14263

0.14247

0.14232

0.14217

0.14201

0.14186

0.14171

0.14156

0.14140

0.14125

0.14110

0.14095

0.14080

0.14065

0.14050

0.14035

0.14019

0.14004

0.13989

0.13974

0.13959

0.13944

0.13930

0.13915

0.13900

0.13885

0.13870

0.13855

0.13840

0.13825

0.13810

0.13796

0.13781

$\begin{array}{ll}15 & 0.31304 \\ 16 & 0.31283 \\ 16 & 0.31262 \\ 15 & 0.31242 \\ 16 & 0.31221 \\ 15 & 0.31201 \\ & \\ 16 & 0.31180 \\ 15 & 0.31160 \\ 16 & 0.31139 \\ 15 & 0.31119 \\ 16 & 0.31099\end{array}$

21

$15 \quad 0.31078$

16

15

15

16

15

15

16

15

15

16

15

15

15

16

15

15

15

15

15

15

15

16

15

15

0.31058

0.31037

0.31017

0.30997

21

20

0.30976

0.30956

0.30935

0.30915

0.30895

0.30874

0.30854

0.30834

0.30814

0.30793

0.30773

0.30753

0.30733

0.30712

0.30692

0.30672

0.30652

0.30631

0.30611

0.30591

15

15

15

14

15

0.30571

0.30551

0.30531

0.30511

0.30490

15

15

15

15

15

0.30470

0.30450

0.30430

0.30410

0.30390

0.30370

0.30350

0.30330

0.30310

0.30290
0.45845

$\Delta$

$\mathrm{Co} / \mathrm{R}$

$\Delta$

0.45809

0.45773

0.45736

0.45700

0.45664

0.45628

0.45592

0.45556

0.45520

0.45484

0.45448

0.45413

0.45377

0.45341

0.45305

0.45269

0.45234

0.45198

0.45162

0.45127

0.45091

0.45055

0.45020

0.44984

0.44949

0.44913

0.44878

0.44843

0.44807

0.44772

0.44737

0.44701

0.44666

0.44631

0.44596

0.44560

0.44525

0.44490

0.44455

0.44420

0.44385

0.44350

0.44315

0.44280

0.44245

0.44210
0.44175

0.44141

0.44106

0.44071
$36 \quad 0.72406$

23

36

0.72383

0.72361

0.72338

0.72315

0.72293

22

23

23

22

23

$\begin{array}{lll}36 & 0.72270 \quad 23\end{array}$

$\begin{array}{lll}36 & 0.72247 & 22\end{array}$

36

36

0.72225

0.72202

0.72179

23

23

22

\section{5}

0.72157

0.72134

0.72111

0.72089

0.72066

23

23

22

23

23

35

0.72043

0.72020

0.71998

0.71975

0.71952

23

22

23

23

22

$0.71930 \quad 23$

$0.71907 \quad 23$ 
Table I Harmonic Oscillator Contributions (in dimensionless form) to the

Thermodynamic Functions for Values of $X=h c v / k T$

\begin{tabular}{|c|c|c|c|c|c|c|c|c|}
\hline $\mathbf{X}$ & $-\left(F^{\circ}-E_{O}\right) / R T$ & $\Delta$ & $\left(H^{\circ}-E_{O}^{\circ}\right) / R T$ & $\Delta$ & So/R & $\Delta$ & $C \stackrel{p}{R}$ & $\Delta$ \\
\hline 2.050 & 0.13781 & 15 & 0.30290 & 20 & 0.44071 & 35 & 0.71269 & 22 \\
\hline $\begin{array}{l}2.051 \\
2.052 \\
2.053 \\
2.054 \\
2.055\end{array}$ & $\begin{array}{l}0.13766 \\
0.13751 \\
0.13737 \\
0.13722 \\
0.13707\end{array}$ & $\begin{array}{l}15 \\
14 \\
15 \\
15 \\
14\end{array}$ & $\begin{array}{l}0.30270 \\
0.30250 \\
0.30230 \\
0.30210 \\
0.30190\end{array}$ & $\begin{array}{l}20 \\
20 \\
20 \\
20 \\
20\end{array}$ & $\begin{array}{l}0.44036 \\
0.44001 \\
0.43967 \\
0.43932 \\
0.43897\end{array}$ & $\begin{array}{l}35 \\
34 \\
35 \\
35 \\
34\end{array}$ & $\begin{array}{l}0.71247 \\
0.71224 \\
0.71201 \\
0.71178 \\
0.71155\end{array}$ & $\begin{array}{l}23 \\
23 \\
23 \\
23 \\
22\end{array}$ \\
\hline $\begin{array}{l}2.056 \\
2.057 \\
2.058 \\
2.059 \\
2.060\end{array}$ & $\begin{array}{l}0.13693 \\
0.13678 \\
0.13663 \\
0.13649 \\
0.13634\end{array}$ & $\begin{array}{l}15 \\
15 \\
14 \\
15 \\
15\end{array}$ & $\begin{array}{l}0.30170 \\
0.30150 \\
0.30130 \\
0.30111 \\
0.30091\end{array}$ & $\begin{array}{l}20 \\
20 \\
19 \\
20 \\
20\end{array}$ & $\begin{array}{l}0.43863 \\
0.43828 \\
0.43794 \\
0.43759 \\
0.43725\end{array}$ & $\begin{array}{l}35 \\
34 \\
35 \\
34 \\
35\end{array}$ & $\begin{array}{l}0.71133 \\
0.71110 \\
0.71087 \\
0.71064 \\
0.71041\end{array}$ & $\begin{array}{l}23 \\
23 \\
23 \\
23 \\
22\end{array}$ \\
\hline $\begin{array}{l}2.061 \\
2.062 \\
2.063 \\
2.064 \\
2.065\end{array}$ & $\begin{array}{l}0.13619 \\
0.13605 \\
0.13590 \\
0.13576 \\
0.13561\end{array}$ & $\begin{array}{l}14 \\
15 \\
14 \\
15 \\
14\end{array}$ & $\begin{array}{l}0.30071 \\
0.30051 \\
0.30031 \\
0.30011 \\
0.29991\end{array}$ & $\begin{array}{l}20 \\
20 \\
20 \\
20 \\
19\end{array}$ & $\begin{array}{l}0.43690 \\
0.43656 \\
0.43621 \\
0.43587 \\
0.43553\end{array}$ & $\begin{array}{l}34 \\
35 \\
34 \\
34 \\
35\end{array}$ & $\begin{array}{l}0.71019 \\
0.70996 \\
0.70973 \\
0.70950 \\
0.70927\end{array}$ & $\begin{array}{l}23 \\
23 \\
23 \\
23 \\
23\end{array}$ \\
\hline $\begin{array}{l}2.066 \\
2.067 \\
2.068 \\
2.069 \\
2.070\end{array}$ & $\begin{array}{l}0.13547 \\
0.13532 \\
0.13518 \\
0.13503 \\
0.13489\end{array}$ & $\begin{array}{l}15 \\
14 \\
15 \\
14 \\
15\end{array}$ & $\begin{array}{l}0.29972 \\
0.29952 \\
0.29932 \\
0.29912 \\
0.29892\end{array}$ & $\begin{array}{l}20 \\
20 \\
20 \\
20 \\
19\end{array}$ & $\begin{array}{l}0.43518 \\
0.43484 \\
0.43450 \\
0.43415 \\
0.43381\end{array}$ & $\begin{array}{l}34 \\
34 \\
35 \\
34 \\
34\end{array}$ & $\begin{array}{l}0.70904 \\
0.70882 \\
0.70859 \\
0.70836 \\
0.70813\end{array}$ & $\begin{array}{l}22 \\
23 \\
23 \\
23 \\
23\end{array}$ \\
\hline $\begin{array}{l}2.071 \\
2.072 \\
2.073 \\
2.074 \\
2.075\end{array}$ & $\begin{array}{l}0.13474 \\
0.13460 \\
0.13446 \\
0.13431 \\
0.13417\end{array}$ & $\begin{array}{l}14 \\
14 \\
15 \\
14 \\
15\end{array}$ & $\begin{array}{l}0.29873 \\
0.29853 \\
0.29833 \\
0.29813 \\
0.29794\end{array}$ & $\begin{array}{l}20 \\
20 \\
20 \\
19 \\
20\end{array}$ & $\begin{array}{l}0.43347 \\
0.43313 \\
0.43279 \\
0.43245 \\
0.43211\end{array}$ & $\begin{array}{l}34 \\
34 \\
34 \\
34 \\
35\end{array}$ & $\begin{array}{l}0.70790 \\
0.70767 \\
0.70744 \\
0.70722 \\
0.70699\end{array}$ & $\begin{array}{l}23 \\
23 \\
22 \\
23 \\
23\end{array}$ \\
\hline $\begin{array}{l}2.076 \\
2.077 \\
2.078 \\
2.079 \\
2.080\end{array}$ & $\begin{array}{l}0.13402 \\
0.13388 \\
0.13374 \\
0.13359 \\
0.13345\end{array}$ & $\begin{array}{l}14 \\
14 \\
15 \\
14 \\
14\end{array}$ & $\begin{array}{l}0.29774 \\
0.29754 \\
0.29735 \\
0.29715 \\
0.29695\end{array}$ & $\begin{array}{l}20 \\
19 \\
20 \\
20 \\
19\end{array}$ & $\begin{array}{l}0.43176 \\
0.43142 \\
0.43108 \\
0.43074 \\
0.43040\end{array}$ & $\begin{array}{l}34 \\
34 \\
34 \\
34 \\
33\end{array}$ & $\begin{array}{l}0.70676 \\
0.70653 \\
0.70630 \\
0.70607 \\
0.70584\end{array}$ & $\begin{array}{l}23 \\
23 \\
23 \\
23 \\
22\end{array}$ \\
\hline $\begin{array}{l}2.081 \\
2.082 \\
2.083 \\
2.084 \\
2.085\end{array}$ & $\begin{array}{l}0.13331 \\
0.13317 \\
0.13302 \\
0.13288 \\
0.13274\end{array}$ & $\begin{array}{l}14 \\
15 \\
14 \\
14 \\
14\end{array}$ & $\begin{array}{l}0.29676 \\
0.29656 \\
0.29636 \\
0.29617 \\
0.29597\end{array}$ & $\begin{array}{l}20 \\
20 \\
19 \\
20 \\
19\end{array}$ & $\begin{array}{l}0.43007 \\
0.42973 \\
0.42939 \\
0.42905 \\
0.42871\end{array}$ & $\begin{array}{l}34 \\
34 \\
34 \\
34 \\
34\end{array}$ & $\begin{array}{l}0.70562 \\
0.70539 \\
0.70516 \\
0.70493 \\
0.70470\end{array}$ & $\begin{array}{l}23 \\
23 \\
23 \\
23 \\
23\end{array}$ \\
\hline $\begin{array}{l}2.086 \\
2.087 \\
2.088 \\
2.089 \\
2.090\end{array}$ & $\begin{array}{l}0.13260 \\
0.13246 \\
0.13231 \\
0.13217 \\
0.13203\end{array}$ & $\begin{array}{l}14 \\
15 \\
14 \\
14 \\
14\end{array}$ & $\begin{array}{l}0.29578 \\
0.29558 \\
0.29538 \\
0.29519 \\
0.29499\end{array}$ & $\begin{array}{l}20 \\
20 \\
19 \\
20 \\
19\end{array}$ & $\begin{array}{l}0.42837 \\
0.42804 \\
0.42770 \\
0.42736 \\
0.42703\end{array}$ & $\begin{array}{l}33 \\
34 \\
34 \\
33 \\
34\end{array}$ & $\begin{array}{l}0.70447 \\
0.70424 \\
0.70401 \\
0.70378 \\
0.70356\end{array}$ & $\begin{array}{l}23 \\
23 \\
23 \\
22 \\
23\end{array}$ \\
\hline $\begin{array}{l}2.091 \\
2.092 \\
2.093 \\
2.094 \\
2.095\end{array}$ & $\begin{array}{l}0.13189 \\
0.13175 \\
0.13161 \\
0.13147 \\
0.13133\end{array}$ & $\begin{array}{l}14 \\
14 \\
14 \\
14 \\
14\end{array}$ & $\begin{array}{l}0.29480 \\
0.29460 \\
0.29441 \\
0.29421 \\
0.29402\end{array}$ & $\begin{array}{l}20 \\
19 \\
20 \\
19 \\
20\end{array}$ & $\begin{array}{l}0.42669 \\
0.42635 \\
0.42602 \\
0.42568 \\
0.42535\end{array}$ & $\begin{array}{l}34 \\
33 \\
34 \\
33 \\
34\end{array}$ & $\begin{array}{l}0.70333 \\
0.70310 \\
0.70287 \\
0.70264 \\
0.70241\end{array}$ & $\begin{array}{l}23 \\
23 \\
23 \\
23 \\
23\end{array}$ \\
\hline $\begin{array}{l}2.096 \\
2.097 \\
2.098 \\
2.099 \\
2.100\end{array}$ & $\begin{array}{l}0.13119 \\
0.13105 \\
0.13091 \\
0.13077 \\
0.13063\end{array}$ & $\begin{array}{l}14 \\
14 \\
14 \\
14\end{array}$ & $\begin{array}{l}0.29382 \\
0.29363 \\
0.29343 \\
0.29324 \\
0.29304\end{array}$ & $\begin{array}{l}19 \\
20 \\
19 \\
20\end{array}$ & $\begin{array}{l}0.42501 \\
0.42468 \\
0.42434 \\
0.42401 \\
0.42367\end{array}$ & $\begin{array}{l}33 \\
34 \\
33 \\
34\end{array}$ & $\begin{array}{l}0.70218 \\
0.70195 \\
0.70172 \\
0.70150 \\
0.70127\end{array}$ & $\begin{array}{l}23 \\
23 \\
22 \\
23\end{array}$ \\
\hline
\end{tabular}


Table I Harmonic Oscillator Contributions (in dimensionless form) to the Thermodynamic Functions for Values of $\mathrm{X}=\mathrm{hcv} / \mathrm{kT}$

\begin{tabular}{|c|c|c|c|c|c|c|c|}
\hline $\mathbf{I}$ & $-\left(F^{\circ}-E_{0}^{O}\right) / R T$ & $\Delta$ & $\left(\mathrm{H}^{\circ}-\mathrm{E}_{\mathrm{O}} \mathrm{O}\right) / \mathrm{RT}$ & $\Delta$ & $\mathrm{S} / \mathrm{R}$ & $\Delta$ & $\mathrm{C} / \mathrm{p}$ \\
\hline 2.100 & 0.13063 & 14 & 0.29304 & 19 & 0.42367 & 33 & 0.70127 \\
\hline $\begin{array}{l}2.101 \\
2.102 \\
2.103 \\
2.104 \\
2.105\end{array}$ & $\begin{array}{l}0.13049 \\
0.13035 \\
0.13021 \\
0.13007 \\
0.12993\end{array}$ & $\begin{array}{l}14 \\
14 \\
14 \\
14 \\
14\end{array}$ & $\begin{array}{l}0.29285 \\
0.29265 \\
0.29246 \\
0.29227 \\
0.29207\end{array}$ & $\begin{array}{l}20 \\
19 \\
19 \\
20 \\
19\end{array}$ & $\begin{array}{l}0.42334 \\
0.42300 \\
0.42267 \\
0.42234 \\
0.42201\end{array}$ & $\begin{array}{l}34 \\
33 \\
33 \\
33 \\
34\end{array}$ & $\begin{array}{l}0.70104 \\
0.70081 \\
0.70058 \\
0.70035 \\
0.70012\end{array}$ \\
\hline $\begin{array}{l}2.106 \\
2.107 \\
2.108 \\
2.109 \\
2.110\end{array}$ & $\begin{array}{l}0.12979 \\
0.12966 \\
0.12952 \\
0.12938 \\
0.12924\end{array}$ & $\begin{array}{l}13 \\
14 \\
14 \\
14 \\
14\end{array}$ & $\begin{array}{l}0.29188 \\
0.29169 \\
0.29149 \\
0.29130 \\
0.29111\end{array}$ & $\begin{array}{l}19 \\
20 \\
19 \\
19 \\
20\end{array}$ & $\begin{array}{l}0.42167 \\
0.42134 \\
0.42101 \\
0.42068 \\
0.42035\end{array}$ & $\begin{array}{l}33 \\
33 \\
33 \\
33 \\
33\end{array}$ & $\begin{array}{l}0.69989 \\
0.69966 \\
0.69943 \\
0.69920 \\
0.69897\end{array}$ \\
\hline $\begin{array}{l}2.111 \\
2.112 \\
2.113 \\
2.114 \\
2.115\end{array}$ & $\begin{array}{l}0.12910 \\
0.12897 \\
0.12883 \\
0.12869 \\
0.12855\end{array}$ & $\begin{array}{l}13 \\
14 \\
14 \\
14 \\
13\end{array}$ & $\begin{array}{l}0.29091 \\
0.29072 \\
0.29053 \\
0.29033 \\
0.29014\end{array}$ & $\begin{array}{l}19 \\
19 \\
20 \\
19 \\
19\end{array}$ & $\begin{array}{l}0.42002 \\
0.41968 \\
0.41935 \\
0.41902 \\
0.41869\end{array}$ & $\begin{array}{l}34 \\
33 \\
33 \\
33 \\
33\end{array}$ & $\begin{array}{l}0.69874 \\
0.69852 \\
0.69829 \\
0.69806 \\
0.69783\end{array}$ \\
\hline $\begin{array}{l}2.116 \\
2.117 \\
2.118 \\
2.119 \\
2.120\end{array}$ & $\begin{array}{l}0.12842 \\
0.12828 \\
0.12814 \\
0.12801 \\
0.12787\end{array}$ & $\begin{array}{l}14 \\
14 \\
13 \\
14 \\
14\end{array}$ & $\begin{array}{l}0.28995 \\
0.28975 \\
0.28956 \\
0.28937 \\
0.28918\end{array}$ & $\begin{array}{l}20 \\
19 \\
19 \\
19 \\
19\end{array}$ & $\begin{array}{l}0.41836 \\
0.41803 \\
0.41770 \\
0.41738 \\
0.41705\end{array}$ & $\begin{array}{l}33 \\
33 \\
32 \\
33 \\
33\end{array}$ & $\begin{array}{l}0.69760 \\
0.69737 \\
0.69714 \\
0.69691 \\
0.69668\end{array}$ \\
\hline $\begin{array}{l}2.121 \\
2.122 \\
2.123 \\
2.124 \\
2.125\end{array}$ & $\begin{array}{l}0.12773 \\
0.12760 \\
0.12746 \\
0.12732 \\
0.12719\end{array}$ & $\begin{array}{l}13 \\
14 \\
14 \\
13 \\
14\end{array}$ & $\begin{array}{l}0.28899 \\
0.28879 \\
0.28860 \\
0.28841 \\
0.28822\end{array}$ & $\begin{array}{l}20 \\
19 \\
19 \\
19 \\
19\end{array}$ & $\begin{array}{l}0.41672 \\
0.41639 \\
0.41606 \\
0.41573 \\
0.41541\end{array}$ & $\begin{array}{l}33 \\
33 \\
33 \\
32 \\
33\end{array}$ & $\begin{array}{l}0.69645 \\
0.69622 \\
0.69599 \\
0.69576 \\
0.69553\end{array}$ \\
\hline $\begin{array}{l}2 \cdot 126 \\
2 \cdot 127 \\
2 \cdot 128 \\
2.129 \\
2 \cdot 130\end{array}$ & $\begin{array}{l}0.12705 \\
0.12692 \\
0.12678 \\
0.12665 \\
0.12651\end{array}$ & $\begin{array}{l}13 \\
14 \\
13 \\
14 \\
13\end{array}$ & $\begin{array}{l}0.28803 \\
0.28783 \\
0.28764 \\
0.28745 \\
0.28726\end{array}$ & $\begin{array}{l}20 \\
19 \\
19 \\
19 \\
19\end{array}$ & $\begin{array}{l}0.41508 \\
0.41475 \\
0.41443 \\
0.41410 \\
0.41377\end{array}$ & $\begin{array}{l}33 \\
32 \\
33 \\
33 \\
32\end{array}$ & $\begin{array}{l}0.69530 \\
0.69507 \\
0.69484 \\
0.69461 \\
0.69438\end{array}$ \\
\hline $\begin{array}{l}2.131 \\
2.132 \\
2.133 \\
2.134 \\
2.135\end{array}$ & $\begin{array}{l}0.12638 \\
0.12624 \\
0.12611 \\
0.12597 \\
0.12584\end{array}$ & $\begin{array}{l}14 \\
13 \\
14 \\
13 \\
13\end{array}$ & $\begin{array}{l}0.28707 \\
0.28688 \\
0.28669 \\
0.28650 \\
0.28631\end{array}$ & $\begin{array}{l}19 \\
19 \\
19 \\
19 \\
19\end{array}$ & $\begin{array}{l}0.41345 \\
0.41312 \\
0.41280 \\
0.41247 \\
0.41215\end{array}$ & $\begin{array}{l}33 \\
32 \\
33 \\
32 \\
33\end{array}$ & $\begin{array}{l}0.69415 \\
0.69392 \\
0.69369 \\
0.69347 \\
0.69324\end{array}$ \\
\hline $\begin{array}{l}2.136 \\
2.137 \\
2.138 \\
2.139 \\
2.140\end{array}$ & $\begin{array}{l}0.12571 \\
0.12557 \\
0.12544 \\
0.12531 \\
0.12517\end{array}$ & $\begin{array}{l}14 \\
13 \\
13 \\
14 \\
13\end{array}$ & $\begin{array}{l}0.28612 \\
0.28593 \\
0.28574 \\
0.28554 \\
0.28535\end{array}$ & $\begin{array}{l}19 \\
19 \\
20 \\
19 \\
19\end{array}$ & $\begin{array}{l}0.41182 \\
0.41150 \\
0.41117 \\
0.41085 \\
0.41053\end{array}$ & $\begin{array}{l}32 \\
33 \\
32 \\
32 \\
33\end{array}$ & $\begin{array}{l}0.69301 \\
0.69278 \\
0.69255 \\
0.69232 \\
0.69209\end{array}$ \\
\hline $\begin{array}{l}2.141 \\
2.142 \\
2.143 \\
2.144 \\
2.145\end{array}$ & $\begin{array}{l}0.12504 \\
0.12491 \\
0.12477 \\
0.12464 \\
0.12451\end{array}$ & $\begin{array}{l}13 \\
14 \\
13 \\
13 \\
14\end{array}$ & $\begin{array}{l}0.28516 \\
0.28497 \\
0.28479 \\
0.28460 \\
0.28441\end{array}$ & $\begin{array}{l}19 \\
18 \\
19 \\
19 \\
19\end{array}$ & $\begin{array}{l}0.41020 \\
0.40988 \\
0.40956 \\
0.40924 \\
0.40891\end{array}$ & $\begin{array}{l}32 \\
32 \\
32 \\
33 \\
32\end{array}$ & $\begin{array}{l}0.69186 \\
0.69163 \\
0.69140 \\
0.69117 \\
0.69094\end{array}$ \\
\hline $\begin{array}{l}2.146 \\
2.147 \\
2.148 \\
2.149 \\
2.150\end{array}$ & $\begin{array}{l}0.12437 \\
0.12424 \\
0.12411 \\
0.12398 \\
0.12385\end{array}$ & $\begin{array}{l}13 \\
13 \\
13 \\
13\end{array}$ & $\begin{array}{l}0.28422 \\
0.28403 \\
0.28384 \\
0.28365 \\
0.28346\end{array}$ & $\begin{array}{l}19 \\
19 \\
19 \\
19\end{array}$ & $\begin{array}{l}0.40859 \\
0.40827 \\
0.40795 \\
0.40763 \\
0.40731\end{array}$ & $\begin{array}{l}32 \\
32 \\
32 \\
32\end{array}$ & $\begin{array}{l}0.69071 \\
0.69048 \\
0.69025 \\
0.69002 \\
0.68979\end{array}$ \\
\hline
\end{tabular}


Table I Harmonic Oscillator Contributions (in dimensionless form) to the

Thermodynamic Functions for Values of $\mathrm{X}=\mathrm{hc} / \mathrm{kT}$

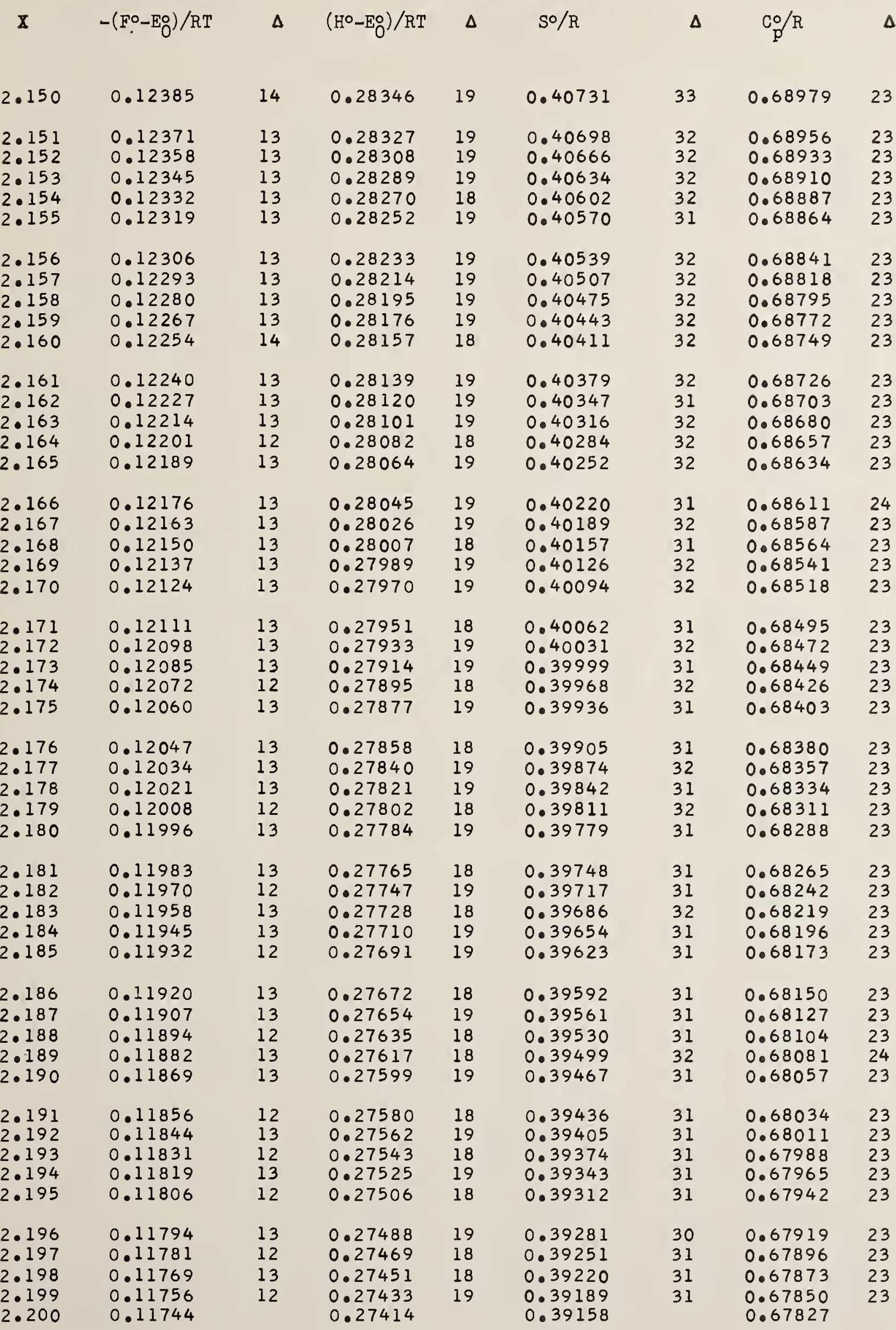


Table I Harmonic Oscillator Contributions (in dimensionless form) to the

Thermodynamic Functions for Values of $X=h c v / k T$

$\begin{array}{llllllll} & -\left(\mathrm{F}^{\circ}-\mathrm{E}_{\mathrm{O}}^{\circ}\right) / \mathrm{RT} & \Delta & \left(\mathrm{H}^{\circ}-\mathrm{E}_{\mathrm{O}}^{\circ}\right) / \mathrm{RT} & \Delta & \mathrm{S} / \mathrm{R} & \Delta \quad \mathrm{CO} / \mathrm{R}\end{array}$

\begin{tabular}{|c|c|c|c|c|c|c|c|}
\hline 2.200 & 0.11744 & 13 & 0.27414 & 18 & 0.39158 & 31 & 0.67827 \\
\hline $\begin{array}{l}2.201 \\
2.202 \\
2.203 \\
2.204 \\
2.205\end{array}$ & $\begin{array}{l}0.11731 \\
0.11719 \\
0.11706 \\
0.11694 \\
0.11682\end{array}$ & $\begin{array}{l}12 \\
13 \\
12 \\
12 \\
13\end{array}$ & $\begin{array}{l}0.27396 \\
0.27378 \\
0.27359 \\
0.27341 \\
0.27323\end{array}$ & $\begin{array}{l}18 \\
19 \\
18 \\
18 \\
19\end{array}$ & $\begin{array}{l}0.39127 \\
0.39096 \\
0.39066 \\
0.39035 \\
0.39004\end{array}$ & $\begin{array}{l}31 \\
30 \\
31 \\
31 \\
31\end{array}$ & $\begin{array}{l}0.67804 \\
0.67781 \\
0.67758 \\
0.67735 \\
0.67711\end{array}$ \\
\hline $\begin{array}{l}2.206 \\
2.207 \\
2.208 \\
2.209 \\
2.210\end{array}$ & $\begin{array}{l}0.11669 \\
0.11657 \\
0.11644 \\
0.11632 \\
0.11620\end{array}$ & $\begin{array}{l}12 \\
13 \\
12 \\
12 \\
13\end{array}$ & $\begin{array}{l}0.27304 \\
0.27286 \\
0.27268 \\
0.27249 \\
0.27231\end{array}$ & $\begin{array}{l}18 \\
18 \\
19 \\
18 \\
18\end{array}$ & $\begin{array}{l}0.38973 \\
0.38943 \\
0.38912 \\
0.38881 \\
0.38851\end{array}$ & $\begin{array}{l}30 \\
31 \\
31 \\
30 \\
31\end{array}$ & $\begin{array}{l}0.67688 \\
0.67665 \\
0.67642 \\
0.67619 \\
0.67596\end{array}$ \\
\hline $\begin{array}{l}2.211 \\
2.212 \\
2.213 \\
2.214 \\
2.215\end{array}$ & $\begin{array}{l}0.11607 \\
0.11595 \\
0.11583 \\
0.11571 \\
0.11558\end{array}$ & $\begin{array}{l}12 \\
12 \\
12 \\
13 \\
12\end{array}$ & $\begin{array}{l}0.27213 \\
0.27195 \\
0.27176 \\
0.27158 \\
0.27140\end{array}$ & $\begin{array}{l}18 \\
19 \\
18 \\
18 \\
18\end{array}$ & $\begin{array}{l}0.38820 \\
0.38790 \\
0.38759 \\
0.38729 \\
0.38698\end{array}$ & $\begin{array}{l}30 \\
31 \\
30 \\
31 \\
30\end{array}$ & $\begin{array}{l}0.67573 \\
0.67550 \\
0.67527 \\
0.67504 \\
0.67481\end{array}$ \\
\hline $\begin{array}{l}2.216 \\
2.217 \\
2.218 \\
2.219 \\
2.220\end{array}$ & $\begin{array}{l}0.11546 \\
0.11534 \\
0.11522 \\
0.11509 \\
0.11497\end{array}$ & $\begin{array}{l}12 \\
12 \\
13 \\
12 \\
12\end{array}$ & $\begin{array}{l}0.27122 \\
0.27104 \\
0.27085 \\
0.27067 \\
0.27049\end{array}$ & $\begin{array}{l}18 \\
19 \\
18 \\
18 \\
18\end{array}$ & $\begin{array}{l}0.38668 \\
0.38637 \\
0.38607 \\
0.38577 \\
0.38546\end{array}$ & $\begin{array}{l}31 \\
30 \\
30 \\
31 \\
30\end{array}$ & $\begin{array}{l}0.67458 \\
0.67435 \\
0.67411 \\
0.67388 \\
0.67365\end{array}$ \\
\hline $\begin{array}{l}2.221 \\
2.222 \\
2.223 \\
2.224 \\
2.225\end{array}$ & $\begin{array}{l}0.11485 \\
0.11473 \\
0.11461 \\
0.11449 \\
0.11436\end{array}$ & $\begin{array}{l}12 \\
12 \\
12 \\
13 \\
12\end{array}$ & $\begin{array}{l}0.27031 \\
0.27013 \\
0.26995 \\
0.26976 \\
0.26958\end{array}$ & $\begin{array}{l}18 \\
18 \\
19 \\
18 \\
18\end{array}$ & $\begin{array}{l}0.38516 \\
0.38486 \\
0.38455 \\
0.38425 \\
0.38395\end{array}$ & $\begin{array}{l}30 \\
31 \\
30 \\
30 \\
30\end{array}$ & $\begin{array}{l}0.67342 \\
0.67319 \\
0.67296 \\
0.67273 \\
0.67250\end{array}$ \\
\hline $\begin{array}{l}2.226 \\
2.227 \\
2.228 \\
2.229 \\
2.230\end{array}$ & $\begin{array}{l}0.11424 \\
0.11412 \\
0.11400 \\
0.11388 \\
0.11376\end{array}$ & $\begin{array}{l}12 \\
12 \\
12 \\
12 \\
12\end{array}$ & $\begin{array}{l}0.26940 \\
0.26922 \\
0.26904 \\
0.26886 \\
0.26868\end{array}$ & $\begin{array}{l}18 \\
18 \\
18 \\
18 \\
18\end{array}$ & $\begin{array}{l}0.38365 \\
0.38334 \\
0.38304 \\
0.38274 \\
0.38244\end{array}$ & $\begin{array}{l}31 \\
30 \\
30 \\
30 \\
30\end{array}$ & $\begin{array}{l}0.67227 \\
0.67204 \\
0.67180 \\
0.67157 \\
0.67134\end{array}$ \\
\hline $\begin{array}{l}2.231 \\
2.232 \\
2.233 \\
2.234 \\
2.235\end{array}$ & $\begin{array}{l}0.11364 \\
0.11352 \\
0.11340 \\
0.11328 \\
0.11316\end{array}$ & $\begin{array}{l}12 \\
12 \\
12 \\
12 \\
12\end{array}$ & $\begin{array}{l}0.26850 \\
0.26832 \\
0.26814 \\
0.26796 \\
0.26778\end{array}$ & $\begin{array}{l}18 \\
18 \\
18 \\
18 \\
18\end{array}$ & $\begin{array}{l}0.38214 \\
0.38184 \\
0.38154 \\
0.38124 \\
0.38094\end{array}$ & $\begin{array}{l}30 \\
30 \\
30 \\
30 \\
30\end{array}$ & $\begin{array}{l}0.67111 \\
0.67088 \\
0.67065 \\
0.67042 \\
0.67019\end{array}$ \\
\hline $\begin{array}{l}2.236 \\
2.237 \\
2.238 \\
2.239 \\
2.240\end{array}$ & $\begin{array}{l}0.11304 \\
0.11292 \\
0.11280 \\
0.11268 \\
0.11256\end{array}$ & $\begin{array}{l}12 \\
12 \\
12 \\
12 \\
12\end{array}$ & $\begin{array}{l}0.26760 \\
0.26742 \\
0.26724 \\
0.26706 \\
0.26688\end{array}$ & $\begin{array}{l}18 \\
18 \\
18 \\
18 \\
18\end{array}$ & $\begin{array}{l}0.38064 \\
0.38034 \\
0.38004 \\
0.37974 \\
0.37944\end{array}$ & $\begin{array}{l}30 \\
30 \\
30 \\
30 \\
30\end{array}$ & $\begin{array}{l}0.66996 \\
0.66973 \\
0.66949 \\
0.66926 \\
0.66903\end{array}$ \\
\hline $\begin{array}{l}2.241 \\
2.242 \\
2.243 \\
2.244 \\
2.245\end{array}$ & $\begin{array}{l}0.111244 \\
0.111232 \\
0.111221 \\
0.111209 \\
0.11197\end{array}$ & $\begin{array}{l}12 \\
11 \\
12 \\
12 \\
12\end{array}$ & $\begin{array}{l}0.26670 \\
0.26652 \\
0.26634 \\
0.26616 \\
0.26598\end{array}$ & $\begin{array}{l}18 \\
18 \\
18 \\
18 \\
18\end{array}$ & $\begin{array}{l}0.37914 \\
0.37884 \\
0.37855 \\
0.37825 \\
0.37795\end{array}$ & $\begin{array}{l}30 \\
29 \\
30 \\
30 \\
30\end{array}$ & $\begin{array}{l}0.66880 \\
0.66857 \\
0.66834 \\
0.66811 \\
0.66788\end{array}$ \\
\hline $\begin{array}{l}2.246 \\
2.247 \\
2.248 \\
2.249 \\
2.250\end{array}$ & $\begin{array}{l}0.111185 \\
0.111173 \\
0.111161 \\
0.111150 \\
0.111138\end{array}$ & $\begin{array}{l}12 \\
12 \\
11 \\
12\end{array}$ & $\begin{array}{l}0.26580 \\
0.26562 \\
0.26545 \\
0.26527 \\
0.26509\end{array}$ & $\begin{array}{l}18 \\
17 \\
18 \\
18\end{array}$ & $\begin{array}{l}0.37765 \\
0.37736 \\
0.37706 \\
0.37676 \\
0.37647\end{array}$ & $\begin{array}{l}29 \\
30 \\
30 \\
29\end{array}$ & $\begin{array}{l}0.66765 \\
0.66741 \\
0.66718 \\
0.66695 \\
0.66672\end{array}$ \\
\hline
\end{tabular}


Table I Harmonic Oscillator Contributions (in dimensionless form) to the Thermodynamic Functions for Values of $\mathrm{X}=\mathrm{hcv} / \mathrm{kT}$

\begin{tabular}{|c|c|c|c|c|c|c|c|c|}
\hline $\mathbf{X}$ & $-\left(F^{\circ}-E_{O}^{\circ}\right) / R T$ & $\Delta$ & $\left(\mathrm{H}^{\circ}-\mathrm{E} \mathrm{O}\right) / \mathrm{RT}$ & $\Delta$ & $S \circ / R$ & $\Delta$ & $\mathrm{Co}_{\mathrm{p}} / \mathrm{R}$ & $\Delta$ \\
\hline 2.250 & 0.11138 & 12 & 0.26509 & 18 & 0.37647 & 30 & 0.66672 & 23 \\
\hline $\begin{array}{l}2.251 \\
2.252\end{array}$ & $\begin{array}{l}0.11126 \\
0.11114\end{array}$ & $\begin{array}{l}12 \\
12\end{array}$ & $\begin{array}{l}0.26491 \\
0.26473\end{array}$ & $\begin{array}{l}18 \\
18\end{array}$ & $\begin{array}{l}0.37617 \\
0.37587\end{array}$ & $\begin{array}{l}30 \\
29\end{array}$ & $\begin{array}{l}0.66649 \\
0.66626\end{array}$ & $\begin{array}{l}23 \\
23\end{array}$ \\
\hline $\begin{array}{l}2.253 \\
2.254 \\
2.255\end{array}$ & $\begin{array}{l}0.11102 \\
0.11091 \\
0.11079\end{array}$ & $\begin{array}{l}11 \\
12 \\
12\end{array}$ & $\begin{array}{l}0.26455 \\
0.26438 \\
0.26420\end{array}$ & $\begin{array}{l}17 \\
18 \\
18\end{array}$ & $\begin{array}{l}0.37558 \\
0.37528 \\
0.37499\end{array}$ & $\begin{array}{l}30 \\
29 \\
30\end{array}$ & $\begin{array}{l}0.66603 \\
0.66580 \\
0.66556\end{array}$ & $\begin{array}{l}23 \\
24 \\
23\end{array}$ \\
\hline $\begin{array}{l}2.256 \\
2.257 \\
2.258 \\
2.259 \\
2.260\end{array}$ & $\begin{array}{l}0.11067 \\
0.11056 \\
0.11044 \\
0.11032 \\
0.11021\end{array}$ & $\begin{array}{l}11 \\
12 \\
12 \\
11 \\
12\end{array}$ & $\begin{array}{l}0.26402 \\
0.26384 \\
0.26366 \\
0.26349 \\
0.26331\end{array}$ & $\begin{array}{l}18 \\
18 \\
17 \\
18 \\
18\end{array}$ & $\begin{array}{l}0.37469 \\
0.37440 \\
0.37410 \\
0.37381 \\
0.37351\end{array}$ & $\begin{array}{l}29 \\
30 \\
29 \\
30 \\
29\end{array}$ & $\begin{array}{l}0.66533 \\
0.66510 \\
0.66487 \\
0.66464 \\
0.66441\end{array}$ & $\begin{array}{l}23 \\
23 \\
23 \\
23 \\
23\end{array}$ \\
\hline $\begin{array}{l}2.261 . \\
2.262 \\
2.263 \\
2.264 \\
2.265\end{array}$ & $\begin{array}{l}0.11009 \\
0.10997 \\
0.10986 \\
0.10974 \\
0.10963\end{array}$ & $\begin{array}{l}12 \\
11 \\
12 \\
11 \\
12\end{array}$ & $\begin{array}{l}0.26313 \\
0.26295 \\
0.26278 \\
0.26260 \\
0.26242\end{array}$ & $\begin{array}{l}18 \\
17 \\
18 \\
18 \\
17\end{array}$ & $\begin{array}{l}0.37322 \\
0.37293 \\
0.37263 \\
0.37234 \\
0.37205\end{array}$ & $\begin{array}{l}29 \\
30 \\
29 \\
29 \\
30\end{array}$ & $\begin{array}{l}0.66418 \\
0.66395 \\
0.66371 \\
0.66348 \\
0.66325\end{array}$ & $\begin{array}{l}23 \\
24 \\
23 \\
23 \\
23\end{array}$ \\
\hline $\begin{array}{l}2.266 \\
2.267 \\
2.268 \\
2.269 \\
2.270\end{array}$ & $\begin{array}{l}0.10951 \\
0.10939 \\
0.10928 \\
0.10916 \\
0.10905\end{array}$ & $\begin{array}{l}12 \\
11 \\
12 \\
11 \\
12\end{array}$ & $\begin{array}{l}0.26225 \\
0.26207 \\
0.26189 \\
0.26172 \\
0.26154\end{array}$ & $\begin{array}{l}18 \\
18 \\
17 \\
18 \\
18\end{array}$ & $\begin{array}{l}0.37175 \\
0.37146 \\
0.37117 \\
0.37088 \\
0.37059\end{array}$ & $\begin{array}{l}29 \\
29 \\
29 \\
29 \\
30\end{array}$ & $\begin{array}{l}0.66302 \\
0.66279 \\
0.66256 \\
0.66233 \\
0.66210\end{array}$ & $\begin{array}{l}23 \\
23 \\
23 \\
23 \\
24\end{array}$ \\
\hline $\begin{array}{l}2.271 \\
2.272 \\
2.273 \\
2.274 \\
2.275\end{array}$ & $\begin{array}{l}0.10893 \\
0.10882 \\
0.10870 \\
0.10859 \\
0.10847\end{array}$ & $\begin{array}{l}11 \\
12 \\
11 \\
12 \\
11\end{array}$ & $\begin{array}{l}0.26136 \\
0.26119 \\
0.26101 \\
0.26083 \\
0.26066\end{array}$ & $\begin{array}{l}17 \\
18 \\
18 \\
17 \\
18\end{array}$ & $\begin{array}{l}0.37029 \\
0.37000 \\
0.36971 \\
0.36942 \\
0.36913\end{array}$ & $\begin{array}{l}29 \\
29 \\
29 \\
29 \\
29\end{array}$ & $\begin{array}{l}0.66186 \\
0.66163 \\
0.66140 \\
0.66117 \\
0.66094\end{array}$ & $\begin{array}{l}23 \\
23 \\
23 \\
23 \\
23\end{array}$ \\
\hline $\begin{array}{l}2.276 \\
2.277 \\
2 \cdot 278 \\
2.279 \\
2.280\end{array}$ & $\begin{array}{l}0.10836 \\
0.10824 \\
0.10813 \\
0.10802 \\
0.10790\end{array}$ & $\begin{array}{l}12 \\
11 \\
11 \\
12 \\
11\end{array}$ & $\begin{array}{l}0.26048 \\
0.26031 \\
0.26013 \\
0.25995 \\
0.25978\end{array}$ & $\begin{array}{l}17 \\
18 \\
18 \\
17 \\
18\end{array}$ & $\begin{array}{l}0.36884 \\
0.36855 \\
0.36826 \\
0.36797 \\
0.36768\end{array}$ & $\begin{array}{l}29 \\
29 \\
29 \\
29 \\
29\end{array}$ & $\begin{array}{l}0.66071 \\
0.66048 \\
0.66024 \\
0.66001 \\
0.65978\end{array}$ & $\begin{array}{l}23 \\
24 \\
23 \\
23 \\
23\end{array}$ \\
\hline $\begin{array}{l}2.281 \\
2.282 \\
2.283 \\
2.284 \\
2.285\end{array}$ & $\begin{array}{l}0.10779 \\
0.10767 \\
0.10756 \\
0.10745 \\
0.10733\end{array}$ & $\begin{array}{l}12 \\
11 \\
11 \\
12 \\
11\end{array}$ & $\begin{array}{l}0.25960 \\
0.25943 \\
0.25925 \\
0.25908 \\
0.25890\end{array}$ & $\begin{array}{l}17 \\
18 \\
17 \\
18 \\
17\end{array}$ & $\begin{array}{l}0.36739 \\
0.36710 \\
0.36681 \\
0.36653 \\
0.36624\end{array}$ & $\begin{array}{l}29 \\
29 \\
28 \\
29 \\
29\end{array}$ & $\begin{array}{l}0.65955 \\
0.65932 \\
0.65909 \\
0.65886 \\
0.65863\end{array}$ & $\begin{array}{l}23 \\
23 \\
23 \\
23 \\
24\end{array}$ \\
\hline $\begin{array}{l}2.286 \\
2.287 \\
2.288 \\
2.289 \\
2.290\end{array}$ & $\begin{array}{l}0.10722 \\
0.10711 \\
0.10699 \\
0.10688 \\
0.10677\end{array}$ & $\begin{array}{l}11 \\
12 \\
11 \\
11 \\
11\end{array}$ & $\begin{array}{l}0.25873 \\
0.25855 \\
0.25838 \\
0.25820 \\
0.25803\end{array}$ & $\begin{array}{l}18 \\
17 \\
18 \\
17 \\
17\end{array}$ & $\begin{array}{l}0.36595 \\
0.36566 \\
0.36537 \\
0.36509 \\
0.36480\end{array}$ & $\begin{array}{l}29 \\
29 \\
28 \\
29 \\
29\end{array}$ & $\begin{array}{l}0.65839 \\
0.65816 \\
0.65793 \\
0.65770 \\
0.65747\end{array}$ & $\begin{array}{l}23 \\
23 \\
23 \\
23 \\
23\end{array}$ \\
\hline $\begin{array}{l}2.291 \\
2.292 \\
2.293 \\
2.294 \\
2.295\end{array}$ & $\begin{array}{l}0.10666 \\
0.10654 \\
0.10643 \\
0.10632 \\
0.10621\end{array}$ & $\begin{array}{l}12 \\
11 \\
11 \\
11 \\
12\end{array}$ & $\begin{array}{l}0.25786 \\
0.25768 \\
0.25751 \\
0.25733 \\
0.25716\end{array}$ & $\begin{array}{l}18 \\
17 \\
18 \\
17 \\
17\end{array}$ & $\begin{array}{l}0.36451 \\
0.36422 \\
0.36394 \\
0.36365 \\
0.36337\end{array}$ & $\begin{array}{l}29 \\
28 \\
29 \\
28 \\
29\end{array}$ & $\begin{array}{l}0.65724 \\
0.65701 \\
0.65677 \\
0.65654 \\
0.65631\end{array}$ & $\begin{array}{l}23 \\
24 \\
23 \\
23 \\
23\end{array}$ \\
\hline $\begin{array}{l}2.296 \\
2.297 \\
2.298 \\
2.299 \\
2.300\end{array}$ & $\begin{array}{l}0.10609 \\
0.10598 \\
0.10587 \\
0.10576 \\
0.10565\end{array}$ & $\begin{array}{l}11 \\
11 \\
11 \\
11\end{array}$ & $\begin{array}{l}0.25699 \\
0.25681 \\
0.25664 \\
0.25646 \\
0.25629\end{array}$ & $\begin{array}{l}18 \\
17 \\
18 \\
17\end{array}$ & $\begin{array}{l}0.36308 \\
0.36279 \\
0.36251 \\
0.36222 \\
0.36194\end{array}$ & $\begin{array}{l}29 \\
28 \\
29 \\
28\end{array}$ & $\begin{array}{l}0.65608 \\
0.65585 \\
0.65562 \\
0.65539 \\
0.65515\end{array}$ & $\begin{array}{l}23 \\
23 \\
23 \\
24\end{array}$ \\
\hline
\end{tabular}


Table I Harmonic Oscillator Contributions (in dimensionless form) to the

Thermodynamic Functions for Values of $X=h c v / k T$

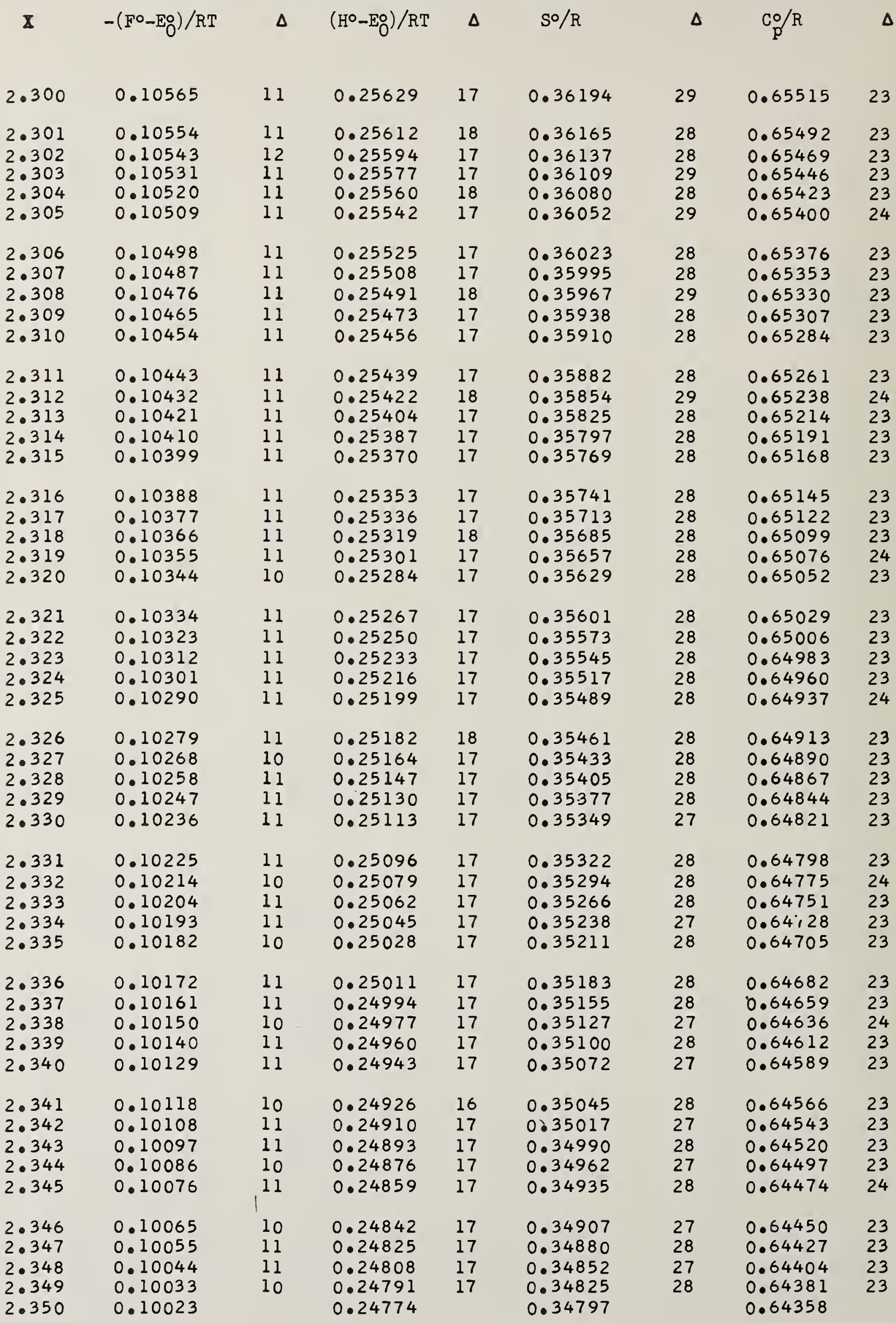


Table I Harmonic Oscillator Contributions (in dimensionless form) to the

Thermodynamic Functions for Values of $X=h c v / k T$

$\begin{array}{llllllll}\mathbf{L} & -\left(\mathrm{F}^{\circ}-\mathrm{E}_{\mathrm{O}}^{\circ}\right) / \mathrm{RT} & \Delta & \left(\mathrm{H}^{\circ}-\mathrm{E}_{\mathrm{O}}^{\circ}\right) / \mathrm{RT} & \Delta & \mathrm{S} \% / \mathrm{R} & \Delta \quad \mathrm{C}_{\mathrm{p}}^{\circ} / \mathrm{R}\end{array}$

\begin{tabular}{|c|c|c|c|c|c|c|c|}
\hline 2.350 & 0.10023 & 11 & 0.24774 & 16 & 0.34797 & 27 & 0.64358 \\
\hline $\begin{array}{l}2.351 \\
2.352\end{array}$ & $\begin{array}{l}0.10012 \\
0.10002\end{array}$ & $\begin{array}{l}10 \\
11\end{array}$ & $\begin{array}{l}0.24758 \\
0.24741\end{array}$ & $\begin{array}{l}17 \\
17\end{array}$ & $\begin{array}{l}0.34770 \\
0.34743\end{array}$ & $\begin{array}{l}27 \\
28\end{array}$ & $\begin{array}{l}0.64335 \\
0.64311\end{array}$ \\
\hline $\begin{array}{l}2.353 \\
2.354 \\
2.355\end{array}$ & $\begin{array}{l}0.09991 \\
0.09981 \\
0.09970\end{array}$ & $\begin{array}{l}10 \\
11 \\
10\end{array}$ & $\begin{array}{l}0.24724 \\
0.24707 \\
0.24690\end{array}$ & $\begin{array}{l}17 \\
17 \\
16\end{array}$ & $\begin{array}{l}0.34715 \\
0.34688 \\
0.34661\end{array}$ & $\begin{array}{l}27 \\
27 \\
28\end{array}$ & $\begin{array}{l}0.64288 \\
0.64265 \\
0.64242\end{array}$ \\
\hline $\begin{array}{l}2.356 \\
2.357 \\
2.358 \\
2.359 \\
2.360\end{array}$ & $\begin{array}{l}0.09960 \\
0.09949 \\
0.09939 \\
0.09928 \\
0.09918\end{array}$ & $\begin{array}{l}11 \\
10 \\
11 \\
10 \\
10\end{array}$ & $\begin{array}{l}0.24674 \\
0.24657 \\
0.24640 \\
0.24623 \\
0.24607\end{array}$ & $\begin{array}{l}17 \\
17 \\
17 \\
16 \\
17\end{array}$ & $\begin{array}{l}0.34633 \\
0.34606 \\
0.34579 \\
0.34552 \\
0.34525\end{array}$ & $\begin{array}{l}27 \\
27 \\
27 \\
27 \\
28\end{array}$ & $\begin{array}{l}0.64219 \\
0.64196 \\
0.64173 \\
0.64149 \\
0.64126\end{array}$ \\
\hline $\begin{array}{l}2.361 \\
2.362 \\
2.363 \\
2.364 \\
2.365\end{array}$ & $\begin{array}{l}0.09908 \\
0.09897 \\
0.09887 \\
0.09876 \\
0.09866\end{array}$ & $\begin{array}{l}11 \\
10 \\
11 \\
10 \\
10\end{array}$ & $\begin{array}{l}0.24590 \\
0.24573 \\
0.24556 \\
0.24540 \\
0.24523\end{array}$ & $\begin{array}{l}17 \\
17 \\
16 \\
17 \\
17\end{array}$ & $\begin{array}{l}0.34497 \\
0.34470 \\
0.34443 \\
0.34416 \\
0.34389\end{array}$ & $\begin{array}{l}27 \\
27 \\
27 \\
27 \\
27\end{array}$ & $\begin{array}{l}0.64103 \\
0.64080 \\
0.64057 \\
0.64034 \\
0.64010\end{array}$ \\
\hline $\begin{array}{l}2.366 \\
2.367 \\
2.368 \\
2.369 \\
2.370\end{array}$ & $\begin{array}{l}0.09856 \\
0.09845 \\
0.09835 \\
0.09825 \\
0.09814\end{array}$ & $\begin{array}{l}11 \\
10 \\
10 \\
11 \\
10\end{array}$ & $\begin{array}{l}0.24506 \\
0.24490 \\
0.24473 \\
0.24456 \\
0.24440\end{array}$ & $\begin{array}{l}16 \\
17 \\
17 \\
16 \\
17\end{array}$ & $\begin{array}{l}0.34362 \\
0.34335 \\
0.34308 \\
0.34281 \\
0.34254\end{array}$ & $\begin{array}{l}27 \\
27 \\
27 \\
27 \\
27\end{array}$ & $\begin{array}{l}0.63987 \\
0.63964 \\
0.63941 \\
0.63918 \\
0.63895\end{array}$ \\
\hline $\begin{array}{l}2 \cdot 371 \\
2 \cdot 372 \\
2 \cdot 373 \\
2.374 \\
2 \cdot 375\end{array}$ & $\begin{array}{l}0.09804 \\
0.09794 \\
0.09783 \\
0.09773 \\
0.09763\end{array}$ & $\begin{array}{l}10 \\
11 \\
10 \\
10 \\
10\end{array}$ & $\begin{array}{l}0.24423 \\
0.24406 \\
0.24390 \\
0.24373 \\
0.24356\end{array}$ & $\begin{array}{l}17 \\
16 \\
17 \\
17 \\
16\end{array}$ & $\begin{array}{l}0.34227 \\
0.34200 \\
0.34173 \\
0.34146 \\
0.34119\end{array}$ & $\begin{array}{l}27 \\
27 \\
27 \\
27 \\
27\end{array}$ & $\begin{array}{l}0.63872 \\
0.63848 \\
0.63825 \\
0.63802 \\
0.63779\end{array}$ \\
\hline $\begin{array}{l}2 \cdot 376 \\
2 \cdot 377 \\
2 \cdot 378 \\
2 \cdot 379 \\
2 \cdot 380\end{array}$ & $\begin{array}{l}0.09753 \\
0.09742 \\
0.09732 \\
0.09722 \\
0.09712\end{array}$ & $\begin{array}{l}11 \\
10 \\
10 \\
10 \\
10\end{array}$ & $\begin{array}{l}0.24340 \\
0.24323 \\
0.24307 \\
0.24290 \\
0.24274\end{array}$ & $\begin{array}{l}17 \\
16 \\
17 \\
16 \\
17\end{array}$ & $\begin{array}{l}0.34092 \\
0.34066 \\
0.34039 \\
0.34012 \\
0.33985\end{array}$ & $\begin{array}{l}26 \\
27 \\
27 \\
27 \\
26\end{array}$ & $\begin{array}{l}0.63756 \\
0.63733 \\
0.63709 \\
0.63686 \\
0.63663\end{array}$ \\
\hline $\begin{array}{l}2 \cdot 381 \\
2 \cdot 382 \\
2 \cdot 383 \\
2.384 \\
2.385\end{array}$ & $\begin{array}{l}0.09702 \\
0.09691 \\
0.09681 \\
0.09671 \\
0.09661\end{array}$ & $\begin{array}{l}11 \\
10 \\
10 \\
10 \\
10\end{array}$ & $\begin{array}{l}0.24257 \\
0.24240 \\
0.24224 \\
0.24207 \\
0.24191\end{array}$ & $\begin{array}{l}17 \\
16 \\
17 \\
16 \\
17\end{array}$ & $\begin{array}{l}0.33959 \\
0.33932 \\
0.33905 \\
0.33878 \\
0.33852\end{array}$ & $\begin{array}{l}27 \\
27 \\
27 \\
26 \\
27\end{array}$ & $\begin{array}{l}0.63640 \\
0.63617 \\
0.63594 \\
0.63571 \\
0.63547\end{array}$ \\
\hline $\begin{array}{l}2 \cdot 386 \\
2 \cdot 387 \\
2 \cdot 388 \\
2 \cdot 389 \\
2 \cdot 390\end{array}$ & $\begin{array}{l}0.09651 \\
0.09641 \\
0.09631 \\
0.09620 \\
0.09610\end{array}$ & $\begin{array}{l}10 \\
10 \\
11 \\
10 \\
10\end{array}$ & $\begin{array}{l}0.24174 \\
0.24158 \\
0.24141 \\
0.24125 \\
0.24109\end{array}$ & $\begin{array}{l}16 \\
17 \\
16 \\
16 \\
17\end{array}$ & $\begin{array}{l}0.33825 \\
0.33799 \\
0.33772 \\
0.33745 \\
0.33719\end{array}$ & $\begin{array}{l}26 \\
27 \\
27 \\
26 \\
27\end{array}$ & $\begin{array}{l}0.63524 \\
0.63501 \\
0.63478 \\
0.63455 \\
0.63432\end{array}$ \\
\hline $\begin{array}{l}2.391 \\
2.392 \\
2.393 \\
2.394 \\
2.395\end{array}$ & $\begin{array}{l}0.09600 \\
0.09590 \\
0.09580 \\
0.09570 \\
0.09560\end{array}$ & $\begin{array}{l}10 \\
10 \\
10 \\
10 \\
10\end{array}$ & $\begin{array}{l}0.24092 \\
0.24076 \\
0.24059 \\
0.24043 \\
0.24026\end{array}$ & $\begin{array}{l}16 \\
17 \\
16 \\
17 \\
16\end{array}$ & $\begin{array}{l}0.33692 \\
0.33666 \\
0.33639 \\
0.33613 \\
0.33586\end{array}$ & $\begin{array}{l}26 \\
27 \\
26 \\
27 \\
26\end{array}$ & $\begin{array}{l}0.63409 \\
0.63385 \\
0.63362 \\
0.63339 \\
0.63316\end{array}$ \\
\hline $\begin{array}{l}2.396 \\
2.397 \\
2.398 \\
2.399 \\
2.400\end{array}$ & $\begin{array}{l}0.09550 \\
0.09540 \\
0.09530 \\
0.09520 \\
0.09510\end{array}$ & $\begin{array}{l}10 \\
10 \\
10 \\
10\end{array}$ & $\begin{array}{l}0.24010 \\
0.23994 \\
0.23977 \\
0.23961 \\
0.23945\end{array}$ & $\begin{array}{l}16 \\
17 \\
16 \\
16\end{array}$ & $\begin{array}{l}0.33560 \\
0.33534 \\
0.33507 \\
0.33481 \\
0.33455\end{array}$ & $\begin{array}{l}26 \\
27 \\
26 \\
26\end{array}$ & $\begin{array}{l}0.63293 \\
0.63270 \\
0.63246 \\
0.63223 \\
0.63200\end{array}$ \\
\hline
\end{tabular}


Table I Harmonic Oscillator Contributions (in dimensionless form) to the

Thermodynamic Functions for Values of $X=h c v / k T$

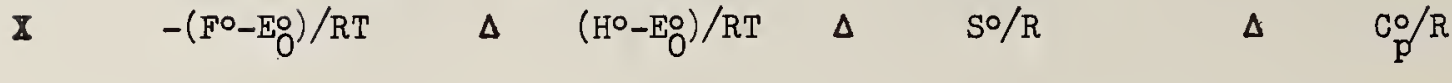

\begin{tabular}{|c|c|c|c|c|c|}
\hline 2.400 & 0.09510 & 10 & 0.23945 & 17 & 0.33455 \\
\hline $\begin{array}{l}2.401 \\
2.402 \\
2.403 \\
2.404 \\
2.405\end{array}$ & $\begin{array}{l}0.09500 \\
0.09490 \\
0.09480 \\
0.09470 \\
0.09460\end{array}$ & $\begin{array}{l}10 \\
10 \\
10 \\
10 \\
10\end{array}$ & $\begin{array}{l}0.23928 \\
0.23912 \\
0.23895 \\
0.23879 \\
0.23863\end{array}$ & $\begin{array}{l}16 \\
17 \\
16 \\
16 \\
16\end{array}$ & $\begin{array}{l}0.33428 \\
0.33402 \\
0.33376 \\
0.33349 \\
0.33323\end{array}$ \\
\hline $\begin{array}{l}2.406 \\
2.407 \\
2.408 \\
2.409 \\
2.410\end{array}$ & $\begin{array}{l}0.09450 \\
0.09440 \\
0.09431 \\
0.09421 \\
0.09411\end{array}$ & $\begin{array}{r}10 \\
9 \\
10 \\
10 \\
10\end{array}$ & $\begin{array}{l}0.23847 \\
0.23830 \\
0.23814 \\
0.23798 \\
0.23781\end{array}$ & $\begin{array}{l}17 \\
16 \\
16 \\
17 \\
16\end{array}$ & $\begin{array}{l}0.33297 \\
0.33271 \\
0.33244 \\
0.33218 \\
0.33192\end{array}$ \\
\hline $\begin{array}{l}2.411 \\
2.412 \\
2.413 \\
2.414 \\
2.415\end{array}$ & $\begin{array}{l}0.09401 \\
0.09391 \\
0.09381 \\
0.09371 \\
0.09362\end{array}$ & $\begin{array}{r}10 \\
10 \\
10 \\
9 \\
10\end{array}$ & $\begin{array}{l}0.23765 \\
0.23749 \\
0.23733 \\
0.23716 \\
0.23700\end{array}$ & $\begin{array}{l}16 \\
16 \\
17 \\
16 \\
16\end{array}$ & $\begin{array}{l}0.33166 \\
0.33140 \\
0.33114 \\
0.33088 \\
0.33062\end{array}$ \\
\hline $\begin{array}{l}2.416 \\
2.417 \\
2.418 \\
2.419 \\
2.420\end{array}$ & $\begin{array}{l}0.09352 \\
0.09342 \\
0.09332 \\
0.09322 \\
0.09313\end{array}$ & $\begin{array}{r}10 \\
10 \\
10 \\
9 \\
10\end{array}$ & $\begin{array}{l}0.23684 \\
0.23668 \\
0.23652 \\
0.23635 \\
0.23619\end{array}$ & $\begin{array}{l}16 \\
16 \\
17 \\
16 \\
16\end{array}$ & $\begin{array}{l}0.33036 \\
0.33010 \\
0.32984 \\
0.32958 \\
0.32932\end{array}$ \\
\hline $\begin{array}{l}2.421 \\
2.422 \\
2.423 \\
2.424 \\
2.425\end{array}$ & $\begin{array}{l}0.09303 \\
0.09293 \\
0.09283 \\
0.09274 \\
0.09264\end{array}$ & $\begin{array}{r}10 \\
10 \\
9 \\
10 \\
10\end{array}$ & $\begin{array}{l}0.23603 \\
0.23587 \\
0.23571 \\
0.23555 \\
0.23539\end{array}$ & $\begin{array}{l}16 \\
16 \\
16 \\
16 \\
17\end{array}$ & $\begin{array}{l}0.32906 \\
0.32880 \\
0.32854 \\
0.32828 \\
0.32803\end{array}$ \\
\hline $\begin{array}{l}2.426 \\
2.427 \\
2.428 \\
2.429 \\
2.430\end{array}$ & $\begin{array}{l}0.09254 \\
0.09245 \\
0.09235 \\
0.09225 \\
0.09216\end{array}$ & $\begin{array}{r}9 \\
10 \\
10 \\
9 \\
10\end{array}$ & $\begin{array}{l}0.23522 \\
0.23506 \\
0.23490 \\
0.23474 \\
0.23458\end{array}$ & $\begin{array}{l}16 \\
16 \\
16 \\
16 \\
16\end{array}$ & $\begin{array}{l}0.32777 \\
0.32751 \\
0.32725 \\
0.32699 \\
0.32674\end{array}$ \\
\hline $\begin{array}{l}2.431 \\
2.432 \\
2.433 \\
2.434 \\
2.435\end{array}$ & $\begin{array}{l}0.09206 \\
0.09196 \\
0.09187 \\
0.09177 \\
0.09167\end{array}$ & $\begin{array}{r}10 \\
9 \\
10 \\
10 \\
9\end{array}$ & $\begin{array}{l}0.23442 \\
0.23426 \\
0.23410 \\
0.23394 \\
0.23378\end{array}$ & $\begin{array}{l}16 \\
16 \\
16 \\
16 \\
16\end{array}$ & $\begin{array}{l}0.32648 \\
0.32622 \\
0.32597 \\
0.32571 \\
0.32545\end{array}$ \\
\hline $\begin{array}{l}2.436 \\
2.437 \\
2.438 \\
2.439 \\
2.440\end{array}$ & $\begin{array}{l}0.09158 \\
0.09148 \\
0.09139 \\
0.09129 \\
0.09120\end{array}$ & $\begin{array}{r}10 \\
9 \\
10 \\
9 \\
10\end{array}$ & $\begin{array}{l}0.23362 \\
0.23346 \\
0.23330 \\
0.23314 \\
0.23298\end{array}$ & $\begin{array}{l}16 \\
16 \\
16 \\
16 \\
16\end{array}$ & $\begin{array}{l}0.32520 \\
0.32494 \\
0.32469 \\
0.32443 \\
0.32417\end{array}$ \\
\hline $\begin{array}{l}2 \cdot 441 \\
2 \cdot 442 \\
2 \cdot 443 \\
2.444 \\
2.445\end{array}$ & $\begin{array}{l}0.09110 \\
0.09100 \\
0.09091 \\
0.09081 \\
0.09072\end{array}$ & $\begin{array}{r}10 \\
9 \\
10 \\
9 \\
10\end{array}$ & $\begin{array}{l}0.23282 \\
0.23266 \\
0.23250 \\
0.23234 \\
0.23218\end{array}$ & $\begin{array}{l}16 \\
16 \\
16 \\
16 \\
16\end{array}$ & $\begin{array}{l}0.32392 \\
0.32366 \\
0.32341 \\
0.32316 \\
0.32290\end{array}$ \\
\hline $\begin{array}{l}2.446 \\
2.447 \\
2.448 \\
2.449 \\
2.450\end{array}$ & $\begin{array}{l}0.09062 \\
0.09053 \\
0.09044 \\
0.09034 \\
0.09025\end{array}$ & $\begin{array}{r}9 \\
9 \\
10 \\
9\end{array}$ & $\begin{array}{l}0.23202 \\
0.23186 \\
0.23170 \\
0.23155 \\
0.23139\end{array}$ & $\begin{array}{l}16 \\
16 \\
15 \\
16\end{array}$ & $\begin{array}{l}0.32265 \\
0.32239 \\
0.32214 \\
0.32189 \\
0.32163\end{array}$ \\
\hline
\end{tabular}

$\Delta$

$\begin{array}{ll}0.63200 & 23 \\ 0.63177 & 23 \\ 0.63154 & 23 \\ 0.63131 & 23 \\ 0.63108 & 24 \\ 0.63084 & 23\end{array}$

$0.63061 \quad 23$

$0.63038 \quad 23$

$0.63015 \quad 23$

$0.62992 \quad 23$

$0.62969 \quad 23$

$0.62946 \quad 23$

$0.62923 \quad 24$

$0.62899 \quad 23$

$0.62876 \quad 23$

$0.62853 \quad 23$

$0.62830 \quad 23$

$0.62807 \quad 23$

$0.62784 \quad 23$

$0.62761 \quad 24$

$0.62737 \quad 23$

$0.62714 \quad 23$

$0.62691 \quad 23$

$0.62668 \quad 23$

$0.62645 \quad 23$

$0.62622 \quad 23$

$0.62599 \quad 24$

$0.62575 \quad 23$

$0.62552 \quad 23$

$0.62529 \quad 23$

$0.62506 \quad 23$

$0.62483 \quad 23$

$0.62460 \quad 23$

$0.62437 \quad 23$

$0.62414 \quad 24$

0.6239023

0.6236723

0.6234423

$0.62321 \quad 23$

$0.62298 \quad 23$

0.6227523

$0.62252 \quad 23$

$0.62229 \quad 24$

$0.62205 \quad 23$

$0.62182 \quad 23$

$0.62159 \quad 23$

$26 \quad 0.62136 \quad 23$

$25 \quad 0.62113 \quad 23$

$25 \quad 0.62090 \quad 23$

$26 \quad 0.62067 \quad 23$

0.62044 
Table I Harmonic Oscillator Contributions (in dimensionless form) to the

Thermodynamic Functions for Values of $\mathrm{X}=\mathrm{hcv} / \mathrm{kT}$
$\mathbf{x}$
$-\left(F^{\circ}-E_{O}^{\circ}\right) / R T$
$\Delta \quad\left(H^{\circ}-E_{O}^{\circ}\right) / R T$
$\Delta$
$\mathrm{S} / \mathrm{R}$
$\Delta \quad \mathrm{C} \circ / \mathrm{R}$

$\Delta$

\begin{tabular}{|c|c|}
\hline 2.450 & 0.09025 \\
\hline $\begin{array}{l}2.451 \\
2.452 \\
2.453 \\
2.454 \\
2.455\end{array}$ & $\begin{array}{l}0.09015 \\
0.09006 \\
0.08996 \\
0.08987 \\
0.08978\end{array}$ \\
\hline $\begin{array}{l}2.456 \\
2.457 \\
2.458 \\
2.459 \\
2.460\end{array}$ & $\begin{array}{l}0.08968 \\
0.08959 \\
0.08949 \\
0.08940 \\
0.08931\end{array}$ \\
\hline $\begin{array}{l}2.461 \\
2.462 \\
2.463 \\
2.464 \\
2.465\end{array}$ & $\begin{array}{l}0.08921 \\
0.08912 \\
0.08903 \\
0.08893 \\
0.08884\end{array}$ \\
\hline $\begin{array}{l}2.466 \\
2.467 \\
2.468 \\
2.469 \\
2.470\end{array}$ & $\begin{array}{l}0.08875 \\
0.08866 \\
0.08856 \\
0.08847 \\
0.08838\end{array}$ \\
\hline $\begin{array}{l}2.471 \\
2.472 \\
2.473 \\
2.474 \\
2.475\end{array}$ & $\begin{array}{l}0.08829 \\
0.08819 \\
0.08810 \\
0.08801 \\
0.08792\end{array}$ \\
\hline $\begin{array}{l}2.476 \\
2.477 \\
2.478 \\
2.479 \\
2.480\end{array}$ & $\begin{array}{l}0.08783 \\
0.08773 \\
0.08764 \\
0.08755 \\
0.08746\end{array}$ \\
\hline $\begin{array}{l}2.481 \\
2.482 \\
2.483 \\
2.484 \\
2.485\end{array}$ & $\begin{array}{l}0.08737 \\
0.08728 \\
0.08718 \\
0.08709 \\
0.08700\end{array}$ \\
\hline $\begin{array}{l}2.486 \\
2.437 \\
2.488 \\
2.489 \\
2.490\end{array}$ & $\begin{array}{l}0.08691 \\
0.08682 \\
0.08673 \\
0.08664 \\
0.08655\end{array}$ \\
\hline $\begin{array}{l}2.491 \\
2.492 \\
2.493 \\
2.494 \\
2.495\end{array}$ & $\begin{array}{l}0.08646 \\
0.08637 \\
0.08628 \\
0.08619 \\
0.08610\end{array}$ \\
\hline $\begin{array}{l}2.496 \\
2.497 \\
2.498 \\
2.499 \\
2.500\end{array}$ & $\begin{array}{l}0.08601 \\
0.08592 \\
0.08583 \\
0.08574 \\
0.08565\end{array}$ \\
\hline
\end{tabular}

$\begin{array}{rll}10 & 0.23139 & 16 \\ 9 & 0.23123 & 16 \\ 10 & 0.23107 & 16 \\ 9 & 0.23091 & 16 \\ 9 & 0.23075 & 16 \\ 10 & 0.23059 & 15\end{array}$

0.32163

$25 \quad 0.62044$

23

10

0.23044

16

0.32138

25

0.62021

0.32113

0.32087

0.32062

0.32037

0.61997

0.61974

0.61951

0.61928

24

25

25

0.32012

0.61905

\section{6}

0.31986

0.31961

0.23012

0.22996

0.31936

0.22980

0.31911

0.61882

0.61859

0.61836

0.61813

23

23

23

23

$9 \quad 0.22965 \quad 16$

0.31886

$0.22949 \quad 16$

0.31861

0.31836

0.31811

$\begin{array}{ll}0.22933 & 16 \\ 0.22917 & 15\end{array}$

0.22902

0.31786

23

25

25

25

0.61789

25
25

0.61766

0.61743

0.61720

25

25

0.61697

0.31761

0.22870

0.22854

0.22839

0.22823

10

$0.22807 \quad 15$

0.22792

0.22776

0.22760

0.22745

16
16

15

16

16

15
16

16

15

16

0.22729

0.22713

0.22698

0.22682

0.22666

16

15

16

16

15

0.31736

0.31711

0.31686

0.31661

0.31636

0.31611

0.31586

0.31561

0.31536

0.22651

16

0.22635

0.22620

0.22604

0.22589

0.31511

0.31487

0.31462

0.31437

0.31412

0.31388

0.31363

0.31338

0.31314

0.31289

0.22573

0.22558

0.22542

0.22526

0.22511

0.31264

0.31240

0.31215

0.31190

0.31166

0.22495

0.22480

0.22464

0.22449

0.22434

0.31141

0.31117

0.31092

0.31068

0.31043

0.22418

0.22403

0.22387

0.22372

0.22356

0.30946
25

25

25

25

25

25

25

25

25

25

24

25

25

25

24

25

25

24

25

25

24

25

25

24

25

24

25

24

25

24

24

25

24

25

$0.61674 \quad 23$

$0.61651 \quad 23$

$0.61628 \quad 23$

$0.61605 \quad 23$

$0.61582 \quad 24$

$0.61558 \quad 23$

$0.61535 \quad 23$

$0.61512 \quad 23$

$0.61489 \quad 23$

$0.61466 \quad 23$

$0.61443 \quad 23$

$0.61420 \quad 23$

$0.61397 \quad 23$

$0.61374 \quad 23$

$0.61351 \quad 24$

$0.61327 \quad 23$

$0.61304 \quad 23$

$0.61281 \quad 23$

$0.61258 \quad 23$

$0.61235 \quad 23$

$0.61212 \quad 23$

$0.61189 \quad 23$

$0.61166 \quad 23$

$0.61143 \quad 23$

$0.61120 \quad 23$

$0.61097 \quad 23$

$0.61074 \quad 23$

$0.61051 \quad 24$

$0.61027 \quad 23$

0.6100423

0.6098123

$0.60958 \quad 23$

$0.60935 \quad 23$

0.6091223

0.60889 
Table I Harmonic Oscillator Contributions (in dimensionless form) to the Thermodynamic Functions for Values of $X=h c v / k T$

\begin{tabular}{|c|c|c|c|c|c|c|c|}
\hline $\mathbb{Z}$ & $-\left(F^{\circ}-E_{O} \circ\right) / R T$ & $\Delta$ & $\left(H^{\circ}-E_{O}^{O}\right) / R T$ & $\Delta$ & So/R & $\Delta$ & $\mathrm{CO}_{\mathrm{p}} / \mathrm{R}$ \\
\hline 2.500 & 0.08565 & 9 & 0.22356 & 15 & 0.30921 & 24 & 0.60889 \\
\hline $\begin{array}{l}2.501 \\
2.502 \\
2.503 \\
2.504 \\
2.505\end{array}$ & $\begin{array}{l}0.08556 \\
0.08547 \\
0.08538 \\
0.08529 \\
0.08520\end{array}$ & $\begin{array}{l}9 \\
9 \\
9 \\
9 \\
8\end{array}$ & $\begin{array}{l}0.22341 \\
0.22326 \\
0.22310 \\
0.22295 \\
0.22279\end{array}$ & $\begin{array}{l}15 \\
16 \\
15 \\
16 \\
15\end{array}$ & $\begin{array}{l}0.30897 \\
0.30873 \\
0.30848 \\
0.30824 \\
0.30800\end{array}$ & $\begin{array}{l}24 \\
25 \\
24 \\
24 \\
24\end{array}$ & $\begin{array}{l}0.60866 \\
0.60843 \\
0.60820 \\
0.60797 \\
0.60774\end{array}$ \\
\hline $\begin{array}{l}2.506 \\
2.507 \\
2.508 \\
2.509 \\
2.510\end{array}$ & $\begin{array}{l}0.08512 \\
0.08503 \\
0.08494 \\
0.08485 \\
0.08476\end{array}$ & $\begin{array}{l}9 \\
9 \\
9 \\
9 \\
9\end{array}$ & $\begin{array}{l}0.22264 \\
0.22249 \\
0.22233 \\
0.22218 \\
0.22203\end{array}$ & $\begin{array}{l}15 \\
16 \\
15 \\
15 \\
16\end{array}$ & $\begin{array}{l}0.30776 \\
0.30751 \\
0.30727 \\
0.30703 \\
0.30679\end{array}$ & $\begin{array}{l}25 \\
24 \\
24 \\
24 \\
24\end{array}$ & $\begin{array}{l}0.60751 \\
0.60728 \\
0.60704 \\
0.60681 \\
0.60658\end{array}$ \\
\hline $\begin{array}{l}2.511 \\
2.512 \\
2.513 \\
2.514 \\
2.515\end{array}$ & $\begin{array}{l}0.08467 \\
0.08458 \\
0.08450 \\
0.08441 \\
0.08432\end{array}$ & $\begin{array}{l}9 \\
8 \\
9 \\
9 \\
9\end{array}$ & $\begin{array}{l}0.22187 \\
0.22172 \\
0.22157 \\
0.22141 \\
0.22126\end{array}$ & $\begin{array}{l}15 \\
15 \\
16 \\
15 \\
15\end{array}$ & $\begin{array}{l}0.30655 \\
0.30631 \\
0.30606 \\
0.30582 \\
0.30558\end{array}$ & $\begin{array}{l}24 \\
25 \\
24 \\
24 \\
24\end{array}$ & $\begin{array}{l}0.60635 \\
0.60612 \\
0.60589 \\
0.60566 \\
0.60543\end{array}$ \\
\hline $\begin{array}{l}2.516 \\
2.517 \\
2.518 \\
2.519 \\
2.520\end{array}$ & $\begin{array}{l}0.08423 \\
0.08414 \\
0.08406 \\
0.08397 \\
0.08388\end{array}$ & $\begin{array}{l}9 \\
8 \\
9 \\
9 \\
9\end{array}$ & $\begin{array}{l}0.22111 \\
0.22096 \\
0.22080 \\
0.22065 \\
0.22050\end{array}$ & $\begin{array}{l}15 \\
16 \\
15 \\
15 \\
15\end{array}$ & $\begin{array}{l}0.30534 \\
0.30510 \\
0.30486 \\
0.30462 \\
0.30438\end{array}$ & $\begin{array}{l}24 \\
24 \\
24 \\
24 \\
24\end{array}$ & $\begin{array}{l}0.60520 \\
0.60497 \\
0.60474 \\
0.60451 \\
0.60428\end{array}$ \\
\hline $\begin{array}{l}2.521 \\
2.522 \\
2.523 \\
2.524 \\
2.525\end{array}$ & $\begin{array}{l}0.08379 \\
0.08371 \\
0.08362 \\
0.08353 \\
0.08344\end{array}$ & $\begin{array}{l}8 \\
9 \\
9 \\
9 \\
8\end{array}$ & $\begin{array}{l}0.22035 \\
0.22020 \\
0.22004 \\
0.21989 \\
0.21974\end{array}$ & $\begin{array}{l}15 \\
16 \\
15 \\
15 \\
15\end{array}$ & $\begin{array}{l}0.30414 \\
0.30390 \\
0.30366 \\
0.30342 \\
0.30318\end{array}$ & $\begin{array}{l}24 \\
24 \\
24 \\
24 \\
23\end{array}$ & $\begin{array}{l}0.60405 \\
0.60382 \\
0.60359 \\
0.60336 \\
0.60313\end{array}$ \\
\hline $\begin{array}{l}2.526 \\
2.527 \\
2.528 \\
2.529 \\
2.530\end{array}$ & $\begin{array}{l}0.08336 \\
0.08327 \\
0.08318 \\
0.08310 \\
0.08301\end{array}$ & $\begin{array}{l}9 \\
9 \\
8 \\
9 \\
9\end{array}$ & $\begin{array}{l}0.21959 \\
0.21944 \\
0.21928 \\
0.21913 \\
0.21898\end{array}$ & $\begin{array}{l}15 \\
16 \\
15 \\
15 \\
15\end{array}$ & $\begin{array}{l}0.30295 \\
0.30271 \\
0.30247 \\
0.30223 \\
0.30199\end{array}$ & $\begin{array}{l}24 \\
24 \\
24 \\
24 \\
24\end{array}$ & $\begin{array}{l}0.60290 \\
0.60267 \\
0.60244 \\
0.60221 \\
0.60198\end{array}$ \\
\hline $\begin{array}{l}2.531 \\
2.532 \\
2.533 \\
2.534 \\
2.535\end{array}$ & $\begin{array}{l}0.08292 \\
0.08284 \\
0.08275 \\
0.08267 \\
0.08258\end{array}$ & $\begin{array}{l}8 \\
9 \\
8 \\
9 \\
9\end{array}$ & $\begin{array}{l}0.21883 \\
0.21868 \\
0.21853 \\
0.21838 \\
0.21823\end{array}$ & $\begin{array}{l}15 \\
15 \\
15 \\
15 \\
16\end{array}$ & $\begin{array}{l}0.30175 \\
0.30152 \\
0.30128 \\
0.30104 \\
0.30080\end{array}$ & $\begin{array}{l}23 \\
24 \\
24 \\
24 \\
23\end{array}$ & $\begin{array}{l}0.60174 \\
0.60151 \\
0.60128 \\
0.60105 \\
0.60082\end{array}$ \\
\hline $\begin{array}{l}2.536 \\
2.537 \\
2.538 \\
2.539 \\
2.540\end{array}$ & $\begin{array}{l}0.08249 \\
0.08241 \\
0.08232 \\
0.08224 \\
0.08215\end{array}$ & $\begin{array}{l}8 \\
9 \\
8 \\
9 \\
9\end{array}$ & $\begin{array}{l}0.21807 \\
0.21792 \\
0.21777 \\
0.21762 \\
0.21747\end{array}$ & $\begin{array}{l}15 \\
15 \\
15 \\
15 \\
15\end{array}$ & $\begin{array}{l}0.30057 \\
0.30033 \\
0.30009 \\
0.29986 \\
0.29962\end{array}$ & $\begin{array}{l}24 \\
24 \\
23 \\
24 \\
23\end{array}$ & $\begin{array}{l}0.60059 \\
0.60036 \\
0.60013 \\
0.59990 \\
0.59967\end{array}$ \\
\hline $\begin{array}{l}2.541 \\
2.542 \\
2.543 \\
2.544 \\
2.545\end{array}$ & $\begin{array}{l}0.08206 \\
0.08198 \\
0.08189 \\
0.08181 \\
0.08172\end{array}$ & $\begin{array}{l}8 \\
9 \\
8 \\
9 \\
8\end{array}$ & $\begin{array}{l}0.21732 \\
0.21717 \\
0.21702 \\
0.21687 \\
0.21672\end{array}$ & $\begin{array}{l}15 \\
15 \\
15 \\
15 \\
15\end{array}$ & $\begin{array}{l}0.29939 \\
0.29915 \\
0.29891 \\
0.29868 \\
0.29844\end{array}$ & $\begin{array}{l}24 \\
24 \\
23 \\
24 \\
23\end{array}$ & $\begin{array}{l}0.59944 \\
0.59921 \\
0.59898 \\
0.59875 \\
0.59852\end{array}$ \\
\hline $\begin{array}{l}2.546 \\
2.547 \\
2.548 \\
2.549 \\
2.550\end{array}$ & $\begin{array}{l}0.08164 \\
0.08155 \\
0.08147 \\
0.08138 \\
0.08130\end{array}$ & $\begin{array}{l}9 \\
8 \\
9 \\
8\end{array}$ & $\begin{array}{l}0.21657 \\
0.21642 \\
0.21627 \\
0.21612 \\
0.21597\end{array}$ & $\begin{array}{l}15 \\
15 \\
15 \\
15\end{array}$ & $\begin{array}{l}0.29821 \\
0.29797 \\
0.29774 \\
0.29750 \\
0.29727\end{array}$ & $\begin{array}{l}24 \\
23 \\
24 \\
23\end{array}$ & $\begin{array}{l}0.59829 \\
0.59806 \\
0.59783 \\
0.59760 \\
0.59737\end{array}$ \\
\hline
\end{tabular}


Table I Harmonic Oscillator Contributions (in dimensionless form) to the

Thermodynamic Functions for Values of $X=h c v / k T$

$\begin{array}{llllllll} & -\left(\mathrm{F}^{\circ}-\mathrm{E}_{\mathrm{O}}^{\circ}\right) / \mathrm{RT} & \Delta & \left(\mathrm{H}^{\circ}-\mathrm{E}_{\mathrm{O}}^{\circ}\right) / \mathrm{RT} & \Delta & \mathrm{S} \% / \mathrm{R} & \Delta \quad \mathrm{C}_{\mathrm{p}}^{\circ} / \mathrm{R}\end{array}$

\begin{tabular}{|c|c|c|c|c|c|c|c|}
\hline 2.550 & 0.08130 & 9 & 0.21597 & 15 & 0.29727 & 23 & 0.59737 \\
\hline $\begin{array}{l}2.551 \\
2.552 \\
2.553 \\
2.554 \\
2.555\end{array}$ & $\begin{array}{l}0.08121 \\
0.08113 \\
0.08104 \\
0.08096 \\
0.08088\end{array}$ & $\begin{array}{l}8 \\
9 \\
8 \\
8 \\
9\end{array}$ & $\begin{array}{l}0.21582 \\
0.21567 \\
0.21552 \\
0.21537 \\
0.21522\end{array}$ & $\begin{array}{l}15 \\
15 \\
15 \\
15 \\
14\end{array}$ & $\begin{array}{l}0.29704 \\
0.29680 \\
0.29657 \\
0.29633 \\
0.29610\end{array}$ & $\begin{array}{l}24 \\
23 \\
24 \\
23 \\
23\end{array}$ & $\begin{array}{l}0.59714 \\
0.59691 \\
0.59668 \\
0.59645 \\
0.59622\end{array}$ \\
\hline $\begin{array}{l}2.556 \\
2.557 \\
2.558 \\
2.559 \\
2.560\end{array}$ & $\begin{array}{l}0.08079 \\
0.08071 \\
0.08062 \\
0.08054 \\
0.08046\end{array}$ & $\begin{array}{l}8 \\
9 \\
8 \\
8 \\
9\end{array}$ & $\begin{array}{l}0.21508 \\
0.21493 \\
0.21478 \\
0.21463 \\
0.21448\end{array}$ & $\begin{array}{l}15 \\
15 \\
15 \\
15 \\
15\end{array}$ & $\begin{array}{l}0.29587 \\
0.29563 \\
0.29540 \\
0.29517 \\
0.29494\end{array}$ & $\begin{array}{l}24 \\
23 \\
23 \\
23 \\
24\end{array}$ & $\begin{array}{l}0.59599 \\
0.59576 \\
0.59553 \\
0.59530 \\
0.59507\end{array}$ \\
\hline $\begin{array}{l}2.561 \\
2.562 \\
2.563 \\
2.564 \\
2.565\end{array}$ & $\begin{array}{l}0.08037 \\
0.08029 \\
0.08021 \\
0.08012 \\
0.08004\end{array}$ & $\begin{array}{l}8 \\
8 \\
9 \\
8 \\
8\end{array}$ & $\begin{array}{l}0.21433 \\
0.21418 \\
0.21403 \\
0.21389 \\
0.21374\end{array}$ & $\begin{array}{l}15 \\
15 \\
14 \\
15 \\
15\end{array}$ & $\begin{array}{l}0.29470 \\
0.29447 \\
0.29424 \\
0.29401 \\
0.29378\end{array}$ & $\begin{array}{l}23 \\
23 \\
23 \\
23 \\
23\end{array}$ & $\begin{array}{l}0.59484 \\
0.59461 \\
0.59438 \\
0.59415 \\
0.59392\end{array}$ \\
\hline $\begin{array}{l}2.566 \\
2.567 \\
2.568 \\
2.569 \\
2.570\end{array}$ & $\begin{array}{l}0.07996 \\
0.07987 \\
0.07979 \\
0.07971 \\
0.07962\end{array}$ & $\begin{array}{l}9 \\
8 \\
8 \\
9 \\
8\end{array}$ & $\begin{array}{l}0.21359 \\
0.21344 \\
0.21329 \\
0.21315 \\
0.21300\end{array}$ & $\begin{array}{l}15 \\
15 \\
14 \\
15 \\
15\end{array}$ & $\begin{array}{l}0.29355 \\
0.29331 \\
0.29308 \\
0.29285 \\
0.29262\end{array}$ & $\begin{array}{l}24 \\
23 \\
23 \\
23 \\
23\end{array}$ & $\begin{array}{l}0.59369 \\
0.59346 \\
0.59323 \\
0.59300 \\
0.59277\end{array}$ \\
\hline $\begin{array}{l}2.571 \\
2.572 \\
2.573 \\
2.574 \\
2.575\end{array}$ & $\begin{array}{l}0.07954 \\
0.07946 \\
0.07937 \\
0.07929 \\
0.07921\end{array}$ & $\begin{array}{l}8 \\
9 \\
8 \\
8 \\
8\end{array}$ & $\begin{array}{l}0.21285 \\
0.21270 \\
0.21256 \\
0.21241 \\
0.21226\end{array}$ & $\begin{array}{l}15 \\
14 \\
15 \\
15 \\
15\end{array}$ & $\begin{array}{l}0.29239 \\
0.29216 \\
0.29193 \\
0.29170 \\
0.29147\end{array}$ & $\begin{array}{l}23 \\
23 \\
23 \\
23 \\
23\end{array}$ & $\begin{array}{l}0.59254 \\
0.59231 \\
0.59208 \\
0.59186 \\
0.59163\end{array}$ \\
\hline $\begin{array}{l}2.576 \\
2.577 \\
2.578 \\
2.579 \\
2.580\end{array}$ & $\begin{array}{l}0.07913 \\
0.07905 \\
0.07896 \\
0.07888 \\
0.07880\end{array}$ & $\begin{array}{l}8 \\
9 \\
8 \\
8 \\
8\end{array}$ & $\begin{array}{l}0.21211 \\
0.21197 \\
0.21182 \\
0.21167 \\
0.21153\end{array}$ & $\begin{array}{l}14 \\
15 \\
15 \\
14 \\
15\end{array}$ & $\begin{array}{l}0.29124 \\
0.29101 \\
0.29078 \\
0.29055 \\
0.29032\end{array}$ & $\begin{array}{l}23 \\
23 \\
23 \\
23 \\
23\end{array}$ & $\begin{array}{l}0.59140 \\
0.59117 \\
0.59094 \\
0.59071 \\
0.59048\end{array}$ \\
\hline $\begin{array}{l}2.581 \\
2.582 \\
2.583 \\
2.584 \\
2.585\end{array}$ & $\begin{array}{l}0.07872 \\
0.07863 \\
0.07855 \\
0.07847 \\
0.07839\end{array}$ & $\begin{array}{l}9 \\
8 \\
8 \\
8 \\
8\end{array}$ & $\begin{array}{l}0.211138 \\
0.21123 \\
0.21108 \\
0.21094 \\
0.21079\end{array}$ & $\begin{array}{l}15 \\
15 \\
14 \\
15 \\
14\end{array}$ & $\begin{array}{l}0.29009 \\
0.28987 \\
0.28964 \\
0.28941 \\
0.28918\end{array}$ & $\begin{array}{l}22 \\
23 \\
23 \\
23 \\
23\end{array}$ & $\begin{array}{l}0.59025 \\
0.59002 \\
0.58979 \\
0.58956 \\
0.58933\end{array}$ \\
\hline $\begin{array}{l}2.586 \\
2.587 \\
2.588 \\
2.589 \\
2.590\end{array}$ & $\begin{array}{l}0.07831 \\
0.07823 \\
0.07815 \\
0.07806 \\
0.07798\end{array}$ & $\begin{array}{l}8 \\
8 \\
9 \\
8 \\
8\end{array}$ & $\begin{array}{l}0.21065 \\
0.21050 \\
0.21035 \\
0.21021 \\
0.21006\end{array}$ & $\begin{array}{l}15 \\
15 \\
14 \\
15 \\
15\end{array}$ & $\begin{array}{l}0.28895 \\
0.28873 \\
0.28850 \\
0.28827 \\
0.28804\end{array}$ & $\begin{array}{l}22 \\
23 \\
23 \\
23 \\
22\end{array}$ & $\begin{array}{l}0.58910 \\
0.58887 \\
0.58864 \\
0.58841 \\
0.58818\end{array}$ \\
\hline $\begin{array}{l}2.591 \\
2.592 \\
2.593 \\
2.594 \\
2.595\end{array}$ & $\begin{array}{l}0.07790 \\
0.07782 \\
0.07774 \\
0.07766 \\
0.07758\end{array}$ & $\begin{array}{l}8 \\
8 \\
8 \\
8 \\
8\end{array}$ & $\begin{array}{l}0.20991 \\
0.20977 \\
0.20962 \\
0.20948 \\
0.20933\end{array}$ & $\begin{array}{l}14 \\
15 \\
14 \\
15 \\
14\end{array}$ & $\begin{array}{l}0.28782 \\
0.28759 \\
0.28736 \\
0.28714 \\
0.28691\end{array}$ & $\begin{array}{l}23 \\
23 \\
22 \\
23 \\
23\end{array}$ & $\begin{array}{l}0.58795 \\
0.58772 \\
0.58749 \\
0.58727 \\
0.58704\end{array}$ \\
\hline $\begin{array}{l}2.596 \\
2.597 \\
2.598 \\
2.599 \\
2.600\end{array}$ & $\begin{array}{l}0.07750 \\
0.07742 \\
0.07734 \\
0.07726 \\
0.07718\end{array}$ & $\begin{array}{l}8 \\
8 \\
8 \\
8\end{array}$ & $\begin{array}{l}0.20919 \\
0.20904 \\
0.20890 \\
0.20875 \\
0.20861\end{array}$ & $\begin{array}{l}15 \\
14 \\
15 \\
14\end{array}$ & $\begin{array}{l}0.28668 \\
0.28646 \\
0.28623 \\
0.28601 \\
0.28578\end{array}$ & $\begin{array}{l}22 \\
23 \\
22 \\
23\end{array}$ & $\begin{array}{l}0.58681 \\
0.58658 \\
0.58635 \\
0.58612 \\
0.58589\end{array}$ \\
\hline
\end{tabular}


Table I Harmonic Oscillator Contributions (in dimensionless form) to the Thermodynamic Functions for Values of $X=h c v / k T$

$\mathbf{x}$ $-\left(F^{\circ}-E_{0} \circ\right) / R T$

$\Delta \quad\left(\mathrm{H}^{\circ}-\mathrm{E}_{\mathrm{O}}\right) / \mathrm{RT}$

$\Delta$

So/R

\begin{tabular}{|c|c|}
\hline 2.600 & 0.07718 \\
\hline $\begin{array}{l}2.601 \\
2.602 \\
2.603 \\
2.604 \\
2.605\end{array}$ & $\begin{array}{r}0.07710 \\
0.07702 \\
0.07694 \\
0.07686 \\
0.07678\end{array}$ \\
\hline $\begin{array}{l}2.606 \\
2.607 \\
2.608 \\
2.609 \\
2.610\end{array}$ & $\begin{array}{l}0.07670 \\
0.07662 \\
0.07654 \\
0.07646 \\
0.07638\end{array}$ \\
\hline $\begin{array}{l}2.611 \\
2.612 \\
2.613 \\
2.614 \\
2.615\end{array}$ & $\begin{array}{l}0.07630 \\
0.07622 \\
0.07614 \\
0.07606 \\
0.07598\end{array}$ \\
\hline $\begin{array}{l}2.616 \\
2.617 \\
2.618 \\
2.619 \\
2.620\end{array}$ & $\begin{array}{l}0.07590 \\
0.07583 \\
0.07575 \\
0.07567 \\
0.07559\end{array}$ \\
\hline $\begin{array}{l}2.621 \\
2.622 \\
2.623 \\
2.624 \\
2.625\end{array}$ & $\begin{array}{l}0.07551 \\
0.07543 \\
0.07535 \\
0.07528 \\
0.07520\end{array}$ \\
\hline $\begin{array}{l}2.626 \\
2.627 \\
2.628 \\
2.629 \\
2.630\end{array}$ & $\begin{array}{l}0.07512 \\
0.07504 \\
0.07496 \\
0.07489 \\
0.07481\end{array}$ \\
\hline $\begin{array}{l}2.631 \\
2.632 \\
2.633 \\
2.634 \\
2.635\end{array}$ & $\begin{array}{l}0.07473 \\
0.07465 \\
0.07458 \\
0.07450 \\
0.07442\end{array}$ \\
\hline $\begin{array}{l}2.636 \\
2.637 \\
2.638 \\
2.639 \\
2.640\end{array}$ & $\begin{array}{l}0.07434 \\
0.07427 \\
0.07419 \\
0.07411 \\
0.07404\end{array}$ \\
\hline $\begin{array}{l}2.641 \\
2.642 \\
2.643 \\
2.644 \\
2.645\end{array}$ & $\begin{array}{l}0.07396 \\
0.07388 \\
0.07381 \\
0.07373 \\
0.07365\end{array}$ \\
\hline $\begin{array}{l}2.646 \\
2.647 \\
2.648 \\
2.649 \\
2.650\end{array}$ & $\begin{array}{l}0.07358 \\
0.07350 \\
0.07342 \\
0.07335 \\
0.07327\end{array}$ \\
\hline
\end{tabular}

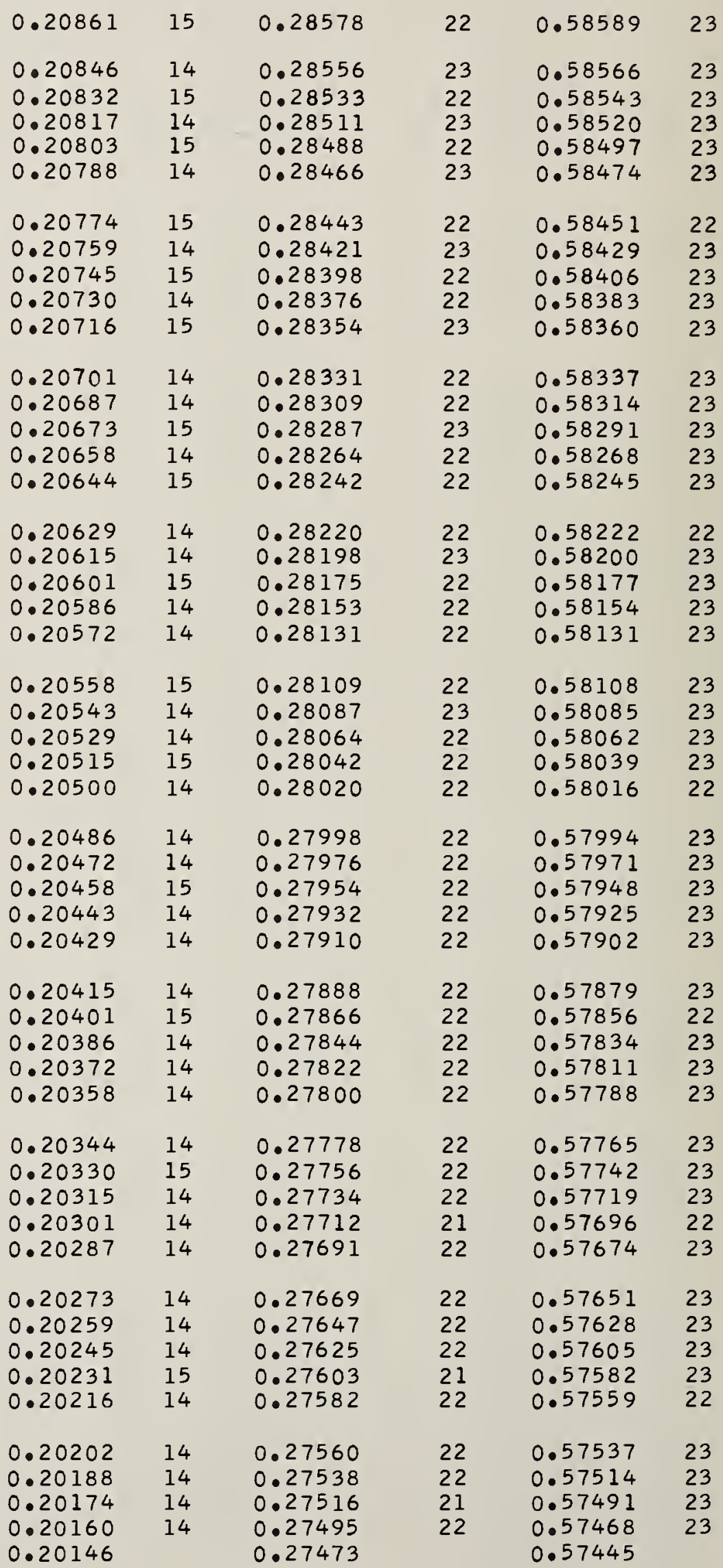


Table I Harmonic Oscillator Contributions (in dimensionless form) to the

Thermodynamic Functions for Values of $X=h c v / k T$

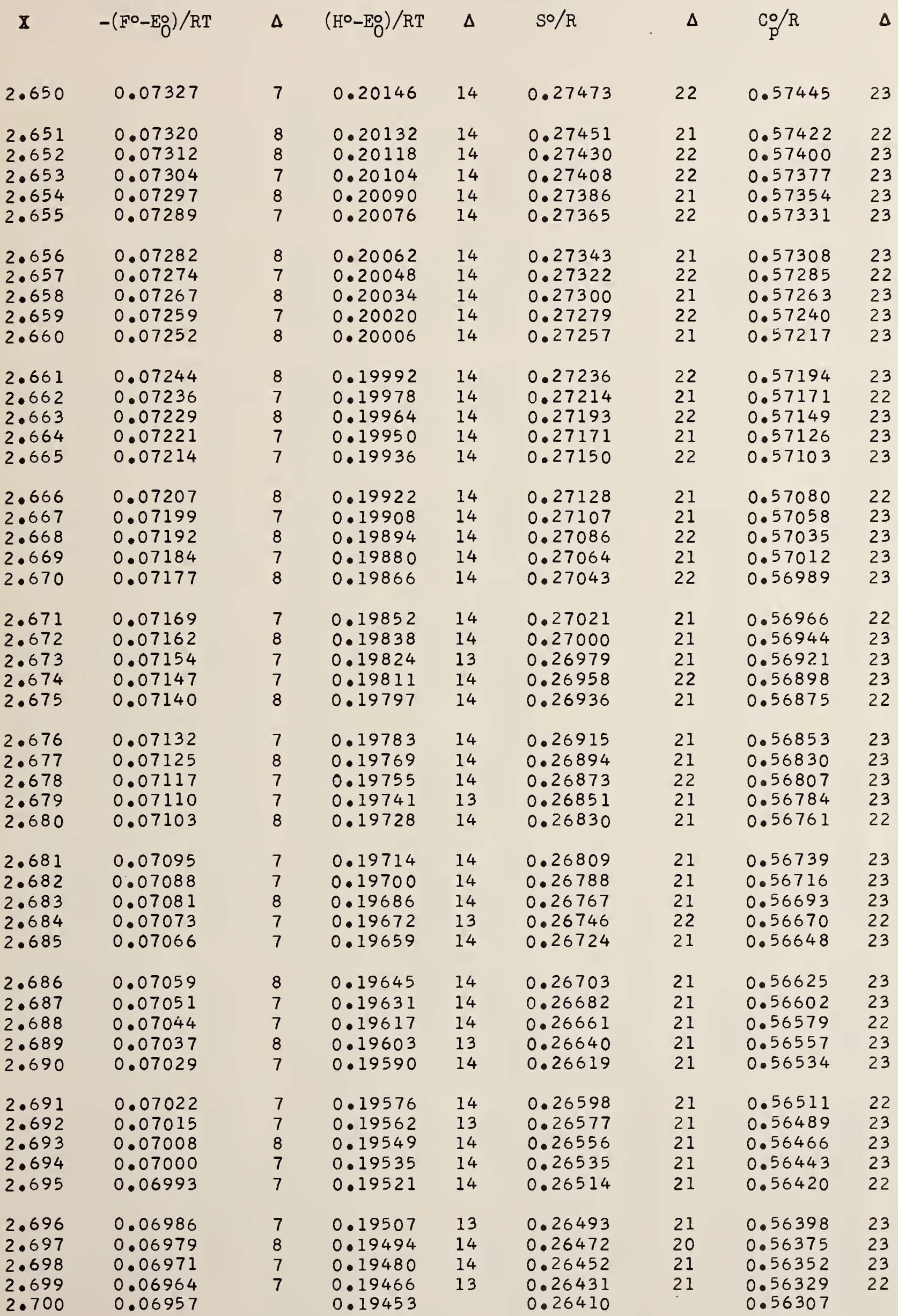


Table I Harmonic Oscillator Contributions (in dimensionless form) to the

Thermodynamic Functions for Values of $\mathrm{X}=\mathrm{hcv} / \mathrm{kT}$

$\mathbf{X}$ $-\left(F^{\circ}-E_{O}^{\circ}\right) / R T$
$\Delta \quad\left(H^{\circ}-E_{0}^{\circ}\right) / R T \quad \Delta \quad S \circ / R$

2.700

2.701

2.702

2.703

2.704

2.705

2.706

2.707

2.708

2.709

2.710

2.711

2.712

2.713

2.714

2.715

2.716

2.717

2.718

2.719

2.720

2.721

2.722

2.723

2.724

2.725

2.726

2.727

2.728

2.729

2.730

2.731

2.732

2.733

2.734

2.735

2.736

2.737

2.738

2.739

2.740

2.741

2.742

2.743

2.744

2.745

2.746

2.747

2.748

2.749

2.750

0.06957
0.06950
0.06943
0.06935
0.06928
0.06921

0.06914

0.06907

0.06900

0.06893

0.06885

0.06878

0.06864

0.06857

0.06850

0.06843

0.06836

0.06829

0.06822

0.06814

0.06807

0.06800

0.06793

0.06786

0.06779

0.06772

0.06765

0.06758

0.06751

0.06744

0.06737

0.06730

0.06723

0.06716

0.06710

0.06703

0.06696

0.06689

0.06682

0.06675

0.06668

0.06661

0.06654

0.06647

0.06641

0.06634

0.06627

0.06620

0.06613

0.06606
0.06871
$0.19439 \quad 13$

0.19412

0.19398

0.19385

0.19371

0.19357

0.19344

0.19330

0.19317

$0.19303 \quad 13$

$0.19290 \quad 14$

0.19276

0.19263

0.19249

$0.19236 \quad 14$

$0.19222 \quad 14$

0.19208

0.19195

0.19182

0.19168

0.19155

0.19141

0.19128

0.19114

0.19101

0.19087

0.19074

0.19061

0.19047

0.19034

0.19020

0.19007

0.18994

0.18980

0.18967

0.18954

0.18940

0.18927

0.18914

0.18900

0.18887

0.18874

0.18860

0.18847

0.18834

0.18821

0.18807

0.18794

0.18781

14

13
14

14

13

14

13
0.19426
$\Delta \quad C \circ / R$

$\Delta$
14

14

0.26410

0.26389

0.26368

0.26347

0.26327

0.26306

0.26285

0.26264

0.26244

0.26223

0.26202

0.26181

0.26161

0.26140

0.26119

0.26099

0.26078

0.26058

0.26037

0.26017

0.25996

0.25975

0.25955

0.25934

0.25914

0.25894

0.25873

0.25853

0.25832

0.25812

0.25791

0.25771

0.25751

0.25730

0.25710

0.25690

0.25669

0.25649

0.25629

0.25609

0.25588

0.25568

0.25548

0.25528

0.25508

0.25488

0.25467

0.25447

0.25427

0.25407

0.25387
21.

21

20

21

21

21

20

21

21

21

20

21

21

20

21

20

21

20

21

21

20

21

20

20

21

20

21

20

21

20

20

21

20

20

21

20

20

20

21

20

20

20

20

20

21

20

20

20

20

0.56307

23

0.5628423

$0.56261 \quad 22$

$0.56239 \quad 23$

$0.56216 \quad 23$

$0.56193 \quad 22$

$0.56171 \quad 23$

$0.56148 \quad 23$

$0.56125 \quad 23$

$0.56102 \quad 22$

$0.56080 \quad 23$

$0.56057 \quad 23$

$0.56034 \quad 22$

$0.56012 \quad 23$

0.5598923

$0.55966 \quad 22$

0.5594423

$0.55921 \quad 23$

$0.55898 \quad 22$ 
Table 1 Harmonic Oscillator Contributions (in dimensionless form) to the

Thermodynamic Functions for Values of $\mathrm{X}=\mathrm{hcv} / \mathrm{kT}$
$\mathbf{X} \quad-\left(\mathrm{F}^{\circ}-\mathrm{E}_{\mathrm{O}}^{\circ}\right) / \mathrm{RT}$
$\Delta \quad\left(\mathrm{H}^{\circ}-\mathrm{E}_{\mathrm{O}}^{\circ}\right) / \mathrm{RT} \quad \Delta \quad \mathrm{S} / \mathrm{R}$
$\Delta \quad \mathrm{Co} / \mathrm{R}$

$\Delta$

\begin{tabular}{|c|c|c|c|c|c|c|c|}
\hline 2.750 & 0.06606 & 7 & 0.18781 & 13 & 0.25387 & 20 & 0.55174 \\
\hline $\begin{array}{l}2.751 \\
2.752 \\
2.753 \\
2.754 \\
2.755\end{array}$ & $\begin{array}{l}0.06599 \\
0.06593 \\
0.06586 \\
0.06579 \\
0.06572\end{array}$ & $\begin{array}{l}6 \\
7 \\
7 \\
7 \\
7\end{array}$ & $\begin{array}{l}0.18768 \\
0.18754 \\
0.18741 \\
0.18728 \\
0.18715\end{array}$ & $\begin{array}{l}14 \\
13 \\
13 \\
13 \\
13\end{array}$ & $\begin{array}{l}0.25367 \\
0.25347 \\
0.25327 \\
0.25307 \\
0.25287\end{array}$ & $\begin{array}{l}20 \\
20 \\
20 \\
20 \\
20\end{array}$ & $\begin{array}{l}0.55152 \\
0.55129 \\
0.55107 \\
0.55084 \\
0.55061\end{array}$ \\
\hline $\begin{array}{l}2.756 \\
2.757 \\
2.758 \\
2.759 \\
2.760\end{array}$ & $\begin{array}{l}0.06565 \\
0.06559 \\
0.06552 \\
0.06545 \\
0.06538\end{array}$ & $\begin{array}{l}6 \\
7 \\
7 \\
7 \\
6\end{array}$ & $\begin{array}{l}0.18702 \\
0.18688 \\
0.18675 \\
0.18662 \\
0.18649\end{array}$ & $\begin{array}{l}14 \\
13 \\
13 \\
13 \\
13\end{array}$ & $\begin{array}{l}0.25267 \\
0.25247 \\
0.25227 \\
0.25207 \\
0.25187\end{array}$ & $\begin{array}{l}20 \\
20 \\
20 \\
20 \\
20\end{array}$ & $\begin{array}{l}0.55039 \\
0.55016 \\
0.54994 \\
0.54971 \\
0.54949\end{array}$ \\
\hline $\begin{array}{l}2.761 \\
2.762 \\
2.763 \\
2.764 \\
2.765\end{array}$ & $\begin{array}{l}0.06532 \\
0.06525 \\
0.06518 \\
0.06511 \\
0.06505\end{array}$ & $\begin{array}{l}7 \\
7 \\
7 \\
6 \\
7\end{array}$ & $\begin{array}{l}0.18636 \\
0.18623 \\
0.18609 \\
0.18596 \\
0.18583\end{array}$ & $\begin{array}{l}13 \\
14 \\
13 \\
13 \\
13\end{array}$ & $\begin{array}{l}0.25167 \\
0.25147 \\
0.25128 \\
0.25108 \\
0.25088\end{array}$ & $\begin{array}{l}20 \\
19 \\
20 \\
20 \\
20\end{array}$ & $\begin{array}{l}0.54926 \\
0.54904 \\
0.54881 \\
0.54858 \\
0.54836\end{array}$ \\
\hline $\begin{array}{l}2.766 \\
2.767 \\
2.768 \\
2.769 \\
2.770\end{array}$ & $\begin{array}{l}0.06498 \\
0.06491 \\
0.06485 \\
0.06478 \\
0.06471\end{array}$ & $\begin{array}{l}7 \\
6 \\
7 \\
7 \\
7\end{array}$ & $\begin{array}{l}0.18570 \\
0.18557 \\
0.18544 \\
0.18531 \\
0.18518\end{array}$ & $\begin{array}{l}13 \\
13 \\
13 \\
13 \\
13\end{array}$ & $\begin{array}{l}0.25068 \\
0.25048 \\
0.25028 \\
0.25009 \\
0.24989\end{array}$ & $\begin{array}{l}20 \\
20 \\
19 \\
20 \\
20\end{array}$ & $\begin{array}{l}0.54813 \\
0.54791 \\
0.54768 \\
0.54746 \\
0.54723\end{array}$ \\
\hline $\begin{array}{l}2.771 \\
2.772 \\
2.773 \\
2.774 \\
2.775\end{array}$ & $\begin{array}{l}0.06464 \\
0.06458 \\
0.06451 \\
0.06444 \\
0.06438\end{array}$ & $\begin{array}{l}6 \\
7 \\
7 \\
6 \\
7\end{array}$ & $\begin{array}{l}0.18505 \\
0.18492 \\
0.18479 \\
0.18466 \\
0.18452\end{array}$ & $\begin{array}{l}13 \\
13 \\
13 \\
14 \\
13\end{array}$ & $\begin{array}{l}0.24969 \\
0.24949 \\
0.24930 \\
0.24910 \\
0.24890\end{array}$ & $\begin{array}{l}20 \\
19 \\
20 \\
20 \\
19\end{array}$ & $\begin{array}{l}0.54701 \\
0.54678 \\
0.54656 \\
0.54633 \\
0.54611\end{array}$ \\
\hline $\begin{array}{l}2.776 \\
2.777 \\
2.778 \\
2.779 \\
2.780\end{array}$ & $\begin{array}{l}0.06431 \\
0.06425 \\
0.06418 \\
0.06411 \\
0.06405\end{array}$ & $\begin{array}{l}6 \\
7 \\
7 \\
6 \\
7\end{array}$ & $\begin{array}{l}0.18439 \\
0.18426 \\
0.18413 \\
0.18400 \\
0.18387\end{array}$ & $\begin{array}{l}13 \\
13 \\
13 \\
13 \\
13\end{array}$ & $\begin{array}{l}0.24871 \\
0.24851 \\
0.24831 \\
0.24812 \\
0.24792\end{array}$ & $\begin{array}{l}20 \\
20 \\
19 \\
20 \\
20\end{array}$ & $\begin{array}{l}0.54588 \\
0.54566 \\
0.54543 \\
0.54521 \\
0.54498\end{array}$ \\
\hline $\begin{array}{l}2.781 \\
2.782 \\
2.783 \\
2.784 \\
2.785\end{array}$ & $\begin{array}{l}0.06398 \\
0.06391 \\
0.06385 \\
0.06378 \\
0.06372\end{array}$ & $\begin{array}{l}7 \\
6 \\
7 \\
6 \\
7\end{array}$ & $\begin{array}{l}0.18374 \\
0.18361 \\
0.18349 \\
0.18336 \\
0.18323\end{array}$ & $\begin{array}{l}13 \\
12 \\
13 \\
13 \\
13\end{array}$ & $\begin{array}{l}0.24772 \\
0.24753 \\
0.24733 \\
0.24714 \\
0.24694\end{array}$ & $\begin{array}{l}19 \\
20 \\
19 \\
20 \\
19\end{array}$ & $\begin{array}{l}0.54476 \\
0.54453 \\
0.54431 \\
0.54408 \\
0.54386\end{array}$ \\
\hline $\begin{array}{l}2.786 \\
2.787 \\
2.788 \\
2.789 \\
2.790\end{array}$ & $\begin{array}{l}0.06365 \\
0.06359 \\
0.06352 \\
0.06345 \\
0.06339\end{array}$ & $\begin{array}{l}6 \\
7 \\
7 \\
6 \\
7\end{array}$ & $\begin{array}{l}0.18310 \\
0.18297 \\
0.18284 \\
0.18271 \\
0.18258\end{array}$ & $\begin{array}{l}13 \\
13 \\
13 \\
13 \\
13\end{array}$ & $\begin{array}{l}0.24675 \\
0.24655 \\
0.24636 \\
0.24616 \\
0.24597\end{array}$ & $\begin{array}{l}20 \\
19 \\
20 \\
19 \\
20\end{array}$ & $\begin{array}{l}0.54363 \\
0.54341 \\
0.54318 \\
0.54296 \\
0.54273\end{array}$ \\
\hline $\begin{array}{l}2.791 \\
2.792 \\
2.793 \\
2.794 \\
2.795\end{array}$ & $\begin{array}{l}0.06332 \\
0.06326 \\
0.06319 \\
0.06313 \\
0.06306\end{array}$ & $\begin{array}{l}6 \\
7 \\
6 \\
7 \\
6\end{array}$ & $\begin{array}{l}0.18245 \\
0.18232 \\
0.18219 \\
0.18206 \\
0.18194\end{array}$ & $\begin{array}{l}13 \\
13 \\
13 \\
12 \\
13\end{array}$ & $\begin{array}{l}0.24577 \\
0.24558 \\
0.24538 \\
0.24519 \\
0.24500\end{array}$ & $\begin{array}{l}19 \\
20 \\
19 \\
19 \\
20\end{array}$ & $\begin{array}{l}0.54251 \\
0.54228 \\
0.54206 \\
0.54183 \\
0.54161\end{array}$ \\
\hline $\begin{array}{l}2.796 \\
2.797 \\
2.798 \\
2.799 \\
2.800\end{array}$ & $\begin{array}{l}0.06300 \\
0.06293 \\
0.06287 \\
0.06280 \\
0.06274\end{array}$ & $\begin{array}{l}7 \\
6 \\
7 \\
6\end{array}$ & $\begin{array}{l}0.18181 \\
0.18168 \\
0.18155 \\
0.18142 \\
0.18129\end{array}$ & $\begin{array}{l}13 \\
13 \\
13 \\
13\end{array}$ & $\begin{array}{l}0.24480 \\
0.24461 \\
0.24442 \\
0.24422 \\
0.24403\end{array}$ & $\begin{array}{l}19 \\
19 \\
20 \\
19\end{array}$ & $\begin{array}{l}0.54138 \\
0.54116 \\
0.54094 \\
0.54071 \\
0.54049\end{array}$ \\
\hline
\end{tabular}


Table I Harmonic Oscillator Contributions (in dimensionless form) to the Thermodynamic Functions for Values of $\mathrm{X}=\mathrm{hcv} / \mathrm{kT}$

$\begin{array}{llllllllll}\mathrm{C} & -\left(\mathrm{F}^{\circ}-\mathrm{E}_{\mathrm{O}}^{\circ}\right) / \mathrm{RT} & \Delta & \left(\mathrm{H}^{\circ}-\mathrm{E}_{\mathrm{O}}^{\circ}\right) / \mathrm{RT} & \Delta & \mathrm{R} & \mathrm{R} / \mathrm{R} & \Delta\end{array}$

\begin{tabular}{|c|c|c|c|c|c|c|c|}
\hline 2.800 & 0.06274 & 7 & 0.18129 & 13 & 0.24403 & 19 & 0.54049 \\
\hline $\begin{array}{l}2.801 \\
2.802 \\
2.803 \\
2.804 \\
2.805\end{array}$ & $\begin{array}{l}0.06267 \\
0.06261 \\
0.06254 \\
0.06248 \\
0.06241\end{array}$ & $\begin{array}{l}6 \\
7 \\
6 \\
7 \\
6\end{array}$ & $\begin{array}{l}0.18116 \\
0.18104 \\
0.18091 \\
0.18078 \\
0.18065\end{array}$ & $\begin{array}{l}12 \\
13 \\
13 \\
13 \\
13\end{array}$ & $\begin{array}{l}0.24384 \\
0.24364 \\
0.24345 \\
0.24326 \\
0.24307\end{array}$ & $\begin{array}{l}20 \\
19 \\
19 \\
19 \\
20\end{array}$ & $\begin{array}{l}0.54026 \\
0.54004 \\
0.53981 \\
0.53959 \\
0.53936\end{array}$ \\
\hline $\begin{array}{l}2.806 \\
2.807 \\
2.808 \\
2.809 \\
2.810\end{array}$ & $\begin{array}{l}0.06235 \\
0.06229 \\
0.06222 \\
0.06216 \\
0.06209\end{array}$ & $\begin{array}{l}6 \\
7 \\
6 \\
7 \\
6\end{array}$ & $\begin{array}{l}0.18052 \\
0.18040 \\
0.18027 \\
0.18014 \\
0.18001\end{array}$ & $\begin{array}{l}12 \\
13 \\
13 \\
13 \\
12\end{array}$ & $\begin{array}{l}0.24287 \\
0.24268 \\
0.24249 \\
0.24230 \\
0.24211\end{array}$ & $\begin{array}{l}19 \\
19 \\
19 \\
19 \\
19\end{array}$ & $\begin{array}{l}0.53914 \\
0.53892 \\
0.53869 \\
0.53847 \\
0.53824\end{array}$ \\
\hline $\begin{array}{l}2.811 \\
2.812 \\
2.813 \\
2.814 \\
2.815\end{array}$ & $\begin{array}{l}0.06203 \\
0.06197 \\
0.06190 \\
0.06184 \\
0.06177\end{array}$ & $\begin{array}{l}6 \\
7 \\
6 \\
7 \\
6\end{array}$ & $\begin{array}{l}0.17989 \\
0.17976 \\
0.17963 \\
0.17950 \\
0.17938\end{array}$ & $\begin{array}{l}13 \\
13 \\
13 \\
12 \\
13\end{array}$ & $\begin{array}{l}0.24192 \\
0.24172 \\
0.24153 \\
0.24134 \\
0.24115\end{array}$ & $\begin{array}{l}20 \\
19 \\
19 \\
19 \\
19\end{array}$ & $\begin{array}{l}0.53802 \\
0.53780 \\
0.53757 \\
0.53735 \\
0.53712\end{array}$ \\
\hline $\begin{array}{l}2.816 \\
2.817 \\
2.818 \\
2.819 \\
2.820\end{array}$ & $\begin{array}{l}0.06171 \\
0.06165 \\
0.06158 \\
0.06152 \\
0.06146\end{array}$ & $\begin{array}{l}6 \\
7 \\
6 \\
6 \\
7\end{array}$ & $\begin{array}{l}0.17925 \\
0.17912 \\
0.17900 \\
0.17887 \\
0.17874\end{array}$ & $\begin{array}{l}13 \\
12 \\
13 \\
13 \\
12\end{array}$ & $\begin{array}{l}0.24096 \\
0.24077 \\
0.24058 \\
0.24039 \\
0.24020\end{array}$ & $\begin{array}{l}19 \\
19 \\
19 \\
19 \\
19\end{array}$ & $\begin{array}{l}0.53690 \\
0.53668 \\
0.53645 \\
0.53623 \\
0.53600\end{array}$ \\
\hline $\begin{array}{l}2.821 \\
2.822 \\
2.823 \\
2.824 \\
2.825\end{array}$ & $\begin{array}{l}0.06139 \\
0.06133 \\
0.06127 \\
0.06120 \\
0.06114\end{array}$ & $\begin{array}{l}6 \\
6 \\
7 \\
6 \\
6\end{array}$ & $\begin{array}{l}0.17862 \\
0.17849 \\
0.17836 \\
0.17824 \\
0.17811\end{array}$ & $\begin{array}{l}13 \\
13 \\
12 \\
13 \\
13\end{array}$ & $\begin{array}{l}0.24001 \\
0.23982 \\
0.23963 \\
0.23944 \\
0.23925\end{array}$ & $\begin{array}{l}19 \\
19 \\
19 \\
19 \\
19\end{array}$ & $\begin{array}{l}0.53578 \\
0.53556 \\
0.53533 \\
0.53511 \\
0.53489\end{array}$ \\
\hline $\begin{array}{l}2.826 \\
2.827 \\
2.828 \\
2.829 \\
2.830\end{array}$ & $\begin{array}{l}0.06108 \\
0.06101 \\
0.06095 \\
0.06089 \\
0.06083\end{array}$ & $\begin{array}{l}7 \\
6 \\
6 \\
6 \\
7\end{array}$ & $\begin{array}{l}0.17798 \\
0.17786 \\
0.17773 \\
0.17761 \\
0.17748\end{array}$ & $\begin{array}{l}12 \\
13 \\
12 \\
13 \\
13\end{array}$ & $\begin{array}{l}0.23906 \\
0.23887 \\
0.23868 \\
0.23849 \\
0.23831\end{array}$ & $\begin{array}{l}19 \\
19 \\
19 \\
18 \\
19\end{array}$ & $\begin{array}{l}0.53466 \\
0.53444 \\
0.53421 \\
0.53399 \\
0.53377\end{array}$ \\
\hline $\begin{array}{l}2.831 \\
2.832 \\
2.833 \\
2.834 \\
2.835\end{array}$ & $\begin{array}{l}0.06076 \\
0.06070 \\
0.06064 \\
0.06058 \\
0.06051\end{array}$ & $\begin{array}{l}6 \\
6 \\
6 \\
7 \\
6\end{array}$ & $\begin{array}{l}0.17735 \\
0.17723 \\
0.17710 \\
0.17698 \\
0.17685\end{array}$ & $\begin{array}{l}12 \\
13 \\
12 \\
13 \\
12\end{array}$ & $\begin{array}{l}0.23812 \\
0.23793 \\
0.23774 \\
0.23755 \\
0.23736\end{array}$ & $\begin{array}{l}19 \\
19 \\
19 \\
19 \\
18\end{array}$ & $\begin{array}{l}0.53354 \\
0.53332 \\
0.53310 \\
0.53287 \\
0.53265\end{array}$ \\
\hline $\begin{array}{l}2.836 \\
2.837 \\
2.838 \\
2.839 \\
2.840\end{array}$ & $\begin{array}{l}0.06045 \\
0.06039 \\
0.06033 \\
0.06026 \\
0.06020\end{array}$ & $\begin{array}{l}6 \\
6 \\
7 \\
6 \\
6\end{array}$ & $\begin{array}{l}0.17673 \\
0.17660 \\
0.17648 \\
0.17635 \\
0.17622\end{array}$ & $\begin{array}{l}13 \\
12 \\
13 \\
13 \\
12\end{array}$ & $\begin{array}{l}0.23718 \\
0.23699 \\
0.23680 \\
0.23661 \\
0.23643\end{array}$ & $\begin{array}{l}19 \\
19 \\
19 \\
18 \\
19\end{array}$ & $\begin{array}{l}0.53243 \\
0.53220 \\
0.53198 \\
0.53176 \\
0.53153\end{array}$ \\
\hline $\begin{array}{l}2.841 \\
2.842 \\
2.843 \\
2.844 \\
2.845\end{array}$ & $\begin{array}{l}0.06014 \\
0.06008 \\
0.06002 \\
0.05995 \\
0.05989\end{array}$ & $\begin{array}{l}6 \\
6 \\
7 \\
6 \\
6\end{array}$ & $\begin{array}{l}0.17610 \\
0.17597 \\
0.17585 \\
0.17573 \\
0.17560\end{array}$ & $\begin{array}{l}13 \\
12 \\
12 \\
13 \\
12\end{array}$ & $\begin{array}{l}0.23624 \\
0.23605 \\
0.23587 \\
0.23568 \\
0.23549\end{array}$ & $\begin{array}{l}19 \\
18 \\
19 \\
19 \\
18\end{array}$ & $\begin{array}{l}0.53131 \\
0.53109 \\
0.53086 \\
0.53064 \\
0.53042\end{array}$ \\
\hline $\begin{array}{l}2.846 \\
2.847 \\
2.848 \\
2.849 \\
2.850\end{array}$ & $\begin{array}{l}0.05983 \\
0.05977 \\
0.05971 \\
0.05965 \\
0.05958\end{array}$ & $\begin{array}{l}6 \\
6 \\
6 \\
7\end{array}$ & $\begin{array}{l}0.17548 \\
0.17535 \\
0.17523 \\
0.17510 \\
0.17498\end{array}$ & $\begin{array}{l}13 \\
12 \\
13 \\
12\end{array}$ & $\begin{array}{l}0.23531 \\
0.23512 \\
0.23493 \\
0.23475 \\
0.23456\end{array}$ & $\begin{array}{l}19 \\
19 \\
18 \\
19\end{array}$ & $\begin{array}{l}0.53020 \\
0.52997 \\
0.52975 \\
0.52953 \\
0.52 .930\end{array}$ \\
\hline
\end{tabular}


Table I Harmonic Oscillator Contributions (in dimensionless form) to the

Thermodynamic Functions for Values of $X=h c v / k T$

$\mathbf{X}$

$-\left(F^{\circ}-E_{O}^{\circ}\right) / R T$

$\Delta \quad\left(\mathrm{H}^{\circ}-\mathrm{E}_{\mathrm{O}}^{\mathrm{O}}\right) / \mathrm{RT}$

$\Delta$

$\mathrm{S} \% / \mathrm{R}$

0.17498

2.850

2.851

2.852

2.853

2.854

2.855

2.856

2.857

2.858

2.859

2.860

2.861

2.862

2.863

2.864

2. 865

2.866

2.867

2.868

2.869

2.870

2.871

2.872

2.873

2.874

2.875

2.876

2.877

2.878

2.879

2.880

2.881

2.882

2.883

2. 884

2.885

2.886

2.887

2.888

2.889

2.890

2.891

2.892

2.893

2.894

2.895

2.896

2.897

2.898

2.899

2.900

\begin{abstract}
0.05958
\end{abstract}
0.05952

0.05946

0.05940

0.05934

0.05928

0.05922

0.05916

0.05910

0.05903

0.05897

0.05891

0.05885

0.05879

0.05873

0.05867

0.05861

0.05855

0.05849

0.05843

0.05837

0.05831

0.05825

0.05819

0.05813

0.05807

0.05801

0.05795

0.05789

0.05783

0.05777

0.05771

0.05765

0.05759

0.05753

0.05748

0.05742

0.05736

0.05730

0.05724

0.05718

0.05712

0.05706

0.05700

0.05695

0.05689

0.05683

0.05677

0.05671

0.05665

0.05659
6

6

6
6

$\begin{array}{ll}0.17177 & 12 \\ 0.17165 & 12 \\ 0.17153 & 12 \\ 0.17141 & 13 \\ 0.17128 & 12\end{array}$

$0.17116 \quad 12$

0.17104

0.17092

0.17080

$0.17055 \quad 12$

$0.17031 \quad 12$

$0.17019 \quad 12$

$0.17007 \quad 12$

0.16995

0.16982

0.16970

0.16946

0.16934

12
12

0.16910

0.16886

\begin{tabular}{|c|c|c|c|c|c|}
\hline 6 & 0.17498 & 13 & 0.23456 & 18 & 0.52930 \\
\hline $\begin{array}{l}6 \\
6\end{array}$ & $\begin{array}{l}0.17485 \\
0.17473\end{array}$ & $\begin{array}{l}12 \\
12\end{array}$ & $\begin{array}{l}0.23438 \\
0.23419\end{array}$ & $\begin{array}{l}19 \\
18\end{array}$ & $\begin{array}{l}0.52908 \\
0.52886\end{array}$ \\
\hline 6 & 0.17461 & 13 & 0.23401 & 19 & 0.52864 \\
\hline 6 & 0.17448 & 12 & 0.23382 & 18 & 0.52841 \\
\hline 6 & 0.17436 & 13 & 0.23364 & 19 & 0.52819 \\
\hline 6 & 0.17423 & 12 & 0.23345 & 18 & 0.52797 \\
\hline 6 & 0.17411 & 12 & 0.23327 & 19 & 0.52774 \\
\hline 7 & 0.17399 & 13 & 0.23308 & 18 & 0.52752 \\
\hline 6 & 0.17386 & 12 & 0.23290 & 19 & 0.52730 \\
\hline 6 & 0.17374 & 13 & 0.23271 & 18 & 0.52708 \\
\hline 6 & 0.17361 & 12 & 0.23253 & 19 & 0.52685 \\
\hline 6 & 0.17349 & 12 & 0.23234 & 18 & 0.52663 \\
\hline 6 & 0.17337 & 13 & 0.23216 & 18 & 0.52641 \\
\hline 6 & 0.17324 & 12 & 0.23198 & 19 & 0.52619 \\
\hline 6 & 0.17312 & 12 & 0.23179 & 18 & 0.52596 \\
\hline 6 & 0.17300 & 12 & 0.23161 & 18 & 0.52574 \\
\hline 6 & 0.17288 & 13 & 0.23143 & 19 & 0.52552 \\
\hline 6 & 0.17275 & 12 & 0.23124 & 18 & 0.52530 \\
\hline 6 & 0.17263 & 12 & 0.23106 & 18 & 0.52508 \\
\hline 6 & 0.17251 & 13 & 0.23088 & 19 & 0.52485 \\
\hline 6 & 0.17238 & 12 & 0.23069 & 18 & 0.52463 \\
\hline 6 & 0.17226 & 12 & 0.23051 & 18 & 0.52441 \\
\hline 6 & 0.17214 & 12 & 0.23033 & 18 & 0.52419 \\
\hline 6 & 0.17202 & 13 & 0.23015 & 19 & 0.52396 \\
\hline 6 & 0.17189 & 12 & 0.22996 & 18 & 0.52374 \\
\hline
\end{tabular}

0.22978

18

0.22960

0.22942

0.22924

0.22905

\section{8}

18

19

18

0.22887

0.22869

0.22851

0.22833

0.22815

\section{8}

18

18

18

18

0.22797

18

$0.17043 \quad 12$

0.22779

0.22761

0.22743

0.22725

\section{8}

18

18

18

0.22707

18

0.22689

0.22671

0.22653

0.22635

\section{8}

18

18

18

0.22617

0.16922

0.22599

0.22581

0.22563 $\begin{array}{ll}0.52352 & 22 \\ 0.52330 & 22 \\ 0.52308 & 23 \\ 0.52285 & 22 \\ 0.52263 & 22\end{array}$

$0.52241 \quad 22$

$0.52219 \quad 22$

$0.52175 \quad 23$

$0.52152 \quad 22$

$0.52130 \quad 22$

$0.52108 \quad 22$

$0.52086 \quad 22$

0.5206422

0.5204223

$0.51997 \quad 22$

$0.51975 \quad 22$

$0.51953 \quad 22$

$0.51931 \quad 22$

18

18

18

18

$0.51909 \quad 22$

$0.51887 \quad 22$

$0.51865 \quad 23$

$0.51842 \quad 22$
$0.52197 \quad 22$

$0.52019 \quad 22$

0.51820
$\Delta \quad \mathrm{co} / \mathrm{R}$

$\Delta$ 
Table I Harmonic Oscillator Contributions (in dimensionless form) to the

Thermodynamic Functions for Values of $\mathrm{X}=\mathrm{hcv} / \mathrm{kT}$

$\mathbf{X}$

$-\left(F^{\circ}-E_{O}^{\circ}\right) / R T$

$\Delta \quad\left(\mathrm{H}^{\circ}-\mathrm{E}_{\mathrm{O}}^{\circ}\right) / \mathrm{RT}$

$\Delta$

2.900

2.902

2.903

2.904

2.905

2.906

2.907

2.908

2.909

2.910

2. 911

2. 912

2. 913

2. 914

2. 915

2.916

2.917

2.918

2.919

2.920

2. 921

2. 922

2.923

2.924

2. 925

2.926

2.927

2. 928

2.929

2.930

2.931

2. 932

2.933

2.934

2.935

2.936

2.937

2. 938

2.939

2. 940

2.941

2.942

2. 943

2. 944

2. 945

2. 946

2.947

2. 948

2. 949

2.950

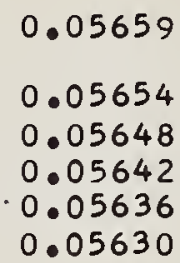

0.05625

0.05619

0.05607

0.05602

0.05596

0.05590

0.05584

0.05579

0.05573

0.05567

0.05561

0.05556

0.05550

0.05544

0.05539

0.05533

0.05527

0.05522

0.05516

0.05510

0.05505

0.05499

0.05493

0.05488

0.05482

0.05476

0.05471

0.05465

0.05459

0.05454

0.05448

0.05443

0.05437

0.05431

0.05426

0.05420

0.05415

0.05409

0.05404

0.05398

0.05393

0.05387

0.05381

0.05376
0.05613

\subsection{6 \\ 0.16862 \\ 0.16850 \\ 0.16838 \\ 0.16826}

12

12

12

12

12

12

0.16814

0.16802

0.16790

0.16778

0.16766

12

12

12

12

12

0.16754

0.16742

0.16730

0.16718

0.16706

0.16694

0.16682

0.16670

0.16658

0.16646

12

12

12

12

12

12

12

12

12

11

0.16635

12

0.16623

0.16611

0.16599

0.16587

0.16575

0.16563

0.16552

0.16540

0.16528

0.16516

0.16504

0.16492

0.16481

0.16469

0.16457

0.16445

0.16434

0.16422

0.16410

12

12

12

12

12

11

12

12

12

12

12

11

12

12

12

11

12

12

12

$0.16398 \quad 11$

0.16387

12

0.16375

0.16363

0.16351

12

12

11

$0.16340 \quad 12$

12

0.16316

0.16305

0.16293
0.16328
$S \circ / R$

0.22545
0.22527
0.22510
0.22492
0.22474
0.22456

18

$\Delta$

$\mathrm{Co} / \mathrm{R}$

$\Delta$

0.22438

0.22421

0.22403

0.22385

0.22367

0.22350

0.22332

0.22314

0.22297

0.22279

0.22261

0.22244

0.22226

0.22208

0.22191

0.22173

0.22156

0.22138

0.22120

0.22103

0.22085

0.22068

0.22050

0.22033

0.22015

0.21998

0.21981

0.21963

0.21946

0.21928

0.21911

0.21894

0.21876

0.21859

0.21841

0.21824

0.21807

0.21790

0.21772

0.21755

0.21738

0.21721

0.21703

0.21686

0.21669

0.51820

22

17

0.51798

22

18

18

18

18

0.51776

0.51754

22

0.51732

$0.51710 \quad 22$

17

0.51688

22

18

18

18

17

0.51666

0.51643

0.51621

0.51599

23

22

22

22

18

0.51577

0.51555

22

17

18 
Table I Harmonic Oscillator Contributions (in dimensionless form) to the

Thermodynamic Functions for Values of $X=h c \nu / k T$

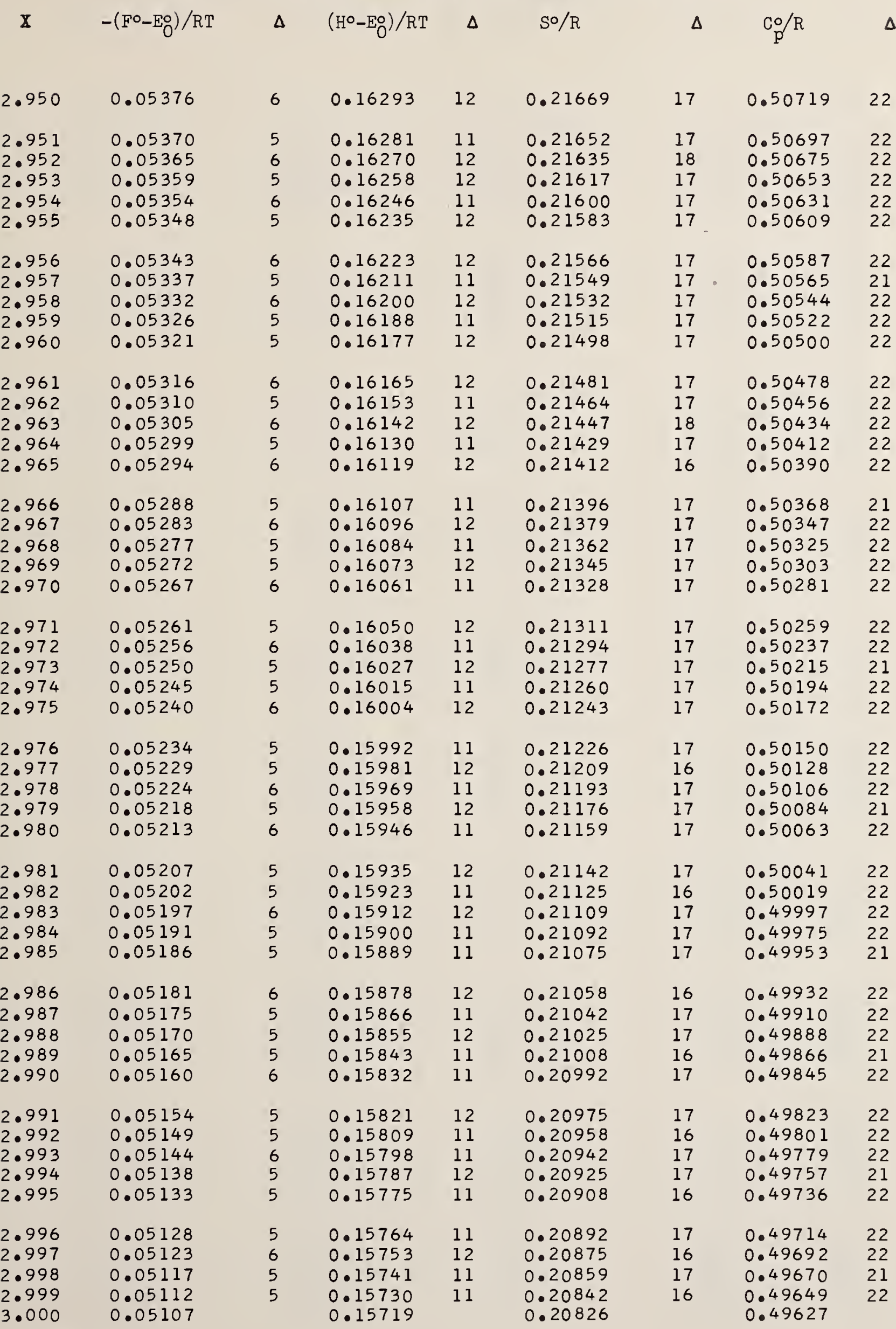


Table I Harmonic Oscillator Contributions (in dimensionless form) to the

Thermodynamic Functions for Values of $\mathrm{X}=\mathrm{hc} / \mathrm{kT}$

$\mathbf{X}$ $-\left(F^{\circ}-E_{O}^{\circ}\right) / R T$

$\Delta \quad\left(\mathrm{H}^{\circ}-\mathrm{E}_{\mathrm{O}}\right) / \mathrm{RT}$

0.05107
0.05102
0.05096
0.05091
0.05086
0.05081
0.05076
0.05070
0.05065
0.05060
0.05055

3.011

3.012

3.013

3.014

3.015

3.016

3.017

3.018

3.019

3.020

3.021

3.022

3.023

3.024

3.025

3.026

3.027

3.028

3.029

3.030

3.031

3.032

3.033

3.034

3.035

3.036

3.037

3.038

3.039

3.040

3.041

3.042

3.043

3.044

3.045

3.046

3.047

3.048

3.049

3.050
0.05050

0.05044

0.05039

0.05034

0.05029

0.05024

0.05019

0.05013

0.05008

0.05003

0.04998

0.04993

0.04988

0.04983

0.04978

0.04973

0.04967

0.04962

0.04957

0.04952

0.04947

0.04942

0.04937

0.04932

0.04927

0.04922

0.04917

0.04912

0.04907

0.04902

0.04897

0.04892

0.04887

0.04882

0.04877

0.04872

0.04867

0.04862

0.04857

0.04852
$\Delta$

So/R

$\begin{array}{lll}0.15719 & 12 & 0.20826 \\ 0.15707 & 11 & 0.20809 \\ 0.15696 & 11 & 0.20793 \\ 0.15685 & 11 & 0.20776 \\ 0.15674 & 12 & 0.20760 \\ 0.15662 & 11 & 0.20743 \\ 0.15651 & 11 & 0.20727 \\ 0.15640 & 11 & 0.20710 \\ 0.15629 & 12 & 0.20694 \\ 0.15617 & 11 & 0.20677 \\ 0.15606 & 11 & 0.20661\end{array}$

17

$\Delta$

$\mathrm{Co} / \mathrm{R}$

$0.49627 \quad 22$

$16 \quad 0.49605 \quad 22$

$\begin{array}{lll}17 & 0.49583 & 21\end{array}$

$16 \quad 0.49562 \quad 22$

$17 \quad 0.49540 \quad 22$

$16 \quad 0.49518 \quad 21$

$\begin{array}{lll}17 & 0.49497 & 22\end{array}$

$\begin{array}{lll}16 & 0.49475 & 22\end{array}$

$17 \quad 0.49453 \quad 22$

$\begin{array}{lll}16 & 0.49431 & 21\end{array}$

$17 \quad 0.49410 \quad 22$

$\begin{array}{llllll}0.15595 & 11 & 0.20644 & 16 & 0.49388 & 22 \\ 0.15584 & 12 & 0.20628 & 16 & 0.49366 & 21 \\ 0.15572 & 11 & 0.20612 & 17 & 0.49345 & 22 \\ 0.15561 & 11 & 0.20595 & 16 & 0.49323 & 22 \\ 0.15550 & 11 & 0.20579 & 16 & 0.49301 & 21\end{array}$

\section{$0.15539 \quad 11 \quad 0.20563$}

0.15528

0.15516

0.15505

0.15494

11

11

0.15483

0.15472

0.15461

0.15450

0.15438

11

11

0.15427

0.15416

0.15405

0.15394

0.15383

0.15372

0.15361

0.15350

0.15339

0.15328

0.15316

0.15305

0.15294

0.15283

0.15272

0.15261

0.15250

0.15239

0.15228

0.15217

0.15206

0.15195

0.15184

0.15174

0.15163
12

11

11

11

12

11

11

11

11

11

11

11

11

11

12

11

11

11

11

11

11

11

11

11

11

11

11

0.20546

0.20530

0.20514

0.20497

0.20481

0.20465

0.20449

0.20432

0.20416

0.20400

0.20384

0.20367

0.20351

0.20335

0.20319

0.20303

0.20287

0.20270

0.20254

0.20238

0.20222

0.20206

0.20190

0.20174

0.20158

0.20142

0.20126

0.20110

0.20094

0.20078

0.20062

0.20046

11

0.20030
0.20014 $\begin{array}{lll}17 & 0.49280 & 22\end{array}$

$\begin{array}{lll}16 & 0.49258 & 22\end{array}$

$\begin{array}{lll}16 & 0.49236 & 21\end{array}$

$17 \quad 0.49215 \quad 22$

$\begin{array}{lll}16 & 0.49193 \quad 22\end{array}$

$16 \quad 0.49171 \quad 21$

$\begin{array}{lll}16 & 0.49150 & 22\end{array}$

$17 \quad 0.49128 \quad 22$

$16 \quad 0.49106 \quad 21$

$16 \quad 0.49085 \quad 22$

$16 \quad 0.49063 \quad 22$

$17 \quad 0.49041 \quad 21$

$16 \quad 0.49020 \quad 22$

$\begin{array}{lll}16 & 0.48998 & 22\end{array}$

$16 \quad 0.48976 \quad 21$

$\begin{array}{lll}16 & 0.48955 & 22\end{array}$

$16 \quad 0.48933 \quad 21$

$17 \quad 0.48912 \quad 22$

$16 \quad 0.48890 \quad 22$

$\begin{array}{lll}16 & 0.48868 & 21\end{array}$

$\begin{array}{lll}16 & 0.48847 & 22\end{array}$

$\begin{array}{lll}16 & 0.48825 & 21\end{array}$

$16 \quad 0.48804 \quad 22$

$16 \quad 0.48782 \quad 22$

$\begin{array}{lll}16 & 0.48760 \quad 21\end{array}$

$\begin{array}{lll}16 & 0.48739 & 22\end{array}$

$\begin{array}{lll}16 & 0.48717 & 21\end{array}$

$16 \quad 0.48696 \quad 22$

$\begin{array}{lll}16 & 0.48674 & 21\end{array}$

$\begin{array}{lll}16 & 0.48653 & 22\end{array}$

$16 \quad 0.48631$

22

0.4860921

$0.48588 \quad 22$

16

$0.48566 \quad 21$

0.48545 
Table I Harmonic Oscillator Contributions (in dimensionless form) to the

Thermodynamic Functions for Values of $\mathrm{X}=\mathrm{hc} / \mathrm{kT}$

$\begin{array}{llllllll} & -\left(\mathrm{F}^{\circ}-\mathrm{E}_{\mathrm{O}}^{\circ}\right) / \mathrm{RT} & \Delta & \left(\mathrm{H}^{\circ}-\mathrm{E}_{\mathrm{O}}\right) / \mathrm{RT} & \Delta & \mathrm{S} \circ / \mathrm{R} & \Delta \quad \mathrm{C}_{\mathrm{p}} / \mathrm{R}\end{array}$

\begin{tabular}{|c|c|c|c|c|c|c|c|}
\hline 3.050 & 0.04852 & 5 & 0.15163 & 11 & 0.20014 & 16 & 0.48545 \\
\hline $\begin{array}{l}3.051 \\
3.052\end{array}$ & $\begin{array}{l}0.04847 \\
0.04842\end{array}$ & $\begin{array}{l}5 \\
5\end{array}$ & $\begin{array}{l}0.15152 \\
0.15141\end{array}$ & $\begin{array}{l}11 \\
11\end{array}$ & $\begin{array}{l}0.19998 \\
0.19982\end{array}$ & $\begin{array}{l}16 \\
15\end{array}$ & $\begin{array}{l}0.48523 \\
0.48502\end{array}$ \\
\hline $\begin{array}{l}3.053 \\
3.054 \\
3.055\end{array}$ & $\begin{array}{l}0.04837 \\
0.04832 \\
0.04827\end{array}$ & $\begin{array}{l}5 \\
5 \\
5\end{array}$ & $\begin{array}{l}0.15130 \\
0.15119 \\
0.15108\end{array}$ & $\begin{array}{ll}1 & 1 \\
1 & 1 \\
1 & 1\end{array}$ & $\begin{array}{l}0.19967 \\
0.19951 \\
0.19935\end{array}$ & $\begin{array}{l}16 \\
16 \\
16\end{array}$ & $\begin{array}{l}0.48480 \\
0.48459 \\
0.48437\end{array}$ \\
\hline $\begin{array}{l}3.056 \\
3.057 \\
3.058 \\
3.059 \\
3.060\end{array}$ & $\begin{array}{l}0.04822 \\
0.04817 \\
0.04812 \\
0.04807 \\
0.04802\end{array}$ & $\begin{array}{l}5 \\
5 \\
5 \\
5 \\
5\end{array}$ & $\begin{array}{l}0.15097 \\
0.15086 \\
0.15075 \\
0.15064 \\
0.15053\end{array}$ & $\begin{array}{ll}1 & 1 \\
1 & 1 \\
1 & 1 \\
1 & 1 \\
1 & 0\end{array}$ & $\begin{array}{l}0.19919 \\
0.19903 \\
0.19887 \\
0.19872 \\
0.19856\end{array}$ & $\begin{array}{l}16 \\
16 \\
15 \\
16 \\
16\end{array}$ & $\begin{array}{l}0.48416 \\
0.48394 \\
0.48373 \\
0.48351 \\
0.48330\end{array}$ \\
\hline $\begin{array}{l}3.061 \\
3.062 \\
3.063 \\
3.064 \\
3.065\end{array}$ & $\begin{array}{l}0.04797 \\
0.04792 \\
0.04788 \\
0.04783 \\
0.04778\end{array}$ & $\begin{array}{l}5 \\
4 \\
5 \\
5 \\
5\end{array}$ & $\begin{array}{l}0.15043 \\
0.15032 \\
0.15021 \\
0.15010 \\
0.14999\end{array}$ & $\begin{array}{l}11 \\
11 \\
11 \\
11 \\
11\end{array}$ & $\begin{array}{l}0.19840 \\
0.19824 \\
0.19808 \\
0.19793 \\
0.19777\end{array}$ & $\begin{array}{l}16 \\
16 \\
15 \\
16 \\
16\end{array}$ & $\begin{array}{l}0.48308 \\
0.48287 \\
0.48265 \\
0.48244 \\
0.48222\end{array}$ \\
\hline $\begin{array}{l}3.066 \\
3.067 \\
3.068 \\
3.069 \\
3.070\end{array}$ & $\begin{array}{l}0.04773 \\
0.04768 \\
0.04763 \\
0.04758 \\
0.04753\end{array}$ & $\begin{array}{l}5 \\
5 \\
5 \\
5 \\
5\end{array}$ & $\begin{array}{l}0.14988 \\
0.14978 \\
0.14967 \\
0.14956 \\
0.14945\end{array}$ & $\begin{array}{l}10 \\
11 \\
11 \\
11 \\
11\end{array}$ & $\begin{array}{l}0.19761 \\
0.19745 \\
0.19730 \\
0.19714 \\
0.19698\end{array}$ & $\begin{array}{l}16 \\
15 \\
16 \\
16 \\
15\end{array}$ & $\begin{array}{l}0.48201 \\
0.48179 \\
0.48158 \\
0.48136 \\
0.48115\end{array}$ \\
\hline $\begin{array}{l}3.071 \\
3.072 \\
3.073 \\
3.074 \\
3.075\end{array}$ & $\begin{array}{l}0.04748 \\
0.04744 \\
0.04739 \\
0.04734 \\
0.04729\end{array}$ & $\begin{array}{l}4 \\
5 \\
5 \\
5 \\
5\end{array}$ & $\begin{array}{l}0.14934 \\
0.14923 \\
0.14913 \\
0.14902 \\
0.14891\end{array}$ & $\begin{array}{l}11 \\
10 \\
11 \\
11 \\
11\end{array}$ & $\begin{array}{l}0.19683 \\
0.19667 \\
0.19651 \\
0.19636 \\
0.19620\end{array}$ & $\begin{array}{l}16 \\
16 \\
15 \\
16 \\
15\end{array}$ & $\begin{array}{l}0.48093 \\
0.48072 \\
0.48051 \\
0.48029 \\
0.48008\end{array}$ \\
\hline $\begin{array}{l}3.076 \\
3.077 \\
3.078 \\
3.079 \\
3.080\end{array}$ & $\begin{array}{l}0.04724 \\
0.04719 \\
0.04715 \\
0.04710 \\
0.04705\end{array}$ & $\begin{array}{l}5 \\
4 \\
5 \\
5 \\
5\end{array}$ & $\begin{array}{l}0.14880 \\
0.14870 \\
0.14859 \\
0.14848 \\
0.14837\end{array}$ & $\begin{array}{ll}10 \\
1 & 1 \\
1 & 1 \\
1 & 1 \\
10\end{array}$ & $\begin{array}{l}0.19605 \\
0.19589 \\
0.19573 \\
0.19558 \\
0.19542\end{array}$ & $\begin{array}{l}16 \\
16 \\
15 \\
16 \\
15\end{array}$ & $\begin{array}{l}0.47986 \\
0.47965 \\
0.47943 \\
0.47922 \\
0.47901\end{array}$ \\
\hline $\begin{array}{l}3.081 \\
3.082 \\
3.083 \\
3.084 \\
3.085\end{array}$ & $\begin{array}{l}0.04700 \\
0.04695 \\
0.04690 \\
0.04686 \\
0.04681\end{array}$ & $\begin{array}{l}5 \\
5 \\
4 \\
5 \\
5\end{array}$ & $\begin{array}{l}0.14827 \\
0.14816 \\
0.14805 \\
0.14794 \\
0.14784\end{array}$ & $\begin{array}{ll}1 & 1 \\
1 & 1 \\
1 & 1 \\
1 & 0 \\
1 & 1\end{array}$ & $\begin{array}{l}0.19527 \\
0.19511 \\
0.19496 \\
0.19480 \\
0.19465\end{array}$ & $\begin{array}{l}16 \\
15 \\
16 \\
15 \\
16\end{array}$ & $\begin{array}{l}0.47879 \\
0.47858 \\
0.47836 \\
0.47815 \\
0.47794\end{array}$ \\
\hline $\begin{array}{l}3.086 \\
3.087 \\
3.088 \\
3.089 \\
3.090\end{array}$ & $\begin{array}{l}0.04676 \\
0.04671 \\
0.04667 \\
0.04662 \\
0.04657\end{array}$ & $\begin{array}{l}5 \\
4 \\
5 \\
5 \\
5\end{array}$ & $\begin{array}{l}0.14773 \\
0.14762 \\
0.14752 \\
0.14741 \\
0.14730\end{array}$ & $\begin{array}{ll}11 \\
10 \\
11 \\
11 \\
10\end{array}$ & $\begin{array}{l}0.19449 \\
0.19434 \\
0.19418 \\
0.19403 \\
0.19387\end{array}$ & $\begin{array}{l}15 \\
16 \\
15 \\
16 \\
15\end{array}$ & $\begin{array}{l}0.47772 \\
0.47751 \\
0.47729 \\
0.47708 \\
0.47687\end{array}$ \\
\hline $\begin{array}{l}3.091 \\
3.092 \\
3.093 \\
3.094 \\
3.095\end{array}$ & $\begin{array}{l}0.04652 \\
0.04647 \\
0.04643 \\
0.04638 \\
0.04633\end{array}$ & $\begin{array}{l}5 \\
4 \\
5 \\
5 \\
5\end{array}$ & $\begin{array}{l}0.14720 \\
0.14709 \\
0.14698 \\
0.14688 \\
0.14677\end{array}$ & $\begin{array}{l}11 \\
11 \\
10 \\
11 \\
11\end{array}$ & $\begin{array}{l}0.19372 \\
0.19356 \\
0.19341 \\
0.19326 \\
0.19310\end{array}$ & $\begin{array}{l}16 \\
15 \\
15 \\
16 \\
15\end{array}$ & $\begin{array}{l}0.47665 \\
0.47644 \\
0.47623 \\
0.47601 \\
0.47580\end{array}$ \\
\hline $\begin{array}{l}3.096 \\
3.097 \\
3.098 \\
3.099 \\
3.100\end{array}$ & $\begin{array}{l}0.04628 \\
0.04624 \\
0.04619 \\
0.04614 \\
0.04610\end{array}$ & $\begin{array}{l}4 \\
5 \\
5 \\
4\end{array}$ & $\begin{array}{l}0.14666 \\
0.14656 \\
0.14645 \\
0.14635 \\
0.14624\end{array}$ & $\begin{array}{ll}10 \\
11 \\
10 \\
11\end{array}$ & $\begin{array}{l}0.19295 \\
0.19280 \\
0.19264 \\
0.19249 \\
0.19234\end{array}$ & $\begin{array}{l}15 \\
16 \\
15 \\
15\end{array}$ & $\begin{array}{l}0.47559 \\
0.47537 \\
0.47516 \\
0.47495 \\
0.47473\end{array}$ \\
\hline
\end{tabular}


Table I Harmonic Oscillator Contributions (in dimensionless form) to the

Thermodynamic Functions for Values of $X=h c v / k T$

\begin{tabular}{|c|c|c|c|c|c|c|c|}
\hline $\mathbf{X}$ & $-\left(F^{\circ}-E_{0}^{\circ}\right) / R T$ & $\Delta$ & $\left(\mathrm{H}^{\circ}-\mathrm{E}_{\mathrm{O}}^{\circ}\right) / \mathrm{RT}$ & $\Delta$ & So/R & $\Delta$ & $\mathrm{C}_{\mathrm{p}} / \mathrm{R}$ \\
\hline 3.100 & 0.04610 & 5 & 0.14624 & 11 & 0.19234 & 16 & 0.47473 \\
\hline $\begin{array}{l}3.101 \\
3.102 \\
3.103 \\
3.104 \\
3.105\end{array}$ & $\begin{array}{l}0.04605 \\
0.04600 \\
0.04595 \\
0.04591 \\
0.04586\end{array}$ & $\begin{array}{l}5 \\
5 \\
4 \\
5 \\
5\end{array}$ & $\begin{array}{l}0.14613 \\
0.14603 \\
0.14592 \\
0.14582 \\
0.14571\end{array}$ & $\begin{array}{l}10 \\
11 \\
10 \\
11 \\
10\end{array}$ & $\begin{array}{l}0.19218 \\
0.19203 \\
0.19188 \\
0.19172 \\
0.11157\end{array}$ & $\begin{array}{l}15 \\
15 \\
16 \\
15 \\
15\end{array}$ & $\begin{array}{l}0.47452 \\
0.47431 \\
0.47409 \\
0.47388 \\
0.47367\end{array}$ \\
\hline $\begin{array}{l}3.106 \\
3.107 \\
3.108 \\
3.109 \\
3.110\end{array}$ & $\begin{array}{l}0.04581 \\
0.04577 \\
0.04572 \\
0.04567 \\
0.04563\end{array}$ & $\begin{array}{l}4 \\
5 \\
5 \\
4 \\
5\end{array}$ & $\begin{array}{l}0.14561 \\
0.14550 \\
0.14540 \\
0.14529 \\
0.14518\end{array}$ & $\begin{array}{l}11 \\
10 \\
11 \\
11 \\
10\end{array}$ & $\begin{array}{l}0.19142 \\
0.19127 \\
0.19111 \\
0.19096 \\
0.19081\end{array}$ & $\begin{array}{l}15 \\
16 \\
15 \\
15 \\
15\end{array}$ & $\begin{array}{l}0.47345 \\
0.47324 \\
0.47303 \\
0.47281 \\
0.47260\end{array}$ \\
\hline $\begin{array}{l}3.111 \\
3.112 \\
3.113 \\
3.114 \\
3.115\end{array}$ & $\begin{array}{l}0.04558 \\
0.04553 \\
0.04549 \\
0.04544 \\
0.04539\end{array}$ & $\begin{array}{l}5 \\
4 \\
5 \\
5 \\
4\end{array}$ & $\begin{array}{l}0.14508 \\
0.14497 \\
0.14487 \\
0.14476 \\
0.14466\end{array}$ & $\begin{array}{l}11 \\
10 \\
11 \\
10 \\
11\end{array}$ & $\begin{array}{l}0.19066 \\
0.19051 \\
0.19036 \\
0.19020 \\
0.19005\end{array}$ & $\begin{array}{l}15 \\
15 \\
16 \\
15 \\
15\end{array}$ & $\begin{array}{l}0.47239 \\
0.47218 \\
0.47196 \\
0.47175 \\
0.47154\end{array}$ \\
\hline $\begin{array}{l}3.116 \\
3.117 \\
3.118 \\
3.119 \\
3.120\end{array}$ & $\begin{array}{l}0.04535 \\
0.04530 \\
0.04525 \\
0.04521 \\
0.04516\end{array}$ & $\begin{array}{l}5 \\
5 \\
4 \\
5 \\
4\end{array}$ & $\begin{array}{l}0.14455 \\
0.14445 \\
0.14434 \\
0.14424 \\
0.14413\end{array}$ & $\begin{array}{l}10 \\
11 \\
10 \\
11 \\
10\end{array}$ & $\begin{array}{l}0.18990 \\
0.18975 \\
0.18960 \\
0.18945 \\
0.18930\end{array}$ & $\begin{array}{l}15 \\
15 \\
15 \\
15 \\
15\end{array}$ & $\begin{array}{l}0.47133 \\
0.47111 \\
0.47090 \\
0.47069 \\
0.47048\end{array}$ \\
\hline $\begin{array}{l}3.121 \\
3.122 \\
3.123 \\
3.124 \\
3.125\end{array}$ & $\begin{array}{l}0.04512 \\
0.04507 \\
0.04502 \\
0.04498 \\
0.04493\end{array}$ & $\begin{array}{l}5 \\
5 \\
4 \\
5 \\
4\end{array}$ & $\begin{array}{l}0.14403 \\
0.14393 \\
0.14382 \\
0.14372 \\
0.14361\end{array}$ & $\begin{array}{l}10 \\
11 \\
10 \\
11 \\
10\end{array}$ & $\begin{array}{l}0.18915 \\
0.18900 \\
0.18884 \\
0.18869 \\
0.18854\end{array}$ & $\begin{array}{l}15 \\
16 \\
15 \\
15 \\
15\end{array}$ & $\begin{array}{l}0.47026 \\
0.47005 \\
0.46984 \\
0.46963 \\
0.46941\end{array}$ \\
\hline $\begin{array}{l}3.126 \\
3.127 \\
3.128 \\
3.129 \\
3.130\end{array}$ & $\begin{array}{l}0.04489 \\
0.04484 \\
0.04479 \\
0.04475 \\
0.04470\end{array}$ & $\begin{array}{l}5 \\
5 \\
4 \\
5 \\
4\end{array}$ & $\begin{array}{l}0.14351 \\
0.14340 \\
0.14330 \\
0.14320 \\
0.14309\end{array}$ & $\begin{array}{l}11 \\
10 \\
10 \\
11 \\
10\end{array}$ & $\begin{array}{l}0.18839 \\
0.18824 \\
0.18809 \\
0.18794 \\
0.18779\end{array}$ & $\begin{array}{l}15 \\
15 \\
15 \\
15 \\
14\end{array}$ & $\begin{array}{l}0.46920 \\
0.46899 \\
0.46878 \\
0.46857 \\
0.46835\end{array}$ \\
\hline $\begin{array}{l}3.131 \\
3 \cdot 132 \\
3 \cdot 133 \\
3 \cdot 134 \\
3.135\end{array}$ & $\begin{array}{l}0.04466 \\
0.04461 \\
0.04457 \\
0.04452 \\
0.04447\end{array}$ & $\begin{array}{l}5 \\
4 \\
5 \\
5 \\
4\end{array}$ & $\begin{array}{l}0.14299 \\
0.14288 \\
0.14278 \\
0.14268 \\
0.14257\end{array}$ & $\begin{array}{l}11 \\
10 \\
10 \\
11 \\
10\end{array}$ & $\begin{array}{l}0.18765 \\
0.18750 \\
0.18735 \\
0.18720 \\
0.18705\end{array}$ & $\begin{array}{l}15 \\
15 \\
15 \\
15 \\
15\end{array}$ & $\begin{array}{l}0.46814 \\
0.46793 \\
0.46772 \\
0.46751 \\
0.46730\end{array}$ \\
\hline $\begin{array}{l}3.136 \\
3.137 \\
3.138 \\
3.139 \\
3.140\end{array}$ & $\begin{array}{l}0.04443 \\
0.04438 \\
0.04434 \\
0.04429 \\
0.04425\end{array}$ & $\begin{array}{l}5 \\
4 \\
5 \\
4 \\
5\end{array}$ & $\begin{array}{l}0.14247 \\
0.14237 \\
0.14226 \\
0.14216 \\
0.14206\end{array}$ & $\begin{array}{l}10 \\
11 \\
10 \\
10 \\
11\end{array}$ & $\begin{array}{l}0.18690 \\
0.18675 \\
0.18660 \\
0.18645 \\
0.18630\end{array}$ & $\begin{array}{l}15 \\
15 \\
15 \\
15 \\
14\end{array}$ & $\begin{array}{l}0.46708 \\
0.46687 \\
0.46666 \\
0.46645 \\
0.46624\end{array}$ \\
\hline $\begin{array}{l}3.141 \\
3.142 \\
3.143 \\
3.144 \\
3.145\end{array}$ & $\begin{array}{l}0.04420 \\
0.04416 \\
0.04411 \\
0.04407 \\
0.04402\end{array}$ & $\begin{array}{l}4 \\
5 \\
4 \\
5 \\
4\end{array}$ & $\begin{array}{l}0.14195 \\
0.14185 \\
0.14175 \\
0.14164 \\
0.14154\end{array}$ & $\begin{array}{l}10 \\
10 \\
11 \\
10 \\
10\end{array}$ & $\begin{array}{l}0.18616 \\
0.18601 \\
0.18586 \\
0.18571 \\
0.18556\end{array}$ & $\begin{array}{l}15 \\
15 \\
15 \\
15 \\
14\end{array}$ & $\begin{array}{l}0.46603 \\
0.46581 \\
0.46560 \\
0.46539 \\
0.46518\end{array}$ \\
\hline $\begin{array}{l}.146 \\
.147 \\
.148 \\
.149 \\
.150\end{array}$ & $\begin{array}{l}0.04398 \\
0.04393 \\
0.04389 \\
0.04384 \\
0.04380\end{array}$ & $\begin{array}{l}5 \\
4 \\
5 \\
4\end{array}$ & $\begin{array}{l}0.14144 \\
0.14134 \\
0.14123 \\
0.14113 \\
0.14103\end{array}$ & $\begin{array}{l}10 \\
11 \\
10 \\
10\end{array}$ & $\begin{array}{l}0.18542 \\
0.18527 \\
0.18512 \\
0.18497 \\
0.18482\end{array}$ & $\begin{array}{l}15 \\
15 \\
15 \\
15\end{array}$ & $\begin{array}{l}0.46497 \\
0.46476 \\
0.46455 \\
0.46434 \\
0.46413\end{array}$ \\
\hline
\end{tabular}


Table I Harmonic Oscillator Contributions (in dimensionless form) to the

Thermodynamic Functions for Values of $\mathrm{X}=\mathrm{hc} / \mathrm{kT}$
X $\quad-\left(\mathrm{F}^{\circ}-\mathrm{E}_{\mathrm{O}}^{\circ}\right) / \mathrm{RT}$
$\Delta \quad\left(H^{\circ}-E_{0}^{\circ}\right) / R T \quad \Delta \quad$ So/R
$\Delta \quad \mathrm{CO} / \mathrm{R}$

$\Delta$

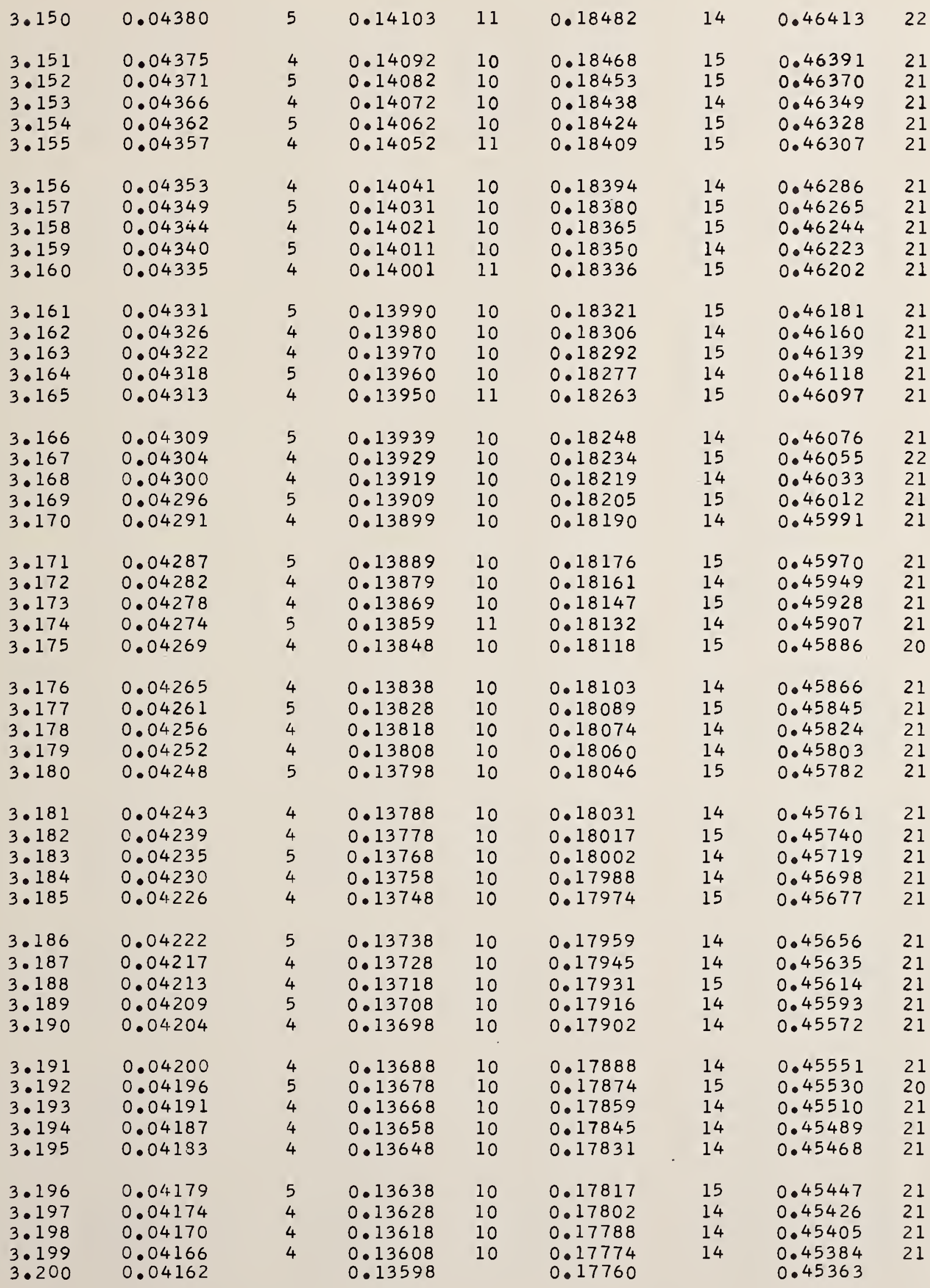


Table I Harmonic Oscillator Contributions (in dimensionless form) to the

Thermodynamic Functions for Values of $\mathrm{X}=\mathrm{hc} / \mathrm{kT}$

$\mathbb{x}$ $-\left(F^{\circ}-E_{0}^{\circ}\right) / R T$

$\Delta \quad\left(\mathrm{H}^{\circ}-\mathrm{E}_{\mathrm{O}}^{\mathrm{O}}\right) / \mathrm{RT} \quad \Delta \quad \mathrm{S} / \mathrm{R}$

0.04162
0.04157
0.04153
0.04149
0.04145
0.04140$$
5
$$

3.200

3.201

3.202

3.203

3.204

3.205

0.04136

3.206

3.207

3.208

3.209

3.210

0.04132

0.04128

0.04124

0.04119

3.211

3. 212

3.213

3. 214

3.215

0.04115

0.04111

0.04107

0.04103

0.04098

3.216

3.217

3.218

3.219

3.220

0.04094

0.04090

0.04086

0.04082

3.221

0.04078

3. 222

3. 223

3.224

3.225

3. 226

3.227

3.228

3.229

3.230

3. 231

3. 232

3. 233

3. 234

3.235

3. 236

3.237

3. 238

3.239

3.240

0.04073

0.04069

0.04065

0.04061

0.04057

0.04053

0.04048

0.04044

0.04040

0.04036

0.04032

0.04028

0.04024

0.04020

0.04016

3.241

3.242

3. 243

3.244

3.245

0.04011

0.04007

0.04003

0.03999

0.03995

0.03991

0.03987

0.03983

0.03979

0.03975

3. 246

3.247

3. 248

3. 249

3.250

0.03971

0.03967

0.03963

0.03959

0.03955

\subsection{8 \\ 0.13588 \\ 0. 13578 \\ 0.13568 \\ 0.13559 \\ 0.13549}

0.13539

0.13529

0.13519

0.13509

0.13499

0.13489

0.13480

0.13470

0.13460

0.13450

0.13440

0.13430

0.13421

0.13411

0.13401

10

10

10

9
10

10

10

10

10

10

10

9

10

10

10

10

10

9

10

10

10

0.13391

0.13381

0.13372

0.13362

0.13352

10
9
10
10
10

0.13342

0.13333

0.13323

0.13313

0.13303

9
10
10
10
9

0.13294

0.13284

0.13274

0.13264

0.13255

10

10

10

9

10

0.13245

0.13226

0.13216

0.13206

10
9
10
10
9

0.13197

0.13187

0.13177

0.13168

0.13158

10

9

10

10

0.13148

0.13139

0.13129

0.13120

0.13110
0.13235
$\Delta \quad \mathrm{Co} / \mathrm{R}$

$\Delta$
14

15

14

14

14

14

0.17675

0.17661

0.17647

0.17633

0.17619

0.17605

0.17590

0.17576

0.17562

0.17548

0.17534

0.17520

0.17506

0.17492

0.17478

0.17465

0.17451

0.17437

0.17423

0.17409

0.17395

0.17381

0.17367

0.17353

0.17339

0.17326

0.17312

0.17298

0.17284

0.17270

\subsection{7}

0.17243

0.17229

0.17215

0.17201

0.17188

0.17174

0.17160

0.17147

0.17133

90.17119

0.17106

0.17092

0.17078

0.17065
14

14

14

14

14

15

14

14

14

14

14

14

14

14

13

14

14

14

14

14

14

14

14

14

13

14

14

14

14

13

14

14

14

14

13

14

14

13

14

14

13

14

14

13

$0.45363 \quad 21$

$0.45342 \quad 20$ 
Table I Harmonic Oscillator Contributions (in dimensionless form) to the

Thermodynamic Functions for Values of $\mathrm{X}=\mathrm{hc} \nu / \mathrm{kT}$

$\mathbf{X}$ $-\left(F^{\circ}-E_{O}^{O}\right) / R T$

$\Delta \quad\left(\mathrm{H}^{\circ}-\mathrm{E}_{\mathrm{O}}^{\circ}\right) / \mathrm{RT} \quad \Delta \quad \mathrm{S} \% / \mathrm{R}$

$\begin{array}{ll}3.250 & 0.03955 \\ 3.251 & 0.03951 \\ 3.252 & 0.03947 \\ 3.253 & 0.03943 \\ 3.254 & 0.03938 \\ 3.255 & 0.03934 \\ 3.256 & 0.03930 \\ 3.257 & 0.03926 \\ 3.258 & 0.03922 \\ 3.259 & 0.03918 \\ 3.260 & 0.03914 \\ 3.261 & 0.03910 \\ 3.262 & 0.03906 \\ 3.263 & 0.03903 \\ 3.264 & 0.03899 \\ 3.265 & 0.03895 \\ 3.266 & 0.03891 \\ 3.267 & 0.03887 \\ 3.268 & 0.03883 \\ 3.269 & 0.03879 \\ 3.270 & 0.03875 \\ & \\ 3.271 & 0.03871 \\ 3.272 & 0.03867 \\ 3.273 & 0.03863 \\ 3.274 & 0.03859 \\ 3.275 & 0.03855\end{array}$

3.276

3.277

3.278

3.279

3.280

3.281

3.282

3.283

3.284

3.285

3.286

3.287

3.288

3.289

3.290

3.291

3.292

3.293

3.294

3.295

3.296

3.297

3.298

3.299

3.300
0.03851

0.03847

0.03843

0.03839

0.03835

0.03832

0.03828

0.03824

0.03820

0.03816

0.03812

0.03808

0.03804

0.03800

0.03797

0.03793

0.03789

0.03785

0.03781

0.03777

0.03773

0.03770

0.03766

0.03762

0.03758

$\begin{array}{llr}4 & 0.13110 & 10 \\ 4 & 0.13100 & \\ 4 & 0.13091 & 10 \\ 5 & 0.13081 & \\ 4 & 0.13072 & 10 \\ 4 & 0.13062 & 10 \\ & & \\ 4 & 0.13052 & \\ 4 & 0.13043 & 10 \\ 4 & 0.13033 & \\ 4 & 0.13024 & 10 \\ 4 & 0.13014 & \\ & & \\ 4 & 0.13005 & 10 \\ 3 & 0.12995 & \\ 4 & 0.12986 & 10 \\ 4 & 0.12976 & \\ 4 & 0.12967 & 10 \\ & & \\ 4 & 0.12957 & \\ 4 & 0.12948 & 10 \\ 4 & 0.12938 & 9 \\ 4 & 0.12929 & 10 \\ 4 & 0.12919 & \\ & & \\ 4 & 0.12910 & 10 \\ 4 & 0.12900 & 9 \\ 4 & 0.12891 & 10 \\ 4 & 0.12881 & 9 \\ 4 & 0.12872 & 10 \\ & & \end{array}$

0.12862

0.12853

0.12843

0.12834

0.12825

0.12815

0.12806

0.12796

0.12787

0.12778

0.12768

0.12750

0.12740

0.12731

0.12721

0.12712

0.12703

0.12693

0.12684

0.12675

0.12665

0.12656

0.12647

0.12638

10
0.12759

90.16647

$10 \quad 0.16594$

90.16580
$\Delta \quad \mathrm{C}_{\mathrm{p}}^{\circ} / \mathrm{R}$

$\begin{array}{rllll}10 & 0.17065 & 14 & 0.44326 & 21 \\ 9 & 0.17051 & 14 & 0.44305 & 20 \\ 10 & 0.17037 & 13 & 0.44285 & 21 \\ 9 & 0.17024 & 14 & 0.44264 & 20 \\ 10 & 0.17010 & 14 & 0.44244 & 21 \\ 10 & 0.16996 & 13 & 0.44223 & 21 \\ 9 & 0.16983 & 14 & 0.44202 & 20 \\ 10 & 0.16969 & 13 & 0.44182 & 21 \\ 9 & 0.16956 & 14 & 0.44161 & 20 \\ 10 & 0.16942 & 13 & 0.44141 & 21 \\ 9 & 0.16929 & 14 & 0.44120 & 21 \\ 10 & 0.16915 & 13 & 0.44099 & 20 \\ 9 & 0.16902 & 14 & 0.44079 & 21 \\ 10 & 0.16888 & 13 & 0.44058 & 20 \\ 9 & 0.16875 & 14 & 0.44038 & 21 \\ 10 & 0.16861 & 13 & 0.44017 & 20 \\ 9 & 0.16848 & 14 & 0.43997 & 21 \\ 9 & 0.16834 & 13 & 0.43976 & 20 \\ 10 & 0.16821 & 14 & 0.43956 & 21 \\ 9 & 0.16821 \\ 10 & 0.16807 & 13 & 0.43935 & 20 \\ 9 & 0.16794 & 14 & 0.43915 & 21 \\ & & & & \\ 10 & 0.16780 & 13 & 0.43894 & 21 \\ 9 & 0.16767 & 13 & 0.43873 & 20 \\ 10 & 0.16754 & 14 & 0.43853 & 21 \\ 9 & 0.16740 & 13 & 0.43832 & 20 \\ 10 & 0.16727 & 14 & 0.43812 & 21\end{array}$

0.16713

13

$0.43791 \quad 20$

0.16700

0.16687

0.16673

0.43771

21

0.43750

20

14

0.43730

20

$0.43710 \quad 21$

13

$0.43689 \quad 20$

0.16633

0.16620

0.16607

14

13

13

13

$0.43669 \quad 21$

$0.43648 \quad 20$

$0.43628 \quad 21$

14

$0.43607 \quad 20$

13

0.43587

21

0.16567

0.43566

0.43546

0.16541

0.43525

0.16527

13

14

13

0.43505

20

20

0.16514

13

0.43485

20

0.16501

0.43464

21

0.16488

0.16475

0.43444

20

13

0.16461

$0.43423 \quad 20$

13

0.43403

20

0.16448

13

0.43383

21

0.16435

0.43362

0.16422

0.43342

20

0.16409

13

0.16396

0.43321

21

0.43301 
Table I Harmonic Oscillator Contributions (in dimensionless form) to the

Thermodynamic Functions for Values of $X=h c v / k T$

$\boldsymbol{X}-\left(\mathrm{F}^{\circ}-\mathrm{E} \mathrm{O}\right) / \mathrm{RT} \quad \Delta \quad\left(\mathrm{H}^{\circ}-\mathrm{E}^{\circ}\right) / \mathrm{RT} \quad \Delta \quad \mathrm{S} / \mathrm{R}$

$\begin{array}{ll}3.300 & 0.03758 \\ 3.301 & 0.03754 \\ 3.302 & 0.03750 \\ 3.303 & 0.03747 \\ 3.304 & 0.03743 \\ 3.305 & 0.03739 \\ 3.306 & 0.03735 \\ 3.307 & 0.03731 \\ 3.308 & 0.03728 \\ 3.309 & 0.03724 \\ 3.310 & 0.03720\end{array}$

3.311

3.312

3.313

3.314

3.315

3.316

3.317

3.318

3.319

3.320

3.321

$3 \cdot 322$

3.323

3.324

3.325

3.326

3.327

3. 328

3.329

3.330

3. 331

3.332

3.333

3. 334

3.335

3. 336

3.337

3.338

3.339

3.340

3. 341

3. 342

3.343

3.344

3.345

3.346

3. 347

$3 \cdot 348$

3.349

3.350
0.03716

0.03712

0.03709

0.03705

0.03701

0.03697

0.03690

0.03686

0.03682

0.03675

0.03671

0.03667

0.03664

0.03660

0.03656

0.03652

0.03649

0.03645

0.03641

0.03638

0.03634

0.03630

0.03626

0.03623

0.03619

0.03615

0.03612

0.03608

0.03604

0.03601

0.03597

0.03593

0.03590

0.03586

0.03582

0.03579

0.03575

0.03571
0.03694

0.03679

$\begin{array}{lr}0.12638 & 10 \\ 0.12628 & 9 \\ 0.12619 & 9 \\ 0.12610 & 10 \\ 0.12600 & 9 \\ 0.12591 & 9 \\ 0.12582 & 9 \\ 0.12573 & 10 \\ 0.12563 & 9 \\ 0.12554 & 9 \\ 0.12545 & 9\end{array}$

$0.12536 \quad 10$

0.12526

0.12517

0.12508

0.12499

$0.12490 \quad 10$

0.12480

0.12471

0.12462

0.12453

0.12444

0.12435

0.12416

0.12407

0.12398

0.12389

0.12380

0.12371

0.12362

0.12352

0.12343

0.12334

0.12325

0.12316

0.12307

0.12298

0.12289

0.12280

0.12271

0.12262

0.12253

0.12244

0.12235

0.12226

0.12217

0.12208

0.12199

0.12190

0.12181
0.12425
$\Delta \quad C_{\mathrm{p}}^{\circ} / \mathrm{R}$

$\Delta$

$\begin{array}{rllll}10 & 0.16396 & 14 & 0.43301 & 20 \\ 9 & 0.16382 & 13 & 0.43281 & 21 \\ 9 & 0.16369 & 13 & 0.43260 & 20 \\ 10 & 0.16356 & 13 & 0.43240 & 20 \\ 9 & 0.16343 & 13 & 0.43220 & 21 \\ 9 & 0.16330 & 13 & 0.43199 & 20\end{array}$

0.16330

$0.43199 \quad 20$

$0.43179 \quad 20$

$0.43159 \quad 21$

$0.43138 \quad 20$

$0.43118 \quad 20$

$0.43098 \quad 21$

0.16278

$0.43077 \quad 20$

$0.16252 \quad 13$

0.16239

$0.43057 \quad 20$

0.16226

$0.43037 \quad 21$

0.16213

0.43016

$0.42996 \quad 20$

0.16187

0.16174

$0.42976 \quad 21$

$0.42955 \quad 20$

0.16161

0.42935

0.16148

0.42915

20

0.16135

$0.42895 \quad 21$

$0.42874 \quad 20$

$0.42854 \quad 20$

0.4283420

$0.42814 \quad 21$

$0.42793 \quad 20$

$0.16084 \quad 13$

$0.42773 \quad 20$

$0.16058 \quad 13$

0.16045

0.42753

0.16032

0.42733

0.16019

0.42712

20

0.16006

12

0.42692

20

0.15994

13

0.15981

0.15968

0.42672

20

0.15955

0.15943

0.42652

0.42631

20

13

12

0.42611

21

13

0.15930

13

0.15917

0.42571

0.42551

0.15904

0.15892

0.42530

0.42510

0.15879

13

13

0.42490

13

0.15866

0.42470

0.42450

0.42430

0.15841

12

0.15828

0.42410

0.15815

13

12

0.42389

20

0.15803

13

0.42369

0.15790

0.42349
0.42329

0.15777

13

0.15765

0.42309

20

0.15752

0.42289

20

20

20 
Table I Harmonic Oscillator Contributions (in dimensionless form) to the

Thermodynamic Functions for Values of $\mathrm{X}=\mathrm{hc} / \mathrm{kT}$

X

$-\left(F^{\circ}-E_{0}^{\circ}\right) / R T$

3.356

3.357

3.358

3.359

3.360

3.361

3.362

3.363

3.364

3. 365

3.366

3.367

3. 368

3.369

3.370

3.371

3.372

3. 373

3. 374

3.375

3.376

3.377

3. 378

3.379

3.380

3.381

3.382

3.383

3.384

3.385

3.386

3.387

3.388

3.389

3.390

3.391

3.392

3.393

3.394

3. 395

3.396

3.397

3. 398

3.399

3.400
0.03550

0.03571

0.03568

0.03564

0.03561

0.03557

0.03553

0.03546

0.03542

0.03539

0.03535

0.03532

0.03528

0.03525

0.03521

0.03517

0.03514

0.03510

0.03507

0.03503

0.03499

0.03496

0.03492

0.03489

0.03485

0.03482

0.03478

0.03475

0.03471

0.03468

0.03464

0.03461

0.03457

0.03453

0.03450

0.03446

0.03443

0.03439

0.03436

0.03432

0.03429

0.03426

0.03422

0.03419

0.03415

0.03412

0.03408

0.03405

0.03401

0.03398

0.03394
0.12038
0.12029

0.12020

0.12011

0.12002

9
9
9
9
9
9
9
9
9
9
9
9
9
9
8
9
9
9
9
9

0.15739
0.15727
0.15714
0.15702
0.15689

0.15676

0.15664

0.15651

0.15639

0.15626

0.15614

0.15601

0.15589

0.15576

0.15564

0.15551

0.15539

0.15526

0.15514

0.15502

\subsection{3}

0.11984

0.11975

0.11967

0.11958

0.11949

0.11940

0.11931

0.11922

0.11914

0.11905

0.11896

0.11887

0.11878

0.11870

0.15489

0.15477

0.15464

0.15452

0.15440

0.11861

0.11852

0.11843

0.11835

0.11826

0.15427

0.15415

0.15402

0.15390

0.15378

0.15365

0.15353

0.15341

0.15328

0.15316

0.15304

0.15292

0.15279

0.15267

0.15255

0.11817

0.11808

0.11800

0.11791

0.11782

0.15243

0.15230

0.15218

0.15206

0.15194

0.11773

0.11765

0.11756

0.11747

0.11739
0.15182

0.15169

0.15157

Q. 15145

0.15133
$\Delta \quad\left(\mathrm{H}^{\circ}-\mathrm{E}_{\mathrm{O}}\right) / \mathrm{RT}$

$\Delta$

$\mathrm{S} \% / \mathrm{R}$

0.12181
0.12172
0.12163
0.12154
0.12145
0.12136
0.12127
0.12118
0.12109
0.12100
0.12091
0.12082
0.12073
0.12064
0.12055
0.12046

30.121819

4

3

4

4

3

4

\section{4}

4

90.15752

0.15752

$\Delta \quad \mathrm{C}_{\mathrm{p}}^{\circ} / \mathrm{R}$

$\Delta$
13. 0.4228920

$12 \quad 0.42269 \quad 21$

$13 \quad 0.42248 \quad 20$

$12 \quad 0.42228 \quad 20$

$13 \quad 0.42208 \quad 20$

$13 \quad 0.42188 \quad 20$

12

13

12

13

12

13

12

13

12

13

12

13

12

12

13

\section{2}

13

12

12

13

\section{2}

13

12

12

13

12

12

13

12

12

12

13

12

12

12

13

12

12

12

12

13

12

12

12

$0.42168 \quad 20$

$0.42148 \quad 20$

$0.42128 \quad 20$

$0.42108 \quad 20$

$0.42088 \quad 20$

$0.42068 \quad 20$

$0.42048 \quad 20$

$0.42028 \quad 20$

$0.42008 \quad 20$

$0.41988 \quad 20$

$0.41968 \quad 21$

$0.41947 \quad 20$

$0.41927 \quad 20$

$0.41907 \quad 20$

$0.41887 \quad 20$

$0.41867 \quad 20$

$0.41847 \quad 20$

$0.41827 \quad 20$

$0.41807 \quad 20$

$0.41787 \quad 20$ 
Table I Harmonic Oscillator Contributions (in dimensionless form) to the

Thermodynamic Functions for Values of $\mathrm{X}=\mathrm{hcv} / \mathrm{kT}$

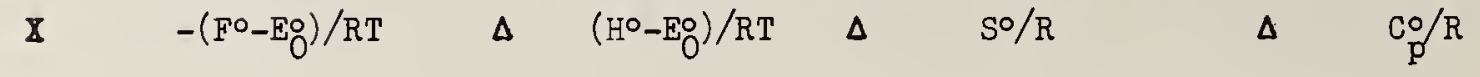

$\begin{array}{ll}3.400 & 0.03394 \\ 3.401 & 0.03391 \\ 3.402 & 0.03387 \\ 3.403 & 0.03384 \\ 3.404 & 0.03381 \\ 3.405 & 0.03377\end{array}$

3.406

3.407

3.408

3.409

3.410

3.411

3.412

3.413

3.414

3.415

3.416

3.417

3.418

3.419

3.420

3.421

3.422

3.423

3.424

3.425

3.426

3.427

3.428

3.429

3.430

3.431

3.432

3.433

3.434

3.435

3.436

3.437

3.438

3.439

3.440

3.441

3.442

3.443

3.444

3.445

3.446

3.447

3.448

3.449

3.450
0.03374

0.03370

0.03363

0.03360

0.03357

0.03353

0.03350

0.03346

0.03343

0.03339

0.03336

0.03333

0.03329

0.03326

0.03323

0.03319

0.03316

0.03312

0.03309

0.03306

0.03302

0.03299

0.03296

0.03292

0.03289

0.03286

0.03282

0.03279

0.03276

0.03272

0.03269

0.03266

0.03262

0.03259

0.03256

0.03252

0.03249

0.03246

0.03242

0.03239

0.03236

0.03233

0.03229

0.03226
0.03367
0.11739
0.11730
0.11721
0.11713
0.11704

0.11695

0.11687

0.11678

0.11669

0.11661

0.11652

0.11643

0.11635

0.11626

0.11618

0.11609

0.11600

0.11592

0.11583

0.11575

0.11566

0.11557

0.11549

0.11540

0.11532

0.11523

0.11515

0.11506

0.11498

0.11489

0.11481

0.11472

0.11464

0.11455

0.11447

0.11438

0.11430

0.11421

0.11413

0.11404

0.11396

0.11387

0.11379

0.11370

0.11362

0.11353

0.11345

0.11337

0.11328

0.11320

0.11311
0.15133

0.15121

0.15109

0.15097

0.15084

0.15072

0.15060

0.15048

0.15036

0.15024

0.15012

0.15000

0.14988

0.14976

0.14964

0.14952

0.14940

0.14928

0.14904

0.14892

0.14880

0.14868

0.14856

0.14844

0.14832

0.14820

0.14808

0.14797

0.14785

0.14773

0.14761

0.14749

0.14737

0.14725

0.14714

0.14702

0.14690

0.14678

0.14666

0.14655

0.14643

0.14631

0.14619

0.14608

0.14596

0.14584

0.14572

0.14561

0.14549

0.14537
0.14916
12

0.41289

19

12

0.41270

0.41250

0.41230

0.41210

0.41190

$\Delta$

$0.41170 \quad 19$

$0.41151 \quad 20$

0.4113120

0.4111120

0.4109120

$0.41071 \quad 19$

$0.41052 \quad 20$

0.4103220

$0.41012 \quad 20$

0.4099220

$0.40972 \quad 19$

0.4095320

0.4093320

0.4091320

$0.40893 \quad 19$

12

0.40874

20

0.40854

0.40834

0.40815

0.40795

20

19

20

20

12

0.40775

20

0.40755

0.40736

0.40716

0.40696

19

20

20

19

12

0.40677

20

0.40657

0.40637

0.40618

0.40598

20

19

20

20

12

0.40578

19

0.40559

0.40539

0.40519

20

20

12

0.40500

20

12

0.40480

0.40460

0.40441

0.40421

0.40402

20

19

20

19

20

$0.40382 \quad 20$

12

0.40362

19

0.40343

0.40323

0.40304 
Table I Harmonic Oscillator Contributions (in dimensionless form) to the

Thermodynamic Functions for Values of $X=h c v / k T$

$\mathbf{z}$

$-\left(F^{\circ}-E_{0}\right) / R T$

$\Delta \quad\left(H^{\circ}-E_{0}^{\circ}\right) / R T$

$\Delta$

$\mathrm{S} \% / \mathrm{R}$

3.450

3.452

3.453

3.454

3.455

3.456

3.457

3.458

3.459

3.460

3.461

3.462

3.463

3.464

3.465

3.466

3.467

3.468

3.469

3.470

3.471

3.472

3.473

3.474

3.475

3.476

3.477

3.478

3.479

3.480

3.481

3.482

3.483

3.484

3.485

3.486

3.487

3.488

3.489

3.490

3.491

3.492

3.493

3.494

3.495

3.496

3.497

3.498

3.499

3.500
0.03226

0.03223

0.03216

0.03213

0.03210

0.03206

0.03203

0.03200

0.03197

0.03193

0.03190

0.03187

0.03184

0.03180

0.03177

0.03174

0.03171

0.03168

0.03164

0.03161

0.03158

0.03152

0.03148

0.03145

0.03142

0.03139

0.03132

0.03129

0.03126

0.03123

0.03120

0.03117

0.03113

0.03110

0.03107

0.03104

0.03101

0.03098

0.03094

0.03091

0.03088

0.03082

0.03079

0.03076

0.03073

0.03066
0.03219

0.03155

0.03136

0.03085

0.03069
3

3

3

3
4

0.11311

0.11303

0.11295

0.11286

0.11278

0.11269

0.11261

0. 11253

0.11244

0.11236

0. 11228

0.11219

0.11211

0.11203

0.11194

0.11186

0.11178

0.11169

0.11161

0.11144

0.11136

0. 11128

0.11120

0.11111

0.11103

0.11095

0.11086

0.11078

0.11070

0.11062

0.11054

0.11045

0.11037

0.11029

0.11021

0.11012

0.11004

0.10996

0.10988

0.10980

0.10972

0.10963

0.10955

0.10947

0.10939

0.10931

0.10923

0.10914

0.10906

0.10898
0.11153
$\Delta \quad \mathrm{Co} / \mathrm{R}$

$\Delta$ $\begin{array}{llll}0.14537 & 11 & 0.40304 & 20\end{array}$

0.14526

0.14514

0.14502

0.14491

0.14479

0.14467

0.14456

0.14444

0.14433

0.14421

0.14409

0.14398

0.14386

0.14375

0.14363

0.14352

0.14340

0.14329

0.14317

0.14306

0.14294

0.14283

0.14271

0.14260

0.14248

0.14237

0.14225

0.14214

0.14202

0.14191

0.14180

0.14168

0.14157

0.14145

0.14134

0.14123

0.14111

0.14100

0.14089

0.14077

0.14066

0.14055

0.14043

0.14032

0.14021

0.14009

0.13998

0.13987

0.13976

0.13964 $\begin{array}{lll}12 & 0.40284 & 20 \\ 12 & 0.40264 & 19\end{array}$

$110.40245 \quad 20$

$12 \quad 0.40225 \quad 19$

$120.40206 \quad 20$

11

12

11

12

12

11

12

11

12

11

12

11

12

11

12

11

12

11

12

11

12

\section{1}

12

11

11

12

11

12

11

11

12

11

11

12

11

11

12

11

11

12

11

11

11

12

$0.40186 \quad 19$

0.4016720

$0.40147 \quad 19$

$0.40108 \quad 20$

$0.40088 \quad 19$

0.4006920

$0.40049 \quad 19$

$0.40030 \quad 20$

$0.40010 \quad 19$

0.3999120

$0.39971 \quad 19$

0.3995220

$0.39932 \quad 19$

$0.39913 \quad 19$

$0.39894 \quad 20$

$0.39874 \quad 19$

$0.39855 \quad 20$

$0.39835 \quad 19$

$0.39816 \quad 20$
$0.40128 \quad 20$ 
Table I Harmonic Oscillator Contributions (in dimensionless form) to the

Thermodynamic Functions for Values of $\mathrm{X}=\mathrm{hc} / \mathrm{kT}$

I $\quad-\left(\mathrm{F}^{\circ}-\mathrm{E}_{\mathrm{O}}^{\circ}\right) / \mathrm{RT} \quad \Delta \quad\left(\mathrm{H}^{\circ}-\mathrm{E}_{\mathrm{O}}^{\circ}\right) / \mathrm{RT} \quad \Delta \quad \mathrm{S} \% / \mathrm{R}$

\begin{tabular}{|c|c|c|c|c|c|c|c|}
\hline 3.500 & 0.03066 & 3 & 0.10898 & 8 & 0.13964 & 11 & 0.39331 \\
\hline $\begin{array}{l}3.501 \\
3.502 \\
3.503 \\
3.504 \\
3.505\end{array}$ & $\begin{array}{l}0.03063 \\
0.03060 \\
0.03057 \\
0.03054 \\
0.03051\end{array}$ & $\begin{array}{l}3 \\
3 \\
3 \\
3 \\
3\end{array}$ & $\begin{array}{l}0.10890 \\
0.10882 \\
0.10874 \\
0.10866 \\
0.10858\end{array}$ & $\begin{array}{l}8 \\
8 \\
8 \\
8 \\
8\end{array}$ & $\begin{array}{l}0.13953 \\
0.13942 \\
0.13931 \\
0.13920 \\
0.13908\end{array}$ & $\begin{array}{l}11 \\
11 \\
11 \\
12 \\
11\end{array}$ & $\begin{array}{l}0.39312 \\
0.39293 \\
0.39273 \\
0.39254 \\
0.39235\end{array}$ \\
\hline $\begin{array}{l}3.506 \\
3.507 \\
3.508 \\
3.509 \\
3.510\end{array}$ & $\begin{array}{l}0.03048 \\
0.03045 \\
0.03041 \\
0.03038 \\
0.03035\end{array}$ & $\begin{array}{l}3 \\
4 \\
3 \\
3 \\
3\end{array}$ & $\begin{array}{l}0.10850 \\
0.10841 \\
0.10833 \\
0.10825 \\
0.10817\end{array}$ & $\begin{array}{l}9 \\
8 \\
8 \\
8 \\
8\end{array}$ & $\begin{array}{l}0.13897 \\
0.13886 \\
0.13875 \\
0.13864 \\
0.13853\end{array}$ & $\begin{array}{l}11 \\
11 \\
11 \\
11 \\
12\end{array}$ & $\begin{array}{l}0.39216 \\
0.39196 \\
0.39177 \\
0.39158 \\
0.39139\end{array}$ \\
\hline $\begin{array}{l}3.511 \\
3.512 \\
3.513 \\
3.514 \\
3.515\end{array}$ & $\begin{array}{l}0.03032 \\
0.03029 \\
0.03026 \\
0.03023 \\
0.03020\end{array}$ & $\begin{array}{l}3 \\
3 \\
3 \\
3 \\
3\end{array}$ & $\begin{array}{l}0.10809 \\
0.10801 \\
0.10793 \\
0.10785 \\
0.10777\end{array}$ & $\begin{array}{l}8 \\
8 \\
8 \\
8 \\
8\end{array}$ & $\begin{array}{l}0.13841 \\
0.13830 \\
0.13819 \\
0.13808 \\
0.13797\end{array}$ & $\begin{array}{l}11 \\
11 \\
11 \\
11 \\
11\end{array}$ & $\begin{array}{l}0.39119 \\
0.39100 \\
0.39081 \\
0.39062 \\
0.39042\end{array}$ \\
\hline $\begin{array}{l}3.516 \\
3.517 \\
3.518 \\
3.519 \\
3.520\end{array}$ & $\begin{array}{l}0.03017 \\
0.03014 \\
0.03011 \\
0.03008 \\
0.03005\end{array}$ & $\begin{array}{l}3 \\
3 \\
3 \\
3 \\
3\end{array}$ & $\begin{array}{l}0.10769 \\
0.10761 \\
0.10753 \\
0.10745 \\
0.10737\end{array}$ & $\begin{array}{l}8 \\
8 \\
8 \\
8 \\
8\end{array}$ & $\begin{array}{l}0.13786 \\
0.13775 \\
0.13764 \\
0.13753 \\
0.13741\end{array}$ & $\begin{array}{ll}1 & 1 \\
1 & 1 \\
1 & 1 \\
12 \\
11\end{array}$ & $\begin{array}{l}0.39023 \\
0.39004 \\
0.38985 \\
0.38966 \\
0.38946\end{array}$ \\
\hline $\begin{array}{l}3.521 \\
3.522 \\
3.523 \\
3.524 \\
3.525\end{array}$ & $\begin{array}{l}0.03002 \\
0.02999 \\
0.02995 \\
0.02992 \\
0.02989\end{array}$ & $\begin{array}{l}3 \\
4 \\
3 \\
3 \\
3\end{array}$ & $\begin{array}{l}0.10729 \\
0.10721 \\
0.10713 \\
0.10705 \\
0.10697\end{array}$ & $\begin{array}{l}8 \\
8 \\
8 \\
8 \\
8\end{array}$ & $\begin{array}{l}0.13730 \\
0.13719 \\
0.13708 \\
0.13697 \\
0.13686\end{array}$ & $\begin{array}{ll}1 & 1 \\
1 & 1 \\
1 & 1 \\
1 & 1 \\
1 & 1\end{array}$ & $\begin{array}{l}0.38927 \\
0.38908 \\
0.38889 \\
0.38870 \\
0.38850\end{array}$ \\
\hline $\begin{array}{l}3.526 \\
3.527 \\
3.528 \\
3.529 \\
3.530\end{array}$ & $\begin{array}{l}0.02986 \\
0.02983 \\
0.02980 \\
0.02977 \\
0.02974\end{array}$ & $\begin{array}{l}3 \\
3 \\
3 \\
3 \\
3\end{array}$ & $\begin{array}{l}0.10689 \\
0.10681 \\
0.10673 \\
0.10665 \\
0.10657\end{array}$ & $\begin{array}{l}8 \\
8 \\
8 \\
8 \\
8\end{array}$ & $\begin{array}{l}0.13675 \\
0.13664 \\
0.13653 \\
0.13642 \\
0.13631\end{array}$ & $\begin{array}{ll}1 & 1 \\
1 & 1 \\
11 \\
11 \\
11\end{array}$ & $\begin{array}{l}0.38831 \\
0.38812 \\
0.38793 \\
0.38774 \\
0.38755\end{array}$ \\
\hline $\begin{array}{l}3.531 \\
3.532 \\
3.533 \\
3.534 \\
3.535\end{array}$ & $\begin{array}{l}0.02971 \\
0.02968 \\
0.02965 \\
0.02962 \\
0.02959\end{array}$ & $\begin{array}{l}3 \\
3 \\
3 \\
3 \\
3\end{array}$ & $\begin{array}{l}0.10649 \\
0.10641 \\
0.10633 \\
0.10625 \\
0.10617\end{array}$ & $\begin{array}{l}8 \\
8 \\
8 \\
8 \\
8\end{array}$ & $\begin{array}{l}0.13620 \\
0.13609 \\
0.13598 \\
0.13587 \\
0.13576\end{array}$ & $\begin{array}{ll}1 & 1 \\
1 & 1 \\
1 & 1 \\
1 & 1 \\
1 & 0\end{array}$ & $\begin{array}{l}0.38736 \\
0.38716 \\
0.38697 \\
0.38678 \\
0.38659\end{array}$ \\
\hline $\begin{array}{l}3.536 \\
3.537 \\
3.538 \\
3.539 \\
3.540\end{array}$ & $\begin{array}{l}0.02956 \\
0.02953 \\
0.02950 \\
0.02947 \\
0.02944\end{array}$ & $\begin{array}{l}3 \\
3 \\
3 \\
3 \\
3\end{array}$ & $\begin{array}{l}0.10609 \\
0.10601 \\
0.10593 \\
0.10586 \\
0.10578\end{array}$ & $\begin{array}{l}8 \\
8 \\
7 \\
8 \\
8\end{array}$ & $\begin{array}{l}0.13566 \\
0.13555 \\
0.13544 \\
0.13533 \\
0.13522\end{array}$ & $\begin{array}{ll}1 & 1 \\
1 & 1 \\
1 & 1 \\
1 & 1 \\
1 & 1\end{array}$ & $\begin{array}{l}0.38640 \\
0.38621 \\
0.38602 \\
0.38583 \\
0.38564\end{array}$ \\
\hline $\begin{array}{l}3.541 \\
3.542 \\
3.543 \\
3.544 \\
3.545\end{array}$ & $\begin{array}{l}0.02941 \\
0.02938 \\
0.02935 \\
0.02932 \\
0.02929\end{array}$ & $\begin{array}{l}3 \\
3 \\
3 \\
3 \\
3\end{array}$ & $\begin{array}{l}0.10570 \\
0.10562 \\
0.10554 \\
0.10546 \\
0.10538\end{array}$ & $\begin{array}{l}8 \\
8 \\
8 \\
8 \\
8\end{array}$ & $\begin{array}{l}0.13511 \\
0.13500 \\
0.13489 \\
0.13478 \\
0.13467\end{array}$ & $\begin{array}{ll}1 & 1 \\
1 & 1 \\
1 & 1 \\
1 & 1 \\
1 & 0\end{array}$ & $\begin{array}{l}0.38545 \\
0.38525 \\
0.38506 \\
0.38487 \\
0.38468\end{array}$ \\
\hline $\begin{array}{l}3.546 \\
3.547 \\
3.548 \\
3.549 \\
3.550\end{array}$ & $\begin{array}{l}0.02926 \\
0.02923 \\
0.02920 \\
0.02917 \\
0.02915\end{array}$ & $\begin{array}{l}3 \\
3 \\
3 \\
2\end{array}$ & $\begin{array}{l}0.10530 \\
0.10522 \\
0.10515 \\
0.10507 \\
0.10499\end{array}$ & $\begin{array}{l}8 \\
7 \\
8 \\
8\end{array}$ & $\begin{array}{l}0.13457 \\
0.13446 \\
0.13435 \\
0.13424 \\
0.13413\end{array}$ & $\begin{array}{ll}1 & 1 \\
1 & 1 \\
1 & 1 \\
1 & 1\end{array}$ & $\begin{array}{l}0.38449 \\
0.38430 \\
0.38411 \\
0.38392 \\
0.38373\end{array}$ \\
\hline
\end{tabular}

$\Delta \quad \mathrm{Co} / \mathrm{R}$

$\Delta$ 
Table I Harmonic Oscillator Contributions (in dimensionless form) to the

Thermodynamic Functions for Values of $\mathrm{X}=\mathrm{hc} v / \mathrm{kT}$

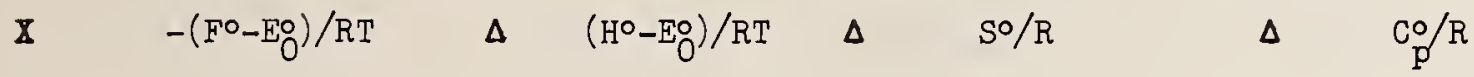

$\Delta$

\begin{tabular}{|c|c|c|c|c|c|c|c|}
\hline 3.550 & 0.02915 & 3 & 0.10499 & 8 & 0.13413 & 10 & 0.38373 \\
\hline $\begin{array}{l}3.551 \\
3.552 \\
3.553 \\
3.554 \\
3.555\end{array}$ & $\begin{array}{l}0.02912 \\
0.02909 \\
0.02906 \\
0.02903 \\
0.02900\end{array}$ & $\begin{array}{l}3 \\
3 \\
3 \\
3 \\
3\end{array}$ & $\begin{array}{l}0.10491 \\
0.10483 \\
0.10475 \\
0.10467 \\
0.10460\end{array}$ & $\begin{array}{l}8 \\
8 \\
8 \\
7 \\
8\end{array}$ & $\begin{array}{l}0.13403 \\
0.13392 \\
0.13381 \\
0.13370 \\
0.13359\end{array}$ & $\begin{array}{l}11 \\
11 \\
11 \\
11 \\
10\end{array}$ & $\begin{array}{l}0.38354 \\
0.38335 \\
0.38316 \\
0.38297 \\
0.38278\end{array}$ \\
\hline $\begin{array}{l}3.556 \\
3.557 \\
3.558 \\
3.559 \\
3.560\end{array}$ & $\begin{array}{l}0.02897 \\
0.02894 \\
0.02891 \\
0.02888 \\
0.02885\end{array}$ & $\begin{array}{l}3 \\
3 \\
3 \\
3 \\
3\end{array}$ & $\begin{array}{l}0.10452 \\
0.10444 \\
0.10436 \\
0.10428 \\
0.10421\end{array}$ & $\begin{array}{l}8 \\
8 \\
8 \\
7 \\
8\end{array}$ & $\begin{array}{l}0.13349 \\
0.13338 \\
0.13327 \\
0.13316 \\
0.13306\end{array}$ & $\begin{array}{l}11 \\
11 \\
11 \\
10 \\
11\end{array}$ & $\begin{array}{l}0.38259 \\
0.38240 \\
0.38221 \\
0.38202 \\
0.38183\end{array}$ \\
\hline $\begin{array}{l}3.561 \\
3.562 \\
3.563 \\
3.564 \\
3.565\end{array}$ & $\begin{array}{l}0.02882 \\
0.02879 \\
0.02876 \\
0.02873 \\
0.02871\end{array}$ & $\begin{array}{l}3 \\
3 \\
3 \\
2 \\
3\end{array}$ & $\begin{array}{l}0.10413 \\
0.10405 \\
0.10397 \\
0.10389 \\
0.10382\end{array}$ & $\begin{array}{l}8 \\
8 \\
8 \\
7 \\
8\end{array}$ & $\begin{array}{l}0.13295 \\
0.13284 \\
0.13274 \\
0.13263 \\
0.13252\end{array}$ & $\begin{array}{l}11 \\
10 \\
11 \\
11 \\
11\end{array}$ & $\begin{array}{l}0.38164 \\
0.38145 \\
0.38126 \\
0.38107 \\
0.38088\end{array}$ \\
\hline $\begin{array}{l}3.566 \\
3.567 \\
3.568 \\
3.569 \\
3.570\end{array}$ & $\begin{array}{l}0.02868 \\
0.02865 \\
0.02862 \\
0.02859 \\
0.02856\end{array}$ & $\begin{array}{l}3 \\
3 \\
3 \\
3 \\
3\end{array}$ & $\begin{array}{l}0.10374 \\
0.10366 \\
0.10358 \\
0.10351 \\
0.10343\end{array}$ & $\begin{array}{l}8 \\
8 \\
7 \\
8 \\
8\end{array}$ & $\begin{array}{l}0.13241 \\
0.13231 \\
0.13220 \\
0.13209 \\
0.13199\end{array}$ & $\begin{array}{l}10 \\
11 \\
11 \\
10 \\
11\end{array}$ & $\begin{array}{l}0.38069 \\
0.38050 \\
0.38032 \\
0.38013 \\
0.37994\end{array}$ \\
\hline $\begin{array}{l}3.571 \\
3.572 \\
3.573 \\
3.574 \\
3.575\end{array}$ & $\begin{array}{l}0.02853 \\
0.02850 \\
0.02847 \\
0.02844 \\
0.02842\end{array}$ & $\begin{array}{l}3 \\
3 \\
3 \\
2 \\
3\end{array}$ & $\begin{array}{l}0.10335 \\
0.10327 \\
0.10320 \\
0.10312 \\
0.10304\end{array}$ & $\begin{array}{l}8 \\
7 \\
8 \\
8 \\
8\end{array}$ & $\begin{array}{l}0.13188 \\
0.13178 \\
0.13167 \\
0.13156 \\
0.13146\end{array}$ & $\begin{array}{l}10 \\
11 \\
11 \\
10 \\
11\end{array}$ & $\begin{array}{l}0.37975 \\
0.37956 \\
0.37937 \\
0.37918 \\
0.37899\end{array}$ \\
\hline $\begin{array}{l}3.576 \\
3.577 \\
3.578 \\
3.579 \\
3.580\end{array}$ & $\begin{array}{l}0.02839 \\
0.02836 \\
0.02833 \\
0.02830 \\
0.02827\end{array}$ & $\begin{array}{l}3 \\
3 \\
3 \\
3 \\
3\end{array}$ & $\begin{array}{l}0.10296 \\
0.10289 \\
0.10281 \\
0.10273 \\
0.10266\end{array}$ & $\begin{array}{l}7 \\
8 \\
8 \\
7 \\
8\end{array}$ & $\begin{array}{l}0.13135 \\
0.13125 \\
0.13114 \\
0.113103 \\
0.13093\end{array}$ & $\begin{array}{l}10 \\
11 \\
11 \\
10 \\
11\end{array}$ & $\begin{array}{l}0.37880 \\
0.37861 \\
0.37843 \\
0.37824 \\
0.37805\end{array}$ \\
\hline $\begin{array}{l}3.581 \\
3.582 \\
3.583 \\
3.584 \\
3.585\end{array}$ & $\begin{array}{l}0.02824 \\
0.02821 \\
0.02819 \\
0.02816 \\
0.02813\end{array}$ & $\begin{array}{l}3 \\
2 \\
3 \\
3 \\
3\end{array}$ & $\begin{array}{l}0.10258 \\
0.10250 \\
0.10243 \\
0.10235 \\
0.10227\end{array}$ & $\begin{array}{l}8 \\
7 \\
8 \\
8 \\
7\end{array}$ & $\begin{array}{l}0.13082 \\
0.13072 \\
0.13061 \\
0.13051 \\
0.13040\end{array}$ & $\begin{array}{l}10 \\
11 \\
10 \\
11 \\
10\end{array}$ & $\begin{array}{l}0.37786 \\
0.37767 \\
0.37748 \\
0.37730 \\
0.37711\end{array}$ \\
\hline $\begin{array}{l}3.586 \\
3.587 \\
3.588 \\
3.589 \\
3.590\end{array}$ & $\begin{array}{l}0.02810 \\
0.02807 \\
0.02804 \\
0.02801 \\
0.02799\end{array}$ & $\begin{array}{l}3 \\
3 \\
3 \\
2 \\
3\end{array}$ & $\begin{array}{l}0.10220 \\
0.10212 \\
0.10204 \\
0.10197 \\
0.10189\end{array}$ & $\begin{array}{l}8 \\
8 \\
7 \\
8 \\
8\end{array}$ & $\begin{array}{l}0.13030 \\
0.13019 \\
0.13009 \\
0.12998 \\
0.12988\end{array}$ & $\begin{array}{l}11 \\
10 \\
11 \\
10 \\
11\end{array}$ & $\begin{array}{l}0.37692 \\
0.37673 \\
0.37654 \\
0.37635 \\
0.37617\end{array}$ \\
\hline $\begin{array}{l}3.591 \\
3.592 \\
3.593 \\
3.594 \\
3.595\end{array}$ & $\begin{array}{l}0.02796 \\
0.02793 \\
0.02790 \\
0.02787 \\
0.02784\end{array}$ & $\begin{array}{l}3 \\
3 \\
3 \\
3 \\
2\end{array}$ & $\begin{array}{l}0.10181 \\
0.10174 \\
0.10166 \\
0.10158 \\
0.10151\end{array}$ & $\begin{array}{l}7 \\
8 \\
8 \\
7 \\
8\end{array}$ & $\begin{array}{l}0.12977 \\
0.12967 \\
0.12956 \\
0.12946 \\
0.12935\end{array}$ & $\begin{array}{l}10 \\
11 \\
10 \\
11 \\
10\end{array}$ & $\begin{array}{l}0.37598 \\
0.37579 \\
0.37560 \\
0.37542 \\
0.37523\end{array}$ \\
\hline $\begin{array}{l}3.596 \\
3.597 \\
3.598 \\
3.599 \\
3.600\end{array}$ & $\begin{array}{l}0.02782 \\
0.02779 \\
0.02776 \\
0.02773 \\
0.02770\end{array}$ & $\begin{array}{l}3 \\
3 \\
3 \\
3\end{array}$ & $\begin{array}{l}0.10143 \\
0.10136 \\
0.10128 \\
0.10120 \\
0.10113\end{array}$ & $\begin{array}{l}7 \\
8 \\
8 \\
7\end{array}$ & $\begin{array}{l}0.12925 \\
0.12914 \\
0.12904 \\
0.112894 \\
0.12883\end{array}$ & $\begin{array}{l}11 \\
10 \\
10 \\
11\end{array}$ & $\begin{array}{l}0.37504 \\
0.37485 \\
0.37466 \\
0.37448 \\
0.37429\end{array}$ \\
\hline
\end{tabular}


Table I Harmonic Oscillator Contributions (in dimensionless form) to the

Thermodynamic Functions for Values of $\mathrm{X}=\mathrm{hc} / \mathrm{kT}$

I $-\left(F^{\circ}-E_{0}^{\circ}\right) / R T$

$\Delta \quad\left(H^{\circ}-E_{0}^{\circ}\right) / R T$

$\Delta$

$S^{\circ} / \mathrm{R}$

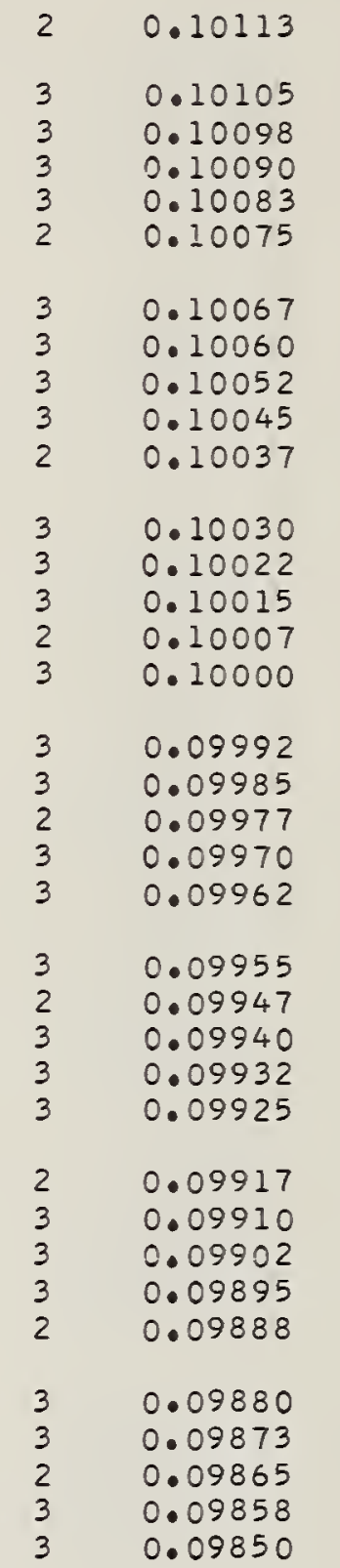

8
7
8
7
8
8

0.12883
0.12873
0.12862
0.12852
0.12842
0.12831

0.10067

3.606

3.607

3.608

3.609

3.610

3.611

3.612

3.613

3.614

3.615

3.616

3.617

3.618

3. 619

3.620

3.621

3.622

3.623

3.624

3.625

3.626

3.627

3.628

3.629

3.630

3.631

3.632

3.633

3.634

3.635

3.636

3.637

3.638

3.639

3.640

3.641

3.642

3.643

3.644

3.645

3.646

3.647

3.648

3.649

3.650

$$
\begin{aligned}
& 0.02770 \\
& 0.02768 \\
& 0.02765 \\
& 0.02762 \\
& 0.02759
\end{aligned}
$$

0.02754

0.02751

0.02748

0.02745

0.02742

0.02740

0.02737

0.02734

0.02731

0.02729

0.02726

0.02723

0.02720

0.02718

0.02715

0.02712

0.02709

0.02707

0.02704

0.02701

0.02698

0.02696

0.02693

0.02690

0.02687

0.02685

0.02679

0.02677

0.02674

0.02671

0.02668

0.02666

0.02663

0.02660

0.02658

0.02655

0.02652

0.02650

0.02647

0.02644

0.02642

0.02636

0.02633
0.02682

0.02639

0.12831

0.12821

0.12811

0.12800

0.12790

0.12780

0.12769

0.12749

0.12738

0.12728

0.12718

0.12708

0.12697

0.12687

0.12677

0.12667

0.12656

0.12646

0.12636

0.12626

0.12616

0.12605

0.12585

0.12575

0.12565

0.12555

0.12545

0.12534

0.12524

0.12514

0.12504

0.12494

0.12484

0.09828

0.09813

0.12474

0.09806

0.09799

0.12464

0.12454

0.12444

0.12434

0.09784

0.09777

0.12423

0.09769

0.09762

0.09755

0.09747

0.09740

0.12393
$\Delta$

$\mathrm{CO} / \mathrm{R}$

$\Delta$

10

0.37429

19

11

0.37410

18

10

10

11

0.37392

0.37373

0.37354

0.37335

19

19

19

10

0.37317

18

10

0.37298

0.37279

19

10

0.37261

0.37242

18

19

11

0.37223

18

10

0.37205

0.37186

11

10

0.37167

0.37149

19

19

18

10

0.37130

19

0.37111

0.37093

0.37074

18

10

10

0.37055

19

11

0.37037

18

10

10

0.37018

0.37000

0.36981

0.36962

19

10

0.36944
0.36925

11

0.36907

0.36888

10

10

0.36869

10

0.36851

10

11

10

0.36832

0.36814

0.36795

0.36777

18

19

19

18

10

0.36758

10

10

0.09821

10

10

0.36740

0.36721

0.36703

0.36684

19

18

19

19

18

10

0.36666

0.36647

0.36629

0.36610

0.36592

19

18

19

18

19
0.12413

0.12403

0.12383

0.12373
0.36573

18

10

0.36555

19

0.36536

18

10

0.36518

0.36499

18

18

19

18

19 
Table I Harmonic Oscillator Contributions (in dimensionless form) to the

Thermodynamic Functions for Values of $X=h c v / k T$

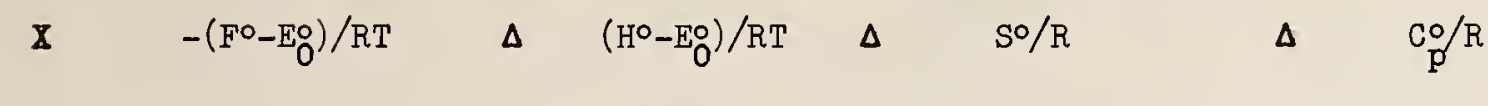

\begin{tabular}{|c|c|c|c|c|c|c|c|}
\hline 3.650 & 0.02633 & 2 & 0.09740 & 7 & 0.12373 & 10 & 0.36499 \\
\hline $\begin{array}{l}3.651 \\
3.652 \\
3.653 \\
3.654 \\
3.655\end{array}$ & $\begin{array}{l}0.02631 \\
0.02628 \\
0.02625 \\
0.02623 \\
0.02620\end{array}$ & $\begin{array}{l}3 \\
3 \\
2 \\
3 \\
2\end{array}$ & $\begin{array}{l}0.09733 \\
0.09725 \\
0.09718 \\
0.09711 \\
0.09703\end{array}$ & $\begin{array}{l}8 \\
7 \\
7 \\
8 \\
7\end{array}$ & $\begin{array}{l}0.12363 \\
0.12353 \\
0.12343 \\
0.12333 \\
0.12323\end{array}$ & $\begin{array}{r}10 \\
10 \\
10 \\
10 \\
9\end{array}$ & $\begin{array}{l}0.36481 \\
0.36462 \\
0.36444 \\
0.36426 \\
0.36407\end{array}$ \\
\hline $\begin{array}{l}3.656 \\
3.657 \\
3.658 \\
3.659 \\
3.660\end{array}$ & $\begin{array}{l}0.02618 \\
0.02615 \\
0.02612 \\
0.02610 \\
0.02607\end{array}$ & $\begin{array}{l}3 \\
3 \\
2 \\
3 \\
3\end{array}$ & $\begin{array}{l}0.09696 \\
0.09689 \\
0.09681 \\
0.09674 \\
0.09667\end{array}$ & $\begin{array}{l}7 \\
8 \\
7 \\
7 \\
7\end{array}$ & $\begin{array}{l}0.12314 \\
0.12304 \\
0.12294 \\
0.12284 \\
0.12274\end{array}$ & $\begin{array}{l}10 \\
10 \\
10 \\
10 \\
10\end{array}$ & $\begin{array}{l}0.36389 \\
0.36370 \\
0.36352 \\
0.36334 \\
0.36315\end{array}$ \\
\hline $\begin{array}{l}3.661 \\
3.662 \\
3.663 \\
3.664 \\
3.665\end{array}$ & $\begin{array}{l}0.02604 \\
0.02602 \\
0.02599 \\
0.02596 \\
0.02594\end{array}$ & $\begin{array}{l}2 \\
3 \\
3 \\
2 \\
3\end{array}$ & $\begin{array}{l}0.09660 \\
0.09652 \\
0.09645 \\
0.09638 \\
0.09631\end{array}$ & $\begin{array}{l}8 \\
7 \\
7 \\
7 \\
8\end{array}$ & $\begin{array}{l}0.12264 \\
0.12254 \\
0.12244 \\
0.12234 \\
0.12224\end{array}$ & $\begin{array}{l}10 \\
10 \\
10 \\
10 \\
10\end{array}$ & $\begin{array}{l}0.36297 \\
0.36278 \\
0.36260 \\
0.36242 \\
0.36223\end{array}$ \\
\hline $\begin{array}{l}3.666 \\
3.667 \\
3.668 \\
3.669 \\
3.670\end{array}$ & $\begin{array}{l}0.02591 \\
0.02589 \\
0.02586 \\
0.02583 \\
0.02581\end{array}$ & $\begin{array}{l}2 \\
3 \\
3 \\
2 \\
3\end{array}$ & $\begin{array}{l}0.09623 \\
0.09616 \\
0.09609 \\
0.09602 \\
0.09594\end{array}$ & $\begin{array}{l}7 \\
7 \\
7 \\
8 \\
7\end{array}$ & $\begin{array}{l}0.12214 \\
0.12205 \\
0.12195 \\
0.12185 \\
0.12175\end{array}$ & $\begin{array}{l}9 \\
10 \\
10 \\
10 \\
10\end{array}$ & $\begin{array}{l}0.36205 \\
0.36187 \\
0.36168 \\
0.36150 \\
0.36132\end{array}$ \\
\hline $\begin{array}{l}3.671 \\
3.672 \\
3.673 \\
3.674 \\
3.675\end{array}$ & $\begin{array}{l}0.02578 \\
0.02575 \\
0.02573 \\
0.02570 \\
0.02568\end{array}$ & $\begin{array}{l}3 \\
2 \\
3 \\
2 \\
3\end{array}$ & $\begin{array}{l}0.09587 \\
0.09580 \\
0.09573 \\
0.09565 \\
0.09558\end{array}$ & $\begin{array}{l}7 \\
7 \\
8 \\
7 \\
7\end{array}$ & $\begin{array}{l}0.12165 \\
0.12155 \\
0.12145 \\
0.12136 \\
0.12126\end{array}$ & $\begin{array}{r}10 \\
10 \\
9 \\
10 \\
10\end{array}$ & $\begin{array}{l}0.36113 \\
0.36095 \\
0.36077 \\
0.36058 \\
0.36040\end{array}$ \\
\hline $\begin{array}{l}3.676 \\
3.677 \\
3.678 \\
3.679 \\
3.680\end{array}$ & $\begin{array}{l}0.02565 \\
0.02562 \\
0.02560 \\
0.02557 \\
0.02555\end{array}$ & $\begin{array}{l}3 \\
2 \\
3 \\
2 \\
3\end{array}$ & $\begin{array}{l}0.09551 \\
0.09544 \\
0.09537 \\
0.09529 \\
0.09522\end{array}$ & $\begin{array}{l}7 \\
7 \\
8 \\
7 \\
7\end{array}$ & $\begin{array}{l}0.12116 \\
0.12106 \\
0.12096 \\
0.12087 \\
0.12077\end{array}$ & $\begin{array}{r}10 \\
10 \\
9 \\
10 \\
10\end{array}$ & $\begin{array}{l}0.36022 \\
0.36003 \\
0.35985 \\
0.35967 \\
0.35949\end{array}$ \\
\hline $\begin{array}{l}3.681 \\
3.682 \\
3.683 \\
3.684 \\
3.685\end{array}$ & $\begin{array}{l}0.02552 \\
0.02549 \\
0.02547 \\
0.02544 \\
0.02542\end{array}$ & $\begin{array}{l}3 \\
2 \\
3 \\
2 \\
3\end{array}$ & $\begin{array}{l}0.09515 \\
0.09508 \\
0.09501 \\
0.09494 \\
0.09486\end{array}$ & $\begin{array}{l}7 \\
7 \\
7 \\
8 \\
7\end{array}$ & $\begin{array}{l}0.12067 \\
0.12057 \\
0.12048 \\
0.12038 \\
0.12028\end{array}$ & $\begin{array}{r}10 \\
9 \\
10 \\
10 \\
10\end{array}$ & $\begin{array}{l}0.35930 \\
0.35912 \\
0.35894 \\
0.35876 \\
0.35857\end{array}$ \\
\hline $\begin{array}{l}3.686 \\
3.687 \\
3.688 \\
3.689 \\
3.690\end{array}$ & $\begin{array}{l}0.02539 \\
0.02537 \\
0.02534 \\
0.02531 \\
0.02529\end{array}$ & $\begin{array}{l}2 \\
3 \\
3 \\
2 \\
3\end{array}$ & $\begin{array}{l}0.09479 \\
0.09472 \\
0.09465 \\
0.09458 \\
0.09451\end{array}$ & $\begin{array}{l}7 \\
7 \\
7 \\
7 \\
7\end{array}$ & $\begin{array}{l}0.12018 \\
0.12009 \\
0.11999 \\
0.11989 \\
0.11980\end{array}$ & $\begin{array}{r}9 \\
10 \\
10 \\
9 \\
10\end{array}$ & $\begin{array}{l}0.35839 \\
0.35821 \\
0.35803 \\
0.35784 \\
0.35766\end{array}$ \\
\hline $\begin{array}{l}3.691 \\
3.692 \\
3.693 \\
3.694 \\
3.695\end{array}$ & $\begin{array}{l}0.02526 \\
0.02524 \\
0.02521 \\
0.02519 \\
0.02516\end{array}$ & $\begin{array}{l}2 \\
3 \\
2 \\
3 \\
2\end{array}$ & $\begin{array}{l}0.09444 \\
0.09436 \\
0.09429 \\
0.09422 \\
0.09415\end{array}$ & $\begin{array}{l}8 \\
7 \\
7 \\
7 \\
7\end{array}$ & $\begin{array}{l}0.11970 \\
0.11960 \\
0.11951 \\
0.11941 \\
0.11931\end{array}$ & $\begin{array}{r}10 \\
9 \\
10 \\
10 \\
9\end{array}$ & $\begin{array}{l}0.35748 \\
0.35730 \\
0.35712 \\
0.35693 \\
0.35675\end{array}$ \\
\hline $\begin{array}{l}3.696 \\
3.697 \\
3.698 \\
3.699 \\
3.700\end{array}$ & $\begin{array}{l}0.02514 \\
0.02511 \\
0.02509 \\
0.02506 \\
0.02503\end{array}$ & $\begin{array}{l}3 \\
2 \\
3 \\
3\end{array}$ & $\begin{array}{l}0.09408 \\
0.09401 \\
0.09394 \\
0.09387 \\
0.09380\end{array}$ & $\begin{array}{l}7 \\
7 \\
7 \\
7\end{array}$ & $\begin{array}{l}0.11922 \\
0.11912 \\
0.11902 \\
0.11893 \\
0.11883\end{array}$ & $\begin{array}{r}10 \\
10 \\
9 \\
10\end{array}$ & $\begin{array}{l}0.35657 \\
0.35639 \\
0.35621 \\
0.35602 \\
0.35584\end{array}$ \\
\hline
\end{tabular}


Table I Harmonic Oscillator Contributions (in dimensionless form) to the

Thermodynamic Functions for Values of $X=h c v / k T$

\begin{tabular}{|c|c|c|c|c|c|c|c|}
\hline $\mathbf{Z}$ & $-\left(F^{\circ}-E_{0}^{\circ}\right) / R T$ & $\Delta$ & $\left(\mathrm{H}^{\circ}-\mathrm{E} \mathrm{O}\right) / \mathrm{RT}$ & $\Delta$ & $\mathrm{SO} / \mathrm{R}$ & $\Delta$ & $c \% / R$ \\
\hline 3.700 & 0.02503 & 2 & 0.09380 & 7 & 0.11883 & 10 & 0.35584 \\
\hline $\begin{array}{l}3.701 \\
3.702 \\
3.703 \\
3.704 \\
3.705\end{array}$ & $\begin{array}{l}0.02501 \\
0.02498 \\
0.02496 \\
0.02493 \\
0.02491\end{array}$ & $\begin{array}{l}3 \\
2 \\
3 \\
2 \\
3\end{array}$ & $\begin{array}{l}0.09373 \\
0.09365 \\
0.09358 \\
0.09351 \\
0.09344\end{array}$ & $\begin{array}{l}8 \\
7 \\
7 \\
7 \\
7\end{array}$ & $\begin{array}{l}0.11873 \\
0.11864 \\
0.11854 \\
0.11845 \\
0.11835\end{array}$ & $\begin{array}{r}9 \\
10 \\
9 \\
10 \\
10\end{array}$ & $\begin{array}{l}0.35566 \\
0.35548 \\
0.35530 \\
0.35512 \\
0.35494\end{array}$ \\
\hline $\begin{array}{l}3.706 \\
3.707 \\
3.708 \\
3.709 \\
3.710\end{array}$ & $\begin{array}{l}0.02488 \\
0.02486 \\
0.02483 \\
0.02481 \\
0.02478\end{array}$ & $\begin{array}{l}2 \\
3 \\
2 \\
3 \\
2\end{array}$ & $\begin{array}{l}0.09337 \\
0.09330 \\
0.09323 \\
0.09316 \\
0.09309\end{array}$ & $\begin{array}{l}7 \\
7 \\
7 \\
7 \\
7\end{array}$ & $\begin{array}{l}0.11825 \\
0.11816 \\
0.11806 \\
0.11797 \\
0.11787\end{array}$ & $\begin{array}{r}9 \\
10 \\
9 \\
10 \\
9\end{array}$ & $\begin{array}{l}0.35475 \\
0.35457 \\
0.35439 \\
0.35421 \\
0.35403\end{array}$ \\
\hline $\begin{array}{l}3.711 \\
3.712 \\
3.713 \\
3.714 \\
3.715\end{array}$ & $\begin{array}{l}0.02476 \\
0.02473 \\
0.02471 \\
0.02468 \\
0.02466\end{array}$ & $\begin{array}{l}3 \\
2 \\
3 \\
2 \\
3\end{array}$ & $\begin{array}{l}0.09302 \\
0.09295 \\
0.09288 \\
0.09281 \\
0.09274\end{array}$ & $\begin{array}{l}7 \\
7 \\
7 \\
7 \\
7\end{array}$ & $\begin{array}{l}0.11778 \\
0.11768 \\
0.11759 \\
0.111749 \\
0.11740\end{array}$ & $\begin{array}{r}10 \\
9 \\
10 \\
9 \\
10\end{array}$ & $\begin{array}{l}0.35385 \\
0.35367 \\
0.35349 \\
0.35331 \\
0.35313\end{array}$ \\
\hline $\begin{array}{l}3.716 \\
3.717 \\
3.718 \\
3.719 \\
3.720\end{array}$ & $\begin{array}{l}0.02463 \\
0.02461 \\
0.02458 \\
0.02456 \\
0.02453\end{array}$ & $\begin{array}{l}2 \\
3 \\
2 \\
3 \\
2\end{array}$ & $\begin{array}{l}0.09267 \\
0.09260 \\
0.09253 \\
0.09246 \\
0.09239\end{array}$ & $\begin{array}{l}7 \\
7 \\
7 \\
7 \\
7\end{array}$ & $\begin{array}{l}0.11730 \\
0.11721 \\
0.11711 \\
0.111702 \\
0.11692\end{array}$ & $\begin{array}{r}9 \\
10 \\
9 \\
10 \\
9\end{array}$ & $\begin{array}{l}0.35295 \\
0.35277 \\
0.35258 \\
0.35240 \\
0.35222\end{array}$ \\
\hline $\begin{array}{l}3.721 \\
3.722 \\
3.723 \\
3.724 \\
3.725\end{array}$ & $\begin{array}{l}0.02451 \\
0.02448 \\
0.02446 \\
0.02443 \\
0.02441\end{array}$ & $\begin{array}{l}3 \\
2 \\
3 \\
2 \\
3\end{array}$ & $\begin{array}{l}0.09232 \\
0.09225 \\
0.09218 \\
0.09211 \\
0.09204\end{array}$ & $\begin{array}{l}7 \\
7 \\
7 \\
7 \\
7\end{array}$ & $\begin{array}{l}0.11683 \\
0.11673 \\
0.11664 \\
0.11654 \\
0.11645\end{array}$ & $\begin{array}{r}10 \\
9 \\
10 \\
9 \\
10\end{array}$ & $\begin{array}{l}0.35204 \\
0.35186 \\
0.35168 \\
0.35150 \\
0.35132\end{array}$ \\
\hline $\begin{array}{l}3.726 \\
3.727 \\
3.728 \\
3.729 \\
3.730\end{array}$ & $\begin{array}{l}0.02438 \\
0.02436 \\
0.02433 \\
0.02431 \\
0.02429\end{array}$ & $\begin{array}{l}2 \\
3 \\
2 \\
2 \\
3\end{array}$ & $\begin{array}{l}0.09197 \\
0.09190 \\
0.09183 \\
0.09176 \\
0.09169\end{array}$ & $\begin{array}{l}7 \\
7 \\
7 \\
7 \\
7\end{array}$ & $\begin{array}{l}0.11635 \\
0.11626 \\
0.11617 \\
0.11607 \\
0.11598\end{array}$ & $\begin{array}{r}9 \\
9 \\
10 \\
9 \\
10\end{array}$ & $\begin{array}{l}0.35114 \\
0.35096 \\
0.35078 \\
0.35060 \\
0.35042\end{array}$ \\
\hline $\begin{array}{l}3.731 \\
3.732 \\
3.733 \\
3.734 \\
3.735\end{array}$ & $\begin{array}{l}0.02426 \\
0.02424 \\
0.02421 \\
0.02419 \\
0.02416\end{array}$ & $\begin{array}{l}2 \\
3 \\
2 \\
3 \\
2\end{array}$ & $\begin{array}{l}0.09162 \\
0.09155 \\
0.09149 \\
0.09142 \\
0.09135\end{array}$ & $\begin{array}{l}7 \\
6 \\
7 \\
7 \\
7\end{array}$ & $\begin{array}{l}0.11588 \\
0.11579 \\
0.11570 \\
0.11560 \\
0.11551\end{array}$ & $\begin{array}{r}9 \\
9 \\
10 \\
9 \\
9\end{array}$ & $\begin{array}{l}0.35024 \\
0.35006 \\
0.34988 \\
0.34970 \\
0.34953\end{array}$ \\
\hline $\begin{array}{l}3.736 \\
3.737 \\
3.738 \\
3.739 \\
3.740\end{array}$ & $\begin{array}{l}0.02414 \\
0.02411 \\
0.02409 \\
0.02407 \\
0.02404\end{array}$ & $\begin{array}{l}3 \\
2 \\
2 \\
3 \\
2\end{array}$ & $\begin{array}{l}0.09128 \\
0.09121 \\
0.09114 \\
0.09107 \\
0.09100\end{array}$ & $\begin{array}{l}7 \\
7 \\
7 \\
7 \\
7\end{array}$ & $\begin{array}{l}0.11542 \\
0.11532 \\
0.11523 \\
0.11514 \\
0.11504\end{array}$ & $\begin{array}{r}10 \\
9 \\
9 \\
10 \\
9\end{array}$ & $\begin{array}{l}0.34935 \\
0.34917 \\
0.34899 \\
0.34881 \\
0.34863\end{array}$ \\
\hline $\begin{array}{l}3.741 \\
3.742 \\
3.743 \\
3.744 \\
3.745\end{array}$ & $\begin{array}{l}0.02402 \\
0.02399 \\
0.02397 \\
0.02394 \\
0.02392\end{array}$ & $\begin{array}{l}3 \\
2 \\
3 \\
2 \\
2\end{array}$ & $\begin{array}{l}0.09093 \\
0.09086 \\
0.09080 \\
0.09073 \\
0.09066\end{array}$ & $\begin{array}{l}7 \\
6 \\
7 \\
7 \\
7\end{array}$ & $\begin{array}{l}0.111495 \\
0.11486 \\
0.11476 \\
0.11467 \\
0.11458\end{array}$ & $\begin{array}{r}9 \\
10 \\
9 \\
9 \\
10\end{array}$ & $\begin{array}{l}0.34845 \\
0.34827 \\
0.34809 \\
0.34791 \\
0.34773\end{array}$ \\
\hline $\begin{array}{l}3.746 \\
3.747 \\
3.748 \\
3.749 \\
3.750\end{array}$ & $\begin{array}{l}0.02390 \\
0.02387 \\
0.02385 \\
0.02382 \\
0.02380\end{array}$ & $\begin{array}{l}3 \\
2 \\
3 \\
2\end{array}$ & $\begin{array}{l}0.09059 \\
0.09052 \\
0.09045 \\
0.09038 \\
0.09032\end{array}$ & $\begin{array}{l}7 \\
7 \\
7 \\
6\end{array}$ & $\begin{array}{l}0.11448 \\
0.11439 \\
0.11430 \\
0.11421 \\
0.11411\end{array}$ & $\begin{array}{r}9 \\
9 \\
9 \\
10\end{array}$ & $\begin{array}{l}0.34756 \\
0.34738 \\
0.34720 \\
0.34702 \\
0.34684\end{array}$ \\
\hline
\end{tabular}


Table I Harmonic Oscillator Contributions (in dimensionless form) to the

Thermodynamic Functions for Values of $\mathrm{X}=\mathrm{hc} v / \mathrm{kT}$

$\bar{x}$ $-\left(F^{\circ}-E_{O} O\right) / R T$

$\Delta \quad\left(H^{\circ}-E_{D}^{\circ}\right) / R T$

$\Delta$

So/R

0.02365

0.02363

0.02361

0.02358

0.02356

3.760

3.761

0.02354

3.762

3.763

3.764

3.765

3.766

3.767

3.768

3.769

3.770

3.771

3.772

3.773

3.774

3.775

3.776

3.777

3.778

3.779

3.780

3.781

3.782

3.783

3.784

3.785

3.786

3.787

3.788

3.789

3.790

3.791

3.792

3.793

3.794

3.795

3.796

3.797

3.798

3.799

3.800
0.02351

0.02349

0.02346

0.02344

0.02342

0.02339

0.02337

0.02335

0.02332

0.02330

0.02327

0.02325

0.02323

0.02320

0.02318

0.02316

0.02313

0.02311

0.02309

0.02306

0.02304

0.02302

0.02299

0.02297

0.02295

0.02292

0.02290

0.02288

0.02285

0.02283

0.02281

0.02279

0.02276

0.02274

0.02272

0.02269

0.02267

0.02265

0.02262

$\begin{array}{llll}3 & 0.09032 & 7 & 0.11411 \\ 2 & 0.09025 & 7 & 0.11402 \\ 2 & 0.09018 & 7 & 0.11393 \\ 3 & 0.09011 & 7 & 0.11384 \\ 2 & 0.09004 & 7 & 0.11374 \\ 3 & 0.08997 & 6 & 0.11365\end{array}$

0.08991

0.08984

0.08970

0.08963

0.08950

0.08943

0.08936

0.08929

0.08923

0.08916

0.08909

0.08902

0.08896

0.08889

0.08882

0.08875

0.08869

0.08862

0.08855

0.08849

0.08842

0.08835

0.08828

0.08822

0.08815

0.08808

0.08802

0.08795

\subsection{8}

0.08782

0.08775

0.08768

0.08762

0.08755

0.08748

0.08742

0.08735

0.08729

0.08722

0.08715

0.08709

0.08702

0.08695
0.11365

0.11356

0.11347

0.11338

0.11328

0.11319

0.11310

0.11301

0.11292

0.11283

0.11273

0.11264

0.11255

0.11246

0.11237

0.11228

0.11219

0.11210

0.11201

0.11192

0.11182

0.11173

0.11164

0.11155

0.11146

0.11137

0.11128

0.11119

0.11110

0.11101

0.11092

0.11083

0.11074

0.11065

0.11056

0.11047

0.11038

0.11029

0.11020

0.11011

0.11002

0.10994

0.10985

0.10976

0.10967

0.10958
0.08977

0.08957
$\Delta \quad \mathrm{C}_{\mathrm{p}}^{\circ} / \mathrm{R}$

$\Delta$

$\begin{array}{rll}9 & 0.34684 & 18 \\ 9 & 0.34666 & 18 \\ 9 & 0.34648 & 18 \\ 10 & 0.34630 & 17 \\ 9 & 0.34613 & 18 \\ 9 & 0.34595 & 18\end{array}$

$\begin{array}{lll}9 & 0.34577 & 18\end{array}$

$90.34559 \quad 18$

$\begin{array}{lll}10 & 0.34541 & 17\end{array}$

$90.34524 \quad 18$

$\begin{array}{lll}9 & 0.34506 & 18\end{array}$

$\begin{array}{lll}9 & 0.34488 & 18\end{array}$

$\begin{array}{lll}9 & 0.34470 \quad 18\end{array}$

$9 \quad 0.34452 \quad 17$

$10 \quad 0.34435 \quad 18$

$\begin{array}{lll}9 & 0.34417 & 18\end{array}$

$9 \quad 0.34399 \quad 18$

$9 \quad 0.34381 \quad 17$

$9 \quad 0.34364 \quad 18$

$9 \quad 0.34346 \quad 18$

$\begin{array}{lll}9 & 0.34328 & 18\end{array}$

$\begin{array}{lll}9 & 0.34310 & 17\end{array}$

$9 \quad 0.34293 \quad 18$

$\begin{array}{lll}9 & 0.34275 & 18\end{array}$

$10 \quad 0.34257 \quad 18$

$\begin{array}{lll}9 & 0.34239 & 17\end{array}$

$9 \quad 0.34222 \quad 18$

$90.34204 \quad 18$

$\begin{array}{lll}9 & 0.34186 & 17\end{array}$

$\begin{array}{lll}9 & 0.34169 & 18\end{array}$

$\begin{array}{lll}9 & 0.34151 & 18\end{array}$

$\begin{array}{lll}9 & 0.34133 & 17\end{array}$

$9 \quad 0.34116 \quad 18$

$9 \quad 0.34098 \quad 18$

$9 \quad 0.34080 \quad 17$

$\begin{array}{lll}9 & 0.34063 & 18\end{array}$

$9 \quad 0.34045$

$9 \quad 0.34027 \quad 17$

$9 \quad 0.34010 \quad 18$

$\begin{array}{lll}9 & 0.33992 & 17\end{array}$

$\begin{array}{lll}9 & 0.33992 & 17 \\ 9 & 0.33975 & 18\end{array}$

$9 \quad 0.33957 \quad 18$

$\begin{array}{lll}9 & 0.33939 & 17\end{array}$

$9 \quad 0.33922 \quad 18$

$\begin{array}{lll}9 & 0.33904 & 17\end{array}$

$\begin{array}{lll}8 & 0.33887 & 18\end{array}$

$\begin{array}{lll}9 & 0.33869 & 18\end{array}$

$\begin{array}{lll}9 & 0.33851 & 17\end{array}$

$9 \quad 0.33834 \quad 18$

$9 \quad 0.33816 \quad 17$

0.33799

\begin{tabular}{l}
18 \\
8 \\
7 \\
8 \\
8 \\
\hline \\
8 \\
7 \\
8 \\
8 \\
8 \\
\hline \\
7 \\
8 \\
8 \\
8 \\
7 \\
7 \\
8 \\
8 \\
7 \\
8 \\
8
\end{tabular}


Table I Harmonic Oscillator Contributions (in dimensionless form) to the

Thermodynamic Functions for Values of $\mathrm{X}=\mathrm{hc} v / \mathrm{kT}$

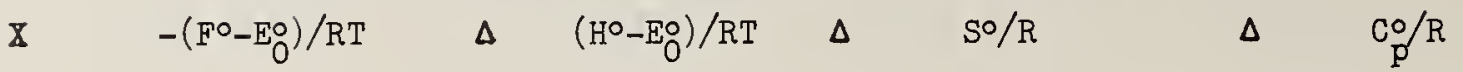

$\Delta$

\begin{tabular}{|c|c|c|c|c|c|c|c|c|}
\hline 3.800 & 0.02262 & 2 & 0.08695 & 6 & 0.10958 & 9 & 0.33799 & 18 \\
\hline 3.801 & 0.02260 & 2 & 0.08689 & 7 & 0.10949 & 9 & 0.33781 & 17 \\
\hline 3.802 & 0.02258 & 2 & 0.08682 & 6 & 0.10940 & 9 & 0.33764 & 18 \\
\hline 3.803 & 0.02256 & 3 & 0.08676 & 7 & 0.10931 & 9 & 0.33746 & 17 \\
\hline 3.804 & 0.02253 & 2 & 0.08669 & 7 & 0.10922 & 8 & 0.33729 & 18 \\
\hline 3.805 & 0.02251 & 2 & 0.08662 & 6 & 0.10914 & 9 & 0.33711 & 18 \\
\hline 3.806 & 0.02249 & 2 & 0.08656 & 7 & 0.10905 & 9 & 0.33693 & 17 \\
\hline 3.807 & 0.02247 & 3 & 0.08649 & 6 & 0.10896 & 9 & 0.33676 & 18 \\
\hline 3.808 & 0.02244 & 2 & 0.08643 & 7 & 0.10887 & 9 & 0.33658 & 17 \\
\hline 3.809 & 0.02242 & 2 & 0.08636 & 6 & 0.10878 & 9 & 0.33641 & 18 \\
\hline 3.810 & 0.02240 & 3 & 0.08630 & 7 & 0.10869 & 9 & 0.33623 & 17 \\
\hline 3.811 & 0.02237 & 2 & 0.08623 & 7 & 0.10860 & 8 & 0.33606 & 18 \\
\hline 3.812 & 0.02235 & 2 & 0.08616 & 6 & 0.10852 & 9 & 0.33588 & 17 \\
\hline 3.813 & 0.02233 & 2 & 0.08610 & 7 & 0.10843 & 9 & 0.33571 & 18 \\
\hline 3.814 & 0.02231 & 3 & 0.08603 & 6 & 0.10834 & 9 & 0.33553 & 17 \\
\hline 3.815 & 0.02228 & 2 & 0.08597 & 7 & 0.10825 & 9 & 0.33536 & 17 \\
\hline 3.816 & 0.02226 & 2 & 0.08590 & 6 & 0.10816 & 8 & 0.33519 & 18 \\
\hline 3.817 & 0.02224 & 2 & 0.08584 & 7 & 0.10808 & 9 & 0.33501 & 17 \\
\hline 3.818 & 0.02222 & 3 & 0.08577 & 6 & 0.10799 & 9 & 0.33484 & 18 \\
\hline 3.819 & 0.02219 & 2 & 0.08571 & 7 & 0.10790 & 9 & 0.33466 & 17 \\
\hline 3.820 & 0.02217 & 2 & 0.08564 & 6 & 0.10781 & 8 & 0.33449 & 18 \\
\hline 3.821 & 0.02215 & 2 & 0.08558 & 7 & 0.10773 & 9 & 0.33431 & 17 \\
\hline 3.822 & 0.02213 & 3 & 0.08551 & 6 & 0.10764 & 9 & 0.33414 & 18 \\
\hline 3.823 & 0.02210 & 2 & 0.08545 & 7 & 0.10755 & 9 & 0.33396 & 17 \\
\hline 3.824 & 0.02208 & 2 & 0.08538 & 6 & 0.10746 & 8 & 0.33379 & 17 \\
\hline 3.825 & 0.02206 & 2 & 0.08532 & 7 & 0.10738 & 9 & 0.33362 & 18 \\
\hline 3.826 & 0.02204 & 2 & 0.08525 & 6 & 0.10729 & 9 & 0.33344 & 17 \\
\hline 3.827 & 0.02202 & 3 & 0.08519 & 7 & 0.10720 & 8 & 0.33327 & 18 \\
\hline 3.828 & 0.02199 & 2 & 0.08512 & 6 & 0.10712 & 9 & 0.33309 & 17 \\
\hline 3.829 & 0.02197 & 2 & 0.08506 & 7 & 0.10703 & 9 & 0.33292 & 17 \\
\hline 3.830 & 0.02195 & 2 & 0.08499 & 6 & 0.10694 & 9 & 0.33275 & 18 \\
\hline 3.831 & 0.02193 & 3 & 0.08493 & 7 & 0.10685 & 8 & 0.33257 & 17 \\
\hline 3.832 & 0.02190 & 2 & 0.08486 & 6 & 0.10677 & 9 & 0.33240 & 17 \\
\hline 3.833 & 0.02188 & 2 & 0.08480 & 7 & 0.10668 & 9 & 0.33223 & 18 \\
\hline 3.834 & 0.02186 & 2 & 0.08473 & 6 & 0.10659 & 8 & 0.33205 & 17 \\
\hline 3.835 & 0.02184 & 2 & 0.08467 & 6 & 0.10651 & 9 & 0.33188 & 17 \\
\hline 3.836 & 0.02182 & 3 & 0.08461 & 7 & 0.10642 & 8 & 0.33171 & 18 \\
\hline 3.837 & 0.02179 & 2 & 0.08454 & 6 & 0.10634 & 9 & 0.33153 & 17 \\
\hline 3.838 & 0.02177 & 2 & 0.08448 & 7 & 0.10625 & 9 & 0.33136 & 17 \\
\hline 3.839 & 0.02175 & 2 & 0.08441 & 6 & 0.10616 & 8 & 0.33119 & 18 \\
\hline 3.840 & 0.02173 & 2 & 0.08435 & 7 & 0.10608 & 9 & 0.33101 & 17 \\
\hline 3.841 & 0.02171 & 3 & 0.08428 & 6 & 0.10599 & 9 & 0.33084 & 17 \\
\hline 3.842 & 0.02168 & 2 & 0.08422 & 6 & 0.10590 & 8 & 0.33067 & 18 \\
\hline 3.843 & 0.02166 & 2 & 0.08416 & 7 & 0.10582 & 9 & 0.33049 & 17 \\
\hline 3.844 & 0.02164 & 2 & 0.08409 & 6 & 0.10573 & 8 & 0.33032 & 17 \\
\hline 3.845 & 0.02162 & 2 & 0.08403 & 7 & 0.10565 & 9 & 0.33015 & 18 \\
\hline 3.846 & 0.02160 & 3 & 0.08396 & 6 & 0.10556 & 9 & 0.32997 & 17 \\
\hline 3.847 & 0.02157 & 2 & 0.08390 & 6 & 0.10547 & 8 & 0.32980 & 17 \\
\hline 3.848 & 0.02155 & 2 & 0.08384 & 7 & 0.10539 & 9 & 0.32963 & 17 \\
\hline 3.849 & 0.02153 & 2 & 0.08377 & 6 & 0.10530 & 8 & 0.32946 & 18 \\
\hline 3.850 & 0.02151 & & 0.08371 & & 0.10522 & & 0.32928 & \\
\hline
\end{tabular}


Table I Harmonic Oscillator Contributions (in dimensionless form) to the

Thermodynamic Functions for Values of $\mathrm{X}=\mathrm{hc} / \mathrm{kT}$

\begin{tabular}{|c|c|c|c|c|c|c|c|c|}
\hline X & $-\left(F^{\circ}-E_{O}^{\circ}\right) / R T$ & $\Delta$ & $\left(\mathrm{H}^{\circ}-\mathrm{E}_{\mathrm{O}}^{\circ}\right) / \mathrm{RT}$ & $\Delta$ & $S \circ / R$ & $\Delta$ & $\mathrm{C}_{\mathrm{p}} / \mathrm{R}$ & $\Delta$ \\
\hline .850 & 0.02151 & 2 & 0.08371 & 7 & 0.10522 & 9 & 0.32928 & 17 \\
\hline $\begin{array}{l}851 \\
852\end{array}$ & $\begin{array}{l}0.02149 \\
0.02147\end{array}$ & $\begin{array}{l}2 \\
3\end{array}$ & $\begin{array}{l}0.08364 \\
0.08358\end{array}$ & $\begin{array}{l}6 \\
6\end{array}$ & $\begin{array}{l}0.10513 \\
0.10505\end{array}$ & $\begin{array}{l}8 \\
9\end{array}$ & $\begin{array}{l}0.32911 \\
0.32894\end{array}$ & $\begin{array}{l}17 \\
17\end{array}$ \\
\hline 853 & 0.02144 & 2 & 0.08352 & 7 & 0.10496 & 8 & 0.32877 & 18 \\
\hline 854 & 0.02142 & 2 & 0.08345 & 6 & 0.10488 & 9 & 0.32859 & 17 \\
\hline 855 & 0.02140 & 2 & 0.08339 & 6 & 0.10479 & 8 & 0.32842 & 17 \\
\hline 856 & 0.02138 & 2 & 0.08333 & 7 & 0.10471 & 9 & 0.32825 & 17 \\
\hline 57 & 0.02136 & 2 & 0.08326 & 6 & 0.10462 & 8 & 0.32808 & 17 \\
\hline 858 & 0.02134 & 3 & 0.08320 & 6 & 0.10454 & 9 & 0.32791 & 18 \\
\hline .859 & 0.02131 & 2 & 0.08314 & 7 & 0.10445 & 8 & 0.32773 & 17 \\
\hline .860 & 0.02129 & 2 & 0.08307 & 6 & 0.10437 & 9 & 0.32756 & 17 \\
\hline 861 & 0.02127 & 2 & 0.08301 & 6 & 0.10428 & 8 & 0.32739 & 17 \\
\hline 862 & 0.02125 & 2 & 0.08295 & 7 & 0.10420 & 9 & 0.32722 & 17 \\
\hline .863 & 0.02123 & 2 & 0.08288 & 6 & 0.10411 & 8 & 0.32705 & 18 \\
\hline 64 & 0.02121 & 2 & 0.08282 & 6 & 0.10403 & 9 & 0.32687 & 17 \\
\hline . 865 & 0.02119 & 3 & 0.08276 & 7 & 0.10394 & 8 & 0.32670 & 17 \\
\hline 3.866 & 0.02116 & 2 & 0.08269 & 6 & 0.10386 & 9 & 0.32653 & 17 \\
\hline $\begin{array}{l}.867 \\
.868\end{array}$ & $\begin{array}{l}0.02114 \\
0.02112\end{array}$ & $\begin{array}{l}2 \\
2\end{array}$ & $\begin{array}{l}0.08263 \\
0.08257\end{array}$ & $\begin{array}{l}6 \\
7\end{array}$ & $\begin{array}{l}0.10377 \\
0.10369\end{array}$ & $\begin{array}{l}8 \\
9\end{array}$ & $\begin{array}{l}0.32636 \\
0.32619\end{array}$ & $\begin{array}{l}17 \\
17\end{array}$ \\
\hline .869 & 0.02110 & 2 & 0.08250 & 6 & 0.10360 & 8 & 0.32602 & 17 \\
\hline & 0.02108 & 2 & 0.08244 & 6 & 0.10352 & 8 & 0.32585 & 18 \\
\hline .871 & 0.02106 & 2 & 0.08238 & 6 & 0.10344 & 9 & 0.32567 & 17 \\
\hline 3.872 & 0.02104 & 2 & 0.08232 & 7 & 0.10335 & 8 & 0.32550 & 17 \\
\hline 3.873 & 0.02102 & 3 & 0.08225 & 6 & 0.10327 & 9 & 0.32533 & 17 \\
\hline 3.874 & 0.02099 & 2 & 0.08219 & 6 & 0.10318 & 8 & 0.32516 & 17 \\
\hline 75 & 0.02097 & 2 & 0.08213 & 7 & 0.10310 & 8 & 0.32499 & 17 \\
\hline
\end{tabular}

$\begin{array}{lllllllll}3.876 & 0.02095 & 2 & 0.08206 & 6 & 0.10302 & 9 & 0.32482 & 17 \\ 3.877 & 0.02093 & 2 & 0.08200 & 6 & 0.10293 & 8 & 0.32465 & 17 \\ 3.878 & 0.02091 & 2 & 0.08194 & 6 & 0.10285 & 8 & 0.32448 & 17 \\ 3.879 & 0.02089 & 2 & 0.08188 & 7 & 0.10277 & 9 & 0.32431 & 18 \\ 3.880 & 0.02087 & 2 & 0.08181 & 6 & 0.10268 & 8 & 0.32413 & 17 \\ 3.881 & 0.02085 & 3 & 0.08175 & 6 & 0.10260 & 9 & 0.32396 & 17 \\ 3.882 & 0.02082 & 2 & 0.08169 & 6 & 0.10251 & 8 & 0.32379 & 17 \\ 3.883 & 0.02080 & 2 & 0.08163 & 6 & 0.10243 & 8 & 0.32362 & 17 \\ 3.884 & 0.02078 & 2 & 0.08157 & 7 & 0.10235 & 9 & 0.32345 & 17 \\ 3.885 & 0.02076 & 2 & 0.08150 & 6 & 0.10226 & 8 & 0.32328 & 17 \\ 3.886 & 0.02074 & 2 & 0.08144 & 6 & 0.10218 & 8 & 0.32311 & 17 \\ 3.887 & 0.02072 & 2 & 0.08138 & 6 & 0.10210 & 8 & 0.32294 & 17 \\ 3.888 & 0.02070 & 2 & 0.08132 & 7 & 0.10202 & 9 & 0.32277 & 17 \\ 3.889 & 0.02068 & 2 & 0.08125 & 6 & 0.10193 & 8 & 0.32260 & 17 \\ 3.890 & 0.02066 & 2 & 0.08119 & 6 & 0.10185 & 8 & 0.32243 & 17 \\ 3.891 & 0.02064 & 2 & 0.08113 & 6 & 0.10177 & 9 & 0.32226 & 17 \\ 3.892 & 0.02062 & 3 & 0.08107 & 6 & 0.10168 & 8 & 0.32209 & 17 \\ 3.893 & 0.02059 & 2 & 0.08101 & 7 & 0.10160 & 8 & 0.32192 & 17 \\ 3.894 & 0.02057 & 2 & 0.08094 & 6 & 0.10152 & 8 & 0.32175 & 17 \\ 3.895 & 0.02055 & 2 & 0.08088 & 6 & 0.10144 & 9 & 0.32158 & 17 \\ 3.896 & 0.02053 & 2 & 0.08082 & 6 & 0.10135 & 8 & 0.32141 & 17 \\ 3.896 & 0.0217 \\ 3.897 & 0.02051 & 2 & 0.08076 & 6 & 0.10127 & 8 & 0.32124 & 17 \\ 3.898 & 0.02049 & 2 & 0.08070 & 6 & 0.10119 & 8 & 0.32107 & 17 \\ 3.899 & 0.02047 & 2 & 0.08064 & 7 & 0.10111 & 9 & 0.32090 & 17 \\ 3.900 & 0.02045 & & 0.08057 & & 0.10102 & & 0.32073 & \end{array}$


Table I Harmonic Oscillator Contributions (in dimensionless form) to the

Thermodynamic Functions for Values of $\mathrm{X}=\mathrm{hc} / \mathrm{kT}$

\begin{tabular}{|c|c|c|c|c|c|c|c|}
\hline$x$ & $-\left(F^{\circ}-E_{0}^{\circ}\right) / R T$ & $\Delta$ & $\left(H^{\circ}-E_{O} O\right) / R T$ & $\Delta$ & $S \% / R$ & $\Delta$ & $\mathrm{Co}_{\mathrm{p}} / \mathrm{R}$ \\
\hline 3.900 & 0.02045 & 2 & 0.08057 & 6 & 0.10102 & 8 & 0.32073 \\
\hline $\begin{array}{l}3.901 \\
3.902 \\
3.903 \\
3.904 \\
3.905\end{array}$ & $\begin{array}{l}0.02043 \\
0.02041 \\
0.02039 \\
0.02037 \\
0.02035\end{array}$ & $\begin{array}{l}2 \\
2 \\
2 \\
2 \\
2\end{array}$ & $\begin{array}{l}0.08051 \\
0.08045 \\
0.08039 \\
0.08033 \\
0.08027\end{array}$ & $\begin{array}{l}6 \\
6 \\
6 \\
6 \\
6\end{array}$ & $\begin{array}{l}0.10094 \\
0.10086 \\
0.10078 \\
0.10070 \\
0.10061\end{array}$ & $\begin{array}{l}8 \\
8 \\
8 \\
9 \\
8\end{array}$ & $\begin{array}{l}0.32056 \\
0.32039 \\
0.32022 \\
0.32005 \\
0.31989\end{array}$ \\
\hline $\begin{array}{l}3.906 \\
3.907 \\
3.908 \\
3.909 \\
3.910\end{array}$ & $\begin{array}{l}0.02033 \\
0.02031 \\
0.02028 \\
0.02026 \\
0.02024\end{array}$ & $\begin{array}{l}2 \\
3 \\
2 \\
2 \\
2\end{array}$ & $\begin{array}{l}0.08021 \\
0.08014 \\
0.08008 \\
0.08002 \\
0.07996\end{array}$ & $\begin{array}{l}7 \\
6 \\
6 \\
6 \\
6\end{array}$ & $\begin{array}{l}0.10053 \\
0.10045 \\
0.10037 \\
0.10029 \\
0.10020\end{array}$ & $\begin{array}{l}8 \\
8 \\
8 \\
9 \\
8\end{array}$ & $\begin{array}{l}0.31972 \\
0.31955 \\
0.31938 \\
0.31921 \\
0.31904\end{array}$ \\
\hline $\begin{array}{l}3.911 \\
3.912 \\
3.913 \\
3.914 \\
3.915\end{array}$ & $\begin{array}{l}0.02022 \\
0.02020 \\
0.02018 \\
0.02016 \\
0.02014\end{array}$ & $\begin{array}{l}2 \\
2 \\
2 \\
2 \\
2\end{array}$ & $\begin{array}{l}0.07990 \\
0.07984 \\
0.07978 \\
0.07972 \\
0.07966\end{array}$ & $\begin{array}{l}6 \\
6 \\
6 \\
6 \\
7\end{array}$ & $\begin{array}{l}0.10012 \\
0.10004 \\
0.09996 \\
0.09988 \\
0.09980\end{array}$ & $\begin{array}{l}8 \\
8 \\
8 \\
8 \\
8\end{array}$ & $\begin{array}{l}0.31887 \\
0.31870 \\
0.31853 \\
0.31837 \\
0.31820\end{array}$ \\
\hline $\begin{array}{l}3.916 \\
3.917 \\
3.918 \\
3.919 \\
3.920\end{array}$ & $\begin{array}{l}0.02012 \\
0.02010 \\
0.02008 \\
0.02006 \\
0.02004\end{array}$ & $\begin{array}{l}2 \\
2 \\
2 \\
2 \\
2\end{array}$ & $\begin{array}{l}0.07959 \\
0.07953 \\
0.07947 \\
0.07941 \\
0.07935\end{array}$ & $\begin{array}{l}6 \\
6 \\
6 \\
6 \\
6\end{array}$ & $\begin{array}{l}0.09972 \\
0.09964 \\
0.09955 \\
0.09947 \\
0.09939\end{array}$ & $\begin{array}{l}8 \\
9 \\
8 \\
8 \\
8\end{array}$ & $\begin{array}{l}0.31803 \\
0.31786 \\
0.31769 \\
0.31752 \\
0.31735\end{array}$ \\
\hline $\begin{array}{l}3.921 \\
3.922 \\
3.923 \\
3.924 \\
3.925\end{array}$ & $\begin{array}{l}0.02002 \\
0.02000 \\
0.01998 \\
0.01996 \\
0.01994\end{array}$ & $\begin{array}{l}2 \\
2 \\
2 \\
2 \\
2\end{array}$ & $\begin{array}{l}0.07929 \\
0.07923 \\
0.07917 \\
0.07911 \\
0.07905\end{array}$ & $\begin{array}{l}6 \\
6 \\
6 \\
6 \\
6\end{array}$ & $\begin{array}{l}0.09931 \\
0.09923 \\
0.09915 \\
0.09907 \\
0.09899\end{array}$ & $\begin{array}{l}8 \\
8 \\
8 \\
8 \\
8\end{array}$ & $\begin{array}{l}0.31719 \\
0.31702 \\
0.31685 \\
0.31668 \\
0.31651\end{array}$ \\
\hline $\begin{array}{l}3.926 \\
3.927 \\
3.928 \\
3.929 \\
3.930\end{array}$ & $\begin{array}{l}0.01992 \\
0.01990 \\
0.01988 \\
0.01986 \\
0.01984\end{array}$ & $\begin{array}{l}2 \\
2 \\
2 \\
2 \\
2\end{array}$ & $\begin{array}{l}0.07899 \\
0.07893 \\
0.07887 \\
0.07881 \\
0.07875\end{array}$ & $\begin{array}{l}6 \\
6 \\
6 \\
6 \\
6\end{array}$ & $\begin{array}{l}0.09891 \\
0.09883 \\
0.09875 \\
0.09867 \\
0.09859\end{array}$ & $\begin{array}{l}8 \\
8 \\
8 \\
8 \\
8\end{array}$ & $\begin{array}{l}0.31635 \\
0.31618 \\
0.31601 \\
0.31584 \\
0.31567\end{array}$ \\
\hline $\begin{array}{l}3.931 \\
3.932 \\
3.933 \\
3.934 \\
3.935\end{array}$ & $\begin{array}{l}0.01982 \\
0.01980 \\
0.01978 \\
0.01976 \\
0.01974\end{array}$ & $\begin{array}{l}2 \\
2 \\
2 \\
2 \\
2\end{array}$ & $\begin{array}{l}0.07869 \\
0.07863 \\
0.07857 \\
0.07851 \\
0.07845\end{array}$ & $\begin{array}{l}6 \\
6 \\
6 \\
6 \\
6\end{array}$ & $\begin{array}{l}0.09851 \\
0.09843 \\
0.09834 \\
0.09826 \\
0.09818\end{array}$ & $\begin{array}{l}8 \\
9 \\
8 \\
8 \\
8\end{array}$ & $\begin{array}{l}0.31551 \\
0.31534 \\
0.31517 \\
0.31500 \\
0.31484\end{array}$ \\
\hline $\begin{array}{l}3.936 \\
3.937 \\
3.938 \\
3.939 \\
3.940\end{array}$ & $\begin{array}{l}0.01972 \\
0.01970 \\
0.01968 \\
0.01966 \\
0.01964\end{array}$ & $\begin{array}{l}2 \\
2 \\
2 \\
2 \\
2\end{array}$ & $\begin{array}{l}0.07839 \\
0.07833 \\
0.07827 \\
0.07821 \\
0.07815\end{array}$ & $\begin{array}{l}6 \\
6 \\
6 \\
6 \\
6\end{array}$ & $\begin{array}{l}0.09810 \\
0.09802 \\
0.09795 \\
0.09787 \\
0.09779\end{array}$ & $\begin{array}{l}8 \\
7 \\
8 \\
8 \\
8\end{array}$ & $\begin{array}{l}0.31467 \\
0.31450 \\
0.31434 \\
0.31417 \\
0.31400\end{array}$ \\
\hline $\begin{array}{l}3.941 \\
3.942 \\
3.943 \\
3.944 \\
3.945\end{array}$ & $\begin{array}{l}0.01962 \\
0.01960 \\
0.01958 \\
0.01956 \\
0.01954\end{array}$ & $\begin{array}{l}2 \\
2 \\
2 \\
2 \\
2\end{array}$ & $\begin{array}{l}0.07809 \\
0.07803 \\
0.07797 \\
0.07791 \\
0.07785\end{array}$ & $\begin{array}{l}6 \\
6 \\
6 \\
6 \\
6\end{array}$ & $\begin{array}{l}0.09771 \\
0.09763 \\
0.09755 \\
0.09747 \\
0.09739\end{array}$ & $\begin{array}{l}8 \\
8 \\
8 \\
8 \\
8\end{array}$ & $\begin{array}{l}0.31383 \\
0.31367 \\
0.31350 \\
0.31333 \\
0.31317\end{array}$ \\
\hline $\begin{array}{l}3.946 \\
3.947 \\
3.948 \\
3.949 \\
3.950\end{array}$ & $\begin{array}{l}0.01952 \\
0.01950 \\
0.01948 \\
0.01946 \\
0.01944\end{array}$ & $\begin{array}{l}2 \\
2 \\
2 \\
2\end{array}$ & $\begin{array}{l}0.07779 \\
0.07773 \\
0.07767 \\
0.07761 \\
0.07755\end{array}$ & $\begin{array}{l}6 \\
6 \\
6 \\
6\end{array}$ & $\begin{array}{l}0.09731 \\
0.09723 \\
0.09715 \\
0.09707 \\
0.09699\end{array}$ & $\begin{array}{l}8 \\
8 \\
8 \\
8\end{array}$ & $\begin{array}{l}0.31300 \\
0.31283 \\
0.31267 \\
0.31250 \\
0.31233\end{array}$ \\
\hline
\end{tabular}


Table I Harmonic Oscillator Contributions (in dimensionless form) to the

Thermodynamic Functions for Values of $X=h c v / k T$

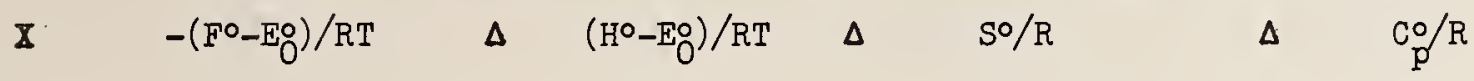

$\Delta$

\begin{tabular}{|c|c|c|c|c|c|c|c|}
\hline 3.950 & 0.01944 & 2 & 0.07755 & 6 & 0.09699 & 8 & 0.31233 \\
\hline $\begin{array}{l}3.951 \\
3.952 \\
3.953 \\
3.954 \\
3.955\end{array}$ & $\begin{array}{l}0.01942 \\
0.01940 \\
0.01938 \\
0.01936 \\
0.01934\end{array}$ & $\begin{array}{l}2 \\
2 \\
2 \\
2 \\
1\end{array}$ & $\begin{array}{l}0.07749 \\
0.07743 \\
0.07737 \\
0.07731 \\
0.07725\end{array}$ & $\begin{array}{l}6 \\
6 . \\
6 \\
6 \\
6\end{array}$ & $\begin{array}{l}0.09691 \\
0.09683 \\
0.09675 \\
0.09668 \\
0.09660\end{array}$ & $\begin{array}{l}8 \\
8 \\
7 \\
8 \\
8\end{array}$ & $\begin{array}{l}0.31217 \\
0.31200 \\
0.31183 \\
0.31167 \\
0.31150\end{array}$ \\
\hline $\begin{array}{l}3.956 \\
3.957 \\
3.958 \\
3.959 \\
3.960\end{array}$ & $\begin{array}{l}0.01933 \\
0.01931 \\
0.01929 \\
0.01927 \\
0.01925\end{array}$ & $\begin{array}{l}2 \\
2 \\
2 \\
2 \\
2\end{array}$ & $\begin{array}{l}0.07719 \\
0.07713 \\
0.07708 \\
0.07702 \\
0.07696\end{array}$ & $\begin{array}{l}6 \\
5 \\
6 \\
6 \\
6\end{array}$ & $\begin{array}{l}0.09652 \\
0.09644 \\
0.09636 \\
0.09628 \\
0.09620\end{array}$ & $\begin{array}{l}8 \\
8 \\
8 \\
8 \\
7\end{array}$ & $\begin{array}{l}0.31134 \\
0.31117 \\
0.31100 \\
0.31084 \\
0.31067\end{array}$ \\
\hline $\begin{array}{l}3.961 \\
3.962 \\
3.963 \\
3.964 \\
3.965\end{array}$ & $\begin{array}{l}0.01923 \\
0.01921 \\
0.01919 \\
0.01917 \\
0.01915\end{array}$ & $\begin{array}{l}2 \\
2 \\
2 \\
2 \\
2\end{array}$ & $\begin{array}{l}0.07690 \\
0.07684 \\
0.07678 \\
0.07672 \\
0.07666\end{array}$ & $\begin{array}{l}6 \\
6 \\
6 \\
6 \\
6\end{array}$ & $\begin{array}{l}0.09613 \\
0.09605 \\
0.09597 \\
0.09589 \\
0.09581\end{array}$ & $\begin{array}{l}8 \\
8 \\
8 \\
8 \\
8\end{array}$ & $\begin{array}{l}0.31051 \\
0.31034 \\
0.31017 \\
0.31001 \\
0.30984\end{array}$ \\
\hline $\begin{array}{l}3.966 \\
3.967 \\
3.968 \\
3.969 \\
3.970\end{array}$ & $\begin{array}{l}0.01913 \\
0.01911 \\
0.01909 \\
0.01907 \\
0.01905\end{array}$ & $\begin{array}{l}2 \\
2 \\
2 \\
2 \\
2\end{array}$ & $\begin{array}{l}0.07660 \\
0.07654 \\
0.07649 \\
0.07643 \\
0.07637\end{array}$ & $\begin{array}{l}6 \\
5 \\
6 \\
6 \\
6\end{array}$ & $\begin{array}{l}0.09573 \\
0.09566 \\
0.09558 \\
0.09550 \\
0.09542\end{array}$ & $\begin{array}{l}7 \\
8 \\
8 \\
8 \\
8\end{array}$ & $\begin{array}{l}0.30968 \\
0.30951 \\
0.30935 \\
0.30918 \\
0.30902\end{array}$ \\
\hline $\begin{array}{l}3.971 \\
3.972 \\
3.973 \\
3.974 \\
3.975\end{array}$ & $\begin{array}{l}0.01903 \\
0.01902 \\
0.01900 \\
0.01898 \\
0.01896\end{array}$ & $\begin{array}{l}1 \\
2 \\
2 \\
2 \\
2\end{array}$ & $\begin{array}{l}0.07631 \\
0.07625 \\
0.07619 \\
0.07613 \\
0.07608\end{array}$ & $\begin{array}{l}6 \\
6 \\
6 \\
5 \\
6\end{array}$ & $\begin{array}{l}0.09534 \\
0.09527 \\
0.09519 \\
0.09511 \\
0.09503\end{array}$ & $\begin{array}{l}7 \\
8 \\
8 \\
8 \\
7\end{array}$ & $\begin{array}{l}0.30885 \\
0.30869 \\
0.30852 \\
0.30836 \\
0.30819\end{array}$ \\
\hline $\begin{array}{l}3.976 \\
3.977 \\
3.978 \\
3.979 \\
3.980\end{array}$ & $\begin{array}{l}0.01894 \\
0.01892 \\
0.01890 \\
0.01888 \\
0.01886\end{array}$ & $\begin{array}{l}2 \\
2 \\
2 \\
2 \\
2\end{array}$ & $\begin{array}{l}0.07602 \\
0.07596 \\
0.07590 \\
0.07584 \\
0.07578\end{array}$ & $\begin{array}{l}6 \\
6 \\
6 \\
6 \\
5\end{array}$ & $\begin{array}{l}0.09496 \\
0.09488 \\
0.09480 \\
0.09472 \\
0.09465\end{array}$ & $\begin{array}{l}8 \\
8 \\
8 \\
7 \\
8\end{array}$ & $\begin{array}{l}0.30803 \\
0.30786 \\
0.30770 \\
0.30753 \\
0.30737\end{array}$ \\
\hline $\begin{array}{l}3.981 \\
3.982 \\
3.983 \\
3.984 \\
3.985\end{array}$ & $\begin{array}{l}0.01884 \\
0.01882 \\
0.01881 \\
0.01879 \\
0.01877\end{array}$ & $\begin{array}{l}2 \\
1 \\
2 \\
2 \\
2\end{array}$ & $\begin{array}{l}0.07573 \\
0.07567 \\
0.07561 \\
0.07555 \\
0.07549\end{array}$ & $\begin{array}{l}6 \\
6 \\
6 \\
6 \\
5\end{array}$ & $\begin{array}{l}0.09457 \\
0.09449 \\
0.09442 \\
0.09434 \\
0.09426\end{array}$ & $\begin{array}{l}8 \\
7 \\
8 \\
8 \\
7\end{array}$ & $\begin{array}{l}0.30720 \\
0.30704 \\
0.30687 \\
0.30671 \\
0.30655\end{array}$ \\
\hline $\begin{array}{l}3.986 \\
3.987 \\
3.988 \\
3.989 \\
3.990\end{array}$ & $\begin{array}{l}0.01875 \\
0.01873 \\
0.01871 \\
0.01869 \\
0.01867\end{array}$ & $\begin{array}{l}2 \\
2 \\
2 \\
2 \\
2\end{array}$ & $\begin{array}{l}0.07544 \\
0.07538 \\
0.07532 \\
0.07526 \\
0.07521\end{array}$ & $\begin{array}{l}6 \\
6 \\
6 \\
5 \\
6\end{array}$ & $\begin{array}{l}0.09419 \\
0.09411 \\
0.09403 \\
0.09395 \\
0.09388\end{array}$ & $\begin{array}{l}8 \\
8 \\
8 \\
7 \\
8\end{array}$ & $\begin{array}{l}0.30638 \\
0.30622 \\
0.30605 \\
0.30589 \\
0.30572\end{array}$ \\
\hline $\begin{array}{l}3.991 \\
3.992 \\
3.993 \\
3.994 \\
3.995\end{array}$ & $\begin{array}{l}0.01865 \\
0.01864 \\
0.01862 \\
0.01860 \\
0.01858\end{array}$ & $\begin{array}{l}1 \\
2 \\
2 \\
2 \\
2\end{array}$ & $\begin{array}{l}0.07515 \\
0.07509 \\
0.07503 \\
0.07497 \\
0.07492\end{array}$ & $\begin{array}{l}6 \\
6 \\
6 \\
5 \\
6\end{array}$ & $\begin{array}{l}0.09380 \\
0.09372 \\
0.09365 \\
0.09357 \\
0.09350\end{array}$ & $\begin{array}{l}8 \\
7 \\
8 \\
7 \\
8\end{array}$ & $\begin{array}{l}0.30556 \\
0.30540 \\
0.30523 \\
0.30507 \\
0.30491\end{array}$ \\
\hline $\begin{array}{l}3.996 \\
3.997 \\
3.998 \\
3.999 \\
4.000\end{array}$ & $\begin{array}{l}0.01856 \\
0.01854 \\
0.01852 \\
0.01850 \\
0.01849\end{array}$ & $\begin{array}{l}2 \\
2 \\
2 \\
1\end{array}$ & $\begin{array}{l}0.07486 \\
0.07480 \\
0.07474 \\
0.07469 \\
0.07463\end{array}$ & $\begin{array}{l}6 \\
6 \\
5 \\
6\end{array}$ & $\begin{array}{l}0.09342 \\
0.09334 \\
0.09327 \\
0.09319 \\
0.09311\end{array}$ & $\begin{array}{l}8 \\
7 \\
8 \\
8\end{array}$ & $\begin{array}{l}0.30474 \\
0.30458 \\
0.30441 \\
0.30425 \\
0.30409\end{array}$ \\
\hline
\end{tabular}


Table I Harmonic Oscillator Contributions (in dimensionless form) to the Thermodynamic Functions for Values of $X=h c v / k T$

$\mathbf{X}$ $-\left(F^{\circ}-E_{O}^{\circ}\right) / R T$

$\Delta$

$\left(\mathrm{H}^{\circ}-\mathrm{E}_{\mathrm{O}}^{\circ}\right) / \mathrm{RT}$
$\mathrm{S} \% / \mathrm{R}$

\begin{tabular}{|c|c|}
\hline $\begin{array}{l}4.01 \\
4.02 \\
4.03 \\
4.04 \\
4.05\end{array}$ & $\begin{array}{l}0.01830 \\
0.01812 \\
0.01793 \\
0.01775 \\
0.01758\end{array}$ \\
\hline $\begin{array}{l}4.06 \\
4.07 \\
4.08 \\
4.09 \\
4.10\end{array}$ & $\begin{array}{l}0.01740 \\
0.01722 \\
0.01705 \\
0.01688 \\
0.01671\end{array}$ \\
\hline $\begin{array}{l}4.11 \\
4.12 \\
4.13 \\
4.14 \\
4.15\end{array}$ & $\begin{array}{l}0.01654 \\
0.01638 \\
0.01621 \\
0.01605 \\
0.01589\end{array}$ \\
\hline $\begin{array}{l}4.16 \\
4.17 \\
4.18 \\
4.19 \\
4.20\end{array}$ & $\begin{array}{l}0.01573 \\
0.01557 \\
0.01542 \\
0.01526 \\
0.01511\end{array}$ \\
\hline $\begin{array}{l}4 \cdot 21 \\
4 \cdot 22 \\
4.23 \\
4.24 \\
4.25\end{array}$ & $\begin{array}{l}0.01496 \\
0.01481 \\
0.01466 \\
0.01451 \\
0.01437\end{array}$ \\
\hline $\begin{array}{l}4.26 \\
4.27 \\
4.28 \\
4.29 \\
4.30\end{array}$ & $\begin{array}{l}0.01422 \\
0.01408 \\
0.01394 \\
0.01380 \\
0.011366\end{array}$ \\
\hline $\begin{array}{l}4.31 \\
4.32 \\
4.33 \\
4.34 \\
4.35\end{array}$ & $\begin{array}{l}0.01352 \\
0.01339 \\
0.01326 \\
0.01312 \\
0.01299\end{array}$ \\
\hline $\begin{array}{l}4.36 \\
4.37 \\
4.38 \\
4.39 \\
4.40\end{array}$ & $\begin{array}{l}0.01286 \\
0.011273 \\
0.01260 \\
0.01248 \\
0.01235\end{array}$ \\
\hline $\begin{array}{l}4.41 \\
4.42 \\
4.43 \\
4.44 \\
4.45\end{array}$ & $\begin{array}{l}0.01223 \\
0.01211 \\
0.01199 \\
0.01187 \\
0.01175\end{array}$ \\
\hline $\begin{array}{l}4.46 \\
4.47 \\
4.48 \\
4.49 \\
4.50\end{array}$ & $\begin{array}{l}0.01163 \\
0.01151 \\
0.011140 \\
0.01128 \\
0.01117\end{array}$ \\
\hline
\end{tabular}

$\begin{array}{ll}19 & 0.07463 \\ 18 & 0.07406 \\ 19 & 0.07349 \\ 18 & 0.07293 \\ 17 & 0.07237 \\ 18 & 0.07181\end{array}$

57

57

56

56

56
55

18

17

17

17

0.07126

0.07071

0.07017

0.06963

0.06909

0.06856

0.06803

0.06751

0.06699

0.06647

0.06596

0.06545

0.06494

0.06444

0.06394

0.06345

0.06295

0.06247

0.06198

0.06150

55

54

54

54

53

53

52

52

52

51

51

51

50

50

49

50

48

49

48

48

0.06102

0.06055

0.06008

0.05961

0.05915

47

47

47

46

46

0.05869

0.05823

0.05778

0.05733

0.05688

46

45

45

45

45

0.05643

0.05599

0.05556

0.05512

0.05469

44

43

44

43

43

0.05426

0.05384

0.05342

0.05300

0.05258

42

42

42

42

41

0.05217

0.05176

0.05136

0.05095

0.05055

41

41
$\Delta \quad \mathrm{Co} / \mathrm{R}$

$\Delta$

$\begin{array}{llll}0.09311 & 75 & 0.3040969591 \\ 0.09236 & 75 & 1.00000 & 0 \\ 0.09161 & 75 & 1.00000 & 0 \\ 0.09086 & 74 & 1.00000 & 0 \\ 0.09012 & 73 & 1.00000 & 0 \\ 0.08939 & 73 & 1.00000 & 0\end{array}$

0.08866

0.08794

0.08722

0.08651

0.08580

0.08510

0.08441

0.08372

0.08304

0.08236

0.08169

0.08102

0.08036

0.07970

0.07905

72

0.07840

0.07776

0.07712

0.07649

0.07587

0.07525

0.07463

0.07402

0.07341

0.07281

0.07221

0.07162

0.07103

0.07045

0.06987

0.06930

0.06873

0.06816

0.06760

0.06705

\subsection{9}

0.06595

0.06540

0.06487

0.06433

0.06380

0.06328

0.06275

0.06224

0.06172
72

71

70

69

69
68

68
68

67

67

66

66

65

65

64

64
63

62

62

62

61

61

60

60

59

59

58

58

57

56

55

56

54
55

53

54

53

$1.00000 \quad 0$

1.0000070879

$0.29121 \quad 158$

$0.28963 \quad 157$

$0.28806 \quad 157$

$0.28649 \quad 157$

$0.28492 \quad 155$

$0.28337 \quad 156$

$0.28181 \quad 154$

$0.28027 \quad 154$

$0.27873 \quad 153$

$0.27720 \quad 153$

$0.27567 \quad 152$

$0.27415 \quad 151$

$0.27264 \quad 151$

$0.27113 \quad 150$

$0.26963,150$

$0.26813 \quad 149$

$0.26664 \quad 148$

$0.2651 \epsilon \quad 148$

$0.26368 \quad 147$

$0.26221 \quad 147$

$0.26074 \quad 145$

$0.25929 \quad 146$

$0.25783 \quad 144$

$0.25639 \quad 145$

$0.25494 \quad 143$

$0.25351 \quad 143$

$0.25208 \quad 142$

$0.25066 \quad 142$

$0.24924 \quad 141$

$0.24783 \quad 140$

$0.24643 \quad 140$

$0.24503 \quad 140$

0.24363138

.52

53

51

52
$0.24225 \quad 138$

$0.24087 \quad 138$

$0.23949 \quad 136$

$0.23813 \quad 137$

$0.23676 \quad 135$

$0.23541 \quad 135$

$0.23406 \quad 135$

$0.23271 \quad 134$

$0.23137 \quad 133$

0.23004 
Table I Harmonic Oscillator Contributions (in dimensionless form) to the

Thermodynamic Functions for Values of $X=h c v / k T$

$\mathbb{Z}$ $-\left(F^{\circ}-E_{O}^{\circ}\right) / R T$

$\left(\mathrm{H}^{\circ}-\mathrm{E}_{\mathrm{O}}^{\circ}\right) / \mathrm{RT}$

0.01117

$4 \cdot 50$

4.51

0.01106

4.52

4.53

4.54

4.55

4.56

4.57

4.58

4.59

4.60

4.61

4.62

4.63

4.64

4.65

4.66

4.67

4.68

4.69

4.70

4. 71

4.72

4.73

4.74

4.75

4.76

4.77

4.78

4.79

4.80

4.81

4.82

4.83

4. 84

4.85

4.86

4.87

4.88

4.89

4.90

4.91

4.92

4.93

4.94

4.95

4.96

4.97

4.98

4. 99

5.00
0.01095

0.01084

0.01073

0.01062

0.01052

0.01041

0.01031

0.01020

0.01000

0.00990

0.00980

0.00970

0.00961

0.00951

0.00942

0.00932

0.00923

0.00914

0.00905

0.00896

0.00887

0.00878

0.00869

0.00860

0.00852

0.00843

0.00835

0.00826

0.00818

0.00810

0.00802

0.00794

0.00786

0.00778

0.00770

0.00763

0.00755

0.00747

0.00740

0.00733

0.00725

0.00718

0.00711

0.00704

0.00697

0.00690

0.00683

0.00676
0.01010

10

11

10

0.04489

0.04418

0.04383

0.04314

0.04080

0.03766

0.03736

0.03706

0.03676

0.03647

0.03618

0.03589

0.03560

0.03531

0.03503

0.03475

0.03447

0.03419

0.03392

$\begin{array}{lll}11 & 0.05055 & 40 \\ 11 & 0.05015 & 39 \\ 11 & 0.04976 & 39 \\ 11 & 0.04937 & 39 \\ 11 & 0.04898 & 39 \\ 10 & 0.04859 & 38\end{array}$

0.06172
0.06121
0.06071
0.06021
0.05971
0.05922

$\Delta$

$$
\mathrm{Co}_{\mathrm{p}} / \mathrm{R}
$$

$11 \quad 0.04821 \quad 38$

$0.04783 \quad 38$

$0.04745 \quad 37$

$0.04708 \quad 37$

0.0467137

0.05873

0.05824

0.05776

0.05728

0.05681

51

0.23004

133

$0.04634 \quad 37$

$0.04597 \quad 36$

0.0456136

$0.04525 \quad 36$

0.05634

0.05587

0.05541

0.05495

0.05450

50

$0.22871 \quad 132$

$50 \quad 0.22739 \quad 131$

50

49

49

0.22608

131

$0.22477 \quad 130$

$0.22347 \quad 130$

49

48

48

47

47

$0.22217 \quad 129$

$0.22088 \quad 129$

$0.21959 \quad 128$

$0.21831 \quad 127$

$0.21704 \quad 127$

0.0445436

0.04349

0.05405

0.05360

0.05315

0.05271

0.05228

47

46

46

45

45

$0.21577 \quad 126$

$0.21451 \quad 126$

$0.21325 \quad 125$

$0.21200 \quad 124$

$0.21076 \quad 124$

$0.04280 \quad 34$

0.05184

45

45

44

43

44

$0.20952 \quad 124$

$0.20828 \quad 122$

$0.20706 \quad 122$

$0.20584 \quad 122$

$0.20462 \quad 121$

$0.04246 \quad 34$

$0.04212 \quad 33$

$0.04179 \quad 34$

$0.04145 \quad 33$

0.05141

0.05099

0.05056

0.05014

43

0.2034

120

$0.20221 \quad 120$

$0.20101 \quad 120$

$0.19981 \quad 118$

42

41

0.19863

119

0.0411232

0.04973

42

$0.19744 \quad 117$

0.04931

0.04890

0.04850

0.0401532

0.04809

41

0.19627

117

$0.19510 \quad 117$

40

40

0.19277

115

0.04769

39

0.04730

0.04690

40

$0.19162 \quad 115$

$0.03920 \quad 31$

$0.03889 \quad 31$

$0.03858 \quad 31$

0.04651

39

0.04613

38

39

$0.19047 \quad 114$

$0.18933 \quad 114$

$0.18819 \quad 113$

$0.18706 \quad 113$

$0.03796 \quad 30$

0.04574

38

0.04536

0.04498

0.18593

112

0.04461

38

37

37

30

0.04424

37

0.04387

0.04350

0.04314

37

0.04278

0.04242

0.1848

111

$0.18370 \quad 111$

$0.18259 \quad 110$

$0.18149 \quad 110$

0.04207

0.04172

0.04137

0.18039

0.18039110

$0.17821 \quad 109$

$0.17712 \quad 107$

$0.17605 \quad 108$ 
Table I Harmonic Oscillator Contributions (in dimensionless form) to the Thermodynamic Functions for Values of $\mathrm{X}=\mathrm{hc} / \mathrm{kT}$

$\mathbf{x}$ $-\left(F^{\circ}-E_{0}^{\circ}\right) / R T$

$\Delta \quad\left(H^{\circ}-E_{0}\right) / R T$

$\triangle$

$\mathrm{S} \% / \mathrm{R}$

$\begin{array}{ll}5.00 & 0.00676 \\ 5.01 & 0.00669 \\ 5.02 & 0.00663 \\ 5.03 & 0.00656 \\ 5.04 & 0.00649 \\ 5.05 & 0.00643 \\ 5.06 & 0.00637 \\ 5.07 & 0.00630 \\ 5.08 & 0.00624 \\ 5.09 & 0.00618 \\ 5.10 & 0.00612\end{array}$

5.11

5.12

5.13

5.14

5.15

5.16

5.17

5.18

5.19

5.20

5.21

5.22

5.23

5.24

5. 25

5.26

5.27

5.28

5.29

5.30

5.31

5.32

5.33

5.34

5.35

5. 36

5.37

5. 38

5.39

5.40

5.41

5.42

5.43

5.44

5.45

5.46

5.47

5.48

5.49

5.50
0.00605

0.00599

0.00593

0.00587

0.00582

0.00576

0.00570

0.00564

0.00559

0.00553

0.00548

0.00542

0.00537

0.00531

0.00526

0.00521

0.00516

0.00511

0.00505

0.00500

0.00495

0.00490

0.00486

0.00481

0.00476

0.00471

0.00467

0.00462

0.00457

0.00453

0.00448

0.00444

0.00439

0.00435

0.00431

0.00426

0.00422

0.00418

0.00414

0.00410
6

$7 \quad 0.03392$

$6 \quad 0.03365$

$7 \quad 0.03338$

0.03311

0.03284

0.03258

27
27
27
27
26
27

6

$0.03103 \quad 25$

$0.03078 \quad 25$

0.03053

0.03029

0.03004

0.0286123

0.02815

23

0.02792

23

23

5

\section{$0.02747 \quad 22$}

0.02703

0.02659

22

$0.02637 \quad 21$

0.02616

$0.02594 \quad 21$

$0.02573 \quad 21$

0.02552

0.02532

0.02511

21

0.02490

0.02450

0.02430

20

0.02371

19

0.02352

20

0.02332

19

0.02313

0.02294

0.02257

0.04068
0.04034
0.04000
0.03967
0.03934
0.03901

34

$\mathrm{C} \circ / \mathrm{R}$

$\Delta$

$\begin{array}{llllll}0.03231 & 26 & 0.03868 & 32 & 0.16455 & 101 \\ 0.03205 & 26 & 0.03836 & 33 & 0.16354 & 101 \\ 0.03179 & 25 & 0.03803 & 31 & 0.16253 & 100 \\ 0.03154 & 26 & 0.03772 & 32 & 0.16153 & 100 \\ 0.03128 & 25 & 0.03740 & 31 & 0.16053 & 100\end{array}$

0.03709

0.03678

31

0.15953

0.03647

0.03616

0.03586

34
33

33

33

33

0.17074

104

$0.16970 \quad 104$

$0.16866 \quad 104$

$0.16762 \quad 103$

$0.16659 \quad 102$

$0.16557 \quad 102$

$\begin{array}{lll}0.02980 & 24 & 0.03556 \\ 0.02956 & 24 & 0.03526 \\ 0.02932 & 24 & 0.03496 \\ 0.02908 & 23 & 0.03467 \\ 0.02885 & 24 & 0.03438\end{array}$

31

0.15855

0.15756

0.15659

0.15561

98

99

97

30

30

98

96

30

0.1546597

30

29

29

29

0.15368

0.15273

0.15177

0.15083

97
95

96

94

0.03409

29

0.14988

0.02838

0.03380

0.03352

0.03324

0.03296

28

28

28

28

0.14895

0.14801

0.14709

0.14616

95

0.02725

0.03268

0.03240

0.03213

0.03186

0.03159

28

0.14525

0.14433

0.14343

0.14252

0.14162

93

93

93

93

91

0.03133

0.03106

0.03080

0.03054

0.03028

0.14073

0.13984

0.13896

0.13808

0.13720

92

90

91

90

89

0.03003
0.02977

0.02952

0.02927

0.02903

0.13633

0.13547

0.13461

0.13375

0.13290

89

88

88

88

87

86

86

86

85

85

0.02878

0.02410

0.02854

0.02830

0.02806

0.02782

0.13205

84

24

0.13121

0.13038

0.12954

0. 12871

83

84

83

82

0.02759

0.12789

82

0.02735

0.02712

0.12707

0.12626

0.12545

0.02666

0.12464

81

81

81 
Table I Harmonic Oscillator Contributions (in dimensionless form) to the

Thermodynamic Functions for Values of $\mathrm{X}=\mathrm{hc} / \mathrm{kT}$

I $\quad-\left(\mathrm{F}^{\circ}-\mathrm{E}_{\mathrm{O}}\right) / \mathrm{RT} \quad \Delta \quad\left(\mathrm{H}^{\circ}-\mathrm{E}_{\mathrm{O}}\right) / \mathrm{RT} \quad \Delta \quad \mathrm{S}^{\circ} / \mathrm{R}$

$\Delta \quad \mathrm{Co} / \mathrm{R}$

$\Delta$

\begin{tabular}{|c|c|c|c|c|c|c|c|c|}
\hline 5.50 & 0.00410 & 5 & 0.02257 & 19 & 0.02666 & 22 & 0.12464 & $8 c$ \\
\hline 5.51 & 0.00405 & 4 & 0.02238 & 18 & 0.02644 & 22 & 0.12384 & \\
\hline 5.52 & 0.00401 & 4 & 0.02220 & 18 & 0.02622 & 23 & 0.12304 & \\
\hline 5.53 & 0.00397 & 4 & 0.02202 & 18 & 0.02599 & 22 & 0.12225 & \\
\hline .54 & 0.00393 & 3 & 0.02184 & 18 & 0.02577 & 22 & 0.12146 & \\
\hline .55 & 0.00390 & 4 & 0.02166 & 18 & 0.02555 & 21 & 0.12068 & \\
\hline 5.56 & 0.00386 & 4 & 0.02148 & 17 & 0.02534 & 22 & 0.11990 & \\
\hline 5.57 & 0.00382 & 4 & 0.02131 & 18 & 0.02512 & 21 & 0.11913 & \\
\hline .58 & 0.00378 & 4 & 0.02113 & 17 & 0.02491 & 21 & 0.11836 & \\
\hline 5.59 & 0.00374 & 4 & 0.02096 & 18 & 0.02470 & 21 & 0.11759 & \\
\hline 5.60 & 0.00370 & 3 & 0.02078 & 17 & 0.02449 & 21 & 0.11683 & \\
\hline 5.61 & 0.00367 & 4 & 0.02061 & 17 & 0.02428 & 20 & 0.11607 & \\
\hline 5.62 & 0.00363 & 3 & 0.02044 & 16 & 0.02408 & 21 & 0.11532 & \\
\hline 5.63 & 0.00360 & 4 & 0.02028 & 17 & 0.02387 & 20 & 0.11457 & \\
\hline 5.64 & 0.00356 & 4 & 0.02011 & 17 & 0.02367 & 20 & 0.11382 & \\
\hline 5.65 & 0.00352 & 3 & 0.01994 & 16 & 0.02347 & 20 & 0.11308 & \\
\hline $\begin{array}{l}5.66 \\
5.67\end{array}$ & $\begin{array}{l}0.00349 \\
0.00345\end{array}$ & $\begin{array}{l}4 \\
3\end{array}$ & $\begin{array}{l}0.01978 \\
0.01962\end{array}$ & $\begin{array}{l}16 \\
16\end{array}$ & $\begin{array}{l}0.02327 \\
0.02307\end{array}$ & $\begin{array}{l}20 \\
20\end{array}$ & $\begin{array}{l}0.11235 \\
0.11161\end{array}$ & \\
\hline 5.68 & 0.00342 & 3 & 0.01946 & 16 & 0.02287 & 19 & 0.11089 & \\
\hline 5.69 & 0.00339 & 4 & 0.01930 & 16 & 0.02268 & 19 & 0.11016 & \\
\hline 5.70 & 0.00335 & 3 & 0.01914 & 16 & 0.02249 & 19 & 0.10944 & \\
\hline 5.71 & 0.00332 & 3 & 0.01898 & 16 & 0.02230 & 19 & 0.10873 & \\
\hline 5.72 & 0.00329 & 4 & 0.01882 & 15 & 0.02211 & 19 & 0.10801 & \\
\hline 5.73 & 0.00325 & 3 & 0.01867 & 16 & 0.02192 & 19 & 0.10731 & \\
\hline 5.74 & 0.00322 & 3 & 0.01851 & 15 & 0.02173 & 18 & 0.10660 & \\
\hline 5.75 & 0.00319 & 3 & 0.01836 & 15 & 0.02155 & 19 & 0.10590 & \\
\hline 5.76 & 0.00316 & 4 & 0.01821 & 15 & 0.02136 & 18 & 0.10521 & \\
\hline 5.77 & 0.00312 & 3 & 0.01806 & 15 & 0.02118 & 18 & 0.10452 & \\
\hline 5.78 & 0.00309 & 3 & 0.01791 & 15 & 0.02100 & 18 & 0.10383 & \\
\hline 5.79 & 0.00306 & 3 & 0.01776 & 15 & 0.02082 & 17 & 0.10315 & \\
\hline 5.80 & 0.00303 & 3 & 0.01761 & 14 & 0.02065 & 18 & 0.10247 & \\
\hline 5.81 & 0.00300 & 3 & 0.01747 & 15 & 0.02047 & 18 & 0.10179 & \\
\hline 5.82 & 0.00297 & 3 & 0.01732 & 14 & 0.02029 & 17 & 0.10112 & \\
\hline 5.83 & 0.00294 & 3 & 0.01718 & $\cdot 14$ & 0.02012 & 17 & 0.10045 & \\
\hline 5.84 & 0.00291 & 3 & 0.01704 & 14 & 0.01995 & 17 & 0.09979 & \\
\hline .85 & 0.00288 & 2 & 0.01690 & 14 & 0.01978 & 17 & 0.09913 & \\
\hline 5.86 & 0.00286 & 3 & 0.01676 & 14 & 0.01961 & 17 & 0.09847 & \\
\hline 5.87 & 0.00283 & 3 & 0.01662 & 14 & 0.01944 & 16 & 0.09782 & \\
\hline 5.88 & 0.00280 & 3 & 0.01648 & 14 & 0.01928 & 17 & 0.09717 & \\
\hline 5.89 & 0.00277 & 3 & 0.01634 & 13 & 0.01911 & 16 & 0.09653 & \\
\hline .90 & 0.00274 & 2 & 0.01621 & 14 & 0.01895 & 16 & 0.09588 & \\
\hline 5.91 & 0.00272 & 3 & 0.01607 & 13 & 0.01879 & 16 & 0.09525 & \\
\hline & 0.00269 & 3 & 0.01594 & 13 & 0.01863 & 16 & 0.09461 & \\
\hline 5.93 & 0.00266 & 2 & 0.01581 & 13 & 0.01847 & 16 & 0.09398 & \\
\hline 94 & 0.00264 & 3 & 0.01568 & 13 & 0.01831 & 16 & 0.09336 & \\
\hline 5.95 & 0.00261 & 3 & 0.01555 & 13 & 0.01815 & 15 & 0.09274 & \\
\hline .96 & 0.00258 & 2 & 0.01542 & 13 & 0.01800 & 15 & 0.09212 & \\
\hline & 0.00256 & 3 & 0.01529 & 13 & 0.01785 & 16 & 0.09150 & \\
\hline 5.98 & 0.00253 & 2 & 0.01516 & 13 & 0.01769 & 15 & 0.09089 & \\
\hline 99 & 0.00251 & 3 & 0.01503 & 12 & 0.01754 & 15 & 0.09028 & \\
\hline D & 0.00248 & & 0.01491 & & 0.01739 & & 0.08968 & \\
\hline
\end{tabular}


Table I Harmonic Oscillator Contributions (in dimensionless form) to the

Thermodynamic Functions for Values of $X=h c v / k T$

$\mathbb{Z}$ $-\left(\mathrm{F}^{\circ}-\mathrm{E}_{\mathrm{O}}^{\circ}\right) / \mathrm{RT}$

$\Delta \quad\left(\mathrm{H}^{\circ}-\mathrm{E}_{\mathrm{O}}^{\circ}\right) / \mathrm{RT}$
0.01491

$$
6 .
$$

6. 01

6.02

6.03

6.04

6.05

6.06

6.07

6.08

6.09

6.10

6. 11

6.12

6.13

6.14

$6 \cdot 15$

6.16

6.17

6.18

6.19

6.20

6.21

6.22

6. 23

6. 24

$6 \cdot 25$

6.26

6.27

6. 28

6.29

6.30

6.31

6. 32

6.33

6.34

6.35

6.36

6. 37

6.38

6.39

6.40

6.41

6.42

6.43

6.44

6.45

6.46

6.47

6.48

6.49

6.50

0.00222

0.00220

0.00218

0.00216

0.00214

0.00211

0.00209

0.00207

0.00205

0.00203

0.00201

0.00199

0.00197

0.00195

0.00193

0.00191

0.00189

0.00188

0.00186

0.00184

0.00182

0.00180

0.00178

0.00177

0.00175

0.00173

0.00171

0.00170

0.00168

0.00166

0.00165

C. 00163

0.00161

0.00160

0.00158

0.00157

c. 00155

0.00154

0.00152

0.00150

2
3
2
3
2
2

0.01466

0.01454

0.01442

0.01430

0.01418

0.01406

0.01394

0.01383

0.01371

0.01360

$0.01348 \quad 11$

0.01337

0.01326

0.01315

12

12

0.01304

0.01293

0.01282

0.01271

0.01261

$0.01250 \quad 10$

0.01240

0.01229

0.01219

0.01209

0.01199

0.01189

0.01179

0.01169

0.01159

0.01149

0.01140

0.01130

0.01111

0.01102

0.01093

0.01083

0.01074

0.01065

0.01056

0.01047

0.01038

0.01030

0.01021

0.01012

0.01004

0.00995

0.00987

0.00979
0.01479

$\Delta \quad \mathrm{S} \% / \mathrm{R}$

12
13
12
12
12
12

0.01739

$\Delta$

$\mathrm{Co}_{\mathrm{p}} / \mathrm{R}$

$\Delta$

12
12

11

0.01724

0.01709

0.01695

0.01680

0.01666

0.01652

0.01638

0.01623

0.01610

0.01596

0.01582

0.01569

0.01555

0.01542

0.01528

0.01515

0.01502

0.01489

0.01477

0.01464

15

0.08968

60

0.08908

0.08848

0.08789

0.08730

0.08671

0.08613

0.08555

0.08497

0.08440

0.08383

0.08327

0.08271

0.08215

0.08159

0.08104

0.08049

0.07995

0.07940

0.07886

0.07833

0.01451

0.01439

0.01427

0.01414

0.01402

0.01390

0.01378

0.01366

0.01354

0.01343

0.01121

0.01331

0.01320

0.01308

0.01297

0.01286

0.01275

0.01264

0.01253

0.01242

0.01231

0.01221

0.01210

0.01200

0.01190

0.01179
0.01169

0.01159

0.01149

0.01139

0.01129
0.07780

0.07727

0.07674

0.07622

0.07570

60

59

59

59

58

58

58

57

57

56

56

56

56

55

55

54

55

54

53

53

53

53

52

52

52

$0.07518 \quad 51$

$0.07467 \quad 51$

$0.07416 \quad 51$

$0.07365 \quad 50$

$0.07315 \quad 50$

0.07265

0.07215

0.07166

0.07117

0.07068

50

49

49

49

49

0.07019

0.06971

0.06923

0.06876

48

11

11 
Table I Harmonic Oscillator Contributions (in dimensionless form) to the

Thermodynamic Functions for Values of $\mathrm{X}=\mathrm{hcv} / \mathrm{kT}$

$\mathbf{x}$

$-\left(F^{\circ}-E_{O}^{\circ}\right) / R T$

$\Delta \quad\left(\mathrm{H}^{\circ}-\mathrm{E}_{\mathrm{O}}^{\circ}\right) / \mathrm{RT}$

$\Delta$

$\mathrm{S} \% / \mathrm{R}$

$\begin{array}{ll}6.50 & 0.00150 \\ 6.51 & 0.00149 \\ 6.52 & 0.00147 \\ 6.53 & 0.00146 \\ 6.54 & 0.00145 \\ 6.55 & 0.00143 \\ 6.56 & 0.00142 \\ 6.57 & 0.00140 \\ 6.58 & 0.00139 \\ 6.59 & 0.00138 \\ 6.60 & 0.00136 \\ 6.61 & 0.00135 \\ 6.62 & 0.00133 \\ 6.63 & 0.00132 \\ 6.64 & 0.00131 \\ 6.65 & 0.00129 \\ & \\ 6.66 & 0.00128 \\ 6.67 & 0.00127 \\ 6.68 & 0.00126 \\ 6.69 & 0.00124 \\ 6.70 & 0.00123\end{array}$

6.71

6.72

6.73

6.74

6.75

6.76

6.77

6.78

6.79

6.80

6.81

6.82

6.83

6.84

6.85

6.86

6.87

6.88

6.89

6.90

6.91

6.92

6.93

6.94

6.95

6.96

6.97

6.98

6.99

7.00

0.00122

0.00121

0.00120

0.00118

0.00117

0.00116

0.00115

0.00114

0.00113

0.00111

0.00110

0.00109

0.00108

0.00107

0.00106

0.00105

0.00104

0.00103

0.00102

0.00101

0.00100

0.00099

0.00098

0.00097

0.00096

0.00095

0.00094

0.00093

0.00092

0.00091
0.00970

0.00962

0.00954

0.00946

0.00938

0.00930

0.00922

0.00914

0.00907

0.00899

0.00891

0.00884

0.00876

0.00869

0.00862

0.00854

0.00847

0.00840

0.00833

0.00826

0.00819

0.00812

0.00805

0.00798

0.00791

0.00785

0.00778

0.00771

0.00765

0.00758

0.00752

0.00745

0.00739

0.00733

0.00726

0.00720

0.00714

0.00708

0.00702

0.00696

0.00690

0.00684

0.00678

0.00673

0.00667

0.00661

0.00656

0.00650

0.00644

0.00639

\subsection{9 \\ 0.01119 \\ 0.01110 \\ 0.01100 \\ 0.01091 \\ 0.01081}

0.01072

0.01063

0.01053

0.01044

0.01035

0.01026

0.01017

0.01009

0.01000

0.00991

0.00983

0.00974

0.00966

0.00957

0.00949

0.00941

0.00933

0.00924

0.00916

0.00908

0.00901

0.00893

0.00885

0.00877

0.00870

0.00862

0.00855

0.00847

0.00840

0.00832

0.00825

0.00818

0.00811

0.00804

0.00797

0.00790

0.00783

0.00776

0.00770

0.00763

0.00756

0.00750

0.00743

0.00737

0.00730
$\Delta \quad \mathrm{Co} / \mathrm{R}$

$\Delta$

$\begin{array}{rll}10 & 0.06371 & 44 \\ & & \\ 9 & 0.06327 & 44 \\ 10 & 0.06283 & 43 \\ 9 & 0.06240 & 44 \\ 10 & 0.06196 & 43 \\ 9 & 0.06153 & 43\end{array}$

$9 \quad 0.06110 \quad 42$

$10 \quad 0.06068 \quad 42$

$9 \quad 0.06026 \quad 42$

$\begin{array}{lll}9 & 0.05984 & 42\end{array}$

$9 \quad 0.05942 \quad 42$

$0.05900 \quad 41$

$0.05859 \quad 41$

$0.05818 \quad 40$

$0.05778 \quad 41$

$0.05737 \quad 40$

$0.05697 \quad 40$

$0.05657 \quad 39$

$0.05618 \quad 40$

$0.05578 \quad 39$

$0.05539 \quad 39$

$0.05500 \quad 38$

$0.05462 \quad 39$

$0.05423 \quad 38$

$0.05385 \quad 38$

$0.05347 \quad 37$

$0.05310 \quad 38$

$0.05272 \quad 37$

$0.05235 \quad 37$

$0.05198 \quad 36$

$0.05162 \quad 37$

$0.05125 \quad 36$

0.0508936

$0.05053 \quad 36$

$0.05017 \quad 35$

$0.04982 \quad 35$

$0.04947 \quad 36$

$0.04911 \quad 34$

$0.04877 \quad 35$

0.0484234

$0.04808 \quad 34$

0.0477434

$0.04740 \quad 34$

$0.04706 \quad 33$

$0.04673 \quad 34$

0.0463933

$0.04606 \quad 32$

0.0457433

$0.04541 \quad 32$

0.0450933

0.04476 
Table I Harmonic Oscillator Contributions (in dimensionless form) to the

Thermodynamic Functions for Values of $X=h c v / k T$

I $\quad-\left(\mathrm{F}^{\circ}-\mathrm{E} \mathrm{O}\right) / \mathrm{RT} \quad \Delta \quad\left(\mathrm{H}^{\circ}-\mathrm{EO} \mathrm{O}\right) / \mathrm{RT} \quad \Delta \quad \mathrm{SO} / \mathrm{R} \quad \Delta \quad \mathrm{CO}_{\mathrm{p}} \mathrm{R}$

$\Delta$

\begin{tabular}{|c|c|c|c|c|c|c|c|}
\hline 7.00 & 0.00091 & 1 & 0.00639 & 6 & 0.00730 & 6 & 0.04476 \\
\hline $\begin{array}{l}7.01 \\
7.02 \\
7.03 \\
7.04 \\
7.05\end{array}$ & $\begin{array}{l}0.00090 \\
0.00089 \\
0.00089 \\
0.00088 \\
0.00087\end{array}$ & $\begin{array}{l}1 \\
0 \\
1 \\
1 \\
1\end{array}$ & $\begin{array}{l}0.00633 \\
0.00628 \\
0.00623 \\
0.00617 \\
0.00612\end{array}$ & $\begin{array}{l}5 \\
5 \\
6 \\
5 \\
5\end{array}$ & $\begin{array}{l}0.00724 \\
0.00717 \\
0.00711 \\
0.00705 \\
0.00699\end{array}$ & $\begin{array}{l}7 \\
6 \\
6 \\
6 \\
6\end{array}$ & $\begin{array}{l}0.04444 \\
0.04413 \\
0.04381 \\
0.04350 \\
0.04319\end{array}$ \\
\hline $\begin{array}{l}7.06 \\
7.07 \\
7.08 \\
7.09 \\
7.10\end{array}$ & $\begin{array}{l}0.00086 \\
0.00085 \\
0.00084 \\
0.00083 \\
0.00083\end{array}$ & $\begin{array}{l}1 \\
1 \\
1 \\
0 \\
1\end{array}$ & $\begin{array}{l}0.00607 \\
0.00602 \\
0.00596 \\
0.00591 \\
0.00586\end{array}$ & $\begin{array}{l}5 \\
6 \\
5 \\
5 \\
5\end{array}$ & $\begin{array}{l}0.00693 \\
0.00687 \\
0.00681 \\
0.00675 \\
0.00669\end{array}$ & $\begin{array}{l}6 \\
6 \\
6 \\
6 \\
6\end{array}$ & $\begin{array}{l}0.04288 \\
0.04257 \\
0.04227 \\
0.04196 \\
0.04166\end{array}$ \\
\hline $\begin{array}{l}7.11 \\
7.12 \\
7.13 \\
7.14 \\
7.15\end{array}$ & $\begin{array}{l}0.00082 \\
0.00081 \\
0.00080 \\
0.00079 \\
0.00079\end{array}$ & $\begin{array}{l}1 \\
1 \\
1 \\
0 \\
1\end{array}$ & $\begin{array}{l}0.00581 \\
0.00576 \\
0.00571 \\
0.00566 \\
0.00562\end{array}$ & $\begin{array}{l}5 \\
5 \\
5 \\
4 \\
5\end{array}$ & $\begin{array}{l}0.00663 \\
0.00657 \\
0.00651 \\
0.00646 \\
0.00640\end{array}$ & $\begin{array}{l}6 \\
6 \\
5 \\
6 \\
5\end{array}$ & $\begin{array}{l}0.04136 \\
0.04107 \\
0.04077 \\
0.04048 \\
0.04019\end{array}$ \\
\hline $\begin{array}{l}7.16 \\
7.17 \\
7.18 \\
7.19 \\
7.20\end{array}$ & $\begin{array}{l}0.00078 \\
0.00077 \\
0.00076 \\
0.00075 \\
0.00075\end{array}$ & $\begin{array}{l}1 \\
1 \\
1 \\
0 \\
1\end{array}$ & $\begin{array}{l}0.00557 \\
0.00552 \\
0.00547 \\
0.00543 \\
0.00538\end{array}$ & $\begin{array}{l}5 \\
5 \\
4 \\
5 \\
5\end{array}$ & $\begin{array}{l}0.00635 \\
0.00629 \\
0.00623 \\
0.00618 \\
0.00613\end{array}$ & $\begin{array}{l}6 \\
6 \\
5 \\
5 \\
6\end{array}$ & $\begin{array}{l}0.03990 \\
0.03961 \\
0.03933 \\
0.03904 \\
0.03876\end{array}$ \\
\hline $\begin{array}{l}7.21 \\
7.22 \\
7.23 \\
7.24 \\
7.25\end{array}$ & $\begin{array}{l}0.00074 \\
0.00073 \\
0.00072 \\
0.00072 \\
0.00071\end{array}$ & $\begin{array}{l}1 \\
1 \\
0 \\
1 \\
1\end{array}$ & $\begin{array}{l}0.00533 \\
0.00529 \\
0.00524 \\
0.00520 \\
0.00515\end{array}$ & $\begin{array}{l}4 \\
5 \\
4 \\
5 \\
4\end{array}$ & $\begin{array}{l}0.00607 \\
0.00602 \\
0.00597 \\
0.00591 \\
0.00586\end{array}$ & $\begin{array}{l}5 \\
5 \\
6 \\
5 \\
5\end{array}$ & $\begin{array}{l}0.03848 \\
0.03820 \\
0.03793 \\
0.03765 \\
0.03738\end{array}$ \\
\hline $\begin{array}{l}7.26 \\
7.27 \\
7.28 \\
7.29 \\
7.30\end{array}$ & $\begin{array}{l}0.00070 \\
0.00070 \\
0.00069 \\
0.00068 \\
0.00068\end{array}$ & $\begin{array}{l}0 \\
1 \\
1 \\
0 \\
1\end{array}$ & $\begin{array}{l}0.00511 \\
0.00506 \\
0.00502 \\
0.00498 \\
0.00493\end{array}$ & $\begin{array}{l}5 \\
4 \\
4 \\
5 \\
4\end{array}$ & $\begin{array}{l}0.00581 \\
0.00576 \\
0.00571 \\
0.00566 \\
0.00561\end{array}$ & $\begin{array}{l}5 \\
5 \\
5 \\
5 \\
5\end{array}$ & $\begin{array}{l}0.03711 \\
0.03684 \\
0.03658 \\
0.03631 \\
0.03605\end{array}$ \\
\hline $\begin{array}{l}7.31 \\
7.32 \\
7.33 \\
7.34 \\
7.35\end{array}$ & $\begin{array}{l}0.00067 \\
0.00066 \\
0.00066 \\
0.00065 \\
0.00064\end{array}$ & $\begin{array}{l}1 \\
0 \\
1 \\
1 \\
0\end{array}$ & $\begin{array}{l}0.00489 \\
0.00485 \\
0.00481 \\
0.00477 \\
0.00473\end{array}$ & $\begin{array}{l}4 \\
4 \\
4 \\
4 \\
4\end{array}$ & $\begin{array}{l}0.00556 \\
0.00551 \\
0.00546 \\
0.00542 \\
0.00537\end{array}$ & $\begin{array}{l}5 \\
5 \\
4 \\
5 \\
5\end{array}$ & $\begin{array}{l}0.03579 \\
0.03553 \\
0.03527 \\
0.03501 \\
0.03476\end{array}$ \\
\hline $\begin{array}{l}7.36 \\
7.37 \\
7.38 \\
7.39 \\
7.40\end{array}$ & $\begin{array}{l}0.00064 \\
0.00063 \\
0.00062 \\
0.00062 \\
0.00061\end{array}$ & $\begin{array}{l}1 \\
1 \\
0 \\
1 \\
0\end{array}$ & $\begin{array}{l}0.00469 \\
0.00465 \\
0.00461 \\
0.00457 \\
0.00453\end{array}$ & $\begin{array}{l}4 \\
4 \\
4 \\
4 \\
4\end{array}$ & $\begin{array}{l}0.00532 \\
0.00528 \\
0.00523 \\
0.00518 \\
0.00514\end{array}$ & $\begin{array}{l}4 \\
5 \\
5 \\
4 \\
5\end{array}$ & $\begin{array}{l}0.03451 \\
0.03426 \\
0.03401 \\
0.03376 \\
0.03351\end{array}$ \\
\hline $\begin{array}{l}7.41 \\
7.42 \\
7.43 \\
7.44 \\
7.45\end{array}$ & $\begin{array}{l}0.00061 \\
0.00060 \\
0.00059 \\
0.00059 \\
0.00058\end{array}$ & $\begin{array}{l}1 \\
1 \\
0 \\
1 \\
0\end{array}$ & $\begin{array}{l}0.00449 \\
0.00445 \\
0.00441 \\
0.00437 \\
0.00433\end{array}$ & $\begin{array}{l}4 \\
4 \\
4 \\
4 \\
3\end{array}$ & $\begin{array}{l}0.00509 \\
0.00505 \\
0.00500 \\
0.00496 \\
0.00492\end{array}$ & $\begin{array}{l}4 \\
5 \\
4 \\
4 \\
5\end{array}$ & $\begin{array}{l}0.03327 \\
0.03303 \\
0.03279 \\
0.03255 \\
0.03231\end{array}$ \\
\hline $\begin{array}{l}7.46 \\
7.47 \\
7.48 \\
7.49 \\
7.50\end{array}$ & $\begin{array}{l}0.00058 \\
0.00057 \\
0.00056 \\
0.00056 \\
0.00055\end{array}$ & $\begin{array}{l}1 \\
1 \\
0 \\
1\end{array}$ & $\begin{array}{l}0.00430 \\
0.00426 \\
0.00422 \\
0.00419 \\
0.00415\end{array}$ & $\begin{array}{l}4 \\
4 \\
3 \\
4\end{array}$ & $\begin{array}{l}0.00487 \\
0.00483 \\
0.00479 \\
0.00475 \\
0.00470\end{array}$ & $\begin{array}{l}4 \\
4 \\
4 \\
5\end{array}$ & $\begin{array}{l}0.03207 \\
0.03184 \\
0.03161 \\
0.03137 \\
0.03115\end{array}$ \\
\hline
\end{tabular}


Table I Harmonic Oscillator Contributions (in dimensionless form) to the

Thermodynamic Functions for Values of $X=h c v / k T$
X $\quad-\left(\mathrm{F}^{\circ}-\mathrm{E}_{\mathrm{O}}^{\mathrm{O}}\right) / \mathrm{RT}$
$\Delta \quad\left(\mathrm{H}^{\circ}-\mathrm{E}_{\mathrm{O}}^{\mathrm{O}}\right) / \mathrm{RT} \quad \Delta \quad \mathrm{S} \% / \mathrm{R}$
$\Delta \quad \mathrm{Co}_{\mathrm{p}} / \mathrm{R}$

$\Delta$

$\begin{array}{ll}7.50 & 0.00055 \\ 7.51 & 0.00055 \\ 7.52 & 0.00054 \\ 7.53 & 0.00054 \\ 7.54 & 0.00053 \\ 7.55 & 0.00053 \\ 7.56 & 0.00052 \\ 7.57 & 0.00052 \\ 7.58 & 0.00051 \\ 7.59 & 0.00051 \\ 7.60 & 0.00050 \\ 7.61 & 0.00050 \\ 7.62 & 0.00049 \\ 7.63 & 0.00049 \\ 7.64 & 0.00048 \\ 7.65 & 0.00048 \\ 7.66 & 0.00047 \\ 7.67 & 0.00047 \\ 7.68 & 0.00046 \\ 7.69 & 0.00046 \\ 7.70 & 0.00045 \\ 7.71 & 0.00045 \\ 7.72 & 0.00044 \\ 7.73 & 0.00044 \\ 7.74 & 0.00044 \\ 7.75 & 0.00043 \\ 7 & \end{array}$

$\begin{array}{ll}0 & 0 \\ 1 & 0 \\ 0 & 0\end{array}$

0.00415
0.00411
0.00408
0.00404
0.00401
0.00397
0.00394
0.00391
0.00387
0.00384
0.00381
0.00377
0.00374
0.00371
0.00368
0.00364
0.00361
0.00358
0.00355
0.00352
0.00349
0.00346
0.00343
0.00340
0.00337
0.00334

$\begin{array}{ll}4 & 0.00470 \\ 3 & 0.00466 \\ 4 & 0.00462 \\ 3 & 0.00458 \\ 4 & 0.00454 \\ 3 & 0.00450\end{array}$

$\begin{array}{lll}4 & 0.03115 & 23 \\ 4 & 0.03092 & 23 \\ 4 & 0.03069 & 22 \\ 4 & 0.03047 & 23 \\ 4 & 0.03024 & 22 \\ 4 & 0.03002 & 22\end{array}$

0.00446
0.00442
0.00438
0.00434
0.00431

0.02980

22

$0.02958 \quad 22$

$0.02936 \quad 21$

$0.02915 \quad 21$

$0.02894 \quad 22$

1

30.00423

$\begin{array}{llll}1 & 0.00371 & 3 & 0.00419\end{array}$

$0 \quad 0.00368$

$1 \quad 0.00364$

0.00416

0.00412

0.02872

0.02851

0.02830

0.02809

0.02789

21

21

21

20

0.00408

0.02768

0.00405

0.00401

0.00398

0.00394

0.02748

0.02727

0.02707

0.02687

0.00391

0.02667

0.00387

0.00384

0.00380

0.00377

0.02648

0.02628

0.02609

0.02589

21

$7.76 \quad 0.00043$

$7.77 \quad 0.00042$

0.00331

0.00374

0.02570

20

0.00328

0.00370

0.00325

0.00042

$7.79 \quad 0.00041$

$7.80 \quad 0.00041$

0.00367

0.00364

0.00323

0.00361

0.02551

0.02532

0.02514

0.02495

$7.81 \quad 0.00041$

$7.82 \quad 0.00040$

$7.83 \quad 0.00040$

$7.84 \quad 0.00039$

$7.85 \quad 0.00039$

0.00317

0.00358

0.00314

0.00311

0.00309

0.00306

0.00354

0.00351

0.00348

0.00345

0.02476

0.02458

0.02440

0.02422

0.00303

7.86

0.00039

7.87

7.88

0.00038

7.89

0.00038

7.90

0.00301

0.00298

0.00296

0.00293

0.00342

0.00339

0.00336

0.00333

0.00330

0.02404

21

20

20

19

20

19

19

7.91

0.00037

7.92

0.00290

0.00327

0.00288

0.00285

0.00283

0.00324

0.00321

0.00319

$7.94 \quad 0.00036$

$7.95 \quad 0.00035$

0.00316

$0.02386 \quad 18$

$0.02368 \quad 18$

$0.02350 \quad 17$

$0.02333 \quad 17$

$0.02316 \quad 18$

$7.96 \quad 0.00035$

$7.97 \quad 0.00035$

$7.98 \quad 0.00034$

$7.99 \quad 0.00034$

$8.00 \quad 0.00034$

0.00280

0.00313

0.00310

0.00276

0.00307

0.00273

0.00271

0.00305

0.00268

0.00302

$0.02298 \quad 17$

$0.02281 \quad 17$

$0.02264 \quad 17$

$0.02247 \quad 17$

$0.02230 \quad 16$

$0.02214 \quad 17$

$0.02197 \quad 16$

$0.02181 \quad 16$

$0.02165 \quad 17$

0.02148 
Table I Harmonic Oscillator Contributions (in dimensionless form) to the

Thermodynamic Functions for Values of $X=h c v / k T$

I $\quad-\left(\mathrm{F}^{\circ}-\mathrm{E}_{\mathrm{O}}^{\circ}\right) / \mathrm{RT} \quad \Delta \quad\left(\mathrm{H}^{\circ}-\mathrm{E}_{\mathrm{O}}^{\circ}\right) / \mathrm{RT} \quad \Delta \quad \mathrm{S} / \mathrm{R}$

$\triangle \quad C O / R$

$\Delta$

\begin{tabular}{|c|c|c|c|c|c|c|c|}
\hline 8.00 & 0.00034 & 1 & 0.00268 & 2 & 0.00302 & 3 & 0.02148 \\
\hline $\begin{array}{l}8.01 \\
8.02 \\
8.03 \\
8.04 \\
8.05\end{array}$ & $\begin{array}{l}0.00033 \\
0.00033 \\
0.00033 \\
0.00032 \\
0.00032\end{array}$ & $\begin{array}{l}0 \\
0 \\
1 \\
0 \\
0\end{array}$ & $\begin{array}{l}0.00266 \\
0.00264 \\
0.00261 \\
0.00259 \\
0.00257\end{array}$ & $\begin{array}{l}2 \\
2 \\
3 \\
2 \\
2\end{array}$ & $\begin{array}{l}0.00299 \\
0.00297 \\
0.00294 \\
0.00291 \\
0.00289\end{array}$ & $\begin{array}{l}2 \\
3 \\
3 \\
2 \\
3\end{array}$ & $\begin{array}{l}0.02132 \\
0.02116 \\
0.02101 \\
0.02085 \\
0.02069\end{array}$ \\
\hline $\begin{array}{l}8.06 \\
8.07 \\
8.08 \\
8.09 \\
8.10\end{array}$ & $\begin{array}{l}0.00032 \\
0.00031 \\
0.00031 \\
0.00031 \\
0.00030\end{array}$ & $\begin{array}{l}1 \\
0 \\
0 \\
1 \\
0\end{array}$ & $\begin{array}{l}0.00255 \\
0.00252 \\
0.00250 \\
0.00248 \\
0.00246\end{array}$ & $\begin{array}{l}3 \\
2 \\
2 \\
2 \\
2\end{array}$ & $\begin{array}{l}0.00286 \\
0.00284 \\
0.00281 \\
0.00279 \\
0.00276\end{array}$ & $\begin{array}{l}2 \\
3 \\
2 \\
3 \\
2\end{array}$ & $\begin{array}{l}0.02054 \\
0.02038 \\
0.02023 \\
0.02008 \\
0.01993\end{array}$ \\
\hline $\begin{array}{l}8.11 \\
8.12 \\
8.13 \\
8.14 \\
8.15\end{array}$ & $\begin{array}{l}0.00030 \\
0.00030 \\
0.00029 \\
0.00029 \\
0.00029\end{array}$ & $\begin{array}{l}0 \\
1 \\
0 \\
0 \\
0\end{array}$ & $\begin{array}{l}0.00244 \\
0.00242 \\
0.00240 \\
0.00237 \\
0.00235\end{array}$ & $\begin{array}{l}2 \\
2 \\
3 \\
2 \\
2\end{array}$ & $\begin{array}{l}0.00274 \\
0.00271 \\
0.00269 \\
0.00267 \\
0.00264\end{array}$ & $\begin{array}{l}3 \\
2 \\
2 \\
3 \\
2\end{array}$ & $\begin{array}{l}0.01978 \\
0.01963 \\
0.01948 \\
0.01934 \\
0.01919\end{array}$ \\
\hline $\begin{array}{l}8.16 \\
8.17 \\
8.18 \\
8.19 \\
8.20\end{array}$ & $\begin{array}{l}0.00029 \\
0.00028 \\
0.00028 \\
0.00028 \\
0.00027\end{array}$ & $\begin{array}{l}1 \\
0 \\
0 \\
1 \\
0\end{array}$ & $\begin{array}{l}0.00233 \\
0.00231 \\
0.00229 \\
0.00227 \\
0.00225\end{array}$ & $\begin{array}{l}2 \\
2 \\
2 \\
2 \\
2\end{array}$ & $\begin{array}{l}0.00262 \\
0.00260 \\
0.00257 \\
0.00255 \\
0.00253\end{array}$ & $\begin{array}{l}2 \\
3 \\
2 \\
2 \\
2\end{array}$ & $\begin{array}{l}0.01905 \\
0.01890 \\
0.01876 \\
0.01862 \\
0.01848\end{array}$ \\
\hline $\begin{array}{l}8.21 \\
8.22 \\
8.23 \\
8.24 \\
8.25\end{array}$ & $\begin{array}{l}0.00027 \\
0.00027 \\
0.00027 \\
0.00026 \\
0.00026\end{array}$ & $\begin{array}{l}0 \\
0 \\
1 \\
0 \\
0\end{array}$ & $\begin{array}{l}0.00223 \\
0.00221 \\
0.00219 \\
0.00217 \\
0.00216\end{array}$ & $\begin{array}{l}2 \\
2 \\
2 \\
1 \\
2\end{array}$ & $\begin{array}{l}0.00251 \\
0.00248 \\
0.00246 \\
0.00244 \\
0.00242\end{array}$ & $\begin{array}{l}3 \\
2 \\
2 \\
2 \\
2\end{array}$ & $\begin{array}{l}0.01834 \\
0.01820 \\
0.01806 \\
0.01793 \\
0.01779\end{array}$ \\
\hline $\begin{array}{l}8.26 \\
8.27 \\
8.28 \\
8.29 \\
8.30\end{array}$ & $\begin{array}{l}0.00026 \\
0.00026 \\
0.00025 \\
0.00025 \\
0.00025\end{array}$ & $\begin{array}{l}0 \\
1 \\
0 \\
0 \\
0\end{array}$ & $\begin{array}{l}0.00214 \\
0.00212 \\
0.00210 \\
0.00208 \\
0.00206\end{array}$ & $\begin{array}{l}2 \\
2 \\
2 \\
2 \\
1\end{array}$ & $\begin{array}{l}0.00240 \\
0.00237 \\
0.00235 \\
0.00233 \\
0.00231\end{array}$ & $\begin{array}{l}3 \\
2 \\
2 \\
2 \\
2\end{array}$ & $\begin{array}{l}0.01766 \\
0.01752 \\
0.01739 \\
0.01726 \\
0.01713\end{array}$ \\
\hline $\begin{array}{l}8.31 \\
8.32 \\
8.33 \\
8.34 \\
8.35\end{array}$ & $\begin{array}{l}0.00025 \\
0.00024 \\
0.00024 \\
0.00024 \\
0.00024\end{array}$ & $\begin{array}{l}1 \\
0 \\
0 \\
0 \\
1\end{array}$ & $\begin{array}{l}0.00205 \\
0.00203 \\
0.00201 \\
0.00199 \\
0.00197\end{array}$ & $\begin{array}{l}2 \\
2 \\
2 \\
2 \\
1\end{array}$ & $\begin{array}{l}0.00229 \\
0.00227 \\
0.00225 \\
0.00223 \\
0.00221\end{array}$ & $\begin{array}{l}2 \\
2 \\
2 \\
2 \\
2\end{array}$ & $\begin{array}{l}0.01700 \\
0.01687 \\
0.01674 \\
0.01662 \\
0.01649\end{array}$ \\
\hline $\begin{array}{l}8.36 \\
8.37 \\
8.38 \\
8.39 \\
8.40\end{array}$ & $\begin{array}{l}0.00023 \\
0.00023 \\
0.00023 \\
0.00023 \\
0.00022\end{array}$ & $\begin{array}{l}0 \\
0 \\
0 \\
1 \\
0\end{array}$ & $\begin{array}{l}0.00196 \\
0.00194 \\
0.00192 \\
0.00191 \\
0.00189\end{array}$ & $\begin{array}{l}2 \\
2 \\
1 \\
2 \\
2\end{array}$ & $\begin{array}{l}0.00219 \\
0.00217 \\
0.00215 \\
0.00213 \\
0.00211\end{array}$ & $\begin{array}{l}2 \\
2 \\
2 \\
2 \\
1\end{array}$ & $\begin{array}{l}0.01636 \\
0.01624 \\
0.01612 \\
0.01600 \\
0.01587\end{array}$ \\
\hline $\begin{array}{l}8.41 \\
8.42 \\
8.43 \\
8.44 \\
8.45\end{array}$ & $\begin{array}{l}0.00022 \\
0.00022 \\
0.00022 \\
0.00022 \\
0.00021\end{array}$ & $\begin{array}{l}0 \\
0 \\
0 \\
1 \\
0\end{array}$ & $\begin{array}{l}0.00187 \\
0.00186 \\
0.00184 \\
0.00182 \\
0.00181\end{array}$ & $\begin{array}{l}1 \\
2 \\
2 \\
1 \\
2\end{array}$ & $\begin{array}{l}0.00210 \\
0.00208 \\
0.00206 \\
0.00204 \\
0.00202\end{array}$ & $\begin{array}{l}2 \\
2 \\
2 \\
2 \\
2\end{array}$ & $\begin{array}{l}0.01575 \\
0.01563 \\
0.01551 \\
0.01540 \\
0.01528\end{array}$ \\
\hline $\begin{array}{l}8.46 \\
8.47 \\
8.48 \\
8.49 \\
8.50\end{array}$ & $\begin{array}{l}0.00021 \\
0.00021 \\
0.00021 \\
0.00021 \\
0.00020\end{array}$ & $\begin{array}{l}0 \\
0 \\
0 \\
1\end{array}$ & $\begin{array}{l}0.00179 \\
0.00178 \\
0.00176 \\
0.00175 \\
0.00173\end{array}$ & $\begin{array}{l}1 \\
2 \\
1 \\
2\end{array}$ & $\begin{array}{l}0.00200 \\
0.00199 \\
0.00197 \\
0.00195 \\
0.00193\end{array}$ & $\begin{array}{l}1 \\
2 \\
2 \\
2\end{array}$ & $\begin{array}{l}0.01516 \\
0.01505 \\
0.01493 \\
0.01482 \\
0.01471\end{array}$ \\
\hline
\end{tabular}


Table I Harmonic Oscillator Contributions (in dimensionless form) to the

Thermodynamic Functions for Values of $X=h c v / k T$

$\begin{array}{llllllll} & -\left(\mathrm{F}^{\circ}-\mathrm{E}_{\mathrm{O}}\right) / \mathrm{RT} & \Delta & \left(\mathrm{H}^{\circ}-\mathrm{E}_{\mathrm{O}}\right) / \mathrm{RT} & \Delta & \mathrm{S} \% / \mathrm{R} & \Delta \quad \mathrm{CO}_{\mathrm{p}} / \mathrm{R}\end{array}$

$\Delta$

\begin{tabular}{|c|c|c|c|c|c|c|c|}
\hline 8.50 & 0.00020 & 0 & 0.00173 & 2 & 0.00193 & 1 & 0.01471 \\
\hline $\begin{array}{l}8.51 \\
8.52 \\
8.53 \\
8.54 \\
8.55\end{array}$ & $\begin{array}{l}0.00020 \\
0.00020 \\
0.00020 \\
0.00020 \\
0.00019\end{array}$ & $\begin{array}{l}0 \\
0 \\
0 \\
1 \\
0\end{array}$ & $\begin{array}{l}0.00171 \\
0.00170 \\
0.00168 \\
0.00167 \\
0.00166\end{array}$ & $\begin{array}{l}1 \\
2 \\
1 \\
1 \\
2\end{array}$ & $\begin{array}{l}0.00192 \\
0.00190 \\
0.00188 \\
0.00187 \\
0.00185\end{array}$ & $\begin{array}{l}2 \\
2 \\
1 \\
2 \\
2\end{array}$ & $\begin{array}{l}0.01459 \\
0.01448 \\
0.01437 \\
0.011426 \\
0.01415\end{array}$ \\
\hline $\begin{array}{l}8.56 \\
8.57 \\
8.58 \\
8.59 \\
8.60\end{array}$ & $\begin{array}{l}0.00019 \\
0.00019 \\
0.00019 \\
0.00019 \\
0.00018\end{array}$ & $\begin{array}{l}0 \\
0 \\
0 \\
1 \\
0\end{array}$ & $\begin{array}{l}0.00164 \\
0.00163 \\
0.00161 \\
0.00160 \\
0.00158\end{array}$ & $\begin{array}{l}1 \\
2 \\
1 \\
2 \\
1\end{array}$ & $\begin{array}{l}0.00183 \\
0.00182 \\
0.00180 \\
0.00178 \\
0.00177\end{array}$ & $\begin{array}{l}1 \\
2 \\
2 \\
1 \\
2\end{array}$ & $\begin{array}{l}0.01405 \\
0.01394 \\
0.01383 \\
0.011373 \\
0.01362\end{array}$ \\
\hline $\begin{array}{l}8.61 \\
8.62 \\
8.63 \\
8.64 \\
8.65\end{array}$ & $\begin{array}{l}0.00018 \\
0.00018 \\
0.00018 \\
0.00018 \\
0.00018\end{array}$ & $\begin{array}{l}0 \\
0 \\
0 \\
0 \\
1\end{array}$ & $\begin{array}{l}0.00157 \\
0.00156 \\
0.00154 \\
0.00153 \\
0.00152\end{array}$ & $\begin{array}{l}1 \\
2 \\
1 \\
1 \\
2\end{array}$ & $\begin{array}{l}0.00175 \\
0.00174 \\
0.00172 \\
0.00171 \\
0.00169\end{array}$ & $\begin{array}{l}1 \\
2 \\
1 \\
2 \\
1\end{array}$ & $\begin{array}{l}0.01352 \\
0.01341 \\
0.01331 \\
0.011321 \\
0.01311\end{array}$ \\
\hline $\begin{array}{l}8.66 \\
8.67 \\
8.68 \\
8.69 \\
8.70\end{array}$ & $\begin{array}{l}0.00017 \\
0.00017 \\
0.00017 \\
0.00017 \\
0.00017\end{array}$ & $\begin{array}{l}0 \\
0 \\
0 \\
0 \\
1\end{array}$ & $\begin{array}{l}0.00150 \\
0.00149 \\
0.00148 \\
0.00146 \\
0.00145\end{array}$ & $\begin{array}{l}1 \\
1 \\
2 \\
1 \\
1\end{array}$ & $\begin{array}{l}0.00168 \\
0.00166 \\
0.00165 \\
0.00163 \\
0.00162\end{array}$ & $\begin{array}{l}2 \\
1 \\
2 \\
1 \\
2\end{array}$ & $\begin{array}{l}0.01301 \\
0.01291 \\
0.01281 \\
0.01271 \\
0.01261\end{array}$ \\
\hline $\begin{array}{l}8.71 \\
8.72 \\
8.73 \\
8.74 \\
8.75\end{array}$ & $\begin{array}{l}0.00016 \\
0.00016 \\
0.00016 \\
0.00016 \\
0.00016\end{array}$ & $\begin{array}{l}0 \\
0 \\
0 \\
0 \\
0\end{array}$ & $\begin{array}{l}0.00144 \\
0.00142 \\
0.00141 \\
0.00140 \\
0.00139\end{array}$ & $\begin{array}{l}2 \\
1 \\
1 \\
1 \\
2\end{array}$ & $\begin{array}{l}0.00160 \\
0.00159 \\
0.00157 \\
0.00156 \\
0.00155\end{array}$ & $\begin{array}{l}1 \\
2 \\
1 \\
1 \\
2\end{array}$ & $\begin{array}{l}0.01252 \\
0.01242 \\
0.011232 \\
0.011223 \\
0.011214\end{array}$ \\
\hline $\begin{array}{l}8.76 \\
8.77 \\
8.78 \\
8.79 \\
8.80\end{array}$ & $\begin{array}{l}0.00016 \\
0.00016 \\
0.00015 \\
0.00015 \\
0.00015\end{array}$ & $\begin{array}{l}0 \\
1 \\
0 \\
0 \\
0\end{array}$ & $\begin{array}{l}0.00137 \\
0.00136 \\
0.00135 \\
0.00134 \\
0.00133\end{array}$ & $\begin{array}{l}1 \\
1 \\
1 \\
1 \\
2\end{array}$ & $\begin{array}{l}0.00153 \\
0.00152 \\
0.00150 \\
0.00149 \\
0.00148\end{array}$ & $\begin{array}{l}1 \\
2 \\
1 \\
1 \\
2\end{array}$ & $\begin{array}{l}0.01204 \\
0.01195 \\
0.01186 \\
0.011177 \\
0.01168\end{array}$ \\
\hline $\begin{array}{l}8.81 \\
8.82 \\
8.83 \\
8.84 \\
8.85\end{array}$ & $\begin{array}{l}0.00015 \\
0.00015 \\
0.00015 \\
0.000114 \\
0.00014\end{array}$ & $\begin{array}{l}0 \\
0 \\
1 \\
0 \\
0\end{array}$ & $\begin{array}{l}0.00131 \\
0.00130 \\
0.00129 \\
0.00128 \\
0.00127\end{array}$ & $\begin{array}{l}1 \\
1 \\
1 \\
1 \\
1\end{array}$ & $\begin{array}{l}0.00146 \\
0.00145 \\
0.00144 \\
0.00143 \\
0.00114\end{array}$ & $\begin{array}{l}1 \\
1 \\
1 \\
2 \\
1\end{array}$ & $\begin{array}{l}0.01159 \\
0.01150 \\
0.011141 \\
0.011132 \\
0.011123\end{array}$ \\
\hline $\begin{array}{l}8.86 \\
8.87 \\
8.88 \\
8.89 \\
8.90\end{array}$ & $\begin{array}{l}0.00014 \\
0.00014 \\
0.00014 \\
0.00014 \\
0.00014\end{array}$ & $\begin{array}{l}0 \\
0 \\
0 \\
0 \\
0\end{array}$ & $\begin{array}{l}0.00126 \\
0.00125 \\
0.00124 \\
0.00122 \\
0.00121\end{array}$ & $\begin{array}{l}1 \\
1 \\
2 \\
1 \\
1\end{array}$ & $\begin{array}{l}0.00140 \\
0.00139 \\
0.00137 \\
0.00136 \\
0.00135\end{array}$ & $\begin{array}{l}1 \\
2 \\
1 \\
1 \\
1\end{array}$ & $\begin{array}{l}0.01115 \\
0.01106 \\
0.011098 \\
0.01089 \\
0.01081\end{array}$ \\
\hline $\begin{array}{l}8.91 \\
8.92 \\
8.93 \\
8.94 \\
8.95\end{array}$ & $\begin{array}{l}0.00014 \\
0.00013 \\
0.00013 \\
0.00013 \\
0.00013\end{array}$ & $\begin{array}{l}1 \\
0 \\
0 \\
0 \\
0\end{array}$ & $\begin{array}{l}0.00120 \\
0.00119 \\
0.00118 \\
0.00117 \\
0.00116\end{array}$ & $\begin{array}{l}1 \\
1 \\
1 \\
1 \\
1\end{array}$ & $\begin{array}{l}0.00134 \\
0.00133 \\
0.00131 \\
0.00130 \\
0.00119\end{array}$ & $\begin{array}{l}1 \\
2 \\
1 \\
1 \\
1\end{array}$ & $\begin{array}{l}0.01072 \\
0.01064 \\
0.01056 \\
0.01048 \\
0.01039\end{array}$ \\
\hline $\begin{array}{l}8.96 \\
8.97 \\
8.98 \\
8.99 \\
9.00\end{array}$ & $\begin{array}{l}0.00013 \\
0.00013 \\
0.00013 \\
0.00012 \\
0.00012\end{array}$ & $\begin{array}{l}0 \\
0 \\
1 \\
0\end{array}$ & $\begin{array}{l}0.00115 \\
0.001114 \\
0.00113 \\
0.001112 \\
0.00111\end{array}$ & $\begin{array}{l}1 \\
1 \\
1 \\
1\end{array}$ & $\begin{array}{l}0.00128 \\
0.00127 \\
0.00126 \\
0.00125 \\
0.00123\end{array}$ & $\begin{array}{l}1 \\
1 \\
1 \\
2\end{array}$ & $\begin{array}{l}0.01031 \\
0.01023 \\
0.01016 \\
0.01008 \\
0.01000\end{array}$ \\
\hline
\end{tabular}


Table I Harmonic Oscillator Contributions (in dimensionless form) to the

Thermodynamic Functions for Values of $X=h c v / k T$

$\mathbb{Z} \quad-\left(\mathrm{F}^{\circ}-\mathrm{E}_{\mathrm{O}}^{\circ}\right) / \mathrm{RT} \quad \Delta \quad\left(\mathrm{H}^{\circ}-\mathrm{E}_{\mathrm{O}} \mathrm{O}\right) / \mathrm{RT} \quad \Delta \quad \mathrm{S} / \mathrm{R} \quad \Delta \quad \mathrm{CO}_{\mathrm{p}} / \mathrm{R}$

\begin{tabular}{|c|c|c|c|c|c|c|c|}
\hline 9.00 & 0.00012 & 0 & 0.00111 & 1 & 0.00123 & 1 & 0.01000 \\
\hline $\begin{array}{l}9.01 \\
9.02 \\
9.03 \\
9.04 \\
9.05\end{array}$ & $\begin{array}{l}0.00012 \\
0.00012 \\
0.00012 \\
0.00012 \\
0.00012\end{array}$ & $\begin{array}{l}0 \\
0 \\
0 \\
0 \\
0\end{array}$ & $\begin{array}{l}0.00110 \\
0.00109 \\
0.00108 \\
0.00107 \\
0.00106\end{array}$ & $\begin{array}{l}1 \\
1 \\
1 \\
1 \\
1\end{array}$ & $\begin{array}{l}0.00122 \\
0.00121 \\
0.00120 \\
0.00119 \\
0.00118\end{array}$ & $\begin{array}{l}1 \\
1 \\
1 \\
1 \\
1\end{array}$ & $\begin{array}{l}0.00992 \\
0.00984 \\
0.00977 \\
0.00969 \\
0.00962\end{array}$ \\
\hline $\begin{array}{l}9.06 \\
9.07 \\
9.08 \\
9.09 \\
9.10\end{array}$ & $\begin{array}{l}0.00012 \\
0.00012 \\
0.00011 \\
0.00011 \\
0.00011\end{array}$ & $\begin{array}{l}0 \\
1 \\
0 \\
0 \\
0\end{array}$ & $\begin{array}{l}0.00105 \\
0.00104 \\
0.00103 \\
0.00103 \\
0.00102\end{array}$ & $\begin{array}{l}1 \\
1 \\
0 \\
1 \\
1\end{array}$ & $\begin{array}{l}0.00117 \\
0.00116 \\
0.00115 \\
0.00114 \\
0.00113\end{array}$ & $\begin{array}{l}1 \\
1 \\
1 \\
1 \\
1\end{array}$ & $\begin{array}{l}0.00954 \\
0.00947 \\
0.00939 \\
0.00932 \\
0.00925\end{array}$ \\
\hline $\begin{array}{l}9.11 \\
9.12 \\
9.13 \\
9.14 \\
9.15\end{array}$ & $\begin{array}{l}0.00011 \\
0.00011 \\
0.00011 \\
0.00011 \\
0.00011\end{array}$ & $\begin{array}{l}0 \\
0 \\
0 \\
0 \\
0\end{array}$ & $\begin{array}{l}0.00101 \\
0.00100 \\
0.00099 \\
0.00098 \\
0.00097\end{array}$ & $\begin{array}{l}1 \\
1 \\
1 \\
1 \\
1\end{array}$ & $\begin{array}{l}0.00112 \\
0.00111 \\
0.00110 \\
0.00109 \\
0.00108\end{array}$ & $\begin{array}{l}1 \\
1 \\
1 \\
1 \\
1\end{array}$ & $\begin{array}{l}0.00918 \\
0.00911 \\
0.00903 \\
0.00896 \\
0.00889\end{array}$ \\
\hline $\begin{array}{l}9.16 \\
9.17 \\
9.18 \\
9.19 \\
9.20\end{array}$ & $\begin{array}{l}0.00011 \\
0.00010 \\
0.00010 \\
0.00010 \\
0.00010\end{array}$ & $\begin{array}{l}1 \\
0 \\
0 \\
0 \\
0\end{array}$ & $\begin{array}{l}0.00096 \\
0.00095 \\
0.00095 \\
0.00094 \\
0.00093\end{array}$ & $\begin{array}{l}1 \\
0 \\
1 \\
1 \\
1\end{array}$ & $\begin{array}{l}0.00107 \\
0.00106 \\
0.00105 \\
0.00104 \\
0.00103\end{array}$ & $\begin{array}{l}1 \\
1 \\
1 \\
1 \\
1\end{array}$ & $\begin{array}{l}0.00883 \\
0.00876 \\
0.00869 \\
0.00862 \\
0.00855\end{array}$ \\
\hline $\begin{array}{l}9.21 \\
9.22 \\
9.23 \\
9.24 \\
9.25\end{array}$ & $\begin{array}{l}0.00010 \\
0.00010 \\
0.00010 \\
0.00010 \\
0.00010\end{array}$ & $\begin{array}{l}0 \\
0 \\
0 \\
0 \\
0\end{array}$ & $\begin{array}{l}0.00092 \\
0.00091 \\
0.00091 \\
0.00090 \\
0.00089\end{array}$ & $\begin{array}{l}1 \\
0 \\
1 \\
1 \\
1\end{array}$ & $\begin{array}{l}0.00102 \\
0.00101 \\
0.00100 \\
0.00099 \\
0.00099\end{array}$ & $\begin{array}{l}1 \\
1 \\
1 \\
0 \\
1\end{array}$ & $\begin{array}{l}0.00849 \\
0.00842 \\
0.00836 \\
0.00829 \\
0.00823\end{array}$ \\
\hline $\begin{array}{l}9.26 \\
9.27 \\
9.28 \\
9.29 \\
9.30\end{array}$ & $\begin{array}{l}0.00010 \\
0.00009 \\
0.00009 \\
0.00009 \\
0.00009\end{array}$ & $\begin{array}{l}1 \\
0 \\
0 \\
0 \\
0\end{array}$ & $\begin{array}{l}0.00088 \\
0.00087 \\
0.00087 \\
0.00086 \\
0.00085\end{array}$ & $\begin{array}{l}1 \\
0 \\
1 \\
1 \\
1\end{array}$ & $\begin{array}{l}0.00098 \\
0.00097 \\
0.00096 \\
0.00095 \\
0.00094\end{array}$ & $\begin{array}{l}1 \\
1 \\
1 \\
1 \\
1\end{array}$ & $\begin{array}{l}0.00816 \\
0.00810 \\
0.00803 \\
0.00797 \\
0.00791\end{array}$ \\
\hline $\begin{array}{l}9.31 \\
9.32 \\
9.33 \\
9.34 \\
9.35\end{array}$ & $\begin{array}{l}0.00009 \\
0.00009 \\
0.00009 \\
0.00009 \\
0.00009\end{array}$ & $\begin{array}{l}0 \\
0 \\
0 \\
0 \\
0\end{array}$ & $\begin{array}{l}0.00084 \\
0.00084 \\
0.00083 \\
0.00082 \\
0.00081\end{array}$ & $\begin{array}{l}0 \\
1 \\
1 \\
1 \\
0\end{array}$ & $\begin{array}{l}0.00093 \\
0.00092 \\
0.00092 \\
0.00091 \\
0.00090\end{array}$ & $\begin{array}{l}1 \\
0 \\
1 \\
1 \\
1\end{array}$ & $\begin{array}{l}0.00785 \\
0.00779 \\
0.00772 \\
0.00766 \\
0.00760\end{array}$ \\
\hline $\begin{array}{l}9.36 \\
9.37 \\
9.38 \\
9.39 \\
9.40\end{array}$ & $\begin{array}{l}0.00009 \\
0.00009 \\
0.00008 \\
0.00008 \\
0.00008\end{array}$ & $\begin{array}{l}0 \\
1 \\
0 \\
0 \\
0\end{array}$ & $\begin{array}{l}0.00081 \\
0.00080 \\
0.00079 \\
0.00078 \\
0.00078\end{array}$ & $\begin{array}{l}1 \\
1 \\
1 \\
0 \\
1\end{array}$ & $\begin{array}{l}0.00089 \\
0.00088 \\
0.00088 \\
0.00087 \\
0.00086\end{array}$ & $\begin{array}{l}1 \\
0 \\
1 \\
1 \\
1\end{array}$ & $\begin{array}{l}0.00754 \\
0.00749 \\
0.00743 \\
0.00737 \\
0.00731\end{array}$ \\
\hline $\begin{array}{l}9.41 \\
9.42 \\
9.43 \\
9.44 \\
9.45\end{array}$ & $\begin{array}{l}0.00008 \\
0.00008 \\
0.00008 \\
0.00008 \\
0.00008\end{array}$ & $\begin{array}{l}0 \\
0 \\
0 \\
0 \\
0\end{array}$ & $\begin{array}{l}0.00077 \\
0.00076 \\
0.00076 \\
0.00075 \\
0.00074\end{array}$ & $\begin{array}{l}1 \\
0 \\
1 \\
1 \\
0\end{array}$ & $\begin{array}{l}0.00085 \\
0.00085 \\
0.00084 \\
0.00083 \\
0.00082\end{array}$ & $\begin{array}{l}0 \\
1 \\
1 \\
1 \\
1\end{array}$ & $\begin{array}{l}0.00725 \\
0.00720 \\
0.00714 \\
0.00708 \\
0.00703\end{array}$ \\
\hline $\begin{array}{l}9.46 \\
9.47 \\
9.48 \\
9.49 \\
9.50\end{array}$ & $\begin{array}{l}0.00008 \\
0.00008 \\
0.00008 \\
0.00008 \\
0.00007\end{array}$ & $\begin{array}{l}0 \\
0 \\
0 \\
1\end{array}$ & $\begin{array}{l}0.00074 \\
0.00073 \\
0.00072 \\
0.00072 \\
0.00071\end{array}$ & $\begin{array}{l}1 \\
1 \\
0 \\
1\end{array}$ & $\begin{array}{l}0.00081 \\
0.00081 \\
0.00080 \\
0.00079 \\
0.00079\end{array}$ & $\begin{array}{l}0 \\
1 \\
1 \\
0\end{array}$ & $\begin{array}{l}0.00697 \\
0.00692 \\
0.00686 \\
0.00681 \\
0.00676\end{array}$ \\
\hline
\end{tabular}


Table I Harmonic Oscillator Contributions (in dimensionless form) to the

Thermodynamic Functions for Values of $X=h c v / k T$

X
$\Delta \quad\left(\mathrm{H}^{\circ}-\mathrm{E}_{\mathrm{O}}^{\circ}\right) / \mathrm{RT} \quad \Delta \quad \mathrm{S} / \mathrm{R}$

0.00071
0.00070
0.00070
0.00069
0.00069
0.00068
0.00067
0.00067
0.00066
0.00066
0.00065
0.00064
0.00064
0.00063
0.00063
0.00062

0.00062

0.00061

0.00061

0.00060

0.00059

0.00059

0.00058

0.00058

0.00057

0.00057

0.00056

0.00056

0.00055

0.00055

0.00054

0.00054

0.00053

0.00053

0.00052

0.00052

0.00051

0.00051

0.00051

0.00050

0.00050

0.00049

0.00049

0.00048

0.00048

0.00047

0.00047

0.00047

0.00046

0.00046

0.00045
0.00078

0.00077

0.00076

0.00076

0.00075

0.00074

0.00074

0.00073

0.00072

0.00072

0.00071

0.00071

0.00070

0.00069

0.00069

0.00068

0.00067

0.00067

0.00066

0.00066

0.00065

0.00064

0.00064

0.00063

0.00063

0.00062

0.00062

0.00061

0.00060

0.00060

0.00059

0.00059

0.00058

0.00058

0.00057

0.00057

0.00056

0.00056

0.00055

0.00055

0.00054

0.00054

0.00053

0.00053

0.00052

0.00052

0.00051

0.00051

0.00050

0.00050 $\triangle \quad C O / R$

$\Delta$

$9.98 \quad 0.00005$

$\begin{array}{rr}9.99 & 0.00005 \\ 10.00 & 0.00005\end{array}$

$6389510-62-9$ 
Table I Harmonic Oscillator Contributions (in dimensionless form) to the

Thermodynamic Functions for Values of $\mathrm{X}=\mathrm{hcv} / \mathrm{kT}$

\begin{tabular}{|c|c|c|c|c|c|c|c|}
\hline $\bar{I}$ & $-\left(F^{\circ}-E_{O}^{O}\right) / R T$ & $\Delta$ & $\left(H^{\circ}-E_{O}^{\circ}\right) / R T$ & $\Delta$ & So/R & $\Delta$ & $\mathrm{C}_{\mathrm{p}}^{\circ} / \mathrm{R}$ \\
\hline 10.0 & 0.00005 & 1 & 0.00045 & 7 & 0.00050 & 8 & 0.00454 \\
\hline 10.2 & 0.00004 & 1 & 0.00038 & 6 & 0.00042 & 7 & 0.00387 \\
\hline 10.4 & 0.00003 & 1 & 0.00032 & 4 & 0.00035 & 6 & 0.00329 \\
\hline 10.6 & 0.00002 & 0 & 0.00026 & 4 & 0.00029 & 5 & 0.00280 \\
\hline 10.8 & 0.00002 & 0 & 0.00022 & 4 & 0.00024 & 4 & 0.00238 \\
\hline 11.0 & 0.00002 & 0 & 0.00018 & 3 & 0.00020 & 3 & 0.00202 \\
\hline $11 \cdot 2$ & 0.00001 & 1 & 0.00015 & 2 & 0.00017 & 3 & 0.00172 \\
\hline 11.4 & 0.00001 & 0 & 0.00013 & 2 & 0.00014 & 2 & 0.00145 \\
\hline 11.6 & 0.00001 & 0 & 0.00011 & 2 & 0.00012 & 2 & 0.00123 \\
\hline $11 \cdot 8$ & 0.00001 & 0 & 0.00009 & 2 & 0.00010 & 2 & 0.00104 \\
\hline 12.0 & 0.00001 & 0 & 0.00007 & 1 & 0.00008 & 1 & 0.00088 \\
\hline $12 \cdot 2$ & 0.00001 & 1 & 0.00006 & 1 & 0.00007 & 1 & 0.00075 \\
\hline 12.4 & 0.00000 & 0 & 0.00005 & 1 & 0.00006 & 1 & 0.00063 \\
\hline 12.6 & 0.00000 & 0 & 0.00004 & 0 & 0.00005 & 1 & 0.00054 \\
\hline $12 \cdot 8$ & 0.00000 & 0 & 0.00004 & 0 & 0.00004 & 1 & 0.00045 \\
\hline 13.0 & 0.00000 & 0 & 0.00003 & 1 & 0.00003 & 0 & 0.00038 \\
\hline 13.2 & 0.00000 & 0 & 0.00002 & 0 & 0.00003 & 1 & 0.00032 \\
\hline 13.4 & 0.00000 & 0 & 0.00002 & 0 & 0.00002 & 0 & 0.00027 \\
\hline $13 \cdot 6$ & 0.00000 & 0 & 0.00002 & 1 & 0.00002 & 1 & 0.00023 \\
\hline 13.8 & 0.00000 & 0 & 0.00001 & 0 & 0.00001 & 0 & 0.00019 \\
\hline 14.0 & 0.00000 & 0 & 0.00001 & 0 & 0.00001 & 0 & 0.00016 \\
\hline 14.2 & 0.00000 & 0 & 0.00001 & 0 & 0.00001 & 0 & 0.00014 \\
\hline 14.4 & 0.00000 & 0 & 0.00001 & 0 & 0.00001 & 0 & 0.00012 \\
\hline 14.6 & 0.00000 & 0 & 0.00001 & 0 & 0.00001 & 0 & 0.00010 \\
\hline $14 \cdot 8$ & 0.00000 & 0 & 0.00001 & 0 & 0.00001 & 0 & 0.00008 \\
\hline 15.0 & 0.00000 & 0 & 0.00001 & 1 & 0.00001 & 0 & 0.00007 \\
\hline 15.2 & 0.00000 & 0 & 0.00000 & 0 & 0.00000 & 1 & 0.00006 \\
\hline 15.4 & 0.00000 & 0 & 0.00000 & 0 & 0.00000 & 0 & 0.00005 \\
\hline 15.6 & 0.00000 & 0 & 0.00000 & 0 & 0.00000 & 0 & 0.00004 \\
\hline $15 \cdot 8$ & 0.00000 & & 0.00000 & & 0.00000 & & 0.00003 \\
\hline 16.0 & 0.00000 & & 0.00000 & & 0.00000 & & 0.00002 \\
\hline
\end{tabular}




\title{
Table II.
}

\section{Harmonic Oscillator Contributions}

\section{to the Thermodynamic Functions}

\author{
(in units of calories, moles, ${ }^{\circ} \mathrm{K}$ )
}



Table II Harmonic Oscillator Contributions to the Thermodynamic

Functions (in units of calories, moles, and ${ }^{\circ} \mathrm{K}$ )
$\nu \quad-\left(\mathrm{F}^{\circ}-\mathrm{E}_{\mathrm{O}}^{\circ}\right) / \mathrm{T} \quad \mathrm{S}^{\circ}$
$\mathrm{cm}^{-1}$
$\mathrm{C}_{\mathrm{p}}^{\circ} \quad-\left(\mathrm{F}^{\circ}-\mathrm{E}_{\mathrm{O}}\right) / \mathrm{T} \quad \mathrm{S}^{\circ}$
$\mathrm{CO}_{\mathrm{p}} \quad-\left(\mathrm{F}^{\circ}-\mathrm{E}_{\mathrm{O}}^{\mathrm{O}}\right) / \mathrm{T}$
So
$\mathrm{cm}^{-1}$

$T=273.15$

$\begin{array}{lllll}100 & 1.77430 & 3.28384 & 1.94185\end{array}$

$110 \quad 1.63244 \quad 3.09920 \quad 1.93250$

$\begin{array}{llll}120 & 1.50662 & 2.93148 & 1.92231\end{array}$

$\begin{array}{lllll}130 & 1.39420 & 2.77804 & 1.91131\end{array}$

$140 \quad 1.29312 \quad 2.63682 \quad 1.89951$

$\begin{array}{llll}150 & 1.20175 & 2.50620 & 1.88694\end{array}$

$160 \quad 1.11880 \quad 2.38484 \quad 1.87361$

$\begin{array}{llll}170 & 1.04317 & 2.27167 & 1.85954\end{array}$

$\begin{array}{llll}180 & 0.97400 & 2.16580 & 1.84476\end{array}$

$190 \quad 0.91053 \quad 2.06647 \quad 1.82929$

$200 \quad 0.85213 \quad 1.97304 \quad 1.81315$

$\begin{array}{lllll}210 & 0.79827 & 1.88498 & 1.79637\end{array}$

$\begin{array}{llll}220 & 0.74849 & 1.80182 & 1.77896\end{array}$

$\begin{array}{lllll}230 & 0.70239 & 1.72313 & 1.76096\end{array}$

$\begin{array}{llll}240 & 0.65962 & 1.64858 & 1.74240\end{array}$

$250 \quad 0.61988 \quad 1.57783 \quad 1.72329$

$260 \quad 0.58290 \quad 1.51063 \quad 1.70367$

$270 \quad 0.54844 \quad 1.44670 \quad 1.68356$

$\begin{array}{lllll}280 & 0.51630 & 1.38585 & 1.66299\end{array}$

$\begin{array}{lllll}290 & 0.48627 & 1.32786 & 1.64199\end{array}$

$\begin{array}{lllll}300 & 0.45820 & 1.27255 & 1.62058\end{array}$

$\begin{array}{lllll}310 & 0.43193 & 1.21977 & 1.59880\end{array}$

$\begin{array}{lllll}320 & 0.40733 & 1.16936 & 1.57667\end{array}$

$\begin{array}{lllll}330 & 0.38427 & 1.12118 & 1.55421\end{array}$

$\begin{array}{llll}340 & 0.36263 & 1.007512 & 1.53147\end{array}$

$\begin{array}{lllll}350 & 0.34233 & 1.03166 & 1.50846\end{array}$

$\begin{array}{lllll}360 & 0.32325 & 0.98889 & 1.48521\end{array}$

$\begin{array}{lllll}370 & 0.30532 & 0.94852 & 1.46175\end{array}$

$\begin{array}{llll}380 & 0.28846 & 0.90985 & 1.43810\end{array}$

$\begin{array}{lllll}390 & 0.27259 & 0.87280 & 1.41430\end{array}$

$400 \quad 0.25766 \quad 0.83729 \quad 1.39036$

$\begin{array}{lllll}410 & 0.24359 & 0.80326 & 1.36631\end{array}$

$\begin{array}{lllll}420 & 0.23034 & 0.77062 & 1.34218\end{array}$

$430 \quad 0.217840 .739321 .3180 \mathrm{C}$

$\begin{array}{llllll}440 & 0.20607 & 0.70930 & 1.29377\end{array}$

$\begin{array}{lllll}450 & 0.19496 & 0.68050 & 1.26954\end{array}$

$\begin{array}{lllll}460 & 0.18447 & 0.65286 & 1.24531\end{array}$

$\begin{array}{lllll}470 & 0.17458 & 0.62634 & 1.22112\end{array}$

$\begin{array}{lllll}480 & 0.16524 & 0.60088 & 1.19697\end{array}$

$\begin{array}{llll}490 & 0.15642 & 0.57645 \quad 1.17290\end{array}$

$\begin{array}{llll}500 & 0.14808 & 0.55299 & 1.14892\end{array}$

$\begin{array}{lllll}510 & 0.14021 & 0.53048 & 1.12506\end{array}$

$\begin{array}{lllll}520 & 0.13277 & 0.50886 & 1.10132\end{array}$

$\begin{array}{lllll}530 & 0.12574 & 0.48811 & 1.07772\end{array}$

$\begin{array}{lllll}540 & 0.11909 & 0.46818 & 1.05429\end{array}$

$\begin{array}{lllll}550 & 0.11280 & 0.44905 & 1.03104\end{array}$ $\begin{array}{lllll}560 & 0.10685 & 0.43068 & 1.00797\end{array}$ $\begin{array}{lllll}570 & 0.10123 & 0.41304 & 0.98512\end{array}$ $\begin{array}{llllll}580 & 0.09591 & 0.39610 & 0.96248\end{array}$ $\begin{array}{lllll}590 & 0.09087 & 0.37984 & 0.94008\end{array}$

$$
\mathrm{T}=298.15
$$

$T=400$.

$1.90812 \quad 3.45423 \quad 1.94905$

1.762643 .268831 .94116 1.633273 .100291 .93256

$1.51737 \quad 2.94596 \quad 1.92326$

$1.41288 \quad 2.80380 \quad 1.91328$

1.318182 .672151 .90263 $1.23196 \quad 2.54972 \quad 1.89132$ $1.153142 .43541 \quad 1.87937$ $\begin{array}{llll}1.08083 & 2.32834 & 1.86680\end{array}$ $1.01430 \quad 2.22776 \quad 1.85362$

$0.952912 .13303 \quad 1.83984$ $\begin{array}{llll}0.89612 & 2.04361 & 1.82549\end{array}$ 0.843481 .959031 .81059 $0.79458 \quad 1.87888 \quad 1.79514$ $0.74908 \quad 1.802821 .77918$

$0.70668 \quad 1.73052 \quad 1.76272$ 0.667091 .661711 .74578 0.630091 .596151 .72838 $0.59547 \quad 1.53362 \quad 1.71055$ $0.56302 \quad 1.47391 \quad 1.69230$

$0.53259 \quad 1.41685 \quad 1.67365$ $0.50402 \quad 1.36228 \quad 1.65462$ $0.47718 \quad 1.31005 \quad 1.63525$ $0.451931 .26004 \quad 1.61554$ $0.42816 \quad 1.21210 \quad 1.59552$

$0.40578 \quad 1.16615 \quad 1.57521$ $0.38469 \quad 1.12206 \quad 1.55463$ $0.364791 .07975 \quad 1.53381$ 0.346011 .039121 .51276 0.328291 .000101 .49151

$0.31154 \quad 0.96261 \quad 1.47008$ $0.29571 \quad 0.92657 \quad 1.44848$ $0.28075 \quad 0.89193 \quad 1.42673$ $0.266590 .85861 \quad 1.40487$ $0.25320 \quad 0.82657 \quad 1.38290$

$0.240510 .79574 \quad 1.36085$ $0.228510 .76607 \quad 1.33873$ 0.217130 .737521 .31657 0.206350 .710031 .29437 $0.196130 .68357 \quad 1.27217$

$\begin{array}{llll}0.18644 & 0.65809 & 1.24997\end{array}$ $0.17726 \quad 0.63356 \quad 1.22780$ $0.168540 .60993 \quad 1.20566$ $\begin{array}{lllll}0.16027 & 0.58717 & 1.18358\end{array}$ $0.15242 \quad 0.56526 \quad 1.16157$

$0.144970 .54414 \quad 1.13964$ $0.13790 \quad 0.52380 \quad 1.11782$ 0.131190 .504211 .09610 $0.12481 \quad 0.48534 \quad 1.07451$ 0.118750 .467151 .05306
$2.37854 \quad 4.02970 \quad 1.96588$

2.222643 .842541 .96145 $2.08302 \quad 3.67207 \quad 1.95660$ $1.95703 \quad 3.51567 \quad 1.95135$ $1.842623 .37126 \quad 1.94570$

$1.73817 \quad 3.23722 \quad 1.93966$ $1.64235 \quad 3.11224 \quad 1.93322$ $1.554112 .99525 \quad 1.92639$ $1.472542 .88534 \quad 1.91918$ $1.39690 \quad 2.78178 \quad 1.91160$

$1.326572 .68392 \quad 1.90364$ $1.26100 \quad 2.59125 \quad 1.89532$ $1.199732 .50327 \quad 1.88664$ 1.142372 .419611 .87760 1.088562 .339891 .86822

$1.03800 \quad 2.26383 \quad 1.85850$ 0.990412 .191131 .84845 $0.94556 \quad 2.12156 \quad 1.83808$ $0.903232 .05491 \quad 1.82738$ 0.863241 .990971 .81638

$0.82540 \quad 1.92958 \quad 1.80507$ 0.789581 .870591 .79346 0.755621 .813831 .78157 $0.72340 \quad 1.759201 .76941$ $0.69280 \quad 1.706561 .75697$

0.663731 .655811 .74427 0.636081 .606851 .73131 0.609771 .559601 .71811 0.584711 .513961 .70467 0.560841 .469851 .69101

$0.53808 \quad 1.42721 \quad 1.67713$ $0.51636 \quad 1.38597 \quad 1.66303$ $0.49564 \quad 1.34607 \quad 1.64874$ 0.475851 .307441 .63425 0.456941 .270041 .61957

$0.43887 \quad 1.23381 \quad 1.60473$ $0.421601 .19870 \quad 1.58971$ $0.40507 \quad 1.16468 \quad 1.57454$ 0.389261 .131691 .55922 $0.374131 .09970 \quad 1.54377$

$0.35964 \quad 1.06867 \quad 1.52818$ $0.34576 \quad 1.03856 \quad 1.51247$ 0.332461 .009341 .49664 $\begin{array}{llll}0.31971 & 0.98099 & 1.48071\end{array}$ $0.30750 \quad 0.95346 \quad 1.46468$

$\begin{array}{llll}0.29578 & 0.92673 & 1.44857\end{array}$ $0.284540 .90077 \quad 1.43238$ $0.27376 \quad 0.87556 \quad 1.41611$ $0.263420 .85108 \quad 1.39979$ $0.253490 .82729 \quad 1.38340$
100

110

120

130

140

150

160

170

180

190

200

210

220

230

240

250

260

270

280

290

300

310

320

330

340

350

360

370

380

390

400

410

420

430

440

450

460

470

480

490

500

510

520

530

540

550

560

570

580

590 
Table II Harmonic Oscillator Contributions to the Thermodynamic

Functions (in units of calories, moles, and ${ }^{\circ} \mathrm{K}$ )

$\begin{array}{lllllllllll}\nu & -\left(F^{\circ}-E_{0}^{\circ}\right) / T & S^{\circ} & C_{p}^{\circ} & -\left(F^{\circ}-E_{O}^{\circ}\right) / T & S^{\circ} & C_{p}^{\circ} & -\left(F^{\circ}-E_{0}^{\circ}\right) / T & S^{\circ} & C_{p}^{\circ} & \nu \\ \mathrm{cm}^{-1} & & & & & & & & & & \end{array}$

$T=273.15$

$600 \quad 0.086110 .364230 .91791$

$\begin{array}{lllll}610 & 0.08160 & 0.34924 & 0.89601\end{array}$

$\begin{array}{lllll}620 & 0.07733 & 0.33484 & 0.87437\end{array}$

$\begin{array}{llll}630 & 0.07329 & 0.32102 & 0.85300\end{array}$

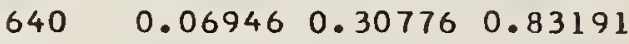

$\begin{array}{llll}650 & 0.06584 & 0.29502 & 0.81112\end{array}$

$\begin{array}{lllll}660 & 0.06240 & 0.28279 & 0.79062\end{array}$

$\begin{array}{llll}670 & 0.05915 & 0.27105 & 0.77043\end{array}$

$\begin{array}{llllll}680 & 0.05607 & 0.25979 & 0.75055\end{array}$

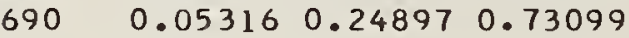

$700 \quad 0.05040 \quad 0.23859 \quad 0.71175$ $\begin{array}{lllll}710 & 0.04778 & 0.22863 & 0.69283\end{array}$

$\begin{array}{llllll}720 & 0.04530 & 0.21907 & 0.67424\end{array}$

$\begin{array}{lllll}730 & 0.04295 & 0.20990 & 0.65598\end{array}$

$\begin{array}{llll}740 & 0.04072 & 0.20109 & 0.63806\end{array}$

$\begin{array}{lllll}750 & 0.03861 & 0.19265 & 0.62047\end{array}$

$\begin{array}{llllll}760 & 0.03661 & 0.18454 & 0.60322\end{array}$

$\begin{array}{llllll}770 & 0.03472 & 0.17677 & 0.58631\end{array}$

$\begin{array}{lllll}780 & 0.03292 & 0.16931 & 0.56973\end{array}$

$\begin{array}{lllll}790 & 0.03122 & 0.16216 & 0.55350\end{array}$

$800 \quad 0.02961 \quad 0.15530 \quad 0.53760$

$\begin{array}{llllll}810 & 0.02808 & 0.14871 & 0.52204\end{array}$

$820 \quad 0.026630 .14240 \quad 0.50682$

$830 \quad 0.025250 .136350 .49193$

$840 \quad 0.023950 .130540 .47738$

$\begin{array}{lllll}850 & 0.02271 & 0.12498 & 0.46315\end{array}$

$860 \quad 0.021540 .119640 .44926$

$870 \quad 0.020430 .114530 .43569$

$880 \quad 0.019380 .109630 .42244$

$890 \quad 0.01838 \quad 0.10493 \quad 0.40951$

$900 \quad 0.017430 .100420 .39690$ $910 \quad 0.016530 .096100 .38460$ $920 \quad 0.01568 \quad 0.09196 \quad 0.37261$ $930 \quad 0.014870 .08800 \quad 0.36092$ $940 \quad 0.01411 \quad 0.08420 \quad 0.34954$

$950 \quad 0.01338 \quad 0.08056 \quad 0.33845$ $960 \quad 0.012690 .07707 \quad 0.32765$ $970 \quad 0.012040 .07373 \quad 0.31714$ $980 \quad 0.011420 .07053 \quad 0.30691$ $990 \quad 0.010830 .06747 \quad 0.29696$

$\begin{array}{llllll}1000 & 0.01027 & 0.06453 & 0.28728\end{array}$ $\begin{array}{llllll}1010 & 0.00975 & 0.06172 & 0.27787\end{array}$ $\begin{array}{lllll}1020 & 0.00924 & 0.05903 & 0.26872\end{array}$ $\begin{array}{llllll}1030 & 0.00877 & 0.05645 & 0.25983\end{array}$ $\begin{array}{llllll}1040 & 0.00832 & 0.05398 & 0.25120\end{array}$

$\begin{array}{lllll}1050 & 0.00789 & 0.05162 & 0.24281\end{array}$ $1060 \quad 0.00748 \quad 0.049350 .23466$ $1070 \quad 0.00710 \quad 0.047190 .22675$ $\begin{array}{llll}1080 & 0.00674 & 0.04511 & 0.21908\end{array}$ $1090 \quad 0.006390 .043130 .21163$

$T=298.15$

$T=400$.

0.112990 .449631 .03176

0.107520 .432751 .01061

0.102320 .416490 .98964

$0.09738 \quad 0.400820 .96885$

0.092690 .385720 .94826

0.088220 .371180 .92786

$\begin{array}{lllll}0.08398 & 0.35717 & 0.90768\end{array}$

$\begin{array}{llll}0.07994 & 0.34367 & 0.88771\end{array}$

0.076100 .330660 .86797

0.072450 .318130 .84846

0.068970 .306060 .82918 0.065670 .294440 .81016 0.062530 .283240 .79138 0.059540 .272450 .77286 0.056690 .262060 .75460

0.053980 .252050 .73661 0.051410 .242410 .71888 $0.04896 \quad 0.233130 .70142$ 0.046620 .224190 .68424 $0.04440 \quad 0.21558 \quad 0.66734$

0.042290 .207290 .65071 0.040270 .199310 .63437 0.038360 .191620 .61831 $0.03653 \quad 0.184220 .60253$ $0.03480 \quad 0.17710 \quad 0.58703$

0.033150 .170240 .57182 0.031570 .163640 .55690 0.030070 .157290 .54225 0.028650 .151170 .52790 0.027290 .145290 .51382

0.025990 .139630 .50003 $0.02476 \quad 0.134180 .48651$ 0.023590 .128930 .47328 $0.02247 \quad 0.123880 .46032$ $0.021410 .11903 \quad 0.44764$

$0.020390 .11436 \quad 0.43523$ 0.019430 .109860 .42309 0.018510 .105540 .41122 0.017630 .101380 .39961 $0.01680 \quad 0.09738 \quad 0.38827$

$0.01600 \quad 0.093540 .37719$ 0.015250 .089840 .36637 $\begin{array}{llll}0.01452 & 0.08628 & 0.35579\end{array}$ 0.013840 .082860 .34547 $\begin{array}{llll}0.01318 & 0.07957 & 0.33540\end{array}$

$0.01256 \quad 0.076410 .32557$ $0.01197 \quad 0.073370 .31598$ $0.01140 \quad 0.070450 .30663$ $0.01086 \quad 0.067640 .29751$ 0.010350 .064930 .28862 $\begin{array}{llll}0.24396 & 0.80417 & 1.36698\end{array}$ 0.234820 .781721 .35051 $0.226030 .75989 \quad 1.33401$ $0.21759 \quad 0.73868 \quad 1.31748$ $0.20948 \quad 0.71806 \quad 1.30094$

$0.201690 .69802 \quad 1.28440$ $0.19420 \quad 0.67853 \quad 1.26785$ 0.187010 .659591 .25130 0.180090 .641171 .23477 $0.173450 .62327 \quad 1.21825$

$0.16705 \quad 0.60586 \quad 1.20177$ $0.16090 \quad 0.58893 \quad 1.18531$ $0.154990 .57246 \quad 1.16889$ $0.14930 \quad 0.55645 \quad 1.15252$ 0.143830 .540881 .13619

$0.138570 .52574 \quad 1.11993$ 0.133510 .511021 .10372 0.128630 .496691 .08758 $0.123940 .48276 \quad 1.07151$ 0.119430 .469211 .05553

$0.115090 .45604 \quad 1.03962$ $0.11090 \quad 0.443221 .02380$ $0.10688 \quad 0.43076 \quad 1.00807$ $0.10300 \quad 0.418630 .99244$ 0.099270 .406840 .97691

$\begin{array}{llll}0.09568 & 0.39537 & 0.96148\end{array}$ 0.092220 .384210 .94616 0.088890 .373360 .93096 $\begin{array}{llll}0.08568 & 0.36281 & 0.91587\end{array}$ 0.082590 .352540 .90090

$0.079610 .34256 \quad 0.88605$ 0.076740 .332850 .87132 $0.07398 \quad 0.323410 .85673$ 0.071320 .314220 .84226 0.068750 .305290 .82793

$0.06628 \quad 0.296610 .81374$ $0.06390 \quad 0.288160 .79969$ $\begin{array}{llll}0.06161 & 0.27994 & 0.78577\end{array}$ $0.05940 \quad 0.27196 \quad 0.77200$ 0.057270 .264190 .75838

0.055220 .256630 .74490 0.053240 .249290 .73156 0.051340 .242140 .71838 0.049500 .235200 .70535 0.047730 .228450 .69247

$0.046020 .22188 \quad 0.67975$ $0.044380 .21550 \quad 0.66717$ $0.04280 \quad 0.209290 .65476$ 0.041270 .203260 .64250 0.039790 .197390 .63039
600

610

620

630

640

650

660

670

680

690

700

710

720

730

740

750

760

770

780

790

800

810

820

830

840

850

860

870

880

890

900

910

920

930

940

950

960

970

980

990

1000

1010

1020

1030

1040

1050

1060

1070

1080

1090 
Table II Harmonic Oscillator Contributions to the Thermodynamic Functions (in units of calories, moles, and ${ }^{\circ} \mathrm{K}$ )
$\mathrm{cm}^{-1}-\left(\mathrm{F}^{\circ}-\mathrm{E}_{\mathrm{O}}^{\circ}\right) / \mathrm{T} \quad \mathrm{S}^{\circ}$
$\mathrm{C}_{\mathrm{p}}^{\circ} \quad-\left(\mathrm{F}^{\circ}-\mathrm{E}_{\mathrm{O}}^{\circ}\right) / \mathrm{T} \quad$ So
$C_{\mathrm{p}}^{\circ} \quad-\left(F^{\circ}-E_{0}^{\circ}\right) / T$
So
$\mathrm{C}_{\mathrm{p}}^{\circ}$ $\mathrm{cm}^{-1}$

$T=273.15$

1100

1110

1120

1130

1140

1150

1160

1170

1180

1190

1200

1210

1220

1230

1240

1250

1260

1270

1280

1290

1300

1310

1320

1330

1340

1350

1360

1370

1380

1390

1400

1410

1420

1430

1440

1450

1460

1470

1480

1490

1500

1510

1520

1530

1540

1550

1560

1570

1580

1590 $\begin{array}{llll}0.00606 & 0.04123 & 0.20440\end{array}$

$\begin{array}{llll}0.00575 & 0.03941 & 0.19740\end{array}$

$\begin{array}{llll}0.00545 & 0.03767 & 0.19060\end{array}$

$\begin{array}{llll}0.00517 & 0.03601 & 0.18401\end{array}$

$\begin{array}{llll}0.00491 & 0.03441 & 0.17762\end{array}$

$\begin{array}{llll}0.00466 & 0.03289 & 0.17144\end{array}$

$\begin{array}{llll}0.00442 & 0.03143 & 0.16544\end{array}$

$0.004190 .03004 \quad 0.15963$

$0.003970 .02870 \quad 0.15401$

0.003770 .027430 .14856

$\begin{array}{llll}0.00358 & 0.02621 & 0.14329\end{array}$

$\begin{array}{llll}0.00339 & 0.02504 & 0.13819\end{array}$

$\begin{array}{lll}0.00322 & 0.02392 & 0.13325\end{array}$

$\begin{array}{llll}0.00305 & 0.02285 & 0.12847\end{array}$

$\begin{array}{llll}0.00290 & 0.02183 & 0.12385\end{array}$

$\begin{array}{llll}0.00275 & 0.02085 & 0.11938\end{array}$

0.002610 .019920 .11506

$\begin{array}{llll}0.00247 & 0.01903 & 0.11088\end{array}$

$\begin{array}{lllll}0.00235 & 0.01817 & 0.10684\end{array}$

$\begin{array}{llll}0.00223 & 0.01736 & 0.10293\end{array}$

$\begin{array}{llll}0.00211 & 0.01658 & 0.09916\end{array}$

$\begin{array}{llll}0.00200 & 0.01583 & 0.09552\end{array}$

$0.00190 \quad 0.015120 .09199$

$\begin{array}{llll}0.00180 & 0.01444 & 0.08859\end{array}$

$\begin{array}{llll}0.00171 & 0.01379 & 0.08531\end{array}$

$0.001620 .01316 \quad 0.08213$

$\begin{array}{llll}0.00154 & 0.01257 & 0.07907\end{array}$

$\begin{array}{llll}0.00146 & 0.01200 & 0.07612\end{array}$

$0.001390 .01146 \quad 0.07326$

$0.001310 .01094 \quad 0.07051$

$0.00125 \quad 0.01044 \quad 0.06785$

$\begin{array}{lllll}0.00118 & 0.00997 & 0.06529\end{array}$

$\begin{array}{lll}0.00112 & 0.00952 & 0.06282\end{array}$

$\begin{array}{llll}0.00106 & 0.00908 & 0.06043\end{array}$

$\begin{array}{lll}0.00101 & 0.00867 & 0.05813\end{array}$

$\begin{array}{llll}0.00096 & 0.00828 & 0.05592\end{array}$

$\begin{array}{llll}0.00091 & 0.00790 & 0.05378\end{array}$

$\begin{array}{llll}0.00086 & 0.00754 & 0.05172\end{array}$

$\begin{array}{llll}0.00082 & 0.00719 & 0.04973\end{array}$

$\begin{array}{llll}0.00078 & 0.00687 & 0.04782\end{array}$

$\begin{array}{llll}0.00074 & 0.00655 & 0.04597\end{array}$

$\begin{array}{llll}0.00070 & 0.00625 & 0.04420\end{array}$

$\begin{array}{llll}0.00066 & 0.00597 & 0.04248\end{array}$

$\begin{array}{lllll}0.00063 & 0.00569 & 0.04084\end{array}$

$\begin{array}{lll}0.00060 & 0.00543 & 0.03925\end{array}$

$\begin{array}{llll}0.00057 & 0.00518 & 0.03772\end{array}$

$0.00054 \quad 0.00495 \quad 0.03624$

$\begin{array}{lll}0.00051 & 0.00472 & 0.03483\end{array}$

$\begin{array}{llll}0.00048 & 0.00450 & 0.03346\end{array}$

$\begin{array}{lll}0.00046 & 0.00430 & 0.03215\end{array}$
$T=298.15$

$T=400$.

$\begin{array}{llll}0.00986 & 0.06234 & 0.27995\end{array}$ $\begin{array}{llll}0.00940 & 0.05984 & 0.27151\end{array}$ $\begin{array}{lllll}0.00895 & 0.05744 & 0.26328\end{array}$ $\begin{array}{lllll}0.00853 & 0.05514 & 0.25527\end{array}$ $\begin{array}{llll}0.00813 & 0.05293 & 0.24747\end{array}$

$\begin{array}{llll}0.00774 & 0.05080 & 0.23987\end{array}$ $\begin{array}{llll}0.00738 & 0.04875 & 0.23248\end{array}$ $\begin{array}{llll}0.00703 & 0.04679 & 0.22528\end{array}$ $\begin{array}{llll}0.00670 & 0.04490 & 0.21828\end{array}$ $\begin{array}{llll}0.00638 & 0.04309 & 0.21147\end{array}$

$\begin{array}{llll}0.00608 & 0.04135 & 0.20485\end{array}$ $\begin{array}{llll}0.00579 & 0.03967 & 0.19840\end{array}$ $\begin{array}{llll}0.00552 & 0.03807 & 0.19214\end{array}$ $\begin{array}{llll}0.00526 & 0.03652 & 0.18605\end{array}$ $\begin{array}{llll}0.00501 & 0.03504 & 0.18014\end{array}$

$\begin{array}{llll}0.00478 & 0.03362 & 0.17439\end{array}$ $\begin{array}{lll}0.00455 & 0.03225 & 0.16881\end{array}$ $\begin{array}{llll}0.00434 & 0.03094 & 0.16338\end{array}$ $0.00413 \quad 0.029680 .15811$ $\begin{array}{llll}0.00394 & 0.02346 & 0.15300\end{array}$

$0.003750 .02730 \quad 0.14803$ $\begin{array}{llll}0.00357 & 0.02619 & 0.14321\end{array}$ $\begin{array}{llll}0.00341 & 0.02512 & 0.13853\end{array}$ $\begin{array}{llll}0.00324 & 0.02409 & 0.13399\end{array}$ $\begin{array}{llll}0.00309 & 0.02310 & 0.12958\end{array}$

$0.00295 \quad 0.022150 .12531$ $\begin{array}{llll}0.00281 & 0.02124 & 0.12117\end{array}$ $0.002670 .02037 \quad 0.11715$ 0.002550 .019530 .11325 . $0.00243 \quad 0.018730 .10947$

$\begin{array}{llll}0.00231 & 0.01796 & 0.10581\end{array}$ $\begin{array}{llll}0.00220 & 0.01722 & 0.10226\end{array}$ $\begin{array}{llll}0.00210 & 0.01651 & 0.09882\end{array}$ $\begin{array}{llll}0.00200 & 0.01582 & 0.09548\end{array}$ $\begin{array}{llll}0.00191 & 0.01517 & 0.09225\end{array}$

$\begin{array}{llll}0.00182 & 0.01454 & 0.08912\end{array}$ $0.00173 \quad 0.013940 .08609$ $\begin{array}{llll}0.00165 & 0.01336 & 0.08316\end{array}$ $\begin{array}{llll}0.00157 & 0.01281 & 0.08031\end{array}$ $\begin{array}{llll}0.00150 & 0.01228 & 0.07756\end{array}$

$0.00143 \quad 0.01177 \quad 0.07490$ $\begin{array}{llll}0.00136 & 0.01128 & 0.07232\end{array}$ $\begin{array}{llll}0.00130 & 0.01081 & 0.06982\end{array}$ $\begin{array}{llll}0.00124 & 0.01036 & 0.06741\end{array}$ $\begin{array}{lll}0.00118 & 0.00993 & 0.06507\end{array}$

$\begin{array}{llll}0.00112 & 0.00951 & 0.06281\end{array}$ $\begin{array}{llll}0.00107 & 0.00912 & 0.06062\end{array}$ $\begin{array}{llll}0.00102 & 0.00874 & 0.05851\end{array}$ $\begin{array}{lll}0.00097 & 0.00837 & 0.05646\end{array}$ $\begin{array}{llll}0.00092 & 0.00802 & 0.05448\end{array}$
0.038380 .191690 .61845 0.037010 .186150 .60666 0.035690 .180760 .59502 0.034420 .175520 .58355 0.033190 .170430 .57223

$0.032010 .16548 \quad 0.56108$ $\begin{array}{llll}0.03087 & 0.16067 & 0.55007\end{array}$ $0.029770 .15599 \quad 0.53923$ 0.028710 .151450 .52855 0.027690 .147030 .51802

$0.02670 \quad 0.142740 .50765$ $0.02575 \quad 0.138570 .49743$ 0.024840 .134520 .48737 $0.02396 \quad 0.13058 \quad 0.47747$ $0.02310 \quad 0.126750 .46772$

$0.022280 .12304 \quad 0.45812$ 0.021490 .119420 .44868 0.020730 .115910 .43938 $0.019990 .11250 \quad 0.43024$ 0.019280 .109190 .42125

$0.01860 \quad 0.10597 \quad 0.41241$ $0.01794 \quad 0.102840 .40371$ $0.01730 \quad 0.099810 .39517$ 0.016690 .096860 .38676 $0.01610 \quad 0.09399 \quad 0.37850$

$\begin{array}{llll}0.01552 & 0.09121 & 0.37039\end{array}$ $\begin{array}{llll}0.01497 & 0.08850 & 0.36241\end{array}$ $\begin{array}{llll}0.01444 & 0.08588 & 0.35458\end{array}$ $\begin{array}{llll}0.01393 & 0.08332 & 0.34689\end{array}$ $\begin{array}{llll}0.01344 & 0.08085 & 0.33933\end{array}$

$\begin{array}{llll}0.01296 & 0.07844 & 0.33191\end{array}$ $\begin{array}{llll}0.01250 & 0.07611 & 0.32462\end{array}$ $\begin{array}{llll}0.01206 & 0.07384 & 0.31747\end{array}$ $\begin{array}{llll}0.01163 & 0.07163 & 0.31045\end{array}$ 0.011220 .069490 .30356

$0.010820 .06742 \quad 0.29679$ $0.010440 .06540 \quad 0.29016$ 0.010070 .063440 .28365 $\begin{array}{llll}0.00971 & 0.06154 & 0.27726\end{array}$ $\begin{array}{lll}0.00937 & 0.05969 & 0.27100\end{array}$

$\begin{array}{llll}0.00904 & 0.05790 & 0.26486\end{array}$ $\begin{array}{lllll}0.00872 & 0.05616 & 0.25883\end{array}$ $\begin{array}{llll}0.00841 & 0.05447 & 0.25293\end{array}$ $\begin{array}{llll}0.00811 & 0.05283 & 0.24714\end{array}$ $\begin{array}{llll}0.00782 & 0.05124 & 0.24147\end{array}$

$\begin{array}{llll}0.00755 & 0.04970 & 0.23590\end{array}$ $\begin{array}{llll}0.00728 & 0.04820 & 0.23045\end{array}$ $\begin{array}{llll}0.00702 & 0.04674 & 0.22511\end{array}$ $\begin{array}{llll}0.00677 & 0.04533 & 0.21988\end{array}$ $\begin{array}{llll}0.00653 & 0.04396 & 0.21475\end{array}$
1100

1110

1120

1130

1140

1150

1160

1170

1180

1190

1200

1210

1220

1230

1240

1250

1260

1270

1280

1290

1300

1310

1320

1330

1340

1350

1360

1370

1380

1390

1400

1410

1420

1430

1440

1450

1460

1470

1480

1490

1500

1510

1520

1530

1540

1550

1560

1570

1580

1590 
Table II Harmonic Oscillator Contributions to the Thermodynamic

Functions (in units of calories, moles, and ${ }^{\circ} \mathrm{K}$ )

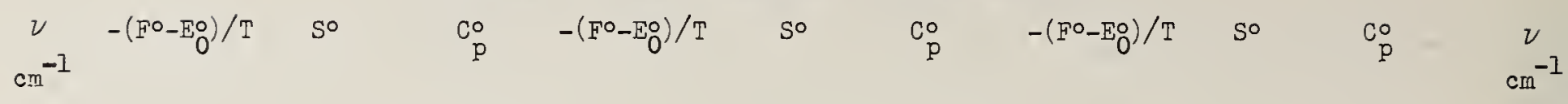

$T=273.15$

1600

1610

1620

1630

1640

$\begin{array}{lllll}1650 & 0.00033 & 0.00324 & 0.02523\end{array}$

$\begin{array}{llll}1660 & 0.00032 & 0.00309 & 0.02423\end{array}$

$\begin{array}{llll}1670 & 0.00030 & 0.00294 & 0.02326\end{array}$

$\begin{array}{lllll}1680 & 0.00029 & 0.00281 & 0.02233\end{array}$

$\begin{array}{lllll}1690 & 0.00027 & 0.00268 & 0.02144\end{array}$

$\begin{array}{lllll}1700 & 0.00026 & 0.00255 & 0.02058\end{array}$

$\begin{array}{llll}1710 & 0.00024 & 0.00244 & 0.01976\end{array}$

$\begin{array}{llll}1720 & 0.00023 & 0.00232 & 0.01896\end{array}$

$\begin{array}{llll}1730 & 0.00022 & 0.00222 & 0.01820\end{array}$

$\begin{array}{lllll}1740 & 0.00021 & 0.00211 & 0.01746\end{array}$

$\begin{array}{lllll}1750 & 0.00020 & 0.00202 & 0.01676\end{array}$

$\begin{array}{lllll}1760 & 0.00019 & 0.00192 & 0.01608\end{array}$

$\begin{array}{lllll}1770 & 0.00018 & 0.00193 & 0.01543\end{array}$

$\begin{array}{lllll}1780 & 0.00017 & 0.00175 & 0.01480\end{array}$

$\begin{array}{llllll}1790 & 0.00016 & 0.00167 & 0.01420\end{array}$

$\begin{array}{lllll}1800 & 0.00015 & 0.00159 & 0.01362\end{array}$

$\begin{array}{llllll}1810 & 0.00014 & 0.00151 & 0.01307\end{array}$

$\begin{array}{llllll}1820 & 0.00014 & 0.00144 & 0.01254\end{array}$

$\begin{array}{lllll}1830 & 0.00013 & 0.00138 & 0.01202\end{array}$

$\begin{array}{lllll}1840 & 0.00012 & 0.00131 & 0.01153\end{array}$

$\begin{array}{llll}1850 & 0.00012 & 0.00125 & 0.01106\end{array}$

$\begin{array}{llllll}1860 & 0.00011 & 0.00119 & 0.01061\end{array}$

$\begin{array}{lllll}1870 & 0.00010 & 0.00114 & 0.01017\end{array}$

$\begin{array}{lllll}1880 & 0.00010 & 0.00108 & 0.00975\end{array}$

$\begin{array}{lllll}1890 & 0.00009 & 0.00103 & 0.00935\end{array}$

$\begin{array}{lllll}1900 & 0.00009 & 0.00099 & 0.00896\end{array}$

$\begin{array}{lllll}1910 & 0.00008 & 0.00094 & 0.00859\end{array}$

$\begin{array}{lllll}1920 & 0.00008 & 0.00090 & 0.00824\end{array}$

$\begin{array}{lllll}1930 & 0.00008 & 0.00085 & 0.00790\end{array}$

$\begin{array}{llllll}1940 & 0.00007 & 0.00081 & 0.00757\end{array}$

$1950 \quad 0.00007 \quad 0.00078 \quad 0.00726$

$\begin{array}{llllll}1960 & 0.00007 & 0.00074 & 0.00695\end{array}$

$\begin{array}{llllll}1970 & 0.00006 & 0.00070 & 0.00666\end{array}$

$\begin{array}{lllll}1980 & 0.00006 & 0.00067 & 0.00639\end{array}$

$\begin{array}{lllll}1990 & 0.00006 & 0.00064 & 0.00612\end{array}$

$\begin{array}{lllll}2000 & 0.00005 & 0.00061 & 0.00587\end{array}$

$\begin{array}{lllll}2010 & 0.00005 & 0.00058 & 0.00562\end{array}$

$\begin{array}{lllll}2020 & 0.00005 & 0.00055 & 0.00538\end{array}$

$2030 \quad 0.000050 .000530 .00516$

$\begin{array}{lllll}2040 & 0.00004 & 0.00050 & 0.00494\end{array}$

$\begin{array}{lllll}2050 & 0.00004 & 0.00048 & 0.00474\end{array}$

2060

2070

2080

2090
$T=298.15$

$\begin{array}{llll}0.00088 & 0.00769 & 0.05257\end{array}$

$\begin{array}{llll}0.00084 & 0.00736 & 0.05072\end{array}$

$\begin{array}{llll}0.00080 & 0.00706 & 0.04893\end{array}$

$\begin{array}{llll}0.00076 & 0.00676 & 0.04720\end{array}$

$\begin{array}{llll}0.00073 & 0.00648 & 0.04553\end{array}$

$0.000690 .00620 \quad 0.04391$

$\begin{array}{lllll}0.00066 & 0.00594 & 0.04235\end{array}$

$\begin{array}{llll}0.00063 & 0.00569 & 0.04084\end{array}$

$\begin{array}{llll}0.00060 & 0.00546 & 0.03938\end{array}$

$\begin{array}{llll}0.00057 & 0.00523 & 0.03797\end{array}$

$\begin{array}{llll}0.00054 & 0.00501 & 0.03661\end{array}$

$0.00052 \quad 0.004790 .03530$

$\begin{array}{llll}0.00049 & 0.00459 & 0.03403\end{array}$

$0.000470 .00440 \quad 0.03280$

$\begin{array}{llll}0.00045 & 0.00421 & 0.03162\end{array}$

$\begin{array}{llll}0.00043 & 0.00404 & 0.03048\end{array}$

$\begin{array}{lllll}0.00041 & 0.00386 & 0.02937\end{array}$

$\begin{array}{llll}0.00039 & 0.00370 & 0.02831\end{array}$

$\begin{array}{lllll}0.00037 & 0.00354 & 0.02728\end{array}$

$\begin{array}{llll}0.00035 & 0.00339 & 0.02629\end{array}$

$\begin{array}{lll}0.00034 & 0.00325 & 0.02533\end{array}$

0.000320 .003110 .02440

$\begin{array}{lllll}0.00030 & 0.00298 & 0.02351\end{array}$

0.000290 .002850 .02265

$\begin{array}{llll}0.00028 & 0.00273 & 0.02182\end{array}$

$\begin{array}{llll}0.00026 & 0.00262 & 0.02102\end{array}$

$\begin{array}{lllll}0.00025 & 0.00251 & 0.02024\end{array}$

$\begin{array}{lll}0.00024 & 0.00240 & 0.01950\end{array}$

$\begin{array}{llll}0.00023 & 0.00230 & 0.01878\end{array}$

$\begin{array}{lll}0.00022 & 0.00220 & 0.01809\end{array}$

$\begin{array}{llll}0.00021 & 0.00211 & 0.01742\end{array}$

$\begin{array}{llll}0.00020 & 0.00202 & 0.01677\end{array}$

$\begin{array}{llll}0.00019 & 0.00193 & 0.01615\end{array}$

$0.00018 \quad 0.001850 .01555$

$\begin{array}{llll}0.00017 & 0.00177 & 0.01497\end{array}$

$0.00016 \quad 0.00169 \quad 0.01441$

$\begin{array}{llll}0.00016 & 0.00162 & 0.01387\end{array}$

0.000150 .001550 .01335

0.000140 .001490 .01286

$\begin{array}{lll}0.00013 & 0.00142 & 0.01237\end{array}$

0.000130 .001360 .01191

0.000120 .001300 .01146

0.000120 .001250 .01103

0.000110 .001190 .01062

0.000110 .001140 .01022

$0.00010 \quad 0.001090 .00983$

$0.00010 \quad 0.00105 \quad 0.00946$

$0.000090 .00100 \quad 0.00910$

$\begin{array}{llll}0.00009 & 0.00096 & 0.00876\end{array}$

$0.00008 \quad 0.00092 \quad 0.00842$
$0.00630 \quad 0.04263 \quad 0.20973$

$\begin{array}{llll}0.00608 & 0.04134 & 0.20481\end{array}$

$\begin{array}{llll}0.00586 & 0.04008 & 0.19999\end{array}$

$\begin{array}{llll}0.00566 & 0.03887 & 0.19528\end{array}$

$0.00546 \quad 0.03769 \quad 0.19066$

0.005260 .036540 .18613

$0.00508 \quad 0.03543 \quad 0.18171$

$0.00490 \quad 0.034350 .17737$

0.004720 .033310 .17313

0.004560 .032290 .16898

$0.00440 \quad 0.031310 .16492$

0.004240 .030350 .16094

0.004090 .029420 .15705

$\begin{array}{llll}0.00395 & 0.02852 & 0.15325\end{array}$

0.003810 .027650 .14953

0.003670 .026810 .14589

$0.003540 .02598 \quad 0.14233$

0.003420 .025190 .13885

$0.00330 \quad 0.024410 .13544$

0.003180 .023670 .13211

0.003070 .022940 .12886

$0.00296 \quad 0.022230 .12568$

0.002850 .021550 .12257

0.002750 .020890 .11953

$0.00266 \quad 0.020240 .11656$

0.002560 .019620 .11365

0.002470 .019010 .11082

$0.00238 \quad 0.018430 .10805$

$0.00230 \quad 0.01786 \quad 0.10534$

0.002220 .017310 .10269

0.002140 .016770 .10011

$0.00206 \quad 0.016250 .09758$

0.001990 .015750 .09511

$0.001920 .01526 \quad 0.09271$

0.001850 .014790 .09035

0.001790 .014330 .08806

0.001720 .013890 .08581

$0.00166 \quad 0.013450 .08362$

0.001600 .013040 .08148

0.001550 .012630 .07940

0.001490 .012240 .07736

$0.001440 .01186 \quad 0.07537$

0.001390 .011490 .07343

0.001340 .011130 .07153

$0.001290 .01078 \quad 0.06969$

$0.00125 \quad 0.01045 \quad 0.06788$

0.001200 .010120 .06612

0.001160 .009810 .06440

$0.001120 .00950 \quad 0.06273$

0.001080 .009200 .06109
1600

1610

1620

1630

1640

1650

1660

1670

1680

1690

1700

1710

1720

1730

1740

1750

1760

1770

1780

1790

1800

1810

1820

1830

1840

1850

1860

1870

1880

1890

1900

1910

1920

1930

1940

1950

1960

1970

1980

1990

2000

2010

2020

2030

2040

2050

2060

2070

2080

2090 
Table II Harmonic Oscillator Contributions to the Thermodynamic Functions (in units of calories, moles, and ${ }^{\circ} \mathrm{K}$ )
$\mathrm{cm}^{-1}$
$-\left(F^{\circ}-E O\right) / T \quad S^{\circ}$
$\mathrm{C}_{\mathrm{p}}^{\circ} \quad-\left(\mathrm{F}^{\circ}-\mathrm{E}_{\mathrm{O}}^{\circ}\right) / \mathrm{T}$
so
$\mathrm{co}_{\mathrm{p}}$
$-\left(F^{\circ}-E_{0}^{\circ}\right) / T$
so
$\mathrm{co}$
$\mathrm{cm}^{-1}$

$T=273.15$

2100

2110

2120

2130

2140

2150

2160

2170

2180

2190

2200

2210

2220

2230

2240

2250

2260

2270

2280

2290

2300

2310

2320

2330

2340

2350

2360

2370

2380

2390

2400

2410

2420

2430

2440

2450

2460

2470

2480

2490

2500

2510

2520

2530

2540

2550

2560

2570

2580

2590
0.000030 .000380 .00382

0.000030 .000360 .00366

0.000030 .000340 .00350

0.000030 .000330 .00335

0.000030 .000310 .00321

$0.00002 \quad 0.00030 \quad 0.00308$

$\begin{array}{llll}0.00002 & 0.00028 & 0.00295\end{array}$

0.000020 .000270 .00282

$0.000020 .00026 \quad 0.00270$

0.000020 .000240 .00258

$0.00002 \quad 0.00023 \quad 0.00247$

0.000020 .000220 .00237

$0.00002 \quad 0.000210 .00227$

$0.00002 \quad 0.00020 \quad 0.00217$

0.000010 .000190 .00208

$\begin{array}{llll}0.00001 & 0.00018 & 0.00199\end{array}$

$0.000010 .00017 \quad 0.00190$

0.000010 .000170 .00182

$0.00001 \quad 0.00016 \quad 0.00174$

0.000010 .000150 .00167

0.000010 .000140 .00160

$0.00001 \quad 0.00014 \quad 0.00153$

$0.000010 .00013 \quad 0.00146$

0.000010 .000120 .00140

0.000010 .000120 .00134

$0.00001 \quad 0.000110 .00128$

0.000010 .000110 .00123

$0.00001 \quad 0.00010 \quad 0.00117$

0.000010 .000100 .00112

$\begin{array}{lll}0.00001 & 0.00009 & 0.00107\end{array}$

$0.00001 \quad 0.00009 \quad 0.00103$

$\begin{array}{llll}0.00001 & 0.00008 & 0.00098\end{array}$

$\begin{array}{llll}0.00001 & 0.00008 & 0.00094\end{array}$

$0.000010 .00008 \quad 0.00090$

0.000010 .000070 .00086

$\begin{array}{llll}0.00000 & 0.00007 & 0.00082\end{array}$ $\begin{array}{llll}0.00000 & 0.00007 & 0.00079\end{array}$

$\begin{array}{llll}0.00000 & 0.00006 & 0.00075\end{array}$

$\begin{array}{llll}0.00000 & 0.00006 & 0.00072\end{array}$

$0.00000 \quad 0.00006 \quad 0.00069$

$0.00000 \quad 0.00005 \quad 0.00066$

$\begin{array}{llll}0.00000 & 0.00005 & 0.00063\end{array}$

$\begin{array}{llll}0.00000 & 0.00005 & 0.00060\end{array}$

$\begin{array}{llll}0.00000 & 0.00005 & 0.00058\end{array}$

$0.00000 \quad 0.00004 \quad 0.00055$

$0.00000 \quad 0.00004 \quad 0.00053$

$\begin{array}{llll}0.00000 & 0.00004 & 0.00050\end{array}$

$\begin{array}{lllll}0.00000 & 0.00004 & 0.00048\end{array}$

$\begin{array}{lllll}0.00000 & 0.00004 & 0.00046\end{array}$

$\begin{array}{llll}0.00000 & 0.00003 & 0.00044\end{array}$
$\mathrm{I}=298.15$

$T=400$.

$\begin{array}{llll}0.00008 & 0.00088 & 0.00810\end{array}$

$\begin{array}{lllll}0.00008 & 0.00084 & 0.00780\end{array}$

$\begin{array}{llll}0.00007 & 0.00080 & 0.00750\end{array}$

$\begin{array}{llllll}0.00007 & 0.00077 & 0.00721\end{array}$

$\begin{array}{lllll}0.00007 & 0.00074 & 0.00694\end{array}$

$\begin{array}{llll}0.00006 & 0.00071 & 0.00667\end{array}$

$0.00006 \quad 0.00067 \quad 0.00642$

$\begin{array}{llll}0.00006 & 0.00065 & 0.00617\end{array}$

$\begin{array}{llll}0.00005 & 0.00062 & 0.00594\end{array}$

0.000050 .000590 .00571

$\begin{array}{llll}0.00005 & 0.00057 & 0.00549\end{array}$

$\begin{array}{lllll}0.00005 & 0.00054 & 0.00528\end{array}$

$\begin{array}{llll}0.00004 & 0.00052 & 0.00507\end{array}$

$\begin{array}{llll}0.00004 & 0.00050 & 0.00488\end{array}$

$\begin{array}{lllll}0.00004 & 0.00047 & 0.00469\end{array}$

$0.00004 \quad 0.00045 \quad 0.00451$

$0.00004 \quad 0.00043 \quad 0.00434$

$\begin{array}{llll}0.00003 & 0.00042 \quad 0.00417\end{array}$

$0.00003 \quad 0.00040 \quad 0.00401$

$0.00003 \quad 0.00038 \quad 0.00385$

$\begin{array}{llll}0.00003 & 0.00036 & 0.00370\end{array}$

$0.00003 \quad 0.00035 \quad 0.00356$

$\begin{array}{llll}0.00003 & 0.00033 & 0.00342\end{array}$

$\begin{array}{llll}0.00003 & 0.00032 & 0.00329\end{array}$

$0.000020 .00030 \quad 0.00316$

$\begin{array}{llll}0.00002 & 0.00029 & 0.00304\end{array}$

$\begin{array}{llll}0.00002 & 0.00028 & 0.00292\end{array}$

$\begin{array}{llll}0.00002 & 0.00027 & 0.00280\end{array}$

$\begin{array}{llll}0.00002 & 0.00026 & 0.00269\end{array}$

$\begin{array}{lll}0.00002 & 0.00024 & 0.00259\end{array}$

$\begin{array}{llll}0.00002 & 0.00023 & 0.00249\end{array}$

$0.00002 \quad 0.00022 \quad 0.00239$

$\begin{array}{llll}0.00002 & 0.00021 & 0.00230\end{array}$

$\begin{array}{lll}0.00002 & 0.00020 & 0.00221\end{array}$

$0.000020 .00020 \quad 0.00212$

$0.00001 \quad 0.00019 \quad 0.00204$

$0.00001 \quad 0.00018 \quad 0.00196$

$\begin{array}{lllll}0.00001 & 0.00017 & 0.00188\end{array}$

$0.00001 \quad 0.00016 \quad 0.00181$

0.000010 .000160 .00173

$0.000010 .00015 \quad 0.00167$

$0.00001 \quad 0.00014 \quad 0.00160$

$\begin{array}{llll}0.00001 & 0.00014 & 0.00154\end{array}$

$0.00001 \quad 0.00013 \quad 0.00148$

0.000010 .000130 .00142

0.000010 .000120 .00136 $\begin{array}{lllll}0.00001 & 0.00011 & 0.00131\end{array}$ 0.000010 .000110 .00126 0.000010 .000100 .00121 0.000010 .000100 .00116
$0.00104 \quad 0.00891 \quad 0.05949$

$\begin{array}{llll}0.00101 & 0.00864 & 0.05794\end{array}$

$\begin{array}{lllll}0.00097 & 0.00836 & 0.05642\end{array}$

$\begin{array}{llll}0.00094 & 0.00810 & 0.05494\end{array}$

$0.00090 \quad 0.007850 .05350$

$\begin{array}{llll}0.00087 & 0.00760 & 0.05209\end{array}$

$\begin{array}{llll}0.00084 & 0.00736 & 0.05071\end{array}$

$\begin{array}{llll}0.00081 & 0.00713 & 0.04938\end{array}$

$\begin{array}{lllll}0.00078 & 0.00691 & 0.04807\end{array}$

$\begin{array}{llll}0.00075 & 0.00669 & 0.04680\end{array}$

$\begin{array}{llll}0.00073 & 0.00648 & 0.04555\end{array}$

$\begin{array}{lllll}0.00070 & 0.00628 & 0.04434\end{array}$

$\begin{array}{lllll}0.00068 & 0.00608 & 0.04316\end{array}$

$\begin{array}{llll}0.00065 & 0.00589 & 0.04201\end{array}$

$0.00063 \quad 0.00570 \quad 0.04089$

$\begin{array}{lll}0.00061 & 0.00552 & 0.03980\end{array}$

$\begin{array}{llll}0.00059 & 0.00535 & 0.03874\end{array}$

$\begin{array}{llll}0.00057 & 0.00518 & 0.03770\end{array}$

$\begin{array}{llll}0.00055 & 0.00502 & 0.03669\end{array}$

$\begin{array}{llll}0.00053 & 0.00486 & 0.03570\end{array}$

$\begin{array}{llll}0.00051 & 0.00471 & 0.03474\end{array}$ $\begin{array}{llll}0.00049 & 0.00456 & 0.03380\end{array}$ $\begin{array}{lllll}0.00047 & 0.00441 & 0.03289\end{array}$ $0.00046 \quad 0.00427 \quad 0.03200$ $0.000440 .00414 \quad 0.03114$

$\begin{array}{llll}0.00042 & 0.00401 & 0.03029\end{array}$

$\begin{array}{lllll}0.00041 & 0.00388 & 0.02947\end{array}$

$\begin{array}{lllll}0.00039 & 0.00376 & 0.02867\end{array}$

$\begin{array}{lllll}0.00038 & 0.00364 & 0.02789\end{array}$

$0.00037 \quad 0.00352 \quad 0.02713$

$\begin{array}{llll}0.00035 & 0.00341 & 0.02639\end{array}$

$\begin{array}{lllll}0.00034 & 0.00330 & 0.02567\end{array}$

$\begin{array}{llll}0.00033 & 0.00320 & 0.02497\end{array}$

$0.000320 .00310 \quad 0.02429$

$\begin{array}{lll}0.00031 & 0.00300 & 0.02362\end{array}$

$\begin{array}{llll}0.00030 & 0.00290 & 0.02298\end{array}$ $\begin{array}{lllll}0.00029 & 0.00281 & 0.02235\end{array}$

$\begin{array}{llll}0.00028 & 0.00272 & 0.02173\end{array}$

$\begin{array}{llll}0.00027 & 0.00263 & 0.02113\end{array}$

$\begin{array}{lll}0.00026 & 0.00255 & 0.02055\end{array}$

$0.00025 \quad 0.00247 \quad 0.01999$

$\begin{array}{llll}0.00024 & 0.00239 & 0.01943\end{array}$

$\begin{array}{lllll}0.00023 & 0.00231 & 0.01890\end{array}$

$\begin{array}{lllll}0.00022 & 0.00224 & 0.01837\end{array}$

$\begin{array}{llllll}0.00021 & 0.00217 & 0.01786\end{array}$

$\begin{array}{llll}0.00021 & 0.00210 & 0.01737\end{array}$

$\begin{array}{llll}0.00020 & 0.00203 & 0.01689\end{array}$

$\begin{array}{llll}0.00019 & 0.00197 & 0.01642\end{array}$

$\begin{array}{llll}0.00019 & 0.00191 & 0.01596\end{array}$

$0.00018 \quad 0.001840 .01552$
2100

2110

2120

2130

2140

2150

2160

2170

2180

2190

2200

2210

2220

7230

2240

2250

2260

2270

2280

2290

2300

2310

2320

2330

2340

2350

2360

2370

2380

2390

2400

2410

2420

2430

2440

2450

2460

2470

2480

2490

2500

2510

2520

2530

2540

2550

2560

2570

2580

2590 
Table II Harmonic Oscillator Contributions to the Thermodynamic

Functions (in units of calories, moles, and ${ }^{\circ} \mathrm{K}$ )

\begin{tabular}{|c|c|c|c|c|c|c|c|c|c|}
\hline $\begin{array}{c}\nu \\
\mathrm{cm}^{-1}\end{array}$ & $-\left(F^{\circ}-E_{O}^{0}\right) / T$ & $\mathrm{~S}^{\circ}$ & $\mathrm{C}_{\mathrm{p}}^{\circ}$ & $-\left(F^{\circ}-E_{O}^{\circ}\right) / T$ & $\mathrm{~S}^{\circ}$ & $\mathrm{C}_{\mathrm{p}}^{0}$ & $-\left(F^{O}-E_{0} O\right) / T$ & $\mathrm{~S}^{\circ}$ & $\mathrm{cm}^{-1}$ \\
\hline
\end{tabular}

$T=273.15$

2600

2610

2620

2630

2640

2650

2660

2670

2680

2690

2700

2710

2720

2730

2740

2750

2760

2770

2780

2790

2800

2810

2820

2830

2840

2850

2860

2870

2880

2890

2900

2910

2920

2930

2940

2950

2960

2970

2980

2990

3000

3010

3020

3030

3040

3050

3060

3070

3080

3090
$\Gamma=298.15$

$0.000010 .00010 \quad 0.00111$

0.000010 .000090 .00107

$0.00001 \quad 0.00009 \quad 0.00103$

$\begin{array}{llll}0.00001 & 0.00008 & 0.00098\end{array}$

$0.000010 .00008 \quad 0.00095$

$0.00001 \quad 0.00008 \quad 0.00091$

$0.000010 .00007 \quad 0.00087$

$0.000010 .00007 \quad 0.00084$

$0.00000 \quad 0.00007 \quad 0.00080$

$\begin{array}{llll}0.00000 & 0.00006 & 0.00077\end{array}$

0.000000 .000020 .00029

$0.00000 \quad 0.000020 .00027$

$0.00000 \quad 0.000020 .00026$

$0.00000 \quad 0.000020 .00024$

$0.00000 \quad 0.00002 \quad 0.00023$

$0.00000 \quad 0.00002 \quad 0.00022$

$0.00000 \quad 0.00002 \quad 0.00021$

$0.00000 \quad 0.000020 .00020$

$0.00000 \quad 0.000010 .00019$

$0.00000 \quad 0.000010 .00019$

$0.00000 \quad 0.000010 .00018$

$\begin{array}{llll}0.00000 & 0.00001 & 0.00017\end{array}$ $0.00000 \quad 0.000010 .00016$ $0.00000 \quad 0.00001 \quad 0.00016$ $0.00000 \quad 0.00001 \quad 0.00015$ $0.00000 \quad 0.000010 .00014$

$0.00000 \quad 0.00001 \quad 0.00014$ $\begin{array}{llll}0.00000 & 0.00001 & 0.00013\end{array}$ $0.00000 \quad 0.00001 \quad 0.00012$ $0.00000 \quad 0.00001 \quad 0.00012$ $0.00000 \quad 0.000010 .00011$

$0.00000 \quad 0.00001 \quad 0.00011$ $0.00000 \quad 0.00001 \quad 0.00010$ $\begin{array}{llll}0.00000 & 0.00001 & 0.00010\end{array}$ $\begin{array}{llll}0.00000 & 0.00001 & 0.00009\end{array}$ $0.00000 \quad 0.00001 \quad 0.00009$

$0.00000 \quad 0.00001 \quad 0.00009$ $\begin{array}{lllll}0.00000 & 0.00001 & 0.00008\end{array}$ $\begin{array}{lllll}0.00000 & 0.00001 & 0.00008\end{array}$ $\begin{array}{llll}0.00000 & 0.00001 & 0.00007\end{array}$ $\begin{array}{lll}0.00000 & 0.00000 & 0.00007\end{array}$

$\begin{array}{llll}0.00000 & 0.00000 & 0.00007\end{array}$ $0.00000 \quad 0.00000 \quad 0.00006$ $0.00000 \quad 0.00000 \quad 0.00006$ $0.00000 \quad 0.00000 \quad 0.00006$ $0.00000 \quad 0.00000 \quad 0.00006$

$0.00000 \quad 0.00000 \quad 0.00005$ $0.00000 \quad 0.00000 \quad 0.00005$ $\begin{array}{llll}0.00000 & 0.00000 & 0.00005\end{array}$ $0.00000 \quad 0.00000 \quad 0.00005$ $0.00000 \quad 0.00000 \quad 0.00004$
$0.00000 \quad 0.00006 \quad 0.00074$ $\begin{array}{lllll}0.00000 & 0.00006 & 0.00071\end{array}$ $\begin{array}{llll}0.00000 & 0.00006 & 0.00068\end{array}$ $0.00000 \quad 0.00005 \quad 0.00065$ $0.00000 \quad 0.000050 .00063$

$0.00000 \quad 0.00005 \quad 0.00060$ $0.00000 \quad 0.00005 \quad 0.00058$ $0.00000 \quad 0.00004 \quad 0.00056$ $\begin{array}{llll}0.00000 & 0.00004 & 0.00053\end{array}$ $0.00000 \quad 0.00004 \quad 0.00051$

$0.00000 \quad 0.00004 \quad 0.00049$ $\begin{array}{llll}0.00000 & 0.00004 & 0.00047\end{array}$ $\begin{array}{llll}0.00000 & 0.00004 & 0.00045\end{array}$ $0.00000 \quad 0.00003 \quad 0.00043$ $0.00000 \quad 0.00003 \quad 0.00042$

$0.00000 \quad 0.00003 \quad 0.00040$ $\begin{array}{llll}0.00000 & 0.00003 & 0.00038\end{array}$ $\begin{array}{llll}0.00000 & 0.00003 & 0.00037\end{array}$ $0.00000 \quad 0.00003 \quad 0.00035$ $0.00000 \quad 0.00003 \quad 0.00034$

$\begin{array}{llll}0.00000 & 0.00002 & 0.00033\end{array}$ $\begin{array}{llll}0.00000 & 0.00002 & 0.00031\end{array}$ $0.00000 \quad 0.000020 .00030$ $\begin{array}{lll}0.00000 & 0.00002 & 0.00029\end{array}$ $0.00000 \quad 0.00002 \quad 0.00028$

$0.00000 \quad 0.00002 \quad 0.00026$ $0.00000 \quad 0.00002 \quad 0.00025$ $\begin{array}{llll}0.00000 & 0.00002 & 0.00024\end{array}$ $0.00000 \quad 0.00002 \quad 0.00023$ $0.00000 \quad 0.00002 \quad 0.00022$

$0.00000 \quad 0.00002 \quad 0.00021$ $\begin{array}{llll}0.00000 & 0.00002 & 0.00021\end{array}$ $\begin{array}{lllll}0.00000 & 0.00001 & 0.00020\end{array}$ $0.00000 \quad 0.00001 \quad 0.00019$ $0.00000 \quad 0.000010 .00018$

$\begin{array}{llll}0.00000 & 0.00001 & 0.00017\end{array}$ $\begin{array}{llll}0.00000 & 0.00001 & 0.00017\end{array}$ $0.00000 \quad 0.000010 .00016$ $0.00000 \quad 0.000010 .00015$ $0.00000 \quad 0.000010 .00015$
$T=400$.

$\begin{array}{llll}0.00017 & 0.00179 & 0.01508\end{array}$ $\begin{array}{llll}0.00017 & 0.00173 & 0.01466\end{array}$ $0.00016 \quad 0.00167 \quad 0.01425$ 0.000150 .001620 .01386 $0.000150 .00157 \quad 0.01347$

0.000140 .001520 .01309 0.000140 .001470 .01272 $\begin{array}{llll}0.00013 & 0.00142 & 0.01237\end{array}$ 0.000130 .001380 .012 .02 0.000120 .001330 .01168

0.000120 .001290 .01135 0.000120 .001250 .01103 0.000110 .001210 .01072 0.000110 .001170 .01042 $0.00010 \quad 0.001130 .01012$

$0.00010 \quad 0.00110 \quad 0.00984$ $0.00010 \quad 0.00106 \quad 0.00956$ 0.000090 .001030 .00929 0.000090 .000990 .00903 $0.000090 .00096 \quad 0.00877$

2600

2610

2620

2630

2640

2650

2660

2670

2680

2690

2700

2710

2720

2730

2740

2750

2760

2770

2780

2790

$\begin{array}{llll}0.00008 & 0.00093 & 0.00852\end{array}$

$\begin{array}{llll}0.00008 & 0.00090 & 0.00828\end{array}$

$\begin{array}{llll}0.00008 & 0.00087 & 0.00804\end{array}$

$\begin{array}{lllll}0.00008 & 0.00084 & 0.00781\end{array}$

$\begin{array}{llll}0.00007 & 0.00082 & 0.00759\end{array}$

$\begin{array}{llll}0.00007 & 0.00079 & 0.00737\end{array}$

$\begin{array}{llll}0.00007 & 0.00076 & 0.00716\end{array}$

$\begin{array}{llll}0.00007 & 0.00074 & 0.00696\end{array}$

$\begin{array}{llll}0.00006 & 0.00072 & 0.00676\end{array}$

$\begin{array}{llll}0.00006 & 0.00069 & 0.00657\end{array}$

$0.00006 \quad 0.000670 .00638$

$\begin{array}{lllll}0.00006 & 0.00065 & 0.00620\end{array}$

$\begin{array}{llll}0.00005 & 0.00063 & 0.00602\end{array}$

$\begin{array}{lllll}0.00005 & 0.00061 & 0.00584\end{array}$

$\begin{array}{llll}0.00005 & 0.00059 & 0.00568\end{array}$

$\begin{array}{llll}0.00005 & 0.00057 & 0.00551\end{array}$

$\begin{array}{llll}0.00005 & 0.00055 & 0.00535\end{array}$

$\begin{array}{llll}0.00005 & 0.00053 & 0.00520\end{array}$

0.000040 .000520 .00505

$0.000040 .00050 \quad 0.00490$

2800

2810

2820

2830

2840

2850

2860

2870

2880

2890

2900

2910

2920

2930

2940

2950

2960

2970

2980

2990

$0.000040 .00048 \quad 0.00476$ 0.000040 .000470 .00463 0.000040 .000450 .00449 0.000040 .000440 .00436 0.000040 .000420 .0042 .4

3000

3010

3020

3030

3040

$0.00003 \quad 0.000410 .00411$

3050

$0.00003 \quad 0.00040 \quad 0.00399$

$0.00003 \quad 0.00038 \quad 0.00388$

$0.00003 \quad 0.000370 .00377$

0.000030 .000360 .00366
3060

3070

3080

3090 
Table II Harmonic Oscillator Contributions to the Thermodynamic

Functions (in units of calories, moles, and ${ }^{\circ} \mathrm{K}$ )

\begin{tabular}{|c|c|c|c|c|c|c|c|c|c|}
\hline $\begin{array}{c}\nu \\
\mathrm{cm}^{-1}\end{array}$ & $-\left(F^{\circ}-E_{0}^{\circ}\right) / T$ & $S^{\circ}$ & $\begin{array}{c}\mathrm{C}_{\mathrm{p}}^{\circ} \\
\mathrm{p}\end{array}$ & $-\left(F^{\circ} \circ-E_{0}^{\circ}\right) / T$ & $\mathrm{~S}^{\circ}$ & $\mathrm{C}_{\mathrm{p}}$ & $-\left(F^{\circ}-E_{0}^{O}\right) / T$ & So & $\mathrm{C}_{\mathrm{p}}^{\circ}$ \\
\hline
\end{tabular}

$T=273.15$

3100

3110

3120

3130

3140

3150

3160

3170

3180

3190

3200

3210

3220

3230

3240

3250

3260

3270

3280

3290

3300

3310

3320

3330

3340

3350

3360

3370

3380

3390

3400

3410

3420

3430

3440

3450

3460

3470

3480

3490

3500

3510

3520

3530

3540

3550

3560

3570

3580

3590
$0.00000 \quad 0.00000 \quad 0.00004$

$0.00000 \quad 0.00000 \quad 0.00004$

$0.00000 \quad 0.00000 \quad 0.00004$

$0.00000 \quad 0.00000 \quad 0.00004$

$0.00000 \quad 0.00000 \quad 0.00004$

$0.00000 \quad 0.00000 \quad 0.00003$

$0.00000 \quad 0.00000 \quad 0.00003$

$0.00000 \quad 0.00000 \quad 0.00003$

$0.00000 \quad 0.00000 \quad 0.00003$

$0.00000 \quad 0.00000 \quad 0.00003$

$0.00000 \quad 0.00000 \quad 0.00003$

$0.00000 \quad 0.00000 \quad 0.00003$

$0.00000 \quad 0.00000 \quad 0.00002$

$0.00000 \quad 0.00000 \quad 0.00002$

$0.00000 \quad 0.00000 \quad 0.00002$

$0.00000 \quad 0.00000 \quad 0.00002$ $0.00000 \quad 0.00000 \quad 0.00002$

$0.00000 \quad 0.00000 \quad 0.00002$

$0.00000 \quad 0.00000 \quad 0.00002$

$0.00000 \quad 0.00000 \quad 0.00002$

$0.00000 \quad 0.00000 \quad 0.00002$

$0.00000 \quad 0.00000 \quad 0.00002$

$0.00000 \quad 0.00000 \quad 0.00002$

$0.00000 \quad 0.00000 \quad 0.00001$

$0.00000 \quad 0.00000 \quad 0.00001$

$0.00000 \quad 0.00000 \quad 0.00001$

$0.00000 \quad 0.00000 \quad 0.00001$

$0.00000 \quad 0.00000 \quad 0.00001$

$0.00000 \quad 0.00000 \quad 0.00001$

$0.00000 \quad 0.00000 \quad 0.00001$

$0.00000 \quad 0.00000 \quad 0.00001$

$0.00000 \quad 0.00000 \quad 0.00001$

$0.00000 \quad 0.00000 \quad 0.00001$

$0.00000 \quad 0.00000 \quad 0.00001$

$0.00000 \quad 0.00000 \quad 0.00001$

$0.00000 \quad 0.00000 \quad 0.00001$

$0.00000 \quad 0.00000 \quad 0.00001$

$0.00000 \quad 0.00000 \quad 0.00001$

$0.00000 \quad 0.00000 \quad 0.00001$

$0.00000 \quad 0.00000 \quad 0.00001$

$\begin{array}{lll}0.00000 & 0.00000 & 0.00001\end{array}$

$\begin{array}{lllll}0.00000 & 0.00000 & 0.00001\end{array}$

$0.00000 \quad 0.00000 \quad 0.00001$

$0.00000 \quad 0.00000 \quad 0.00001$

$0.00000 \quad 0.00000 \quad 0.00001$

$\begin{array}{llll}0.00000 & 0.00000 & 0.00001\end{array}$

$\begin{array}{llll}0.00000 & 0.00000 & 0.00001\end{array}$

$0.00000 \quad 0.00000 \quad 0.00000$

$0.00000 \quad 0.00000 \quad 0.00000$

$0.00000 \quad 0.000000 .00000$

$$
T=298 \cdot 15
$$

$T=400$.

$0.00000 \quad 0.000010 .00014$

$0.00000 \quad 0.000010 .00014$

$0.00000 \quad 0.000010 .00013$

$0.00000 \quad 0.000010 .00012$

$0.00000 \quad 0.000010 .00012$

$\begin{array}{llll}0.00000 & 0.00001 & 0.00011\end{array}$

$0.00000 \quad 0.000010 .00011$

$0.00000 \quad 0.000010 .00011$

$0.00000 \quad 0.000010 .00010$

$0.00000 \quad 0.000010 .00010$

$\begin{array}{llll}0.00000 & 0.00001 & 0.00009\end{array}$

$0.00000 \quad 0.000010 .00009$

$\begin{array}{llll}0.00000 & 0.00001 & 0.00009\end{array}$

$0.00000 \quad 0.00001 \quad 0.00008$

$\begin{array}{llll}0.00000 & 0.00001 & 0.00008\end{array}$

$\begin{array}{lllll}0.00000 & 0.00001 & 0.00008\end{array}$

$\begin{array}{llll}0.00000 & 0.00000 & 0.00007\end{array}$

$\begin{array}{llll}0.00000 & 0.00000 & 0.00007\end{array}$

$\begin{array}{llll}0.00000 & 0.00000 & 0.00007\end{array}$

$0.00000 \quad 0.00000 \quad 0.00006$

$\begin{array}{llll}0.00000 & 0.00000 & 0.00006\end{array}$

$0.00000 \quad 0.00000 \quad 0.00006$

$\begin{array}{llll}0.00000 & 0.00000 & 0.00006\end{array}$

$0.00000 \quad 0.00000 \quad 0.00005$

$0.00000 \quad 0.00000 \quad 0.00005$

$0.00000 \quad 0.00000 \quad 0.00005$

$0.00000 \quad 0.00000 \quad 0.00005$

$0.00000 \quad 0.00000 \quad 0.00005$

$0.00000 \quad 0.00000 \quad 0.00004$

$0.00000 \quad 0.00000 \quad 0.00004$

$0.00000 \quad 0.00000 \quad 0.00004$ $0.00000 \quad 0.00000 \quad 0.00004$ $\begin{array}{llll}0.00000 & 0.00000 & 0.00004\end{array}$ $\begin{array}{llll}0.00000 & 0.00000 & 0.00004\end{array}$ $0.00000 \quad 0.00000 \quad 0.00003$

$\begin{array}{llll}0.00000 & 0.00000 & 0.00003\end{array}$

$\begin{array}{llll}0.00000 & 0.00000 & 0.00003\end{array}$

$\begin{array}{llll}0.00000 & 0.00000 & 0.00003\end{array}$

$0.00000 \quad 0.00000 \quad 0.00003$

$0.00000 \quad 0.00000 \quad 0.00003$

$0.00000 \quad 0.00000 \quad 0.00003$

$\begin{array}{lllll}0.00000 & 0.00000 & 0.00003\end{array}$

$\begin{array}{llll}0.00000 & 0.00000 & 0.00002\end{array}$

$\begin{array}{lll}0.00000 & 0.00000 & 0.00002\end{array}$

$\begin{array}{lll}0.00000 & 0.00000 & 0.00002\end{array}$

$\begin{array}{llll}0.00000 & 0.00000 & 0.00002\end{array}$

$\begin{array}{llll}0.00000 & 0.00000 & 0.00002\end{array}$

$0.00000 \quad 0.00000 \quad 0.00002$

$0.00000 \quad 0.00000 \quad 0.00002$

$0.00000 \quad 0.00000 \quad 0.00002$ $\begin{array}{llll}0.00003 & 0.00035 & 0.00355\end{array}$

$0.00003 \quad 0.00034 \quad 0.00345$

$\begin{array}{llll}0.00003 & 0.00032 & 0.00335\end{array}$

$0.00003 \quad 0.000310 .00325$

$0.000020 .00030 \quad 0.00315$

$0.00002 \quad 0.000290 .00306$

$\begin{array}{llll}0.00002 & 0.00028 & 0.00297\end{array}$

$\begin{array}{lllll}0.00002 & 0.00028 & 0.00289\end{array}$

$\begin{array}{lllll}0.00002 & 0.00027 & 0.00280\end{array}$

$\begin{array}{lll}0.00002 & 0.00026 & 0.00272\end{array}$

$0.00002 \quad 0.000250 .00264$

$0.00002 \quad 0.000240 .00256$

$0.000020 .00023 \quad 0.00249$

$0.00002 \quad 0.00023 \quad 0.00241$

$\begin{array}{lll}0.00002 & 0.00022 & 0.00234\end{array}$

$\begin{array}{llll}0.00002 & 0.00021 & 0.00227\end{array}$

$\begin{array}{lllll}0.00002 & 0.00020 & 0.00221\end{array}$

0.000020 .000200 .00214

$0.00002 \quad 0.000190 .00208$

0.000010 .000190 .00202

$0.00001 \quad 0.00018 \quad 0.00196$

$\begin{array}{lllll}0.00001 & 0.00017 & 0.00190\end{array}$

$\begin{array}{llll}0.00001 & 0.00017 & 0.00185\end{array}$

$0.000010 .00016 \quad 0.00179$

$\begin{array}{llll}0.00001 & 0.00016 & 0.00174\end{array}$

0.000010 .000150 .00169

$\begin{array}{llll}0.00001 & 0.00015 & 0.00164\end{array}$

0.000010 .000140 .00159

0.000010 .000140 .00154

0.000010 .000130 .00150

0.000010 .000130 .00145

0.000010 .000120 .00141

0.000010 .000120 .00137

0.000010 .000120 .00133

0.000010 .000110 .00129

0.000010 .000110 .00125

0.000010 .000110 .00121

$0.00001 \quad 0.00010 \quad 0.00118$

$0.000010 .00010 \quad 0.00114$

0.000010 .000100 .00111

$0.00001 \quad 0.000090 .00107$

$0.000010 .00009 \quad 0.00104$

0.000010 .000090 .00101

$0.000010 .00008 \quad 0.00098$

$\begin{array}{lll}0.00001 & 0.00008 & 0.00095\end{array}$

$\begin{array}{llll}0.00001 & 0.00008 & 0.00092\end{array}$

$\begin{array}{llll}0.00001 & 0.00008 & 0.00089\end{array}$

$\begin{array}{lllll}0.00001 & 0.00007 & 0.00087\end{array}$

$0.000010 .00007 \quad 0.00084$

$\begin{array}{llll}0.00000 & 0.00007 & 0.00082\end{array}$
3100

3110

3120

3130

3140

3150

3160

3170

3180

3190

3200

3210

3220

3230

3240

3250

3260

3270

3280

3290

3300

3310

3320

3330

3340

3350

3360

3370

3380

3390

3400

3410

3420

3430

3440

3450

3460

3470

3480

3490

3500

3510

3520

3530

3540

3550

3560

3570

3580

3590 
Table II Harmonic Oscillator Contributions to the Thermodynamic

Functions (in units of calories, moles, and ${ }^{\circ} \mathrm{K}$ )
$\operatorname{cm}^{-1}-\left(F^{\circ}-E_{0}^{\circ}\right) / T \quad S^{\circ}$
$\mathrm{C}_{\mathrm{p}}^{\circ} \quad-\left(\mathrm{F}^{\circ}-\mathrm{E}_{\mathrm{O}}^{\circ}\right) / \mathrm{T} \quad \mathrm{S}^{\circ}$
$\mathrm{C}_{\mathrm{p}}^{\circ}$
$-\left(F^{\circ}-E \circ\right) / T$
S०
$\mathrm{Co}_{\mathrm{p}}^{\circ}$
$\nu$
$\mathrm{cm}^{-1}$

$T=273.15$

3600

3610

3620

3630

3640

3650

3660

3670

3680

3690

3700

3710

3720

3730

3740

3750

3760

3770

3780

3790

3800

3810

3820

3830

3840

3850

3860

3870

3880

3890

3900

3910

3920

3930

3940

3950

3960

3970

3980

3990

4000

$$
T=298.15
$$

$T=400$.

$\begin{array}{llll}0.00000 & 0.00000 & 0.00002\end{array}$

$\begin{array}{llll}0.00000 & 0.00000 & 0.00002\end{array}$

$\begin{array}{llll}0.00000 & 0.00000 & 0.00002\end{array}$

$\begin{array}{llll}0.00000 & 0.00000 & 0.00002\end{array}$

$\begin{array}{lll}0.00000 & 0.00000 & 0.00001\end{array}$

$\begin{array}{llll}0.00000 & 0.00000 & 0.00001\end{array}$

$\begin{array}{llll}0.00000 & 0.00000 & 0.00001\end{array}$

$\begin{array}{llll}0.00000 & 0.00000 & 0.00001\end{array}$

$\begin{array}{lll}0.00000 & 0.00000 & 0.00001\end{array}$

$0.00000 \quad 0.00000 \quad 0.00001$

$\begin{array}{llll}0.00000 & 0.00000 & 0.00001\end{array}$

$\begin{array}{lllll}0.00000 & 0.00000 & 0.00001\end{array}$

$\begin{array}{llll}0.00000 & 0.00000 & 0.00001\end{array}$

$\begin{array}{llll}0.00000 & 0.00000 & 0.00001\end{array}$

$\begin{array}{lll}0.00000 & 0.00000 & 0.00001\end{array}$

$\begin{array}{llll}0.00000 & 0.00000 & 0.00001\end{array}$

$\begin{array}{lllll}0.00000 & 0.00000 & 0.00001\end{array}$

$\begin{array}{lllll}0.00000 & 0.00000 & 0.00001\end{array}$

$\begin{array}{llll}0.00000 & 0.00000 & 0.00001\end{array}$

$0.00000 \quad 0.00000 \quad 0.00001$

$0.00000 \quad 0.00000 \quad 0.00001$

$\begin{array}{lllll}0.00000 & 0.00000 & 0.00001\end{array}$

$\begin{array}{llll}0.00000 & 0.00000 & 0.00001\end{array}$

$\begin{array}{llll}0.00000 & 0.00000 & 0.00001\end{array}$

$\begin{array}{llll}0.00000 & 0.00000 & 0.00001\end{array}$

$\begin{array}{llll}0.00000 & 0.00000 & 0.00001\end{array}$

$\begin{array}{llll}0.00000 & 0.00000 & 0.00001\end{array}$

$\begin{array}{llll}0.00000 & 0.00000 & 0.00001\end{array}$

$\begin{array}{llll}0.00000 & 0.00000 & 0.00001\end{array}$

$\begin{array}{lll}0.00000 & 0.00000 \quad 0.00000\end{array}$

$0.00000 \quad 0.00000 \quad 0.00000$

$\begin{array}{llll}0.00000 & 0.00000 & 0.00000\end{array}$

$0.00000 \quad 0.00000 \quad 0.00000$

$0.00000 \quad 0.00000 \quad 0.00000$

$0.00000 \quad 0.00000 \quad 0.00000$

$\begin{array}{llll}0.00000 & 0.00000 & 0.00000\end{array}$

$0.00000 \quad 0.00000 \quad 0.00000$

$\begin{array}{llll}0.00000 & 0.00000 & 0.00000\end{array}$

$\begin{array}{llll}0.00000 & 0.00000 & 0.00000\end{array}$

$0.00000 \quad 0.00000 \quad 0.00000$

$0.00000 \quad 0.00000 \quad 0.00000$
$0.00000 \quad 0.00007 \quad 0.00079$

$\begin{array}{lllll}0.00000 & 0.00006 & 0.00077\end{array}$

$\begin{array}{lll}0.00000 & 0.00006 & 0.00075\end{array}$

$\begin{array}{llll}0.00000 & 0.00006 & 0.00072\end{array}$

$0.00000 \quad 0.00006 \quad 0.00070$

$0.00000 \quad 0.00006 \quad 0.00068$

$0.00000 \quad 0.00005 \quad 0.00066$

$\begin{array}{llll}0.00000 & 0.00005 & 0.00064\end{array}$

$\begin{array}{lll}0.00000 & 0.00005 & 0.00062\end{array}$

$0.00000 \quad 0.000050 .00060$

$0.00000 \quad 0.00005 \quad 0.00058$

$0.00000 \quad 0.00005 \quad 0.00057$

$0.00000 \quad 0.00004 \quad 0.00055$

$0.00000 \quad 0.00004 \quad 0.00053$

$0.00000 \quad 0.00004 \quad 0.00052$

$0.00000 \quad 0.00004 \quad 0.00050$

$0.00000 \quad 0.00004 \quad 0.00049$

$0.00000 \quad 0.00004 \quad 0.00047$

$0.00000 \quad 0.00004 \quad 0.00046$

$0.00000 \quad 0.000030 .00044$

$0.00000 \quad 0.000030 .00043$

$0.00000 \quad 0.00003 \quad 0.00042$

$0.00000 \quad 0.00003 \quad 0.00040$

$0.00000 \quad 0.00003 \quad 0.00039$

$0.00000 \quad 0.00003 \quad 0.00038$

$\begin{array}{lll}0.00000 & 0.00003 & 0.00037\end{array}$

$0.00000 \quad 0.00003 \quad 0.00036$

$0.00000 \quad 0.00003 \quad 0.00035$

$0.00000 \quad 0.00003 \quad 0.00034$

$0.00000 \quad 0.00003 \quad 0.00033$

$0.00000 \quad 0.00002 \quad 0.00032$

$\begin{array}{llll}0.00000 & 0.00002 & 0.00031\end{array}$

$0.00000 \quad 0.00002 \quad 0.00030$

$\begin{array}{lll}0.00000 & 0.00002 & 0.00029\end{array}$

$0.00000 \quad 0.00002 \quad 0.00028$

$0.00000 \quad 0.00002 \quad 0.00027$

$0.00000 \quad 0.00002 \quad 0.00026$

$\begin{array}{llll}0.00000 & 0.00002 & 0.00025\end{array}$

$0.00000 \quad 0.00002 \quad 0.00025$

$0.00000 \quad 0.00002 \quad 0.00024$

$0.00000 \quad 0.000020 .00023$
3600

3610

3620

3630

3640

3650

3660

3670

3680

3690

3700

3710

3720

3730

3740

3750

3760

3770

3780

3790

3800

3810

3820

3830

3840

3850

3860

3870

3880

3890

3900

3910

3920

3930

3940

3950

3960

3970

3980

3990

4000 
Table II Harmonic Oscillator Contributions to the Thermodynamic

Functions (in units of calories, moles, and ${ }^{\circ} \mathrm{K}$ )
$\nu_{-1}-\left(F^{\circ}-E_{0}^{\circ}\right) / T \quad S^{\circ}$
$\mathrm{co}_{\mathrm{p}}$
$-\left(\mathrm{F}^{\circ}-\mathrm{E}_{\mathrm{O}}\right) / \mathrm{T}$
So
$\mathrm{C}_{\mathrm{p}}$
$-\left(F^{\circ}-E_{O}^{\circ}\right) / T$
So
$\begin{array}{cc}\mathrm{C}_{\mathrm{p}}^{\circ} & \nu \\ \mathrm{cm}^{-1}\end{array}$

$T=500$.

$T=600$.

$T=700$.

$100 \quad 2.75434 \quad 4.46929 \quad 1.97351$

$110 \quad 2.592094 .281321 .97066$

$120 \quad 2.446214 .109991 .96754$

$130 \quad 2.31403 \quad 3.95263 \quad 1.96416$

$\begin{array}{llll}140 & 2.19351 & 3.80720 \quad 1.96051\end{array}$

$\begin{array}{llll}150 & 2.08302 & 3.67207 & 1.95660\end{array}$

$\begin{array}{lllll}160 & 1.98124 & 3.54593 & 1.95243\end{array}$

$\begin{array}{lllll}170 & 1.88711 & 3.42769 & 1.94801\end{array}$

$\begin{array}{lllll}180 & 1.79972 & 3.31648 & 1.94333\end{array}$

$190 \quad 1.71835 \quad 3.21154 \quad 1.93840$

$200 \quad 1.64235 \quad 3.11224 \quad 1.93322$

$210 \quad 1.571193 .01805 \quad 1.92779$

$220 \quad 1.504412 .92850 \quad 1.92211$

$230 \quad 1.441612 .84319 \quad 1.91619$

$\begin{array}{lll}240 \quad 1.38243 & 2.76177 \quad 1.91003\end{array}$

$\begin{array}{llll}250 & 1.32657 & 2.68392 & 1.90364\end{array}$

$260 \quad 1.273752 .609391 .89701$

$270 \quad 1.22375 \quad 2.53792 \quad 1.89015$

$\begin{array}{lllll}280 & 1.17634 & 2.46931 & 1.88307\end{array}$

$290 \quad 1.13134 \quad 2.40336 \quad 1.87576$

$\begin{array}{lllll}300 & 1.08856 & 2.33989 & 1.86822\end{array}$

$\begin{array}{lllll}310 & 1.04786 & 2.27876 & 1.86048\end{array}$

$\begin{array}{lllll}320 & 1.00910 & 2.21982 & 1.85251\end{array}$

$\begin{array}{llll}330 & 0.97215 & 2.16294 & 1.84434\end{array}$

$340 \quad 0.93690 \quad 2.10800 \quad 1.83596$

$\begin{array}{lllll}350 & 0.90323 & 2.05491 & 1.82738\end{array}$

$\begin{array}{lllll}360 & 0.87106 & 2.00355 & 1.81860\end{array}$

$\begin{array}{llll}370 & 0.84029 & 1.95384 & 1.80963\end{array}$

$\begin{array}{lllll}380 & 0.81084 & 1.90571 & 1.80046\end{array}$

$390 \quad 0.78264 \quad 1.85906 \quad 1.79111$

$\begin{array}{llll}400 & 0.75562 & 1.81383 & 1.78157\end{array}$

$\begin{array}{lllll}410 & 0.72971 & 1.76996 & 1.77186\end{array}$

$\begin{array}{llll}420 & 0.70485 & 1.72738 & 1.76197\end{array}$

$\begin{array}{lllll}430 & 0.68100 & 1.68604 & 1.75192\end{array}$

$\begin{array}{llll}440 & 0.65809 & 1.64588 & 1.74169\end{array}$

$\begin{array}{lllll}450 & 0.63608 & 1.60685 & 1.73131\end{array}$

$\begin{array}{lllll}460 & 0.61493 & 1.56892 & 1.72077\end{array}$

$\begin{array}{lllll}470 & 0.59459 & 1.53202 & 1.71008\end{array}$

$480 \quad 0.57503 \quad 1.496131 .69923$

$\begin{array}{lllll}490 & 0.55620 & 1.46121 & 1.68825\end{array}$

$\begin{array}{lllll}500 & 0.53808 & 1.42721 & 1.67713\end{array}$

$\begin{array}{lllll}510 & 0.52062 & 1.39411 & 1.66587\end{array}$

$\begin{array}{lllll}520 & 0.50381 & 1.36188 & 1.65448\end{array}$

$\begin{array}{lllll}530 & 0.48761 & 1.33047 & 1.64296\end{array}$

$\begin{array}{llllll}540 & 0.47200 & 1.29987 & 1.63133\end{array}$

$\begin{array}{lllll}550 & 0.45694 & 1.27004 & 1.61957\end{array}$

$\begin{array}{lllll}560 & 0.44242 & 1.24097 & 1.60771\end{array}$

$\begin{array}{llll}570 & 0.42842 & 1.21262 & 1.59574\end{array}$

$\begin{array}{lllll}580 & 0.41490 & 1.18497 & 1.58366\end{array}$

$590 \quad 0.401861 .15800 \quad 1.57149$
$3.07108 \quad 4.82950 \quad 1.97767$ 2.904514 .641101 .97569 $2.75434 \quad 4.469291 .97351$ 2.617924 .311411 .97115 2.493194 .165431 .96861

$2.37854 \quad 4.02970 \quad 1.96588$ $2.27265 \quad 3.90292 \quad 1.96297$ $2.17443 \quad 3.78400 \quad 1.95988$ $2.08302 \quad 3.67207 \quad 1.95660$ $1.99765 \quad 3.56638 \quad 1.95315$

$1.917693 .46628 \quad 1.94951$ 1.842623 .371261 .94570 $1.771973 .28084 \quad 1.94172$ $1.70533 \quad 3.194611 .93755$ $1.64235 \quad 3.11224 \quad 1.93322$

$1.58274 \quad 3.03342 \quad 1.92871$ 1.526212 .957861 .92403 $1.472542 .88534 \quad 1.91918$ 1.421492 .815631 .91417 1.372902 .748551 .90899

$\begin{array}{llll}1.32657 & 2.68392 & 1.90364\end{array}$ 1.282362 .621591 .89813 1.240122 .561421 .89246 $1.19973 \quad 2.50327 \quad 1.88664$ $1.161082 .44704 \quad 1.88065$

$1.12406 \quad 2.392611 .87452$ $1.088562 .33989 \quad 1.86822$ $\begin{array}{llll}1.05451 & 2.28879 & 1.86178\end{array}$ 1.021822 .239231 .85519 0.990412 .191131 .84845

$0.96022 \quad 2.14442 \quad 1.84157$ $0.93118 \quad 2.09903 \quad 1.83455$ $0.903232 .05491 \quad 1.82738$ 0.876322 .011991 .82008 $0.85039 \quad 1.97024 \quad 1.81264$

$0.82540 \quad 1.92958 \quad 1.80507$ $0.80130 \quad 1.89000 \quad 1.79736$ $0.77805 \quad 1.85142 \quad 1.78953$ 0.755621 .813831 .78157 $0.73395 \quad 1.77718 \quad 1.77349$

$0.71302 \quad 1.741431 .76529$ $0.69280 \quad 1.70656 \quad 1.75697$ $0.67326 \quad 1.672521 .74853$ $0.654361 .63930 \quad 1.73998$ $0.63608 \quad 1.606851 .73131$

$0.61840 \quad 1.57517 \quad 1.72254$ $0.60128 \quad 1.54421 \quad 1.71366$ $0.58471 \quad 1.51396 \quad 1.70467$ 0.568671 .484391 .69559 $0.553131 .45548 \quad 1.68641$
3.344635 .134571 .98019 $3.174924 .94590 \quad 1.97873$ 3.021634 .773801 .97713 2.882124 .615611 .97539 $2.75434 \quad 4.469291 .97351$

2.636654 .333201 .97150 2.527744 .206031 .96936 $2.42654 \quad 4.08670 \quad 1.96707$ $2.33215 \quad 3.97434 \quad 1.96466$ $2.243843 .86818 \quad 1.96211$

$2.16098 \quad 3.76761 \quad 1.95942$ 2.083023 .672071 .95660 2. $00950 \quad 3.581121 .95365$ $1.940023 .49434 \quad 1.95057$ 1.874233 .411391 .94736

$1.81182 \quad 3.33197 \quad 1.94402$ $1.75253 \quad 3.255791 .94054$ 1.696113 .182621 .93694 1.642353 .112241 .93322 $1.59106 \quad 3.044471 .92936$

$1.54206 \quad 2.97913 \quad 1.92538$ $1.495212 .91606 \quad 1.92128$ 1.450352 .855131 .91705 1.407372 .796211 .91270 $1.366142 .73917 \quad 1.90823$

$\begin{array}{llll}1.32657 & 2.68392 & 1.90364\end{array}$ 1.288552 .630361 .89893 $1.251992 .57840 \quad 1.89410$ $1.21682 \quad 2.52795 \quad 1.88915$ $1.18296 \quad 2.47895 \quad 1.88409$

$1.150342 .43131 \quad 1.87892$ $\begin{array}{llll}1.11889 & 2.38498 & 1.87363\end{array}$ $\begin{array}{lll}1.08856 & 2.33989 & 1.86822\end{array}$ $1.059292 .29600 \quad 1.86271$ $1.03102 \quad 2.25324 \quad 1.85709$

$1.00372 \quad 2.211571 .85136$ $0.97733 \quad 2.17094 \quad 1.84552$ $0.951812 .13132 \quad 1.83958$ $0.92712 \quad 2.092651 .83353$ $0.903232 .05491 \quad 1.82738$

$0.880102 .01805 \quad 1.82113$ $0.85770 \quad 1.98205 \quad 1.81478$ $0.83600 \quad 1.94687 \quad 1.80833$ $0.81497 \quad 1.91249 \quad 1.80178$ $0.79458 \quad 1.87887 \quad 1.79514$

$\begin{array}{llll}0.77480 & 1.84600 & 1.78840\end{array}$ $0.755621 .81383 \quad 1.78157$ $0.73700 \quad 1.78236 \quad 1.77465$ $0.71893 \quad 1.75156 \quad 1.76765$ $0.70138 \quad 1.72140 \quad 1.76055$
100

110

120

130

140

150

160

170

180

190

200

210

220

230

240

250

260

270

280

290

300

310

320

330

340

350

360

370

380

390

400

410

420

430

440

450

460

470

480

490

500

510

520

530

540

550

560

570

580

590 
Table II Harmonic Oscillator Contributions to the Thermodynamic

Functions (in units of calories, moles, and ${ }^{\circ} \mathrm{K}$ )

\begin{tabular}{|c|c|c|c|}
\hline \multirow[t]{2}{*}{$\begin{array}{c}\nu \\
\mathrm{cm}^{-1}\end{array}$} & $-\left(F^{\circ}-E_{O}^{\circ}\right) / T$ & So & $\mathrm{C}_{\mathrm{p}}^{\circ}$ \\
\hline & & $T=500$. & \\
\hline 500 & 0.38926 & 1.13169 & 1.55922 \\
\hline 10 & 0.37710 & 1.10602 & 1.54687 \\
\hline 20 & 0.36536 & 1.08097 & 1.53443 \\
\hline 30 & 0.35401 & 1.05651 & 1.52191 \\
\hline 40 & 0.34305 & 1.03265 & 1.50931 \\
\hline 50 & 0.33246 & 1.00934 & 1.49664 \\
\hline 60 & 0.32222 & 0.98659 & 48390 \\
\hline 7 & 0.31232 & 0.96437 & 1.47111 \\
\hline 8 & 0.30275 & 0.94267 & 1.45825 \\
\hline 9 & 0.29350 & 0.92148 & 1.44534 \\
\hline & 0.28454 & 0.90077 & 1.43238 \\
\hline & 0.27589 & 0.88055 & 1.41937 \\
\hline & 0.26751 & 0.86079 & 1.40632 \\
\hline 73 & 0.25940 & 0.84148 & 1.39324 \\
\hline & 0.25156 & 0.82261 & 1.38012 \\
\hline & & 0.00 & \\
\hline & 0.23662 & 0.78616 & 1.35380 \\
\hline & 0.22950 & 0.76854 & 1.34061 \\
\hline & 0.22261 & 0.75133 & 1.32740 \\
\hline & 0.21594 & 0.73451 & 1.31418 \\
\hline
\end{tabular}

$\begin{array}{llll}800 & 0.20948 \quad 0.71806 \quad 1.30094\end{array}$ $\begin{array}{llll}810 & 0.20322 & 0.70198 & 1.28771\end{array}$ $820 \quad 0.19716 \quad 0.68626 \quad 1.27447$ $830 \quad 0.19129 \quad 0.67089 \quad 1.26123$ $\begin{array}{llll}840 & 0.18560 & 0.65587 & 1.24799\end{array}$

$\begin{array}{llll}850 & 0.18009 & 0.64117 & 1.23477\end{array}$ $860 \quad 0.17475 \quad 0.62681 \quad 1.22156$ $870 \quad 0.16958 \quad 0.61276 \quad 1.20836$ $880 \quad 0.16456 \quad 0.59903 \quad 1.19518$ $890 \quad 0.15970 \quad 0.58560 \quad 1.18202$

$\begin{array}{lllll}900 & 0.15499 & 0.57246 & 1.16889\end{array}$ $\begin{array}{lllll}910 & 0.15042 & 0.55962 & 1.15579\end{array}$ $\begin{array}{lllll}920 & 0.14600 & 0.54706 & 1.14272\end{array}$ $930 \quad 0.14170 \quad 0.53478 \quad 1.12968$ $940 \quad 0.13754 \quad 0.52276 \quad 1.11668$

$\begin{array}{lllll}950 & 0.13351 & 0.51102 & 1.10372\end{array}$ $960 \quad 0.129590 .49953 \quad 1.09080$ $\begin{array}{lllll}970 & 0.12580 & 0.48829 & 1.07793\end{array}$ $980 \quad 0.122120 .47730 \quad 1.06511$ $\begin{array}{lllll}990 & 0.11855 & 0.46655 & 1.05234\end{array}$

$\begin{array}{lllll}1000 & 0.11509 & 0.45604 & 1.03962\end{array}$ $\begin{array}{lllll}1010 & 0.11173 & 0.44576 & 1.02696\end{array}$ $\begin{array}{lllll}1020 & 0.10847 & 0.43570 & 1.01435\end{array}$ $\begin{array}{lllll}1030 & 0.10531 & 0.42586 & 1.00181\end{array}$ $\begin{array}{lllll}1040 & 0.10225 & 0.41625 & 0.98933\end{array}$

$\begin{array}{lllll}1050 & 0.09927 & 0.40684 & 0.97691\end{array}$ $\begin{array}{llllll}1060 & 0.09639 & 0.39764 & 0.96456\end{array}$ $\begin{array}{llllll}1070 & 0.09359 & 0.38864 & 0.95228\end{array}$ $\begin{array}{lllll}1080 & 0.09087 & 0.37984 & 0.94007\end{array}$ $\begin{array}{lllll}1090 & 0.08823 & 0.37123 & 0.92793\end{array}$

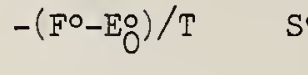

so

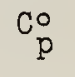

$-\left(F^{\circ}-E_{O}^{\circ}\right) / T$

$S^{\circ}$

p

$\mathrm{m}^{-1}$

$T=600$.

$T=700$.

$0.53808 \quad 1.42721 \quad 1.67713$ $0.523491 .39957 \quad 1.66775$ $0.50935 \quad 1.37253 \quad 1.65829$ $0.49564 \quad 1.34607 \quad 1.64874$ 0.482341 .320181 .63910

$0.46945 \quad 1.29484 \quad 1.62938$ $0.45694 \quad 1.27004 \quad 1.61957$ $0.44481 \quad 1.24576 \quad 1.60970$ $0.43303 \quad 1.22199 \quad 1.59974$ $0.421601 .19870 \quad 1.58971$

$0.41050 \quad 1.17590 \quad 1.57962$ $0.39973 \quad 1.15357 \quad 1.56945$ $0.38926 \quad 1.13169 \quad 1.55922$ $0.37910 \quad 1.11025 \quad 1.54893$ $0.36923 \quad 1.08925 \quad 1.53858$

$0.35964 \quad 1.06867 \quad 1.52818$ $0.35032 \quad 1.04849 \quad 1.51772$ $0.34126 \quad 1.02872 \quad 1.50720$ $0.33246 \quad 1.00934 \quad 1.49664$ $\begin{array}{llll}0.32390 & 0.99034 & 1.48603\end{array}$

$\begin{array}{llll}0.31558 & 0.97172 & 1.47538\end{array}$ $0.30750 \quad 0.95346 \quad 1.46468$ $0.29963 \quad 0.93555 \quad 1.45395$ $0.29198 \quad 0.91799 \quad 1.44318$ $0.28454 \quad 0.90077 \quad 1.43238$

$\begin{array}{llll}0.27731 & 0.88389 & 1.42154\end{array}$ $0.27027 \quad 0.86732 \quad 1.41068$ $\begin{array}{llll}0.26342 & 0.85108 & 1.39979\end{array}$ $0.256760 .83514 \quad 1.38887$ $\begin{array}{llll}0.25027 & 0.81951 & 1.37793\end{array}$

$\begin{array}{llll}0.24396 & 0.80417 & 1.36698\end{array}$ $0.237820 .78913 \quad 1.35600$ $\begin{array}{llll}0.23185 & 0.77437 & 1.34501\end{array}$ $0.22603 \quad 0.75989 \quad 1.33401$ $0.22036 \quad 0.74568 \quad 1.32299$

$0.214850 .73174 \quad 1.31197$ $0.20948 \quad 0.71806 \quad 1.30094$ $0.20425 \quad 0.70463 \quad 1.28991$ $0.19916 \quad 0.69146 \quad 1.27888$ $0.19420 \quad 0.678531 .26785$

$\begin{array}{llll}0.18938 & 0.66585 & 1.25682\end{array}$ $\begin{array}{llll}0.18467 & 0.65339 & 1.24579\end{array}$ $0.18009 \quad 0.64117 \quad 1.23477$ 0.175630 .629181 .22376 $0.17129 \quad 0.61741 \quad 1.21276$

$\begin{array}{llll}0.16705 & 0.60586 \quad 1.20177\end{array}$ $0.16293 \quad 0.59452 \quad 1.19079$ 0.158910 .583391 .17983 $0.15499 \quad 0.57246 \quad 1.16889$ $\begin{array}{lll}0.15118 & 0.56174 & 1.15797\end{array}$
$0.68435 \quad 1.69187 \quad 1.75336$ $0.66779 \quad 1.662951 .74610$ $\begin{array}{llll}0.65171 & 1.63461 & 1.73874\end{array}$ $0.63608 \quad 1.60685 \quad 1.73131$ 0.620891 .579651 .72380

$0.60612 \quad 1.55298 \quad 1.71621$ $0.59175 \quad 1.52684 \quad 1.70854$ $0.57778 \quad 1.50120 \quad 1.70079$ $0.56418 \quad 1.47606 \quad 1.69297$ $0.55095 \quad 1.45140 \quad 1.68509$

$0.53808 \quad 1.42721 \quad 1.67713$ $0.52554 \quad 1.40348 \quad 1.66910$ 0.513341 .380191 .66100 $0.50146 \quad 1.35734 \quad 1.65284$ $0.489891 .33491 \quad 1.64462$

$0.478621 .31289 \quad 1.63633$ $0.46764 \quad 1.29127 \quad 1.62798$ $0.45694 \quad 1.27004 \quad 1.61957$ 0.446521 .249201 .61111 $0.436361 .22873 \quad 1.60259$

$0.42646 \quad 1.20862 \quad 1.59402$ $0.416801 .18887 \quad 1.58540$ $0.407391 .16947 \quad 1.57672$ $0.39821 \quad 1.15042 \quad 1.56800$ $0.38926 \quad 1.131691 .55922$

$0.38053 \quad 1.11329 \quad 1.55041$ $0.37202 \quad 1.09521 \quad 1.54155$ $0.36371 \quad 1.07744 \quad 1.53264$ 0.355611 .059971 .52370 $0.347701 .04281 \quad 1.51472$

$0.33999 \quad 1.02593 \quad 1.50570$ $0.33246 \quad 1.00934 \quad 1.49664$ $0.325110 .99304 \quad 1.48755$ $0.31794 \quad 0.97700 \quad 1.47843$ $0.31093 \quad 0.96124 \quad 1.46927$

$0.30410 \quad 0.94574 \quad 1.46009$ $0.297420 .93050 \quad 1.45088$ $0.290910 .91551 \quad 1.44164$ $\begin{array}{llll}0.28454 & 0.90077 & 1.43238\end{array}$ $\begin{array}{llll}0.27833 & 0.88628 & 1.42309\end{array}$

$\begin{array}{llll}0.27226 & 0.87202 \quad 1.41378\end{array}$ $0.26633 \quad 0.85800 \quad 1.40446$ $0.260540 .84421 \quad 1.39511$ 0.254890 .830641 .38575 $\begin{array}{llll}0.24936 & 0.81730 & 1.37637\end{array}$

$\begin{array}{llll}0.24396 & 0.80417 & 1.36698\end{array}$ $\begin{array}{llll}0.23869 & 0.79126 & 1.35757\end{array}$ $0.233540 .77856 \quad 1.34815$ $0.22850 \quad 0.76606 \quad 1.33872$ $\begin{array}{llll}0.22358 & 0.75377 & 1.32929\end{array}$
600

610

620

630

640

650

660

670

680

690

700

710

720

730

740

750

760

770

780

790

800

810

820

830

840

850

860

870

880

890

900

910

920

930

940

950

960

970

980

990

1000

1010

1020

1030

1040

1050

1060

1070

1080

1090 
Table II Harmonic Oscillator Contributions to the The rmodynamic

Functions (in units of calories, moles, and ${ }^{\circ} \mathrm{K}$ )
$\nu \quad-\left(\mathrm{F}^{\circ}-\mathrm{E}_{0}^{\circ}\right) / \mathrm{T} \quad \mathrm{S}^{\circ}$
$\mathrm{C}_{\mathrm{p}}^{\mathrm{O}} \quad-\left(\mathrm{F}^{\circ}-\mathrm{E}_{\mathrm{O}}^{\mathrm{O}}\right) / \mathrm{T}$
so
$\mathrm{c}_{\mathrm{p}}$
$-(F \circ-E O) / T$
S०
2
$\mathrm{cm}^{-1}$

$T=500$.

1100

$0.08568 \quad 0.36281 \quad 0.91587$

$\begin{array}{llll}1110 & 0.08320 & 0.35457 & 0.90388\end{array}$

$\begin{array}{llll}1120 & 0.08079 & 0.34652 & 0.89197\end{array}$

$\begin{array}{llll}1130 & 0.07845 & 0.33864 & 0.88014\end{array}$

$\begin{array}{llll}1140 & 0.07618 & 0.33094 & 0.86839\end{array}$

$\begin{array}{lllll}1150 & 0.07398 & 0.32341 & 0.85673\end{array}$

$\begin{array}{llllll}1160 & 0.07184 & 0.31604 & 0.84515\end{array}$

$\begin{array}{lllll}1170 & 0.06977 & 0.30884 & 0.83365\end{array}$

$\begin{array}{llllll}1180 & 0.06775 & 0.30179 & 0.82224\end{array}$

$\begin{array}{lllll}1190 & 0.06580 & 0.29490 & 0.81092\end{array}$

$\begin{array}{llllll}1200 & 0.06390 & 0.28816 & 0.79969\end{array}$

$\begin{array}{llllll}1210 & 0.06206 & 0.28157 & 0.78854\end{array}$

$\begin{array}{lllll}1220 & 0.06028 & 0.27512 & 0.77749\end{array}$

$\begin{array}{lllll}1230 & 0.05854 & 0.26882 & 0.76653\end{array}$

$\begin{array}{lllll}1240 & 0.05686 & 0.26266 & 0.75567\end{array}$

$\begin{array}{lllll}1250 & 0.05522 & 0.25663 & 0.74490\end{array}$

$\begin{array}{lllll}1260 & 0.05363 & 0.25074 & 0.73422\end{array}$

$\begin{array}{lllll}1270 & 0.05209 & 0.24498 & 0.72364\end{array}$

$\begin{array}{lllll}1280 & 0.05059 & 0.23934 & 0.71315\end{array}$

$\begin{array}{lllll}1290 & 0.04914 & 0.23383 & 0.70276\end{array}$

$\begin{array}{llll}1300 & 0.04773 & 0.22845 & 0.69247\end{array}$

$\begin{array}{lllll}1310 & 0.04636 & 0.22318 & 0.68228\end{array}$

$\begin{array}{lllll}1320 & 0.04503 & 0.21803 & 0.67218\end{array}$

$\begin{array}{lllll}1330 & 0.04374 & 0.21299 & 0.66219\end{array}$

$\begin{array}{llllll}1340 & 0.04249 & 0.20807 & 0.65229\end{array}$

$\begin{array}{lllll}1350 & 0.04127 & 0.20326 & 0.64250\end{array}$

$\begin{array}{lllll}1360 & 0.04008 & 0.19855 & 0.63280\end{array}$

$\begin{array}{llllll}1370 & 0.03894 & 0.19395 & 0.62321\end{array}$

$\begin{array}{lllll}1380 & 0.03782 & 0.18945 & 0.61371\end{array}$

$\begin{array}{llllll}1390 & 0.03674 & 0.18506 & 0.60432\end{array}$

$\begin{array}{lllll}1400 & 0.03569 & 0.18076 & 0.59502\end{array}$

$\begin{array}{lllll}1410 & 0.03467 & 0.17655 & 0.58583\end{array}$

$\begin{array}{llllll}1420 & 0.03367 & 0.17245 & 0.57674\end{array}$

$\begin{array}{lllll}1430 & 0.03271 & 0.16843 & 0.56775\end{array}$

$\begin{array}{lllll}1440 & 0.03178 & 0.16451 & 0.55886\end{array}$

$\begin{array}{llllll}1450 & 0.03087 & 0.16067 & 0.55007\end{array}$

$\begin{array}{lllll}1460 & 0.02999 & 0.15692 & 0.54139\end{array}$

$\begin{array}{lllll}1470 & 0.02913 & 0.15325 & 0.53280\end{array}$

$\begin{array}{lllll}1480 & 0.02830 & 0.14967 & 0.52432\end{array}$

$\begin{array}{llllll}1490 & 0.02749 & 0.14617 & 0.51593\end{array}$

$\begin{array}{llllll}1500 & 0.02670 & 0.14274 & 0.50765\end{array}$

$\begin{array}{lllll}1510 & 0.02594 & 0.13940 & 0.49946\end{array}$

$\begin{array}{llllll}1520 & 0.02520 & 0.13613 & 0.49138\end{array}$

$1530 \quad 0.02448 \quad 0.13293 .0 .48339$

$\begin{array}{lllll}1540 & 0.02378 & 0.12981 & 0.47550\end{array}$

$\begin{array}{lllll}1550 & 0.02310 & 0.12675 & 0.46772\end{array}$

$\begin{array}{llllll}1560 & 0.02244 & 0.12377 & 0.46003\end{array}$

$\begin{array}{lllll}1570 & 0.02180 & 0.12086 & 0.45244\end{array}$

$\begin{array}{llllll}1580 & 0.02118 & 0.11801 & 0.44494\end{array}$

$\begin{array}{lllll}1590 & 0.02058 & 0.11522 & 0.43754\end{array}$

$T=600$.

$\mathrm{T}=700$.

$0.14746 \quad 0.55122 \quad 1.14707$

0.143830 .540881 .13619

$0.14030 \quad 0.53074 \quad 1.12534$

$0.136860 .52079 \quad 1.11452$

0.133510 .511021 .10372

$0.130240 .50142 \quad 1.09295$

$0.127050 .49201 \quad l .08222$

$0.123940 .48276 \quad 1.07151$

$0.120920 .47369 \quad 1.06085$

$0.11796 \quad 0.46478 \quad 1.05021$

$0.115090 .45604 \quad 1.03962$

$0.112280 .44745 \quad 1.02906$

0.109550 .439031 .01855

$0.10688 \quad 0.43076 \quad 1.00807$

0.104280 .422630 .99764

0.101740 .414660 .98725

0.099270 .406840 .97691

0.096860 .399160 .96661

0.094510 .391610 .95636

0.092220 .384210 .94616

$0.08998 \quad 0.37694 \quad 0.93601$

$\begin{array}{llll}0.08780 & 0.36981 & 0.92591\end{array}$

0.085680 .362810 .91587

$0.08360 \quad 0.355930 .90587$

$0.08158 \quad 0.349180 .89593$

$0.079610 .34256 \quad 0.88605$ $0.077690 .33606 \quad 0.87622$ $0.07581 \quad 0.32967 \quad 0.86644$ $0.07398 \quad 0.323410 .85673$ $0.072190 .31726 \quad 0.84707$

$\begin{array}{llll}0.07045 & 0.31122 & 0.83747\end{array}$ $0.06875 \quad 0.305290 .82793$ $\begin{array}{llll}0.06710 & 0.29947 & 0.81846\end{array}$ $\begin{array}{llll}0.06548 & 0.29376 & 0.80904\end{array}$ $\begin{array}{lll}0.06390 & 0.28816 & 0.79969\end{array}$

$\begin{array}{llll}0.06237 & 0.28266 & 0.79039\end{array}$ $\begin{array}{llll}0.06087 & 0.27726 & 0.78117\end{array}$ $\begin{array}{llll}0.05940 & 0.27196 & 0.77200\end{array}$ $\begin{array}{llll}0.05797 & 0.26675 & 0.76290\end{array}$ $\begin{array}{lll}0.05658 & 0.26165 & 0.75387\end{array}$

$\begin{array}{llll}0.05522 & 0.25663 & 0.74490\end{array}$ $\begin{array}{llll}0.05389 & 0.25171 & 0.73599\end{array}$ $\begin{array}{llll}0.05260 & 0.24688 & 0.72715\end{array}$ $0.05134 \quad 0.24214 \quad 0.71838$ $\begin{array}{lll}0.05011 & 0.23749 & 0.70968\end{array}$

$0.04890 \quad 0.23293 \quad 0.70104$ $\begin{array}{llll}0.04773 & 0.22845 & 0.69247\end{array}$ $\begin{array}{lll}0.04659 & 0.22405 & 0.68397\end{array}$ 0.045470 .219730 .67554 $\begin{array}{lll}0.04438 & 0.21550 & 0.66717\end{array}$
$0.218770 .74167 \quad 1.31985$

$0.214070 .72977 \quad 1.31040$ $0.209480 .71806 \quad 1.30095$

$0.20499 \quad 0.70654 \quad 1.29149$

$0.20060 \quad 0.69520 \quad 1.28203$

$\begin{array}{llll}0.19631 & 0.68404 & 1.27257\end{array}$ $0.192120 .67307 \quad 1.26312$

$0.18802 \quad 0.66226 \quad 1.25366$

0.184010 .651631 .24421

$\begin{array}{llll}0.18009 & 0.64117 & 1.23477\end{array}$

$\begin{array}{llll}0.17626 & 0.63088 & 1.22533\end{array}$ $0.172520 .62075 \quad 1.21590$

$0.168850 .61078 \quad 1.20647$

$0.16527 \quad 0.60097 \quad 1.19706$

$0.161770 .59132 \quad 1.18766$

$\begin{array}{llll}0.15834 & 0.58182 & 1.17827\end{array}$

$0.15499 \quad 0.57246 \quad 1.16889$

$0.15171 \quad 0.56326 \quad 1.15953$

$0.14851 \quad 0.55420 \quad 1.15018$

$0.14538 \quad 0.54529 \quad 1.14085$

$\begin{array}{llll}0.14231 & 0.53651 & 1.13154\end{array}$ $0.13931 \quad 0.52788 \quad 1.12225$

$0.13638 \quad 0.51938 \quad 1.11297$

$0.133510 .51102 \quad 1.10372$

$0.13070 \quad 0.50278 \quad 1.09449$

$0.12795 \quad 0.49468 \quad 1.08528$ $0.125270 .48670 \quad 1.07610$ 0.122640 .478851 .06694 $0.12007 \quad 0.471131 .05781$ $0.117550 .46352 \quad 1.04870$

$\begin{array}{llll}0.11509 & 0.45604 & 1.03962\end{array}$ $0.112680 .44867 \quad 1.03057$ 0.110320 .441421 .02155 $0.10801 \quad 0.43428 \quad 1.01256$ $0.10576 \quad 0.42726 \quad 1.00360$

0.103550 .420340 .99467 0.101390 .413540 .98577 $0.09927 \quad 0.406840 .97691$ $0.09720 \quad 0.40024 \quad 0.96808$ $0.09518 \quad 0.39376 \quad 0.95929$

$\begin{array}{llll}0.09319 & 0.38737 & 0.95053\end{array}$ $0.091250 .38108 \quad 0.94181$ $\begin{array}{llll}0.08935 & 0.37489 & 0.93312\end{array}$ $0.08750 \quad 0.36880 \quad 0.92448$ $\begin{array}{llll}0.08568 & 0.36281 & 0.91587\end{array}$

$\begin{array}{llll}0.08390 & 0.35691 & 0.90730\end{array}$ $\begin{array}{llll}0.08215 & 0.35110 & 0.89877\end{array}$ $\begin{array}{llll}0.08045 & 0.34538 & 0.89028\end{array}$ $\begin{array}{llll}0.07878 & 0.33976 & 0.88183\end{array}$ $\begin{array}{llll}0.07714 & 0.33422 & 0.87342\end{array}$
1100

1110

1120

1130

1140

1150

1160

1170

1180

1190

1200

1210

1220

1230

1240

1250

1260

1270

1280

1290

1300

1310

1320

1330

1340

1350

1360

1370

1380

1390

1400

1410

1420

1430

1440

1450

1460

1470

1480

1490

1500

1510

1520

1530

1540

1550

1560

1570

1580

1590 
Table II Harmonic Oscillator Contributions to the Thermodynamic Functions (in units of calories, moles, and ${ }^{\circ} \mathrm{K}$ )

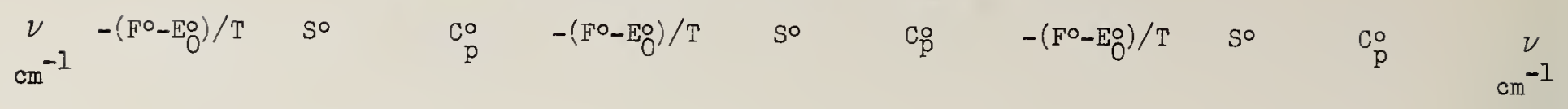

$$
T=500 .
$$

1600

1610

1620

1630

1640

1650

1660

1670

1680

1690

1700

1710

1720

1730

1740

1750

1760

1770

1780

1790

1800

1810

1820

1830

1840

1850

1860

1870

1880

1890

1900

1910

1920

1930

1940

1950

1960

1970

1980

1990

2000

2010

2020

2030

2040

2050

2060

2070

2080

2090
$0.01999 \quad 0.11250 \quad 0.43024$ $0.019420 .10984 \quad 0.42304$ $\begin{array}{lll}0.01887 & 0.10725 & 0.41593\end{array}$ $\begin{array}{llll}0.01833 & 0.10471 & 0.40891\end{array}$ 0.017810 .102230 .40199

$\begin{array}{llll}0.01730 & 0.09981 & 0.39517\end{array}$ $\begin{array}{llll}0.01681 & 0.09744 & 0.38843\end{array}$ $\begin{array}{llll}0.01633 & 0.09513 & 0.38179\end{array}$ $\begin{array}{llll}0.01586 & 0.09287 & 0.37524\end{array}$ $\begin{array}{llll}0.01541 & 0.09066 & 0.36878\end{array}$

$\begin{array}{llll}0.01497 & 0.08850 & 0.36241\end{array}$ $\begin{array}{llll}0.01455 & 0.08639 & 0.35614\end{array}$ $\begin{array}{llll}0.01413 & 0.08434 & 0.34995\end{array}$ $\begin{array}{llll}0.01373 & 0.08233 & 0.34385\end{array}$ $\begin{array}{llll}0.01334 & 0.08036 & 0.33783\end{array}$

$\begin{array}{llll}0.01296 & 0.07844 & 0.33191\end{array}$ $\begin{array}{llll}0.01259 & 0.07657 & 0.32607\end{array}$ $\begin{array}{llll}0.01223 & 0.07474 & 0.32031\end{array}$ 0.011890 .072950 .31464 $\begin{array}{lll}0.01155 & 0.07120 & 0.30906\end{array}$

0.011220 .069490 .30356 $\begin{array}{llll}0.01090 & 0.06783 & 0.29814\end{array}$ $\begin{array}{llll}0.01059 & 0.06620 & 0.29280\end{array}$ $\begin{array}{llll}0.01029 & 0.06461 & 0.28754\end{array}$ $\begin{array}{llll}0.01000 & 0.06306 & 0.28236\end{array}$

$\begin{array}{llll}0.00971 & 0.06154 & 0.27726\end{array}$ $\begin{array}{llll}0.00944 & 0.06006 & 0.27224\end{array}$ $\begin{array}{llll}0.00917 & 0.05861 & 0.26730\end{array}$ $\begin{array}{llll}0.00891 & 0.05720 & 0.26243\end{array}$ $\begin{array}{llll}0.00865 & 0.05582 & 0.25764\end{array}$

$\begin{array}{llll}0.00841 & 0.05447 & 0.25293\end{array}$ $\begin{array}{llll}0.00817 & 0.05316 & 0.24829\end{array}$ $\begin{array}{llll}0.00794 & 0.05187 & 0.24372\end{array}$ $\begin{array}{llll}0.00771 & 0.05062 & 0.23923\end{array}$ $\begin{array}{llll}0.00749 & 0.04939 & 0.23480\end{array}$

$\begin{array}{llll}0.00728 & 0.04820 & 0.23045\end{array}$ $\begin{array}{llll}0.00707 & 0.04703 & 0.22617\end{array}$ $\begin{array}{llll}0.00687 & 0.04589 & 0.22196\end{array}$ $\begin{array}{llll}0.00668 & 0.04478 & 0.21781\end{array}$ $\begin{array}{llll}0.00649 & 0.04369 & 0.21374\end{array}$

$\begin{array}{llll}0.00630 & 0.04263 & 0.20973\end{array}$ $\begin{array}{llll}0.00612 & 0.04159 & 0.20579\end{array}$ $\begin{array}{llll}0.00595 & 0.04058 & 0.20191\end{array}$ $\begin{array}{llll}0.00578 & 0.03959 & 0.19809\end{array}$ $\begin{array}{llll}0.00562 & 0.03863 & 0.19434\end{array}$

$\begin{array}{llll}0.00546 & 0.03769 & 0.19066\end{array}$ $\begin{array}{lllll}0.00530 & 0.03677 & 0.18703\end{array}$ $\begin{array}{llll}0.00515 & 0.03587 & 0.18347\end{array}$ $\begin{array}{llll}0.00500 & 0.03500 & 0.17996\end{array}$ $\begin{array}{llll}0.00486 & 0.03414 & 0.17652\end{array}$

$$
\mathrm{T}=600 \text {. }
$$

$\begin{array}{llll}0.04332 & 0.21134 & 0.65888\end{array}$ $\begin{array}{llll}0.04228 & 0.20726 & 0.65065\end{array}$ 0.041270 .203260 .64250 $\begin{array}{llll}0.04028 & 0.19933 & 0.63441\end{array}$ $\begin{array}{llll}0.03932 & 0.19547 & 0.62639\end{array}$

0.038380 .191690 .61845 $\begin{array}{llll}0.03746 & 0.18798 & 0.61057\end{array}$ $\begin{array}{llll}0.03656 & 0.18433 & 0.60276\end{array}$ $0.03569 \quad 0.18076 \quad 0.59502$ $\begin{array}{lll}0.03483 & 0.17725 & 0.58736\end{array}$

$0.03400 \quad 0.17381 \quad 0.57976$ $0.033190 .17043 \quad 0.57223$ $\begin{array}{llll}0.03240 & 0.16711 & 0.56478\end{array}$ $\begin{array}{llll}0.03162 & 0.16386 & 0.55739\end{array}$ $\begin{array}{lll}0.03087 & 0.16067 & 0.55007\end{array}$

$0.03013 \quad 0.15754 \quad 0.54283$ $\begin{array}{llll}0.02941 & 0.15446 & 0.53565\end{array}$ $\begin{array}{llll}0.02871 & 0.15145 & 0.52855\end{array}$ $\begin{array}{llll}0.02802 & 0.14849 & 0.52151\end{array}$ 0.027360 .145590 .51454

$0.02670 \quad 0.142740 .50765$ $\begin{array}{llll}0.02607 & 0.13995 & 0.50082\end{array}$ $\begin{array}{llll}0.02544 & 0.13721 & 0.49406\end{array}$ $\begin{array}{llll}0.02484 & 0.13452 & 0.48737\end{array}$ $\begin{array}{llll}0.02425 & 0.13188 & 0.48075\end{array}$

0.023670 .129290 .47420 0.023100 .126750 .46772 0.022550 .124260 .46130 0.022020 .121820 .45495 0.021490 .119420 .44868

$0.02098 \quad 0.117070 .44246$ $0.02048 \quad 0.11477 \quad 0.43632$ $0.019990 .11250 \quad 0.43024$ 0.019520 .110280 .42423 0.019050 .108110 .41829

$\begin{array}{llll}0.01860 & 0.10597 & 0.41241\end{array}$ $\begin{array}{llll}0.01816 & 0.10388 & 0.40660\end{array}$ $0.017720 .10182 \quad 0.40085$ 0.017300 .099810 .39517 $\begin{array}{lll}0.01689 & 0.09783 & 0.38955\end{array}$

$\begin{array}{llll}0.01649 & 0.09589 & 0.38399\end{array}$ $\begin{array}{llll}0.01610 & 0.09399 & 0.37850\end{array}$ $\begin{array}{llll}0.01571 & 0.09213 & 0.37308\end{array}$ $0.015340 .09030 \quad 0.36771$ $\begin{array}{llll}0.01497 & 0.08850 & 0.36241\end{array}$

$\begin{array}{llll}0.01462 & 0.08674 & 0.35718\end{array}$ $\begin{array}{llll}0.01427 & 0.08502 & 0.35200\end{array}$ $\begin{array}{llll}0.01393 & 0.08332 & 0.34689\end{array}$ $0.01360 \quad 0.08167 \quad 0.34183$ $\begin{array}{llll}0.01328 & 0.08004 & 0.33684\end{array}$
$T=700$.

$\begin{array}{llll}0.07555 & 0.32877 & 0.86505\end{array}$

$\begin{array}{llll}0.07398 & 0.32341 & 0.85673\end{array}$

$0.07245 \quad 0.318130 .84845$

$0.07095 \quad 0.312930 .84021$

$0.06948 \quad 0.307820 .83202$

$\begin{array}{llll}0.06804 & 0.30279 & 0.82387\end{array}$

$0.06663 \quad 0.29783 \quad 0.81576$

$\begin{array}{llll}0.06525 & 0.29296 & 0.80770\end{array}$

$0.06390 \quad 0.288160 .79969$

$0.06258 \quad 0.28344 \quad 0.79172$

$0.061290 .27879 \quad 0.78380$ 0.060020 .274220 .77592

$\begin{array}{llll}0.05878 & 0.26971 & 0.76809\end{array}$

$\begin{array}{llll}0.05757 & 0.26528 & 0.76031\end{array}$

$\begin{array}{lll}0.05638 & 0.26092 & 0.75258\end{array}$

0.055220 .256630 .74490

$0.05408 \quad 0.252410 .73726$

$\begin{array}{llll}0.05297 & 0.24825 & 0.72967\end{array}$

0.051870 .244160 .72213

0.050810 .240140 .71464

$\begin{array}{llll}0.04976 & 0.23618 & 0.70720\end{array}$

$\begin{array}{llll}0.04873 & 0.23228 & 0.69981\end{array}$

$\begin{array}{llll}0.04773 & 0.22845 & 0.69247\end{array}$

$\begin{array}{llll}0.04675 & 0.22467 & 0.68518\end{array}$

$\begin{array}{llll}0.04579 & 0.22096 & 0.67794\end{array}$

$\begin{array}{llll}0.04484 & 0.21730 & 0.67075\end{array}$

$\begin{array}{llll}0.04392 & 0.21371 & 0.66361\end{array}$

$\begin{array}{lll}0.04302 & 0.21017 & 0.65652\end{array}$

$0.042130 .20668 \quad 0.64948$

$\begin{array}{llll}0.04127 & 0.20326 & 0.64250\end{array}$

0.040420 .199890 .63556 $\begin{array}{llll}0.03959 & 0.19657 & 0.62868\end{array}$ $0.03878 \quad 0.19330 \quad 0.62184$ $0.03798 \quad 0.190090 .61506$ $\begin{array}{lll}0.03720 & 0.18693 & 0.60833\end{array}$

0.036440 .183820 .60165 $0.035690 .18076 \quad 0.59502$ $\begin{array}{llll}0.03495 & 0.17775 & 0.58845\end{array}$ $0.034240 .17478 \quad 0.58192$ $\begin{array}{llll}0.03354 & 0.17187 & 0.57545\end{array}$

$0.032850 .16900 \quad 0.56903$ 0.032170 .166180 .56266 $0.03151 \quad 0.16340 \quad 0.55634$ $\begin{array}{llll}0.03087 & 0.16067 & 0.55007\end{array}$ $\begin{array}{llll}0.03023 & 0.15798 & 0.54386\end{array}$

$0.02961 \quad 0.15534 \quad 0.53770$ $0.02901 \quad 0.152730 .53158$ $0.02841 \quad 0.150170 .52552$ $0.027830 .14766 \quad 0.51951$ $0.02726 \quad 0.145180 .51355$
1600

1610

1620

1630

1640

1650

1660

1670

1680

1690

1700

1710

1720

1730

1740

1750

1760

1770

1780

1790

1800

1810

1820

1830

1840

1850

1860

1870

1880

1890

1900

1910

1920

1930

1940

1950

1960

1970

1980

1990

2000

2010

2020

2030

2040

2050

2060

2070

2080

2090 
Table II Harmonic Oscillator Contributions to the Thermodynamic

Functions (in units of calories, moles, and ${ }^{\circ} \mathrm{K}$ )
$\nu_{-1}-\left(F^{\circ}-E_{O}^{\circ}\right) / T$
S०
$\mathrm{C}_{\mathrm{p}}^{\circ} \quad-\left(\mathrm{F}^{\circ}-\mathrm{E}_{\mathrm{O}}^{\circ}\right) / \mathrm{T} \quad \mathrm{S}^{\circ}$
$\mathrm{C}_{\mathrm{p}} \quad-\left(\mathrm{F}^{\circ}-\mathrm{E}_{\mathrm{O}}^{\circ}\right) / \mathrm{T}$
S०
$\mathrm{C}_{\mathrm{p}}^{\circ}$
$\mathrm{cm}^{-1}$

$\mathrm{T}=500$.

$T=600$.

$T=700$.

$\begin{array}{llll}2100 & 0.00472 & 0.03331 & 0.17313\end{array}$ $2110 \quad 0.004590 .032490 .16980$ $2120 \quad 0.00446 \quad 0.03170 \quad 0.16653$ $2130 \quad 0.00433 \quad 0.03092 \quad 0.16332$ $2140 \quad 0.004210 .030160 .16016$

$\begin{array}{lllll}2150 & 0.00409 & 0.02942 & 0.15705\end{array}$ $\begin{array}{lllll}2160 & 0.00397 & 0.02870 & 0.15400\end{array}$ $\begin{array}{lllll}2170 & 0.00386 & 0.02800 & 0.15101\end{array}$ $\begin{array}{lllll}2180 & 0.00375 & 0.02731 & 0.14806\end{array}$ $\begin{array}{llllll}2190 & 0.00365 & 0.02664 & 0.14517\end{array}$

$\begin{array}{lllll}2200 & 0.00354 & 0.02598 & 0.14233\end{array}$ $\begin{array}{llllll}2210 & 0.00344 & 0.02534 & 0.13954\end{array}$ $\begin{array}{lllll}2220 & 0.00334 & 0.02472 & 0.13680\end{array}$ $2230 \quad 0.003250 .024110 .13410$ $2240 \quad 0.00316 \quad 0.023520 .13146$

$\begin{array}{lllll}2250 & 0.00307 & 0.02294 & 0.12886\end{array}$ $\begin{array}{lllll}2260 & 0.00298 & 0.02237 & 0.12631\end{array}$ $\begin{array}{lllll}2270 & 0.00290 & 0.02182 & 0.12380\end{array}$ $\begin{array}{lllll}2280 & 0.00281 & 0.02128 & 0.12134\end{array}$ $\begin{array}{llllll}2290 & 0.00273 & 0.02076 & 0.11893\end{array}$

$\begin{array}{llll}2300 & 0.00266 & 0.02024 & 0.11656\end{array}$ $\begin{array}{lllll}2310 & 0.00258 & 0.01974 & 0.11423\end{array}$ $\begin{array}{lllll}2320 & 0.00251 & 0.01925 & 0.11194\end{array}$ $\begin{array}{lllll}2330 & 0.00244 & 0.01878 & 0.10970\end{array}$ $\begin{array}{lllll}2340 & 0.00237 & 0.01831 & 0.10750\end{array}$

$\begin{array}{lllll}2350 & 0.00230 & 0.01786 & 0.10534\end{array}$ $\begin{array}{lllll}2360 & 0.00223 & 0.01742 & 0.10322\end{array}$ $\begin{array}{lllll}2370 & 0.00217 & 0.01698 & 0.10113\end{array}$ $\begin{array}{lllll}2380 & 0.00211 & 0.01656 & 0.09909\end{array}$ $\begin{array}{lllll}2390 & 0.00205 & 0.01615 & 0.09708\end{array}$

$\begin{array}{lllll}2400 & 0.00199 & 0.01575 & 0.09511\end{array}$ $2410 \quad 0.00193 \quad 0.01536 \quad 0.09318$ $\begin{array}{lllll}2420 & 0.00188 & 0.01498 & 0.09129\end{array}$ $\begin{array}{lllll}2430 & 0.00183 & 0.01460 & 0.08943\end{array}$ $\begin{array}{lllll}2440 & 0.00177 & 0.01424 & 0.08760\end{array}$

$\begin{array}{llllll}2450 & 0.00172 & 0.01389 & 0.08581\end{array}$ $\begin{array}{lllll}2460 & 0.00168 & 0.01354 & 0.08406\end{array}$ $\begin{array}{llllll}2470 & 0.00163 & 0.01320 & 0.08233\end{array}$ $\begin{array}{llllll}2480 & 0.00158 & 0.01287 & 0.08064\end{array}$ $\begin{array}{lllll}2490 & 0.00154 & 0.01255 & 0.07899\end{array}$

$\begin{array}{lllll}2500 & 0.00149 & 0.01224 & 0.07736\end{array}$ $2510 \quad 0.001450 .011930 .07576$ $\begin{array}{llllll}2520 & 0.00141 & 0.01163 & 0.07420\end{array}$ $\begin{array}{lllll}2530 & 0.00137 & 0.01134 & 0.07267\end{array}$ $2540 \quad 0.001330 .011060 .07116$

$\begin{array}{lllll}2550 & 0.00129 & 0.01078 & 0.06969\end{array}$ $\begin{array}{lllll}2560 & 0.00126 & 0.01051 & 0.06824\end{array}$ $\begin{array}{lllll}2570 & 0.00122 & 0.01025 & 0.06682\end{array}$ $\begin{array}{llllll}2580 & 0.00119 & 0.00999 & 0.06543\end{array}$ $2590 \quad 0.001150 .009740 .06406$ $\begin{array}{llll}0.01296 & 0.07844 & 0.33191\end{array}$ 0.012650 .076880 .32704 0.012350 .075340 .32222 $0.01206 \quad 0.073840 .31747$ $0.01177 \quad 0.07236 \quad 0.31277$

$\begin{array}{lll}0.01149 & 0.07091 & 0.30814\end{array}$ 0.011220 .069490 .30356 0.010950 .068100 .29903 $0.01069 \quad 0.06674 \quad 0.29457$ $0.010440 .06540 \quad 0.29016$

0.010190 .064090 .28580 $\begin{array}{llll}0.00995 & 0.06280 & 0.28151\end{array}$ $0.009710 .06154 \quad 0.27726$ $\begin{array}{llll}0.00948 & 0.06030 & 0.27307\end{array}$ $\begin{array}{lll}0.00926 & 0.05909 & 0.26894\end{array}$

$0.009040 .05790 \quad 0.26486$ 0.008820 .056740 .26083 0.008610 .055590 .25685 0.008410 .054470 .25293 0.008210 .053370 .24906

0.008010 .052300 .24524 0.007820 .051240 .24147 0.007640 .050210 .23774 $0.00746 \quad 0.049190 .23407$ $0.00728 \quad 0.048200 .23045$

0.007110 .047220 .22688 0.006940 .046270 .22335 0.006770 .045330 .21988 0.006610 .044410 .21645 $0.00646 \quad 0.043510 .21307$

$0.00630 \quad 0.042630 .20973$ $0.006150 .04176 \quad 0.20644$ 0.006010 .040910 .20319 $0.00586 \quad 0.04008 \quad 0.19999$ 0.005720 .039270 .19684

0.005590 .038470 .19372 0.005460 .037690 .19066 0.005330 .036920 .18763 $0.00520 \quad 0.036170 .18465$ 0.005080 .035430 .18171

$\begin{array}{llll}0.00496 & 0.03471 & 0.17881\end{array}$ $0.004840 .03400 \quad 0.17595$ 0.004720 .033310 .17313 0.004610 .032630 .17035 $0.00450 \quad 0.03196 \quad 0.16762$

$0.00440 \quad 0.031310 .16492$ 0.004290 .030670 .16226 0.004190 .030040 .15964 0.004090 .029420 .15705 0.003990 .028820 .15451
$0.02670 \quad 0.14274 \quad 0.50765$ 0.026160 .140340 .50179 $\begin{array}{llll}0.02562 & 0.13799 & 0.49598\end{array}$ $0.02510 \quad 0.135670 .49023$ $0.02458 \quad 0.13338 \quad 0.48453$

$\begin{array}{llll}0.02408 & 0.13114 & 0.47887\end{array}$ 0.023590 .128930 .47327 0.023100 .126750 .46772 0.022630 .124620 .46221 0.022170 .122510 .45676

0.021710 .120440 .45136 $0.02127 \quad 0.118410 .44601$ 0.020840 .116410 .44070 0.020410 .114440 .43545 $0.019990 .11250 \quad 0.43024$

$0.01958 \quad 0.110600 .42509$ 0.019180 .108720 .41998 $\begin{array}{llll}0.01879 & 0.10688 & 0.41492\end{array}$ 0.018410 .105070 .40991 0.018030 .103290 .40495

0.017660 .101530 .40003 0.017300 .099810 .39517 $\begin{array}{llll}0.01695 & 0.09811 & 0.39035\end{array}$ $\begin{array}{llll}0.01660 & 0.09644 & 0.38557\end{array}$ $0.01626 \quad 0.09480 \quad 0.38085$

$0.01593 \quad 0.093190 .37617$ $0.01560 \quad 0.09160 \quad 0.37154$ $0.015290 .09004 \quad 0.36695$ $0.014970 .08850 \quad 0.36241$ 0.014670 .086990 .35792

$\begin{array}{llll}0.01437 & 0.08551 & 0.35347\end{array}$ $0.01408 \quad 0.084050 .34907$ $\begin{array}{llll}0.01379 & 0.08261 & 0.34471\end{array}$ 0.013510 .081200 .34040 0.013230 .079810 .33613

0.012960 .078440 .33191 $0.01270 \quad 0.07710 \quad 0.32773$ $0.01244 \quad 0.07578 \quad 0.32359$ $0.01218 \quad 0.07448 \quad 0.31950$ 0.011930 .073200 .31545

0.011690 .071940 .31144 0.011450 .070710 .30748 0.011220 .069490 .30356 $0.010990 .06830 \quad 0.29968$ $\begin{array}{llll}0.01077 & 0.06713 & 0.29584\end{array}$

$\begin{array}{llll}0.01055 & 0.06597 & 0.29204\end{array}$ 0.010330 .064830 .28829 0.010120 .063720 .28457 0.009910 .062620 .28090 0.009710 .061540 .27726
2100

2110

2120

2130

2140

2150

2160

21.70

2180

2190

2200

2.210

2220

2230

2240

2250

2260

2270

2280

2290

2300

2310

2320

2330

2340

2350

2360

2370

2380

2390

2400

2410

2420

2430

2440

2450

2460

2470

2480

2490

2500

2510

2520

2530

2540

2550

2560

2570

2580

2590 
Table II Harmonic Oscillator Contributions to the Thermodynamic

Functions (in units of calories, moles, and ${ }^{\circ} \mathrm{K}$ )

$$
\mathrm{cm}^{-1}-\left(\mathrm{F}^{\circ}-\mathrm{E}_{\mathrm{O}}^{\mathrm{O}}\right) / \mathrm{T} \quad \mathrm{S}^{\circ}
$$$$
T=500 .
$$$$
\mathrm{C}_{\mathrm{p}}^{\mathrm{O}} \quad-\left(\mathrm{F}^{\circ}-\mathrm{E}_{\mathrm{O}}^{\circ}\right) / \mathrm{T}
$$$$
\text { so }
$$

So

$-\left(F^{\circ}-E_{0}^{\circ}\right) / T \quad S^{\circ}$ $v$
2600

2610

2620

2630

2640

2650

2660

2670

2680

2690

2700

2710

2720

2730

2740

2750

2760

2770

2780

2790

2800

2810

2820

2830

2840

2850

2860

2870

2880

2890

2900

2910

2920

2930

2940

2950

2960

2970

2980

2990

3000

3010

3020

3030

3040

3050

3060

3070

3080

3090
$T=600$.

$0.00390 \quad 0.028230 .15200$

0.003810 .027650 .14953

$0.003720 .02708 \quad 0.14709$

0.003630 .026530 .14469

$0.00354 \quad 0.02598 \quad 0.14233$

$0.00346 \quad 0.025450 .14000$ $0.00338 \quad 0.02493 \quad 0.13770$ $0.00330 \quad 0.02441 \quad 0.13544$ 0.003220 .023910 .13322 0.003140 .023420 .13102

$0.00307 \quad 0.02294 \quad 0.12886$ 0.002990 .022470 .12673 $0.002920 .02200 \quad 0.12463$ $\begin{array}{llll}0.00285 & 0.02155 & 0.12257\end{array}$ $0.00279 \quad 0.021110 .12053$

$\begin{array}{llll}0.00272 & 0.02067 & 0.11853\end{array}$ $0.00266 \quad 0.020240 .11656$ 0.002590 .019830 .11462 $0.00253 \quad 0.019420 .11270$ 0.002470 .019010 .11082

0.002410 .018620 .10896 $0.00236 \quad 0.018240 .10714$ $0.00230 \quad 0.01786 \quad 0.10534$ 0.002250 .017490 .10357 0.002190 .017130 .10182

0.002140 .016770 .10011 0.002090 .016420 .09842 $0.002040 .01608 \quad 0.09675$ 0.001990 .015750 .09511 0.001940 .015420 .09350

$0.00190 \quad 0.015100 .09192$ 0.001850 .014790 .09035 $0.001810 .01448 \quad 0.08882$ $0.001770 .01418 \quad 0.08730$ 0.001720 .013890 .08581

$0.001680 .01360 \quad 0.08435$ 0.001640 .013310 .08290 0.001600 .013040 .08148 0.001570 .012760 .08009 $0.001530 .01250 \quad 0.07871$

0.001490 .012240 .07736 $0.00146 \quad 0.01198 \quad 0.07603$ 0.001420 .011730 .07472 0.001390 .011490 .07343 0.001360 .011250 .07216

0.001320 .011010 .07091 $0.001290 .01078 \quad 0.06969$ $0.001260 .01056 \quad 0.06848$ 0.001230 .010340 .06729 $0.00120 \quad 0.010120 .06612$
$T=700$

$0.009510 .06048 \quad 0.27367$ 0.009320 .059430 .27011 0.009130 .058410 .26660 $0.008940 .05740 \quad 0.26312$ $\begin{array}{llll}0.00876 & 0.05641 & 0.25969\end{array}$

$0.00858 \quad 0.05543 \quad 0.25629$ $0.008410 .05447 \quad 0.25293$ 0.008240 .053530 .24961 $0.00807 \quad 0.05260 \quad 0.24632$ $\begin{array}{llll}0.00790 & 0.05169 & 0.24307\end{array}$

$0.00774 \quad 0.05080 \quad 0.23986$ $0.00758 \quad 0.049920 .23669$ 0.007430 .049050 .23355 $0.00728 \quad 0.04820 \quad 0.23045$ 0.007130 .047360 .22739

$0.00699 \quad 0.04654 \quad 0.22436$ 0.006840 .045730 .22136 $0.00670 \quad 0.044930 .21840$ $0.00657 \quad 0.044150 .21548$ $0.00643 \quad 0.04338 \quad 0.21259$

$0.00630 \quad 0.04263 \quad 0.20973$ 0.006170 .041890 .20691 $0.00605 \quad 0.04116 \quad 0.20412$ 0.005920 .040440 .20136 $0.00580 \quad 0.03973 \quad 0.19863$

$0.00569 \quad 0.03904 \quad 0.19594$ $0.00557 \quad 0.038360 .19328$ $0.00546 \quad 0.037690 .19066$ 0.005350 .037030 .18806 $0.00524 \quad 0.03638 \quad 0.18550$

$0.00513 \quad 0.035740 .18296$ 0.005030 .035120 .18046 $0.00492 \quad 0.03450 \quad 0.17799$ $0.00482 \quad 0.03390 \quad 0.17554$ 0.004720 .033310 .17313

$0.00463 \quad 0.032720 .17075$ 0.004530 .032150 .16839 $0.00444 \quad 0.03158 \quad 0.16607$ $0.004350 .03103 \quad 0.16377$ $0.00426 \quad 0.030490 .16151$

$0.00418 \quad 0.02995 \quad 0.15927$ 0.004090 .029420 .15705 $0.00401 \quad 0.028910 .15487$ $0.00393 \quad 0.02840 \quad 0.15271$ $0.003850 .02790 \quad 0.15058$

0.003770 .027410 .14848 $\begin{array}{llll}0.00369 & 0.02692 & 0.14640\end{array}$ 0.003620 .026450 .14435 $0.00354 \quad 0.02598 \quad 0.14233$ 0.003470 .025530 .14033
2600

2610

2620

2630

2640

2650

2660

2670

2680

2690

2700

2710

2720

2730

2740

2750

2760

2770

2780

2790

2800

2810

2820

2830

2840

2850

2860

2870

2880

2890

2900

2910

2920

2930

2940

2950

2960

2970

2980

2990

3000

3010

3020

3030

3040

3050

3060

3070

3080

3090 
Table II Harmonic Oscillator Contributions to the Thermodynamic

Functions (in units of calories, moles, and ${ }^{\circ} \mathrm{K}$ )

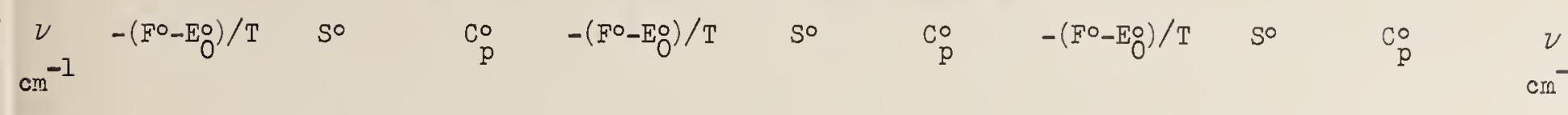

$T=500$.

3100

3110

3120

3130

3140

3150

3160

3170

3180

3190

3200

3210

3220

3230

3240

3250

3260

3270

3280

3290

3300

3310

3320

3330

3340

3350

3360

3370

3380

3390

3400

3410

3420

3430

3440

3450

3460

3470

3480

3490

3500

3510

3520

3530

3540

3550

3560

3570

3580

3590 $\begin{array}{llll}0.00027 & 0.00263 & 0.02113\end{array}$ $\begin{array}{llllll}0.00026 & 0.00257 & 0.02067\end{array}$ $0.00025 \quad 0.00250 \quad 0.02021$ $0.00024 \quad 0.00244 \quad 0.01976$ $0.00024 \quad 0.00238 \quad 0.01933$

$\begin{array}{llll}0.00023 & 0.00231 & 0.01890\end{array}$ $\begin{array}{llll}0.00022 & 0.00226 & 0.01848\end{array}$ $\begin{array}{llll}0.00022 & 0.00220 & 0.01807\end{array}$ $0.00021 \quad 0.00214 \quad 0.01767$ $\begin{array}{lll}0.00021 & 0.00209 & 0.01727\end{array}$

$0.00020 \quad 0.00203 \quad 0.01689$ 0.000190 .001980 .01651 $0.000190 .00193 \quad 0.01614$ $\begin{array}{llll}0.00018 & 0.00188 & 0.01578\end{array}$ $\begin{array}{llll}0.00018 & 0.00183 & 0.01543\end{array}$

0.000170 .001790 .01508 0.000170 .001740 .01475 $0.00016 \quad 0.001690 .01442$ 0.000160 .001650 .01409 $\begin{array}{llll}0.00015 & 0.00161 & 0.01378\end{array}$

$\begin{array}{llll}0.00015 & 0.00157 & 0.01347\end{array}$ $0.000150 .00153 \quad 0.01317$ 0.000140 .001490 .01287 0.000140 .001450 .01258 $\begin{array}{lll}0.00013 & 0.00141 & 0.01230\end{array}$

0.000130 .001380 .01202 0.000130 .001340 .01175 $\begin{array}{lllll}0.00012 & 0.00131 & 0.01148\end{array}$ $\begin{array}{llll}0.00012 & 0.00127 & 0.01122\end{array}$ 0.000120 .001240 .01097

$\begin{array}{llll}0.00011 & 0.00121 & 0.01072\end{array}$ 0.000110 .001180 .01048 $\begin{array}{llll}0.00011 & 0.00115 & 0.01024\end{array}$ 0.000100 .001120 .01001 0.000100 .001090 .00978

$0.00010 \quad 0.00106 \quad 0.00956$ $0.00009 \quad 0.00103 \quad 0.00934$ 0.000090 .001010 .00913 $\begin{array}{llll}0.00009 & 0.00098 & 0.00892\end{array}$ 0.000090 .000950 .00872

$\begin{array}{llll}0.00008 & 0.00093 & 0.00852\end{array}$ $\begin{array}{llll}0.00008 & 0.00091 & 0.00833\end{array}$ $\begin{array}{llll}0.00008 & 0.00088 & 0.00814\end{array}$ $\begin{array}{llll}0.00008 & 0.00086 & 0.00795\end{array}$ $\begin{array}{llll}0.00007 & 0.00084 & 0.00777\end{array}$

$0.00007 \quad 0.00082 \quad 0.00759$ $\begin{array}{llllll}0.00007 & 0.00079 & 0.00742\end{array}$ $\begin{array}{lllll}0.00007 & 0.00077 & 0.00725\end{array}$ $\begin{array}{lllll}0.00007 & 0.00075 & 0.00708\end{array}$ $\begin{array}{lll}0.00006 & 0.00073 \quad 0.00692\end{array}$

$$
T=600 \text {. }
$$

$T=700$.

$\begin{array}{llll}0.00117 & 0.00991 & 0.06497\end{array}$ $0.00115 \quad 0.00970 \quad 0.06384$ $0.001120 .00950 \quad 0.06273$ $0.001090 .00930 \quad 0.06163$ $0.00107 \quad 0.00910 \quad 0.06055$

$\begin{array}{llll}0.00104 & 0.00891 & 0.05949\end{array}$ $\begin{array}{llll}0.00102 & 0.00873 & 0.05845\end{array}$ $\begin{array}{llll}0.00099 & 0.00854 & 0.05743\end{array}$ $\begin{array}{lllll}0.00097 & 0.00836 & 0.05642\end{array}$ $\begin{array}{llll}0.00095 & 0.00819 & 0.05543\end{array}$

$0.000920 .00802 \quad 0.05445$ $\begin{array}{llll}0.00090 & 0.00785 & 0.05350\end{array}$ $\begin{array}{llll}0.00088 & 0.00768 & 0.05255\end{array}$ $0.00086 \quad 0.00752 \quad 0.05163$ $\begin{array}{llll}0.00084 & 0.00736 & 0.05071\end{array}$

$\begin{array}{llll}0.00082 & 0.00721 & 0.04982\end{array}$ $\begin{array}{llll}0.00080 & 0.00706 & 0.04894\end{array}$ $\begin{array}{llll}0.00078 & 0.00691 & 0.04807\end{array}$ $\begin{array}{llll}0.00076 & 0.00676 & 0.04722\end{array}$ $\begin{array}{llll}0.00074 & 0.00662 & 0.04638\end{array}$

$\begin{array}{llll}0.00073 & 0.00648 & 0.04555\end{array}$ $0.00071 \quad 0.00635 \quad 0.04474$ $\begin{array}{llll}0.00069 & 0.00621 & 0.04395\end{array}$ $0.00068 \quad 0.00608 \quad 0.04316$ $\begin{array}{llll}0.00066 & 0.00595 & 0.04239\end{array}$

$0.000640 .00583 \quad 0.04164$ $0.00063 \quad 0.00570 \quad 0.04089$ $0.000610 .00558 \quad 0.04016$ $\begin{array}{llll}0.00060 & 0.00547 & 0.03944\end{array}$ $\begin{array}{llll}0.00059 & 0.00535 & 0.03874\end{array}$

$\begin{array}{llll}0.00057 & 0.00524 & 0.03804\end{array}$ $0.00056 \quad 0.005130 .03736$ $\begin{array}{lll}0.00055 & 0.00502 & 0.03669\end{array}$ $\begin{array}{llll}0.00053 & 0.00491 & 0.03603\end{array}$ $\begin{array}{llll}0.00052 & 0.00481 & 0.03538\end{array}$

$\begin{array}{llll}0.00051 & 0.00471 & 0.03474\end{array}$ $0.00050 \quad 0.004610 .03411$ $\begin{array}{llll}0.00048 & 0.00451 & 0.03350\end{array}$ 0.000470 .004410 .03289 0.000460 .004320 .03230

$0.00045 \quad 0.00423 \quad 0.03171$ 0.000440 .004140 .03114 $0.00043 \quad 0.00405 \quad 0.03057$ $0.000420 .00396 \quad 0.03002$ $\begin{array}{llll}0.00041 & 0.00388 & 0.02947\end{array}$

$\begin{array}{llll}0.00040 & 0.00380 & 0.02894\end{array}$ $\begin{array}{llll}0.00039 & 0.00372 & 0.02841\end{array}$ $0.00038 \quad 0.003640 .02789$ $0.00037 \quad 0.00356 \quad 0.02739$ $\begin{array}{llll}0.00036 & 0.00349 & 0.02689\end{array}$
$0.00340 \quad 0.02508 \quad 0.13836$ 0.003330 .024630 .13641 0.003260 .024200 .13448 $0.00320 \quad 0.023770 .13259$ $\begin{array}{llll}0.00313 & 0.02335 & 0.13071\end{array}$

$0.00307 \quad 0.022940 .12886$ $0.00300 \quad 0.02253 \quad 0.12703$ 0.002940 .022130 .12523 0.002880 .021740 .12345 0.002820 .021360 .12169

$0.002770 .02098 \quad 0.11996$ 0.002710 .020610 .11825 $0.00266 \quad 0.020240 .11656$ $0.00260 \quad 0.01988 \quad 0.11489$ 0.002550 .019530 .11325

$0.00250 \quad 0.019190 .11162$ 0.002450 .018840 .11002 $0.00240 \quad 0.018510 .10844$ $0.00235 \quad 0.01818 \quad 0.10688$ $0.00230 \quad 0.017860 .10534$

0.002250 .017540 .10382 0.002210 .017230 .10232 0.002160 .016920 .10084 0.002120 .016620 .09938 0.002070 .016330 .09794

0.002030 .016040 .09652 0.001990 .015750 .09511 $0.00195 \quad 0.015470 .09373$ 0.001910 .015190 .09237 0.001870 .014920 .09102

0.001830 .014660 .08969 $0.00180 \quad 0.014390 .08838$ $0.00176 \quad 0.014140 .08709$ 0.001720 .013890 .08581 0.001690 .013640 .08456

0.001650 .013390 .08331 0.001620 .013150 .08209 $0.00159 \quad 0.012920 .08088$ $\begin{array}{llll}0.00156 & 0.01269 & 0.07969\end{array}$ $0.001520 .01246 \quad 0.07852$

0.001490 .012240 .07736 $0.00146 \quad 0.012020 .07622$ 0.001430 .011800 .07509 $0.00140 \quad 0.011590 .07398$ $\begin{array}{llll}0.00138 & 0.01138 & 0.07288\end{array}$

0.001350 .011180 .07180 $\begin{array}{llll}0.00132 & 0.01098 & 0.07074\end{array}$ $0.001290 .01078 \quad 0.06969$ $0.00127 \quad 0.010590 .06865$ $0.001240 .01040 \quad 0.06763$
3100

3110

3120

3130

3140

3150

3160

3170

3180

3190

3200

3210

3220

3230

3240

3250

3260

3270

3280

3290

3300

3310

3320

3330

3340

3350

3360

3370

3380

3390

3400

3410

3420

3430

3440

3450

3460

3470

3480

3490

3500

3510

3520

3530

3540

3550

3560

3570

3580

3590 
Table II Harmonic Oscillator Contributions to the Thermodynamic

Functions (in units of calories, moles, and ${ }^{\circ} \mathrm{K}$ )

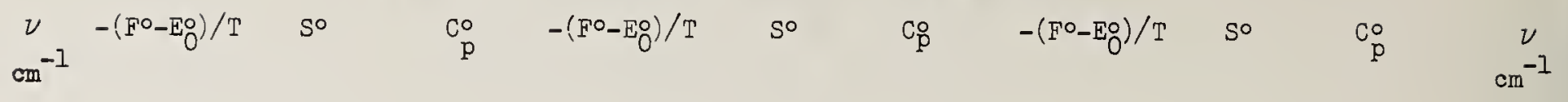

3600

3610

3620

3630

3640

3650

3660

3670

3680

3690

3700

3710

3720

3730

3740

3750

3760

3770

3780

3790

3800

3810

3820

3830

3840

3850

3860

3870

3880

3890

3900

3910

3920

3930

3940

3950

3960

3970

3980

3990

4000

\begin{abstract}
$T=500$.
$0.00006 \quad 0.000720 .00676$

$0.00006 \quad 0.00070 \quad 0.00660$

$0.00006 \quad 0.00068 \quad 0.00645$

$0.00006 \quad 0.00066 \quad 0.00630$

$0.00006 \quad 0.000640 .00616$

$0.00005 \quad 0.00063 \quad 0.00602$

$\begin{array}{lllllll}0.00005 & 0.00061 & 0.00588\end{array}$

$0.00005 \quad 0.00060 \quad 0.00574$

$0.00005 \quad 0.00058 \quad 0.00561$

$\begin{array}{llll}0.00005 & 0.00056 & 0.00548\end{array}$
\end{abstract}

$0.00005 \quad 0.00055 \quad 0.00535$

$\begin{array}{llll}0.00005 & 0.00054 & 0.00523\end{array}$

$0.00004 \quad 0.000520 .00511$

0.000040 .000510 .00499

$\begin{array}{llll}0.00004 & 0.00050 & 0.00488\end{array}$

$\begin{array}{llll}0.00004 & 0.00048 & 0.00476\end{array}$

$0.00004 \quad 0.00047 \quad 0.00465$

$0.000040 .00046 \quad 0.00454$

$0.00004 \quad 0.00045 \quad 0.00444$

$0.00004 \quad 0.00043 \quad 0.00434$

0.000040 .000420 .00424

$0.00003 \quad 0.00041 \quad 0.00414$

$\begin{array}{llll}0.00003 & 0.00040 & 0.00404\end{array}$

$0.00003 \quad 0.000390 .00395$

$\begin{array}{llll}0.00003 & 0.00038 & 0.00386\end{array}$

$\begin{array}{llll}0.00003 & 0.00037 & 0.00377\end{array}$

$\begin{array}{llll}0.00003 & 0.00036 & 0.00368\end{array}$

$0.00003 \quad 0.000350 .00359$

$0.00003 \quad 0.00034 \quad 0.00351$

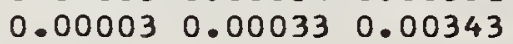

$0.00003 \quad 0.000320 .00335$

$\begin{array}{llll}0.00003 & 0.00032 & 0.00327\end{array}$

$0.00003 \quad 0.000310 .00319$

$0.000020 .00030 \quad 0.00312$

$\begin{array}{lll}0.00002 & 0.00029 & 0.00304\end{array}$

$\begin{array}{llll}0.00002 & 0.00028 & 0.00297\end{array}$

$0.000020 .00028 \quad 0.00290$

$\begin{array}{lllll}0.00002 & 0.00027 & 0.00283\end{array}$

$\begin{array}{lllll}0.00002 & 0.00026 & 0.00277\end{array}$

$0.000020 .00026 \quad 0.00270$

$\begin{array}{llll}0.00002 & 0.00025 & 0.00264\end{array}$

$$
T=600 \text {. }
$$

$\begin{array}{llll}0.00035 & 0.00341 & 0.02639\end{array}$

$\begin{array}{llllll}0.00035 & 0.00334 & 0.02591\end{array}$

0.000340 .003270 .02544

$\begin{array}{llll}0.00033 & 0.00320 & 0.02497\end{array}$

$\begin{array}{lll}0.00032 & 0.00313 & 0.02452\end{array}$

$\begin{array}{llll}0.00031 & 0.00306 & 0.02407\end{array}$

$0.00031 \quad 0.00300 \quad 0.02362$

$\begin{array}{llll}0.00030 & 0.00293 & 0.02319\end{array}$

$\begin{array}{lllll}0.00029 & 0.00287 & 0.02276\end{array}$

$\begin{array}{llll}0.00029 & 0.00281 & 0.02235\end{array}$

$0.00028 \quad 0.00275 \quad 0.02193$

$\begin{array}{lllll}0.00027 & 0.00269 & 0.02153\end{array}$

$\begin{array}{llll}0.00027 & 0.00263 & 0.02113\end{array}$

$\begin{array}{lllll}0.00026 & 0.00258 & 0.02074\end{array}$

$0.000250 .00252 \quad 0.02036$

$\begin{array}{llll}0.00025 & 0.00247 & 0.01999\end{array}$

$0.00024 \quad 0.00242 \quad 0.01962$

$0.000240 .00236 \quad 0.01925$

0.000230 .002310 .01890

$\begin{array}{lll}0.00022 & 0.00226 & 0.01855\end{array}$

0.000220 .002220 .01820

$\begin{array}{lllll}0.00021 & 0.00217 & 0.01786\end{array}$

$\begin{array}{llll}0.00021 & 0.00212 & 0.01753\end{array}$

$\begin{array}{llll}0.00020 & 0.00208 & 0.01721\end{array}$

0.000200 .002030 .01689

$\begin{array}{llll}0.00019 & 0.00199 & 0.01657\end{array}$

$0.000190 .00195 \quad 0.01626$

0.000190 .001910 .01596

$0.00018 \quad 0.00186 \quad 0.01566$

$\begin{array}{lll}0.00018 & 0.00182 & 0.01537\end{array}$

$\begin{array}{llll}0.00017 & 0.00179 & 0.01508\end{array}$

$\begin{array}{llll}0.00017 & 0.00175 & 0.01480\end{array}$

$0.00016 \quad 0.001710 .01453$

0.000160 .001670 .01425

0.000160 .001640 .01399

$0.00015 \quad 0.00160 \quad 0.01373$

$\begin{array}{lllll}0.00015 & 0.00157 & 0.01347\end{array}$

$0.000150 .00153 \quad 0.01322$

$\begin{array}{llll}0.00014 & 0.00150 & 0.01297\end{array}$

$0.00014 \quad 0.00147 \quad 0.01272$

$\begin{array}{llll}0.00014 & 0.00144 & 0.01248\end{array}$
$T=700$.

0.001220 .010210 .06662 0.001190 .010030 .06562 0.001170 .009850 .06464 $\begin{array}{llll}0.00114 & 0.00967 & 0.06368\end{array}$ $0.001120 .00950 \quad 0.06273$

3600

3610

3620

3630

3640

0.001100 .009330 .06179

0.001070 .009160 .06086

$0.00105 \quad 0.00900 \quad 0.05995$

$0.00103 \quad 0.008830 .05905$

0.001010 .008670 .05816

3650

3660

3670

3680

3690

$0.00099 \quad 0.008520 .05728$

$0.00097 \quad 0.00836 \quad 0.05642$

$\begin{array}{llll}0.00095 & 0.00821 & 0.05557\end{array}$

$0.00093 \quad 0.00807 \quad 0.05473$

0.000910 .007920 .05390

$0.000890 .00778 \quad 0.05309$

$0.00087 \quad 0.00764 \quad 0.05229$

$0.00086 \quad 0.00750 \quad 0.05149$

$0.00084 \quad 0.00736 \quad 0.05071$

$\begin{array}{llll}0.00082 & 0.00723 & 0.04994\end{array}$

0.000810 .007100 .04919

$0.00079 \quad 0.00697 \quad 0.04844$

0.000770 .006850 .04770

$\begin{array}{llll}0.00076 & 0.00672 & 0.04698\end{array}$

$0.00074 \quad 0.00660 \quad 0.04626$

$0.00073 \quad 0.00648 \quad 0.04555$

0.000710 .006360 .04486

$0.00070 \quad 0.00625 \quad 0.04417$

$0.00068 \quad 0.006140 .04350$

0.000670 .006020 .04283

3700

3710

3720

3730

3740

3750

3760

3770

3780

3790

3800

3810

3820

3830

3840

3850

3860

3870

3880

3890

$0.00066 \quad 0.00592 \quad 0.04218$

3900

0.000640 .005810 .04153

0.000630 .005700 .04089

0.000620 .005600 .04027

$0.00060 \quad 0.00550 \quad 0.03965$

3910

3920

3930

3940

$\begin{array}{llll}0.00059 & 0.00540 & 0.03904\end{array}$

3950

$\begin{array}{lllll}0.00058 & 0.00530 & 0.03844\end{array}$

$\begin{array}{llll}0.00057 & 0.00520 & 0.03784\end{array}$

$\begin{array}{llll}0.00056 & 0.00511 & 0.03726\end{array}$

$\begin{array}{lll}0.00055 & 0.00502 & 0.03669\end{array}$

3960

3980

3990

$\begin{array}{lll}0.00053 & 0.00493 \quad 0.03612\end{array}$

4000 
Table II Harmonic Oscillator Contributions to the Thermodynamic Functions (in units of calories, moles, and ${ }^{\circ} \mathrm{K}$ )
$\mathrm{cm}^{-1}-\left(\mathrm{F}^{\circ}-\mathrm{E}_{\mathrm{O}}^{\circ}\right) / \mathrm{T} \quad \mathrm{S}^{\circ}$
$\mathrm{C}_{\mathrm{p}}^{\circ} \quad-\left(\mathrm{F}^{\circ}-\mathrm{E}_{\mathrm{O}}^{\circ}\right) / \mathrm{T}$
So
$C_{p}^{\circ} \quad-\left(F^{\circ}-E_{0}^{\circ}\right) / T$
So
$\begin{array}{cc}\mathrm{c}_{\mathrm{p}}^{\circ} & \nu \\ \mathrm{cm}^{-1}\end{array}$

$T=800$.

$T=900$.

$T=1000$

$100 \quad 3.58527 \quad 5.399101 .98182$

$110 \quad 3.41318 \quad 5.21026 \quad 1.98070$

$\begin{array}{lllll}120 & 3.25753 & 5.03797 & 1.97947\end{array}$

$130 \quad 3.115674 .87958 \quad 1.97814$

$140 \quad 2.98555 \quad 4.73304 \quad 1.97670$

$\begin{array}{llll}150 & 2.86555 \quad 4.59671 & 1.97516\end{array}$

$160 \quad 2.75434 \quad 4.46929 \quad 1.97351$

$\begin{array}{llll}170 & 2.65085 \quad 4.34970 \quad 1.97176\end{array}$

$180 \quad 2.554204 .23705 \quad 1.96991$

$190 \quad 2.46364 \quad 4.13059 \quad 1.96795$

$\begin{array}{lllll}200 & 2.37854 & 4.02970 \quad 1.96588\end{array}$

$210 \quad 2.29836 \quad 3.93384 \quad 1.96372$

$220 \quad 2.22264 \quad 3.84254 \quad 1.96145$

$230 \quad 2.15097 \quad 3.75540 \quad 1.95908$

$\begin{array}{lll}240 & 2.08302 \quad 3.67207 \quad 1.95660\end{array}$

$\begin{array}{lllll}250 & 2.01846 \quad 3.59225 & 1.95403\end{array}$

$\begin{array}{lllll}260 & 1.95703 & 3.51567 & 1.95135\end{array}$

$\begin{array}{lllll}270 & 1.89849 & 3.44207 & 1.94858\end{array}$

$280 \quad 1.84262 \quad 3.37126 \quad 1.94570$

$\begin{array}{lllll}290 & 1.78924 & 3.30303 & 1.94273\end{array}$

$\begin{array}{lllll}300 & 1.73817 & 3.23722 & 1.93966\end{array}$

$\begin{array}{llllll}310 & 1.68925 & 3.17367 & 1.93648\end{array}$

$320 \quad 1.64235 \quad 3.11224 \quad 1.93322$

$\begin{array}{lllll}330 & 1.59734 & 3.05281 & 1.92985\end{array}$

$\begin{array}{llll}340 \quad 1.55411 & 2.99525 \quad 1.92639\end{array}$

$\begin{array}{lllll}350 & 1.51254 & 2.93946 & 1.92283\end{array}$

$360 \quad 1.472542 .885341 .91918$

$370 \quad 1.43402 \quad 2.83281 \quad 1.91544$

$\begin{array}{llll}380 & 1.39690 & 2.78178 & 1.91160\end{array}$

$390 \quad 1.361112 .732171 .90766$

$400 \quad 1.326572 .683921 .90364$ $\begin{array}{llll}410 & 1.29322 & 2.63697 & 1.89952\end{array}$ $\begin{array}{lllll}420 & 1.26100 & 2.59125 & 1.89532\end{array}$ $430 \quad 1.229852 .54670 \quad 1.89102$ $\begin{array}{lll}440 \quad 1.19973 & 2.50327 \quad 1.88664\end{array}$

$450 \quad 1.170592 .46093 \quad 1.88216$ $460 \quad 1.142372 .419611 .87760$ $470 \quad 1.115042 .37928 \quad 1.87296$ $\begin{array}{lllll}480 & 1.08856 & 2.33989 & 1.86822\end{array}$ $\begin{array}{lll}490 \quad 1.06289 & 2.30142 \quad 1.86341\end{array}$

$\begin{array}{lllll}500 \quad 1.03800 & 2.26383 & 1.85850\end{array}$ $\begin{array}{lllll}510 & 1.01385 & 2.22707 & 1.85352\end{array}$ $\begin{array}{lllll}520 & 0.99041 & 2.19113 & 1.84845\end{array}$ $\begin{array}{lllll}530 & 0.96766 & 2.15597 & 1.84330\end{array}$ $\begin{array}{lllll}540 & 0.94556 \quad 2.12156 \quad 1.83808\end{array}$

$\begin{array}{lllll}550 & 0.92409 & 2.08788 & 1.83277\end{array}$

$\begin{array}{lllll}560 & 0.90323 & 2.05491 & 1.82738\end{array}$

$\begin{array}{lllll}570 & 0.88295 & 2.02261 & 1.82192\end{array}$

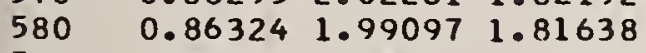

$\begin{array}{lll}590 & 0.84406 \quad 1.95997 & 1.81076\end{array}$
$3.800035 .63259 \quad 1.98294$

$3.62608 \quad 5.44364 \quad 1.98206$

3.468575 .271221 .98109

3.324865 .112691 .98003

$3.19291 \quad 4.96599 \quad 1.97890$

$3.07108 \quad 4.82950 \quad 1.97767$

$2.95806 \quad 4.701911 .97637$

$2.85278 \quad 4.582131 .97498$

$2.75434 \quad 4.46929 \quad 1.97351$

2.662004 .362631 .97196

2.575134 .261521 .97033

2.493194 .165431 .96861

2.415734 .073891 .96681

2.342333 .986501 .96493

$2.27265 \quad 3.90292 \quad 1.96297$

$2.20638 \quad 3.822831 .96093$

$2.143253 .74596 \quad 1.95881$

$2.08302 \quad 3.67207 \quad 1.95660$

2.025473 .600961 .95432

1.970423 .532421 .95196

$1.917693 .46628 \quad 1.94951$

$1.86713 \quad 3.40240 \quad 1.94699$

$1.81860 \quad 3.34063 \quad 1.94439$

$1.771973 .28084 \quad 1.94172$

$1.72712 \quad 3.22291 \quad 1.93896$

$1.68394 \quad 3.16675 \quad 1.93613$ $1.642353 .11224 \quad 1.93322$ 1.602253 .059321 .93023 $1.563573 .00788 \quad 1.92717$ $1.526212 .95786 \quad 1.92403$

1.490132 .909191 .92082 $1.455242 .86180 \quad 1.91753$ 1.421492 .815631 .91417 1.388832 .770631 .91073 $1.357212 .72675 \quad 1.90722$

1.326572 .683921 .90364 1.296872 .642121 .89999 $1.268062 .60130 \quad 1.89626$ 1.240122 .561421 .89246 $1.21300 \quad 2.52244 \quad 1.88860$

$1.18666 \quad 2.48432 \quad 1.88466$ $1.161082 .44704 \quad 1.88065$ $1.13622 \quad 2.41056 \quad 1.87658$ $1.11206 \quad 2.37486 \quad 1.87244$ 1.088562 .339891 .86822

$\begin{array}{llll}1.06570 & 2.30565 \quad 1.86395\end{array}$ $1.04346 \quad 2.27211 \quad 1.85960$ $1.02182 \quad 2.23923 \quad 1.85519$ $1.00074 \quad 2.20701 \quad 1.85072$ $0.98021 \quad 2.17541 \quad 1.84617$
$3.99392 \quad 5.84156 \quad 1.98375$

3.818465 .652521 .98303

$3.65945 \quad 5.48001 \quad 1.98224$

$3.51426 \quad 5.32138 \quad 1.98139$

$3.380835 .17458 \quad 1.98046$

$3.25753 \quad 5.03797 \quad 1.97947$

$3.14304 \quad 4.910251 .97842$

$3.036304 .79034 \quad 1.97729$

2.936424 .677361 .97610

2.842644 .570551 .97484

$2.75434 \quad 4.46929 \quad 1.97351$

$2.67098 \quad 4.37303 \quad 1.97212$

2.592094 .281321 .97066

$2.51729 \quad 4.19376 \quad 1.96913$

2.446214 .109991 .96754

$2.37854 \quad 4.02970 \quad 1.96588$

2.314033 .952631 .96416

$2.25242 \quad 3.87854 \quad 1.96237$

$2.193513 .80720 \quad 1.96051$

$2.137103 .73844 \quad 1.95859$

$2.08302 \quad 3.67207 \quad 1.95660$

$2.031113 .60795 \quad 1.95455$

$1.98124 \quad 3.54593 \quad 1.95243$

$1.93328 \quad 3.48588 \quad 1.95025$

1.887113 .427691 .94801

$1.84262 \quad 3.37126 \quad 1.94570$

1.799723 .316481 .94333

1. $758333.26327 \quad 1.94090$

$1.71835 \quad 3.21154 \quad 1.93840$

1.679713 .161221 .93584

$1.642353 .11224 \quad 1.93322$

1.606203 .064541 .93053

1.571193 .018051 .92779

$1.53728 \quad 2.97272 \quad 1.92498$

1.504412 .928501 .92211

$1.47254 \quad 2.88534 \quad 1.91918$

1.441612 .843191 .91619

1.411582 .802011 .91314

1.382432 .761771 .91003

$1.35410 \quad 2.722421 .90687$

$1.32657 \quad 2.683921 .90364$ $1.29980 \quad 2.64626 \quad 1.90035$ $1.27375 \quad 2.60939 \quad 1.89701$ 1.248422 .573291 .89361 1.223752 .537921 .89015

$1.19973 \quad 2.50327 \quad 1.88664$ 1.176342 .469311 .88307 $1.15355 \quad 2.43601 \quad 1.87944$ 1.131342 .403361 .87576 $1.109682 .37133 \quad 1.87202$
100

110

120

130

140

150

160

170

180

190

200

210

220

230

240

250

260

270

280

290

300

310

320

330

340

350

360

370

380

390

400

410

420

430

440

450

460

470

480

490

500

510

520

530

540

550

560

570

580

590 
Table II Harmonic Oscillator Contributions to the Thermodynamic Functions (in units of calories, moles, and ${ }^{\circ} \mathrm{K}$ )
$\mathrm{cm}^{-1}-\left(\mathrm{F}^{\circ}-\mathrm{E}_{\mathrm{O}}^{\circ}\right) / \mathrm{T} \quad$ So
$\mathrm{co}$
$-\left(F^{\circ}-E_{O}^{\circ}\right) / T \quad S^{\circ}$
$\mathrm{C}_{\mathrm{p}}^{\circ}$
$-\left(F^{\circ}-E_{0}\right) / T$
so
$T=800$.
$T=900$.
$T=1000$.

$\nu$

$\begin{array}{llll}600 & 0.82540 & 1.92958 & 1.80507\end{array}$

$\begin{array}{lllll}610 & 0.80725 & 1.89980 & 1.79930\end{array}$

$\begin{array}{lllll}620 & 0.78958 & 1.87059 & 1.79346\end{array}$

$\begin{array}{lllll}630 & 0.77237 & 1.84194 & 1.78756\end{array}$

$\begin{array}{llll}640 & 0.75562 & 1.81383 & 1.78157\end{array}$

$\begin{array}{llll}650 & 0.73930 & 1.78626 & 1.77552\end{array}$

$\begin{array}{lllll}660 & 0.72340 & 1.75920 & 1.76941\end{array}$

$\begin{array}{lllll}670 & 0.70790 & 1.73263 & 1.76322\end{array}$

$\begin{array}{lllll}680 & 0.69280 & 1.70656 & 1.75697\end{array}$

$\begin{array}{lllll}690 & 0.67808 & 1.68095 & 1.75065\end{array}$

$\begin{array}{lllll}700 & 0.66373 & 1.65581 & 1.74427\end{array}$

$\begin{array}{lllll}710 & 0.64973 & 1.63111 & 1.73782\end{array}$

$720 \quad 0.63608 \quad 1.60685 \quad 1.73131$

$\begin{array}{lllll}730 & 0.62277 & 1.58302 & 1.72474\end{array}$

$\begin{array}{lllll}740 & 0.60977 & 1.55960 & 1.71811\end{array}$

$\begin{array}{lllll}750 & 0.59709 & 1.53658 & 1.71142\end{array}$

$\begin{array}{lllll}760 & 0.58471 & 1.51396 & 1.70467\end{array}$

$\begin{array}{lllll}770 & 0.57263 & 1.49172 & 1.69787\end{array}$

$\begin{array}{lllll}780 & 0.56084 & 1.46985 & 1.69101\end{array}$

$\begin{array}{llll}790 & 0.54932 & 1.44835 & 1.68409\end{array}$

$800 \quad 0.53808 \quad 1.42721 \quad 1.67713$

$810 \quad 0.527091 .406421 .67010$

$\begin{array}{lllll}820 & 0.51636 & 1.38597 & 1.66303\end{array}$

$830 \quad 0.50588 \quad 1.36586 \quad 1.65591$

$\begin{array}{lllll}840 & 0.49564 & 1.34607 & 1.64874\end{array}$

850

860

870

880

890

900

910

920

930

940

950

960

970

980

990

1000

1010

1020

1030

1040

0.485631 .326601 .64151

$0.475851 .30744 \quad 1.63425$

$0.46629 \quad 1.28859 \quad 1.62693$

$0.45694 \quad 1.27004 \quad 1.61957$

$0.44781 \quad 1.25178 \quad 1.61217$

$0.43887 \quad 1.23381 \quad 1.60473$ 0.430141 .216121 .59724 $0.421601 .19870 \quad 1.58971$ $0.413241 .18156 \quad 1.58215$ $0.40507 \quad 1.16468 \quad 1.57454$

$0.39708 \quad 1.14806 \quad 1.56690$ 0.389261 .131691 .55922 $\begin{array}{llll}0.38161 & 1.11557 & 1.55151\end{array}$ $0.374131 .09970 \quad 1.54377$ $0.366801 .08406 \quad 1.53599$

1050

1060

1070

1080

1090
$0.96022 \quad 2.14442 \quad 1.84157$ $0.940742 .11401 \quad 1.83690$ $0.92175 \quad 2.084181 .83217$ $0.903232 .05491 \quad 1.82738$ $0.88518 \quad 2.02617 \quad 1.82253$

$\begin{array}{llll}0.86757 & 1.99795 & 1.81761\end{array}$ $0.850391 .97024 \quad 1.81264$ $0.833631 .94301 \quad 1.80761$ 0.817271 .916271 .80251 $0.80130 \quad 1.89000 \quad 1.79736$

$0.78571 \quad 1.864171 .79216$ 0.770491 .838791 .78689 0.755621 .813831 .78157 0.741091 .789301 .77620 $0.726891 .76517 \quad 1.77077$

0.713021 .741431 .76529 0.699471 .718091 .75975 0.686221 .695121 .75417 0.673261 .672521 .74853 $0.66059 \quad 1.65028 \quad 1.74284$

$0.64820 \quad 1.62840 \quad 1.73710$ $0.63608 \quad 1.606851 .73131$ $\begin{array}{llll}0.62423 & 1.58565 & 1.72547\end{array}$ 0.612631 .564771 .71959 $0.60128 \quad 1.54421 \quad 1.71366$

$0.59018 \quad 1.52396 \quad 1.70768$ $0.57931 \quad 1.504021 .70166$ 0.568671 .484391 .69559 $0.558261 .46504 \quad 1.68948$ $0.54806 \quad 1.44599 \quad 1.68332$

$0.53808 \quad 1.42721 \quad 1.67713$ 0.528301 .408721 .67089 0.518731 .390491 .66461 0.509351 .372531 .65829 0.500161 .354831 .65193

0.491161 .337381 .64553 0.482341 .320181 .63910 0.473701 .303231 .63263 0.465241 .286521 .62612 $0.456941 .27004 \quad 1.61957$

$0.44881 \quad 1.25380 \quad 1.61300$ $0.44084 \quad 1.23778 \quad 1.60639$ $0.43303 \quad 1.22199 \quad 1.59974$ $0.425371 .20641 \quad 1.59306$ $0.41786 \quad 1.19105 \quad 1.58636$

$0.41050 \quad 1.17590 \quad 1.57962$ $0.40328 \quad 1.16096 \quad 1.57285$ 0.396201 .146231 .56605 0.389261 .131691 .55922 0.382451 .117351 .55237
$1.08856 \quad 2.33989 \quad 1.86822$ $1.06796 \quad 2.30905 \quad 1.86438$ $\begin{array}{llll}1.04786 & 2.27876 & 1.86048\end{array}$ 1.028252 .249021 .85652 $1.00910 \quad 2.21982 \quad 1.85251$

$0.990412 .19113 \quad 1.84845$ $0.97215 \quad 2.16294 \quad 1.84434$ $0.95432 \quad 2.13524 \quad 1.84018$ $0.93690 \quad 2.10800 \quad 1.83596$ 0.919872 .081231 .83170

$0.90323 \quad 2.05491 \quad 1.82738$ $0.88696 \quad 2.02902 \quad 1.82302$ $\begin{array}{llll}0.87106 & 2.00355 & 1.81860\end{array}$ $\begin{array}{llll}0.85550 & 1.97850 \quad 1.81414\end{array}$ $0.840291 .95384 \quad 1.80963$

$0.82540 \quad 1.92958 \quad 1.80507$ 0.810841 .905711 .80046 $0.796591 .88220 \quad 1.79581$ $0.782641 .85906 \quad 1.79111$ $0.76898 \quad 1.83627 \quad 1.78636$

$\begin{array}{llll}0.75562 & 1.81383 & 1.78157\end{array}$ $0.74253 \quad 1.79173 \quad 1.77674$ $\begin{array}{llll}0.72971 & 1.76996 & 1.77186\end{array}$ $0.717151 .74851 \quad 1.76694$ $0.70485 \quad 1.72738 \quad 1.76197$

$0.69280 \quad 1.70656 \quad 1.75697$ $0.68100 \quad 1.68604 \quad 1.75192$ $0.669431 .66581 \quad 1.74683$ $0.65809 \quad 1.64588 \quad 1.74169$ $0.64698 \quad 1.62623 \quad 1.73652$

$0.63608 \quad 1.606851 .73131$ 0.625401 .587751 .72606 $0.61493 \quad 1.56892 \quad 1.72077$ $0.60466 \quad 1.55034 \quad 1.71544$ 0.594591 .532021 .71008

$\begin{array}{llll}0.58471 & 1.51396 & 1.70467\end{array}$ 0.575031 .496131 .69923 0.565521 .478551 .69376 $0.55620 \quad 1.46121 \quad 1.68825$ $0.547051 .44410 \quad 1.68270$

$0.53808 \quad 1.42721 \quad 1.67713$ $0.52927 \quad 1.41055 \quad 1.67151$ 0.520621 .394111 .66587 0.512141 .377891 .66019 0.503811 .361881 .65448

$0.49564 \quad 1.34607 \quad 1.64874$ 0.487611 .330471 .64296 $0.479731 .31507 \quad 1.63716$ $0.47200 \quad 1.29987 \quad 1.63133$ 0.464401 .284861 .62547
600

610

620

630

640

650

660

670

680

690

700

710

720

730

740

750

760

770

780

790

800

810

820

830

840

850

860

870

880

890

900

910

920

930

940

950

960

970

980

990

1000

1010

1020

1030

1040

1050

1060

1070

1080

1090 
Table II Harmonic Oscillator Contributions to the Thermodynamic

Functions (in units of calories, moles, and ${ }^{\circ} \mathrm{K}$ )

\section{$\mathrm{cm}^{-1}$}

$-\left(\mathrm{F}^{\circ}-\mathrm{E}_{\mathrm{O}} \mathrm{O}\right) / \mathrm{T} \quad \mathrm{S}^{\circ}$

$T=800$. so

$C_{p}^{\circ} \quad-(F \circ-E O) / T$

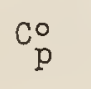

p
$-\left(F^{\circ}-E_{0}^{0}\right) / T$

$S^{\circ}$

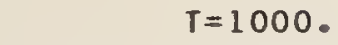

1100

1110

1120

1130

1140

1150

1160

1170

1180

1190

1200

1210

1220

1230

1240

1250

1260

1270

1280

1290

1300

1310

1320

1330

1340

1350

1360

1370

1380

1390

1400

1410

1420

1430

1440

1450

1460

1470

1480

1490

1500

1510

1520

1530

1540

1550

1560

1570

1580

1590 $\begin{array}{llll}0.29578 & 0.92673 & 1.44857\end{array}$

$0.29010 \quad 0.91366 \quad 1.44048$

$0.28454 \quad 0.90077 \quad 1.43238$

$0.27910 \quad 0.88808 \quad 1.42425$

$0.27376 \quad 0.87556 \quad 1.41611$

$0.26854 \quad 0.86323 \quad 1.40796$ $0.263420 .85108 \quad 1.39979$

$\begin{array}{lll}0.25841 & 0.83910 & 1.39160\end{array}$

$0.253490 .82729 \quad 1.38340$

$0.24868 \quad 0.81565 \quad 1.37520$

0.243960 .804171 .36698 $0.239340 .79286 \quad 1.35875$ $0.234820 .78172 \quad 1.35051$ 0.230380 .770721 .34226 0.226030 .759891 .33401

$0.221770 .74921 \quad 1.32575$ $0.21759 \quad 0.73868 \quad 1.31748$ $0.213490 .72829 \quad 1.30922$ $\begin{array}{llll}0.20948 & 0.71806 & 1.30094\end{array}$ $0.205550 .70797 \quad 1.29267$

$0.201690 .69802 \quad 1.28440$ $0.197910 .68821 \quad 1.27612$ 0.194200 .678531 .26785 $\begin{array}{llll}0.19057 & 0.66899 & 1.25957\end{array}$ 0.187010 .659591 .25130

$0.183520 .65032 \quad 1.24303$ $\begin{array}{lllll}0.18009 & 0.64117 & 1.23477\end{array}$ $0.176740 .63216 \quad 1.22651$ $0.17345 \quad 0.62327 \quad 1.21825$ $0.170220 .61450 \quad 1.21001$

$0.16705 \quad 0.60586 \quad 1.20177$ $0.163950 .59733 \quad 1.19353$ $0.16090 \quad 0.58893 \quad 1.18531$ $0.157920 .58064 \quad 1.17710$ $0.15499 \quad 0.57246 \quad 1.16889$

$0.152120 .56440 \quad 1.16070$ $0.14930 \quad 0.55645 \quad 1.15252$ $0.146540 .54861 \quad 1.14435$ $0.143830 .54088 \quad 1.13619$ $0.14118 \quad 0.53326 \quad 1.12805$

$0.13857 \quad 0.52574 \quad 1.11993$ 0.136010 .518331 .11182 $0.13351 \quad 0.51102 \quad 1.10372$ $0.131050 .50380 \quad 1.09564$ $0.128630 .49669 \quad 1.08758$

$\begin{array}{llll}0.12627 & 0.48968 & 1.07954\end{array}$ $0.12394 \quad 0.48276 \quad 1.07151$ $\begin{array}{llll}0.12167 & 0.47594 & 1.06351\end{array}$ $0.119430 .46921 \quad 1.05553$ $\begin{array}{llll}0.11724 & 0.46258 & 1.04756\end{array}$
$0.37578 \quad 1.103201 .54549$ 0.369231 .089251 .53858 $0.362801 .07548 \quad 1.53165$ $0.35650 \quad 1.06190 \quad 1.52470$ $0.35032 \quad 1.04849 \quad 1.51772$

$0.344251 .03527 \quad 1.51071$ $0.33830 \quad 1.02222 \quad 1.50369$ $0.33246 \quad 1.00934 \quad 1.49664$ $0.32673 \quad 0.99664 \quad 1.48957$ $0.32110 \quad 0.98410 \quad 1.48249$

$\begin{array}{llll}0.31558 & 0.97172 & 1.47538\end{array}$ 0.310170 .959501 .46825 0.304850 .947451 .46111 0.299630 .935551 .45395 $\begin{array}{llll}0.29451 & 0.92381 & 1.44678\end{array}$

$0.28948 \quad 0.912221 .43958$ $0.284540 .90077 \quad 1.43238$ $0.27970 \quad 0.88948 \quad 1.42516$ $0.27494 \quad 0.87833 \quad 1.41792$ 0.270270 .867321 .41068

$0.26568 \quad 0.85646 \quad 1.40342$ $\begin{array}{llll}0.26118 & 0.84573 & 1.39615\end{array}$ 0.256760 .835141 .38887 0.252420 .824691 .38158 0.248150 .814371 .37428

$0.24396 \quad 0.80417 \quad 1.36698$ 0.239850 .794111 .35966 $\begin{array}{llll}0.23581 & 0.78418 & 1.35234\end{array}$ 0.231850 .774371 .34501 0.227950 .764691 .33768

0.224120 .755121 .33034 $0.22036 \quad 0.74568 \quad 1.32299$ $0.216670 .73636 \quad 1.31565$ 0.213040 .727151 .30830 $0.209480 .71806 \quad 1.30094$

$0.20598 \quad 0.70908 \quad 1.29359$ $0.202540 .70021 \quad 1.28624$ $0.19916 \quad 0.69146 \quad 1.27888$ 0.195840 .682811 .27152 $0.19258 \quad 0.67428 \quad 1.26417$

$0.18938 \quad 0.66585 \quad 1.25682$ 0.186230 .657521 .24946 0.183130 .649301 .24211 0.180090 .641171 .23477 0.177110 .633151 .22743

0.174170 .625231 .22009 $0.171290 .61741 \quad 1.21276$ $0.168450 .60968 \quad 1.20543$ 0.165660 .602051 .19811 0.162930 .594521 .19079
$0.456941 .27004 \quad 1.61957$ 0.449621 .255411 .61366 0.442421 .240971 .60771 0.435361 .226701 .60174 $0.42842 \quad 1.212621 .59574$

$0.421601 .19870 \quad 1.58971$ $0.41490 \quad 1.18497 \quad 1.58366$ 0.408321 .171401 .57759 $0.401861 .15800 \quad 1.57149$ $0.39550 \quad 1.14476 \quad 1.56537$

$0.38926 \quad 1.131691 .55922$ $0.383131 .11878 \quad 1.55306$ 0.377101 .106021 .54687 0.371181 .093421 .54066 $0.365361 .08097 \quad 1.53443$

0.359641 .068671 .52818 0.354011 .056511 .52191 0.348481 .044511 .51562 0.343051 .032651 .50931 $0.33771 \quad 1.02092 \quad 1.50298$

$0.33246 \quad 1.009341 .49664$ $0.327290 .99790 \quad 1.49028$ 0.322220 .986591 .48390 $0.317230 .97541 \quad 1.47751$ $0.31232 \quad 0.96437 \quad 1.47111$

$0.30750 \quad 0.95346 \quad 1.46468$ $0.302750 .94267 \quad 1.45825$ $0.29808 \quad 0.932011 .45180$ $0.29350 \quad 0.92148 \quad 1.44534$ $0.28898 \quad 0.91106 \quad 1.43886$

$\begin{array}{llll}0.28454 & 0.90077 & 1.43238\end{array}$ 0.280180 .890601 .42588 $0.275890 .88055 \quad 1.41937$ 0.271660 .870611 .41285 $0.267510 .86079 \quad 1.40632$

$0.263420 .85108 \quad 1.39979$ $\begin{array}{llll}0.25940 & 0.84148 & 1.39324\end{array}$ $0.255450 .83199 \quad 1.38668$ $0.251560 .82261 \quad 1.38012$ $0.247730 .81334 \quad 1.37355$

$\begin{array}{llll}0.24396 & 0.80417 & 1.36698\end{array}$ $0.240260 .79511 \quad 1.36039$ $0.236620 .78616 \quad 1.35380$ 0.233030 .777301 .34721 $0.22950 \quad 0.76854 \quad 1.34061$

$\begin{array}{llll}0.22603 & 0.75989 & 1.33401\end{array}$ 0.222610 .751331 .32740 0.219250 .742871 .32079 $0.215940 .73451 \quad 1.31418$ 0.212690 .726231 .30756
1100 1110 1120 1130 1140

1150 1160 1170 1180 1190

1200 1210 1220 1230 1240

1250 1260 1270 1280 1290

1300

1310

1320

1330

1340

1350

1360

1370

1380

1390

1400

1410

1420

1430

1440

1450

1460

1470

1480

1490

1500

1510

1520

1530

1540

1550

1560

1570

1580

1590 
Table II Harmonic Oscillator Contributions to the Thermodynamic

Functions (in units of calories, moles, and ${ }^{\circ} \mathrm{K}$ )

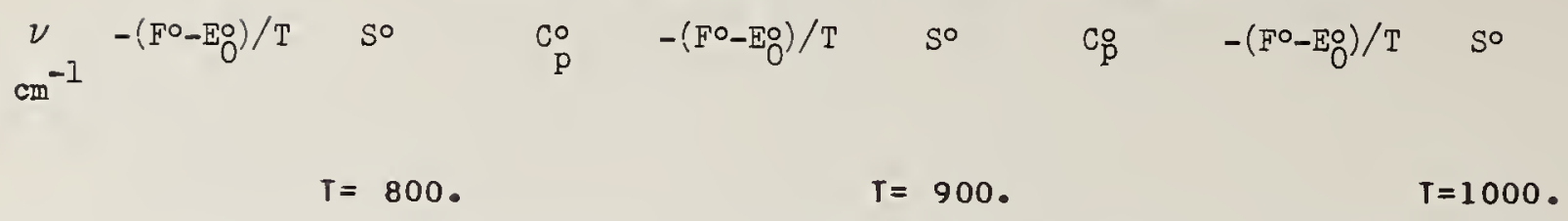

$1600 \quad 0.115090 .45604 \quad 1.03962$

$1610 \quad 0.11298 \quad 0.44958 \quad 1.03170$

$1620 \quad 0.11090 \quad 0.443221 .02380$

$1630 \quad 0.10887 \quad 0.43694 \quad 1.01592$

$1640 \quad 0.10688 \quad 0.43076 \quad 1.00807$

$1650 \quad 0.104920 .424651 .00024$ $1660 \quad 0.103000 .41863 \quad 0.99244$ $\begin{array}{lllll}1670 & 0.10112 & 0.41269 & 0.98466\end{array}$ $\begin{array}{llllll}1680 & 0.09927 & 0.40684 & 0.97691\end{array}$ $\begin{array}{lllll}1690 & 0.09746 & 0.40106 & 0.96918\end{array}$

$\begin{array}{lllll}1700 & 0.09568 & 0.39537 & 0.96148\end{array}$ $\begin{array}{llllll}1710 & 0.09393 & 0.38975 & 0.95381\end{array}$ $\begin{array}{lllll}1720 & 0.09222 & 0.38421 & 0.94616\end{array}$ $\begin{array}{lllll}1730 & 0.09054 & 0.37875 & 0.93855\end{array}$ $\begin{array}{lllll}1740 & 0.08889 & 0.37336 & 0.93096\end{array}$

$\begin{array}{lllll}1750 & 0.08727 & 0.36805 & 0.92340\end{array}$ $\begin{array}{lllll}1760 & 0.08568 & 0.36281 & 0.91587\end{array}$ $\begin{array}{llllll}1770 & 0.08412 & 0.35764 & 0.90837\end{array}$ $\begin{array}{lllll}1780 & 0.08259 & 0.35254 & 0.90090\end{array}$ $\begin{array}{lllll}1790 & 0.08108 & 0.34752 & 0.89346\end{array}$

$\begin{array}{lllll}1800 & 0.07961 & 0.34256 & 0.88605\end{array}$ $\begin{array}{llllll}1810 & 0.07816 & 0.33767 & 0.87867\end{array}$ $\begin{array}{llllll}1820 & 0.07674 & 0.33285 & 0.87132\end{array}$ $\begin{array}{llllll}1830 & 0.07535 & 0.32810 & 0.86401\end{array}$ $\begin{array}{llllll}1840 & 0.07398 & 0.32341 & 0.85673\end{array}$

$\begin{array}{lllll}1850 & 0.07264 & 0.31878 & 0.84948\end{array}$ $\begin{array}{lllll}1860 & 0.07132 & 0.31422 & 0.84226\end{array}$ $\begin{array}{llllll}1870 & 0.07002 & 0.30973 & 0.83508\end{array}$ $\begin{array}{lllll}1880 & 0.06875 & 0.30529 & 0.82793\end{array}$ $\begin{array}{lllll}1890 & 0.06751 & 0.30092 & 0.82082\end{array}$

$\begin{array}{llllll}1900 & 0.06628 & 0.29661 & 0.81374\end{array}$ $1910 \quad 0.06508 \quad 0.292350 .80670$ $\begin{array}{llllll}1920 & 0.06390 & 0.28816 & 0.79969\end{array}$ $\begin{array}{lllll}1930 & 0.06275 & 0.28402 & 0.79271\end{array}$ $\begin{array}{lllll}1940 & 0.06161 & 0.27994 & 0.78577\end{array}$

$\begin{array}{lllll}1950 & 0.06050 & 0.27592 & 0.77887\end{array}$ $\begin{array}{lllll}1960 & 0.05940 & 0.27196 & 0.77200\end{array}$ $\begin{array}{llllll}1970 & 0.05833 & 0.26804 & 0.76517\end{array}$ $\begin{array}{llllll}1980 & 0.05727 & 0.26419 & 0.75838\end{array}$ $\begin{array}{lllll}1990 & 0.05624 & 0.26038 & 0.75162\end{array}$

$\begin{array}{lllll}2000 & 0.05522 & 0.25663 & 0.74490\end{array}$ $\begin{array}{lllll}2010 & 0.05422 & 0.25293 & 0.73821\end{array}$ $2020 \quad 0.053240 .249290 .73156$ $2030 \quad 0.05228 \quad 0.245690 .72495$ $2040 \quad 0.051340 .242140 .71838$

$\begin{array}{lllll}2050 & 0.05041 & 0.23865 & 0.71185\end{array}$ $2060 \quad 0.04950 \quad 0.23520 \quad 0.70535$ $2070 \quad 0.048610 .23180 \quad 0.69889$ $\begin{array}{lllll}2080 & 0.04773 & 0.22845 & 0.69247\end{array}$ $\begin{array}{lllll}2090 & 0.04687 & 0.22514 & 0.68609\end{array}$
$0.160240 .58708 \quad 1.18348$ $0.157590 .57972 \quad 1.17618$ $0.154990 .57246 \quad 1.16889$ 0.152440 .565291 .16161 $0.14993 \quad 0.55821 \quad 1.15433$

$0.14746 \quad 0.55122 \quad 1.14707$ $0.14503 \quad 0.54431 \quad 1.13982$ $0.142650 .53748 \quad 1.13257$ $0.14030 \quad 0.53074 \quad 1.12534$ $0.13800 \quad 0.524091 .11812$

$0.135730 .51751 \quad 1.11092$ 0.133510 .511021 .10372 0.131320 .504601 .09654 $0.129170 .49826 \quad 1.08937$ $0.127050 .49201 \quad 1.08222$

$0.12497 \quad 0.48583 \quad 1.07508$ 0.122930 .479721 .06795 0.120920 .473691 .06085 $0.118940 .46773 \quad 1.05375$ $0.11700 \quad 0.46185 \quad 1.04668$

$0.115090 .45604 \quad 1.03962$ $0.113210 .45030 \quad 1.03258$ 0.111360 .444631 .02555 $0.10955 \quad 0.43903 \quad 1.01855$ $0.10776 \quad 0.43350 \quad 1.01156$

$0.10601 \quad 0.42803 \quad 1.00459$ $0.104280 .42263 \quad 0.99764$ $\begin{array}{llll}0.10258 & 0.41730 & 0.99071\end{array}$ $0.10091 \quad 0.41204 \quad 0.98380$ $\begin{array}{lll}0.09927 & 0.40684 & 0.97691\end{array}$

$0.09766 \quad 0.40170 \quad 0.97004$ $\begin{array}{llll}0.09607 & 0.39663 & 0.96319\end{array}$ $\begin{array}{llll}0.09451 & 0.39161 & 0.95636\end{array}$ $\begin{array}{llll}0.09298 & 0.38666 & 0.94956\end{array}$ $0.09147 \quad 0.38177 \quad 0.94277$

$\begin{array}{lll}0.08998 & 0.37694 & 0.93601\end{array}$ $\begin{array}{llll}0.08852 & 0.37217 & 0.92927\end{array}$ $\begin{array}{lllll}0.08709 & 0.36746 & 0.92256\end{array}$ $\begin{array}{llll}0.08568 & 0.36281 & 0.91587\end{array}$ $\begin{array}{llll}0.08429 & 0.35821 & 0.90920\end{array}$

$\begin{array}{llll}0.08292 & 0.35367 & 0.90255\end{array}$ $\begin{array}{llll}0.08158 & 0.34918 & 0.89593\end{array}$ $\begin{array}{llll}0.08026 & 0.34475 & 0.88934\end{array}$ $\begin{array}{llll}0.07896 & 0.34038 & 0.88276\end{array}$ $\begin{array}{llll}0.07769 & 0.33606 & 0.87622\end{array}$

$\begin{array}{llll}0.07643 & 0.33179 & 0.86970\end{array}$ $\begin{array}{llll}0.07519 & 0.32757 & 0.86320\end{array}$ $\begin{array}{llll}0.07398 & 0.32341 & 0.85673\end{array}$ $\begin{array}{llll}0.07278 & 0.31929 & 0.85028\end{array}$ $\begin{array}{llll}0.07161 & 0.31523 & 0.84387\end{array}$ $\begin{array}{llll}0.20948 & 0.71806 & 1.30094\end{array}$ $0.20633 \quad 0.70997 \quad 1.29433$ $0.203220 .70198 \quad 1.28771$ $0.200170 .69407 \quad 1.28109$ $\begin{array}{lll}0.19716 & 0.68626 & 1.27447\end{array}$

$0.19420 \quad 0.67853 \quad 1.26785$ $0.191290 .67089 \quad 1.26123$ 0.188430 .663341 .25461 $\begin{array}{llll}0.18560 & 0.65587 & 1.24799\end{array}$ $0.182830 .64848 \quad 1.24138$

$0.18009 \quad 0.64117 \quad 1.23477$ $0.17740 \quad 0.633951 .22816$ $0.174750 .62681 \quad 1.22156$ 0.172150 .619751 .21495 $0.169580 .61276 \quad 1.20836$

$\begin{array}{llll}0.16705 & 0.60586 & 1.20177\end{array}$ $0.16456 \quad 0.59903 \quad 1.19518$ $0.162110 .59228 \quad 1.18860$ $\begin{array}{llll}0.15970 & 0.58560 & 1.18202\end{array}$ $0.157330 .57899 \quad 1.17545$

$0.154990 .57246 \quad 1.16889$ 0.152690 .566011 .16234 0.150420 .559621 .15579 0.148190 .553311 .14925 $0.14600 \quad 0.54706 \quad 1.14272$

$0.143830 .54088 \quad 1.13619$ $0.14170 \quad 0.53478 \quad 1.12968$ $0.13961 \quad 0.52874 \quad 1.12318$ $0.137540 .52276 \quad 1.11668$ $0.135510 .51686 \quad 1.11020$

$0.133510 .51102 \quad 1.10372$ 0.131530 .505241 .09726 0.129590 .499531 .09080 $0.12768 \quad 0.49388 \quad 1.08436$ $0.12580 \quad 0.488291 .07793$

$0.123940 .48276 \quad 1.07151$ 0.122120 .477301 .06511 $0.120320 .47189 \quad 1.05872$ 0.118550 .466551 .05234 $0.11680 \quad 0.46126 \quad 1.04597$

$0.115090 .45604 \quad 1.03962$ $0.11339 \quad 0.45087 \quad 1.03328$ $0.111730 .44576 \quad 1.02696$ $0.110090 .44070 \quad 1.02065$ $0.108470 .43570 \quad 1.01435$

$0.10688 \quad 0.43076 \quad 1.00807$ $0.105310 .42586 \quad 1.00181$ 0.103770 .421030 .99556 0.102250 .416250 .98933 $0.10075 \quad 0.411520 .98311$
1600

1610

1620

1630

1640

1650

1660

1670

1680

1690

1700

1710

1720

1730

1740

1750

1760

1770

1780

1790

1800

1810

1820

1830

1840

1850

1860

1870

1880

1890

1900

1910

1920

1930

1940

1950

1960

1970

1980

1990

2000

2010

2020

2030

2040

2050 2060 2070 2080 2090 
Table II Harmonic Oscillator Contributions to the Thermodynamic

Functions (in units of calories, moles, and ${ }^{\circ} \mathrm{K}$ )
$v_{-1}-\left(F^{\circ}-E_{0}^{\circ}\right) / T \quad S^{\circ}$
$\mathrm{C}_{\mathrm{p}}^{\circ} \quad-\left(\mathrm{F}^{\circ}-\mathrm{E}_{\mathrm{O}}^{\circ}\right) / \mathrm{T}$
S०
$\mathrm{co}$
$-\left(\mathrm{F}^{\circ}-\mathrm{E}_{\mathrm{O}}\right) / \mathrm{T}$
So
$\stackrel{\mathrm{p}}{\mathrm{C}} \quad \stackrel{\nu}{\mathrm{cm}^{-1}}$

$T=800$.

$T=900$.

$T=1000$.

$\begin{array}{lllll}2100 & 0.04602 & 0.22188 & 0.67975\end{array}$

2110

2120

2130

2140

2150

2160

2170

2180

2190

2200

2210

2220

2230

2240

2250

2260

2270

2280

2290

2300

2310

2320

2330

2340

2350

2360

2370

2380

2390

2400

2410

2420

2430

2440

2450

2460

2470

2480

2490

2500

2510

2520

2530

2540

2550

2560

2570

2580

2590

0.045190 .218670 .67344

$0.04438 \quad 0.21550 \quad 0.66717$

$0.04358 \quad 0.21237 \quad 0.66095$

$0.04280 \quad 0.209290 .65476$

0.042020 .206250 .64861

0.041270 .203260 .64250

0.040520 .200300 .63643

0.039790 .197390 .63039

$0.03908 \quad 0.194520 .62440$

0.038380 .191690 .61845

$0.037680 .18890 \quad 0.61253$

0.037010 .186150 .60666

0.036340 .183430 .60082

0.035690 .180760 .59502

0.035050 .178120 .58927

0.034420 .175520 .58355

$0.03380 \quad 0.172950 .57787$

0.033190 .170430 .57223

0.032590 .167940 .56663

$0.032010 .16548 \quad 0.56108$ 0.031430 .163060 .55556 0.030870 .160670 .55007 $0.03031 \quad 0.15831 \quad 0.54463$ 0.029770 .155990 .53923

$\begin{array}{llll}0.02923 & 0.15371 & 0.53387\end{array}$ 0.028710 .151450 .52855 0.028190 .149230 .52326 $0.027690 .14703 \quad 0.51802$ $\begin{array}{lll}0.02719 & 0.14487 & 0.51281\end{array}$

$\begin{array}{llll}0.02670 & 0.14274 & 0.50765\end{array}$ 0.026220 .140640 .50252 $0.025750 .13857 \quad 0.49743$ $0.025290 .13653 \quad 0.49238$ $\begin{array}{lll}0.02484 & 0.13452 & 0.48737\end{array}$

$0.02439 \quad 0.13254 \quad 0.48240$ $\begin{array}{llll}0.02396 & 0.13058 & 0.47747\end{array}$ $\begin{array}{llll}0.02353 & 0.12865 & 0.47257\end{array}$ $\begin{array}{llll}0.02310 & 0.12675 & 0.46772\end{array}$ $0.022690 .12488 \quad 0.46290$

$\begin{array}{llll}0.02228 & 0.12304 & 0.45812\end{array}$ $\begin{array}{llll}0.02188 & 0.12122 & 0.45338\end{array}$ $0.021490 .11942 \quad 0.44868$ $\begin{array}{lll}0.02111 & 0.11766 & 0.44401\end{array}$ $0.02073 \quad 0.115910 .43938$

$0.02036 \quad 0.11420 \quad 0.43479$ $\begin{array}{llll}0.01999 & 0.11250 & 0.43024\end{array}$ $0.01963 \quad 0.110830 .42573$ $\begin{array}{llll}0.01928 & 0.10919 & 0.42125\end{array}$ 0.018940 .107570 .41681 $\begin{array}{llll}0.07045 & 0.31122 & 0.83747\end{array}$ $0.069320 .30726 \quad 0.83111$ $\begin{array}{llll}0.06820 & 0.30334 & 0.82477\end{array}$ $\begin{array}{llll}0.06710 & 0.29947 & 0.81846\end{array}$ $\begin{array}{llll}0.06602 & 0.29566 & 0.81217\end{array}$

$\begin{array}{llll}0.06495 & 0.29188 & 0.80592\end{array}$ $0.06390 \quad 0.28816 \quad 0.79969$ $\begin{array}{llll}0.06287 & 0.28448 & 0.79349\end{array}$ $\begin{array}{llll}0.06186 & 0.28085 & 0.78731\end{array}$ $\begin{array}{lll}0.06087 & 0.27726 & 0.78117\end{array}$

$\begin{array}{llll}0.05989 & 0.27371 & 0.77505\end{array}$ $\begin{array}{llll}0.05892 & 0.27021 & 0.76896\end{array}$ $\begin{array}{llll}0.05797 & 0.26675 & 0.76290\end{array}$ $\begin{array}{llll}0.05704 & 0.26334 & 0.75687\end{array}$ $\begin{array}{lll}0.05612 & 0.25996 & 0.75087\end{array}$

$\begin{array}{llll}0.05522 & 0.25663 & 0.74490\end{array}$ $\begin{array}{llll}0.05433 & 0.25334 & 0.73895\end{array}$ $\begin{array}{llll}0.05346 & 0.25009 & 0.73304\end{array}$ $\begin{array}{llll}0.05260 & 0.24688 & 0.72715\end{array}$ $\begin{array}{lll}0.05175 & 0.24371 & 0.72130\end{array}$

$\begin{array}{llll}0.05092 & 0.24058 & 0.71547\end{array}$ $\begin{array}{llll}0.05011 & 0.23749 & 0.70968\end{array}$ $\begin{array}{llll}0.04930 & 0.23444 & 0.70391\end{array}$ $0.048510 .23142 \quad 0.69818$ $\begin{array}{llll}0.04773 & 0.22845 & 0.69247\end{array}$

$\begin{array}{lllll}0.04696 & 0.22551 & 0.68680\end{array}$ $\begin{array}{llll}0.04621 & 0.22260 & 0.68115\end{array}$ $\begin{array}{llll}0.04547 & 0.21973 & 0.67554\end{array}$ $0.04474 \quad 0.21690 \quad 0.66995$ $0.044020 .21410 \quad 0.66440$

$\begin{array}{llll}0.04332 & 0.21134 & 0.65888\end{array}$ $\begin{array}{llll}0.04262 & 0.20861 & 0.65339\end{array}$ $\begin{array}{llll}0.04194 & 0.20592 & 0.64793\end{array}$ 0.041270 .203260 .64250 0.040610 .200630 .63710

$\begin{array}{llll}0.03996 & 0.19804 & 0.63173\end{array}$ $\begin{array}{llll}0.03932 & 0.19547 & 0.62639\end{array}$ $0.038690 .19294 \quad 0.62109$ $\begin{array}{llll}0.03807 & 0.19044 & 0.61581\end{array}$ $\begin{array}{lll}0.03746 & 0.18798 & 0.61057\end{array}$

$\begin{array}{llll}0.03686 & 0.18554 & 0.60536\end{array}$ $\begin{array}{llll}0.03627 & 0.18313 & 0.60017\end{array}$ $\begin{array}{llll}0.03569 & 0.18076 & 0.59502\end{array}$ $\begin{array}{lllll}0.03512 & 0.17841 & 0.58991\end{array}$ 0.034550 .17609
0.58482

$\begin{array}{llll}0.03400 & 0.17381 & 0.57976\end{array}$ $\begin{array}{llll}0.03346 & 0.17155 & 0.57473\end{array}$ $0.032920 .16932 \quad 0.56974$ $\begin{array}{llll}0.03240 & 0.16711 & 0.56478\end{array}$ $\begin{array}{lll}0.03188 & 0.16494 & 0.55985\end{array}$ $\begin{array}{llll}0.09927 & 0.40684 & 0.97691\end{array}$ $\begin{array}{llll}0.09782 & 0.40221 & 0.97073\end{array}$ $\begin{array}{llll}0.09639 & 0.39764 & 0.96456\end{array}$ $\begin{array}{llll}0.09498 & 0.39311 & 0.95841\end{array}$ 0.093590 .388640 .95228

0.092220 .384210 .94616 $0.09087 \quad 0.379840 .94007$ $0.08954 \quad 0.37551 \quad 0.93399$ 0.088230 .371230 .92793 0.086950 .366990 .92189

$\begin{array}{llll}0.08568 & 0.36281 & 0.91587\end{array}$ 0.084430 .358670 .90986 $0.08320 \quad 0.35457 \quad 0.90388$ $0.08198 \quad 0.350520 .89792$

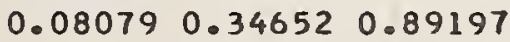

0.079610 .342560 .88605 $0.07845 \quad 0.338640 .88014$ $\begin{array}{llll}0.07731 & 0.33477 & 0.87426\end{array}$ $0.07618 \quad 0.33094 \quad 0.86839$ 0.075070 .327150 .86255

$\begin{array}{llll}0.07398 & 0.32341 & 0.85673\end{array}$ $0.07290 \quad 0.31970 \quad 0.85093$ 0.071840 .316040 .84515 $\begin{array}{llll}0.07080 & 0.31242 & 0.83939\end{array}$ 0.069770 .308840 .83365

$\begin{array}{llll}0.06875 & 0.30529 & 0.82793\end{array}$ $0.06775 \quad 0.301790 .82224$ 0.066770 .298320 .81657 $0.06580 \quad 0.29490 \quad 0.81092$ 0.064850 .291510 .80529

$\begin{array}{llll}0.06390 & 0.28816 & 0.79969\end{array}$ $0.06298 \quad 0.284850 .79410$ $0.06206 \quad 0.28157 \quad 0.78854$ $0.06116 \quad 0.278330 .78301$ $\begin{array}{llll}0.06028 & 0.27512 & 0.77749\end{array}$

$\begin{array}{llll}0.05940 & 0.27196 & 0.77200\end{array}$ $\begin{array}{llll}0.05854 & 0.26882 & 0.76653\end{array}$ $\begin{array}{lll}0.05769 & 0.26572 & 0.76109\end{array}$ $\begin{array}{llll}0.05686 & 0.26266 & 0.75567\end{array}$ $\begin{array}{llll}0.05603 & 0.25963 & 0.75027\end{array}$

0.055220 .256630 .74490 0.054420 .253670 .73954 $0.05363 \quad 0.250740 .73422$ $0.05286 \quad 0.24784 \quad 0.72892$ $0.052090 .24498 \quad 0.72364$

0.051340 .242140 .71838 $0.05059 \quad 0.23934 \quad 0.71315$ $0.04986 \quad 0.23657 \quad 0.70794$ 0.049140 .233830 .70276 0.048430 .231120 .69760
2100

2110

2120

2130

2140

2150

2160

2170

2180

2190

2200

2210

2220

2230

2240

2250

2260

2270

2280

2290

2300

2310

2320

2330

2340

2350

2360

2370

2380

2390

2400

2410

2420

2430

2440

2450

2460

2470

2480

2490

2500

2510

2520

2530

2540

2550

2560

2570

2580

2590 
Table II Harmonic Oscillator Contributions to the Thermodynamic

Functions (in units of calories, moles, and ${ }^{\circ} \mathrm{K}$ )

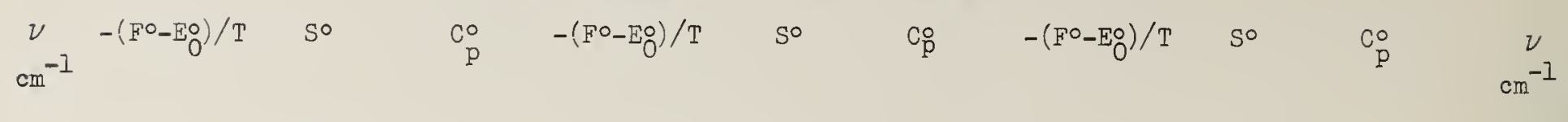

$T=800$

2600

2610

2620

2630

2640

2650

2660

2670

2680

2690

2700

2710

2720

2730

2740

2750

2760

2770

2780

2790

2800

2810

2820

2830

2840

2850

2860

2870

2880

2890

2900

2910

2920

2930

2940

2950

2960

2970

2980

2990

3000

3010

3020

3030

3040

3050

3060

3070

3080

3090
$\mathrm{T}=900$.

$0.01860 \quad 0.10597 \quad 0.41241$ $\begin{array}{llll}0.01826 & 0.10440 & 0.40804\end{array}$ $\begin{array}{lllll}0.01794 & 0.10284 & 0.40371\end{array}$ $0.01762 \quad 0.10131 \quad 0.39942$ $\begin{array}{llll}0.01730 & 0.09981 & 0.39517\end{array}$

$\begin{array}{llll}0.01699 & 0.09832 & 0.39095\end{array}$ $\begin{array}{llll}0.01669 & 0.09686 & 0.38676\end{array}$ $\begin{array}{lllll}0.01639 & 0.09541 & 0.38261\end{array}$ $\begin{array}{llll}0.01610 & 0.09399 & 0.37850\end{array}$ $\begin{array}{llll}0.01581 & 0.09259 & 0.37443\end{array}$

$\begin{array}{llll}0.01552 & 0.09121 & 0.37039\end{array}$ $\begin{array}{llll}0.01525 & 0.08984 & 0.36638\end{array}$ $\begin{array}{llll}0.01497 & 0.08850 & 0.36241\end{array}$ $\begin{array}{llll}0.01471 & 0.08718 & 0.35848\end{array}$ $\begin{array}{llll}0.01444 & 0.08588 & 0.35458\end{array}$

$\begin{array}{llll}0.01418 & 0.08459 & 0.35072\end{array}$ $\begin{array}{llll}0.01393 & 0.08332 & 0.34689\end{array}$ $\begin{array}{llll}0.01368 & 0.08208 & 0.34309\end{array}$ $\begin{array}{llll}0.01344 & 0.08085 & 0.33933\end{array}$ $\begin{array}{llll}0.01320 & 0.07964 & 0.33560\end{array}$

$\begin{array}{llll}0.01296 & 0.07844 & 0.33191\end{array}$ $\begin{array}{lllll}0.01273 & 0.07727 & 0.32825\end{array}$ $\begin{array}{llll}0.01250 & 0.07611 & 0.32462\end{array}$ $\begin{array}{llll}0.01228 & 0.07496 & 0.32103\end{array}$ $\begin{array}{llll}0.01206 & 0.07384 & 0.31747\end{array}$

$\begin{array}{llll}0.01184 & 0.07273 & 0.31394\end{array}$ $\begin{array}{llll}0.01163 & 0.07163 & 0.31045\end{array}$ $\begin{array}{llll}0.01142 & 0.07056 & 0.30698\end{array}$ $\begin{array}{llll}0.01122 & 0.06949 & 0.30356\end{array}$ $\begin{array}{lll}0.01102 & 0.06845 & 0.30016\end{array}$

$\begin{array}{llll}0.01082 & 0.06742 & 0.29679\end{array}$ $\begin{array}{llll}0.01063 & 0.06640 & 0.29346\end{array}$ $\begin{array}{llll}0.01044 & 0.06540 & 0.29016\end{array}$ $\begin{array}{llll}0.01025 & 0.06441 & 0.28689\end{array}$ $\begin{array}{llll}0.01007 & 0.06344 & 0.28365\end{array}$

$\begin{array}{llll}0.00989 & 0.06248 & 0.28044\end{array}$ $\begin{array}{llll}0.00971 & 0.06154 & 0.27726\end{array}$ $\begin{array}{llll}0.00954 & 0.06061 & 0.27412\end{array}$ $\begin{array}{llll}0.00937 & 0.05969 & 0.27100\end{array}$ $\begin{array}{llll}0.00920 & 0.05879 & 0.26791\end{array}$

$\begin{array}{llll}0.00904 & 0.05790 & 0.26486\end{array}$ $\begin{array}{llll}0.00887 & 0.05703 & 0.26183\end{array}$ $\begin{array}{llll}0.00872 & 0.05616 & 0.25883\end{array}$ $\begin{array}{llll}0.00856 & 0.05531 & 0.25587\end{array}$ $\begin{array}{llll}0.00841 & 0.05447 & 0.25293\end{array}$

$\begin{array}{llll}0.00826 & 0.05365 & 0.25002\end{array}$ $\begin{array}{llll}0.00811 & 0.05283 & 0.24714\end{array}$ $\begin{array}{llll}0.00797 & 0.05203 & 0.24429\end{array}$ $\begin{array}{llll}0.00782 & 0.05124 & 0.24147\end{array}$ $\begin{array}{llll}0.00768 & 0.05046 & 0.23867\end{array}$
$0.03137 \quad 0.16279 \quad 0.55494$ $\begin{array}{llll}0.03087 & 0.16067 & 0.55007\end{array}$ $\begin{array}{lllll}0.03037 & 0.15857 & 0.54524\end{array}$ $\begin{array}{lllll}0.02989 & 0.15651 & 0.54043\end{array}$ $\begin{array}{llll}0.02941 & 0.15446 & 0.53565\end{array}$

0.028940 .152450 .53091 $\begin{array}{lllll}0.02848 & 0.15046 & 0.52619\end{array}$ $\begin{array}{llll}0.02802 & 0.14849 & 0.52151\end{array}$ $\begin{array}{llll}0.02758 & 0.14655 & 0.51686\end{array}$ 0.027140 .144630 .51224

$0.02670 \quad 0.142740 .50765$ 0.026280 .140870 .50309 $\begin{array}{llll}0.02586 & 0.13903 & 0.49856\end{array}$ 0.025440 .137210 .49406 $\begin{array}{llll}0.02504 & 0.13541 & 0.48959\end{array}$

0.024640 .133630 .48516 $0.024250 .13188 \quad 0.48075$ $\begin{array}{llll}0.02386 & 0.13015 & 0.47638\end{array}$ $\begin{array}{llll}0.02348 & 0.12844 & 0.47203\end{array}$ $\begin{array}{llll}0.02310 & 0.12675 & 0.46772\end{array}$

$0.022740 .12509 \quad 0.46343$ $0.02237 \quad 0.123440 .45918$ $0.022020 .12182 \quad 0.45495$ $0.02166 \quad 0.12022 \quad 0.45076$ 0.021320 .118630 .44660

$0.02098 \quad 0.11707 \quad 0.44246$ $0.020640 .11553 \quad 0.43836$ 0.020320 .114010 .43429 $\begin{array}{llll}0.01999 & 0.11250 & 0.43024\end{array}$ $0.01967 \quad 0.11102 \quad 0.42623$

$\begin{array}{llll}0.01936 & 0.10955 & 0.42224\end{array}$ $0.01905 \quad 0.108110 .41829$ $0.018750 .10668 \quad 0.41436$ 0.018450 .105270 .41046 $\begin{array}{llll}0.01816 & 0.10388 & 0.40660\end{array}$

$\begin{array}{llll}0.01787 & 0.10250 & 0.40276\end{array}$ $0.01758 \quad 0.10115 \quad 0.39895$ $\begin{array}{llll}0.01730 & 0.09981 & 0.39517\end{array}$ $\begin{array}{llll}0.01703 & 0.09848 & 0.39141\end{array}$ $\begin{array}{llll}0.01675 & 0.09718 & 0.38769\end{array}$

$\begin{array}{llll}0.01649 & 0.09589 & 0.38399\end{array}$ $\begin{array}{llll}0.01623 & 0.09462 & 0.38033\end{array}$ $\begin{array}{llll}0.01597 & 0.09336 & 0.37669\end{array}$ $\begin{array}{llll}0.01571 & 0.09213 & 0.37308\end{array}$ $\begin{array}{llll}0.01546 & 0.09090 & 0.36950\end{array}$

$\begin{array}{llll}0.01522 & 0.08969 & 0.36594\end{array}$ $\begin{array}{llll}0.01497 & 0.08850 & 0.36241\end{array}$ $\begin{array}{llll}0.01474 & 0.08733 & 0.35892\end{array}$ $\begin{array}{llll}0.01450 & 0.08616 & 0.35544\end{array}$ $\begin{array}{lll}0.01427 & 0.08502 & 0.35200\end{array}$
$T=1000$

$\begin{array}{llll}0.04773 & 0.22845 & 0.69247\end{array}$ $\begin{array}{llll}0.04704 & 0.22580 & 0.68736\end{array}$ $\begin{array}{llll}0.04636 & 0.22318 & 0.68228\end{array}$ $\begin{array}{llll}0.04569 & 0.72059 & 0.67722\end{array}$ $\begin{array}{llll}0.04503 & 0.21803 & 0.67218\end{array}$

$0.04438 \quad 0.21550 \quad 0.66717$ 0.043740 .212990 .66219 0.043110 .210520 .65723 $\begin{array}{llll}0.04249 & 0.20807 & 0.65229\end{array}$ 0.041870 .205650 .64738

$0.041270 .20326 \quad 0.64250$ $0.04067 \quad 0.200890 .63764$ $0.04008 \quad 0.198550 .63280$ 0.039510 .196240 .62799 $0.03894 \quad 0.19395 \quad 0.62321$

0.038380 .191690 .61845 0.037820 .189450 .61371 0.037280 .187240 .60900 $0.03674 \quad 0.18506 \quad 0.60432$ $\begin{array}{llll}0.03621 & 0.18289 & 0.59966\end{array}$

$0.035690 .18076 \quad 0.59502$ 0.035170 .178640 .59042 0.034670 .176550 .58583 $0.034170 .17449 \quad 0.58127$ $0.033670 .17245 \quad 0.57674$

$0.033190 .17043 \quad 0.57223$ $0.03271 \quad 0.168430 .56775$ $0.032240 .16646 \quad 0.56329$ $0.03178 \quad 0.16451 \quad 0.55886$ $0.031320 .16258 \quad 0.55446$

$\begin{array}{llll}0.03087 & 0.16067 & 0.55007\end{array}$ $0.030420 .15878 \quad 0.54572$ 0.029990 .156920 .54139 0.029550 .155070 .53708 0.029130 .153250 .53280

0.028710 .151450 .52855 $0.02830 \quad 0.14967 \quad 0.52432$ 0.027890 .147910 .52011 0.027490 .146170 .51593 $\begin{array}{llll}0.02709 & 0.14444 & 0.51178\end{array}$

$0.02670 \quad 0.142740 .50765$ $0.026320 .14106 \quad 0.50354$ $0.025940 .13940 \quad 0.49946$ $\begin{array}{llll}0.02557 & 0.13775 & 0.49541\end{array}$ $0.02520 \quad 0.136130 .49138$

$\begin{array}{llll}0.02484 & 0.13452 & 0.48737\end{array}$ $0.02448 \quad 0.132930 .48339$ 0.024130 .131360 .47944 0.023780 .129810 .47550 0.023440 .128270 .47160
2600

2610

2620

2630

2640

2650

2660

2670

2680

2690

2700

2710

2720

2730

2740

2750

2760

2770

2780

2790

2800

2810

2820

2830

2840

2850

2860

2870

2880

2890

2900

2910

2920

2930

2940

2950

2960

2970

2980

2990

3000

3010

3020

3030

3040

3050

3060

3070

3080

3090 
Table II Harmonic Oscillator Contributions to the Thermodynamic

Functions (in units of calories, moles, and ${ }^{\circ} \mathrm{K}$ )
$\nu_{-1}-\left(F^{\circ}-E_{0}^{\circ}\right) / T \quad$ So
$\mathrm{C}_{\mathrm{p}}^{\circ} \quad-\left(\mathrm{F}^{\circ}-\mathrm{E}_{\mathrm{O}} \mathrm{O}\right) / \mathrm{T}$
So
$\mathrm{cop}_{\mathrm{p}}$
$-(F \circ-E O) / T$

so

$\nu$

$\mathrm{cm}^{-1}$

$T=800$.

$\begin{array}{llll}3100 & 0.00755 & 0.04970 & 0.23590\end{array}$

$\begin{array}{lllll}3110 & 0.00741 & 0.04894 & 0.23316\end{array}$

$\begin{array}{llll}3120 & 0.00728 & 0.04820 & 0.23045\end{array}$

$\begin{array}{lllll}3130 & 0.00715 & 0.04746 & 0.22777\end{array}$

$\begin{array}{llll}3140 & 0.00702 & 0.04674 & 0.22511\end{array}$

$\begin{array}{llll}3150 & 0.00690 & 0.04603 & 0.22248\end{array}$

$\begin{array}{lllll}3160 & 0.00677 & 0.04533 & 0.21988\end{array}$

$\begin{array}{llll}3170 & 0.00665 & 0.04464 & 0.21730\end{array}$

$\begin{array}{lllll}3180 & 0.00653 & 0.04396 & 0.21475\end{array}$

$\begin{array}{lllll}3190 & 0.00642 & 0.04329 & 0.21223\end{array}$

$\begin{array}{lllll}3200 & 0.00630 & 0.04263 & 0.20973\end{array}$

$\begin{array}{lllll}3210 & 0.00619 & 0.04198 & 0.20726\end{array}$

$\begin{array}{llllll}3220 & 0.00608 & 0.04134 & 0.20481\end{array}$

$\begin{array}{llllll}3230 & 0.00597 & 0.04071 & 0.20239\end{array}$

$\begin{array}{lllll}3240 & 0.00586 & 0.04008 & 0.19999\end{array}$

$\begin{array}{lllll}3250 & 0.00576 & 0.03947 & 0.19762\end{array}$

$\begin{array}{llllll}3260 & 0.00566 & 0.03887 & 0.19528\end{array}$

$\begin{array}{llllll}3270 & 0.00556 & 0.03827 & 0.19295\end{array}$

$\begin{array}{llllll}3280 & 0.00546 & 0.03769 & 0.19066\end{array}$

$\begin{array}{lllll}3290 & 0.00536 & 0.03711 & 0.18838\end{array}$

$\begin{array}{lllll}3300 & 0.00526 & 0.03654 & 0.18613\end{array}$

$\begin{array}{llllll}3310 & 0.00517 & 0.03598 & 0.18391\end{array}$

$\begin{array}{lllll}3320 & 0.00508 & 0.03543 & 0.18171\end{array}$

$\begin{array}{llllll}3330 & 0.00499 & 0.03489 & 0.17953\end{array}$

$\begin{array}{lllll}3340 & 0.00490 & 0.03435 & 0.17737\end{array}$

$\begin{array}{lllll}3350 & 0.00481 & 0.03383 & 0.17524\end{array}$

$\begin{array}{lllll}3360 & 0.00472 & 0.03331 & 0.17313\end{array}$

$\begin{array}{lllll}3370 & 0.00464 & 0.03279 & 0.17104\end{array}$

$\begin{array}{lllll}3380 & 0.00456 & 0.03229 & 0.16898\end{array}$

$\begin{array}{lllll}3390 & 0.00448 & 0.03179 & 0.16694\end{array}$

$\begin{array}{lllll}3400 & 0.00440 & 0.03131 & 0.16492\end{array}$

$\begin{array}{lllll}3410 & 0.00432 & 0.03082 & 0.16292\end{array}$

$\begin{array}{lllll}3420 & 0.00424 & 0.03035 & 0.16094\end{array}$

$\begin{array}{lllll}3430 & 0.00416 & 0.02988 & 0.15899\end{array}$

$\begin{array}{llll}3440 & 0.00409 & 0.02942 & 0.15705\end{array}$

$\begin{array}{lllll}3450 & 0.00402 & 0.02897 & 0.15514\end{array}$

$\begin{array}{lllll}3460 & 0.00395 & 0.02852 & 0.15325\end{array}$

$\begin{array}{lllll}3470 & 0.00388 & 0.02808 & 0.15138\end{array}$

$\begin{array}{lllll}3480 & 0.00381 & 0.02765 & 0.14953\end{array}$

$\begin{array}{lllll}3490 & 0.00374 & 0.02723 & 0.14770\end{array}$

$\begin{array}{llllll}3500 & 0.00367 & 0.02681 & 0.14589\end{array}$

$\begin{array}{llllll}3510 & 0.00361 & 0.02639 & 0.14410\end{array}$

$\begin{array}{lllll}3520 & 0.00354 & 0.02598 & 0.14233\end{array}$

$\begin{array}{lllll}3530 & 0.00348 & 0.02558 & 0.14058\end{array}$

$\begin{array}{llllll}3540 & 0.00342 & 0.02519 & 0.13885\end{array}$

$\begin{array}{lllll}3550 & 0.00336 & 0.02480 & 0.13714\end{array}$ $\begin{array}{llllll}3560 & 0.00330 & 0.02441 & 0.13544\end{array}$

$\begin{array}{llllll}3570 & 0.00324 & 0.02404 & 0.13377\end{array}$

$\begin{array}{llllll}3580 & 0.00318 & 0.02367 & 0.13211\end{array}$

3590

$$
T=900
$$

0.014040 .083890 .34858 0.013820 .082770 .34519

0.013600 .081670 .34183

0.013380 .080580 .33850

$0.013170 .07950 \quad 0.33519$

$0.01296 \quad 0.07844 \quad 0.33191$

$0.012750 .07740 \quad 0.32865$

0.012550 .076360 .32542

$0.01235 \quad 0.075340 .32222$

0.012160 .074340 .31905

$0.01196 \quad 0.073340 .31590$ $0.01177 \quad 0.07236 \quad 0.31277$ $0.01158 \quad 0.071390 .30967$ $0.01140 \quad 0.070440 .30660$ 0.011220 .069490 .30356

$0.01104 \quad 0.06856 \quad 0.30053$ $0.01087 \quad 0.06764 \quad 0.29754$ $0.010690 .06674 \quad 0.29457$ 0.010520 .065840 .29162 $0.010350 .06496 \quad 0.28870$

0.010190 .064090 .28580 $0.01003 \quad 0.063230 .28293$ 0.009870 .062380 .28009 0.009710 .061540 .27726 0.009560 .060710 .27446

$0.009410 .05990 \quad 0.27169$ 0.009260 .059090 .26894 $0.009110 .05830 \quad 0.26621$ $\begin{array}{llll}0.00896 & 0.05751 & 0.26351\end{array}$ 0.008820 .056740 .26083

$\begin{array}{llll}0.00868 & 0.05597 & 0.25817\end{array}$ 0.008540 .055220 .25554 0.008410 .054470 .25293 0.008270 .053740 .25034 0.008140 .053010 .24778

0.008010 .052300 .24524 0.007890 .051590 .24272 $\begin{array}{llll}0.00776 & 0.05090 & 0.24022\end{array}$ 0.007640 .050210 .23774 $\begin{array}{llll}0.00752 & 0.04953 & 0.23529\end{array}$

$0.00740 \quad 0.048860 .23286$ 0.007280 .048200 .23045 $0.00716 \quad 0.047550 .22807$ $0.007050 .04690 \quad 0.22570$ 0.006940 .046270 .22335

0.006830 .045640 .22103 0.006720 .045020 .21873 0.006610 .044410 .21645 0.006510 .043810 .21419 $0.00640 \quad 0.043210 .21195$
$T=1000$.

$0.02310 \quad 0.126750 .46772$ $0.02277 \quad 0.125250 .46386$ $0.02244 \quad 0.123770 .46003$ 0.022120 .122310 .45622 $0.02180 \quad 0.12086 \quad 0.45244$

0.021490 .119420 .44868 $0.02118 \quad 0.118010 .44494$ 0.020880 .116610 .44123 $0.02058 \quad 0.115220 .43754$ $0.02028 \quad 0.11386 \quad 0.43388$

3100 3110 3120 3130 3140

3150

3160

3170

3180

3190

$\begin{array}{llll}0.01999 & 0.11250 & 0.43024\end{array}$ $\begin{array}{lllll}0.01971 & 0.11117 & 0.42663\end{array}$ $0.01942 \quad 0.10984 \quad 0.42304$ $0.019140 .10854 \quad 0.41947$ $\begin{array}{lll}0.01887 & 0.10725 & 0.41593\end{array}$

3200

3210

3220

3230

3240

$0.01860 \quad 0.10597 \quad 0.41241$ 0.018330 .104710 .40891 $0.01807 \quad 0.10346 \quad 0.40544$ 0.017810 .102230 .40199 0.017550 .101010 .39857

3250

3260

3270

3280

3290

$\begin{array}{llll}0.01730 & 0.09981 & 0.39517\end{array}$ $\begin{array}{llll}0.01705 & 0.09862 & 0.39179\end{array}$ $\begin{array}{llll}0.01681 & 0.09744 & 0.38843\end{array}$ $\begin{array}{llll}0.01657 & 0.09628 & 0.38510\end{array}$ $\begin{array}{llll}0.01633 & 0.09513 & 0.38179\end{array}$

3300 3310 3320 3330 3340

$\begin{array}{llll}0.01610 & 0.09399 & 0.37850\end{array}$ $\begin{array}{llll}0.01586 & 0.09287 & 0.37524\end{array}$ $\begin{array}{llll}0.01564 & 0.09176 & 0.37200\end{array}$ $\begin{array}{llll}0.01541 & 0.09066 & 0.36878\end{array}$ $\begin{array}{llll}0.01519 & 0.08957 & 0.36559\end{array}$

3350 3360 3370 3380 3390

$\begin{array}{llll}0.01497 & 0.08850 & 0.36241\end{array}$ $\begin{array}{llll}0.01476 & 0.08744 & 0.35926\end{array}$ $\begin{array}{llll}0.01455 & 0.08639 & 0.35614\end{array}$ $\begin{array}{llll}0.01434 & 0.08536 & 0.35303\end{array}$ 0.014130 .084340 .34995

3400 3410 3420 3430 3440

$\begin{array}{llll}0.01393 & 0.08332 & 0.34689\end{array}$ $\begin{array}{llll}0.01373 & 0.08233 & 0.34385\end{array}$ $\begin{array}{llll}0.01353 & 0.08134 & 0.34083\end{array}$ $\begin{array}{llll}0.01334 & 0.08036 & 0.33783\end{array}$ $\begin{array}{llll}0.01315 & 0.07940 & 0.33486\end{array}$

3450 3460 3470 3480 3490

$\begin{array}{llll}0.01296 & 0.07844 & 0.33191\end{array}$ $\begin{array}{llll}0.01278 & 0.07750 & 0.32898\end{array}$ $\begin{array}{llll}0.01259 & 0.07657 & 0.32607\end{array}$ $\begin{array}{llll}0.01241 & 0.07565 & 0.32318\end{array}$ $\begin{array}{llll}0.01223 & 0.07474 & 0.32031\end{array}$

3500 3510 3520 3530 3540

$\begin{array}{llll}0.01206 & 0.07384 & 0.31747\end{array}$ $\begin{array}{llll}0.01189 & 0.07295 & 0.31464\end{array}$ 0.011720 .072070 .31184 $\begin{array}{llll}0.01155 & 0.07120 & 0.30906\end{array}$ $\begin{array}{lll}0.01138 & 0.07034 & 0.30630\end{array}$
3550 3560 3570 3580 3590 
Table II Harmonic Oscillator Contributions to the Thermodynamic Functions (in units of calories, moles, and ${ }^{\circ} \mathrm{K}$ )

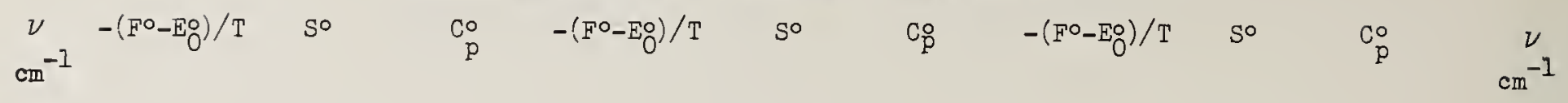

$T=800 . \quad T=900$.

$\begin{array}{llll}3600 & 0.00307 & 0.02294 & 0.12886\end{array}$

$\begin{array}{llll}3610 & 0.00301 & 0.02258 & 0.12726\end{array}$

$\begin{array}{llll}3620 & 0.00296 & 0.02223 & 0.12568\end{array}$

$\begin{array}{llll}3630 & 0.00291 & 0.02189 & 0.12411\end{array}$

$\begin{array}{llll}3640 & 0.00285 & 0.02155 & 0.12257\end{array}$

$\begin{array}{llll}3650 & 0.00280 & 0.02122 & 0.12104\end{array}$

$\begin{array}{llll}3660 & 0.00275 & 0.02089 & 0.11953\end{array}$

$\begin{array}{llll}3670 & 0.00270 & 0.02056 & 0.11804\end{array}$

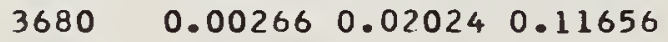

$\begin{array}{llll}3690 & 0.00261 & 0.01993 & 0.11510\end{array}$

$\begin{array}{lllll}3700 & 0.00256 & 0.01962 & 0.11365\end{array}$

$\begin{array}{lllll}3710 & 0.00252 & 0.01931 & 0.11223\end{array}$

$\begin{array}{lllll}3720 & 0.00247 & 0.01901 & 0.11082\end{array}$

$\begin{array}{lllll}3730 & 0.00243 & 0.01872 & 0.10942\end{array}$

$\begin{array}{lllll}3740 & 0.00238 & 0.01843 & 0.10805\end{array}$

$\begin{array}{llllll}3750 & 0.00234 & 0.01814 & 0.10668\end{array}$

$\begin{array}{lllll}3760 & 0.00230 & 0.01786 & 0.10534\end{array}$

$\begin{array}{lllll}3770 & 0.00226 & 0.01758 & 0.10401\end{array}$

$\begin{array}{lllll}3780 & 0.00222 & 0.01731 & 0.10269\end{array}$

$\begin{array}{lllll}3790 & 0.00218 & 0.01704 & 0.10139\end{array}$

$\begin{array}{lllll}3800 & 0.00214 & 0.01677 & 0.10011\end{array}$

$\begin{array}{llllll}3810 & 0.00210 & 0.01651 & 0.09884\end{array}$

$\begin{array}{lllll}3820 & 0.00206 & 0.01625 & 0.09758\end{array}$

$\begin{array}{lllll}3830 & 0.00203 & 0.01600 & 0.09634\end{array}$

$\begin{array}{lllll}3840 & 0.00199 & 0.01575 & 0.09511\end{array}$

3850

3860

3870

3880

3890

3900

3910

3920

3930

3940

3950

3960

3970

3980

3990

4000

$\begin{array}{llll}0.00630 & 0.04263 & 0.20973\end{array}$

$\begin{array}{llll}0.00620 & 0.04205 & 0.20753\end{array}$

$\begin{array}{llll}0.00610 & 0.04148 & 0.20535\end{array}$

$\begin{array}{llll}0.00601 & 0.04091 & 0.20319\end{array}$

0.005910 .040360 .20105

$\begin{array}{llll}0.00582 & 0.03981 & 0.19894\end{array}$

$0.00572 \quad 0.03927 \quad 0.19684$

$0.00563 \quad 0.038730 .19476$

$\begin{array}{llll}0.00554 & 0.03821 & 0.19270\end{array}$

$\begin{array}{lll}0.00546 & 0.03769 & 0.19066\end{array}$

0.005370 .037170 .18863

0.005280 .036670 .18663

$0.00520 \quad 0.036170 .18465$

0.005120 .035670 .18268

$0.00504 \quad 0.035190 .18074$

$0.00496 \quad 0.034710 .17881$

0.004880 .034230 .17690

$0.00480 \quad 0.033770 .17501$

0.004720 .033310 .17313

0.004650 .032850 .17128

$\begin{array}{llll}0.00458 & 0.03240 & 0.16944\end{array}$

$\begin{array}{llll}0.00450 & 0.03196 & 0.16762\end{array}$

$\begin{array}{llll}0.00443 & 0.03152 & 0.16581\end{array}$

0.004360 .031090 .16403

0.004290 .030670 .16226

$\begin{array}{llll}0.00196 & 0.01550 & 0.09390\end{array}$

$\begin{array}{llll}0.00192 & 0.01526 & 0.09271\end{array}$

$\begin{array}{lll}0.00189 & 0.01502 & 0.09152\end{array}$

$\begin{array}{lll}0.00185 & 0.01479 & 0.09035\end{array}$

$\begin{array}{lll}0.00182 & 0.01456 & 0.08920\end{array}$

$0.00179 \quad 0.014330 .08806$

$\begin{array}{lllll}0.00176 & 0.01411 & 0.08693\end{array}$

$\begin{array}{llll}0.00172 & 0.01389 & 0.08581\end{array}$

0.001690 .013670 .08471

0.001660 .013450 .08362

$\begin{array}{llll}0.00163 & 0.01324 & 0.08255\end{array}$

$\begin{array}{llll}0.00160 & 0.01304 & 0.08148\end{array}$

0.001580 .012830 .08043

$0.001550 .01263 \quad 0.07940$

0.001520 .012430 .07837

0.001490 .012240 .07736 $\begin{array}{llll}0.00422 & 0.03025 & 0.16051\end{array}$

$\begin{array}{llll}0.00416 & 0.02983 & 0.15877\end{array}$

$\begin{array}{llll}0.00409 & 0.02942 & 0.15705\end{array}$

$\begin{array}{llll}0.00403 & 0.02902 & 0.15535\end{array}$

$\begin{array}{llll}0.00396 & 0.02862 & 0.15367\end{array}$

$0.00390 \quad 0.028230 .15200$ $\begin{array}{llll}0.00384 & 0.02784 & 0.15035\end{array}$

$\begin{array}{llll}0.00378 & 0.02746 & 0.14871\end{array}$

$\begin{array}{llll}0.00372 & 0.02708 & 0.14709\end{array}$

$\begin{array}{llll}0.00366 & 0.02671 & 0.14549\end{array}$

0.003600 .026350 .14390

0.003540 .025980 .14233

0.003490 .025630 .14077

0.003430 .025270 .13923

0.003380 .024930 .13770

0.003320 .024580 .13619
$T=1000$

0.011220 .069490 .30356

$0.01106 \quad 0.06866 \quad 0.30084$

$0.01090 \quad 0.067830 .29814$

$0.01074 \quad 0.067010 .29546$

0.010590 .066200 .29280

3600

3610

3620

3630

3640

$0.010440 .06540 \quad 0.29016$

$\begin{array}{llllll}0.01029 & 0.06461 & 0.28754\end{array}$

$\begin{array}{llll}0.01014 & 0.06383 & 0.28494\end{array}$

$0.01000 \quad 0.06306 \quad 0.28236$

$\begin{array}{llll}0.00985 & 0.06229 & 0.27980\end{array}$

3650

3660

3670

3680

3690

$\begin{array}{lll}0.00971 & 0.06154 & 0.27726\end{array}$ $0.00957 \quad 0.06080 \quad 0.27474$

$0.009440 .06006 \quad 0.27224$

$0.00930 \quad 0.059330 .26976$

$\begin{array}{llll}0.00917 & 0.05861 & 0.26730\end{array}$

3700

3710

3720

3730

3740

$\begin{array}{llll}0.00904 & 0.05790 & 0.26486\end{array}$

$\begin{array}{llll}0.00891 & 0.05720 & 0.26243\end{array}$

$\begin{array}{llll}0.00878 & 0.05651 & 0.26003\end{array}$

$\begin{array}{lllll}0.00865 & 0.05582 & 0.25764\end{array}$

$\begin{array}{llllll}0.00853 & 0.05514 & 0.25528\end{array}$

3750

3760

3770

3780

3790

$\begin{array}{llll}0.00841 & 0.05447 & 0.25293\end{array}$

$\begin{array}{llll}0.00829 & 0.05381 & 0.25060\end{array}$

$\begin{array}{lllll}0.00817 & 0.05316 & 0.24829\end{array}$

$\begin{array}{llll}0.00805 & 0.05251 & 0.24600\end{array}$

$\begin{array}{llll}0.00794 & 0.05187 & 0.24372\end{array}$

3800

3810

3820

3830

3840

$\begin{array}{llll}0.00782 & 0.05124 & 0.24147\end{array}$

$\begin{array}{llll}0.00771 & 0.05062 & 0.23923\end{array}$

3850

3860

3870

0.007490 .049390 .23480

$\begin{array}{llll}0.00738 & 0.04879 & 0.23262\end{array}$

3880

3890

$\begin{array}{llll}0.00728 & 0.04820 & 0.23045\end{array}$

$\begin{array}{lllll}0.00717 & 0.04761 & 0.22830\end{array}$

$\begin{array}{llll}0.00707 & 0.04703 & 0.22617\end{array}$

$0.00697 \quad 0.04646 \quad 0.22406$

$\begin{array}{llll}0.00687 & 0.04589 & 0.22196\end{array}$

$\begin{array}{llll}0.00677 & 0.04533 & 0.21988\end{array}$

$\begin{array}{llll}0.00668 & 0.04478 & 0.21781\end{array}$

$\begin{array}{llll}0.00658 & 0.04423 & 0.21577\end{array}$

$\begin{array}{llll}0.00649 & 0.04369 & 0.21374\end{array}$

$0.00639 \quad 0.04316 \quad 0.21173$

3900

3910

3920

3930

3940

3950

3960

3970

3980

3990

0.006300 .042630 .20973

4000 
Table II Harmonic Oscillator Contributions to the Thermodynamic

Functions (in units of calories, moles, and ${ }^{\circ} \mathrm{K}$ )
$\nu_{-1}-\left(F^{\circ}-E_{0}^{\circ}\right) / T$ So
So
$\mathrm{C}_{\mathrm{p}}^{\circ} \quad-\left(\mathrm{F}^{\circ}-\mathrm{E}_{\mathrm{O}}^{\circ}\right) / \mathrm{T} \quad$ So $\quad \mathrm{C}_{\mathrm{p}}^{\circ}$
$-\left(F^{\circ}-E_{0}^{\circ}\right) / T$
So

$\mathrm{C}_{\mathrm{p}}^{\circ}$

$\nu$

$T=1100$.

$T=1200$.

$\mathrm{T}=1300$.

$100 \quad 4.170626 .03066 \quad 1.98434$

$110 \quad 3.99392 \quad 5.84156 \quad 1.98375$

$120 \quad 3.83368 \quad 5.66898 \quad 1.98310$

$130 \quad 3.68727 \quad 5.51027 \quad 1.98239$

$140 \quad 3.552625 .36339 \quad 1.98163$

$150 \quad 3.42810 \quad 5.22670 \quad 1.98081$

$160,3.312415 .098891 .97993$

$170 \quad 3.20447 \quad 4.97888 \quad 1.97900$

$180 \quad 3.10338 \quad 4.86580 \quad 1.97802$

$190 \quad 3.00842 \quad 4.75888 \quad 1.97697$

$200 \quad 2.918934 .65750 \quad 1.97588$

$210 \quad 2.834394 .56112 \quad 1.97472$

$220 \quad 2.754344 .46929 \quad 1.97351$

$230 \quad 2.67836 \quad 4.38159 \quad 1.97225$

$240 \quad 2.606124 .29768 \quad 1.97093$

$250 \quad 2.53731 \quad 4.21725 \quad 1.96956$

$260 \quad 2.471644 .140031 .96813$

$270 \quad 2.408894 .06578 \quad 1.96664$

$280 \quad 2.348843 .99428 \quad 1.96511$

$290 \quad 2.29130 \quad 3.92535 \quad 1.96351$

$\begin{array}{llll}300 \quad 2.23609 & 3.85882 & 1.96187\end{array}$

$\begin{array}{llll}310 & 2.18307 & 3.79451 & 1.96017\end{array}$

$\begin{array}{lllll}320 & 2.13209 & 3.73231 & 1.95841\end{array}$

$\begin{array}{llll}330 & 2.08302 & 3.67207 & 1.95660\end{array}$

$\begin{array}{lll}340 \quad 2.03574 & 3.61369 & 1.95474\end{array}$

$\begin{array}{llll}350 & 1.99016 & 3.55705 & 1.95282\end{array}$

$\begin{array}{llll}360 & 1.94618 & 3.50207 & 1.95086\end{array}$

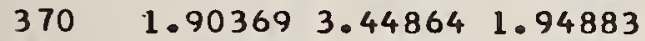

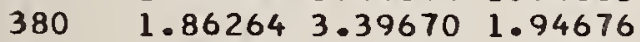

$390 \quad 1.82293 \quad 3.34616 \quad 1.94463$

$400 \quad 1.78450 \quad 3.29695 \quad 1.94245$

$410 \quad 1.74729 \quad 3.24902 \quad 1.94022$

$\begin{array}{lllll}420 & 1.71123 & 3.20229 & 1.93794\end{array}$

$430 \quad 1.676273 .156721 .93560$

$\begin{array}{llll}440 & 1.64235 & 3.11224 & 1.93322\end{array}$

$\begin{array}{lllll}450 & 1.60944 & 3.06883 & 1.93078\end{array}$

$460 \quad 1.57748 \quad 3.02642 \quad 1.92829$

$\begin{array}{llll}470 & 1.54643 & 2.98497 & 1.92575\end{array}$

$480 \quad 1.516252 .944461 .92316$

$\begin{array}{lllll}490 & 1.48691 & 2.90483 & 1.92052\end{array}$

$\begin{array}{lllll}500 & 1.45836 & 2.86606 & 1.91783\end{array}$

$\begin{array}{lllll}510 & 1.43059 & 2.82811 & 1.91509\end{array}$

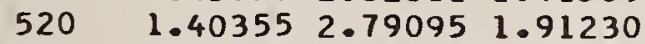

$\begin{array}{llll}530 & 1.37722 & 2.75455 & 1.90946\end{array}$

$\begin{array}{lllll}540 & 1.35156 & 2.71888 & 1.90658\end{array}$

$\begin{array}{lllll}550 & 1.32657 & 2.68392 & 1.90364\end{array}$

$\begin{array}{lllll}560 & 1.30220 & 2.64965 & 1.90066\end{array}$

$570 \quad 1.278442 .616041 .89762$

$\begin{array}{lllll}580 & 1.25526 & 2.58306 & 1.89454\end{array}$

$590 \quad 1.232642 .550701 .89142$
$4.33292 \quad 6.20334 \quad 1.98479$

4.155196 .014191 .98429

$3.99392 \quad 5.84156 \quad 1.98375$

$3.84648 \quad 5.68280 \quad 1.98315$

$3.71080 \quad 5.53585 \quad 1.98251$

3.585275 .399101 .98182

$3.468575 .27122 \quad 1.98109$

3.359625 .151141 .98030

$3.257535 .03797 \quad 1.97947$

$3.16156 \quad 4.93097 \quad 1.97860$

$3.07108 \quad 4.82950 \quad 1.97767$

2.98555 4.73304 1.97670

2.904514 .641101 .97569

$2.82756 \quad 4.55330 \quad 1.97462$

$2.75434 \quad 4.469291 .97351$

$2.68455 \quad 4.38875 \quad 1.97236$

$2.617924 .31141 \quad 1.97115$

2.554204 .237051 .96991

$2.493194 .16543 \quad 1.96861$

$2.43470 \quad 4.09637 \quad 1.96727$

$2.37854 \quad 4.02970 \quad 1.96588$

$2.324573 .96526 \quad 1.96445$

$2.272653 .90292 \quad 1.96297$

2.222643 .842541 .96145

$2.17443 \quad 3.78400 \quad 1.95988$

$2.12793 \quad 3.72722 \quad 1.95826$

$2.08302 \quad 3.67207 \quad 1.95660$

2.039623 .618491 .95490

$1.997653 .56638 \quad 1.95315$

$1.95703 \quad 3.515671 .95135$

$1.917693 .46628 \quad 1.94951$

$1.87958 \quad 3.41817 \quad 1.94763$

$1.842623 .37126 \quad 1.94570$

1.806773 .325501 .94373

$1.771973 .28084 \quad 1.94172$

$1.73817 \quad 3.23722 \quad 1.93966$

$1.70533 \quad 3.19461 \quad 1.93755$

$1.67340 \quad 3.15297 \quad 1.93541$

$1.642353 .11224 \quad 1.93322$

$1.61214 \quad 3.07241 \quad 1.93098$

$1.58274 \quad 3.03342 \quad 1.92871$

$1.55411 \quad 2.99525 \quad 1.92639$

$1.52621 \quad 2.95786 \quad 1.92403$

$1.49903 \quad 2.92124 \quad 1.92163$

$1.47254 \quad 2.88534 \quad 1.91918$

$1.44670 \quad 2.85015 \quad 1.91670$ $1.42149 \quad 2.81563 \quad 1.91417$ $1.396902 .78178 \quad 1.91160$ $1.372902 .74855 \quad 1.90899$ $1.34946 \quad 2.71594 \quad 1.90633$
$4.482996 .36222 \quad 1.98514$

4.304386 .173041 .98472

$4.142246 .00037 \quad 1.98425$

3.993925 .841561 .98375

$3.85738 \quad 5.69457 \quad 1.98320$

$3.73098 \quad 5.55776 \quad 1.98261$

3.613415 .429831 .98199

3.503605 .309691 .98132

$3.400665 .19646 \quad 1.98061$

3.303845 .089391 .97986

$3.21252 \quad 4.98786 \quad 1.97908$

3.126144 .891321 .97825

$3.044264 .79931 \quad 1.97738$

2.966474 .711431 .97647

$2.892424 .62734 \quad 1.97553$

$2.82180 \quad 4.54671 \quad 1.97454$

$2.75434 \quad 4.469291 .97351$

2.689804 .394831 .97245

2. $62797 \quad 4.323111 .97134$

$2.56866 \quad 4.25396 \quad 1.97020$

2.511694 .187181 .96901

2.456914 .122641 .96779

2.404184 .060181 .96653

$2.35337 \quad 3.999691 .96523$

2. 304373.941041 .96389

$2.25706 \quad 3.884131 .96251$

$2.21136 \quad 3.82887 \quad 1.96109$

$2.167173 .77516 \quad 1.95963$

$2.12442 \quad 3.72292 \quad 1.95814$

$2.08302 \quad 3.67207 \quad 1.95660$

$2.04290 \quad 3.62256 \quad 1.95503$

2.004013 .574301 .95342

$\begin{array}{llll}1.96628 & 3.52725 & 1.95177\end{array}$

$1.92966 \quad 3.481341 .95008$

1.894103 .436531 .94836

$1.85954 \quad 3.39276 \quad 1.94660$

$1.82594 \quad 3.35000 \quad 1.94480$

$1.79326 \quad 3.308191 .94296$

$1.76146 \quad 3.267311 .94109$

$1.73050 \quad 3.22730 \quad 1.93917$

$1.70036 \quad 3.18815 \quad 1.93723$

$1.67098 \quad 3.14980 \quad 1.93524$

1.642353 .112241 .93322

1.614443 .075441 .93116

$1.587213 .03936 \quad 1.92906$

$1.560653 .00398 \quad 1.92693$

$1.53472 \quad 2.96928 \quad 1.92476$

1.509402 .935231 .92256

1.484682 .901821 .92032

1.460532 .869011 .91804
100

110

120

130

140

150

160

170

180

190

200

210

220

230

240

250

260

270

280

290

300

310

320

330

340

350

360

370

380

390

400

410

420

430

440

450

460

470

480

490

500

510

520

530

540

550

560

570

580

590 
Table II Harmonic Oscillator Contributions to the Thermodynamic

Functions (in units of calories, moles, and ${ }^{\circ} \mathrm{K}$ )

\begin{abstract}
$v$
$\mathrm{cm}^{-1}$

$-\left(F^{\circ}-E_{O}^{O}\right) / T \quad S^{\circ}$

so

$\mathrm{C}_{\mathrm{p}}^{\circ}$

$$
T=1100 \text {. }
$$

600

610

620

630

640

$1.210572 .51894 \quad 1.88824$ 1.189022 .487751 .88502 $1.16798 \quad 2.457131 .88175$ $1.14743 \quad 2.42705 \quad 1.87844$ $1.127362 .39749 \quad 1.87508$
\end{abstract}

$-\left(F^{\circ}-E_{0}^{\circ}\right) / T$

So

$\mathrm{c}_{\mathrm{p}}^{\circ}$

$-\left(F^{\circ}-E_{O}^{O}\right) / T \quad S^{\circ}$

$\mathrm{C}_{\mathrm{p}}^{\circ}$

$\nu$

$\mathrm{cin}^{-1}$

650

660

670

680

690

700

710

720

730

740

750

760

770

780

790

800

810

820

830

840

850

860

870

880

890

900

910

920

930

940

950

960

970

980

990

1000

1010

1020

1030

1040

1050

1060

1070

1080

1090
1. $10774 \quad 2.36844 \quad 1.87167$ 1.088562 .339891 .86822 $\begin{array}{lll}1.06981 & 2.31183 & 1.86473\end{array}$ $1.05148 \quad 2.284231 .86119$ 1.033552 .257081 .85760

$\begin{array}{llll}1.01601 & 2.23038 & 1.85398\end{array}$ $0.998852 .20411 \quad 1.85031$ 0.982062 .178251 .84659 0.965622 .152811 .84283 $0.949532 .12776 \quad 1.83903$

0.933772 .103101 .83519 $0.918342 .07882 \quad 1.83131$ $0.903232 .05491 \quad 1.82738$ 0.888432 .031351 .82341 0.873922 .008151 .81941

$0.85971 \quad 1.98529 \quad 1.81536$ $0.84578 \quad 1.96276 \quad 1.81127$ 0.832131 .940561 .80715 $0.81874 \quad 1.918681 .80298$ 0.805621 .897121 .79877

$\begin{array}{llll}0.79275 & 1.87585 & 1.79453\end{array}$ 0.780131 .854891 .79025 0.767761 .834221 .78593 0.755621 .813831 .78157 0.743701 .793731 .77718

$0.73202 \quad 1.77389 \quad 1.77275$ 0.720551 .754331 .76829 $0.709301 .73503 \quad 1.76379$ $0.698251 .71598 \quad 1.75925$ $0.68741 \quad 1.69719 \quad 1.75468$

$0.67676 \quad 1.67865 \quad 1.75007$ $0.66631 \quad 1.660351 .74543$ $0.656051 .64228 \quad 1.74076$ $0.64598 \quad 1.624451 .73605$ 0.636081 .606851 .73131

$0.62636 \quad 1.58948 \quad 1.72654$ 0.616821 .572321 .72173 0.607441 .555381 .71690 $0.598231 .53866 \quad 1.71203$ 0.589181 .522141 .70713

$0.580291 .50582 \quad 1.70221$ 0.571551 .489711 .69725 0.562961 .473801 .69226 $0.554521 .45808 \quad 1.68724$ 0.546231 .442551 .68220

$$
T=1200
$$

1.326572 .683921 .90364 $1.304212 .65248 \quad 1.90091$ $1.28236 \quad 2.62159 \quad 1.89813$ 1.261002 .591251 .89532 1.240122 .561421 .89246

$1.21970 \quad 2.53210 \quad 1.88957$ $1.199732 .50327 \quad 1.88664$ $1.18020 \quad 2.47493 \quad 1.88367$ $1.16108 \quad 2.44704 \quad 1.88065$ $1.142372 .41961 \quad 1.87760$

$1.12406 \quad 2.39261 \quad 1.87452$ 1.106122 .366051 .87139 $1.08856 \quad 2.33989 \quad 1.86822$ $1.071362 .31415 \quad 1.86502$ $\begin{array}{llll}1.05451 & 2.28879 & 1.86178\end{array}$

$\begin{array}{llll}1.03800 & 2.26383 & 1.85850\end{array}$ 1.021822 .239231 .85519 $1.00596 \quad 2.21500 \quad 1.85184$ 0.990412 .191131 .84845 $0.975172 .16760 \quad 1.84503$

$0.96022 \quad 2.144421 .84157$ $0.945562 .12156 \quad 1.83808$ $0.93118 \quad 2.09903 \quad 1.83455$ $0.917072 .07681 \quad 1.83098$ $0.903232 .05491 \quad 1.82738$

$0.889652 .03330 \quad 1.82375$ 0.876322 .011991 .82008 0.863241 .990971 .81638 $0.850391 .97024 \quad 1.81264$ $0.83778 \quad 1.94977 \quad 1.80887$

$\begin{array}{llll}0.82540 & 1.92958 & 1.80507\end{array}$ 0.813241 .909661 .80123 $0.801301 .89000 \quad 1.79736$ $0.78958 \quad 1.870591 .79346$ $0.778051 .85142 \quad 1.78953$

$0.76674 \quad 1.83251 \quad 1.78557$ $0.75562 \quad 1.813831 .78157$ $0.74469 \quad 1.79539 \quad 1.77755$ $0.73395 \quad 1.77718 \quad 1.77349$ $0.72340 \quad 1.75920 \quad 1.76941$

$0.71302 \quad 1.741431 .76529$ 0.702831 .723891 .76114 0.692801 .706561 .75697 0.682951 .689441 .75276 $0.67326 \quad 1.672521 .74853$

$0.66373 \quad 1.65581 \quad 1.74427$ $0.654361 .63930 \quad 1.73998$ $0.64515 \quad 1.62298 \quad 1.73566$ $0.63608 \quad 1.60685 \quad 1.73131$ 0.627171 .590921 .72694

$$
T=1300 \text {. }
$$

$1.436932 .83679 \quad 1.91573$ $1.41386 \quad 2.80515 \quad 1.91338$ 1.391312 .774051 .91100 $\begin{array}{llll}1.36925 & 2.74350 & 1.90858\end{array}$ $1.347682 .71346 \quad 1.90613$

600 610 620 630 640

$1.326572 .68392 \quad 1.90364$ 1.305912 .654881 .90112 $1.28568 \quad 2.62631 \quad 1.89856$ $1.26588 \quad 2.59820 \quad 1.89597$ $1.246492 .57054 \quad 1.89335$

650

660

670

680

690

$1.22750 \quad 2.54332 \quad 1.89069$

700

1.208902 .516521 .88800

$1.190662 .49013 \quad 1.88527$

$1.172802 .46415 \quad 1.88251$

$1.15528 \quad 2.43855 \quad 1.87972$

710

720

730

740

$\begin{array}{llll}1.13811 & 2.41334 & 1.87689\end{array}$

750

$1.12127 \quad 2.38850 \quad 1.87404$

$1.10476 \quad 2.364021 .87115$

$1.088562 .33989 \quad 1.86822$

$\begin{array}{llll}1.07267 & 2.31611 & 1.86527\end{array}$

760

770

780

790

$\begin{array}{llll}1.05708 & 2.29267 & 1.86228\end{array}$ $\begin{array}{llll}1.04178 & 2.26955 & 1.85926\end{array}$

$1.026762 .24676 \quad 1.85621$

$1.01202 \quad 2.22428 \quad 1.85313$

0.997552 .202101 .85002

$0.98334 \quad 2.18023 \quad 1.84688$ $0.96938 \quad 2.15865 \quad 1.84370$

$0.95568 \quad 2.13735 \quad 1.84050$ $0.94222 \quad 2.116331 .83726$ 0.928992 .095591 .83400

800

810

820

830

840

850

860

870

880

890

$0.91600 \quad 2.07512 \quad 1.83071$

$0.90323 \quad 2.05491 \quad 1.82738$

$0.890692 .03495 \quad 1.82403$

0.878352 .015251 .82064

$0.866231 .99580 \quad 1.81723$

900

910

920

930

940

$0.85432 \quad 1.97659 \quad 1.81379$

950

$0.842611 .95761 \quad 1.81032$

0.831091 .938871 .80683

$0.81976 \quad 1.92036 \quad 1.80330$

0.808631 .902071 .79975

$0.79767 \quad 1.88400 \quad 1.79617$ $0.78690 \quad 1.866141 .79256$ $0.77630 \quad 1.84850 \quad 1.78893$ $0.76587 \quad 1.83106 \quad 1.78526$ 0.755621 .813831 .78157

960

970

980

990

1000

1010

1020

1030

1040

$0.745521 .79680 \quad 1.77786$

1050

$0.73559 \quad 1.77997 \quad 1.77412$

1060

$\begin{array}{llll}0.72582 & 1.76333 & 1.77035\end{array}$

$0.716201 .74688 \quad 1.76656$

$0.70673 \quad 1.73061 \quad 1.76274$
1070

1080

1090 
Table II Harmonic Oscillator Contributions to the Thermodynamic

Functions (in units of calories, moles, and ${ }^{\circ} \mathrm{K}$ )
$\mathrm{cm}^{-1}$
$-\left(F^{\circ}-E_{0}^{\circ}\right) / T \quad S^{\circ}$
So $\quad c_{0}^{\circ}$
$\stackrel{0}{p} \quad-\left(F^{\circ}-E O\right) / T$
so
$\mathrm{C}_{\mathrm{p}}^{\circ}$
$-(F \circ-E O) / T$
So
$\mathrm{c}_{\mathrm{p}} \quad \mathrm{cm}^{-1}$

$T=1100$.

$\mathrm{T}=1200$.

$T=1300$.

$1100 \quad 0.53808 \quad 1.42721 \quad 1.67713$

$\begin{array}{llll}1110 & 0.53006 & 1.41206 & 1.67202\end{array}$

$1120 \quad 0.52218 \quad 1.39709 \quad 1.66690$

$1130 \quad 0.51444 \quad 1.38229 \quad 1.66174$

$1140 \quad 0.50682 \quad 1.36767 \quad 1.65656$

$\begin{array}{lllll}1150 & 0.49933 & 1.35323 & 1.65135\end{array}$

$\begin{array}{lllll}1160 & 0.49197 & 1.33895 & 1.64612\end{array}$

$1170 \quad 0.48473 \quad 1.32485 \quad 1.64086$

$\begin{array}{lllll}1180 & 0.47761 & 1.31090 & 1.63557\end{array}$

$\begin{array}{llll}1190 \quad 0.47061 & 1.29712 & 1.63026\end{array}$

$1200 \quad 0.46372 \quad 1.28350 \quad 1.62493$

$\begin{array}{lllll}1210 & 0.45694 & 1.27004 & 1.61957\end{array}$

$\begin{array}{lllll}1220 & 0.45028 & 1.25673 & 1.61420\end{array}$

$1230 \quad 0.44372 \quad 1.24358 \quad 1.60879$

$\begin{array}{lllll}1240 & 0.43727 & 1.23057 & 1.60337\end{array}$

$\begin{array}{lllll}1250 & 0.43093 & 1.21772 & 1.59792\end{array}$

$1260 \quad 0.42468 \quad 1.20501 \quad 1.59246$

$\begin{array}{lllll}1270 & 0.41854 & 1.19244 & 1.58697\end{array}$

$1280 \quad 0.41249 \quad 1.18001 \quad 1.58146$

$1290 \quad 0.406551 .16773 \quad 1.57593$

$\begin{array}{lllll}1300 & 0.40069 & 1.15558 & 1.57038\end{array}$

$\begin{array}{lllll}1310 & 0.39493 & 1.14357 & 1.56481\end{array}$

$\begin{array}{llll}1320 & 0.38926 & 1.13169 & 1.55922\end{array}$

$\begin{array}{lllll}1330 & 0.38368 & 1.11994 & 1.55362\end{array}$

$1340 \quad 0.378191 .108331 .54800$

$\begin{array}{lllll}1350 & 0.37278 & 1.09684 & 1.54235\end{array}$

$\begin{array}{lllll}1360 & 0.36746 & 1.08548 & 1.53670\end{array}$

$\begin{array}{llll}1370 & 0.36222 & 1.07424 & 1.53102\end{array}$

$\begin{array}{lllll}1380 & 0.35707 & 1.06312 & 1.52533\end{array}$

$\begin{array}{lllll}1390 & 0.35199 & 1.05213 & 1.51962\end{array}$

$\begin{array}{lllll}1400 & 0.34699 & 1.04126 & 1.51390\end{array}$

$1410 \quad 0.34207 \quad 1.030501 .50816$

$\begin{array}{llllll}1420 & 0.33723 & 1.01987 & 1.50241\end{array}$

$\begin{array}{lllll}1430 & 0.33246 & 1.00934 & 1.49664\end{array}$

$\begin{array}{lllll}1440 & 0.32776 & 0.99893 & 1.49086\end{array}$

$\begin{array}{lllll}1450 & 0.32314 & 0.98864 & 1.48506\end{array}$ $\begin{array}{lllll}1460 & 0.31858 & 0.97845 & 1.47926\end{array}$

$\begin{array}{llllll}1470 & 0.31410 & 0.96837 & 1.47344\end{array}$

$\begin{array}{llll}1480 & 0.30968 & 0.95840 & 1.46761\end{array}$

$\begin{array}{lllll}1490 & 0.30533 & 0.94854 & 1.46176\end{array}$

$\begin{array}{lllll}1500 & 0.30104 & 0.93878 & 1.45591\end{array}$

$\begin{array}{lllll}1510 & 0.29683 & 0.92913 & 1.45004\end{array}$

$\begin{array}{llllll}1520 & 0.29267 & 0.91957 & 1.44416\end{array}$

$\begin{array}{lllll}1530 & 0.28858 & 0.91012 & 1.43828\end{array}$

$\begin{array}{lllll}1540 & 0.28454 & 0.90077 & 1.43238\end{array}$

$\begin{array}{lllll}1550 & 0.28057 & 0.89152 & 1.42647\end{array}$

$\begin{array}{lllll}1560 & 0.27666 & 0.88237 & 1.42056\end{array}$

$\begin{array}{lllll}1570 & 0.27281 & 0.87331 & 1.41463\end{array}$

$\begin{array}{lllll}1580 & 0.26901 & 0.86435 & 1.40870\end{array}$

$\begin{array}{lllll}1590 & 0.26527 & 0.85548 & 1.40276\end{array}$
$0.61840 \quad 1.57517 \quad 1.72254$ 0.609771 .559601 .71811 $0.601281 .54421 \quad 1.71366$ 0.592931 .528991 .70918 $0.58471 \quad 1.513961 .70467$

0.576631 .499091 .70014 0.568671 .484391 .69559 0.560841 .469851 .69101 0.553131 .455481 .68641 0.545541 .441271 .68178

$0.538081 .42721 \quad 1.67713$ 0.530731 .413321 .67245 0.523491 .399571 .66775 0.516361 .385971 .66303 0.509351 .372531 .65829

0.502441 .359231 .65352 0.495641 .346071 .64874 0.488941 .333061 .64393 0.482341 .320181 .63910 $0.475851 .30744 \quad 1.63425$

$0.46945 \quad 1.29484 \quad 1.62938$ 0.463151 .282381 .62449 $0.456941 .27004 \quad 1.61957$ $0.450831 .25784 \quad 1.61464$ 0.444811 .245761 .60970

$0.438871 .23381 \quad 1.60473$ 0.433031 .221991 .59974 0.427271 .210281 .59474 0.421601 .198701 .58971 0.416011 .187241 .58467

$0.41050 \quad 1.17590 \quad 1.57962$ 0.405071 .164681 .57454 0.399731 .153571 .56945 $0.394461 .14257 \quad 1.56435$ 0.389261 .131691 .55922

$0.38414 \quad 1.120921 .55409$ 0.379101 .110251 .54893 0.374131 .099701 .54377 0.369231 .089251 .53858 0.364401 .078911 .53339

$0.359641 .06867 \quad 1.52818$ 0.354941 .058531 .52295 0.350321 .048491 .51772 $0.34576 \quad 1.03856 \quad 1.51247$ $0.34126 \quad 1.028721 .50720$

$0.336831 .01898 \quad 1.50193$ $0.33246 \quad 1.00934 \quad 1.49664$ $0.328150 .99980 \quad 1.49134$ $0.32390 \quad 0.99034 \quad 1.48603$ $\begin{array}{llll}0.31971 & 0.98099 & 1.48071\end{array}$
$0.69741 \quad 1.714531 .75890$ 0.688231 .698631 .75503 $0.67920 \quad 1.68291 \quad 1.75114$ $0.67031 \quad 1.667361 .74722$ $0.66155 \quad 1.65198 \quad 1.74328$

$0.652931 .63677 \quad 1.73931$ 0.644441 .621731 .73532 $0.63608 \quad 1.606851 .73131$ $0.627851 .59214 \quad 1.72728$ $0.61974 \quad 1.57758 \quad 1.72322$

$0.61175 \quad 1.563171 .71913$ $0.60388 \quad 1.548921 .71503$ 0.596131 .534831 .71090 0.588491 .520881 .70676 $0.58097 \quad 1.50707 \quad 1.70259$

$\begin{array}{llll}0.57355 & 1.49341 & 1.69839\end{array}$ $0.566251 .47990 \quad 1.69418$ 0.559051 .466521 .68995 $0.55196 \quad 1.453281 .68569$ $0.54497 \quad 1.440181 .68142$

$\begin{array}{llll}0.53808 & 1.42721 & 1.67713\end{array}$ 0.531291 .414381 .67281 0.524591 .401671 .66848 $0.51800 \quad 1.38910 \quad 1.66412$ 0.511491 .376651 .65975

$0.50508 \quad 1.364331 .65536$ $0.49876 \quad 1.352131 .65095$ 0.492531 .340051 .64652 $0.486391 .32809 \quad 1.64207$ 0.480331 .316251 .63761

$0.47436 \quad 1.304521 .63312$ 0.468471 .292921 .62863 0.462671 .281421 .62411 $0.45694 \quad 1.27004 \quad 1.61957$ $0.45130 \quad 1.25877 \quad 1.61502$

$0.44573 \quad 1.24761 \quad 1.61046$ 0.440241 .236561 .60588 0.434821 .225611 .60128 $0.42948 \quad 1.21477 \quad 1.59666$ 0.424211 .204031 .59203

$0.41901 \quad 1.193401 .58739$ $0.41388 \quad 1.18287 \quad 1.58273$ 0.408821 .172441 .57806 0.403831 .162101 .57337 $0.398911 .15187 \quad 1.56867$

0.394051 .141731 .56395 0.389261 .131691 .55922 $0.384531 .12174 \quad 1.55448$ 0.379871 .111891 .54973 0.375271 .102121 .54496
1100

1110

1120

1130

1140

1150

1160

1170

1180

1190

1200

1210

1220

1230

1240

1250

1260

1270

1280

1290

1300

1310

1320

1330

1340

1350

1360

1370

1380

1390

1400

1410

1420

1430

1440

1450

1460

1470

1480

1490

1500

1510

1520

1530

1540

1550

1560

1570

1580

1590 
Table II Harmonic Oscillator Contributions to the Thermodynamic

Functions (in units of calories, moles, and ${ }^{\circ} \mathrm{K}$ )

\begin{tabular}{|c|c|c|c|c|c|c|c|c|}
\hline $\mathrm{cm}^{-1}-\left(\mathrm{F}^{\circ}-\mathrm{E}_{\mathrm{O}}^{\circ}\right) / \mathrm{T}$ & so & $\mathrm{C}_{\mathrm{p}}^{\circ}$ & $-\left(F^{\circ}-E_{O}^{\circ}\right) / T$ & $\mathrm{~S}^{\circ}$ & $\mathrm{C}_{\mathrm{p}}$ & $-\left(\mathrm{F}^{\circ}-\mathrm{E}_{\mathrm{O}}^{\circ}\right) / \mathrm{T}$ & $S^{\circ}$ & $\mathrm{C}_{\mathrm{p}}^{\circ}$ \\
\hline
\end{tabular}

$$
T=1100 \text {. }
$$

1600

1610

1620

1630

1640

1650

1660

1670

1680

1690

$\begin{array}{lllll}1700 & 0.22760 & 0.76381 & 1.33701\end{array}$

1710

1720

1730

1740

1750

1760

1770

1780

1790

1800

1810

1820

1830

1840

1850

1860

1870

1880

1890

1900

1910

1920

1930

1940

1950

1960

1970

1980

1990

2000

2010

2020

2030

2040

2050

2060

2070

2080

2090

$\begin{array}{llll}0.26159 & 0.84670 & 1.39681\end{array}$ $0.25796 \quad 0.83802 \quad 1.39086$ $\begin{array}{llll}0.25438 & 0.82942 & 1.38490\end{array}$ $0.25086 \quad 0.82092 \quad 1.37893$ $\begin{array}{llll}0.24739 & 0.81250 \quad 1.37295\end{array}$

$0.24396 \quad 0.80417 \quad 1.36698$ $\begin{array}{llll}0.24059 & 0.79593 & 1.36099\end{array}$ $\begin{array}{lllll}0.23727 & 0.78778 & 1.35500\end{array}$ $\begin{array}{lllll}0.23400 & 0.77971 & 1.34901\end{array}$ $\begin{array}{llll}0.23078 & 0.77172 & 1.34301\end{array}$ $0.224470 .75599 \quad 1.33101$ $0.22138 \quad 0.74824 \quad 1.32500$ $0.218340 .74058 \quad 1.31899$ 0.215350 .732991 .31298

$0.212390 .72549 \quad 1.30696$ $0.20948 \quad 0.71806 \quad 1.30095$ $0.206610 .71070 \quad 1.29493$ $0.20378 \quad 0.70343 \quad 1.28891$ $0.20100 \quad 0.69622 \quad 1.28289$

$\begin{array}{llll}0.19825 & 0.68909 & 1.27687\end{array}$ $0.19554 \quad 0.68203 \quad 1.27086$ $0.19288 \quad 0.67505 \quad 1.26484$ $0.190250 .66813 \quad 1.25882$ $0.18765 \quad 0.66129 \quad 1.25281$

0.185100 .654521 .24679 $0.18258 \quad 0.64781 \quad 1.24078$ $0.18009 \quad 0.64117 \quad 1.23477$ $0.17765 \quad 0.63461 \quad 1.22876$ $0.175230 .62810 \quad 1.22276$

$0.172850 .62167 \quad 1.21675$ $0.170510 .61529 \quad 1.21076$ $0.16820 \quad 0.60899 \quad 1.20476$ $0.16592 \quad 0.60274 \quad 1.19877$ $0.163670 .59656 \quad 1.19279$

0.161450 .590451 .18680 $0.15927 \quad 0.58439 \quad 1.18083$ $0.157120 .57840 \quad 1.17486$ $0.154990 .57246 \quad 1.16889$ $0.15290 \quad 0.56659 \quad 1.16293$

$\begin{array}{llll}0.15083 & 0.56078 \quad 1.15698\end{array}$ $0.14880 \quad 0.55502 \quad 1.15103$ $0.146790 .54932 \quad 1.14509$ $0.144810 .54368 \quad 1.13916$ $0.14286 \quad 0.53810 \quad 1.13323$

$0.14094 \quad 0.53257 \quad 1.12731$ $0.139040 .52710 \quad 1.12140$ $0.137170 .52169 \quad 1.11550$ $0.135330 .51632 \quad 1.10961$ $0.133510 .51102 \quad 1.10372$

$T=1200$.

$T=1300$.

$\begin{array}{llll}0.31558 & 0.97172 & 1.47538\end{array}$ $0.31151 \quad 0.96254 \quad 1.47004$ $0.30750 \quad 0.95346 \quad l .46468$ $0.30354 \quad 0.94446 \quad 1.45932$ $0.29963 \quad 0.93555 \quad 1.45395$

$\begin{array}{llll}0.29578 & 0.92673 & 1.44857\end{array}$ $\begin{array}{llll}0.29198 & 0.91799 & 1.44318\end{array}$ $\begin{array}{llll}0.28824 & 0.90934 & 1.43778\end{array}$ $\begin{array}{llll}0.28454 & 0.90077 & 1.43238\end{array}$ $0.28090 \quad 0.89229 \quad 1.42696$

$0.27731 \quad 0.88389 \quad 1.42154$ $0.27376 \quad 0.87556 \quad 1.41611$ $0.27027 \quad 0.86732 \quad 1.41068$ $0.266820 .85916 \quad 1.40524$ $0.263420 .85108 \quad 1.39979$

$0.26007 \quad 0.84307 \quad 1.39433$ $\begin{array}{llll}0.25676 & 0.83514 & 1.38887\end{array}$ $\begin{array}{llll}0.25349 & 0.82729 & 1.38340\end{array}$ 0.250270 .819511 .37793 $0.24710 \quad 0.81181 \quad 1.37246$

$0.24396 \quad 0.80417 \quad 1.36698$ $0.240870 .79662 \quad 1.36149$ 0.237820 .789131 .35600 $0.234820 .78172 \quad 1.35051$ $0.231850 .77437 \quad 1.34501$

$0.228920 .76710 \quad 1.33951$ $0.22603 \quad 0.759891 .33401$ 0.223180 .752751 .32850 $0.22036 \quad 0.74568 \quad 1.32299$ $\begin{array}{llll}0.21759 & 0.73868 & 1.31748\end{array}$

$0.21485 \quad 0.73174 \quad 1.31197$ $0.212150 .72487 \quad 1.30646$ $0.20948 \quad 0.71806 \quad 1.30094$ $0.206850 .71131 \quad 1.29543$ 0.204250 .704631 .28991

$0.20169 \quad 0.69802 \quad 1.28440$ $\begin{array}{llll}0.19916 & 0.69146 & 1.27888\end{array}$ $\begin{array}{llll}0.19667 & 0.68497 & 1.27336\end{array}$ $0.19420 \quad 0.67853 \quad 1.26785$ $0.191770 .67216 \quad 1.26233$

$\begin{array}{llll}0.18938 & 0.66585 & 1.25682\end{array}$ $\begin{array}{llll}0.18701 & 0.65959 & 1.25130\end{array}$ $0.18467 \quad 0.65339 \quad 1.24579$ $\begin{array}{llll}0.18237 & 0.64726 & 1.24028\end{array}$ $0.18009 \quad 0.64117 \quad 1.23477$

$0.177850 .63515 \quad 1.22926$ $0.17563 \quad 0.62918 \quad 1.22376$ $0.17345 \quad 0.62327 \quad 1.21825$ 0.171290 .617411 .21276 $0.16916 \quad 0.611611 .20726$
$0.37073 \quad 1.09245 \quad 1.54018$

$0.366251 .08287 \quad 1.53539$

$0.36182 \quad 1.07338 \quad 1.53058$

$0.35746 \quad 1.06398 \quad 1.52577$

$0.353161 .05466 \quad 1.52094$

$0.34891 \quad 1.045431 .51610$

$0.34471 \quad 1.03628 \quad 1.51125$

0.340571 .027221 .50639

$0.336491 .01824 \quad 1.50152$

0.332461 .009341 .49664

$0.32848 \quad 1.00053 \quad 1.49175$

$0.324550 .99179 \quad 1.48685$

$0.320670 .98314 \quad 1.48194$

$\begin{array}{llll}0.31685 & 0.97456 & 1.47702\end{array}$

$0.31307 \quad 0.96606 \quad 1.47209$

$0.30934 \quad 0.95764 \quad 1.46716$

$0.30566 \quad 0.94929 \quad 1.46221$

$0.30203 \quad 0.94102 \quad 1.45726$

0.298440 .932831 .45230

$0.29490 \quad 0.92471 \quad 1.44733$

$0.29140 \quad 0.91666 \quad 1.44235$

$0.28795 \quad 0.90868 \quad 1.43737$

$\begin{array}{lllll}0.28454 & 0.90077 & 1.43238\end{array}$

$\begin{array}{llll}0.28118 & 0.89294 & 1.42738\end{array}$

$\begin{array}{llll}0.27786 & 0.88517 & 1.42238\end{array}$

$0.27458 \quad 0.87748 \quad 1.41737$

$0.271340 .86985 \quad 1.41235$

0.268140 .862291 .40733

$0.26499 \quad 0.85480 \quad 1.40230$

$0.261870 .84737 \quad 1.39727$

$\begin{array}{llll}0.25879 & 0.84001 & 1.39223\end{array}$

$0.255750 .83272 \quad 1.38719$

0.252750 .825491 .38214

$0.24978 \quad 0.81832 \quad 1.37709$

0.246860 .811221 .37203

$\begin{array}{llll}0.24396 & 0.80417 & 1.36698\end{array}$

0.241110 .797201 .36191

$\begin{array}{llll}0.23829 & 0.79028 & 1.35685\end{array}$

$0.235510 .78342 \quad 1.35178$

$0.23276 \quad 0.776621 .34670$

0.230040 .769891 .34163

$0.22736 \quad 0.76321 \quad 1.33655$

$0.224710 .75659 \quad 1.33147$

$0.222090 .75002 \quad 1.32638$

0.219510 .743521 .32130

$0.216950 .73707 \quad 1.31621$

$0.214430 .73068 \quad 1.31112$

0.211940 .724341 .30604

$0.20948 \quad 0.71806 \quad 1.30094$

0.207050 .711831 .29585
1600

1610

1620

1630

1640

1650

1660

1670

1680

1690

1700

1710

1720

1730

1740

1750

1760

1770

1780

1790

1800

1810

1820

1830

1840

1850

1860

1870

1880

1890

1900

1910

1920

1930

1940

1950

1960

1970

1980

1990

2000

2010

2020

2030

2040

2050

2060

2070

2080

2090 
Table II Harmonic Oscillator Contributions to the Thermodynamic

Functions (in units of calories, moles, and ${ }^{\circ} \mathrm{K}$ )
$\mathrm{cm}^{-1}$
So
$\mathrm{C}_{\mathrm{p}}^{\circ} \quad-\left(\mathrm{F}^{\circ}-\mathrm{E}_{\mathrm{O}}^{\circ}\right) / \mathrm{T} \quad$ So
$\mathrm{C}_{\mathrm{p}}^{\circ} \quad-\left(\mathrm{F}^{\circ}-\mathrm{E} \mathrm{O}\right) / \mathrm{T}$
So
$T=1100$.

$$
T=1200
$$
$T=1300$.

$\mathrm{c}_{\mathrm{p}}^{\circ}$

$v$

$\mathrm{cm}^{-1}$

2100

2110

2120

2130

2140

$2150 \quad 0.12311 \quad 0.48027 \quad 1.06860$

$\begin{array}{lllll}2160 & 0.12146 & 0.47533 & 1.06278\end{array}$

$2170 \quad 0.11983 \quad 0.47043 \quad 1.05698$

2180

2190

$2200 \quad 0.115090 .45604 \quad 1.03962$

$2210 \quad 0.113550 .45134 \quad 1.03386$

$\begin{array}{lllll}2220 & 0.11203 & 0.44668 & 1.02810\end{array}$

$\begin{array}{lllll}2230 & 0.11053 & 0.44207 & 1.02237\end{array}$

$\begin{array}{lllll}2240 & 0.10906 & 0.43751 & 1.01664\end{array}$

$\begin{array}{lllll}2250 & 0.10760 & 0.43300 & 1.01092\end{array}$

$\begin{array}{lllll}2260 & 0.10616 & 0.42853 & 1.00522\end{array}$

$\begin{array}{lllll}2270 & 0.10475 & 0.42410 & 0.99953\end{array}$

$\begin{array}{lllll}2280 & 0.10335 & 0.41972 & 0.99386\end{array}$

$\begin{array}{llllll}2290 & 0.10197 & 0.41538 & 0.98819\end{array}$

$\begin{array}{llllll}2300 & 0.10061 & 0.41109 & 0.98254\end{array}$

$\begin{array}{llllll}2310 & 0.09927 & 0.40684 & 0.97691\end{array}$

$\begin{array}{lllll}2320 & 0.09795 & 0.40263 & 0.97129\end{array}$

$\begin{array}{lllll}2330 & 0.09665 & 0.39846 & 0.96568\end{array}$

$\begin{array}{lllll}2340 & 0.09536 & 0.39434 & 0.96008\end{array}$

$\begin{array}{lllll}2350 & 0.09409 & 0.39026 & 0.95451\end{array}$

$\begin{array}{lllll}2360 & 0.09284 & 0.38622 & 0.94894\end{array}$

$\begin{array}{lllll}2370 & 0.09160 & 0.38222 & 0.94339\end{array}$

$\begin{array}{lllll}2380 & 0.09039 & 0.37826 & 0.93785\end{array}$

$\begin{array}{llllll}2390 & 0.08918 & 0.37434 & 0.93233\end{array}$

$\begin{array}{lllll}2400 & 0.08800 & 0.37045 & 0.92683\end{array}$

$\begin{array}{lllll}2410 & 0.08683 & 0.36661 & 0.92134\end{array}$

$\begin{array}{lllll}2420 & 0.08568 & 0.36281 & 0.91587\end{array}$

$\begin{array}{llllll}2430 & 0.08454 & 0.35904 & 0.91041\end{array}$

$\begin{array}{lllll}2440 & 0.08342 & 0.35531 & 0.90497\end{array}$

$\begin{array}{lllll}2450 & 0.08231 & 0.35162 & 0.89954\end{array}$ $\begin{array}{lllll}2460 & 0.08122 & 0.34797 & 0.89413\end{array}$ $\begin{array}{llllll}2470 & 0.08014 & 0.34435 & 0.88874\end{array}$ $\begin{array}{lllll}2480 & 0.07908 & 0.34077 & 0.88336\end{array}$ $\begin{array}{llll}2490 & 0.07803 & 0.33723 & 0.87800\end{array}$

$\begin{array}{lllll}2500 & 0.07700 & 0.33372 & 0.87266\end{array}$ $\begin{array}{lllll}2510 & 0.07598 & 0.33025 & 0.86733\end{array}$ $\begin{array}{lllll}2520 & 0.07497 & 0.32681 & 0.86202\end{array}$ $\begin{array}{lllll}2530 & 0.07398 & 0.32341 & 0.85673\end{array}$ $\begin{array}{lllll}2540 & 0.07300 & 0.32004 & 0.85145\end{array}$

$2550 \quad 0.072030 .31670 \quad 0.84620$ $2560 \quad 0.07108 \quad 0.31340 \quad 0.84096$ $2570 \quad 0.070140 .310130 .83573$ $\begin{array}{lllll}2580 & 0.06921 & 0.30690 & 0.83053\end{array}$ $\begin{array}{lllll}2590 & 0.06830 & 0.30370 & 0.82534\end{array}$
$0.167050 .60586 \quad 1.20177$ $0.16498 \quad 0.60016 \quad 1.19628$ 0.162930 .594521 .19079 $0.16090 \quad 0.588931 .18531$ 0.158910 .583391 .17983

$0.156940 .57790 \quad 1.17436$ $0.154990 .57246 \quad 1.16889$ $0.153070 .56708 \quad 1.16343$ $0.15118 \quad 0.56174 \quad 1.15797$ $0.149300 .55645 \quad 1.15252$

$0.14746 \quad 0.55122 \quad 1.14707$ 0.145630 .546031 .14163 $0.143830 .54088 \quad 1.13619$ $0.14206 \quad 0.53579 \quad 1.13077$ $0.14030 \quad 0.53074 \quad 1.12534$

$0.13857 \quad 0.52574 \quad 1.11993$ $0.13686 \quad 0.52079 \quad 1.11452$ $0.135170 .51588 \quad 1.10912$ 0.133510 .511021 .10372 $0.131860 .50620 \quad 1.09833$

$0.130240 .50142 \quad 1.09295$ $0.12863 \quad 0.49669 \quad 1.08758$ $0.12705 \quad 0.49201 \quad 1.08222$ $0.125490 .48736 \quad 1.07686$ $0.12394 \quad 0.48276 \quad 1.07151$

$0.122420 .47821 \quad 1.06618$ 0.120920 .473691 .06085 0.119430 .469211 .05553 $0.11796 \quad 0.46478 \quad 1.05021$ 0.116520 .460391 .04491

$0.11509 \quad 0.45604 \quad 1.03962$ $0.11367 \quad 0.45173 \quad 1.03434$ $0.112280 .44745 \quad 1.02906$ $0.11090 \quad 0.44322 \quad 1.02380$ 0.109550 .439031 .01855

$0.10820 \quad 0.43487 \quad 1.01330$ $0.106880 .43076 \quad 1.00807$ $0.105570 .42668 \quad 1.00285$ 0.104280 .422630 .99764 $0.10300 \quad 0.41863 \quad 0.99244$

$0.101740 .41466 \quad 0.98725$ $0.10050 \quad 0.41073 \quad 0.98207$ $\begin{array}{llll}0.09927 & 0.40684 & 0.97691\end{array}$ $\begin{array}{llll}0.09806 & 0.40298 & 0.97175\end{array}$ $0.096860 .39916 \quad 0.96661$

$\begin{array}{llll}0.09568 & 0.39537 & 0.96148\end{array}$ $\begin{array}{llll}0.09451 & 0.39161 & 0.95636\end{array}$ $\begin{array}{llll}0.09336 & 0.38790 & 0.95126\end{array}$ 0.092220 .384210 .94616 $\begin{array}{lll}0.09109 & 0.38056 & 0.94108\end{array}$
$0.204650 .70566 \quad 1.29076$ $0.20228 \quad 0.69954 \quad 1.28567$ $0.19994 \quad 0.69347 \quad 1.28058$ $0.197620 .68746 \quad 1.27548$ 0.195340 .681491 .27039

$0.19308 \quad 0.67558 \quad 1.26530$ $0.190850 .66972 \quad 1.26021$ $0.188650 .66391 \quad 1.25512$ $0.186470 .65816 \quad 1.25003$ $0.184320 .65245 \quad 1.24494$

$0.18219 \quad 0.64679 \quad 1.23985$ $0.18009 \quad 0.64117 \quad 1.23477$ $0.17802 \quad 0.63561 \quad 1.22969$ 0.175970 .630101 .22460 0.173950 .624631 .21952

$0.17195 \quad 0.61921 \quad 1.21445$ 0.169970 .613831 .20937 $0.168020 .60850 \quad 1.20430$ 0.166090 .603221 .19923 $0.164180 .59799 \quad 1.19417$

$0.16230 \quad 0.59279 \quad 1.18910$ $0.160440 .58765 \quad 1.18405$ $0.15860 \quad 0.58254 \quad 1.17899$ $0.156790 .57748 \quad 1.17394$ $0.154990 .57246 \quad 1.16889$

$0.153220 .56749 \quad 1.16385$ $0.151470 .56256 \quad 1.15881$ 0.149730 .557671 .15378 0.148020 .552821 .14875 0.146330 .548021 .14372

0.144660 .543251 .13870 0.143010 .538531 .13369 $0.14138 \quad 0.53384 \quad 1.12868$ $0.139770 .52920 \quad 1.12368$ 0.138170 .524591 .11868

$0.13660 \quad 0.52003 \quad 1.11369$ 0.135040 .515501 .10870 0.133510 .511021 .10372 0.131990 .50657 .1 .09875 0.130490 .502151 .09378

$0.12900 \quad 0.49778 \quad 1.08882$ $0.127540 .49344 \quad 1.08387$ $0.126090 .48914 \quad 1.07892$ $0.124650 .48488 \quad 1.07398$ 0.123240 .480651 .06905

$0.121840 .47646 \quad 1.06413$ 0.120460 .472311 .05921 0.119090 .468191 .05430 0.117740 .464101 .04940 0.116410 .460051 .04450
2100 2110 2120

2130

2140

2150

2160

2170

2180

2190

2200

2210

2220

2230

2240

2250

2260

2270

2280

2290

2300

2310

2320

2330

2340

2350

2360

2370

2380

2390

2400

2410

2420

2430

2440

2450

2460

2470

2480

2490

2500

2510

2520

2530

2540

2550

2560

2570

2580 2590 
Table II Harmonic Oscillator Contributions to the Thermodynamic

Functions (in units of calories, moles, and ${ }^{\circ} \mathrm{K}$ )

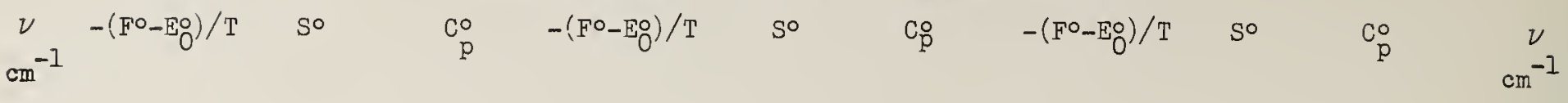

$T=1100$

2600

2610

2620

2630

2640

2650

2660

2670

2680

2690

2700

2710

2720

2730

2740

2750

2760

2770

2780

2790

2800

2810

2820

2830

2840

2850

2860

2870

2880

2890

2900

2910

2920

2930

2940

2950

2960

2970

2980

2990

3000

3010

3020

3030

3040

3050

3060

3070

3080

3090 $\begin{array}{llll}0.06740 & 0.30052 & 0.82018\end{array}$

$\begin{array}{llll}0.06650 & 0.29739 & 0.81503\end{array}$

$0.065630 .29428 \quad 0.80989$

$\begin{array}{llll}0.06476 & 0.29120 & 0.80478\end{array}$

$\begin{array}{lll}0.06390 & 0.28816 & 0.79969\end{array}$

$\begin{array}{llll}0.06306 & 0.28515 & 0.79461\end{array}$

$\begin{array}{llll}0.06223 & 0.28216 & 0.78955\end{array}$

$\begin{array}{llll}0.06141 & 0.27921 & 0.78451\end{array}$

$\begin{array}{llll}0.06060 & 0.27629 & 0.77950\end{array}$

$\begin{array}{llll}0.05980 & 0.27339 & 0.77449\end{array}$

0.059010 .270530 .76951

0.058230 .267690 .76455

$0.05746 \quad 0.26488 \quad 0.75961$

$0.05670 \quad 0.262110 .75468$

$0.05596 \quad 0.25936 \quad 0.74978$

0.055220 .256630 .74490 0.054490 .253940 .74003

0.053770 .251270 .73518

$\begin{array}{llll}0.05307 & 0.24863 & 0.73036\end{array}$

0.052370 .246020 .72555

$0.05168 \quad 0.243430 .72077$

$0.05100 \quad 0.240870 .71600$

0.050330 .238330 .71126

$0.04966 \quad 0.235820 .70653$

0.049010 .233340 .70182

$\begin{array}{llll}0.04837 & 0.23088 & 0.69714\end{array}$ $\begin{array}{llll}0.04773 & 0.22845 & 0.69247\end{array}$ $0.04710 \quad 0.22604 \quad 0.68783$ 0.046480 .223650 .68320 $\begin{array}{lll}0.04587 & 0.22129 & 0.67860\end{array}$

$0.04527 \quad 0.21896 \quad 0.67401$ 0.044670 .216640 .66945 0.044090 .214360 .66491 0.043510 .212090 .66038 0.042940 .209850 .65588

0.042370 .207630 .65140 0.041820 .205430 .64694 0.041270 .203260 .64250 0.040730 .201110 .63808 0.040190 .198970 .63368

$0.03966 \quad 0.19687 \quad 0.62930$ $0.039140 .19478 \quad 0.62494$ $\begin{array}{llll}0.03863 & 0.19271 & 0.62061\end{array}$ $\begin{array}{llll}0.03812 & 0.19067 & 0.61629\end{array}$ 0.037620 .188650 .61200

$\begin{array}{llll}0.03713 & 0.18664 & 0.60772\end{array}$ $\begin{array}{llll}0.03664 & 0.18466 & 0.60347\end{array}$ $\begin{array}{llll}0.03616 & 0.18270 & 0.59924\end{array}$ $\begin{array}{llll}0.03569 & 0.18076 & 0.59502\end{array}$ $\begin{array}{lll}0.03522 & 0.17883 & 0.59083\end{array}$

$T=1200$.

$T=1300$.

$\begin{array}{llll}0.08998 & 0.37694 & 0.93601\end{array}$ $\begin{array}{llll}0.08889 & 0.37336 & 0.93096\end{array}$ $\begin{array}{llll}0.08780 & 0.36981 & 0.92591\end{array}$ $\begin{array}{llll}0.08673 & 0.36629 & 0.92088\end{array}$ $\begin{array}{llll}0.08568 & 0.36281 & 0.91587\end{array}$

0.084630 .359350 .91086 $\begin{array}{llll}0.08360 & 0.35593 & 0.90587\end{array}$ $\begin{array}{llll}0.08259 & 0.35254 & 0.90090\end{array}$ $0.08158 \quad 0.349180 .89593$ $\begin{array}{llll}0.08059 & 0.34586 & 0.89098\end{array}$

$\begin{array}{llll}0.07961 & 0.34256 & 0.88605\end{array}$ 0.078640 .339290 .88112 $0.077690 .33606 \quad 0.87622$ 0.076740 .332850 .87132 0.075810 .329670 .86644

$0.074890 .32653 \quad 0.86158$ $0.07398 \quad 0.323410 .85673$ 0.073080 .320320 .85189 $\begin{array}{llll}0.07219 & 0.31726 & 0.84707\end{array}$ 0.071320 .314220 .84226

$\begin{array}{lll}0.07045 & 0.31122 & 0.83747\end{array}$ $\begin{array}{llll}0.06960 & 0.30824 & 0.83270\end{array}$ $\begin{array}{llll}0.06875 & 0.30529 & 0.82793\end{array}$ $\begin{array}{llll}0.06792 & 0.30237 & 0.82319\end{array}$ $\begin{array}{llll}0.06710 & 0.29947 & 0.81846\end{array}$

$\begin{array}{llll}0.06628 & 0.29661 & 0.81374\end{array}$ $\begin{array}{llll}0.06548 & 0.29376 & 0.80904\end{array}$ $\begin{array}{llll}0.06469 & 0.29095 & 0.80436\end{array}$ $\begin{array}{llll}0.06390 & 0.28816 & 0.79969\end{array}$ $\begin{array}{llll}0.06313 & 0.28540 & 0.79503\end{array}$

$\begin{array}{llll}0.06237 & 0.28266 & 0.79039\end{array}$ $\begin{array}{llll}0.06161 & 0.27994 & 0.78577\end{array}$ $\begin{array}{llll}0.06087 & 0.27726 & 0.78117\end{array}$ $\begin{array}{llll}0.06013 & 0.27459 & 0.77658\end{array}$ $\begin{array}{llll}0.05940 & 0.27196 & 0.77200\end{array}$

$0.05868 \quad 0.26934 \quad 0.76744$ $\begin{array}{llll}0.05797 & 0.26675 & 0.76290\end{array}$ $\begin{array}{llll}0.05727 & 0.26419 & 0.75838\end{array}$ $\begin{array}{llll}0.05658 & 0.26165 & 0.75387\end{array}$ $\begin{array}{llll}0.05590 & 0.25913 & 0.74937\end{array}$

$\begin{array}{llll}0.05522 & 0.25663 & 0.74490\end{array}$ $\begin{array}{llll}0.05455 & 0.25416 & 0.74044\end{array}$ $\begin{array}{lllll}0.05389 & 0.25171 & 0.73599\end{array}$ $\begin{array}{llll}0.05324 & 0.24929 & 0.73156\end{array}$ $\begin{array}{llll}0.05260 & 0.24688 & 0.72715\end{array}$

$\begin{array}{llll}0.05196 & 0.24450 & 0.72276\end{array}$ $\begin{array}{lllll}0.05134 & 0.24214 & 0.71838\end{array}$ $\begin{array}{lllll}0.05072 & 0.23981 & 0.71402\end{array}$ $\begin{array}{llll}0.05011 & 0.23749 & 0.70968\end{array}$ $\begin{array}{llll}0.04950 & 0.23520 & 0.70535\end{array}$
$0.11509 \quad 0.45604 \quad 1.03962$ $0.11378 \quad 0.45206 \quad 1.03474$ $0.11249 \quad 0.44811 \quad 1.02987$ 0.111220 .444191 .02501 $0.10996 \quad 0.440311 .02016$

$0.108720 .43647 \quad 1.01532$ 0.107490 .432651 .01049 $0.106270 .42887 \quad 1.00566$ 0.105070 .425121 .00084 $0.10388 \quad 0.42140 \quad 0.99604$

0.102710 .417710 .99124 $0.101550 .41406 \quad 0.98645$ $0.10040 \quad 0.410430 .98168$ $0.09927 \quad 0.40684 \quad 0.97691$ $0.09815 \quad 0.40327 \quad 0.97215$

$0.09704 \quad 0.39974 \quad 0.96740$ $0.09595 \quad 0.39624 \quad 0.96266$ $0.094870 .39277 \quad 0.95794$ $\begin{array}{llll}0.09380 & 0.38932 & 0.95322\end{array}$ $0.09274 \quad 0.38591 \quad 0.94851$

$0.09170 \quad 0.38252 \quad 0.94382$ $0.09066 \quad 0.379170 .93913$ $0.08964 \quad 0.37584 \quad 0.93446$ $0.08864 \quad 0.37254 \quad 0.92979$ $0.087640 .36927 \quad 0.92514$

$0.08665 \quad 0.36602 \quad 0.92050$ $0.08568 \quad 0.36281 \quad 0.91587$ $0.08471 \quad 0.359620 .91125$ $\begin{array}{llll}0.08376 & 0.35646 & 0.90664\end{array}$ 0.082820 .353320 .90204

0.081890 .350210 .89746 $\begin{array}{llll}0.08097 & 0.34713 & 0.89288\end{array}$ $\begin{array}{llll}0.08006 & 0.34408 & 0.88832\end{array}$ $\begin{array}{llll}0.07916 & 0.34105 & 0.88377\end{array}$ $0.078270 .33805 \quad 0.87924$

$0.07739 \quad 0.33507 \quad 0.87471$ $\begin{array}{llll}0.07653 & 0.33212 & 0.87020\end{array}$ $\begin{array}{llll}0.07567 & 0.32919 & 0.86569\end{array}$ 0.074820 .326290 .86121 $0.07398 \quad 0.323410 .85673$

$\begin{array}{llll}0.07315 & 0.32055 & 0.85226\end{array}$ $\begin{array}{llll}0.07233 & 0.31773 & 0.84781\end{array}$ $0.07152 \quad 0.31492 \quad 0.84337$ 0.070720 .312140 .83895 $0.06993 \quad 0.30938 \quad 0.83453$

0.069140 .306650 .83013 $0.06837 \quad 0.30394 \quad 0.82574$ $\begin{array}{lll}0.06760 & 0.30125 & 0.82137\end{array}$ $0.066850 .29859 \quad 0.81700$ $0.06610 \quad 0.29595 \quad 0.81266$
2600

2610

2620

2630

2640

2650

2660

2670

2680

2690

2700

2710

2720

2730

2740

2750

2760

2770

2780

2790

2800

2810

2820

2830

2840

2850

2860

2870

2880

2890

2900

2910

2920

2930

2940

2950

2960

2970

2980

2990

3000

3010

3020

3030

3040

3050

3060

3070

3080

3090 
Table II Harmonic Oscillator Contributions to the Thermodynamic

Functions (in units of calories, moles, and ${ }^{\circ} \mathrm{K}$ )
$\mathrm{cm}^{-1}-\left(\mathrm{F}^{\circ}-\mathrm{E}_{\mathrm{O}}^{\circ}\right) / \mathrm{T} \quad$ So

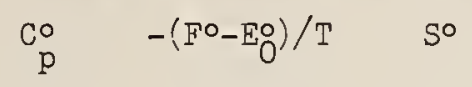
$\mathrm{C}_{\mathrm{p}}^{\circ} \quad-\left(\mathrm{F}^{\circ}-\mathrm{E}_{\mathrm{O}}\right) / \mathrm{T}$
So
$m^{-1}$

$T=1100$.

$T=1200$.

$T=1300$.

$\begin{array}{lllll}3100 & 0.03476 & 0.17693 & 0.58666\end{array}$

3110

3120

3130

3140

3150

3160

3170

3180

3190

3200

3210

3220

3230

3240

3250

3260

3270

3280

3290

3300

3310

3320

3330

3340

3350

3360

3370

3380

3390

3400

3410

3420

3430

3440

3450

3460

3470

3480

3490

3500

3510

3520

3530

3540

3550

3560

3570

3580

3590

$0.03430 \quad 0.175050 .58251$

0.033850 .173190 .57839

0.033410 .171340 .57428

0.032970 .169520 .57019

0.032540 .167710 .56613 0.032110 .165920 .56208 0.031690 .164150 .55806 $0.031280 .16240 \quad 0.55406$ 0.030870 .160670 .55007

$0.03046 \quad 0.15895 \quad 0.54611$ $0.03006 \quad 0.15725 \quad 0.54217$ $\begin{array}{llll}0.02967 & 0.15557 & 0.53825\end{array}$ $\begin{array}{llll}0.02928 & 0.15391 & 0.53436\end{array}$ $\begin{array}{llll}0.02890 & 0.15227 & 0.53048\end{array}$

0.028520 .150640 .52662 0.028150 .149030 .52278 $0.02778 \quad 0.147430 .51897$ 0.027420 .145850 .51517 0.027060 .144290 .51140

$\begin{array}{llll}0.02670 & 0.14274 & 0.50765\end{array}$ 0.026350 .141210 .50391 $0.026010 .13970 \quad 0.50020$ $\begin{array}{llll}0.02567 & 0.13820 & 0.49651\end{array}$ 0.025330 .136720 .49284

$0.02500 \quad 0.135250 .48919$ 0.024670 .133790 .48556 $\begin{array}{llll}0.02435 & 0.13236 & 0.48195\end{array}$ 0.024030 .130930 .47836 $0.023720 .12953 \quad 0.47479$

0.023410 .128130 .47124 $\begin{array}{llll}0.02310 & 0.12675 & 0.46772\end{array}$ $0.02280 \quad 0.125390 .46421$ $\begin{array}{llll}0.02250 & 0.12404 & 0.46072\end{array}$ $0.022210 .12270 \quad 0.45726$

$\begin{array}{llll}0.02192 & 0.12138 & 0.45381\end{array}$ $\begin{array}{llll}0.02163 & 0.12007 & 0.45038\end{array}$ $\begin{array}{llll}0.02135 & 0.11878 & 0.44698\end{array}$ $\begin{array}{llll}0.02107 & 0.11750 & 0.44359\end{array}$ $0.02080 \quad 0.116230 .44022$

$\begin{array}{llll}0.02052 & 0.11497 & 0.43688\end{array}$ 0.020260 .113730 .43355 $0.019990 .11250 \quad 0.43024$ 0.019730 .111290 .42696 $0.019470 .11008 \quad 0.42369$

$\begin{array}{llll}0.01922 & 0.10889 & 0.42044\end{array}$ $\begin{array}{llll}0.01897 & 0.10772 & 0.41721\end{array}$ $\begin{array}{llll}0.01872 & 0.10655 & 0.41400\end{array}$ $\begin{array}{lll}0.01848 & 0.10540 & 0.41082\end{array}$ $\begin{array}{lll}0.01823 & 0.10425 & 0.40765\end{array}$ $\begin{array}{llll}0.04890 & 0.23293 & 0.70104\end{array}$ $\begin{array}{llll}0.04831 & 0.23068 & 0.69675\end{array}$ $\begin{array}{llll}0.04773 & 0.22845 & 0.69247\end{array}$ $\begin{array}{llll}0.04715 & 0.22624 & 0.68821\end{array}$ 0.046590 .224050 .68397

$\begin{array}{llll}0.04602 & 0.22188 & 0.67975\end{array}$ $0.045470 .21973 \quad 0.67554$ 0.044920 .217610 .67135 $\begin{array}{llll}0.04438 & 0.21550 & 0.66717\end{array}$ $\begin{array}{llll}0.04385 & 0.21341 & 0.66302\end{array}$

0.043320 .211340 .65888 0.042800 .209290 .65476 0.042280 .207260 .65065 $\begin{array}{llll}0.04177 & 0.20525 & 0.64657\end{array}$ 0.041270 .203260 .64250

0.040770 .201280 .63845 0.040280 .199330 .63441 $0.03979 \quad 0.197390 .63039$ $\begin{array}{llll}0.03932 & 0.19547 & 0.62639\end{array}$ 0.038840 .193570 .62241

$0.03838 \quad 0.191690 .61845$ $\begin{array}{llll}0.03791 & 0.18982 & 0.61450\end{array}$ $\begin{array}{llll}0.03746 & 0.18798 & 0.61057\end{array}$ 0.037010 .186150 .60666 $0.03656 \quad 0.18433 \quad 0.60276$

$\begin{array}{llll}0.03612 & 0.18254 & 0.59888\end{array}$ $\begin{array}{lllll}0.03569 & 0.18076 & 0.59502\end{array}$ $\begin{array}{llll}0.03526 & 0.17899 & 0.59118\end{array}$ $\begin{array}{lll}0.03483 & 0.17725 & 0.58736\end{array}$ $\begin{array}{lll}0.03442 & 0.17552 & 0.58355\end{array}$

$\begin{array}{llll}0.03400 & 0.17381 & 0.57976\end{array}$ $\begin{array}{llll}0.03359 & 0.17211 & 0.57599\end{array}$ $\begin{array}{llll}0.03319 & 0.17043 & 0.57223\end{array}$ $\begin{array}{llll}0.03279 & 0.16876 & 0.56850\end{array}$ $\begin{array}{llll}0.03240 & 0.16711 & 0.56478\end{array}$

$\begin{array}{llll}0.03201 & 0.16548 & 0.56108\end{array}$ $\begin{array}{llll}0.03162 & 0.16386 & 0.55739\end{array}$ 0.031240 .162260 .55372 $\begin{array}{lllll}0.03087 & 0.16067 & 0.55007\end{array}$ $\begin{array}{llll}0.03050 & 0.15910 & 0.54644\end{array}$

$\begin{array}{llll}0.03013 & 0.15754 & 0.54283\end{array}$ $0.029770 .15599 \quad 0.53923$ $\begin{array}{llll}0.02941 & 0.15446 & 0.53565\end{array}$ $\begin{array}{llll}0.02906 & 0.15295 & 0.53209\end{array}$ $\begin{array}{lll}0.02871 & 0.15145 & 0.52855\end{array}$

$0.028360 .14996 \quad 0.52502$ $\begin{array}{llll}0.02802 & 0.14849 & 0.52151\end{array}$ $\begin{array}{llll}0.02769 & 0.14703 & 0.51802\end{array}$ $0.02736 \quad 0.145590 .51454$ 0.027030 .144160 .51109
$0.06536 \quad 0.293330 .80832$

0.064630 .290730 .80400

$0.06390 \quad 0.28816 \quad 0.79969$

0.063190 .285610 .79539

$0.06248 \quad 0.28308 \quad 0.79111$

$\begin{array}{llll}0.06178 & 0.28057 & 0.78684\end{array}$ $0.061090 .27808 \quad 0.78258$ 0.060410 .275610 .77834 0.059740 .273170 .77411 0.059070 .270750 .76990

$\begin{array}{llll}0.05841 & 0.26834 & 0.76569\end{array}$ $0.057760 .26596 \quad 0.76151$ 0.057110 .263600 .75733 $\begin{array}{llll}0.05647 & 0.26126 & 0.75317\end{array}$ 0.055840 .258930 .74903

0.055220 .256630 .74490 $0.05460 \quad 0.254350 .74078$ 0.053990 .252090 .73667 0.053390 .249840 .73258 $0.05280 \quad 0.247620 .72851$

0.052210 .245420 .72445 0.051630 .243230 .72040 $0.051050 .24106 \quad 0.71637$ $0.05048 \quad 0.238910 .71235$ 0.049920 .236780 .70834

$0.04936 \quad 0.234670 .70435$ 0.048810 .232580 .70038 $0.048270 .23050 \quad 0.69642$ 0.047730 .228450 .69247 $0.04720 \quad 0.226410 .68854$

0.046670 .224380 .68462 $0.04615 \quad 0.22238 \quad 0.68072$ $\begin{array}{llll}0.04564 & 0.22039 & 0.67683\end{array}$ 0.045130 .218420 .67296 0.044630 .216470 .66910

0.044130 .214530 .66525 0.043640 .212610 .66142 0.043160 .210710 .65761 0.042680 .208820 .65381 $0.04220 \quad 0.206950 .65002$

0.041730 .205100 .64625 0.041270 .203260 .64250 0.040810 .201430 .63876 0.040350 .199630 .63503 0.039910 .197840 .63132

$0.039460 .19606 \quad 0.62762$ $0.039020 .19430 \quad 0.62394$ $0.038590 .19256 \quad 0.62027$ 0.038160 .190830 .61662 0.037740 .189110 .61299
3100

3110

3120

3130

3140

3150

3160

3170

3180

3190

3200

3210

3220

3230

3240

3250

3260

3270

3280

3290

3300

3310

3320

3330

3340

3350

3360

3370

3380

3390

3400

3410

3420

3430

3440

3450

3460

3470

3480

3490

3500

3510

3520

3530

3540

3550

3560

3570

3580

3590 
Table II Harmonic Oscillator Contributions to the Thermodynamic

Functions (in units of calories, moles, and ${ }^{\circ} \mathrm{K}$ )

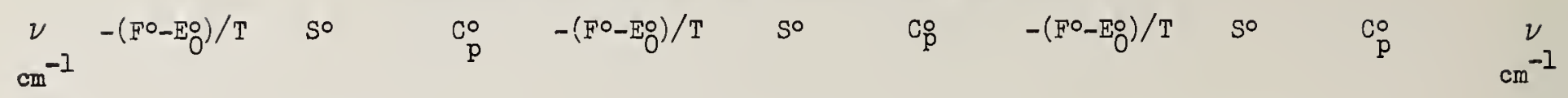

$$
T=1100
$$

3600

3610

3620

3630

3640

3650

3660

3670

3680

3690

3700

3710

3720

3730

3740

3750

3760

3770

3780

3790

3800

3810

3820

3830

3840

3850

3860

3870

3880

3890

3900

3910

3920

3930

3940

3950

3960

3970

3980

3990

4000
$0.01800 \quad 0.103120 .40450$

0.017760 .102010 .40137

$0.017530 .10090 \quad 0.39826$

$\begin{array}{llll}0.01730 & 0.09981 & 0.39517\end{array}$

$0.01708 \quad 0.098720 .39209$

0.016850 .097650 .38904

0.016630 .096590 .38601

$0.016420 .09554 \quad 0.38299$

$0.016200 .09450 \quad 0.37999$

$\begin{array}{llll}0.01599 & 0.09348 & 0.37702\end{array}$

$0.01578 \quad 0.092460 .37406$

$0.01558 \quad 0.091460 .37112$

0.015370 .090460 .36820

$0.015170 .08948 \quad 0.36530$

$\begin{array}{llll}0.01497 & 0.08850 & 0.36241\end{array}$

$\begin{array}{llll}0.01478 & 0.08754 & 0.35955\end{array}$

$\begin{array}{llll}0.01459 & 0.08658 & 0.35670\end{array}$

$0.01440 \quad 0.085640 .35388$

0.014210 .084710 .35107

$\begin{array}{llll}0.01402 & 0.08378 & 0.34827\end{array}$

$\begin{array}{llll}0.01384 & 0.08287 & 0.34550\end{array}$ 0.013660 .081960 .34275 0.013480 .081070 .34001 $\begin{array}{llll}0.01331 & 0.08018 & 0.33729\end{array}$ 0.013130 .079310 .33459

$\begin{array}{llll}0.01296 & 0.07844 & 0.33191\end{array}$ $0.012790 .07758 \quad 0.32924$ $\begin{array}{llll}0.01263 & 0.07674 & 0.32660\end{array}$ $0.012460 .07590 \quad 0.32397$ $0.01230 \quad 0.07507 \quad 0.32135$

0.012140 .074240 .31876 0.011980 .073430 .31618 0.011820 .072630 .31362 $0.011670 .07183 \quad 0.31108$ 0.011520 .071040 .30855

$0.011370 .07026 \quad 0.30605$ 0.011220 .069490 .30356 $\begin{array}{llll}0.01107 & 0.06873 & 0.30108\end{array}$ $\begin{array}{llll}0.01093 & 0.06798 & 0.29862\end{array}$ $\begin{array}{llll}0.01079 & 0.06723 & 0.29618\end{array}$

0.010650 .066490 .29376
$T=1200$.

$0.02670 \quad 0.14274 \quad 0.50765$ 0.026380 .141340 .50422 0.026070 .139950 .50082 0.025750 .138570 .49743 0.025440 .137210 .49406

$0.02514 \quad 0.13586 \quad 0.49071$ 0.024840 .134520 .48737 0.024540 .133190 .48405 $0.024250 .13188 \quad 0.48075$ $0.02396 \quad 0.13058 \quad 0.47747$

0.023670 .129290 .47420 0.023380 .128020 .47095 $\begin{array}{llll}0.02310 & 0.12675 & 0.46772\end{array}$ $0.022830 .12550 \quad 0.46450$ 0.022550 .124260 .46130

$0.02228 \quad 0.123040 .45812$ 0.022020 .121820 .45495 0.021750 .120620 .45181 0.021490 .119420 .44868 0.021230 .118240 .44556

0.020980 .117070 .44246 0.020730 .115910 .43938 0.020480 .114770 .43632 $\begin{array}{llll}0.02023 & 0.11363 & 0.43327\end{array}$ $0.019990 .11250 \quad 0.43024$

$0.01975 \quad 0.111390 .42723$ $0.019520 .11028 \quad 0.42423$ 0.019280 .109190 .42125 0.019050 .108110 .41829 $0.018820 .10703 \quad 0.41534$

0.018600 .105970 .41241 0.018380 .104920 .40949 $0.01816 \quad 0.103880 .40660$ 0.017940 .102840 .40371 0.017720 .101820 .40085

$\begin{array}{llll}0.01751 & 0.10081 & 0.39800\end{array}$ $\begin{array}{llll}0.01730 & 0.09981 & 0.39517\end{array}$ $\begin{array}{llll}0.01709 & 0.09881 & 0.39235\end{array}$ $\begin{array}{llll}0.01689 & 0.09783 & 0.38955\end{array}$ $\begin{array}{lll}0.01669 & 0.09686 & 0.38676\end{array}$

0.016490 .095890 .38399
$T=1300$.

$0.03732 \quad 0.18741 \quad 0.60936$ $0.03690 \quad 0.18573 \quad 0.60576$ 0.036490 .184050 .60216 $0.036090 .18240 \quad 0.59859$ $0.035690 .18076 \quad 0.59502$

0.035290 .179130 .59148 $0.03490 \quad 0.17752 \quad 0.58794$ 0.034510 .175920 .58443 0.034130 .174330 .58092 $0.03375 \quad 0.17276 \quad 0.57744$

$0.033370 .17120 \quad 0.57396$ $0.03300 \quad 0.16966 \quad 0.57051$ 0.032640 .168130 .56706 $0.03228 \quad 0.16661 \quad 0.56364$ 0.031920 .165100 .56022

$\begin{array}{llll}0.03156 & 0.16361 & 0.55683\end{array}$ $0.03121 \quad 0.16213 \quad 0.55344$ 0.030870 .160670 .55007 0.030530 .159220 .54672 $\begin{array}{llll}0.03019 & 0.15778 & 0.54338\end{array}$

0.029850 .156350 .54006 0.029520 .154930 .53675 0.029190 .153530 .53346 0.028870 .152140 .53018 0.028550 .150760 .52692

$0.02823 \quad 0.14940 \quad 0.52367$ 0.027920 .148040 .52043 $0.027610 .14670 \quad 0.51721$ 0.027300 .145370 .51401 $0.02700 \quad 0.14405 \quad 0.51082$

$0.02670 \quad 0.14274 \quad 0.50765$ 0.026410 .141450 .50449 $0.026110 .14016 \quad 0.50134$ $0.02583 \quad 0.138890 .49821$ 0.025540 .137630 .49510

$0.025260 .13638 \quad 0.49200$ $0.02498 \quad 0.135130 .48891$ $0.02470 \quad 0.133910 .48584$ $0.02443 \quad 0.132690 .48278$ $0.024160 .13148 \quad 0.47974$

$0.02389 \quad 0.13028 \quad 0.47671$
3600

3610

3620

3630

3640

3650

3660

3670

3680

3690

3700

3710

3720

3730

3740

3750

3760

3770

3780

3790

3800

3810

3820

3830

3840

3850

3860

3870

3880

3890

3900

3910

3920

3930

3940

3950

3960

3970

3980

3990

4000 
Table II Harmonic Oscillator Contributions to the Thermodynamic

Functions (in units of calories, moles, and ${ }^{\circ} \mathrm{K}$ )

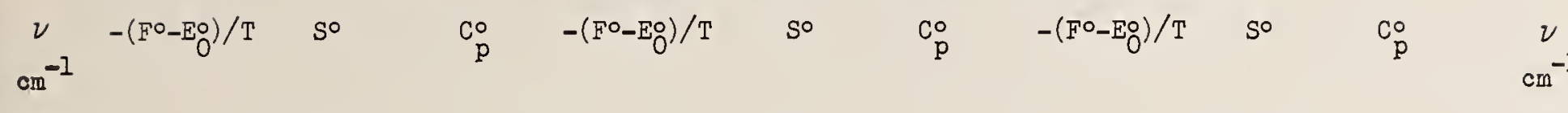

$T=1400$.

$T=1500$.

$T=1600$.

$100 \quad 4.62254 \quad 6.509351 .98542$

$110 \quad 4.443176 .320131 .98505$

$120 \quad 4.280286 .14743 \quad 1.98465$

$130 \quad 4.13121 \quad 5.98859 \quad 1.98422$

$140 \quad 3.993925 .84156 \quad 1.98375$

$\begin{array}{lllll}150 & 3.86678 & 5.70471 & 1.98324\end{array}$

$160 \quad 3.748475 .57673 \quad 1.98270$

$170 \quad 3.637925 .456551 .98212$

$180 \quad 3.534245 .34327 \quad 1.98151$

$190 \quad 3.436695 .236161 .98087$

$200 \quad 3.344635 .13457 \quad 1.98019$

$210 \quad 3.257535 .03797 \quad 1.97947$

$220 \quad 3.17492 \quad 4.94590 \quad 1.97873$

$230 \quad 3.096414 .85796 \quad 1.97794$

$240 \quad 3.021634 .773801 .97713$

$250 \quad 2.95030 \quad 4.69311 \quad 1.97627$

$260 \quad 2.882124 .615611 .97539$

$270 \quad 2.81688 \quad 4.54108 \quad 1.97447$

$280 \quad 2.754344 .469291 .97351$

$290 \quad 2.694324 .400051 .97253$

$300 \quad 2.63665 \quad 4.33320 \quad 1.97150$

$\begin{array}{llll}310 & 2.58117 & 4.26857 & 1.97045\end{array}$

$320 \quad 2.527744 .206031 .96936$

$330 \quad 2.476234 .145441 .96823$

$340 \quad 2.426544 .08670 \quad 1.96707$

$\begin{array}{llll}350 \quad 2.37854 & 4.02970 \quad 1.96588\end{array}$

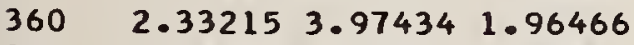

$\begin{array}{llll}370 & 2.28728 & 3.92052 & 1.96340\end{array}$

$\begin{array}{lllll}380 & 2.24384 & 3.86818 & 1.96211\end{array}$

$390 \quad 2.201773 .817231 .96078$

$400 \quad 2.16098 \quad 3.76761 \quad 1.95942$

$410 \quad 2.12141 \quad 3.71924 \quad 1.95803$

$420 \quad 2.08302 \quad 3.67207 \quad 1.95660$

$430 \quad 2.04573 \quad 3.626051 .95514$

$440 \quad 2.00950 \quad 3.581121 .95365$

$\begin{array}{lllll}450 & 1.97427 & 3.53723 & 1.95213\end{array}$

$460 \quad 1.940023 .49434 \quad 1.95057$

$470 \quad 1.90668 \quad 3.45241 \quad 1.94898$

$480 \quad 1.87423 \quad 3.411391 .94736$

$490 \quad 1.842623 .37126 \quad 1.94570$

$\begin{array}{llll}500 \quad 1.81182 & 3.33197 & 1.94402\end{array}$

$\begin{array}{llll}510 & 1.78180 & 3.29349 & 1.94230\end{array}$

$\begin{array}{llll}520 & 1.75253 & 3.25579 & 1.94054\end{array}$

$\begin{array}{lllll}530 & 1.72398 & 3.21884 & 1.93876\end{array}$

$\begin{array}{lll}540 \quad 1.69611 & 3.18262 & 1.93694\end{array}$

$\begin{array}{llll}550 & 1.66891 & 3.14709 & 1.93510\end{array}$

$560 \quad 1.642353 .112241 .93322$

$570 \quad 1.616413 .078041 .93131$

$\begin{array}{llllll}580 & 1.59106 & 3.04447 & 1.92936\end{array}$

$590 \quad 1.56628 \quad 3.011511 .92739$
$4.75295 \quad 6.64634 \quad 1.98565$ $4.572926 .45710 \quad 1.98533$ $4.409376 .28437 \quad 1.98498$ 4.259656 .125501 .98460 4.121715 .978441 .98419

$3.993925 .84156 \quad 1.98375$ 3.874975 .713551 .98327 3.763775 .593331 .98277 $3.65945 \quad 5.48001 \quad 1.98224$ 3.561265 .372851 .98168

3.468575 .271221 .98109 $3.380835 .17458 \quad 1.98046$ 3.297595 .082461 .97981 $3.218454 .99447 \quad 1.97913$ 3.143044 .910251 .97842

$3.07108 \quad 4.82950 \quad 1.97767$ $3.00229 \quad 4.751951 .97690$ 2.936424 .677361 .97610 $2.87326 \quad 4.60551 \quad 1.97527$ 2.812624 .536211 .97441

$2.75434 \quad 4.46929 \quad 1.97351$ 2.698254 .404591 .97259 2.644214 .341981 .97164 2.592094 .281321 .97066 $2.541794 .22251 \quad 1.96965$

2.493194 .165431 .96861 2.446214 .109991 .96754 2.400734 .056091 .96644 $2.35670 \quad 4.00366 \quad 1.96531$ 2.314033 .952631 .96416

$2.27265 \quad 3.90292 \quad 1.96297$ $2.23249 \quad 3.85446 \quad 1.96175$ $2.193513 .80720 \quad 1.96051$ $2.15563 \quad 3.76108 \quad 1.95924$ $2.11882 \quad 3.71606 \quad 1.95793$

$2.08302 \quad 3.672071 .95660$ $2.04818 \quad 3.62908 \quad 1.95524$ 2.014273 .587051 .95385 1.981243 .545931 .95243 $1.94906 \quad 3.505681 .95099$

$1.91769 \quad 3.46628 \quad 1.94951$ $1.887113 .42769 \quad 1.94801$ $1.85727 \quad 3.38988 \quad 1.94648$ $1.82815 \quad 3.35282 \quad 1.94492$ $1.799723 .31648 \quad 1.94333$

$1.77197 \quad 3.28084 \quad 1.94172$ $1.74485 \quad 3.24586 \quad 1.94007$ $1.71835 \quad 3.21154 \quad 1.93840$ $1.69245 \quad 3.17784 \quad 1.93670$ 1.667123 .144751 .93497
4.875336 .774491 .98583 $4.69473 \quad 6.585241 .98555$ $4.53060 \quad 6.412481 .98524$ $4.380316 .25359 \quad 1.98491$ $4.241806 .10651 \quad 1.98455$

4.113445 .969601 .98416 3.993925 .841561 .98375 $3.88216 \quad 5.721311 .98330$ $3.77728 \quad 5.60796 \quad 1.98284$ 3.678535 .500771 .98234

$3.58527 \quad 5.39910 \quad 1.98182$ $3.49698 \quad 5.302421 .98128$ 3.413185 .210261 .98070 $3.33348 \quad 5.122231 .98010$ $3.257535 .03797 \quad 1.97947$

$3.18502 \quad 4.95718 \quad 1.97882$ $3.11567 \quad 4.87958 \quad 1.97814$ $3.04926 \quad 4.80494 \quad 1.97744$ $2.985554 .73304 \quad 1.97670$ 2.924374 .663681 .97595

$2.86555 \quad 4.59671 \quad 1.97516$ $2.808914 .53196 \quad 1.97435$ $2.75434 \quad 4.46929 \quad 1.97351$ 2.701694 .408571 .97265 2.650854 .349701 .97176

2.601724 .292551 .97085 $2.55420 \quad 4.23705 \quad 1.96991$ $2.50820 \quad 4.18308 \quad 1.96894$ $2.46364 \quad 4.130591 .96795$ $2.42045 \quad 4.07948 \quad 1.96693$

$2.378544 .02970 \quad 1.96588$ 2.337873 .981171 .96481 $2.29836 \quad 3.93384 \quad 1.96372$ $2.25997 \quad 3.887641 .96259$ $2.222643 .84254 \quad 1.96145$

$2.18632 \quad 3.79847 \quad 1.96027$ $2.150973 .75540 \quad 1.95908$ $2.11655 \quad 3.71328 \quad 1.95785$ 2.083023 .672071 .95660 2. $05033 \quad 3.63174 \quad 1.95533$

$2.01846 \quad 3.592251 .95403$ $1.98737 \quad 3.55357 \quad 1.95270$ 1.957033 .515671 .95135 1.927413 .478511 .94998 $1.898493 .44207 \quad 1.94858$

1.870233 .406331 .94715 $1.842623 .37126 \quad 1.94570$ 1.815633 .336831 .94423 1.789243 .303031 .94273 1.763423 .269841 .94120
100

110

120

130

140

150

160

170

180

190

200

210

220

230

240

250

260

270

280

290

300

310

320

330

340

350

360

370

380

390

400

410

420

430

440

450

460

470

480

490

500

510

520

530

540

550

560

570

580

590 
Table II Harmonic Oscillator Contributions to the Thermodynamic

Functions (in units of calories, moles, and ${ }^{\circ} \mathrm{K}$ )
$\mathrm{cm}^{-1}-\left(F^{\circ}-E_{0}^{\circ}\right) / T \quad$ So
$\stackrel{\mathrm{c}}{\mathrm{p}}$
$-\left(F^{\circ}-E_{O}^{O}\right) / T \quad$ SO
$\mathrm{C}_{\mathrm{p}} \quad-\left(\mathrm{F}^{\circ}-\mathrm{E}_{\mathrm{O}}^{\circ}\right) / \mathrm{T}$
$\mathrm{S}^{\circ}$

$$
T=1400 \text {. }
$$
$T=1500$.
$T=1600$.

$\nu$

$\mathrm{cm}^{-1}$

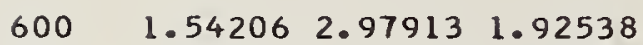

$\begin{array}{lll}610 & 1.51838 \quad 2.94732 \quad 1.92335\end{array}$

$\begin{array}{llll}620 \quad 1.49521 & 2.91606 \quad 1.92128\end{array}$

$\begin{array}{lll}630 & 1.47254 & 2.88534 \quad 1.91918\end{array}$

$\begin{array}{llll}640 \quad 1.45035 & 2.85513 \quad 1.91705\end{array}$

$\begin{array}{llll}650 \quad 1.42863 & 2.82543 \quad 1.91489\end{array}$

$\begin{array}{llll}660 & 1.40737 & 2.79621 & 1.91270\end{array}$

$\begin{array}{lllll}670 & 1.38654 & 2.76746 & 1.91048\end{array}$

$\begin{array}{llllll}680 & 1.36614 & 2.73917 & 1.90823\end{array}$

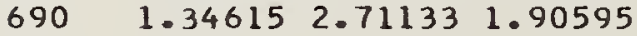

$\begin{array}{lllll}700 & 1.32657 & 2.68392 & 1.90364\end{array}$

$\begin{array}{lllll}710 & 1.30737 & 2.65694 & 1.90130\end{array}$

$\begin{array}{lllll}720 & 1.28855 & 2.63036 & 1.89893\end{array}$

$\begin{array}{lllll}730 & 1.27009 & 2.60419 & 1.89653\end{array}$

$\begin{array}{lll}740 & 1.25199 & 2.57840 \quad 1.89410\end{array}$

$\begin{array}{lllll}750 & 1.23424 & 2.55299 & 1.89164\end{array}$

$\begin{array}{llll}760 & 1.21682 & 2.52795 & 1.88915\end{array}$

$\begin{array}{lllll}770 & 1.19973 & 2.50327 & 1.88664\end{array}$

$\begin{array}{llll}780 & 1.18296 & 2.47895 & 1.88409\end{array}$

$\begin{array}{llll}790 & 1.16650 & 2.45496 & 1.88152\end{array}$

$\begin{array}{lllll}800 & 1.15034 & 2.43131 & 1.87892\end{array}$

$\begin{array}{lllll}810 & 1.13447 & 2.40799 & 1.87629\end{array}$

$\begin{array}{lllll}820 & 1.11889 & 2.38498 & 1.87363\end{array}$

$\begin{array}{lllll}830 & 1.10359 & 2.36229 & 1.87094\end{array}$

$\begin{array}{llll}840 & 1.08856 & 2.33989 & 1.86822\end{array}$

$\begin{array}{lllll}850 & 1.07380 & 2.31780 & 1.86548\end{array}$

$\begin{array}{lllll}860 & 1.05929 & 2.29600 & 1.86271\end{array}$

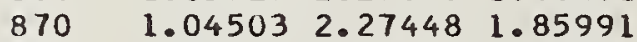

$\begin{array}{lllll}880 & 1.03102 & 2.25324 & 1.85709\end{array}$

$\begin{array}{lllll}890 & 1.01725 & 2.23227 & 1.85424\end{array}$

$\begin{array}{lllll}900 \quad 1.00372 & 2.21157 & 1.85136\end{array}$

$910 \quad 0.990412 .19113 \quad 1.84845$

$920 \quad 0.97733 \quad 2.17094 \quad 1.84552$

$930 \quad 0.96446 \quad 2.15101 \quad 1.84256$

$940 \quad 0.95181$
$2.13132 \quad 1.83958$

$950 \quad 0.93936 \quad 2.11187 \quad 1.83657$

$960 \quad 0.92712 \quad 2.09265 \quad 1.83353$

$970 \quad 0.91508 \quad 2.07367 \quad 1.83047$

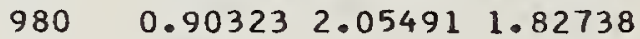

$990 \quad 0.89157 \quad 2.03637 \quad 1.82427$

$\begin{array}{llll}1000 & 0.88010 & 2.01805 & 1.82113\end{array}$

$\begin{array}{llll}1010 & 0.86881 & 1.99995 & 1.81797\end{array}$

$\begin{array}{lllll}1020 & 0.85770 & 1.98205 & 1.81478\end{array}$

$\begin{array}{lllll}1030 & 0.84677 & 1.96436 & 1.81157\end{array}$

$\begin{array}{lllll}1040 & 0.83600 & 1.94687 & 1.80833\end{array}$

$\begin{array}{lllll}1050 & 0.82540 & 1.92958 & 1.80507\end{array}$

$\begin{array}{lllll}1060 & 0.81497 & 1.91249 & 1.80178\end{array}$

$\begin{array}{lllll}1070 & 0.80469 & 1.89559 & 1.79847\end{array}$

$1080 \quad 0.79458 \quad 1.87887 \quad i .79514$

$\begin{array}{lllll}1090 & 0.78461 & 1.86234 & 1.79178\end{array}$
$1.64235 \quad 3.11224 \quad 1.93322$

1.618123 .080301 .93143

$1.594413 .04891 \quad 1.92962$

1.571193 .013051 .92779

$1.54847 \quad 2.98771 \quad 1.92592$

$\begin{array}{llll}1.52 .621 & 2.95786 & 1.92403\end{array}$

1.504412 .928501 .92211

$1.483052 .89961 \quad 1.92016$

$1.46212 \quad 2.871181 .91819$

1.441612 .843191 .91619

$1.421492 .81563 \quad 1.91417$

$1.401772 .78850 \quad 1.91211$

1.382432 .761771 .91003

$1.36345 \quad 2.73544 \quad 1.90793$

1.344842 .709491 .90580

$1.32657 \quad 2.68392 \quad 1.90364$ $1.308642 .65872 \quad 1.90146$ $1.291042 .63388 \quad 1.89925$ $1.27375 \quad 2.60939 \quad 1.89701$ $1.256792 .58524 \quad 1.89475$

1.240122 .561421 .89246 $1.223752 .53792 \quad 1.89015$ $1.207672 .51475 \quad 1.88782$ $1.191872 .49188 \quad 1.88545$ $1.176342 .46931 \quad 1.88307$

$1.16108 \quad 2.44704 \quad 1.88065$ $1.14608 \quad 2.42506 \quad 1.87822$ $1.13134 \quad 2.40336 \quad 1.87576$ $1.11684 \quad 2.38194 \quad 1.87327$ $1.10258 \quad 2.36078 \quad 1.87076$

$1.08856 \quad 2.33989 \quad 1.86822$ 1.074772 .319271 .86567 $\begin{array}{llll}1.06121 & 2.29889 & 1.86308\end{array}$ $1.04786 \quad 2.27876 \quad 1.86048$ $\begin{array}{lll}1.03474 & 2.25888 & 1.85784\end{array}$

$1.02182 \quad 2.23923 \quad 1.85519$ $1.009102 .21982 \quad 1.85251$ $0.996592 .20064 \quad 1.84981$ $0.98428 \quad 2.18168 \quad 1.84709$ $0.97215 \quad 2.16294 \quad 1.84434$

$\begin{array}{llll}0.96022 & 2.14442 & 1.84157\end{array}$ $0.94847 \quad 2.12611 \quad 1.83878$ $0.936902 .10800 \quad 1.83596$ $0.92550 \quad 2.09011 \quad 1.83312$ $0.91428 \quad 2.07241 \quad 1.83026$

$\begin{array}{llll}0.90323 & 2.05491 & 1.82738\end{array}$ $0.89235 \quad 2.03760 \quad 1.82448$ $\begin{array}{llll}0.88162 & 2.02048 & 1.82155\end{array}$ $0.87106 \quad 2.00355 \quad 1.81860$ 0.860651 .986801 .81563
$1.73817 \quad 3.23722 \quad 1.93966$

$1.71345 \quad 3.20517 \quad 1.93808$ $\begin{array}{llll}1.68925 & 3.17367 & 1.93648\end{array}$

$1.665563 .14270 \quad 1.93486$

$1.64235 \quad 3.11224 \quad 1.93322$

$1.619623 .08228 \quad 1.93155$

$\begin{array}{llll}1.59734 & 3.05281 & 1.92985\end{array}$

$1.575513 .02380 \quad 1.92813$

$1.554112 .99525 \quad 1.92639$

$1.533122 .96714 \quad 1.92462$

$1.512542 .93946 \quad 1.92283$

1.492352 .912191 .92102

$1.472542 .88534 \quad 1.91918$

$1.45310 \quad 2.85888 \quad 1.91732$

1.434022 .832811 .91544

$1.415292 .80711 \quad 1.91353$

$\begin{array}{llll}1.39690 & 2.78178 \quad 1.91160\end{array}$

$1.378842 .75680 \quad 1.90964$

1.361112 .732171 .90766

$\begin{array}{llll}1.34368 & 2.70788 & 1.90566\end{array}$

$1.326572 .68392 \quad 1.90364$

$1.30975 \quad 2.66029 \quad 1.90159$

$1.29322 \quad 2.63697 \quad 1.89952$

$1.27697 \quad 2.61396 \quad 1.89743$

$1.26100 \quad 2.59125 \quad 1.89532$

1. $24530 \quad 2.56883 \quad 1.89318$

$1.229852 .54670 \quad 1.89102$

1. 214672.524851 .88884

$\begin{array}{lllll}1.19973 & 2.50327 & 1.88664\end{array}$

$1.18504 \quad 2.48197 \quad 1.88441$

1.170592 .460931 .88216

1.156372 .440141 .87990

$1.142372 .41961 \quad 1.87760$

$1.128602 .39932 \quad 1.87529$

$1.115042 .37928 \quad 1.87296$

$\begin{array}{llll}1.10170 \quad 2.35947 & 1.87060\end{array}$

1.088562 .339891 .86822

$1.075632 .32055 \quad 1.86583$

1.062892 .301421 .86341

1.050352 .282521 .86097

$1.03800 \quad 2.26383 \quad 1.85850$

1.025832 .245351 .85602

$1.013852 .22707 \quad 1.85352$

1.002042 .209001 .85100

0.990412 .191131 .84845

$0.97895 \quad 2.17345 \quad 1.84589$

$\begin{array}{llll}0.96766 & 2.15597 & 1.84330\end{array}$

$0.956532 .13867 \quad 1.84070$

$0.945562 .12156 \quad 1.83808$

0.934752 .104631 .83543
600

610

620

630

640

650

660

670

680

690

700

710

720

730

740

750

760

770

780

790

800

810

820

830

840

850

860

870

880

890

900

910

920

930

940

950

960

970

980

990

1000

1010

1020

1030

1040

1050

1060

1070

1080

1090 
Table II Harmonic Oscillator Contributions to the Thermodynamic

Functions (in units of calories, moles, and ${ }^{\circ} \mathrm{K}$ )
$\mathrm{cm}^{-1}$
S०
$\mathrm{C}_{\mathrm{p}}^{\circ} \quad-\left(\mathrm{F}^{\circ}-\mathrm{E}_{\mathrm{O}} \mathrm{O}\right) / \mathrm{T}$
So
$\mathrm{C}_{\mathrm{p}}^{\circ}$
$-\left(F^{\circ}-E_{O}^{O}\right) / T$
So

$\mathrm{cm}^{-1}$

$T=1400$.

$r=1500$.

$T=1600$.

$1100 \quad 0.77480 \quad 1.84600 \quad 1.78840$

$\begin{array}{llll}1110 & 0.76514 & 1.82983 & 1.78500\end{array}$

$\begin{array}{llll}1120 & 0.75562 & 1.81383 \quad 1.78157\end{array}$

$\begin{array}{llll}1130 & 0.74624 & 1.79801 & 1.77813\end{array}$

$\begin{array}{lllll}1140 & 0.73700 & 1.78236 & 1.77465\end{array}$

$\begin{array}{llll}1150 & 0.72790 & 1.76688 & 1.77116\end{array}$

$\begin{array}{lllll}1160 & 0.71893 & 1.75156 & 1.76765\end{array}$

$\begin{array}{lllll}1170 & 0.71009 & 1.73640 & 1.76411\end{array}$

$\begin{array}{lllll}1180 & 0.70138 & 1.72140 & 1.76055\end{array}$

$\begin{array}{llll}1190 & 0.69280 & 1.70656 & 1.75697\end{array}$

$\begin{array}{lllll}1200 & 0.68435 & 1.69187 & 1.75336\end{array}$

$\begin{array}{lllll}1210 & 0.67601 & 1.67733 & 1.74974\end{array}$

$\begin{array}{lllll}1220 & 0.66779 & 1.66295 & 1.74610\end{array}$

$\begin{array}{lllll}1230 & 0.65970 & 1.64871 & 1.74243\end{array}$

$\begin{array}{lllll}1240 & 0.65171 & 1.63461 & 1.73874\end{array}$

$\begin{array}{llll}1250 & 0.64384 & 1.62066 & 1.73504\end{array}$

1260

1270

1280

1290

1300

1310

1320

1330

1340

1350

1360

1370

1380

1390

1400

1410

1420

1430

1440

1450

1460

1470

1480

1490

1500

1510

1520

1530

1540

1550

1560

1570

1580

1590
0.636081 .606851 .73131

$0.62843 \quad 1.59318 \quad 1.72756$

$0.620891 .57965 \quad 1.72380$

0.613451 .566251 .72001

$0.60612 \quad 1.55298 \quad 1.71621$ $0.59888 \quad 1.53984 \quad 1.71238$ $0.59175 \quad 1.52684 \quad 1.70854$ $0.58471 \quad 1.51396 \quad 1.70467$ $0.57778 \quad 1.50120 \quad 1.70079$

$0.57093 \quad 1.48857 \quad 1.69689$ $0.56418 \quad 1.47606 \quad 1.69297$ $0.55752 \quad 1.46367 \quad 1.68904$ $0.550951 .45140 \quad 1.68509$ 0.544471 .439251 .68111

$0.53808 \quad 1.42721 \quad 1.67713$ $0.53177 \quad 1.41529 \quad 1.67312$ $0.52554 \quad 1.40348 \quad 1.66910$ $0.51940 \quad 1.39178 \quad 1.66506$ 0.513341 .380191 .66100

$\begin{array}{llll}0.50736 & 1.36871 & 1.65693\end{array}$ $0.50146 \quad 1.35734 \quad 1.65284$ $0.49564 \quad 1.34607 \quad 1.64874$ $0.48989 \quad 1.33491 \quad 1.64462$ $0.48422 \quad 1.32385 \quad 1.64048$

$0.478621 .31289 \quad 1.63633$ 0.473091 .302031 .63216 $0.46764 \quad 1.29127 \quad 1.62798$ 0.462261 .280611 .62379 $0.45694 \quad 1.27004 \quad 1.61957$

$0.45170 \quad 1.25957 \quad 1.61535$ 0.446521 .249201 .61111 $0.44141 \quad 1.23892 \quad 1.60686$ $0.436361 .22873 \quad 1.60259$ 0.431381 .218631 .59831
$0.85039 \quad 1.97024 \quad 1.81264$ $0.840291 .95384 \quad 1.80963$ $0.830331 .93763 \quad 1.80659$ $0.82051 \quad 1.92158 \quad 1.80354$ $0.810841 .90571 \quad 1.80046$

$0.80130 \quad 1.89000 \quad 1.79736$ $0.79190 \quad 1.87445 \quad 1.79425$ $0.78264 \quad 1.85906 \quad 1.79111$ $0.77350 \quad 1.84383 \quad 1.78795$ $0.76450 \quad 1.82875 \quad 1.78477$

$\begin{array}{llll}0.75562 & 1.81383 & 1.78157\end{array}$ $\begin{array}{llll}0.74686 & 1.79906 & 1.77836\end{array}$ $0.73822 \quad 1.78444 \quad 1.77512$ $0.72971 \quad 1.76996 \quad 1.77186$ $0.72131 \quad 1.75563 \quad 1.76859$

0.713021 .741431 .76529 $0.704851 .72738 \quad 1.76197$ $0.69679 \quad 1.71346 \quad 1.75864$ $0.68884 \quad 1.69968 \quad 1.75529$ $0.68100 \quad 1.68604 \quad 1.75192$

$0.67326 \quad 1.67252 \quad 1.74853$ 0.665621 .659141 .74512 0.658091 .645881 .74169 $0.650661 .63275 \quad 1.73825$ 0.643321 .619741 .73479

0.636081 .606851 .73131 $0.62894 \quad 1.594091 .72781$ $0.621891 .58144 \quad 1.72430$ 0.614931 .568921 .72077 0.608061 .556501 .71722

$0.601281 .54421 \quad 1.71366$ 0.594591 .532021 .71008 0.587991 .519951 .70648 $0.58146 \quad 1.507991 .70286$ 0.575031 .496131 .69923

$0.56867 \quad 1.48439 \quad 1.69559$ $0.56240 \quad 1.472751 .69193$ $0.55620 \quad 1.46121 \quad 1.68825$ $0.55008 \quad 1.44978 \quad 1.68456$ $0.54404 \quad 1.43844 \quad 1.68085$

$0.53808 \quad 1.42721 \quad 1.67713$ $0.53219 \quad 1.41608 \quad 1.67339$ $0.52637 \quad 1.40505 \quad 1.66963$ $0.520621 .39411 \quad 1.66587$ $0.514951 .38327 \quad 1.66208$

$0.509351 .37253 \quad 1.65829$ $0.50381 \quad 1.36188 \quad 1.65448$ $0.493351 .35132 \quad 1.65065$ $0.49295 \quad 1.34085 \quad 1.64681$ $0.487611 .33047 \quad 1.64296$
$0.92409 \quad 2.08788 \quad 1.83277$ $0.913592 .07131 \quad 1.83008$ $0.903232 .05491 \quad 1.82738$ 0.893022 .038681 .82466 $0.88295 \quad 2.02261 \quad 1.82192$

$0.873032 .00671 \quad 1.81916$ $0.86324 \quad 1.99097 \quad 1.81638$ $\begin{array}{llll}0.85358 & 1.97539 & 1.81358\end{array}$ $0.84406 \quad 1.95997 \quad 1.81076$ $0.83467 \quad 1.94470 \quad 1.80792$

$0.82540 \quad 1.92958 \quad 1.80507$ $0.81626 \quad 1.91462 \quad 1.80219$ $0.807251 .89980 \quad 1.79930$ 0.798351 .885121 .79639 $0.78958 \quad 1.87059 \quad 1.79346$

$0.78092 \quad 1.85619 \quad 1.79052$ $0.77237 \quad 1.84194 \quad 1.78756$ $0.76394 \quad 1.827821 .78457$ 0.755621 .813831 .78157 $0.74740 \quad 1.79998 \quad 1.77856$

$\begin{array}{llll}0.73930 & 1.78626 & 1.77552\end{array}$ $0.73129 \quad 1.77266 \quad 1.77247$ 0.723401 .759201 .76941 $0.71560 \quad 1.74585 \quad 1.76632$ $0.70790 \quad 1.732631 .76322$

$0.70031 \quad 1.719541 .76010$ $0.69280 \quad 1.70656 \quad 1.75697$ $0.68540 \quad 1.69370 \quad 1.75382$ $0.67808 \quad 1.680951 .75065$ $0.67086 \quad 1.668331 .74747$

$0.66373 \quad 1.655811 .74427$ $0.656691 .64341 \quad 1.74105$ 0.649731 .631111 .73782 $0.64287 \quad 1.618931 .73457$ $0.63608 \quad 1.606851 .73131$

$0.62938 \quad 1.59488 \quad 1.72803$ $0.62277 \quad 1.583021 .72474$ $0.616231 .57126 \quad 1.72143$ $0.60977 \quad 1.559601 .71811$ $0.603391 .54804 \quad 1.71477$

$0.59709 \quad 1.53658 \quad 1.71142$ $0.59087 \quad 1.52522 \quad 1.70805$ $0.58471 \quad 1.51396 \quad 1.70467$ 0.578641 .502791 .70128 $0.572631 .49172 \quad 1.69787$

$0.56670 \quad 1.48074 \quad 1.69445$ $0.56084 \quad 1.469851 .69101$ $0.55505 \quad 1.45906 \quad 1.68756$ 0.549321 .448351 .68409 $0.543671 .43774 \quad 1.68062$
1100

1110

1120

1130

1140

1150

1160

1170

1180

1190

1200

1210

1220

1230

1240

1250

1260

1270

1280

1290

1300

1310

1320

1330

1340

1350

1360

1370

1380

1390

1400

1410

1420

1430

1440

1450

1460

1470

1480

1490

1500

1510

1520

1530

1540

1550

1560

1570

1580

1590 
Table II Harmonic Oscillator Contributions to the Thermodynamic

Functions (in units of calories, moles, and ${ }^{\circ} \mathrm{K}$ )

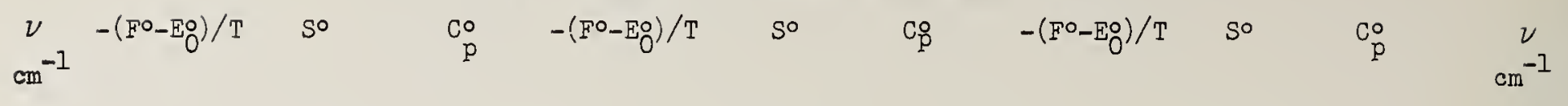

$T=1400$.

1600

1610

1620

1630

1640

1650

1660

1670

1680

1690

1700

1710

1720

1730

1740

1750

1760

1770

1780

1790

1800

1810

1820

1830

1840

1850

1860

1870

1880

1890

1900

1910

1920

1930

1940

1950

1960

1970

1980

1990

2000

2010

2020

2030

2040

2050

2060

2070

2080

2090
$T=1500$.

$0.482341 .32018 \quad 1.63910$

$\begin{array}{lllll}0.47714 & 1.30998 & 1.63522\end{array}$

$0.472001 .29987 \quad 1.63133$

$0.46692 \quad 1.28984 \quad 1.62742$

$0.46190 \quad 1.27990 \quad 1.62350$

0.412071 .179131 .58106

$0.407391 .16947 \quad 1.57672$

$0.40277 \quad 1.15990 \quad 1.57236$ 0.398211 .150421 .56800 $0.39371 \quad 1.14101 \quad 1.56362$ $0.389261 .13169 \quad 1.55922$ $0.38487 \quad 1.12245 \quad 1.55482$

$0.38053 \quad 1.11329 \quad 1.55041$ $0.376251 .10421 \quad 1.54598$ $0.372021 .09521 \quad 1.54155$ $0.367841 .08628 \quad 1.53710$ 0.363711 .077441 .53264

$0.35964 \quad 1.06867 \quad 1.52818$ $0.355611 .05997 \quad 1.52370$ 0.351631 .051351 .51921 $0.34770 \quad 1.04281 \quad 1.51472$ $0.343821 .03433 \quad 1.51021$

$0.33999 \quad 1.02593 \quad 1.50570$ $\begin{array}{llll}0.33620 & 1.01760 & 1.50117\end{array}$ $0.33246 \quad 1.009341 .49664$ $0.32876 \quad 1.00115 \quad 1.49210$ $0.325110 .99304 \quad 1.48755$

$\begin{array}{llll}0.32150 & 0.98499 & 1.48299\end{array}$ $\begin{array}{llll}0.31794 & 0.97700 & 1.47843\end{array}$ 0.314410 .969091 .47385 $\begin{array}{llll}0.31093 & 0.96124 & 1.46927\end{array}$ $0.30750 \quad 0.95346 \quad 1.46468$

$0.30410 \quad 0.94574 \quad 1.46009$ 0.300740 .938091 .45549 $0.29742 \quad 0.93050 \quad 1.45088$ $\begin{array}{lll}0.29415 & 0.92297 & 1.44626\end{array}$ 0.290910 .915511 .44164

$\begin{array}{lllll}0.28771 & 0.90811 & 1.43701\end{array}$ $0.284540 .90077 \quad 1.43238$ $\begin{array}{llll}0.28142 & 0.89350 & 1.42774\end{array}$ $\begin{array}{llll}0.27833 & 0.88628 & 1.42309\end{array}$ $\begin{array}{lll}0.27528 & 0.87912 & 1.41844\end{array}$

$\begin{array}{llll}0.27226 & 0.87202 & 1.41378\end{array}$ $\begin{array}{llll}0.26928 & 0.86498 & 1.40912\end{array}$ $0.266330 .85800 \quad 1.40446$ 0.263420 .851081 .39979 $0.260540 .84421 \quad 1.39511$

$\begin{array}{llll}0.25770 & 0.83740 & 1.39043\end{array}$ $\begin{array}{llll}0.25489 & 0.83064 & 1.38575\end{array}$ $0.252110 .82395 \quad 1.38106$ $0.24936 \quad 0.81730 \quad 1.37637$ $0.246650 .81071 \quad 1.37167$
$0.45694 \quad 1.27004 \quad 1.61957$ $0.45204 \quad 1.26027 \quad 1.61563$ $0.44720 \quad 1.25058 \quad 1.61168$ $0.44242 \quad 1.24097 \quad 1.60771$ $0.437701 .23144 \quad 1.60373$

$0.43303 \quad 1.22199 \quad 1.59974$ $0.42842 \quad 1.21262 \quad 1.59574$ $0.42386 \quad 1.20332 \quad 1.59173$ $0.419351 .19411 \quad 1.58770$ $0.414901 .18497 \quad 1.58366$

$0.41050 \quad 1.17590 \quad 1.57962$ $0.406151 .16691 \quad 1.57556$ $0.401861 .15800 \quad 1.57149$ $0.39761 \quad 1.14916 \quad 1.56741$ $0.39341 \quad 1.140391 .56332$

$0.38926 \quad 1.13169 \quad 1.55922$ $0.38516 \quad 1.12306 \quad 1.55512$ 0.381111 .114511 .55100 $0.37710 \quad 1.10602 \quad 1.54687$ $0.37314 \quad 1.09760 \quad 1.54273$

$0.36923 \quad 1.08925 \quad 1.53858$ $0.36536 \quad 1.08097 \quad 1.53443$ 0.361531 .072751 .53026 $0.35775 \quad 1.06460 \quad 1.52609$ 0.354011 .056511 .52191

$0.35032 \quad 1.04849 \quad 1.51772$ $0.346661 .04054 \quad 1.51352$ $0.34305 \quad 1.03265 \quad 1.50931$ $0.33948 \quad 1.024821 .50509$ $0.33595 \quad 1.01705 \quad 1.50087$

$0.33246 \quad 1.00934 \quad 1.49664$ $0.32901 \quad 1.001701 .49240$ $\begin{array}{llll}0.32559 & 0.99411 & 1.48816\end{array}$ $0.32222 \quad 0.98659 \quad 1.48390$ $\begin{array}{llll}0.31888 & 0.97913 & 1.47965\end{array}$

$0.31558 \quad 0.97172 \quad 1.47538$ 0.312320 .964371 .47111 0.309090 .957081 .46683 $0.30590 \quad 0.949851 .46254$ $0.302750 .94267 \quad 1.45825$

0.299630 .935551 .45395 0.296550 .928491 .44965 $0.29350 \quad 0.92148 \quad 1.44534$ 0.290480 .914521 .44102 0.287490 .907621 .43670
$\mathrm{T}=1600$

$0.53808 \quad 1.42721 \quad 1.67713$

$\begin{array}{llll}0.53255 & 1.41678 & 1.67362\end{array}$ 0.527091 .406421 .67010 $0.52170 \quad 1.39616 \quad 1.66657$ $0.51636 \quad 1.38597 \quad 1.66303$

$\begin{array}{llll}0.51109 & 1.37588 & 1.65948\end{array}$ $0.505881 .36586 \quad 1.65591$ 0.500731 .355921 .65233 $0.49564 \quad 1.34607 \quad 1.64874$ 0.490601 .336301 .64513

0.485631 .326601 .64151 $0.48071 \quad 1.316981 .63789$ $0.47585 \quad 1.30744 \quad 1.63425$ 0.471041 .297981 .63060 0.466291 .288591 .62693

$0.461591 .27928 \quad 1.62326$ 0.456941 .270041 .61957 $0.45235 \quad 1.26088 \quad 1.61588$ 0.447811 .251781 .61217 0.443321 .242761 .60846

$0.43887 \quad 1.23381 \quad 1.60473$ $0.43448 \quad 1.22493 \quad 1.60099$ 0.430141 .216121 .59724 $0.42584 \quad 1.20738 \quad 1.59348$ $0.421601 .19870 \quad 1.58971$

$0.417401 .19010 \quad 1.58594$ 0.413241 .181561 .58215 0.409141 .173091 .57835 $0.40507 \quad 1.16468 \quad 1.57454$ 0.401061 .156341 .57073

$0.39708 \quad 1.14806 \quad 1.56690$ 0.393151 .139841 .56307 0.389261 .131691 .55922 $0.385421 .12360 \quad 1.55537$ 0.381611 .115571 .55151

$0.37785 \quad 1.10760 \quad 1.54764$ $0.374131 .09970 \quad 1.54377$ 0.370451 .091851 .53988 0.366801 .084061 .53599 $0.36320 \quad 1.07634 \quad 1.53209$

$0.35964 \quad 1.06867 \quad 1.52818$ 0.356111 .061051 .52426 $0.352621 .05350 \quad 1.52034$ 0.349171 .046001 .51640 0.345761 .038561 .51247

$0.342381 .03117 \quad 1.50852$ 0.339041 .023841 .50457 0.335731 .016571 .50061 $0.332461 .00934 \quad 1.49664$ 0.329221 .002171 .49267
1600

1610

1620

1630

1640

1650

1660

1670

1680

1690

1700

1710

1720

1730

1740

1750

1760

1770

1780

1790

1800

1810

1820

1830

1840

1850

1860

1870

1880

1890

1900

1910

1920

1930

1940

1950

1960

1970

1980

1990

2000

2010

2020

2030

2040

2050

2060

2070

2080

2090 
Table II Harmonic Oscillator Contributions to the Thermodynamic

Functions (in units of calories, moles, and ${ }^{\circ} \mathrm{K}$ )
$\mathrm{cm}^{-1}$
So
$\mathrm{C}_{\mathrm{p}}^{\circ} \quad-\left(\mathrm{F}^{\circ}-\mathrm{E}_{\mathrm{O}}^{\circ}\right) / \mathrm{T}$
So
$\mathrm{C}_{\mathrm{p}}^{\circ}$
$-\left(F^{\circ}-E_{O}^{\circ}\right) / T$
So
$\mathrm{C}_{\mathrm{p}}^{\circ}$
$\mathrm{cm}^{-1}$

$r=1400$.

2100

2110

2120

2130

2140

$\begin{array}{llll}2150 & 0.23101 & 0.77228 & 1.34344\end{array}$

2160

2170

2180

2190

$\begin{array}{lllll}2200 & 0.21877 & 0.74167 & 1.31985\end{array}$

2210

2220

2230

2240

2250

2260

2270

2280

2290

2300

2310

2320

2330

2340

2350

2360

2370

2380

2390

2400

2410

2420

2430

2440

2450

2460

2470

2480

2490

2500

2510

2520

2530

2540

2550

2560

2570

2580

2590

$\begin{array}{llll}0.24396 & 0.80417 & 1.36698\end{array}$

$\begin{array}{llll}0.24131 & 0.79769 & 1.36227\end{array}$

$\begin{array}{llll}0.23869 & 0.79126 & 1.35757\end{array}$

$0.23610 \quad 0.78488 \quad 1.35286$

$0.233540 .77856 \quad 1.34815$

$\begin{array}{llll}0.22850 & 0.76606 & 1.33872\end{array}$

0.226030 .759891 .33401

$\begin{array}{llll}0.22358 & 0.75377 & 1.32929\end{array}$

$\begin{array}{llll}0.22116 & 0.74769 & 1.32457\end{array}$

$\begin{array}{lll}0.21641 & 0.73569 & 1.31512 \\ 0.21407 & 0.72977 & 1.31040\end{array}$

$\begin{array}{llll}0.21176 & 0.72389 & 1.30567\end{array}$

$\begin{array}{llll}0.20948 & 0.71806 & 1.30095\end{array}$

$0.207220 .71227 \quad 1.29622$ $\begin{array}{llll}0.20499 & 0.70654 & 1.29149\end{array}$ $0.20278 \quad 0.70084 \quad 1.28676$ $0.20060 \quad 0.69520 \quad 1.28203$ $0.19845 \quad 0.68960 \quad 1.27730$

$\begin{array}{llll}0.19631 & 0.68404 & 1.27257\end{array}$ $0.19420 \quad 0.678531 .26785$ $0.192120 .67307 \quad 1.26312$ $0.19006 \quad 0.66764 \quad 1.25839$ $0.18802 \quad 0.66226 \quad 1.25366$

$0.18601 \quad 0.65693 \quad 1.24894$ $\begin{array}{llll}0.18401 & 0.65163 & 1.24421\end{array}$ $\begin{array}{llll}0.18204 & 0.64638 & 1.23949\end{array}$ $\begin{array}{lllll}0.18009 & 0.64117 & 1.23477\end{array}$ $\begin{array}{llll}0.17817 & 0.63601 & 1.23005\end{array}$

$\begin{array}{llll}0.17626 & 0.63088 & 1.22533\end{array}$ $\begin{array}{llll}0.17438 & 0.62580 & 1.22061\end{array}$ $0.172520 .62075 \quad 1.21590$ $0.170670 .61575 \quad 1.21118$ $\begin{array}{llll}0.16885 & 0.61078 & 1.20647\end{array}$

$\begin{array}{llll}0.16705 & 0.60586 \quad 1.20177\end{array}$ $0.16527 \quad 0.60097 \quad 1.19706$ 0.163510 .596131 .19236 $0.161770 .59132 \quad 1.18766$ $0.160050 .58655 \quad 1.18296$

$\begin{array}{llll}0.15834 & 0.58182 & 1.17827\end{array}$ $0.15666 \quad 0.57712 \quad 1.17358$ $0.154990 .57246 \quad 1.16889$ $0.153340 .56784 \quad 1.16421$ $0.151710 .56326 \quad 1.15953$

$0.15010 \quad 0.55871 \quad 1.15485$ $\begin{array}{llll}0.14851 & 0.55420 & 1.15018\end{array}$ $\begin{array}{llll}0.14693 & 0.54973 & 1.14552\end{array}$ $0.14538 \quad 0.54529 \quad 1.14085$ 0.143830 .540881 .13619

$$
T=1500 \text {. }
$$

$T=1600$.

$0.284540 .90077 \quad 1.43238$ $\begin{array}{llll}0.28163 & 0.89398 & 1.42805\end{array}$ $0.278740 .88724 \quad 1.42371$ $0.275890 .88055 \quad 1.41937$ $0.27306 \quad 0.87391 \quad 1.41503$

$0.27027 \quad 0.86732 \quad 1.41068$ $0.26751 \quad 0.86079 \quad 1.40632$ $0.264780 .85430 \quad 1.40197$ $0.262070 .84787 \quad 1.39761$ $0.25940 \quad 0.84148 \quad 1.39324$

$\begin{array}{llll}0.25676 & 0.83514 & 1.38887\end{array}$ 0.254140 .828851 .38450 $0.25156 \quad 0.82261 \quad 1.38012$ $0.24900 \quad 0.81642 \quad 1.37574$ $0.246470 .81027 \quad 1.37136$

$0.24396 \quad 0.80417 \quad 1.36698$ 0.241490 .798121 .36259 0.239040 .792121 .35820 $0.236620 .78616 \quad 1.35380$ $0.234220 .78024 \quad 1.34941$

$0.231850 .77437 \quad 1.34501$ $0.22950 \quad 0.768541 .34061$ $0.227180 .76276 \quad 1.33621$ $0.224880 .75703 \quad 1.33181$ 0.222610 .751331 .32740

$\begin{array}{llll}0.22036 & 0.74568 & 1.32299\end{array}$ $0.218140 .74007 \quad 1.31859$ $0.21594 \quad 0.73451 \quad 1.31418$ $0.21376 \quad 0.72898 \quad 1.30977$ 0.211610 .723501 .30536

$\begin{array}{llll}0.20948 & 0.71806 \quad 1.30094\end{array}$ $0.7 .0737 \quad 0.71266 \quad 1.29653$ $0.205290 .70730 \quad 1.29212$ $0.203220 .70198 \quad 1.28771$ $0.201180 .69670 \quad 1.28329$

$\begin{array}{llll}0.19916 & 0.69146 \quad 1.27888\end{array}$ $\begin{array}{llll}0.19716 & 0.68626 & 1.27447\end{array}$ $0.195190 .68110 \quad 1.27005$ $0.193230 .67598 \quad 1.26564$ $0.191290 .67089 \quad 1.26123$

$0.18938 \quad 0.66585 \quad 1.25682$ $0.18748 \quad 0.66084 \quad 1.25240$ $0.18560 \quad 0.65587 \quad 1.24799$ 0.183750 .650931 .24358 $0.181910 .64604 \quad 1.23918$

$\begin{array}{llll}0.18009 & 0.64117 & 1.23477\end{array}$ $0.17830 \quad 0.63635 \quad 1.23036$ $0.17652 \quad 0.63156 \quad 1.22596$ $0.174750 .62681 \quad 1.22156$ $0.17301 \quad 0.62209 \quad 1.21715$
$0.326020 .99506 \quad 1.48869$ $0.322850 .98800 \quad 1.48470$ $0.31971 \quad 0.98099 \quad 1.48071$ $0.316610 .97403 \quad 1.47671$ $0.313540 .96712 \quad 1.47271$

$0.31050 \quad 0.96026 \quad 1.46870$ $0.30750 \quad 0.95346 \quad 1.46468$ 0.304520 .946701 .46066 $0.30158 \quad 0.93999 \quad 1.45664$ $0.29866 \quad 0.93334 \quad 1.45261$

$0.29578 \quad 0.92673 \quad 1.44857$ $0.292930 .92017 \quad 1.44453$ $0.290100 .91366 \quad 1.44048$ 0.287310 .907191 .43643 $0.284540 .90077 \quad 1.43238$

$\begin{array}{llll}0.28181 & 0.89440 & 1.42832\end{array}$ $0.27910 \quad 0.88808 \quad 1.42425$ $0.276420 .88180 \quad 1.42019$ $0.27376 \quad 0.87556 \quad 1.41611$ $0.271140 .86938 \quad 1.41204$

0.268540 .863231 .40796 $0.26597 \quad 0.857131 .40387$ $0.263420 .85108 \quad 1.39979$ $0.26090 \quad 0.84507 \quad 1.39570$ 0.258410 .839101 .39160

$\begin{array}{llll}0.25594 & 0.83317 & 1.38750\end{array}$ 0.253490 .827291 .38340 $0.25108 \quad 0.821451 .37930$ 0.248680 .815651 .37520 0.246310 .809891 .37109

$\begin{array}{llll}0.24396 & 0.80417 & 1.36698\end{array}$ $0.241640 .79850 \quad 1.36286$ $\begin{array}{llll}0.23934 & 0.79286 & 1.35875\end{array}$ $0.23707 \quad 0.78727 \quad 1.35463$ 0.234820 .781721 .35051

$0.23258 \quad 0.77620 \quad 1.34638$ $0.230380 .77072 \quad 1.34226$ $0.228190 .76529 \quad 1.33814$ $0.22603 \quad 0.759891 .33401$ $\begin{array}{llll}0.22389 & 0.75453 & 1.32988\end{array}$

$\begin{array}{llll}0.22177 & 0.74921 & 1.32575\end{array}$ $0.21967 \quad 0.74392 \quad 1.32162$ $0.217590 .73868 \quad 1.31748$ $\begin{array}{llll}0.21553 & 0.73347 & 1.31335\end{array}$ $0.21349 \quad 0.72829 \quad 1.30922$

$0.21148 \quad 0.72316 \quad 1.30508$ $0.20948 \quad 0.71806 \quad 1.30094$ $0.20750 \quad 0.71299 \quad 1.29681$ $0.20555 \quad 0.70797 \quad 1.29267$ $0.203610 .70297 \quad 1.28853$
2100

2110

2120

2130

2140

2150

2160

2170

2180

2190

2200

2210

2220

2230

2240

2250

2260

2270

2280

2290

2300

2310

2320

2330

2340

2350

2360

2370

2380

2390

2400

2410

2420

2430

2440

2450

2460

2470

2480

2490

2500

2510

2520

2530

2540

2550

2560

2570

2580

2590 
Table II Harmonic Oscillator Contributions to the Thermodynamic

Functions (in units of calories, moles, and ${ }^{\circ} \mathrm{K}$ )
$\mathrm{cm}^{-1}$
$c_{p}^{0}$
so
$\mathrm{co}_{\mathrm{p}}$
$-\left(F^{\circ}-E_{O}^{\circ}\right) / T$ So
$\mathrm{c}_{\mathrm{p}}^{\circ}$
$v$
$\mathrm{cm}^{-1}$

$\mathrm{T}=1400$

$T=1500$

2600

2610

2620

2630

2640

2650

2660

2670

2680

2690

2700

2710

2720

2730

2740

2750

2760

2770

2780

2790

2800

2810

2820

2830

2840

2850

2860

2870

2880

2890

2900

2910

2920

2930

2940

2950

2960

2970

2980

2990

3000

3010

3020

3030

3040

3050

3060

3070

3080

3090
$0.142310 .53651 \quad 1.13154$ $0.14080 \quad 0.53218 \quad 1.12689$ $0.13931 \quad 0.52788 \quad 1.12225$ $\begin{array}{llll}0.13784 & 0.52361 & 1.11761\end{array}$ $0.13638 \quad 0.51938 \quad 1.11297$

$0.134930 .51518 \quad 1.10834$ 0.133510 .511021 .10372 $0.13210 \quad 0.50688 \quad 1.09910$ $0.13070 \quad 0.50278 \quad 1.09449$ $\begin{array}{lllll}0.12932 & 0.49871 & 1.08988\end{array}$

$0.12795 \quad 0.49468 \quad 1.08528$ $\begin{array}{llll}0.12660 & 0.49068 & 1.08069\end{array}$ $0.12527 \quad 0.48670 \quad 1.07610$ $0.123940 .48276 \quad 1.07151$ $0.12264 \quad 0.47885 \quad 1.06694$

$0.12134 \quad 0.47498 \quad 1.06237$ 0.120070 .471131 .05781 $\begin{array}{llll}0.11880 & 0.46731 & 1.05325\end{array}$ $0.11755 \quad 0.46352 \quad 1.04870$ $0.116310 .45977 \quad 1.04416$

$0.11509 \quad 0.45604 \quad 1.03962$ 0.113880 .452341 .03509 $0.11268 \quad 0.44867 \quad 1.03057$ 0.111490 .445031 .02605 0.110320 .441421 .02155

$0.10916 \quad 0.43784 \quad 1.01705$ $0.10801 \quad 0.43428 \quad 1.01256$ $\begin{array}{llll}0.10688 & 0.43076 & 1.00807\end{array}$ $\begin{array}{llll}0.10576 & 0.42726 & 1.00360\end{array}$ 0.104650 .423790 .99913

0.103550 .420340 .99467 $0.10246 \quad 0.41693 \quad 0.99021$ 0.101390 .413540 .98577 0.100320 .410170 .98134 $\begin{array}{llll}0.09927 & 0.40684 & 0.97691\end{array}$

$\begin{array}{llll}0.09823 & 0.40353 & 0.97249\end{array}$ $0.09720 \quad 0.400240 .96808$ 0.096180 .396990 .96368 $\begin{array}{llll}0.09518 & 0.39376 & 0.95929\end{array}$ 0.094180 .390550 .95490

$\begin{array}{llll}0.09319 & 0.38737 & 0.95053\end{array}$ 0.092220 .384210 .94616 $0.091250 .38108 \quad 0.94181$ $0.09030 \quad 0.37797 \quad 0.93746$ 0.089350 .374890 .93312

$\begin{array}{llll}0.08842 & 0.37184 & 0.92879\end{array}$ $\begin{array}{llll}0.08750 & 0.36880 & 0.92448\end{array}$ $0.08658 \quad 0.365790 .92017$ 0.085680 .362810 .91587 0.084780 .359850 .91158
$0.17129 \quad 0.61741 \quad 1.21276$ $0.169580 .61276 \quad 1.20836$ 0.167890 .608151 .20396 $0.166220 .60357 \quad 1.19957$ $0.16456 \quad 0.59903 \quad 1.19518$

0.162930 .594521 .19079 $0.161310 .59004 \quad 1.18641$ $0.15970 \quad 0.58560 \quad 1.18202$ 0.158120 .581191 .17764 $0.156550 .57681 \quad 1.17327$

$0.154990 .57246 \quad 1.16889$ 0.153450 .568151 .16452 $\begin{array}{llll}0.15193 & 0.56387 & 1.16015\end{array}$ 0.150420 .559621 .15579 $0.14893 \quad 0.55540 \quad 1.15143$

$0.14746 \quad 0.55122 \quad 1.14707$ $0.14600 \quad 0.54706 \quad 1.14272$ $0.14455 \quad 0.54294 \quad 1.13837$ $0.143120 .53884 \quad 1.13402$ $0.14170 \quad 0.53478 \quad 1.12968$

$0.14030 \quad 0.53074 \quad 1.12534$ $0.138920 .52674 \quad 1.12101$ $0.137540 .52276 \quad 1.11668$ 0.136180 .518821 .11236 $0.134840 .51490 \quad 1.10804$

$0.133510 .51102 \quad 1.10372$ 0.132190 .507161 .09941 0.130880 .503331 .09510 0.129590 .499531 .09080 $0.128320 .49575 \quad 1.08651$

$0.12705 \quad 0.49201 \quad 1.08222$ $0.12580 \quad 0.48829 \quad 1.07793$ $0.12456 \quad 0.48460 \quad 1.07365$ 0.123330 .480931 .06938 0.122120 .477301 .06511

0.120920 .473691 .06085 $0.119730 .47011 \quad 1.05659$ $0.118550 .46655 \quad 1.05234$ 0.117380 .463021 .04809 0.116230 .459521 .04385

$0.11509 \quad 0.45604 \quad 1.03962$ 0.113960 .452581 .03539 $0.112840 .44916 \quad 1.03117$ $0.111730 .44576 \quad 1.02696$ $0.110630 .44238 \quad 1.02275$

$0.109550 .43903 \quad 1.01855$ $0.108470 .43570 \quad 1.01435$ $0.107410 .43240 \quad 1.01016$ 0.106350 .429121 .00598 $0.105310 .42586 \quad 1.00181$
$T=1600$.

$0.201690 .69802 \quad 1.28440$ $0.19979 \quad 0.69309 \quad 1.28026$ $0.19791 \quad 0.68821 \quad 1.27612$ $0.19605 \quad 0.68335 \quad 1.27198$ $0.19420 \quad 0.67853 \quad 1.26785$

$0.19238 \quad 0.67375 \quad 1.26371$ $0.19057 \quad 0.66899 \quad 1.25957$ $0.18878 \quad 0.66428 \quad 1.25544$ $0.18701 \quad 0.65959 \quad 1.25130$ $0.18526 \quad 0.65494 \quad 1.24717$

$0.183520 .65032 \quad 1.24303$ $0.18180 \quad 0.64573 \quad 1.23890$ 0.180090 .641171 .23477 0.178410 .636651 .23064 $0.176740 .63216 \quad 1.22651$

$\begin{array}{llll}0.17508 & 0.62770 & 1.22238\end{array}$ $0.17345 \quad 0.62327 \quad 1.21825$ $0.17182 \quad 0.61887 \quad 1.21413$ 0.170220 .614501 .21001 0.168630 .610171 .20589

$\begin{array}{llll}0.16705 & 0.60586 & 1.20177\end{array}$ $0.165490 .60158 \quad 1.19765$ $0.16395 \quad 0.59733 \quad 1.19353$ $0.16242 \quad 0.593121 .18942$ $0.16090 \quad 0.58893 \quad 1.18531$

$0.15940 \quad 0.58477 \quad 1.18120$ 0.157920 .580641 .17710 $0.156450 .57654 \quad 1.17299$ $0.15499 \quad 0.57246 \quad 1.16889$ $0.153550 .56842 \quad 1.16479$

$0.152120 .56440 \quad 1.16070$ $0.15071 \quad 0.56041 \quad 1.15661$ $0.14930 \quad 0.55645 \quad 1.15252$ 0.147920 .552521 .14843 $0.146540 .54861 \quad 1.14435$

$0.14518 \quad 0.54474 \quad 1.14027$ 0.143830 .540881 .13619 $0.14250 \quad 0.53706 \quad 1.13212$ $\begin{array}{llll}0.14118 & 0.53326 & 1.12805\end{array}$ 0.139870 .529491 .12399

$0.13857 \quad 0.52574 \quad 1.11993$ 0.137290 .522021 .11587 0.136010 .518331 .11182 $0.13475 \quad 0.51466 \quad 1.10777$ $0.133510 .51102 \quad 1.10372$

$0.13227 \quad 0.50740 \quad 1.09968$ $0.13105 \quad 0.50380 \quad 1.09564$ 0.129830 .500241 .09161 0.128630 .496691 .08758 $0.12744 \quad 0.49317 \quad 1.08356$
2600

2610

2620

2630

2640

2650

2660

2670

2680

2690

2700

2710

2720

2730

2740

2750

2760

2770

2780

2790

2800

2810

2820

2830

2840

2850

2860

2870

2.880

2890

2900

2910

2920

2930

2940

2950

2960

2970

2980

2990

3000

3010

3020

3030

3040

3050

3060

3070

3080

3090 
Table II Harmonic Oscillator Contributions to the Thermodynamic

Functions (in units of calories, moles, and ${ }^{\circ} \mathrm{K}$ )
V
$\mathrm{cm}^{-1}$
So
so
$-\left(F^{\circ}-E O\right) / T$
$\mathrm{C}_{\mathrm{p}}^{\circ}$

$$
T=1400 \text {. }
$$
3100
3110
3120
3130
3140
$0.08390 \quad 0.356910 .90730$ $0.08302 \quad 0.353990 .90303$ 0.082150 .351100 .89877 $0.08130 \quad 0.348230 .89452$ $0.08045 \quad 0.34538 \quad 0.89028$

$$
T=1500
$$
0.104280 .422630 .99764 $0.103260 .41943 \quad 0.99348$ 0.102250 .416250 .98933 0.101240 .413090 .98518 0.100250 .409950 .98104

$-\left(F^{\circ}-E \circ\right) / T$

So $v$ $\mathrm{cm}^{-1}$
3150

3160

3170

3180

3190

3200

3210

3220

3230

3240

3250

3260

3270

3280

3290

3300

3310

3320

3330

3340

3350

3360

3370

3380

3390

3400

3410

3420

3430

3440

3450

3460

3470

3480

3490

3500

3510

3520

3530

3540

3550

3560

3570

3580

3590
$0.079610 .34256 \quad 0.88605$ $\begin{array}{llll}0.07878 & 0.33976 & 0.88183\end{array}$ $\begin{array}{llll}0.07796 & 0.33698 & 0.87762\end{array}$ $\begin{array}{llll}0.07714 & 0.33422 & 0.87342\end{array}$ 0.076340 .331490 .86923

$\begin{array}{llll}0.07555 & 0.32877 & 0.86505\end{array}$ $0.07476 \quad 0.32608 \quad 0.86088$ $\begin{array}{llll}0.07398 & 0.32341 & 0.85673\end{array}$ $\begin{array}{llll}0.07321 & 0.32076 & 0.85258\end{array}$ 0.072450 .318130 .84845

$\begin{array}{lll}0.07169 & 0.31552 & 0.84432\end{array}$ $\begin{array}{llll}0.07095 & 0.31293 & 0.84021\end{array}$ $0.070210 .31037 \quad 0.83611$ $0.06948 \quad 0.30782 \quad 0.83202$ $\begin{array}{llll}0.06875 & 0.30529 & 0.82793\end{array}$

$\begin{array}{llll}0.06804 & 0.30279 & 0.82387\end{array}$ $\begin{array}{llll}0.06733 & 0.30030 & 0.81981\end{array}$ $\begin{array}{llll}0.06663 & 0.29783 & 0.81576\end{array}$ $\begin{array}{llll}0.06594 & 0.29539 & 0.81172\end{array}$ $\begin{array}{llll}0.06525 & 0.29296 & 0.80770\end{array}$

$\begin{array}{llll}0.06458 & 0.29055 & 0.80369\end{array}$ $\begin{array}{llll}0.06390 & 0.28816 & 0.79969\end{array}$ $\begin{array}{llll}0.06324 & 0.28579 & 0.79570\end{array}$ $\begin{array}{llll}0.06258 & 0.28344 & 0.79172\end{array}$ $\begin{array}{lll}0.06193 & 0.28110 & 0.78775\end{array}$

$\begin{array}{llll}0.06129 & 0.27879 & 0.78380\end{array}$ $\begin{array}{llll}0.06065 & 0.27649 & 0.77985\end{array}$ $\begin{array}{llll}0.06002 & 0.27422 & 0.77592\end{array}$ $\begin{array}{llll}0.05940 & 0.27196 & 0.77200\end{array}$ $\begin{array}{llll}0.05878 & 0.26971 & 0.76809\end{array}$

$\begin{array}{llll}0.05817 & 0.26749 & 0.76420\end{array}$ $\begin{array}{llll}0.05757 & 0.26528 & 0.76031\end{array}$ $\begin{array}{llll}0.05697 & 0.26309 & 0.75644\end{array}$ $\begin{array}{llll}0.05638 & 0.26092 & 0.75258\end{array}$ $\begin{array}{llll}0.05580 & 0.25877 & 0.74873\end{array}$

$\begin{array}{llll}0.05522 & 0.25663 & 0.74490\end{array}$ $\begin{array}{llll}0.05465 & 0.25451 & 0.74107\end{array}$ $\begin{array}{llll}0.05408 & 0.25241 & 0.73726\end{array}$ $\begin{array}{llll}0.05352 & 0.25032 & 0.73346\end{array}$ $\begin{array}{llll}0.05297 & 0.24825 & 0.72967\end{array}$

0.052420 .246200 .72590 $\begin{array}{lllll}0.05187 & 0.24416 & 0.72213\end{array}$ $\begin{array}{llll}0.05134 & 0.24214 & 0.71838\end{array}$ $\begin{array}{llll}0.05081 & 0.24014 & 0.71464\end{array}$ $\begin{array}{lll}0.05028 & 0.23815 & 0.71092\end{array}$

$T=1600$.

0.099270 .406840 .97691 $0.09830 \quad 0.403750 .97278$ $0.097340 .40068 \quad 0.96867$ 0.096390 .397640 .96456 0.095440 .394610 .96046

0.094510 .391610 .95636 0.093590 .388640 .9522 .8 0.092670 .385680 .94820 0.091770 .382750 .94413 0.090870 .379840 .94007

$0.08998 \quad 0.37694 \quad 0.93601$ $0.08910 \quad 0.37408 \quad 0.93197$ 0.088230 .371230 .92793 $0.087370 .36840 \quad 0.92390$ 0.086520 .365590 .91988

$0.08568 \quad 0.362810 .91587$ $0.084840 .36004 \quad 0.91186$ $0.084010 .35730 \quad 0.90787$ $0.08320 \quad 0.35457 \quad 0.90388$ 0.082380 .351870 .89990

$\begin{array}{llll}0.08158 & 0.34918 & 0.89593\end{array}$ 0.080790 .346520 .89197 $0.08000 \quad 0.34388 \quad 0.88802$ 0.079220 .341250 .88408 0.078450 .338640 .88014

$\begin{array}{llll}0.07769 & 0.33606 & 0.87622\end{array}$ 0.076930 .333490 .87230 0.076180 .330940 .86839 0.075440 .328410 .86450 $0.074710 .32590 \quad 0.86061$

$\begin{array}{llll}0.07398 & 0.32341 & 0.85673\end{array}$ 0.073260 .320930 .85286 $0.072550 .31848 \quad 0.84900$ $0.071840 .31604 \quad 0.84515$ 0.071140 .313620 .84131

$0.07045 \quad 0.311220 .83747$ 0.069770 .308840 .83365 0.069090 .3064 .70 .82984 0.068420 .304120 .82603 0.067750 .301790 .82224

$0.06710 \quad 0.29947 \quad 0.81846$ 0.066450 .297180 .81468 $0.06580 \quad 0.29490 \quad 0.81092$ $0.06516 \quad 0.292630 .80717$ 0.064530 .290390 .80342
$0.12627 \quad 0.48968 \quad 1.07954$ $0.12510 \quad 0.48621 \quad 1.07552$ $0.123940 .48276 \quad 1.07151$ $0.12280 \quad 0.47934 \quad 1.06751$ 0.121670 .475941 .06351

$0.12054 \quad 0.47257 \quad 1.05952$ 0.119430 .469211 .05553 $0.118330 .46589 \quad 1.05154$ 0.117240 .462581 .04756 $0.11616 \quad 0.459301 .04359$

0.115090 .456041 .03962 $0.114030 .45280 \quad 1.03566$ $0.112980 .44958 \quad 1.03170$ 0.111940 .446391 .02775 $0.11090 \quad 0.443221 .02380$

$0.109880 .44007 \quad 1.01986$ 0.108870 .436941 .01592 0.107870 .433841 .01199 $0.106880 .43076 \quad 1.00807$ $0.10590 \quad 0.427691 .00415$

0.104920 .424651 .00024 $0.10396 \quad 0.421630 .99634$ $0.10300 \quad 0.418630 .99244$ $0.10206 \quad 0.415650 .98855$ 0.101120 .412690 .98466

0.100190 .409760 .98078 0.099270 .406840 .97691 $0.09836 \quad 0.403940 .97304$ $0.09746 \quad 0.40106 \quad 0.96918$ $0.09656 \quad 0.398210 .96533$

0.095680 .395370 .96148 $0.09480 \quad 0.392550 .95764$ 0.093930 .389750 .95381 $0.093070 .38697 \quad 0.94998$ 0.092220 .384210 .94616

0.091370 .381470 .94235 0.090540 .378750 .93855 0.089710 .376050 .93475 0.08889 .0 .373360 .93096 $0.08807 \quad 0.37070 \quad 0.92717$

0.087270 .368050 .92340 0.086470 .365420 .91963 $\begin{array}{llll}0.08568 & 0.36281 & 0.91587\end{array}$ 0.084890 .360210 .91211 0.084120 .357640 .90837

$\begin{array}{llll}0.08335 & 0.35508 & 0.90463\end{array}$ 0.082590 .352540 .90090 $\begin{array}{llll}0.08183 & 0.35002 & 0.89717\end{array}$ $0.08108 \quad 0.347520 .89346$ 0.080340 .345030 .88975
3100

3110

3120

3130

3140

3150

3160

3170

3180

3190

3200

3210

3220

3230

3240

3250

3260

3270

3280

3290

3300

3310

3320

3330

3340

3350

3360

3370

3380

3390

3400

3410

3420

3430

3440

3450

3460

3470

3480

3490

3500

3510

3520

3530

3540

3550

3560

3570

3580

3590 
Table II Harmonic Oscillator Contributions to the Thermodynamic Functions (in units of calories, moles, and ${ }^{\circ} \mathrm{K}$ )
$\mathrm{cm}^{-1}-\left(F^{\circ}-E_{0}^{0}\right) / T \quad S^{\circ}$
$\mathrm{C}_{\mathrm{p}}^{\circ} \quad-\left(\mathrm{F}^{\circ}-\mathrm{E}_{\mathrm{O}}\right) / \mathrm{T} \quad \mathrm{S}^{\circ}$
$\mathrm{C}_{\mathrm{p}}$
$-\left(F^{\circ}-E_{O}^{\circ}\right) / T$
So
$\mathrm{c}_{\mathrm{p}}^{\circ}$
$\nu$
$\mathrm{cm}^{-1}$

$$
T=1400 \text {. }
$$

$\begin{array}{llll}0.04976 & 0.23618 & 0.70720\end{array}$ $0.049240 .23422 \quad 0.70350$ $\begin{array}{llll}0.04873 & 0.23228 & 0.69981\end{array}$ $0.04823 \quad 0.23036 \quad 0.69614$ $\begin{array}{lll}0.04773 & 0.22845 & 0.69247\end{array}$

$0.04724 \quad 0.22655 \quad 0.68882$ 0.046750 .224670 .68518 $0.04626 \quad 0.222810 .68155$ $0.04579 \quad 0.22096 \quad 0.67794$ 0.045310 .219120 .67434

3680

3690

3700

3710

3720

3730

3740

3750

3760

3770

3780

3790

3800

3810

3820

3830

3840

3850

3860

3870

3880

3890

3900

3910

3920

3930

3940

3950

3960

3970

3980

3990

4000 $\begin{array}{llll}0.04484 & 0.21730 & 0.67075\end{array}$

$\begin{array}{llll}0.04438 & 0.21550 & 0.66717\end{array}$

0.043920 .213710 .66361

$\begin{array}{llll}0.04347 & 0.21193 & 0.66006\end{array}$

$\begin{array}{lll}0.04302 & 0.21017 & 0.65652\end{array}$

0.042570 .208420 .65300 $\begin{array}{llll}0.04213 & 0.20668 & 0.64948\end{array}$ $\begin{array}{llll}0.04170 & 0.20496 & 0.64598\end{array}$ $\begin{array}{llll}0.04127 & 0.20326 & 0.64250\end{array}$ $\begin{array}{llll}0.04084 & 0.20156 & 0.63902\end{array}$

$0.04042 \quad 0.199890 .63556$ $\begin{array}{llll}0.04000 & 0.19822 & 0.63211\end{array}$ $\begin{array}{llll}0.03959 & 0.19657 & 0.62868\end{array}$ $\begin{array}{llll}0.03918 & 0.19493 & 0.62525\end{array}$ $\begin{array}{lll}0.03878 & 0.19330 & 0.62184\end{array}$

0.038380 .191690 .61845 $\begin{array}{llll}0.03798 & 0.19009 & 0.61506\end{array}$ $0.037590 .18850 \quad 0.61169$ $0.037200 .18693 \quad 0.60833$ $\begin{array}{lll}0.03682 & 0.18537 & 0.60499\end{array}$

0.036440 .183820 .60165 $\begin{array}{llll}0.03606 & 0.18228 & 0.59833\end{array}$ $\begin{array}{llll}0.03569 & 0.18076 & 0.59502\end{array}$ $\begin{array}{llll}0.03532 & 0.17925 & 0.59173\end{array}$ $\begin{array}{lll}0.03495 & 0.17775 & 0.58845\end{array}$

$\begin{array}{llll}0.03459 & 0.17626 & 0.58518\end{array}$ $\begin{array}{llll}0.03424 & 0.17478 & 0.58192\end{array}$ $\begin{array}{llll}0.03388 & 0.17332 & 0.57868\end{array}$ $\begin{array}{lllll}0.03354 & 0.17187 & 0.57545\end{array}$ $\begin{array}{llll}0.03319 & 0.17043 & 0.57223\end{array}$

$\begin{array}{llll}0.03285 & 0.16900 & 0.56903\end{array}$

$$
T=1500 \text {. }
$$

$\begin{array}{llll}0.06390 & 0.28816 & 0.79969\end{array}$ $\begin{array}{llll}0.06328 & 0.28595 & 0.79596\end{array}$ $\begin{array}{llll}0.06267 & 0.28375 & 0.79225\end{array}$ $\begin{array}{llll}0.06206 & 0.28157 & 0.78854\end{array}$ $\begin{array}{llll}0.06146 & 0.27940 & 0.78485\end{array}$

$\begin{array}{llll}0.06087 & 0.27726 & 0.78117\end{array}$ $\begin{array}{llll}0.06028 & 0.27512 & 0.77749\end{array}$ $\begin{array}{lllll}0.05969 & 0.27301 & 0.77383\end{array}$ $\begin{array}{llll}0.05911 & 0.27091 & 0.77018\end{array}$ $\begin{array}{llll}0.05854 & 0.26882 & 0.76653\end{array}$

$\begin{array}{llll}0.05797 & 0.26675 & 0.76290\end{array}$ $\begin{array}{llll}0.05741 & 0.26470 & 0.75928\end{array}$ $\begin{array}{llll}0.05686 & 0.26266 & 0.75567\end{array}$ $\begin{array}{llll}0.05631 & 0.26064 & 0.75207\end{array}$ $\begin{array}{llll}0.05576 & 0.25863 & 0.74848\end{array}$

$\begin{array}{llll}0.05522 & 0.25663 & 0.74490\end{array}$ $\begin{array}{llll}0.05469 & 0.25465 & 0.74133\end{array}$ $\begin{array}{llll}0.05416 & 0.25269 & 0.73777\end{array}$ $\begin{array}{llll}0.05363 & 0.25074 & 0.73422\end{array}$ $\begin{array}{llll}0.05311 & 0.24880 & 0.73068\end{array}$

$\begin{array}{llll}0.05260 & 0.24688 & 0.72715\end{array}$ $\begin{array}{llll}0.05209 & 0.24498 & 0.72364\end{array}$ $\begin{array}{llll}0.05159 & 0.24309 & 0.72013\end{array}$ $\begin{array}{llll}0.05109 & 0.24121 & 0.71664\end{array}$ $\begin{array}{lll}0.05059 & 0.23934 & 0.71315\end{array}$

$\begin{array}{llll}0.05011 & 0.23749 & 0.70968\end{array}$ $\begin{array}{llll}0.04962 & 0.23566 & 0.70621\end{array}$ $\begin{array}{llll}0.04914 & 0.23383 & 0.70276\end{array}$ $\begin{array}{llll}0.04867 & 0.23202 & 0.69932\end{array}$ $\begin{array}{lll}0.04820 & 0.23023 & 0.69589\end{array}$

$\begin{array}{llll}0.04773 & 0.22845 & 0.69247\end{array}$ $\begin{array}{llll}0.04727 & 0.22668 & 0.68906\end{array}$ $\begin{array}{llll}0.04681 & 0.22492 & 0.68567\end{array}$ $\begin{array}{llll}0.04636 & 0.22318 & 0.68228\end{array}$ $\begin{array}{lll}0.04591 & 0.22145 & 0.67890\end{array}$

$\begin{array}{llll}0.04547 & 0.21973 & 0.67554\end{array}$ $\begin{array}{llll}0.04503 & 0.21803 & 0.67218\end{array}$ $\begin{array}{llll}0.04460 & 0.21634 & 0.66884\end{array}$ $\begin{array}{llll}0.04417 & 0.21466 & 0.66551\end{array}$ $\begin{array}{lll}0.04374 & 0.21299 & 0.66219\end{array}$

$0.043320 .21134 \quad 0.65888$
$T=1600$.

$\begin{array}{llll}0.07961 & 0.34256 & 0.88605\end{array}$ $\begin{array}{llll}0.07888 & 0.34011 & 0.88235\end{array}$ $\begin{array}{lllll}0.07816 & 0.33767 & 0.87867\end{array}$ $\begin{array}{llll}0.07745 & 0.33525 & 0.87499\end{array}$ $\begin{array}{llll}0.07674 & 0.33285 & 0.87132\end{array}$

$\begin{array}{llll}0.07604 & 0.33047 & 0.86766\end{array}$ $\begin{array}{lllll}0.07535 & 0.32810 & 0.86401\end{array}$ $\begin{array}{llllll}0.07466 & 0.32574 & 0.86036\end{array}$ $\begin{array}{llll}0.07398 & 0.32341 & 0.85673\end{array}$ $0.07330 \quad 0.321090 .85310$

$\begin{array}{llll}0.07264 & 0.31878 & 0.84948\end{array}$ $\begin{array}{llll}0.07197 & 0.31650 & 0.84587\end{array}$ $\begin{array}{llll}0.07132 & 0.31422 & 0.84226\end{array}$ $\begin{array}{llll}0.07067 & 0.31197 & 0.83867\end{array}$ $\begin{array}{llll}0.07002 & 0.30973 & 0.83508\end{array}$

$\begin{array}{llll}0.06939 & 0.30750 & 0.83150\end{array}$ $\begin{array}{llll}0.06875 & 0.30529 & 0.82793\end{array}$ $\begin{array}{llll}0.06813 & 0.30310 & 0.82437\end{array}$ $\begin{array}{lll}0.06751 & 0.30092 & 0.82082\end{array}$ $\begin{array}{llll}0.06689 & 0.29876 & 0.81728\end{array}$

$\begin{array}{llll}0.06628 & 0.29661 & 0.81374\end{array}$ $\begin{array}{llll}0.06568 & 0.29447 & 0.81021\end{array}$ $\begin{array}{llll}0.06508 & 0.29235 & 0.80670\end{array}$ $\begin{array}{llll}0.06449 & 0.29025 & 0.80319\end{array}$ $\begin{array}{llll}0.06390 & 0.28816 & 0.79969\end{array}$

$\begin{array}{llll}0.06332 & 0.28608 & 0.79619\end{array}$ $\begin{array}{llll}0.06275 & 0.28402 & 0.79271\end{array}$ $\begin{array}{llll}0.06218 & 0.28198 & 0.78924\end{array}$ $\begin{array}{llll}0.06161 & 0.27994 & 0.78577\end{array}$ $\begin{array}{llll}0.06105 & 0.27793 & 0.78232\end{array}$

$\begin{array}{llll}0.06050 & 0.27592 & 0.77887\end{array}$ $\begin{array}{llll}0.05995 & 0.27393 & 0.77543\end{array}$ $\begin{array}{llll}0.05940 & 0.27196 & 0.77200\end{array}$ $\begin{array}{llll}0.05886 & 0.26999 & 0.76858\end{array}$ $\begin{array}{llllll}0.05833 & 0.26804 & 0.76517\end{array}$

$\begin{array}{llll}0.05780 & 0.26611 & 0.76177\end{array}$ $\begin{array}{lllll}0.05727 & 0.26419 & 0.75838\end{array}$ $\begin{array}{llll}0.05675 & 0.26228 & 0.75499\end{array}$ $\begin{array}{llll}0.05624 & 0.26038 & 0.75162\end{array}$ $\begin{array}{llll}0.05573 & 0.25850 & 0.74825\end{array}$

$\begin{array}{llll}0.05522 & 0.25663 & 0.74490\end{array}$
3600 3610 3620 3630 3640

3650

3660

3670

3680

3690

3700

3710

3720

3730

3740

3750

3760

3770

3780

3790

3800

3810

3820

3830

3840

3850

3860

3870

3880

3890

3900

3910

3920

3930

3940

3950

3960

3970

3980

3990

4000 
Table II Harmonic Oscillator Contributions to the Thermodynamic

Functions (in units of calories, moles, and ${ }^{\circ} \mathrm{K}$ )
$-\left(F^{\circ}-E \circ\right) / T \quad S \circ$
co
$-\left(F^{\circ}-E_{O} O\right) / T \quad S^{\circ}$
$\mathrm{C}_{\mathrm{p}}^{\circ}$
$-\left(F^{\circ}-E O\right) / T$
So
$\mathrm{c}_{\mathrm{p}}^{\circ}$
2

$\mathrm{cm}^{-1}$

$T=1700$.

$100 \quad 4.99062 \quad 6.89489 \quad 1.98598$ $110 \quad 4.80951 \quad 6.70561 \quad 1.98573$ $120 \quad 4.64488 \quad 6.53284 \quad 1.98546$ $130 \quad 4.49408 .6 .37393 \quad 1.98517$ $140 \quad 4.35506 \quad 6.22683 \quad 1.98485$

$150 \quad 4.22620 \quad 6.08990 \quad 1.98450$ $\begin{array}{lllll}160 & 4.210618 & 5.96184 & 1.98414\end{array}$ $\begin{array}{lllll}170 & 3.99392 & 5.84156 & 1.98375\end{array}$ $\begin{array}{lllll}180 & 3.88854 & 5.72818 & 1.98333\end{array}$ $190 \quad 3.78929 \quad 5.62096 \quad 1.98289$

$200 \quad 3.69554 \quad 5.51926 \quad 1.98243$ $210 \quad 3.60675 \quad 5.42255 \quad 1.98195$ $220 \quad 3.52246 \quad 5.33036 \quad 1.98144$ $\begin{array}{lllll}230 & 3.44227 & 5.24230 & 1.98091\end{array}$ $240 \quad 3.36583 \quad 5.15800 \quad 1.98035$

250 260

270

280

290

300

310

320

330

340

350

360

370

380

390

400

410

420

430

440

450

460

470

480

490

500

510

520

530

540

550

560

570

580

590
$3.29283 \quad 5.07717 \quad 1.97977$ $\begin{array}{llll}3.22299 & 4.99954 & 1.97917\end{array}$ $3.156094 .92485 \quad 1.97855$ $3.09191 \quad 4.85291 \quad 1.97790$ $3.030254 .78351 \quad 1.97722$

$2.97094 \quad 4.71650 \quad 1.97653$ $2.91383 \quad 4.65170 \quad 1.97581$ $2.85877 \quad 4.58898 \quad 1.97507$ $2.80565 \quad 4.52821 \quad 1.97430$ $2.75434 \quad 4.46929 \quad 1.97351$

$2.70474 \quad 4.412091 .97270$ $2.65674 \quad 4.35653 \quad 1.97187$ 2.610274 .302521 .97101 $2.56525 \quad 4.24996 \quad 1.97013$ $2.52158 \quad 4.19880 \quad 1.96923$

$2.47921 \quad 4.14896 \quad 1.96830$ $2.43807 \quad 4.10036 \quad 1.96735$ $2.398114 .05297 \quad 1.96638$ $2.35925 \quad 4.00671 \quad 1.96538$ $2.32146 \quad 3.96154 \quad 1.96436$

$2.284693 .91740 \quad 1.96332$ $2.24888 \quad 3.87426 \quad 1.96226$ $2.21401 \quad 3.83208 \quad 1.96117$ $2.18002 \quad 3.79080 \quad 1.96006$ $2.14688 \quad 3.75039 \quad 1.95893$

$2.11456 \quad 3.71083 \quad 1.95778$ $2.08302 \quad 3.67207 \quad 1.95660$ $2.05223 \quad 3.63409 \quad 1.95540$ $2.02217 \quad 3.59686 \quad 1.95418$ $1.99280 \quad 3.56034 \quad 1.95294$

$1.96410 \quad 3.52452 \quad 1.95167$ $1.93605 \quad 3.48936 \quad 1.95038$ $1.908623 .45485 \quad 1.94907$ $1.88179 \quad 3.42097 \quad 1.94774$ $1.855543 .38768 \quad 1.94639$

$T=1800$.

$T=1900$

$5.099607 .00841 \quad 1.98611$ $4.91803 \quad 6.81912 \quad 1.98589$ $4.75295 \quad 6.646341 .98565$ $4.60170 \quad 6.48741 \quad 1.98538$ 4.462236 .340291 .98510

$4.332926 .20334 \quad 1.98479$ $4.21245 \quad 6.07526 \quad 1.98446$ $4.09975 \quad 5.95496 \quad 1.98412$ $3.99392 \quad 5.84156 \quad 1.98375$ $3.894235 .73431 \quad 1.98335$

$3.80003 \quad 5.63259 \quad 1.98294$ $3.71080 \quad 5.53585 \quad 1.98251$ $3.62608 \quad 5.44364 \quad 1.98206$ $3.54545 \quad 5.35554 \quad 1.98158$ $3.468575 .27122 \quad 1.98109$

$3.39513 \quad 5.19036 \quad 1.98057$ $3.324865 .11269 \quad 1.98003$ $3.25753 \quad 5.03797 \quad 1.97947$ $3.19291 \quad 4.96599 \quad 1.97890$ $3.130824 .89656 \quad 1.97830$

$3.07108 \quad 4.82950 \quad 1.97767$ $3.01354 \quad 4.76467 \quad 1.97703$ $2.95806 \quad 4.70191 \quad 1.97637$ 2.904514 .641101 .97569 $2.85278 \quad 4.58213 \quad 1.97498$

$2.80275 \quad 4.52489 \quad 1.97426$ $2.75434 \quad 4.46929 \quad 1.97351$ $2.70745 \quad 4.41523 \quad 1.97275$ 2.662004 .362631 .97196 2.617924 .311411 .97115

$2.57513 \quad 4.26152 \quad 1.97033$ $2.53358 \quad 4.21288 \quad 1.96948$ $2.493194 .16543 \quad 1.96861$ $2.45393 \quad 4.119121 .96772$ $2.41573 \quad 4.07389 \quad 1.96681$

$2.37854 \quad 4.02970 \quad 1.96588$ $2.34233 \quad 3.98650 \quad 1.96493$ $2.307043 .94425 \quad 1.96396$ $2.272653 .90292 \quad 1.96297$ $2.23910 \quad 3.86245 \quad 1.96196$

$2.20638 \quad 3.82283 \quad 1.96093$ $2.17443 \quad 3.78400 \quad 1.95988$ $2.14325 \quad 3.74596 \quad 1.95881$ $2.11278 \quad 3.70866 \quad 1.95771$ 2.083023 .672071 .95660

$2.05392 \quad 3.63618 \quad 1.95547$ $2.025473 .60096 \quad 1.95432$ $1.997653 .56638 \quad 1.95315$ $1.970423 .53242 \quad 1.95196$ $1.94378 \quad 3.49906 \quad 1.95074$
$5.202927 .11579 \quad 1.98622$ $5.02094 \quad 6.92650 \quad 1.98602$ $4.855456 .75370 \quad 1.98580$ $4.70380 \quad 6.59476 \quad 1.98557$ $4.563936 .44762 \quad 1.98531$

$4.43421 \quad 6.31066 \quad 1.98503$ $4.31334 \quad 6.18256 \quad 1.98474$ $4.200246 .06224 \quad 1.98443$ $4.094015 .94883 \quad 1.98410$ $3.99392 \quad 5.84156 \quad 1.98375$

3.899335 .739821 .98338 $3.809715 .64306 \quad 1.98299$ $3.72458 \quad 5.550821 .98258$ $3.64356 \quad 5.46270 \quad 1.98215$ $3.566295 .37835 \quad 1.98171$

$3.49246 \quad 5.29746 \quad 1.98125$ $3.421805 .21976 \quad 1.98076$ $3.35408 \quad 5.14502 \quad 1.98026$ $3.289075 .07301 \quad 1.97974$ 3.226595 .003551 .97920

$3.16647 \quad 4.93646 \quad 1.97865$ $3.10855 \quad 4.87159 \quad 1.97807$ $\begin{array}{llll}3.05268 & 4.80880 & 1.97747\end{array}$ $2.99875 \quad 4.74796 \quad 1.97686$ $2.946634 .68895 \quad 1.97623$

2.896234 .631671 .97558 2.847434 .576031 .97491 $2.80016 \quad 4.52193 \quad 1.97422$ $2.75434 \quad 4.469291 .97351$ 2.709884 .418031 .97279

$2.666724 .36810 \quad 1.97205$ $2.624794 .31941 \quad 1.97128$ $2.58403 \quad 4.271921 .97050$ $2.54440 \quad 4.22556 \quad 1.96970$ $2.50582 \quad 4.18029 \quad 1.96889$

2.468274 .136051 .96805 $2.43169 \quad 4.09280 \quad 1.96720$ 2.396034 .050511 .96633 $2.36127 \quad 4.009121 .96543$ $2.32736 \quad 3.96860 \quad 1.96453$

$2.29427 \quad 3.92892 \quad 1.96360$ $2.26196 \quad 3.890051 .96265$ 2.230413 .851941 .96169 $2.199593 .81459 \quad 1.96071$ $2.16946 \quad 3.77795 \quad 1.95971$

$2.140013 .74200 \quad 1.95869$ $2.11120 \quad 3.70671 \quad 1.95766$ $2.08302 \quad 3.67207 \quad 1.95660$ $2.05544 \quad 3.63805 \quad 1.95553$ 2.028443 .604631 .95444
100

110

120

130

140

150

160

170

180

190

200

210

220

230

240

250

260

270

280

290

300

310

320

330

340

350

360

370

380

390

400

410

420

430

440

450

460

470

480

490

500

510

520

530

540

550

560

570

580

590 
Table II Harmonic Oscillator Contributions to the Thermodynamic

Functions (in units of calories, moles, and ${ }^{\circ} \mathrm{K}$ )

$\mathrm{cm}_{-1}^{-1}-\left(F^{\circ}-E_{0}^{0}\right) / T \quad$ So

$T=1700$.
So

$\mathrm{C}_{\mathrm{p}}^{\circ} \quad-\left(F^{\circ}-\mathrm{E}_{\mathrm{O}}^{\circ}\right) / \mathrm{T}$
$T=1800$

$1.917693 .46628 \quad 1.94951$

$1.892153 .43407 \quad 1.94826$

$1.86713 \quad 3.40240 \quad 1.94699$

$1.842623 .37126 \quad 1.94570$

1.818603 .340631 .94439

$1.79505 \quad 3.31049 \quad 1.94306$ $1.77197 \quad 3.28084 \quad 1.94172$ 1.749323 .251651 .94035 $1.72712 \quad 3.22291 \quad 1.93896$ $1.70533 \quad 3.19461 \quad 1.93755$

$1.68394 \quad 3.16675 \quad 1.93613$ $1.66296 \quad 3.13929 \quad 1.93468$ $1.64235 \quad 3.11224 \quad 1.93322$ $1.62212 \quad 3.08559 \quad 1.93173$ 1.602253 .059321 .93023

$1.58274 \quad 3.03342 \quad 1.92871$ $1.563573 .00788 \quad 1.92717$ $1.54473 \quad 2.98270 \quad 1.92561$ $1.52621 \quad 2.95786 \quad 1.92403$ $1.50802 \quad 2.93336 \quad 1.92243$

1.490132 .909191 .92082 $1.472542 .88534 \quad 1.91918$ $1.45524 \quad 2.86180 \quad 1.91753$ $1.43823 \quad 2.83857 \quad 1.91586$ 1.421492 .815631 .91417

$1.40503 \quad 2.79299 \quad 1.91246$ $1.388832 .77063 \quad 1.91073$ 1.372902 .748551 .90899 $1.35721 \quad 2.72675 \quad 1.90722$ $1.341772 .70520 \quad 1.90544$

$1.326572 .68392 \quad 1.90364$ $1.311602 .66290 \quad 1.90182$ 1.296872 .642121 .89999 $1.28236 \quad 2.62159 \quad 1.89813$ 1.268062 .601301 .89626

$1.25399 \quad 2.58125 \quad 1.89437$ 1.240122 .561421 .89246 $1.22646 \quad 2.54182 \quad 1.89054$ $1.21300 \quad 2.52244 \quad 1.88860$ $1.19973 \quad 2.50327 \quad 1.88664$

$1.18666 \quad 2.48432 \quad 1.88466$ $\begin{array}{llll}1.17378 & 2.46558 & 1.88267\end{array}$ $1.16108 \quad 2.44704 \quad 1.88065$ $1.148562 .42870 \quad 1.87862$ $1.13622 \quad 2.41056 \quad 1.87658$

$1.12406 \quad 2.39261 \quad 1.87452$ $1.11206 \quad 2.37486 \quad 1.87244$ $1.100232 .35728 \quad 1.87034$ $1.08856 \quad 2.33989 \quad 1.86822$

$1.07705 \quad 2.322691 .86609$
$-\left(F^{0}-E_{0}^{0}\right) / T \quad S^{\circ}$

$\mathrm{c}_{\mathrm{p}}^{\circ}$ $v$ $\mathrm{cm}^{-1}$
600
610
620
630
64
650
660
670
680
690
700
710
720
730
740
750
760
770
780
790
800
810
820

1010

1020

1030

1040

1050

1060

1070

1080

1090
$1.05255 \quad 2.28584 \quad 1.86140$ $1.040892 .26820 \quad 1.85909$ $1.029392 .25076 \quad 1.85675$ $1.01806 \quad 2.23350 \quad 1.85441$ $1.00688 \quad 2.21642 \quad 1.85204$
$T=1900$.

$2.00200 \quad 3.57179 \quad 1.95333$

$1.97610 \quad 3.53952 \quad 1.95221$

$1.95073 \quad 3.50778 \quad 1.95107$

$1.925873 .47657 \quad 1.94990$

$1.90150 \quad 3.44587 \quad 1.94873$

$1.87760 \quad 3.41567 \quad 1.94753$

$1.854173 .38595 \quad 1.94632$

1.831183 .356691 .94508

$1.80863 \quad 3.32788 \quad 1.94384$

$1.786493 .29951 \quad 1.94257$

$1.764773 .27157 \quad 1.94129$

1.743443 .244041 .93998

$1.72249 \quad 3.21692 \quad 1.93867$

$1.70192 \quad 3.19019 \quad 1.93733$

1.681723 .163841 .93598

$1.66186 \quad 3.13786 \quad 1.93460$

1.642353 .112241 .93322

$1.62318 \quad 3.08698 \quad 1.93181$

1.604333 .062061 .93039

$1.58580 \quad 3.03748 \quad 1.92895$

1.567573 .013231 .92749

$\begin{array}{llll}1.54965 & 2.98929 & 1.92602\end{array}$

$1.53203 \quad 2.96567 \quad 1.92453$

$1.51468 \quad 2.942351 .92302$

$1.49762 \quad 2.91933 \quad 1.92150$

1.480832 .896601 .91996 $1.464312 .87415 \quad 1.91840$ 1.448042 .851981 .91683

$1.432032 .83008 \quad 1.91524$

$1.41627 \quad 2.80845 \quad 1.91363$

$1.40074 \quad 2.78708 \quad 1.91201$

$1.38546 \quad 2.76596 \quad 1.91036$

$\begin{array}{llll}1.37040 & 2.74509 & 1.90871\end{array}$

1.355572 .724471 .90704

$1.34096 \quad 2.704081 .90535$

$1.326572 .68392 \quad 1.90364$

$1.31238 \quad 2.664001 .90192$

1.298412 .644301 .90018

$1.28463 \quad 2.62482 \quad 1.89843$

$1.27105 \quad 2.60555 \quad 1.89666$

$1.257672 .58650 \quad 1.89487$ $1.24448 \quad 2.56766 \quad 1.89307$ 1.231472 .549011 .89125

$1.218642 .53057 \quad 1.88942$

$\begin{array}{llll}1.20599 & 2.51232 & 1.88757\end{array}$

$1.19352 \quad 2.49427 \quad 1.88570$

$1.18122 \quad 2.47641 \quad 1.88382$

$1.16908 \quad 2.45873 \quad 1.88193$

1.157112 .441231 .88002

$1.14530 \quad 2.42391 \quad 1.87809$
600

610

620

630

640

650

660

670

680

690

700

710

720

730

740

750

760

770

780

790

800

810

820

830

840

850

860

870

880

890

900

910

920

930

940

950

960

970

980

990

1000

1010

1020

1030

1040

1050

1060

1070

1080

1090 
Table II Harmonic Oscillator Contributions to the Thermodynamic

Functions (in units of calories, moles, and ${ }^{\circ} \mathrm{K}$ )
$\mathrm{cm}^{-1}-\left(\mathrm{F}^{\circ}-\mathrm{E}_{\mathrm{O}} \mathrm{O}\right) / \mathrm{T}$
So
$\mathrm{c}_{\mathrm{p}}^{\circ}$
$-\left(F^{\circ}-E_{O}^{\circ}\right) / T \quad S^{\circ}$
$\mathrm{C} \stackrel{\mathrm{p}}{\mathrm{p}}$
$-\left(F^{\circ}-E_{O}\right) / T$
So
$\mathrm{C}_{\mathrm{p}}^{\circ}$
$\mathrm{cm}^{-1}$

$$
T=1700 \text {. }
$$

$\begin{array}{llll}1100 & 0.99586 & 2.19951 & 1.84965\end{array}$

$\begin{array}{lllll}1110 & 0.98500 & 2.18279 & 1.84725\end{array}$

$\begin{array}{lllll}1120 & 0.97428 & 2.16623 & 1.84483\end{array}$

$\begin{array}{llllll}1130 & 0.96371 & 2.14984 & 1.84239\end{array}$

$\begin{array}{lllll}1140 & 0.95329 & 2.13362 \quad 1.83993\end{array}$

$1150 \quad 0.94300 \quad 2.11756 \quad 1.83746$

$\begin{array}{llll}1160 & 0.93286 \quad 2.10166 \quad 1.83496\end{array}$

$1170 \quad 0.922852 .08592 \quad 1.83245$

$\begin{array}{lllll}1180 & 0.91297 & 2.07034 & 1.82993\end{array}$

$\begin{array}{lllll}1190 & 0.90323 & 2.05491 & 1.82738\end{array}$

$\begin{array}{llll}1200 & 0.89362 & 2.03963 & 1.82482\end{array}$ $\begin{array}{lllll}1210 & 0.88413 & 2.02449 & 1.82224\end{array}$ $\begin{array}{lllll}1220 & 0.87477 & 2.00951 & 1.81964\end{array}$ $\begin{array}{lllll}1230 & 0.86553 & 1.99466 & 1.81703\end{array}$ $1240 \quad 0.856411 .97996 \quad 1.81440$

$\begin{array}{lllll}1250 & 0.84741 & 1.96540 & 1.81176\end{array}$ $\begin{array}{llll}1260 & 0.83852 & 1.95097 & 1.80909\end{array}$ $\begin{array}{lllll}1270 & 0.82975 & 1.93668 & 1.80641\end{array}$ $\begin{array}{lllll}1280 & 0.82109 & 1.92252 & 1.80372\end{array}$ $1290 \quad 0.812541 .908501 .80101$

$\begin{array}{lllll}1300 & 0.80409 & 1.89460 & 1.79828\end{array}$ $\begin{array}{llll}1310 & 0.79576 & 1.88083 & 1.79553\end{array}$ $\begin{array}{lllll}1320 & 0.78753 & 1.86719 & 1.79277\end{array}$ $\begin{array}{lllll}1330 & 0.77940 & 1.85367 & 1.79000\end{array}$ $\begin{array}{lllll}1340 & 0.77137 & 1.84027 & 1.78721\end{array}$

$\begin{array}{lllll}1350 & 0.76345 & 1.82699 & 1.78440\end{array}$ $\begin{array}{llll}1360 & 0.75562 & 1.81383 & 1.78157\end{array}$ $\begin{array}{lllll}1370 & 0.74788 & 1.80079 & 1.77874\end{array}$ $\begin{array}{lllll}1380 & 0.74024 & 1.78786 & 1.77588\end{array}$ $\begin{array}{lllll}1390 & 0.73270 & 1.77505 & 1.77301\end{array}$

$\begin{array}{llll}1400 & 0.72525 & 1.76235 & 1.77013\end{array}$ $\begin{array}{lllll}1410 & 0.71788 & 1.74976 & 1.76723\end{array}$ $\begin{array}{lllll}1420 & 0.71061 & 1.73729 & 1.76432\end{array}$ $\begin{array}{lllll}1430 & 0.70342 & 1.72491 & 1.76139\end{array}$ $\begin{array}{lllll}1440 & 0.69632 & 1.71265 & 1.75844\end{array}$

$\begin{array}{lllll}1450 & 0.68931 & 1.70049 & 1.75549\end{array}$

$\begin{array}{lllll}1460 & 0.68237 & 1.68844 & 1.75251\end{array}$ $\begin{array}{lllll}1470 & 0.67552 & 1.67648 & 1.74953\end{array}$ $\begin{array}{lllll}1480 & 0.66876 & 1.66463 & 1.74653\end{array}$

$\begin{array}{lllll}1490 & 0.66207 & 1.65288 & 1.74351\end{array}$

$\begin{array}{lllll}1500 & 0.65546 & 1.64123 & 1.74048\end{array}$ $\begin{array}{lllll}1510 & 0.64892 & 1.62967 & 1.73744\end{array}$ $\begin{array}{lllll}1520 & 0.64246 & 1.61822 & 1.73438\end{array}$ $\begin{array}{lllll}1530 & 0.63608 & 1.60685 & 1.73131\end{array}$ $\begin{array}{lllll}1540 & 0.62977 & 1.59558 & 1.72823\end{array}$

$\begin{array}{lllll}1550 & 0.62354 & 1.58441 & 1.72513\end{array}$ $\begin{array}{lllll}1560 & 0.61738 & 1.57332 & 1.72202\end{array}$ $\begin{array}{llll}1570 & 0.61128 & 1.56233 & 1.71889\end{array}$ $\begin{array}{lllll}1580 & 0.60526 & 1.55143 & 1.71576\end{array}$ $\begin{array}{llllll}1590 & 0.59931 & 1.54061 & 1.71261\end{array}$

$$
T=1800
$$

$T=1900$

$1.06570 \quad 2.30565 \quad 1.86395$ $\begin{array}{llll}1.05451 & 2.28879 & 1.86178\end{array}$ $1.04346 \quad 2.27211 \quad 1.85960$ $1.03257 \quad 2.25559 \quad 1.85740$ $1.02182 \quad 2.239231 .85519$

$1.01121 \quad 2.22304 \quad 1.85296$ $1.00074 \quad 2.207011 .85072$ 0.990412 .191131 .84845 $0.980212 .17541 \quad 1.84617$ $0.97015 \quad 2.15984 \quad 1.84388$

$0.96022 \quad 2.144421 .84157$ $0.950412 .12914 \quad 1.83924$ $0.940742 .11401 \quad 1.83690$ $0.93118 \quad 2.099031 .83455$ $0.92175 \quad 2.084181 .83217$

$0.91243 \quad 2.06948 \quad 1.82978$ $0.90323 \quad 2.05491 \quad 1.82738$ $0.89415 \quad 2.04047 \quad 1.82496$ $0.88518 \quad 2.02617 \quad 1.82253$ 0.876322 .011991 .82008

$0.86757 \quad 1.99795 \quad 1.81761$ 0.858931 .984031 .81513 $0.850391 .97024 \quad 1.81264$ 0.841961 .956561 .81013 0.833631 .943011 .80761

$0.82540 \quad 1.92958 \quad 1.80507$ $0.81727 \quad 1.916271 .80251$ 0.809241 .903081 .79995 $0.80130 \quad 1.890001 .79736$ $0.79346 \quad 1.87703 \quad 1.79477$

$\begin{array}{llll}0.78571 & 1.86417 & 1.79216\end{array}$ $0.77805 \quad 1.851421 .78953$ $0.770491 .83879 \quad 1.78689$ 0.763011 .826261 .78424 0.755621 .813831 .78157

0.748311 .801511 .77889 0.741091 .789301 .77620 $0.73395 \quad 1.77718 \quad 1.77349$ 0.726891 .765171 .77077 $0.71992 \quad 1.75325 \quad 1.76804$

0.713021 .741431 .76529 $0.70621 \quad 1.72971 \quad 1.76253$ $0.69947 \quad 1.718091 .75975$ $0.69280 \quad 1.70656 \quad 1.75697$ 0.686221 .695121 .75417

$0.67970 \quad 1.68378 \quad 1.75135$ $0.67326 \quad 1.672521 .74853$ 0.666891 .661361 .74569 $0.66059 \quad 1.65028 \quad 1.74284$ $0.65436 \quad 1.63930 \quad 1.73998$
$1.13365 \quad 2.40677 \quad 1.87615$ $1.122152 .38980 \quad 1.87419$ 1.110812 .373001 .87222 $1.099612 .35636 \quad 1.87023$ $1.08856 \quad 2.33989 \quad 1.86822$

$1.07766 \quad 2.32359 \quad 1.86621$ $1.066892 .30744 \quad 1.86417$ $1.056272 .29145 \quad 1.86212$ $\begin{array}{llll}1.04578 & 2.27561 & 1.86006\end{array}$ 1.035422 .259921 .85798

$1.02520 \quad 2.24438 \quad 1.85589$ $1.01510 \quad 2.22899 \quad 1.85378$ $\begin{array}{llll}1.00513 & 2.21374 & 1.85166\end{array}$ 0.995292 .198631 .84953 $0.98556 \quad 2.18366 \quad 1.84738$

$0.97596 \quad 2.168831 .84521$ $0.96648 \quad 2.15414 \quad 1.84303$ $0.957112 .13958 \quad 1.84084$ $0.94786 \quad 2.12515 \quad 1.83863$ 0.938712 .110851 .83641

$0.92968 \quad 2.09668 \quad 1.83417$ 0.920762 .082631 .83192 $0.911942 .06871 \quad 1.82966$ 0.903232 .054911 .82738 0.894622 .041231 .82509

$\begin{array}{llll}0.88612 & 2.02767 & 1.82278\end{array}$ $0.87771 \quad 2.014221 .82047$ $0.86940 \quad 2.000891 .81813$ $0.86119 \quad 1.98768 \quad 1.81579$ $0.853081 .97458 \quad 1.81343$

$0.84506 \quad 1.96159 \quad 1.81106$ 0.837131 .948711 .80867 $0.82929 \quad 1.93593 \quad 1.80627$ 0.821541 .923261 .80386 $0.81388 \quad 1.91070 \quad 1.80143$

$\begin{array}{llll}0.80631 & 1.89824 & 1.79900\end{array}$ $0.79882 \quad 1.88589 \quad 1.79655$ $0.79141 \quad 1.873631 .79408$ 0.784091 .861481 .79161 $0.776851 .84942 \quad 1.78912$

$0.76970 \quad 1.83746 \quad 1.78662$ $0.762621 .82560 \quad 1.78410$ 0.755621 .813831 .78157 0.748691 .802161 .77904 $0.74184 \quad 1.79058 \quad 1.77648$

$0.73507 \quad 1.77909 \quad 1.77392$ 0.728371 .767691 .77135 $0.72175 \quad 1.75638 \quad 1.76876$ $0.71519 \quad 1.74515 \quad 1.76616$ 0.708711 .734021 .76355
1100

1110

1120

1130

1140

1150

1160

1170

1180

1190

1200

1210

1220

1230

1240

1250

1260

1270

1280

1290

1300

1310

1320

1330

1340

1350

1360

1370

1380

1390

1400

1410

1420

1430

1440

1450

1460

470

1480

1490

1500

1510

1520

1530

1540

1550

1560

1570

1580

1590 
Table II Harmonic Oscillator Contributions to the Thermodynamic Functions (in units of calories, moles, and ${ }^{\circ} \mathrm{K}$ )

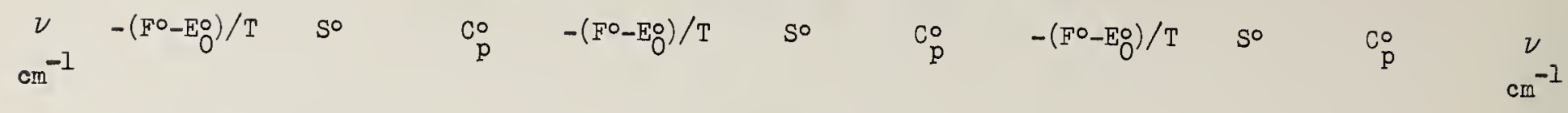

$T=1700$.

1600

1610

1620

1630

1640

1650

1660

1670

1680

1690

1700

1710

1720

1730

1740

1750

1760

1770

1780

1790

1800

1810

1820

1830

1840

1850

1860

1870

1880

1890

1900

1910

1920

1930

1940

1950

1960

1970

1980

1990

2000

2010

2020

2030

2040

2050

2060

2070

2080

2090
$T=1800$.

$0.59342 \quad 1.52988 \quad 1.70944$ $\begin{array}{llll}0.58760 & 1.51924 & 1.70627\end{array}$ $0.581851 .50869 \quad 1.70308$ $0.57616 \quad 1.49822 \quad 1.69988$ 0.570531 .487831 .69666

$0.56497 \quad 1.47753 \quad 1.69344$ $0.55947 \quad 1.46730 \quad 1.69020$ $0.55403 \quad 1.45716 \quad 1.68695$ 0.548651 .447101 .68369 $0.543341 .43712 \quad 1.68041$

$0.53808 \quad 1.42721 \quad 1.67713$ $0.53288 \quad 1.41739 \quad 1.67383$ $0.52773 \quad 1.40764 \quad 1.67052$ $0.522641 .39796 \quad 1.66720$ $0.517611 .38836 \quad 1.66387$

$0.512631 .37884 \quad 1.66052$ $0.50771 \quad 1.36939 \quad 1.65717$ $0.50284 \quad 1.36001 \quad 1.65380$ $0.49803 \quad 1.35070 \quad 1.65043$ $0.49326 \quad 1.34146 \quad 1.64704$

$0.48855 \quad 1.33229 \quad 1.64364$ $0.48389 \quad 1.32320 \quad 1.64024$ $0.47927 \quad 1.31417 \quad 1.63682$ $0.47471 \quad 1.30521 \quad 1.63339$ $0.47020 \quad 1.29632 \quad 1.62995$

$0.46573 \quad 1.28749 \quad 1.62650$ $0.46131 \quad 1.27873 \quad 1.62304$ $0.45694 \quad 1.27004 \quad 1.61957$ $0.452621 .26141 \quad 1.61610$ 0.448341 .252851 .61261

$0.44410 \quad 1.24435 \quad 1.60911$ $0.43991 \quad 1.23591 \quad 1.60561$ $0.43577 \quad 1.22754 \quad 1.60209$ $0.431671 .21922 \quad 1.59856$ 0.427611 .210971 .59503

$0.42359 \quad 1.20278 \quad 1.59149$ $0.41962 \quad 1.19465 \quad 1.58794$ $\begin{array}{llll}0.41568 & 1.18657 & 1.58438\end{array}$ $0.411791 .17856 \quad 1.58081$ $0.40794 \quad 1.17061 \quad 1.57723$

$0.40412 \quad 1.16271 \quad 1.57365$ $0.400351 .15487 \quad 1.57005$ 0.396621 .147091 .56645 $0.39292 \quad 1.13936 \quad 1.56284$ $0.38926 \quad 1.13169 \quad 1.55922$

$0.385641 .12407 \quad 1.55560$ $0.38206 \quad 1.11651 \quad 1.55197$ $0.378511 .10901 \quad 1.54833$ $0.37500 \quad 1.10155 \quad 1.54468$ $0.371521 .09415 \quad 1.54102$
$0.64820 \quad 1.62840 \quad 1.73710^{\circ}$ 0.642111 .617581 .73421 $0.63608 \quad 1.60685 \quad 1.73131$ $0.630121 .59621 \quad 1.72840$ $0.624231 .58565 \quad 1.72547$

$0.61840 \quad 1.57517 \quad 1.72254$ 0.612631 .564771 .71959 0.606931 .554451 .71663 $0.60128 \quad 1.54421 \quad 1.71366$ $0.59570 \quad 1.53405 \quad 1.71067$

$0.59018 \quad 1.52396 \quad 1.70768$ $0.58471 \quad 1.51396 \quad 1.70467$ $0.57931 \quad 1.50402 \quad 1.70166$ $0.57396 \quad 1.49417 \quad 1.69863$ $0.56867 \quad 1.484391 .69559$

$0.56344 \quad 1.47468 \quad 1.69254$ $0.558261 .46504 \quad 1.68948$ $0.553131 .45548 \quad 1.68641$ $0.54806 \quad 1.445991 .68332$ 0.543041 .436571 .68023

$0.53808 \quad 1.42721 \quad 1.67713$ $0.53316 \quad 1.417931 .67401$ $0.528301 .40872 \quad 1.67089$ 0.523491 .399571 .66775 $0.51873 \quad 1.390491 .66461$

$0.51401 \quad 1.38148 \quad 1.66145$ $0.509351 .37253 \quad 1.65829$ 0.504731 .363641 .65511 0.500161 .354831 .65193 $0.495641 .34607 \quad 1.64874$

$0.49116 \quad 1.33738 \quad 1.64553$ 0.486731 .328751 .64232 $0.482341 .32018 \quad 1.63910$ $0.47800 \quad 1.31167 \quad 1.63587$ 0.473701 .303231 .63263

$0.46945 \quad 1.29484 \quad 1.62938$ $0.46524 \quad 1.28652 \quad 1.62612$ 0.461071 .278251 .62285 0.456941 .270041 .61957 $0.45286 \quad 1.26189 \quad 1.61629$

$0.44881 \quad 1.25380 \quad 1.61300$ $0.444811 .24576 \quad 1.60970$ $0.440841 .23778 \quad 1.60639$ 0.436921 .229861 .60307 $0.43303 \quad 1.221991 .59974$

$0.429181 .21417 \quad 1.59641$ 0.425371 .206411 .59306 $0.42160 \quad 1.19870 \quad 1.58971$ 0.417861 .191051 .58636 0.414161 .183451 .58299
$T=1900$.

$0.70230 \quad 1.72297 \quad 1.76092$

1600

0.695951 .712011 .75829

0.689671 .701131 .75564

0.683461 .690331 .75298

$0.67732 \quad 1.67962 \quad 1.75031$

1610

1620

1630

1640

$0.67124 \quad 1.66899 \quad 1.74763$

$0.66522 \quad 1.65844 \quad 1.74494$

$0.659271 .64796 \quad 1.74224$

$\begin{array}{llll}0.65338 & 1.63757 & 1.73952\end{array}$

0.647561 .627251 .73680

1650

1660

1670

1680

1690

0.641791 .617021 .73406

0.636081 .606851 .73131

$0.63044 \quad 1.59677 \quad 1.72855$

$0.624851 .58675 \quad 1.72578$

0.619311 .576811 .72300

1700

1710

1720

1730

1740

$0.61384 \quad 1.56695 \quad 1.72021$

1750

$0.60842 \quad 1.55716 \quad 1.71741$

$0.60306 \quad 1.54743 \quad 1.71460$

$0.59775 \quad 1.53778 \quad 1.71177$

$0.59250 \quad 1.52820 \quad 1.70894$

1760

1770

1780

1790

$0.58730 \quad 1.51869 \quad 1.70610$

1800

$0.58215 \quad 1.50924 \quad 1.70325$

$0.57705 \quad 1.49987 \quad 1.70038$

$0.57201 \quad 1.49056 \quad 1.69751$

$0.56701 \quad 1.48131 \quad 1.69463$

1810

1820

1830

1840

$0.56207 \quad 1.47214 \quad 1.69173$

1850

$0.55717 \quad 1.46302 \quad 1.68883$

$0.552331 .45398 \quad 1.68592$

$0.54753 \quad 1.44499 \quad 1.68300$

$0.54278 \quad 1.43607 \quad 1.68007$

$0.53808 \quad 1.42721 \quad 1.67713$

0.533421 .418421 .67418

0.528811 .409681 .67122

$0.524241 .40101 \quad 1.66825$

$0.51972 \quad 1.39240 \quad 1.66527$

$0.515251 .38384 \quad 1.66228$

$0.510821 .37535 \quad 1.65929$

$0.506431 .36691 \quad 1.65628$

$0.50208 \quad 1.358531 .65327$

$0.49777 \quad 1.350211 .65025$

$0.49351 \quad 1.34195 \quad 1.64722$

$0.489291 .33374 \quad 1.64418$

0.485111 .325591 .64113

0.480971 .317491 .63808

$0.47687 \quad 1.309451 .63501$

$0.47281 \quad 1.30146 \quad 1.63194$ $0.46878 \quad 1.293521 .62886$ $0.46480 \quad 1.28564 \quad 1.62577$ $0.46085 \quad 1.27782 \quad 1.62268$ $0.45694 \quad 1.27004 \quad 1.61957$
1860

1870

1880

1890

1900

1910

1920

1930

1940

1950

1960

1970

1980

1990

2000

2010

2020

2030

2040

2050

2060

2070

2080

2090 
Table II Harmonic Oscillator Contributions to the Thermodynamic

Functions (in units of calories, moles, and ${ }^{\circ} \mathrm{K}$ )
$\mathrm{cm}^{-1}$
so
$C_{p}^{\circ} \quad-\left(F^{\circ}-E_{O}^{\circ}\right) / T$
$\mathrm{S}^{\circ}$
$\mathrm{C}_{\mathrm{p}}$
$-\left(F^{\circ}-E_{O}^{\circ}\right) / T$
So
$\mathrm{C}_{\mathrm{p}}^{\circ}$
$\mathrm{cm}^{-1}$

$T=1700$.

$\mathrm{T}=1800$.

$T=1900$.

2100

2110

2120

2130

2140

2150

2160

2170

2180

2190

2200

2210

2220

2230

2240

2250

2260

2270

2280

2290

2300

2310

2320

2330

2340

2350

2360

2370

2380

2390

2400

2410

2420

2430

2440

2450

2460

2470

2480

2490

2500

2510

2520

2530

2540

2550

2560

2570

2580

2590
$0.36808 \quad 1.08681 \quad 1.53736$ $0.36468 \quad 1.07951 \quad 1.53369$ $0.361311 .07227 \quad 1.53002$ $0.35797 \quad 1.06508 \quad 1.52633$ $0.35467 \quad 1.05794 \quad 1.52264$

$0.35140 \quad 1.05085 \quad 1.51895$ $0.34816 \quad 1.04381 \quad 1.51525$ $0.34496 \quad 1.03682 \quad 1.51154$ $0.34179 \quad 1.02988 \quad 1.50782$ $0.33864 \quad 1.02298 \quad 1.50410$

$0.335541 .01614 \quad 1.50037$ $0.33246 \quad 1.00934 \quad 1.49664$ 0.329411 .002591 .49290 $0.326390 .99589 \quad 1.48916$ $\begin{array}{llll}0.32341 & 0.98924 & 1.48541\end{array}$

$0.32045 \quad 0.982631 .48165$ $0.31752 \quad 0.97607 \quad 1.47789$ $0.314620 .96955 \quad 1.47412$ $0.31175 \quad 0.96308 \quad 1.47035$ $\begin{array}{lll}0.30891 & 0.95665 & 1.46657\end{array}$

$\begin{array}{llll}0.30609 & 0.95027 & 1.46279\end{array}$ $0.30330 \quad 0.94393 \quad 1.45901$ $0.300540 .93764 \quad 1.45522$ $0.29781 \quad 0.931391 .45142$ $0.29511 \quad 0.92518 \quad 1.44762$

$0.29243 \quad 0.91902 \quad 1.44382$ $0.289770 .91289 \quad 1.44001$ $0.28715 \quad 0.90681 \quad 1.43619$ $0.28454 \quad 0.90077 \quad 1.43238$ $0.28197 \quad 0.89478 \quad 1.42856$

$0.27942 \quad 0.88882 \quad 1.42473$ $\begin{array}{llll}0.27689 & 0.88290 & 1.42090\end{array}$ $\begin{array}{llll}0.27439 & 0.87703 & 1.41707\end{array}$ $0.27191 \quad 0.87119 \quad 1.41324$ $0.26945 \quad 0.86540 \quad 1.40940$

$0.267020 .85964 \quad 1.40556$ $0.264620 .85392 \quad 1.40171$ $0.262230 .84824 \quad 1.39786$ $\begin{array}{llll}0.25987 & 0.84260 & 1.39401\end{array}$ 0.257530 .837001 .39016

$0.255220 .83144 \quad 1.38630$ $0.25292 \quad 0.82591 \quad 1.38244$ $\begin{array}{lll}0.25065 & 0.82042 & 1.37858\end{array}$ $0.24840 \quad 0.81497 \quad 1.37471$ 0.246170 .809551 .37084

$\begin{array}{llll}0.24396 & 0.80417 & 1.36698\end{array}$ $0.241780 .79883 \quad 1.36310$ $\begin{array}{llll}0.23961 & 0.79353 & 1.35923\end{array}$ $\begin{array}{llll}0.23747 & 0.78825 & 1.35535\end{array}$ $0.235340 .78302 \quad 1.35148$
$0.41050 \quad 1.17590 \quad 1.57962$ 0.406871 .168411 .57624 $0.40328 \quad 1.16096 \quad 1.57285$ $0.39973 \quad 1.15357 \quad 1.56945$ 0.396201 .146231 .56605

$0.39272 \quad 1.13893 \quad 1.56264$ $0.389261 .13169 \quad 1.55922$ $0.38584 \quad 1.12450 \quad 1.55580$ $0.38245 \quad 1.11735 \quad 1.55237$ 0.379101 .110251 .54893

$0.37578 \quad 1.103201 .54549$ 0.372491 .096201 .54204 0.369231 .089251 .53858 $0.366001 .08234 \quad 1.53512$ $0.362801 .07548 \quad 1.53165$

0.359641 .068671 .52818 0.356501 .061901 .52470 $0.353391 .05517 \quad 1.52121$ $0.35032 \quad 1.04849 \quad 1.51772$ $0.34727 \quad 1.04186 \quad 1.51422$

$0.344251 .03527 \quad 1.51071$ $0.34126 \quad 1.02872 \quad 1.50720$ $0.33830 \quad 1.02222 \quad 1.50369$ $0.335361 .01576 \quad 1.50017$ $0.33246 \quad 1.00934 \quad 1.49664$

$0.32958 \quad 1.00297 \quad 1.49311$ $\begin{array}{llll}0.32673 & 0.99664 & 1.48957\end{array}$ $0.32390 \quad 0.99034 \quad 1.48603$ $0.321100 .98410 \quad 1.48249$ $\begin{array}{llll}0.31833 & 0.97789 & 1.47893\end{array}$

$\begin{array}{llll}0.31558 & 0.97172 & 1.47538\end{array}$ $\begin{array}{llll}0.31286 & 0.96559 & 1.47182\end{array}$ $0.310170 .95950 \quad 1.46825$ $0.30750 \quad 0.95346 \quad 1.46468$ $0.304850 .94745 \quad 1.46111$

$0.302230 .94148 \quad 1.45753$ $0.29963 \quad 0.93555 \quad 1.45395$ $0.29706 \quad 0.92966 \quad 1.45037$ $\begin{array}{llll}0.29451 & 0.92381 & 1.44678\end{array}$ $0.29198 \quad 0.91799 \quad 1.44318$

$0.28948 \quad 0.91222 \quad 1.43958$ $0.28700 \quad 0.90648 \quad 1.43598$ $0.28454 \quad 0.90077 \quad 1.43238$ 0.282110 .895111 .42877 $0.27970 \quad 0.88948 \quad 1.42516$

$0.27731 \quad 0.88389 \quad 1.42154$ $0.27494 \quad 0.87833 \quad 1.41792$ $0.27259 \quad 0.87281 \quad 1.41430$ $0.27027 \quad 0.867321 .41068$ $0.26797 \quad 0.86187 \quad 1.40705$
$0.45307 \quad 1.26232 \quad 1.61646$ 0.449241 .254651 .61334 0.445441 .247031 .61022 $0.44167 \quad 1.23946 \quad 1.60708$ $0.43795 \quad 1.23194 \quad 1.60394$

$0.434251 .22447 \quad 1.60079$ 0.430591 .217041 .59764 $0.42697 \quad 1.20967 \quad 1.59447$ $0.42338 \quad 1.202351 .59130$ $0.419821 .19507 \quad 1.58812$

$0.41630 \quad 1.18785 \quad 1.58494$ 0.412811 .180661 .58175 0.409351 .173531 .57855 0.405921 .166441 .57535 0.402531 .159401 .57213

0.399171 .152411 .56892 0.395831 .145461 .56569 0.392531 .138551 .56246 $0.38926 \quad 1.131691 .55922$ $0.386021 .12487 \quad 1.55598$

$0.38281 \quad 1.118101 .55273$ 0.379631 .111371 .54948 0.376471 .104681 .54622 0.373351 .098041 .54295 $0.370251 .09144 \quad 1.53968$

$0.36718 \quad 1.084881 .53640$ 0.364141 .078361 .53311 0.361131 .071891 .52982 0.358151 .065451 .52653 0.355191 .059061 .52323

$0.35226 \quad 1.05271 \quad 1.51992$ 0.349351 .046391 .51661 0.346471 .040121 .51329 0.343621 .033891 .50997 0.340791 .027691 .50665

$0.33799 \quad 1.02154 \quad 1.50332$ 0.335211 .015421 .49998 0.332461 .009341 .49664 0.329731 .003301 .49330 0.327030 .997301 .48995

$0.324350 .99134 \quad 1.48659$ 0.321690 .985411 .48323 $0.31906 \quad 0.97952 \quad 1.47987$ $0.31645 \quad 0.97366 \quad 1.47650$ $0.31386 \quad 0.96784 \quad 1.47313$

$0.31130 \quad 0.96206 \quad 1.46976$ $0.30876 \quad 0.956321 .46638$ 0.306240 .950611 .46299 0.303740 .944931 .45961 $0.30127 \quad 0.939291 .45621$
2100

2110

2120

2130

2140

2150

2160

2170

2180

2190

2200

2210

2220

2230

2240

2250

2260

2270

2280

2290

2300

2310

2320

2330

2340

2350

2360

2370

2380

2390

2400

2410

2420

2430

2440

2450

2460

2470

2480

2490

2500

2510

2520

2530

2540

2550

2560

2570

2580

2590 
Table II Harmonic Oscillator Contributions to the Thermodynamic

Functions (in units of calories, moles, and ${ }^{\circ} \mathrm{K}$ )

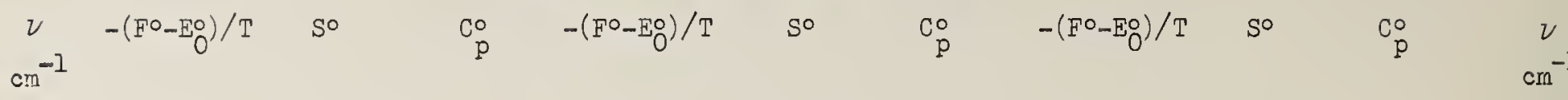

$T=1700$.

2600

2610

2620

2630

2640

2650

2660

2670

2680

2690

2700

2710

2720

2730

2740

2750

2760

2770

2780

2790

2800

2810

2820

2830

2840

2850

2860

2870

2880

2890

2900

2910

2920

2930

2940

2950

2960

2970

2980

2990

3000

3010

3020

3030

3040

3050

3060

3070

3080

3090

$$
T=1800 .
$$

$0.233240 .77782 \quad 1.34760$ $\begin{array}{llll}0.23115 & 0.77265 & 1.34372\end{array}$ $0.22909 \quad 0.767521 .33983$ $\begin{array}{llll}0.22704 & 0.76242 & 1.33595\end{array}$ $0.225020 .75736 \quad 1.33206$

$\begin{array}{llll}0.22301 & 0.75233 & 1.32818\end{array}$ $0.221020 .74734 \quad 1.32429$ $0.21905 \quad 0.74238 \quad 1.32040$ $0.21710 \quad 0.73745 \quad 1.31651$ $0.215170 .73255 \quad 1.31262$

$\begin{array}{llll}0.21326 & 0.72769 & 1.30873\end{array}$ $\begin{array}{llll}0.21136 & 0.72286 & 1.30484\end{array}$ $\begin{array}{lll}0.20948 & 0.71806 & 1.30095\end{array}$ 0.207620 .713291 .29705 $\begin{array}{lll}0.20578 & 0.70856 \quad 1.29316\end{array}$

$\begin{array}{llll}0.20395 & 0.70385 & 1.28926\end{array}$ $\begin{array}{llll}0.20214 & 0.69918 & 1.28537\end{array}$ $0.20035 \quad 0.69454 \quad 1.28148$ $\begin{array}{llll}0.19857 & 0.68993 & 1.27758\end{array}$ $\begin{array}{llll}0.19681 & 0.68535 & 1.27369\end{array}$

$\begin{array}{llll}0.19507 & 0.68080 & 1.26979\end{array}$ $\begin{array}{llll}0.19334 & 0.67628 & 1.26590\end{array}$ $\begin{array}{llll}0.19163 & 0.67179 & 1.26201\end{array}$ $\begin{array}{llll}0.18994 & 0.66733 & 1.25811\end{array}$ $\begin{array}{llll}0.18826 & 0.66289 & 1.25422\end{array}$

$\begin{array}{llll}0.18660 & 0.65849 & 1.25033\end{array}$ $0.18495 \quad 0.65412 \quad 1.24644$ $\begin{array}{llll}0.18331 & 0.64978 & 1.24255\end{array}$ $0.18170 \quad 0.64546 \quad 1.23866$ $\begin{array}{lll}0.18009 & 0.64117 & 1.23477\end{array}$

$\begin{array}{llll}0.17851 & 0.63692 & 1.23088\end{array}$ $0.17693 \quad 0.63269 \quad 1.22699$ $0.17537 \quad 0.62848 \quad 1.22311$ $\begin{array}{llll}0.17383 & 0.62431 & 1.21923\end{array}$ $0.17230 \quad 0.62016 \quad 1.21534$

$0.17078 \quad 0.61604 \quad 1.21146$ $0.16928 \quad 0.61195 \quad 1.20758$ $\begin{array}{llll}0.16779 & 0.60788 & 1.20370\end{array}$ $0.166320 .60384 \quad 1.19983$ $\begin{array}{lll}0.16485 & 0.59983 & 1.19595\end{array}$

$0.163410 .59584 \quad 1.19208$ $0.16197 \quad 0.59188 \quad 1.18821$ $0.160550 .58795 \quad 1.18434$ $0.159140 .58404 \quad 1.18048$ $0.157750 .58015 \quad 1.17661$

$0.15636 \quad 0.57630 \quad 1.17275$ $0.154990 .57246 \quad 1.16889$ $0.153630 .56866 \quad 1.16503$ $0.152290 .56487 \quad 1.16118$ 0.150950 .561121 .15733
$0.26568 \quad 0.85646 \quad 1.40342$ $0.263420 .85108 \quad 1.39979$ $0.261180 .84573 \quad 1.39615$ $0.25896 \quad 0.84042 \quad 1.39251$ $0.25676 \quad 0.83514 \quad 1.38887$

$\begin{array}{llll}0.25458 & 0.82990 & 1.38523\end{array}$ $0.252420 .82469 \quad 1.38158$ $0.250270 .81951 \quad 1.37793$ $0.248150 .81437 \quad 1.37428$ $0.24605 \quad 0.80925 \quad 1.37063$

$0.24396 \quad 0.80417 \quad 1.36698$ $0.24190 \quad 0.799131 .36332$ $0.239850 .79411 \quad 1.35966$ 0.237820 .789131 .35600 $0.235810 .78418 \quad 1.35234$

$\begin{array}{llll}0.23382 & 0.77926 & 1.34868\end{array}$ $0.231850 .77437 \quad 1.34501$ $0.229890 .76951 \quad 1.34134$ $0.227950 .76469 \quad 1.33768$ 0.226030 .759891 .33401

0.224120 .755121 .33034 $0.222240 .75039 \quad 1.32667$ $0.220360 .74568 \quad 1.32299$ $0.218510 .74100 \quad 1.31932$ $0.21667 \quad 0.73636 \quad 1.31565$

$0.214850 .73174 \quad 1.31197$ 0.213040 .727151 .30830 $0.211250 .72259 \quad 1.30462$ $0.20948 \quad 0.71806 \quad 1.30094$ $0.207720 .71355 \quad 1.29727$

$0.20598 \quad 0.70908 \quad 1.29359$ 0.204250 .704631 .28991 $0.20254 \quad 0.70021 \quad 1.28624$ 0.200840 .695821 .28256 $0.19916 \quad 0.69146 \quad 1.27888$

$0.19750 \quad 0.687121 .27520$ $0.195840 .68281 \quad 1.27152$ $0.19420 \quad 0.67853 \quad 1.26785$ $\begin{array}{llll}0.19258 & 0.67428 & 1.26417\end{array}$ 0.190970 .670051 .26049

$\begin{array}{llll}0.18938 & 0.66585 & 1.25682\end{array}$ $0.18780 \quad 0.66167 \quad 1.25314$ 0.186230 .657521 .24946 $0.18467 \quad 0.653391 .24579$ 0.183130 .649301 .24211

$0.18161 \quad 0.64522 \quad 1.23844$ $0.18009 \quad 0.64117 \quad 1.23477$ 0.178590 .637151 .23110 $0.177110 .63315 \quad 1.22743$ $0.175630 .62918 \quad 1.22376$

$$
T=1900 \text {. }
$$

$0.298820 .93369 \quad 1.45282$ $0.29638 \quad 0.928121 .44942$ $0.29397 \quad 0.92258 \quad 1.44602$ $0.291590 .91708 \quad 1.44261$ 0.289220 .911611 .43921

0.286870 .906171 .43579 $0.284540 .90077 \quad 1.43238$ $0.282240 .89540 \quad 1.42896$ $0.27995 \quad 0.89007 \quad 1.42554$ $0.27768 \quad 0.88477 \quad 1.42211$

$0.27544 \quad 0.87950 \quad 1.41869$ $0.273210 .87426 \quad 1.41526$ $0.27100 \quad 0.86905 \quad 1.41182$ $\begin{array}{llll}0.26881 & 0.86388 & 1.40839\end{array}$ $0.26664 \quad 0.85873 \quad 1.40495$

0.264490 .853621 .40151 $0.26236 \quad 0.848541 .39806$ $0.260240 .84349 \quad 1.39462$ $0.258150 .83847 \quad 1.39117$ $0.25607 \quad 0.83348 \quad 1.38772$

0.254010 .828521 .38427 $0.25196 \quad 0.823591 .38081$ $0.24994 \quad 0.81870 \quad 1.37736$ 0.247930 .813831 .37390 $\begin{array}{llll}0.24594 & 0.80899 & 1.37044\end{array}$

$\begin{array}{llll}0.24396 & 0.80417 & 1.36698\end{array}$ 0.242010 .799391 .36351 $0.24007 \quad 0.79464 \quad 1.36005$ $\begin{array}{llll}0.23814 & 0.78991 & 1.35658\end{array}$ 0.236240 .785221 .35311

$0.23434 \quad 0.78055 \quad 1.34964$ 0.232470 .775911 .34617 $0.230610 .77130 \quad 1.34270$ 0.228760 .766711 .33922 $0.22694 \quad 0.76216 \quad 1.33575$

0.225120 .757631 .33227 0.223330 .753121 .32879 0.221540 .748651 .32531 $0.21978 \quad 0.74420 \quad 1.32183$ $0.218020 .73978 \quad 1.31835$

$0.216290 .73538 \cdot 1.31487$ 0.214560 .731011 .31139 $0.21286 \quad 0.72667 \quad 1.30791$ $\begin{array}{llll}0.21116 & 0.72235 & 1.30443\end{array}$ $0.20948 \quad 0.71806 \quad 1.30094$

$0.207810 .71379 \quad 1.29746$ $0.206160 .70955 \quad 1.29398$ 0.204520 .705331 .29049 $0.20290 \quad 0.70114 \quad 1.28701$ $0.201290 .69698 \quad 1.28353$
2600 2610 2620 2630 2640

2650

2660

2670

2680

2690

2700

2710

2720

2730

2740

2750

2760

2770

2780

2790

2800

2810

2820

2830

2840

2850

2860

2870

2880

2890

2900

2910

2920

2930

2940

2950

2960

2970

2980

2990

3000

3010

3020

3030

3040

3050

3060

3070

3080

3090 
Table II Harmonic Oscillator Contributions to the Thermodynamic

Functions (in units of calories, moles, and ${ }^{\circ} \mathrm{K}$ )
$v_{\mathrm{cm}^{-1}}-\left(\mathrm{F}^{\circ}-\mathrm{E}_{\mathrm{O}}^{\circ}\right) / \mathrm{T} \quad$ So
$\mathrm{C}_{\mathrm{p}}^{\circ} \quad-\left(\mathrm{F}^{\circ}-\mathrm{E} \mathrm{O}\right) / \mathrm{T}$
so
$\mathrm{C}_{\mathrm{p}}^{\circ}$
$-\left(F^{\circ}-E_{0}^{\circ}\right) / T$
So
$\mathrm{cm}^{-1}$

$\mathrm{T}=1700$.

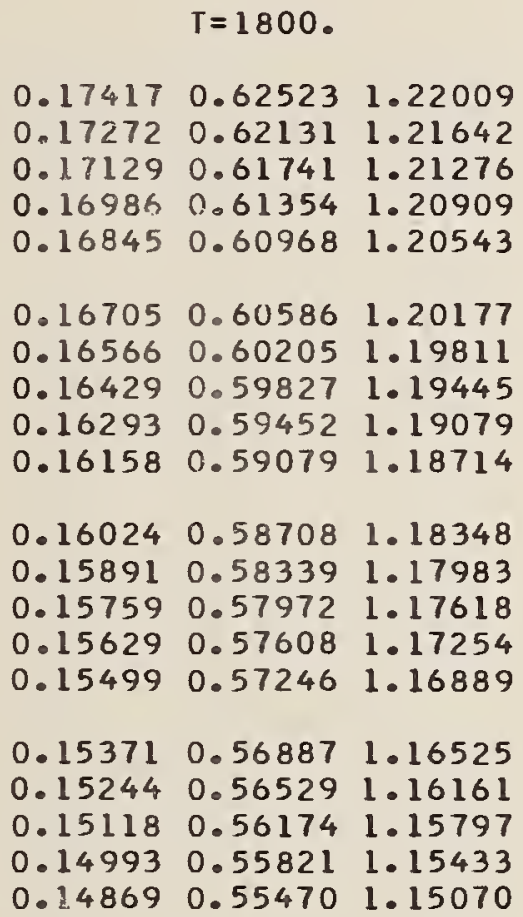

$T=1900$.

3100

$0.14963 \quad 0.55738 \quad 1.15348$

$\begin{array}{llll}3110 & 0.14832 & 0.55367 & 1.14963\end{array}$

3120

3130

3140

3150

3160

3170

3180

3190

3200

3210

3220

3230

3240

3250

3260

3270

3280

3290

3300

3310

3320

3330

3340

3350

3360

3370

3380

3390

3400

3410

3420

3430

3440

3450

3460

3470

3480

3490

3500

3510

3520

3530

3540

3550

3560

3570

3580

3590

$0.14703 \quad 0.54999 \quad 1.14579$

$0.14574 \quad 0.54633 \quad 1.14195$

0.144470 .542691 .13811

$0.14320 \quad 0.53908 \quad 1.13428$

$0.141950 .53549 \quad 1.13045$

$0.14071 \quad 0.53193 \quad 1.12662$

$0.13948 \quad 0.52838 \quad 1.12279$

$0.13827 \quad 0.52486 \quad 1.11897$

$0.13706 \quad 0.52137 \quad 1.11515$

0.135870 .517891 .11134

$0.13468 \quad 0.51444 \quad 1.10753$

0.133510 .511021 .10372

$0.132340 .50761 \quad 1.09992$

$0.131190 .50423 \quad 1.09612$ $0.13005 \quad 0.50086 \quad 1.09232$ $0.12892 \quad 0.49752 \quad 1.08853$ $0.127790 .49421 \quad 1.08474$ $0.12668 \quad 0.49091 \quad 1.08096$

$0.12558 \quad 0.48764 \quad 1.07718$ $0.124490 .48438 \quad 1.07340$ $0.12340 \quad 0.48115 \quad 1.06963$ $0.12233 \quad 0.47794 \quad 1.06586$ $0.121270 .47475 \quad 1.06210$

$0.120210 .47158 \quad 1.05834$ $0.119170 .46843 \quad 1.05459$ $0.11814 \quad 0.46530 \quad 1.05084$ 0.117110 .462191 .04709 $0.116090 .45911 \quad 1.04335$

$0.115090 .45604 \quad 1.03962$ $0.114090 .45299 \quad 1.03589$ $0.113100 .44996 \quad 1.03216$ 0.112120 .446951 .02844 $0.111150 .44396 \quad 1.02473$

$0.11018 \quad 0.44100 \quad 1.02102$ $0.10923 \quad 0.43805 \quad 1.01731$ $0.10828 \quad 0.43512 \quad 1.01361$ $0.10734 \quad 0.43220 \quad 1.00992$ $0.106420 .42931 \quad 1.00623$

$0.105490 .42644 \quad 1.00254$ $0.10458 \quad 0.42358 \quad 0.99886$ $\begin{array}{llll}0.10368 & 0.42075 & 0.99519\end{array}$ $\begin{array}{llll}0.10278 & 0.41793 & 0.99152\end{array}$ $\begin{array}{llll}0.10189 & 0.41513 & 0.98786\end{array}$

0.101010 .412350 .98420 $0.100140 .40958 \quad 0.98055$ $\begin{array}{llll}0.09927 & 0.40684 & 0.97691\end{array}$ $\begin{array}{lllll}0.09841 & 0.40411 & 0.97327\end{array}$ $0.09756 \quad 0.40140 \quad 0.96964$
$0.14746 \quad 0.55122 \quad 1.14707$ $0.14624 \quad 0.54775 \quad 1.14344$ $0.14503 \quad 0.54431 \quad 1.13982$ $0.143830 .54088 \quad 1.13619$ $\begin{array}{lll}0.14265 & 0.53748 & 1.13257\end{array}$

$0.14147 \quad 0.534101 .12896$ $0.14030 \quad 0.53074 \quad 1.12534$ $0.139150 .52740 \quad 1.12173$ $0.13800 \quad 0.524091 .11812$ $0.13686 \quad 0.52079 \quad 1.11452$

$0.13573 \quad 0.51751 \quad 1.11092$ 0.134620 .514251 .10732 $0.13351 \quad 0.51102 \quad 1.10372$ $0.13241 \quad 0.50780 \quad 1.10013$ $\begin{array}{lll}0.13132 & 0.50460 \quad 1.09654\end{array}$

$0.13024 \quad 0.50142 \quad 1.09295$ $0.12917 \quad 0.49826 \quad 1.08937$ $0.12810 \quad 0.495131 .08579$ $\begin{array}{llll}0.12705 & 0.49201 & 1.08222\end{array}$ $0.12601 \quad 0.48891 \quad 1.07865$

$\begin{array}{llll}0.12497 & 0.48583 & 1.07508\end{array}$ $0.12394 \quad 0.48276 \quad 1.07151$ $0.12293 \quad 0.47972 \quad 1.06795$ $0.121920 .47670 \quad 1.06440$ $0.12092 \quad 0.47369 \quad 1.06085$

$0.119920 .47070 \quad 1.05730$ $0.11894 \quad 0.46773 \quad 1.05375$ $\begin{array}{llll}0.11796 & 0.46478 & 1.05021\end{array}$ $0.11700 \quad 0.46185 \quad 1.04668$ $0.11604 \quad 0.45893 \quad 1.04315$ $\begin{array}{llll}0.19969 & 0.69283 & 1.28004\end{array}$ $0.198110 .68872 \quad 1.27656$ $\begin{array}{llll}0.19654 & 0.68463 & 1.27307\end{array}$ $0.19498 \quad 0.68056 \quad 1.26959$ $0.19343 \quad 0.67651 \quad 1.26610$

$0.19190 \quad 0.67249 \quad 1.26262$ $0.19038 \quad 0.66850 \quad 1.25914$ $0.18888 \quad 0.66452 \quad 1.25565$ $\begin{array}{llll}0.18738 & 0.66057 & 1.25217\end{array}$ $\begin{array}{llll}0.18590 & 0.65665 & 1.24869\end{array}$

$0.18443 \quad 0.65275 \quad 1.24521$ $\begin{array}{llll}0.18297 & 0.64887 & 1.24173\end{array}$ $0.18153 \quad 0.64501 \quad 1.23825$ $\begin{array}{llll}0.18009 & 0.64117 & 1.23477\end{array}$ $0.178670 .63736 \quad 1.23129$

$\begin{array}{llll}0.17726 & 0.63357 & 1.22781\end{array}$ $\begin{array}{llll}0.17586 & 0.62981 & 1.22434\end{array}$ $0.17448 \quad 0.62606 \quad 1.22086$ $0.17310 \quad 0.62234 \quad 1.21739$ $0.17174 \quad 0.61864 \quad 1.21391$

$0.17039 \quad 0.61496 \quad 1.21044$ $0.16904 \quad 0.61130 \quad 1.20697$ $0.167710 .60767 \quad 1.20350$ $0.166390 .60405 \quad 1.20003$ $0.16508 \quad 0.60046 \quad 1.19657$

$0.163790 .59689 \quad 1.19310$ $0.16250 \quad 0.59334 \quad 1.18964$ $0.161220 .58981 \quad 1.18618$ $\begin{array}{llll}0.15996 & 0.58630 & 1.18271\end{array}$ $\begin{array}{llll}0.15870 & 0.58281 & 1.17926\end{array}$

$0.15745 \quad 0.57934 \quad 1.17580$ $0.156220 .57589 \quad 1.17234$ $\begin{array}{llll}0.15499 & 0.57246 & 1.16889\end{array}$ $0.15378 \quad 0.56906 \quad 1.16544$ $0.152570 .56567 \quad 1.16199$

$\begin{array}{llll}0.15137 & 0.56230 & 1.15854\end{array}$ $0.150190 .55895 \quad 1.15510$ 0.149010 .555621 .15166 $\begin{array}{llll}0.14784 & 0.55231 & 1.14822\end{array}$ $0.146690 .54902 \quad 1.14478$

$0.14554 \quad 0.54575 \quad 1.14134$ $0.14440 \quad 0.54250 \quad 1.13791$ $\begin{array}{llll}0.14327 & 0.53927 & 1.13448\end{array}$ $0.142150 .53606 \quad 1.13105$ $0.14104 \quad 0.53286 \quad 1.12762$

$\begin{array}{llll}0.13994 & 0.52969 & 1.12420\end{array}$ $\begin{array}{llll}0.13884 & 0.52653 & 1.12078\end{array}$ $\begin{array}{llll}0.13776 & 0.52339 & 1.11736\end{array}$ $0.136680 .52027 \quad 1.11395$ $0.135620 .51717 \quad 1.11054$
3100

3110

3120

3130

3140

3150

3160

3170

3180

3190

3200

3210

3220

3230

3240

3250

3260

3270

3280

3290

3300

3310

3320

3330

3340

3350

3360

3370

3380

3390

3400

3410

3420

3430

3440

3450

3460

3470

3480

3490

3500

3510

3520

3530

3540

3550

3560 3570

3580

3590 
Table II Harmonic Oscillator Contributions to the Thermodynamic

Functions (in units of calories, moles, and ${ }^{\circ} \mathrm{K}$ )

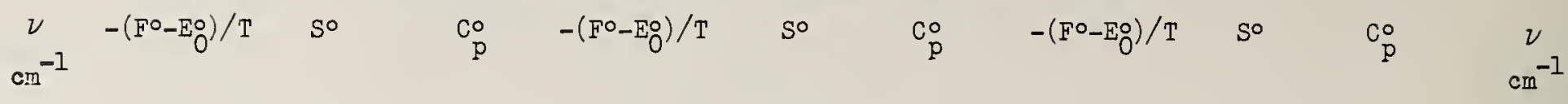

$T=1700$.

3600

3610

3620

3630

3640

3650

3660

3670

3680

3690

3700

3710

3720

3730

3740

3750

3760

3770

3780

3790

3800

3810

3820

3830

3840

3850

3860

3870

3880

3890

3900

3910

3920

3930

3940

3950

3.960

3970

3980

3990

4000 $\begin{array}{llll}0.09672 & 0.39871 & 0.96601\end{array}$ $0.095890 .39603 \quad 0.96239$ $0.09506 \quad 0.39338 \quad 0.95877$ $0.09424 \quad 0.39074 \quad 0.95516$ 0.093420 .388110 .95156

$\begin{array}{llll}0.09262 & 0.38551 & 0.94796\end{array}$ $\begin{array}{llll}0.09182 & 0.38292 & 0.94437\end{array}$ $\begin{array}{llll}0.09103 & 0.38035 & 0.94078\end{array}$ $\begin{array}{llll}0.09024 & 0.37779 & 0.93720\end{array}$ $\begin{array}{llll}0.08947 & 0.37525 & 0.93363\end{array}$

$\begin{array}{llll}0.08869 & 0.37273 & 0.93007\end{array}$ $\begin{array}{llll}0.08793 & 0.37023 & 0.92651\end{array}$ $\begin{array}{llll}0.08717 & 0.36774 & 0.92295\end{array}$ $0.086420 .36526 \quad 0.91941$ $\begin{array}{llll}0.08568 & 0.36281 & 0.91587\end{array}$

$\begin{array}{llll}0.08494 & 0.36037 & 0.91233\end{array}$ $\begin{array}{llll}0.08421 & 0.35794 & 0.90881\end{array}$ $\begin{array}{llll}0.08348 & 0.35553 & 0.90529\end{array}$ $\begin{array}{llll}0.08276 & 0.35314 & 0.90177\end{array}$ $\begin{array}{llll}0.08205 & 0.35076 & 0.89827\end{array}$

$\begin{array}{llll}0.08135 & 0.34840 & 0.89477\end{array}$ $0.080650 .34605 \quad 0.89127$ $\begin{array}{llll}0.07995 & 0.34372 & 0.88779\end{array}$ $\begin{array}{llll}0.07927 & 0.34140 & 0.88431\end{array}$ $0.07858 \quad 0.33910 \quad 0.88084$

$\begin{array}{llll}0.07791 & 0.33682 & 0.87737\end{array}$ $0.07724 \quad 0.33454 \quad 0.87391$ $\begin{array}{llll}0.07658 & 0.33229 & 0.87046\end{array}$ 0.075920 .330050 .86702 $\begin{array}{llll}0.07527 & 0.32782 & 0.86358\end{array}$

0.074620 .325610 .86015 $\begin{array}{llll}0.07398 & 0.32341 & 0.85673\end{array}$ 0.073340 .321220 .85331 $0.072710 .31905 \quad 0.84991$ $0.072090 .31690 \quad 0.84650$

$0.071470 .31476 \quad 0.84311$ $\begin{array}{llll}0.07086 & 0.31263 & 0.83973\end{array}$ $\begin{array}{llll}0.07025 & 0.31052 & 0.83635\end{array}$ $\begin{array}{llll}0.06965 & 0.30842 & 0.83298\end{array}$ $\begin{array}{llll}0.06905 & 0.30633 & 0.82961\end{array}$

$0.06846 \quad 0.30426 \quad 0.82626$
$T=1800$

$0.11509 \quad 0.45604 \quad 1.03962$ 0.114140 .453161 .03610 $0.113210 .45030 \quad 1.03258$ $0.112280 .44745 \quad 1.02906$ $0.11136 \quad 0.444631 .02555$

$0.11045 \quad 0.44182 \quad 1.02205$ $0.109550 .43903 \quad 1.01855$ $0.108650 .43625 \quad 1.01505$ 0.107760 .433501 .01156 $0.106880 .43076 \quad 1.00807$

$0.106010 .42803 \quad 1.00459$ $0.10514 \quad 0.425321 .00111$ $0.10428 \quad 0.422630 .99764$ $\begin{array}{llll}0.10343 & 0.41996 & 0.99417\end{array}$ $0.102580 .41730 \quad 0.99071$

$0.10174 \quad 0.41466 \quad 0.98725$ $0.10091 \quad 0.41204 \quad 0.98380$ $0.10009 \quad 0.409430 .98035$ $0.09927 \quad 0.406840 .97691$ $0.09846 \quad 0.40426 \quad 0.97347$

$\begin{array}{llll}0.09766 & 0.40170 & 0.97004\end{array}$ $0.096860 .39916 \quad 0.96661$ 0.096070 .396630 .96319 $\begin{array}{llll}0.09529 & 0.39411 & 0.95977\end{array}$ 0.094510 .391610 .95636

0.093740 .389130 .95296 $0.09298 \quad 0.38666 \quad 0.94956$ 0.092220 .384210 .94616 0.091470 .381770 .94277 0.090720 .379350 .93939

$\begin{array}{llll}0.08998 & 0.37694 & 0.93601\end{array}$ 0.089250 .374550 .93264 $\begin{array}{llll}0.08852 & 0.37217 & 0.92927\end{array}$ $\begin{array}{llll}0.08780 & 0.36981 & 0.92591\end{array}$ $\begin{array}{llll}0.08709 & 0.36746 & 0.92256\end{array}$

$0.08638 \quad 0.365130 .91921$ $\begin{array}{llll}0.08568 & 0.36281 & 0.91587\end{array}$ $\begin{array}{llll}0.08498 & 0.36050 & 0.91253\end{array}$ 0.084290 .358210 .90920 $\begin{array}{llll}0.08360 & 0.35593 & 0.90587\end{array}$

$\begin{array}{llll}0.08292 & 0.35367 & 0.90255\end{array}$
$T=1900$

$0.13456 \quad 0.51408 \quad 1.10713$ 0.133510 .511021 .10372 $0.132470 .50797 \quad 1.10032$ 0.131430 .504941 .09692 0.130410 .501921 .09352

0.129390 .498931 .09013 $0.12838 \quad 0.49595 \quad 1.08673$ $0.12738 \quad 0.49299 \quad 1.08335$ 0.126390 .490051 .07996 0.125410 .487121 .07658

$0.12443 \quad 0.48421 \quad 1.07320$ $0.12346 \quad 0.48132 \quad 1.06983$ $0.12250 \quad 0.47844 \quad 1.06646$ $0.12155 \quad 0.47559 \quad 1.06309$ $0.12060 \quad 0.47274 \quad 1.05973$

$0.11966 \quad 0.46992 \quad 1.05637$ $\begin{array}{llll}0.11873 & 0.46711 & 1.05301\end{array}$ 0.117810 .464321 .04966 $0.11690 \quad 0.46154 \quad 1.04631$

$0.11599 \quad 0.45878 \quad 1.04296$

$0.115090 .45604 \quad 1.03962$ 0.114190 .45331 .1 .03628 $0.113310 .45060 \quad 1.03295$ $0.112430 .44790 \quad 1.02962$ 0.111550 .445221 .02629

$0.110690 .44256 \quad 1.02297$ 0.109830 .439911 .01965 $0.10898 \quad 0.43727 \quad 1.01634$

0.108130 .434651 .01303

$0.10730 \quad 0.432051 .00972$

$0.10646 \quad 0.42946 \quad 1.00642$ 0.105640 .426891 .00312

0.104820 .424330 .99983

0.104010 .421790 .99654

$0.10320 \quad 0.41926 \quad 0.99326$

$0.10240 \quad 0.416750 .98998$ 0.101610 .414250 .98671 $0.10083 \quad 0.41176 \quad 0.98344$ $\begin{array}{llll}0.10005 & 0.40929 & 0.98017\end{array}$ $\begin{array}{llll}0.09927 & 0.40684 & 0.97691\end{array}$

$0.09850 \quad 0.40440 \quad 0.97365$
3600

3610

3620

3630

3640

3650

3660

3670

3680

3690

3700

3710

3720

3730

3740

3750

3760

3770

3780

3790

3800

3810

3820

3830

3840

3850

3860

3870

3880

3890

3900

3910

3920

3930

3940

3950

3960

3970

3980

3990

4000 
Table II Harmonic Oscillator Contributions to the Thermodynamic

Functions (in units of calories, moles, and ${ }^{\circ} \mathrm{K}$ )
$\operatorname{cm}^{-1}-\left(F^{\circ}-E_{O}^{\circ}\right) / T \quad$ So
$\mathrm{C}_{\mathrm{p}}^{\circ} \quad-\left(\mathrm{F}^{\circ}-\mathrm{E}_{\mathrm{O}}^{\circ}\right) / \mathrm{T} \quad \mathrm{S}^{\circ}$
$C_{p} \quad-\left(F^{\circ}-E_{0}^{\circ}\right) / T$
So
$v$
-1

$r=2000$.

$\mathrm{T}=2100$

$T=2200$.

100

110

120

130

140

150

160

170

180

190

200

210

220

230

240

250

260

270

280

290

300

310

320

330

340

350

360

370

380

390

400

410

420

430

440

450

460

470

480

490

500

510

520

530

540

550

560

570

580

590 $\begin{array}{llll}5.30113 & 7.21768 & 1.98631\end{array}$

5.118797 .028371 .98613

$4.95293 \quad 6.85556 \quad 1.98594$

$\begin{array}{llll}4.80091 & 6.69661 & 1.98572\end{array}$

$4.66068 \quad 6.54946 \quad 1.98549$

$4.53060 \quad 6.41248 \quad 1.98524$

$\begin{array}{lllll}4.40937 & 6.28437 & 1.98498\end{array}$

$4.295906 .16404 \quad 1.98470$

$4.18932 \quad 6.05060 \quad 1.98440$

$\begin{array}{llll}4.08887 & 5.94332 & 1.98408\end{array}$

$3.993925 .84156 \quad 1.98375$

$3.90394 \quad 5.74478 \quad 1.98339$

$3.81846 \quad 5.65252 \quad 1.98303$

$3.73708 \quad 5.56438 \quad 1.98264$

$3.65945 \quad 5.48001 \quad 1.98224$

$3.58527 \quad 5.39910 \quad 1.98182$

$3.51426 \quad 5.32138 \quad 1.98139$

$3.446195 .24661 \quad 1.98093$

$3.380835 .17458 \quad 1.98046$

$3.31800 \quad 5.10509 \quad 1.97998$

$\begin{array}{llll}3.25753 & 5.03797 & 1.97947\end{array}$

$3.19926 \quad 4.97307 \quad 1.97895$

$3.14304 \quad 4.91025 \quad 1.97842$

$3.08877 \quad 4.84938 \quad 1.97786$

$3.03630 \quad 4.79034 \quad 1.97729$

$2.985554 .73304 \quad 1.97670$

2.936424 .677361 .97610

$2.88881 \quad 4.62322 \quad 1.97548$

$2.842644 .57055 \quad 1.97484$

$2.79784 \quad 4.51926 \quad 1.97419$

$2.75434 \quad 4.46929 \quad 1.97351$

$2.71207 \quad 4.42056 \quad 1.97283$

2.670984 .373031 .97212

$2.63100 \quad 4.32664 \quad 1.97140$

2.592094 .281321 .97066

$2.55420 \quad 4.23705 \quad 1.96991$

$2.51729 \quad 4.19376 \quad 1.96913$

$2.48130 \quad 4.151421 .96835$

2.446214 .109991 .96754

2.411964 .069421 .96672

$2.37854 \quad 4.02970 \quad 1.96588$ $2.34591 \quad 3.99078 \quad 1.96503$ $2.31403 \quad 3.952631 .96416$ $2.28287 \quad 3.91522 \quad 1.96327$ $2.25242 \quad 3.87854 \quad 1.96237$

$2.22264 \quad 3.84254 \quad 1.96145$ $2.19351 \quad 3.80720 \quad 1.96051$ $2.16500 \quad 3.77251 \quad 1.95956$ $2.137103 .73844 \quad 1.95859$ $2.109773 .70497 \quad 1.95760$
$5.39472 \quad 7.31459 \quad 1.98639$

$5.212057 .12528 \quad 1.98623$

$5.04586 \quad 6.95246 \quad 1.98605$

$4.893516 .79350 \quad 1.98586$

$4.75295 \quad 6.646341 .98565$

$4.62254 \quad 6.50935 \quad 1.98542$

4. $50098 \quad 6.38122 \quad 1.98518$

$4.387196 .26088 \quad 1.98492$

4.280286 .147431 .98465

$4.17950 \quad 6.04013 \quad 1.98437$

$4.08423 \quad 5.93836 \quad 1.98406$

$3.99392 \quad 5.84156 \quad 1.98375$

$3.90812 \quad 5.74928 \quad 1.98341$

3.826425 .661131 .98306

$3.74847 \quad 5.57673 \quad 1.98270$

$3.67397 \quad 5.49580 \quad 1.98232$

$3.602645 .41806 \quad 1.98192$

$3.534245 .34327 \quad 1.98151$

3.468575 .271221 .98109

3.405425 .201711 .98065

3.344635 .134571 .98019

$3.28604 \quad 5.06964 \quad 1.97972$

$3.22951 \quad 5.00680 \quad 1.97923$

$3.17492 \quad 4.94590 \quad 1.97873$

$3.122154 .88684 \quad 1.97821$

$3.07108 \quad 4.82950 \quad 1.97767$

3.021634 .773801 .97713

2.973714 .719631 .97656

2.927234 .666931 .97598

$2.882124 .61561 \quad 1.97539$

$2.838314 .56561 \quad 1.97478$

$2.79574 \quad 4.516851 .97415$

$2.75434 \quad 4.469291 .97351$

$2.71406 \quad 4.42286 \quad 1.97286$

$2.67484 \quad 4.37751 \quad 1.97219$

$2.636654 .33320 \quad 1.97150$

$2.59943 \quad 4.28987 \quad 1.97080$

$2.56314 \quad 4.247501 .97009$

$2.52774 \quad 4.206031 .96936$

2.493194 .165431 .96861

$2.45947 \quad 4.12566 \quad 1.96785$

$2.42654 \quad 4.08670 \quad 1.96707$

$2.39436 \quad 4.04851 \quad 1.96628$

$2.362914 .01107 \quad 1.96548$

$2.33215 \quad 3.97434 \quad 1.96466$

$2.30208 \quad 3.93829 \quad 1.96382$

2.272653 .902921 .96297

$\begin{array}{llll}2.24384 & 3.86818 & 1.96211\end{array}$

$2.215653 .83406 \quad 1.96123$

$2.18803 \quad 3.800551 .96033$
$5.484107 .40700 \quad 1.98646$

$5.301137 .21768 \quad 1.98631$

$5.13464 \quad 7.04485 \quad 1.98615$

$4.98199 \quad 6.88588 \quad 1.98597$

$4.841136 .73871 \quad 1.98578$

$4.710426 .60171 \quad 1.98558$

$4.58856 \quad 6.47357 \quad 1.98536$

$4.47447 \quad 6.35322 \quad 1.98512$

$4.36726 \quad 6.23976 \quad 1.98488$

4.266196 .132451 .98461

$4.170626 .03066 \quad 1.98434$

4.080025 .933851 .98405

$3.99392 \quad 5.84156 \quad 1.98375$

3.911935 .753391 .98343

$3.83368 \quad 5.66898 \quad 1.98310$

$3.758895 .58803 \quad 1.98275$

$\begin{array}{llll}3.68727 & 5.51027 & 1.98239\end{array}$

$3.61858 \quad 5.43547 \quad 1.98201$

3.552625 .363391 .98163

$3.48918 \quad 5.29386 \quad 1.98122$

$3.428105 .22670 \quad 1.98081$

$3.36922 \quad 5.16176 \quad 1.98038$

3.312415 .098891 .97993

$3.257535 .03797 \quad 1.97947$

$3.20447 \quad 4.97888 \quad 1.97900$

3.153124 .921531 .97852

$3.10338 \quad 4.86580 \quad 1.97802$

$3.05518 \quad 4.811611 .97750$

$3.00842 \quad 4.75888 \quad 1.97697$

$2.96302 \quad 4.707531 .97643$

$2.91893 \quad 4.65750 \quad 1.97588$

$2.87607 \quad 4.60872 \quad 1.97531$

2.834394 .561121 .97472

$2.793834 .51466 \quad 1.97413$

$2.75434 \quad 4.469291 .97351$

$2.71586 \quad 4.42494 \quad 1.97289$

$2.67836 \quad 4.381591 .97225$

$2.64180 \quad 4.33918 \quad 1.97160$

$2.606124 .29768 \quad 1.97093$

$2.57130 \quad 4.257051 .97025$

$2.537314 .21725 \quad 1.96956$

2.504104 .178251 .96885

2.471644 .140031 .96813

$2.43992 \quad 4.102551 .96739$

2.408894 .065781 .96664

$2.37854 \quad 4.02970 \quad 1.96588$

2.34884 3.99428 1.96511

$2.31977 \quad 3.95951 \quad 1.96432$

$2.29130 \quad 3.925351 .96351$

$2.263423 .89180 \quad 1.96270$
100

110

120

130

140

150

160

170

180

190

200

210

220

230

240

250

260

270

280

290

300

310

320

330

340

350

360

370

380

390

400

410

420

430

440

450

460

470

480

490

500

510

520

530

540

550

560

570

580

590 
Table II Harmonic Oscillator Contributions to the Thermodynamic

Functions (in units of calories, moles, and ${ }^{\circ} \mathrm{K}$ )

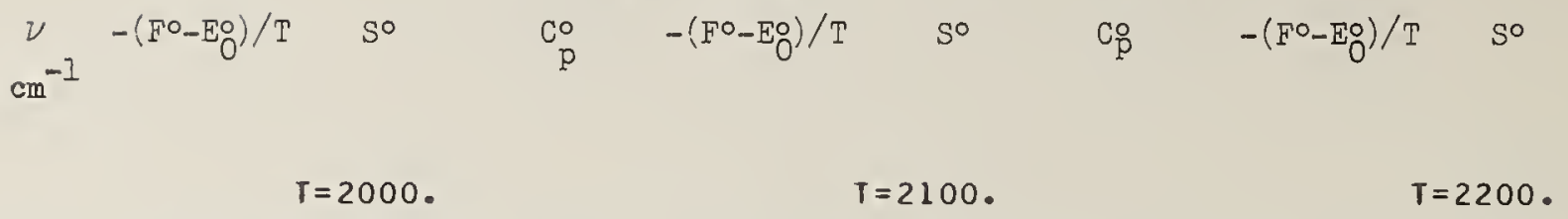

$\begin{array}{lllll}600 & 2.08302 & 3.67207 & 1.95660\end{array}$

$610 \quad 2.05680 \quad 3.63974 \quad 1.95558$

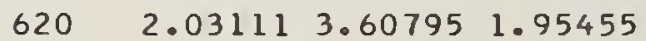

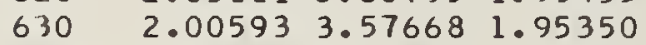

$\begin{array}{lll}640 \quad 1.98124 & 3.54593 & 1.95243\end{array}$

$\begin{array}{lllll}650 & 1.95703 & 3.51567 & 1.95135\end{array}$

$\begin{array}{lllll}660 & 1.93328 & 3.48588 & 1.95025\end{array}$

$\begin{array}{llll}670 & 1.90997 & 3.45656 & 1.94914\end{array}$

$\begin{array}{lllll}680 & 1.88711 & 3.42769 & 1.94801\end{array}$

$690 \quad 1.86466 \quad 3.39926 \quad 1.94686$

$700 \quad 1.84262 \quad 3.37126 \quad 1.94570$

$710 \quad 1.82098 \quad 3.34367 \cdot 1.94452$

$\begin{array}{lllll}720 & 1.79972 & 3.31648 & 1.94333\end{array}$

$\begin{array}{lllll}730 & 1.77884 & 3.28968 & 1.94212\end{array}$

$\begin{array}{lll}740 & 1.75833 & 3.26327 \quad 1.94090\end{array}$

$\begin{array}{lllll}750 & 1.73817 & 3.23722 & 1.93966\end{array}$

$\begin{array}{llll}760 & 1.71835 & 3.21154 & 1.93840\end{array}$

$\begin{array}{lllll}770 & 1.69887 & 3.18621 & 1.93713\end{array}$

$\begin{array}{llll}780 & 1.67971 & 3.16122 & 1.93584\end{array}$

$\begin{array}{llll}790 \quad 1.66088 & 3.13657 & 1.93454\end{array}$

$800 \quad 1.64235 \quad 3.11224 \quad 1.93322$

$810 \quad 1.62413 \quad 3.088241 .93188$

$820 \quad 1.60620 \quad 3.06454 \quad 1.93053$

$830 \quad 1.588563 .041151 .92917$

$840 \quad 1.57119 \quad 3.01805 \quad 1.92779$

$\begin{array}{lllll}850 & 1.55411 & 2.99525 & 1.92639\end{array}$ $8601.53728 \quad 2.97272 \quad 1.92498$ $870 \quad 1.520722 .95048 \quad 1.92355$ $880 \quad 1.504412 .92850 \quad 1.92211$ $890 \quad 1.48835 \quad 2.90679 \quad 1.92065$

$900 \quad 1.47254 \quad 2.88534 \quad 1.91918$ $910 \quad 1.456962 .86414 \quad 1.91769$ $920 \quad 1.441612 .84319 \quad 1.91619$ $9301.426492 .82248 \quad 1.91468$ $940 \quad 1.411582 .802011 .91314$

$950 \quad 1.39690 \quad 2.78178 \quad 1.91160$ $9601.382432 .76177 \quad 1.91003$ $970 \quad 1.36816 \quad 2.74198 \quad 1.90846$ $980 \quad 1.35410 \quad 2.722421 .90687$ $9901.340242 .70306 \quad 1.90526$

$1000 \quad 1.32657 \quad 2.68392 \quad 1.90364$ $1010 \quad 1.313092 .664991 .90200$ $1020 \quad 1.29980 \quad 2.64626 \quad 1.90035$ $1030 \quad 1.286692 .62773 \quad 1.89869$ $1040 \quad 1.27375 \quad 2.60939 \quad 1.89701$

$1050 \quad 1.26100 \quad 2.59125 \quad 1.89532$ $1060 \quad 1.24842 \quad 2.57329 \quad 1.89361$ $1070 \quad 1.236002 .55552 \quad 1.89189$ $\begin{array}{llll}1080 & 1.22375 & 2.53792 & 1.89015\end{array}$ $1090 \quad 1.21166 \quad 2.52051 \quad 1.88840$
$2.16098 \quad 3.76761 \quad 1.95942$ 2.134473 .735221 .95850 2.108493 .703391 .95756 $2.08302 \quad 3.67207 \quad 1.95660$ $2.058043 .64127 \quad 1.95563$

$2.03353 \quad 3.61095 \quad 1.95465$ $2.00950 \quad 3.581121 .95365$ 1.985913 .551751 .95264 1.962753 .522831 .95161 1.940023 .494341 .95057

$1.917693 .46628 \quad 1.94951$ $1.895773 .43864 \quad 1.94844$ $1.87423 \quad 3.411391 .94736$ $1.85306 \quad 3.38454 \quad 1.94626$ 1.832273 .358071 .94514

$1.81182 \quad 3.33197 \quad 1.94402$ $1.79173 \quad 3.306231 .94287$ $1.771973 .28084 \quad 1.94172$ $1.75253 \quad 3.25579 \quad 1.94054$ $1.73342 \quad 3.23108 \quad 1.93936$

$1.71461 \quad 3.206691 .93816$ 1.696113 .182621 .93694 $1.67791 \quad 3.15886 \quad 1.93572$ 1.659993 .135401 .93447 $1.64235 \quad 3.11224 \quad 1.93322$

$1.62499 \quad 3.08937 \quad 1.93195$ $1.607893 .06678 \quad 1.93066$ $1.59106 \quad 3.044471 .92936$ 1.574483 .022431 .92805 $1.55815 \quad 3.00065 \quad 1.92672$

$1.54206 \quad 2.97913 \quad 1.92538$ 1.526212 .957861 .92403 $1.51060 \quad 2.93684 \quad 1.92266$ $1.495212 .91606 \quad 1.92128$ $1.480042 .89552 \quad 1.91988$

$1.465092 .87521 \quad 1.91848$ 1.450352 .855131 .91705 $1.435822 .83527 \quad 1.91562$ 1.421492 .815631 .91417 1.407372 .796211 .91270

$1.39344 \quad 2.77699 \quad 1.91123$ $1.379692 .75798 \quad 1.90974$ $1.366142 .73917 \quad 1.90823$ 1.352772 .720561 .90671 $\begin{array}{llll}1.33958 & 2.70215 & 1.90518\end{array}$

$1.326572 .68392 \quad 1.90364$ $1.313732 .66589 \quad 1.90208$ 1.301052 .648041 .90051 1.288552 .630361 .89893 $1.27620 \quad 2.61287 \quad 1.89733$ $\begin{array}{llll}2.23609 & 3.85882 & 1.96187\end{array}$

$2.20932 \quad 3.826391 .96102$

$2.18307 \quad 3.79451 \quad 1.96017$

$2.15733 \quad 3.763161 .95930$

$2.132093 .73231 \quad 1.95841$

$2.10732 \quad 3.70195 \quad 1.95751$

$2.083023 .67207 \quad 1.95660$

$2.05916 \quad 3.642661 .95568$

$2.03574 \quad 3.61369 \quad 1.95474$

$2.01275 \quad 3.58516 \quad 1.95379$

$1.99016 \quad 3.55705 \quad 1.95282$

$1.967973 .52936 \quad 1.95185$

$1.94618 \quad 3.50207 \quad 1.95086$

$1.92475 \quad 3.47517 \quad 1.94985$

1.903693 .448641 .94883

$1.88299 \quad 3.42249 \quad 1.94780$

$1.862643 .39670 \quad 1.94676$

$1.842623 .37126 \quad 1.94570$

1.822933 .346161 .94463

$1.80356 \quad 3.32139 \quad 1.94355$

$1.78450 \quad 3.29695 \quad 1.94245$

$1.76575 \quad 3.27283 \quad 1.94134$

$1.74729 \quad 3.24902 \quad 1.94022$

1.729123 .225501 .93909

1.711233 .202291 .93794

$1.693613 .17936 \quad 1.93678$

1.676273 .156721 .93560

1.659183 .134341 .93442

$1.64235 \quad 3.11224 \quad 1.93322$

1.625773 .090411 .93200

$1.609443 .06883 \quad 1.93078$

$1.593343 .04750 \quad 1.92954$

1.577483 .026421 .92829

$1.561843 .00558 \quad 1.92703$

$1.546432 .98497 \quad 1.92575$

$1.531232 .96460 \quad 1.92446$ 1.516252 .944461 .92316 $1.50148 \quad 2.924531 .92185$ 1.486912 .904831 .92052 $1.472542 .88534 \quad 1.91918$

$1.45836 \quad 2.86606 \quad 1.91783$ $1.444382 .84698 \quad 1.91647$ 1.430592 .828111 .91509 1.416982 .809431 .91370 1.403552 .790951 .91230

$1.39030 \quad 2.77265 \quad 1.91089$ $1.37722 \quad 2.75455 \quad 1.90946$ 1.364312 .736621 .90803 $1.35156 \quad 2.71888 \quad 1.90658$ 1.338992 .701321 .90511
600

610

620

630

640

650

660

670

680

690

700

710

720

730

740

750

760

770

780

790

800

810

820

830

840

850

860

870

880

890

900

910

920

930

940

950

960

970

980

990

1000

1010

1020

1030

1040

1050

1060

1070

1080

1090 
Table II Harmonic Oscillator Contributions to the Thermodynamic

Functions (in units of calories, moles, and ${ }^{\circ} \mathrm{K}$ )
$v_{-1}^{-1}-\left(F^{\circ}-E O\right) / T$ So
$\mathrm{Co}_{\mathrm{p}}^{\mathrm{O}} \quad-\left(\mathrm{F}^{\circ}-\mathrm{E}_{\mathrm{O}} \mathrm{O}\right) / \mathrm{T} \quad \mathrm{S}^{\circ}$
$\mathrm{C}_{\mathrm{p}} \quad-\left(\mathrm{F}^{\circ}-\mathrm{E}_{\mathrm{O}} \mathrm{O}\right) / \mathrm{T}$
So
$T=2000$.
$T=2100$.
$T=2200$.

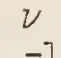

$\mathrm{cm}^{-1}$

1100

1110

1120

1130

1140

1150

1160

1170

1180

1190

1200

1210

1220

1230

1240

1250

1260

1270

1280

1290

1300

1310

1320

1330

1340

1350

1360

1370

1380

1390

1400

1410

1420

1430

1440

1450

1460

1470

1480

1490

1500

1510

1520

1530

1540

1550

1560

1570

1580

1590
$1.19973 \quad 2.50327 \quad 1.88664$

$1.18796 \quad 2.48621 \quad 1.88486$

$1.176342 .46931 \quad 1.88307$

$1.164872 .45258 \quad 1.88126$

$1.153552 .43601 \quad 1.87944$

$1.14237 \quad 2.41961 \quad 1.87760$

$1.13134 \quad 2.40336 \quad 1.87576$

$1.120442 .38727 \quad 1.87389$

$\begin{array}{llll}1.10968 & 2.37133 \quad 1.87202\end{array}$

$1.09905 \quad 2.35554 \quad 1.87013$

$\begin{array}{llll}1.08856 & 2.33989 & 1.86822\end{array}$

$\begin{array}{llll}1.07820 & 2.32440 & 1.86631\end{array}$

$\begin{array}{lll}1.06796 & 2.30905 & 1.86438\end{array}$

$\begin{array}{llll}1.05785 & 2.29383 & 1.86243\end{array}$

$\begin{array}{llll}1.04786 & 2.27876 & 1.86048\end{array}$

$\begin{array}{llll}1.03800 & 2.26383 & 1.85850\end{array}$

$\begin{array}{llll}1.02825 & 2.24902 & 1.85652\end{array}$

$\begin{array}{llll}1.01862 & 2.23436 & 1.85452\end{array}$

$1.00910 \cdot 2.21982 \quad 1.85251$

$0.99970 \quad 2.20541 \quad 1.85049$

$0.990412 .19113 \quad 1.84845$

$0.981232 .17697 \quad 1.84640$

$0.97215 \quad 2.16294 \quad 1.84434$

$0.963192 .14903 \quad 1.84227$

$0.95432 \quad 2.13524 \quad 1.84018$

$\begin{array}{llll}0.94556 & 2.12156 \quad 1.83808\end{array}$ $\begin{array}{llll}0.93690 & 2.10800 \quad 1.83596\end{array}$ $0.92834 \quad 2.09456 \quad 1.83384$ $\begin{array}{llll}0.91987 & 2.08123 & 1.83170\end{array}$

$0.91150 \quad 2.06801 \quad 1.82955$

$\begin{array}{llll}0.90323 & 2.05491 & 1.82738\end{array}$ $0.89505 \quad 2.04191 \quad 1.82520$ $0.88696 \quad 2.02902 \quad 1.82302$ $\begin{array}{llll}0.87897 & 2.01623 & 1.82081\end{array}$ $0.87106 \quad 2.00355 \quad 1.81860$

$\begin{array}{llll}0.86324 & 1.99097 \quad 1.81638\end{array}$ $0.85550 \quad 1.97850 \quad 1.81414$ $0.847851 .96612 \quad 1.81189$ $0.840291 .95384 \quad 1.80963$ $0.832801 .94167 \quad 1.80735$

$\begin{array}{llll}0.82540 & 1.92958 & 1.80507\end{array}$ $\begin{array}{llll}0.81808 & 1.91760 & 1.80277\end{array}$ $0.81084 \quad 1.90571 \quad 1.80046$ $0.803671 .89391 \quad 1.79814$ $0.79659 \quad 1.88220 \quad 1.79581$

$0.78958 \quad 1.87059 \quad 1.79346$ $0.782641 .85906 \quad 1.79111$ $\begin{array}{llll}0.77578 & 1.84762 & 1.78874\end{array}$ $\begin{array}{llll}0.76898 & 1.83627 & 1.78636\end{array}$ $\begin{array}{llll}0.76226 & 1.82501 & 1.78398\end{array}$ $\begin{array}{llll}1.26402 & 2.59555 \quad 1.89572\end{array}$

$\begin{array}{llll}1.25199 & 2.57840 \quad 1.89410\end{array}$

$1.24012 \quad 2.56142 \quad 1.89246$

$\begin{array}{llll}1.22840 & 2.54460 & 1.89082\end{array}$

1.216822 .527951 .88915

$1.205392 .51146 \quad 1.88748$ $\begin{array}{llll}1.19411 & 2.49513 & 1.88579\end{array}$ $1.18296 \quad 2.47895 \quad 1.88409$ $1.17195 \quad 2.46292 \quad 1.88238$ $1.16108 \quad 2.44704 \quad 1.88065$

$1.150342 .43131 \quad 1.87892$ $\begin{array}{llll}1.13973 & 2.41572 & 1.87717\end{array}$ $1.129252 .40028 \quad 1.87540$ $\begin{array}{lll}1.11889 & 2.38498 & 1.87363\end{array}$ $1.10866 \quad 2.36982 \quad 1.87184$

$1.09855 \quad 2.35479 \quad 1.87004$ $\begin{array}{llll}1.08856 & 2.33989 & 1.86822\end{array}$ $1.078692 .32513 \quad 1.86640$ $1.068932 .31050 \quad 1.86456$ $\begin{array}{llll}1.05929 & 2.29600 \quad 1.86271\end{array}$

$1.04976 \quad 2.28162 \quad 1.86085$ $\begin{array}{llll}1.04034 & 2.26737 & 1.85898\end{array}$ $1.03102 \quad 2.25324 \quad 1.85709$ $1.02182 \quad 2.23923 \quad 1.85519$ $1.01272 \quad 2.22534 \quad 1.85328$

$1.00372 \quad 2.21157 \quad 1.85136$ $0.994822 .19791 \quad 1.84942$ $0.98602 \quad 2.18437 \quad 1.84748$ $0.97733 \quad 2.17094 \quad 1.84552$ $0.96872 \quad 2.15762 \quad 1.84355$

$0.96022 \quad 2.14442 \quad 1.84157$ 0.951812 .131321 .83958 0.943492 .118321 .83757 $\begin{array}{llll}0.93526 & 2.10543 & 1.83556\end{array}$ 0.927122 .092651 .83353

$0.91907 \quad 2.07997 \quad 1.83149$ $0.91111 \quad 2.06739 \quad 1.82944$ $0.90323 \quad 2.05491 \quad 1.82738$ 0.895442 .042521 .82531 $0.88773 \quad 2.03024 \quad 1.82322$

$0.88010 \quad 2.01805 \quad 1.82113$ $\begin{array}{lll}0.87256 & 2.00596 \quad 1.81902\end{array}$

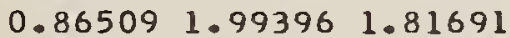
0.857701 .982051 .81478 $0.85039 \quad 1.97024 \quad 1.81264$

$0.84316 \quad 1.95851 \quad 1.81049$ $0.83600 \quad 1.94687 \quad 1.80833$ 0.828921 .935331 .80616 $0.82191 \quad 1.923871 .80398$ 0.814971 .912491 .80178
$1.326572 .68392 \quad 1.90364$ 1.314312 .666701 .90215 1.302202 .649651 .90066 $1.290242 .63276 \quad 1.89915$ 1.278442 .616041 .89762

$1.26678 \quad 2.59947 \quad 1.89609$ $1.25526 \quad 2.58306 \quad 1.89454$ 1.243882 .566801 .89299 $1.232642 .55070 \quad 1.89142$ $1.221542 .53475 \quad 1.88984$

1. $210572.51894 \quad 1.88824$ $1.19973 \quad 2.50327 \quad 1.88664$ $1.18902 \quad 2.48775 \quad 1.88502$ $1.178442 .47237 \quad 1.88339$ 1.167982 .457131 .88175

$1.15765 \quad 2.442021 .88010$ $1.147432 .42705 \quad 1.87844$ 1.137342 .412201 .87677 $\begin{array}{llll}1.12736 & 2.39749 & 1.87508\end{array}$ $1.117492 .38290 \quad 1.87338$

$1.107742 .36844 \quad 1.87167$ $1.098092 .35411 \quad 1.86996$ $1.08856 \quad 2.33989 \quad 1.86822$ $\begin{array}{llll}1.07913 & 2.32580 & 1.86648\end{array}$ 1.069812 .311831 .86473

$\begin{array}{llll}1.06060 & 2.29797 & 1.86296\end{array}$ $1.05148 \quad 2.284231 .86119$ $1.042472 .27060 \quad 1.85940$ $1.03355 \quad 2.25708 \quad 1.85760$ $1.024732 .24368 \quad 1.85580$

$\begin{array}{llll}1.01601 & 2.23038 & 1.85398\end{array}$ $1.007392 .21719 \quad 1.85215$ $0.99885 \quad 2.20411 \quad 1.85031$ 0.990412 .191131 .84845 $0.98206 \quad 2.178251 .84659$

$0.97380 \quad 2.16548 \quad 1.84472$ $0.965622 .15281 \quad 1.84283$ $0.95753 \quad 2.14024 \quad 1.84094$ 0.949532 .127761 .83903 $0.94161 \quad 2.11538 \quad 1.83712$

$0.933772 .10310 \quad 1.83519$ $0.926022 .09091 \quad 1.83325$ $\begin{array}{llll}0.91834 & 2.07882 & 1.83131\end{array}$ 0.910752 .066821 .82935 $0.903232 .05491 \quad 1.82738$

0.895792 .043091 .82540 0.888432 .031351 .82341 0.881142 .019711 .82142 0.873922 .008151 .81941 $0.86678 \quad 1.99668 \quad 1.81739$
1100

1110

1120

1130

1140

1150

1160

1170

1180

1190

1200

1210

1220

1230

1240

1250

1260

1270

1280

1290

1300

1310

1320

1330

1340

1350

1360

1370

1380

1390

1400

1410

1420

1430

1440

1450

1460

1470

1480

1490

1500

1510

1520

1530

1540

1550

1560

1570

1580

1590 
Table II Harmonic Oscillator Contributions to the Thermodynamic

Functions (in units of calories, moles, and ${ }^{\circ} \mathrm{K}$ )

\begin{tabular}{lccc}
$\mathrm{cm}^{-1}$ & $-\left(\mathrm{F}^{\circ}-E_{0} \mathrm{O}\right) / \mathrm{T}$ & So & $\mathrm{C}_{\mathrm{p}}^{\circ}$ \\
& & & \\
\multicolumn{5}{c}{$\mathrm{T}=2000}$. \\
1600 & 0.75562 & 1.81383 & 1.78157 \\
1610 & 0.74904 & 1.80274 & 1.77916 \\
1620 & 0.74253 & 1.79173 & 1.77674 \\
1630 & 0.73608 & 1.78080 & 1.77431 \\
1640 & 0.72971 & 1.76996 & 1.77186
\end{tabular}

$\begin{array}{lllll}1650 & 0.72340 & 1.75920 & 1.76941\end{array}$

$\begin{array}{llll}1660 & 0.71715 & 1.74851 & 1.76694\end{array}$

$\begin{array}{lllll}1670 & 0.71097 & 1.73791 & 1.76446\end{array}$

$\begin{array}{llll}1680 & 0.70485 & 1.72738 & 1.76197\end{array}$

$1690 \quad 0.69880 \quad 1.71693 \quad 1.75948$

$\begin{array}{llll}1700 \quad 0.69280 & 1.70656 \quad 1.75697\end{array}$

$\begin{array}{llll}1710 & 0.68637 & 1.69626 & 1.75445\end{array}$

$\begin{array}{llll}1720 & 0.68100 & 1.68604 & 1.75192\end{array}$

$\begin{array}{lllll}1730 & 0.67518 & 1.67589 & 1.74938\end{array}$

$\begin{array}{lllll}1740 & 0.66943 & 1.66581 & 1.74683\end{array}$

$\begin{array}{lllll}1750 & 0.66373 & 1.65581 & 1.74427\end{array}$

$\begin{array}{lllll}1760 & 0.65809 & 1.64588 & 1.74169\end{array}$

$\begin{array}{lllll}1770 & 0.65251 & 1.63602 & 1.73911\end{array}$

$\begin{array}{lllll}1780 & 0.64698 & 1.62623 & 1.73652\end{array}$

$\begin{array}{lllll}1790 & 0.64150 & 1.61651 & 1.73392\end{array}$

$\begin{array}{lllll}1800 & 0.63608 & 1.60685 & 1.73131\end{array}$

$\begin{array}{lllll}1810 & 0.63072 & 1.59727 & 1.72869\end{array}$

$\begin{array}{lllll}1820 & 0.62540 & 1.58775 & 1.72606\end{array}$

$\begin{array}{lllll}1830 & 0.62014 & 1.57830 & 1.72342\end{array}$

$\begin{array}{lllll}1840 & 0.61493 & 1.56892 & 1.72077\end{array}$

$\begin{array}{lllll}1850 & 0.60977 & 1.55960 & 1.71811\end{array}$ $\begin{array}{lllll}1860 & 0.60466 & 1.55034 & 1.71544\end{array}$

$\begin{array}{lllll}1870 & 0.59960 & 1.54115 & 1.71276\end{array}$

$\begin{array}{llll}1880 & 0.59459 & 1.53202 & 1.71008\end{array}$

$\begin{array}{lllll}1890 & 0.58963 & 1.52296 & 1.70738\end{array}$

$\begin{array}{lllll}1900 & 0.58471 & 1.51396 & 1.70467\end{array}$

$\begin{array}{lllll}1910 & 0.57985 & 1.50501 & 1.70196\end{array}$

$\begin{array}{lllll}1920 & 0.57503 & 1.49613 & 1.69923\end{array}$

$1930 \quad 0.570251 .487311 .69650$

$\begin{array}{lllll}1940 & 0.56552 & 1.47855 & 1.69376\end{array}$

$\begin{array}{lllll}1950 & 0.56084 & 1.46985 & 1.69101\end{array}$

$1960 \quad 0.55620 \quad 1.46121 \quad 1.68825$

$\begin{array}{lllll}1970 & 0.55160 & 1.45262 & 1.68548\end{array}$

$1980 \quad 0.54705 \quad 1.44410 \quad 1.68270$

$1990 \quad 0.54254 \quad 1.43563 \quad 1.67992$

$\begin{array}{lllll}2000 & 0.53808 & 1.42721 & 1.67713\end{array}$ $2010 \quad 0.53365 \quad 1.41886 \quad 1.67432$ $2020 \quad 0.52927 \quad 1.41055 \quad 1.67151$ $2030 \quad 0.52493 \quad 1.40231 \quad 1.66869$ $\begin{array}{lllll}2040 \quad 0.52062 & 1.39411 & 1.66587\end{array}$

$2050 \quad 0.51636 \quad 1.38597 \quad 1.66303$ $2060 \quad 0.51214 \quad 1.37789 \quad 1.66019$ $2070 \quad 0.50796 \quad 1.36986 \quad 1.65734$ $2080 \quad 0.503811 .361881 .65448$ $2090 \quad 0.49971 \quad 1.35395 \quad 1.65161$

$-\left(F^{\circ}-E_{0}^{\circ}\right) / T \quad$ So $\quad C_{p}^{\circ} \quad-\left(F^{\circ}-E_{O}^{\circ}\right) / T \quad S^{\circ}$

$\mathrm{T}=2200$

0.808101 .901201 .79958 $0.80130 \quad 1.89000 \quad 1.79736$ $0.79458 \quad 1.87887 \quad 1.79514$ $0.78792 \quad 1.86783 \quad 1.79290$ 0.781331 .856871 .79066

$0.77480 \quad 1.84600 \quad 1.78840$ $0.768341 .83520 \quad 1.78614$ $0.761951 .82448 \quad 1.78386$ 0.755621 .813831 .78157 0.749351 .803271 .77928

$0.74314 \quad 1.79278 \quad 1.77697$ $0.73700 \quad 1.78236 \quad 1.77465$ $0.73092 \quad 1.77202 \quad 1.77233$ $0.72489 \quad 1.76175 \quad 1.76999$ $0.71893 \quad 1.75156 \quad 1.76765$

$0.71302 \quad 1.741431 .76529$ $0.70718 \quad 1.73138 \quad 1.76292$ 0.701381 .721401 .76055 0.695651 .711491 .75816 $0.68997 \quad 1.70164 \quad 1.75577$

$0.68435 \quad 1.69187 \quad 1.75336$ $0.67878 \quad 1.682161 .75095$ $0.67326 \quad 1.67252 \quad 1.74853$ $0.66779 \quad 1.66295 \quad 1.74610$ $0.66238 \quad 1.65344 \quad 1.74365$

$0.65702 \quad 1.643991 .74120$ $0.65171 \quad 1.634611 .73874$ 0.646451 .625301 .73628 $0.641241 .61604 \quad 1.73380$ 0.636081 .606851 .73131

$\begin{array}{llll}0.63097 & 1.59772 & 1.72882\end{array}$ $0.62591 \quad 1.58866 \quad 1.72631$ $0.620891 .57965 \quad 1.72380$ 0.615921 .570701 .72128 0.610991 .561811 .71874

$0.606121 .55298 \quad 1.71621$ $0.60128 \quad 1.54421 \quad 1.71366$ 0.596491 .535491 .71110 $0.59175 \quad 1.52684 \quad 1.70854$ $0.58705 \quad 1.51824 \quad 1.70596$

0.582391 .509691 .70338 $0.57778 \quad 1.501201 .70079$ $0.57320 \quad 1.49277 \quad 1.69819$ $\begin{array}{llll}0.56867 & 1.48439 & 1.69559\end{array}$ $0.564181 .47606 \quad 1.69298$

$\begin{array}{llll}0.55973 & 1.46779 & 1.69035\end{array}$ $0.55532 \quad 1.45957 \quad 1.68772$ 0.550951 .451401 .68509 0.546621 .443291 .68244 0.542331 .435231 .67979
$0.85971 \quad 1.98529 \quad 1.81536$ $\begin{array}{llll}0.85271 & 1.97399 & 1.81332\end{array}$ $0.84578 \quad 1.96276 \quad 1.81127$ $0.838921 .95162 \quad 1.80921$ 0.832131 .940561 .80715

$\begin{array}{llll}0.82540 & 1.92958 & 1.80507\end{array}$ $0.818741 .91868 \quad 1.80298$ $\begin{array}{llll}0.81215 & 1.90786 & 1.80088\end{array}$ 0.805621 .897121 .79877 $0.79916 \quad 1.88645 \quad 1.79666$

$0.79275 \quad 1.87585 \quad 1.79453$ $0.786411 .86534 \quad 1.79240$ $\begin{array}{llll}0.78013 & 1.85489 & 1.79025\end{array}$ $0.77392 \quad 1.84452 \quad 1.78810$ $\begin{array}{llll}0.76776 & 1.83422 & 1.78593\end{array}$

$0.76166 \quad 1.82399 \quad 1.78376$ $0.75562 \quad 1.81383 \quad 1.78157$ $0.74963 \quad 1.80374 \quad 1.77938$ $0.74370 \quad 1.79373 \quad 1.77718$ $\begin{array}{llll}0.73783 & 1.78378 & 1.77497\end{array}$

$\begin{array}{llll}0.73202 & 1.77389 & 1.77275\end{array}$ $0.72626 \quad 1.76408 \quad 1.77052$ 0.720551 .754331 .76829 $0.71490 \quad 1.74465 \quad 1.76604$ 0.709301 .735031 .76379

$\begin{array}{llll}0.70375 & 1.72547 & 1.76152\end{array}$ $0.698251 .71598 \quad 1.75925$ $\begin{array}{llll}0.69280 & 1.70656 & 1.75697\end{array}$ $0.68741 \quad 1.69719 \quad 1.75468$ $0.68206 \quad 1.687891 .75238$

$0.67676 \quad 1.67865 \quad 1.75007$ 0.671511 .669471 .74776 $0.66631 \quad 1.660351 .74543$ 0.661161 .651291 .74310 $0.65605 \quad 1.64228 \quad 1.74076$

$0.65099 \quad 1.63334 \quad 1.73841$ $0.64598 \quad 1.62445 \quad 1.73605$ 0.641011 .615631 .73368 $0.63608 \quad 1.606851 .73131$ $0.631201 .59814 \quad 1.72893$

$0.62636 \quad 1.58948 \quad 1.72654$ $0.62157 \quad 1.58087 \quad 1.72414$ $0.616821 .57232 \quad 1.72173$ 0.612111 .563821 .71932 0.607441 .555381 .71690

$0.60282 \quad 1.54699 \quad 1.71447$ $0.59823 \quad 1.53866 \quad 1.71203$ $\begin{array}{llll}0.59369 & 1.53037 & 1.70959\end{array}$ $0.58918 \quad 1.52214 \quad 1.70713$ $0.584711 .51396 \quad 1.70467$ $\nu$

$\mathrm{cm}^{-1}$

1600

1610

1620

1630

1640

1650

1660

1670

1680

1690

1700

1710

1720

1730

1740

1750

1760

1770

1780

1790

1800

1810

1820

1830

1840

1850

1860

1870

1880

1890

1900

1910

1920

1930

1940

1950

1960

1970

1980

1990

2000

2010

2020

2030

2040

2050

2060

2070

2080

2090 
Table II Harmonic Oscillator Contributions to the Thermodynamic

Functions (in units of calories, moles, and ${ }^{\circ} \mathrm{K}$ )
$\sum_{-1}-\left(F^{\circ}-E_{0}^{\circ}\right) / T \quad$ So
$\mathrm{CO}^{\circ} \quad-\left(\mathrm{F}^{\circ}-\mathrm{E}_{\mathrm{O}}\right) / \mathrm{T} \quad \mathrm{S}^{\circ}$
$\mathrm{C}_{\mathrm{p}}^{\circ}$
$-\left(\mathrm{F}^{\circ}-\mathrm{E}_{0}^{\circ}\right) / \mathrm{T}$
S०
$\mathrm{cm}^{-1}$

$T=2000$.

2100

2110

2120

2130

2140

2150

2160

2170

2180

2190

2200

2210

2220

2230

2240

2250

2260

2270

2280

2290

2300

2310

2320

2330

2340

2350

2360

2370

2380

2390

2400

2410

2420

2430

2440

2450

2460

2470

2480

2490

2500

2510

2520

2530

2540

2550

2560

2570

2580

2590
$0.49564 \quad 1.34607 \quad 1.64874$ $0.49161 \quad 1.33824 \quad 1.64585$ $0.48761 \quad 1.33047 \quad 1.64296$ $0.48366 \quad 1.32275 \quad 1.64006$ $0.479731 .31507 \quad 1.63716$

$0.47585 \quad 1.30744 \quad 1.63425$ $0.47200 \quad 1.29987 \quad 1.63133$ $0.46818 \quad 1.29234 \quad 1.62840$ $0.46440 \quad 1.28486 \quad 1.62547$ $0.46066 \quad 1.27743 \quad 1.62252$

$\begin{array}{llll}0.45694 & 1.27004 & 1.61957\end{array}$ $0.45326 \quad 1.26270 \quad 1.61662$ $0.44962 \quad 1.25541 \quad 1.61366$ 0.446001 .248171 .61069 $0.442421 .24097 \quad 1.60771$

$0.438871 .23381 \quad 1.60473$ $0.43536 \quad 1.22670 \quad 1.60174$ $0.43187 \quad 1.21964 \quad 1.59874$ $0.42842 \quad 1.21262 \quad 1.59574$ $0.424991 .20564 \quad 1.59273$

$0.42160 \quad 1.19870 \quad 1.58971$ $0.41823 \quad 1.19181 \quad 1.58669$ $0.41490 \quad 1.18497 \quad 1.58366$ $0.411601 .17816 \quad 1.58063$ $0.40832 \quad 1.17140 \quad 1.57759$

$0.40507 \quad 1.16468 \quad 1.57454$ $0.40186 \quad 1.15800 \quad 1.57149$ $0.398671 .15136 \quad 1.56843$ $\begin{array}{llll}0.39550 & 1.14476 & 1.56537\end{array}$ $0.392371 .13821 \quad 1.56230$

$0.38926 \quad 1.13169 \quad 1.55922$ $0.38618 \quad 1.12521 \quad 1.55614$ $0.383131 .11878 \quad 1.55306$ $0.380101 .11238 \quad 1.54997$ $0.37710 \quad 1.10602 \quad 1.54687$

$0.37413 \quad 1.09970 \quad 1.54377$ $0.371181 .09342 \quad 1.54066$ $0.36826 \quad 1.08717 \quad 1.53755$ $0.36536 \quad 1.08097 \quad 1.53443$ 0.362481 .074801 .53130

$0.35964 \quad 1.06867 \quad 1.52818$ $0.35681 \quad 1.06257 \quad 1.52504$ $0.35401 \quad 1.05651 \quad 1.52191$ $0.35124 \quad 1.05049 \quad 1.51876$ $0.348481 .04451 \quad 1.51562$

$\begin{array}{llll}0.34576 & 1.03856 & 1.51247\end{array}$ $0.34305 \quad 1.03265 \quad 1.50931$ 0.340371 .026771 .50615 $0.33771 \quad 1.02092 \quad 1.50298$ $0.33507 \quad 1.01512 \quad 1.49981$

$T=2100$.

$T=2200$

$0.53808 \quad 1.42721 \quad 1.67713$ $0.53386 \quad 1.41925 \quad 1.67446$ $0.529681 .41134 \quad 1.67178$ $0.52554 \quad 1.40348 \quad 1.66910$ $0.52144 \quad 1.395671 .66641$

$0.51737 \quad 1.38791 \quad 1.66371$ 0.513341 .380191 .66100 0.509351 .372531 .65829 $0.505391 .36491 \quad 1.65557$ 0.501461 .357341 .65284

$0.49757 \quad 1.349821 .65011$ $0.49371 \quad 1.342341 .64736$ $0.489891 .33491 \quad 1.64462$ 0.486101 .327521 .64186 0.482341 .320181 .63910

0.478621 .312891 .63633 0.474931 .305641 .63355 $0.47127 \quad 1.298431 .63077$ $0.467641 .29127 \quad 1.62798$ 0.464041 .284151 .62519

$0.46048 \quad 1.27707 \quad 1.62238$ 0.456941 .270041 .61957 $0.45344 \quad 1.263051 .61676$ $0.44996 \quad 1.25610 \quad 1.61394$ 0.446521 .249201 .61111

0.443101 .242331 .60828 $0.439721 .23551 \quad 1.60544$ 0.436361 .228731 .60259 0.433031 .221991 .59974 $0.42973 \quad 1.215281 .59688$

$0.42646 \quad 1.208621 .59402$ 0.423211 .202001 .59115 0.419991 .195421 .58828 $0.41680 \quad 1.18887 \quad 1.58540$ $0.413641 .18237 \quad 1.58251$

$0.41050 \quad 1.17590 \quad 1.57962$ 0.407391 .169471 .57672 $0.40430 \quad 1.16308 \quad 1.57382$ 0.401251 .156731 .57091 $0.39821 \quad 1.15042 \quad 1.56800$

$0.395201 .14414 \quad 1.56508$ $0.39222 \quad 1.137891 .56215$ $0.389261 .13169 \quad 1.55922$ $0.386331 .12552 \quad 1.55629$ $0.38342 \quad 1.119391 .55335$

0.380531 .113291 .55041 0.377671 .107231 .54746 $0.374831 .10120 \quad 1.54451$

$0.372021 .09521 \quad 1.54155$ $0.369231 .08925 \quad 1.53858$
$0.58029 \quad 1.50582 \quad 1.70221$ $\begin{array}{llll}0.57590 & 1.49774 & 1.69973\end{array}$ $0.57155 \quad 1.48971 \quad 1.69725$ $0.56724 \quad 1.48173 \quad 1.69476$ $0.562961 .47380 \quad 1.69226$

$0.55873 \quad 1.46592 \quad 1.68976$ $0.55452 \quad 1.45808 \quad 1.68724$ $0.55036 \quad 1.450291 .68473$ 0.546231 .442551 .68220 $0.54214 \quad 1.43486 \quad 1.67967$

$0.53808 \quad 1.42721 \quad 1.67713$ $0.53405 \quad 1.41961 \quad 1.67458$ 0.530061 .412061 .67202 0.526111 .404551 .66946 $0.52218 \quad 1.39709 \quad 1.66690$

$0.51829 \quad 1.38967 \quad 1.66432$ 0.514441 .382291 .66174 0.510611 .374961 .65915 0.506821 .367671 .65656 $0.50306 \quad 1.360431 .65396$

0.499331 .353231 .65135 $0.495641 .34607 \quad 1.64874$ 0.491971 .338951 .64612 $0.488341 .33188 \quad 1.64349$ $0.48473 \quad 1.324851 .64086$

$0.48116 \quad 1.31786 \quad 1.63822$ $0.47761 \quad 1.31090 \quad 1.63557$ 0.474091 .303991 .63292 0.470611 .297121 .63026 $0.467151 .29029 \quad 1.62760$

$0.46372 \quad 1.28350 \quad 1.62493$ $0.46032 \quad 1.27675 \quad 1.62226$ $0.456941 .27004 \quad 1.61957$ $0.453601 .26337 \quad 1.61689$ 0.450281 .256731 .61420

$0.44699 \quad 1.25014 \quad 1.61150$ $0.44372 \quad 1.243581 .60879$ $0.440481 .23706 \quad 1.60608$ $0.43727 \quad 1.23057 \quad 1.60337$ 0.434091 .224131 .60065

0.430931 .217721 .59792 0.427791 .211341 .59519 0.424681 .205011 .59246 $0.42160 \quad 1.19870 \quad 1.58971$ $0.418541 .19244 \quad 1.58697$

$0.41550 \quad 1.18621 \quad 1.58422$ 0.412491 .180011 .58146 $\begin{array}{llll}0.40951 & 1.17385 & 1.57870\end{array}$ 0.406551 .167731 .57593 0.403611 .161641 .57316
2100

2110

2120

2130

2140

2150

2160

2170

2180

2190

2200

2210

2220

2230

2240

2250

2260

2270

2280

2290

2300

2310

2320

2330

2340

2350

2360

2370

2380

2390

2400

2410

2420

2430

2440

2450

2460

2470

2480

2490

2500

2510

2520

2530

2540

2550

2560

2570

2580

2590 
Table II Harmonic Oscillator Contributions to the Thermodynamic

Functions (in units of calories, moles, and ${ }^{\circ} \mathrm{K}$ )

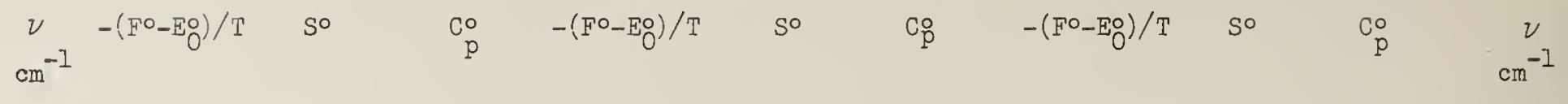

$T=2000$

$2600 \quad 0.33246 \quad 1.00934 \quad 1.49664$

$2610 \quad 0.329871 .003601 .49346$

$\begin{array}{lllll}2620 & 0.32729 & 0.99790 & 1.49028\end{array}$

$2630 \quad 0.32475 \quad 0.99223 \quad 1.48709$

$2640 \quad 0.322220 .98659 \quad 1.48390$

$\begin{array}{lllll}2650 & 0.31971 & 0.98099 & 1.48071\end{array}$

$\begin{array}{lllll}2660 & 0.31723 & 0.97541 & 1.47751\end{array}$

$\begin{array}{lllll}2670 & 0.31476 & 0.96988 & 1.47431\end{array}$

$2680 \quad 0.312320 .96437 \quad 1.47111$

$\begin{array}{lllll}2690 & 0.30990 & 0.95890 & 1.46790\end{array}$

$2700 \quad 0.30750 \quad 0.95346 \quad 1.46468$

$\begin{array}{lllll}2710 & 0.30511 & 0.94805 & 1.46147\end{array}$

$\begin{array}{lllll}2720 & 0.30275 & 0.94267 & 1.45825\end{array}$

$\begin{array}{lllll}2730 & 0.30041 & 0.93733 & 1.45503\end{array}$

$\begin{array}{lllll}2740 & 0.29808 & 0.93201 & 1.45180\end{array}$

$\begin{array}{lllll}2750 & 0.29578 & 0.92673 & 1.44857\end{array}$ $\begin{array}{lllll}2760 & 0.29350 & 0.92148 & 1.44534\end{array}$ $\begin{array}{lllll}2770 & 0.29123 & 0.91626 & 1.44210\end{array}$ $\begin{array}{lllll}2780 & 0.28898 & 0.91106 & 1.43886\end{array}$ $\begin{array}{lllll}2790 & 0.28675 & 0.90590 & 1.43562\end{array}$

$\begin{array}{lllll}2800 & 0.28454 & 0.90077 & 1.43238\end{array}$ $\begin{array}{lllll}2810 & 0.28235 & 0.89567 & 1.42913\end{array}$ $\begin{array}{lllll}2820 & 0.28018 & 0.89060 & 1.42588\end{array}$ $\begin{array}{lllll}2830 & 0.27802 & 0.88556 & 1.42263\end{array}$ $\begin{array}{lllll}2840 & 0.27589 & 0.88055 & 1.41937\end{array}$

$\begin{array}{lllll}2850 & 0.27376 & 0.87556 & 1.41611\end{array}$

$\begin{array}{lllll}2860 & 0.27166 & 0.87061 & 1.41285\end{array}$

$\begin{array}{lllll}2870 & 0.26958 & 0.86568 & 1.40959\end{array}$

$\begin{array}{lllll}2880 & 0.26751 & 0.86079 & 1.40632\end{array}$

$\begin{array}{lllll}2890 & 0.26546 \quad 0.85592 & 1.40306\end{array}$

$2900 \quad 0.263420 .85108 \quad 1.39979$

$2910 \quad 0.26140 \quad 0.84626 \quad 1.39651$

$2920 \quad 0.25940 \quad 0.84148 \quad 1.39324$

$\begin{array}{lllll}2930 & 0.25742 & 0.83672 & 1.38996\end{array}$

$\begin{array}{lllll}2940 & 0.25545 & 0.83199 & 1.38668\end{array}$

$\begin{array}{lllll}2950 & 0.25349 & 0.82729 & 1.38340\end{array}$ $\begin{array}{lllll}2960 & 0.25156 & 0.82261 & 1.38012\end{array}$ $\begin{array}{lllll}2970 & 0.24964 & 0.81796 & 1.37684\end{array}$ $2980 \quad 0.24773 \quad 0.81334 \quad 1.37355$ $\begin{array}{llllll}2990 & 0.24584 & 0.80874 & 1.37026\end{array}$

$\begin{array}{llllll}3000 & 0.24396 & 0.80417 & 1.36698\end{array}$ $\begin{array}{lllll}3010 & 0.24211 & 0.79963 & 1.36368\end{array}$ $\begin{array}{llllll}3020 & 0.24026 & 0.79511 & 1.36039\end{array}$ $3030 \quad 0.23843 \quad 0.79062 \quad 1.35710$ $3040 \quad 0.236620 .786161 .35380$

$\begin{array}{lllll}3050 & 0.23482 & 0.78172 & 1.35051\end{array}$ $\begin{array}{llllll}3060 & 0.23303 & 0.77730 & 1.34721\end{array}$ $\begin{array}{lllll}3070 & 0.23126 & 0.77291 & 1.34391\end{array}$ $3080 \quad 0.229500 .76854 \quad 1.34061$ $3090 \quad 0.22776 \quad 0.76420 \quad 1.33731$

$T=2100$

$T=2200$

0.366461 .083331 .53562 $0.36371 \quad 1.07744 \quad 1.53264$ $0.36099 \quad 1.07158 \quad 1.52967$ $0.358291 .06576 \quad 1.52669$ $0.35561 \quad 1.05997 \quad 1.52370$

$0.352951 .05422 \quad 1.52071$ 0.350321 .048491 .51772 $0.34770 \quad 1.04281 \quad 1.51472$ 0.345111 .037151 .51171 0.342541 .031521 .50871

$0.33999 \quad 1.02593 \quad 1.50570$ $0.33746 \quad 1.020371 .50268$ 0.334951 .014841 .49966 $0.33246 \quad 1.00934 \quad 1.49664$ 0.329991 .003881 .49361

$\begin{array}{llll}0.32754 & 0.99844 & 1.49058\end{array}$ $0.325110 .99304 \quad 1.48755$ $0.32270 \quad 0.98766 \quad 1.48451$ 0.320310 .982321 .48147 $0.317940 .97700 \quad 1.47843$

$\begin{array}{llll}0.31558 & 0.97172 & 1.47538\end{array}$ $0.31325 \quad 0.96646 \quad 1.47233$ $0.310930 .96124 \quad 1.46927$ $0.308640 .95604 \quad 1.46621$ $0.30636 \quad 0.95088 \quad 1.46315$

$0.30410 \quad 0.94574 \quad 1.46009$ $0.30186 \quad 0.940631 .45702$ 0.299630 .935551 .45395 0.297420 .930501 .45088 $0.295230 .92548 \quad 1.44780$

$0.29306 \quad 0.92048 \quad 1.44472$ 0.290910 .915511 .44164 0.288770 .910571 .43856 $0.286650 .90566 \quad 1.43547$ $0.284540 .90077 \quad 1.43238$

$\begin{array}{llll}0.28246 & 0.89591 & 1.42929\end{array}$ $0.28038 \quad 0.89108 \quad 1.42619$ $0.278330 .88628 \quad 1.42309$ $0.276290 .88150 \quad 1.41999$ $0.274270 .87675 \quad 1.41689$

$0.27226 \quad 0.87202 \quad 1.41378$ 0.270270 .867321 .41068 $0.26829 \quad 0.862651 .40757$ $0.266330 .85800 \quad 1.40446$ $0.26439 \quad 0.85338 \quad 1.40134$

$0.26246 \quad 0.84878 \quad 1.39823$ $0.260540 .84421 \quad 1.39511$ $0.25864 \quad 0.83966 \quad 1.39199$ $0.25676 \quad 0.835141 .38887$ $0.254890 .83064 \quad 1.38575$
$0.40069 \quad 1.15558 \quad 1.57038$ 0.397801 .149561 .56760 0.394931 .143571 .56481 0.392091 .137611 .56202 0.389261 .131691 .55922

$0.386461 .12580 \quad 1.55642$ 0.383681 .119941 .55362 0.380921 .114121 .55081 0.378191 .108331 .54800 $0.37548 \quad 1.10257 \quad 1.54518$

$\begin{array}{llll}0.37278 & 1.09684 & 1.54235\end{array}$ 0.370111 .091141 .53953 0.367461 .085481 .53670 $0.364831 .07984 \quad 1.53386$ 0.362221 .074241 .53102

$0.359641 .06867 \quad 1.52818$ 0.357071 .063121 .52533 $0.354521 .05761 \quad 1.52248$ $0.35199 \quad 1.052131 .51962$ 0.349481 .046681 .51676

$0.346991 .04126 \quad 1.51390$ 0.344521 .035871 .51103 0.342071 .030501 .50816 $0.339641 .02517 \quad 1.50529$ $0.337231 .01987 \quad 1.50241$

$0.33483 \quad 1.01459 \quad 1.49953$ 0.332461 .009341 .49664 0.330101 .004121 .49375 $0.32776 \quad 0.998931 .49086$ $0.325440 .99377 \quad 1.48796$

$0.32314 \quad 0.98864 \quad 1.48506$ 0.320850 .983531 .48216 $0.31858 \quad 0.978451 .47926$ $0.316330 .97340 \quad 1.47635$ 0.314100 .968371 .47344

$0.31188 \quad 0.96337 \quad 1.47052$ $0.309680 .95840 \quad 1.46761$ $0.30750 \quad 0.95346 \quad 1.46468$ 0.305330 .948541 .46176 $0.303180 .94365 \quad 1.45883$

$0.30104 \quad 0.93878 \quad 1.45591$ $0.298930 .93394 \quad 1.45297$ 0.296830 .929131 .45004 $0.294740 .92434 \quad 1.44710$ $0.292670 .91957 \quad 1.44416$

$0.29061 \quad 0.91484 \quad 1.44122$ 0.288580 .910121 .43828 $0.286550 .90544 \quad 1.43533$ $0.28454 \quad 0.90077 \quad 1.43238$ $0.282550 .89614 \quad 1.42943$
2600

2610

2620

2630

2640

2650

2660

2670

2680

2690

2700

2710

2720

2730

2740

2750

2760

2770

2780

2790

2800

2810

2820

2830

2840

2850

2860

2870

2880

2890

2900

2910

2920

2930

2940

2950

2960

2970

2980

2990

3000

3010

3020

3030

3040

3050

3060

3070

3080

3090 
Table II Harmonic Oscillator Contributions to the Thermodynamic

Functions (in units of calories, moles, and ${ }^{\circ} \mathrm{K}$ )
$v_{-1}-\left(F^{\circ}-E_{0}^{\circ}\right) / T \quad S O$
$\mathrm{C}_{\mathrm{p}}^{\circ} \quad-\left(\mathrm{F}^{\circ}-\mathrm{E}_{\mathrm{O}}^{\circ}\right) / \mathrm{T}$
S०
$\mathrm{Co}_{\mathrm{p}}$
$-\left(\mathrm{F}^{\circ}-\mathrm{E}_{0}\right) / \mathrm{T}$
$S^{\circ}$

$\mathrm{cm}^{-1}$

$T=2000$.

$T=2100$ 。

$0.25303 \quad 0.82617 \quad 1.38262$ $0.251190 .82172 \quad 1.37950$ 0.249360 .817301 .37637 0.247550 .812901 .37324 $0.24575 \quad 0.80853 \quad 1.37011$

$0.243960 .80417 \quad 1.36698$ $0.242190 .79985 \quad 1.36384$ $0.240440 .79554 \quad 1.36071$ $\begin{array}{llll}0.23869 & 0.79126 & 1.35757\end{array}$ $0.23696 \quad 0.78700 \quad 1.35443$

$0.23524 \quad 0.79277 \quad 1.35129$ $0.233540 .77356 \quad 1.34815$ $0.231850 .77437 \quad 1.34501$ $0.230170 .77020 \quad 1.34187$ $0.22850 \quad 0.76606 \quad 1.33872$

3240

3250

3260

3270

3280

3290

3300

3310

3320

3330

3340

3350

3360

3370

3380

3390

3400

3410

3420

3430

3440

3450

3460

3470

3480

3490

3500

3510

3520

3530

3540

3550

3560

3570

3580

3590 $\begin{array}{llll}0.20948 & 0.71806 \quad 1.30094\end{array}$ $0.20790 \quad 0.71400 \quad 1.29764$ $0.20633 \quad 0.70997 \quad 1.29433$ $0.204770 .70596 \quad 1.29102$ $0.203220 .70198 \quad 1.28771$

$0.20169 \quad 0.69802 \quad 1.28440$ $0.200170 .69407 \quad 1.28109$ $\begin{array}{llll}0.19866 & 0.69016 & 1.27778\end{array}$ $\begin{array}{llll}0.19716 & 0.68626 & 1.27447\end{array}$ 0.195680 .682391 .27116

$0.19420 \quad 0.67853 \quad 1.26785$ $0.19274 \quad 0.67470 \quad 1.26454$ 0.191290 .670891 .26123 $0.189850 .66710 \quad 1.25792$ 0.188430 .663341 .25461

0.187010 .659591 .25130 $\begin{array}{llll}0.18560 & 0.65587 & 1.24799\end{array}$ $0.18421 \quad 0.65216 \quad 1.24469$ $\begin{array}{llll}0.18283 & 0.64848 & 1.24138\end{array}$ $\begin{array}{lll}0.18146 & 0.64482 & 1.23807\end{array}$

$\begin{array}{lllll}0.18009 & 0.64117 & 1.23477\end{array}$ $0.17874 \quad 0.63755 \quad 1.23146$ $0.17740 \quad 0.63395 \quad 1.22816$ $0.17607 \quad 0.63037 \quad 1.22486$ $\begin{array}{lll}0.17475 & 0.62681 & 1.22156\end{array}$

$0.17345 \quad 0.62327 \quad 1.21825$ $\begin{array}{llll}0.17215 & 0.61975 & 1.21495\end{array}$ $0.17086 \quad 0.61625 \quad 1.21166$ $0.16958 \quad 0.61276 \quad 1.20836$ $0.168310 .60930 \quad 1.20506$

$\begin{array}{llll}0.16705 & 0.60586 & 1.20177\end{array}$ $\begin{array}{llll}0.16580 & 0.60243 & 1.19847\end{array}$ $0.16456 \quad 0.59903 \quad 1.19518$ $0.163330 .59564 \quad 1.19189$ $\begin{array}{llll}0.16211 & 0.59228 & 1.18860\end{array}$

$\begin{array}{llll}0.16090 & 0.58893 & 1.18531\end{array}$ $\begin{array}{llll}0.15970 & 0.58560 & 1.18202\end{array}$ $0.15851 \quad 0.58229 \quad 1.17874$ $\begin{array}{llll}0.15733 & 0.57899 & 1.17545\end{array}$ $\begin{array}{lll}0.15616 & 0.57572 \quad 1.17217\end{array}$ $\begin{array}{llll}0.22685 & 0.76194 & 1.33558\end{array}$ $0.22521 \quad 0.75784 \quad 1.33243$ $0.223580 .75377 \quad 1.32929$ $0.22197 \quad 0.74971 \quad 1.32614$ $0.22036 \quad 0.74568 \quad 1.32299$

$\begin{array}{llll}0.21877 & 0.74167 & 1.31985\end{array}$ $\begin{array}{llll}0.21720 & 0.73768 & 1.31670\end{array}$ $0.215630 .73371 \quad 1.31355$ $0.214070 .72977 \quad 1.31040$ $0.21253 \quad 0.72584 \quad 1.30725$

$0.21100 \quad 0.72194 \quad 1.30410$ $0.20948 \quad 0.71806 \quad 1.30095$ $\begin{array}{llll}0.20797 & 0.71420 & 1.29779\end{array}$ c.20648 $0.71036 \quad 1.29464$ 0.204990 .706541 .29149

$0.20352 \quad 0.70274 \quad 1.28834$ $\begin{array}{llll}0.20205 & 0.69896 & 1.28518\end{array}$ $\begin{array}{llll}0.20060 & 0.69520 & 1.28203\end{array}$ $0.19916 \quad 0.69146 \quad 1.27888$ $\begin{array}{llll}0.19773 & 0.68774 & 1.27573\end{array}$

$0.19631 \quad 0.68404 \quad 1.27257$ $0.19491 \quad 0.68036 \quad 1.26942$ $\begin{array}{llll}0.19351 & 0.67671 & 1.26627\end{array}$ $0.192120 .67307 \quad 1.26312$ $0.19074 \quad 0.669451 .25997$

$\begin{array}{llll}0.18938 & 0.66585 & 1.25682\end{array}$ $0.18802 \quad 0.66226 \quad 1.25366$ $\begin{array}{llll}0.18667 & 0.65870 & 1.25051\end{array}$ $0.185340 .65516 \quad 1.24736$ $0.18401 \quad 0.651631 .24421$

$0.18270 \quad 0.64813 \quad 1.24106$ $0.181390 .64464 \quad 1.23792$ $\begin{array}{lllll}0.18009 & 0.64117 & 1.23477\end{array}$ $0.17881 \quad 0.63773 \quad 1.23162$ $\begin{array}{llll}0.17753 & 0.63429 & 1.22848\end{array}$
$T=2200$

0.280570 .891521 .42647 0.278610 .886931 .42351 $0.27666 \quad 0.882371 .42056$ 0.274730 .877831 .41759 0.272610 .873311 .41463

$0.27090 \quad 0.86882 \quad 1.41167$ 0.269010 .864351 .40870 $0.267130 .85990 \quad 1.40573$ $0.26527 \quad 0.85548 \quad 1.40276$ 0.263420 .851081 .39979

$0.261590 .84670 \quad 1.39681$ 0.259760 .842351 .39384 $0.25796 \quad 0.83802 \quad 1.39086$ $\begin{array}{llll}0.25616 & 0.83371 & 1.38788\end{array}$ $0.25438 \quad 0.829421 .38490$

$0.252610 .82516 \quad 1.38191$ $0.25086 \quad 0.820921 .37893$ 0.24911 .0 .816701 .37594 0.247390 .812501 .37295 0.245670 .808331 .36997

$0.24396 \quad 0.80417 \quad 1.36698$ $0.24227 \quad 0.80004 \quad 1.36398$ 0.240590 .795931 .36099 $0.238930 .79184 \quad 1.35800$ $0.23727 \quad 0.78778 \quad 1.35500$

0.235630 .783731 .35201 $0.23400 \quad 0.77971 \quad 1.34901$ $0.232380 .77570 \quad 1.34601$ $0.23078 \quad 0.77172 \quad 1.34301$ $0.229180 .76775 \quad 1.34001$

$0.22760 \quad 0.763811 .33701$ 0.226030 .759891 .33401 0.224470 .755991 .33101 0.222920 .752111 .32800 $0.221380 .74824 \quad 1.32500$

$0.219860 .74440 \quad 1.32199$ $0.218340 .74058 \quad 1.31899$ $0.216840 .73678 \quad 1.31598$ $0.215350 .73299 \quad 1.31298$ 0.213860 .729231 .30997

$0.212390 .72549 \quad 1.30696$ $0.210930 .72176 \quad 1.30395$ $0.209480 .71806 \quad 1.30095$ 0.208040 .714371 .29794 $0.206610 .71070 \quad 1.29493$

$0.205190 .70706 \quad 1.29192$ $0.203780 .70343 \quad 1.28891$ 0.202390 .699811 .28590 $0.20100 \quad 0.696221 .28289$ $0.199620 .69265 \quad 1.27988$
3100

3110

3120

3130

3140

3150

3160

3170

3180

3190

3200

3210

3220

3230

3240

3250

3260

3270

3280

3290

3300

3310

3320

3330

3340

3350

3360

3370

3380

3390

3400

3410

3420

3430

3440

3450

3460

3470

3480

3490

3500

3510

3520

3530

3540

3550

3560

3570

3580

3590 
Table II Harmonic Oscillator Contributions to the Thermodynamic

Functions (in units of calories, moles, and ${ }^{\circ} \mathrm{K}$ )
$\mathrm{cm}^{-1}-\left(F^{\circ}-E_{O}^{\circ}\right) / T \quad$ So
$\mathrm{C}_{\mathrm{p}}^{\circ} \quad-\left(\mathrm{F}^{\circ}-\mathrm{E}_{\mathrm{O}}^{\circ}\right) / \mathrm{T} \quad \mathrm{S}^{\circ}$
$\mathrm{C}_{\mathrm{p}}^{\circ} \quad-\left(\mathrm{F}^{\circ}-\mathrm{E}_{\mathrm{O}}^{\circ}\right) / \mathrm{T} \quad \mathrm{S}^{\circ}$
$\mathrm{C}_{\mathrm{p}}^{\circ}$
$\nu$
$\mathrm{cm}^{-1}$

$T=2000$

3600

3610

3620

3630

3640

3650

3660

3670

3680

3690

3700

3710

3720

3730

3740

3750

3760

3770

3780 .

3790

3800

3810

3820

3830

3840

3850

3860

3870

3880

3890

3900

3910

3920

3930

3940

3950

3960

3970

3980

3990

4000
$0.154990 .57246 \quad 1.16889$

$0.153840 .56923 \quad 1.16561$

$0.15269 \quad 0.56601 \quad 1.16234$

0.151550 .562801 .15906

0.150420 .559621 .15579

$0.14930 \quad 0.55645 \quad 1.15252$

0.148190 .553311 .14925

0.147090 .550171 .14598

$0.14600 \quad 0.54706 \quad 1.14272$

$0.144910 .54396 \quad 1.13945$

$0.143830 .54088 \quad 1.13619$

$0.14276 \quad 0.53782 \quad 1.13294$

$\begin{array}{lllll}0.14170 & 0.53478 & 1.12968\end{array}$

$0.140650 .53175 \quad 1.12643$

$0.139610 .52874 \quad 1.12318$

$0.138570 .52574 \quad 1.11993$

$0.137540 .52276 \quad 1.11668$

$0.136520 .51980 \quad 1.11344$

$0.135510 .51686 \quad 1.11020$

$0.13450 \quad 0.51393 \quad 1.10696$

$0.133510 .51102 \quad 1.10372$

0.132520 .508121 .10049

$0.131530 .50524 \quad 1.09726$

$\begin{array}{llll}0.13056 & 0.50237 & 1.09403\end{array}$

0.129590 .499531 .09080

$0.12863 \quad 0.49669 \quad 1.08758$

$0.12768 \quad 0.49388 \quad 1.08436$

$0.12674 \quad 0.49107 \quad 1.08115$

$0.12580 \quad 0.488291 .07793$

$0.124870 .48552 \quad 1.07472$

$0.123940 .48276 \quad 1.07151$

$0.12303 \quad 0.48002 \quad 1.06831$

$0.122120 .47730 \quad 1.06511$

$0.121220 .47459 \quad 1.06191$

$0.120320 .47189 \quad 1.05872$

0.119430 .469211 .05553

$0.11855 \quad 0.46655 \quad 1.05234$

$0.117670 .46390 \quad 1.04915$

$0.11680 \quad 0.46126 \quad 1.04597$

$0.115940 .45864 \quad 1.04279$

$0.11509 \quad 0.45604 \quad 1.03962$
$\mathrm{T}=2100$

$0.17626 \quad 0.63088 \quad 1.22533$

$0.17500 \quad 0.62749 \quad 1.22218$

$0.17376 \quad 0.62411 \quad 1.21904$

$0.172520 .62075 \quad 1.21590$

0.171290 .617411 .21276

$\begin{array}{llll}0.17007 & 0.61409 & 1.20961\end{array}$

$0.168850 .61078 \quad 1.20647$

$0.16765 \quad 0.60750 \quad 1.20334$

$0.16646 \quad 0.604231 .20020$

$0.16527 \quad 0.60097 \quad 1.19706$

$0.164090 .59774 \quad 1.19393$

$0.16293 \quad 0.59452 \quad 1.19079$

0.161770 .591321 .18766

0.160620 .588131 .18453

$0.15948 \quad 0.58497 \quad 1.18140$

$0.158340 .58182 \quad 1.17827$

$0.157220 .57868 \quad 1.17514$

$0.15610 \quad 0.57556 \quad 1.17202$

$0.15499 \quad 0.57246 \quad 1.16889$

$0.153890 .56938 \quad 1.16577$

$0.15280 \quad 0.56631 \quad 1.16265$

$0.15171 \quad 0.56326 \quad 1.15953$

$0.150640 .56023 \quad 1.15641$

0.149570 .557211 .15330

$0.148510 .55420 \quad 1.15018$

$0.14746 \quad 0.55122 \quad 1.14707$

0.146410 .548241 .14396

$0.145380 .54529 \quad 1.14085$

0.144350 .542351 .13775

0.143320 .534421 .13464

0.142310 .536511 .13154

0.141300 .533621 .12844

$0.14030 \quad 0.53074 \quad 1.12534$

$0.139310 .52788 \quad 1.12225$

0.138330 .525031 .11915

$0.137350 .52220 \quad 1.11606$

$0.136380 .51938 \quad 1.11297$

$0.135410 .51658 \quad 1.10989$

$0.13446 \quad 0.51379 \quad 1.10680$

$0.133510 .51102 \quad 1.10372$

$0.13256 \quad 0.50826 \quad 1.10064$
$T=2200$.

$0.19825 \quad 0.68909 \quad 1.27687$

$0.196890 .68555 \quad 1.27386$

$0.195540 .68203 \quad 1.27086$

$0.19420 \quad 0.67853 \quad 1.26785$

$0.19288 \quad 0.67505 \quad 1.26484$

$\begin{array}{llll}0.19156 & 0.67158 & 1.26183\end{array}$

0.190250 .668131 .25882

$0.188940 .66470 \quad 1.25581$

0.187650 .661291 .25281

$0.18637 \quad 0.65789 \quad 1.24980$

0.185100 .654521 .24679

$0.183830 .65116 \quad 1.24378$

$0.182580 .64781 \quad 1.24078$

$\begin{array}{llll}0.18133 & 0.64448 & 1.23777\end{array}$

$0.18009 \quad 0.64117 \quad 1.23477$

3600

3610

3620

3630

3640

3650

3660

3670

3680

3690

3700

3710

3720

3730

3740

$\begin{array}{llll}0.17887 & 0.63788 & 1.23176\end{array}$

$\begin{array}{llll}0.17765 & 0.63461 & 1.22876\end{array}$

0.176430 .631351 .22576

$0.17523 \quad 0.62810 \quad 1.22276$

$0.17404 \quad 0.62488 \quad 1.21976$

$\begin{array}{lll}0.17285 & 0.62167 & 1.21675\end{array}$

$0.17168 \quad 0.61847 \quad 1.21376$

$0.17051 \quad 0.61529 \quad 1.21076$

$0.169350 .61213 \quad 1.20776$

$0.16820 \quad 0.60899 \quad 1.20476$

$\begin{array}{llll}0.16705 & 0.60586 & 1.20177\end{array}$

$0.165920 .60274 \quad 1.19877$

$0.164790 .59965 \quad 1.19578$

0.163670 .596561 .19279

$0.162560 .59350 \quad 1.18979$

$0.16145 \quad 0.59045 \quad 1.18680$

$0.16036 \quad 0.58741 \quad 1.18382$

0.159270 .584391 .18083

$\begin{array}{llll}0.15819 & 0.58139 & 1.17784\end{array}$

$0.15712 \quad 0.57840 \quad 1.17486$

$0.15605 \quad 0.57542 \quad 1.17187$

$0.154990 .57246 \quad 1.16889$

0.153940 .569521 .16591

$0.15290 \quad 0.56659 \quad 1.16293$

$0.15186 \quad 0.56368 \quad 1.15995$

$0.150830 .56078 \quad 1.15698$
3750

3760

3770

3780

3790

3800

3810

3820

3830

3840

3850

3860

3870

3880

3890

3900

3910

3920

3930

3940

3950

3960

3970

3980

3990

4000 
Table II Harmonic Oscillator Contributions to the Thermodynamic

Functions (in units of calories, moles, and ${ }^{\circ} \mathrm{K}$ )
$\mathrm{cm}^{-1}$
$-\left(F^{\circ}-E_{0}^{\circ}\right) / T \quad S^{\circ}$$$
T=2300 \text {. }
$$

\begin{abstract}
100
110

120

130
\end{abstract}
140

$$
\begin{array}{ccc}
C_{p}^{\circ} & -\left(F^{\circ}-E_{0}^{\circ}\right) / T & S^{\circ}
\end{array}
$$

so

$\mathrm{C}_{\mathrm{p}}^{\circ}$

$$
-\left(F^{\circ}-E_{O}^{O}\right) / T
$$

$\mathrm{S}^{\circ}$

$\mathrm{Co}_{\mathrm{p}}$

$\mathrm{cm}^{-1}$

$\begin{array}{lllll}150 & 4.79458 & 6.68998 & 1.98571\end{array}$

$160 \quad 4.672456 .56183 \quad 1.98551$

$170 \quad 4.558096 .44147 \quad 1.98530$

$\begin{array}{lllll}180 & 4.45061 & 6.32800 \quad 1.98507\end{array}$

$190 \quad 4.34926 \quad 6.22068 \quad 1.98483$

$200 \quad 4.253426 .11887 \quad 1.98458$ $210 \quad 4.162556 .02205 \quad 1.98431$ $220 \quad 4.07618 \quad 5.92975 \quad 1.98404$ $230 \quad 3.993925 .84156 \quad 1.98375$ $240 \quad 3.915415 .75714 \quad 1.98344$

$250 \quad 3.84035 \quad 5.67618 \quad 1.98312$ $260 \quad 3.76846 \quad 5.59840 \quad 1.98279$ $270 \quad 3.69951 \quad 5.52358 \quad 1.98245$ $280 \quad 3.633285 .451491 .98210$ $290 \quad 3.569575 .38194 \quad 1.98173$

$300 \quad 3.50823 \quad 5.31476 \quad 1.98135$ $310 \quad 3.449095 .24980 \quad 1.98095$ $320 \quad 3.392015 .18691 \quad 1.98055$ $330 \quad 3.336875 .12598 \quad 1.98013$ $340 \quad 3.28354 \quad 5.06687 \quad 1.97970$

$350 \quad 3.23193 \quad 5.00949 \quad 1.97925$ $360 \cdot 3.181944 .95374 \quad 1.97879$ $370 \quad 3.133474 .899531 .97832$ $380 \quad 3.08645 \quad 4.84678 \quad 1.97784$ $390 \quad 3.04080 \quad 4.79541 \quad 1.97734$

$400 \quad 2.996454 .745351 .97683$ $410 \quad 2.953334 .696551 .97631$ $420 \quad 2.911394 .648931 .97578$ $430 \quad 2.870574 .60244 \quad 1.97523$ $440 \quad 2.830824 .55704 \quad 1.97467$

$450 \quad 2.792094 .51267 \quad 1.97410$ $460 \quad 2.75434 \quad 4.469291 .97351$ $470 \quad 2.717524 .42685 \quad 1.97292$ $\begin{array}{lllll}480 & 2.68159 & 4.38532 & 1.97231\end{array}$ $490 \quad 2.646524 .34466 \quad 1.97168$

$500 \quad 2.61226 \quad 4.30483 \quad 1.97105$ $\begin{array}{lllll}510 & 2.57880 & 4.26581 & 1.97040\end{array}$ $\begin{array}{lllll}520 & 2.54610 & 4.22755 & 1.96974\end{array}$ $530 \quad 2.514124 .190041 .96907$ $540 \quad 2.482854 .15324 \quad 1.96838$

$\begin{array}{llll}550 & 2.45225 & 4.11713 & 1.96768\end{array}$ $560 \quad 2.42230 \quad 4.08168 \quad 1.96697$ $\begin{array}{lllll}570 & 2.39298 & 4.04687 & 1.96625\end{array}$ $580 \quad 2.36426 \quad 4.01268 \quad 1.96551$ $590 \quad 2.33613 \quad 3.97909$
1.96476
$T=2400$

$5.651657 .57985 \quad 1.98657$ 5.468157 .390521 .98645 5.301137 .217681 .98631 $5.14795 \quad 7.058691 .98616$ $5.00656 \quad 6.911511 .98600$

$4.875336 .77449 \quad 1.98583$ $4.75295 \quad 6.64634 \quad 1.98565$ $4.638336 .52596 \quad 1.98545$ $4.53060 \quad 6.41248 \quad 1.98524$ 4.429016 .305151 .98502

$4.33292 \quad 6.20334 \quad 1.98479$ $4.241806 .10651 \quad 1.98455$ 4.155196 .014191 .98429 $4.07268 \quad 5.92599 \quad 1.98402$ $3.993925 .84156 \quad 1.98375$

$3.91861 \quad 5.76059 \quad 1.98345$ $3.84648 \quad 5.68280 \quad 1.98315$ $3.77728 \quad 5.60796 \quad 1.98284$ $3.71080 \quad 5.535851 .98251$ 3.646865 .466291 .98217

$3.585275 .39910 \quad 1.98182$ 3.525895 .334121 .98146 3.468575 .271221 .98109 $3.41318 \quad 5.21026 \quad 1.98070$ 3.359625 .151141 .98030

$3.30776 \quad 5.09374 \quad 1.97990$ $3.257535 .03797 \quad 1.97947$ $3.208824 .98374 \quad 1.97904$ $3.16156 \quad 4.93097 \quad 1.97860$ $3.115674 .87958 \quad 1.97814$

$\begin{array}{llll}3.07108 & 4.82950 & 1.97767\end{array}$ $3.02773 \quad 4.78068 \quad 1.97720$ $2.98555 \quad 4.73304 \quad 1.97670$ $2.94450 \quad 4.686531 .97620$ $2.90451 \quad 4.641101 .97569$

$2.86555 \quad 4.59671 \quad 1.97516$ $2.82756 \quad 4.55330 \quad 1.97462$ $2.79050 \quad 4.51084 \quad 1.97407$ $2.75434 \quad 4.46929 \quad 1.97351$ 2.719034 .428601 .97294

$2.68455 \quad 4.38875 \quad 1.97236$ $2.65085 \quad 4.34970 \quad 1.97176$ 2.617924 .311411 .97115 $2.585714 .27387 \quad 1.97054$ 2.554204 .237051 .96991

$2.523374 .20091 \quad 1.96926$ 2.493194 .165431 .96861 $2.46364 \quad 4.130591 .96795$ $2.43470 \quad 4.09637 \quad 1.96727$ $2.40634 \quad 4.06275 \quad 1.96658$ $\mathrm{r}=2500$.

$5.730417 .66095 \quad 1.98662$ $5.546677 .47161 \quad 1.98651$ $5.379427 .29876 \quad 1.98638$ 5.226017 .139771 .98624 $5.084396 .99258 \quad 1.98610$

$4.95293 \quad 6.85556 \quad 1.98594$ $4.830326 .72740 \quad 1.98577$ $4.715476 .60702 \quad 1.98559$ 4.607516 .493531 .98539 4.505696 .386191 .98519

$4.40937 \quad 6.28437 \quad 1.98498$ 4.318026 .187531 .98475 $4.23118 \quad 6.09520 \quad 1.98452$ $4.148446 .00699 \quad 1.98427$ 4. 069465.922551 .98401

$3.993925 .84156 \quad 1.98375$ $3.92156 \quad 5.76376 \quad 1.98347$ $3.852145 .68891 \quad 1.98318$ 3.785445 .616791 .98288

3.721275 .547221 .98256

$3.65945 \quad 5.48001 \quad 1.98224$ 3.599855 .415021 .98191 3.542305 .352101 .98156 3.486695 .291131 .98121 $3.43290 \quad 5.23199 \quad 1.98084$

$3.38083 \quad 5.17458 \quad 1.98046$ 3.330375 .118791 .98008 $3.28145 \quad 5.06454 \quad 1.97968$ $3.23396 \quad 5.011751 .97927$ $3.18785 \quad 4.96035 \quad 1.97885$

3. $14304 \quad 4.91025 \quad 1.97842$ $3.09947 \quad 4.86140 \quad 1.97798$ $3.05708 \quad 4.81375 \quad 1.97752$ 3. $01580 \quad 4.76722 \quad 1.97706$ $2.97560 \quad 4.72177 \quad 1.97658$

$2.936424 .67736 \quad 1.97610$ 2.898214 .633931 .97560 $2.86094 \quad 4.591451 .97510$ $2.82456 \quad 4.54987 \quad 1.97458$ 2.789044 .509161 .97405

$2.75434 \quad 4.46929 \quad 1.97351$ 2.720434 .430211 .97296 $2.68728 \quad 4.39191 \quad 1.97240$ $2.65486 \quad 4.35434 \quad 1.97183$ $2.62314 \quad 4.317491 .97125$

2.592094 .281321 .97066 2.561704 .245821 .97006 $2.53194 \quad 4.21096 \quad 1.96944$ $2.50278 \quad 4.17671 \quad 1.96882$ 2.474214 .143061 .96819
100

110

120

130

140

150

160

170

180

190

200

210

220

230

240

250

260

270

280

290

300

310

320

330

340

350

360

370

380

390

400

410

420

430

440

450

460

470

480

490

500

510

520

530

540

550

560

570

580

590 
Table II Harmonic Oscillator Contributions to the Thermodynamic

Functions (in units of calories, moles, and ${ }^{\circ} \mathrm{K}$ )

\begin{abstract}
$\nu \quad-\left(F^{\circ}-E_{0}^{\circ}\right) / T \quad S^{\circ}$
$\mathrm{cm}^{-1}$

$T=2300$

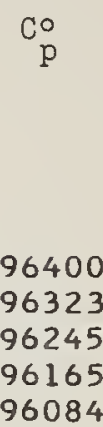

600

$2.30856 \quad 3.94607 \quad 1.96400$

610

620

630

$2.28154 \quad 3.91361 \quad 1.96323$

$2.255043 .88170 \quad 1.96245$

$2.22906 \quad 3.850301 .96165$

$\begin{array}{lll}640 & 2.20357 & 3.81942 \quad 1.96084\end{array}$

$650 \quad 2.17856 \quad 3.78902 \quad 1.96002$

$660 \quad 2.15401 \quad 3.75910 \quad 1.95918$

$670 \quad 2.12991 \quad 3.72965 \quad 1.95833$

$\begin{array}{lllll}680 & 2.10625 & 3.70064 & 1.95747\end{array}$

$690 \quad 2.083023 .67207 \quad 1.95660$
\end{abstract}

$\begin{array}{lllll}700 & 2.06019 & 3.64393 & 1.95572\end{array}$

$\begin{array}{ll}710 & 2.03776 \quad 3.61619 \quad 1.95482\end{array}$

$\begin{array}{lllll}720 & 2.01572 & 3.58886 & 1.95391\end{array}$

$\begin{array}{lllll}730 & 1.99406 & 3.56191 & 1.95299\end{array}$

$\begin{array}{lllll}740 & 1.97276 & 3.53535 & 1.95206\end{array}$

750

760

770

780

790

$1.95183 \quad 3.50915 \quad 1.95112$

$1.931233 .48331 \quad 1.95016$

$1.91098 \quad 3.457831 .94919$

$1.89105 \quad 3.43268 \quad 1.94821$

$1.87145 \quad 3.40787 \quad 1.94722$

800

810

820

830

840

850

860

870

880

890

900

910

920

930

940

$1.85215 \quad 3.38338 \quad 1.94621$ $1.83316 \quad 3.35921 \quad 1.94519$ 1.814473 .335351 .94416 $1.796073 .31179 \quad 1.94312$ $1.77794 \quad 3.28853 \quad 1.94207$

1.760103 .26555 .1 .94100 $1.742523 .24285^{\circ} 1.93993$ $1.72520 \quad 3.22043 \quad 1.93884$ 1.708143 .198281 .93774 $1.691343 .17639 \quad 1.93662$

$1.674773 .15476 \quad 1.93550$ 1.658443 .133381 .93436 $1.642353 .11224 \quad 1.93322$ 1.626493 .091351 .93206 1.610853 .070691 .93089

950

960

970

980

990

$1.59543 \quad 3.05027 \quad 1.92970$

$1.580223 .03007 \quad 1.92851$

1.565223 .010091 .92730

1.550432 .990331 .92608

$1.535832 .97078 \quad 1.92486$

1000

1010

1020

1030

$1.521442 .95144 \quad 1.92361$

$1.507232 .93230 \quad 1.92236$

$1.49322 \quad 2.913371 .92110$

1040

$1.47938 \quad 2.89463 \quad 1.91982$

1.465732 .876091 .91854

$\begin{array}{lllll}1050 & 1.45226 & 2.85774 & 1.91724\end{array}$

$1060 \quad 1.43896 \quad 2.83957 \quad 1.91593$

$1070 \quad 1.42583 \quad 2.82159 \quad 1.91461$

$1080 \quad 1.41287 \quad 2.80378 \quad 1.91328$

$109 \mathrm{C} \quad 1.400072 .786161 .91193$
$-\left(F \circ-E_{O}^{O}\right) / T$

so

$\mathrm{C}_{\mathrm{p}}^{\circ}$

$-\left(F^{\circ}-E_{O} O\right) / T$

So

v

$\mathrm{cm}^{-1}$

$T=2400$.

$\mathrm{T}=2500$.

$2.37854 \quad 4.02970 \quad 1.96588$ $2.35129 \quad 3.99721 \quad 1.96517$ $2.324573 .96526 \quad 1.96445$ $2.29836 \quad 3.93384 \quad 1.96372$ 2.272653 .902721 .96297

$2.247413 .87249 \quad 1.96221$ $2.222643 .84254 \quad 1.96145$ $2.19832 \quad 3.81305 \quad 1.96067$ $2.17443 \quad 3.78400 \quad 1.95988$ $2.150973 .75540 \quad 1.95908$

$2.12793 \quad 3.72722 \quad 1.95826$ $2.10528 \quad 3.69944 \quad 1.95744$ $2.08302 \quad 3.67207 \quad 1.95660$ $2.06113 \quad 3.64509 \quad 1.95576$ $2.03962 \quad 3.618491 .95490$

$2.01846 \quad 3.59225 \quad 1.95403$ $1.99765 \quad 3.56638 \quad 1.95315$ $1.97717 \quad 3.54085 \quad 1.95226$ $1.95703 \quad 3.51567 \quad 1.95135$ $1.93720 \quad 3.49081 \quad 1.95044$

$1.917693 .46628 \quad 1.94951$ $1.898493 .44207 \quad 1.94858$ 1.879583 .418171 .94763 $1.86096 \quad 3.39457 \quad 1.94667$ $1.842623 .37126 \quad 1.94570$

$1.82456 \quad 3.34824 \quad 1.94472$ 1.806773 .325501 .94373 $1.789243 .30303 \quad 1.94273$ 1.771973 .280841 .94172 1.754943 .258901 .94069

$\begin{array}{llll}1.73817 & 3.23722 & 1.93966\end{array}$ $1.72163 \quad 3.21580 \quad 1.93861$ $1.70533 \quad 3.19461 \quad 1.93755$ $1.689253 .17367 \quad 1.93648$ $1.67340 \quad 3.15297 \quad 1.93541$

$\begin{array}{llll}1.65777 & 3.13249 & 1.93432\end{array}$ $1.64235 \quad 3.11224 \quad 1.93322$ $1.62715 \quad 3.09222 \quad 1.93211$ $1.612143 .07241 \quad 1.93098$ $1.59734 \quad 3.05281 \quad 1.92985$

$1.58274 \quad 3.03342 \quad 1.92871$ $1.56833 \quad 3.01423 \quad 1.92755$ $1.554112 .99525 \quad 1.92639$ $1.540072 .97646 \quad 1.92522$ $1.526212 .95786 \quad 1.92403$

$1.51254 \quad 2.93946 \quad 1.92283$ 1.499032 .921241 .92163 $\begin{array}{llll}1.48570 & 2.90320 & 1.92041\end{array}$ $1.47254 \quad 2.88534 \quad 1.91918$ $1.459542 .86766 \quad 1.91794$
$2.446214 .10999 \quad 1.96754$ $2.41875 \quad 4.07747 \quad 1.96689$ 2.391824 .045491 .96622 $2.36540 \quad 4.01404 \quad 1.96554$ $2.339473 .98309 \quad 1.96486$

$2.31403 \quad 3.952631 .96416$ $2.28905 \quad 3.92265 \quad 1.96345$ $2.26452 \quad 3.89313 \quad 1.96273$ $2.24043 \quad 3.86405 \quad 1.96200$ $2.21676 \quad 3.83542 \quad 1.96126$

$2.19351 \quad 3.80720 \quad 1.96051$ $2.17065 \quad 3.77940 \quad 1.95975$ $2.148193 .75199 \quad 1.95898$ $2.126103 .72498 \quad 1.95820$ $2.10438 \quad 3.69834 \quad 1.95740$

$2.08302 \quad 3.67207 \quad 1.95660$ $2.06200 \quad 3.64616 \quad 1.95579$ $2.04132 \quad 3.62060 \quad 1.95497$ 2.02098 3.59538 1.95413 $2.000953 .57049 \quad 1.95329$

$1.98124 \quad 3.54593 \quad 1.95243$ $1.96183 \quad 3.52168 \quad 1.95157$ $1.94272 \quad 3.49774 \quad 1.95070$ $1.92390 \quad 3.47410 \quad 1.94981$ $1.905373 .45075 \quad 1.94892$

$1.887113 .42769 \quad 1.94801$ $1.869113 .40491 \quad 1.94710$ $1.851393 .38241 \quad 1.94617$ $1.83392 \quad 3.36017 \quad 1.94523$ $1.81670 \quad 3.33820 \quad 1.94429$

$1.79972 \quad 3.31648 \quad 1.94333$ 1.782993 .295011 .94236 $1.76649 \quad 3.27379 \quad 1.94139$ $1.750223 .25281 \quad 1.94040$ $1.73418 \quad 3.23206 \quad 1.93941$

$1.71835 \quad 3.21154 \quad 1.93840$ $1.702743 .19125 \quad 1.93738$ 1.687343 .171181 .93636 1.672143 .151321 .93532 $1.657153 .13168 \quad 1.93427$

$1.54235 \quad 3.11224 \quad 1.93322$ 1.627753 .093011 .93215 $1.613343 .07398 \quad 1.93107$ $1.599113 .05515 \quad 1.92999$ 1.585063 .036511 .92889

$1.571193 .01805 \quad 1.92779$ $1.55750 \quad 2.99978 \quad 1.92667$ $1.54398 \quad 2.98170 \quad 1.92555$ $1.530632 .96379 \quad 1.92441$ $1.517442 .94606 \quad 1.92327$
600

610

620

630

640

650

660

670

680

690

700

710

720

730

740

750

760

770

780

790

800

810

820

830

840

850

860

870

880

890

900

910

920

930

940

950

960

970

980

990

1000

1010

1020

1030

1040

1050

1060

1070

1080

1090 
Table II Harmonic Oscillator Contributions to the Thermodynamic

Functions (in units of calories, moles, and ${ }^{\circ} \mathrm{K}$ )
$\mathrm{cm}^{-1}$
S०
$\mathrm{C}_{\mathrm{p}}^{\mathrm{O}} \quad-\left(\mathrm{F} O-\mathrm{E}_{\mathrm{O}} \mathrm{O}\right) / \mathrm{T} \quad \mathrm{S}^{\circ}$
$\mathrm{C}_{\mathrm{p}}^{\circ}$
$-\left(F^{\circ}-E_{0}^{\circ}\right) / T$
So
$\mathrm{c}_{\mathrm{p}}^{\circ}$
$\mathrm{m}^{-1}$

$T=2300$.

$T=2400$.

$T=2500$.

$1100 \quad 1.38744 \quad 2.76870 \quad 1.91058$ $1110 \quad 1.37496 \quad 2.75142 \quad 1.90921$ $1120 \quad 1.36264 \quad 2.73430 \quad 1.90784$ $1130 \quad 1.35046 \quad 2.71735 \quad 1.90645$ $1140 \quad 1.338442 .70056 \quad 1.90505$

$\begin{array}{lllll}1150 & 1.32657 & 2.68392 & 1.90364\end{array}$ $1160 \quad 1.314842 .66745 \quad 1.90222$ $1170 \quad 1.30325 \quad 2.65113 \quad 1.90079$ $\begin{array}{lllll}1180 & 1.29179 & 2.63496 & 1.89934\end{array}$ $1190 \quad 1.28048 \quad 2.61893 \quad 1.89789$

$\begin{array}{lllll}1200 \quad 1.26930 & 2.60306 & 1.89642\end{array}$ $1210 \quad 1.25825 \quad 2.58733 \quad 1.89495$ 1220

1230

1240

1250

1260

1270

1280

1290

1300

1310

1320

1330

1340

1350

1360

1370

1380

1390

1400

1410

1420

1430

1440

1450

1460

1470

1480

1490

1500

1510

1520

1530

1540

1550

1560

1570

1580

1590

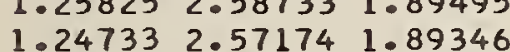
1.236542 .556281 .89196 $1.22587 \quad 2.54097 \quad 1.89046$

$1.21532 \quad 2.52579 \quad 1.88894$ $1.20490 \quad 2.51075 \quad 1.88741$ $1.19460 \quad 2.49583 \quad 1.88587$ $1.184412 .48105 \quad 1.88431$ $1.17434 \quad 2.46639 \quad 1.88275$

$1.16438 \quad 2.45186 \quad 1.88118$ $1.154532 .43745 \quad 1.87960$ $1.144792 .42316 \quad 1.87800$ $1.13516 \quad 2.40899 \quad 1.87640$ $1.125632 .39494 \quad 1.87479$

$1.116212 .38101 \quad 1.87316$ $1.106892 .36719 \quad 1.87153$ $1.09768 \quad 2.35349 \quad 1.86988$ $\begin{array}{llll}1.08856 & 2.33989 & 1.86822\end{array}$ $1.07954 \quad 2.32641 \quad 1.86656$

$1.070622 .31304 \quad 1.86488$ $\begin{array}{llll}1.06179 & 2.29977 & 1.86320\end{array}$ $1.05306 \quad 2.28661 \quad 1.86150$ $1.04442 \quad 2.27355 \quad 1.85979$ $1.035872 .26060 \quad 1.85807$

$\begin{array}{llll}1.02741 & 2.24774 & 1.85635\end{array}$ 1.019042 .234991 .85461 1.010752 .222341 .85286 $1.00255 \quad 2.20978 \quad 1.85111$ $0.99444 \quad 2.19732 \quad 1.84934$

$0.98640 \quad 2.18496 \quad 1.84756$ $0.97846 \quad 2.17269 \quad 1.84578$ $\begin{array}{llll}0.97059 & 2.16051 & 1.84398\end{array}$ $0.96280 \quad 2.14842 \quad 1.84217$ 0.955092 .136431 .84036

$0.94746 \quad 2.1 .2452 \quad 1.83853$ $0.93990 \quad 2.11271 \quad 1.83670$ $\begin{array}{llll}0.93242 & 2.10098 & 1.83485\end{array}$ 0.925012 .089331 .83300 $0.91768 \quad 2.07777 \quad 1.83114$
$1.44670 \quad 2.85015 \quad 1.91670$ 1.434022 .832811 .91544 1.421492 .815631 .91417 1.409122 .798621 .91289 $1.39690 \quad 2.78178 \quad 1.91160$

$1.384832 .76509 \quad 1.91030$ $1.37290 \quad 2.74855 \quad 1.90899$ $\begin{array}{llll}1.36111 & 2.73217 & 1.90766\end{array}$ $1.34946 \quad 2.71594 \quad 1.90633$ $1.33794 \quad 2.69986 \quad 1.90499$

$1.326572 .68392 \quad 1.90364$ $1.31532 \quad 2.668131 .90228$ $\begin{array}{llll}1.30421 & 2.65248 & 1.90091\end{array}$ $1.29322 \quad 2.63697 \quad 1.89952$ $1.28236 \quad 2.62159 \quad 1.89813$

1.271622 .606351 .89673 $1.26100 \quad 2.59125 \quad 1.89532$ $\begin{array}{llll}1.25050 & 2.57627 & 1.89390\end{array}$ 1.240122 .561421 .89246 $1.229852 .54670 \quad 1.89102$

$\begin{array}{llll}1.21970 & 2.53210 & 1.88957\end{array}$ 1.209662 .517631 .88811 $\begin{array}{llll}1.19973 & 2.50327 & 1.88664\end{array}$ $1.189912 .48904 \quad 1.88516$ $1.18020 \quad 2.47493 \quad 1.88367$

1.170592 .460931 .88216 $1.16108 \quad 2.44704 \quad 1.88065$ $1.151682 .43327 \quad 1.87913$ $1.142372 .41961 \quad 1.87760$ $1.133162 .40606 \quad 1.87606$

$1.12406 \quad 2.39261 \quad 1.87452$ $1.11504 \quad 2.37928 \quad 1.87296$ 1.106122 .366051 .87139 $1.09730 \quad 2.35292 \quad 1.86981$ $1.08856 \quad 2.33989 \quad 1.86822$

$1.07992 \quad 2.32697 \quad 1.86663$ $1.07136 \quad 2.314151 .86502$ $1.062892 .30142 \quad 1.86341$ $\begin{array}{llll}1.05451 & 2.28879 & 1.86178\end{array}$ $1.046212 .27626 \quad 1.86015$

$1.03800 \quad 2.26383 \quad 1.85850$ $1.029872 .25148 \quad 1.85685$ $1.021822 .23923 \quad 1.85519$ $1.01385 \quad 2.22707 \quad 1.85352$ $1.00596 \quad 2.21500 \quad 1.85184$

$0.99815 \quad 2.20302 \quad 1.85015$ 0.990412 .191131 .84845 $0.98275 \quad 2.17932 \quad 1.84675$ 0.975172 .167601 .84503 $0.96766 \quad 2.15597 \quad 1.84330$
$1.504412 .92850 \quad 1.92211$ 1.491552 .911111 .92095 $1.478832 .89389 \quad 1.91977$ $1.46628 \quad 2.876831 .91859$ 1.453872 .859931 .91740

$1.441612 .84319 \quad 1.91619$ 1.429492 .826601 .91498 $1.41752 \quad 2.81017 \quad 1.91376$ $1.405692 .79389 \quad 1.91253$ $1.393992 .77776 \quad 1.91129$

$1.382432 .76177 \quad 1.91003$ $\begin{array}{llll}1.37100 & 2.74592 & 1.90877\end{array}$ $1.35970 \quad 2.73022 \quad 1.90751$ 1.348532 .714651 .90623 $1.337492 .69922 \quad 1.90494$

$1.326572 .68392 \quad 1.90364$ $1.315772 .66876 \quad 1.90233$ 1.305092 .653731 .90102 1.294532 .638821 .89969 $1.284092 .62404 \quad 1.89836$

$1.27375 \quad 2.60939 \quad 1.89701$ $1.26354 \quad 2.59486 \quad 1.89566$ $1.25343 \quad 2.58045 \quad 1.89430$ $1.243432 .56616 \quad 1.89292$ $1.233542 .55198 \quad 1.89154$

$1.22375 \quad 2.53792 \quad 1.89015$ $1.214072 .52398 \quad 1.88875$ 1.204492 .510151 .88735 1.195012 .496431 .88593 $1.18562 \quad 2.48282 \quad 1.88450$

$\begin{array}{llll}1.17634 & 2.46931 & 1.88307\end{array}$ 1.167152 .455911 .88162 $1.15806 \quad 2.44262 \quad 1.88017$ $1.14906 \quad 2.42943 \quad 1.87871$ $1.140152 .41635 \quad 1.87724$

$1.13134 \quad 2.40336 \quad 1.87576$ $1.122612 .39047 \quad 1.87427$ $\begin{array}{llll}1.11397 & 2.37768 & 1.87277\end{array}$ $1.105412 .36499 \quad 1.87126$ $1.096942 .35240 \quad 1.86975$

$\begin{array}{lll}1.08856 & 2.33989 & 1.86822\end{array}$ $1.080262 .32749 \quad 1.86669$ $1.072042 .31517 \quad 1.86515$ $1.06390 \quad 2.30294 \quad 1.86360$ 1.055842 .290811 .86204

$\begin{array}{llll}1.04786 & 2.27876 & 1.86048\end{array}$ $1.03996 \quad 2.26680 \quad 1.85890$ $1.03213 \quad 2.25493 \quad 1.85732$ $1.02438 \quad 2.24314 \quad 1.85572$ $1.016712 .23144 \quad 1.85412$
1100

1110

1120

1130

1140

1150

1160

1170

1180

1190

1200

1210

1220

1230

1240

1250

1260

1270

1280

1290

1300

1310

1320

1330

1340

1350

1360

1370

1380

1390

1400

1410

1420

1430

1440

1450

1460

1470

1480

1490

1500

1510

1520

1530

1540

1550

1560

1570

1580

1590 
Table II Harmonic Oscillator Contributions to the Thermodynamic

Functions (in units of calories, moles, and ${ }^{\circ} \mathrm{K}$ )
$\nu \quad-(F \circ-E O) / T$
So
$\mathrm{C}_{\mathrm{p}}^{\circ}$
$-\left(F^{\circ}-E_{O}\right) / T \quad S^{\circ}$
$\mathrm{C} \stackrel{\mathrm{p}}{\mathrm{P}}$
$-\left(F^{\circ}-E_{O}^{O}\right) / T$
So
$\nu$
$\mathrm{cm}^{-1}$

$T=2300$.

1600

1610

1620

1630

1640

1650

1660

1670

1680

1690

1700

1710

1720

1730

1740

1750

1760

1770

1780

1790

$\begin{array}{lllll}1800 & 0.77905 & 1.85308 & 1.78988\end{array}$ $\begin{array}{lllll}1810 & 0.77311 & 1.84317 & 1.78781\end{array}$

$\begin{array}{lllll}1820 & 0.76722 & 1.83333 & 1.78574\end{array}$

$\begin{array}{lllll}1830 & 0.76139 & 1.82355 & 1.78366\end{array}$

$\begin{array}{llll}1840 & 0.75562 & 1.81383 & 1.78157\end{array}$

$\begin{array}{llll}1850 & 0.74989 & 1.80418 & 1.77948\end{array}$

$\begin{array}{lllll}1860 & 0.74422 & 1.79459 & 1.77737\end{array}$

$\begin{array}{lllll}1870 & 0.73860 & 1.78507 & 1.77526\end{array}$

$\begin{array}{lllll}1880 & 0.73303 & 1.77561 & 1.77314\end{array}$

$\begin{array}{lllll}1890 & 0.72750 & 1.76621 & 1.77101\end{array}$

$\begin{array}{lllll}1900 & 0.72203 & 1.75687 & 1.76887\end{array}$

$\begin{array}{lllll}1910 & 0.71661 & 1.74759 & 1.76672\end{array}$

$\begin{array}{lllll}1920 & 0.71124 & 1.73837 & 1.76457\end{array}$

$\begin{array}{lllll}1930 & 0.70591 & 1.72921 & 1.76241\end{array}$

$\begin{array}{llll}1940 & 0.70063 & 1.72010 & 1.76024\end{array}$

$\begin{array}{lllll}1950 & 0.69540 & 1.71106 & 1.75806\end{array}$

$\begin{array}{lllll}1960 & 0.69022 & 1.70207 & 1.75587\end{array}$

$\begin{array}{llllll}1970 & 0.68508 & 1.69314 & 1.75368\end{array}$

$\begin{array}{lllll}1980 & 0.67998 & 1.68427 & 1.75148\end{array}$

$\begin{array}{lllll}1990 & 0.67493 & 1.67545 & 1.74927\end{array}$

$\begin{array}{lllll}2000 & 0.66993 & 1.66669 & 1.74705\end{array}$

$\begin{array}{lllll}2010 & 0.66496 & 1.65798 & 1.74482\end{array}$

$\begin{array}{llll}2020 & 0.66005 & 1.64932 & 1.74259\end{array}$

$2030 \quad 0.65517 \quad 1.64072 \quad 1.74035$

$2040 \quad 0.65034 \quad 1.63218 \quad 1.73810$

$\begin{array}{lllll}2050 & 0.64554 & 1.62368 & 1.73585\end{array}$

$\begin{array}{lllll}2060 & 0.64079 & 1.61524 & 1.73358\end{array}$

$2070 \quad 0.63608 \quad 1.60685 \quad 1.73131$

$2080 \quad 0.63141 \quad 1.59852 \quad 1.72903$

$2090 \quad 0.62678 \quad 1.59023 \quad 1.72675$

$T=2400$.

$$
T=2500 \text {. }
$$

$0.96022 \quad 2.14442 \quad 1.84157$ $0.952852 .13295 \quad 1.83983$ $0.94556 \quad 2.12156 \quad 1.83808$ $0.93833 \quad 2.11026 \quad 1.83632$ 0.931182 .099031 .83455

$0.924092 .08788 \quad 1.83277$ 0.917072 .076811 .83098 0.910122 .065821 .82919 $0.903232 .05491 \quad 1.82738$ $\begin{array}{lllll}0.89641 & 2.04407 & 1.82557\end{array}$

$0.889652 .03330 \quad 1.82375$ 0.882952 .022611 .82192 $0.876322 .01199 \quad 1.82008$ $0.869752 .00145 \quad 1.81823$ 0.863241 .990971 .81638

$0.85679 \quad 1.98057 \quad 1.81451$ $0.850391 .97024 \quad 1.81264$ $0.84406 \quad 1.95997 \quad 1.81076$ $\begin{array}{llll}0.83778 & 1.94977 & 1.80887\end{array}$ $0.83157 \quad 1.93965 \quad 1.80697$

$0.82540 \quad 1.92958 \quad 1.80507$ 0.819301 .919591 .80315 $0.813241 .90966 \quad 1.80123$ $0.807251 .89980 \quad 1.79930$ 0.801301 .890001 .79736

$0.79541 \quad 1.88026 \quad 1.79542$ 0.789581 .870591 .79346 $0.783791 .86097 \quad 1.79150$ 0.778051 .851421 .78953 $0.77237 \quad 1.84194 \quad 1.78756$

$0.76674 \quad 1.83251 \quad 1.78557$. $0.761151 .82314 \quad 1.78358$ $0.75562 \quad 1.81383 \quad 1.78157$ $0.75013 \quad 1.80458 \quad 1.77957$ $0.74469 \quad 1.79539 \quad 1.77755$

$0.73930 \quad 1.78626 \quad 1.77552$ $0.73395 \quad 1.777181 .77349$ 0.728651 .768161 .77145 $0.72340 \quad 1.759201 .76941$ 0.718191 .750291 .76735

$0.71302 \quad 1.74143 \quad 1.76529$ 0.707901 .732631 .76322 $0.70283 \quad 1.72389 \quad 1.76114$ $0.697791 .71520 \quad 1.75906$ $0.69280 \quad 1.70656 \quad 1.75697$

$0.68786 \quad 1.69797 \quad 1.75487$ 0.682951 .689441 .75276 $0.67808 \quad 1.68095 \quad 1.75065$ $0.67326 \quad 1.67252 \quad 1.74853$ 0.668471 .664141 .74640 $\begin{array}{llll}1.00910 & 2.21982 & 1.85251\end{array}$ $1.001572 .20828 \quad 1.85090$ 0.994112 .196831 .84927 $0.98672 \quad 2.18545 \quad 1.84763$ $0.97940 \quad 2.17416 \quad 1.84599$

$0.97215 \quad 2.16294 \quad 1.84434$ $0.96497 \quad 2.15180 \quad 1.84268$ $0.95785 \quad 2.14074 \quad 1.84101$ $0.95080 \quad 2.12975 \quad 1.83934$ $0.94382 \quad 2.11884 \quad 1.83765$

$0.93690 \quad 2.10800 \quad 1.83596$ $0.93004 \quad 2.09724 \quad 1.83426$ 0.923252 .086551 .83255 $0.916512 .07593 \quad 1.83084$ 0.909842 .065381 .82911

$0.90323 \quad 2.05491 \quad 1.82738$ $0.89668 \quad 2.04450 \quad 1.82564$ $0.890192 .03416 \quad 1.82389$ $0.88375 \quad 2.02389 \quad 1.82214$ $0.87738 \quad 2.013691 .82037$

$0.87106 \quad 2.00355 \quad 1.81860$ $0.864791 .99348 \quad 1.81682$ $0.858591 .98348 \quad 1.81503$ $0.852431 .97353 \quad 1.81324$ 0.846331 .963661 .81144

$0.84029 \quad 1.95384 \quad 1.80963$ 0.834291 .944091 .80781 $0.828351 .93441 \quad 1.80598$ $0.822461 .92478 \quad 1.80415$ 0.816631 .915211 .80231

$0.810841 .90571 \quad 1.80046$ $0.80510 \quad 1.89626 \quad 1.79861$ $\begin{array}{llll}0.79941 & 1.88687 & 1.79674\end{array}$ $\begin{array}{llll}0.79377 & 1.87754 & 1.79487\end{array}$ $\begin{array}{llll}0.78818 & 1.86827 & 1.79299\end{array}$

$0.782641 .85906 \quad 1.79111$ $0.77714 \quad 1.84990 \quad 1.78922$ $0.77169 \quad 1.84080 \quad 1.78732$ $0.76629 \quad 1.83176 \quad 1.78541$ $0.76093 \quad 1.82277 \quad 1.78350$

$0.75562 \quad 1.81383 \quad 1.78157$ $0.750351 .80495 \quad 1.77965$ 0.745121 .796121 .77771 $0.73994 \quad 1.787351 .77577$ $\begin{array}{llll}0.73480 & 1.77863 & 1.77382\end{array}$

$0.72971 \quad 1.76996 \quad 1.77186$ 0.724651 .761341 .76990 $0.71964 \quad 1.75278 \quad 1.76793$ $\begin{array}{llll}0.71467 & 1.74426 & 1.76595\end{array}$ $0.70974 \quad 1.73580 \quad 1.76397$
1600

1610

1620

1630

1640

1650

1660

1670

1680

1690

1700

1710

1720

1730

1740

1750

1760

1770

1780

1790

1800

1810

1820

1830

1840

1850

1860

1870

1880

1890

1900

1910

1920

1930

1940

1950

1960

1970

1980

1990

2000 2010 2020 2030 2040

2050 2060 2070 2080 2090 
Table II Harmonic Oscillator Contributions to the Thermodynamic

Functions (in units of calories, moles, and ${ }^{\circ} \mathrm{K}$ )
$\mathrm{cm}^{-1}$
So
$\mathrm{C}_{\mathrm{p}}^{\circ} \quad-\left(\mathrm{F} \circ-\mathrm{E}_{\mathrm{O}}^{\circ}\right) / \mathrm{T} \quad \mathrm{S}$
$\mathrm{C}_{\mathrm{p}} \quad-\left(\mathrm{F} \circ-\mathrm{E}_{\mathrm{O}} \mathrm{O}\right) / \mathrm{T}$
So
$\stackrel{\mathrm{p}}{\mathrm{p}}$
$\mathrm{cm}^{-1}$

$T=2300$.

$T=2400$.

$T=2500$.

$\begin{array}{lllll}2100 & 0.62219 & 1.58199 & 1.72445\end{array}$

$\begin{array}{lllll}2110 & 0.61764 & 1.57380 & 1.72215\end{array}$

$\begin{array}{lllll}2120 & 0.61313 & 1.56567 & 1.71985\end{array}$

$\begin{array}{lllll}2130 & 0.60866 & 1.55758 & 1.71753\end{array}$

$\begin{array}{lllll}2140 & 0.60422 & 1.54954 & 1.71521\end{array}$

$\begin{array}{lllll}2150 & 0.59982 & 1.54155 & 1.71288\end{array}$

$\begin{array}{llllll}2160 & 0.59546 & 1.53361 & 1.71054\end{array}$

$\begin{array}{lllll}2170 & 0.59113 & 1.52571 & 1.70820\end{array}$

$\begin{array}{lllll}2180 & 0.58685 & 1.51786 & 1.70585\end{array}$

$\begin{array}{lllll}2190 \quad 0.58259 & 1.51006 & 1.70349\end{array}$

$\begin{array}{lllll}2200 & 0.57838 & 1.50231 & 1.70113\end{array}$

$2210 \cdot 0.574191 .494601 .69876$

$\begin{array}{lllll}2220 & 0.57005 & 1.48693 & 1.69638\end{array}$

$\begin{array}{lllll}2230 & 0.56593 & 1.47931 & 1.69400\end{array}$

$\begin{array}{lllll}2240 & 0.56185 & 1.47174 & 1.69161\end{array}$

$\begin{array}{llllll}2250 & 0.55781 & 1.46421 & 1.68921\end{array}$

2260

2270

2280

2290

2300

2310

2320

2330

2340

2350

2360

2370

2380

2390

2400

2410

2420

2430

2440

2450

2460

2470

2480

2490

2500

2510

2520

2530

2540

2550

2560

2570

2580

2590
$0.55380 \quad 1.45672 \quad 1.68681$

$0.54982 \quad 1.44928 \quad 1.68440$

$0.54587 \quad 1.44188 \quad 1.68198$

$0.54196 \quad 1.43453 \quad 1.67956$

$0.53808 \quad 1.42721 \quad 1.67713$ $0.534231 .41994 \quad 1.67469$ 0.530411 .412711 .67225 0.526621 .405531 .66980 $0.52286 \quad 1.39838 \quad 1.66734$

$0.51914 \quad 1.39128 \quad 1.66488$ $0.515441 .38421 \quad 1.66241$ $0.51177 \quad 1.37719 \quad 1.65994$ $0.50814 \quad 1.370201 .65746$ 0.504531 .363261 .65498

$0.50095 \quad 1.35635 \quad 1.65248$ $0.49740 \quad 1.349491 .64999$ $0.49388 \quad 1.34266 \quad 1.64748$ $0.49039 \quad 1.33587 \quad 1.64497$ 0.486921 .329121 .64246

$\begin{array}{llll}0.48348 & 1.32241 & 1.63994\end{array}$ $0.48007 \quad 1.31574 \quad 1.63741$ 0.476691 .309101 .63488 0.473331 .302501 .63234 0.470001 .295931 .62980

$0.46670 \quad 1.28941 \quad 1.62725$ 0.463421 .282921 .62470 $0.46017 \quad 1.27646 \quad 1.62214$ 0.456941 .270041 .61957 $0.45374 \quad 1.26366 \quad 1.61700$

$0.450571 .25731 \quad 1.61443$ 0.447411 .251001 .61185 0.444291 .244721 .60926 $0.441181 .23847 \quad 1.60667$ 0.438111 .232261 .60408
$0.66373 \quad 1.65581 \quad 1.74427$ 0.659031 .647531 .74212 $0.65436 \quad 1.639301 .73998$ 0.649731 .631111 .73782 0.645151 .622981 .73566

$0.640601 .61489 \quad 1.73349$ $0.63608 \quad 1.606851 .73131$ 0.631611 .598861 .72913 0.627171 .590921 .72694 $0.62277 \quad 1.583021 .72474$

$0.61840 \quad 1.57517 \quad 1.72254$ $0.61407 \quad 1.56736 \quad 1.72033$ 0.609771 .559601 .71811 $0.60551 \quad 1.55188 \quad 1.71589$ $0.60128 \quad 1.54421 \quad 1.71366$

$0.59709 \quad 1.53658 \quad 1.71142$ 0.592931 .528991 .70918 $0.58881 \quad 1.521451 .70693$ $0.58471 \quad 1.51396 \quad 1.70467$ 0.580661 .506501 .70241

0.576631 .499091 .70014 $0.572631 .49172 \quad 1.69787$ 0.568671 .484391 .69559 $0.56474 \quad 1.477101 .69330$ 0.560841 .469851 .69101

$0.55697 \quad 1.462651 .68871$ $0.553131 .45548 \quad 1.68641$ 0.549321 .448351 .68409 $0.54554 \quad 1.44127 \quad 1.68178$ 0.541801 .434221 .67945

$0.53808 \quad 1.42721 \quad 1.67713$ 0.534391 .420251 .67479 $0.53073 \quad 1.413321 .67245$ 0.527091 .406421 .67010 $0.523491 .39957 \quad 1.66775$

$0.51991 \quad 1.39275 \quad 1.66539$ $0.51636 \quad 1.38597 \quad 1.66303$ 0.512841 .379231 .66066 0.509351 .372531 .65829 $0.50588 \quad 1.36586 \quad 1.65591$

$0.50244 \quad 1.35923 \quad 1.65352$ 0.499031 .352631 .65113 $0.495641 .34607 \quad 1.64874$ 0.492281 .339551 .64633 $0.48894 \quad 1.33306 \quad 1.64393$

0.485631 .326601 .64151 0.482341 .320181 .63910 0.479081 .313801 .63667 $0.475851 .30744 \quad 1.63425$ 0.472641 .301131 .63181
$0.70485 \quad 1.72738 \quad 1.76197$

0.700001 .719011 .75998 0.695191 .710701 .75797 0.690421 .702431 .75596 0.685691 .694211 .75394

$0.68100 \quad 1.68604 \quad 1.75192$ $0.676341 .67791 \quad 1.74989$ 0.671721 .669831 .74785 0.667141 .661801 .74580 0.662601 .653821 .74375

$0.65809 \quad 1.64588 \quad 1.74169$ $0.653621 .63798 \quad 1.73963$ 0.649181 .630141 .73756 0.644781 .622331 .73548 $0.64041 \quad 1.61457 \quad 1.73340$

$0.63608 \quad 1.606851 .73131$ $0.63179 \quad 1.59918 \quad 1.72922$ 0.627521 .591551 .72711 0.623291 .583961 .72500 $0.619091 .57642 \quad 1.72289$

$0.61493 \quad 1.56892 \quad 1.72077$ $0.61080 \quad 1.56146 \quad 1.71864$ 0.606701 .554041 .71651 $0.602631 .54666 \quad 1.71437$ 0.598601 .539321 .71223

0.594591 .532021 .71008 $0.590621 .52477 \quad 1.70792$ 0.586671 .517551 .70576 $0.58276 \quad 1.51037 \quad 1.70359$ $0.57888 \quad 1.503231 .70141$

$0.57503 \quad 1.496131 .69923$ $0.571201 .48907 \quad 1.69705$ 0.567411 .482051 .69486 $0.563641 .47507 \quad 1.69266$ 0.559911 .468121 .69046

0.556201 .461211 .68825 0.552521 .454341 .68604 $0.54887 \quad 1.44750 \quad 1.68382$ 0.545241 .440701 .68159 0.541651 .433941 .67936

$0.53808 \quad 1.42721 \quad 1.67713$ 0.534531 .420521 .67488 $0.53102 \quad 1.413871 .67264$ 0.527531 .407251 .67039 0.524061 .400661 .66813

0.520621 .394111 .66587 $0.51721 \quad 1.387601 .66360$ 0.513821 .381121 .66133 0.510461 .374671 .65905 $0.507131 .36826 \quad 1.65677$
2100

2110

2120

2130

2140

2150

2160

2170

2180

2190

2200

2210

2220

2230

2240

2250

2260

2270

2280

2290

2300

2310

2320

2330

2340

2350

2360

2370

2380

2390

2400

2410

2420

2430

2440

2450

2460

2470

2480

2490

2500

2510

2520

2530

2540

2550

2560

2570

2580

2590 
Table II Harmonic Oscillator Contributions to the Thermodynamic Functions (in units of calories, moles, and ${ }^{\circ} \mathrm{K}$ )

$$
\begin{aligned}
& \mathrm{cm}^{-1} \\
& \text { So } \\
& -\left(F^{\circ}-E_{0}^{\circ}\right) / T \\
& T=2300 \text {. } \\
& \mathrm{co}
\end{aligned}
$$$$
-\left(F^{\circ}-E O\right) / T \quad S^{\circ}
$$$$
\mathrm{C}_{\mathrm{p}}
$$$$
-\left(\mathrm{FO}^{\circ}-\mathrm{E}_{\mathrm{O}}^{\circ}\right) / \mathrm{T}
$$$$
S^{\circ}
$$

2650

2660

2670

2680

2690

2700

2710

2720

2730

2740

2750

2760

2770

2780

2790

2800

2810

2820

2830

2840

2850

2860

2870

2880

2890

2900

2910

2920

2930

2940

2950

2960

2970

2980

2990

3000

3010

3020

3030

3040

3050

3060

3070

3080

3090
$0.420131 .19570 \quad 1.58840$ $0.41722 \quad 1.18973 \quad 1.58577$ 0.414321 .183781 .58314 $0.41145 \quad 1.17787 \quad 1.58050$ $0.408601 .17199 \quad 1.57785$

$0.405781 .16614 \quad 1.57521$ 0.402971 .160321 .57255 0.400191 .154531 .56990 0.397421 .148771 .56723 $0.39468 \quad 1.14305 \quad 1.56457$

$0.39196 \quad 1.137351 .56190$ $0.38926 \quad 1.131691 .55922$ $0.386581 .12606 \quad 1.55655$ 0.383921 .120451 .55386 $0.38128 \quad 1.11488 \quad 1.55118$

0.378661 .109331 .54848 $0.37606 \quad 1.103821 .54579$ 0.373481 .098331 .54309 0.370921 .092871 .54039 $0.368381 .08744 \quad 1.53768$

$0.36586 \quad 1.08204 \quad 1.53497$ $0.36336 \quad 1.07667 \quad 1.53226$ $0.36087 \quad 1.07133 \quad 1.52954$ $0.358411 .06601 \quad 1.52682$ $0.35596 \quad 1.06072 \quad 1.52409$

$0.353531 .05546 \quad 1.52136$ 0.351121 .050231 .51863 0.348721 .045031 .51589 0.346351 .039851 .51315 $0.34399 \quad 1.03470 \quad 1.51041$

$0.341651 .02957 \quad 1.50766$ $0.339321 .02448 \quad 1.50491$ 0.337021 .019411 .50216 $0.33473 \quad 1.01436 \quad 1.49940$ $0.33246 \quad 1.00934 \quad 1.49664$

$0.33020 \quad 1.00435 \quad 1.49388$ $0.32796 \quad 0.99938 \quad 1.49111$ $0.32574 \quad 0.99444 \quad 1.48834$ 0.323530 .989531 .48557 0.321340 .984641 .48279

$0.319170 .97977 \quad 1.48002$ $0.31701 \quad 0.97493 \quad 1.47723$ $0.31487 \quad 0.97012 \quad 1.47445$ $0.31274 \quad 0.96533 \quad 1.47166$ $0.31063 \quad 0.96056 \quad 1.46887$
$T=2400$

$0.469451 .29484 \quad 1.62938$ 0.466291 .288591 .62693

0.463151 .282381 .62449

0.460031 .276191 .62203

$0.456941 .27004 \quad 1.61957$

0.453871 .263921 .61711

$0.450831 .25784 \quad 1.61464$

$0.44781 \quad 1.25178 \quad 1.61217$

$0.444811 .24576 \quad 1.60970$

$0.441831 .23977 \quad 1.60721$

$0.43887 \quad 1.233811 .60473$

$0.43594 \quad 1.22788 \quad 1.60224$

0.433031 .221991 .59974

0.430141 .216121 .59724

$0.42727 \quad 1.21028 \quad 1.59474$

$0.424421 .20448 \quad 1.59223$

$0.421601 .19870 \quad 1.58971$

$0.41879 \quad 1.19296 \quad 1.58720$

$0.41601 \quad 1.187241 .58467$

$0.413241 .18156 \quad 1.58215$

$0.41050 \quad 1.17590 \quad 1.57962$ $0.40778 \quad 1.17028 \quad 1.57708$ $0.40507 \quad 1.16468 \quad 1.57454$ 0.402391 .159111 .57200 $0.399731 .15357 \quad 1.56945$

$0.39708 \quad 1.14806 \quad 1.56690$ $0.39446 \quad 1.14257 \quad 1.56435$ 0.391851 .137121 .56179 0.389261 .131691 .55922 0.386691 .126291 .55666

0.384141 .120921 .55409 $0.381611 .11557 \quad 1.55151$ $0.379101 .11025 \quad 1.54893$ 0.376601 .104961 .54635 $0.374131 .09970 \quad 1.54377$

$0.371671 .09446 \quad 1.54118$ 0.369231 .089251 .53858 $0.366801 .08406 \quad 1.53599$ $0.36440 \quad 1.07891 \quad 1.53339$ $0.36201 \quad 1.07377 \quad 1.53078$

$0.359641 .06867 \quad 1.52818$ 0.357281 .063591 .52557 0.354941 .058531 .52295 0.352621 .053501 .52034 0.350321 .048491 .51772

$0.348031 .04351 \quad 1.51509$ $0.34576 \quad 1.03856 \quad 1.51247$ $0.34350 \quad 1.03363 \quad 1.50984$ 0.341261 .028721 .50720 $0.339041 .02384 \quad 1.50457$
$T=2500$.

$0.50381 \quad 1.36188 \quad 1.65448$ 0.500521 .355531 .65218 0.497261 .349221 .64989 0.494021 .342931 .64758 $0.49081 \quad 1.336691 .64528$

$0.48761 \quad 1.33047 \quad 1.64296$ 0.484441 .324291 .64065 0.481301 .318131 .63832 0.478181 .312011 .63600 0.475081 .305931 .63366

$0.47200 \quad 1.29987 \quad 1.63133$ $0.46894 \quad 1.29384 \quad 1.62899$ $0.46591 \quad 1.287851 .62664$ 0.462901 .281881 .62429 0.459911 .275951 .62193

$0.45694 \quad 1.27004 \quad 1.61957$ 0.454001 .264171 .61721 0.451071 .258321 .61484 0.448171 .252511 .61247 0.445291 .246721 .61009

$0.442421 .24097 \quad 1.60771$ 0.439581 .235241 .60532 $0.43676 \quad 1.22954 \quad 1.60293$ $0.43396 \quad 1.223871 .60054$ 0.431181 .218231 .59814

0.428421 .212621 .59574 $0.42567 \quad 1.20703 \quad 1.59333$ $0.422951 .20147 \quad 1.59092$ $0.420251 .19594 \quad 1.58851$ 0.417561 .190441 .58609

$0.41490 \quad 1.18497 \quad 1.58366$ 0.412251 .179521 .58124 0.409631 .174101 .57881 0.407021 .168711 .57637 0.404431 .163341 .57393

$0.40186 \quad 1.15800 \quad 1.57149$ 0.399301 .152691 .56905 $0.39676 \quad 1.14740 \quad 1.56660$ $0.39425 \quad 1.14214 \quad 1.56414$ 0.391751 .136901 .56169

$0.38926 \quad 1.13169 \quad 1.55922$ 0.386801 .126501 .55676 0.384351 .121351 .55429 0.381911 .116211 .55182 $0.379501 .11110 \quad 1.54935$

$0.37710 \quad 1.10602 \quad 1.54687$ 0.374721 .100961 .54439 $0.372351 .09592 \quad 1.54190$ $0.37001 \quad 1.090911 .53941$ $0.36767 \quad 1.085931 .53692$
2600

2610

2620

2630

2640

2650

2660

2670

2680

2690

2700

2710

2720

2730

2740

2750

2760

2770

2780

2790

2800

2810

2820

2830

2840

2850

2860

2870

2880

2890

2900

2910

2920

2930

2940

2950

2960

2970

2980

2990

3000

3010

3020

3030

3040

3050

3060

3070

3080

3090 
Table II Harmonic Oscillator Contributions to the Thermodynamic

Functions (in units of calories, moles, and ${ }^{\circ} \mathrm{K}$ )
$2 \quad-\left(\mathrm{F}^{\circ}-\mathrm{E}_{\mathrm{O}}^{\circ}\right) / \mathrm{T}$ So
$\mathrm{Co}_{\mathrm{p}} \quad-\left(\mathrm{F}^{\circ}-\mathrm{E}_{\mathrm{O}} \mathrm{O}\right) / \mathrm{T}$
So
$\mathrm{C}_{\mathrm{p}}^{\circ}$
$-\left(F^{\circ}-E_{0} O\right) / T$
so
$\mathrm{cm}^{-1}$

$T=2300$.

\begin{abstract}
$T=2400$.
$0.33683 \quad 1.01898 \quad 1.50193$

0.334631 .014151 .49929

$0.33246 \quad 1.00934 \quad 1.49664$

$0.33030 \quad 1.00456 \quad 1.49399$

$0.32815 \quad 0.999801 .49134$

$0.32602 \quad 0.99506 \quad 1.48869$

$0.32390 \quad 0.99034 \quad 1.48603$

$\begin{array}{llll}0.32180 & 0.98565 & 1.48337\end{array}$

$0.31971 \quad 0.98099 \quad 1.48071$

$0.31764 \quad 0.97634 \quad 1.47805$

$\begin{array}{llll}0.31558 & 0.97172 & 1.47538\end{array}$

$0.313540 .96712 \quad 1.47271$

0.311510 .962541 .47004

$0.30950 \quad 0.95799 \quad 1.46736$

$0.30750 \quad 0.95346 \quad 1.46468$

$0.30551 \quad 0.94895 \quad 1.46201$ $0.30354 \quad 0.94446 \quad 1.45932$ $0.30158 \quad 0.93999 \quad 1.45664$ $0.29963 \quad 0.93555 \quad 1.45395$ $0.29770 \quad 0.93113 \quad 1.45126$
\end{abstract}

$0.29578 \quad 0.92673 \quad 1.44857$ $0.29387 \quad 0.92235 \quad 1.44588$ $0.291980 .91799 \quad 1.44318$ $0.29010 \quad 0.91366 \quad 1.44048$ $0.28824 \quad 0.90934 \quad 1.43778$

$\begin{array}{llll}0.28638 & 0.90505 & 1.43508\end{array}$ $0.28454 \quad 0.90077 \quad 1.43238$ $\begin{array}{llll}0.28272 & 0.89652 & 1.42967\end{array}$ $0.28090 \quad 0.89229 \quad 1.42696$ $\begin{array}{llll}0.27910 & 0.88808 & 1.42425\end{array}$

$\begin{array}{llll}0.27731 & 0.88389 & 1.42154\end{array}$ $0.27553 \quad 0.87971 \quad 1.41883$ $0.27376 \quad 0.87556 \quad 1.41611$ $0.272010 .87143 \quad 1.41340$ $0.27027 \quad 0.86732 \quad 1.41068$

$\begin{array}{llll}0.26854 & 0.86323 & 1.40796\end{array}$ $0.266820 .85916 \quad 1.40524$ $\begin{array}{llll}0.26512 & 0.85511 & 1.40251\end{array}$ $0.263420 .85108 \quad 1.39979$ $0.26174 \quad 0.84706 \quad 1.39706$

$\begin{array}{llll}0.26007 & 0.84307 & 1.39433\end{array}$ $0.258410 .83910 \quad 1.39160$ $0.25676 \quad 0.83514 \quad 1.38887$ $0.255120 .83121 \quad 1.38614$ $0.253490 .82729 \quad 1.38340$

$\begin{array}{llll}0.25188 & 0.82339 & 1.38067\end{array}$ $0.25027 \quad 0.81951 \quad 1.37793$ $0.24868 \quad 0.81565 \quad 1.37520$ 0.247100 .811811 .37246 $\begin{array}{llll}0.24553 & 0.80798 & 1.36972\end{array}$
$T=2500$.

$0.36536 \quad 1.08097 \quad 1.53443$ $0.36306 \quad 1.07603 \quad 1.53193$ $0.36077 \quad 1.07111 \quad 1.52943$ $0.35850 \quad 1.06622 \quad 1.52692$ $0.35625 \quad 1.06136 \quad 1.52442$

$0.35401 \quad 1.05651 \quad 1.52191$ $0.35179 \quad 1.05169 \quad 1.51939$ $0.34958 \quad 1.04690 \quad 1.51688$ 0.347391 .042121 .51436 $0.34521 \quad 1.03737 \quad 1.51183$

$0.34305 \quad 1.03265 \quad 1.50931$ $\begin{array}{llll}0.34090 & 1.02794 & 1.50678\end{array}$ $0.33877 \quad 1.02326 \quad 1.50425$ $0.33665 \quad 1.01860 \quad 1.50172$ $0.33455 \quad 1.01396 \quad 1.49918$

$0.33246 \quad 1.00934 \quad 1.49664$ $0.33038 \quad 1.00475 \quad 1.49410$ $0.328321 .00018 \quad 1.49155$ $\begin{array}{llll}0.32627 & 0.99563 & 1.48901\end{array}$ $0.32424 \quad 0.99110 \quad 1.48646$

$\begin{array}{llll}0.32222 & 0.98659 & 1.48390\end{array}$ $\begin{array}{llll}0.32021 & 0.98210 & 1.48135\end{array}$ $0.31822 \quad 0.97764 \quad 1.47879$ $0.31624 \quad 0.97320 \quad 1.47623$ $\begin{array}{llll}0.31427 & 0.96877 & 1.47367\end{array}$

$\begin{array}{llll}0.31232 & 0.96437 & 1.47111\end{array}$ $0.310380 .95999 \quad 1.46854$ $\begin{array}{llll}0.30845 & 0.95563 & 1.46597\end{array}$ $0.306540 .95129 \quad 1.46340$ $0.304640 .94697 \quad 1.46083$

$\begin{array}{llll}0.30275 & 0.94267 & 1.45825\end{array}$ $0.30087 \quad 0.93839 \quad 1.45567$ $0.29901 \quad 0.93413 \quad 1.45309$ $\begin{array}{llll}0.29716 & 0.92990 & 1.45051\end{array}$ $0.29532 \quad 0.92568 \quad 1.44792$

$\begin{array}{llll}0.29350 & 0.92148 & 1.44534\end{array}$ $0.291680 .91730 \quad 1.44275$ $0.289880 .91314 \quad 1.44016$ $\begin{array}{llll}0.28809 & 0.90900 & 1.43757\end{array}$ $\begin{array}{llll}0.28631 & 0.90488 & 1.43497\end{array}$

$\begin{array}{llll}0.28454 & 0.90077 & 1.43238\end{array}$ $0.282790 .89669 \quad 1.42978$ $0.28105 \quad 0.89263 \quad 1.42718$ $0.279310 .88858 \quad 1.42458$ $\begin{array}{llll}0.27759 & 0.88456 & 1.42198\end{array}$

$\begin{array}{llll}0.27589 & 0.88055 & 1.41937\end{array}$ $\begin{array}{llll}0.27419 & 0.87656 & 1.41677\end{array}$ $0.27250 \quad 0.87259 \quad 1.41416$ $0.270830 .86864 \quad 1.41155$ $\begin{array}{llll}0.26916 & 0.86470 & 1.40894\end{array}$
3100

3110

3120

3130

3140

3150

3160

3170

3180

3190

3200

3210

3220

3230

3240

3250

3260

3270

3280

3290

3300

3310

3320

3330

3340

3350

3360

3370

3380

3390

3400

3410

3420

3430

3440

3450

3460

3470

3480

3490

3500

3510

3520

3530

3540

3550

3560

3570

3580

3590 
Table II Harmonic Oscillator Contributions to the Thermodynamic Functions (in units of calories, moles, and ${ }^{\circ} \mathrm{K}$ )

$$
\mathrm{cm}^{-1} \quad-\left(F^{\circ}-E_{0}^{\circ}\right) / T \quad \text { So }
$$

$$
T=2300 \text {. }
$$

$$
\mathrm{C}_{\mathrm{p}}^{\circ} \quad-\left(\mathrm{F}^{\circ}-\mathrm{E}_{\mathrm{O}} \mathrm{O}\right) / \mathrm{T} \quad \text { So }
$$$$
\mathrm{C}_{\mathrm{p}}^{\circ}
$$

$$
T=2400 \text {. }
$$

3600

3610

3620

3630

3640

3650

3660

3670

3680

3690

3700

3710

3720

3730

3740

3750

3760

3770

3780

3790

3800

3810

3820

3830

3840

3850

3860

3870

3880

3890

3900

3910

3920

3930

3940

3950

3960

3970

3980

3990

4000
$0.220850 .74690 \quad 1.32395$

0.219390 .743241 .32108

$\begin{array}{llll}0.21795 & 0.73959 & 1.31820\end{array}$

$0.216510 .73595 \quad 1.31533$

0.215090 .732341 .31245

$\begin{array}{llll}0.21367 & 0.72874 & 1.30958\end{array}$

$0.212260 .72516 \quad 1.30670$

$\begin{array}{lll}0.21087 & 0.72160 & 1.30382\end{array}$

$0.20948 \quad 0.71806 \quad 1.30095$

$0.20810 \quad 0.714531 .29807$

$0.20674 \quad 0.71102 \quad 1.29519$ $0.20538 \quad 0.70753 \quad 1.29231$ $0.20403 \quad 0.70406 \quad 1.28943$ $0.202690 .70060 \quad 1.28656$ $0.20136 \quad 0.69716 \quad 1.28368$

$0.20004 \quad 0.69373 \quad 1.28080$ $0.19873 \quad 0.69033 \quad 1.27792$ $\begin{array}{llll}0.19742 & 0.68694 & 1.27504\end{array}$ $0.196130 .68356 \quad 1.27216$ $0.194840 .68020 \quad 1.26929$

$0.19357 \quad 0.67686 \quad 1.26641$ $0.19230 \quad 0.67354 \quad 1.26353$ $0.19104 \quad 0.67023 \quad 1.26065$ $\begin{array}{llll}0.18979 & 0.66694 & 1.25777\end{array}$ $\begin{array}{llll}0.18855 & 0.66366 & 1.25490\end{array}$

$0.18732 \quad 0.66040 \quad 1.25202$ $\begin{array}{llll}0.18609 & 0.65716 & 1.24914\end{array}$ $\begin{array}{llll}0.18488 & 0.65393 & 1.24627\end{array}$ $0.18367 \quad 0.65072 \quad 1.24339$ $\begin{array}{llll}0.18247 & 0.64752 \quad 1.24052\end{array}$

$0.18128 \quad 0.64434 \quad 1.23764$ $\begin{array}{llll}0.18009 & 0.64117 & 1.23477\end{array}$ $0.178920 .63802 \quad 1.23190$ $\begin{array}{llll}0.17775 & 0.63489 & 1.22902\end{array}$ 0.176590 .631771 .22615

$\begin{array}{llll}0.17544 & 0.62867 & 1.22328\end{array}$ $\begin{array}{llll}0.17430 & 0.62558 & 1.22041\end{array}$ $0.17316 \quad 0.62250 \quad 1.21754$ $0.17203 \quad 0.61944 \quad 1.21467$ 0.170910 .616401 .21180

$0.16980 \quad 0.61337 \quad 1.20893$
$0.24396 \quad 0.80417 \quad 1.36698$ $\begin{array}{llll}0.24241 & 0.80039 & 1.36423\end{array}$ $\begin{array}{llll}0.24087 & 0.79662 & 1.36149\end{array}$ $\begin{array}{llll}0.23934 & 0.79286 & 1.35875\end{array}$ $0.23782 \quad 0.78913 \quad 1.35600$

$\begin{array}{llll}0.23631 & 0.78541 & 1.35325\end{array}$ $\begin{array}{llll}0.23482 & 0.78172 & 1.35051\end{array}$ $\begin{array}{llll}0.23333 & 0.77803 & 1.34776\end{array}$ $0.231850 .77437 \quad 1.34501$ $\begin{array}{lll}0.23038 & 0.77072 \quad 1.34226\end{array}$

$0.22892 \quad 0.76710 \quad 1.33951$ $\begin{array}{llll}0.22747 & 0.76348 & 1.33676\end{array}$ $0.22603 \quad 0.759891 .33401$ $0.224600 .75631 \quad 1.33126$ $\begin{array}{lll}0.22318 & 0.75275 & 1.32850\end{array}$

$\begin{array}{llll}0.22177 & 0.74921 & 1.32575\end{array}$ $\begin{array}{llll}0.22036 & 0.74568 & 1.32299\end{array}$ $\begin{array}{lllll}0.21897 & 0.74217 & 1.32024\end{array}$ $\begin{array}{llll}0.21759 & 0.73868 & 1.31748\end{array}$ 0.216210 .735201 .31473

$\begin{array}{llll}0.21485 & 0.73174 & 1.31197\end{array}$ $0.213490 .72829 \quad 1.30922$ $\begin{array}{llll}0.21215 & 0.72487 & 1.30646\end{array}$ $0.21081 \quad 0.72145 \quad 1.30370$ $\begin{array}{llll}0.20948 & 0.71806 \quad 1.30094\end{array}$

$0.20816 \quad 0.71468 \quad 1.29819$ $\begin{array}{llll}0.20685 & 0.71131 & 1.29543\end{array}$ $\begin{array}{lll}0.20555 & 0.70797 & 1.29267\end{array}$ $\begin{array}{llll}0.20425 & 0.70463 & 1.28991\end{array}$ 0.202970 .701321 .28715

$0.201690 .69802 \quad 1.28440$ $0.20042 \quad 0.69473 \quad 1.28164$ $\begin{array}{llll}0.19916 & 0.69146 & 1.27888\end{array}$ $\begin{array}{llll}0.19791 & 0.68821 & 1.27612\end{array}$ $0.196670 .68497 \quad 1.27336$

$0.19543 \quad 0.68174 \quad 1.27060$ $\begin{array}{llll}0.19420 & 0.67853 & 1.26785\end{array}$ $0.192990 .67534 \quad 1.26509$ $\begin{array}{llll}0.19177 & 0.67216 & 1.26233\end{array}$ $\begin{array}{llll}0.19057 & 0.66899 & 1.25957\end{array}$

$0.18938 \quad 0.66585 \quad 1.25682$
$-\left(\mathrm{F}^{\circ}-\mathrm{E}_{\mathrm{O}}\right) / \mathrm{T} \quad \mathrm{S}^{\circ}$

$\mathrm{C}_{\mathrm{p}}$ $\nu$ $\mathrm{cm}^{-1}$
$T=2500$.

$\begin{array}{llll}0.26751 & 0.86079 & 1.40632\end{array}$ $0.26587 \quad 0.856891 .40371$ 0.264230 .853011 .40109 0.262610 .849151 .39848 $0.26100 \quad 0.84530 \quad 1.39586$

$0.25940 \quad 0.84148 \quad 1.39324$ $0.257810 .83767 \quad 1.39062$ 0.256230 .833881 .38800 $0.25466 \quad 0.830111 .38537$ 0.253110 .826351 .38275

$0.25156 \quad 0.82261 \quad 1.38012$ 0.250020 .818891 .37749 0.248490 .815191 .37487 0.246970 .811501 .37224 0.245460 .807831 .36961

$\begin{array}{llll}0.24396 & 0.80417 & 1.36698\end{array}$ $0.24248 \quad 0.800541 .36434$ $0.24100 \quad 0.796921 .36171$ $0.23953 \quad 0.79331 \quad 1.35907$ $\begin{array}{llll}0.23807 & 0.78973 & 1.35644\end{array}$

$0.236620 .78616 \quad 1.35380$ 0.235170 .78260 .1 .35117 $0.233740 .77906 \quad 1.34853$ 0.232320 .775541 .34589 0.230910 .772031 .34325

$0.22950 \quad 0.76854 \quad 1.34061$ $0.22810 \quad 0.76507 \quad 1.33797$ 0.226720 .761611 .33533 $0.225340 .75817 \quad 1.33269$ $0.223970 .75474 \quad 1.33004$

0.222610 .751331 .32740 $0.22126 \quad 0.74794 \quad 1.32476$ 0.219920 .744551 .32211 $0.21858 \quad 0.741191 .31947$ $0.21726 \quad 0.73784 \quad 1.31682$

$\begin{array}{llll}0.21594 & 0.73451 & 1.31418\end{array}$ 0.214630 .731191 .31153 0.213330 .727881 .30889 0.212040 .724591 .30624 0.210760 .721321 .30359

$0.20948 \quad 0.71806 \quad 1.30094$
3600

3610

3620

3630

3640

3650

3660

3670

3680

3690

3700

3710

3720

3730

3740

3750

3760

3770

3780

3790

3800

3810

3820

3830

3840

3850

3860

3870

3880

3890

3900

3910

3920

3930

3940

3950

3960

3970

3980

3990

4000 
Table II Harmonic Oscillator Contributions to the Thermodynamic

Functions (in units of calories, moles, and ${ }^{\circ} \mathrm{K}$ )
$\mathrm{cm}^{-1}$
$-\left(F^{\circ}-E O\right) / T$ SO
$\mathrm{C}_{\mathrm{P}}^{O} \quad-\left(\mathrm{F}^{\circ}-\mathrm{E}_{\mathrm{O}}^{\circ}\right) / \mathrm{T} \quad \mathrm{S}^{\circ}$
co
$-\left(F^{\circ}-E_{O}^{O}\right) / T$
So

$$
T=2600 \text {. }
$$

$$
T=2700
$$
$T=2800$.

$\mathrm{c}_{\mathrm{p}}^{\circ}$

$\nu$

$\mathrm{cm}^{-1}$

$100 \quad 5.80617 \quad 7.73886 \quad 1.98666$

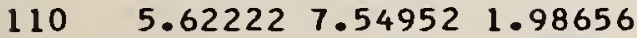

$\begin{array}{lllll}120 & 5.45475 & 7.37667 & 1.98644\end{array}$

$\begin{array}{lllll}130 & 5.30113 & 7.21768 & 1.98631\end{array}$

$\begin{array}{lll}140 & 5.15929 & 7.07048 \quad 1.98618\end{array}$

$150 \quad 5.027626 .93345 \quad 1.98603$

$\begin{array}{lllll}160 & 4.90479 & 6.80528 & 1.98587\end{array}$

$170 \quad 4.78973 \quad 6.68489 \quad 1.98570$

$\begin{array}{lllll}180 & 4.68156 & 6.57140 & 1.98553\end{array}$

$190 \quad 4.579526 .46405 \quad 1.98534$

$200 \quad 4.482996 .36222 \quad 1.98514$

$210 \quad 4.391436 .26537 \quad 1.98493$

$220 \quad 4.30438 \quad 6.17304 \quad 1.98472$

$230 \quad 4.221436 .08482 \quad 1.98449$

$240 \quad 4.142246 .00037 \quad 1.98425$

$250 \quad 4.06649 \quad 5.91937 \quad 1.98400$

$260 \quad 3.99392 \quad 5.84156 \quad 1.98375$

$\begin{array}{lllll}270 & 3.92429 & 5.76670 & 1.98348\end{array}$

$\begin{array}{lllll}280 & 3.85738 & 5.69457 & 1.98320\end{array}$

$290 \quad 3.79300 \quad 5.62498 \quad 1.98291$

$\begin{array}{lllll}300 & 3.73098 & 5.55776 & 1.98261\end{array}$

$310 \quad 3.67116 \quad 5.49276 \quad 1.98230$

$320 \quad 3.613415 .429831 .98199$

$330 \quad 3.557605 .368841 .98166$

$340 \quad 3.50360 \quad 5.309691 .98132$

$\begin{array}{llll}350 & 3.45132 & 5.25226 & 1.98097\end{array}$

$360 \quad 3.400665 .19646 \quad 1.98061$

$370 \quad 3.35153 \quad 5.142201 .98024$

$\begin{array}{llll}380 & 3.30384 & 5.08939 & 1.97986\end{array}$

$\begin{array}{lllll}390 & 3.25753 \quad 5.03797 & 1.97947\end{array}$

$\begin{array}{llll}400 \quad 3.21252 \quad 4.98786 & 1.97908\end{array}$

$410 \quad 3.16874 \quad 4.93900 \quad 1.97867$

$420 \quad 3.126144 .891321 .97825$

$430 \quad 3.08467 \quad 4.84478 \quad 1.97782$

$440 \quad 3.04426 \quad 4.79931 \quad 1.97738$

$\begin{array}{lllll}450 & 3.00488 \quad 4.75488 & 1.97693\end{array}$

$460 \quad 2.966474 .711431 .97647$

$470 \quad 2.929004 .668931 .97601$

$480 \quad 2.892424 .62734 \quad 1.97553$

$490 \quad 2.856694 .58661 \quad 1.97504$

$\begin{array}{lllll}500 & 2.82180 & 4.54671 & 1.97454\end{array}$

$\begin{array}{llll}510 & 2.78769 & 4.50761 & 1.97403\end{array}$

$\begin{array}{lllll}520 & 2.75434 & 4.46929 & 1.97351\end{array}$

$530 \quad 2.721724 .43170 \quad 1.97299$

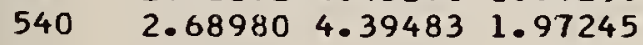

$\begin{array}{llll}550 & 2.65856 & 4.35864 & 1.97190\end{array}$ $\begin{array}{llll}560 & 2.62797 & 4.32311 & 1.97134\end{array}$ $\begin{array}{lllll}570 & 2.59801 & 4.28823 & 1.97078\end{array}$ $\begin{array}{llll}580 & 2.56866 & 4.25396 & 1.97020\end{array}$ $\begin{array}{llll}590 & 2.53989 & 4.22028 & 1.96961\end{array}$ $\begin{array}{llll}5.87915 & 7.81384 & 1.98670\end{array}$ $5.69500 \quad 7.62449 \quad 1.98660$ 5.527337 .451641 .98649 $5.373517 .29264 \quad 1.98638$ $5.231477 .14544 \quad 1.98625$

$5.099607 .00841 \quad 1.98611$ $4.97657 \quad 6.88023 \quad 1.98597$ 4.861326 .759841 .98581 $4.75295 \quad 6.64634 \quad 1.98565$ $\begin{array}{llll}4.65071 & 6.53898 & 1.98547\end{array}$

4.553996 .437151 .98529 4.462236 .340291 .98510 $4.37498 \quad 6.24795 \quad 1.98490$ $4.29184 \quad 6.15972 \quad 1.98468$ $4.212456 .07526 \quad 1.98446$

$4.13651 \quad 5.99425 \quad 1.98423$ $4.06375 \quad 5.916431 .98399$ $3.99392 \quad 5.84156 \quad 1.98375$ $3.926825 .76942 \quad 1.98349$ $3.862245 .69982 \quad 1.98322$

$3.80003 \quad 5.63259 \quad 1.98294$ $3.74003 \quad 5.56758 \quad 1.98266$ 3.682085 .504631 .98236 $3.62608 \quad 5.443641 .98206$ $3.571895 .38447 \quad 1.98174$

3.519425 .327031 .98142 3.468575 .271221 .98109 3.419245 .216941 .98074 3.371375 .164121 .98039 3.324865 .112691 .98003

$3.27966 \quad 5.06256 \quad 1.97966$ $3.23570 \quad 5.01368 \quad 1.97928$ 3.192914 .965991 .97890 $3.151254 .91943 \quad 1.97850$ 3.110654 .873951 .97809

$3.07108 \quad 4.82950 \quad 1.97767$ 3.032494 .786041 .97725 2.994834 .743521 .97681 2.95806 4.70191 1.97637 2. 922154.661161 .97592

$2.88707 \quad 4.62125 \quad 1.97546$ $2.85278 \quad 4.582131 .97498$ $2.81924 \quad 4.543791 .97450$ $2.78644 \quad 4.506181 .97401$ $2.75434 \quad 4.469291 .97351$

$2.722914 .43308 \quad 1.97301$ 2.692144 .397531 .97249 2.662004 .362631 .97196 $2.63246 \quad 4.328341 .97143$ 2.603514 .294641 .97088
$5.94954 \quad 7.88609 \quad 1.98673$ $5.765207 .69674 \quad 1.98664$ 5.597357 .523891 .98654 $5.443357 .36488 \quad 1.98643$ 5.301137 .217681 .98631

5.169077 .080641 .98619 $5.04586 \quad 6.952461 .98605$ 4.930426 .832061 .98591 $4.82187 \quad 6.718551 .98575$ 4.719456 .611191 .98559

4.622546 .509351 .98542 $4.53060 \quad 6.41248 \quad 1.98524$ 4.443176 .320131 .98505 4.359856 .231901 .98486 4.280286 .147431 .98465

$4.20415 \quad 6.06642 \quad 1.98444$ $4.131215 .98859 \quad 1.98422$ $4.06120 \quad 5.91371 \quad 1.98399$ $3.993925 .84156 \quad 1.98375$ 3.929175 .771951 .98350

$3.86678 \quad 5.70471 \quad 1.98324$ $3.80659 \quad 5.63969 \quad 1.98297$ 3.748475 .576731 .98270 3.692285 .515731 .98242 3.637925 .456551 .98212

$3.585275 .39910 \quad 1.98182$ 3.534245 .343271 .98151 3.484745 .288991 .98119 $3.436695 .23616 \quad 1.98087$ 3.390015 .184711 .98053

3.344635 .134571 .98019 3.300495 .085681 .97984 $3.257535 .03797 \quad 1.97947$ 3.215694 .991401 .97910 $3.174924 .94590 \quad 1.97873$

$3.135184 .90144 \quad 1.97834$ 3.096414 .857961 .97794 3.058574 .815431 .97754 $3.021634 .77380 \quad 1.97713$ $2.98555 \quad 4.73304 \quad 1.97670$

$2.95030 \quad 4.693111 .97627$ 2.915834 .653971 .97584 2.882124 .615611 .97539 2.849154 .577991 .97493 2.816884 .541081 .97447

$2.78528 \quad 4.50485 \quad 1.97400$ 2.754344 .469291 .97351 $2.72402 \quad 4.434361 .97302$ 2.694324 .400051 .97253 $2.66520 \quad 4.366341 .97202$
100

110

120

130

140

150

160

170

180

190

200

210

220

230

240

250

260

270

280

290

300

310

320

330

340

350

360

370

380

390

400

410

420

430

440

450

460

470

480

490

500

510

520

530

540

550

560

570

580

590 
Table II Harmonic Oscillator Contributions to the Thermodynamic Functions (in units of calories, moles, and ${ }^{\circ} \mathrm{K}$ )

\begin{tabular}{llcc}
$\mathrm{cm}^{-1}$ & $-\left(\mathrm{F}^{\circ}-\mathrm{E}_{\mathrm{O}}^{\circ}\right) / \mathrm{T}$ & $\mathrm{S}^{\circ}$ & $\mathrm{C}_{\mathrm{p}}^{\circ}$ \\
\multicolumn{5}{c}{$\mathrm{T}=2600}$. \\
\multicolumn{5}{c}{} \\
600 & 2.51169 & 4.18718 & 1.96901 \\
610 & 2.48404 & 4.15464 & 1.96841 \\
620 & 2.45691 & 4.12264 & 1.96779 \\
630 & 2.43030 & 4.09116 & 1.96716 \\
640 & 2.40418 & 4.06018 & 1.96653 \\
650 & 2.37854 & 4.02970 & 1.96588 \\
660 & 2.35337 & 3.99969 & 1.96523 \\
670 & 2.32865 & 3.97014 & 1.96456 \\
680 & 2.30437 & 3.94104 & 1.96389 \\
690 & 2.28051 & 3.91238 & 1.96320
\end{tabular}

$700 \quad 2.25706 \quad 3.88413 \quad 1.96251$

$\begin{array}{lll}710 & 2.23402 \quad 3.85630 \quad 1.96180\end{array}$

$\begin{array}{llll}720 & 2.21136 \quad 3.82887 & 1.96109\end{array}$

$730 \quad 2.18908 \quad 3.80182 \quad 1.96036$

$\begin{array}{lllll}740 & 2.16717 & 3.77516 \quad 1.95963\end{array}$

$\begin{array}{llll}750 & 2.14562 & 3.74886 \quad 1.95889\end{array}$

$760 \quad 2.12442 \quad 3.72292 \quad 1.95814$

$\begin{array}{lllll}770 & 2.10355 & 3.69732 & 1.95737\end{array}$

$\begin{array}{lllll}780 & 2.08302 & 3.67207 & 1.95660\end{array}$

$\begin{array}{lllll}790 & 2.06280 & 3.64715 & 1.95582\end{array}$

800

810

820

830

840

850

860

870

880

890

900

910

920

930

940

$2.04290 \quad 3.62256 \quad 1.95503$

$2.02331 \quad 3.59827 \quad 1.95423$

$2.004013 .57430 \quad 1.95342$

1.985013 .550631 .95260

$1.96628 \quad 3.52725 \quad 1.95177$

$1.947843 .50415 \quad 1.95093$ $1.929663 .48134 \quad 1.95008$ $1.91175 \quad 3.45880 \quad 1.94923$ $1.89410 \quad 3.43653 \quad 1.94836$ $1.876693 .41452 \quad 1.94748$

$1.859543 .39276 \quad 1.94660$ $1.842623 .37126 \quad 1.94570$ $1.82594 \quad 3.35000 \quad 1.94480$ $1.809493 .32898 \quad 1.94388$ $1.79326 \quad 3.30819 \quad 1.94296$

950

960

$1.77725 \quad 3.28764 \quad 1.94203$

970

980

990

$1.76146 \quad 3.26731 \quad 1.94109$

$1.74588 \quad 3.24720 \quad 1.94013$

$1.73050 \quad 3.227301 .93917$

$1.71533 \quad 3.207621 .93820$

1000

1010

1020

1030

1040

1050

1060

1070

1080

$1.70036 \quad 3.18815 \quad 1.93723$

$\begin{array}{llll}1.68557 & 3.16887 & 1.93624\end{array}$

$1.67098 \quad 3.14980 \quad 1.93524$

1.656583 .130931 .93423

$1.642353 .11224 \quad 1.93322$

1.628313 .093751 .93219

1.614443 .075441 .93116

$1.60074 \quad 3.05731 \quad 1.93011$

$1.58721 \quad 3.03936 \quad 1.92906$

1090
$-\left(F^{\circ}-E_{O}^{O}\right) / T$

so

$-\left(F^{\circ}-E_{0}^{0}\right) / T$

So

$\mathrm{C}_{\mathrm{p}}^{\circ}$

$\mathrm{cm}^{-1}$

$T=2700$.

$2.575134 .26152 \quad 1.97033$ $2.54730 \quad 4.22896 \quad 1.96976$ 2.519994 .196931 .96919 2.493194 .165431 .96861 2.466904 .134431 .96802

$2.44108 \quad 4.103921 .96742$ $2.41573 \quad 4.073891 .96681$ 2.390834 .044321 .96619 $2.36637 \quad 4.01519 \quad 1.96557$ $2.34233 \quad 3.93650 \quad 1.96493$

$2.31870 \quad 3.95823 \quad 1.96429$ $2.29548 \quad 3.93038 \quad 1.96363$ $2.27265 \quad 3.90292 \quad 1.96297$ $2.25019 \quad 3.87585 \quad 1.96230$ 2.228113 .849151 .96162

$2.20638 \quad 3.82283 \quad 1.96093$ 2. $18500 \quad 3.79686 \quad 1.96023$ $2.16396 \quad 3.77124 \quad 1.95952$ $2.14325 \quad 3.74596 \quad 1.95881$ $2.12286 \quad 3.72101 \quad 1.95808$

$2.10278 \quad 3.69638 \quad 1.95735$ $2.08302 \quad 3.67207 \quad 1.95660$ $2.06355 \quad 3.64807 \quad 1.95585$ $2.044373 .62437 \quad 1.95509$ $2.025473 .60096 \quad 1.95432$

$2.006853 .57783 \quad 1.95354$ $1.98850 \quad 3.55499 \quad 1.95275$ 1.970423 .532421 .95196 $1.952593 .51011 \quad 1.95115$ $1.935023 .48807 \quad 1.95034$

$1.917693 .46628 \quad 1.94951$ 1.900613 .444751 .94868 $1.88375 \quad 3.42345 \quad 1.94784$ 1.867133 .402401 .94699 $1.850743 .38158 \quad 1.94613$

$1.83456 \quad 3.36099 \quad 1.94527$ 1.818603 .340631 .94439 $1.802853 .32048 \quad 1.94351$ 1.787313 .300551 .94262 $1.771973 .28084 \quad 1.94172$

$1.75682 \quad 3.261331 .94081$ $1.741873 .24202 \quad 1.93989$ $1.727123 .22291 \quad 1.93896$ $1.712543 .20400 \quad 1.93802$ $1.698153 .18528 \quad 1.93708$

$1.68394 \quad 3.16675 \quad 1.93613$ 1.669913 .148401 .93517 1.656053 .130231 .93420 $1.64235 \quad 3.11224 \quad 1.93322$ 1.628823 .094431 .93223

\author{
$T=2800$.
}

$2.636654 .33320 \quad 1.97150$

$2.608644 .30061 \quad 1.97098$

$2.58117 \quad 4.26857 \quad 1.97045$

$2.55420 \quad 4.23705 \quad 1.96991$

$2.52774 \quad 4.206031 .96936$

$2.50175 \quad 4.17550 \quad 1.96880$

$2.476234 .14544 \quad 1.96823$

2.451174 .115851 .96766

$2.42654 \quad 4.08670 \quad 1.96707$

$2.402334 .05799 \quad 1.96648$

$2.37854 \quad 4.02970 \quad 1.96588$

$2.35515 \quad 4.00182 \quad 1.96527$

$2.33215 \quad 3.97434 \quad 1.96466$

$2.30953 \quad 3.94724 \quad 1.96403$

$2.28728 \quad 3.920521 .96340$

600

610

620

630

640

650

660

670

680

690

700

710

720

730

740

$2.265393 .89417 \quad 1.96276$

750

$2.24384 \quad 3.86818 \quad 1.96211$

$2.22264 \quad 3.84254 \quad 1.96145$

2.201773 .817231 .96078

$2.181213 .79226 \quad 1.96010$

760

770

780

790

$2.16098 \quad 3.76761 \quad 1.95942$

800

$2.14105 \quad 3.74327 \quad 1.95873$

$2.121413 .71924 \quad 1.95803$

$2.10207 \quad 3.69551 \quad 1.95732$

$2.08302 \quad 3.67207 \quad 1.95660$

810

820

830

840

$2.06424 \quad 3.64892 \quad 1.95588$

$2.04573 \quad 3.62605 \quad 1.95514$

$2.02748 \quad 3.60345 \quad 1.95440$

$2.00950 \quad 3.58112 \quad 1.95365$

$1.99176 \quad 3.55905 \quad 1.95289$

$1.97427 \quad 3.53723 \quad 1.95213$

$1.95703 \quad 3.51567 \quad 1.95135$

$1.940023 .49434 \quad 1.95057$

$1.923243 .47326 \quad 1.94978$

$1.906683 .45241 \quad 1.94898$

$1.89035 \quad 3.43179 \quad 1.94817$

$1.87423 \quad 3.411391 .94736$

1.858323 .391221 .94653

1.842623 .371261 .94570

1.827123 .351511 .94486

$1.81182 \quad 3.33197 \quad 1.94402$

1.796723 .312631 .94316

$1.78180 \quad 3.29349 \quad 1.94230$

$1.76708 \quad 3.27454 \quad 1.94142$

$1.75253 \quad 3.25579 \quad 1.94054$

$1.73817 \quad 3.23722 \quad 1.93966$ $1.72398 \quad 3.21884 \quad 1.93876$ $1.70996 \quad 3.200641 .93786$ 1.696113 .182621 .93694 1.682433 .164771 .93602
850

860

870

880

890

900

910

920

930

940

950

960

970

980

990

1000

1010

1020

1030

1040

1050

1060

1070

1080

1090 
Table II Harmonic Oscillator Contributions to the Thermodynamic

Functions (in units of calories, moles, and ${ }^{\circ} \mathrm{K}$ )
$\mathrm{cm}^{-1}-\left(\mathrm{F} O-E_{0}^{\circ}\right) / T \quad$ So
$\mathrm{C}_{\mathrm{p}}^{\circ} \quad-\left(\mathrm{F}^{\circ}-\mathrm{E}_{\mathrm{O}}\right) / \mathrm{T}$
So
$\mathrm{C}_{\mathrm{p}}^{\circ} \quad-\left(\mathrm{F}^{\circ}-\mathrm{E}_{\mathrm{O}}^{\circ}\right) / \mathrm{T}$
$\mathrm{S}^{\circ}$
$T=2700$
$\mathrm{T}=2800$.

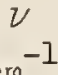

$\mathrm{T}=2600$.

$1100 \quad 1.56065 \quad 3.00398 \quad 1.92693$ $\begin{array}{lllll}1110 & 1.54760 & 2.98655 & 1.92585\end{array}$ $1120 \quad 1.534722 .969281 .92476$ $\begin{array}{lllll}1130 & 1.52199 & 2.95218 & 1.92366\end{array}$ $1140 \quad 1.50940 \quad 2.93523 \quad 1.92256$

$\begin{array}{llll}1150 & 1.49697 & 2.91845 & 1.92144\end{array}$ $\begin{array}{llll}1160 \quad 1.48468 \quad 2.90182 & 1.92032\end{array}$ $\begin{array}{lllll}1170 & 1.47254 & 2.88534 & 1.91918\end{array}$ $\begin{array}{lllll}1180 & 1.46053 & 2.86901 & 1.91804\end{array}$ $1190 \quad 1.44866 \quad 2.85283 \quad 1.91689$

$1200 \quad 1.43693 \quad 2.83679 \quad 1.91573$ $\begin{array}{lllll}1210 & 1.42533 & 2.82090 & 1.91456\end{array}$ $\begin{array}{lllll}1220 & 1.41386 & 2.80515 & 1.91338\end{array}$ $1230 \quad 1.40252 \quad 2.78953 \quad 1.91219$ $1240 \quad 1.391312 .774051 .91100$

$\begin{array}{lllll}1250 & 1.38022 & 2.75871 & 1.90979\end{array}$ $\begin{array}{lllll}1260 & 1.36925 & 2.74350 & 1.90858\end{array}$ $\begin{array}{lllll}1270 & 1.35841 & 2.72841 & 1.90736\end{array}$ $\begin{array}{llll}1280 & 1.34768 & 2.71346 & 1.90613\end{array}$ $1290 \quad 1.33706 \quad 2.69863 \quad 1.90489$

$\begin{array}{lllll}1300 \quad 1.32657 & 2.68392 & 1.90364\end{array}$ $\begin{array}{lllll}1310 & 1.31618 & 2.66934 & 1.90238\end{array}$ $\begin{array}{lllll}1320 & 1.30591 & 2.65488 & 1.90112\end{array}$ $\begin{array}{llll}1330 & 1.29574 \quad 2.64054 \quad 1.89984\end{array}$ $\begin{array}{lllll}1340 & 1.28568 & 2.62631 & 1.89856\end{array}$

$\begin{array}{lllll}1350 & 1.27573 & 2.61220 & 1.89727\end{array}$ $\begin{array}{lllll}1360 & 1.26588 & 2.59820 & 1.89597\end{array}$ $1370 \quad 1.25614 \quad 2.58432 \quad 1.89466$ $1380 \quad 1.246492 .57054 \quad 1.89335$ $\begin{array}{lllll}1390 \quad 1.23695 \quad 2.55688 & 1.89202\end{array}$

$\begin{array}{llll}1400 & 1.22750 & 2.54332 & 1.89069\end{array}$ $\begin{array}{lllll}1410 & 1.21815 & 2.52987 & 1.88935\end{array}$ $1420 \quad 1.20890 \quad 2.51652 \quad 1.88800$ $\begin{array}{lllll}1430 & 1.19973 & 2.50327 & 1.88664\end{array}$ $1440 \quad 1.19066 \quad 2.49013 \quad 1.88527$

1450

1460

1470

1480

1490

1500

1510

1520

1530

1540

1550

1560

1570

1580

1590
$1.181692 .47709 \quad 1.88390$

$1.17280 \quad 2.46415 \quad 1.88251$

1.164002 .451301 .88112 $1.15528 \quad 2.43855 \quad 1.87972$ $1.146652 .42590 \quad 1.87831$

$\begin{array}{llll}1.13811 & 2.41334 & 1.87689\end{array}$ $1.12965 \quad 2.40087 \quad 1.87547$ $1.121272 .38850 \quad 1.87404$ $1.11298 \quad 2.37621 \quad 1.87260$ 1.104762 .364021 .87115

$\begin{array}{llll}1.09662 & 2.35191 & 1.86969\end{array}$ $1.088562 .33989 \quad 1.86822$ $1.08058 \quad 2.32796 \quad 1.86675$ $1.072672 .31611 \quad 1.86527$ 1.064842 .304351 .86378
$1.61546 \quad 3.076791 .93123$ 1.602253 .059321 .93023 1.589213 .042011 .92922 $1.576313 .02487 \quad 1.92820$ $1.563573 .00788 \quad 1.92717$

$1.550972 .99105 \quad 1.92613$ $1.53852 \quad 2.97438 \quad 1.92508$ $1.526212 .95786 \quad 1.92403$ $\begin{array}{lllll}1.51405 & 2.94149 & 1.92297\end{array}$ $1.502022 .92527 \quad 1.92190$

1.490132 .909191 .92082 $1.478372 .89325 \quad 1.91973$ $1.466742 .87746 \quad 1.91863$ $1.455242 .86180 \quad 1.91753$ 1.443872 .846281 .91642

1.432622 .830891 .91530 1.421492 .815631 .91417 $1.410492 .80051 \quad 1.91303$ $\begin{array}{llll}1.39960 & 2.78551 & 1.91188\end{array}$ 1.388832 .770631 .91073

$\begin{array}{llll}1.37818 & 2.75588 & 1.90957\end{array}$ $1.367642 .74125 \quad 1.90840$ $\begin{array}{llll}1.35721 & 2.72675 & 1.90722\end{array}$ $\begin{array}{llll}1.34689 & 2.71236 & 1.90604\end{array}$ $1.336672 .69808 \quad 1.90484$

$\begin{array}{llll}1.32657 & 2.68392 & 1.90364\end{array}$ $\begin{array}{llll}1.31656 & 2.66988 & 1.90243\end{array}$ 1.306662 .655951 .90121 1.296872 .642121 .89999 1.287172 .628411 .89875

$\begin{array}{llll}1.27757 & 2.61480 & 1.89751\end{array}$ $1.26806 \quad 2.601301 .89626$ $\begin{array}{llll}1.25866 & 2.58791 & 1.89500\end{array}$ $1.249342 .57461 \quad 1.89374$ 1.240122 .561421 .89246

1.230992 .548331 .89118 1.221952 .535331 .88989 $1.21300 \quad 2.52244 \quad 1.88860$ $1.204132 .50964 \quad 1.88729$ $1.19536 \quad 2.49693 \quad 1.88598$

$1.18666 \quad 2.48432 \quad 1.88466$ $1.17805 \quad 2.47180 \quad 1.88333$ 1.169532 .459381 .88200 $1.16108 \quad 2.44704 \quad 1.88065$ $1.15272 \quad 2.43479 \quad 1.87930$

1.144432 .422631 .87794 $1.13622 \quad 2.41056 \quad 1.87658$ $\begin{array}{llll}1.12809 & 2.39857 & 1.87521\end{array}$ $1.12004 \quad 2.38667 \quad 1.87382$ $1.11206 \quad 2.37486 \quad 1.87244$
1.668913 .147091 .93510 1.655553 .129591 .93416 $1.642353 .11224 \quad 1.93322$ $1.629313 .09506 \quad 1.93227$ 1.616413 .078041 .93131

$1.60366 \quad 3.06118 \quad 1.93034$ $1.59106 \quad 3.04447 \quad 1.92936$ $\begin{array}{llll}1.57860 & 3.02791 & 1.92838\end{array}$ $1.56628 \quad 3.01151 \quad 1.92739$ 1.554112 .995251 .92639

$1.54206 \quad 2.979131 .92538$ $1.530152 .96316 \quad 1.92437$ 1.518382 .947321 .92335 1.506732 .931621 .92232 $1.495212 .91606 \quad 1.92128$

$1.483812 .90064 \quad 1.92023$ $1.472542 .88534 \quad 1.91918$ 1.461382 .870171 .91812 1.450352 .855131 .91705 1.439432 .840221 .91598

1.428632 .825431 .91489 $1.417942 .81076 \quad 1.91380$ 1.407372 .796211 .91270 $1.39690 \quad 2.78178 \quad 1.91160$ $1.386542 .76746 \quad 1.91048$

$1.376292 .75326 \quad 1.90936$ $1.366142 .73917 \quad 1.90823$ $1.35610 \quad 2.72520 \quad 1.90710$ 1.346152 .711331 .90595 $1.336312 .69757 \quad 1.90480$

$1.326572 .68392 \quad 1.90364$ $1.31692 \quad 2.67038 \quad 1.90247$ $1.307372 .65694 \quad 1.90130$ 1.297912 .643601 .90012 $1.288552 .63036 \quad 1.89893$

$1.279272 .61723 \quad 1.89773$ $1.270092 .60419 \quad 1.89653$ $1.26100 \quad 2.59125 \quad 1.89532$. $\begin{array}{llll}1.25199 & 2.57840 & 1.89410\end{array}$ $1.24307 \quad 2.56565 \quad 1.89287$

$1.23424 \quad 2.55299 \quad 1.89164$ $1.225492 .54043 \quad 1.89040$ 1.216822 .527951 .88915 $1.208242 .51557 \quad 1.88790$ $1.19973 \quad 2.50327 \quad 1.88664$

$\begin{array}{llll}1.19131 & 2.49107 & 1.88537\end{array}$ $1.18296 \quad 2.47895 \quad 1.88409$ $1.174692 .46691 \quad 1.88281$ $1.16650 \quad 2.45496 \quad 1.88152$ $1.15838 \quad 2.44309 \quad 1.88022$
1100

1110

1120

1130

1140

1150

1160

1170

1180

1190

1200

1210

1220

1230

1240

1250

1260

1270

1280

1290

1300

1310

1320

1330

1340

1350

1360

1370

1380

1390

1400

1410

1420

1430

1440

1450

1460

1470

1480

1490

1500

1510

1520

1530

1540

1550 1560 1570 1580 1590 
Table II Harmonic Oscillator Contributions to the Thermodynamic

Functions (in units of calories, moles, and ${ }^{\circ} \mathrm{K}$ )

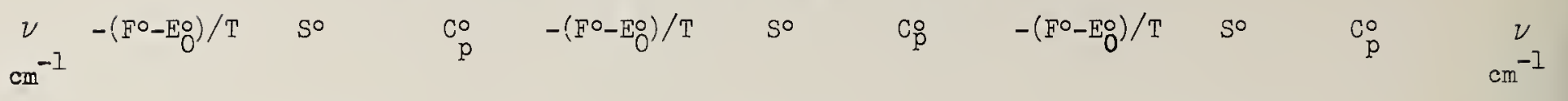

$$
T=2600
$$

$\begin{array}{llll}1.05708 \quad 2.29267 & 1.86228\end{array}$ $\begin{array}{llll}1.04939 & 2.28107 \quad 1.86078\end{array}$ $1.04178 \quad 2.26955 \quad 1.85926$ 1.034232 .258121 .85774 $1.026762 .24676 \quad 1.85621$

1600

1620

1630

1640

1650

1660

1670

1680

1690

1700

1710

1720

1730

1740

1750

1760

1770

1780

1790

1800

1810

1820

1830

1840

1850

1860

1870

1880

1890

1900

1910

1920

1930

1940

1950

1960

1970

1980

1990

2000

2010

2020

2030

2040

2050

2060

2070

2080

2090

$1.01936 \quad 2.23548 \quad 1.85468$ $1.01202 \quad 2.22428 \quad 1.85313$ $1.004752 .21315 \quad 1.85158$ $0.99755 \quad 2.20210 \quad 1.85002$ 0.990412 .191131 .84845

$0.98334 \quad 2.18023 \quad 1.84688$ $0.976332 .16940 \quad 1.84529$ $0.96938 \quad 2.15865 \quad 1.84370$ $0.96250 \quad 2.14796 \quad 1.84211$ 0.955682 .137351 .84050

$0.94892 \quad 2.12681 \quad 1.83889$ 0.942222 .116331 .83726 0.935572 .105931 .83564 $0.928992 .09559 \quad 1.83400$ 0.922472 .085321 .83236

$0.91600 \quad 2.075121 .83071$ $0.909592 .06498 \quad 1.82905$ $0.903232 .05491 \quad 1.82738$ $0.896932 .04490 \quad 1.82571$ 0.890692 .034951 .82403

$0.884492 .02507 \quad 1.82234$ 0.878352 .015251 .82064 0.872272 .005491 .81894 0.866231 .995801 .81723 $0.860251 .98616 \quad 1.81552$

$0.85432 \quad 1.97659 \quad 1.81379$ $0.84844 \quad 1.96707 \quad 1.81206$ 0.842611 .957611 .81032 $0.83682 \quad 1.94821 \quad 1.80858$ $0.831091 .93887 \quad 1.80683$

$0.82540 \quad 1.92958 \quad 1.80507$ $0.81976 \quad 1.92036 \quad 1.80330$ $0.81417 \quad 1.91118 \quad 1.80153$ $0.80863 \quad 1.90207 \quad 1.79975$ $0.80313 \quad 1.89300 \quad 1.79796$

$\begin{array}{llll}0.79767 & 1.88400 & 1.79617\end{array}$ $0.79226 \quad 1.87504 \quad 1.79437$ $0.78690 \quad 1.86614 \quad 1.79256$ $0.78158 \quad 1.85729 \quad 1.79075$ $0.77630 \quad 1.84850 \quad 1.78893$

$0.77107 \quad 1.83976 \quad 1.78710$ $0.76587 \quad 1.83106 \quad 1.78526$ $0.76072 \quad 1.82242 \quad 1.78342$ $0.75562 \quad 1.81383 \quad 1.78157$ $0.75055 \quad 1.80529 \quad 1.77972$

$$
T=2700
$$

$$
T=2800
$$

$1.10415 \quad 2.363121 .87104$ $1.096322 .35147 \quad 1.86964$ $1.08856 \quad 2.33989 \quad 1.86822$ $1.080872 .32840 \quad 1.86681$ $1.073252 .31699 \quad 1.86538$

$1.06570 \quad 2.30565 \quad 1.86395$ $1.05822 \quad 2.29440 \quad 1.86251$ $1.050812 .28321 \quad 1.86106$ $1.04346 \quad 2.27211 \quad 1.85960$ $1.03618 \quad 2.26107 \quad 1.85814$

$\begin{array}{llll}1.02897 & 2.25012 & 1.85667\end{array}$ $\begin{array}{lll}1.02182 & 2.23923 & 1.85519\end{array}$ 1.014732 .228421 .85371 $1.00770 \quad 2.21768 \quad 1.85221$ $1.00074 \quad 2.20701 \quad 1.85072$

$0.99384 \quad 2.19640 \quad 1.84921$ $0.98700 \quad 2.18587 \quad 1.84770$ 0.980212 .175411 .84617 $0.973492 .16501 \quad 1.84465$ 0.966832 .154681 .84311

$0.96022 \quad 2.144421 .84157$ $0.953672 .13422 \quad 1.84002$ $0.947172 .12408 \quad 1.83847$ $0.940742 .11401 \quad 1.83690$ $0.934352 .10401 \quad 1.83533$

$0.92802 \quad 2.09407 \quad 1.83376$ $0.92175 \quad 2.08418 \quad 1.83217$ $0.91552 \quad 2.07436 \quad 1.83058$ $0.909352 .06461 \quad 1.82899$ $0.90323 \quad 2.05491 \quad 1.82738$

$\begin{array}{llll}0.89716 & 2.04527 & 1.82577\end{array}$ 0.891152 .035691 .82415 $0.88518 \quad 2.02617 \quad 1.82253$ $0.87926 \quad 2.01670 \quad 1.82090$ $0.873392 .00730 \quad 1.81926$

$0.86757 \quad 1.99795 \quad 1.81761$ $0.86180 \quad 1.988651 .81596$ $0.85607 \quad 1.979421 .81430$ $0.850391 .97024 \quad 1.81264$ $0.84476 \quad 1.96111 \quad 1.81097$

$0.83917 \quad 1.95203 \quad 1.80929$ $0.833631 .94301 \quad 1.80761$ 0.828131 .934051 .80592 0.822681 .925131 .80422 $0.81727 \quad 1.91627 \quad 1.80251$

$0.81191 \quad 1.90746 \quad 1.80080$ 0.806581 .898701 .79909 $0.801301 .89000 \quad 1.79736$ 0.796071 .881341 .79564 $0.79087 \quad 1.87273 \quad 1.79390$
$1.15034 \quad 2.43131 \quad 1.87892$ 1.142372 .419611 .87760 $1.134472 .40799 \quad 1.87629$ $1.126652 .39644 \quad 1.87496$ $\begin{array}{lll}1.11889 & 2.38498 & 1.87363\end{array}$

$\begin{array}{llll}1.11121 & 2.37359 & 1.87229\end{array}$ $1.103592 .36229 \quad 1.87094$ 1.096042 .351051 .86959 1.088562 .339891 .86822 $1.081152 .32881 \quad 1.86686$

$\begin{array}{llll}1.07380 & 2.31780 \quad 1.86548\end{array}$ 1.066512 .306861 .86410 $1.059292 .29600 \quad 1.86271$ 1.052132 .285201 .86132 $\begin{array}{llll}1.04503 & 2.27448 & 1.85991\end{array}$

$1.03800 \quad 2.263831 .85850$ 1.031022 .253241 .85709 1.024112 .242721 .85567 $\begin{array}{llll}1.01725 & 2.23227 & 1.85424\end{array}$ $1.01046 \quad 2.221891 .85280$

$1.00372 \quad 2.21157 \quad 1.85136$ $0.99704 \quad 2.201321 .84991$ 0.990412 .191131 .84845 $0.983842 .18100 \quad 1.84699$ $0.977332 .17094 \quad 1.84552$

$0.970872 .16094 \quad 1.84405$ 0.964462 .151011 .84256 $0.958112 .14113 \quad 1.84107$ 0.951812 .131321 .83958 $0.94556 \quad 2.12156 \quad 1.83808$

$\begin{array}{llll}0.93936 & 2.11187 & 1.83657\end{array}$ $0.93322 \quad 2.102231 .83505$ $0.927122 .09265 \quad 1.83353$ 0.921082 .083131 .83200 $0.91508 \quad 2.07367 \quad 1.83047$

$0.90913 \quad 2.06426 \quad 1.82893$ $0.90323 \quad 2.05491 \quad 1.82738$ $\begin{array}{llll}0.89738 & 2.04561 & 1.82583\end{array}$ $\begin{array}{llll}0.89157 & 2.03637 & 1.82427\end{array}$ $0.88582 \quad 2.02718 \quad 1.82270$

$0.88010 \quad 2.01805 \quad 1.82113$ $0.874442 .00397 \quad 1.81955$ $0.86881 \quad 1.999951 .81797$ $0.86324 \quad 1.99097 \quad 1.81638$ $0.85770 \quad 1.982051 .81478$

$0.85221 \quad 1.97318 \quad 1.81318$ $0.84677 \quad 1.96436 \quad 1.81157$ $0.84136 \quad 1.95559 \quad 1.80995$ 0.836001 .946871 .80833 0.830681 .938201 .80670
1600

1610

1620

1630

1640

1650

1660

1670

1680

1690

1700

1710

1720

1730

1740

1750

1760

1770

1780

1790

1800

1810

1820

1830

1840

1850

1860

1870

1880

1890

1900

1910 1920 1930

1940

1950

1960

1970

1980 1990

2000

2010

2020

2030

2040

2050 2060 2070 2080 2090 
Table II Harmonic Oscillator Contributions to the Thermodynamic

Functions (in units of calories, moles, and ${ }^{\circ} \mathrm{K}$ )
$\mathrm{cm}^{-1}$
$-\left(F^{\circ}-E_{O} O\right) / T \quad S^{\circ}$
$\mathrm{C}_{\mathrm{p}} \quad-\left(\mathrm{F}^{\circ}-\mathrm{E}_{\mathrm{O}}^{\circ}\right) / \mathrm{T}$
So
$\mathrm{C}_{\mathrm{p}}^{\circ}$
$-\left(\mathrm{F}^{\circ}-\mathrm{E}_{\mathrm{O}}\right) / \mathrm{T}$
So

$\mathrm{cn}^{-1}$

$T=2600$.

$T=2700$.

$\mathrm{T}=2800$.

$\begin{array}{llll}2100 & 0.74552 & 1.79680 & 1.77786\end{array}$ $2110 \quad 0.740541 .788361 .77599$ $\begin{array}{llll}2120 & 0.73559 & 1.77997 & 1.77412\end{array}$ $\begin{array}{llll}2130 & 0.73068 & 1.77162 & 1.77224\end{array}$ $2140 \quad 0.725821 .76333 \quad 1.77035$

$\begin{array}{lllll}2150 & 0.72099 & 1.75508 & 1.76846\end{array}$ $\begin{array}{lllll}2160 & 0.71620 & 1.74688 & 1.76656\end{array}$ $\begin{array}{llll}2170 & 0.71144 & 1.73872 & 1.76465\end{array}$ $\begin{array}{lllll}2180 & 0.70673 & 1.73061 & 1.76274\end{array}$ $\begin{array}{llll}2190 & 0.70205 & 1.72255 & 1.76082\end{array}$

$\begin{array}{lllll}2200 & 0.69741 & 1.71453 & 1.75890\end{array}$ $\begin{array}{llll}2210 & 0.69280 & 1.70656 & 1.75697\end{array}$ $\begin{array}{llll}2220 & 0.68823 & 1.69863 & 1.75503\end{array}$ $\begin{array}{llll}2230 & 0.68370 & 1.69075 & 1.75309\end{array}$ $\begin{array}{llll}2240 & 0.67920 & 1.68291 & 1.75114\end{array}$

$\begin{array}{lllll}2250 & 0.67474 & 1.67511 & 1.74918\end{array}$ $\begin{array}{lllll}2260 & 0.67031 & 1.66736 & 1.74722\end{array}$ $\begin{array}{lllll}2270 & 0.66592 & 1.65965 & 1.74525\end{array}$ $2280 \quad 0.661551 .651981 .74328$ $2290 \quad 0.65723 \quad 1.64436 \quad 1.74130$

$\begin{array}{lllll}2300 & 0.65293 & 1.63677 & 1.73931\end{array}$ $\begin{array}{lllll}2310 & 0.64867 & 1.62923 & 1.73732\end{array}$ $\begin{array}{lllll}2320 & 0.64444 & 1.62173 & 1.73532\end{array}$ $\begin{array}{lllll}2330 & 0.64025 & 1.61427 & 1.73332\end{array}$ $2340 \quad 0.63608 \quad 1.606851 .73131$

$\begin{array}{lllll}2350 & 0.63195 & 1.59947 & 1.72930\end{array}$ $\begin{array}{lllll}2360 & 0.62785 & 1.59214 & 1.72728\end{array}$ $\begin{array}{lllll}2370 & 0.62378 & 1.58484 & 1.72525\end{array}$ $\begin{array}{lllll}2380 & 0.61974 & 1.57758 & 1.72322\end{array}$ $2390 \quad 0.615731 .57036 \quad 1.72118$

$\begin{array}{lllll}2400 \quad 0.61175 & 1.56317 & 1.71913\end{array}$ $2410 \quad 0.60780 \quad 1.556031 .71709$ $\begin{array}{lllll}2420 & 0.60388 & 1.54892 & 1.71503\end{array}$ $\begin{array}{lllll}2430 & 0.59999 & 1.54186 & 1.71297\end{array}$ $\begin{array}{llll}2440 & 0.59613 & 1.53483 & 1.71090\end{array}$

$\begin{array}{lllll}2450 & 0.59230 & 1.52783 & 1.70883\end{array}$ $2460 \quad 0.58849 \quad 1.52088 \quad 1.70676$ $\begin{array}{llllll}2470 & 0.58471 & 1.51396 & 1.70467\end{array}$ $\begin{array}{llllll}2480 & 0.58097 & 1.50707 & 1.70259\end{array}$ $\begin{array}{lllll}2490 & 0.57725 & 1.50023 & 1.70049\end{array}$

$\begin{array}{lllll}2500 & 0.57355 & 1.49341 & 1.69839\end{array}$ $\begin{array}{lllll}2510 & 0.56989 & 1.48664 & 1.69629\end{array}$ $\begin{array}{lllll}2520 & 0.56625 & 1.47990 & 1.69418\end{array}$ $\begin{array}{lllll}2530 & 0.56264 & 1.47319 & 1.69207\end{array}$ $\begin{array}{llll}2540 & 0.55905 & 1.46652 & 1.68995\end{array}$

2550

2560

2570

2580

2590
$0.78571 \quad 1.86417 \quad 1.79216$ 0.780601 .855661 .79041 $0.77552 \quad 1.84720 \quad 1.78865$ $0.770491 .83879 \quad 1.78689$ 0.765491 .830421 .78513

0.760531 .822101 .78335 0.755621 .813831 .78157 $0.75074 \quad 1.80561 \quad 1.77979$ 0.745891 .797431 .77800 0.741091 .789301 .77620

0.736321 .781211 .77440 0.731591 .773161 .77259 $0.726891 .76517 \quad 1.77077$ $0.722241 .75721 \quad 1.76895$ $0.71761 \quad 1.749301 .76712$

0.713021 .741431 .76529 $0.70847 \quad 1.733611 .76345$ 0.703951 .725831 .76161 0.699471 .718091 .75975 0.695021 .710391 .75790

$0.69060 \quad 1.70273 \quad 1.75604$ 0.686221 .695121 .75417 0.681861 .687551 .75229 0.677551 .680011 .75041 $0.67326 \quad 1.672521 .74853$

$0.66900 \quad 1.66507 \quad 1.74664$ 0.664781 .657661 .74474 0.660591 .650281 .74284 0.656431 .642951 .74093 0.652301 .635651 .73902

$0.64820 \quad 1.62840 \quad 1.73710$ 0.644131 .621181 .73518 0.640091 .614001 .73325 0.636081 .606851 .73131 $0.632101 .59975 \quad 1.72937$

$0.628151 .59268 \quad 1.72742$ 0.624231 .585651 .72547 0.620331 .578651 .72352 $0.61647 \quad 1.57169 \quad 1.72156$ 0.612631 .564771 .71959

$0.60882 \quad 1.55788 \quad 1.71762$ $0.60504 \quad 1.55103 \quad 1.71564$ $0.60128 \quad 1.54421 \quad 1.71366$ $0.59755 \quad 1.53742 \quad 1.71167$ $0.59385 \quad 1.53068 \quad 1.70968$

$0.59018 \quad 1.52396 \quad 1.70768$ $0.586531 .51728 \quad 1.70568$ $0.58291 \quad 1.51064 \quad 1.70367$ 0.579311 .504021 .70166 $0.57574 \quad 1.497451 .69964$
$0.82540 \quad 1.92958 \quad 1.80507$ $0.82017 \quad 1.921011 .80343$ 0.814971 .912491 .80178 0.809811 .904021 .80013 $0.804691 .89559 \quad 1.79847$

$0.799621 .88721 \quad 1.79681$ $0.79458 \quad 1.878871 .79514$ 0.789581 .870591 .79346 0.784611 .862341 .79178 0.779691 .854151 .79010

$0.77480 \quad 1.84600 \quad 1.78840$ $0.769951 .83789 \quad 1.78671$ $0.76514 \quad 1.829831 .78500$ 0.760361 .821811 .78329 $0.75562 \quad 1.813831 .78157$

$0.75091 \quad 1.80590 \quad 1.77985$

0.746241 .798011 .77813 $0.741601 .79016 \quad 1.77639$ 0.737001 .782361 .77465 0.732431 .774601 .77291

$0.72790 \quad 1.76688 \quad 1.77116$ 0.723401 .759201 .76941 $0.71893 \quad 1.75156 \quad 1.76765$ $0.714491 .74396 \quad 1.76588$ $0.710091 .73640 \quad 1.76411$

$0.70572 \quad 1.72888 \quad 1.76233$ $0.701381 .72140 \quad 1.76055$ 0.697081 .713961 .75876 $0.69280 \quad 1.70656 \quad 1.75697$ 0.688561 .699191 .75517

$0.684351 .69187 \quad 1.75336$ 0.680161 .684581 .75156 $0.67601 \quad 1.677331 .74974$ $0.67189 \quad 1.670121 .74792$ 0.667791 .662951 .74610

$0.66373 \quad 1.65581 \quad 1.74427$ $0.65970 \quad 1.64871 \quad 1.74243$ 0.655691 .641641 .74059 $0.65171 \quad 1.634611 .73874$ 0.647761 .627621 .73689

$0.64384 \quad 1.62066 \quad 1.73504$ 0.639951 .613741 .73318 0.636081 .606851 .73131 $0.63224 \quad 1.600001 .72944$ 0.628431 .593181 .72756

$0.624651 .58640 \quad 1.72568$ 0.620891 .579651 .72380 0.617161 .572931 .72191 0.613451 .566251 .72001 $0.60977 \quad 1.559601 .71811$
2100

2110

2120

2130

2140

2150

2160

2170

2180

2190

2200

2210

2220

2230

2240

2250

2260

2270

2280

2290

2300

2310

2320

2330

2340

2350

2360

2370

2380

2390

2400

2410

2420

2430

2440

2450

2460

2470

2480

2490

2500

2510

2520

2530

2540

2550

2560

2570

2580

2590 
Table II Harmonic Oscillator Contributions to the Thermodynamic

Functions (in units of calories, moles, and ${ }^{\circ} \mathrm{K}$ )

$\mathrm{cm}^{-1}-\left(\mathrm{F}^{\circ}-\mathrm{E}_{\mathrm{O}}^{0}\right) / \mathrm{T} \quad \mathrm{S}^{\circ}$ so

$T=2600$.

2600

2610

2620

2630

2640

2650

2660

2670

2680

2690

2700

2710

2720

2730

2740

2750

2760

2770

2780

2790

2800

2810

2820

2830

2840

2850

2860

2870

2880

2890

2900

2910

2920

2930

2940

2950

2960

2970

2980

2990

3000

3010

3020

3030

3040

3050

3060

3070

3080

3090
$0.53808 \quad 1.42721 \quad 1.67713$ $0.53467 \quad 1.42078 \quad 1.67497$ $0.531291 .41438 \quad 1.67281$ $0.527931 .40801 \quad 1.67065$ $0.524591 .40167 \quad 1.66848$

$0.52128 \quad 1.39537 \quad 1.66630$ $0.51800 \quad 1.38910 \quad 1.66412$ $0.514731 .38286 \quad 1.66194$ $0.51149 \quad 1.37665 \quad 1.65975$ $0.50828 \quad 1.37047 \quad 1.65756$

$0.50508 \quad 1.364331 .65536$ 0.501911 .358211 .65316 $0.49876 \quad 1.352131 .65095$ $0.495641 .34607 \quad 1.64874$ 0.492531 .340051 .64652

$0.489451 .33405 \quad 1.64430$ 0.486391 .328091 .64207 0.483351 .322151 .63984 0.480331 .316251 .63761 $0.47734 \quad 1.31037 \quad 1.63537$

$0.47436 \quad 1.30452 \quad 1.63312$ $0.47141 \quad 1.29871 \quad 1.63088$ $0.46847 \quad 1.29292 \quad 1.62863$ $\begin{array}{llll}0.46556 & 1.28716 & 1.62637\end{array}$ 0.462671 .281421 .62411

$0.45980 \quad 1.27572 \quad 1.62184$ $0.456941 .27004 \quad 1.61957$ 0.454111 .264391 .61730 $0.45130 \quad 1.25877 \quad 1.61502$ $0.44850 \quad 1.25318 \quad 1.61274$

$0.445731 .24761 \quad 1.61046$ 0.442971 .242071 .60817 $0.440241 .23656 \quad 1.60588$ $0.437521 .23107 \quad 1.60358$ 0.434821 .225611 .60128

$0.43214 \quad 1.22018 \quad 1.59897$ $0.42948 \quad 1.21477 \quad 1.59666$ 0.424211 .204031 .59203 $0.421601 .19870 \quad 1.58971$

$0.41901 \quad 1.19340 \quad 1.58739$ 0.416441 .188121 .58506 $0.41388 \quad 1.18287 \quad 1.58273$ 0.411341 .177641 .58040 0.408821 .172441 .57806

$0.406321 .16726 \quad 1.57572$ 0.403831 .162101 .57337 $0.40136 \quad 1.15698 \quad 1.57102$ $0.39891 \quad 1.15187 \quad 1.56867$ $0.39647 \quad 1.14679 \quad 1.56631$ 0.426831 .209391 .59435

$$
T=2700
$$

$\mathrm{co}_{\mathrm{p}}$

$-(F \circ-E O) / T$

So

$\mathrm{c}_{\mathrm{p}}^{\circ}$ $v$

$\mathrm{cm}^{-1}$

$0.57219 \quad 1.49090 \quad 1.69762$ 0.568671 .484391 .69559 0.565181 .477911 .69356 $0.56170 \quad 1.47146 \quad 1.69152$ $0.558261 .46504 \quad 1.68948$

$0.554831 .45866 \quad 1.68743$ $0.55144 \quad 1.45231 \quad 1.68538$ $0.54806 \quad 1.44599 \quad 1.68332$ $0.544711 .43970 \quad 1.68126$ 0.541381 .433441 .67920

$0.53808 \quad 1.42721 \quad 1.67713$ $0.53480 \quad 1.42102 \quad 1.67505$ $0.53154 \quad 1.41485 \quad 1.67297$ 0.528301 .408721 .67089 0.525091 .402611 .66880

$0.52189 \quad 1.39654 \quad 1.66670$ 0.518731 .390491 .66461 $0.515581 .38447 \quad 1.66251$

0.512451 .378491 .66040 0.509351 .372531 .65829

0.506261 .366601 .65617 0.503201 .360701 .65405 $0.50016 \quad 1.354831 .65193$ $0.497141 .34898 \quad 1.64980$ 0.494141 .343171 .64767

$0.49116 \quad 1.33738 \quad 1.64553$ 0.488201 .331621 .64339 $0.485261 .32589 \quad 1.64125$ 0.482341 .320181 .63910 0.479441 .314501 .63694

0.476571 .308851 .63479 0.47370 .1 .303231 .63263 0.470861 .297631 .63046 $0.468041 .29206 \quad 1.62829$ 0.465241 .286521 .62612

$0.46245 \quad 1.28100 \quad 1.62394$ 0.459691 .275511 .62176 $0.456941 .27004 \quad 1.61957$ $0.45421 \quad 1.264601 .61739$ $0.45150 \quad 1.259191 .61519$

0.448811 .253801 .61300 0.446141 .248431 .61080 0.443481 .243091 .60859 0.440841 .237781 .60639 0.438221 .232491 .60417

0.435621 .227231 .60196 0.433031 .221991 .59974 $0.43046 \quad 1.21677 \quad 1.59752$ 0.427911 .211581 .59529 0.425371 .206411 .59306
$T=2800$.

$0.606121 .55298 \quad 1.71621$ $0.602491 .54640 \quad 1.71430$ $0.59888 \quad 1.53984 \quad 1.71238$ $0.59530 \quad 1.53332 \quad 1.71046$ $0.591751 .52684 \quad 1.70854$

$0.58822 \quad 1.52038 \quad 1.70661$ $0.58471 \quad 1.51396 \quad 1.70467$ $0.58123 \quad 1.50756 \quad 1.70274$ $0.57778 \quad 1.50120 \quad 1.70079$ 0.574341 .494871 .69884

$0.57093 \quad 1.48857 \quad 1.69689$ $0.56754 \quad 1.48230 \quad 1.69494$ $0.56418 \quad 1.47606 \quad 1.69297$ 0.560841 .469851 .69101 $0.55752 \quad 1.46367 \quad 1.68904$

$0.554231 .45752 \quad 1.68706$ $0.55095 \quad 1.45140 \quad 1.68509$ $0.54770 \quad 1.445311 .68310$ 0.544471 .439251 .68111 $0.54126 \quad 1.43322 \quad 1.67912$

$0.53808 \quad 1.42721 \quad 1.67713$ $0.53491 \quad 1.42124 \quad 1.67512$ $0.53177 \quad 1.415291 .67312$ $0.528651 .40937 \quad 1.67111$ 0.525541 .403481 .66910

$0.52246 \quad 1.39762 \quad 1.66708$ $0.51940 \quad 1.39178 \quad 1.66506$ $0.51636 \quad 1.38597 \quad 1.66303$ 0.513341 .380191 .66100 $0.510341 .37444 \quad 1.65897$

$0.50736 \quad 1.36871 \quad 1.65693$ $0.50440 \quad 1.36301 \quad 1.65489$ $0.50146 \quad 1.35734 \quad 1.65284$ 0.498541 .351691 .65079 0.495641 .346071 .64874

$0.49275 \quad 1.34048 \quad 1.64668$ $0.489891 .33491 \quad 1.64462$ $0.487051 .32936 \quad 1.64255$ 0.484221 .323851 .64048 $0.48141 \quad 1.31835 \quad 1.63841$

$0.47862 \quad 1.31289 \quad 1.63633$ $0.475851 .30744 \quad 1.63425$ 0.473091 .302031 .63216 $0.47036 \quad 1.29664 \quad 1.63007$ $0.467641 .29127 \quad 1.62798$

$0.46494 \quad 1.28592 \quad 1.62588$ 0.462261 .280611 .62379 $0.459591 .27531 \quad 1.62168$ 0.456941 .270041 .61957 $0.45431 \quad 1.264801 .61746$
2600

2610

2620

2630

2640

2650

2660

2670

2680

2690

2700

2710

2720

2730

2740

2750

2760

2770

2780

2790

2800

2810

2820

2830

2840

2850

2860

2870

2880

2890

2900

2910

2920

2930

2940

2950

2960

2970

2980

2990

3000

3010

3020

3030

3040

3050

3060

3070

3080

3090 
Table II Harmonic Oscillator Contributions to the Thermodynamic

Functions (in units of calories, moles, and ${ }^{\circ} \mathrm{K}$ )
$\mathrm{cm}^{-1}-\left(\mathrm{F}^{\circ}-\mathrm{E}_{\mathrm{O}}^{\circ}\right) / \mathrm{T} \quad$ So
$\mathrm{C}_{\mathrm{p}}^{\circ} \quad-\left(\mathrm{F}^{\circ}-\mathrm{E}_{\mathrm{O}}^{\circ}\right) / \mathrm{T}$
so
$\mathrm{C}_{\mathrm{p}}$
$-\left(F^{\circ}-E_{O}^{\circ}\right) / T$
so
$\stackrel{\mathrm{p}}{\mathrm{c}} \quad \stackrel{\nu}{\mathrm{cm}^{-1}}$

$T=2600$.

$T=2700$.

$T=2800$.

$\begin{array}{lllll}3100 & 0.39405 & 1.14173 & 1.56395\end{array}$

$3110 \quad 0.391651 .136701 .56159$

$\begin{array}{lllll}3120 & 0.38926 & 1.13169 & 1.55922\end{array}$

$\begin{array}{lllll}3130 & 0.38689 & 1.12670 & 1.55686\end{array}$

$\begin{array}{llll}3140 & 0.38453 & 1.12174 & 1.55448\end{array}$

$\begin{array}{lllll}3150 & 0.38219 & 1.11680 & 1.55211\end{array}$

$\begin{array}{lllll}3160 & 0.37987 & 1.11189 & 1.54973\end{array}$

$\begin{array}{lllll}3170 & 0.37756 & 1.10699 & 1.54735\end{array}$

$3180 \quad 0.37527 \quad 1.102121 .54496$

$\begin{array}{lllll}3190 & 0.37299 & 1.09728 & 1.54257\end{array}$

$\begin{array}{lllll}3200 & 0.37073 & 1.09245 & 1.54018\end{array}$

$\begin{array}{lllll}3210 & 0.36848 & 1.08765 & 1.53779\end{array}$

$\begin{array}{lllll}3220 & 0.36625 & 1.08287 & 1.53539\end{array}$

$\begin{array}{lllll}3230 & 0.36403 & 1.07811 & 1.53299\end{array}$

$\begin{array}{lllll}3240 & 0.36182 & 1.07338 & 1.53058\end{array}$

$\begin{array}{lllll}3250 & 0.35964 & 1.06867 & 1.52818\end{array}$

$\begin{array}{lllll}3260 & 0.35746 & 1.06398 & 1.52577\end{array}$

$\begin{array}{lllll}3270 & 0.35530 & 1.05931 & 1.52335\end{array}$

$\begin{array}{lllll}3280 & 0.35316 & 1.05466 & 1.52094\end{array}$

$\begin{array}{lllll}3290 & 0.35102 & 1.05003 & 1.51852\end{array}$

$\begin{array}{llll}3300 & 0.34891 & 1.04543 & 1.51610\end{array}$

$\begin{array}{lllll}3310 & 0.34680 & 1.04084 & 1.51368\end{array}$

$\begin{array}{lllll}3320 & 0.34471 & 1.03628 & 1.51125\end{array}$

$\begin{array}{llllll}3330 & 0.34264 & 1.03174 & 1.50882\end{array}$

$\begin{array}{lllll}3340 & 0.34057 & 1.02722 & 1.50639\end{array}$

$\begin{array}{lllll}3350 & 0.33852 & 1.02272 & 1.50396\end{array}$

$\begin{array}{lllll}3360 & 0.33649 & 1.01824 & 1.50152\end{array}$

$\begin{array}{lllll}3370 & 0.33447 & 1.01378 & 1.49908\end{array}$

$\begin{array}{lllll}3380 & 0.33246 & 1.00934 & 1.49664\end{array}$

$\begin{array}{lllll}3390 & 0.33046 & 1.00493 & 1.49420\end{array}$

$\begin{array}{lllll}3400 & 0.32848 & 1.00053 & 1.49175\end{array}$

$\begin{array}{llllll}3410 & 0.32651 & 0.99615 & 1.48930\end{array}$

$3420 \quad 0.324550 .99179 \quad 1.48685$

$\begin{array}{lllll}3430 & 0.32261 & 0.98746 & 1.48440\end{array}$

$3440 \quad 0.320670 .983141 .48194$

$\begin{array}{lllll}3450 & 0.31875 & 0.97884 & 1.47948\end{array}$

$\begin{array}{lllll}3460 & 0.31685 & 0.97456 & 1.47702\end{array}$

$\begin{array}{llll}3470 & 0.31495 & 0.97030 & 1.47456\end{array}$

$\begin{array}{lllll}3480 & 0.31307 & 0.96606 & 1.47209\end{array}$

$\begin{array}{lllll}3490 & 0.31120 & 0.96184 & 1.46963\end{array}$

$\begin{array}{llll}3500 & 0.30934 & 0.95764 & 1.46716\end{array}$ $\begin{array}{lllll}3510 & 0.30750 & 0.95346 & 1.46468\end{array}$ $\begin{array}{llllll}3520 & 0.30566 & 0.94929 & 1.46221\end{array}$ $\begin{array}{lllll}3530 & 0.30384 & 0.94515 & 1.45974\end{array}$ $3540 \quad 0.302030 .941021 .45726$

$\begin{array}{lllll}3550 & 0.30023 & 0.93692 & 1.45478\end{array}$ $\begin{array}{lllll}3560 & 0.29844 & 0.93283 & 1.45230\end{array}$ $\begin{array}{llllll}3570 & 0.29666 & 0.92876 & 1.44981\end{array}$ $\begin{array}{llllll}3580 & 0.29490 & 0.92471 & 1.44733\end{array}$ $\begin{array}{lllll}3590 & 0.29315 & 0.92067 & 1.44484\end{array}$
$0.422851 .20127 \quad 1.59083$ 0.420351 .196151 .58860 0.417861 .191051 .58636 $0.415391 .18598 \quad 1.58411$ 0.412941 .180931 .58187

$0.41050 \quad 1.17590 \quad 1.57962$ 0.408081 .170901 .57736 0.405671 .165921 .57511 0.403281 .160961 .57285 $0.40091 \quad 1.156031 .57059$

0.398551 .151121 .56832 0.396201 .146231 .56605 $0.39387 \quad 1.14136 \quad 1.56378$ $0.391561 .13651 \quad 1.56150$ 0.389261 .131691 .55922

$0.38698 \quad 1.126891 .55694$ $0.38471 \quad 1.122111 .55466$ 0.382451 .117351 .55237 0.380211 .112611 .55008 $0.377991 .10790 \quad 1.54779$

0.375781 .103201 .54549 0.373581 .098531 .54319 0.371401 .093881 .54089 0.369231 .089251 .53858 0.367071 .084641 .53628

0.364931 .080051 .53397 $0.362801 .07548 \quad 1.53165$ 0.360691 .070931 .52934 $0.358591 .06640 \quad 1.52702$ 0.356501 .061901 .52470

$0.354431 .05741 \quad 1.52237$ 0.352361 .052941 .52004 0.350321 .048491 .51772 $0.348281 .04407 \quad 1.51538$ 0.346261 .039661 .51305

$0.344251 .03527 \quad 1.51071$ 0.342251 .030901 .50837 0.340271 .026551 .50603 0.338301 .022221 .50369 0.336341 .017911 .50134

0.334391 .013621 .49899 0.332461 .009341 .49664 0.330541 .005091 .49429 0.328621 .000851 .49193 0.326730 .996641 .48957

0.324840 .992441 .48721 0.322970 .988261 .48485 0.321100 .984101 .48249 0.319250 .979951 .48012 0.317410 .975831 .47775
$0.45170 \quad 1.25957 \quad 1.61535$

$0.449101 .25437 \quad 1.61323$

0.446521 .249201 .61111

0.443951 .244051 .60899

0.441411 .238921 .60686

$0.43887 \quad 1.233811 .60473$

$0.43636 \quad 1.22873 \quad 1.60259$

$0.43386 \quad 1.22367 \quad 1.60045$

0.431381 .218631 .59831

0.428911 .213621 .59617

0.426461 .208621 .59402

0.424021 .203651 .59187

$0.42160 \quad 1.19870 \quad 1.58971$

$0.419191 .19378 \quad 1.58756$

$0.416801 .18887 \quad 1.58540$

0.414431 .183991 .58323

0.412071 .179131 .58106

0.409721 .174291 .57889

$0.407391 .16947 \quad 1.57672$

$0.40507 \quad 1.16468 \quad 1.57454$

$0.40277 \quad 1.15990 \quad 1.57236$

$0.40048 \quad 1.155151 .57018$

$0.39821 \quad 1.150421 .56800$

$0.395951 .14570 \quad 1.56581$

$0.39371 \quad 1.14101 \quad 1.56362$

$0.39148 \quad 1.136341 .56142$ 0.389261 .131691 .55922

$0.387061 .12706 \quad 1.55702$

$0.38487 \quad 1.122451 .55482$

$0.382691 .11786 \quad 1.55262$

0.380531 .113291 .55041 $0.37838 \quad 1.10874 \quad 1.54820$

$0.376251 .10421 \quad 1.54598$

$0.374131 .09970 \quad 1.54377$

0.372021 .095211 .54155

$0.36992 \quad 1.09074 \quad 1.53932$ $0.36784 \quad 1.086281 .53710$ 0.365771 .081851 .53487 $0.36371 \quad 1.07744 \quad 1.53264$ 0.361671 .073041 .53041

$0.35964 \quad 1.06867 \quad 1.52818$ 0.357621 .064311 .52594 0.355611 .059971 .52370 0.353611 .055651 .52146 0.351631 .051351 .51921

$0.34966 \quad 1.04707 \quad 1.51697$ 0.347701 .042811 .51472 $0.34576 \quad 1.03856 \quad 1.51247$ 0.343821 .034331 .51021 0.341901 .030121 .50795
3100

3110

3120

3130

3140

3150

3160

3170

3180

3190

3200

3210

3220

3230

3240

3250

3260

3270

3280

3290

3300

3310

3320

3330

3340

3350

3360

3370

3380

3390

3400

3410

3420

3430

3440

3450

3460

3470

3480

3490

3500

3510

3520

3530

3540

3550

3560

3570

3580

3590 
Table II Harmonic Oscillator Contributions to the Thermodynamic Functions (in units of calories, moles, and ${ }^{\circ} \mathrm{K}$ )

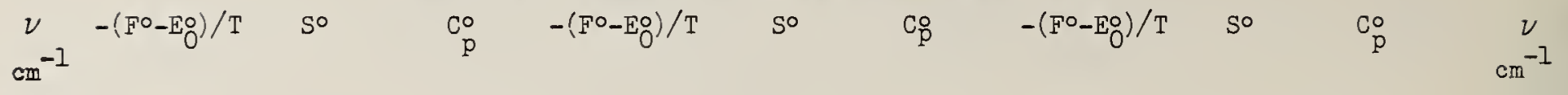

$$
T=2600 \text {. }
$$

3600

3610

3620

3630

3640

3650

3660

3670

3680

3690

3700

3710

3720

3730

3740

3750

3760

3770

3780

3790

3800

3810

3820

3830

3840

3850

3860

3870

3880

3890

3900

3910

3920

3930

3940

3950

3960

3970

3980

3990

4000
$0.29140 \quad 0.91666 \quad 1.44235$ 0.289670 .912661 .43986 $0.28795 \quad 0.90868 \quad 1.43737$ 0.286240 .904721 .43487 $\begin{array}{llll}0.28454 & 0.90077 & 1.43238\end{array}$

$0.28286 \quad 0.89685 \quad 1.42988$ $0.28118 \quad 0.89294 \quad 1.42738$ $\begin{array}{llll}0.27951 & 0.889 C 5 & 1.42488\end{array}$ $0.27786 \quad 0.88517 \quad 1.42238$ $0.276210 .88132 \quad 1.41987$

$0.27458 \quad 0.87748 \quad 1.41737$ $0.272950 .87366 \quad 1.41486$ $0.271340 .86985 \quad 1.41235$ $0.26974 \quad 0.86606 \quad 1.40984$ 0.268140 .862291 .40733

$0.26656 \quad 0.858541 .40482$ $0.264990 .85480 \quad 1.40230$ $0.263420 .85108 \quad 1.39979$ $\begin{array}{llll}0.26187 & 0.84737 & 1.39727\end{array}$ $0.260320 .84368 \quad 1.39475$

$0.258790 .84001 \quad 1.39223$ $0.25726 \quad 0.83636 \quad 1.38971$ $\begin{array}{llll}0.25575 & 0.83272 & 1.38719\end{array}$ 0.254240 .829091 .38467 0.252750 .825491 .38214

$0.25126 \quad 0.82189 \quad 1.37962$ $0.24978 \quad 0.818321 .37709$ $0.248310 .81476 \quad 1.37456$ $0.24686 \quad 0.811221 .37203$ $0.245410 .80769 \quad 1.36951$

$0.24396 \quad 0.804171 .36698$ $0.24253 \quad 0.80068 \quad 1.36444$ 0.241110 .797201 .36191 $\begin{array}{llll}0.23970 & 0.79373 & 1.35938\end{array}$ $0.23829 \quad 0.79028 \quad 1.35685$

$0.236890 .78684 \quad 1.35431$ 0.235510 .783421 .35178 $0.234130 .78001 \quad 1.34924$ $\begin{array}{llll}0.23276 & 0.77662 & 1.34670\end{array}$ 0.231390 .773251 .34416 $0.23004 \quad 0.76989 \quad 1.34163$

$$
T=2700 \text {. }
$$

$$
T=2800
$$

$0.31558 \quad 0.97172 \quad 1.47538$ 0.313770 .967631 .47301 $0.31196 \quad 0.96356 \quad 1.47063$ $0.310170 .95950 \quad 1.46825$ $0.30838 \quad 0.95547 \quad 1.46588$

0.306610 .951451 .46349 0.304850 .947451 .46111 $0.30310 \quad 0.94347 \quad 1.45873$ $0.30136 \quad 0.93950 \quad 1.45634$ $0.29963 \quad 0.93555 \quad 1.45395$

0.297910 .931621 .45156 $0.296210 .92771 \quad 1.44917$ $0.29451 \quad 0.923811 .44678$ $0.292820 .91993 \quad 1.44438$ $0.291150 .91606 \quad 1.44198$

$0.28948 \quad 0.91222 \quad 1.43958$ $0.287820 .90838 \quad 1.43718$ $0.28618 \quad 0.90457 \quad 1.43478$ 0.284540 .900771 .43238 $0.282920 .89699 \quad 1.42997$

$0.28130 \quad 0.89323 \quad 1.42757$ $0.27970 \quad 0.88948 \quad 1.42516$ $0.27810 \quad 0.88575 \quad 1.42275$ 0.276520 .882031 .42034 $0.27494 \quad 0.878331 .41792$

$0.273370 .87464 \quad 1.41551$ $0.271820 .87098 \quad 1.41309$ 0.270270 .867321 .41068 $0.268730 .86369 \quad 1.40826$ $0.26720 \quad 0.86006 \quad 1.40584$

$0.26568 \quad 0.85646 \quad 1.40342$ $0.264170 .85287 \quad 1.40100$ $0.26267 \quad 0.84929 \quad 1.39857$ 0.261180 .845731 .39615 $0.25970 \quad 0.84219 \quad 1.39372$

$\begin{array}{llll}0.25822 & 0.83866 & 1.39130\end{array}$ $0.25676 \quad 0.835141 .38887$ $0.25530 \quad 0.83164 \quad 1.38644$ 0.253850 .828161 .38401 0.252420 .824691 .38158

$0.25099 \quad 0.82123 \quad 1.37915$
$0.33999 \quad 1.02593 \quad 1.50570$ $0.33809 \quad 1.02176 \quad 1.50344$ 0.336201 .017601 .50117 0.334321 .013461 .49891 0.332461 .009341 .49664

$\begin{array}{llll}0.33060 & 1.00524 & 1.49437\end{array}$ 0.328761 .001151 .49210 $0.326930 .99709 \quad 1.48983$ $0.325110 .99304 \quad 1.48755$ $0.32330 \quad 0.98900 \quad 1.48527$

$0.32150 \quad 0.98499 \quad 1.48299$ $\begin{array}{llll}0.31971 & 0.98099 & 1.48071\end{array}$ $0.31794 \quad 0.97700 \quad 1.47843$ $0.316170 .97304 \quad 1.47614$ 0.314410 .969091 .47385

$0.31267 \quad 0.96516 \quad 1.47156$ $0.31093 \quad 0.96124 \quad 1.46927$ $0.30921 \quad 0.95734 \quad 1.46698$ $0.30750 \quad 0.95346 \quad 1.46468$ $0.305790 .94959 \quad 1.46239$

$0.30410 \quad 0.94574 \quad 1.46009$ $0.302410 .94191 \quad 1.45779$ $0.30074 \quad 0.93809 \quad 1.45549$ $0.29908 \quad 0.93429 \quad 1.45318$ $0.297420 .93050 \quad 1.45088$

$\begin{array}{llll}0.29578 & 0.92673 & 1.44857\end{array}$ $0.29415 \quad 0.92297 \quad 1.44626$ $0.292520 .91924 \quad 1.44395$ $0.290910 .91551 \quad 1.44164$ $0.28930 \quad 0.91180 \quad 1.43933$

0.287710 .908111 .43701 0.286120 .904431 .43470 $0.284540 .90077 \quad 1.43238$ $0.28298 \quad 0.897131 .43006$ $0.281420 .89350 \quad 1.42774$

$\begin{array}{llll}0.27987 & 0.88988 & 1.42542\end{array}$ $0.278330 .88628 \quad 1.42309$ $0.27680 \quad 0.88269 \quad 1.42077$ $0.27528 \quad 0.87912 \quad 1.41844$ $0.27376 \quad 0.87556 \quad 1.41611$

$0.27226 \quad 0.87202 \quad 1.41378$
3600

3610

3620

3630

3640

3650

3660

3670

3680

3690

3700

3710

3720

3730

3740

3750

3760

3770

3780

3790

3800

3810

3820

3830

3840

3850

3860

3870

3880

3890

3900

3910

3920

3930

3940

3950 3960 3970 3980 3990

4000 
Table II Harmonic Oscillator Contributions to the Thermodynamic

Functions (in units of calories, moles, and ${ }^{\circ} \mathrm{K}$ )
$\nu$
$-\left(F^{\circ}-E_{0}^{O}\right) / T$
so
$c_{p}^{\circ}$
$-\left(F^{\circ}-E_{O}^{O}\right) / T$
so
$\mathrm{C}_{\mathrm{p}}^{\circ}$
$-\left(F^{\circ}-E_{O}^{\circ}\right) / T$
So
$\mathrm{co}_{\mathrm{p}}$
$\mathrm{cn}^{-1}$

$\mathrm{T}=2900$.

$100 \quad 6.017537 .95581 \quad 1.98676$

$110 \quad 5.833027 .76646 \quad 1.98668$

$120 \quad 5.66499 \quad 7.59360 \quad 1.98658$

$130 \quad 5.510827 .43459 \quad 1.98648$

$140 \quad 5.368427 .28738 \quad 1.98637$

$150 \quad 5.23619 \quad 7.15034 \quad 1.98625$

$160 \quad 5.112817 .022151 .98613$

$170 \quad 4.997206 .90175 \quad 1.98599$

$180 \quad 4.88848 \quad 6.78824 \quad 1.98585$

$190 \quad 4.785896 .68087 \quad 1.98570$

$200 \quad 4.688816 .57902 \quad 1.98554$

$210 \quad 4.59670 \quad 6.48215 \quad 1.98537$

$220 \quad 4.509106 .38980 \quad 1.98520$

$230 \quad 4.425616 .30155 \quad 1.98501$

$240 \quad 4.34587 \quad 6.21708 \quad 1.98482$

$250 \quad 4.26958 \quad 6.13606 \quad 1.98462$

$260 \quad 4.196476 .05822 \quad 1.98442$

$270 \quad 4.126295 .98333 \quad 1.98420$

$280 \quad 4.058845 .911181 .98398$

$290 \quad 3.99392 \quad 5.84156 \quad 1.98375$

$300 \quad 3.93136 \quad 5.77431 \quad 1.98351$

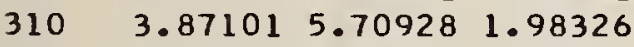

$320 \quad 3.81272 \quad 5.64632 \quad 1.98300$

$330 \quad 3.756375 .58530 \quad 1.98274$

$340 \quad 3.701845 .526111 .98246$

$350 \quad 3.64902 \quad 5.46865 \quad 1.98218$

$360 \quad 3.597835 .412811 .98190$

$370 \quad 3.54816 \quad 5.35852 \quad 1.98160$

$380 \quad 3.499945 .30567 \quad 1.98129$

$390 \quad 3.45310 \quad 5.25421 \quad 1.98098$

$400 \quad 3.40756 \quad 5.20406 \quad 1.98066$

$410 \quad 3.36325 \quad 5.15516 \quad 1.98033$

$420 \quad 3.320135 .107441 .97999$

$430 \quad 3.278135 .06086 \quad 1.97965$

$440 \quad 3.237195 .01535 \quad 1.97930$

$\begin{array}{lllll}450 & 3.19728 & 4.97087 & 1.97894\end{array}$

$460 \quad 3.15835 \quad 4.92738 \quad 1.97857$

$470 \quad 3.120364 .884831 .97819$

$480 \quad 3.08326 \quad 4.84319 \quad 1.97780$

$490 \quad 3.047014 .80241 \quad 1.97741$

$\begin{array}{lllll}500 \quad 3.01160 & 4.76247 & 1.97701\end{array}$

$\begin{array}{llll}510 & 2.97697 & 4.72332 & 1.97660\end{array}$

$520 \quad 2.943104 .68494 \quad 1.97618$

$530 \quad 2.90997 \quad 4.64731 \quad 1.97576$

$540 \quad 2.877534 .61038 .1 .97533$

$\begin{array}{lllll}550 & 2.84578 & 4.57414 & 1.97489\end{array}$

$560 \quad 2.81468 \quad 4.53856 \quad 1.97444$

$570 \quad 2.784204 .50361 \quad 1.97398$

$580 \quad 2.75434 \quad 4.46929 \quad 1.97351$

$590 \quad 2.72506 \quad 4.43556 \quad 1.97304$

$$
T=3000
$$

$6.08327 \quad 8.02317 \quad 1.98679$ $5.898607 .83381 \quad 1.98671$

$5.730417 .66095 \quad 1.98662$

$5.57607 \quad 7.50194 \quad 1.98653$

5.433527 .354721 .98642

$5.301137 .21768 \quad 1.98631$

5.177597 .089491 .98620

$5.06182 \quad 6.96908 \quad 1.98607$

$4.95293 \quad 6.85556 \quad 1.98594$

4.850196 .748191 .98579

$4.75295 \quad 6.64634 \quad 1.98565$

$4.660686 .54946 \quad 1.98549$

$4.572926 .45710 \quad 1.98533$

$4.48927 \quad 6.368851 .98516$

4.409376 .284371 .98498

$4.33292 \quad 6.20334 \quad 1.98479$ $4.259656 .12550 \quad 1.98460$ $4.189326 .05060 \quad 1.98440$ 4.121715 .978441 .98419 4.056645 .908821 .98397

$3.99392 \quad 5.84156 \quad 1.98375$

3.933415 .776521 .98351

3. $874975.71355 \quad 1.98327$

$3.81846 \quad 5.652521 .98303$

3.763775 .593331 .98277

$3.71080 \quad 5.53585 \quad 1.98251$

$3.65945 \quad 5.48001 \quad 1.98224$

3.609635 .425701 .98196

$3.56126 \quad 5.372851 .98168$

$3.51426 \quad 5.321381 .98139$

$3.468575 .27122 \quad 1.98109$

$3.42411 \quad 5.22230 \quad 1.98078$

$3.38083 \quad 5.17458 \quad 1.98046$

$3.338675 .12798 \quad 1.98014$

$3.297595 .08246 \quad 1.97981$

$3.257535 .03797 \quad 1.97947$

$3.21845 \quad 4.99447 \quad 1.97913$

$3.18030 \quad 4.95191 \quad 1.97878$

$3.14304 \quad 4.910251 .97842$

$3.106654 .86946 \quad 1.97805$

$3.07108 \quad 4.82950 \quad 1.97767$

$3.03630 \quad 4.79034 \quad 1.97729$

$3.00229 \quad 4.75195 \quad 1.97690$

$2.96900 \quad 4.71430 \quad 1.97650$

$2.93642 \quad 4.67736 \quad 1.97610$

$2.90451 \quad 4.64110 \quad 1.97569$

$2.87326 \quad 4.60551 \quad 1.97527$

$2.84264 \quad 4.570551 .97484$

$2.812624 .53621 \quad 1.97441$

$2.78320 \quad 4.50246 \quad 1.97396$
$T=3100$.

$6.14690 \quad 8.08831 \quad 1.98681$ 5.96208 7.89895 1.98674 5.793747 .726091 .98666 $5.639257 .56707 \quad 1.98657$ $5.49655 \quad 7.41986 \quad 1.98647$

$5.364017 .28281 \quad 1.98637$ 5.240327 .154611 .98626 $5.12440 \quad 7.03420 \quad 1.98614$ $5.01536 \quad 6.92068 \quad 1.98601$ 4.912476 .813311 .98588

$4.81508 \quad 6.71145 \quad 1.98574$ $4.72266 \quad 6.61457 \quad 1.98560$ $4.63476 \quad 6.52220 \quad 1.98544$ $4.55095 \quad 6.43395 \quad 1.98528$ 4.470916 .349461 .98512

$4.394316 .26842 \quad 1.98494$ $4.320906 .19058 \quad 1.98476$ $4.250426 .11567 \quad 1.98457$ $4.18266 \quad 6.04350 \quad 1.98438$ $4.117445 .97387 \quad 1.98417$

$4.05458 \quad 5.90661 \quad 1.98396$ $3.99392 \quad 5.84156 \quad 1.98375$ $3.935335 .77858 \quad 1.98352$ $3.87868 \quad 5.71755 \quad 1.98329$ $3.82385 \quad 5.658351 .98305$

$3.77073 \quad 5.60087 \quad 1.98281$ 3.719245 .545011 .98255 $3.669275 .49070 \quad 1.98229$ 3.620755 .437831 .98203 3.573615 .386351 .98175

$3.52777 \quad 5.33618 \quad 1.98147$ $3.483175 .28726 \quad 1.98118$ $3.43975 \quad 5.239521 .98089$ $3.39745 \quad 5.192911 .98059$ $3.35622 \quad 5.14738 \quad 1.98028$

$3.31602 \quad 5.10289 \quad 1.97996$ $3.27679 \quad 5.05937 \quad 1.97964$ 3.238505 .016801 .97931 3.201104 .975131 .97897 $3.16457 \quad 4.934331 .97863$

$3.12886 \quad 4.89436 \quad 1.97828$ $3.09394 \quad 4.85519 \quad 1.97792$ $3.05978 \quad 4.81679 \quad 1.97755$ $3.02635 \quad 4.77912 \quad 1.97718$ 2.993634 .742171 .97680

$2.96158 \quad 4.70590 \quad 1.97641$ 2.930194 .670291 .97602 2.899434 .635321 .97562 $2.869274 .60096 \quad 1.97521$ 2.839714 .567201 .97480
100

110

120

130

140

150

160

170

180

190

200

210

220

230

240

250

260

270

280

290

300

310

320

330

340

350

360

370

380

390

400

410

420

430

440

450

460

470

480

490

500

510

520

530

540

550

560

570

580

590 
Table II Harmonic Oscillator Contributions to the Thermodynamic Functions (in units of calories, moles, and ${ }^{\circ} \mathrm{K}$ )

\begin{tabular}{|c|c|c|c|}
\hline $\mathrm{cm}^{-1}$ & $-\left(\mathrm{FO}^{\circ}-\mathrm{E} O\right) / \mathrm{T}$ & $S^{\circ}$ & ${ }_{\mathrm{p}}^{\mathrm{Co}}$ \\
\hline & & $T=2900$. & \\
\hline $\begin{array}{l}600 \\
610 \\
620 \\
630 \\
640\end{array}$ & $\begin{array}{l}2.69635 \\
2.66819 \\
2.64055 \\
2.61343 \\
2.58681\end{array}$ & $\begin{array}{l}4.40240 \\
4.36980 \\
4.33773 \\
4.30619 \\
4.27516\end{array}$ & $\begin{array}{l}1.97256 \\
1.97207 \\
1.97157 \\
1.97107 \\
1.97056\end{array}$ \\
\hline $\begin{array}{l}650 \\
660 \\
670 \\
680 \\
690\end{array}$ & $\begin{array}{l}2.56067 \\
2.53499 \\
2.50977 \\
2.48498 \\
2.46062\end{array}$ & $\begin{array}{l}4.24461 \\
4.21453 \\
4.18492 \\
4.15575 \\
4.12702\end{array}$ & $\begin{array}{l}1.97004 \\
1.96951 \\
1.96897 \\
1.96843 \\
1.96788\end{array}$ \\
\hline
\end{tabular}

$700 \quad 2.43668 \quad 4.09871 \quad 1.96732$

$710 \quad 2.413134 .07081 \quad 1.96675$

$\begin{array}{llll}720 & 2.38998 \quad 4.04331 & 1.96617\end{array}$

$730 \quad 2.36720 \quad 4.016191 .96559$

$\begin{array}{llll}740 & 2.34480 \quad 3.98945 \quad 1.96500\end{array}$

$\begin{array}{llll}750 & 2.32275 \quad 3.96308 \quad 1.96440\end{array}$

$\begin{array}{lll}760 & 2.30105 \quad 3.93706 \quad 1.96379\end{array}$

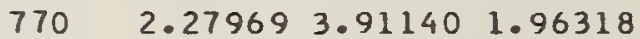

$\begin{array}{lllll}780 & 2.25867 & 3.88607 & 1.96255\end{array}$

$\begin{array}{lllll}790 & 2.23796 \quad 3.86107 & 1.96192\end{array}$

$\begin{array}{lll}800 \quad 2.21757 & 3.83640 \quad 1.96129\end{array}$

$810 \quad 2.19749 \quad 3.81204 \quad 1.96064$

$\begin{array}{lllll}820 & 2.17770 & 3.78798 & 1.95999\end{array}$

$\begin{array}{lllll}830 & 2.15821 & 3.76423 & 1.95933\end{array}$

$\begin{array}{lllll}840 & 2.13900 \quad 3.74077 & 1.95866\end{array}$

$\begin{array}{lllll}850 & 2.12007 & 3.71759 & 1.95798\end{array}$

$860 \quad 2.101413 .69470 \quad 1.95729$

$870 \quad 2.083023 .672071 .95660$

$\begin{array}{lllll}880 & 2.06488 & 3.64971 & 1.95590\end{array}$

$890 \quad 2.04699 \quad 3.62762 \quad 1.95519$

$900 \quad 2.02936 \quad 3.60578 \quad 1.95448$

$910 \quad 2.01196 \quad 3.58418 \quad 1.95376$

$920 \quad 1.99480 \quad 3.56283 \quad 1.95303$

$930 \quad 1.97787 \quad 3.54172 \quad 1.95229$

$940 \quad 1.96117 \quad 3.52085 \quad 1.95154$

$950 \quad 1.94469 \quad 3.50020 \quad 1.95079$

$960 \quad 1.92842 \quad 3.47978 \quad 1.95003$

$970 \quad 1.91236 \quad 3.45957 \quad 1.94926$

$980 \quad 1.89652 \quad 3.43959 \quad 1.94848$

$990 \quad 1.880873 .41981 \quad 1.94770$

$1000 \quad 1.86543 \quad 3.40024 \quad 1.94690$

$\begin{array}{lllll}1010 & 1.85017 & 3.38087 & 1.94611\end{array}$

$1020 \quad 1.835113 .361701 .94530$

$1030 \quad 1.820243 .34272 \quad 1.94448$

$1040 \quad 1.80555 \quad 3.32394 \quad 1.94366$

$1050 \quad 1.79104 \quad 3.30534 \quad 1.94283$ $1060 \quad 1.77670 \quad 3.28693 \quad 1.94200$ $1070 \quad 1.76254 \quad 3.26870 \quad 1.94115$ $1080 \quad 1.74855 \quad 3.25065 \quad 1.94030$ $1090 \quad 1.73473 \quad 3.23277 \quad 1.93944$
$-\left(\mathrm{F}^{\circ}-\mathrm{E}_{\mathrm{O}}\right) / \mathrm{T} \quad \mathrm{S}^{\circ}$

so

$-\left(F^{\circ}-E_{O}^{O}\right) / T$

So

$\mathrm{cm}^{-1}$

$T=3000$

$T=3100$

$2.75434 \quad 4.46929 \quad 1.97351$ $2.72603 \quad 4.43667 \quad 1.97306$ $2.69825 \quad 4.40459 \quad 1.97259$ $2.67098 \quad 4.37303 \quad 1.97212$ $2.644214 .34198 \quad 1.97164$

$2.61792 \quad 4.31141 \quad 1.97115$ 2.592094 .281321 .97066 2.566724 .251691 .97016 $2.54179 \quad 4.22251 \quad 1.96965$ $2.517294 .19376 \quad 1.96913$

$2.493194 .16543 \quad 1.96861$ $2.469504 .13751 \quad 1.96808$ $2.44621 \quad 4.10999 \quad 1.96754$ 2.423294 .082851 .96700 2.400734 .056091 .96644

$2.37854 \quad 4.02970 \quad 1.96588$ $2.35670 \quad 4.00366 \quad 1.96531$ $2.33520 \quad 3.97798 \quad 1.96474$ $2.31403 \quad 3.952631 .96416$ $2.29318 \quad 3.92761 \quad 1.96357$

$2.27265 \quad 3.90292 \quad 1.96297$ $2.25242 \quad 3.87854 \quad 1.96237$ $2.232493 .85446 \quad 1.96175$ $2.21286 \quad 3.83069 \quad 1.96114$ $2.19351 \quad 3.80720 \quad 1.96051$

$2.17443 \quad 3.78400 \quad 1.95988$ $2.15563 \quad 3.76108 \quad 1.95924$ $2.13710 \quad 3.73844 \quad 1.95859$ $2.11882 \quad 3.71606 \quad 1.95793$ $2.10079 \quad 3.69394 \quad 1.95727$

$2.08302 \quad 3.67207 \quad 1.95660$ $2.06548 \quad 3.65046 \quad 1.95593$ $2.04818 \quad 3.62908 \quad 1.95524$ 2.031113 .607951 .95455 $2.01427 \quad 3.58705 \quad 1.95385$

$1.99765 \quad 3.56638 \quad 1.95315$ $1.98124 \quad 3.54593 \quad 1.95243$ $1.96505 \quad 3.52570 \quad 1.95172$ $1.94906 \quad 3.50568 \quad 1.95099$ $1.93328 \quad 3.48588 \quad 1.95025$

$1.917693 .46628 \quad 1.94951$ $1.90230 \quad 3.44689 \quad 1.94877$ 1.887113 .427691 .94801 $1.87209 \quad 3.40869 \quad 1.94725$ $1.85727 \quad 3.38988 \quad 1.94648$

$1.84262 \quad 3.37126 \quad 1.94570$ 1.828153 .352821 .94492 $1.81385 \quad 3.33456 \quad 1.94413$ $1.799723 .31648 \quad 1.94333$ $1.78576 \quad 3.29857 \quad 1.94253$ $\begin{array}{llll}2.81071 & 4.53401 & 1.97438\end{array}$ $2.78226 \quad 4.50138 \quad 1.97395$ $2.75434 \quad 4.46929 \quad 1.97351$ $2.72693 \quad 4.43771 \quad 1.97307$ 2.700024 .406651 .97262

$2.67359 \quad 4.37606 \quad 1.97217$ 2.647634 .345961 .97170 2.622134 .316311 .97123 $2.59706 \quad 4.28711 \quad 1.97076$ $2.57241 \quad 4.25834 \quad 1.97027$

$2.54818 \quad 4.23000 \quad 1.96978$ $2.52436 \quad 4.20206 \quad 1.96928$ $2.50092 \quad 4.17452 \quad 1.96878$ 2.477874 .147371 .96827 $2.45518 \quad 4.12059 \quad 1.96775$

$2.43285 \quad 4.09418 \quad 1.96723$ $2.41087 \quad 4.068131 .96669$ $2.38924 \quad 4.042421 .96615$ $2.367934 .01706 \quad 1.96561$ 2.346953 .992021 .96506

$2.32628 \quad 3.96731 \quad 1.96450$ $2.30592 \quad 3.94291 \quad 1.96393$ $2.28586 \quad 3.918811 .96336$ $2.26609 \quad 3.89502 \quad 1.96278$ 2.246613 .871511 .96219

$2.22740 \quad 3.84830 \quad 1.96160$ $2.20846 \quad 3.825361 .96100$ $2.18979 \quad 3.80269 \quad 1.96039$ $\begin{array}{llll}2.17138 & 3.78029 & 1.95977\end{array}$ $2.15323 \quad 3.75815 \quad 1.95915$

$2.13532 \quad 3.73626 \quad 1.95853$ $2.11765 \quad 3.71462 \quad 1.95789$ $2.10022 \quad 3.69323 \quad 1.95725$

2.083023 .672071 .95660

$2.06604 \quad 3.65115 \quad 1.95595$

$2.04929 \quad 3.63045 \quad 1.95529$ $2.03275 \quad 3.60998 \quad 1.95462$ $2.01643 \quad 3.58973 \quad 1.95394$ $2.000313 .56969 \quad 1.95326$ $1.98440 \quad 3.54987 \quad 1.95257$

$1.96868 \quad 3.53025 \quad 1.95188$ 1.953173 .510831 .95118 $1.93784 \quad 3.49161 \quad 1.95047$ $1.92270 \quad 3.47258 \quad 1.94975$ $1.907743 .45375 \quad 1.94903$

$1.89297 \quad 3.43510 \quad 1.94830$ $1.878373 .41664 \quad 1.94757$ 1.863943 .398351 .94683 1.849693 .380251 .94608 $1.835603 .36231 \quad 1.94532$
600

610

620

630

640

650

660

670

680

690

700

710

720

730

740

750

760

770

780

790

800

810

820

830

840

850

860

870

880

890

900

910

920

930

940

950

960

970

980

990

1000

1010

1020

1030

1040

1050

1060

1070

1080

1090 
Table II Harmonic Oscillator Contributions to the Thermodynamic

Functions (in units of calories, moles, and ${ }^{\circ} \mathrm{K}$ )
$\mathrm{cm}^{-1}$
So
$\begin{array}{cc}\mathrm{C}_{\mathrm{p}}^{\circ} & -\left(\mathrm{F}^{\circ}-\mathrm{E}_{\mathrm{O}}^{\circ}\right) / \mathrm{T}\end{array}$
$\mathrm{C}_{\mathrm{p}}^{\circ} \quad-\left(\mathrm{F}^{\circ}-\mathrm{E}_{\mathrm{O}}^{\circ}\right) / \mathrm{T}$
So
$\begin{array}{cc}C_{\mathrm{p}}^{\circ} & \nu \\ \mathrm{cm}^{-1}\end{array}$

$T=2900$.

$T=3000$.

$T=3100$.

$1100 \quad 1.72106 \quad 3.21506 \quad 1.93857$

$1110 \quad 1.70756 \quad 3.19752 \quad 1.93770$

$1120 \quad 1.69422 \quad 3.18015 \quad 1.93682$

$1130 \quad 1.68103 \quad 3.16293 \quad 1.93593$

$1140 \quad 1.66799 \quad 3.14588 \quad 1.93503$

$\begin{array}{llll}1150 \quad 1.65510 & 3.12899 & 1.93413\end{array}$

$1160 \quad 1.64235 \quad 3.11224 \quad 1.93322$

$\begin{array}{llll}1170 & 1.62975 & 3.09565 & 1.93230\end{array}$

$\begin{array}{lllll}1180 & 1.61729 & 3.07921 & 1.93137\end{array}$

$\begin{array}{llll}1190 \quad 1.60497 & 3.06292 & 1.93044\end{array}$

$\begin{array}{lllll}1200 & 1.59279 & 3.04677 & 1.92950\end{array}$

$\begin{array}{lllll}1210 & 1.58074 & 3.03076 & 1.92855\end{array}$

$\begin{array}{lllll}1220 & 1.56882 & 3.01489 & 1.92759\end{array}$

$\begin{array}{lllll}1230 & 1.55703 & 2.99916 & 1.92663\end{array}$

$\begin{array}{lllll}1240 & 1.54537 & 2.98356 & 1.92566\end{array}$

$\begin{array}{lllll}1250 & 1.53384 & 2.96810 & 1.92469\end{array}$

$\begin{array}{lllll}1260 & 1.52242 & 2.95277 & 1.92370\end{array}$

$\begin{array}{lllll}1270 & 1.51113 & 2.93756 & 1.92271\end{array}$

$\begin{array}{lllll}1280 & 1.49996 & 2.92249 & 1.92171\end{array}$

$\begin{array}{lllll}1290 & 1.48890 & 2.90754 & 1.92070\end{array}$

$\begin{array}{llll}1300 & 1.47796 & 2.89271 & 1.91969\end{array}$

$\begin{array}{lllll}1310 & 1.46714 & 2.87800 & 1.91867\end{array}$

$\begin{array}{lllll}1320 & 1.45642 & 2.86341 & 1.91764\end{array}$

$\begin{array}{lllll}1330 & 1.44582 & 2.84895 & 1.91661\end{array}$

$\begin{array}{lllll}1340 & 1.43532 & 2.83459 & 1.91557\end{array}$

$\begin{array}{llll}1350 \quad 1.42493 & 2.82035 & 1.91452\end{array}$

$\begin{array}{llll}1360 & 1.41465 & 2.80623 & 1.91346\end{array}$

$\begin{array}{lllll}1370 & 1.40447 & 2.79221 & 1.91240\end{array}$

$\begin{array}{lllll}1380 & 1.39439 & 2.77831 & 1.91133\end{array}$

$\begin{array}{lllll}1390 \quad 1.38441 & 2.76451 & 1.91025\end{array}$

$\begin{array}{llll}1400 \quad 1.37453 & 2.75082 & 1.90917\end{array}$

$\begin{array}{lllll}1410 & 1.36475 & 2.73724 & 1.90808\end{array}$

$\begin{array}{llll}1420 & 1.35506 & 2.72376 & 1.90698\end{array}$

$\begin{array}{llllll}1430 & 1.34547 & 2.71038 & 1.90587\end{array}$

$1440 \quad 1.33597 \quad 2.69710 \quad 1.90476$

$\begin{array}{lllll}1450 & 1.32657 & 2.68392 & 1.90364\end{array}$ $\begin{array}{llll}1460 & 1.31725 & 2.67084 & 1.90251\end{array}$ $\begin{array}{llll}1470 & 1.30802 & 2.65786 & 1.90138\end{array}$ $\begin{array}{lllll}1480 & 1.29889 & 2.64498 & 1.90024\end{array}$ $\begin{array}{llll}1490 \quad 1.28983 & 2.63218 & 1.89909\end{array}$

$\begin{array}{lllll}1500 \quad 1.28087 & 2.61948 & 1.89794\end{array}$ $\begin{array}{lllll}1510 & 1.27198 & 2.60688 & 1.89678\end{array}$ $\begin{array}{lllll}1520 & 1.26319 & 2.59436 & 1.89561\end{array}$ $\begin{array}{llll}1530 & 1.25447 & 2.58193 & 1.89444\end{array}$ $1540 \quad 1.245832 .56960 \quad 1.89326$

$\begin{array}{lllll}1550 & 1.23728 & 2.55735 & 1.89207\end{array}$ $\begin{array}{lllll}1560 & 1.22880 & 2.54518 & 1.89087\end{array}$ $\begin{array}{llllll}1570 & 1.22040 & 2.53310 & 1.88967\end{array}$ $\begin{array}{llll}1580 & 1.21208 & 2.52111 & 1.88846\end{array}$ $1590 \quad 1.203832 .50920 \quad 1.88725$
$1.77197 \quad 3.28084 \quad 1.94172$ $1.758333 .26327 \quad 1.94090$ $1.744853 .24586 \quad 1.94007$ $1.73152 \quad 3.22862 \quad 1.93924$ $1.718353 .21154 \quad 1.93840$

$1.70533 \quad 3.194611 .93755$ $1.692453 .17784 \quad 1.93670$ 1.679713 .161221 .93584 1.667123 .144751 .93497 1.654673 .128431 .93410

$1.64235 \quad 3.11224 \quad 1.93322$ 1.630173 .096201 .93233 $1.618123 .08030 \quad 1.93143$ $1.606203 .06454 \quad 1.93053$ $1.594413 .04891 \quad 1.92962$

$1.58274 \quad 3.03342 \quad 1.92871$ 1.571193 .018051 .92779 1.559773 .002821 .92686 $\begin{array}{llll}1.54847 & 2.98771 & 1.92592\end{array}$ $\begin{array}{llll}1.53728 & 2.97272 \quad 1.92498\end{array}$

$1.526212 .95786 \quad 1.92403$ $\begin{array}{llll}1.51526 & 2.94312 & 1.92307\end{array}$ 1.504412 .928501 .92211 1.493682 .914001 .92114 1.483052 .899611 .92016

$1.472542 .88534 \quad 1.91918$ 1.462122 .871181 .91819 1.451812 .857131 .91720 1.441612 .843191 .91619 $1.43150 \quad 2.82936 \quad 1.91518$

1.421492 .815631 .91417 $1.41158 \quad 2.802011 .91314$ 1.401772 .788501 .91211 $1.39205 \quad 2.77508 \quad 1.91108$ 1.382432 .761771 .91003

$1.37290 \quad 2.74855 \quad 1.90899$ $1.36345 \quad 2.73544 \quad 1.90793$ $1.35410 \quad 2.722421 .90687$ 1. 344842.709491 .90580 $1.33566 \quad 2.69666 \quad 1.90472$

$1.32657 \quad 2.68392 \quad 1.90364$ $1.31756 \quad 2.67128 \quad 1.90255$ 1.308642 .658721 .90146 $1.29980 \quad 2.64626 \quad 1.90035$ $1.291042 .63388 \quad 1.89925$

$1.28236 \quad 2.62159 \quad 1.89813$ $1.27375 \quad 2.609391 .89701$ $1.26523 \quad 2.59727 \quad 1.89588$ $1.256792 .58524 \quad 1.89475$ $1.24842 \quad 2.57329 \quad 1.89361$
$1.821673 .34455 \quad 1.94456$ $1.807913 .32696 \quad 1.94380$ $1.79430 \quad 3.30953 \quad 1.94302$ $1.78085 \quad 3.29226 \quad 1.94224$ $1.767553 .27515 \quad 1.94145$

$1.75440 \quad 3.25820 \quad 1.94066$ $1.741393 .24140 \quad 1.93986$ $1.72854 \quad 3.22475 \quad 1.93905$ $1.71582 \quad 3.20825 \quad 1.93824$ $1.703243 .19190 \quad 1.93742$

$1.69080 \quad 3.17569 \quad 1.93659$ $1.67849 \quad 3.15962 \quad 1.93576$ $1.666313 .14369 \quad 1.93492$ $1.654273 .12790 \quad 1.93407$ $1.642353 .11224 \quad 1.93322$

$1.63056 \quad 3.09672 \quad 1.93236$ 1.618893 .081331 .93149 $1.607353 .06606 \quad 1.93062$ 1.595923 .050921 .92974 $1.584613 .03591 \quad 1.92886$

1.573423 .021021 .92797 $1.562343 .00625 \quad 1.92707$ 1.551372 .991591 .92616 $\begin{array}{llll}1.54052 & 2.97706 & 1.92525\end{array}$ $1.529772 .96264 \quad 1.92434$

$1.519132 .94834 \quad 1.92341$ $1.508602 .93415 \quad 1.92248$ 1.498172 .920071 .92155 1.487842 .906091 .92061 1.477612 .892231 .91966

$\begin{array}{llll}1.46749 & 2.87847 & 1.91870\end{array}$ $1.45746 \quad 2.86482 \quad 1.91774$ 1.447522 .851271 .91678 $1.437682 .83782 \quad 1.91580$ $1.427942 .82448 \quad 1.91482$

1.418292 .811231 .91384 $1.408732 .79808 \quad 1.91285$ $1.399252 .78502 \quad 1.91185$ $1.389872 .77207 \quad 1.91084$ $1.38058 \quad 2.75920 \quad 1.90983$

$1.371372 .74643 \quad 1.90882$ $1.362242 .73375 \quad 1.90779$ $1.35320 \quad 2.72116 \quad 1.90676$ $1.344242 .70866 \quad 1.90573$ $1.33536 \quad 2.69625 \quad 1.90469$

$1.326572 .68392 \quad 1.90364$ 1.317852 .671691 .90259 $1.309212 .65953 \quad 1.90153$ $1.300652 .64746 \quad 1.90046$ $1.29216 \quad 2.63548 \quad 1.89939$
1100

1110

1120

1130

1140

1150

1160

1170

1180

1190

1200

1210

1220

1230

1240

1250

1260

1270

1280

1290

1300

1310

1320

1330

1340

1350

1360

1370

1380

1390

1400

1410

1420

1430

1440

1450

1460

1470

1480

1490

1500

1510

1520

1530

1540

1550

1560

1570

1580

1590 
Table II Harmonic Oscillator Contributions to the Thermodynamic

Functions (in units of calories, moles, and ${ }^{\circ} \mathrm{K}$ )
$\mathrm{cm}^{-1}$
$-\left(\mathrm{F}^{\circ}-\mathrm{E}_{\mathrm{O}}^{\mathrm{O}}\right) / \mathrm{T}$
So
co
$-\left(\mathrm{F}^{\circ}-\mathrm{E}_{\mathrm{O}}^{\circ}\right) / \mathrm{T} \quad \mathrm{S}^{\circ}$
$\mathrm{c} \stackrel{\mathrm{p}}{\mathrm{p}}$
$-(F \circ-E O) / T$
So
$\nu$

$T=2900$.

1600

1610

1620

1630

1640

1650

1660

1670

1680

1690

1700

1710

1720

1730

1740

1750

1760

1770

1780

1790

1800

1810

1820

1830

1840

1850

1860

1870

1880

1890

1900

1910

1920

1930

1940

1950

1960

1970

1980

1990

2000

2010

2020

2030

2040

2050

2060

2070

2080

2090
$\mathrm{T}=3000$.

$1.240122 .56142 \quad 1.89246$

1.231902 .549631 .89131

$1.223752 .53792 \quad 1.89015$

$\begin{array}{llll}1.21567 & 2.52630 & 1.88899\end{array}$

$1.20767 \quad 2.51475 \quad 1.88782$

$1.19973 \quad 2.50327 \quad 1.88664$ $1.191872 .49188 \quad 1.88545$

$1.184072 .48056 \quad 1.88426$

$\begin{array}{llll}1.17634 & 2.46931 & 1.88307\end{array}$

$1.168682 .45814 \quad 1.88186$

$1.16108 \quad 2.44704 \quad 1.88065$

$\begin{array}{llll}1.15355 & 2.43601 & 1.87944\end{array}$

$1.14608 \quad 2.42506 \quad 1.87822$

$1.13868 \quad 2.41417 \quad 1.87699$

$1.131342 .40336 \quad 1.87576$

$1.12406 \quad 2.39261 \quad 1.87452$ $1.11684 \quad 2.38194 \quad 1.87327$ $1.10968 \quad 2.37133 \quad 1.87202$ $1.10258 \quad 2.36078 \quad 1.87076$ 1.095542 .350311 .86949

$\begin{array}{lll}1.08856 & 2.33989 & 1.86822\end{array}$ $\begin{array}{llll}1.08164 & 2.32955 & 1.86695\end{array}$ $\begin{array}{llll}1.07477 & 2.31927 & 1.86567\end{array}$ $1.067962 .30905 \quad 1.86438$ 1.061212 .298891 .86308

$\begin{array}{llll}1.05451 & 2.28879 & 1.86178\end{array}$ $\begin{array}{llll}1.04786 & 2.27876 & 1.86048\end{array}$ 1.041272 .268791 .85916 $1.03474 \quad 2.25888 \quad 1.85784$ $1.02825 \quad 2.24902 \quad 1.85652$

$1.02182 \quad 2.239231 .85519$ $1.015432 .22950 \quad 1.85385$ $\begin{array}{lll}1.00910 & 2.21982 \quad 1.85251\end{array}$ $1.00282 \quad 2.210201 .85117$ $0.996592 .20064 \quad 1.84981$

0.990412 .191131 .84845 $0.98428 \quad 2.18168 \quad 1.84709$ $0.97819 \quad 2.17228 \quad 1.84572$ $0.97215 \quad 2.16294 \quad 1.84434$ $0.96616 \quad 2.153651 .84296$

$0.96022 \quad 2.14442 \quad 1.84157$ $0.95432 \quad 2.13524 \quad 1.84018$ $0.948472 .12611 \quad 1.83878$ $0.942662 .11703 \quad 1.83737$ 0.936902 .108001 .83596

$0.93118 \quad 2.09903 \quad 1.83455$ $0.925502 .09011 \quad 1.83312$ 0.919872 .081231 .83170 $0.91428 \quad 2.07241 \quad 1.83026$ $0.90874 \quad 2.06363 \quad 1.82883$
$T=3100$

$1.28375 \quad 2.62357 \quad 1.89831$

$1.275412 .61175 \quad 1.89723$

$1.267152 .60000 \quad 1.89614$

$1.25896 \quad 2.588341 .89504$

$1.250842 .57675 \quad 1.89394$

$1.242792 .56524 \quad 1.89283$

$\begin{array}{llll}1.23481 & 2.55381 & 1.89172\end{array}$

$1.226902 .54245 \quad 1.89060$

$1.21905 \quad 2.53116 \quad 1.88948$

$1.21128 \quad 2.51995 \quad 1.88835$

$1.20356 \quad 2.50882 \quad 1.88721$

$1.19592 \quad 2.49775 \quad 1.88607$

$1.188342 .48676 \quad 1.88492$

1.180822 .475831 .88376

1.173372 .464981 .88260

$1.165972 .45419 \quad 1.88143$

1.158642 .443481 .88026

$1.151372 .43283 \quad 1.87908$

$1.14416 \quad 2.422241 .87790$

1.137012 .411731 .87671

$1.12992 \quad 2.40127 \quad 1.87552$

$1.122892 .39089 \quad 1.87431$

$1.11591 \quad 2.38056 \quad 1.87311$

1.108992 .370301 .87190

$1.10213 \quad 2.36011 \quad 1.87068$

$1.09532 \quad 2.34997 \quad 1.86945$

$\begin{array}{llll}1.08856 & 2.33989 & 1.86822\end{array}$

$1.08186 \quad 2.32988 \quad 1.86699$

1.075212 .319931 .86575

1.068622 .310031 .86450

$1.06208 \quad 2.30020 \quad 1.86325$

1.055592 .290421 .86199

1.049152 .280701 .86073

$1.04276 \quad 2.27104 \quad 1.85946$

1.036422 .261431 .85819

$1.03013 \quad 2.25188 \quad 1.85691$

1.023892 .242381 .85562

$1.017692 .23294 \quad 1.85433$

$1.011552 .22356 \quad 1.85303$

$1.00545 \quad 2.214231 .85173$

$0.99940 \quad 2.20495 \quad 1.85042$ $0.99340 \quad 2.19572 \quad 1.84911$ 0.987442 .186551 .84779 $0.98152 \quad 2.17743 \quad 1.84647$ $0.975652 .16836 \quad 1.84514$

$0.969832 .15934 \quad 1.84381$ $0.96405 \quad 2.15037 \quad 1.84247$ 0.958312 .141451 .84112 $0.95262 \quad 2.13258 \quad 1.83977$ 0.946972 .123761 .83842
1600

1610

1620

1630

1640

1650

1660

1670

1680

1690

1700

1710

1720

1730

1740

1750

1760

1770

1780

1790

1800

1810

1820

1830

1840

1850

1860

1870

1880

1890

1900

1910

1920

1930

1940

1950

1960

1970

1980

1990

2000

2010

2020

2030

2040

2050

2060

2070

2080

2090 
Table II Harmonic Oscillator Contributions to the Thermodynamic

Functions (in units of calories, moles, and ${ }^{\circ} \mathrm{K}$ )
$\mathrm{cm}^{-1}$
so
$-(F \circ-E O) / T$
$\mathrm{co}$
$-\left(F^{\circ}-E_{0}^{\circ}\right) / T \quad S^{\circ}$
$\mathrm{C}_{\mathrm{p}}$
$-(F \circ-E O) / T$
So
$\stackrel{\circ}{\mathrm{p}} \quad \begin{gathered}\nu \\ \mathrm{cm}^{-1}\end{gathered}$

$T=2900$.

$\mathrm{T}=3000$.

$T=3100$.

$\begin{array}{lllll}2100 & 0.86458 & 1.99313 & 1.81676\end{array}$ $\begin{array}{lllll}2110 & 0.85923 & 1.98451 & 1.81522\end{array}$ $\begin{array}{lllll}2120 & 0.85391 & 1.97593 & 1.81367\end{array}$ $\begin{array}{lllll}2130 & 0.84864 & 1.96740 & 1.81212\end{array}$ $2140 \quad 0.84341 \quad 1.95891 \quad 1.81056$

$\begin{array}{lllll}2150 & 0.83821 & 1.95048 & 1.80900\end{array}$ $2160 \quad 0.83306 \quad 1.94208 \quad 1.80743$ $\begin{array}{lllll}2170 & 0.82795 & 1.93374 & 1.80586\end{array}$ $\begin{array}{lllll}2180 & 0.82287 & 1.92544 & 1.80428\end{array}$ $\begin{array}{lllll}2190 \quad 0.81783 & 1.91719 & 1.80269\end{array}$

$\begin{array}{lllll}2200 & 0.81283 & 1.90898 & 1.80110\end{array}$ $\begin{array}{llllll}2210 & 0.80787 & 1.90081 & 1.79950\end{array}$ $\begin{array}{lllll}2220 & 0.80294 & 1.89269 & 1.79790\end{array}$ $\begin{array}{lllll}2230 & 0.79805 & 1.88462 & 1.79629\end{array}$ $\begin{array}{lllll}2240 & 0.79319 & 1.87658 & 1.79468\end{array}$

$\begin{array}{lllll}2250 & 0.78837 & 1.86859 & 1.79306\end{array}$ $2260 \quad 0.78359 \quad 1.86064 \quad 1.79143$ 2270 2280 2290

2300

2310

2320

2330

2340

2350

2360

2370

2380

2390

2400

2410

2420

2430

2440

2450

2460

2470

2480

2490

2500

2510

2520

2530

2540

2550

2560

2570

2580

2590
0.774131 .844871 .78817

0.769451 .837051 .78653

$\begin{array}{llll}0.76481 & 1.82927 & 1.78488\end{array}$

$0.76019 \quad 1.821531 .78323$

0.755621 .813831 .78157

$0.751071 .80617 \quad 1.77991$

$0.74656 \quad 1.79855 \quad 1.77825$

$\begin{array}{llll}0.74208 & 1.79097 & 1.77657\end{array}$ 0.737631 .783431 .77490 $0.733221 .77593 \quad 1.77321$ 0.728831 .768471 .77152 $0.72448 \quad 1.76105 \quad 1.76983$

$0.72016 \quad 1.75366 \quad 1.76813$ $0.715871 .74631 \quad 1.76643$ 0.711611 .739001 .76472 $0.70738 \quad 1.73173 \quad 1.76301$ 0.703181 .724491 .76129

$0.69901 \quad 1.71729 \quad 1.75956$ $0.69486 \quad 1.710131 .75783$ $0.69075 \quad 1.703001 .75610$ $0.686671 .69591 \quad 1.75436$ 0.682611 .688851 .75262

$\begin{array}{llll}0.67858 & 1.68183 & 1.75087\end{array}$ $0.674591 .67484 \quad 1.74911$ 0.670611 .667891 .74736 0.666671 .660981 .74559 0.662751 .654091 .74382

$0.65886 \quad 1.64724 \quad 1.74205$ $0.65500 \quad 1.64043 \quad 1.74027$ $0.65117 \quad 1.63365 \quad 1.73849$ $0.647361 .62690 \quad 1.73670$ 0.643571 .620191 .73491
$0.77884 \quad 1.85274 \quad 1.78981$
$0.90323 \quad 2.05491 \quad 1.82738$ 0.897772 .046231 .82593 0.892352 .037601 .82448 $0.886962 .02902 \quad 1.82302$ 0.881622 .020481 .82155

$0.87632 \quad 2.01199 \quad 1.82008$ $0.87106 \quad 2.00355 \quad 1.81860$ 0.865831 .995151 .81712 0.860651 .986801 .81563 $0.85550 \quad 1.97850 \quad 1.81414$

$0.85039 \quad 1.97024 \quad 1.81264$ 0.845321 .962021 .81114 $0.840291 .95384 \quad 1.80963$ 0.835291 .945721 .80811 0.830331 .937631 .80659

$\begin{array}{llll}0.82540 & 1.92958 \quad 1.80507\end{array}$ $0.82051 \quad 1.92158 \quad 1.80354$ $0.81566 \quad 1.91362 \quad 1.80200$ $0.810841 .90571 \quad 1.80046$ $0.80605 \quad 1.89783 \quad 1.79892$

$0.80130 \quad 1.89000 \quad 1.79736$ $0.79659 \quad 1.88220 \quad 1.79581$ $0.79190 \quad 1.87445 \quad 1.79425$ $0.78726 \quad 1.86673 \quad 1.79268$ $0.782641 .85906 \quad 1.79111$

$0.77805 \quad 1.85142 \quad 1.78953$ $0.77350 \quad 1.843831 .78795$ $0.768981 .83627 \quad 1.78636$ $0.76450 \quad 1.82875 \quad 1.78477$ $0.760041 .82127 \quad 1.78318$

0.755621 .813831 .78157 0.751221 .806431 .77997 $0.746861 .79906 \quad 1.77836$ $0.742531 .79173 \quad 1.77674$ $0.73822 \quad 1.78444 \quad 1.77512$

$0.73395 \quad 1.77718 \quad 1.77349$ 0.729711 .769961 .77186 $0.725491 .76277 \quad 1.77023$ $0.72131 \quad 1.755631 .76859$ $0.717151 .74851 \quad 1.76694$

0.713021 .741431 .76529 $0.70892 \quad 1.73439 \quad 1.76363$ $0.704851 .72738 \quad 1.76197$ $0.70081 \quad 1.72041 \quad 1.76031$ 0.696791 .713461 .75864

$\begin{array}{llll}0.69280 & 1.70656 & 1.75697\end{array}$ $0.68884 \quad 1.69968 \quad 1.75529$ 0.684911 .692841 .75361 0.681001 .686041 .75192 $0.677121 .67926 \quad 1.75023$
$0.941362 .11499 \quad 1.83705$ $0.93579 \quad 2.10626 \quad 1.83569$ $0.93026 \quad 2.09759 \quad 1.83432$ $0.92478 \quad 2.08896 \quad 1.83294$ 0.919332 .080381 .83156

$\begin{array}{llll}0.91392 & 2.07184 & 1.83017\end{array}$ $0.90856 \quad 2.06335 \quad 1.82878$ $0.90323 \quad 2.05491 \quad 1.82738$ $0.89794 \quad 2.04651 \quad 1.82598$ $0.892692 .03815 \quad 1.82457$

$0.88748 \quad 2.02985 \quad 1.82316$ $0.882312 .02158 \quad 1.82174$ $0.877172 .01336 \quad 1.82032$ 0.872072 .005181 .81889 $0.867011 .99705 \quad 1.81745$

$0.86198 \quad 1.98895 \quad 1.81602$ 0.856991 .980901 .81457 0.852041 .972901 .81312 0.847121 .964931 .81167 0.842231 .957001 .81021

$0.83738 \quad 1.949121 .80875$ $0.83256 \quad 1.94128 \quad 1.80728$ $0.827781 .93347 \quad 1.80581$ $0.82303 \quad 1.92571 \quad 1.80433$ $0.818321 .91798 \quad 1.80284$

0.813631 .910301 .80136 $0.80898 \quad 1.902651 .79986$ $0.804361 .89505 \quad 1.79837$ $0.79978 \quad 1.88748 \quad 1.79686$ $0.79522 \quad 1.87995 \quad 1.79536$

$0.79070 \quad 1.87245 \quad 1.79384$ $0.78621 \quad 1.86500 \quad 1.79233$ $0.78175 \quad 1.85758 \quad 1.79080$ 0.777321 .850201 .78928 0.772921 .842851 .78775

$0.768551 .83554 \quad 1.78621$ $0.764211 .82827 \quad 1.78467$ $0.75990 \quad 1.82103 \quad 1.78312$ 0.755621 .813831 .78157 0.751361 .806671 .78002

$0.74714 \quad 1.799531 .77846$ 0.742941 .792441 .77690 $0.73878 \quad 1.785381 .77533$ $0.73464 \quad 1.77835 \quad 1.77376$ 0.730531 .771351 .77218

$0.72644 \quad 1.76439 \quad 1.77060$ $0.722391 .75747 \quad 1.76901$ $0.71836 \quad 1.75057 \quad 1.76742$ $0.714351 .74371 \quad 1.76582$ $0.71038 \quad 1.73688 \quad 1.76422$
2100

2110

2120

2130

2140

2150

2160

2170

2180

2190

2200

2210

2220

2230

2240

2250

2260

2270

2280

2290

2300

2310

2320

2330

2340

2350

2360

2370

2380

2390

2400

2410

2420

2430

2440

2450

2460

2470

2480

2490

2500

2510

2520

2530

2540

2550

2560

2570

2580

2590 
Table II Harmonic Oscillator Contributions to the Thermodynamic

Functions (in units of calories, moles, and ${ }^{\circ} \mathrm{K}$ )

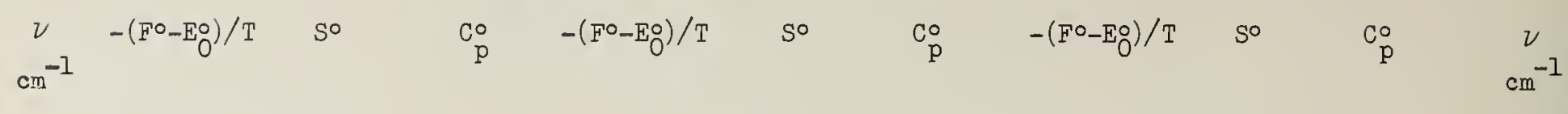

2600

2610

2620

2630

2640

2650

2660

2670

2680

2690

2700

2710

2720

2730

2740

2750

2760

2770

2780

2790

2800

2810

2820

2830

2840

2850

2860

2870

2880

2890

2900

2910

2920

2930

2940

2950

2960

2970

2980

2990

3000

3010

3020

3030

3040

3050

3060

3070

3080

3090

\begin{abstract}
$T=2900$.
$\begin{array}{llll}0.63981 & 1.61350 & 1.73311\end{array}$ 0.636081 .606851 .73131 $0.63238 \quad 1.60024 \quad 1.72950$ 0.628691 .593651 .72769 $0.62504 \quad 1.58710 \quad 1.72588$

$0.62141 \quad 1.58058 \quad 1.72406$ $0.61780 \quad 1.57409 \quad 1.72223$ $0.61422 \quad 1.56763 \quad 1.72040$ $0.61066 \quad 1.561201 .71857$ $0.607121 .55480 \quad 1.71673$
\end{abstract}

$0.60361 \quad 1.54844 \quad 1.71489$ $0.60012 \quad 1.54210 \quad 1.71304$ $0.59666 \quad 1.53579 \quad 1.71119$ $0.59322 \quad 1.52952 \quad 1.70933$ $0.58980 \quad 1.52327 \quad 1.70747$

$0.58640 \quad 1.51705 \quad 1.70561$ $0.58303 \quad 1.51087 \quad 1.70374$ $0.57968 \quad 1.50471 \quad 1.70187$ 0.576351 .498581 .69999 $0.57305 \quad 1.49248 \quad 1.69811$

$0.56976 \quad 1.48640 \quad 1.69622$ $0.56650 \quad 1.48036 \quad 1.69433$ 0.563261 .474351 .69243 $0.56004 \quad 1.468361 .69053$ $0.55684 \quad 1.46240 \quad 1.68863$

$0.55366 \quad 1.45647 \quad 1.68672$ $0.55050 \quad 1.45056 \quad 1.68481$ 0.547371 .444681 .68290 $0.544251 .43883 \quad 1.68098$ 0.541151 .433011 .67905

$0.53808 \quad 1.42721 \quad 1.67713$ $0.535021 .42144 \quad 1.67519$ $0.53198 \quad 1.41570 \quad 1.67326$ $0.52897 \quad 1.40998 \quad 1.67132$ 0.525971 .404291 .66937

$\begin{array}{llll}0.52299 & 1.39863 & 1.66743\end{array}$ $0.52003 \quad 1.39299 \quad 1.66548$ $0.51709 \quad 1.38737 \quad 1.66352$ 0.514171 .381791 .66156 $0.51127 \quad 1.37622 \quad 1.65960$

$0.50839 \quad 1.37068 \quad 1.65763$ $0.50552 \quad 1.36517 \quad 1.65566$ $0.50268 \quad 1.35968 \quad 1.65369$ $0.49985 \quad 1.35422 \quad 1.65171$ $0.49704 \quad 1.34878 \quad 1.64973$

0.494241 .343371 .64774 $0.49147 \quad 1.33798 \quad 1.64575$ 0.488711 .332611 .64376 0.485971 .327271 .64176 0.483251 .321951 .63976

$$
T=3000 \text {. }
$$

$0.67326 \quad 1.67252 \quad 1.74853$

$0.66943 \quad 1.66581 \quad 1.74683$

$0.665621 .65914 \quad 1.74512$

$0.66184 \quad 1.65249 \quad 1.74341$

$0.658091 .64588 \quad 1.74169$

$0.65436 \quad 1.63930 \quad 1.73998$

$0.65066 \quad 1.63275 \quad 1.73825$

$0.64698 \quad 1.626231 .73652$

$0.643321 .61974 \quad 1.73479$

0.639691 .613281 .73305

$0.63608 \quad 1.606851 .73131$

$0.632501 .60046 \quad 1.72956$

$0.628941 .59409 \quad 1.72781$

$0.62540 \quad 1.58775 \quad 1.72606$

0.621891 .581441 .72430

$0.61840 \quad 1.57517 \quad 1.72254$

0.614931 .568921 .72077

0.611491 .562701 .71900

$0.60806 \quad 1.556501 .71722$

0.604661 .550341 .71544

$0.60128 \quad 1.54421 \quad 1.71366$

$0.597931 .53810 \quad 1.71187$

0.594591 .532021 .71008

$0.59128 \quad 1.52597 \quad 1.70828$

$0.587991 .51995 \quad 1.70648$

$0.58471 \quad 1.51396 \quad 1.70467$ $0.58146 \quad 1.50799 \quad 1.70286$ 0.578241 .502051 .70105 $0.57503 \quad 1.49613 \quad 1.69923$ 0.571841 .490251 .69741

$0.56867 \quad 1.48439 \quad 1.69559$ 0.565521 .478551 .69376 $0.56240 \quad 1.47275 \quad 1.69193$ $0.559291 .46696 \quad 1.69009$ $0.556201 .46121 \quad 1.68825$

$0.55313 \quad 1.45548 \quad 1.68641$ $0.55008 \quad 1.44978 \quad 1.68456$ $0.54705 \quad 1.44410 \quad 1.68270$ $0.544041 .43844 \quad 1.68085$ $0.54105 \quad 1.43282 \quad 1.67899$

$0.53808 \quad 1.42721 \quad 1.67713$ 0.535121 .421641 .67526 $0.53219 \quad 1.41608 \quad 1.67339$ $0.52927 \quad 1.410551 .67151$ 0.526371 .405051 .66963

$0.52349 \quad 1.39957 \quad 1.66775$ 0.520621 .394111 .66587 $0.51778 \quad 1.38868 \quad 1.66398$ $0.514951 .38327 \quad 1.66208$ 0.512141 .377891 .66019
$T=3100$.

$0.70643 \quad 1.73009 \quad 1.76262$

2600

$0.70250 \quad 1.72333 \quad 1.76101$

$\begin{array}{llll}0.69860 & 1.71659 & 1.75940\end{array}$

$0.69473 \quad 1.70990 \quad 1.75778$

0.690881 .703231 .75616

$\begin{array}{llll}0.68706 & 1.69659 & 1.75453\end{array}$

$0.68326 \quad 1.68999 \quad 1.75290$

$0.67949 \quad 1.68341 \quad 1.75126$

$0.67574 \quad 1.67687 \quad 1.74962$

$\begin{array}{llll}0.67202 & 1.67035 & 1.74798\end{array}$

$0.66832 \quad 1.66387 \quad 1.74633$

$0.664651 .65742 \quad 1.74468$

$0.66099 \quad 1.65100 \quad 1.74302$

$0.65737 \quad 1.64460 \quad 1.74136$

$0.65376 \quad 1.63824 \quad 1.73970$

$0.65018 \quad 1.63190 \quad 1.73803$

$0.646621 .62560 \quad 1.73636$

$0.64309 \quad 1.61932 \quad 1.73468$

0.639571 .613071 .73300

$0.63608 \quad 1.606851 .73131$

$0.63261 \quad 1.60066 \quad 1.72962$

$\begin{array}{llll}0.62917 & 1.59450 & 1.72793\end{array}$

$0.62574 \quad 1.58836 \quad 1.72623$

$0.62234 \quad 1.58226 \quad 1.72453$

$0.61896 \quad 1.57618 \quad 1.72282$

$0.61560 \quad 1.570121 .72111$

0.612261 .564101 .71940

$0.608941 .55810 \quad 1.71768$

$0.605651 .55213 \quad 1.71596$

$0.602371 .54618 \quad 1.71423$

$0.59912 \quad 1.54026 \quad 1.71250$

$0.59588 \quad 1.53437 \quad 1.71077$

$0.59267 \quad 1.52851 \quad 1.70903$

0.589471 .522671 .70729

0.586301 .516851 .70555

$0.58314 \quad 1.51106 \quad 1.70380$

0.580001 .505301 .70205

$\begin{array}{llll}0.57689 & 1.49956 & 1.70029\end{array}$

$0.57379 \quad 1.49385 \quad 1.69853$

$0.57071 \quad 1.48816 \quad 1.69677$

$0.567651 .48250 \quad 1.69500$

$0.56461 \quad 1.47686 \quad 1.69323$

0.561591 .471251 .69145

$0.558591 .46566 \quad 1.68968$

0.555601 .460101 .68789

$0.552641 .45456 \quad 1.68611$ $0.549691 .44904 \quad 1.68432$ $0.54676 \quad 1.44355 \quad 1.68253$ $0.543851 .43808 \quad 1.68073$

0.540951 .432641 .67893
2610

2620

2630

2640

2650

2660

2670

2680

2690

2700

2710

2720

2730

2740

2750

2760

2770

2780

2790

2800

2810

2820

2830

2840

2850

2860

2870

2880

2890

2900

2910

2920

2930

2940

2950

2960

2970

2980

2990

3000

3010

3020

3030

3040

3050

3060

3070

3080

3090 
Table II Harmonic Oscillator Contributions to the Thermodynamic

Functions (in units of calories, moles, and ${ }^{\circ} \mathrm{K}$ )
$\mathrm{cm}^{-1}$
So
$\mathrm{c}_{\mathrm{p}}^{\circ} \quad-\left(\mathrm{F}^{\circ}-\mathrm{E}_{0}^{\circ}\right) / \mathrm{T}$
So
$\mathrm{C}_{\mathrm{p}}^{\circ}$
$-\left(\mathrm{F} \circ-\mathrm{E}_{\mathrm{O}}\right) / \mathrm{T}$
So
$\begin{array}{cc}\mathrm{C} & \nu \\ \mathrm{p} & \mathrm{cm}^{-1}\end{array}$

$\mathrm{T}=2900$.

$T=3000$.

$T=3100$

3100

3110

3120

3130

3140

3150

3160

3170

3180

3190

$\begin{array}{llll}3200 & 0.45440 & 1.26498 & 1.61754\end{array}$

3210

3220

3230

3240

$\begin{array}{llll}3250 & 0.44193 & 1.23998 & 1.60730\end{array}$

$\begin{array}{lllll}3260 & 0.43948 & 1.23504 & 1.60524\end{array}$

$\begin{array}{llll}3270 & 0.43705 & 1.23013 & 1.60318\end{array}$

$3280 \quad 0.43463 \quad 1.22524 \quad 1.60112$

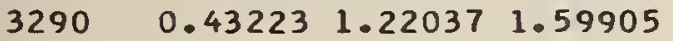

$\begin{array}{lllll}3300 & 0.42984 & 1.21552 & 1.59698\end{array}$

$\begin{array}{lllll}3310 & 0.42747 & 1.21069 & 1.59491\end{array}$

$\begin{array}{lllll}3320 & 0.42511 & 1.20588 & 1.59283\end{array}$

$3330 \quad 0.422761 .201091 .59075$

$\begin{array}{llll}3340 & 0.42043 & 1.19632 & 1.58867\end{array}$

$\begin{array}{llll}3350 & 0.41812 & 1.19158 & 1.58659\end{array}$

$\begin{array}{llll}3360 & 0.41582 & 1.18685 & 1.58450\end{array}$

$\begin{array}{llll}3370 & 0.41353 & 1.18215 & 1.58241\end{array}$

$3380 \quad 0.41126 \quad 1.17746 \quad 1.58032$

$\begin{array}{lllll}3390 & 0.40900 & 1.17280 & 1.57822\end{array}$

$\begin{array}{llll}3400 & 0.40675 & 1.16815 & 1.57612\end{array}$

$\begin{array}{llll}3410 & 0.40452 & 1.16352 & 1.57402\end{array}$

$\begin{array}{lllll}3420 & 0.40230 & 1.15892 & 1.57191\end{array}$

$\begin{array}{lllll}3430 & 0.40009 & 1.15433 & 1.56980\end{array}$

$\begin{array}{lllll}3440 & 0.39790 & 1.14976 & 1.56769\end{array}$

$3450 \quad 0.39572 \quad 1.145221 .56558$

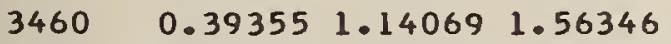

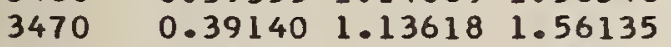
$\begin{array}{llll}3480 & 0.38926 & 1.13169 & 1.55922\end{array}$ $3490 \quad 0.387131 .127221 .55710$

3500

3510

3520

3530

3540

3550

3560

3570

3580

3590
$0.38502 \quad 1.12277 \quad 1.55497$ $0.382921 .11833 \quad 1.55284$ $0.380831 .11392 \quad 1.55071$ $0.37875 \quad 1.10952 \quad 1.54858$ $0.376691 .10514 \quad 1.54644$

$0.37464 \quad 1.10078 \quad 1.54430$ $0.372601 .09644 \quad 1.54216$ $0.37057 \quad 1.09212 \quad 1.54001$ $\begin{array}{llll}0.36856 & 1.08782 & 1.53787\end{array}$ $0.366551 .08353 \quad 1.53572$
$0.50935 \quad 1.37253 \quad 1.65829$ 0.506571 .367191 .65638 $0.503811 .36188 \quad 1.65448$ $0.50107 \quad 1.35658 \quad 1.65257$ 0.498351 .351321 .65065

$0.49564 \quad 1.34607 \quad 1.64874$ 0.492951 .340851 .64681 $0.49027 \quad 1.33565 \quad 1.64489$ $0.487611 .33047 \quad 1.64296$ 0.484971 .325311 .64103

$0.48234 \quad 1.32018 \quad 1.63910$ 0.479731 .315071 .63716 $0.47714 \quad 1.30998 \quad 1.63522$ 0.474561 .304911 .63327 0.472001 .299871 .63133

$0.46945 \quad 1.29484 \quad 1.62938$ $0.466921 .28984 \quad 1.62742$ $0.46440 \quad 1.28486 \quad 1.62547$ $0.46190 \quad 1.27990 \quad 1.62350$ $0.45941 \quad 1.27496 \quad 1.62154$

$0.45694 \quad 1.27004 \quad 1.61957$ $0.45449 \quad 1.26514 \quad 1.61760$ $0.45204 \quad 1.26027 \quad 1.61563$ 0.449621 .255411 .61366 $0.44720 \quad 1.250581 .61168$

$0.44481 \quad 1.24576 \quad 1.60970$ $0.44242 \quad 1.24097 \quad 1.60771$ $0.440051 .23619 \quad 1.60572$ $0.43770 \quad 1.23144 \quad 1.60373$ $0.43536 \quad 1.22670 \quad 1.60174$

$0.43303 \quad 1.22199 \quad 1.59974$ $0.43072 \quad 1.217291 .59774$ 0.428421 .212621 .59574 $0.426131 .20796 \quad 1.59373$ $0.42386 \quad 1.20332 \quad 1.59173$

$0.42160 \quad 1.19870 \quad 1.58971$ 0.419351 .194111 .58770 0.417121 .189531 .58568 $0.41490 \quad 1.18497 \quad 1.58366$ 0.412691 .180431 .58164

$0.41050 \quad 1.17590 \quad 1.57962$ $0.408321 .17140 \quad 1.57759$ $0.40615 \quad 1.16691 \quad 1.57556$ 0.404001 .162451 .57353 $0.40186 \quad 1.158001 .57149$

$0.399731 .15357 \quad 1.56945$ 0.397611 .149161 .56741 $0.39550 \quad 1.14476 \quad 1.56537$ 0.393411 .140391 .56332 0.391331 .136031 .56128
$0.53808 \quad 1.42721 \quad 1.67713$ 0.535221 .421821 .67532 0.532381 .416441 .67351 0.529551 .411091 .67169 $0.526741 .40576 \quad 1.66988$

0.523951 .400451 .66806 0.521181 .395171 .66623 $0.51842 \cdot 1.389911 .66440$ $0.51568 \quad 1.38467 \quad 1.66257$ 0.512951 .379451 .66074

$0.510251 .37425 \quad 1.65890$ 0.507551 .369081 .65706 $0.50488 \quad 1.363931 .65522$ $0.502221 .35880 \quad 1.65337$ $0.49957 \quad 1.353691 .65152$

0.496951 .348611 .64966 0.494331 .343541 .64781 0.491741 .338501 .64595 0.489151 .333471 .64408 $0.48659 \quad 1.32847 \quad 1.64222$

$0.48404 \quad 1.32349 \quad 1.64035$ 0.481501 .318531 .63847 $0.47898 \quad 1.313591 .63660$ $0.47647 \quad 1.30867 \quad 1.63472$ $0.473981 .30377 \quad 1.63283$

$0.47150 \quad 1.29889 \quad 1.63095$ $0.46904 \quad 1.29404 \quad 1.62906$ $0.46659 \quad 1.28920 \quad 1.62717$ $0.46416 \quad 1.28438 \quad 1.62528$ $0.46174 \quad 1.27958 \quad 1.62338$

$0.459331 .27480 \quad 1.62148$ $0.45694 \quad 1.27004 \quad 1.61957$ $0.45457 \quad 1.265301 .61767$ $0.452201 .26058 \quad 1.61576$ $0.449851 .25588 \quad 1.61385$

0.447521 .251201 .61193 0.445191 .246541 .61002 0.442881 .241891 .60809 $0.440591 .23727 \quad 1.60617$ 0.438301 .232661 .60425

$0.436031 .22807 \quad 1.60232$ $0.433781 .22351 \quad 1.60039$ 0.431541 .218951 .59845 0.429301 .214421 .59651 0.427091 .209911 .59457

$0.42488 \quad 1.20541 \quad 1.59263$ $0.422691 .20094 \quad 1.59069$ $0.42051 \quad 1.19648 \quad 1.58874$ 0.418341 .192041 .58679 0.416191 .187611 .58484
3100

3110

3120

3130

3140

3150

3160

3170

3180

3190

3200

3210

3220

3230

3240

3250

3260

3270

3280

3290

3300

3310

3320

3330

3340

3350

3360

3370

3380

3390

3400

3410

3420

3430

3440

3450

3460

3470

3480

3490

3500

3510

3520

3530

3540

3550

3560

3570

3580

3590 
Table II Harmonic Oscillator Contributions to the Thermodynamic Functions (in units of calories, moles, and ${ }^{\circ} \mathrm{K}$ )
$\mathrm{cm}^{-1}-\left(\mathrm{FO}-\mathrm{E}_{\mathrm{O}} \mathrm{O}\right) / \mathrm{T} \quad \mathrm{S}$
$\mathrm{C}_{\mathrm{p}}^{\circ} \quad-\left(\mathrm{F}^{\circ}-\mathrm{E}_{\mathrm{O}}^{\circ}\right) / \mathrm{T} \quad \mathrm{S}^{\circ}$
$\mathrm{co}_{\mathrm{p}}$
$-\left(F^{\circ}-E_{O}^{\circ}\right) / T$
so
$\stackrel{\mathrm{p}}{\mathrm{p}^{\circ}} \quad \begin{gathered}\nu \\ \mathrm{cm}^{-1}\end{gathered}$

$$
T=2900
$$

3600

3610

3620

3630

3640

3650

3660

3670

3680

3690

3700

3710

3720

3730

3740

3750

3760

3770

3780

3790

3800

3810

3820

3830

3840

3850

3860

3870

3880

3890

3900

3910

3920

3930

3940

3950

3960

3970

3980

3990

4000
$0.36456 \quad 1.07926 \quad 1.53357$ $0.362581 .07501 \quad 1.53141$ 0.360621 .070781 .52926 0.358661 .066561 .52710 0.356711 .062361 .52494

$\begin{array}{llll}0.35478 & 1.05818 & 1.52277\end{array}$ 0.352861 .054021 .52061 0.350951 .049871 .51844 $0.349051 .04574 \quad 1.51627$ 0.347161 .041631 .51410

$0.345291 .03754 \quad 1.51192$ $0.343421 .03346 \quad 1.50974$ $0.341571 .02940 \quad 1.50757$ 0.339721 .025351 .50538 0.337891 .021331 .50320

0.336071 .017321 .50102 0.334261 .013321 .49883 0.332461 .009341 .49664 0.330671 .005381 .49445 $0.328891 .00144 \quad 1.49226$

$0.327120 .99751 \quad 1.49006$ $0.32536 \quad 0.99359 \quad 1.48786$ $0.323610 .98970 \quad 1.48567$ $0.32187 \quad 0.98582 \quad 1.48346$ 0.320140 .981951 .48126

$0.318420 .97810 \quad 1.47906$ $0.316720 .97427 \quad 1.47685$ $0.315020 .97045 \quad 1.47464$ 0.313330 .966651 .47243 $0.31165 \quad 0.96286 \quad 1.47022$

$0.30998 \quad 0.95909 \quad 1.46801$ $0.30832 \quad 0.95533 \quad 1.46579$ $0.30667 \quad 0.95159 \quad 1.46358$ $0.30503 \quad 0.94786 \quad 1.46136$ $0.30340 \quad 0.94415 \quad 1.45914$

$0.30178 \quad 0.94046 \quad 1.45692$ $0.30017 \quad 0.93677 \quad 1.45469$ $0.29856 \quad 0.93311 \quad 1.45247$ $0.29697 \quad 0.92946 \quad 1.45024$ $\begin{array}{lll}0.29538 & 0.92582 & 1.44801\end{array}$

$\begin{array}{llll}0.29381 & 0.92220 & 1.44578\end{array}$
$T=3000$.

$0.38926 \quad 1.131691 .55922$ $0.38721 \quad 1.127371 .55717$ 0.385161 .123061 .55512 0.383131 .118781 .55306 0.381111 .114511 .55100

$0.37910 \quad 1.11025 \quad 1.54893$ $0.37710 \quad 1.10602 \quad 1.54687$ 0.375121 .101801 .54480 0.373141 .097601 .54273 0.371181 .093421 .54066

$0.36923 \quad 1.089251 .53858$ 0.367291 .085101 .53651 $0.365361 .08097 \quad 1.53443$ 0.363441 .076851 .53235 0.361531 .072751 .53026

$0.35964 \quad 1.06867 \quad 1.52818$ $0.35775 \quad 1.06460 \quad 1.52609$ 0.355881 .060551 .52400 $0.35401 \quad 1.05651 \quad 1.52191$ 0.352161 .052501 .51981

$0.35032 \quad 1.04849 \quad 1.51772$ $0.34848 \quad 1.04451 \quad 1.51562$ 0.346661 .040541 .51352 $0.34485 \quad 1.03658 \quad 1.51141$ 0.343051 .032651 .50931

$0.34126 \quad 1.028721 .50720$ $0.33948 \quad 1.024821 .50509$ $0.33771 \quad 1.020921 .50298$ 0.335951 .017051 .50087 0.334201 .013191 .49876

$0.33246 \quad 1.00934 \quad 1.49664$ 0.330731 .005511 .49452 0.329011 .001701 .49240 $0.327290 .99790 \quad 1.49028$ $0.325590 .99411 \quad 1.48816$

$0.32390 \quad 0.99034 \quad 1.48603$ 0.322220 .986591 .48390 0.320550 .982851 .48178 0.318880 .979131 .47965 0.317230 .975411 .47751

$0.31558 \quad 0.97172 \quad 1.47538$
$T=3100$.

$0.41404 \quad 1.18321 \quad 1.58288$

0.411911 .178821 .58092

0.409801 .174451 .57896

$0.407691 .17010 \quad 1.57700$

$0.405601 .16576 \quad 1.57504$

$0.403511 .16144 \quad 1.57307$

$0.40144 \quad 1.15714 \quad 1.57110$

$0.39938 \quad 1.15286 \quad 1.56912$

0.397341 .148591 .56715

$0.39530 \quad 1.14434 \quad 1.56517$

$0.39328 \quad 1.14011 \quad 1.56319$

$0.39126 \quad 1.13589 \quad 1.56121$

0.389261 .131691 .55922

$0.38727 \quad 1.12751 \quad 1.55724$

$0.38529 \quad 1.12334 \quad 1.55525$

$0.38332 \quad 1.11919 \quad 1.55326$

$0.38137 \quad 1.11506 \quad 1.55126$

0.379421 .110941 .54927

$0.37749 \quad 1.10684 \quad 1.54727$

0.375561 .102751 .54527

$0.37365 \quad 1.09868 \quad 1.54327$

0.371751 .094631 .54126

$0.36986 \quad 1.090591 .53925$

0.367971 .086571 .53724

0.366101 .082561 .53523

$0.36424 \quad 1.07857 \quad 1.53322$

0.362391 .074601 .53120

$0.36055 \quad 1.07064 \quad 1.52919$

$0.358721 .06670 \quad 1.52717$

$0.35690 \quad 1.06277 \quad 1.52514$

$0.35509 \quad 1.05886 \quad 1.52312$

$0.35329 \quad 1.05496 \quad 1.52110$

$0.35150 \quad 1.05107 \quad 1.51907$

$0.349721 .04721 \quad 1.51704$

0.347951 .043351 .51501

0.346191 .039521 .51297 $0.34444 \quad 1.03569 \quad 1.51094$ 0.342701 .031891 .50890 $0.34097 \quad 1.02809 \quad 1.50686$ 0.339251 .024311 .50482

$0.33754 \quad 1.02055 \quad 1.50278$
3600

3610

3620

3630

3640

3650

3660

3670

3680

3690

3700

3710

3720

3730

3740

3750

3760

3770

3780

3790

3800

3810

3820

3830

3840

3850

3860

3870

3880

3890

3900

3910

3920

3930

3940

3950

3960

3970

3980

3990

4000 
Table II Harmonic Oscillator Contributions to the Thermodynamic

Functions (in units of calories, moles, and ${ }^{\circ} \mathrm{K}$ )
$\nu$
$-\left(F^{\circ}-E_{O}^{\circ}\right) / T \quad S^{\circ}$
$\mathrm{cm}^{-1}$
$\mathrm{C}_{\mathrm{p}}^{\circ} \quad-\left(\mathrm{F}^{\circ}-\mathrm{E}_{\mathrm{O}}^{\circ}\right) / \mathrm{T}$
So
$\mathrm{C}_{\mathrm{p}}^{\circ}$
$-\left(F^{\circ}-E_{O}^{\circ}\right) / T$
So
$\mathrm{c}_{\mathrm{p}}^{\circ}$
$\mathrm{cm}^{-1}$

$\mathrm{T}=3200$.

$T=3300$.

$T=3400$

$100 \quad 6.20856 \quad 8.15139 \quad 1.98683$

$110 \quad 6.023607 .962031 .98676$

$120 \quad 5.855127 .78916 \quad 1.98669$

$130 \quad 5.700497 .63015 \quad 1.98660$

$140 \quad 5.557647 .482931 .98651$

$150 \quad 5.424967 .34587 \quad 1.98642$

$160 \quad 5.301137 .21768 \quad 1.98631$

$\begin{array}{lllll}170 & 5.18507 & 7.09726 & 1.98620\end{array}$

$180 \quad 5.075896 .98373 \quad 1.98609$

$190 \quad 4.97286 \quad 6.87636 \quad 1.98596$

$200 \quad 4.875336 .77449 \quad 1.98583$

$210 \cdot 4.78278 \quad 6.67761 \quad 1.98569$

$220 \quad 4.69473 \quad 6.58524 \quad 1.98555$

$230 \quad 4.610796 .496981 .98540$

$240 \quad 4.53060 \quad 6.41248 \quad 1.98524$

$250 \quad 4.45387 \quad 6.33145 \quad 1.98508$

$260 \quad 4.380316 .25359 \quad 1.98491$

$270 \quad 4.309696 .17868 \quad 1.98473$

$280 \quad 4.24180 \quad 6.10651 \quad 1.98455$

$290 \quad 4.176446 .03687 \quad 1.98436$

$300 \quad 4.113445 .96960 \quad 1.98416$ $310 \quad 4.05265 \quad 5.904541 .98396$

$320 \quad 3.993925 .84156 \quad 1.98375$

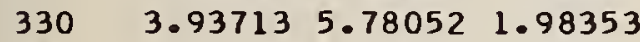

$\begin{array}{lllll}340 \quad 3.88216 & 5.72131 & 1.98330\end{array}$

$\begin{array}{lllll}350 & 3.82891 & 5.66382 & 1.98307\end{array}$ $\begin{array}{lllll}360 & 3.77728 & 5.60796 & 1.98284\end{array}$ $\begin{array}{lllll}370 & 3.72718 & 5.55364 & 1.98259\end{array}$ $\begin{array}{lllll}380 & 3.67853 & 5.50077 & 1.98234\end{array}$ $390 \quad 3.63125 \quad 5.44928 \quad 1.98209$

$\begin{array}{lllll}400 & 3.58527 & 5.39910 & 1.98182\end{array}$

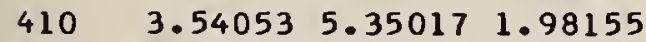

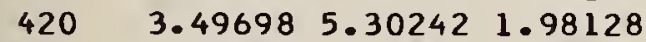
$\begin{array}{lllll}430 & 3.45454 & 5.25580 & 1.98099\end{array}$ $\begin{array}{lll}440 \quad 3.41318 & 5.21026 \quad 1.98070\end{array}$

$\begin{array}{lllll}450 & 3.37284 & 5.16575 & 1.98040\end{array}$ $460 \quad 3.33348 \quad 5.122231 .98010$ $\begin{array}{lllll}470 & 3.29506 & 5.07965 & 1.97979\end{array}$ $\begin{array}{lllll}480 & 3.25753 & 5.03797 & 1.97947\end{array}$ $490 \quad 3.22086 \quad 4.99716 \quad 1.97915$

$\begin{array}{llll}500 & 3.18502 & 4.95718 & 1.97882\end{array}$ $510 \quad 3.14996 \quad 4.91800 \quad 1.97849$ $\begin{array}{llll}520 & 3.11567 & 4.87958 & 1.97814\end{array}$ $\begin{array}{llll}530 & 3.08211 & 4.84190 & 1.97779\end{array}$ $\begin{array}{llll}540 & 3.04926 & 4.80494 & 1.97744\end{array}$

$\begin{array}{lllll}550 & 3.01708 & 4.76866 & 1.97707\end{array}$ $560 \quad 2.985554 .73304 \quad 1.97670$ $\begin{array}{lll}570 & 2.95466 \quad 4.69805 & 1.97633\end{array}$

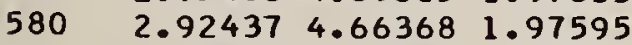
$590 \quad 2.89468 \quad 4.62991 \quad 1.97556$
6.268378 .212531 .98685 6.083278 .023171 .98679 $5.91466 \quad 7.85030 \quad 1.98672$ $5.759897 .69128 \quad 1.98664$ 5.616927 .544051 .98655

$5.484107 .40700 \quad 1.98646$ 5.360147 .278801 .98636 $5.24394 \quad 7.15838 \quad 1.98626$ 5.134647 .044851 .98615 5.031476 .937471 .98603

$\begin{array}{llll}4.93381 & 6.83560 & 1.98591\end{array}$ 4.841136 .738711 .98578 $4.75295 \quad 6.64634 \quad 1.98565$ $4.66888 \quad 6.55807 \quad 1.98551$ $4.58856 \quad 6.47357 \quad 1.98536$

$4.51170 \quad 6.39253 \quad 1.98520$ 4.438016 .314671 .98504 $4.36726 \quad 6.23976 \quad 1.98488$ $4.29924 \quad 6.16758 \quad 1.98470$ $4.23375 \quad 6.09794 \quad 1.98452$

$4.17062 \quad 6.03066 \quad 1.98434$ $4.10970 \quad 5.96560 \quad 1.98415$ 4. $05084 \quad 5.90261 \quad 1.98395$ $3.993925 .84156 \quad 1.98375$ $3.938825 .78234 \quad 1.98353$

$3.88544 \quad 5.72485 \quad 1.98332$ $3.83368 \quad 5.66898 \quad 1.98310$ 3.783455 .614651 .98287 $3.734675 .56177 \quad 1.98263$ $3.687275 .51027 \quad 1.98239$

3.641165 .460091 .98214 $3.59630 \quad 5.41115 \quad 1.98189$ 3.552625 .363391 .98163 $3.51006 \quad 5.31677 \quad 1.98136$ 3.468575 .271221 .98109

$3.42810 \quad 5.22670 \quad 1.98081$ $3.388615 .18317 \quad 1.98052$ $3.35006 \quad 5.14058 \quad 1.98023$ 3.312415 .098891 .97993 3.275615 .058071 .97963

$3.23965 \quad 5.01808 \quad 1.97932$ $3.204474 .97888 \quad 1.97900$ $3.17005 \quad 4.94046 \quad 1.97868$ $3.13636 \quad 4.90277 \quad 1.97835$ $3.10338 \quad 4.86580 \quad 1.97802$

$3.07108 \quad 4.82950 \quad 1.97767$ 3.039434 .793871 .97733 $\begin{array}{llll}3.00842 & 4.75888 & 1.97697\end{array}$ 2.978014 .724501 .97661 2.948194 .690711 .97625
$6.32642 \quad 8.27184 \quad 1.98687$ 6.141208 .082481 .98681 5.972467 .909611 .98674 $5.81757 \quad 7.750591 .98667$ 5.674477 .603361 .98659

$5.541537 .46630 \quad 1.98650$ $5.417447 .33810 \quad 1.98641$ 5.301137 .217681 .98631 $5.191707 .10414 \quad 1.98621$ $5.088416 .99676 \quad 1.98610$

$4.99062 \quad 6.89489 \quad 1.98598$ $4.897816 .79799 \quad 1.98586$ $4.809516 .70561 \quad 1.98573$ 4.725326 .617351 .98560 $4.64488 \quad 6.53284 \quad 1.98546$

$4.567896 .45180 \quad 1.98532$ $4.49408 \quad 6.37393 \quad 1.98517$ 4.423216 .299021 .98501 $4.35506 \quad 6.226831 .98485$ $4.289456 .15718 \quad 1.98468$

$4.22620 \quad 6.08990 \quad 1.98450$ $4.16516 \quad 6.024831 .98432$ 4.106185 .961841 .98414 $4.049145 .90078 \quad 1.98394$ $3.993925 .84156 \quad 1.98375$

$3.940425 .78406 \quad 1.98354$ 3.888545 .728181 .98333 3.838195 .673851 .98312 $3.789295 .62096 \quad 1.98289$ $3.741765 .56946 \quad 1.98267$

$3.695545 .51926 \quad 1.98243$ 3.650555 .470321 .98219 $3.60675 \quad 5.422551 .98195$ 3.564075 .375921 .98170 3.522465 .330361 .98144

$3.48188 \quad 5.28584 \quad 1.98118$ 3.442275 .242301 .98091 $3.403605 .19970 \quad 1.98063$ 3.365835 .158001 .98035 $3.328915 .11717 \quad 1.98007$

$3.29283 \quad 5.07717 \quad 1.97977$ $3.257535 .03797 \quad 1.97947$ $3.222994 .99954 \quad 1.97917$ 3.189194 .961841 .97886 3.156094 .924851 .97855

$3.12367 \quad 4.88855 \quad 1.97822$ 3.091914 .852911 .97790 $3.06077 \quad 4.81790 \quad 1.97756$ $3.030254 .78351 \quad 1.97722$ 3.000314 .749721 .97688
100

110

120

130

140

150

160

170

180

190

200

210

220

230

240

250

260

270

280

290

300

310

320

330

340

350

360

370

380

390

400

410

420

430

440

450

460

470

480

490

500

510

520

530

540

550

560

570

580

590 
Table II Harmonic Oscillator Contributions to the Thermodynamic

Functions (in units of calories, moles, and ${ }^{\circ} \mathrm{K}$ )

$\mathrm{cm}^{-1}-\left(F^{\circ}-E_{O}^{\circ}\right) / \mathrm{T} \quad$ So

$\mathrm{C}_{\mathrm{p}}^{\circ} \quad-\left(\mathrm{F}^{\circ}-\mathrm{E}_{\mathrm{O}}\right) / \mathrm{T}$

So

$T=3200$

$600 \quad 2.86555 \quad 4.59671 \quad 1.97516$

$610 \quad 2.83697 \quad 4.56406 \quad 1.97476$

$\begin{array}{llll}620 & 2.80891 & 4.53196 & 1.97435\end{array}$

$\begin{array}{lll}630 & 2.78138 \quad 4.50037 \quad 1.97394\end{array}$

$640 \quad 2.75434 \quad 4.469291 .97351$

$\begin{array}{llll}650 & 2.72778 & 4.43869 & 1.97309\end{array}$

$\begin{array}{lllll}660 & 2.70169 & 4.40857 & 1.97265\end{array}$

$\begin{array}{llll}670 & 2.67605 & 4.37891 & 1.97221\end{array}$

$\begin{array}{llll}680 & 2.65085 & 4.34970 & 1.97176\end{array}$

$690 \quad 2.62608 \quad 4.32091 \quad 1.97131$

$700 \quad 2.601724 .29255 \quad 1.97085$

$\begin{array}{llll}710 & 2.57777 & 4.26460 & 1.97038\end{array}$

$\begin{array}{lllll}720 & 2.55420 & 4.23705 & 1.96991\end{array}$

$\begin{array}{llll}730 & 2.53102 & 4.20988 & 1.96943\end{array}$

$\begin{array}{llll}740 & 2.50820 & 4.18308 & 1.96894\end{array}$

$\begin{array}{lllll}750 & 2.48575 & 4.15666 & 1.96845\end{array}$

$760 \quad 2.463644 .130591 .96795$

$\begin{array}{llll}770 & 2.44188 & 4.10487 & 1.96744\end{array}$

$\begin{array}{lll}780 & 2.42045 \quad 4.07948 & 1.96693\end{array}$

$790 \quad 2.39934 \quad 4.05443 \quad 1.96641$

$800 \quad 2.37854 \quad 4.02970 \quad 1.96588$ $810 \quad 2.35806 \quad 4.00528 \quad 1.96535$ $\begin{array}{llll}820 & 2.33787 & 3.98117 & 1.96481\end{array}$ $830 \quad 2.31797 \quad 3.95736 \quad 1.96427$ $\begin{array}{lllll}840 & 2.29836 & 3.93384 & 1.96372\end{array}$

$\begin{array}{llll}850 & 2.27903 \quad 3.91060 \quad 1.96316\end{array}$ $\begin{array}{lllll}860 & 2.25997 & 3.88764 & 1.96259\end{array}$ $870 \quad 2.24118 \quad 3.86496 \quad 1.96202$
$880 \quad 2.2264$ $\begin{array}{lllll}880 & 2.22264 & 3.84254 & 1.96145\end{array}$ $890 \quad 2.20436 \quad 3.82038 \quad 1.96086$

$900 \quad 2.18632 \quad 3.79847 \quad 1.96027$ $910 \quad 2.16853 \quad 3.77681 \quad 1.95968$ $920 \quad 2.15097 \quad 3.75540 \quad 1.95908$ $930 \quad 2.13365 \quad 3.73422 \quad 1.95847$ $940 \quad 2.11655 \quad 3.71328 \quad 1.95785$

$\begin{array}{lllll}950 & 2.09968 & 3.69256 & 1.95723\end{array}$ $960 \quad 2.083023 .67207 \quad 1.95660$ $970 \quad 2.06657 \quad 3.65180 \quad 1.95597$ $980 \quad 2.05033 \quad 3.63174 \quad 1.95533$ $990 \quad 2.03429 \quad 3.61189 \quad 1.95468$

$1000 \quad 2.01846 \quad 3.59225 \quad 1.95403$ $\begin{array}{lllll}1010 & 2.00282 & 3.57281 & 1.95337\end{array}$ $\begin{array}{llllll}1020 & 1.98737 & 3.55357 & 1.95270\end{array}$ $1030 \quad 1.972113 .534521 .95203$ $1040 \quad 1.95703 \quad 3.51567 \quad 1.95135$

$1050 \quad 1.94213 \quad 3.49700 \quad 1.95067$ $1060 \quad 1.92741 \quad 3.47851 \quad 1.94998$ $1070 \quad 1.91286 \quad 3.46020 \quad 1.94928$ $\begin{array}{lllll}1080 & 1.89849 & 3.44207 & 1.94858\end{array}$ $1090 \quad 1.88428 \quad 3.42412 \quad 1.94787$

\begin{abstract}
$T=3300$
$2.91893 \quad 4.65750 \quad 1.97588$

2.890234 .624841 .97550

2. $86205 \quad 4.592721 .97511$

2.834394 .561121 .97472

2.807234 .530031 .97433

$2.78055 \quad 4.49942 \quad 1.97392$

$2.75434 \quad 4.469291 .97351$

$2.72858 \quad 4.43961 \quad 1.97310$

$2.70326 \quad 4.41038 \quad 1.97268$

$2.67836 \quad 4.38159 \quad 1.97225$

$2.653894 .35321 \quad 1.97182$

$2.629814 .32525 \quad 1.97138$

$2.606124 .29768 \quad 1.97093$

$2.58282 \quad 4.27050 \quad 1.97048$

$2.55988 \quad 4.243691 .97002$
\end{abstract}

$2.537314 .21725 \quad 1.96956$

$2.51508 \quad 4.191161 .96909$

2.493194 .165431 .96861

$2.47164 \quad 4.140031 .96813$

2.450414 .114961 .96764

$2.42950 \quad 4.09021 \quad 1.96715$

$2.408894 .06578 \quad 1.96664$

2.388594 .041651 .96614

2.368574 .017821 .96562

$2.348843 .99428 \quad 1.96511$

$2.329393 .97103 \quad 1.96458$

$2.310213 .94806 \quad 1.96405$

$2.29130 \quad 3.92535 \quad 1.96351$

$2.27265 \quad 3.90292 \quad 1.96297$

$2.25425 \quad 3.88074 \quad 1.96242$

$2.23609 \quad 3.85882 \quad 1.96187$

$2.21818 \quad 3.83714 \quad 1.96131$

$2.200513 .81571 \quad 1.96074$

$2.18307 \quad 3.79451 \quad 1.96017$

$2.16586 \quad 3.77355 \quad 1.95959$

$2.14886 \quad 3.75282 \quad 1.95900$ 2.132093 .732311 .95841

2.115523 .712021 .95781

$2.09917 \quad 3.69194 \quad 1.95721$

2.08302 3.67207 1.95660

$2.06706 \quad 3.65241 \quad 1.95599$

$2.051313 .63295 \quad 1.95537$

$2.03574 \quad 3.61369 \quad 1.95474$

2.020373 .594621 .95411

$2.00517 \quad 3.57574 \quad 1.95347$

$1.99016 \quad 3.55705 \quad 1.95282$ $1.97533 \quad 3.53855 \quad 1.95217$ 1.960673 .520221 .95152 $1.946183 .50207 \quad 1.95086$ $1.93185 \quad 3.48409 \quad 1.95019$
$-\left(F^{\circ}-E_{0}^{\circ}\right) / T \quad S^{\circ}$

$\stackrel{c o}{p}$

$v$

$\mathrm{cm}^{-1}$

$T=3400$.

$2.97094 \quad 4.71650 \quad 1.97653$

$2.94212 \quad 4.68383 \quad 1.97617$

$2.91383 \quad 4.65170 \quad 1.97581$

$2.88605 \quad 4.62009 \quad 1.97544$

$2.85877 \quad 4.58898 \quad 1.97507$

$2.83198 \quad 4.55836 \quad 1.97469$

$2.80565 \quad 4.52821 \quad 1.97430$

$2.77977 \quad 4.49853 \quad 1.97391$

$2.75434 \quad 4.469291 .97351$

$2.729334 .44048 \quad 1.97311$

$2.70474 \quad 4.41209 \quad 1.97270$

$2.68054 \quad 4.384111 .97229$

2.656744 .356531 .97187

2.633324 .329341 .97144

$2.61027 \quad 4.302521 .97101$

$2.58758 \quad 4.27606 \quad 1.97057$

$2.56525 \quad 4.24996 \quad 1.97013$

$2.54325 \quad 4.22421 \quad 1.96968$

$2.52158 \quad 4.19880 \quad 1.96923$

$2.50024 \quad 4.173721 .96877$

$2.47921 \quad 4.14896 \quad 1.96830$

$2.45849 \quad 4.12451 \quad 1.96783$

2.438074 .100361 .96735

$2.41795 \quad 4.07652 \quad 1.96687$

2.398114 .052971 .96638

$2.37854 \quad 4.02970 \quad 1.96588$

$2.35925 \quad 4.006711 .96538$

$2.34023 \quad 3.98399 \quad 1.96488$

$2.32146 \quad 3.96154 \quad 1.96436$

$2.30295 \quad 3.93934 \quad 1.96385$

$2.28469 \quad 3.91740 \quad 1.96332$

2.266673 .895711 .96279

$2.24888 \quad 3.87426 \quad 1.96226$

$2.23133 \quad 3.85305 \quad 1.96172$

2.214013 .832081 .96117

$2.19690 \quad 3.81132 \quad 1.96062$

2.180023 .790801 .96006

$2.163343 .77049 \quad 1.95950$

$2.14688 \quad 3.750391 .95893$

2.130623 .730511 .95836

$2.11456 \quad 3.71083 \quad 1.95778$

2.09869 3.69135 1.95719

$2.08302 \quad 3.67207 \quad 1.95660$

$2.06753 \quad 3.652991 .95601$

2.052233 .634091 .95540

$2.037113 .61538 \quad 1.95480$

$2.022173 .59686 \quad 1.95418$

$2.00740 \quad 3.57851 \quad 1.95356$

$1.99280 \quad 3.56034 \quad 1.95294$

$1.978373 .54234 \quad 1.95231$
600

610

620

630

640

650

660

670

680

690

700

710

720

730

740

750

760

770

780

790

800

810

820

830

840

850

860

870

880

890

900

910

920

930

940

950

960

970

980

990

1000

1010

1020

1030

1040

1050

1060

1070

1080

1090 
Table II Harmonic Oscillator Contributions to the Thermodynamic

Functions (in units of calories, moles, and ${ }^{\circ} \mathrm{K}$ )
$\mathrm{cm}^{-1}-\left(\mathrm{F}^{\circ}-\mathrm{E}_{\mathrm{O}}^{\circ}\right) / \mathrm{T} \quad \mathrm{S}^{\circ}$
$\begin{array}{cc}C_{0}^{\circ} & -\left(F^{\circ}-E_{O}^{\circ}\right) / T\end{array}$
So
$\mathrm{co}_{\mathrm{p}}$
$-\left(F^{\circ}-E O O\right) / T$
So

$c_{p}^{\circ}$

$\mathrm{cm}^{-1}$

$T=3200$.

$1100 \quad 1.87023 \quad 3.40633 \quad 1.94715$

$1110 \quad 1.856353 .38871 \quad 1.94643$

$1120 \quad 1.84262 \quad 3.37126 \quad 1.94570$

$1130 \quad 1.82905 \quad 3.35397 \quad 1.94497$

$1140 \quad 1.81563 \quad 3.33683 \quad 1.94423$

$\begin{array}{lllll}1150 & 1.80236 & 3.31986 & 1.94348\end{array}$

$\begin{array}{lllll}1160 & 1.78924 & 3.30303 & 1.94273\end{array}$

$\begin{array}{lllll}1170 & 1.77626 & 3.28636 & 1.94197\end{array}$

$1180 \quad 1.763423 .269841 .94120$

$1190 \quad 1.75073 \quad 3.25346 \quad 1.94043$

$\begin{array}{lllll}1200 \quad 1.73817 & 3.23722 & 1.93966\end{array}$

$\begin{array}{lllll}1210 & 1.72574 & 3.22113 & 1.93887\end{array}$

$\begin{array}{lllll}1220 & 1.71345 & 3.20517 & 1.93808\end{array}$

$1230 \quad 1.701293 .189361 .93729$

$\begin{array}{llll}1240 & 1.68925 & 3.17367 & 1.93648\end{array}$

$\begin{array}{lllll}1250 \quad 1.67734 & 3.15812 & 1.93568\end{array}$

$1260 \quad 1.66556 \quad 3.14270 \quad 1.93486$

$1270 \quad 1.65390 \quad 3.12741 \quad 1.93404$

$1280 \quad 1.64235 \quad 3.11224 \quad 1.93322$

$1290 \quad 1.63093 \quad 3.09720 \quad 1.93238$

$1300 \quad 1.61962 \quad 3.08228 \quad 1.93155$

$1310 \quad 1.608423 .06749 \quad 1.93070$

$1320 \quad 1.59734 \quad 3.05281 \quad 1.92985$

$1330 \quad 1.58637 \quad 3.03825 \quad 1.92900$

$1340 \quad 1.575513 .02380 \quad 1.92813$

$\begin{array}{lllll}1350 & 1.56475 & 3.00947 & 1.92726\end{array}$

$\begin{array}{lllll}1360 & 1.55411 & 2.99525 & 1.92639\end{array}$

$1370 \quad 1.543562 .98114 \quad 1.92551$

$\begin{array}{lllll}1380 & 1.53312 & 2.96714 & 1.92462\end{array}$

$\begin{array}{lllll}1390 \quad 1.52278 & 2.95324 & 1.92373\end{array}$

$1400 \quad 1.51254 \quad 2.93946 \quad 1.92283$

$\begin{array}{lllll}1410 & 1.50239 & 2.92577 & 1.92193\end{array}$

$1420 \quad 1.492352 .912191 .92102$

$1430 \quad 1.482392 .898721 .92010$

$1440 \quad 1.472542 .88534 \quad 1.91918$

$\begin{array}{lllll}1450 & 1.46277 & 2.87206 & 1.91825\end{array}$

$\begin{array}{lllll}1460 & 1.45310 & 2.85888 & 1.91732\end{array}$

$\begin{array}{lllll}1470 & 1.44351 & 2.84580 & 1.91638\end{array}$

$\begin{array}{lllll}1480 & 1.43402 & 2.83281 & 1.91544\end{array}$

$\begin{array}{lllll}1490 & 1.42461 & 2.81991 & 1.91448\end{array}$

$\begin{array}{lllll}1500 \quad 1.41529 & 2.80711 & 1.91353\end{array}$

$\begin{array}{llll}1510 \quad 1.40605 & 2.79440 & 1.91257\end{array}$

$\begin{array}{lllll}1520 & 1.39690 & 2.78178 & 1.91160\end{array}$

$\begin{array}{lllll}1530 & 1.38783 & 2.76924 & 1.91062\end{array}$

$\begin{array}{lllll}1540 & 1.37884 & 2.75680 & 1.90964\end{array}$

$\begin{array}{lllll}1550 & 1.36993 & 2.74444 & 1.90866\end{array}$

$\begin{array}{lllll}1560 & 1.36111 & 2.73217 & 1.90766\end{array}$

$\begin{array}{lllll}1570 & 1.35236 & 2.71999 & 1.90667\end{array}$

$\begin{array}{lllll}1580 & 1.34368 & 2.70788 & 1.90566\end{array}$

$\begin{array}{llll}1590 & 1.33509 & 2.69586 & 1.90465\end{array}$
$\mathrm{T}=3300$

$1.917693 .46628 \quad 1.94951$

1.903693 .448641 .94883

$1.88985 \quad 3.43117 \quad 1.94815$

1.876173 .413861 .94746

1.862643 .396701 .94676

$1.84926 \quad 3.37970 \quad 1.94606$

1.836023 .362851 .94535

$1.822933 .34616 \quad 1.94463$

1.809983 .329611 .94391

$1.797173 .31321 \quad 1.94319$

$1.78450 \quad 3.29695 \quad 1.94245$

$1.771973 .28084 \quad 1.94172$

$1.75956 \quad 3.26486 \quad 1.94097$

$1.747293 .24902 \quad 1.94022$

$1.73514 \quad 3.23331 \quad 1.93947$

$1.723123 .21773 \quad 1.93871$

$1.71123 \quad 3.202291 .93794$

$\begin{array}{llll}1.69945 & 3.18697 & 1.93717\end{array}$

$1.68780 \quad 3.17178 \quad 1.93639$

1.676273 .156721 .93560

$1.66485 \quad 3.14177 \quad 1.93481$

1.653543 .126951 .93402

$1.64235 \quad 3.11224 \quad 1.93322$

$1.63127 \quad 3.09766 \quad 1.93241$

$1.62030 \quad 3.08318 \quad 1.93160$

$1.60944 \quad 3.06883 \quad 1.93078$

$1.59868 \quad 3.05458 \quad 1.92996$

1.588033 .040441 .92913

$1.57748 \quad 3.02642 \quad 1.92829$

1.567033 .012501 .92745

$\begin{array}{llll}1.55668 & 2.99868 & 1.92660\end{array}$ 1.546432 .984971 .92575 1.536272 .971371 .92489 $1.526212 .95786 \quad 1.92403$ $1.51625 \quad 2.94446 \quad 1.92316$

$1.50638 \quad 2.931151 .92229$ 1.496602 .917941 .92141 1.486912 .904831 .92052 $1.47730 \quad 2.89181 \quad 1.91963$ $1.467792 .87889 \quad 1.91873$

$1.458362 .86606 \quad 1.91783$ 1.449022 .853321 .91692 $1.43976 \quad 2.84067 \quad 1.91601$ 1.430592 .828111 .91509 1.421492 .815631 .91417

$1.41248 \quad 2.80325 \quad 1.91324$ 1.403552 .790951 .91230 1.394692 .778731 .91136 1.385922 .766601 .91041 1.377222 .754551 .90946
$\mathrm{T}=3400$.

$1.96410 \quad 3.524521 .95167$ $1.94999 \quad 3.50686 \quad 1.95103$ $1.93605 \quad 3.48936 \quad 1.95038$ 1.922263 .472031 .94973 1.908623 .454851 .94907

$1.89513 \quad 3.437831 .94841$ $1.881793 .42097 \quad 1.94774$ 1.868593 .404251 .94707 $1.855543 .38768 \quad 1.94639$ $1.84262 \quad 3.37126 \quad 1.94570$

$1.82984 \quad 3.35498 \quad 1.94501$ $1.81720 \quad 3.33884 \quad 1.94432$ $1.804693 .32284 \quad 1.94361$ 1.792313 .306981 .94291 $1.78006 \quad 3.291251 .94219$

$1.76794 \quad 3.27565 \quad 1.94148$ $1.75 .594 \quad 3.26018 \quad 1.94075$ $1.74406 \quad 3.244851 .94002$ $1.73230 \quad 3.229631 .93929$ $1.72066 \quad 3.21454 \quad 1.93855$

$1.70914 \quad 3.19958 \quad 1.93780$ $1.69773 \quad 3.184731 .93705$ 1.686443 .170001 .93630 1.675253 .155391 .93553 $1.66418 \quad 3.14090 \quad 1.93477$

$\begin{array}{llll}1.65321 & 3.12651 & 1.93399\end{array}$ 1.642353 .112241 .93322 1.631603 .098081 .93243 1.620943 .084031 .93165 1.610393 .070091 .93085

$1.59994 \quad 3.05625 \quad 1.93005$ 1.589593 .042521 .92925 $1.57933 \quad 3.02888 \quad 1.92844$ 1.569173 .015351 .92762 $1.55910 \quad 3.001921 .92680$

$1.54913 \quad 2.98859 \quad 1.92598$ $1.539252 .97536 \quad 1.92515$ $1.52946 \quad 2.96222 \quad 1.92431$ $1.51976 \quad 2.94918 \quad 1.92347$ 1.510142 .936231 .92262

$\begin{array}{llll}1.50061 & 2.92337 & 1.92177\end{array}$ 1.491172 .910601 .92091 1.481812 .897931 .92005 1.472542 .885341 .91918 1.463342 .872841 .91831

$1.454232 .86043 \quad 1.91743$ $1.44520 \quad 2.84810 \quad 1.91655$ 1.436242 .835851 .91566 1.427372 .823691 .91477 1.418572 .811621 .91387
1100

1110

1120

1130

1140

1150

1160

1170

1180

1190

1200

1210

1220

1230

1240

1250

1260

1270

1280

1290

1300

1310

1320

1330

1340

1350

1360

1370

1380

1390

1400

1410

1420

1430

1440

1450

1460

1470

1480

1490

1500

1510

1520

1530

1540

1550

1560

1570

1580

1590 
Table II Harmonic Oscillator Contributions to the Thermodynamic

Functions (in units of calories, moles, and ${ }^{\circ} \mathrm{K}$ )

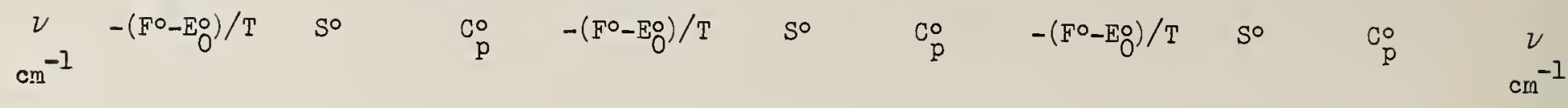

$T=3200$

$T=3300$.

$T=3400$.

$1600 \quad 1.32657 \quad 2.68392 \quad 1.90364$ $1610 \quad 1.318122 .67207 \quad 1.90262$ $\begin{array}{lll}1620 \quad 1.30975 & 2.66029 & 1.90159\end{array}$ $1630 \quad 1.30145 \quad 2.648591 .90056$ $\begin{array}{lllll}1640 \quad 1.29322 & 2.63697 & 1.89952\end{array}$

$\begin{array}{llll}1650 \quad 1.28506 \quad 2.62542 & 1.89848\end{array}$ $\begin{array}{lllll}1660 & 1.27697 & 2.61396 & 1.89743\end{array}$ $\begin{array}{llll}1670 \quad 1.26895 & 2.60256 & 1.89638\end{array}$ $\begin{array}{lllll}1680 & 1.26100 & 2.59125 & 1.89532\end{array}$ $\begin{array}{lllll}1690 & 1.25311 & 2.58000 & 1.89425\end{array}$

$\begin{array}{lllll}1700 \quad 1.24530 & 2.56883 & 1.89318\end{array}$ $1710 \quad 1.237542 .55773 \quad 1.89210$ $1720 \quad 1.22985 \quad 2.54670 \quad 1.89102$ $\begin{array}{lllll}1730 & 1.22223 & 2.53574 & 1.88993\end{array}$ $1740 \quad 1.214672 .52485 \quad 1.88884$

$\begin{array}{lllll}1750 & 1.20717 & 2.51403 & 1.88774\end{array}$ $\begin{array}{lllll}1760 \quad 1.19973 & 2.50327 \quad 1.88664\end{array}$ $\begin{array}{lllll}1770 \quad 1.19236 & 2.49259 & 1.88553\end{array}$ $\begin{array}{lllll}1780 & 1.18504 & 2.48197 & 1.88441\end{array}$ $1790 \quad 1.17779 \quad 2.47141 \quad 1.88329$

$\begin{array}{llll}1800 \quad 1.17059 & 2.46093 \quad 1.88216\end{array}$ $1810 \quad 1.16345 \quad 2.45050 \quad 1.88103$ $1820 \quad 1.156372 .44014 \quad 1.87990$ $\begin{array}{lllll}1830 & 1.14934 & 2.42984 & 1.87875\end{array}$ $1840 \quad 1.142372 .419611 .87760$

1850

1860

1870

1880

1890

1900

1910

1920

1930

1940

1950

1960

1970

1980

1990

2000

2010

2020

2030

2040

2050

2060

2070

2080

2090
$1.368592 .74258 \quad 1.90851$ $1.36004 \quad 2.73069 \quad 1.90754$ $\begin{array}{llll}1.35157 & 2.71888 & 1.90658\end{array}$ $1.34316 \quad 2.70715 \quad 1.90560$ $1.334832 .69550 \quad 1.90462$

$1.326572 .68392 \quad 1.90364$ $1.31838 \quad 2.67243 \quad 1.90265$ 1.310252 .661001 .90166 $1.30220 \quad 2.64965 \quad 1.90066$ $1.294212 .63837 \quad 1.89965$

$1.28629 \quad 2.62717 \quad 1.89864$ $1.278442 .61604 \quad 1.89762$ $1.270652 .60497 \quad 1.89660$ $1.26292 \quad 2.59398 \quad 1.89558$ $1.25526 \quad 2.58306 \quad 1.89454$

$\begin{array}{llll}1.24766 & 2.57221 & 1.89351\end{array}$ $1.240122 .56142 \quad 1.89246$ $1.232642 .55070 \quad 1.89142$ $1.22523 \quad 2.54005 \quad 1.89036$ $1.21787 \quad 2.52946 \quad 1.88931$

$\begin{array}{llll}1.21057 & 2.51894 & 1.88824\end{array}$ $1.203332 .50848 \quad 1.88717$ $1.19615 \quad 2.49808 \quad 1.88610$ $1.18902 \quad 2.48775 \quad 1.88502$ $1.18196 \quad 2.47748 \quad 1.88394$

$1.17494 \quad 2.46728 \quad 1.88285$ $1.16798 \quad 2.45713 \quad 1.88175$ $1.16108 \quad 2.44704 \quad 1.88065$ $1.15423 \quad 2.43701 \quad 1.87955$ $1.14743 \quad 2.42705 \quad 1.87844$

$1.140692 .41714 \quad 1.87732$ $1.13400 \quad 2.40728 \quad 1.87620$ $1.12736 \quad 2.39749 \quad 1.87508$ $\begin{array}{llll}1.12077 & 2.38775 & 1.87395\end{array}$ $1.114232 .37807 \quad 1.87281$

$1.10774 \quad 2.36844 \quad 1.87167$ $1.101302 .35887 \quad 1.87053$ $\begin{array}{llll}1.09490 & 2.34936 & 1.86938\end{array}$ $1.08856 \quad 2.33989 \quad 1.86822$ $1.08226 \quad 2.33049 \quad 1.86706$

$1.07602 \quad 2.321131 .86590$ 1.069812 .311831 .86473 $1.06366 \quad 2.30258 \quad 1.86355$ 1.057552 .293381 .86237 $1.05148 \quad 2.284231 .86119$

$1.04546 \quad 2.27513 \quad 1.86000$ $1.03948 \quad 2.26608 \quad 1.85880$ 1.033552 .257081 .85760 $1.02766 \quad 2.24813 \quad 1.85640$ $1.02182 \quad 2.239231 .85519$
$1.40985 \quad 2.79962 \quad 1.91296$ $1.401202 .78770 \quad 1.91205$ $1.39262 \quad 2.77587 \quad 1.91114$ 1.384122 .764111 .91022 $1.375692 .75243 \quad 1.90929$

$\begin{array}{lll}1.36733 & 2.74082 & 1.90837\end{array}$ $1.35904 \quad 2.72930 \quad 1.90743$ 1.350822 .717841 .90649 $1.342672 .70646 \quad 1.90555$ $1.33458 \quad 2.69516 \quad 1.90460$

$1.32657 \quad 2.68392 \quad 1.90364$ $1.318622 .67276 \quad 1.90268$ 1.310732 .661671 .90171 1.302912 .650651 .90074 $1.29515 \quad 2.63970 \quad 1.89977$

$\begin{array}{llll}1.28745 & 2.62881 & 1.89879\end{array}$ $1.27982 \quad 2.61800 \quad 1.89780$ $1.27224 \quad 2.60725 \quad 1.89681$ $1.264732 .59656 \quad 1.89582$ $1.25728 \quad 2.58594 \quad 1.89482$

$1.249892 .57539 \quad 1.89381$ $1.242552 .56490 \quad 1.89280$ $1.23528 \quad 2.55448 \quad 1.89179$ $1.22806 \quad 2.544111 .89077$ $1.220892 .53381 \quad 1.88974$

$\begin{array}{llll}1.21378 & 2.52357 & 1.88871\end{array}$ 1.206732 .513391 .88768 $1.19973 \quad 2.50327 \quad 1.88664$ $1.192792 .49321 \quad 1.88559$ $1.18590 \quad 2.48321 \quad 1.88454$

$\begin{array}{llll}1.17906 & 2.47327 & 1.88349\end{array}$ $\begin{array}{llll}1.17228 & 2.46339 & 1.88243\end{array}$ $1.165542 .45356 \quad 1.88137$ $1.15886 \quad 2.44379 \quad 1.88030$ $1.152232 .43408 \quad 1.87922$

$1.14564 \quad 2.42442 \quad 1.87815$ 1.139112 .414811 .87706 $1.132632 .40526 \quad 1.87597$ $1.126192 .39577 \quad 1.87488$ $1.11980 \quad 2.38632 \quad 1.87378$

$1.11346 \quad 2.37693 \quad 1.87268$ $1.107172 .36760 \quad 1.87157$ $1.10092 \quad 2.358311 .87046$ 1.094722 .349081 .86935 $1.08856 \quad 2.33989 \quad 1.86822$

$\begin{array}{llll}1.08245 & 2.33076 \quad 1.86710\end{array}$ $\begin{array}{llll}1.07638 & 2.32168 & 1.86597\end{array}$ $1.07036 \quad 2.312651 .86483$ $1.06438 \quad 2.30366 \quad 1.86369$ 1.058442 .294731 .86255
1600

1610

1620

1630

1640

1650

1660

1670

1680

1690

1700

1710

1720

1730

1740

1750

1760

1770

1780

1790

1800

1810

1820

1830

1840

1850

1860

1870

1880

1890

1900

1910

1920

1930

1940

1950

1960

1970

1980

1990

2000

2010

2020

2030

2040

2050 2060 2070 2080 2090 
Table II Harmonic Oscillator Contributions to the Thermodynamic

Functions (in units of calories, moles, and ${ }^{\circ} \mathrm{K}$ )
$\nu_{-1}-\left(\mathrm{F}^{\circ}-\mathrm{E}_{\mathrm{O}}^{\circ}\right) / \mathrm{T} \quad$ So
$\mathrm{Co}_{\mathrm{p}}^{\mathrm{O}} \quad-\left(\mathrm{F}^{\circ}-\mathrm{EO}\right) / \mathrm{T} \quad \mathrm{S}^{\circ}$
$\mathrm{C}_{\mathrm{p}}^{\circ} \quad-\left(\mathrm{F}^{\circ}-\mathrm{E}_{\mathrm{O}}^{\circ}\right) / \mathrm{T}$
So
$\mathrm{C}_{\mathrm{p}}^{\circ}$
$\nu$
$\mathrm{cm}^{-1}$

$\mathrm{T}=3200$.

2100

2110

2120

2130

2140

2150

2160

2170

2180

2190

2200

2210

2220

2230

2240

2250

2260

2270

2280

2290

2300

2310

2320

2330

2340

2350

2360

2370

2380

2390

2400

2410

2420

2430

2440

2450

2460

2470

2480

2490

2500

2510

2520

2530

2540

2550

2560

2570

2580

2590
$0.97895 \quad 2.17345 \quad 1.84589$

$0.97328 \quad 2.16469 \quad 1.84460$

$0.96766 \quad 2.15597 \quad 1.84330$

$0.962072 .14730 \quad 1.84201$

$0.956532 .13867 \quad 1.84070$

$0.95102 \quad 2.13009 \quad 1.83939$

$0.94556 \quad 2.12156 \quad 1.83808$

0.940132 .113071 .83676

$0.934752 .10463 \quad 1.83543$

0.929402 .096241 .83410

$\begin{array}{llll}0.92409 & 2.08788 & 1.83277\end{array}$

$0.91882 \quad 2.07957 \quad 1.83143$

$0.913592 .07131 \quad 1.83008$

$0.908392 .06309 \quad 1.82873$

$0.90323 \quad 2.05491 \quad 1.82738$

$0.89811 \quad 2.04677 \quad 1.82602$

$0.89302 \quad 2.03868 \quad 1.82466$

0.887972 .030621 .82329

$0.88295 \quad 2.02261 \quad 1.82192$

0.877972 .014641 .82054

$0.87303 \quad 2.00671 \quad 1.81916$

$\begin{array}{llll}0.86811 & 1.99882 & 1.81777\end{array}$

$0.86324 \quad 1.99097 \quad 1.81638$

$0.858391 .98316 \quad 1.81498$

$0.85358 \quad 1.97539 \quad 1.81358$

$0.84880 \quad 1.96766 \quad 1.81217$ $0.84406 \quad 1.959971 .81076$

0.839351 .952321 .80934

$0.83467 \quad 1.94470 \quad 1.80792$

0.830021 .937121 .80650

$0.82540 \quad 1.92958 \quad 1.80507$

$0.820821 .92208 \quad 1.80363$

0.816261 .914621 .80219

$0.81174 \quad 1.907191 .80075$

$0.807251 .89980 \quad 1.79930$

$0.80278 \quad 1.89244 \quad 1.79785$

$0.79835 \quad 1.88512 \quad 1.79639$

$0.79395 \quad 1.87783 \quad 1.79493$

$0.78958 \quad 1.870591 .79346$

0.785231 .863371 .79199

$0.78092 \quad 1.85619 \quad 1.79052$

$0.77663 \quad 1.84905 \quad 1.78904$

$0.77237 \quad 1.84194 \quad 1.78756$

$0.76814 \quad 1.83486 \quad 1.78607$

$0.76394 \quad 1.82782 \quad 1.78457$

$\begin{array}{llll}0.75976 & 1.82081 & 1.78308\end{array}$

$0.75562 \quad 1.81383 \quad 1.78157$

$\begin{array}{llll}0.75150 & 1.80689 & 1.78007\end{array}$

$\begin{array}{llll}0.74740 & 1.79998 & 1.77856\end{array}$

$0.74334 \quad 1.79310 \quad 1.77704$
$T=3300$.

$T=3400$.

$\begin{array}{llll}1.01601 & 2.23038 & 1.85398\end{array}$

$1.010252 .22157 \quad 1.85276$

$1.004532 .21282 \quad 1.85153$

$0.99885 \quad 2.20411 \quad 1.85031$

$0.993212 .19544 \quad 1.84907$

$0.98762 \quad 2.18683 \quad 1.84783$

$0.98206 \quad 2.17825 \quad 1.84659$

$0.976542 .16973 \quad 1.84534$

$0.97106 \quad 2.16125 \quad 1.84409$

$0.96562 \quad 2.15281 \quad 1.84283$

$0.96022 \quad 2.14442 \quad 1.84157$

$0.95486 \quad 2.13607 \quad 1.84030$

$0.949532 .12776 \quad 1.83903$

$0.944242 .11950 \quad 1.83776$

$0.938992 .11128 \quad 1.83648$

0.933772 .103101 .83519

$\begin{array}{llll}0.92859 & 2.09497 & 1.83390\end{array}$

$0.92345 \quad 2.08687 \quad 1.83261$

$0.91834 \quad 2.07882 \quad 1.83131$

0.913272 .070811 .83000

$0.90823 \quad 2.06284 \quad 1.82869$

$0.903232 .05491 \quad 1.82738$

$0.89826 \quad 2.04702 \quad 1.82606$

$0.89333 \quad 2.03916 \quad 1.82474$

0.888432 .031351 .82341

$\begin{array}{llll}0.88356 & 2.02358 & 1.82208\end{array}$

$0.87873 \quad 2.01585 \quad 1.82075$

$0.87392 \quad 2.008151 .81941$

$0.869152 .00049 \quad 1.81806$

$0.864421 .99287 \quad 1.81671$

$0.85971 \quad 1.98529 \quad 1.81536$

$0.85504 \quad 1.97774 \quad 1.81400$

$0.850391 .97024 \quad 1.81264$

$0.84578 \quad 1.96276 \quad 1.81127$

0.841201 .955331 .80990

$0.83665 \quad 1.94793 \quad 1.80853$

$0.832131 .94056 \quad 1.80715$

$0.82764 \quad 1.93324 \quad 1.80576$

$\begin{array}{llll}0.82318 & 1.92594 & 1.80437\end{array}$

$0.81874 \quad 1.91868 \quad 1.80298$

$0.81434 \quad 1.91146 \quad 1.80158$

$0.80997 \quad 1.90427 \quad 1.80018$

$0.80562 \quad 1.89712 \quad 1.79877$

$0.801301 .89000 \quad 1.79736$

$0.79701 \quad 1.88291 \quad 1.79595$

$0.79275 \quad 1.87585 \quad 1.79453$

$0.78852 \quad 1.86883 \quad 1.79311$

$0.78431 \quad 1.86185 \quad 1.79168$

$0.78013 \quad 1.854891 .79025$

$0.77598 \quad 1.84797 \quad 1.78881$
$1.05255 \quad 2.28584 \quad 1.86140$

$1.04670 \quad 2.27700 \quad 1.86024$

$\begin{array}{lll}1.04089 & 2.26820 & 1.85909\end{array}$

$1.03512 \quad 2.25946 \quad 1.85792$

$1.029392 .25076 \quad 1.85675$

$\begin{array}{llll}1.02370 & 2.24211 & 1.85558\end{array}$ $1.01806 \quad 2.23350 \quad 1.85441$

1.012452 .224941 .85322

$1.00688 \quad 2.21642 \quad 1.85204$

$1.00135 \quad 2.20794 \quad 1.85085$

$0.99586 \quad 2.19951 \quad 1.84965$ 0.990412 .191131 .84845 $0.98500 \quad 2.18279 \quad 1.84725$ $0.97962 \quad 2.17449 \quad 1.84604$ $0.97428 \quad 2.166231 .84483$

$0.96898 \quad 2.15801 \quad 1.84361$ $0.963712 .14984 \quad 1.84239$ $0.95848 \quad 2.14171 \quad 1.84116$ $0.953292 .13362 \quad 1.83993$ $0.948132 .12557 \quad 1.83870$

$0.94300 \quad 2.11756 \quad 1.83746$ $0.937912 .10959 \quad 1.83621$ $0.93286 \quad 2.10166 \quad 1.83496$ $0.92784 \quad 2.09377 \quad 1.83371$ $0.922852 .08592 \quad 1.83245$

$0.91790 \quad 2.078111 .83119$ 0.912972 .070341 .82993 0.908092 .062601 .82866 $0.90323 \quad 2.05491 \quad 1.82738$ 0.898412 .047251 .82610

$0.89362 \quad 2.039631 .82482$ 0.888862 .032041 .82353 0.884132 .024491 .82224 $0.879432 .01698 \quad 1.82094$ 0.874772 .009511 .81964

0.870132 .002071 .81834 0.865531 .994661 .81703 $0.860951 .98729 \quad 1.81572$ $0.856411 .97996 \quad 1.81440$ $0.851891 .97266 \quad 1.81308$

$0.84741 \quad 1.96540 \quad 1.81176$ $0.84295 \quad 1.95817 \quad 1.81043$ 0.838521 .950971 .80909 $0.834121 .94381 \quad 1.80776$ $0.82975 \quad 1.93668 \quad 1.80641$

$0.82540 \quad 1.92958 \quad 1.80507$ 0.821091 .922521 .80372 0.816801 .915491 .80236 $0.812541 .90850 \quad 1.80101$ 0.808301 .901531 .79964
2100

2110

2120

2130

2140

2150

2160

2170

2180

2190

2200

2210

2220

2230

2240

2250

2260

2270

2280

2290

2300

2310

2320

2330

2340

2350

2360

2370

2380

2390

2400

2410

2420

2430

2440

2450

2460

2470

2480

2490

2500

2510

2520

2530

2540

2550

2560

2570

2580

2590 
Table II Harmonic Oscillator Contributions to the Thermodynamic

Functions (in units of calories, moles, and ${ }^{\circ} \mathrm{K}$ )
$\mathrm{Cm}^{-1}-\left(\mathrm{F}^{\circ}-\mathrm{E}_{\mathrm{O}}^{\circ}\right) / \mathrm{T} \quad \mathrm{S}^{\circ}$
co
$-\left(F^{\circ}-E_{O}^{\circ}\right) / T$
so
$\mathrm{C}_{\mathrm{p}}$
$-\left(F^{\circ}-E_{O}^{\circ}\right) / T$
So
$\mathrm{T}=3200$.
$T=3300$.
$T=3400$

$\mathrm{cm}^{-1}$

2600

2610

2620

2630

2640

2650

2660

2670

2680

2690

2700

2710

2720

2730

2740

2750

2760

2770

2780

2790

2800

2810

2820

2830

2840

2850

2860

2870

2880

2890

2900

2910

2920

2930

2940

2950

2960

2970

2980

2990

3000

3010

3020

3030

3040

3050

3060

3070

3080

3090 $\begin{array}{llll}0.73930 & 1.78626 & 1.77552\end{array}$ $0.73528 \quad 1.77944 \quad 1.77400$ $\begin{array}{llll}0.73129 & 1.77266 & 1.77247\end{array}$ $\begin{array}{llll}0.72733 & 1.76591 & 1.77094\end{array}$ 0.723401 .759201 .76941

$\begin{array}{llll}0.71949 & 1.75251 & 1.76787\end{array}$ $\begin{array}{llll}0.71560 & 1.74585 & 1.76632\end{array}$ $\begin{array}{llll}0.71174 & 1.73923 & 1.76477\end{array}$ 0.707901 .732631 .76322 $0.70409 \quad 1.72607 \quad 1.76166$

$0.70031 \quad 1.71954 \quad 1.76010$ $0.696541 .71303 \quad 1.75854$ $0.692801 .70656 \quad 1.75697$ 0.689091 .700111 .75539 $0.685401 .69370 \quad 1.75382$

$0.68173 \quad 1.68731 \quad 1.75223$ $0.67808 \quad 1.680951 .75065$ $0.67446 \quad 1.67463 \quad 1.74906$ $0.67086 \quad 1.66833 \quad 1.74747$ $\begin{array}{llll}0.66729 & 1.66205 & 1.74587\end{array}$

$\begin{array}{llll}0.66373 & 1.65581 & 1.74427\end{array}$ $0.66020 \quad 1.64959 \quad 1.74266$ $0.656691 .64341 \quad 1.74105$ $0.65320 \quad 1.63725 \quad 1.73944$ $0.649731 .63111 \quad 1.73782$

$\begin{array}{llll}0.64629 & 1.62501 & 1.73620\end{array}$ $0.64287 \quad 1.61893 \quad 1.73457$ $\begin{array}{llll}0.63946 & 1.61288 & 1.73294\end{array}$ 0.636081 .606851 .73131 $0.632721 .60086 \quad 1.72967$

$\begin{array}{llll}0.62938 & 1.59488 & 1.72803\end{array}$ $0.62606 \quad 1.58894 \quad 1.72639$ $0.622771 .58302 \quad 1.72474$ $0.61949 \quad 1.57712 \quad 1.72309$ $0.616231 .57126 \quad 1.72143$

$\begin{array}{llll}0.61299 & 1.56541 & 1.71977\end{array}$ 0.609771 .559601 .71811 0.606571 .553811 .71644 $0.603391 .54804 \quad 1.71477$ 0.600231 .542301 .71310

$0.59709 \quad 1.53658 \quad 1.71142$ $0.59397 \quad 1.53089 \quad 1.70974$ $0.59087 \quad 1.52522 \quad 1.70805$ $\begin{array}{llll}0.58778 & 1.51958 & 1.70637\end{array}$ $0.584711 .51396 \quad 1.70467$

$0.58167 \quad 1.50836 \quad 1.70298$ $0.57864 \quad 1.50279 \quad 1.70128$ $0.575631 .49724 \quad 1.69958$ $0.57263 \quad 1.49172 \quad 1.69787$ $0.56966 \quad 1.48622 \quad 1.69616$ $\begin{array}{llll}0.77186 & 1.84108 \quad 1.78737\end{array}$ $0.76776 \quad 1.83422 \quad 1.78593$ $\begin{array}{llll}0.76368 & 1.82739 & 1.78448\end{array}$ $0.75964 \quad 1.82060 \quad 1.78303$ $\begin{array}{lll}0.75562 & 1.81383 & 1.78157\end{array}$

$0.75162 \quad 1.80710 \quad 1.78011$ $0.74765 \quad 1.80040 \quad 1.77865$ $0.74370 \quad 1.79373 \quad 1.77718$ $0.73978 \quad 1.78708 \quad 1.77571$ $\begin{array}{llll}0.73589 & 1.78047 & 1.77423\end{array}$

$0.73202 \quad 1.77389 \quad 1.77275$ $0.72817 \quad 1.76734 \quad 1.77127$ $0.724351 .76082 \quad 1.76978$ $0.720551 .75433 \quad 1.76829$ $\begin{array}{lll}0.71678 & 1.74787 & 1.76679\end{array}$

0.713021 .741431 .76529 $0.709301 .73503 \quad 1.76379$ $\begin{array}{llll}0.70559 & 1.72865 & 1.76228\end{array}$ $0.70191 \quad 1.72230 \quad 1.76076$ $0.698251 .71598 \quad 1.75925$

$0.69461 \quad 1.70969 \quad 1.75773$ 0.691001 .703431 .75620 $0.68741 \quad 1.69719 \quad 1.75468$ $0.68384 \quad 1.69098 \quad 1.75315$ 0.680291 .684801 .75161

$\begin{array}{llll}0.67676 & 1.67865 & 1.75007\end{array}$ $0.67326 \quad 1.67252 \quad 1.74853$ $0.66978 \quad 1.66642 \quad 1.74698$ $0.66631 \quad 1.66035 \quad 1.74543$ $0.66287 \quad 1.65430 \quad 1.74388$

$\begin{array}{llll}0.65945 & 1.64828 & 1.74232\end{array}$ $0.65605 \quad 1.64228 \quad 1.74076$ 0.652671 .636321 .73919 $0.64932 \quad 1.63037 \quad 1.73762$ $0.64598 \quad 1.62445 \quad 1.73605$

$0.64266 \quad 1.61856 \quad 1.73447$ 0.639361 .612701 .73289 $0.63608 \quad 1.60685 \quad 1.73131$ $0.63282 \quad 1.60104 \quad 1.72972$ $0.629581 .59524 \quad 1.72813$

$0.62636 \quad 1.58948 \quad 1.72654$ $0.62316 \quad 1.58373 \quad 1.72494$ $0.61998 \quad 1.57802 \quad 1.72334$ 0.616821 .572321 .72173 0.613681 .566651 .72013

$0.61055 \quad 1.56100 \quad 1.71851$ $0.60744 \quad 1.55538 \quad 1.71690$ $0.60435 \quad 1.54978 \quad 1.71528$ $0.60128 \quad 1.54421 \quad 1.71366$ $0.59823 \quad 1.53866 \quad 1.71203$
$0.80409 \quad 1.89460 \quad 1.79828$ $0.799911 .88770 \quad 1.79691$ $0.79576 \quad 1.88083 \quad 1.79553$ $0.791631 .87399 \quad 1.79416$ $0.78753 \quad 1.86719 \quad 1.79277$

$0.78345 \quad 1.86041 \quad 1.79139$ $0.77940 \quad 1.85367 \quad 1.79000$ 0.775371 .846951 .78860 $0.771371 .84027 \quad 1.78721$ $0.76740 \quad 1.833611 .78580$

$0.76345 \quad 1.82699 \quad 1.78440$ $0.75952 \quad 1.82040 \quad 1.78299$ $0.75562 \quad 1.81383 \quad 1.78157$ $0.75174 \quad 1.80730 \quad 1.78016$ 0.747881 .800791 .77874

$\begin{array}{llll}0.74405 & 1.79431 & 1.77731\end{array}$ 0.740241 .787861 .77588 $0.73646 \quad 1.78144 \quad 1.77445$ $0.73270 \quad 1.77505 \quad 1.77301$ 0.728961 .768691 .77157

$0.72525 \quad 1.76235 \quad 1.77013$ $0.721551 .75604 \quad 1.76868$ $0.71788 \quad 1.74976 \quad 1.76723$ 0.714241 .743511 .76578 0.710611 .737291 .76432

$0.70700 \quad 1.73109 \quad 1.76285$ 0.703421 .724911 .76139 $0.699861 .71877 \quad 1.75992$ 0.696321 .712651 .75844 $0.692801 .70656 \quad 1.75697$

$0.68931 \quad 1.70049 \quad 1.75549$ 0.685831 .694451 .75400 $0.682371 .68844 \quad 1.75251$ 0.678941 .682451 .75102 0.675521 .676481 .74953

0.672131 .670551 .74803 0.668761 .664631 .74653 $0.665401 .65874 \quad 1.74502$ $0.66207 \quad 1.65288 \quad 1.74351$ 0.658751 .647041 .74200

0.655461 .641231 .74048 0.652181 .635441 .73896 $0.648921 .62967 \quad 1.73744$ 0.645681 .623931 .73591 0.642461 .618221 .73438

$0.63926 \quad 1.612521 .73285$ 0.636081 .606851 .73131 $0.632921 .60121 \quad 1.72977$ $0.629771 .59558 \quad 1.72823$ 0.626651 .589991 .72668
2600

2610

2620

2630

2640

2650

2660

2670

2680

2690

2700

2710

2720

2730

2740

2750

2760

2770

2780

2790

2800

2810

2820

2830

2840

2850

2860

2870

2880

2890

2900

2910

2920

2930

2940

2950

2960

2970

2980

2990

3000

3010

3020

3030

3040

3050

3060

3070

3080

3090 
Table II Harmonic Oscillator Contributions to the Thermodynamic

Functions (in units of calories, moles, and ${ }^{\circ} \mathrm{K}$ )
$\underset{\mathrm{cm}^{-I}}{\nu}-\left(\mathrm{F}^{\circ}-\mathrm{E}_{\mathrm{O}} \mathrm{O}\right) / \mathrm{T} \quad$ SO
$C_{p}^{\circ} \quad-\left(F^{\circ}-E_{0}^{\circ}\right) / T$
So
$\mathrm{C}_{\mathrm{p}}$
$-\left(F^{\circ}-E_{O}^{O}\right) / T$
So

$$
\mathrm{T}=3200 \text {. }
$$
$T=3300$.
$T=3400$.

$\stackrel{\mathrm{C}_{\mathrm{p}}^{\circ}}{v} \quad \mathrm{~cm}^{-1}$

3100

3110

3120

3130

3140

$\begin{array}{llll}3150 & 0.55218 & 1.45369 & 1.68583\end{array}$

3160

3170

3180

3190

3200

3210

3220

3230

3240

3250

3260

3270

3280

3290

3300

3310

3320

3330

3340

3350

3360

3370

3380

3390

3400

3410

3420

3430

3440

3450

3460

3470

3480

3490

3500

3510

3520

3530

3540

3550

3560

3570

3580

3590

$\begin{array}{llll}0.56670 & 1.48074 & 1.69445\end{array}$

$0.56376 \quad 1.47528 \quad 1.69273$

$0.56084 \quad 1.46985 \quad 1.69101$

$0.55793 \quad 1.46444 \quad 1.68929$

$0.55505 \quad 1.45906 \quad 1.68756$

$0.54932 \quad 1.448351 .68409$

$0.546491 .44304 \quad 1.68236$

$0.54367 \quad 1.43774 \quad 1.68062$

$\begin{array}{lll}0.54086 & 1.43247 & 1.67887\end{array}$

$\begin{array}{llll}0.53808 & 1.42721 & 1.67713\end{array}$

$\begin{array}{llll}0.53531 & 1.42198 & 1.67538\end{array}$

$0.53255 \quad 1.41678 \quad 1.67362$

$0.52981 \quad 1.41159 \quad 1.67186$

0.527091 .406421 .67010

$0.52439 \quad 1.40128 \quad 1.66834$

$\begin{array}{llll}0.52170 & 1.39616 & 1.66657\end{array}$

$0.51902 \quad 1.39106 \quad 1.66480$

$0.51636 \quad 1.38597 \quad 1.66303$

$0.513721 .38091 \quad 1.66126$

$\begin{array}{llll}0.51109 & 1.37588 & 1.65948\end{array}$

$\begin{array}{llll}0.50848 & 1.37086 & 1.65769\end{array}$

$\begin{array}{llll}0.50588 & 1.36586 & 1.65591\end{array}$

$0.50330 \quad 1.36088 \quad 1.65412$

$\begin{array}{llll}0.50073 & 1.35592 & 1.65233\end{array}$

$\begin{array}{llll}0.49818 & 1.35099 & 1.65053\end{array}$

$0.49564 \quad 1.34607 \quad 1.64874$

$0.493111 .34117 \quad 1.64693$

$0.49060 \quad 1.33630 \quad 1.64513$

$0.48811 \quad 1.33144 \quad 1.64332$

$0.48563 \quad 1.32660 \quad 1.64151$ $\begin{array}{llll}0.48316 & 1.32178 & 1.63970\end{array}$ $0.48071 \quad 1.31698 \quad 1.63789$ $0.47827 \quad 1.31220 \quad 1.63607$ 0.475851 .307441 .63425

$0.47344 \quad 1.30270 \quad 1.63242$ 0.471041 .297981 .63060 $0.46866 \quad 1.293281 .62877$ 0.466291 .288591 .62693 0.463931 .283931 .62510

$\begin{array}{llll}0.46159 & 1.27928 & 1.62326\end{array}$ $0.45926 \quad 1.27465 \quad 1.62142$ $0.45694 \quad 1.27004 \quad 1.61957$ $0.454641 .26545 \quad 1.61773$ $0.45235 \quad 1.26088 \quad 1.61588$

$0.45007 \quad 1.25632 \quad 1.61403$ $\begin{array}{llll}0.44781 & 1.25178 & 1.61217\end{array}$ $0.44555 \quad 1.24726 \quad 1.61032$ $0.443321 .24276 \quad 1.60846$ $\begin{array}{llll}0.44109 & 1.23828 & 1.60659\end{array}$ $\begin{array}{llll}0.59520 & 1.53313 & 1.71040\end{array}$ $0.59218 \quad 1.52762 \quad 1.70877$ $0.58918 \quad 1.52214 \quad 1.70713$ $0.58620 \quad 1.51668 \quad 1.70549$ 0.583241 .511241 .70385

$\begin{array}{llll}0.58029 & 1.50582 & 1.70221\end{array}$

$0.57736 \quad 1.500431 .70056$ 0.574451 .495061 .69890 0.571551 .489711 .69725 $0.56867 \quad 1.484391 .69559$

$0.56581 \quad 1.47908 \quad 1.69393$ $0.56296 \quad 1.473801 .69226$ 0.560131 .468541 .69059 $0.557321 .46330 \quad 1.68892$ $0.55452 \quad 1.45808 \quad 1.68724$

$\begin{array}{llll}0.55174 & 1.45288 & 1.68557\end{array}$ $0.54898 \quad 1.44771 \quad 1.68388$ 0.546231 .442551 .68220 0.543501 .437421 .68051 $0.54078 \quad 1.43231 \quad 1.67882$

$0.53808 \quad 1.42721 \quad 1.67713$ $0.53539 \quad 1.42214 \quad 1.67543$ 0.532721 .417091 .67373 0.530061 .412061 .67202 0.527421 .407051 .67032

$0.52480 \quad 1.40206 \quad 1.66861$ 0.522181 .397091 .66690 $0.519591 .39214 \quad 1.66518$ 0.517011 .387201 .66346 0.514441 .382291 .66174

$0.51189 \quad 1.37740 \quad 1.66002$ 0.509351 .372531 .65829 0.506821 .367671 .65656 $0.50431 \quad 1.36284 \quad 1.65482$ 0.501821 .358031 .65309

0.499331 .353231 .65135 0.496871 .348451 .64961 0.494411 .343691 .64786 0.491971 .338951 .64612 0.489541 .334231 .64436

$0.48713 \quad 1.32953 \quad 1.64261$ $0.48473 \quad 1.32485 \quad 1.64086$ $0.48234 \quad 1.32018 \quad 1.63910$ $0.47997 \quad 1.31553 \quad 1.63734$ $\begin{array}{llll}0.47761 & 1.31090 & 1.63557\end{array}$

$\begin{array}{llll}0.47526 & 1.30629 & 1.63381\end{array}$ $0.472931 .30170 \quad 1.63204$ $0.47061 \quad 1.29712 \quad 1.63026$ $0.468301 .29257 \quad 1.62849$ 0.466001 .288031 .62671
$0.62354 \quad 1.58441 \quad 1.72513$ $0.62045 \quad 1.57886 \quad 1.72358$ $0.61738 \quad 1.57332 \quad 1.72202$ $0.61432 \quad 1.56782 \quad 1.72046$ $0.61128 \quad 1.56233 \quad 1.71889$

$\begin{array}{llll}0.60826 & 1.55687 & 1.71733\end{array}$ $0.60526 \quad 1.55143 \quad 1.71576$ $0.60227 \quad 1.54601 \quad 1.71418$ $0.59931 \quad 1.54061 \quad 1.71261$ $0.59635 \quad 1.53524 \quad 1.71103$

$\begin{array}{llll}0.59342 & 1.52988 & 1.70944\end{array}$ $0.59050 \quad 1.52455 \quad 1.70786$ $0.58760 \quad 1.51924 \quad 1.70627$ $\begin{array}{llll}0.58471 & 1.51396 & 1.70467\end{array}$ 0.581851 .508691 .70308

$\begin{array}{llll}0.57899 & 1.50344 & 1.70148\end{array}$ $0.57616 \quad 1.49822 \quad 1.69988$ $0.57334 \quad 1.49301 \quad 1.69827$ $0.57053 \quad 1.48783 \quad 1.69666$ $0.56774 \quad 1.48267 \quad 1.69505$

$0.56497 \quad 1.47753 \quad 1.69344$ $0.56221 \quad 1.47241 \quad 1.69182$ $0.559471 .46730 \quad 1.69020$ 0.556741 .462221 .68857 $0.554031 .45716 \quad 1.68695$

$0.55134 \quad 1.45212 \quad 1.68532$ 0.548651 .447101 .68369 0.545991 .442101 .68205 0.543341 .437121 .68041 $0.54070 \quad 1.43216 \quad 1.67877$

$0.53808 \quad 1.42721 \quad 1.67713$ $0.53547 \quad 1.422291 .67548$ $0.53288 \quad 1.41739 \quad 1.67383$ $0.53030 \quad 1.41250 \quad 1.67217$ 0.527731 .407641 .67052

$0.52518 \quad 1.40279 \quad 1.66886$ $0.522641 .39796 \quad 1.66720$ 0.520121 .393151 .66553 $0.517611 .38836 \quad 1.66387$ 0.515121 .383591 .66220

$0.512631 .37884 \quad 1.66052$ 0.510171 .374101 .65885 $0.50771 \quad 1.36939 \quad 1.65717$ $0.50527 \quad 1.364691 .65549$ 0.502841 .360011 .65380

$0.50043 \quad 1.35534 \quad 1.65212$ 0.498031 .350701 .65043 $0.49564 \quad 1.34607 \quad 1.64874$ $0.493261 .34146 \quad 1.64704$ $0.49090 \quad 1.33687 \quad 1.64534$
3100

3110

3120

3130

3140

3150

3160

3170

3180

3190

3200

3210

3220

3230

3240

3250

3260

3270

3280

3290

3300

3310

3320

3330

3340

3350

3360

3370

3380

3390

3400

3410

3420

3430

3440

3450

3460

3470

3480

3490

3500

3510

3520

3530

3540

3550

3560

3570

3580

3590 
Table II Harmonic Oscillator Contributions to the Thermodynamic

Functions (in units of calories, moles, and ${ }^{\circ} \mathrm{K}$ )
$\mathrm{cm}^{-1}$
$\mathrm{C}_{\mathrm{p}}^{\circ} \quad-\left(\mathrm{F}^{\circ}-\mathrm{E}_{\mathrm{O}}^{\circ}\right) / \mathrm{T} \quad \mathrm{S}^{\circ}$
$\mathrm{C}_{\mathrm{p}}$
$-\left(F^{\circ}-E_{O}^{\circ}\right) / T$
S०
$\mathrm{c}_{\mathrm{p}}^{\circ}$
$\nu$
$\mathrm{cm}^{-1}$

$T=3200$.

3600

3610

3620

3630

3640

3650

3660

3670

3680

3690

3700

3710

3720

3730

3740

3750

3760

3770

3780

3790

3800

3810

3820

3830

3840

3850

3860

3870

3880

3890

3900

3910

3920

3930

3940

3950

3960

3970

3980

3990

4000
$0.43887 \quad 1.23381 \quad 1.60473$ $0.436671 .22936 \quad 1.60286$ $0.43448 \quad 1.22493 \quad 1.60099$ 0.432301 .220521 .59912 0.430141 .216121 .59724

$0.42799 \quad 1.21174 \quad 1.59536$ 0.425841 .207381 .59348 0.423721 .203031 .59160 0.421601 .198701 .58971 0.419491 .194391 .58783

$0.41740 \quad 1.19010 \quad 1.58594$ $0.415321 .18582 \quad 1.58404$ $0.413241 .18156 \quad 1.58215$ 0.411181 .177311 .58025 0.409141 .173091 .57835

$0.40710 \quad 1.16887 \quad 1.57645$ $0.40507 \quad 1.16468 \quad 1.57454$ $0.40306 \quad 1.160501 .57264$ $0.40106 \quad 1.15634 \quad 1.57073$ 0.399061 .152191 .56882

$0.39708 \quad 1.14806 \quad 1.56690$ $0.39511 \quad 1.14394 \quad 1.56499$ $0.39315 \quad 1.13984 \quad 1.56307$ $0.391201 .13576 \quad 1.56115$ 0.389261 .131691 .55922

0.387331 .127641 .55730 $0.385421 .12360 \quad 1.55537$ $0.38351 \quad 1.11958 \quad 1.55344$ 0.381611 .115571 .55151 $0.379731 .11158 \quad 1.54958$

$0.37785 \quad 1.10760 \quad 1.54764$ $0.37598 \quad 1.10364 \quad 1.54571$ $0.374131 .09970 \quad 1.54377$ $0.372281 .09577 \quad 1.54182$ 0.370451 .091851 .53988

$0.368621 .08795 \quad 1.53793$ $0.366801 .08406 \quad 1.53599$ $0.36500 \quad 1.08019 \quad 1.53404$ $0.363201 .07634 \quad 1.53209$ 0.361411 .072491 .53013

$0.35964 \quad 1.06867 \quad 1.52818$
$T=3300$.

$0.46372 \quad 1.28350 \quad 1.62493$ 0.461451 .279001 .62315 $0.459191 .27451 \quad 1.62136$ $0.45694 \quad 1.27004 \quad 1.61957$ 0.454711 .265591 .61778

0.452491 .261151 .61599 $0.450281 .25673 \quad 1.61420$ 0.448081 .252331 .61240 0.445891 .247951 .61060 $0.44372 \quad 1.24358 \quad 1.60879$

0.441561 .239231 .60699 $0.43941 \quad 1.23489 \quad 1.60518$ $0.43727 \quad 1.23057 \quad 1.60337$ $0.435141 .22627 \quad 1.60156$ 0.433031 .221991 .59974

$0.430931 .21772 \quad 1.59792$ 0.428831 .213461 .59610 0.426751 .209231 .59428 $0.424681 .20501 \quad 1.59246$ 0.422621 .200801 .59063

$0.42058 \quad 1.196611 .58880$ $0.41854 \quad 1.19244 \quad 1.58697$ $0.41651 \quad 1.188281 .58513$ 0.414501 .184141 .58330 0.412491 .180011 .58146

$0.410501 .17590 \quad 1.57962$ $0.408521 .17181 \quad 1.57777$ $0.406551 .16773 \quad 1.57593$ 0.404581 .163661 .57408 0.402631 .159611 .57223

$0.400691 .15558 \quad 1.57038$ $0.39876 \quad 1.15156 \quad 1.56853$ $0.39684 \quad 1.14756 \quad 1.56667$ $0.394931 .14357 \quad 1.56481$ 0.393031 .139591 .56295

$0.39114 \quad 1.135631 .56109$ 0.389261 .131691 .55922 $0.387391 .12776 \quad 1.55736$ 0.385531 .123841 .55549 $0.383681 .11994 \quad 1.55362$

$0.38184 \quad 1.11606 \quad 1.55175$
$T=3400$.

$0.48855 \quad 1.33229 \quad 1.64364$ 0.486211 .327741 .64194 $0.483891 .32320 \quad 1.64024$ $0.48157 \quad 1.318681 .63853$ 0.479271 .314171 .63682

$0.476991 .30968 \quad 1.63510$ $0.47471 \quad 1.305211 .63339$ $0.472451 .30076 \quad 1.63167$ 0.470201 .296321 .62995 0.467961 .291901 .62823

$0.46573 \quad 1.28749 \quad 1.62650$ $0.46352 \quad 1.283111 .62477$ 0.461311 .278731 .62304 0.459121 .274381 .62131 $0.45694 \quad 1.27004 \quad 1.61957$

$0.45477 \quad 1.26572 \quad 1.61784$ 0.452621 .261411 .61610 $0.45047 \quad 1.257121 .61435$ $0.44834 \quad 1.25285 \quad 1.61261$

0.446221 .248591 .61086

0.444101 .244351 .60911 0.442001 .240121 .60736 0.439911 .235911 .60561 $0.43784 \quad 1.23172 \quad 1.60385$ $0.43577 \quad 1.22754 \quad 1.60209$

$\begin{array}{llll}0.43371 & 1.22337 & 1.60033\end{array}$ $0.43167 \quad 1.21922 \quad 1.59856$ 0.429631 .215091 .59680 0.427611 .210971 .59503 $0.42559 \quad 1.20687 \quad 1.59326$

$0.42359 \quad 1.20278 \quad 1.59149$ $0.421601 .19870 \quad 1.58971$ 0.419621 .194651 .58794 0.417641 .190601 .58616 $0.415681 .18657 \quad 1.58438$

$0.413731 .18256 \quad 1.58259$ 0.411791 .178561 .58081 0.409861 .174581 .57902 0.407941 .170611 .57723 0.406031 .166651 .57544

$0.404121 .16271 \quad 1.57365$
3600

3610

3620

3630

3640

3650

3660

3670

3680

3690

3700

3710

3720

3730

3740

3750

3760

3770

3780

3790

3800

3810

3820

3830

3840

3850

3860

3870

3880

3890

3900

3910

3920

3930

3940

3950 3960 3970 3980 3990

4000 
Table II Harmonic Oscillator Contributions to the Thermodynamic

Functions (in units of calories, moles, and ${ }^{\circ} \mathrm{K}$ )
$\mathrm{cm}^{-1}-\left(\mathrm{F}^{\circ}-\mathrm{E}_{\mathrm{O}}^{\circ}\right) / \mathrm{T} \quad \mathrm{S}^{\circ}$
$C_{p}^{\circ} \quad-(F \circ-E O) / T$
so
$\underset{p}{c o}$
$-\left(F^{\circ}-E_{O}^{\circ}\right) / T$
So
$\mathrm{C}_{\mathrm{p}}^{\circ}$

$\mathrm{cm}^{-1}$

$\mathrm{T}=3500$.

$T=3600$.

$T=3700$.

$100 \quad 6.38283 \quad 8.32944 \quad 1.98689$

$\begin{array}{lllll}110 & 6.19749 & 8.14007 & 1.98683\end{array}$

$\begin{array}{llll}120 & 6.02864 & 7.96720 \quad 1.98677\end{array}$

$130 \quad 5.873637 .80817 \quad 1.98670$

$140 \quad 5.730417 .660951 .98662$

$\begin{array}{llll}150 \quad 5.59735 & 7.52389 & 1.98654\end{array}$

$160 \quad 5.47315 \quad 7.39568 \quad 1.98645$

$170 \quad 5.356717 .27525 \quad 1.98636$

$180 \quad 5.247167 .161721 .98626$

$190 \quad 5.14376 \quad 7.054331 .98616$

$200 \quad 5.04586 \quad 6.95246 \quad 1.98605$

$210 \quad 4.95293 \quad 6.85556 \quad 1.98594$

$220 \quad 4.864516 .76318 \quad 1.98582$

$\begin{array}{lllll}230 & 4.78020 & 6.67491 & 1.98569\end{array}$

$240 \quad 4.699656 .59040 \quad 1.98556$

$250 \quad 4.62254 \quad 6.50935 \quad 1.98542$

$260 \quad 4.54862 \quad 6.43148 \quad 1.98528$

$270 \quad 4.47763 \quad 6.35656 \quad 1.98513$

$280 \quad 4.409376 .28437 \quad 1.98498$

$290 \quad 4.343646 .21471 \quad 1.98482$

$300 \quad 4.28028 \quad 6.14743 \quad 1.98465$

$310 \quad 4.219126 .08236 \quad 1.98448$

$320 \quad 4.160026 .01935 \quad 1.98431$

$330 \quad 4.10287 \quad 5.95830 \quad 1.98413$

$\begin{array}{lll}340 \quad 4.04754 & 5.89907 & 1.98394\end{array}$

$\begin{array}{llll}350 & 3.99392 & 5.84156 \quad 1.98375\end{array}$

$360 \quad 3.94192 \quad 5.78568 \quad 1.98355$

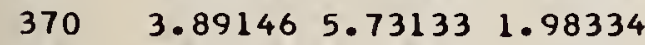

$380 \quad 3.84245 \quad 5.67844 \quad 1.98313$

$390 \quad 3.794815 .62693 \quad 1.98292$

$\begin{array}{lllll}400 & 3.74847 & 5.57673 & 1.98270\end{array}$

$\begin{array}{lllll}410 & 3.70337 & 5.52778 & 1.98247\end{array}$

$420 \quad 3.65945 \quad 5.48001 \quad 1.98224$

$430 \quad 3.616665 .433371 .98200$

$440 \quad 3.57494 \quad 5.38781 \quad 1.98176$

$\begin{array}{lllll}450 & 3.53424 & 5.34327 & 1.98151\end{array}$

$460 \quad 3.494525 .299721 .98126$

$\begin{array}{ll}470 \quad 3.45574 & 5.257121 .98100\end{array}$

$\begin{array}{lllll}480 & 3.41786 & 5.21541 & 1.98073\end{array}$

$490 \quad 3.380835 .17458 \quad 1.98046$

$\begin{array}{lllll}500 & 3.34463 & 5.13457 & 1.98019\end{array}$

$510 \quad 3.309225 .095361 .97991$

$\begin{array}{lllll}520 & 3.27458 & 5.05691 & 1.97962\end{array}$

$530 \quad 3.24066 \quad 5.01921 \quad 1.97933$

$540 \quad 3.20745 \quad 4.98221$
1.97903

$550 \quad 3.17492 \quad 4.94590 \quad 1.97873$

$\begin{array}{lllll}560 & 3.14304 & 4.91025 & 1.97842\end{array}$

$570 \quad 3.111804 .87524 \quad 1.97810$

$580 \quad 3.08116 \quad 4.84084 \quad 1.97778$

590
$6.437698 .38541 \quad 1.98691$

$6.252238 .19604 \quad 1.98685$

$6.083278 .02317 \quad 1.98679$

5.928157 .864141 .98672

$5.784827 .71691 \quad 1.98665$

$\begin{array}{llll}5.65165 & 7.57985 & 1.98657\end{array}$

5.527337 .451641 .98649

$5.410797 .33121 \quad 1.98641$

5.301137 .217681 .98631

$5.19761 \quad 7.11028 \quad 1.98622$

$5.099607 .00841 \quad 1.98611$

$5.00656 \quad 6.91151 \quad 1.98600$

4.918036 .819121 .98589

4.833616 .730851 .98577

$4.75295 \quad 6.64634 \quad 1.98565$

$4.675736 .56528 \quad 1.98552$ $4.60170 \quad 6.48741 \quad 1.98538$ $4.530606 .41248 \quad 1.98524$ 4.462236 .340291 .98510 $4.39640 \quad 6.270631 .98495$

$4.33292 \quad 6.20334 \quad 1.98479$ 4.271656 .138261 .98463 $4.21245 \quad 6.07526 \quad 1.98446$ 4.155196 .014191 .98429 $4.09975 \quad 5.95496 \quad 1.98412$

$4.04602 \quad 5.89745 \quad 1.98393$ $3.993925 .84156 \quad 1.98375$ $3.94335 \quad 5.787211 .98355$ $3.894235 .73431 \quad 1.98335$ $3.84648 \quad 5.682801 .98315$

$3.80003 \quad 5.63259 \quad 1.98294$ 3.754835 .583631 .98273 $3.71080 \quad 5.53585 \quad 1.98251$ $3.66790 \quad 5.48921 \quad 1.98229$ $3.62608 \quad 5.443641 .98206$

$3.58527 \quad 5.39910 \quad 1.98182$ $3.54545 \quad 5.35554 \quad 1.98158$ $3.50656 \quad 5.312931 .98134$ 3.468575 .271221 .98109 $3.43144 \quad 5.23037 \quad 1.98083$

$3.39513 \quad 5.19036 \quad 1.98057$ 3.359625 .151141 .98030 $3.32486 \quad 5.112691 .98003$ 3.290845 .074971 .97976 $3.25753 \quad 5.03797 \quad 1.97947$

3.224895 .001651 .97919 $3.192914 .96599 \quad 1.97890$ $3.16156 \quad 4.93097 \quad 1.97860$ $3.130824 .89656 \quad 1.97830$ 3.100674 .862751 .97799
$6.49107 \quad 8.439851 .98692$

$6.305518 .25048 \quad 1.98687$

$6.136448 .07760 \quad 1.98681$

$5.981217 .91858 \quad 1.98675$

$5.83778 \quad 7.77134 \quad 1.98668$

$5.70450 \quad 7.63428 \quad 1.98661$

$5.580087 .50607 \quad 1.98653$

5.463437 .385641 .98645

5.353677 .272101 .98636

$5.25004 \quad 7.16471 \quad 1.98627$

5.151937 .062831 .98617

$\begin{array}{llll}5.05879 & 6.96592 & 1.98607\end{array}$

4.970156 .873531 .98596

$4.885636 .78526 \quad 1.98585$

$4.80486 \quad 6.70074 \quad 1.98573$

$4.72754 \quad 6.61968 \quad 1.98561$ $4.65340 \quad 6.541811 .98548$ $4.58220 \quad 6.46688 \quad 1.98535$ $4.513736 .39468 \quad 1.98521$

4.447796 .325021 .98507

4.384216 .257721 .98492 4.322846 .192641 .98477 4.263536 .129631 .98461 $4.206176 .06856 \quad 1.98444$ 4.150636 .009321 .98428

$4.09680 \quad 5.95181 \quad 1.98411$ $4.044595 .89591 \quad 1.98393$ $3.99392 \quad 5.84156 \quad 1.98375$ $3.94470 \quad 5.78866 \quad 1.98356$ $3.89685 \quad 5.73714 \quad 1.98337$

$3.85030 \quad 5.68693 \quad 1.98317$ $3.80499 \quad 5.63796 \quad 1.98297$ $3.760875 .59018 \quad 1.98276$ 3.717875 .543521 .98255 3.675945 .497951 .98233

$3.635035 .45340 \quad 1.98211$ $3.59510 \quad 5.40984 \quad 1.98188$ 3. 556125.367221 .98165 3.518025 .325501 .98141 3.480795 .284651 .98117

3.444395 .244631 .98092 $3.408775 .20540 \quad 1.98067$ $3.373925 .16694 \quad 1.98041$ 3.339805 .129221 .98015 $3.306395 .09221 \quad 1.97988$

$3.27365 \quad 5.055891 .97961$ 3.241575 .020221 .97934 3.210124 .985191 .97905 $3.17928 \quad 4.95077 \quad 1.97877$ $3.149034 .91695 \quad 1.97848$
100

110

120

130

140

150

160

170

180

190

200

210

220

230

240

250

260

270

280

290

300

310

320

330

340

350

360

370

380

390

400

410

420

430

440

450

460

470

480

490

500

510

520

530

540

550

560

570

580

590 
Table II Harmonic Oscillator Contributions to the Thermodynamic

Functions (in units of calories, moles, and ${ }^{\circ} \mathrm{K}$ )
$\mathrm{cm}^{-1}-\left(F^{\circ}-E_{0}^{\circ}\right) / T$
So
$\begin{array}{cc}c_{p}^{\circ} & -\left(F^{\circ}-E_{O}^{\circ}\right) / T\end{array}$
$\mathrm{c}_{\mathrm{p}}$
$-\left(F^{\circ}-E_{0}^{\circ}\right) / T$
So
$T=3500$.
$T=3600$.
$T=3700$.

$\mathrm{C}_{\mathrm{p}}^{\circ}$

$v$

$\mathrm{cm}^{-1}$

$600 \quad 3.02163 \quad 4.77380 \quad 1.97713$ $\begin{array}{lll}610 & 2.99270 \quad 4.74112 \quad 1.97679\end{array}$ $\begin{array}{lll}620 & 2.96430 \quad 4.70898 & 1.97645\end{array}$ $\begin{array}{lll}630 & 2.93642 \quad 4.67736 \quad 1.97610\end{array}$ $640 \quad 2.90903 \quad 4.646241 .97575$

$650 \quad 2.882124 .61561 \quad 1.97539$ $\begin{array}{ll}660 \quad 2.85569 & 4.58546 \quad 1.97502\end{array}$ $\begin{array}{llll}670 \quad 2.82970 & 4.55576 & 1.97466\end{array}$ $\begin{array}{ll}680 \quad 2.80416 \quad 4.52651 & 1.97428\end{array}$ $690 \quad 2.779044 .49769 \quad 1.97390$

$700 \quad 2.75434 \quad 4.46929 \quad 1.97351$ $710 \quad 2.73004 \quad 4.44130 \quad 1.97312$ $\begin{array}{ll}720 & 2.706134 .41370 \quad 1.97273\end{array}$

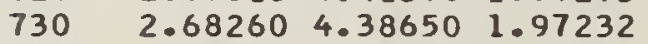
$740 \quad 2.65944 \quad 4.35966 \quad 1.97192$

$\begin{array}{lllll}750 & 2.63665 & 4.33320 & 1.97150\end{array}$ $\begin{array}{ll}760 \quad 2.61420 \quad 4.30709 & 1.97108\end{array}$ $770 \quad 2.592094 .281321 .97066$ $780 \quad 2.570324 .25590 \quad 1.97023$ $\begin{array}{llll}790 & 2.54887 & 4.23080 & 1.96980\end{array}$

$800 \quad 2.52774 \quad 4.20603 \quad 1.96936$ $810 \quad 2.506914 .18157 \quad 1.96891$ $820 \quad 2.486394 .15741 \quad 1.96846$ $830 \quad 2.466154 .133551 .96800$ $840 \quad 2.446214 .109991 .96754$

$850 \quad 2.42654 \quad 4.08670 \quad 1.96707$ $860 \quad 2.407144 .063701 .96660$ $870 \quad 2.388014 .04097 \quad 1.96612$ $880 \quad 2.369144 .018501 .96564$ $890 \quad 2.350523 .996291 .96515$

$900 \quad 2.33215 \quad 3.97434 \quad 1.96466$ $910 \quad 2.31403 \quad 3.952631 .96416$ $920 \quad 2.296143 .93117 \quad 1.96365$ $930 \quad 2.27848 \quad 3.90994 \quad 1.96314$ $940 \quad 2.26105 \quad 3.88895 \quad 1.96263$

$950 \quad 2.24384 \quad 3.86818 \quad 1.96211$ $960 \quad 2.22685 \quad 3.84764 \quad 1.96158$ $970 \quad 2.21008 \quad 3.82731 \quad 1.96105$ $980 \quad 2.19351 \quad 3.80720 \quad 1.96051$ $990 \quad 2.177143 .78730 \quad 1.95997$

$1000 \quad 2.16098 \quad 3.76761 \quad 1.95942$ $\begin{array}{lllll}1010 & 2.14501 & 3.74811 & 1.95887\end{array}$ $1020 \quad 2.12923 \quad 3.72881 \quad 1.95831$ $1030 \quad 2.11364 \quad 3.70971 \quad 1.95775$

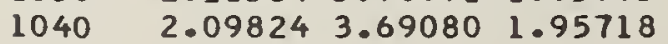

$\begin{array}{lllll}1050 & 2.08302 & 3.67207 & 1.95660\end{array}$ $\begin{array}{lllll}1060 & 2.06797 & 3.65353 & 1.95602\end{array}$ $\begin{array}{lllll}1070 \quad 2.05310 & 3.63517 & 1.95544\end{array}$ $1080 \quad 2.03840 \quad 3.61698 \quad 1.95485$ $1090 \quad 2.02386 \quad 3.59896 \quad 1.95425$
$3.07108 \quad 4.82950 \quad 1.97767$ 3.04205 4.79682 1.97736 $3.01354 \quad 4.76467 \quad 1.97703$ 2. $98555 \quad 4.73304 \quad 1.97670$ $2.95806 \quad 4.70191 \quad 1.97637$

$2.93105 \quad 4.67127 \quad 1.97603$ 2.904514 .641101 .97569 $2.878424 .61140 \quad 1.97534$ $2.85278 \quad 4.582131 .97498$ $2.82756 \quad 4.55330 \quad 1.97462$

$2.80275 \quad 4.52489 \quad 1.97426$ 2.778354 .496891 .97389 $2.75434 \quad 4.46929 \quad 1.97351$ 2.730714 .442071 .97313 2.707454 .415231 .97275

$2.68455 \quad 4.38875 \quad 1.97236$ $2.66200 \quad 4.36263 \quad 1.97196$ $2.63979 \quad 4.336851 .97156$ $2.61792 \quad 4.311411 .97115$ $2.596374 .28631 \quad 1.97074$

$2.575134 .26152 \quad 1.97033$ 2. 554204.237051 .96991 2. $53358 \quad 4.21288 \quad 1.96948$ $2.51324 \quad 4.18901 \quad 1.96905$ 2.493194 .165431 .96861

2.473434 .142131 .96817 2.453934 .119121 .96772 $2.43470 \quad 4.09637 \quad 1.96727$ 2.415734 .073891 .96681 $2.397014 .05167 \quad 1.96635$

$2.37854 \quad 4.02970 \quad 1.96588$ $2.36032 \quad 4.00798 \quad 1.96541$ $2.34233 \quad 3.98650 \quad 1.96493$ $2.32457 \quad 3.96526 \quad 1.96445$ 2. 307043.944251 .96396

$2.28974 \quad 3.92347 \quad 1.96347$ $2.27265 \quad 3.90292 \quad 1.96297$ $2.255773 .88258 \quad 1.96247$ $2.23910 \quad 3.86245 \quad 1.96196$ 2.222643 .842541 .96145

2. $20638 \quad 3.822831 .96093$ $2.19031 \quad 3.80332 \quad 1.96041$ $2.17443 \quad 3.784001 .95988$ $2.15875 \quad 3.76489 \quad 1.95934$ $2.143253 .74596 \quad 1.95881$

$2.12793 \quad 3.72722 \quad 1.95826$ $2.11278 \quad 3.70866 \quad 1.95771$ $2.097813 .69028 \quad 1.95716$ $2.08302 \quad 3.67207 \quad 1.95660$ $2.068393 .65404 \quad 1.95604$
$3.11934 \quad 4.88370 \quad 1.97818$ $3.09021 \quad 4.85100 \quad 1.97788$ $3.061614 .81884 \quad 1.97757$ $3.033524: 787201.97726$ $3.005934 .75607 \quad 1.97694$

$2.97882 \quad 4.72542 \quad 1.97662$ $2.95218 \quad 4.69524 \quad 1.97630$ $2.92600 \quad 4.66553 \quad 1.97597$ $2.900254 .63625 \quad 1.97563$ 2.874934 .607421 .97529

$2.85003 \quad 4.57900 \quad 1.97495$ $2.82553 \quad 4.55098 \quad 1.97459$ $2.801424 .52337 \quad 1.97424$ $2.777694 .49614 \quad 1.97388$ 2.754344 .469291 .97351

$2.73134 \quad 4.44280 \quad 1.97314$ $2.70870 \quad 4.41667 \quad 1.97277$ $2.686394 .39088 \quad 1.97239$ 2.664424 .365431 .97200 $2.642774 .34031 \quad 1.97162$

2.621444 .315521 .97122 2.600424 .291031 .97082 $2.57970 \quad 4.26685 \quad 1.97042$ $2.559274 .24297 \quad 1.97001$ $2.53912 \quad 4.21938 \quad 1.96959$

$2.51926 \quad 4.19607 \quad 1.96918$ 2.499674 .173041 .96875 $2.48034 \quad 4.150291 .96832$ 2.461274 .127791 .96789 $2.44246 \quad 4.10556 \quad 1.96745$

$2.42390 \quad 4.08358 \quad 1.96701$ $2.40558 \quad 4.06185 \quad 1.96656$ $2.38750 \quad 4.04036 \quad 1.96611$ 2.369654 .019101 .96565 2.352023 .998081 .96519

$2.33462 \quad 3.97729 \quad 1.96472$ $2.31744 \quad 3.95672 \quad 1.96425$ $2.300473 .93636 \quad 1.96378$ $2.283713 .91623 \quad 1.96329$ $2.267153 .89630 \quad 1.96281$

$2.25079 \quad 3.87657 \quad 1.96232$ 2.234633 .857051 .96182 2.218673 .837721 .96132 $2.202893 .81859 \quad 1.96082$ $2.187293 .79965 \quad 1.96031$

$\begin{array}{llll}2.17188 & 3.78089 & 1.95979\end{array}$ 2.156643 .762321 .95927 $2.14158 \quad 3.74392 \quad 1.95875$ $2.126693 .72570 \quad 1.95822$ $2.111973 .70766 \quad 1.95768$
600 610 620 630 640

650 660 670 680 690

700

710

720

730

740

750

760

770

780

790

800

810

820

830

840

850

860

870

880

890

900

910

920

930

940

950

960

970

980

990

1000

1010

1020

1030

1040

1050

1060

1070

1080

1090 
Table II Harmonic Oscillator Contributions to the Thermodynamic

Functions (in units of calories, moles, and ${ }^{\circ} \mathrm{K}$ )
$\mathrm{cm}^{-1}$
$-\left(F^{\circ}-E_{O}^{\circ}\right) / T \quad S^{\circ}$
So $\quad \mathrm{co}$
$\mathrm{C}_{\mathrm{p}}^{\circ} \quad-\left(\mathrm{F}^{\circ}-\mathrm{E}_{\mathrm{O}}^{\circ}\right) / \mathrm{T}$
So
$\mathrm{co}$
$-\left(F^{\circ}-E_{O}^{\circ}\right) / T$
So
$\stackrel{\mathrm{C}_{\mathrm{p}}^{\circ}}{\nu} \quad \mathrm{cm}^{-1}$

$T=3500$.

1100

1110

1120

1130

1140

$1150 \quad 1.94002 \quad 3.49434 \quad 1.95057$

$\begin{array}{llll}1160 & 1.92657 & 3.47746 & 1.94994\end{array}$

$1170 \quad 1.91328 \quad 3.46072 \quad 1.94930$

$1180 \quad 1.90012 \quad 3.44414 \quad 1.94866$

$1190 \quad 1.887113 .42769 \quad 1.94801$

$\begin{array}{lllll}1200 & 1.87423 & 3.41139 & 1.94736\end{array}$

$\begin{array}{lllll}1210 & 1.86149 & 3.39524 & 1.94670\end{array}$

$\begin{array}{llll}1220 & 1.84888 & 3.37922 & 1.94604\end{array}$

$\begin{array}{lllll}1230 & 1.83640 & 3.36333 & 1.94537\end{array}$

$\begin{array}{llll}1240 & 1.82405 & 3.34758 & 1.94469\end{array}$

$\begin{array}{lllll}1250 & 1.81182 & 3.33197 & 1.94402\end{array}$

$\begin{array}{lllll}1260 & 1.79972 & 3.31648 & 1.94333\end{array}$

$\begin{array}{lllll}1270 & 1.78775 & 3.30112 & 1.94264\end{array}$

$\begin{array}{lllll}1280 & 1.77589 & 3.28589 & 1.94195\end{array}$

$\begin{array}{lllll}1290 & 1.76415 & 3.27078 & 1.94125\end{array}$

$\begin{array}{lllll}1300 & 1.75253 & 3.25579 & 1.94054\end{array}$

$\begin{array}{lllll}1310 & 1.74103 & 3.24092 & 1.93983\end{array}$

$\begin{array}{lllll}1320 & 1.72963 & 3.22617 & 1.93912\end{array}$

$\begin{array}{llll}1330 & 1.71835 & 3.21154 & 1.93840\end{array}$

$\begin{array}{llll}1340 & 1.70718 & 3.19702 & 1.93767\end{array}$

$\begin{array}{lllll}1350 & 1.69611 & 3.18262 & 1.93694\end{array}$

$\begin{array}{lllll}1360 & 1.68515 & 3.16833 & 1.93621\end{array}$

$\begin{array}{lllll}1370 & 1.67430 & 3.15414 & 1.93547\end{array}$

$\begin{array}{lllll}1380 & 1.66355 & 3.14007 & 1.93472\end{array}$

$\begin{array}{lllll}1390 & 1.65290 & 3.12610 & 1.93397\end{array}$

$\begin{array}{lllll}1400 & 1.64235 & 3.11224 & 1.93322\end{array}$

$\begin{array}{lllll}1410 & 1.63190 & 3.09849 & 1.93246\end{array}$

$1420 \quad 1.621553 .08483 \quad 1.93169$

$\begin{array}{llll}1430 & 1.61129 & 3.07128 & 1.93092\end{array}$

$\begin{array}{llll}1440 & 1.60113 & 3.05783 & 1.93014\end{array}$

$\begin{array}{lllll}1450 & 1.59106 & 3.04447 & 1.92936\end{array}$

$\begin{array}{lllll}1460 & 1.58108 & 3.03121 & 1.92858\end{array}$

$1470 \quad 1.571193 .01805 \quad 1.92779$

$1480 \quad 1.56140 \quad 3.00499 \quad 1.92699$

$1490 \quad 1.551692 .99201 \quad 1.92619$

$\begin{array}{lllll}1500 & 1.54206 & 2.97913 & 1.92538\end{array}$

$\begin{array}{lllll}1510 & 1.53253 & 2.96634 & 1.92457\end{array}$

$\begin{array}{lllll}1520 & 1.52307 & 2.95364 & 1.92376\end{array}$

$1530 \quad 1.51370 \quad 2.94103 \quad 1.92294$

$\begin{array}{lllll}1540 \quad 1.50441 & 2.92850 & 1.92211\end{array}$

$\begin{array}{lllll}1550 \quad 1.49521 & 2.91606 & 1.92128\end{array}$

$\begin{array}{lllll}1560 & 1.48608 & 2.90371 & 1.92044\end{array}$

$\begin{array}{llllll}1570 & 1.47703 & 2.891 .44 & 1.91960\end{array}$

$\begin{array}{llll}1580 & 1.46806 & 2.87926 & 1.91876\end{array}$

$\begin{array}{lllll}1590 \quad 1.45917 & 2.86715 & 1.91791\end{array}$
$T=3600$.

$T=3700$.

$2.05392 \quad 3.63618 \quad 1.95547$

$2.03962 \quad 3.61849 \quad 1.95490$

$2.02547 \quad 3.60096 \quad 1.95432$

$2.01148 \quad 3.58359 \quad 1.95374$

$1.99765 \quad 3.56638 \quad 1.95315$

$\begin{array}{lll}1.98396 & 3.54932 \quad 1.95255\end{array}$

1.970423 .532421 .95196

$1.95703 \quad 3.51567 \quad 1.95135$

$1.94378 \quad 3.49906 \quad 1.95074$

1.930673 .482601 .95013

$\begin{array}{llll}1.91769 & 3.46628 & 1.94951\end{array}$

$1.90486 \quad 3.45011 \quad 1.94889$

$1.89215 \quad 3.43407 \quad 1.94826$

$1.87958 \quad 3.418171 .94763$

$1.86713 \quad 3.402401 .94699$

$1.85481 \quad 3.38676 \quad 1.94635$

$1.84262 \quad 3.37126 \quad 1.94570$

$1.83055 \quad 3.35588 \quad 1.94505$

1.818603 .340631 .94439

$1.80677 \quad 3.32550 \quad 1.94373$

$1.79505 \quad 3.31049 \quad 1.94306$

$1.78345 \quad 3.29560 \quad 1.94239$

$1.77197 \quad 3.28084 \quad 1.94172$

$1.760593 .26618 \quad 1.94103$

$1.74932 \quad 3.25165 \quad 1.94035$

$\begin{array}{llll}1.73817 & 3.23722 \quad 1.93966\end{array}$

$\begin{array}{llll}1.72712 & 3.22291 & 1.93896\end{array}$

$1.71617 \quad 3.20871 \quad 1.93826$

$1.70533 \quad 3.19461 \quad 1.93755$

$1.69458 \quad 3.180631 .93684$

$1.68394 \quad 3.16675 \quad 1.93613$

$1.67340 \quad 3.15297 \quad 1.93541$

$\begin{array}{lll}1.66296 & 3.13929 & 1.93468\end{array}$

$1.652613 .12572 \quad 1.93395$

$1.642353 .11224 \quad 1.93322$

$\begin{array}{llll}1.63219 & 3.09887 & 1.93248\end{array}$ $1.62212 \quad 3.08559 \quad 1.93173$ $\begin{array}{llll}1.61214 & 3.07241 & 1.93098\end{array}$ $1.60225 \quad 3.05932 \quad 1.93023$

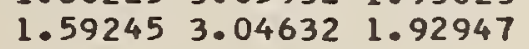

$\begin{array}{llll}1.58274 & 3.03342 & 1.92871\end{array}$ $\begin{array}{llll}1.57311 & 3.02060 & 1.92794\end{array}$ $\begin{array}{llll}1.56357 & 3.00788 & 1.92717\end{array}$ $\begin{array}{lll}1.55411 & 2.99525 & 1.92639\end{array}$ $\begin{array}{ll}1.54473 & 2.98270 \quad 1.92561\end{array}$

$\begin{array}{lll}1.53543 & 2.97024 & 1.92482\end{array}$ $1.526212 .95786 \quad 1.92403$ $1.51708 \quad 2.94557 \quad 1.92323$ $1.50802 \quad 2.93336 \quad 1.92243$ $\begin{array}{llll}1.49903 & 2.92124 & 1.92163\end{array}$
$2.09741 \quad 3.68978 \quad 1.95715$

$2.08302 \quad 3.67207 \quad 1.95660$

$2.06878 \quad 3.65453 \quad 1.95605$

$2.05470 \quad 3.63714 \quad 1.95550$

$2.040773 .61992 \quad 1.95494$

$2.02699 \quad 3.60284 \quad 1.95438$

$2.01336 \quad 3.58592 \quad 1.95381$

$\begin{array}{llll}1.99988 & 3.56916 & 1.95324\end{array}$

$\begin{array}{llll}1.98654 & 3.55254 & 1.95267\end{array}$

$1.973343 .53606 \quad 1.95209$

$1.96027 \quad 3.51973 \quad 1.95150$

$1.947343 .50353 \quad 1.95091$

$\begin{array}{llll}1.93455 & 3.48748 & 1.95031\end{array}$

$1.921893 .47156 \quad 1.94971$

$1.909353 .45578 \quad 1.94911$

$\begin{array}{lll}1.89694 & 3.44012 & 1.94850\end{array}$

$1.88466 \quad 3.42460 \quad 1.94789$

$1.87250 \quad 3.40920 \quad 1.94727$

$1.86046 \quad 3.39393 \quad 1.94665$

$1.84854 \quad 3.37879 \quad 1.94602$

$\begin{array}{llll}1.83673 & 3.36376 & 1.94539\end{array}$

$1.82504 \quad 3.34886 \quad 1.94475$

$1.813473 .33407 \quad 1.94411$

$1.802003 .31940 \quad 1.94346$

$1.790653 .30484 \quad 1.94281$

$\begin{array}{llll}1.77940 & 3.29040 & 1.94215\end{array}$

$\begin{array}{llll}1.76826 & 3.27607 & 1.94149\end{array}$

$1.75723 \quad 3.261851 .94083$

$1.74630 \quad 3.24774 \quad 1.94016$

$1.735473 .23373 \quad 1.93949$

$1.72474 \quad 3.219831 .93881$

$1.714113 .20603 \quad 1.93813$

$1.70358 \quad 3.192341 .93744$

$1.693143 .17875 \quad 1.93675$

1.682803 .165251 .93605

$1.67255 \quad 3.15186 \quad 1.93535$

$1.662393 .13856 \quad 1.93464$

1.652333 .125351 .93393

$1.64235 \quad 3.11224 \quad 1.93322$

1.632463 .099231 .93250

$1.62266 \quad 3.08630 \quad 1.93177$

$1.61295 \quad 3.07347 \quad 1.93104$

1.603323 .060731 .93031

$1.593773 .04807 \quad 1.92957$

1.584313 .035501 .92883

$1.57493 \quad 3.023021 .92809$

1.565623 .010621 .92734

$1.55640 \quad 2.99831 \quad 1.92658$

$1.547252 .98608 \quad 1.92582$

1.538192 .973931 .92506
1100

1110

1120

1130

1140

1150

1160

1170

1180

1190

1200

1210

1220

1230

1240

1250

1260

1270

1280

1290

1300

1310

1320

1330

1340

1350

1360

1370

1380

1390

1400

1410

1420

1430

1440

1450

1460

1470

1480

1490

1500

1510

1520

1530

1540

1550

1560

1570

1580

1590 
Table II Harmonic Oscillator Contributions to the Thermodynamic

Functions (in units of calories, moles, and ${ }^{\circ} \mathrm{K}$ )
$\mathrm{cm}^{-1}$
So
$-\left(F^{\circ}-E_{O}^{\circ}\right) / T$
$\mathrm{C}_{\mathrm{p}}^{\circ}$
$-\left(F^{\circ}-E_{O}^{\circ}\right) / T \quad S^{\circ}$
$\mathrm{c}_{\mathrm{p}}$
$-\left(F^{\circ}-E_{O}^{\circ}\right) / T$
So
$\mathrm{C}_{\mathrm{p}}^{\circ}$
$\nu$
$\mathrm{cm}^{-1}$

$\mathrm{T}=3500$.

$T=3600$.

$T=3700$

$\begin{array}{llll}1600 \quad 1.45035 & 2.85513 \quad 1.91705\end{array}$

$1610 \quad 1.441612 .843191 .91619$

$1620 \quad 1.432942 .831331 .91533$

$1630 \quad 1.424342 .819541 .91446$

$1640 \quad 1.41582 \quad 2.80784 \quad 1.91358$

$\begin{array}{lllll}1650 & 1.40737 & 2.79621 & 1.91270\end{array}$

$\begin{array}{llll}1660 & 1.39899 & 2.78465 & 1.91182\end{array}$

$\begin{array}{lllll}1670 & 1.39067 & 2.77317 & 1.91093\end{array}$

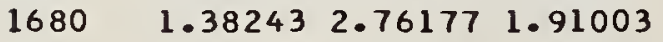

$\begin{array}{lll}1690 & 1.37425 & 2.75043 \quad 1.90914\end{array}$

$\begin{array}{lllll}1700 & 1.36614 & 2.73917 & 1.90823\end{array}$

$\begin{array}{lllll}1710 & 1.35810 & 2.72798 & 1.90732\end{array}$

$1720 \quad 1.350122 .71687 \quad 1.90641$

$\begin{array}{lllll}1730 & 1.34221 & 2.70582 & 1.90549\end{array}$

$\begin{array}{llll}1740 \quad 1.33435 & 2.69484 & 1.90457\end{array}$

$\begin{array}{lllll}1750 & 1.32657 & 2.68392 & 1.90364\end{array}$

$\begin{array}{lllll}1760 & 1.31884 & 2.67308 & 1.90271\end{array}$

$\begin{array}{lllll}1770 & 1.31118 & 2.66230 & 1.90177\end{array}$

$\begin{array}{lllll}1780 & 1.30357 & 2.65159 & 1.90083\end{array}$

$\begin{array}{lll}1790 \quad 1.29603 \quad 2.64094 & 1.89988\end{array}$

$\begin{array}{lllll}1800 & 1.28855 & 2.63036 & 1.89893\end{array}$

$\begin{array}{lllll}1810 & 1.28112 & 2.61985 & 1.89797\end{array}$

$\begin{array}{lllll}1820 & 1.27375 & 2.60939 & 1.89701\end{array}$

$\begin{array}{lllll}1830 & 1.26644 & 2.59900 & 1.89605\end{array}$

$\begin{array}{lllll}1840 & 1.25919 & 2.58867 & 1.89508\end{array}$

$\begin{array}{lllll}1850 & 1.25199 & 2.57840 & 1.89410\end{array}$

$\begin{array}{llll}1860 & 1.24485 & 2.56819 & 1.89312\end{array}$

$\begin{array}{lllll}1870 & 1.23776 & 2.55804 & 1.89214\end{array}$

$1880 \quad 1.230732 .54795 \quad 1.89115$

$\begin{array}{lllll}1890 & 1.22375 & 2.53792 & 1.89015\end{array}$

1900

1910

1920

1930

1940

1950

1960

1970

1980

1990

2000

2010

2020

2030

2040

2050

2060

2070

2080

2090
$1.490132 .90919 \quad 1.92082$

$1.481292 .89723 \quad 1.92000$

$1.472542 .88534 \quad 1.91918$

$\begin{array}{llll}1.46385 & 2.87353 & 1.91836\end{array}$

1.455242 .861801 .91753

$1.44670 \quad 2.85015 \quad 1.91670$

$\begin{array}{llll}1.43823 & 2.83857 & 1.91586\end{array}$

$1.429832 .82706 \quad 1.91501$

$1.421492 .81563 \quad 1.91417$

1.413232 .804281 .91331

1.405032 .792991 .91246 $1.39690 \quad 2.78178 \quad 1.91160$ 1.388832 .770631 .91073 $1.380832 .75956 \quad 1.90986$ 1.372902 .748551 .90899

1.365022 .737621 .90811 1.357212 .726751 .90722 $1.34946 \quad 2.71594 \quad 1.90633$ $\begin{array}{llll}1.34177 & 2.70520 & 1.90544\end{array}$ 1.334142 .694531 .90454

$1.326572 .68392 \quad 1.90364$ $1.31906 \quad 2.67338 \quad 1.90273$ 1.311602 .662901 .90182 1.304212 .652481 .90091 1.296872 .642121 .89999

$\begin{array}{llll}1.28958 & 2.63183 & 1.89906\end{array}$ $1.282362 .62159 \quad 1.89813$ $1.27518 \quad 2.61142 \quad 1.89720$ 1.268062 .601301 .89626 1.261002 .591251 .89532

$\begin{array}{llll}1.25399 & 2.58125 & 1.89437\end{array}$ $1.24703 \quad 2.57130 \quad 1.89342$ $1.24012 \quad 2.56142 \quad 1.89246$ $1.23326 \quad 2.55159 \quad 1.89150$ $1.22646 \quad 2.54182 \quad 1.89054$

$\begin{array}{llll}1.21970 & 2.53210 & 1.88957\end{array}$ 1.213002 .522441 .88860 1.206342 .512831 .88762 $1.19973 \quad 2.50327 \quad 1.88664$ $1.193172 .49377 \quad 1.88565$

$1.18666 \quad 2.48432 \quad 1.88466$ $1.180202 .47493 \quad 1.88367$ $1.17378 \quad 2.46558 \quad 1.88267$ $1.167412 .45628 \quad 1.88166$ $1.161082 .44704 \quad 1.88065$

$\begin{array}{llll}1.15480 & 2.43785 & 1.87964\end{array}$ $\begin{array}{llll}1.14856 & 2.42870 & 1.87862\end{array}$ $1.142372 .41961 \quad 1.87760$ $1.13622 \quad 2.41056 \quad 1.87658$ $1.130122 .40156 \quad 1.87555$
$1.529192 .96187 \quad 1.92429$ $1.52028 \quad 2.94988 \quad 1.92351$ $\begin{array}{llll}1.51143 & 2.93797 & 1.92274\end{array}$ 1.502672 .926141 .92195 $1.49397 \quad 2.914391 .92117$

$\begin{array}{lll}1.48534 & 2.90271 & 1.92038\end{array}$ $\begin{array}{llll}1.47679 & 2.89111 & 1.91958\end{array}$ $1.468302 .87959 \quad 1.91878$ 1.459892 .868131 .91798 $\begin{array}{llll}1.45154 & 2.85675 \quad 1.91717\end{array}$

$1.44326 \quad 2.84544 \quad 1.91636$ $1.435042 .83421 \quad 1.91554$ 1.426892 .823041 .91472 1.418812 .811941 .91389 1.410792 .800911 .91306

$1.402832 .78995 \quad 1.91223$ $1.394932 .77906 \quad 1.91139$ 1.387102 .768231 .91054 $1.379332 .75747 \quad 1.90970$ $1.371612 .74677 \quad 1.90884$

$1.36396 \quad 2.73614 \quad 1.90799$ 1.356372 .725571 .90713 1.348832 .715071 .90626 1.341352 .704631 .90539 1.333932 .694241 .90452

$1.326572 .68392 \quad 1.90364$ 1.319262 .673661 .90276 $1.312002 .66346 \quad 1.90187$ 1.304802 .653321 .90098 $1.29766 \quad 2.64324 \quad 1.90009$

$1.29056 \quad 2.63322 \quad 1.89919$ $1.28352 \quad 2.62325 \quad 1.89828$ $1.27654 \quad 2.61334 \quad 1.89738$ 1.269602 .603491 .89646 1.262712 .593691 .89555

$1.25588 \quad 2.58394 \quad 1.89463$ 1.249092 .574251 .89370 1.242352 .564621 .89277 $1.23567 \quad 2.55504 \quad 1.89184$ $1.229032 .54551 \quad 1.89091$

$1.22244 \quad 2.53603 \quad 1.88996$ 1.215892 .526611 .88902 $1.209392 .51724 \quad 1.88807$ $1.202942 .50792 \quad 1.88712$ $1.196542 .49864 \quad 1.88616$

$1.19018 \quad 2.48942 \quad 1.88520$ $1.18386 \quad 2.48025 \quad 1.88423$ 1.177592 .471131 .88326 $1.171362 .46206 \quad 1.88229$ $1.165182 .45303 \quad 1.88131$
1600

1610

1620

1630

1640

1650

1660

1670

1680

1690

1700

1710

1720

1730

1740

17.50

1760

1770

1780

1790

1800

1810

1820

1830

1840

1850

1860

1870

1880

1890

1900

1910

1920

1930

1940

1950

1960

1970

1980

1990

2000

2010

2020

2030

2040

2050

2060

2070

2080

2090 
Table II Harmonic Oscillator Contributions to the Thermodynamic

Functions (in units of calories, moles, and ${ }^{\circ} \mathrm{K}$ )
$\mathrm{cm}^{-1}$
So
$\mathrm{C}_{\mathrm{p}}^{\circ} \quad-\left(\mathrm{F}^{\circ}-\mathrm{E}_{\mathrm{O}}^{\circ}\right) / \mathrm{T}$
So
$\mathrm{C}_{\mathrm{p}}^{\circ}$
$-\left(F^{\circ}-E_{O} O\right) / T$
So
$\nu$

$\mathrm{T}=3500$.

$T=3600$.

$T=3700$.

$\begin{array}{llll}2100 & 1.08856 & 2.33989 & 1.86822\end{array}$

$2110 \quad 1.082622 .331021 .86713$

$2120 \quad 1.076732 .322201 .86603$

$\begin{array}{llll}2130 & 1.07087 & 2.31342 & 1.86493\end{array}$

$2140 \quad 1.065062 .30469 \quad 1.86382$

$\begin{array}{lllll}2150 & 1.05929 & 2.29600 & 1.86271\end{array}$

$2160 \quad 1.05356 \quad 2.28736 \quad 1.86160$

$2170 \quad 1.04786 \quad 2.27876 \quad 1.86048$

$\begin{array}{lllll}2180 & 1.04221 & 2.27021 & 1.85935\end{array}$

$2190 \quad 1.03660 \quad 2.26170 \quad 1.85822$

$\begin{array}{llll}2200 \quad 1.03102 & 2.25324 & 1.85709\end{array}$

$\begin{array}{lllll}2210 & 1.02549 & 2.24482 & 1.85595\end{array}$

2220

2230

2240

2250

2260

2270

2280

2290

2300

2310

2320

2330

2340

2350

2360

2370

2380

2390

2400

2410

2420

2430

2440

2450

2460

2470

2480

2490

2500

2510

2520

2530

2540

2550

2560

2570

2580

2590
$1.019992 .23644 \quad 1.85481$

1.014532 .228111 .85366

1.009102 .219821 .85251

$1.003722 .21157 \quad 1.85136$

$0.99837 \quad 2.20336 \quad 1.85020$ $0.99305 \quad 2.19520 \quad 1.84904$ $0.98778 \quad 2.18707 \quad 1.84787$ $0.982532 .17899 \quad 1.84670$

$0.97733 \quad 2.17094 \quad 1.84552$ $0.97215 \quad 2.16294 \quad 1.84434$ $0.96702 \quad 2.15497 \quad 1.84316$ 0.961912 .147051 .84197 $0.95684 \quad 2.13916 \quad 1.84078$

$0.951812 .13132 \quad 1.83958$ $\begin{array}{llll}0.94680 & 2.12351 & 1.83838\end{array}$ $0.94184 \quad 2.11574 \quad 1.83717$ 0.936902 .108001 .83596 $0.931992 .10031 \quad 1.83475$

$0.92712 \quad 2.09265 \quad 1.83353$ $0.92228 \quad 2.08503 \quad 1.83231$ $\begin{array}{llll}0.91747 & 2.07744 & 1.83108\end{array}$ $0.912692 .06990 \quad 1.82985$ $0.90795 \quad 2.06238 \quad 1.82862$

$\begin{array}{llll}0.90323 & 2.05491 & 1.82738\end{array}$ $\begin{array}{llll}0.89855 & 2.04747 & 1.82614\end{array}$ $0.893892 .04006 \quad 1.82489$

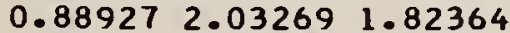
0.884672 .025351 .82239

$0.880102 .01805 \quad 1.82113$ $0.875572 .01078 \quad 1.81987$ $0.87106 \quad 2.00355 \quad 1.81860$ 0.866581 .996351 .81733 0.862131 .989181 .81606

$\begin{array}{llll}0.85770 & 1.98205 & 1.81478\end{array}$ $0.853311 .97495 \quad 1.81350$ $0.84894 \quad 1.96788 \quad 1.81221$ 0.844601 .960851 .81092 $0.840291 .95384 \quad 1.80963$
$1.12406 \quad 2.39261 \quad 1.87452$ 1.118042 .383711 .87348 $1.11206 \quad 2.37486 \quad 1.87244$ 1.106122 .366051 .87139 $1.10023 \quad 2.35728 \quad 1.87034$ $\begin{array}{llll}1.09437 & 2.34857 \quad 1.86928\end{array}$ $1.088562 .33989 \quad 1.86822$ $1.082792 .33127 \quad 1.86716$ 1.077052 .322691 .86609 $1.07136 \quad 2.31415 \quad 1.86502$

$1.06570 \quad 2.30565 \quad 1.86395$ $1.060092 .29720 \quad 1.86287$ 1.054512 .288791 .86178 1.048972 .280431 .86069 $1.04346 \quad 2.272111 .85960$

$1.03800 \quad 2.26383 \quad 1.85850$ 1.032572 .255591 .85740 1.027172 .247391 .85630 $1.02182 \quad 2.239231 .85519$ 1.016492 .231111 .85408

$1.011212 .22304 \quad 1.85296$ $1.00596 \quad 2.21500 \quad 1.85184$ $1.00074 \quad 2.20701 \quad 1.85072$ $0.995562 .19905 \quad 1.84959$ 0.990412 .191131 .84845

$0.98530 \quad 2.18325 \quad 1.84732$ $0.980212 .17541 \quad 1.84617$ 0.975172 .167601 .84503 $0.970152 .15984 \quad 1.84388$ $0.965172 .15211 \quad 1.84273$

$0.96022 \quad 2.14442 \quad 1.84157$ $0.95530 \quad 2.13676 \quad 1.84041$ $0.950412 .12914 \quad 1.83924$ $0.94556 \quad 2.12156 \quad 1.83808$ 0.940742 .114011 .83690

0.935942 .106501 .83573 0.931182 .099031 .83455 0.926452 .091591 .83336 $0.921752 .08418 \quad 1.83217$ 0.917072 .076811 .83098

$0.912432 .06948 \quad 1.82978$ $0.907822 .06218 \quad 1.82858$ $0.903232 .05491 \quad 1.82738$ $0.89868 \quad 2.04767 \quad 1.82617$ 0.894152 .040471 .82496 $\begin{array}{llll}0.88965 & 2.03330 & 1.82375\end{array}$ $0.88518 \quad 2.026171 .82253$ 0.880742 .019061 .82130 0.876322 .011991 .82008 $0.871932 .00495 \quad 1.81885$
$1.15904 \quad 2.44405 \quad 1.88033$

1.152942 .435121 .87934

1.146892 .426241 .87835

1.140872 .417401 .87736

$1.13490 \quad 2.40861 \quad 1.87636$

$\begin{array}{llll}1.12897 & 2.39987 & 1.87535\end{array}$

$1.12308 \quad 2.391171 .87435$

$1.11723 \quad 2.38251 \quad 1.87334$

$1.111412 .37390 \quad 1.87232$

1.105642 .365331 .87130

$\begin{array}{lll}1.09991 & 2.35681 & 1.87028\end{array}$

$1.09422 \quad 2.348331 .86925$

$1.08856 \quad 2.33989 \quad 1.86822$

1.082942 .331501 .86719

$1.07736 \quad 2.32315 \quad 1.86615$

$1.07182 \quad 2.31484 \quad 1.86511$

1.066312 .306571 .86406 $1.060842 .29834 \quad 1.86301$

1.055412 .290161 .86196

1.050012 .282011 .86090

$1.04465 \quad 2.27390 \quad 1.85984$ $1.03932 \quad 2.26584 \quad 1.85877$ $\begin{array}{llll}1.03403 & 2.25781 & 1.85770\end{array}$ 1.028772 .249821 .85663 $1.02355 \quad 2.24187 \quad 1.85555$

$1.01836 \quad 2.23396 \quad 1.85447$ $1.01320 \quad 2.22609 \quad 1.85338$ $\begin{array}{llll}1.00808 & 2.21826 & 1.85230\end{array}$ $1.002992 .21046 \quad 1.85120$ $0.99794 \quad 2.20270 \quad 1.85011$

$0.992912 .19498 \quad 1.84900$ $0.98792 \quad 2.18729 \quad 1.84790$ 0.982962 .179641 .84679 0.978032 .172031 .84568 0.973132 .164451 .84456

$0.96826 \quad 2.15691 \quad 1.84344$ 0.963432 .149401 .84232 0.958622 .141931 .84119 0.953842 .134491 .84006 0.949102 .127091 .83893

$0.94438 \quad 2.11972 \quad 1.83779$ 0.939702 .112391 .83665 $0.935042 .10509 \quad 1.83550$ 0.930412 .097821 .83435 0.925812 .090591 .83320

$0.92124 \quad 2.08339 \quad 1.83204$ $0.91670 \quad 2.07622 \quad 1.83088$ $0.91218 \quad 2.06908 \quad 1.82972$ $0.907692 .06198 \quad 1.82855$ $0.90323 \quad 2.05491 \quad 1.82738$
2100

2110

2120

2130

2140

2150

2160

2170

2180

2190

2200

2210

2220

2230

2240

2250

2260

2270

2280

2290

2300

2310

2320

2330

2340

2350

2360

2370

2380

2390

2400

2410

2420

2430

2440

2450

2460

2470

2480

2490

2500

2510

2520

2530

2540

2550

2560

2570

2580

2590 
Table II Harmonic Oscillator Contributions to the Thermodynamic

Functions (in units of calories, moles, and ${ }^{\circ} \mathrm{K}$ )

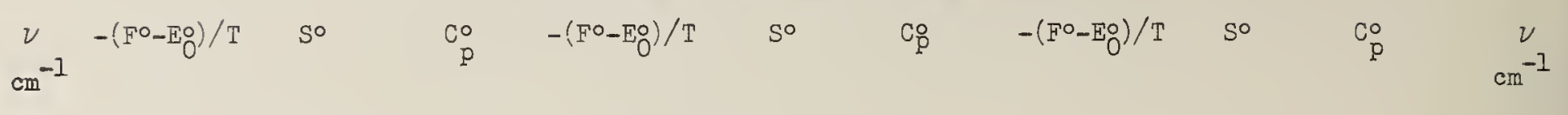

$T=3500$

2600

2610

2620

2630

2640

2650

2660

2670

2680

2690

2700

2710

2720

2730

2740

2750

2760

2770

2780

2790

2800

2810

2820

2830

2840

2850

2860

2870

2880

2890

2900

2910

2920

2930

2940

2950

2960

2970

2980

2990

3000

3010

3020

3030

3040

3050

3060

3070

3080

3090
$T=3600$.

$0.86757 \quad 1.99795 \quad 1.81761$ $0.86324 \quad 1.99097 \quad 1.81638$ 0.858931 .984031 .81513 0.854651 .977121 .81389 $0.850391 .97024 \quad 1.81264$

$0.84616 \quad 1.96338 \quad 1.81139$ $0.841961 .95656 \quad 1.81013$ $0.83778 \quad 1.94977 \quad 1.80887$ $0.833631 .94301 \quad 1.80761$ $0.829501 .93628 \quad 1.80634$

$\begin{array}{llll}0.82540 & 1.92958 & 1.80507\end{array}$ $0.821331 .92291 \quad 1.80379$ $0.817271 .91627 \quad 1.80251$ $0.813241 .90966 \quad 1.80123$ $0.80924 \quad 1.90308 \quad 1.79995$

$0.80526 \quad 1.89652 \quad 1.79866$ $0.80130 \quad 1.89000 \quad 1.79736$ $0.79737 \quad 1.88350 \quad 1.79607$ 0.793461 .877031 .79477 $0.78958 \quad 1.87059 \quad 1.79346$

$0.78571 \quad 1.86417 \quad 1.79216$ $0.78187 \quad 1.85778 \quad 1.79085$ $0.77805 \quad 1.85142 \quad 1.78953$ $0.77426 \quad 1.84509 \quad 1.78822$ $0.77049 \quad 1.83879 \quad 1.78689$

$0.76674 \quad 1.83251 \quad 1.78557$ $0.76301 \quad 1.82626 \quad 1.78424$ $0.75930 \quad 1.82003 \quad 1.78291$ 0.755621 .813831 .78157 $0.75195 \quad 1.80766 \quad 1.78024$

$0.74831 \quad 1.80151 \quad 1.77889$ 0.744691 .795391 .77755 0.741091 .789301 .77620 $0.73751 \quad 1.78322 \quad 1.77485$ $0.73395 \quad 1.777181 .77349$

$0.73041 \quad 1.77116 \quad 1.77213$ $0.726891 .76517 \quad 1.77077$ $0.72340 \quad 1.759201 .76941$ 0.719921 .753251 .76804 0.716461 .747331 .76667

0.713021 .741431 .76529 0.709611 .735561 .76391 $0.70621 \quad 1.72971 \quad 1.76253$ 0.702831 .723891 .76114 0.699471 .718091 .75975

0.696131 .712311 .75836 0.692801 .706561 .75697 $0.68950 \quad 1.700831 .75557$ 0.686221 .695121 .75417 0.682951 .689441 .75276
$T=3700$

$0.89880 \quad 2.04787 \quad 1.82621$ $0.894392 .04086 \quad 1.82503$ $\begin{array}{llll}0.89001 & 2.03388 & 1.82385\end{array}$ $0.88566 \quad 2.02694 \quad 1.82266$ 0.881332 .020021 .82147

$0.87703 \quad 2.01314 \quad 1.82028$ $0.87276 \quad 2.00628 \quad 1.81908$ $0.86851 \quad 1.99946 \quad 1.81788$ $0.864291 .99267 \quad 1.81668$ $0.860091 .98590 \quad 1.81547$

$0.85592 \quad 1.97917 \quad 1.81426$ $0.85177 \quad 1.97246 \quad 1.81305$ $0.847651 .96579 \quad 1.81183$ $0.843551 .95914 \quad 1.81061$ $0.83947 \quad 1.952521 .80938$

0.835421 .945931 .80815 $0.83140 \quad 1.93937 \quad 1.80692$ $0.82740 \quad 1.932841 .80569$ 0.823421 .926341 .80445 $0.81946 \quad 1.91986 \quad 1.80321$

$0.81553 \quad 1.91341 \quad 1.80196$ 0.811621 .906991 .80071 $0.80773 \quad 1.900591 .79946$ 0.803871 .894231 .79820 $0.80003 \quad 1.88788 \quad 1.79694$

$\begin{array}{llll}0.79621 & 1.88157 & 1.79568\end{array}$ $0.792411 .87528 \quad 1.79442$ 0.788631 .869021 .79315 $0.784881 .86279 \quad 1.79187$ $0.781151 .85658 \quad 1.79060$

$0.77744 \quad 1.85040 \quad 1.78932$ $0.77375 \quad 1.84424 \quad 1.78804$ 0.770081 .838111 .78675 $0.766431 .83200 \quad 1.78546$ 0.762811 .825921 .78417

$0.75920 \quad 1.81986 \quad 1.78287$ 0.755621 .813831 .78157 $0.75205 \quad 1.80783 \quad 1.78027$ $0.748511 .80184 \quad 1.77897$ $0.74498 \quad 1.79589 \quad 1.77766$

$0.74148 \quad 1.78995 \quad 1.77635$ $0.73799 \quad 1.78404 \quad 1.77503$ $0.734531 .77816 \quad 1.77371$ $0.73108 \quad 1.77230 \quad 1.77239$ $0.727651 .76646 \quad 1.77107$

$0.72425 \quad 1.76065 \quad 1.76974$ 0.720861 .754851 .76841 $0.71749 \quad 1.74909 \quad 1.76707$ $0.71414 \quad 1.74334 \quad 1.76574$ $0.71080 \quad 1.73762 \quad 1.76440$
2600

2610

2620

2630

2640

2650

2660

2670

2680

2690

2700

2710

2720

2730

2740

2750

2760

2770

2780

2790

2800

2810

2820

2830

2840

2850

2860

2870

2880

2890

2900

2910

2920

2930

2940

2950

2960

2970

2980

2990

3000

3010

3020

3030

3040

3050

3060

3070

3080

3090 
Table II Harmonic Oscillator Contributions to the Thermodynamic

Functions (in units of calories, moles, and ${ }^{\circ} \mathrm{K}$ )
$\mathrm{cm}^{-1}$
$-\left(F^{\circ}-E_{O}^{\circ}\right) / T \quad S^{\circ}$
$\quad \stackrel{\mathrm{C}}{\mathrm{p}}$
$\mathrm{C}_{\mathrm{p}}^{\circ} \quad-\left(\mathrm{F}^{\circ}-\mathrm{E}_{\mathrm{O}} \mathrm{O}\right) / \mathrm{T}$
So
$\mathrm{C}_{\mathrm{p}}$
$-\left(\mathrm{F}^{\circ}-\mathrm{E}_{\mathrm{O}}\right) / \mathrm{T}$
So
$\stackrel{\mathrm{C}_{\mathrm{p}}^{\circ}}{ }$

$\mathrm{cm}^{-1}$

$T=3500$.

$T=3600$.

$T=3700$.

3100

3110

3120

3130

3140

3150

3160

3170

3180

3190

3200

3210

3220

3230

3240

3250

3260

3270

3280

3290

3300

3310

3320

3330

3340

3350

3360

3370

3380

3390

3400

3410

3420

3430

3440

3450

3460

3470

3480

3490

3500

3510

3520

3530

3540

3550

3560

3570

3580

3590
$0.65171 \quad 1.634611 .73874$

0.648551 .629021 .73726

$0.64541 \quad 1.62344 \quad 1.73578$

0.642281 .617891 .73429

$0.639171 .61236 \quad 1.73280$

$0.63608 \quad 1.606851 .73131$

$0.63301 \quad 1.601371 .72981$

$0.62995 \quad 1.59591 \quad 1.72832$

0.626921 .590461 .72681

$0.623891 .58504 \quad 1.72531$

$0.620891 .57965 \quad 1.72380$

$0.61790 \quad 1.57427 \quad 1.72229$

0.614931 .568921 .72077

0.611981 .563581 .71925

$0.60904 \quad 1.55827 \quad 1.71773$

$0.606121 .55298 \quad 1.71621$

$0.60321 \quad 1.54771 \quad 1.71468$

0.600321 .542461 .71315

$0.597451 .53723 \quad 1.71161$

0.594591 .532021 .71008

$0.59175 \quad 1.52684 \quad 1.70854$ 0.588921 .521671 .70699

0.586111 .516521 .70545

0.583321 .511391 .70390

0.580541 .506291 .70235

$0.57778 \quad 1.50120 \quad 1.70079$

0.575031 .496131 .69923

0.572291 .491091 .69767

$0.56957 \quad 1.48606 \quad 1.69611$

0.566871 .481051 .69454

$0.56418 \quad 1.47606 \quad 1.69297$

$0.56151 \quad 1.47109 \quad 1.69140$

$0.55885 \quad 1.46614 \quad 1.68983$

$0.556201 .46121 \quad 1.68825$

$0.55357 \quad 1.45630 \quad 1.68667$

$0.55095 \quad 1.45140 \quad 1.68509$

$0.548351 .44653 \quad 1.68350$

$0.54576 \quad 1.44167 \quad 1.68191$

$0.543191 .43683 \quad 1.68032$

0.540621 .432021 .67872

$0.53808 \quad 1.42721 \quad 1.67713$

$0.53554 \quad 1.42243 \quad 1.67553$

$0.53302 \quad 1.41767 \quad 1.67392$

$0.53052 \quad 1.41292 \quad 1.67232$

$0.52802 \quad 1.40819 \quad 1.67071$

$0.52554 \quad 1.40348 \quad 1.66910$

$0.52308 \quad 1.39879 \quad 1.66748$

0.520621 .394111 .66587

$0.518181 .38946 \quad 1.66425$

$0.51576 \quad 1.38482 \quad 1.66263$
$0.67970 \quad 1.68378 \quad 1.75135$

0.676471 .678141 .74994

$0.67326 \quad 1.672521 .74853$

0.670071 .666931 .74711

0.666891 .661361 .74569

$0.663731 .65581 \quad 1.74427$

0.660591 .650281 .74284

$0.657471 .64478 \quad 1.74141$

0.654361 .639301 .73998

$0.65127 \quad 1.63384 \quad 1.73854$

0.648201 .628401 .73710

0.645151 .622981 .73566

$0.642111 .61758 \quad 1.73421$

$0.639091 .61221 \quad 1.73276$

0.636081 .606851 .73131

0.633091 .601521 .72986

$0.630121 .59621 \quad 1.72840$

0.627171 .590921 .72694

$0.62423 \quad 1.58565 \quad 1.72547$

0.621311 .580401 .72401

$0.618401 .57517 \quad 1.72254$ 0.615511 .569961 .72106 0.612631 .564771 .71959 0.609771 .559601 .71811 0.606931 .554451 .71663

$0.60410 \quad 1.54932 \quad 1.71514$ $0.60128 \quad 1.54421 \quad 1.71366$ $0.59848 \quad 1.53912 \quad 1.71217$ $0.59570 \quad 1.53405 \quad 1.71067$ $0.59293 \quad 1.52899 \quad 1.70918$

$\begin{array}{llll}0.59018 & 1.52396 & 1.70768\end{array}$ $0.58744 \quad 1.51895 \quad 1.70618$ $0.58471 \quad 1.51396 \quad 1.70467$ $\begin{array}{llll}0.58201 & 1.50898 & 1.70317\end{array}$ $0.57931 \quad 1.504021 .70166$

$0.576631 .49909 \quad 1.70014$ $0.57396 \quad 1.49417 \quad 1.69863$ $0.57131 \quad 1.48927 \quad 1.69711$ $0.56867 \quad 1.48439 \quad 1.69559$ $0.566051 .47952 \quad 1.69407$

$0.56344 \quad 1.47468 \quad 1.69254$ $0.56084 \quad 1.46985 \quad 1.69101$ $0.55826 \quad 1.46504 \quad 1.68948$ $0.555691 .46025 \quad 1.68794$ $0.55313 \quad 1.45548 \quad 1.68641$

$\begin{array}{llll}0.55059 & 1.45073 & 1.68487\end{array}$ $0.54806 \quad 1.44599 \quad 1.68332$ $0.54554 \quad 1.44127 \quad 1.68178$ $0.54304 \quad 1.43657 \quad 1.68023$ $0.54055 \quad 1.43188 \quad 1.67868$
$0.707491 .73192 \quad 1.76305$ 0.704201 .726251 .76171 0.700921 .720591 .76036 $0.697661 .71496 \quad 1.75900$ 0.694421 .709351 .75765

$0.691191 .70377 \quad 1.75629$ $0.68799 \quad 1.698201 .75493$ 0.684801 .692661 .75356 0.681631 .687141 .75219 $0.67848 \quad 1.681641 .75082$

$0.675341 .67616 \quad 1.74945$ $0.672221 .67071 \quad 1.74807$ $0.669121 .66527 \quad 1.74669$ $0.666031 .65986 \quad 1.74531$ $0.66297 \quad 1.65446 \quad 1.74392$

$0.65991 \quad 1.649091 .74253$ $0.65688 \quad 1.64374 \quad 1.74114$ $0.65386 \quad 1.63841 \quad 1.73974$ $0.65086 \quad 1.633101 .73834$ 0.647871 .627811 .73694

$0.64490 \quad 1.62254 \quad 1.73554$ 0.641941 .617291 .73413 $0.63901 \quad 1.61206 \quad 1.73272$ 0.636081 .606851 .73131 0.633181 .601661 .72990

$0.63028 \quad 1.59649 \quad 1.72848$ $0.62741 \quad 1.591351 .72706$ $0.62455 \quad 1.58621 \quad 1.72563$ $0.62170 \quad 1.581101 .72421$ $0.61887 \quad 1.57601 \quad 1.72278$

0.616051 .570941 .72134 0.613251 .565891 .71991 $0.61047 \quad 1.560851 .71847$ 0.607691 .555841 .71703 0.604941 .550841 .71559

$0.602191 .54586 \quad 1.71414$ $0.59947 \quad 1.54090 \quad 1.71269$ $0.59675 \quad 1.53596 \quad 1.71124$ 0.594051 .531041 .70979 $0.59137 \quad 1.526141 .70833$

$0.58870 \quad 1.52125 \quad 1.70687$ $0.58604 \quad 1.51638 \quad 1.70541$ 0.583391 .511531 .70394 $0.58076 \quad 1.50670 \quad 1.70247$ 0.578151 .501891 .70100

$0.57555 \quad 1.49709 \quad 1.69953$ $0.57296 \quad 1.49231 \quad 1.69805$ 0.570381 .487551 .69658 $0.567821 .48281 \quad 1.69510$ $0.56527 \quad 1.47808 \quad 1.69361$
3100

3110

3120

3130

3140

3150

3160

3170

3180

3190

3200

3210

3220

3230

3240

3250

3260

3270

3280

3290

3300

3310

3320

3330

3340

3350

3360

3370

3380

3390

3400

3410

3420

3430

3440

3450

3460

3470

3480

3490

3500

3510

3520

3530

3540

3550

3560

3570

3580

3590 
Table II Harmonic Oscillator Contributions to the Thermodynamic Functions (in units of calories, moles, and ${ }^{\circ} \mathrm{K}$ )
$\mathrm{cm}^{-1}-(\mathrm{F} \circ-\mathrm{EO}) / \mathrm{T} \quad \mathrm{S}^{\circ}$
$\mathrm{C}_{\mathrm{P}}^{\circ} \quad-\left(\mathrm{F}^{\circ}-\mathrm{E}_{\mathrm{O}} \mathrm{O}\right) / \mathrm{T} \quad \mathrm{S}^{\circ}$
$\mathrm{C}_{\mathrm{p}}$
$-\left(F^{\circ}-E_{O} \circ\right) / T$ SO
v
$\mathrm{cm}^{-1}$

$T=3500$.

3600

3610

3620

3630

3640

3650

3660

3670

3680

3690

3700

3710

3720

3730

3740

3750

3760

3770

3780

3790

3800

3810

3820

3830

3840

3850

3860

3870

3880

3890

3900

3910

3920

3930

3940

3950

3960

3970

3980

3990

4000
$0.51334 \quad 1.380191 .66100$ $0.51094 \quad 1.37559 \quad 1.65937$ $0.50855 \quad 1.37100 \quad 1.65774$ $0.50618 \quad 1.36643 \quad 1.65611$ $\begin{array}{llll}0.50381 & 1.36188 & 1.65448\end{array}$

$0.50146 \quad 1.35734 \quad 1.65284$ $0.499121 .35282 \quad 1.65120$ $0.49680 \quad 1.34832 \quad 1.64956$ $0.49448 \quad 1.34383 \quad 1.64791$ $0.49218 \quad 1.33936 \quad 1.64627$

$\begin{array}{llll}0.48989 & 1.33491 & 1.64462\end{array}$ $0.487611 .33047 \quad 1.64296$ 0.485351 .326051 .64131 $0.48309 \quad 1.32165 \quad 1.63965$ $0.480851 .31726 \quad 1.63799$

$0.47862 \quad 1.31289 \quad 1.63633$ $0.47640 \quad 1.30853 \quad 1.63466$ $0.47419 \quad 1.30419 \quad 1.63300$ $0.47200 \quad 1.29987 \quad 1.63133$ 0.469811 .295561 .62966

$0.46764 \quad 1.29127 \quad 1.62798$ $0.46548 \quad 1.28699 \quad 1.62630$ $0.46333 \quad 1.28273 \quad 1.62463$ 0.461191 .278491 .62294 $\begin{array}{llll}0.45906 & 1.27426 & 1.62126\end{array}$

$0.45694 \quad 1.27004 \quad 1.61957$ $0.45484 \quad 1.26584 \quad 1.61789$ $0.45274 \quad 1.26166 \quad 1.61620$ 0.450661 .257491 .61450 $0.448581 .25334 \quad 1.61281$

$0.44652 \quad 1.24920 \quad 1.61111$ $0.44447 \quad 1.24507 \quad 1.60941$ $0.44242 \quad 1.24097 \quad 1.60771$ $0.440391 .23687 \quad 1.60601$ $0.43837 \quad 1.23279 \quad 1.60430$

$0.43636 \quad 1.22873 \quad 1.60259$ $\begin{array}{llll}0.43436 & 1.22468 & 1.60088\end{array}$ $0.43237 \quad 1.22064 \quad 1.59917$ $0.43039 \quad 1.21662 \quad 1.59746$ 0.428421 .212621 .59574

$0.42646 \quad 1.20862 \quad 1.59402$

$$
T=3600 \text {. }
$$

$0.53808 \quad 1.42721 \quad 1.67713$ $0.53561 \quad 1.42256 \quad 1.67557$ $0.53316 \quad 1.41793 \quad 1.67401$ $0.53073 \quad 1.41332 \quad 1.67245$ $0.528301 .40872 \quad 1.67089$

$0.52589 \quad 1.40413 \quad 1.66932$ $0.523491 .39957 \quad 1.66775$ $0.521101 .39502 \quad 1.66618$ $0.518731 .39049 \quad 1.66461$ $0.51636 \quad 1.38597 \quad 1.66303$

$0.51401 \quad 1.38148 \quad 1.66145$ $\begin{array}{llll}0.51167 & 1.37699 & 1.65987\end{array}$ 0.509351 .372531 .65829 $0.50703 \quad 1.36808 \quad 1.65670$ $0.50473 \quad 1.363641 .65511$

$0.50244 \quad 1.359231 .65352$ 0.500161 .354831 .65193 $0.497891 .35044 \quad 1.65033$ $0.49564 \quad 1.34607 \quad 1.64874$ 0.493391 .341721 .64713

$0.49116 \quad 1.33738 \quad 1.64553$ $0.48894 \quad 1.33306 \quad 1.64393$ $0.48673 \quad 1.32875 \quad 1.64232$ $0.484531 .32446 \quad 1.64071$ 0.482341 .320181 .63910

$0.48017 \quad 1.31592 \quad 1.63748$ $0.47800 \quad 1.31167 \quad 1.63587$ $0.475851 .30744 \quad 1.63425$ $0.47370 \quad 1.30323 \quad 1.63263$ 0.471571 .299031 .63100

$0.46945 \quad 1.29484 \quad 1.62938$ $0.467341 .29067 \quad 1.62775$ $0.46524 \quad 1.28652 \quad 1.62612$ $0.46315 \quad 1.28238 \quad 1.62449$ $0.46107 \quad 1.27825 \quad 1.62285$

$0.45900 \quad 1.27414 \quad 1.62121$ $0.45694 \quad 1.27004 \quad 1.61957$ $0.45489 \quad 1.26596 \quad 1.61793$ $0.45286 \quad 1.26189 \quad 1.61629$ $0.45083 \quad 1.25784 \quad 1.61464$

$0.44881 \quad 1.25380 \quad 1.61300$
$T=3700$.

$0.56273 \quad 1.47337 \quad 1.69213$ $0.56021 \quad 1.46868 \quad 1.69064$ $0.55770 \quad 1.46401 \quad 1.68915$ $0.55520 \quad 1.45935 \quad 1.68765$ $0.55272 \quad 1.45471 \quad 1.68616$

$0.55025 \quad 1.45008 \quad 1.68466$ $0.54779 \quad 1.44548 \quad 1.68316$ 0.545341 .440891 .68165 0.542911 .436311 .68015 $0.540491 .43176 \quad 1.67864$

$0.53808 \quad 1.427211 .67713$ $\begin{array}{llll}0.53568 & 1.42269 & 1.67561\end{array}$ 0.533301 .418181 .67410 0.530921 .413691 .67258 0.528561 .409211 .67106

$0.52621 \quad 1.40475 \quad 1.66953$ $0.523881 .40031 \quad 1.66801$ $0.521551 .39588 \quad 1.66648$ $0.51924 \quad 1.39147 \quad 1.66495$ 0.516941 .387071 .66341

$0.514651 .38269 \quad 1.66188$ 0.512371 .378321 .66034 $0.510101 .37397 \quad 1.65880$ 0.507841 .369641 .65726 $0.50560 \quad 1.36532 \quad 1.65571$

$0.50337 \quad 1.36102 \quad 1.65417$ 0.501141 .356731 .65262 0.498931 .352451 .65107 0.496731 .348191 .64951 0.494541 .343951 .64796

$0.49237 \quad 1.33972 \quad 1.64640$ 0.490201 .335511 .64484 0.488041 .331311 .64328 $0.48590 \quad 1.327121 .64171$ $0.48376 \quad 1.32295 \quad 1.64014$

$0.48164 \quad 1.31880 \quad 1.63857$ 0.479521 .314661 .63700 0.477421 .310531 .63543 0.475331 .306421 .63385 0.473241 .302321 .63227

$0.47117 \quad 1.29824 \quad 1.63069$
3600

3610

3620

3630

3640

3650

3660

3670

3680

3690

3700

3710

3720

3730

3740

3750

3760

3770

3780

3790

3800

3810

3820

3830

3840

3850

3860

3870

3880

'3890

3900

3910

3920

3930

3940

3950

3960

3970

3980

3990

4000 
Table II Harmonic Oscillator Contributions to the Thermodynamic

Functions (in units of calories, moles, and ${ }^{\circ} \mathrm{K}$ )
$\mathrm{cm}^{-1}$
$-\left(F^{\circ}-E O\right) / T \quad S^{\circ}$
$\mathrm{C}_{\mathrm{p}}^{\circ}$
$-\left(F^{\circ}-E_{0}^{O}\right) / T$
So
$\mathrm{C}_{\mathrm{p}}^{\circ}$
$-\left(F^{\circ}-E_{O}^{\circ}\right) / T$
So
$\mathrm{c}_{\mathrm{p}}^{\mathrm{o}}$
$\mathrm{cm}^{-1}$

$T=3800$.

100

110

120

130

140

150

160

170

180

190

200

210

220

230

240

$\begin{array}{lllll}250 & 4.77803 & 6.67264 & 1.98569\end{array}$

$\begin{array}{llllll}260 & 4.70380 & 6.59476 & 1.98557\end{array}$

$\begin{array}{lllll}270 & 4.63250 & 6.51983 & 1.98544\end{array}$

$280 \quad 4.56393 \quad 6.44762 \quad 1.98531$

$290 \quad 4.49789 \quad 6.37796 \quad 1.98517$

$300 \quad 4.43421 \quad 6.31066 \quad 1.98503$

$\begin{array}{lllll}310 & 4.37274 & 6.24557 & 1.98489\end{array}$

$\begin{array}{lllll}320 & 4.31334 & 6.18256 & 1.98474\end{array}$

$\begin{array}{lllll}330 & 4.25588 & 6.12149 & 1.98459\end{array}$

$\begin{array}{llll}340 & 4.20024 & 6.06224 & 1.98443\end{array}$

$\begin{array}{lllll}350 & 4.14631 & 6.00472 & 1.98426\end{array}$

$\begin{array}{lllll}360 & 4.09401 & 5.94883 & 1.98410\end{array}$

$\begin{array}{lllll}370 & 4.04324 & 5.89447 & 1.98392\end{array}$

$\begin{array}{lllll}380 & 3.99392 & 5.84156 & 1.98375\end{array}$

$\begin{array}{lllll}390 & 3.94597 & 5.79003 & 1.98356\end{array}$

$\begin{array}{lllll}400 \quad 3.89933 & 5.73982 & 1.98338\end{array}$

$\begin{array}{lllll}410 & 3.85393 & 5.69084 & 1.98318\end{array}$

$420 \quad 3.809715 .64306 \quad 1.98299$

$\begin{array}{lllll}430 & 3.76661 & 5.59640 & 1.98279\end{array}$

$\begin{array}{lllll}440 & 3.72458 & 5.55082 & 1.98258\end{array}$

$\begin{array}{lllll}450 & 3.68358 & 5.50627 & 1.98237\end{array}$

$\begin{array}{lllll}460 & 3.64356 & 5.46270 & 1.98215\end{array}$

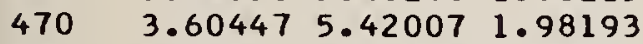

$\begin{array}{lllll}480 & 3.56629 & 5.37835 & 1.98171\end{array}$

$\begin{array}{llll}490 \quad 3.52896 & 5.33749 & 1.98148\end{array}$

$\begin{array}{llll}500 & 3.49246 & 5.29746 \quad 1.98125\end{array}$

$\begin{array}{lllll}510 & 3.45675 & 5.25823 & 1.98101\end{array}$

$\begin{array}{lllll}520 & 3.42180 & 5.21976 & 1.98076\end{array}$

$\begin{array}{lllll}530 & 3.38759 & 5.18203 & 1.98051\end{array}$

$\begin{array}{lll}540 & 3.35408 & 5.14502 \quad 1.98026\end{array}$

$\begin{array}{lllll}550 & 3.32125 & 5.10868 & 1.98000\end{array}$

$\begin{array}{lllll}560 & 3.28907 & 5.07301 & 1.97974\end{array}$

$\begin{array}{lllll}570 & 3.25753 & 5.03797 & 1.97947\end{array}$

$\begin{array}{llll}580 & 3.22659 & 5.00355 & 1.97920\end{array}$

$\begin{array}{lll}590 & 3.19625 & 4.96972 \quad 1.97893\end{array}$

$T=3900$.

$T=4000$.

$6.59371 \quad 8.54445 \quad 1.98695$ $6.40796 \quad 8.35508 \quad 1.98690$ $6.238698 .18220 \quad 1.98685$ 6.083278 .023171 .98679 $5.939647 .87593 \quad 1.98673$

5.806177 .738861 .98666 5.681557 .610651 .98659 5.564717 .490221 .98652 $5.45475 \quad 7.37667 \quad 1.98644$ $5.350937 .26927 \quad 1.98636$

$5.252637 .16739 \quad 1.98627$ $5.159297 .07048 \quad 1.98618$ 5.070476 .978091 .98608 4. $98575 \quad 6.88980 \quad 1.98598$ 4. $90479 \quad 6.80528 \quad 1.98587$

$4.82728 \quad 6.72422 \quad 1.98576$ $4.75295 \quad 6.64634 \quad 1.98565$ $4.68156 \quad 6.57140 \quad 1.98553$ 4.612896 .499191 .98540 $4.54676 \quad 6.429521 .98528$

4.482996 .362221 .98514 4.421436 .297131 .98501 4.361936 .234111 .98486 $4.304386 .17304 \quad 1.98472$ $4.248656 .11379 \quad 1.98457$

$4.194636 .05627 \quad 1.98441$ $4.142246 .00037 \quad 1.98425$ 4.091375 .946001 .98409 $4.041965 .89309 \quad 1.98392$ 3.993925 .841561 .98375

$3.94719 \quad 5.79134 \quad 1.98357$ 3.901695 .742361 .98339 $3.85738 \quad 5.69457 \quad 1.98320$ 3.814195 .647901 .98301 3.772075 .602321 .98281

$3.73098 \quad 5.55776 \quad 1.98261$ 3.690875 .514191 .98241 3.651695 .471561 .98220 3.613415 .429831 .98199 $3.57600 \quad 5.38896 \quad 1.98177$

$3.53940 \quad 5.34893 \quad 1.98154$ 3.503605 .309691 .98132 3.468575 .271221 .98109 $3.43426 \quad 5.23348 \quad 1.98085$ 3.400665 .196461 .98061

$3.36774 \quad 5.16012 \quad 1.98037$ 3. $33548 \quad 5.12444 \quad 1.98012$ 3.303845 .089391 .97986 $3.272825 .05496 \quad 1.97961$ 3.242385 .021121 .97934
$6.643118 .59476 \quad 1.98696$ $6.45726 \quad 8.40538 \quad 1.98691$ $\begin{array}{llll}6.28791 & 8.23250 & 1.98686\end{array}$ $6.13240 \quad 8.07347 \quad 1.98681$ 5.988687 .926231 .98675

5.855127 .789161 .98669 5.730417 .660951 .98662 $5.61348 \quad 7.54051 \quad 1.98655$ 5.503437 .426961 .98648 5.399527 .319561 .98640

5.301137 .217681 .98631 $5.207707 .12077 \quad 1.98622$ $5.11879 \quad 7.02837 \quad 1.98613$ $5.03398 \quad 6.94008 \quad 1.98604$ $4.952936 .85556 \quad 1.98594$

$4.875336 .77449 \quad 1.98583$ $4.800916 .69661 \quad 1.98572$ $4.729436 .62167 \quad 1.98561$ $4.660686 .54946 \quad 1.98549$ $\begin{array}{llll}4.59446 & 6.47979 & 1.98537\end{array}$

$4.53060 \quad 6.41248 \quad 1.98524$ $4.46895 \quad 6.347391 .98511$ $4.409376 .28437 \quad 1.98498$ $4.35173 \quad 6.223291 .98484$ $4.295906 .16404 \quad 1.98470$

$4.24180 \quad 6.10651 \quad 1.98455$ 4.189326 .050601 .98440 $4.138375 .99624 \quad 1.98424$ 4. 088875.943321 .98408 $4.04074 \quad 5.891791 .98391$

$3.99392 \quad 5.84156 \quad 1.98375$ $3.948345 .79258 \quad 1.98357$ 3.903945 .744781 .98339 3.860665 .698111 .98321 3.818465 .652521 .98303

$3.77728 \quad 5.60796 \quad 1.98284$ $3.73708 \quad 5.56438 \quad 1.98264$ 3.697825 .521741 .98244 3.659455 .480011 .98224 3.621955 .439141 .98203

$\begin{array}{llll}3.58527 & 5.39910 & 1.98182\end{array}$ $3.549395 .35986 \quad 1.98161$ $3.514265 .32138 \quad 1.98139$ 3.479875 .283641 .98116 3.446195 .246611 .98093

3.413185 .210261 .98070 $3.380835 .17458 \quad 1.98046$ 3.349115 .139521 .98022 $3.31800 \quad 5.10509 \quad 1.97998$ $3.28748 \quad 5.07124 \quad 1.97973$
100

110

120

130

140

150

160

170

180

190

200

210

220

230

240

250

260

270

280

290

300

310

320

330

340

350

360

370

380

390

400

410

420

430

440

450

460

470

480

490

500

510

520

530

540

550

560

570

580

590 
Table II Harmonic Oscillator Contributions to the Thermodynamic

Functions (in units of calories, moles, and ${ }^{\circ} \mathrm{K}$ )

\section{$\mathrm{cm}^{-1}-\left(\mathrm{F}^{\circ}-\mathrm{E}_{0}^{\circ}\right) / \mathrm{T} \quad \mathrm{S}^{\circ}$

\author{
$\mathrm{C}_{\mathrm{p}}$
}

$$
T=3800
$$ \\ 600 \\ 610 \\ 620 \\ 630 \\ 640 \\ 650 \\ 660 \\ 670 \\ 680 \\ 690 \\ $3.16647 \quad 4.93646 \quad 1.97865$ $3.13724 \quad 4.90375 \quad 1.97836$ $3.10855 \quad 4.87159 \quad 1.97807$ $3.08036 \quad 4.83994 \quad 1.97777$ $3.05268 \quad 4.80880 \quad 1.97747$ \\ $3.02548 \quad 4.77814 \quad 1.97717$ $2.99875 \quad 4.74796 \quad 1.97686$ $2.972474 .71823 \quad 1.97655$ 2.946634 .688951 .97623 $2.921224 .66010 \quad 1.97591$}

$-\left(F^{\circ}-E_{0}^{\circ}\right) / T$ so
$\mathrm{C}_{\mathrm{p}}^{\circ}$
$-\left(F^{\circ}-E_{0}\right) / T \quad S^{\circ}$ $v$

$\mathrm{cm}^{-1}$
700

710

720

730

740

750

760

770

780

790

800

810

820

830

840

850

860

870

880

890

900

910

920

930

940

950

960

970

980

990

1000

1010

1020

1030

1040

1050

1060

1070

1080

1090
$2.896234 .63167 \quad 1.97558$ 2.871634 .603651 .97525 $2.847434 .57603 \quad 1.97491$ 2.823614 .548791 .97457 2.800164 .521931 .97422

$2.77708 \quad 4.49543 \quad 1.97387$ $2.75434 \quad 4.46929 \quad 1.97351$ $2.73194 \quad 4.44349 \quad 1.97315$ 2.709884 .418031 .37279 $2.68814 \quad 4.39290 \quad 1.97242$

2.666724 .368101 .97205 $2.64560 \quad 4.34360 \quad 1.97167$ $2.624794 .31941 \quad 1.97128$ $2.604274 .29552 \quad 1.97090$ $2.58403 \quad 4.27192 \quad 1.97050$

$2.56408 \quad 4.24860 \quad 1.97011$ $2.54440 \quad 4.22556 \quad 1.96970$ $2.52498 \quad 4.20279 \quad 1.96930$ $2.505824 .18029 \quad 1.96889$ 2.486924 .156041 .96847

2.468274 .136051 .96805 $2.449864 .11430 \quad 1.96763$ 2.431694 .092801 .96720 2.413754 .071541 .96676 $2.396034 .05051 \quad 1.96633$

$2.37854 \quad 4.02970 \quad 1.96588$ 2.361274 .009121 .96543 $2.34421 \quad 3.98875 \quad 1.96498$ $2.32736 \quad 3.96860 \quad 1.96453$ $2.310713 .94866 \quad 1.96400$

$2.29427 \quad 3.92892 \quad 1.96360$ $2.278023 .90938 \quad 1.96313$ $2.26196 \quad 3.89005 \quad 1.96265$ $2.24610 \quad 3.87090 \quad 1.96217$ $2.230413 .85194 \quad 1.96169$

$2.21491 \quad 3.83317 \quad 1.96120$ $2.19959 \quad 3.81459 \quad 1.96071$ $2.18444 \quad 3.79618 \quad 1.96021$ $2.16946 \quad 3.77795 \quad 1.95971$ $2.15465 \quad 3.75989 \quad 1.95920$
$T=3900$.

$\begin{array}{llll}3.21252 & 4.98786 & 1.97908\end{array}$

3.183204 .955151 .97880

3.154414 .922981 .97853

$3.12614 \quad 4.89132 \quad 1.97825$

$3.09837 \quad 4.86017 \quad 1.97796$

$3.07108 \quad 4.82950 \quad 1.97767$

$3.04426 \quad 4.79931 \quad 1.97738$

$3.01789 \quad 4.76758 \quad 1.97708$

2.941974 .740291 .97678

$2.96647 \quad 4.71143 \quad 1.97647$

$2.94138 \quad 4.68300 \quad 1.97616$

$2.91670 \quad 4.65497 \quad 1.97585$

$2.89242 \quad 4.62734 \quad 1.97553$

$2.86851 \quad 4.60009 \quad 1.97520$

$2.84497 \quad 4.57322 \quad 1.97487$

$2.82180 \quad 4.54671 \quad 1.97454$ $2.79897 \quad 4.52056 \quad 1.97420$ $2.77649 \quad 4.49475 \quad 1.97386$ $2.75434 \quad 4.46929 \quad 1.97351$ 2.732514 .444151 .97316

2.711004 .419331 .97281 $2.68980 \quad 4.39483 \quad 1.97245$ 2.668904 .370631 .97208 $2.64829 \quad 4.34672 \quad 1.97172$ 2.627974 .323111 .97134

$2.60793 \quad 4.29779 \quad 1.97097$ $2.58816 \quad 4.27674 \quad 1.97058$ $2.568664 .25396 \quad 1.97020$ $2.549424 .23144 \quad 1.96981$ 2.530434 .209191 .96941

$2.511694 .18718 \quad 1.96901$ 2.493194 .165431 .96861 $2.47494 \quad 4.14391 \quad 1.96820$ $2.456914 .12264 \quad 1.96779$ $2.43911 \quad 4.10159 \quad 1.96737$

$2.42154 \quad 4.08078 \quad 1.96695$ $2.40418 \quad 4.06018 \quad 1.96653$ $2.38704 \quad 4.03981 \quad 1.96610$ $2.37010 \quad 4.019641 .96566$ $2.35337 \quad 3.99969 \quad 1.96523$

$2.33684 \quad 3.97994 \quad 1.96478$ 2.320513 .960391 .96434 2.304373 .941041 .96389 $2.28841 \quad 3.92188 \quad 1.96343$ $2.27265 \quad 3.90292 \quad 1.96297$

2.257063 .884131 .96251 $2.241653 .86553 \quad 1.96204$ $2.22642 \quad 3.847111 .96157$ $2.21136 \quad 3.82887 \quad 1.96109$ $2.196473 .81080 \quad 1.96061$
$T=4000$

$\begin{array}{llll}3.25753 & 5.03797 & 1.97947\end{array}$

$3.228135 .00525 \quad 1.97922$

$3.19926 \quad 4.97307 \quad 1.97895$

$3.17090 \quad 4.94141 \quad 1.97869$

$3.14304 \quad 4.91025 \quad 1.97842$

$3.115574 .87958 \quad 1.97814$ $3.08877 \quad 4.84938 \quad 1.97786$ $3.06231 \quad 4.81964 \quad 1.97758$ $3.03630 \quad 4.79034 \quad 1.97729$

$3.010 / 24.76148 \quad 1.97700$

$2.98555 \quad 4.73304 \quad 1.97670$ 2.960794 .105001 .97640 2.936424 .677361 .97610 2.912434 .650101 .97579 $2.888814 .62322 \quad 1.97548$

$2.86555 \quad 4.59671 \quad 1.97516$ 2.842644 .570551 .97484 $2.82007 \quad 4.54474 \quad 1.97452$ $2.79784 \quad 4.51926 \quad 1.97419$ $2.77593 \quad 4.49411 \quad 1.97385$

$2.75434 \quad 4.46929 \quad 1.97351$ $2.73305 \quad 4.44477 \quad 1.97317$ $2.71207 \quad 4.42056 \quad 1.97283$ $2.69138 \quad 4.396651 .97248$ $2.67078 \quad 4.37303 \quad 1.97212$

$2.65035 \quad 4.34970 \quad 1.97176$ $2.63100 \quad 4.32664 \quad 1.97140$ $2.611424 .30385 \quad 1.97103$ 2.592094 .281321 .97066 $2.57302 \quad 4.25906 \quad 1.97029$

$2.55420 \quad 4.23705 \quad 1.96991$ $2.535634 .21528 \quad 1.96952$ $2.517294 .19376 \quad 1.96913$ $2.49918 \quad 4.17247 \quad 1.96874$ 2.481304 .151421 .96835

$2.46364 \quad 4.13059 \quad 1.96795$ $2.44621 \quad 4.10999 \quad 1.96754$ $2.42898 \quad 4.08960 \quad 1.96713$ $2.41196 \quad 4.06942 \quad 1.96672$ $2.39515 \quad 4.04946 \quad 1.96630$

$2.37854 \quad 4.02970 \quad 1.96588$ $2.362134 .01014 \quad 1.96546$ $2.34591 \quad 3.99078 \quad 1.96503$ $2.32988 \quad 3.97161 \quad 1.96459$ $2.31403 \quad 3.952631 .96416$

$2.29836 \quad 3.93384 \quad 1.96372$ $2.28287 \quad 3.91522 \quad 1.96327$ $2.26756 \quad 3.89679 \quad 1.96282$ $2.25242 \quad 3.87854 \quad 1.96237$ $2.23745 \quad 3.860451 .96191$
600

610

620

630

640

650

660

670

680

690

700

710

720

730

740

750

760

770

780

790

800

810

820

830

840

850

860

870

880

890

900

910

920

930

940

950

960

970

980

990

1000

1010

1020

1030

1040

1050

1060

1070

1080

1090 
Table II Harmonic Oscillator Contributions to the Thermodynamic

Functions (in units of calories, moles, and ${ }^{\circ} \mathrm{K}$ )
$\mathrm{cm}^{-1}-\left(F^{\circ}-E_{0}^{\circ}\right) / T \quad S^{\circ}$
$\mathrm{C}_{\mathrm{p}}^{\circ} \quad-\left(\mathrm{F}^{\circ}-\mathrm{E}_{\mathrm{O}}^{\circ}\right) / \mathrm{T} \quad \mathrm{S}$
$\mathrm{C}_{\mathrm{p}}$
$-\left(F^{\circ}-E_{0}^{\circ}\right) / T$
$S^{\circ}$
$-1$

$T=3800$.

$\Gamma=3900$.

$r=4000$.

$1100 \quad 2.140013 .74200 \quad 1.95869$ $\begin{array}{llll}1110 & 2.12552 & 3.72427 & 1.95818\end{array}$ $1120 \quad 2.11120 \quad 3.70671 \quad 1.95766$ $1130 \quad 2.09703 \quad 3.68931 \quad 1.95713$ $\begin{array}{lllll}1140 \quad 2.08302 & 3.67207 & 1.95660\end{array}$

$\begin{array}{llll}1150 & 2.06915 \quad 3.65499 & 1.95607\end{array}$ $\begin{array}{llll}1160 & 2.05544 & 3.63805 & 1.95553\end{array}$ $1170 \quad 2.04186 \quad 3.62127 \quad 1.95499$ $1180 \quad 2.028443 .60463 \quad 1.95444$ $\begin{array}{lll}1190 \quad 2.01515 \quad 3.58814 & 1.95389\end{array}$

$1200 \quad 2.00200 \quad 3.57179 \quad 1.95333$ $\begin{array}{lllll}1210 & 1.98898 & 3.55559 & 1.95277\end{array}$ $\begin{array}{lllll}1220 & 1.97610 & 3.53952 & 1.95221\end{array}$ $1230 \quad 1.963353 .52358 \quad 1.95164$ $\begin{array}{lllll}1240 & 1.95073 & 3.50778 & 1.95107\end{array}$

$\begin{array}{lllll}1250 & 1.93824 & 3.49211 & 1.95049\end{array}$ $\begin{array}{lllll}1260 & 1.92587 & 3.47657 & 1.94990\end{array}$ $1270 \quad 1.913623 .46116 \quad 1.94932$ $\begin{array}{lllll}1280 & 1.90150 & 3.44587 & 1.94873\end{array}$ $1290 \quad 1.88949 \quad 3.43071 \quad 1.94813$

$\begin{array}{lllll}1300 & 1.87760 & 3.41567 & 1.94753\end{array}$ $1310 \quad 1.86583 \quad 3.40075 \quad 1.94693$ $\begin{array}{llll}1320 & 1.85417 & 3.38595 & 1.94632\end{array}$ $1330 \quad 1.842623 .37126 \quad 1.94570$ $\begin{array}{lllll}1340 \quad 1.83118 & 3.35669 & 1.94508\end{array}$

$1350 \quad 1.81985 \quad 3.34223 \quad 1.94446$ $\begin{array}{lllll}1360 & 1.80863 & 3.32788 & 1.94384\end{array}$ $\begin{array}{lllll}1370 & 1.79751 & 3.31364 & 1.94320\end{array}$ $\begin{array}{lllll}1380 & 1.78649 & 3.29951 & 1.94257\end{array}$ $\begin{array}{lllll}1390 \quad 1.77558 & 3.28549 & 1.94193\end{array}$

$\begin{array}{lllll}1400 & 1.76477 & 3.27157 & 1.94129\end{array}$ $1410 \quad 1.75405 \quad 3.25775 \quad 1.94064$ $1420 \quad 1.743443 .24404 \quad 1.93998$ $1430 \quad 1.73292 \quad 3.230431 .93933$ $1440 \quad 1.72249 \quad 3.21692 \quad 1.93867$

$1450 \quad 1.71216 \quad 3.20350 \quad 1.93800$ 1460 1470 1480 1490

1500

1510

1520

1530

1540

1550

1560

1570

1580

1590
1.701923 .190191 .93733

$1.691773 .17696 \quad 1.93665$

1.681723 .163841 .93598

$1.671753 .15080 \quad 1.93529$

$1.66186 \quad 3.13786 \quad 1.93460$ $1.652073 .12501 \quad 1.93391$ $1.642353 .11224 \quad 1.93322$ $1.632723 .09957 \quad 1.93252$ 1.623183 .086981 .93181

$1.613713 .07448 \quad 1.93110$ 1.604333 .062061 .93039 1.595023 .049731 .92967 $1.58580 \quad 3.03748 \quad 1.92895$ $1.576653 .02531 \quad 1.92822$
$2.18174 \quad 3.79289 \quad 1.96012$ $2.16717 \quad 3.77516 \quad 1.95963$ $2.15276 \quad 3.75758 \quad 1.95914$ 2.138513 .740171 .95864 2.124423 .722921 .95814

$2.110473 .70582 \quad 1.95763$ 2.096673 .688871 .95712 $2.08302 \quad 3.67207 \quad 1.95660$ $2.06951 \quad 3.655421 .95608$ $2.05614 \quad 3.638921 .95556$

$2.04290 \quad 3.62256 \quad 1.95503$ 2.029813 .606331 .95450 2. $01684 \quad 3.59025 \quad 1.95396$ 2.004013 .574301 .95342 $1.991313 .55848 \quad 1.95287$

$1.97873 \quad 3.54280 \quad 1.95232$ $1.96628 \quad 3.52725 \quad 1.95177$ $1.95396 \quad 3.51182 \quad 1.95121$ $1.94175 \quad 3.496521 .95065$ 1.929663 .481341 .95008

$1.917693 .46628 \quad 1.94951$ $1.905843 .45135 \quad 1.94894$ 1.894103 .436531 .94836 $1.88247 \quad 3.42183 \quad 1.94778$ 1.870953 .407241 .94719

$1.85954 \quad 3.39276 \quad 1.94660$ $1.84823 \quad 3.37840 \quad 1.94600$ $1.83703 \quad 3.364151 .94540$ $1.825943 .35000 \quad 1.94480$ $1.81495 \quad 3.33596 \quad 1.94419$

$1.80405 \quad 3.32202 \quad 1.94358$ $1.79326 \quad 3.308191 .94296$ $1.78256 \quad 3.29446 \quad 1.94234$ $1.771973 .28084 \quad 1.94172$ 1.761463 .267311 .94109

$1.75105 \quad 3.25388 \quad 1.94045$ $1.74073 \quad 3.24054 \quad 1.93982$ $1.73050 \quad 3.22730 \quad 1.93917$ 1.720373 .214161 .93853 1.710323 .201111 .93788

$1.70036 \quad 3.188151 .93723$ $1.69048 \quad 3.17528 \quad 1.93657$ $1.680693 .16250 \quad 1.93591$ $1.67098 \quad 3.14980 \quad 1.93524$ 1.661363 .137201 .93457

$1.65182 \quad 3.12468 \quad 1.93390$ 1.642353 .112241 .93322 1.632973 .099891 .93253 1.623673 .087631 .93185 1.614443 .075441 .93116
$2.22264 \quad 3.84254 \quad 1.96145$ $2.20799 \quad 3.82479 \quad 1.96098$ $2.193513 .80720 \quad 1.96051$ $2.17918 \quad 3.78978 \quad 1.96004$ $2.16500 \quad 3.77251 \quad 1.95956$

$2.15097 \quad 3.75540 \quad 1.95908$ $2.13710 \quad 3.73844 \quad 1.95859$ $2.12336 \quad 3.721631 .95810$ $2.109773 .70497 \quad 1.95760$ 2.096333 .688451 .95711

$2.08302 \quad 3.67207 \quad 1.95660$ $2.06984 \quad 3.65584 \quad 1.95610$ $2.05680 \quad 3.63974 \quad 1.95558$ $2.043893 .62378 \quad 1.95507$ 2.031113 .607951 .95455

$2.01846 \quad 3.59225 \quad 1.95403$ 2.00593 3.57668 1.95350 $1.993523 .56124 \quad 1.95297$ 1.981243 .545931 .95243 $1.969073 .53074 \quad 1.95190$

$1.95703 \quad 3.51567 \quad 1.95135$ $1.94510 \quad 3.50071 \quad 1.95081$ $1.93328 \quad 3.48588 \quad 1.95025$ $1.92157 \quad 3.47116 \quad 1.94970$ $1.909973 .45656 \quad 1.94914$

$1.89849 \quad 3.44207 \quad 1.94858$ 1.887113 .427691 .94801 $\begin{array}{llll}1.87583 & 3.41342 & 1.94744\end{array}$ $1.864663 .39926 \quad 1.94686$ 1.853593 .385211 .94629

$1.84262 \quad 3.37126 \quad 1.94570$ $1.83175 \quad 3.35741 \quad 1.94512$ $1.82098 \quad 3.343671 .94452$ 1.810303 .330021 .94393 1.799723 .316481 .94333

$1.789243 .30303 \quad 1.94273$ $1.77884 \quad 3.289681 .94212$ 1.768543 .276431 .94151 $1.75833 \quad 3.26327 \quad 1.94090$ $1.74820 \quad 3.250201 .94028$

$1.73817 \quad 3.23722 \quad 1.93966$ 1.728223 .224341 .93903 $1.71835 \quad 3.21154 \quad 1.93840$ 1.708573 .198831 .93777 1.698873 .186211 .93713

$\begin{array}{llll}1.68925 & 3.17367 & 1.93648\end{array}$ 1.679713 .161221 .93584 $1.67026 \quad 3.148851 .93519$ $1.66088 \quad 3.13657 \quad 1.93454$ 1.651583 .124371 .93388
1100

1110

1120

1130

1140

1150

1160

1170

1180

1190

1200

1210

1220

1230

1240

1250

1260

1270

1280

1290

1300

1310

1320

1330

1340

1350

1360

1370

1380

1390

1400

1410

1420

1430

1440

1450

1460

1470

1480

1490

1500

1510

1520

1530

1540

1550

1560

1570

1580

1590 
Table II Harmonic Oscillator Contributions to the Thermodynamic

Functions (in units of calories, moles, and ${ }^{\circ} \mathrm{K}$ )

$\operatorname{cm}^{-1}-\left(F^{\circ}-E_{0}^{0}\right) / T \quad S^{\circ}$

$T=380 C$.
$\mathrm{S}^{\circ}$

$\mathrm{C}_{\mathrm{p}}^{\circ}$

$\mathrm{C}_{\mathrm{p}}^{\circ}$

$-\left(F^{\circ}-E_{0}^{\circ}\right) / T$

So
$T=3900$.

1.605293 .063331 .93046

$1.596213 .05131 \quad 1.92976$

$1.58721 \quad 3.03936 \quad 1.92906$

$1.57828 \quad 3.02749 \quad 1.92835$

$1.56943 \quad 3.01570 \quad 1.92764$

$1.56065 \quad 3.00398 \quad 1.92693$

1.551932 .992341 .92621

$\begin{array}{lll}1.54329 & 2.98078 & 1.92549\end{array}$

$1.53472 \quad 2.96928 \quad 1.92476$

$1.526212 .95786 \quad 1.92403$

$\begin{array}{llll}1.51778 & 2.94651 & 1.92329\end{array}$

$1.50940 \quad 2.93523 \quad 1.92256$

$1.50110 \quad 2.92403 \quad 1.92181$

$1.49286 \quad 2.91289 \quad 1.92107$

1.484682 .901821 .92032

1.476572 .890821 .91956

$1.468522 .87988 \quad 1.91880$

$1.460532 .86901 \quad 1.91804$

$\begin{array}{llll}1.45260 & 2.85821 & 1.91727\end{array}$

$1.444742 .84747 \quad 1.91650$

$\begin{array}{llll}1.43693 & 2.83679 & 1.91573\end{array}$

1.429182 .826181 .91495

1.421492 .815631 .91417

1.413862 .805151 .91338

$1.406292 .79472 \quad 1.91259$

$1.37790 \quad 2.75550 \quad 1.90954$ $1.37040 \quad 2.74509 \quad 1.90871$

$\begin{array}{lllll}1850 \quad 1.36296 & 2.73475 & 1.90787\end{array}$ $\begin{array}{lllll}1860 & 1.35557 & 2.72447 & 1.90704\end{array}$ $\begin{array}{lllll}1870 & 1.34824 & 2.71424 & 1.90619\end{array}$ $\begin{array}{lllll}1880 & 1.34096 \quad 2.70408 \quad 1.90535\end{array}$ $\begin{array}{lllll}1890 & 1.33374 \quad 2.69397 & 1.90450\end{array}$

1900

1910

1920

1930

1940

$\begin{array}{lllll}1950 & 1.29149 & 2.63453 & 1.89931\end{array}$ $\begin{array}{lllll}1960 & 1.28463 & 2.62482 & 1.89843\end{array}$ $\begin{array}{lllll}1970 & 1.27782 & 2.61516 & 1.89754\end{array}$ $\begin{array}{lllll}1980 & 1.27105 & 2.60555 \quad 1.89666\end{array}$ $\begin{array}{lllll}1990 & 1.26434 & 2.59600 & 1.89577\end{array}$

$\begin{array}{lllll}2000 & 1.25767 & 2.58650 & 1.89487\end{array}$ $\begin{array}{lllll}2010 & 1.25105 & 2.57705 & 1.89397\end{array}$ $2020 \quad 1.24448 \quad 2.56766 \quad 1.89307$ $2030 \quad 1.237952 .55831 \quad 1.89216$ $2040 \quad 1.231472 .54901 \quad 1.89125$

$\begin{array}{llllll}2050 & 1.22503 & 2.53977 & 1.89034\end{array}$ $2060 \quad 1.218642 .53057 \quad 1.88942$ $2070 \quad 1.212292 .52142 \quad 1.88849$ $\begin{array}{lllll}2080 & 1.20599 & 2.51232 & 1.88757\end{array}$ $2090 \quad 1.19973 \quad 2.50327 \quad 1.88664$
$1.398772 .78436 \quad 1.91180$ $\begin{array}{llll}1.39131 & 2.77405 & 1.91100\end{array}$ 1.383902 .763811 .91020 1.376552 .753621 .90939 $1.36925 \quad 2.74350 \quad 1.90858$

$1.362012 .73343 \quad 1.90777$ $1.35482 \quad 2.72341 \quad 1.90695$ $1.347682 .71346 \quad 1.90613$ $1.340592 .70356 \quad 1.90530$ $1.333552 .69371 \quad 1.90447$

$1.32657 \quad 2.68392 \quad 1.90364$ 1.319632 .674191 .90280 $1.31274 \quad 2.66451 \quad 1.90196$ $1.305912 .65488 \quad 1.90112$ $1.299122 .64530 \quad 1.90027$

$1.29238 \quad 2.63578 \quad 1.89942$ 1.285682 .626311 .89856 1.279042 .616891 .89770 1.272442 .607521 .89684 $1.26588 \quad 2.59820 \quad 1.89597$

$1.25938 \quad 2.58893 \quad 1.89510$ $\begin{array}{llll}1.25291 & 2.57971 & 1.89423\end{array}$ $1.246492 .57054 \quad 1.89335$ 1.240122 .561421 .89246 $1.233792 .55235 \quad 1.89158$
$T=4000$.

$1.64235 \quad 3.11224 \quad 1.93322$ 1.633203 .100201 .93255 $1.62413 \quad 3.088241 .93188$ 1.615133 .076351 .93121 $1.60620 \quad 3.06454 \quad 1.93053$

$1.59734 \quad 3.05281 \quad 1.92985$

$1.58856 \quad 3.041151 .92917$ $1.579843 .02956 \quad 1.92848$ 1.571193 .018051 .92779 1.562623 .006611 .92709

$1.554112 .99525 \quad 1.92639$ 1.545662 .983951 .92569 $1.53728 \quad 2.97272 \quad 1.92498$ $1.52897 \quad 2.96157 \quad 1.92427$ 1.520722 .950481 .92355

$1.51254 \quad 2.93946 \quad 1.92283$ 1.504412 .928501 .92211 $1.49635 \quad 2.91761 \quad 1.92138$ $1.488352 .90679 \quad 1.92065$ 1.480422 .896031 .91992

$1.472542 .88534 \quad 1.91918$ $1.464722 .87471 \quad 1.91844$ $1.45696 \quad 2.86414 \quad 1.91769$ 1.449252 .853631 .91695 1.441612 .843191 .91619

$1.43402 \quad 2.83281 \quad 1.91544$ $1.426492 .82248 \quad 1.91468$ 1.419012 .812221 .91391 $1.41158 \quad 2.802011 .91314$ $1.40422 \quad 2.79187 \quad 1.91237$

$1.39690 \quad 2.78178 \quad 1.91160$ $1.389642 .77174 \quad 1.91082$ $1.382432 .76177 \quad 1.91003$ $\begin{array}{llll}1.37527 & 2.75185 & 1.90925\end{array}$ $1.36816 \quad 2.74198 \quad 1.90846$

$1.361112 .73217 \quad 1.90766$ $1.354102 .72242 \quad 1.90687$ $1.347142 .71271 \quad 1.90607$ $1.340242 .70306 \quad 1.90526$ $1.33338 \quad 2.69347 \quad 1.90445$

$1.32657 \quad 2.68392 \quad 1.90364$ $1.31980 \quad 2.67443 \quad 1.90282$ $1.313092 .66499 \quad 1.90200$ 1.306422 .655601 .90118 $1.29980 \quad 2.64626 \quad 1.90035$

$\begin{array}{llll}1.29322 & 2.63697 & 1.89952\end{array}$ 1.286692 .627731 .89869 1.280202 .618541 .89785 1.273752 .609391 .89701 $1.26736 \quad 2.60029 \quad 1.89617$
1600

1610

1620

1630

1640

1650

1660

1670

1680

1690

1700

1710

1720

1730

1740

1750

1760

1770

1780

1790

1800

1810

1820

1830

1840

1850

1860

1870

1880

1890

1900

1910

1920

1930

1940

1950

1960

1970

1980

1990

2000

2010

2020

2030

2040

2050

2060

2070

2080

2090 
Table II Harmonic Oscillator Contributions to the Thermodynamic

Functions (in units of calories, moles, and ${ }^{\circ} \mathrm{K}$ )
$\mathrm{cm}^{-1}-\left(\mathrm{F}^{\circ}-\mathrm{E}_{\mathrm{O}}^{\circ}\right) / \mathrm{T} \quad \mathrm{S}^{\circ}$
$\mathrm{C}_{\mathrm{p}}^{\circ} \quad-\left(\mathrm{F} \circ-\mathrm{E}_{\mathrm{O}}^{\circ}\right) / \mathrm{T} \quad \mathrm{SO}^{\circ}$
$\mathrm{C}_{\mathrm{p}}^{\circ}$
$-\left(\mathrm{F}^{\circ}-\mathrm{E}_{0}^{\circ}\right) / \mathrm{T}$
So
$\mathrm{Co}_{\mathrm{p}}$

$\mathrm{cm}^{-1}$

$T=3800$

$T=3900$

$T=4000$

$\begin{array}{lllll}2100 & 1.19352 & 2.49427 & 1.88570\end{array}$ $2110 \quad 1.18735 \quad 2.48531 \quad 1.88476$ $2120 \quad 1.18122 \quad 2.47641 \quad 1.88382$ $2130 \quad 1.17513 \quad 2.46754 \quad 1.88288$ $\begin{array}{lllll}2140 \quad 1.16908 \quad 2.45873 & 1.88193\end{array}$

$\begin{array}{lllll}2150 & 1.16307 & 2.44995 & 1.88097\end{array}$ $2160 \quad 1.157112 .441231 .88002$ $\begin{array}{lllll}2170 & 1.15118 & 2.43255 & 1.87905\end{array}$ $2180 \quad 1.14530 \quad 2.42391 \quad 1.87809$ $2190 \quad 1.13945 \quad 2.41532 \quad 1.87712$ $\begin{array}{lllll}2200 \quad 1.13365 & 2.40677 & 1.87615\end{array}$ $\begin{array}{lllll}2210 & 1.12788 & 2.39826 & 1.87517\end{array}$ $\begin{array}{lllll}2220 \quad 1.12215 & 2.38980 & 1.87419\end{array}$ $2230 \quad 1.11646 \quad 2.38138 \quad 1.87320$ $\begin{array}{lllll}2240 & 1.11081 & 2.37300 & 1.87222\end{array}$

$2250 \quad 1.105192 .36466 \quad 1.87122$ $\begin{array}{lllll}2260 \quad 1.09961 & 2.35636 & 1.87023\end{array}$ $\begin{array}{llllll}2270 & 1.09407 & 2.34811 & 1.86923\end{array}$ $\begin{array}{lllll}2280 & 1.08856 & 2.33989 & 1.86822\end{array}$ $\begin{array}{lllll}2290 & 1.08309 & 2.33172 & 1.86722\end{array}$

$\begin{array}{lllll}2300 & 1.07766 & 2.32359 & 1.86621\end{array}$ $\begin{array}{lllll}2310 & 1.07226 & 2.31549 & 1.86519\end{array}$ $\begin{array}{lllll}2320 & 1.06689 & 2.30744 & 1.86417\end{array}$ $2330 \quad 1.061562 .29942 \quad 1.86315$ $\begin{array}{llll}2340 \quad 1.05627 & 2.29145 \quad 1.86212\end{array}$

2350 2360 2370 2380 2390

2400 2410 2420

2430

2440

2450

2460

2470

2480

2490

2500

2510

2520

2530

2540

2550

2560

2570

2580

2590 $\begin{array}{llll}1.05100 & 2.28351 & 1.86110\end{array}$ $1.04578 \quad 2.27561 \quad 1.86006$ $1.04058 \quad 2.26774 \quad 1.85902$ 1.035422 .259921 .85798 1.030292 .252131 .85694

$1.02520 \quad 2.24438 \quad 1.85589$ $1.020132 .23666 \quad 1.85484$ $1.01510 \quad 2.22899 \quad 1.85378$ $1.010102 .22134 \quad 1.85273$ $1.005132 .21374 \quad 1.85166$

$1.000192 .20617 \quad 1.85060$ 0.995292 .198631 .84953 0.990412 .191131 .84845 $0.985562 .18366 \quad 1.84738$ 0.980752 .176231 .84630

$0.97596 \quad 2.16883 \quad 1.84521$ $0.971202 .16147 \quad 1.84412$ $0.96648 \quad 2.15414 \quad 1.84303$ $0.96178 \quad 2.14684 \quad 1.84194$ 0.957112 .139581 .84084

$0.952472 .13235 \quad 1.83974$ 0.947862 .125151 .83863 $0.943272 .11793 \quad 1.83752$ 0.938712 .110851 .83641 0.934182 .103751 .83529 $\begin{array}{llll}1.22750 & 2.54332 & 1.89069\end{array}$ $1.22126 \quad 2.53434 \quad 1.88979$ $1.215062 .52541 \quad 1.88890$ $\begin{array}{llll}1.20890 & 2.51652 & 1.89800\end{array}$ $1.20278 \quad 2.50768 \quad 1.88709$

$1.19670 \quad 2.49888 \quad 1.88618$ 1.190662 .490131 .88527 1.184672 .481431 .88435 $1.178712 .47276 \quad 1.88344$ 1.172802 .464151 .88251

$1.16692 \quad 2.45557 \quad 1.88158$ $1.161082 .44704 \quad 1.88065$ $1.15528 \quad 2.43855 \quad 1.87972$ $1.149522 .43011 \quad 1.87878$ $1.14380 \quad 2.42170 \quad 1.87784$

$1.138112 .41334 \quad 1.87689$ $1.13246 \quad 2.40502 \quad 1.87595$ $1.126852 .39674 \quad 1.87499$ $1.121272 .38850 \quad 1.87404$ $1.11573 \quad 2.38030 \quad 1.87308$

$1.110232 .37214 \quad 1.87211$ $1.10476 \quad 2.36402 \quad 1.87115$ $1.09932 \quad 2.35594 \quad 1.87018$ $1.093932 .34790 \quad 1.86920$ $1.08856 \quad 2.33989 \quad 1.86822$

$1.083232 .33193 \quad 1.86724$ $1.077932 .32400 \quad 1.86626$ 1.072672 .316111 .86527 $1.067442 .30826 \quad 1.86428$ $1.06224 \quad 2.30045 \quad 1.86328$

$\begin{array}{llll}1.05708 & 2.29267 & 1.86228\end{array}$ 1.051952 .284931 .86128 $1.046852 .27722 \quad 1.86027$ $\begin{array}{llll}1.04178 & 2.26955 & 1.85926\end{array}$ $1.03674 \quad 2.261921 .85825$

$\begin{array}{lll}1.03174 & 2.25432 & 1.85723\end{array}$ $1.02676 \quad 2.24676 \quad 1.85621$ 1.021822 .239231 .85519 $1.016902 .23174 \quad 1.85416$ 1.012022 .224281 .85313

1.007172 .216851 .85210 $1.002342 .20946 \quad 1.85106$ 0.997552 .202101 .85002 $0.99278 \quad 2.19478 \quad 1.84898$ $0.98805 \quad 2.18749 \quad 1.84793$

$0.983342 .18023 \quad 1.84688$ $0.97866 \quad 2.173001 .84582$ $0.97401 \quad 2.16581 \quad 1.84477$ $0.96938 \quad 2.15865 \quad 1.84370$ $0.964792 .15152 \quad 1.84264$
$1.26100 \quad 2.59125 \quad 1.89532$ $\begin{array}{llll}1.25469 & 2.58224 & 1.89447\end{array}$ $1.248422 .57329 \quad 1.89361$ 1.242192 .564381 .89275 $1.23600 \quad 2.55552 \quad 1.89189$

$\begin{array}{llll}1.22985 & 2.54670 & 1.89102\end{array}$ 1.223752 .337921 .89015 $1.217692 .52920 \quad 1.88928$ 1.211662 .520511 .88840 $1.205682 .51187 \quad 1.88752$

$1.19973 \quad 2.50327 \quad 1.88664$ 1.193832 .494721 .88575 $1.18796 \quad 2.48621 \quad 1.88486$ 1. 182132.477741 .88396 $1.17634 \quad 2.46931 \quad 1.88307$

1.170592 .460931 .88216 1.164872 .452581 .88126 $1.159192 .44428 \quad 1.88035$ $1.153552 .43601 \quad 1.87944$ $1.147942 .42779 \quad 1.87852$

1.142372 .419611 .87760 $1.136842 .41146 \quad 1.87668$ $1.13134 \quad 2.40336 \quad 1.87576$ $1.125872 .39529 \quad 1.87483$ $1.120442 .38727 \quad 1.87389$

$1.11504 \quad 2.37928 \quad 1.87296$ 1.109682 .371331 .87202 $1.10435 \quad 2.36341 \quad 1.87107$ $1.09905 \quad 2.35554 \quad 1.87013$ $1.093792 .34770 \quad 1.86918$

$1.08856 \quad 2.33989 \quad 1.86822$ $1.08336 \quad 2.332131 .86727$ $1.07820 \quad 2.32440 \quad 1.86631$ $1.07306 \quad 2.31670 \quad 1.86534$ 1.067962 .309051 .86438

$1.06289 \quad 2.30142 \quad 1.86341$ 1.057852 .293831 .86243 $1.052842 .28628 \quad 1.86146$ $1.04786 \quad 2.27976 \quad 1.86048$ 1.042922 .271281 .85949

$1.03800 \quad 2.26383 \quad 1.85850$ 1.033112 .256411 .85751 1.028252 .249021 .85652 1.023422 .241671 .85552 1.018622 .234361 .85452

$\begin{array}{llll}1.01385 & 2.22707 & 1.85352\end{array}$ 1.009102 .219821 .85251 1.004392 .212601 .85150 $0.99970 \quad 2.20541 \quad 1.85049$ $0.995042 .19825 \quad 1.84947$
2100

2110

2120

2130

2140

2150

2160

2170

2180

2190

2200

2210

2220

2230

2240

2250

2260

2270

2280

2290

2300

2310

2320

2330

2340

2350

2360

2370

2380

2390

2400

2410

2420

2430

2440

2450

2460

2470

2480

2490

2500

2510

2520

2530

2540

2550

2560

2570

2580

2590 
Table II Harmonic Oscillator Contributions to the Thermodynamic

Functions (in units of calories, moles, and ${ }^{\circ} \mathrm{K}$ )

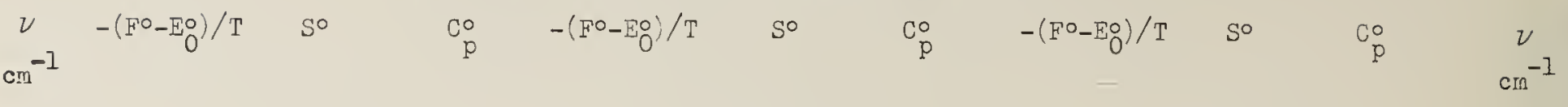

$T=380 \mathrm{C}$.

2600

2610

2620

2630

2640

2650

2660

2670

2680

2690

2700

2710

2720

2730

2740

2750

2760

2770

2780

2790

2800

2810

2820

2830

2840

2850

2860

2870

2880

2890

2900

2910

2920

2930

2940

2950

2960

2970

2980

2990

3000

3010

3020

3030

3040

3050

3060

3070

3080

3090
$T=3900$.

$0.96022 \quad 2.14442 \quad 1.84157$ $0.95568 \quad 2.13735 \quad 1.84050$ $0.95116 \quad 2.13031 \quad 1.83942$ $0.946682 .12331 \quad 1.838335$ 0.942222 .116331 .83726

$0.93778 \quad 2.10939 \quad 1.83618$ $0.933372 .10248 \quad 1.83507$ 0.928972 .095591 .83400 $0.924642 .08874 \quad 1.83290$ $0.920302 .08191 \quad 1.83181$

$0.91600 \quad 2.075121 .83071$ $0.91172 \quad 2.068351 .82960$ $0.90746 \quad 2.06162 \quad 1.82849$ $0.90323 \quad 2.05491 \quad 1.82738$ 0.899032 .048231 .82627

$0.89484 \quad 2.04158 \quad 1.82515$ $0.89069 \quad 2.03495 \quad 1.82403$ 0.886552 .028301 .82290 $0.88244 \quad 2.02179 \quad 1.82178$ $0.87835 \quad 2.01525 \quad 1.82064$

$0.87429 \quad 2.00874 \quad 1.81951$ $0.87025 \quad 2.00226 \quad 1.81837$ $0.86623 \quad 1.99580 \quad 1.81723$ $0.86224 \quad 1.98937 \quad 1.81609$ $0.85827 \quad 1.98296 \quad 1.81494$

$0.85432 \quad 1.97659 \quad 1.81379$ $0.85039 \quad 1.97024 \quad 1.81264$ $0.846491 .96391 \quad 1.81148$ $0.842611 .95 / 611.81032$ 0.838751 .951341 .80916

$0.83491 \quad 1.94509 \quad 1.80800$ $0.831091 .93887 \quad 1.80683$ $0.82729 \quad 1.93267 \quad 1.80565$ $0.823521 .92650 \quad 1.80448$ $0.81976 \quad 1.920361 .80330$

0.816031 .914231 .80212 0.812321 .908141 .80094 0.808631 .902071 .79975 $0.80496 \quad 1.89602 \quad 1.79856$ 0.801301 .890001 .79736

$0.79767 \quad 1.88400 \quad 1.79617$ $0.79406 \quad 1.87802 \quad 1.79497$ $0.79047 \quad 1.87207 \quad 1.79377$ $0.78690 \quad 1.86614 \quad 1.79256$ $0.783351 .86024 \quad 1.79135$

$0.77981 \quad 1.85436 \quad 1.79014$ $0.776301 .84850 \quad 1.78893$ $0.77281 \quad 1.84266 \quad 1.78771$ 0.769331 .836851 .78649 $0.76587 \quad 1.83106 \quad 1.78526$
$T=4000$.

$0.990412 .19113 \quad 1.84845$ $0.985812 .18403 \quad 1.84743$ $0.981232 .17697 \quad 1.84640$ $0.97668 \quad 2.16994 \quad 1.84537$ $0.97215 \quad 2.16294 \quad 1.84434$

$0.96766 \quad 2.15597 \quad 1.84330$ $0.963192 .14903 \quad 1.84227$ $0.958142 .14212 \quad 1.84122$ $0.954322 .13524 \quad 1.84018$ $0.94993 \quad 2.12838 \quad 1.83913$

$0.94556 \quad 2.12156 \quad 1.83808$ $0.94122 \quad 2.11477 \quad 1.83702$ $0.93690 \quad 2.10800 \quad 1.83596$ $0.932612 .10127 \quad 1.83490$ $0.928342 .09456 \quad 1.83384$

$0.92409 \quad 2.08788 \quad 1.83277$ 0.919872 .081231 .83170 $0.91568 \quad 2.07461 \quad 1.83062$ $0.91150 \quad 2.06801 \quad 1.82955$ 0.907362 .061451 .82846

$0.903232 .05491 \quad 1.82738$ 0.899132 .048391 .82629 $0.89505 \quad 2.04191 \quad 1.82520$ $0.89100 \quad 2.03545 \quad 1.82411$ $0.886962 .02902 \quad 1.82302$

$0.88295 \quad 2.02261 \quad 1.82192$ 0.878972 .016231 .82081 $\begin{array}{llll}0.87500 & 2.00988 & 1.81971\end{array}$ $0.87106 \quad 2.00355 \quad 1.51860$ $0.86714 \quad 1.99725 \quad 1.81749$

$0.86324 \quad 1.99097 \quad 1.81638$ $0.85936 \quad 1.98472 \quad 1.81526$ $0.85550 \quad 1.97850 \quad 1.81414$ $0.85167 \quad 1.97230 \quad 1.81301$ 0.847851 .966121 .81189

$0.84406 \quad 1.95997 \quad 1.81076$ $0.840291 .95384 \quad 1.80963$ $0.83654 \quad 1.94774 \quad 1.80849$ $0.83280 \quad 1.94167 \quad 1.80735$ 0.829091 .935611 .80621

$0.82540 \quad 1.92958 \quad 1.80507$ 0.821731 .923581 .80392 $0.81808 \quad 1.91760 \quad 1.80277$ 0.814451 .911641 .30162 $0.81084 \quad 1.90571 \quad 1.80046$

$0.80725 \quad 1.89980 \quad 1.79930$ $0.803671 .39391 \quad 1.79814$ 0.800121 .888041 .79698 0.796591 .882201 .79581 $0.79307 \quad 1.87638 \quad 1.79464$
2600

2610

2620

2630

2640

2650

2660

2670

2680

2690

2700

2710

2720

2730

2740

2750

2760

2770

2780

2790

2800

2810

2820

2830

2840

2850

2860

2870

2880

2890

2900

2910

2920

2930

2940

2950

2960

2970

2980

2990

3000

3010

3020

3030

3040

3050

3060

3070

3080

3090 
Table II Harmonic Oscillator Contributions to the Thermodynamic

Functions (in units of calories, moles, and ${ }^{\circ} \mathrm{K}$ )

$\mathrm{cm}^{-1}-\left(\mathrm{F}^{\circ}-\mathrm{E}_{0}^{\circ}\right) / \mathrm{T} \quad \mathrm{S}^{\circ}$

$\mathrm{T}=3800$.

$\begin{array}{cc}\mathrm{C}_{\mathrm{p}}^{\circ} & -\left(\mathrm{F} \circ-\mathrm{E}_{\mathrm{O}} \mathrm{O}\right) / \mathrm{T}\end{array}$

So

$\mathrm{C}_{\mathrm{p}}^{\circ}$

$-\left(F^{\circ}-E_{O} O\right) / T$

So

$\stackrel{\mathrm{P}}{\mathrm{p}} \quad \begin{gathered}\nu \\ \mathrm{Cn}^{-1}\end{gathered}$

$T=3900$.

$0.76244 \quad 1.82530 \quad 1.73404$ $0.75902 \quad 1.81955 \quad 1.78281$ $0.755621 .81 .383 \quad 1.78157$ $0.75223 \quad 1.80813 \quad 1.78034$ $0.74887 \quad 1.80246 \quad 1.77910$

$0.74552 \quad 1.79680 \quad 1.77786$ $0.742191 .79117 \quad 1.77662$ $0.73888 \quad 1.78556 \quad 1.77537$ $0.735591 .77997 \quad 1.77412$ $\begin{array}{llll}0.73231 & 1.77440 & 1.77287\end{array}$

$0.72906 \quad 1.76885 \quad 1.77161$ 0.725821 .763331 .77035 $0.722591 .75782 \quad 1.76909$ $0.719391 .75234 \quad 1.76783$ $\begin{array}{llll}0.71620 & 1.74688 & 1.76656\end{array}$

$0.71302 \quad 1.74143 \quad 1.76529$ $0.709871 .73601 \quad 1.76402$ $0.70673 \quad 1.73061 \quad 1.76274$ $0.70361 \quad 1.72523 \quad 1.76146$ 0.700501 .719871 .76018

$0.69741 \quad 1.71453 \quad 1.75890$ $0.694331 .70921 \quad 1.75761$ $0.69128 \quad 1.70391 \quad 1.75632$ $0.688231 .69863 \quad 1.75503$ 0.685211 .693371 .75374

$0.68220 \quad 1.68813 \quad 1.75244$ $0.67920 \quad 1.68291 \quad 1.75114$ $0.67622 \quad 1.67770 \quad 1.74983$ $0.67326 \quad 1.67252 \quad 1.74853$ $0.67031 \quad 1.66736 \quad 1.74722$

$0.66738 \quad 1.66221 \quad 1.74591$ $0.66446 \quad 1.65709 \quad 1.74459$ $0.66155 \quad 1.65198 \quad 1.74328$ $0.65867 \quad 1.64689 \quad 1.74196$ $0.65579 \quad 1.64182 \quad 1.74064$

$0.652931 .63677 \quad 1.73931$ $0.65009 \quad 1.63174 \quad 1.73799$ $0.64726 \quad 1.62673 \quad 1.73666$ $0.64444 \quad 1.62173 \quad 1.73532$ 0.641641 .616751 .73399

$0.63886 \quad 1.61180 \quad 1.73265$ $0.636081 .60685 \quad 1.73131$ $0.63332 \quad 1.601931 .72997$ $0.63058 \quad 1.59702 \quad 1.72862$ $0.627851 .59214 \quad 1.72728$

0.625131 .587271 .72592 $0.622431 .58241 \quad 1.72457$ $0.61974 \quad 1.57758 \quad 1.72322$ $0.61706 \quad 1.57276 \quad 1.72186$ $0.614401 .56796 \quad 1.72050$
$\mathrm{T}=4000$.

$0.78958 \quad 1.870591 .79346$ $0.78610 \quad 1.86481 \quad 1.79229$ $0.782641 .85906 \quad 1.79111$ 0.779201 .853331 .78993 0.775781 .847621 .78874

$0.77237 \quad 1.84194 \quad 1.78756$ $0.76898 \quad 1.83627 \quad 1.78636$ 0.765621 .830631 .78517 $0.762261 .82501 \quad 1.78398$ $0.758931 .81941 \quad 1.78278$

0.755621 .813831 .78157 $0.75232 \quad 1.808281 .78037$ $0.74904 \quad 1.80274 \quad 1.77916$ $0.74577 \quad 1.79722 \quad 1.77795$ $0.742531 .79173 \quad 1.77674$

3100 3110

3120

3130

3140

3150

3160

3170

3180

3190

3200

3210

3220

3230

3240

$\begin{array}{llll}0.73930 & 1.78626 & 1.77552\end{array}$

$\begin{array}{llll}0.73608 & 1.78080 & 1.77431\end{array}$

$0.732891 .77537 \quad 1.77309$

$0.72971 \quad 1.76996 \quad 1.77186$

$\begin{array}{llll}0.72654 & 1.76457 & 1.77064\end{array}$

3250

3260

3270

3280

3290

$\begin{array}{llll}0.72340 & 1.75920 & 1.76941\end{array}$

$0.72027 \quad 1.75384 \quad 1.76817$

$0.71715 \quad 1.74851 \quad 1.76694$

0.714051 .743201 .76570

$0.710971 .73791 \quad 1.76446$

3300

3310

3320

3330

3340

$0.70790 \quad 1.73263 \quad 1.76322$ $\begin{array}{llll}0.70485 & 1.72738 & 1.76197\end{array}$ $0.70182 \quad 1.72215 \quad 1.76073$ $0.698801 .71693 \quad 1.75948$ 0.695791 .711731 .75822

3350

3360

3370

3380

3390

$\begin{array}{llll}0.69280 & 1.70656 & 1.75697\end{array}$ $0.689831 .70140 \quad 1.75571$ $0.68687 \quad 1.69626 \quad 1.75445$ $0.683931 .69114 \quad 1.75318$ $0.681001 .68604 \quad 1.75192$

3400

3410

3420

3430

3440

$0.67808 \quad 1.68095 \quad 1.75065$

$0.67518 \quad 1.67589 \quad 1.74938$

0.672301 .670841 .74810

0.669431 .665811 .74683

$0.66657 \quad 1.66080 \quad 1.74555$

$\begin{array}{llll}0.66373 & 1.65581 & 1.74427\end{array}$

$0.660901 .65084 \quad 1.74298$

$0.65809 \quad 1.64588 \quad 1.74169$

$0.65529 \quad 1.64094 \quad 1.74041$

0.652511 .636021 .73911

$0.64973 \quad 1.63111 \quad 1.73782$ $\begin{array}{llll}0.64698 & 1.62623 & 1.73652\end{array}$ $0.644231 .62136 \quad 1.73522$ $0.64150 \quad 1.61651 \quad 1.73392$ $0.63879 \quad 1.61167 \quad 1.73262$
3450

3460

3470

3480

3490

3500

3510

3520

3530

3540

3550

3560

3570

3580

3590 
Table II Harmonic Oscillator Contributions to the Thermodynamic

Functions (in units of calories, moles, and ${ }^{\circ} \mathrm{K}$ )
$\mathrm{cm}^{-1}$
So

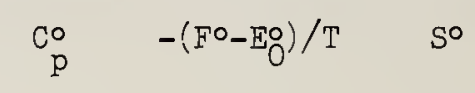
$\mathrm{C}_{\mathrm{p}}$
$-\left(F^{\circ}-E_{O}^{\circ}\right) / T$
So
$\mathrm{C}_{\mathrm{p}}^{\circ}$
$\nu$
$\mathrm{cm}^{-1}$

$\mathrm{T}=3800$.

3600

3610

3620

3630

3640

3650

3660

3670

3680

3690

$\begin{array}{lllll}3700 & 0.56207 & 1.47214 & 1.69173\end{array}$

$\begin{array}{lllll}3710 & 0.55961 & 1.46757 & 1.69028\end{array}$

$\begin{array}{lllll}3720 & 0.55717 & 1.46302 & 1.68883\end{array}$

$\begin{array}{llll}3730 & 0.55474 & 1.45849 & 1.68738\end{array}$

$\begin{array}{llll}3740 & 0.55233 & 1.45398 & 1.68592\end{array}$

$\begin{array}{lllll}3750 & 0.54992 & 1.44948 & 1.68446\end{array}$

$\begin{array}{lllll}3760 & 0.54753 & 1.44499 & 1.68300\end{array}$

$\begin{array}{llll}3770 & 0.54515 & 1.44052 & 1.68153\end{array}$

$\begin{array}{lllll}3780 & 0.54278 & 1.43607 & 1.68007\end{array}$

$\begin{array}{lllll}3790 & 0.54042 & 1.43164 & 1.67860\end{array}$

$\begin{array}{lllll}3800 & 0.53808 & 1.42721 & 1.67713\end{array}$

$\begin{array}{lllll}3810 & 0.53574 & 1.42281 & 1.67565\end{array}$

$\begin{array}{lllll}3820 & 0.53342 & 1.41842 & 1.67418\end{array}$

$\begin{array}{lllll}3830 & 0.53111 & 1.41404 & 1.67270\end{array}$

$\begin{array}{lllll}3840 & 0.52881 & 1.40968 & 1.67122\end{array}$

$\begin{array}{llll}3850 & 0.52652 & 1.40534 & 1.66973\end{array}$

$\begin{array}{llll}3860 & 0.52424 & 1.40101 & 1.66825\end{array}$

$\begin{array}{llll}3870 & 0.52198 & 1.39669 & 1.66676\end{array}$

$\begin{array}{lllll}3880 & 0.51972 & 1.39240 & 1.66527\end{array}$

$\begin{array}{lllll}3890 & 0.51748 & 1.38811 & 1.66378\end{array}$

$\begin{array}{lllll}3900 & 0.51525 & 1.38384 & 1.66228\end{array}$

$\begin{array}{lllll}3910 & 0.51303 & 1.37959 & 1.66079\end{array}$

$\begin{array}{llll}3920 & 0.51082 & 1.37535 & 1.65929\end{array}$

$\begin{array}{llll}3930 & 0.50861 & 1.37112 & 1.65779\end{array}$

$\begin{array}{lllll}3940 & 0.50643 & 1.36691 & 1.65628\end{array}$

$\begin{array}{llllll}3950 & 0.50425 & 1.36271 & 1.65478\end{array}$

$\begin{array}{lllll}3960 & 0.50208 & 1.35853 & 1.65327\end{array}$

$\begin{array}{lllll}3970 & 0.49992 & 1.35436 & 1.65176\end{array}$

$\begin{array}{lllll}3980 & 0.49777 & 1.35021 & 1.65025\end{array}$

$\begin{array}{lllll}3990 & 0.49564 & 1.34607 & 1.64874\end{array}$

$4000 \quad 0.49351 \quad 1.34195 \quad 1.64722$
$T=3900$.

$0.61175 \quad 1.563171 .71913$ 0.609111 .558411 .71777 0.606491 .553661 .71640 $0.60388 \quad 1.54892 \quad 1.71503$ $0.601281 .54421 \quad 1.71366$

$0.59870 \quad 1.53951 \quad 1.71228$ 0.596131 .534831 .71090 $0.59357 \quad 1.53016 \quad 1.70952$ 0.591021 .525511 .70814 $0.588491 .52088 \quad 1.70676$

$\begin{array}{llll}0.58597 & 1.51626 & 1.70537\end{array}$ $0.58346 \quad 1.511661 .70398$ $0.58097 \quad 1.50707 \quad 1.70259$ $0.57848 \quad 1.50250 \quad 1.70119$ 0.576011 .497951 .69979

$0.57355 \quad 1.49341 \quad 1.69839$ 0.571111 .488891 .69699 0.568671 .484391 .69559 0.566251 .479901 .69418 $0.56384 \quad 1.47542 \quad 1.69277$

$0.56144 \quad 1.470961 .69136$ $0.55905 \quad 1.466521 .68995$ 0.556671 .462091 .68853 $0.554311 .45768 \quad 1.68712$ $0.55196 \quad 1.453281 .68569$

$0.549621 .44890 \quad 1.68427$ $0.547291 .44453 \quad 1.68285$ $0.54497 \quad 1.440181 .68142$ 0.542661 .435841 .67999 $0.54036 \quad 1.431521 .67856$

$0.53808 \quad 1.42721 \quad 1.67713$ 0.535801 .422921 .67569 $0.53354 \quad 1.418641 .67425$ 0.531291 .414381 .67281 0.529041 .410131 .67137

$0.52681 \quad 1.405891 .66992$ 0.524591 .401671 .66848 $0.522381 .39747 \quad 1.66703$ 0.520191 .393281 .66558 $0.518001 .38910 \quad 1.66412$

$0.515821 .38493 \quad 1.66267$
$T=4000$.

$0.63608 \quad 1.606851 .73131$ 0.633391 .602051 .73000 $0.63072 \quad 1.59727 \quad 1.72869$ $0.62805 \quad 1.59250 \quad 1.72738$ $0.625401 .58775 \quad 1.72606$

$0.62277 \quad 1.58302 \quad 1.72474$ 0.620141 .578301 .72342 0.617531 .573601 .72210 0.614931 .568921 .72077 0.612341 .564251 .71944

$0.60977 \quad 1.55960 \quad 1.71811$

$0.607211 .55496 \quad 1.71678$ $0.60466 \quad 1.55034 \quad 1.71544$ 0.602131 .545741 .71410 $0.59960 \quad 1.54115 \quad 1.71276$

$0.59709 \quad 1.53658 \quad 1.71142$ 0.594591 .532021 .71008 $0.59210 \quad 1.52748 \quad 1.70873$ $0.589631 .52296 \quad 1.70738$ $0.58717 \quad 1.51845 \quad 1.70603$

$0.58471 \quad 1.51396 \quad 1.70467$ $0.58228 \quad 1.50948 \quad 1.70332$ $0.579851 .50501 \quad 1.70196$ $0.577431 .50057 \quad 1.70060$ 0.575031 .496131 .69923

$0.572631 .49172 \quad 1.69787$ 0.570251 .487311 .69650 0.567881 .482931 .69513 $0.56552 \quad 1.478551 .69376$ 0.563181 .474201 .69239

0.560841 .469851 .69101 $0.55851 \quad 1.465521 .68963$ $0.556201 .46121 \quad 1.68825$ $0.55390 \quad 1.45691 \quad 1.68687$ 0.551601 .452621 .68548

$0.54932 \quad 1.448351 .68409$ $0.54705 \quad 1.44410 \quad 1.68270$ $0.544791 .43986 \quad 1.68131$ 0.542541 .435631 .67992 $0.540301 .43141 \quad 1.67852$

$0.53808 \quad 1.42721 \quad 1.67713$
3600

3610

3620

3630

3640

3650

3660

3670

3680

3690

3700

3710

3720

3730

3740

3750

3760

3770

3780

3790

3800

3810

3820

3830

3840

3850

3860

3870

3880

3890

3900

3910

3920

3930

3940

3950

3960

3970

3980

3990

4000 
Table II Harmonic Oscillator Contributions to the Thermodynamic

Functions (in units of calories, moles, and ${ }^{\circ} \mathrm{K}$ )
$\mathrm{cm}^{-1}-\left(\mathrm{F}^{\circ}-\mathrm{E}_{0}^{\circ}\right) / \mathrm{T} \quad$ So
$\mathrm{C}_{\mathrm{p}}^{\circ} \quad-\left(\mathrm{F}^{\circ}-\mathrm{E}_{\mathrm{O}}^{\circ}\right) / \mathrm{T} \quad \mathrm{S}^{\circ}$
$\mathrm{C}_{\mathrm{p}}^{\circ}$
$\mathrm{C}_{\mathrm{p}}^{\circ} \quad-\left(\mathrm{F}^{\circ}-\mathrm{E}_{\mathrm{O}}^{0}\right) / \mathrm{T}$
So

$\mathrm{cm}^{-1}$

\author{
$T=4100$
}

$\mathrm{T}=4200$.

$T=4300$.

$100 \quad 6.691318 .64382 \quad 1.98696$

$110 \quad 6.50538 \quad 8.45444 \quad 1.98692$

$120 \quad 6.33594 \quad 8.28156 \quad 1.98688$

$130 \quad 6.18034 \quad 8.12253 \quad 1.98682$

$\begin{array}{lll}140 \quad 6.03653 \quad 7.97529 & 1.98677\end{array}$

$\begin{array}{llll}150 & 5.90289 & 7.83822 & 1.98671\end{array}$

$160 \quad 5.778107 .71000 \quad 1.98665$

$170 \quad 5.66108 \quad 7.58956 \quad 1.98658$

$130 \quad 5.550957 .47602 \quad 1.98651$

$190 \quad 5.44696 \quad 7.36861 \quad 1.98643$

$200 \quad 5.34847 \quad 7.26672 \quad 1.98635$

$210 \quad 5.254967 .16981 \quad 1.98627$

$220 \quad 5.16596 \quad 7.07741 \quad 1.98618$

$230 \quad 5.08107 \quad 6.98912 \quad 1.98609$

$240 \quad 4.99994 \quad 6.90460 \quad 1.98600$

$250 \quad 4.922256 .82353 \quad 1.98590$

$260 \quad 4.847756 .74564 \quad 1.98579$

$270 \quad 4.776196 .670701 .98568$

$\begin{array}{lllll}280 & 4.70735 & 6.59849 & 1.98557\end{array}$

$290 \quad 4.641046 .52881 \quad 1.98546$

$300 \quad 4.57710 \quad 6.46151 \quad 1.98534$ $\begin{array}{lllll}310 & 4.51537 & 6.39641 & 1.98521\end{array}$

$320 \quad 4.45570 \quad 6.33338 \quad 1.98508$

$\begin{array}{lllll}330 & 4.39797 & 6.27230 & 1.98495\end{array}$

$340 \quad 4.342076 .21305 \quad 1.98481$

$\begin{array}{lllll}350 & 4.28788 & 6.15551 & 1.98467\end{array}$

$\begin{array}{llll}360 & 4.23532 & 6.09960 \quad 1.98453\end{array}$

$\begin{array}{lllll}370 & 4.18428 & 6.04523 & 1.98438\end{array}$

$\begin{array}{lllll}380 & 4.13470 & 5.99232 & 1.98423\end{array}$

$\begin{array}{lll}390 & 4.08649 \quad 5.94078 \quad 1.98407\end{array}$

$400 \quad 4.03958 \quad 5.89055 \quad 1.98391$

$410 \quad 3.993925 .84156 \quad 1.98375$

$\begin{array}{lllll}420 & 3.94944 & 5.79376 & 1.98358\end{array}$

$430 \quad 3.90608 \quad 5.74709 \quad 1.98340$

$\begin{array}{lll}440 \quad 3.86379 & 5.70149 & 1.98323\end{array}$

$\begin{array}{llll}450 & 3.82253 & 5.65692 \quad 1.98305\end{array}$

$460 \quad 3.78225 \quad 5.61334 \quad 1.98286$

$\begin{array}{llllll}470 & 3.74290 & 5.57070 & 1.98267\end{array}$

$\begin{array}{llll}480 & 3.70446 & 5.52896 & 1.98248\end{array}$

$\begin{array}{lllll}490 & 3.66687 & 5.48808 & 1.98228\end{array}$

$\begin{array}{lllll}500 & 3.63011 & 5.44804 & 1.98208\end{array}$

$\begin{array}{lllll}510 & 3.59414 & 5.40879 & 1.98187\end{array}$

$\begin{array}{lllll}520 & 3.55894 & 5.37031 & 1.98166\end{array}$

$530 \quad 3.52447 \quad 5.33256 \quad 1.98145$

$540 \quad 3.49070$
$5.29553 \quad 1.98123$

$\begin{array}{lllll}550 & 3.45761 & 5.25918 & 1.98101\end{array}$

$\begin{array}{lllll}560 & 3.42518 & 5.22348 & 1.98079\end{array}$

$\begin{array}{lllll}570 & 3.39338 & 5.18843 & 1.98056\end{array}$

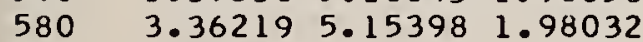

$590 \quad 3.331595 .12013 \quad 1.98009$
$6.738378 .69170 \quad 1.98698$ $6.55236 \quad 8.50232 \quad 1.98694$ 6.382838 .329441 .98689 $6.22716 \quad 8.17041 \quad 1.98684$ $6.083278 .02317 \quad 1.98679$

$5.949547 .88609 \quad 1.98673$ $5.82467 \quad 7.75787 \quad 1.98667$ $5.707577 .63744 \quad 1.98661$ $5.59735 \quad 7.52389 \quad 1.98654$ $5.49328 \quad 7.41648 \quad 1.98647$

$5.39472 \quad 7.31459 \quad 1.98639$ 5.301137 .217681 .98631 $5.21205 \quad 7.12528 \quad 1.98623$ 5.127077 .036991 .98614 5.045866 .952461 .98605

$4.968096 .87139 \quad 1.98596$ 4.893516 .793501 .98586 4.821876 .718551 .98575 $4.75295 \quad 6.64634 \quad 1.98565$ $4.68656 \quad 6.57666 \quad 1.98554$

$4.62254 \quad 6.50935 \quad 1.98542$ 4.560736 .444251 .98530 $4.50098 \quad 6.38122 \quad 1.98518$ 4.443176 .320131 .98505 $4.387196 .26088 \quad 1.98492$

$4.33292 \quad 6.20334 \quad 1.98479$ 4.280286 .147431 .98465 $4.22916 \quad 6.093051 .98451$ $4.17950 \quad 6.04013 \quad 1.98437$ 4.131215 .988591 .98422

4.084235 .938361 .98406 $4.03848 \quad 5.889371 .98391$ 3.993925 .841561 .98375 $3.95048 \quad 5.79488 \quad 1.98358$ $3.908125 .74928 \quad 1.98341$

$\begin{array}{llll}3.86678 & 5.70471 & 1.98324\end{array}$ 3.826425 .661131 .98306 $3.78699 \quad 5.61848 \quad 1.98288$ $3.748475 .57673 \quad 1.98270$ $3.71080 \quad 5.53585 \quad 1.98251$

$3.67397 \quad 5.49580 \quad 1.98232$ 3.637925 .456551 .98212 3.602645 .418061 .98192 $3.568095 .38031 \quad 1.98172$ 3.534245 .343271 .98151

$3.501085 .30692 \quad 1.98130$ $3.468575 .27122 \quad 1.98109$ $3.436695 .23616 \quad 1.98087$ 3.405425 .201711 .98065 3.374745 .167851 .98042
$6.78435 \quad 8.73846 \quad 1.98699$

$6.598258 .54908 \quad 1.98695$

$6.428658 .37619 \quad 1.98690$

6.272898 .217161 .98686

$6.128938 .06992 \quad 1.98681$

5.99512 $7.93284 \quad 1.98675$

5.870177 .804621 .98669

5.752997 .684181 .98663

5.642707 .570631 .98657

5.538557 .463221 .98650

5.439917 .361331 .98643

5.346247 .264421 .98635

5.257097 .172011 .98627

5.172047 .083721 .98619

5.090746 .999191 .98610

$5.01290 \quad 6.91812 \quad 1.98601$ 4.938246 .840231 .98592

$4.866526 .76528 \quad 1.98582$

$4.797536 .69306 \quad 1.98572$

$4.73107 \quad 6.62338 \quad 1.98561$

$4.666976 .55607 \quad 1.98550$ 4.605086 .490961 .98539 $4.545256 .42793 \quad 1.98527$ 4.487376 .366851 .98515 4.431316 .307581 .98503

$4.37696 \quad 6.25004 \quad 1.98490$ 4.324246 .194131 .98477 $4.273056 .13975 \quad 1.98463$ 4.223316 .086831 .98449 $4.174956 .03528 \quad 1.98435$

$4.127895 .98504 \quad 1.98421$ 4.082075 .936051 .98406 $4.037435 .88824 \quad 1.98390$ 3.993925 .841561 .98375 $3.95148 \quad 5.79596 \quad 1.98358$

$3.910065 .75138 \quad 1.98342$ 3.869635 .707791 .98325 3.830135 .665141 .98308 3.791535 .623391 .98290 $3.753795 .58251 \quad 1.98272$

$3.71688 \quad 5.54245 \quad 1.98254$ $3.68016 \quad 5.50319 \quad 1.98235$ $3.64540 \quad 5.46470 \quad 1.98216$ $3.610785 .42695 \quad 1.98197$ $3.576865 .38990 \quad 1.98177$

$3.54362 \quad 5.35354 \quad 1.98157$ $3.511035 .31784 \quad 1.98137$ $3.47908 \quad 5.28277 \quad 1.98116$ 3.447745 .248321 .98094 3.416995 .214451 .98073
100

110

120

130

140

150

160

170

180

190

200

210

220

230

240

250

260

270

280

290

300

310

320

330

340

350

360

370

380

390

400

410

420

430

440

450

460

470

480

490

500

510

520

530

540

550

560

570

580

590 
Table II Harmonic Oscillator Contributions to the Thermodynamic

Functions (in units of calories, moles, and ${ }^{\circ} \mathrm{K}$ )
$\mathrm{cm}^{-1}$
$\mathrm{co}_{\mathrm{p}}$
$-\left(\mathrm{F}^{\circ}-\mathrm{E}_{\mathrm{O}} \mathrm{O}\right) / \mathrm{T} \quad \mathrm{S}^{\circ}$
$\mathrm{C}_{\mathrm{p}}$
$-\left(F^{\circ}-E_{O}^{\circ}\right) / T$
so
$\stackrel{\mathrm{c}}{\mathrm{p}} \quad \begin{gathered}\nu \\ \mathrm{cm}^{-1}\end{gathered}$

$T=4100$.

600

610

620

630

640

650

660

670

680

690

700

710

720

730

740

750

760

770

780

790

$800 \quad 2.79676 \quad 4.51803 \quad 1.97417$ $810 \quad 2.77540 \quad 4.49350 \quad 1.97384$ $820 \quad 2.75434 \quad 4.46929 \quad 1.97351$ $830 \quad 2.73357 \quad 4.44537 \quad 1.97318$ $840 \quad 2.713094 .42174 \quad 1.97284$

$\begin{array}{lllll}850 & 2.69289 & 4.39839 & 1.97250\end{array}$ $860 \quad 2.67296 \quad 4.37532 \quad 1.97216$ $870 \quad 2.653294 .35253 \quad 1.97181$ $880 \quad 2.633894 .329991 .97145$ $890 \quad 2.61474 \quad 4.30772 \quad 1.97109$

$900 \quad 2.59584 \quad 4.28570 \quad 1.97073$

$910 \quad 2.57719 \quad 4.26392 \quad 1.97037$

$920 \quad 2.55877 \quad 4.24239 \quad 1.97000$

$930 \quad 2.540594 .22110 \quad 1.96963$

$940 \quad 2.522634 .20003 \quad 1.96925$

$950 \quad 2.50490 \quad 4.17920 \quad 1.96887$ $960 \quad 2.48738 \quad 4.15858 \quad 1.96848$ $970 \quad 2.47008 \quad 4.13818 \quad 1.96809$ $980 \quad 2.45298 \quad 4.11800 \quad 1.96770$ $990 \quad 2.436104 .098031 .96730$

$1000 \quad 2.41941 \quad 4.07825 \quad 1.96690$ $1010 \quad 2.402924 .058691 .96650$ $1020 \quad 2.386624 .039311 .96609$ $1030 \quad 2.370514 .020131 .96568$ $1040 \quad 2.354594 .001141 .96526$

$\begin{array}{lllll}1050 & 2.33885 & 3.98234 & 1.96484\end{array}$ $1060 \quad 2.32328 \quad 3.96372 \quad 1.96441$ $\begin{array}{lllll}1070 & 2.30789 & 3.94527 & 1.96399\end{array}$ $1080 \quad 2.29268 \quad 3.92701 \quad 1.96355$ $1090 \quad 2.27763 \quad 3.90891 \quad 1.96312$

$$
r=4200
$$

$3.344635 .13457 \quad 1.98019$ 3.315075 .101841 .97995 $3.28604 \quad 5.06964 \quad 1.97972$ 3.257535 .037971 .97947 3.229515 .006801 .97923

$3.20198 \quad 4.97611 \quad 1.97898$ $3.17492 \quad 4.94590 \quad 1.97873$ $3.14831 \quad 4.91615 \quad 1.97847$ $3.12215 \cdot 4.886841 .97821$ $3.09641 \quad 4.85796 \quad 1.97794$

$3.07108 \quad 4.82950 \quad 1.97767$ $3.04616 \quad 4.801451 .97740$ 3.021634 .773801 .97713 $2.99749 \quad 4.74653 \quad 1.97685$ 2.973714 .719631 .97656

2. $95030 \quad 4.69311 \quad 1.97627$ 2.927234 .666931 .97598 2.904514 .641101 .97569 2.882124 .615611 .97539 2. $86006 \quad 4.59045 \quad 1.97509$

$2.83831 \quad 4.56561 \quad 1.97478$ $2.81688 \quad 4.54108 \quad 1.97447$ $2.79574 \quad 4.51685 \quad 1.97415$ $2.77490 \quad 4.49292 \quad 1.97384$ $2.75434 \quad 4.46929 \quad 1.97351$

$2.73406 \quad 4.445931 .97319$ $2.71406 \quad 4.42286 \quad 1.97286$ 2.694324 .400051 .97253 $2.67484 \quad 4.37751 \quad 1.97219$ 2.655624 .355231 .97185

2.636654 .333201 .97150 $2.617924 .31141 \quad 1.97115$ $2.599434 .28987 \quad 1.97080$ 2.581174 .268571 .97045 2.563144 .247501 .97009

2.545334 .226651 .96972 $2.52774 \quad 4.206031 .96936$ $2.51036 \quad 4.185621 .96899$ 2.493194 .165431 .96861 2.476234 .145441 .96823

$2.45947 \quad 4.12566 \quad 1.96785$ 2.442914 .106091 .96746 $2.42654 \quad 4.08670 \quad 1.96707$ $2.410354 .06751 \quad 1.96668$ $2.39436 \quad 4.04851 \quad 1.96628$

$2.37854 \quad 4.02970 \quad 1.96588$ 2. 362914.011071 .96548 $2.34744 \quad 3.99261 \quad 1.96507$ $2.33215 \quad 3.97434 \quad 1.96466$ 2.317033 .956231 .96424
$T=4300$.

$3.38680 \quad 5.18117 \quad 1.98051$ 3.357175 .148431 .98029 3.328075 .116231 .98006 $3.29948 \quad 5.08455 \quad 1.97983$ $3.271395 .05338 \quad 1.97959$

$3.243795 .02269 \quad 1.97936$ 3.216654 .992471 .97911 $3.18997 \quad 4.96271 \quad 1.97887$ 3.163734 .933391 .97862 $3.137924 .90451 \quad 1.97837$

$3.11252 \quad 4.87604 \quad 1.97811$ $3.08753 \quad 4.84799 \quad 1.97785$ 3.062924 .820331 .97759 3.038714 .793051 .97732 $3.01486 \quad 4.766151 .97705$

$2.99137 \quad 4.739611 .97677$ 2.968234 .713431 .97650 2.945444 .687601 .97621 $2.92298 \quad 4.66210 \quad 1.97593$ 2.900844 .636931 .97564

$2.87903 \quad 4.61208 \quad 1.97535$ $2.857524 .58754 \quad 1.97505$ 2.836314 .563311 .97475 $2.815394 .53938 \quad 1.97445$ $2.79476 \quad 4.51573 \quad 1.97414$

$2.77441 \quad 4.49237 \quad 1.97383$ $2.75434 \quad 4.469291 .97351$ $2.734534 .44647 \quad 1.97320$ $2.71498 \quad 4.42392 \quad 1.97287$ 2.695694 .401631 .97255

$2.676644 .37960 \quad 1.97222$ $2.65784 \quad 4.35780 \quad 1.97189$ $2.63928 \quad 4.33626 \quad 1.97155$ 2.620954 .314941 .97121 $2.60285 \quad 4.29386 \quad 1.97087$

$2.58497 \quad 4.27301 \quad 1.97052$ 2.567314 .252381 .97017 $2.54986 \quad 4.23196 \quad 1.96982$ $2.532624 .21176 \quad 1.96946$ $2.515594 .19177 \quad 1.96910$

$2.49876 \quad 4.17198 \quad 1.96873$ 2.482134 .152391 .96836 2.465694 .133001 .96799 $2.449434 .11380 \quad 1.96762$ $2.433374 .09479 \quad 1.96724$

$2.41748 \quad 4.07597 \quad 1.96685$ $2.40178 \quad 4.057331 .96647$ $2.38624 \quad 4.03887 \quad 1.96608$ $2.37088 \quad 4.02058 \quad 1.96568$ $2.355694 .00246 \quad 1.96529$
600

610

620

630

640

650

660

670

680

690

700

710

720

730

740

750

760

770

780

790

800

810

820

830

840

850

860

870

880

890

900

910

920

930

940

950

960

970

980

990

1000

1010

1020

1030

1040

1050

1060

1070

1080

1090 
Table II Harmonic Oscillator Contributions to the Thermodynamic

Functions (in units of calories, moles, and ${ }^{\circ} \mathrm{K}$ )
$\mathrm{cm}^{-1}$
$-\left(F^{\circ}-E_{O}^{\circ}\right) / T \quad S^{\circ}$
$\stackrel{\mathrm{C}}{\mathrm{O}} \mathrm{p}$
$-\left(\mathrm{F} O-\mathrm{E}_{\mathrm{O}}\right) / \mathrm{T}$
So
$\mathrm{C}_{\mathrm{p}}^{\circ}$
$-\left(\mathrm{F}^{\circ}-\mathrm{E}_{\mathrm{O}}\right) / \mathrm{T}$
So
$\mathrm{C}_{\mathrm{p}}^{\circ}$

v

$T=4100$

$\mathrm{T}=4200$.

$\mathrm{T}=4300$.

$1100 \quad 2.26274 \quad 3.89098 \quad 1.96268$ $1110 \quad 2.24802 \quad 3.87322 \quad 1.96223$

$1120 \quad 2.23346 \quad 3.85563 \quad 1.96178$

$1130 \quad 2.21905 \quad 3.83819 \quad 1.96133$

$\begin{array}{llll}1140 & 2.20480 & 3.82091 & 1.96088\end{array}$

$\begin{array}{lllll}1150 & 2.19070 & 3.80379 & 1.96042\end{array}$

$\begin{array}{llll}1160 & 2.17675 & 3.78682 & 1.95995\end{array}$

$1170 \quad 2.16294 \quad 3.77000 \quad 1.95949$

$\begin{array}{lllll}1180 & 2.14927 & 3.75332 & 1.95902\end{array}$

$1190 \quad 2.13575 \quad 3.73679 \quad 1.95854$

$1200 \quad 2.12237 \quad 3.72040 \quad 1.95806$

$1210 \quad 2.109123 .70416 \quad 1.95758$

$\begin{array}{lllll}1220 & 2.09600 & 3.68805 & 1.95709\end{array}$

$1230 \quad 2.083023 .67207 \quad 1.95660$

$\begin{array}{lllll}1240 \quad 2.07016 \quad 3.65623 & 1.95611\end{array}$

$1250 \quad 2.05743 \quad 3.64052 \quad 1.95561$

$\begin{array}{lllll}1260 & 2.04483 & 3.62494 & 1.95511\end{array}$

$1270 \quad 2.03235 \quad 3.60949 \quad 1.95460$

$\begin{array}{lllll}1280 & 2.01999 & 3.59416 & 1.95409\end{array}$

$1290 \quad 2.00775 \quad 3.57895 \quad 1.95358$

$\begin{array}{lllll}1300 & 1.99563 & 3.56387 & 1.95306\end{array}$

$\begin{array}{lllll}1310 & 1.98363 & 3.54891 & 1.95254\end{array}$

$\begin{array}{llllll}1320 & 1.97174 & 3.53406 & 1.95201\end{array}$

$\begin{array}{llll}1330 & 1.95996 & 3.51933 & 1.95149\end{array}$

$1340 \quad 1.948293 .50471 \quad 1.95095$

$\begin{array}{lllll}1350 & 1.93672 & 3.49021 & 1.95042\end{array}$

$\begin{array}{lllll}1360 & 1.92527 & 3.47582 & 1.94988\end{array}$

$\begin{array}{llllll}1370 & 1.91392 & 3.46154 & 1.94933\end{array}$

$\begin{array}{lllll}1380 & 1.90268 & 3.44736 & 1.94878\end{array}$

$1390 \quad 1.89153 \quad 3.43329 \quad 1.94823$

$1400 \quad 1.880493 .41933 \quad 1.94768$

$1410 \quad 1.86955 \quad 3.40547 \quad 1.94712$

$1420 \quad 1.858713 .39171 \quad 1.94655$

$\begin{array}{lllll}1430 & 1.84796 & 3.37805 & 1.94599\end{array}$

$1440 \quad 1.837313 .364491 .94542$

$1450 \quad 1.82675 \quad 3.351031 .94484$

$1460 \quad 1.81628 \quad 3.33767 \quad 1.94426$

$\begin{array}{lllll}1470 & 1.80591 & 3.32440 & 1.94368\end{array}$

$1480 \quad 1.795623 .311221 .94310$

$1490 \quad 1.78542 \quad 3.29814 \quad 1.94251$

1500

1510

1520

1530

1540

1550

1560

1570

1580

1590
$1.77532 \quad 3.28515 \quad 1.94191$

$1.76529 \quad 3.27225 \quad 1.94132$

$1.75536 \quad 3.259431 .94072$

$1.74550 \quad 3.24671 \quad 1.94011$

$1.73573 \quad 3.23407 \quad 1.93950$

$1.72604 \quad 3.22152 \quad 1.93889$ $1.71643 \quad 3.20905 \quad 1.93828$ 1.706913 .196671 .93766 $1.69746 \quad 3.18437 \quad 1.93703$ 1.688083 .172151 .93641
$2.30208 \quad 3.93829 \quad 1.96382$ $2.28728 \quad 3.92052 \quad 1.96340$ $2.27265 \quad 3.90292 \quad 1.96297$ $2.25817 \quad 3.88547 \quad 1.96254$ 2.243843 .868181 .96211

$2.229673 .85105 \quad 1.96167$ $2.21565 \quad 3.83406 \quad 1.96123$ $2.20177 \quad 3.81723 \quad 1.96078$ $2.18803 \quad 3.80055 \quad 1.96033$ $2.17443 \quad 3.78400 \quad 1.95988$

$2.16098 \quad 3.76761 \quad 1.95942$ $2.14766 \quad 3.751351 .95896$ 2.134473 .735221 .95850 $2.12141 \quad 3.71924 \quad 1.95803$ 2.108493 .703391 .95756

$2.095693 .68766 \quad 1.95708$ 2.083023 .672071 .95660 $2.070473 .65661 \quad 1.95612$ $2.05804 \quad 3.64127 \quad 1.95563$ $2.04573 \quad 3.626051 .95514$

$2.03353 \quad 3.61095 \quad 1.95465$ $2.02146 \quad 3.59598 \quad 1.95415$ 2. $00950 \quad 3.581121 .95365$ $1.99765 \quad 3.56638 \quad 1.95315$ 1.985913 .551751 .95264

$1.97427 \quad 3.53723 \quad 1.95213$ $1.96275 \quad 3.522831 .95161$ 1.951333 .508531 .95109 1.940023 .494341 .95057 $1.92880 \quad 3.48026 \quad 1.95004$

$1.917693 .46628 \quad 1.94951$ $1.90668 \quad 3.45241 \quad 1.94898$ $1.89577 \quad 3.43864 \quad 1.94844$ 1.884953 .424971 .94790 $1.87423 \quad 3.411391 .94736$

$1.86360 \quad 3.39792 \quad 1.94681$ $1.85306 \quad 3.38454 \quad 1.94626$ $1.842623 .37126 \quad 1.94570$ $1.83227 \quad 3.35807 \quad 1.94514$ $1.82200 \quad 3.34497 \quad 1.94458$

$1.811823 .33197 \quad 1.94402$ $1.80173 \quad 3.319051 .94345$ $1.79173 \quad 3.306231 .94287$ $1.78180 \quad 3.29349 \quad 1.94230$ $1.77197 \quad 3.28084 \quad 1.94172$

$1.762213 .26827 \quad 1.94113$ $1.75253 \quad 3.25579 \quad 1.94054$ 1.742943 .243391 .93995 $1.733423 .23108 \quad 1.93936$ $1.72398 \quad 3.21884 \quad 1.93876$
2.340673 .984521 .96489 $2.32580 \quad 3.96674 \quad 1.96448$ $2.31110 \quad 3.949121 .96408$ $2.29655 \quad 3.93166 \quad 1.96366$ $2.28216 \quad 3.91436 \quad 1.96325$

$2.26792 \quad 3.89722 \quad 1.96283$ $2.25382 \quad 3.88023 \quad 1.96241$ $2.23987 \quad 3.86338 \quad 1.96198$ $2.22607 \quad 3.84669 \quad 1.96155$ $2.212413 .83014 \quad 1.96112$

2. $19888 \quad 3.81373 \quad 1.96069$ $2.18549 \quad 3.79746 \quad 1.96025$ $2.17223 \quad 3.781321 .95980$ 2.159113 .765331 .95936 $2.146123 .74946 \quad 1.95891$

$2.13325 \quad 3.73373 \quad 1.95845$ 2.120513 .718131 .95800 $2.107893 .70265 \quad 1.95753$ $2.09539 \quad 3.68730 \quad 1.95707$ 2.083023 .672071 .95660

$2.07076 \quad 3.65697 \quad 1.95613$ $2.05861 \quad 3.64198 \quad 1.95566$ $2.04658 \quad 3.627111 .95518$ $2.03466 \quad 3.612351 .95470$ $2.02286 \quad 3.59771 \quad 1.95421$

$2.01116 \quad 3.58319 \quad 1.95372$ $1.99957 \quad 3.56877 \quad 1.95323$ $1.98808 \quad 3.55446 \quad 1.95273$ $1.97670 \quad 3.54026 \quad 1.95223$ $1.965423 .52617 \quad 1.95173$

1.954243 .512181 .95123 $1.94316 \quad 3.498291 .95072$ $1.93218 \quad 3.48451 \quad 1.95020$ $1.92130 \quad 3.470821 .94969$ $1.910513 .45724 \quad 1.94917$

$1.89982 \quad 3.443751 .94864$ $1.88922 \quad 3.43036 \quad 1.94812$ $1.87870 \quad 3.41706 \quad 1.94759$ 1.868283 .403861 .94705 $1.85795 \quad 3.39075 \quad 1.94652$

$1.84771 \quad 3.37773 \quad 1.94597$ $1.83755 \quad 3.364811 .94543$ $1.82748 \quad 3.35197 \quad 1.94488$ 1.817493 .339211 .94433 $1.80759 \quad 3.32655 \quad 1.94378$

$1.79777 \quad 3.31397 \quad 1.94322$ $1.78802 \quad 3.30148 \quad 1.94266$ $1.77836 \quad 3.28906 \quad 1.94209$ $1.76878 \quad 3.27674 \quad 1.94153$ $1.75927 \quad 3.264491 .94095$
1100

1110

1120

1130

1140

1150

1160

1170

1180

1190

1200

1210

1220

1230

1240

1250

1260

1270

1280

1290

1300

1310

1320

1330

1340

1350

1360

1370

1380

1390

1400

1410

1420

1430

1440

1450

1460

1470

1480

1490

1500

1510

1520

1530

1540

1550

1560

1570

1580

1590 
Table II Harmonic Oscillator Contributions to the Thermodynamic Functions (in units of calories, moles, and ${ }^{\circ} \mathrm{K}$ )
$\mathrm{cm}^{-1}-\left(F^{\circ}-E_{0}^{\circ}\right) / T \quad S^{\circ}$
$C_{\mathrm{P}}^{\circ} \quad-\left(F^{\circ}-E_{0}^{\circ}\right) / T \quad$ So
$\mathrm{co}$
$-\left(F^{\circ}-E_{O}^{\circ}\right) / T$
So
$\mathrm{c}_{\mathrm{p}}^{\circ}$
$\mathrm{m}^{-1}$

$\mathrm{T}=4100$.

$\begin{array}{lllll}1600 \quad 1.67879 & 3.16001 & 1.93578\end{array}$

$\begin{array}{lllll}1610 & 1.66957 & 3.14795 & 1.93514\end{array}$

$1620 \quad 1.660423 .13597 \quad 1.93450$

$\begin{array}{lllll}1630 & 1.65135 & 3.12407 & 1.93386\end{array}$

$\begin{array}{llll}1640 \quad 1.64235 & 3.11224 & 1.93322\end{array}$

$\begin{array}{llll}1650 \quad 1.63343 & 3.10049 & 1.93257\end{array}$

$\begin{array}{lllll}1660 & 1.62457 & 3.08882 & 1.93192\end{array}$

$\begin{array}{llll}1670 \quad 1.61578 & 3.07722 & 1.93126\end{array}$

$\begin{array}{llll}1680 & 1.60707 & 3.06569 & 1.93060\end{array}$

$\begin{array}{llll}1690 & 1.59842 & 3.05423 & 1.92993\end{array}$

$\begin{array}{llll}1700 \quad 1.58984 & 3.04285 & 1.92927\end{array}$

$\begin{array}{llll}1710 & 1.58132 \quad 3.03154 & 1.92860\end{array}$

$\begin{array}{lllll}1720 & 1.57288 & 3.02029 & 1.92792\end{array}$

$\begin{array}{llll}1730 & 1.56449 & 3.00912 & 1.92724\end{array}$

$\begin{array}{llll}1740 \quad 1.55618 & 2.99801 & 1.92656\end{array}$

$\begin{array}{lllll}1750 & 1.54792 & 2.98697 & 1.92588\end{array}$

$\begin{array}{lllll}1760 & 1.53973 & 2.97600 & 1.92519\end{array}$

$\begin{array}{llll}1770 & 1.53160 & 2.96510 & 1.92449\end{array}$

$\begin{array}{llll}1780 & 1.52353 & 2.95426 & 1.92380\end{array}$

$\begin{array}{llll}1.790 & 1.51552 & 2.94348 & 1.92310\end{array}$

$\begin{array}{lllll}1800 & 1.50758 & 2.93277 & 1.92239\end{array}$

$\begin{array}{llll}1810 & 1.49969 & 2.92212 & 1.92169\end{array}$

$\begin{array}{llll}1820 & 1.49186 & 2.91153 \quad 1.92098\end{array}$

$\begin{array}{llll}1830 & 1.48409 & 2.90101 & 1.92026\end{array}$

$\begin{array}{lllll}1840 & 1.47637 & 2.89055 & 1.91954\end{array}$

$\begin{array}{llll}1850 & 1.46871 & 2.88015 & 1.91882\end{array}$

$\begin{array}{lllll}1860 & 1.46111 & 2.86980 & 1.91810\end{array}$

$\begin{array}{llll}1870 & 1.45357 & 2.85952 & 1.91737\end{array}$

$\begin{array}{lllll}1880 & 1.44608 & 2.84930 & 1.91663\end{array}$

$\begin{array}{lllll}1890 & 1.43864 & 2.83913 & 1.91590\end{array}$

$1900 \quad 1.43126 \quad 2.82902 \quad 1.91516$

$\begin{array}{lllll}1910 & 1.42393 & 2.81897 & 1.91441\end{array}$

$\begin{array}{llll}1920 & 1.41665 & 2.80898 & 1.91367\end{array}$

$\begin{array}{lllll}1930 & 1.40942 & 2.79904 & 1.91292\end{array}$

$1940 \quad 1.402252 .789151 .91216$

$\begin{array}{llll}1950 & 1.39512 & 2.77932 & 1.91141\end{array}$

$\begin{array}{llll}1960 & 1.38805 & 2.76955 & 1.91065\end{array}$

$\begin{array}{lllll}1970 & 1.38103 & 2.75983 & 1.90988\end{array}$

$\begin{array}{lllll}1980 & 1.37405 & 2.75016 & 1.90911\end{array}$

$1990 \quad 1.36713 \quad 2.74054 \quad 1.90834$

$\begin{array}{lllll}2000 & 1.36025 & 2.73098 & 1.90757\end{array}$

$\begin{array}{llll}2010 & 1.35342 & 2.72147 & 1.90679\end{array}$

$20201.346642 .71201 \quad 1.90601$

$\begin{array}{lllll}2030 & 1.33990 & 2.70260 & 1.90522\end{array}$

$\begin{array}{lllll}2040 \quad 1.33321 & 2.69324 & 1.90443\end{array}$

$\begin{array}{llll}2050 & 1.32657 & 2.68392 & 1.90364\end{array}$

$\begin{array}{lllll}2060 & 1.31997 & 2.67466 & 1.90284\end{array}$

$2070 \quad 1.313412 .66545 \quad 1.90204$

$2080 \quad 1.306912 .656291 .90124$

$\begin{array}{lllll}2090 & 1.30044 & 2.64717 & 1.90044\end{array}$ $\mathrm{r}=4200$.

$1.714613 .20669 \quad 1.93816$

$1.70533 \quad 3.19461 \quad 1.93755$

$1.69611 \quad 3.18262 \quad 1.93694$

1.686973 .170701 .93633

$1.67791 \quad 3.15886 \quad 1.93572$

$1.66891 \quad 3.14709 \quad 1.93510$

$1.65999 \quad 3.13540 \quad 1.93447$

$1.65114 \quad 3.12379 \quad 1.93385$

$1.642353 .11224 \quad 1.93322$

$1.633643 .10077 \quad 1.93258$

$1.624993 .08937 \quad 1.93195$

1.616413 .078041 .93131

$1.607893 .06678 \quad 1.93066$

$1.59945 \quad 3.05559 \quad 1.93001$

$1.59106 \quad 3.044471 .92936$

$1.58274 \quad 3.03342 \quad 1.92871$

$1.57448 \quad 3.022431 .92805$

$1.56628 \quad 3.01151 \quad 1.92739$

$1.55815 \quad 3.00065 \quad 1.92672$

$1.55008 \quad 2.989861 .92606$

$\begin{array}{llll}1.54206 & 2.97913 & 1.92538\end{array}$ $1.534112 .96846 \quad 1.92471$ $1.52621 \quad 2.95786 \quad 1.92403$ $1.518382 .94732 \quad 1.92335$ $1.510602 .93684 \quad 1.92266$

$\begin{array}{lll}1.50287 & 2.92642 \quad 1.92197\end{array}$ $\begin{array}{llll}1.49521 & 2.91606 & 1.92128\end{array}$ $\begin{array}{llll}1.48760 & 2.90576 & 1.92058\end{array}$ $\begin{array}{lll}1.48004 & 2.89552 & 1.91988\end{array}$ $1.472542 .88534 \quad 1.91918$

$\begin{array}{llll}1.46509 & 2.87521 & 1.91848\end{array}$ $1.457692 .86514 \quad 1.91777$ $1.45035 \quad 2.85513 \quad 1.91705$ $1.44306 \quad 2.84517 \quad 1.91634$ $1.435822 .83527 \quad 1.91562$

1.428632 .825431 .91489 $1.421492 .81563 \quad 1.91417$ $1.414412 .80589 \quad 1.91344$ $1.407372 .79621 \quad 1.91270$ $1.40038 \quad 2.78657 \quad 1.91197$

$1.393442 .77699 \quad 1.91123$ $1.386542 .76746 \quad 1.91048$ $1.379692 .75798 \quad 1.90974$ $1.372902 .74855 \quad 1.90899$ 1.366142 .739171 .90823

$\begin{array}{llll}1.35943 & 2.72984 & 1.90747\end{array}$ $1.35277 \quad 2.72056 \quad 1.90671$ 1.346152 .711331 .90595 $\begin{array}{llll}1.33958 & 2.70215 & 1.90518\end{array}$ $1.333052 .69301 \quad 1.90441$
$T=4300$.

$1.74985 \quad 3.25232 \quad 1.94038$ $1.740493 .24023 \quad 1.93980$ $1.73121 \quad 3.228221 .93922$ $1.72201 \quad 3.21629 \quad 1.93863$ $1.71288 \quad 3.20444 \quad 1.93805$

$1.70382 \quad 3.19266 \quad 1.93745$ $1.69483 \quad 3.18095 \quad 1.93686$ 1.685923 .169321 .93626 $1.677073 .15776 \quad 1.93566$ $1.668293 .14628 \quad 1.93505$

$1.65958 \quad 3.13486 \quad 1.93444$ $1.65093 \quad 3.123521 .93383$ 1.642353 .112241 .93322 1.633843 .101041 .93260 1.625393 .089901 .93198

1.617013 .078831 .93135 $1.60868 \quad 3.067831 .93072$ 1.600423 .056891 .93009 1.592233 .046021 .92945 $1.584093 .03521 \quad 1.92882$

$\begin{array}{llll}1.57601 & 3.02447 & 1.92817\end{array}$ $1.56800 \quad 3.01379 \quad 1.92753$ $1.560043 .00317 \quad 1.92688$ 1.552142 .992611 .92623 1.544292 .982121 .92557

$\begin{array}{llll}1.53651 & 2.97168 & 1.92491\end{array}$ $\begin{array}{llll}1.52878 & 2.96131 & 1.92425\end{array}$ 1.521102 .950991 .92359 $1.51348 \quad 2.94073 \quad 1.92292$ 1.505922 .930531 .92225

$1.49841 \quad 2.92039 \quad 1.92157$ $1.490952 .91031 \quad 1.92089$ $1.483552 .90028 \quad 1.92021$ 1.476192 .890311 .91953 1.468892 .880391 .91884

1.461642 .870521 .91815 $1.454442 .86071 \quad 1.91745$ $1.447292 .85096 \cdot 1.91675$ 1.440192 .841251 .91605 1.433142 .831601 .91535

$1.426142 .82200 \quad 1.91464$ 1.419182 .812461 .91393 $1.412272 .80296 \quad 1.91322$ $\begin{array}{llll}1.40541 & 2.79351 & 1.91250\end{array}$ 1.398602 .784121 .91178

$1.391832 .77477 \quad 1.91105$ $1.38510 \quad 2.76547 \quad 1.91033$ 1.378432 .756221 .90960 $1.371792 .74702 \quad 1.90886$ $1.365202 .73787 \quad 1.90813$
1600

1610

1620

1630

1640

1650

1660

1670

1680

1690

1700

1710

1720

1730

1740

1750

1760

1770

1780

1790

1800

1810

1820

1830

1840

1850

1860

1870

1880

1890

1900

1910

1920

1930

1940

1950

1960

1970

1980

1990

2000

2010

2020

2030

2040

2050

2060

2070

2080

2090 
Table II Harmonic Oscillator Contributions to the Thermodynamic

Functions (in units of calories, moles, and ${ }^{\circ} \mathrm{K}$ )
$v_{\mathrm{cm}^{-1}}-(\mathrm{F} \circ-\mathrm{EO}) / \mathrm{T} \quad$ So
$C_{p}^{\circ} \quad-\left(F^{\circ}-E_{O}^{O}\right) / T \quad S^{\circ}$
$\mathrm{C}_{\mathrm{p}}^{\circ}$
$-\left(\mathrm{F}^{\circ}-\mathrm{E}_{\mathrm{O}}\right) / \mathrm{T}$
So
$c_{p}^{\circ}$
$\mathrm{cm}^{-1}$

$T=4100$.

$2100 \quad 1.29402 \quad 2.63810 \quad 1.89963$

$2110 \quad 1.287642 .62908 \quad 1.89881$

$2120 \quad 1.28130 \quad 2.62010 \quad 1.89800$

$\begin{array}{lllll}2130 & 1.27501 & 2.61117 & 1.89718\end{array}$

$2140 \quad 1.26876 \quad 2.60229 \quad 1.89635$

$2150 \quad 1.262552 .59345 \quad 1.89553$

$2160 \quad 1.25638 \quad 2.58465 \quad 1.89470$

$2170 \quad 1.25025 \quad 2.57590 \quad 1.89386$

$2180 \quad 1.24416 \quad 2.56720 \quad 1.89302$

$2190 \quad 1.238112 .558541 .89218$

$2200 \quad 1.23210 \quad 2.54992 \quad 1.89134$

$\begin{array}{lllll}-2210 & 1.22613 & 2.54134 & 1.89049\end{array}$

$2220 \quad 1.22020 \quad 2.53281 \quad 1.88964$

$\begin{array}{llll}2230 & 1.21430 & 2.52432 & 1.88879\end{array}$

$2240 \quad 1.20845 \quad 2.51587 \quad 1.88793$

$2250 \quad 1.202632 .50746 \quad 1.88707$

$2260 \quad 1.196852 .49910 \quad 1.88621$

$2270 \quad 1.19110 \quad 2.49077 \quad 1.88534$

$2280 \quad 1.18540 \quad 2.48248 \quad 1.88447$

$2290 \quad 1.179732 .47424 \quad 1.88359$

$2300 \quad 1.17409 \quad 2.46603 \quad 1.88271$

$2310 \quad 1.168492 .45787 \quad 1.88183$

$2320 \quad 1.162932 .44974 \quad 1.88095$

$2330 \quad 1.15740 \quad 2.44165 \quad 1.88006$

$2340 \quad 1.15190 \quad 2.43360 \quad 1.87917$

$2350 \quad 1.14644 \quad 2.42559 \quad 1.87828$

$2360 \quad 1.14102 \quad 2.41762 \quad 1.87738$

$2370 \quad 1.135632 .409681 .87648$

$2380 \quad 1.13027 \quad 2.40178 \quad 1.87557$

$2390 \quad 1.124942 .39392 \quad 1.87467$

$2400 \quad 1.119652 .38609 \quad 1.87376$

$\begin{array}{lllll}2410 & 1.11439 & 2.37831 & 1.87284\end{array}$

$2420 \quad 1.10916 \quad 2.37055 \quad 1.87193$

$2430 \quad 1.103962 .362831 .87100$

$\begin{array}{lllll}2440 & 1.09880 & 2.35515 & 1.87008\end{array}$

$\begin{array}{lllll}2450 & 1.09366 & 2.34751 & 1.86915\end{array}$ $2460 \quad 1.088562 .33989 \quad 1.86822$

$\begin{array}{lllll}2470 & 1.08349 & 2.33232 & 1.86729\end{array}$

$\begin{array}{lllll}2480 & 1.07845 & 2.32477 & 1.86635\end{array}$

$\begin{array}{lllll}2490 & 1.07344 & 2.31727 & 1.86541\end{array}$

$\begin{array}{lllll}2500 & 1.06846 & 2.30979 & 1.86447\end{array}$ $2510 \quad 1.063512 .30235 \quad 1.86352$ $\begin{array}{lllll}2520 & 1.05859 & 2.29494 & 1.86258\end{array}$ $\begin{array}{lllll}2530 & 1.05370 & 2.28757 & 1.86162\end{array}$ $\begin{array}{lllll}2540 \quad 1.04883 & 2.28023 & 1.86067\end{array}$

$\begin{array}{lllll}2550 & 1.04400 & 2.27292 & 1.85971\end{array}$ $\begin{array}{lllll}2560 & 1.03919 & 2.26564 & 1.85875\end{array}$ $\begin{array}{lllll}2570 & 1.03442 & 2.25840 & 1.85778\end{array}$ $\begin{array}{lllll}2580 & 1.02967 & 2.25118 & 1.85681\end{array}$ $\begin{array}{llll}2590 \quad 1.02495 & 2.24400 & 1.85584\end{array}$

$$
T=4200 \text {. }
$$

$T=4300$.

1.326572 .683921 .90364 $1.320122 .67488 \quad 1.90286$ $1.313732 .66589 \quad 1.90208$ 1.307372 .656941 .90130 $1.301052 .64804 \quad 1.90051$

$1.29478 \quad 2.63918 \quad 1.89972$ $1.288552 .63036 \quad 1.89893$ $1.28236 \quad 2.621591 .89813$ $1.27620 \quad 2.61287 \quad 1.89733$ 1.270092 .604191 .89653

$1.26402 \quad 2.59555 \quad 1.89572$ $1.257992 .58695 \quad 1.89491$ 1.251992 .578401 .89410 1.246042 .569891 .89328 1.240122 .561421 .89246

$1.234242 .55299 \quad 1.89164$ $1.22840 \quad 2.54460 \quad 1.89082$ $1.222592 .53626 \quad 1.88999$ $1.21682 \quad 2.527951 .88915$ 1.211092 .519691 .88832

$1.20539 \quad 2.51146 \quad 1.88748$ $1.19973 \quad 2.50327 \quad 1.88664$ 1.194112 .495131 .88579 $1.18852 \quad 2.48702 \quad 1.88494$ $1.182962 .47895 \quad 1.88409$

1.177442 .470911 .88324 1.171952 .462921 .88238 $1.166502 .45496 \quad 1.88152$ $1.16108 \quad 2.44704 \quad 1.88065$ $1.155692 .43916 \quad 1.87979$

$1.150342 .43131 \quad 1.87892$ 1.145022 .423501 .87804 1.139732 .415721 .87717 1.134472 .407991 .87629 $1.129252 .40028 \quad 1.87540$

$1.12406 \quad 2.39261 \quad 1.87452$ $1.118892 .38498 \quad 1.87363$ $\begin{array}{llll}1.11376 & 2.37738 & 1.87273\end{array}$ $1.10866 \quad 2.36982 \quad 1.87184$ 1.103592 .362291 .87094

$1.098552 .35479 \quad 1.87004$ 1.093542 .347321 .86913 $1.08856 \quad 2.33989 \quad 1.86822$ 1.083612 .332501 .86731 1.078692 .325131 .86640

$1.07380 \quad 2.31780 \quad 1.86548$ 1.068932 .310501 .86456 1.064102 .303231 .86364 $1.059292 .29600 \quad 1.86271$ $\begin{array}{lll}1.05451 & 2.28879 & 1.86178\end{array}$
$1.35866 \quad 2.72876 \quad 1.90739$ $1.352152 .71970 \quad 1.90664$ 1.345692 .710691 .90590 $1.33928 \quad 2.701721 .90515$ $1.332902 .69280 \quad 1.90440$

1.326572 .683921 .90364 1.320272 .675091 .90288 $1.314022 .66631 \quad 1.90212$ 1.307812 .657561 .90135 1.301642 .648861 .90059

$1.295512 .64020 \quad 1.89981$ 1.289412 .631591 .89904 $1.28336 \quad 2.62302 \quad 1.89826$ $1.277352 .61449 \quad 1.89748$ $1.271372 .60600 \quad 1.89670$

$1.265432 .59755 \quad 1.89591$ 1.259532 .589151 .89512 $1.25366 \quad 2.58078 \quad 1.89433$ $1.247832 .57246 \quad 1.89353$ $1.242042 .56417 \quad 1.89273$

$1.236292 .55593 \quad 1.89193$ 1.230572 .547721 .89112 $1.224882 .53955 \quad 1.89031$ 1.219232 .531421 .88950 1.213622 .523331 .88869

$1.20804 \quad 2.51528 \quad 1.88787$ $1.202492 .50727 \quad 1.88705$ 1.196982 .499291 .88623 $1.19150 \quad 2.49135 \quad 1.88540$ 1.186062 .483451 .88457

$1.180652 .47558 \quad 1.88373$ $1.175272 .46775 \quad 1.88290$ 1.169922 .459951 .88206 1.164612 .452191 .88122 $1.159322 .44447 \quad 1.88037$

$1.15407 \quad 2.43678 \quad 1.87952$ 1.148852 .429131 .87867 $1.143662 .42151 \quad 1.87782$ 1.138512 .413921 .87696 1.133382 .406371 .87610

$\begin{array}{llll}1.12828 & 2.39885 & 1.87524\end{array}$ $\begin{array}{llll}1.12321 & 2.39137 & 1.87437\end{array}$ $\begin{array}{lll}1.118: 8 & 2.38392 & 1.87350\end{array}$ $1.113172 .37650 \quad 1.87263$ 1.108192 .369111 .87175

$1.103242 .36176 \quad 1.87088$ $1.098322 .35444 \quad 1.87000$ 1.093432 .347151 .86911 $1.088562 .33989 \quad 1.86822$ $1.08372 \quad 2.33267 \quad 1.86733$
2100

2110

2120

2130

2140

2150

2160

2170

2180

2190

2200

2210

2220

2230

2240

2250

2260

2270

2280

2290

2300

2310

2320

2330

2340

2350

2360

2370

2380

2390

2400

2410

2420

2430

2440

2450

2460

2470

2480

2490

2500

2510

2520

2530

2540

2550

2560

2570

2580

2590 
Table II Harmonic Oscillator Contributions to the Thermodynamic

Functions (in units of calories, moles, and ${ }^{\circ} \mathrm{K}$ )
$\mathrm{cm}^{-1}$
$-\left(F^{\circ}-E O\right) / T$
so

\begin{abstract}
$\mathrm{C}_{\mathrm{p}}^{\circ}$
\end{abstract}

$$
\mathrm{T}=4100 \text {. }
$$
2600
2610
2620
2630
2640
$1.02026 \quad 2.23685 \quad 1.85487$
$1.015592 .22973 \quad 1.85389$
$1.01095 \quad 2.22265 \quad 1.85291$
$1.00634 \quad 2.21559 \quad 1.85192$
$1.00176 \quad 2.20856 \quad 1.85093$

$-\left(F^{\circ}-E_{O}^{\circ}\right) / T$

so

$\mathrm{C}_{\mathrm{p}}^{\circ}$

$-\left(F^{\circ}-E_{O}^{\circ}\right) / T$

So

2650

2660

2670

2680

2690

2700

2710

2720

2730

2740

2750

2760

2770

2780

2790

2800

2810

2820

2830

2840

2850

2860

2870

2880

2890

2900

2910

2920

2930

2940

2950

2960

2970

2980

2990

3000

3010

3020

3030

3040

3050

3060

3070

3080

3090
$0.99720 \quad 2.20157 \quad 1.84994$

$0.99267 \quad 2.19460 \quad 1.84895$

$0.98816 \quad 2.18766 \quad 1.84795$

$0.983682 .18076 \quad 1.84695$

$0.97923 \quad 2.17388 \quad 1.84595$

$0.97480 \quad 2.16703 \quad 1.84495$

$0.97040 \quad 2.16021 \quad 1.84394$

$0.966022 .15342 \quad 1.84292$

$0.96166 \quad 2.14666 \quad 1.84191$

$0.95734 \quad 2.13993 \quad 1.84089$

$\begin{array}{llll}0.95303 & 2.13323 \quad 1.83987\end{array}$

$0.94875 \quad 2.12655 \quad 1.83885$

$0.94450 \quad 2.11990 \quad 1.83782$

$0.94027 \quad 2.11328 \quad 1.83679$

$0.93606 \quad 2.106691 .83576$

0.931872 .100121 .83472

$0.92771 \quad 2.09358 \quad 1.83368$

$0.92358 \quad 2.08707 \quad 1.83264$

$0.91946 \quad 2.08058 \quad 1.83159$

0.915372 .074131 .83054

$0.91130 \quad 2.06769 \quad 1.82949$ $0.90726 \quad 2.06129 \quad 1.82844$ $0.90323 \quad 2.05491 \quad 1.82738$ $0.899232 .04855 \quad 1.82632$ $0.89525 \quad 2.04222 \quad 1.82526$

$\begin{array}{lll}0.89129 & 2.03592 \quad 1.82419\end{array}$ $0.88736 \quad 2.02964 \quad 1.82312$ $0.88344 \quad 2.02339 \quad 1.82205$ $0.879552 .01716 \quad 1.82098$ $0.87568 \quad 2.01096 \quad 1.81990$

$0.87183 \quad 2.00478 \quad 1.81882$ $0.86800 \quad 1.99863 \quad 1.81773$ 0.864191 .992501 .81665 $0.860401 .98640 \quad 1.81556$ $0.85663 \quad 1.98032 \quad 1.81447$

$\begin{array}{llll}0.85288 & 1.97426 & 1.81337\end{array}$ $0.84915 \quad 1.96823 \quad 1.81227$ $0.84544 \quad 1.96222 \quad 1.81117$ $0.84176 \quad 1.95623 \quad 1.81007$ $0.838091 .95027 \quad 1.80896$

$0.83444 \quad 1.94433 \quad 1.80785$ $0.830811 .93842 \quad 1.80674$ $0.827201 .93252 \quad 1.80563$ 0.823611 .926651 .80451 $0.82004 \quad 1.92080 \quad 1.80339$

$$
T=4200 \text {. }
$$

$1.04976 \quad 2.28162 \quad 1.86085$

$1.04503 \quad 2.27448 \quad 1.85991$

$1.04034 \quad 2.26737 \quad 1.85898$

$1.035672 .26029 \quad 1.85803$

$1.03102 \quad 2.25324 \quad 1.85709$

$\begin{array}{lll}1.02641 & 2.24622 & 1.85614\end{array}$ $1.02182 \quad 2.239231 .85519$ $\begin{array}{lll}1.01725 & 2.23227 & 1.85424\end{array}$ $\begin{array}{llll}1.01272 & 2.22534 & 1.85328\end{array}$ $1.00820 \quad 2.21844 \quad 1.85232$

$1.003722 .21157 \quad 1.85136$ $0.999262 .20473 \quad 1.85039$ $0.99482 \quad 2.19791 \quad 1.84942$ 0.990412 .191131 .84845 $0.986022 .18437 \quad 1.84748$

$0.98166 \quad 2.17764 \quad 1.84650$ $0.977332 .17094 \quad 1.84552$ 0.973012 .164271 .84454 $\begin{array}{llll}0.96872 & 2.15762 & 1.84355\end{array}$ 0.964462 .151011 .84256

$0.96022 \quad 2.14442 \quad 1.84157$ $0.95600 \quad 2.13785 \quad 1.84058$ $0.951812 .13132 \quad 1.83958$ $0.947642 .12481 \quad 1.83858$ 0.943492 .118321 .83757

$0.93936 \quad 2.11187 \quad 1.83657$ $0.93526 \quad 2.10543 \quad 1.83556$ $0.93118 \quad 2.09903 \quad 1.83455$ $0.927122 .09265 \quad 1.83353$ $0.92309 \quad 2.08630 \quad 1.83251$

$\begin{array}{llll}0.91907 & 2.07997 & 1.83149\end{array}$ $0.91508 \quad 2.07367 \quad 1.83047$ $0.911112 .06739 \quad 1.82944$ $0.90716 \quad 2.06113 \quad 1.82841$ $0.90323 \quad 2.05491 \quad 1.82738$

$0.89932 \quad 2.04870 \quad 1.82635$ $0.89544 \quad 2.04252 \quad 1.82531$ $0.891572 .03637 \quad 1.82427$ $0.88773 \quad 2.03024 \quad 1.82322$ $0.883912 .02413 \quad 1.82218$

0.880102 .018051 .82113 $0.876322 .01199 \quad 1.82008$ $0.87256 \quad 2.00596 \quad 1.81902$ $0.86881 \quad 1.99995 \quad 1.81797$ $0.865091 .99396 \quad 1.81691$

$0.86139 \quad 1.98799 \quad 1.81584$ $0.85770 \quad 1.98205 \quad 1.81478$ $0.85404 \quad 1.976131 .81371$ $0.85039 \quad 1.97024 \quad 1.81264$ 0.846771 .964361 .81157
$T=4300$

$1.07892 \quad 2.32547 \quad 1.86644$ $1.07414 \quad 2.31831 \quad 1.86555$ $1.06938 \quad 2.31118 \quad 1.86465$ $1.06466 \quad 2.30408 \quad 1.86375$ $1.05996 \quad 2.29701 \quad 1.86284$

$\begin{array}{llll}1.05528 & 2.28997 & 1.86193\end{array}$ $1.050642 .28295 \quad 1.86102$ $1.046022 .27597 \quad 1.86011$ 1.041432 .269021 .85919 $1.03686 \quad 2.26210 \quad 1.85827$

$1.03232 \quad 2.25520 \quad 1.85735$ $1.02780 \quad 2.24834 \quad 1.85643$ $1.023312 .24150 \quad 1.85550$ $1.01884 \quad 2.23470 \quad 1.85457$ $1.01440 \quad 2.22792 \quad 1.85364$

$\begin{array}{lll}1.00998 & 2.22117 & 1.85270\end{array}$ $1.00559 \quad 2.21444 \quad 1.85176$ 1.001222 .207751 .85082 $\begin{array}{llll}0.99688 & 2.20108 & 1.84988\end{array}$ $0.99256 \quad 2.19444 \quad 1.84893$

$0.98827 \quad 2.18783 \quad 1.84798$ $0.983992 .18124 \quad 1.84702$ $0.97974 \quad 2.17468 \quad 1.84607$ $0.97552 \quad 2.16815 \quad 1.84511$ $0.97132 \quad 2.16164 \quad 1.84415$

$0.96714 \quad 2.15516 \quad 1.84318$ $0.96298 \quad 2.14870 \quad 1.84222$ $0.958842 .14228 \quad 1.84125$ $0.954732 .13587 \quad 1.84027$ $0.950642 .12950 \quad 1.83930$

$0.94657 \quad 2.12315 \quad 1.83832$ $0.94253 \quad 2.11682 \quad 1.83734$ $0.93850 \quad 2.110521 .83636$ $0.93450 \quad 2.104241 .83537$ $0.93052 \quad 2.09799 \quad 1.83438$

$0.92656 \quad 2.09176 \quad 1.83339$ $0.92262 \quad 2.08556 \quad 1.83239$ $0.91870 \quad 2.07938 \quad 1.83140$ $0.91480 \quad 2.07323 \quad 1.83040$ $0.91092 \quad 2.06710 \quad 1.82939$

$0.90707 \quad 2.06099 \quad 1.82839$ $0.903232 .05491 \quad 1.82738$ $0.89942 \quad 2.04885 \quad 1.82637$ 0.895622 .042811 .82536 $0.89184 \quad 2.03680 \quad 1.82434$

$0.88809 \quad 2.03081 \quad 1.82332$ $0.88435 \quad 2.02484 \quad 1.82230$ $0.880632 .01890 \quad 1.82128$ 0.876932 .012981 .82025 $0.87326 \quad 2.00708 \quad 1.81922$
2600 2610 2620 2630 2640

2650

2660

2670

2680

2690

2700

2710

2720

2730

2740

2750

2760

2770

2780

2790

2800

2810

2820

2830

2840

2850

2860

2870

2880

2890

2900

2910

2920

2930

2940

2950

2960

2970

2980

2990

3000

3010

3020

3030

3040

3050

3060

3070

3080

3090 
Table II Harmonic Oscillator Contributions to the Thermodynamic

Functions (in units of calories, moles, and ${ }^{\circ} \mathrm{K}$ )
$\mathrm{cm}^{-1}-\left(\mathrm{F}^{\circ}-\mathrm{E}_{\mathrm{O}}^{\circ}\right) / \mathrm{T} \quad \mathrm{S}^{\circ}$
$\mathrm{C}_{\mathrm{p}}^{\circ} \quad-\left(\mathrm{F}^{\circ}-\mathrm{E}_{\mathrm{O}}\right) / \mathrm{T} \quad \mathrm{S}^{\circ}$
so $\quad \mathrm{C}_{\mathrm{p}}$
$-\left(F^{\circ}-E_{O}^{\circ}\right) / T$
So
$\mathrm{c}_{\mathrm{p}}^{\circ}$

$\mathrm{cm}^{-1}$

$T=4100$.

$3100 \quad 0.81648 \quad 1.91498 \quad 1.80226$

$3110 \quad 0.81295 \quad 1.90918 \quad 1.80114$

$3120 \quad 0.80944 \quad 1.90340 \quad 1.80001$

$\begin{array}{llll}3130 & 0.80594 \quad 1.89764 \quad 1.79888\end{array}$

$\begin{array}{llll}3140 & 0.80246 \quad 1.89190 & 1.79774\end{array}$

$\begin{array}{llll}3150 & 0.79900 \quad 1.88619 & 1.79661\end{array}$

$\begin{array}{llll}3160 & 0.79556 \quad 1.88050 & 1.79547\end{array}$

$\begin{array}{llll}3170 & 0.79213 & 1.87482 & 1.79432\end{array}$

$\begin{array}{llll}3180 & 0.78873 \quad 1.86917 & 1.79318\end{array}$

$\begin{array}{llll}3190 & 0.78534 & 1.86355 & 1.79203\end{array}$

$\begin{array}{lllll}3200 & 0.78197 & 1.85794 & 1.79088\end{array}$

$\begin{array}{lllll}3210 & 0.77861 & 1.85235 & 1.78973\end{array}$

$\begin{array}{lllll}3220 & 0.77528 & 1.84679 & 1.78857\end{array}$

$\begin{array}{lllll}3230 & 0.77196 & 1.84124 & 1.78741\end{array}$

$\begin{array}{llll}3240 & 0.76865 & 1.83572 & 1.78625\end{array}$

$\begin{array}{lllll}3250 & 0.76537 & 1.83022 & 1.78508\end{array}$

$\begin{array}{lllll}3260 & 0.76210 & 1.82474 & 1.78392\end{array}$

$\begin{array}{llll}3270 & 0.75885 & 1.81927 & 1.78275\end{array}$

$\begin{array}{llll}3280 & 0.75562 & 1.81383 & 1.78157\end{array}$

$\begin{array}{lllll}3290 & 0.75240 & 1.80841 & 1.78040\end{array}$

$\begin{array}{llll}3300 & 0.74920 & 1.80301 & 1.77922\end{array}$

$\begin{array}{llll}3310 & 0.74601 & 1.79763 & 1.77804\end{array}$

$\begin{array}{lllll}3320 & 0.74284 & 1.79227 & 1.77686\end{array}$

$\begin{array}{lllll}3330 & 0.73969 & 1.78692 & 1.77567\end{array}$

$\begin{array}{llll}3340 & 0.73655 & 1.78160 & 1.77449\end{array}$

$\begin{array}{llll}3350 & 0.73343 & 1.77630 & 1.77329\end{array}$

$\begin{array}{llll}3360 & 0.73033 & 1.77101 & 1.77210\end{array}$

$\begin{array}{lllll}3370 & 0.72724 & 1.76575 & 1.77090\end{array}$

$\begin{array}{lllll}3380 & 0.72416 & 1.76050 & 1.76971\end{array}$

$3390 \quad 0.72110 \quad 1.75528 \quad 1.76851$

$\begin{array}{llll}3400 & 0.71806 & 1.75007 & 1.76730\end{array}$

$\begin{array}{llll}3410 & 0.71503 & 1.74488 & 1.76610\end{array}$

$\begin{array}{llll}3420 & 0.71202 & 1.73971 & 1.76489\end{array}$

$\begin{array}{llll}3430 & 0.70902 & 1.73456 & 1.76367\end{array}$

$\begin{array}{llll}3440 & 0.70604 & 1.72943 & 1.76246\end{array}$

$\begin{array}{lllll}3450 & 0.70307 & 1.72431 & 1.76124\end{array}$

$\begin{array}{llll}3460 & 0.70012 & 1.71922 & 1.76003\end{array}$

$\begin{array}{lllll}3470 & 0.69718 & 1.71414 & 1.75880\end{array}$

$\begin{array}{lllll}3480 & 0.69426 & 1.70908 & 1.75758\end{array}$

$\begin{array}{llll}3490 & 0.69135 & 1.70404 & 1.75635\end{array}$

$\begin{array}{llll}3500 & 0.68846 & 1.69902 & 1.75512\end{array}$

$\begin{array}{lllll}3510 & 0.68558 & 1.69401 & 1.75389\end{array}$

$\begin{array}{llll}3520 & 0.68271 & 1.68902 & 1.75266\end{array}$

$\begin{array}{lllll}3530 & 0.67986 & 1.68405 & 1.75142\end{array}$

$\begin{array}{llll}3540 & 0.67702 & 1.67910 & 1.75018\end{array}$

$\begin{array}{lllll}3550 & 0.67420 & 1.67416 & 1.74894\end{array}$

$\begin{array}{lllll}3560 & 0.67139 & 1.66925 & 1.74770\end{array}$

$\begin{array}{llll}3570 & 0.66859 & 1.66434 & 1.74645\end{array}$

$\begin{array}{lllll}3580 & 0.66581 & 1.65946 & 1.74520\end{array}$

$\begin{array}{lllll}3590 & 0.66304 & 1.65460 & 1.74395\end{array}$

$$
\mathrm{T}=4200 \text {. }
$$

$T=4300$.

$0.84316 \quad 1.95851 \quad 1.81049$

$0.83957 \quad 1.95268 \quad 1.80941$

$0.83600 \quad 1.94687 \quad 1.80833$

0.832451 .941091 .80724

0.828921 .935331 .80616

$0.82540 \quad 1.92958 \quad 1.80507$

0.821911 .923871 .80398

0.818431 .918171 .80288

$0.81497 \quad 1.91249 \quad 1.80178$

0.811531 .906841 .80068

$0.80810 \quad 1.901201 .79958$ $0.804691 .89559 \quad 1.79847$ 0.801301 .890001 .79736 0.797931 .884421 .79625 $0.79458 \quad 1.87887 \quad 1.79514$

$0.79124 \quad 1.87334 \quad 1.79402$ 0.787921 .867831 .79290 $0.78461 \quad 1.86234 \quad 1.79178$ $0.781331 .85687 \quad 1.79066$ 0.778051 .851421 .78953

$0.77480 \quad 1.84600 \quad 1.78840$ $0.77156 \quad 1.84059 \quad 1.78727$ 0.768341 .835201 .78614 0.765141 .829831 .78500 0.761951 .824481 .78386

$0.75877 \quad 1.81914 \quad 1.78272$ $0.75562 \quad 1.81383 \quad 1.78157$ $0.75247 \quad 1.80854 \quad 1.78043$ $\begin{array}{llll}0.74935 & 1.80327 & 1.77928\end{array}$ $0.74624 \quad 1.79801 \quad 1.77813$

$\begin{array}{llll}0.74314 & 1.79278 & 1.77697\end{array}$ 0.740061 .787561 .77581 $0.73700 \quad 1.78236 \quad 1.77465$ 0.733951 .777181 .77349 0.730921 .772021 .77233

$0.72790 \quad 1.76688 \quad 1.77116$ $0.72489 \quad 1.76175 \quad 1.76999$ $0.721901 .75664 \quad 1.76882$ $0.71893 \quad 1.75156 \quad 1.76765$ $\begin{array}{llll}0.71597 & 1.74649 & 1.76647\end{array}$

$0.71302 \quad 1.74143 \quad 1.76529$ $0.71009 \quad 1.73640 \quad 1.76411$ $0.70718 \quad 1.73138 \quad 1.76292$ $0.70427 \quad 1.72638 \quad 1.76174$ $0.70138 \quad 1.72140 \quad 1.76055$

$0.69851 \quad 1.71644 \quad 1.75936$ $0.695651 .71149 \quad 1.75816$ $0.69280 \quad 1.70656 \quad 1.75697$ 0.689971 .701641 .75577 $\begin{array}{llll}0.68715 & 1.69675 & 1.75457\end{array}$
$0.86960 \quad 2.00120 \quad 1.81819$ $0.86595 \quad 1.99535 \quad 1.81715$ 0.862331 .989521 .81612

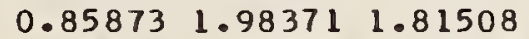
$0.85514 \quad 1.97792 \quad 1.81403$

$0.85158 \quad 1.97215 \quad 1.81299$ $0.848031 .96641 \quad 1.81194$ $0.84450 \quad 1.960681 .81089$ $0.84099 \quad 1.95498 \quad 1.80984$ $0.83749 \quad 1.949301 .80878$

$0.83402 \quad 1.94364 \quad 1.80772$ 0.830561 .938001 .80666 0.827121 .932391 .80560 $0.823691 .92679 \quad 1.80453$ 0.820291 .921211 .80347

$0.81690 \quad 1.91566 \quad 1.80240$ 0.813521 .910121 .80132 $0.81017 \quad 1.90461 \quad 1.80025$ $0.806831 .89911 \quad 1.79917$ 0.803511 .893631 .79809

$0.80020 \quad 1.88818 \quad 1.79700$ $0.796921 .88274 \quad 1.79592$ $0.79364 \quad 1.87733 \quad 1.79483$ 0.790391 .871931 .79374 $0.787151 .86655 \quad 1.79264$

$0.78392 \quad 1.86120 \quad 1.79155$ $0.78072 \quad 1.85586 \quad 1.79045$ $0.77752 \quad 1.85054 \quad 1.78935$ $0.77435 \quad 1.84524 \quad 1.78825$ $0.77119 \quad 1.83996 \quad 1.78714$

$0.76804 \quad 1.83470 \quad 1.78603$ $0.76491 \quad 1.82945 \quad 1.78492$ $0.76180 \quad 1.824231 .78381$ 0.758701 .819021 .78269 0.755621 .813831 .78157

$0.75255 \quad 1.80866 \quad 1.78045$ $0.74949 \quad 1.80351 \quad 1.77933$ $\begin{array}{llll}0.74645 & 1.79838 & 1.77821\end{array}$ $0.74343 \quad 1.79326 \quad 1.77708$ $0.74042 \quad 1.78816 \quad 1.77595$

$\begin{array}{lll}0.73743 & 1.78308 & 1.77482\end{array}$ $0.73445 \quad 1.77802 \quad 1.77368$ $0.73148 \quad 1.77298 \quad 1.77255$ $0.728531 .76795 \quad 1.77141$ $0.72559 \quad 1.76294 \quad 1.77026$

$0.72267 \quad 1.75795 \quad 1.76912$ $\begin{array}{llll}0.71976 & 1.75297 & 1.76797\end{array}$ $0.71686 \quad 1.74802 \quad 1.76682$ $\begin{array}{llll}0.71398 & 1.74308 & 1.76567\end{array}$ $0.71111 \quad 1.738151 .76452$
3100

3110

3120

3130

3140

3150

3160

3170

3180

3190

3200

3210

3220

3230

3240

3250

3260

3270

3280

3290

3300

3310

3320

3330

3340

3350

3360

3370

3380

3390

3400

3410

3420

3430

3440

3450

3460

3470

3480

3490

3500

3510

3520

3530

3540

3550

3560

3570

3580

3590 
Table II Harmonic Oscillator Contributions to the Thermodynamic Functions (in units of calories, moles, and ${ }^{\circ} \mathrm{K}$ )
$\mathrm{cm}^{-1}$
So
$-\left(F^{\circ}-E_{O}^{O}\right) / T$
$\mathrm{C}_{\mathrm{p}}^{\circ} \quad-\left(\mathrm{F}^{\circ}-\mathrm{E}_{\mathrm{O}}^{\circ}\right) / \mathrm{T} \quad$ So
$\mathrm{C}_{\mathrm{p}}^{\circ}$
$-\left(F^{\circ}-E_{g}\right) / T$
So
$\underset{\mathrm{p}}{\mathrm{O}}$
$\nu$
$\mathrm{cm}^{-1}$

$\mathrm{T}=4100$.

3600

3610

3620

3630

3640

3650

3660

3670

3680

3690

3700

3710

3720

3730

3740

3750

3760

3770

3780

3790

3800

3810

3820

3830

3840

3850

3860

3870

3880

3890

3900

3910

3920

3930

3940

3950

3960

3970

3980

3990

4000
$0.66028 \quad 1.64975 \quad 1.74270$ $0.65754 \quad 1.64491 \quad 1.74144$ 0.654811 .640101 .74019 $0.65210 \quad 1.635301 .73892$ 0.649401 .630521 .73766

$0.64671 \quad 1.625751 .73640$ 0.644031 .621001 .73513 $0.641371 .61627 \quad 1.73386$ 0.638721 .611551 .73259 $0.63608 \quad 1.606851 .73131$

$0.63346 \quad 1.60217 \quad 1.73003$ $0.630851 .59750 \quad 1.72875$ $\begin{array}{llll}0.62825 & 1.59285 & 1.72747\end{array}$ $0.62566 \quad 1.58821 \quad 1.72619$ 0.623091 .583591 .72490

$0.620521 .57899 \quad 1.72361$ 0.617971 .574401 .72232 0.615441 .569831 .72103 $0.612911 .56527 \quad 1.71973$ 0.610401 .560731 .71844

$0.60790 \quad 1.55620 \quad 1.71714$ 0.605411 .551691 .71583 0.602931 .547201 .71453 0.600461 .542721 .71322 0.598011 .538251 .71191

$0.59557 \quad 1.53380 \quad 1.71060$ 0.593131 .529361 .70929 $0.59071 \quad 1.52494 \quad 1.70797$ $0.58831 \quad 1.52054 \quad 1.70665$ 0.585911 .516151 .70533

$0.583521 .51177 \quad 1.70401$ $0.581151 .50741 \quad 1.70269$ $0.578791 .50306 \quad 1.70136$ $0.576431 .49873 \quad 1.70003$ $0.574091 .49441 \quad 1.69870$

$0.57176 \quad 1.49010 \quad 1.69737$ $0.56944 \quad 1.48581 \quad 1.69603$ $0.56713 \quad 1.48154 \quad 1.69470$ $0.56484 \quad 1.47728 \quad 1.69336$ 0.562551 .473031 .69202 $\begin{array}{llll}0.56027 & 1.46880 & 1.69067\end{array}$

$$
T=4200 .
$$

$0.684351 .69187 \quad 1.75336$ 0.681551 .687011 .75216 $0.67878 \quad 1.68216 \quad 1.75095$ 0.676011 .677331 .74974 0.673261 .672521 .74853

$\begin{array}{llll}0.67052 & 1.66773 & 1.74731\end{array}$ $0.667791 .66295 \quad 1.74610$ $0.66508 \quad 1.65819 \quad 1.74488$ $0.66238 \quad 1.65344 \quad 1.74365$ $0.65970 \quad 1.64871 \quad 1.74243$

$0.65702 \quad 1.643991 .74120$ $0.65436 \quad 1.63930 \quad 1.73998$ $0.65171 \quad 1.634611 .73874$ $0.64908 \quad 1.629951 .73751$ 0.646451 .625301 .73628

$0.64384 \quad 1.62066 \quad 1.73504$ 0.641241 .616041 .73380 $0.63866 \quad 1.61144 \quad 1.73256$ 0.636081 .606851 .73131 0.633521 .602281 .73006

$0.63097 \quad 1.59772 \quad 1.72882$ 0.628431 .593181 .72756 $0.62591 \quad 1.58866 \quad 1.72631$ 0.623391 .584141 .72506 0.620891 .579651 .72380

$0.61840 \quad 1.57517 \quad 1.72254$ 0.615921 .570701 .72128 0.613451 .566251 .72001 0.610991 .561811 .71874 $0.608551 .55739 \quad 1.71748$

0.606121 .552981 .71621 0.603691 .548591 .71493 0.601281 .544211 .71366 $0.59888 \quad 1.539841 .71238$ 0.596491 .535491 .71110

0.594121 .531161 .70982 $0.59175 \quad 1.52684 \quad 1.70854$ $0.589391 .52253 \quad 1.70725$ $0.58705 \quad 1.51824 \quad 1.70596$ $0.584711 .51396 \quad 1.70467$ 0.582391 .509691 .70338

$$
T=4300 \text {. }
$$

$0.708261 .73325 \quad 1.76336$ $\begin{array}{llll}0.70542 & 1.72836 & 1.76221\end{array}$ $0.702591 .72348 \quad 1.76105$ $\begin{array}{llll}0.69978 & 1.71863 & 1.75988\end{array}$ $0.69698 \quad 1.71379 \quad 1.75872$

$0.69419 \quad 1.70896 \quad 1.75755$ 0.691421 .704161 .75638 $0.68866 \quad 1.69937 \quad 1.75521$ $0.68591 \quad 1.69459 \quad 1.75404$ 0.683181 .689831 .75286

$0.680451 .68509 \quad 1.75168$ 0.677751 .680361 .75050 0.675051 .675651 .74932 0.672371 .670961 .74813 $0.669691 .66628 \quad 1.74695$

0.667041 .661621 .74576 0.664391 .656971 .74456 $0.66176 \quad 1.652341 .74337$ 0.659141 .647721 .74217 0.656531 .643121 .74098

0.653931 .638531 .73978 $0.65134 \quad 1.63396 .1 .73857$ 0.648771 .629411 .73737 0.646211 .624871 .73616 0.643661 .620341 .73495

0.641121 .615831 .73374 0.638601 .611331 .73253 0.636081 .606851 .73131 $0.63358 \quad 1.602391 .73009$ 0.631091 .597941 .72887

$0.62861 \quad 1.59350 \quad 1.72765$ 0.626141 .589081 .72643 $0.62368 \quad 1.58467 \quad 1.72520$ 0.621241 .580271 .72397 0.618801 .575891 .72274

$0.61638 \quad 1.57153 \quad 1.72151$ 0.613971 .567181 .72028 0.611571 .562841 .71904 $0.60917 \quad 1.55852 \quad 1.71780$ 0.606791 .554211 .71656

$0.604431 .54991 \quad 1.71532$
3600 3610 3620 3630 3640

3650

3660 3670 3680 3690

3700

3710 3720 3730 3740

3750 3760 3770 3780 3790

3800 3810

3820

3830

3840

3850

3860 3870 3880 3890

3900 3910 3920 3930 3940

3950 3960 3970 3980 3990 4000 
-Table II Harmonic Oscillator Contributions to the Thermodynamic

Functions (in units of calories, moles, and ${ }^{\circ} \mathrm{K}$ )

$\mathrm{cm}^{-1}-\left(\mathrm{F}^{\circ}-\mathrm{E}_{\mathrm{O}}^{\mathrm{O}}\right) / \mathrm{T} \quad$ So

$T=4400$
$\mathrm{C}_{\mathrm{p}}^{\circ} \quad-\left(\mathrm{F}^{\circ}-\mathrm{E}_{\mathrm{O}}^{\mathrm{O}}\right) / \mathrm{T} \quad \mathrm{S}^{\circ}$

$\mathrm{S}^{\circ}$

$$
-\left(F^{\circ}-E_{O}^{O}\right) / T
$$

$\begin{array}{lllll}100 & 6.82928 & 8.78414 & 1.98699\end{array}$ $110 \quad 6.643118 .59476 \quad 1.98696$ $120 \quad 6.47343 \quad 8.42187 \quad 1.98692$ $\begin{array}{lllll}130 & 6.31760 & 8.26283 & 1.98687\end{array}$ $140 \quad 6.17356 \quad 8.115591 .98682$

$\begin{array}{lllll}150 & 6.03969 & 7.97852 & 1.98677\end{array}$

$160 \quad 5.914667 .85030 \quad 1.98672$

$\begin{array}{lllll}170 & 5.79741 & 7.72985 & 1.98666\end{array}$

$180 \quad 5.687047 .616301 .98660$

$190 \quad 5.582827 .508891 .98653$

$200 \quad 5.484107 .40700 \quad 1.98646$

$210 \quad 5.39036 \quad 7.31008 \quad 1.98639$

$220 \quad 5.301137 .21768 \quad 1.98631$

$230 \quad 5.216007 .12938 \quad 1.98623$

$240 \quad 5.134647 .044851 .98615$

$\begin{array}{lllll}250 & 5.05672 & 6.96377 & 1.98606\end{array}$ $260 \quad 4.98199 \quad 6.88588 \quad 1.98597$ $270 \quad 4.91020 \quad 6.81093 \quad 1.98588$ $280 \quad 4.841136 .73871 \quad 1.98578$ $290 \quad 4.774596 .66903 \quad 1.98568$

$\begin{array}{llll}300 & 4.71042 & 6.60171 & 1.98558\end{array}$ $\begin{array}{lllll}310 & 4.64846 & 6.53661 & 1.98547\end{array}$ $\begin{array}{lllll}320 & 4.58856 & 6.47357 & 1.98536\end{array}$ $\begin{array}{lllll}330 & 4.53060 & 6.41248 & 1.98524\end{array}$ $\begin{array}{lllll}340 & 4.47447 & 6.35322 & 1.98512\end{array}$

$\begin{array}{lllll}350 & 4.42006 & 6.29568 & 1.98500\end{array}$ $\begin{array}{lllll}360 & 4.36726 & 6.23976 & 1.98488\end{array}$ $370 \quad 4.31600 \quad 6.18538 \quad 1.98475$ $380 \quad 4.266196 .13245 \quad 1.98461$ $390 \quad 4.217756 .08090 \quad 1.98448$

$400 \quad 4.17062 \quad 6.03066 \quad 1.98434$ $410 \quad 4.124735 .981661 .98420$ $420 \quad 4.08002 \quad 5.933851 .98405$ $430 \quad 4.03643 \quad 5.88717 \quad 1.98390$ $\begin{array}{lll}440 \quad 3.99392 & 5.84156 & 1.98375\end{array}$

$\begin{array}{lllll}450 & 3.95243 & 5.79698 & 1.98359\end{array}$ $460 \quad 3.91193 \quad 5.753391 .98343$ $470 \quad 3.87236 \quad 5.71073 \quad 1.98326$ $\begin{array}{lllll}480 & 3.83368 & 5.66898 & 1.98310\end{array}$ $490 \quad 3.79587 \quad 5.62809 \quad 1.98292$

$\begin{array}{llll}500 & 3.75889 & 5.58803 & 1.98275\end{array}$ $\begin{array}{lllll}510 & 3.72270 & 5.54877 & 1.98257\end{array}$ $\begin{array}{lllll}520 & 3.68727 & 5.51027 & 1.98239\end{array}$ $\begin{array}{lllll}530 & 3.65257 & 5.47251 & 1.98220\end{array}$ $\begin{array}{lllll}540 & 3.61858 & 5.43547 & 1.98201\end{array}$

$\begin{array}{llll}550 & 3.58527 & 5.39910 & 1.98182\end{array}$ $\begin{array}{llll}560 & 3.55262 & 5.36339 & 1.98163\end{array}$ $570 \quad 3.520595 .328321 .98143$ $\begin{array}{llll}580 & 3.48918 & 5.29386 & 1.98122\end{array}$ $590 \quad 3.45836 \quad 5.25999 \quad 1.98102$
$T=4500$.

$6.87322 \quad 8.82879 \quad 1.98700$ $6.68698 \quad 8.63941 \quad 1.98697$ $6.51723 \quad 8.46652 \quad 1.98693$ $6.361338 .30748 \quad 1.98688$ $6.217228 .16024 \quad 1.98684$

$6.083278 .02317 \quad 1.98679$ 5.958177 .894941 .98674 $5.84085 \quad 7.77450 \quad 1.98668$ 5.730417 .660951 .98662 5.626127 .553541 .98656

5.527337 .451641 .98649 5.433527 .354721 .98642 5.344227 .262321 .98635 $5.25902 \quad 7.17402 \quad 1.98627$ 5.177597 .089491 .98620

5.099607 .008411 .98611 $5.02480 \quad 6.93051 \quad 1.98603$ 4.952936 .855561 .98594 $4.883796 .78334 \quad 1.98584$ 4.817196 .713651 .98575

$4.75295 \quad 6.64634 \quad 1.98565$ 4.690916 .581231 .98554 $4.63095 \quad 6.518191 .98544$ $4.57292 \quad 6.45710 \quad 1.98533$ 4.516726 .397831 .98521

4.462236 .340291 .98510 $4.40937 \quad 6.28437 \quad 1.98498$ $4.358046 .22998 \quad 1.98485$ $4.30816 \quad 6.17705 \quad 1.98473$ 4.259656 .125501 .98460

$4.212456 .07526 \quad 1.98446$ $4.166496 .02626 \quad 1.98433$ 4.121715 .978441 .98419 $4.07806 \quad 5.93175 \quad 1.98404$ $4.03548 \quad 5.88614 \quad 1.98390$

3. 993925.841561 .98375 3.953345 .797961 .98359 3.913715 .755301 .98343 $3.874975 .71355 \quad 1.98327$ 3.837095 .672651 .98311 $3.800035 .63259 \quad 1.98294$ 3.763775 .593331 .98277 $3.728285 .55483 \quad 1.98260$ $3.693515 .51706 \quad 1.98242$ 3.659455 .480011 .98224

$3.62608 \quad 5.44364 \quad 1.98206$ 3.593355 .407931 .98187 $3.56126 \quad 5.372851 .98168$ $3.52978 \quad 5.338391 .98148$ 3.498895 .304521 .98129
$6.916218 .87246 \quad 1.98701$ $6.72990 \quad 8.68308 \quad 1.98697$ $6.56008 \quad 8.510191 .98694$ 6.404118 .351151 .98690 6.259938 .203911 .98685

6.125928 .066831 .98681 $6.00075 \quad 7.938611 .98676$ 5.883367 .818161 .98670 5.772867 .704611 .98665 $5.668497 .59720 \quad 1.98658$

$5.569647 .49530 \quad 1.98652$ $5.47576 \quad 7.39838 \quad 1.98646$ $5.386397 .30597 \quad 1.98639$ $5.301137 .21768 \quad 1.98631$ $5.219627 .13314 \quad 1.98624$

5.141577 .052061 .98616 $5.06670 \quad 6.97416 \quad 1.98607$ 4.994776 .899211 .98599 $4.92556 \quad 6.82699 \quad 1.98590$ $4.858896 .75730 \quad 1.98581$

$4.79458 \quad 6.68998 \quad 1.98571$ $4.73248 \quad 6.62487 \quad 1.98561$ 4.672456 .561831 .98551 $4.61436 \quad 6.500731 .98541$ $4.558096 .44147 \quad 1.98530$

$4.503546 .38392 \quad 1.98519$ 4.450616 .328001 .98507 4.399216 .273611 .98495 $4.34926 \quad 6.22068 \quad 1.98483$ 4.300696 .169121 .98471

$4.25342 \quad 6.11887 \quad 1.98458$ $4.20740 \quad 6.06987 \quad 1.98445$ 4.162556 .022051 .98431 $4.118835 .97536 \quad 1.98418$ 4.076185 .929751 .98404

$4.03456 \quad 5.88516 \quad 1.98389$ $3.993925 .84156 \quad 1.98375$ 3.954225 .798901 .98359 $3.91541 \quad 5.75714 \quad 1.98344$ $3.87747 \quad 5.71624 \quad 1.98328$

$3.84035 \quad 5.67618 \quad 1.98312$ 3.804025 .636911 .98296 $3.76846 \quad 5.59840 \quad 1.98279$ 3.733635 .560641 .98263 $3.699515 .52358 \quad 1.98245$

$3.66606 \quad 5.48720 \quad 1.98228$ $3.63328 \quad 5.451491 .98210$ 3.601125 .416411 .98191 $3.569575 .38194 \quad 1.98173$ 3.538625 .348071 .98154
100

110

120

130

140

150

160

170

180

190

200

210

220

230

240

250

260

270

280

290

300

310

320

330

340

350

360

370

380

390

400

410

420

430

440

450

460

470

480

490

500

510

520

530

540

550

560

570

580

590 
Table II Harmonic Oscillator Contributions to the Thermodynamic

Functions (in units of calories, moles, and ${ }^{\circ} \mathrm{K}$ )
$\mathrm{cm}^{-1}-\left(\mathrm{F}^{\circ}-\mathrm{E}_{\mathrm{O}}\right) / \mathrm{T}$, So
$\mathrm{C}_{\mathrm{p}}^{\circ} \quad-\left(\mathrm{F} \circ-\mathrm{E}_{0}^{\circ}\right) / \mathrm{T} \quad \mathrm{S}^{\circ}$
$\mathrm{C}_{\mathrm{P}} \quad-\left(\mathrm{F}^{\circ}-\mathrm{E}_{\mathrm{O}}\right) / \mathrm{T}$
So
$T=4400$
$T=4500$
$T=4600$

$\mathrm{co}_{\mathrm{p}}^{\circ}$

$600 \quad 3.42810 \quad 5.22670 \quad 1.98081$

$\begin{array}{llll}610 & 3.39840 & 5.19396 \quad 1.98059\end{array}$

$\begin{array}{lll}620 & 3.36922 & 5.16176 \quad 1.98038\end{array}$

$630 \quad 3.340575 .13007 \quad 1.98016$

$\begin{array}{lllll}640 & 3.31241 & 5.09889 & 1.97993\end{array}$

$650 \quad 3.28473 \quad 5.06819 \quad 1.97971$

$\begin{array}{lllll}660 & 3.25753 & 5.03797 & 1.97947\end{array}$

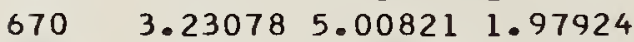

$\begin{array}{lllll}680 & 3.20447 & 4.97888 & 1.97900\end{array}$

$690 \quad 3.17858 \quad 4.95000 \quad 1.97876$

$700 \quad 3.153124 .92153 \quad 1.97852$

$\begin{array}{lllll}710 & 3.12805 & 4.89346 & 1.97827\end{array}$

$720 \quad 3.10338 \quad 4.86580 \quad 1.97802$

$\begin{array}{lllll}730 & 3.07910 & 4.83851 & 1.97776\end{array}$

$740 \quad 3.05518 \quad 4.81161 \quad 1.97750$

$\begin{array}{llll}750 & 3.03162 & 4.78506 & 1.97724\end{array}$ $\begin{array}{llll}760 & 3.00842 & 4.75888 & 1.97697\end{array}$ $770 \quad 2.985554 .73304 \quad 1.97670$ $780 \quad 2.963024 .70753 \quad 1.97643$ $790 \quad 2.940824 .682361 .97616$

$800 \quad 2.91893 \quad 4.65750 \quad 1.97588$ $810 \quad 2.897354 .63296 \quad 1.97559$ $820 \quad 2.87607 \quad 4.60872 \quad 1.97531$ $830 \quad 2.855094 .58478 \quad 1.97502$ $840 \quad 2.834394 .561121 .97472$

$850 \quad 2.81398 \quad 4.53776 \quad 1.97443$ $860 \quad 2.793834 .51466 \quad 1.97413$ $870 \quad 2.77395 \quad 4.49184 \quad 1.97382$ $880 \quad 2.75434 \quad 4.46929 \quad 1.97351$ $890 \quad 2.73498 \quad 4.44699 \quad 1.97320$

$900 \quad 2.71586 \quad 4.42494 \quad 1.97289$ $910 \quad 2.69700 \quad 4.40315 \quad 1.97257$ $920 \quad 2.678364 .38159 \quad 1.97225$ $930 \quad 2.65997 \quad 4.36027 \quad 1.97193$ $940 \quad 2.641804 .33918 \quad 1.97160$

$950 \quad 2.623854 .31832 \quad 1.97127$ $960 \quad 2.606124 .29768 \quad 1.97093$ $970 \quad 2.588614 .27726 \quad 1.97059$ $980 \quad 2.57130 \quad 4.25705 \quad 1.97025$ $990 \quad 2.55420 \quad 4.23705 \quad 1.96991$

$1000 \quad 2.53731 \quad 4.21725 \quad 1.96956$ $1010 \quad 2.520604 .197651 .96920$ $1020 \quad 2.504104 .17825 \quad 1.96885$ $1030 \quad 2.48778 \quad 4.15905 \quad 1.96849$ $1040 \quad 2.471644 .140031 .96813$

$1050 \quad 2.45569 \quad 4.12120 \quad 1.96776$ $1060 \quad 2.439924 .102551 .96739$ $1070 \quad 2.424324 .08407 \quad 1.96702$

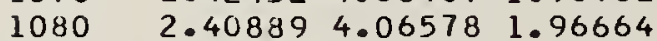
$1090 \quad 2.393634 .04765 \quad 1.96626$
$3.468575 .27122 \quad 1.98109$ $3.43879 \quad 5.23847 \quad 1.98088$ $3.409555 .20627 \quad 1.98068$ $3.380835 .17458 \quad 1.98046$ 3.352615 .143391 .98025

$3.32486 \quad 5.112691 .98003$ $3.297595 .08246 \quad 1.97981$ $3.270775 .05269 \quad 1.97959$ 3.244395 .023361 .97936 $3.21845 \quad 4.99447 \quad 1.97913$

$3.192914 .96599 \quad 1.97890$ $3.16778 \quad 4.93792 \quad 1.97866$ 3.143044 .910251 .97842 $3.11869 \quad 4.88296 \quad 1.97817$ 3.094714 .856051 .97793

$3.07108 \quad 4.82950 \quad 1.97767$ 3.047814 .803311 .97742 $3.02488 \quad 4.77746 \quad 1.97716$ 3.00229 4.75195 1.97690 $2.980024 .72677 \quad 1.97664$ $2.95806 \quad 4.70191 \quad 1.97637$ $2.936424 .67736 \quad 1.97610$ 2.91507 4.65311 1.97583 $2.89402 \quad 4.62917 \quad 1.97555$ $2.87326 \quad 4.60551 \quad 1.97527$

$2.85278 \quad 4.582131 .97498$ $2.832574 .55904 \quad 1.97470$ $2.812624 .53621 \quad 1.97441$ $2.79294 \quad 4.51364 \quad 1.97411$ $2.773524 .49134 \quad 1.97381$

$2.75434 \quad 4.469291 .97351$ $2.73540 \quad 4.44748 \quad 1.97321$ 2.716714 .425921 .97290 $2.69825 \quad 4.404591 .97259$ $2.680014 .38350 \quad 1.97228$

2.662004 .362631 .97196 $2.644214 .34198 \quad 1.97164$ 2.626634 .321551 .97132 2.609264 .301331 .97099 2.592094 .281321 .97066

$2.57513 \quad 4.26152 \quad 1.97033$ 2.558374 .241921 .96999 $2.541794 .22251 \quad 1.96965$ 2.525414 .203291 .96931 $2.50921 \quad 4.18427 \quad 1.96896$

2.493194 .165431 .96861 $2.47736 \quad 4.14677 \quad 1.96826$ 2.461704 .128291 .96790 2.446214 .109991 .96754 $2.43088 \quad 4.091851 .96718$
$3.50823 \quad 5.31476 \quad 1.98135$ 3.478395 .282011 .98115 $3.449095 .24980 \quad 1.98095$ $3.42030 \quad 5.21811 \quad 1.98075$ 3.392015 .186911 .98055

$3.36420 \quad 5.15621 \quad 1.98034$ 3.336875 .125981 .98013 $3.30998 \quad 5.09620 \quad 1.97991$ $3.283545 .06687 \quad 1.97970$ $3.257535 .03797 \quad 1.97947$

$3.23193 \quad 5.009491 .97925$ $3.20674 \quad 4.98142 \quad 1.97902$ $3.18194 \quad 4.953741 .97879$ 3.157524 .926451 .97856 $3.133474 .89953 \quad 1.97832$

$3.10978 \quad 4.87298 \quad 1.97808$ $3.08645 \quad 4.84678 \quad 1.97784$ $3.06346 \quad 4.82092 \quad 1.97759$ $3.040804 .79541 \quad 1.97734$ $3.01846 \quad 4.770221 .97709$ 600 610 620 630 640

650 660 670 680 690

700

710

720

730

740

750

760

770

780

790

$2.99645 \quad 4.74535 \quad 1.97683$ $2.97474 \quad 4.72080 \quad 1.97657$ 2.953334 .696551 .97631 $2.93222 \quad 4.672591 .97605$ 2.911394 .648931 .97578

800

810

820

830

840

$2.89085 \quad 4.62555 \quad 1.97551$ $2.87057 \quad 4.60244 \quad 1.97523$ $2.85057 \quad 4.57961 \quad 1.97495$ $2.830824 .55704 \quad 1.97467$ 2.811334 .534731 .97439

$2.79209 \quad 4.51267 \quad 1.97410$ $2.77310 \quad 4.49086 \quad 1.97381$ $2.75434 \quad 4.46929 \quad 1.97351$ $2.735814 .44795 \quad 1.97322$ 2.717524 .426851 .97292

$2.69944 \quad 4.40598 \quad 1.97261$ $2.68159 \quad 4.38532 \quad 1.97231$ $2.66395 \quad 4.36488 \quad 1.97200$ $2.646524 .34466 \quad 1.97168$ 2.629294 .324641 .97137

$2.61226 \quad 4.30483 \quad 1.97105$ $2.59544 \quad 4.285221 .97073$ $2.57880 \quad 4.265811 .97040$ $2.56236 \quad 4.246591 .97007$ $2.54610 \quad 4.22755 \quad 1.96974$

$2.53002 \quad 4.20870 \quad 1.96940$ $2.514124 .19004 \quad 1.96907$ $2.49840 \quad 4.17155 \quad 1.96872$ 2.482854 .153241 .96838 $2.46746 \quad 4.13510 \quad 1.96803$
850

860

870

880

890

900

910

920

930

940

950

960

970

980

990

1000

1010

1020

1030

1040

1050

1060

1070

1080

1090 
Table II Harmonic Oscillator Contributions to the Thermodynamic

Functions (in units of calories, moles, and ${ }^{\circ} \mathrm{K}$ )
$\mathrm{cm}^{-1}$
S०
$\mathrm{C}_{\mathrm{p}}^{\circ} \quad-\left(\mathrm{F}^{\circ}-\mathrm{E}_{\mathrm{O}}^{\circ}\right) / \mathrm{T} \quad \mathrm{S}^{\circ}$
$\mathrm{C}_{\mathrm{p}}^{\circ} \quad-\left(\mathrm{F}^{\circ}-\mathrm{E}_{\mathrm{O}}^{\circ}\right) / \mathrm{T}$
S०
$\stackrel{\mathrm{C}}{\mathrm{p}} \quad \begin{gathered}\nu \\ \mathrm{cm}^{-1}\end{gathered}$

$\mathrm{T}=4400$.

$\mathrm{T}=4500$.

$T=4600$.

$1100 \quad 2.37854 \quad 4.02970 \quad 1.96588$

$1110 \quad 2.363614 .01191 \quad 1.96550$

$\begin{array}{lllll}1120 & 2.34884 & 3.99428 & 1.96511\end{array}$

$1130 \quad 2.33423 \quad 3.97682 \quad 1.96471$

$\begin{array}{lllll}1140 \quad 2.31977 & 3.95951 & 1.96432\end{array}$

$\begin{array}{llll}1150 & 2.30546 & 3.94236 & 1.96392\end{array}$

$\begin{array}{lllll}1160 & 2.29130 & 3.92535 & 1.96351\end{array}$

$1170 \quad 2.27729 \quad 3.90850 \quad 1.96311$

$\begin{array}{llll}1180 & 2.26342 & 3.89180 & 1.96270\end{array}$

$\begin{array}{lllll}1190 & 2.24969 & 3.87523 & 1.96228\end{array}$

$\begin{array}{llll}1200 \quad 2.23609 & 3.85882 & 1.96187\end{array}$

$1210 \quad 2.22264 \quad 3.84254 \quad 1.96145$

$1220 \quad 2.209323 .826391 .96102$

$1230 \quad 2.19613 \quad 3.810391 .96060$

$\begin{array}{lllll}1240 & 2.18307 & 3.79451 & 1.96017\end{array}$

$\begin{array}{lllll}1250 & 2.17014 & 3.77877 & 1.95973\end{array}$

$\begin{array}{lllll}1260 & 2.15733 & 3.76316 & 1.95930\end{array}$

$\begin{array}{llllll}1270 & 2.14465 & 3.74767 & 1.95885\end{array}$

$\begin{array}{lllll}1280 & 2.13209 & 3.73231 & 1.95841\end{array}$

$\begin{array}{lllll}1290 & 2.11964 & 3.71707 & 1.95796\end{array}$

$1300 \quad 2.10732 \quad 3.70195 \quad 1.95751$

$\begin{array}{lllll}1310 & 2.09511 & 3.68695 & 1.95706\end{array}$

$\begin{array}{lllll}1320 & 2.08302 & 3.67207 & 1.95660\end{array}$

$\begin{array}{lllll}1330 & 2.07103 & 3.65731 & 1.95614\end{array}$

$\begin{array}{llll}1340 \quad 2.05916 & 3.64266 & 1.95568\end{array}$

$\begin{array}{lllll}1350 & 2.04740 & 3.62812 & 1.95521\end{array}$

$\begin{array}{lllll}1360 & 2.03574 & 3.61369 & 1.95474\end{array}$

$\begin{array}{lllll}1370 & 2.02419 & 3.59937 & 1.95427\end{array}$

$\begin{array}{lllll}1380 & 2.01275 & 3.58516 & 1.95379\end{array}$

$1390 \quad 2.00140 \quad 3.57105 \quad 1.95331$

$\begin{array}{llll}1400 & 1.99016 & 3.55705 & 1.95282\end{array}$

$1410 \quad 1.979023 .543161 .95234$

$\begin{array}{lllll}1420 & 1.96797 & 3.52936 & 1.95185\end{array}$

$\begin{array}{llllll}1430 & 1.95703 & 3.51567 & 1.95135\end{array}$

$\begin{array}{lllll}1440 & 1.94618 & 3.50207 & 1.95086\end{array}$

$\begin{array}{lllll}1450 & 1.93542 & 3.48857 & 1.95036\end{array}$

$1460 \quad 1.92475 \quad 3.47517 \quad 1.94985$

$1470 \quad 1.91418 \quad 3.46186 \quad 1.94934$

$\begin{array}{lllll}1480 & 1.90369 & 3.44864 & 1.94883\end{array}$

$1490 \quad 1.89330 \quad 3.43552 \quad 1.94832$

$\begin{array}{lllll}1500 & 1.88299 & 3.42249 & 1.94780\end{array}$

$\begin{array}{lllll}1510 & 1.87277 & 3.40955 & 1.94728\end{array}$

$\begin{array}{llll}1520 & 1.86264 & 3.39670 & 1.94676\end{array}$

$\begin{array}{lllll}1530 & 1.85259 & 3.38394 & 1.94623\end{array}$

$\begin{array}{llll}1540 \quad 1.84262 & 3.37126 & 1.94570\end{array}$

1550

1560

1570

1580

1590
2.415734 .073891 .96681

2.400734 .056091 .96644

$2.38590 \quad 4.03846 \quad 1.96607$

$2.37122 \quad 4.02098 \quad 1.96569$

$2.35670 \quad 4.00366 \quad 1.96531$

$2.34233 \quad 3.98650 \quad 1.96493$

2.328113 .969491 .96455

$2.31403 \quad 3.952631 .96416$

$2.300093 .93592 \quad 1.96376$

$2.28630 \quad 3.91934 \quad 1.96337$

$2.27265 \quad 3.90292 \quad 1.96297$ $2.25913 \quad 3.88663 \quad 1.96257$

$2.24575 \quad 3.87048 \quad 1.96216$

$2.23249 \quad 3.85446 \quad 1.96175$

$2.21937 \quad 3.83858 \quad 1.96134$

$2.20638 \quad 3.82283 \quad 1.96093$

$2.19351 \quad 3.80720 \quad 1.96051$

$2.180763 .79171 \quad 1.96009$

2.168143 .776331 .95966

2.155633 .761081 .95924

$2.14325 \quad 3.74596 \quad 1.95881$ $2.13098 \quad 3.73095 \quad 1.95837$ $2.118823 .71606 \quad 1.95793$ $2.10677 \quad 3.70128 \quad 1.95749$ 2.094843 .686621 .95705

$2.08302 \quad 3.67207 \quad 1.95660$ 2.071303 .657631 .95615 2.059693 .643301 .95570 $2.04818 \quad 3.62908 \quad 1.95524$ 2.036773 .614971 .95478

$2.02547 \quad 3.60096 \quad 1.95432$ $2.01427 \quad 3.58705 \quad 1.95385$ $2.00316 \quad 3.57324 \quad 1.95338$ $1.99215 \quad 3.55954 \quad 1.95291$ $1.981243 .54593 \quad 1.95243$

$1.97042 \quad 3.53242 \quad 1.95196$ $1.95969 \quad 3.51900 \quad 1.95147$ $1.94906 \quad 3.50568 \quad 1.95099$ $1.93852 \quad 3.49246 \quad 1.95050$ $1.92806 \quad 3.479331 .95001$

$1.91769 \quad 3.46628 \quad 1.94951$ $1.90741 \quad 3.45333 \quad 1.94902$ $1.89722 \quad 3.44047 \quad 1.94851$ $1.887113 .42769 \quad 1.94801$ $1.87708 \quad 3.41500 \quad 1.94750$

$1.86713 \quad 3.40240 \quad 1.94699$ $1.85727 \quad 3.38988 \quad 1.94648$ $1.84748 \quad 3.37745 \quad 1.94596$ $1.83778 \quad 3.36509 \quad 1.94544$ $1.82815 \quad 3.35282 \quad 1.94492$
$2.45225 \quad 4.11713 \quad 1.96768$ 2.437194 .099321 .96733 $2.42230 \quad 4.08168 \quad 1.96697$ $2.40756 \quad 4.064201 .96661$ $2.39298 \quad 4.04687 \quad 1.96625$

$2.378544 .02970 \quad 1.96588$ $2.36426 \quad 4.01268 \quad 1.96551$ $2.35012 \quad 3.99581 \quad 1.96514$ $2.33613 \quad 3.979091 .96476$ $2.32227 \quad 3.962511 .96439$

$2.30856 \quad 3.94607 \quad 1.96400$ $\begin{array}{llll}2.29498 & 3.92977 & 1.96362\end{array}$ 2.281543 .913611 .96323 $2.26822 \quad 3.89759 \quad 1.96284$ $2.255043 .88170 \quad 1.96245$

$2.24199 \quad 3.86594 \quad 1.96205$ $2.22906 \quad 3.85030 \quad 1.96165$ $2.216253 .83480 \quad 1.96124$ $2.20357 \quad 3.81942 \quad 1.96084$ $2.19100 \quad 3.80416 \quad 1.96043$

$2.17856 \quad 3.78902 \quad 1.96002$ $2.16623 \quad 3.77401 \quad 1.95960$ 2.154013 .759101 .95918 2.141913 .744321 .95876 2.129913 .729651 .95833

$2.11803 \quad 3.715091 .95791$ $2.10625 \quad 3.700641 .95747$ $2.09458 \quad 3.68630 \quad 1.95704$ $2.08302 \quad 3.67207 \quad 1.95660$ 2.071553 .657951 .95616

2.060193 .643931 .95572 $2.04893 \quad 3.63001 \quad 1.95527$ $2.03776 \quad 3.616191 .95482$ 2.026693 .602471 .95437 $2.01572 \quad 3.58886 \quad 1.95391$

$2.00485 \quad 3.57534 \quad 1.95346$ $1.99406 \quad 3.56191 \quad 1.95299$ $1.983373 .54858 \quad 1.95253$ $1.97276 \quad 3.535351 .95206$ 1.962253 .522201 .95159

$1.95183 \quad 3.50915 \quad 1.95112$ $1.94149 \quad 3.49619 \quad 1.95064$ 1.931233 .483311 .95016 1.921063 .470531 .94968 1.910983 .457831 .94919

$1.90097 \quad 3.44521 \quad 1.94870$ $1.891053 .43268 \quad 1.94821$ $1.88121 \quad 3.420241 .94771$ $1.87145 \quad 3.40787 \quad 1.94722$ $1.86176 \quad 3.39559 \quad 1.94671$
1100

1110

1120

1130

1140

1150

1160

1170

1180

1190

1200

1210

1220

1230

1240

1250

1260

1270

1280

1290

1300

1310

1320

1330

1340

1350

1360

1370

1380

1390

1400

1410

1420

1430

1440

1450

1460

1470

1480

1490

1500

1510

1520

1530

1540

1550

1560

1570

1580

1590 
Table II Harmonic Oscillator Contributions to the Thermodynamic

Functions (in units of calories, moles, and ${ }^{\circ} \mathrm{K}$ )

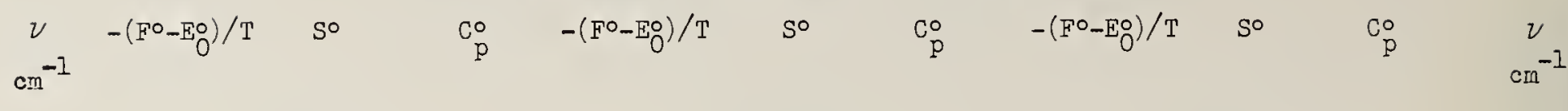

$T=4400$.

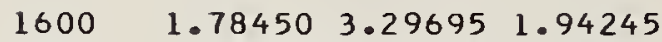

$\begin{array}{llll}1610 & 1.77509 & 3.28485 & 1.94190\end{array}$

$1620 \quad 1.76575 \quad 3.27283 \quad 1.94134$

$\begin{array}{llll}1630 & 1.75648 & 3.26088 & 1.94078\end{array}$

$1640 \quad 1.74729 \quad 3.24902 \quad 1.94022$

$1650 \quad 1.73817 \quad 3.23722 \quad 1.93966$ $1660 \quad 1.72912 \quad 3.22550 \quad 1.93909$ $1670 \quad 1.72014 \quad 3.21386 \quad 1.93851$ $1680 \quad 1.71123 \quad 3.202291 .93794$ $\begin{array}{lllll}1690 & 1.70239 & 3.19079 & 1.93736\end{array}$

$\begin{array}{lllll}1700 & 1.69361 & 3.17936 & 1.93678\end{array}$ $\begin{array}{lllll}1710 & 1.68491 & 3.16800 & 1.93619\end{array}$ $\begin{array}{lllll}1720 & 1.67627 & 3.15672 & 1.93560\end{array}$ $\begin{array}{lllll}1730 & 1.66769 & 3.14550 & 1.93501\end{array}$ $1740 \quad 1.65918 \quad 3.13434 \quad 1.93442$

$\begin{array}{lllll}1750 & 1.65074 & 3.12326 & 1.93382\end{array}$ $\begin{array}{lllll}1760 & 1.64235 & 3.11224 & 1.93322\end{array}$ $\begin{array}{llll}1770 & 1.63403 & 3.10129 & 1.93261\end{array}$ $\begin{array}{lllll}1780 & 1.62577 & 3.09041 & 1.93200\end{array}$ $\begin{array}{lllll}1790 & 1.61758 & 3.07958 & 1.93139\end{array}$

$\begin{array}{lllll}1800 & 1.60944 & 3.06883 & 1.93078\end{array}$ $\begin{array}{lllll}1810 & 1.60136 & 3.05813 \quad 1.93016\end{array}$ $\begin{array}{lllll}1820 & 1.59334 & 3.04750 & 1.92954\end{array}$ $\begin{array}{llll}1830 & 1.58538 & 3.03693 & 1.92892\end{array}$ $\begin{array}{llll}1840 & 1.57748 & 3.02642 \quad 1.92829\end{array}$

$\begin{array}{lllll}1850 & 1.56963 & 3.01597 & 1.92766\end{array}$ $\begin{array}{lllll}1860 & 1.56184 & 3.00558 & 1.92703\end{array}$ $\begin{array}{lllll}1870 & 1.55411 & 2.99525 & 1.92639\end{array}$ $\begin{array}{lllll}1880 & 1.54643 & 2.98497 & 1.92575\end{array}$ $\begin{array}{lllll}1890 & 1.53880 & 2.97476 & 1.92511\end{array}$

$\begin{array}{llll}1900 & 1.53123 & 2.96460 & 1.92446\end{array}$ $\begin{array}{lllll}1910 & 1.52371 & 2.95450 & 1.92381\end{array}$ $\begin{array}{lllll}1920 & 1.51625 & 2.94446 & 1.92316\end{array}$ $\begin{array}{lllll}1930 & 1.50884 & 2.93447 & 1.92251\end{array}$ $\begin{array}{llll}1940 & 1.50148 & 2.92453 & 1.92185\end{array}$

$1950 \quad 1.49417 \quad 2.91466 \quad 1.92119$ $\begin{array}{lllll}1960 & 1.48691 & 2.90483 & 1.92052\end{array}$ $\begin{array}{lllll}1970 & 1.47970 & 2.89506 & 1.91985\end{array}$ $\begin{array}{lllll}1980 & 1.47254 & 2.88534 & 1.91918\end{array}$ $\begin{array}{llllll}1990 & 1.46543 & 2.87567 & 1.91851\end{array}$

$\begin{array}{lllll}2000 & 1.45836 & 2.86606 & 1.91783\end{array}$ $2010 \quad 1.45135 \quad 2.85649 \quad 1.91715$ $\begin{array}{lllll}2020 & 1.44438 & 2.84698 & 1.91647\end{array}$ $\begin{array}{lllll}2030 & 1.43746 & 2.83752 & 1.91578\end{array}$ $2040 \quad 1.430592 .82811 \quad 1.91509$

$2050 \quad 1.42376 \quad 2.81874 \quad 1.91440$ $\begin{array}{lllll}2060 & 1.41698 & 2.80943 & 1.91370\end{array}$ $2070 \quad 1.41024 \quad 2.80016 \quad 1.91300$ $\begin{array}{llll}2080 & 1.40355 & 2.79095 & 1.91230\end{array}$ $2090 \quad 1.39690 \quad 2.78178 \quad 1.91160$
$T=4500$

$$
\mathrm{T}=4600 \text {. }
$$

$1.818603 .34063 \quad 1.94439$

$1.809123 .32851 \quad 1.94386$

$1.79972 \quad 3.31648 \quad 1.94333$

$1.79040 \quad 3.30452 \quad 1.94280$

$1.78115 \quad 3.29264 \quad 1.94226$

$1.77197 \quad 3.28084 \quad 1.94172$

$1.76286 \quad 3.26911 \quad 1.94117$

$1.75382 \quad 3.25745 \quad 1.94062$

$1.74485 \quad 3.24586 \quad 1.94007$

1.735953 .234351 .93952

$1.72712 \quad 3.22291 \quad 1.93896$

$1.718353 .21154 \quad 1.93840$

1.709653 .200241 .93784

$1.70102 \quad 3.18901 \quad 1.93727$

$1.692453 .17784 \quad 1.93670$

$1.68394 \quad 3.16675 \quad 1.93613$

1.675503 .155721 .93555

$1.667123 .14475 \quad 1.93497$

$1.65881 \quad 3.133851 .93439$

$1.65055 \quad 3.12302 \quad 1.93380$

$1.64235 \quad 3.11224 \quad 1.93322$

1.634223 .101541 .93263

1.626143 .090891 .93203

1.618123 .080301 .93143

$1.61016 \quad 3.06978 \quad 1.93083$

1.602253 .059321 .93023

$\begin{array}{llll}1.59441 & 3.04891 & 1.92962\end{array}$

$1.586613 .03857 \quad 1.92901$

$1.57888 \quad 3.02828 \quad 1.92840$

$1.571193 .01805 \quad 1.92779$

$\begin{array}{llll}1.56357 & 3.00788 & 1.92717\end{array}$ $\begin{array}{llll}1.55599 & 2.99777 & 1.92655\end{array}$ $1.548472 .98771 \quad 1.92592$ $1.54100 \quad 2.97770 \quad 1.92529$ $1.53358 \quad 2.96776 \quad 1.92466$

$\begin{array}{llll}1.52621 & 2.95786 & 1.92403\end{array}$ 1.518902 .948021 .92339 $1.511632 .93824 \quad 1.92275$ $1.504412 .92850 \quad 1.92211$ $1.497252 .91882 \quad 1.92147$

$1.49013 \quad 2.90919 \quad 1.92082$ 1.483052 .899611 .92016 $1.476032 .89008 \quad 1.91951$ $1.469052 .88061 \quad 1.91885$ $1.46212 \quad 2.87118 \quad 1.91819$

$1.45524 \quad 2.86180 \quad 1.91753$ $1.44840 \quad 2.85247 \quad 1.91686$ 1.441612 .843191 .91619 $1.434862 .83396 \quad 1.91532$ 1.428152 .824771 .91484
$1.85215 \quad 3.38338 \quad 1.94621$

$1.842623 .37126 \quad 1.94570$ $1.83316 \quad 3.35921 \quad 1.94519$ $1.82378 \quad 3.34724 \quad 1.94468$ 1.814473 .335351 .94416

$1.80523 \quad 3.32353 \quad 1.94364$ $1.796073 .31179 \quad 1.94312$ 1.786973 .300121 .94260 $1.77794 \quad 3.28853 \quad 1.94207$ $1.76899 \quad 3.27700 \quad 1.94154$

$1.76010 \quad 3.26555 \quad 1.94100$ $1.75128 \quad 3.25417 \quad 1.94047$ $1.742523 .24285 \quad 1.93993$ $1.73383 \quad 3.23161 \quad 1.93938$

$1.72520 \quad 3.22043 \quad 1.93884$

$1.716643 .20932 \quad 1.93829$ $1.70814 \quad 3.198281 .93774$ $1.699713 .18730 \quad 1.93718$ 1.691343 .176391 .93662 1.683023 .165541 .93606

$1.67477 \quad 3.15476 \quad 1.93550$ $1.66658 \quad 3.14404 \quad 1.93493$ $1.65844 \quad 3.13338 \quad 1.93436$ $1.650373 .12278 \quad 1.93379$ $1.64235 \quad 3.11224 \quad 1.93322$

$1.634393 .10177 \quad 1.93264$ 1.626493 .091351 .93206 1.618643 .080991 .93147

$1.610853 .07069 \quad 1.93089$

1.603113 .060451 .93030

$\begin{array}{llll}1.59543 & 3.05027 & 1.92970\end{array}$ $1.58780 \quad 3.04014 \quad 1.92911$ $1.580223 .03007 \quad 1.92851$ $1.572693 .02005 \quad 1.92791$

1.565223 .010091 .92730

$1.55780 \quad 3.00018 \quad 1.92670$ $1.55043 \quad 2.990331 .92608$ 1.543102 .980531 .92547 $\begin{array}{llll}1.53583 & 2.97078 & 1.92486\end{array}$ $1.528612 .96108 \quad 1.92424$

$1.52144 \quad 2.95144 \quad 1.92361$ $1.514312 .94185 \quad 1.92299$ $1.50723 \quad 2.93230 \quad 1.92236$ $1.500202 .92281 \quad 1.92173$ $1.49322 \quad 2.91337 \quad 1.92110$

$1.48628 \quad 2.90398 \quad 1.92046$ $1.47938 \quad 2.89463 \quad 1.91982$ $1.472542 .88534 \quad 1.91918$ $1.46513 \quad 2.87609 \quad 1.91854$ $1.45898 \quad 2.866891 .91789$
1600

1610

1620

1630

1640

1650

1660

1670

1680

1690

1700

1710

1720

1730

1740

1750

1760

1770

1780

1790

1800

1810

1820

1830

1840

1850

1860

1870

1880

1890

1900

1910

1920

1930

1940

1950

1960

1970

1980

1990

2000

2010

2020

2030

2040

2050

2060

2070

2080

2090 
Table II Harmonic Oscillator Contributions to the Thermodynamic

Functions (in units of calories, moles, and ${ }^{\circ} \mathrm{K}$ )
$\mathrm{cm}^{-1}-\left(F^{\circ}-E O\right) / T$ So
$\begin{array}{cc}C_{0}^{\circ} & -\left(F^{\circ}-E_{O}\right) / T\end{array}$
So
$C O \quad-\left(F^{\circ}-E_{0}^{\circ}\right) / T$
so

$\mathrm{cm}^{-1}$

$T=4400$

2100

2110

2120

2130

2140

2150

2160

2170

2180

2190

2200

2210

2220

2230

2240

2250

2260

2270

2280

2290

2300

2310

2320

2330

2340

2350

2360

2370

2380

2390

2400

2410

2420

2430

2440

2450

2460

2470

2480

2490

2500

2510

2520

2530

2540

2550

2560

2570

2580

2590
$1.39030 \quad 2.77265 \quad 1.91089$

$\begin{array}{llll}1.38373 & 2.76358 & 1.91018\end{array}$

$1.37722 \quad 2.75455 \quad 1.90946$

$1.37074 \quad 2.74556 \quad 1.90875$

1.364312 .736621 .90803

$\begin{array}{llll}1.35792 & 2.72773 & 1.90730\end{array}$ $\begin{array}{llll}1.35156 & 2.71888 & 1.90658\end{array}$ 1. 345262.710081 .90585 1.338992 .701321 .90511 1.332762 .692601 .90438

$1.326572 .68392 \quad 1.90364$ 1.320422 .675291 .90290 $1.314312 .66670 \quad 1.90215$ $1.308232 .65816 \quad 1.90141$ 1.302202 .649651 .90066

$1.29620 \quad 2.64119 \quad 1.89990$ $1.29024 \quad 2.63276 \quad 1.89915$ $1.28432 \quad 2.62438 \quad 1.89839$ $1.278442 .61604 \quad 1.89762$ $1.272592 .60773 \quad 1.89686$

$\begin{array}{llll}1.26678 & 2.59947 & 1.89609\end{array}$ $1.26100 \quad 2.59125 \quad 1.89532$ $1.25526 \quad 2.58306 \quad 1.89454$ $\begin{array}{llll}1.24955 & 2.57491 & 1.89377\end{array}$ 1. $24388 \quad 2.56680 \quad 1.89299$

$\begin{array}{llll}1.23825 & 2.55873 \quad 1.89220\end{array}$ $1.232642 .55070 \quad 1.89142$ $1.22708 \quad 2.54270 \quad 1.89063$ $1.22154 \quad 2.53475 \quad 1.88984$ $1.216042 .52682 \quad 1.88904$

$\begin{array}{llll}1.21057 & 2.51894 & 1.88824\end{array}$ $1.205142 .51109 \quad 1.88744$ $1.199732 .50327 \quad 1.88664$ $1.19436 \quad 2.49550 \quad 1.88583$ $1.18902 \quad 2.48775 \quad 1.88502$

$1.183722 .48004 \quad 1.88421$ $1.17844 \quad 2.47237 \quad 1.88339$ $1.17320 \quad 2.46473 \quad 1.88257$ $1.167982 .45713 \quad 1.88175$ $1.162802 .44956 \quad 1.88093$

$1.15765 \quad 2.44202 \quad 1.88010$ $\begin{array}{llll}1.15253 & 2.43452 \quad 1.87927\end{array}$ 1.147432 .427051 .87844 1.142372 .419611 .87760 $1.13734 \quad 2.41220 \quad 1.87677$

$1.13233 \quad 2.40483 \quad 1.87592$ $1.12736 \quad 2.39749 \quad 1.87508$ $\begin{array}{llll}1.12241 & 2.39018 & 1.87423\end{array}$ $1.11749 \quad 2.38290 \quad 1.87338$ $1.11260 \quad 2.37566 \quad 1.87253$

\author{
$T=4500$.
}

$\mathrm{T}=4600$.

1.421492 .815631 .91417

$1.41488 \quad 2.80654 \quad 1.91349$

$1.40830 \quad 2.79750 \quad 1.91280$

1.401772 .788501 .91211

$1.39528 \quad 2.77954 \quad 1.91142$

1.388832 .770631 .91073 $1.382432 .76177 \quad 1.91003$ $1.37606 \quad 2.75295 \quad 1.90934$ 1.369742 .744171 .90863 $1.36345 \quad 2.73544 \quad 1.90793$

$\begin{array}{llll}1.35721 & 2.72675 & 1.90722\end{array}$ 1.351002 .718101 .90651 1.344842 .709491 .90580 $\begin{array}{llll}1.33871 & 2.70093 & 1.90508\end{array}$ $1.332622 .69241 \quad 1.90436$

1.326572 .683921 .90364 $1.320552 .67548 \quad 1.90292$ $1.31458 \quad 2.66708 \quad 1.90219$ 1.308642 .658721 .90146 1.302732 .650401 .90072

$\begin{array}{llll}1.29687 & 2.64212 & 1.89999\end{array}$ $1.291042 .63388 \quad 1.89925$ $1.285242 .62568 \quad 1.89850$ $1.27948 \quad 2.61752 \quad 1.89776$ $1.27375 \quad 2.60939 \quad 1.89701$

$1.26306 \quad 2.601301 .89626$ 1.262412 .593251 .89551 $1.25679 \quad 2.58524 \quad 1.89475$ $1.25120 \quad 2.57726 \quad 1.89399$ 1.245642 .569321 .89323

1.240122 .561421 .89246 1.234632 .553551 .89170 $1.229172 .54572 \quad 1.89093$ 1.223752 .537921 .89015 $1.218362 .53016 \quad 1.88938$

$1.21300 \quad 2.52244 \quad 1.88860$ $1.20767 \quad 2.51475 \quad 1.88782$ 1.202372 .507091 .88703 $1.197102 .49947 \quad 1.88624$ $1.19187 \quad 2.49188 \quad 1.88545$

$1.18666 \quad 2.48432 \quad 1.88466$ 1.181492 .476801 .88386 $\begin{array}{llll}1.17634 & 2.46931 & 1.88307\end{array}$ $1.17122 \quad 2.46186 \quad 1.88226$ 1.166142 .454431 .88146

$1.16108 \quad 2.44704 \quad 1.88065$ $1.15605 \quad 2.43968 \quad 1.87984$ 1.151052 .432351 .87903 $1.14608 \quad 2.42506 \quad 1.87822$ 1.141142 .417791 .87740
$1.45226 \quad 2.85774 \quad 1.91724$ 1.445592 .848631 .91639 $1.43896 \quad 2.83957 \quad 1.91593$ $1.43238 \quad 2.83056 \quad 1.91527$ 1.425832 .821591 .91461

1.419332 .812661 .91394 $\begin{array}{llll}1.41287 & 2.80378 & 1.91328\end{array}$ $1.406452 .79495 \quad 1.91261$ 1.400072 .786161 .91193 1.393742 .777411 .91126

$1.387442 .76870 \quad 1.91058$ $1.381182 .76004 \quad 1.90990$ 1.374962 .751421 .90921 $1.36878 \quad 2.74284 \quad 1.90853$ $1.362642 .73430 \quad 1.90784$

$1.35653 \quad 2.72580 \quad 1.90714$ $1.35046 \quad 2.717351 .90645$ 1.344442 .708931 .90575 1.338442 .700561 .90505 1.332492 .692221 .90435

$1.32657 \quad 2.68392 \quad 1.90364$ $1.32068 \quad 2.67567 \quad 1.90293$ 1.314842 .667451 .90222 1.309022 .659271 .90150 1.303252 .651131 .90079

$1.29750 \quad 2.64302 \quad 1.90007$ $1.291792 .63496 \quad 1.89934$ 1.286122 .626931 .89862 $1.28048 \quad 2.61893 \quad 1.89789$ 1.274872 .610981 .89716

$1.26930 \quad 2.60306 \quad 1.89642$ $1.26376 \quad 2.59517 \quad 1.89569$ $1.25825 \quad 2.587331 .89495$ $1.252772 .57951 \quad 1.89421$ $1.247332 .57174 \quad 1.89346$

$1.24192 \quad 2.56399 \quad 1.89271$ $1.236542 .55628 \quad 1.89196$ 1.231192 .548611 .89121 1.225872 .540971 .89046 $1.22058 \quad 2.53337 \quad 1.88970$

$\begin{array}{llll}1.21532 & 2.52579 & 1.88894\end{array}$ 1.210102 .518251 .88817 $1.204902 .51075 \quad 1.88741$ $1.19973 \quad 2.50327 \quad 1.88664$ $1.19460 \quad 2.49583 \quad 1.88587$

$1.189492 .48842 \quad 1.88509$ $1.184412 .48105 \quad 1.88431$ $1.17936 \quad 2.47370 \quad 1.88354$ 1.174342 .466391 .88275 $1.169342 .45911 \quad 1.88197$
2100

2110

2120

2130

2140

2150

2160

2170

2180

2190

2200

2210

2220

2230

2240

2250

2260

2270

2280

2290

2300

2310

2320

2330

2340

2350

2360

2370

2380

2390

2400

2410

2420

2430

2440

2450

2460

2470

2480

2490

2500

2510

2520

2530

2540

2550

2560

2570

2580

2590 
Table II Harmonic Oscillator Contributions to the Thermodynamic

Functions (in units of calories, moles, and ${ }^{\circ} \mathrm{K}$ )

$\mathrm{cm}^{-1}-\left(F^{\circ}-E_{0}^{\circ}\right) / T \quad S^{\circ}$

$T=4400$.
$\begin{array}{llll}C_{p}^{\circ} & -\left(F^{\circ}-E_{O}^{O}\right) / T & \text { So } & C_{p}^{\circ}\end{array}$

$T=4600$.
$2600 \quad 1.10774 \quad 2.36844 \quad 1.87167$

$2610 \quad 1.102902 .36126 \quad 1.87082$

$\begin{array}{lllll}2620 \quad 1.09809 & 2.35411 & 1.86996\end{array}$

$2630 \quad 1.093312 .34699 \quad 1.86909$

$\begin{array}{lllll}2640 \quad 1.08856 & 2.33989 & 1.86822\end{array}$

$\begin{array}{lllll}2650 & 1.08383 & 2.33283 & 1.86735\end{array}$

$\begin{array}{lllll}2660 & 1.07913 & 2.32580 & 1.86648\end{array}$

$2670 \quad 1.07446 \quad 2.31880 \quad 1.86561$

$\begin{array}{lllll}2680 & 1.06981 & 2.31183 & 1.86473\end{array}$

$\begin{array}{lllll}2690 \quad 1.06519 & 2.30488 & 1.86385\end{array}$

$\begin{array}{lllll}2700 & 1.06060 & 2.29797 & 1.86296\end{array}$ $\begin{array}{lllll}2710 & 1.05603 & 2.29108 & 1.86208\end{array}$ $\begin{array}{lllll}2720 & 1.05148 & 2.28423 & 1.86119\end{array}$ $2730 \quad 1.04696 \quad 2.27740 \quad 1.86030$ $2740 \quad 1.04247 \quad 2.270601 .85940$

$\begin{array}{lllll}2750 \quad 1.03800 & 2.26383 & 1.85850\end{array}$ $\begin{array}{llllll}2760 \quad 1.03355 & 2.25708 & 1.85760\end{array}$ $\begin{array}{lllll}2770 & 1.02913 & 2.25036 & 1.85670\end{array}$ $\begin{array}{lllll}2780 & 1.02473 & 2.24368 & 1.85580\end{array}$ $2790 \quad 1.02036 \quad 2.23701 \quad 1.85489$

$\begin{array}{lllll}2800 \quad 1.01601 & 2.23038 & 1.85398\end{array}$ $\begin{array}{lllll}2810 & 1.01169 & 2.22377 & 1.85306\end{array}$ $\begin{array}{lllll}2820 & 1.00739 & 2.21719 & 1.85215\end{array}$

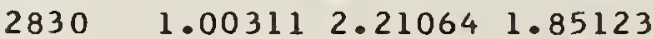
$\begin{array}{lllll}2840 & 0.99885 & 2.20411 & 1.85031\end{array}$

$\begin{array}{lllll}2850 & 0.99462 & 2.19760 & 1.84938\end{array}$ $\begin{array}{lllll}2860 & 0.99041 & 2.19113 & 1.84845\end{array}$ $\begin{array}{lllll}2870 & 0.98622 & 2.18468 & 1.84752\end{array}$ $\begin{array}{lllll}2880 & 0.98206 & 2.17825 & 1.84659\end{array}$ $\begin{array}{llll}2890 & 0.97792 \quad 2.17185 & 1.84565\end{array}$

2900

2910

2920

2930

2940

2950

2960

2970

2980

2990

3000

3010

3020

3030

3040

3050

3060

3070

3080

3090
$\mathrm{T}=4500$.

$1.13622 \quad 2.41056 \quad 1.87658$ 1.131342 .403361 .87576 $1.12648 \quad 2.39619 \quad 1.87493$ 1.121642 .389051 .87410 $1.11684 \quad 2.38194 \quad 1.87327$

$1.11206 \quad 2.37486 \quad 1.87244$ $\begin{array}{lll}1.10731 & 2.36780 \quad 1.87160\end{array}$ $1.10258 \quad 2.36078 \quad 1.87076$

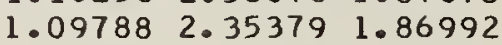
$1.09321 \quad 2.34683 \quad 1.86907$

$1.08856 \quad 2.33989 \quad 1.86822$ $1.08394 \quad 2.33299 \quad 1.86737$ $1.079342 .32611 \quad 1.86652$ $1.07477 \quad 2.31927 \quad 1.86567$ $1.07023 \quad 2.31245 \quad 1.86481$

$1.06570 \quad 2.30565 \quad 1.86395$ 1.061212 .298891 .86308 $1.05674 \quad 2.29215 \quad 1.86222$ $1.052292 .28544 \quad 1.86135$ $1.04786 \quad 2.27876 \quad 1.86048$

$1.04346 \quad 2.27211 \quad 1.85960$ $1.039092 .26548 \quad 1.85872$ $1.034742 .25888 \quad 1.85784$ $1.03041 \quad 2.25230 \quad 1.85696$ $1.02610 \quad 2.24575 \quad 1.85608$

$1.02182 \quad 2.23923 \quad 1.85519$ $1.01756 \quad 2.23273 \quad 1.85430$ $1.01332 \quad 2.22626 \quad 1.85341$ $1.00910 \quad 2.21982 \quad 1.85251$ $1.004912 .21340 \quad 1.85162$

$1.000742 .20701 \quad 1.85072$ $0.996592 .20064 \quad 1.84981$ 0.992472 .194291 .84891 $0.98836 \quad 2.18797 \quad 1.84800$ $0.98428 \quad 2.18168 \quad 1.84709$

$\begin{array}{llll}0.98021 & 2.17541 & 1.84617\end{array}$ $0.976172 .16916 \quad 1.84526$ $0.972152 .16294 \quad 1.84434$ 0.968152 .156741 .84342 $0.96418 \quad 2.15057 \quad 1.84250$

$0.96022 \quad 2.14442 \quad 1.84157$ $0.95628 \quad 2.138291 .84064$ $0.952372 .13219 \quad 1.83971$ 0.948472 .126111 .83878 $0.944592 .12005 \quad 1.83784$

$0.94074 \quad 2.11401 \quad 1.83690$ $0.93690 \quad 2.10800 \quad 1.83596$ $0.93308 \quad 2.102021 .83502$ $0.92928 \quad 2.096051 .83407$ $0.92550 \quad 2.09011 \quad 1.83312$ $\begin{array}{llll}1.16438 & 2.45186 & 1.88118\end{array}$ 1.159442 .444641 .88039 $1.154532 .43745 \quad 1.87960$ $1.149642 .43029 \quad 1.87880$ 1.144792 .423161 .87800

$1.13996 \quad 2.41606 \quad 1.87720$ $1.13516 \quad 2.40899 \quad 1.87640$ $1.13038 \quad 2.40195 \quad 1.87559$ 1.125632 .394941 .87479 $1.120912 .38796 \quad 1.87397$

$1.116212 .38101 \quad 1.87316$ $1.11134 \quad 2.37409 \quad 1.87234$ $1.106892 .36719 \quad 1.87153$ 1.102272 .360331 .87070 $1.09768 \quad 2.35349 \quad 1.86988$

$1.093112 .34668 \quad 1.86905$ $1.08856 \quad 2.33989 \quad 1.86822$ 1.084042 .333141 .86739 $1.07954 \quad 2.32641 \quad 1.86656$ $1.07507 \quad 2.319711 .86572$

$1.07062 \quad 2.31304 \quad 1.86488$ 1.066192 .306391 .86404 $1.06179 \quad 2.29977 \quad 1.86320$ $\begin{array}{llll}1.05741 & 2.29318 & 1.86235\end{array}$ $1.05306 \quad 2.28661 \quad 1.86150$

$\begin{array}{llll}1.04873 & 2.28007 & 1.86065\end{array}$ $1.04442 \quad 2.27355 \quad 1.85979$ $1.040132 .26706 \quad 1.85893$ 1.035872 .260601 .85807 $1.031632 .25416 \quad 1.85721$

$1.02741 \quad 2.24774 \quad 1.85635$ $1.023212 .24136 \quad 1.85548$ $1.01904 \quad 2.23499 \quad 1.85461$ $1.01488 \quad 2.22865 \quad 1.85374$ $1.01075 \quad 2.22234 \quad 1.85286$

$1.006642 .21605 \quad 1.85199$ $1.002552 .20978 \quad 1.85111$ $0.99848 \quad 2.20354 \quad 1.85022$ $0.99444 \quad 2.19732 \quad 1.84934$ 0.990412 .191131 .84845

$0.98640 \quad 2.18496 \quad 1.84756$ $0.982422 .17881 \quad 1.84667$ $0.97846 \quad 2.17269 \quad 1.84578$ 0.974512 .166591 .84488 0.970592 .160511 .84398

$0.966682 .15446 \quad 1.84308$ $0.96280 \quad 2.14842 \quad 1.84217$ $0.95893 \quad 2.14242 \quad 1.84127$ 0.955092 .136431 .84036 $0.95126 \quad 2.13047 \quad 1.83945$
$V$

$\mathrm{Cm}^{-1}$

2600

2610

2620

2630

2640

2650

2660

2670

2680

2690

2700

2710

2720

2730

2740

2750

2760

2770

2780

2790

2800

2810

2820

2830

2840

2850

2860

2870

2880

2890

2900

2910

2920

2930

2940

2950

2960

2970

2980

2990

3000

3010

3020

3030

3040

3050

3060

3070

3080

3090 
Table II Harmonic Oscillator Contributions to the Thermodynamic

Functions (in units of calories, moles, and ${ }^{\circ} \mathrm{K}$ )
$\mathrm{cm}^{-1}$
So
$-\left(F^{\circ}-E_{0}\right) / T$
$\mathrm{C}_{\mathrm{p}}^{\circ}$
$-\left(\mathrm{F}^{\circ}-\mathrm{E}_{\mathrm{O}}\right) / \mathrm{T} \quad \mathrm{S}^{\circ}$
$\mathrm{C}_{\mathrm{p}}$
$-\left(\mathrm{F}^{\circ}-\mathrm{E}_{\mathrm{O}}^{\circ}\right) / \mathrm{T}$
So
$\mathrm{C}_{\mathrm{p}}^{\circ}$
$\mathrm{cm}^{-1}$

$T=4400$.

$T=4500$.

$T=4600$.

3100

3110

3120

3130

3140

3150

3160

3170

3180

3190

3200

3210

3220

3230

3240

3250

3260

3270

3280

3290

3300

3310

3320

3330

3340

3350

3360

3370

3380

3390

3400

3410

3420

3430

3440

3450

3460

3470

3480

3490

3500

3510

3520

3530

3540

3550

3560

3570

3580

3590
$0.895792 .04309 \quad 1.82540$ $0.89210 \quad 2.03721 \quad 1.82441$ 0.888432 .031351 .82341 0.884772 .025521 .82242 $0.88114 \quad 2.01971 \quad 1.82142$

$0.87752 \quad 2.01392 \quad 1.82041$ $0.87392 \quad 2.00815 \quad 1.81941$ $0.870342 .00240 \quad 1.81840$ $0.86678 \quad 1.99668 \quad 1.81739$ $0.86324 \quad 1.99097 \quad 1.81638$

0.859711 .985291 .81536 $0.856201 .97963 \quad 1.81434$ $0.85271 \quad 1.97399 \quad 1.81332$ $0.84924 \quad 1.96836 \quad 1.81230$ $0.845781 .96276 \quad 1.81127$

$0.84234 \quad 1.95718 \quad 1.81024$ 0.838921 .951621 .80921 $0.835521 .94608 \quad 1.80818$ 0.832131 .940561 .80715 $0.828761 .93506 \quad 1.80611$

$0.82540 \quad 1.92958 \quad 1.80507$ 0.822061 .924121 .80402 $0.818741 .91868 \quad 1.80298$ $0.81544 \quad 1.91326 \quad 1.80193$ $0.812151 .90786 \quad 1.80088$

$0.80888 \quad 1.90248 \quad 1.79983$ 0.805621 .897121 .79877 $0.80238 \quad 1.89177 \quad 1.79772$ 0.799161 .886451 .79666 $0.79595 \quad 1.881141 .79560$

$\begin{array}{llll}0.79275 & 1.87585 & 1.79453\end{array}$ $0.78958 \quad 1.87059 \quad 1.79346$ $0.786411 .86534 \quad 1.79240$ 0.783271 .860101 .79132 0.780131 .854891 .79025

$0.77702 \quad 1.84969 \quad 1.78917$ 0.773921 .844521 .78810 0.770831 .839361 .78701 $0.76776 \quad 1.83422 \quad 1.78593$ 0.764701 .829101 .78485

$0.76166 \quad 1.82399 \quad 1.78376$ $0.75863 \quad 1.81890 \quad 1.78267$ 0.755621 .813831 .78157 $0.75262 \quad 1.80878 \quad 1.78048$ $0.749631 .80374 \quad 1.77938$

$0.746661 .79873 \quad 1.77828$ $0.74370 \quad 1.79373 \quad 1.77718$ $0.74076 \quad 1.78874 \quad 1.77608$ $0.737831 .78378 \quad 1.77497$ 0.734921 .778831 .77386
$0.92175 \quad 2.08418 \quad 1.83217$ $0.91800 \quad 2.07829 \quad 1.83122$ $0.91428 \quad 2.07241 \quad 1.83026$ $0.91058 \quad 2.066551 .82931$ $0.90690 \quad 2.06072 \quad 1.82834$

$\begin{array}{llll}0.90323 & 2.05491 & 1.82738\end{array}$ $0.89958 \quad 2.04912 \quad 1.82642$ $0.89596 \quad 2.04335 \quad 1.82545$ $0.892352 .03760 \quad 1.82448$ $0.88875 \quad 2.03187 \quad 1.82350$

$\begin{array}{llll}0.88518 & 2.02617 & 1.82253\end{array}$ $0.88162 \quad 2.02048 \quad 1.82155$ $0.87808 \quad 2.014821 .82057$ $0.87456 \quad 2.00917 \quad 1.81959$ $0.87106 \quad 2.003551 .81860$ $0.86757 \quad 1.99795 \quad 1.81761$ 0.864101 .992371 .81662 0.860651 .986801 .81563 $0.85721 \quad 1.98126 \quad 1.81464$ $0.85379 \quad 1.97574 \quad 1.81364$ $0.85039 \quad 1.97024 \quad 1.81264$ 0.847011 .964751 .81164 0.843641 .959291 .81063 0.840291 .953841 .80963 0.836951 .948421 .80862

$0.833631 .94301 \quad 1.80761$ 0.830331 .937631 .80659 $0.82704 \quad 1.93226 \quad 1.80558$ $0.82377 \quad 1.92691 \quad 1.80456$ $0.82051 \quad 1.92158 \quad 1.80354$

$0.81727 \quad 1.91627 \quad 1.80251$ 0.814051 .910981 .80149 $0.810841 .90571 \quad 1.80046$ 0.807651 .900451 .79943 $0.80447 \quad 1.895211 .79840$

$0.80130 \quad 1.89000 \quad 1.79736$ $0.79816 \quad 1.88479 \quad 1.79633$ 0.795021 .879611 .79529 $0.79190 \quad 1.87445 \quad 1.79425$ $0.78880 \quad 1.86930 \quad 1.79320$

$0.78571 \quad 1.86417 \quad 1.79216$ $0.782641 .85906 \quad 1.79111$ $0.77958 \quad 1.85397 \quad 1.79006$ $0.77653 \quad 1.84889 \quad 1.78901$ $0.77350 \quad 1.843831 .78795$

$0.77049 \quad 1.83879 \quad 1.78689$ $0.76748 \quad 1.83376 \quad 1.78583$ $0.76450 \quad 1.82875 \quad 1.78477$ $0.761521 .82376 \quad 1.78371$ $0.758561 .81879 \quad 1.78264$
$0.947462 .12452 \quad 1.83853$ 0.943672 .118601 .83762 $0.93990 \quad 2.112711 .83670$ 0.936152 .106831 .83578 $0.93242 \quad 2.10098 \quad 1.83485$

$0.928712 .09514 \quad 1.83393$ 0.925012 .089331 .83300 $0.92134 \quad 2.08354 \quad 1.83207$ $0.91768 \quad 2.07777 \quad 1.83114$ 0.914042 .072031 .83020

$0.91042 \quad 2.06630 \quad 1.82926$ $0.90682 \quad 2.06059 \quad 1.82832$ 0.903232 .054911 .82738 $0.89966 \quad 2.04924 \quad 1.82644$ 0.896112 .043601 .82549

$\begin{array}{llll}0.89258 & 2.03797 & 1.82454\end{array}$ $0.88906 \quad 2.03237 \quad 1.82359$ $0.885572 .02679 \quad 1.82263$ 0.882082 .021221 .82168 0.878622 .015681 .82072

$0.875172 .01015 \quad 1.81976$ 0.871742 .004651 .81879 $0.868331 .99916 \quad 1.81783$ $0.864931 .99370 \quad 1.81686$ $0.861551 .98825 \quad 1.81589$

0.858181 .982821 .81492 $0.854831 .97742 \quad 1.81394$ $0.85150 \quad 1.97203 \quad 1.81297$ 0.848181 .966661 .81199 0.844881 .961311 .81100

$0.84160 \quad 1.95597 \quad 1.81002$ 0.838331 .950661 .80903 $0.83507 \quad 1.945361 .80805$ 0.831831 .940091 .80706 0.828611 .934831 .80606

$0.82540 \quad 1.92958 \quad 1.80507$ $0.82221 \quad 1.92436 \quad 1.80407$ 0.819031 .919161 .80307 $\begin{array}{llll}0.81587 & 1.91397 & 1.80207\end{array}$ 0.812721 .908801 .80106

0.809591 .903651 .80006 0.806471 .898511 .79905 0.803371 .893401 .79804 $0.80028 \quad 1.88830 \quad 1.79703$ 0.797201 .883221 .79601

$0.794141 .87815 \quad 1.79499$ $0.79109 \quad 1.87310 \quad 1.79398$ $0.78806 \quad 1.86807 \quad 1.79295$ $0.78504 \quad 1.86306 \quad 1.79193$ $0.782041 .85806 \quad 1.79090$
3100

3110

3120

3130

3140

3150

3160

3170

3180

3190

3200

3210

3220

3230

3240

3250

3260

3270

3280

3290

3300

3310

3320

3330

3340

3350

3360

3370

3380

3390

3400

3410

3420

3430

3440

3450

3460

3470

3480

3490

3500

3510

3520

3530

3540

3550 3560 3570 3580 3590 
Table II Harmonic Oscillator Contributions to the Thermodynamic

Functions (in units of calories, moles, and ${ }^{\circ} \mathrm{K}$ )

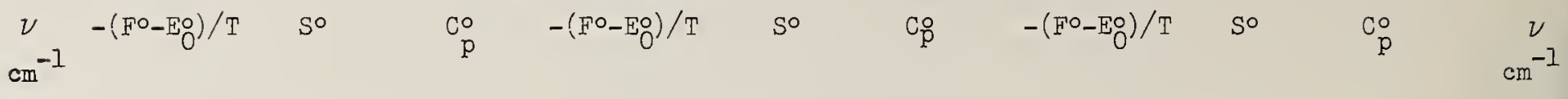

$T=4400$.

3600

3610

3620

3630

3640

3650

3660

3670

3680

3690

3700

3710

3720

3730

3740

3750

3760

3770

3780

3790

3800

3810

3820

3830

3840

3850

3860

3870

3880

3890

3900

3910

3920

3930

3940

3950

3960

3970

3980

3990

4000
$T=4500$.

$T=4600$.

$\begin{array}{llll}0.73202 & 1.77389 & 1.77275\end{array}$

$0.729131 .76898 \quad 1.77164$

$0.72626 \quad 1.76408 \quad 1.77052$

$0.72340 \quad 1.75920 \quad 1.76941$

$0.720551 .75433 \quad 1.76829$

$0.71772 \quad 1.74948 \quad 1.76716$

$0.71490 \quad 1.74465 \quad 1.76604$

$0.71209 \quad 1.73983 \quad 1.76491$

$0.70930 \quad 1.73503 \quad 1.76379$

$\begin{array}{llll}0.70652 & 1.73024 & 1.76265\end{array}$

$0.70375 \quad 1.72547 \quad 1.76152$

$0.70099 \quad 1.72072 \quad 1.76039$

$\begin{array}{llll}0.69825 & 1.71598 & 1.75925\end{array}$

$0.69552 \quad 1.71126 \quad 1.75811$

$0.69280 \quad 1.70656 \quad 1.75697$

$0.69010 \quad 1.70187 \quad 1.75582$

$0.68741 \quad 1.697191 .75468$

$0.68473 \quad 1.69253 \quad 1.75353$

$0.68206 \quad 1.68789 \quad 1.75238$

$0.67941 \quad 1.68326 \quad 1.75123$

$\begin{array}{llll}0.67676 & 1.67865 & 1.75007\end{array}$

$0.67413 \quad 1.67405 \quad 1.74891$

$0.67151 \quad 1.66947 \quad 1.74776$

0.668911 .664901 .74659

$0.66631 \quad 1.06035 \quad 1.74543$

$0.66373 \quad 1.65581 \quad 1.74427$

$0.66116 \quad 1.65129 \quad 1.74310$

$0.65860 \quad 1.64678 \quad 1.74193$

$0.65605 \quad 1.64228 \quad 1.74076$

$\begin{array}{llll}0.65352 & 1.63781 & 1.73958\end{array}$

$0.65099 \quad 1.63334 \quad 1.73841$

$0.64848 \quad 1.62889 \quad 1.73723$

$0.64598 \quad 1.62445 \quad 1.73605$

$0.64349 \quad 1.62003 \quad 1.73487$

$0.64101 \quad 1.615631 .73368$

$0.63854 \quad 1.611231 .73250$

$0.63608 \quad 1.60685 \quad 1.73131$

$0.63364 \quad 1.60249 \quad 1.73012$

$0.631201 .59814 \quad 1.72893$

$0.62878 \quad 1.59380 \quad 1.72773$

$0.62636 \quad 1.58948 \quad 1.72654$
$0.75562 \quad 1.81383 \quad 1.78157$

$0.75268 \quad 1.80889 \quad 1.78050$

$\begin{array}{llll}0.74976 & 1.80397 & 1.77943\end{array}$

$0.74686 \quad 1.79906 \quad 1.77836$

$0.74397 \quad 1.79417 \quad 1.77728$

0.741091 .789301 .77620

$0.738221 .78444 \quad 1.77512$

$0.73537 \quad 1.77960 \quad 1.77404$

0.732531 .774771 .77295

0.729711 .769961 .77186

$0.726891 .76517 \quad 1.77077$

0.724091 .760391 .76968

$0.72131 \quad 1.755631 .76859$

$0.718531 .75088 \quad 1.76749$

0.715771 .746151 .76639

$0.71302 \quad 1.741431 .76529$

0.710291 .736731 .76419

$0.70756 \quad 1.73205 \quad 1.76308$

0.704851 .727381 .76197

$0.702151 .72273 \quad 1.76087$

$0.69947 \quad 1.71809 \quad 1.75975$

$0.69679 \quad 1.71346 \quad 1.75864$

$0.69413 \quad 1.70886 \quad 1.75753$

$0.69148 \quad 1.704261 .75641$

$0.68884 \quad 1.69968 \quad 1.75529$

$0.68622 \quad 1.69512 \quad 1.75417$

$0.683601 .69057 \quad 1.75304$

$0.68100 \quad 1.68604 \quad 1.75192$

$0.67841 \quad 1.68152 \quad 1.75079$

$0.675831 .67701 \quad 1.74966$

$0.67326 \quad 1.67252 \quad 1.74853$

0.670701 .668051 .74739

0.668161 .663581 .74626

0.665621 .659141 .74512

0.663101 .654701 .74398

$0.66059 \quad 1.650281 .74284$

0.658091 .645881 .74169

0.655601 .641491 .74055

0.653121 .637111 .73940

$0.650661 .63275 \quad 1.73825$

$0.64820 \quad 1.62840 \quad 1.73710$ $\begin{array}{llll}0.77905 & 1.85308 & 1.78988\end{array}$

$0.77607 \quad 1.84812 \quad 1.78885$

0.773111 .843171 .78781

$0.77016 \quad 1.838241 .78678$

$0.76722 \quad 1.83333 \quad 1.78574$

$0.76430 \quad 1.82843 \quad 1.78470$

$0.761391 .82355 \quad 1.78366$

0.758501 .818681 .78262

0.755621 .813831 .78157

$0.75275 \quad 1.80900 \quad 1.78053$

$0.74989 \quad 1.80418 \quad 1.77948$

$0.74705 \quad 1.79938 \quad 1.77843$

$0.74422 \quad 1.79459 \quad 1.77737$

$0.74140 \quad 1.789821 .77632$

$0.73860 \quad 1.78507 \quad 1.77526$

$\begin{array}{llll}0.73580 & 1.78033 & 1.77420\end{array}$

$0.73303 \quad 1.775611 .77314$

$0.73026 \quad 1.77090 \quad 1.77207$

$0.72750 \quad 1.76621 \quad 1.77101$

0.724761 .761531 .76994

$\begin{array}{llll}0.72203 & 1.75687 & 1.76887\end{array}$

0.719321 .752221 .76780

$0.716611 .74759 \quad 1.76672$

0.713921 .742971 .76565

$0.71124 \quad 1.73837 \quad 1.76457$

$0.70857 \quad 1.73378 \quad 1.76349$

$0.70591 \quad 1.72921 \quad 1.76241$

$0.70327 \quad 1.72465 \quad 1.76132$

$0.700631 .72010 \quad 1.76024$

$0.69801 \quad 1.71557 \quad 1.75915$

$0.69540 \quad 1.71106 \quad 1.75806$ $0.692801 .70656 \quad 1.75697$

$0.690221 .70207 \quad 1.75587$

$0.68764 \quad 1.69760 \quad 1.75478$

$0.68508 \quad 1.693141 .75368$

$0.68252 \quad 1.68870 \quad 1.75258$

$0.67998 \quad 1.68427 \quad 1.75148$

$0.67745 \quad 1.679851 .75037$

0.674931 .675451 .74927

$0.672421 .67106 \quad 1.74816$

0.669931 .666691 .74705
3600

3610

3620

3630

3640

3650

3660

3670

3680

3690

3700

3710

3720

3730

3740

3750

3760

3770

3780

3790

3800

3810

3820

3830

3840

3850

3860

3870

3880

3890

3900

3910

3920

3930

3940

3950

3960

3970

3980

3990

4000 
Table II Harmonic Oscillator Contributions to the Thermodynamic

Functions (in units of calories, moles, and ${ }^{\circ} \mathrm{K}$ )
$v_{\mathrm{cm}^{-1}}-\left(\mathrm{F}^{\circ}-\mathrm{E}_{\mathrm{O}}^{\circ}\right) / \mathrm{T} \quad \mathrm{S}^{\circ}$
$\underset{p}{c o}$
$-\left(F^{\circ}-E_{O}^{O}\right) / T \quad S^{\circ}$
$\mathrm{C}_{\mathrm{p}}^{\circ}$
$T=4700$.
$T=4800$
$100 \quad 6.95829 \quad 8.91520 \quad 1.98702$ $\begin{array}{llll}110 & 6.77191 & 8.72581 & 1.98698\end{array}$ $120 \quad 6.602038 .55292 \quad 1.98695$ $\begin{array}{lllll}130 & 6.44599 & 8.39388 & 1.98691\end{array}$ $140 \quad 6.30175 \quad 8.24664 \quad 1.98687$
$6.99949 \quad 8.95703 \quad 1.98702$ $6.81306 \quad 8.767651 .98699$ $6.643118 .59476 \quad 1.98696$ 6.487018 .435721 .98692 $6.342718 .28847 \quad 1.98688$

$-\left(F^{\circ}-E_{0}\right) / T$

$S^{\circ}$

$T=4900$

$150 \quad 6.167678 .10956 \quad 1.98682$ $160 \quad 6.04244 \quad 7.981341 .98677$

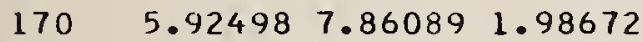
$180 \quad 5.814417 .747341 .98667$ $190 \quad 5.709987 .639921 .98661$

$200 \quad 5.61107 \quad 7.53803 \quad 1.98655$ $210 \quad 5.517127 .441101 .98649$ $220 \quad 5.427697 .34869 \quad 1.98642$ $230 \quad 5.34236 \quad 7.26039 \quad 1.98635$ $\begin{array}{lll}240 \quad 5.26079 & 7.17586 & 1.98628\end{array}$

$250 \quad 5.18268 \quad 7.09478 \quad 1.98620$ $260 \quad 5.10774 \quad 7.01688 \quad 1.98612$ $270 \quad 5.035756 .941921 .98604$ $280 \quad 4.96648 \quad 6.86970 \quad 1.98595$ $290 \quad 4.89974 \quad 6.80001 \quad 1.98587$

$\begin{array}{lllll}300 & 4.83537 & 6.73269 & 1.98577\end{array}$ $\begin{array}{lllll}310 & 4.77320 & 6.66757 & 1.98568\end{array}$

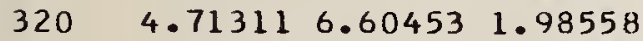

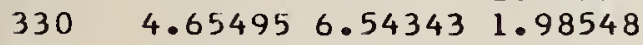
$\begin{array}{llll}340 & 4.59862 & 6.48416 & 1.98538\end{array}$

$\begin{array}{lllll}350 & 4.54400 & 6.42661 & 1.98527\end{array}$ $360 \cdot 4.491016 .370691 .98516$ $370 \quad 4.439556 .31630 \quad 1.98505$ $380 \quad 4.38953 \quad 6.26336 \quad 1.98493$ $390 \quad 4.340906 .21180 \quad 1.98481$

$400 \quad 4.293576 .16156 \quad 1.98469$ $410 \quad 4.247486 .112551 .98456$ $420 \quad 4.202576 .06473 \quad 1.98443$ $\begin{array}{llll}430 & 4.15879 & 6.01803 & 1.98430\end{array}$ $440 \quad 4.11608 \quad 5.97242 \quad 1.98417$

$\begin{array}{llll}450 & 4.07439 & 5.92783 & 1.98403\end{array}$ $\begin{array}{lllll}460 & 4.03369 & 5.88422 & 1.98389\end{array}$ $470 \quad 3.993925 .84156 \quad 1.98375$

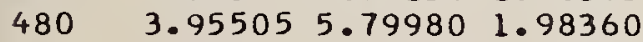
$490 \quad 3.91704 \quad 5.75890 \quad 1.98345$

$\begin{array}{lllll}500 & 3.87986 & 5.71883 & 1.98329\end{array}$ $\begin{array}{lllll}510 & 3.84347 & 5.67956 & 1.98314\end{array}$ $\begin{array}{lllll}520 & 3.80785 & 5.64105 & 1.98298\end{array}$ $\begin{array}{lllll}530 & 3.77296 & 5.60328 & 1.98282\end{array}$ $\begin{array}{lllll}540 & 3.73877 & 5.56622 & 1.98265\end{array}$

$\begin{array}{lllll}550 & 3.70527 & 5.52984 & 1.98248\end{array}$ $\begin{array}{lllll}560 & 3.67242 & 5.49412 & 1.98231\end{array}$ $570 \quad 3.64020 \quad 5.45903 \quad 1.98214$ $\begin{array}{llll}580 & 3.60859 & 5.42456 & 1.98196\end{array}$ $\begin{array}{llll}590 & 3.57757 & 5.39068 & 1.98178\end{array}$
$6.20856 \quad 8.15139 \quad 1.98683$ 6.083278 .023171 .98679 $5.96575 \quad 7.90272 \quad 1.98674$ 5.855127 .789161 .98669 $5.750637 .68175 \quad 1.98663$

$5.65165 \quad 7.57985 \quad 1.98657$ 5.557647 .482931 .98651 5.468157 .390521 .98645 $5.38276 \quad 7.30221 \quad 1.98638$ 5.301137 .217681 .98631

$5.222957 .13659 \quad 1.98624$ 5.147957 .058691 .98616 $5.075896 .98374 \quad 1.98609$ $5.00656 \quad 6.911511 .98600$ $4.939776 .84182 \quad 1.98592$

$4.875336 .77449 \quad 1.98583$ 4.813116 .709381 .98574 $4.75295 \quad 6.64634 \quad 1.93565$ $4.694736 .58524 \quad 1.98555$ 4.638336 .525961 .98545

$4.58366 \quad 6.468411 .98535$ $4.530606 .41248 \quad 1.98524$ 4.479086 .358091 .98513 4.429016 .305151 .98502 $4.380316 .25359 \quad 1.98491$

$4.33292 \quad 6.20334 \quad 1.98479$ 4.286776 .154331 .98467 4.241806 .106511 .98455 $4.19796 \quad 6.059811 .98442$ 4.155196 .014191 .98429

4.113445 .969601 .98416 $4.07268 \quad 5.92599 \quad 1.98402$ $4.03285 \quad 5.883331 .98389$ $3.99392 \quad 5.84156 \quad 1.98375$ 3.955855 .800661 .98360

3.918615 .760591 .98345 $3.88216 \quad 5.721311 .98330$ 3.846485 .682801 .98315 3.811535 .645021 .98300 $3.77728 \quad 5.60796 \quad 1.98284$

$\begin{array}{llll}3.74371 & 5.57158 & 1.98268\end{array}$ 3.710805 .535851 .98251 3.678535 .500771 .98234 $3.64686 \quad 5.46629 \quad 1.98217$ 3.615785 .432411 .98200
$7.03986 \quad 8.99800 \quad 1.98703$ 6.853378 .808621 .98700 $6.68336 \quad 8.63573 \quad 1.98696$ $6.52720 \quad 8.47669 \quad 1.98693$ 6.382838 .329441 .98689

$6.248638 .19236 \quad 1.98685$ $6.12328 \quad 8.064131 .98680$ $6.00570 \quad 7.94368 \quad 1.98676$ 5.895017 .830131 .98671 $5.79046 \quad 7.72271 \quad 1.98665$

$\begin{array}{llll}5.69142 & 7.62081 & 1.98660\end{array}$ $\begin{array}{llll}5.59735 & 7.52389 & 1.98654\end{array}$ $5.50780 \quad 7.43147 \quad 1.98648$ 5.422357 .343171 .98641 5.340667 .258631 .98635

$5.262427 .17755 \quad 1.98628$ 5.187377 .099651 .98621 5.115257 .024691 .98613 $5.04586 \quad 6.95246 \quad 1.98605$ $4.97900 \quad 6.88277 \quad 1.98597$

$\begin{array}{llll}4.91451 & 6.81544 & 1.98589\end{array}$ 4.852236 .750321 .98580 $4.792016 .68728 \quad 1.98571$ $4.733736 .62618 \quad 1.98562$ $4.67728 \quad 6.56690 \quad 1.98552$

4.622546 .509351 .98542 4.569436 .453421 .98532 4.517856 .399021 .98522 $4.467726 .34608 \quad 1.98511$ $4.41896 \quad 6.294521 .98500$

$4.371516 .24427 \quad 1.98489$ $4.325306 .19526 \quad 1.98477$ $4.280286 .14743 \quad 1.98465$ $4.236376 .10073 \quad 1.98453$ 4.193546 .055111 .98441

$4.151746 .01051 \quad 1.98428$ 4.110925 .966901 .98415 4.071035 .924231 .98402 $4.032055 .88246 \quad 1.98388$ $3.993925 .84156 \quad 1.98375$

$3.956625 .80148 \quad 1.98360$ 3.920115 .762201 .98346 3.884375 .723691 .98331 $3.849365 .68591 \quad 1.98316$ 3.815065 .648851 .98301

$3.781445 .61246 \quad 1.98286$ $3.748475 .57673 \quad 1.98270$ 3.716135 .541641 .98254 $3.684415 .50716 \quad 1.98237$ $3.653275 .47328 \quad 1.98221$
100

110

120

130

140

150

160

170

180

190

200

210

220

230

240

250

260

270

280

290

300

310

320

330

340

350

360

370

380

390

400

410

420

430

440

450

460

470

480

490

500

510

520

530

540

550

560

570

580

590 
Table II Harmonic Oscillator Contributions to the Thermodynamic

Functions (in units of calories, moles, and ${ }^{\circ} \mathrm{K}$ )

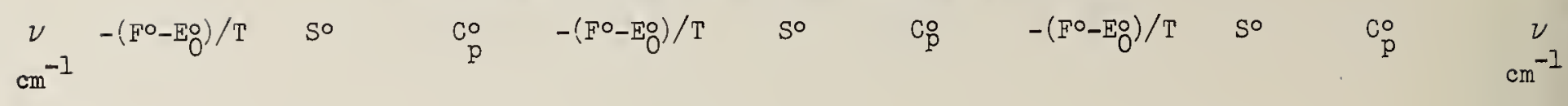

$$
T=4700 . \quad T=4800 .
$$

$600 \quad 3.547125 .35738 \quad 1.98159$ $610 \quad 3.51722 \quad 5.32462 \quad 1.98141$ $\begin{array}{lllll}620 & 3.48786 & 5.29241 & 1.98122\end{array}$ $630 \quad 3.459015 .26071 \quad 1.98102$ $640 \quad 3.43065 \quad 5.22951 \quad 1.98083$

$\begin{array}{lllll}650 & 3.40279 & 5.19880 & 1.98063\end{array}$ $6603.375395 .16856 \quad 1.98042$

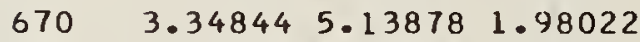
$\begin{array}{lll}680 & 3.32194 \quad 5.10945 \quad 1.98001\end{array}$ $690 \quad 3.29587$
$5.08055 \quad 1.97980$

$\begin{array}{lllll}700 \quad 3.27021 & 5.05206 \quad 1.97958\end{array}$ $\begin{array}{lllll}710 & 3.24495 & 5.02398 & 1.97937\end{array}$ $\begin{array}{lllll}720 & 3.22009 & 4.99630 & 1.97914\end{array}$ $\begin{array}{llll}730 & 3.19561 & 4.96900 & 1.97892\end{array}$ $\begin{array}{llll}740 & 3.17150 \quad 4.94208 & 1.97869\end{array}$

$\begin{array}{llll}750 & 3.14775 & 4.91552 \quad 1.97846\end{array}$ $\begin{array}{lllll}760 & 3.12436 & 4.88932 & 1.97823\end{array}$ $\begin{array}{llll}770 & 3.10130 & 4.86346 \quad 1.97799\end{array}$ $\begin{array}{lllll}780 & 3.07858 & 4.83794 & 1.97776\end{array}$ $790 \quad 3.056194 .81274 \quad 1.97751$

$\begin{array}{lllll}800 & 3.03411 & 4.78787 & 1.97727\end{array}$ $810 \quad 3.01234 \quad 4.76331 \quad 1.97702$ $\begin{array}{llll}820 & 2.99087 \quad 4.73905 & 1.97677\end{array}$ $830 \quad 2.96970 \quad 4.71509 \quad 1.97651$ $840 \quad 2.948814 .691421 .97626$

$\begin{array}{llll}850 \quad 2.92821 & 4.66804 & 1.97600\end{array}$ $860 \quad 2.907874 .64493 \quad 1.97573$ $\begin{array}{lllll}870 & 2.88781 & 4.62209 & 1.97547\end{array}$ $\begin{array}{lllll}880 & 2.86800 & 4.59951 & 1.97520\end{array}$ $890 \quad 2.84845 \quad 4.57720 \quad 1.97492$

$900 \quad 2.82915 \quad 4.55513 \quad 1.97465$ $910 \quad 2.81010 \quad 4.53331 \quad 1.97437$ $920 \quad 2.79128 \quad 4.51174 \quad 1.97409$ $930 \quad 2.772694 .49040 \quad 1.97380$ $940 \quad 2.75434 \quad 4.46929 \quad 1.97351$

$950 \quad 2.73620 \quad 4.44841 \quad 1.97322$ $960 \quad 2.718294 .42774 \quad 1.97293$ $970 \quad 2.70059 \quad 4.40730 \quad 1.97263$ $980 \quad 2.68310 \quad 4.38707 \quad 1.97233$ $990 \quad 2.665814 .367051 .97203$

$1000 \quad 2.648734 .34723 \quad 1.97172$ $1010 \quad 2.63184 \quad 4.32761 \quad 1.97141$ $1020 \quad 2.615154 .30819 \quad 1.97110$ $1030 \quad 2.59864 \quad 4.28896 \quad 1.97079$

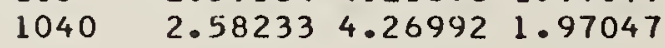

$1050 \quad 2.566194 .25107 \quad 1.97015$ $1060 \quad 2.550234 .232391 .96982$ $1070 \quad 2.53445 \quad 4.21390 \quad 1.96950$ $1080 \quad 2.51884 \quad 4.19558 \quad 1.96917$ - 10902.503404 .177431 .96883
$3.58527 \quad 5.39910 \quad 1.98182$ 3.555315 .366341 .98164 3.525895 .334121 .99146 3.496985 .302421 .98128 $3.468575 .27122 \quad 1.98109$

3.440645 .240501 .98090 3.413185 .210261 .98070 $3.38618 \quad 5.18048 \quad 1.98050$ 3.359625 .151141 .98030 $3.33348 \quad 5.122231 .98010$

$3.30776 \quad 5.09374 \quad 1.97990$ $3.28245 \quad 5.06566 \quad 1.97969$ $3.257535 .03797 \quad 1.97947$ $3.232995 .01067 \quad 1.97926$ $3.20882 \quad 4.98374 \quad 1.97904$

$3.18502 \quad 4.95718 \quad 1.97882$ $3.16156 \quad 4.93097 \quad 1.97860$ $3.13845 \quad 4.905111 .97837$ $3.115674 .87958 \quad 1.97814$ $3.09322 \quad 4.85438 \quad 1.97791$

$3.07108 \quad 4.82950 \quad 1.97767$ $3.04926 \quad 4.80494 \quad 1.97744$ $3.02773 \quad 4.78068 \quad 1.97720$ $3.00650 \quad 4.75671 \quad 1.97695$ $2.98555 \quad 4.73304 \quad 1.97670$

2.964894 .709641 .97645 $2.94450 \quad 4.686531 .97620$ 2.924374 .663681 .97595 2.904514 .641101 .97569 $2.88490 \quad 4.61878 \quad 1.97543$

$2.865554 .59671 \quad 1.97516$ $2.846434 .57489 \quad 1.97489$ $2.82756 \quad 4.55330 \quad 1.97462$ $2.808914 .53196 \quad 1.97435$ $2.79050 \quad 4.51084 \quad 1.97407$

$2.772314 .48995 \quad 1.97380$ $2.75434 \quad 4.469291 .97351$ $2.73658 \quad 4.44884 \quad 1.97323$ $2.71903 \quad 4.42860 \quad 1.97294$ $2.701694 .40857 \quad 1.97265$

$2.68455 \quad 4.38875 \quad 1.97236$ 2.667604 .369121 .97206 $2.650854 .34970 \quad 1.97176$ $2.634294 .33046 \quad 1.97146$ 2.617924 .311411 .97115

2.601724 .292551 .97085 $2.585714 .27387 \quad 1.97054$ 2.569874 .255371 .97022 $2.55420 \quad 4.237051 .96991$ 2.538714 .218891 .96959
$T=4900$

$3.622715 .43996 \quad 1.98204$ 3.592695 .407201 .98187 3.563215 .374981 .98169 $3.534245 .34327 \quad 1.98151$ 3.505775 .312071 .98133

$3.47779 \quad 5.281351 .98115$ 3.450275 .251111 .98096 3.423215 .221321 .98077 $3.39660 \quad 5.191971 .98058$ $3.370415 .16306 \quad 1.98039$

$3.344635 .13457 \quad 1.98019$ 3.319265 .106481 .97999 $3.29428 \quad 5.07879 \quad 1.97978$ $3.269695 .05148 \quad 1.97958$ $3.24546 \quad 5.024551 .97937$

$3.22160 \quad 4.99798 \quad 1.97916$ $3.198094 .97177 \quad 1.97894$ $3.17492 \quad 4.94590 \quad 1.97873$ $3.15209 \quad 4.920371 .97851$ $3.12958 \quad 4.89517 \quad 1.97828$

3.107394 .870291 .97806 $3.08550 \quad 4.845711 .97783$ $3.06392 \quad 4.821451 .97760$ $3.042634 .79748 \quad 1.97736$ 3.021634 .773801 .97713

$3.000914 .75040 \quad 1.97689$ $2.98047 \quad 4.727281 .97664$ $2.96029 \quad 4.704431 .97640$ 2.940374 .681841 .97615 2.920714 .659521 .97590

$2.90129 \quad 4.63744 \quad 1.97565$ 2.882124 .615611 .97539 2.863194 .594021 .97513 $2.84450 \quad 4.57267 \quad 1.97487$ 2.826034 .551551 .97460

$2.80778 \quad 4.53066 \quad 1.97433$ $2.78975 \quad 4.50999 \quad 1.97406$ $2.77194 \quad 4.489531 .97379$ $2.75434 \quad 4.46929 \quad 1.97351$ $2.73694 \quad 4.44925 \quad 1.97324$

$2.71974 \quad 4.42942 \quad 1.97295$ $2.70275 \quad 4.40979 \quad 1.97267$ $2.68594 \quad 4.39036 \quad 1.97238$ 2.669324 .371121 .97209 $2.652894 .35206 \quad 1.97180$

$2.636654 .33320 \quad 1.97150$ $2.620584 .31451 \quad 1.97120$ $2.60468 \quad 4.29600 \quad 1.97090$ $2.58896 \quad 4.27767 \quad 1.97060$ 2.573414 .259511 .97029
600

610

620

630

640

650

660

670

680

690

700

710

720

730

740

750

760

770

780

790

800

810

820

830

840

850

860

870

880

890

900

910

920

930

940

950

960

970

980

990

1000

1010

1020

1030

1040

1050

1060

1070

1080

1090 
Table II Harmonic Oscillator Contributions to the Thermodynamic

Functions (in units of calories, moles, and ${ }^{\circ} \mathrm{K}$ )
$\mathrm{cm}^{-1} \quad-\left(F^{\circ}-\mathrm{E}_{\mathrm{O}}^{\circ}\right) / \mathrm{T} \quad$ So
$\mathrm{C}_{\mathrm{p}}^{\circ} \quad-\left(\mathrm{F}^{\circ}-\mathrm{EO}\right) / \mathrm{T}$
So
$\mathrm{C}_{\mathrm{p}}^{\circ} \quad-\left(F^{\circ}-\mathrm{E}_{\mathrm{O}}^{\circ}\right) / \mathrm{T}$
so
$\mathrm{c}_{\mathrm{p}}^{\circ}$
$\mathrm{cm}^{-1}$

$T=4700$.

$1100 \quad 2.488124 .15945 \quad 1.96850$

$1110 \quad 2.473014 .141641 .96816$

$1120 \quad 2.45805 \quad 4.12399 \quad 1.96782$

$1130 \quad 2.443264 .10650 \quad 1.96747$

$1140 \quad 2.428624 .08917 \quad 1.96712$

$\begin{array}{lllll}1150 & 2.41412 & 4.07199 & 1.96677\end{array}$

$1160 \quad 2.39978 \quad 4.05496 \quad 1.96642$

$1170 \quad 2.38559 \quad 4.03808 \quad 1.96606$

$\begin{array}{lllll}1180 & 2.37153 & 4.02135 & 1.96570\end{array}$

$1190 \quad 2.357624 .00477 \quad 1.96534$

$\begin{array}{lllll}1200 & 2.34385 & 3.98832 & 1.96497\end{array}$

$1210 \quad 2.33021 \quad 3.97202 \quad 1.96460$

$\begin{array}{llll}1220 & 2.31671 & 3.95585 & 1.96423\end{array}$

$\begin{array}{lllll}1230 & 2.30334 & 3.93981 & 1.96386\end{array}$

$1240 \quad 2.29010 \quad 3.92391 \quad 1.96348$

$\begin{array}{llll}1250 \quad 2.27699 & 3.90814 & 1.96310\end{array}$ $1260 \quad 2.26400 \quad 3.89250 \quad 1.96271$ $1270 \quad 2.25114 \quad 3.87699 \quad 1.96233$ $1280 \quad 2.23840 \quad 3.86160 \quad 1.96194$ $1290 \quad 2.22578 \quad 3.84633 \quad 1.96155$

$\begin{array}{llll}1300 & 2.21327 & 3.83119 & 1.96115\end{array}$ $1310 \quad 2.200893 .816161 .96075$ $1320 \quad 2.188613 .80125 \quad 1.96035$ $\begin{array}{lllll}1330 & 2.17645 & 3.78646 & 1.95994\end{array}$ $\begin{array}{lllll}1340 \quad 2.16440 & 3.77178 & 1.95954\end{array}$

$\begin{array}{lllll}1350 & 2.15246 & 3.75721 & 1.95913\end{array}$ $1360 \quad 2.140633 .74275 \quad 1.95871$ $\begin{array}{lllll}1370 & 2.12890 & 3.72841 & 1.95830\end{array}$ $\begin{array}{lllll}1380 & 2.11727 & 3.71417 & 1.95788\end{array}$ $1390 \quad 2.10575 \quad 3.70003 \quad 1.95746$

$1400 \quad 2.09434 \quad 3.68600 \quad 1.95703$ $1410 \quad 2.08302 \quad 3.67207 \quad 1.95660$ $\begin{array}{lllll}1420 & 2.07179 & 3.65825 & 1.95617\end{array}$ $\begin{array}{lllll}1430 & 2.06067 & 3.64452 & 1.95574\end{array}$ $1440 \quad 2.049643 .63089 \quad 1.95530$

$\begin{array}{lllll}1450 & 2.03871 & 3.61736 & 1.95486\end{array}$ $\begin{array}{lllll}1460 & 2.02787 & 3.60393 & 1.95442\end{array}$ $\begin{array}{lllll}1470 & 2.01712 & 3.59059 & 1.95397\end{array}$ $\begin{array}{lllll}1480 & 2.00646 & 3.57734 & 1.95352\end{array}$ $\begin{array}{lllll}1490 & 1.99589 & 3.56419 & 1.95307\end{array}$

$\begin{array}{lllll}1500 & 1.98541 & 3.55113 & 1.95262\end{array}$ $\begin{array}{lllll}1510 & 1.97501 & 3.53815 & 1.95216\end{array}$ $\begin{array}{lllll}1520 & 1.96470 & 3.52527 & 1.95170\end{array}$ $1530 \quad 1.95448 \quad 3.51247 \quad 1.95124$ $\begin{array}{llllll}1540 \quad 1.94434 & 3.49976 & 1.95077\end{array}$

$\begin{array}{lllll}1550 & 1.93428 & 3.48714 & 1.95030\end{array}$ $1560 \quad 1.92430 \quad 3.47460 \quad 1.94983$ $\begin{array}{llllll}1570 & 1.91440 & 3.46214 & 1.94936\end{array}$ $\begin{array}{llllll}1580 & 1.90458 & 3.44977 & 1.94888\end{array}$ $\begin{array}{llllll}1590 & 1.89484 & 3.43747 & 1.94840\end{array}$

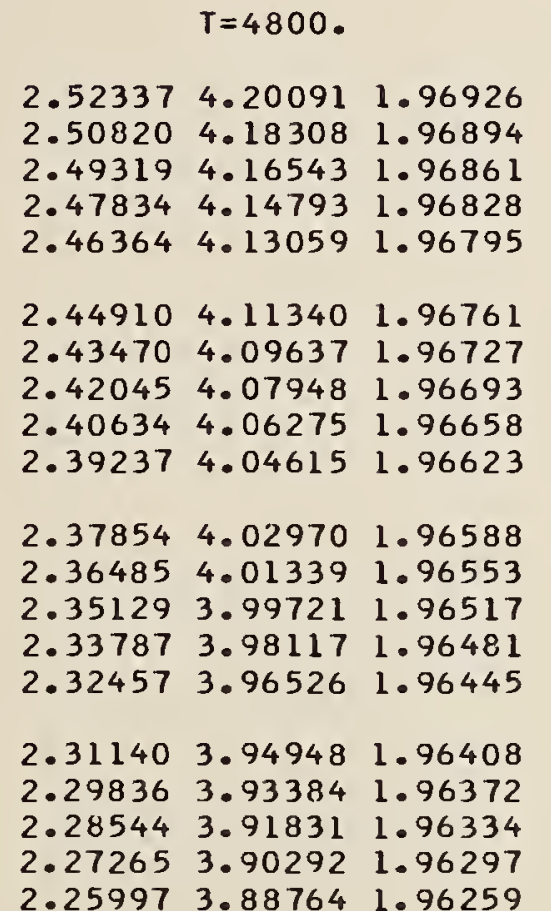

$2.247413 .87249 \quad 1.96221$

2.234973 .857451 .96183

2.222643 .842541 .96145

2.210423 .827731 .96106

$2.19832 \quad 3.81305 \quad 1.96067$

$2.18632 \quad 3.79847 \quad 1.96027$

$2.17443 \quad 3.78400 \quad 1.95988$

2.162653 .769651 .95948

$2.150973 .75540 \quad 1.95908$

$2.13940 \quad 3.74125 \quad 1.95867$

$2.12793 \quad 3.72722 \quad 1.95826$

2. $116553.71328 \quad 1.95785$

2. $10528 \quad 3.69944 \quad 1.95744$

$2.094103 .68571 \quad 1.95702$

2.083023 .672071 .95660

$2.07203 \quad 3.658531 .95618$

2.061133 .645091 .95576

$2.05033 \quad 3.63174 \quad 1.95533$

2.039623 .618491 .95490

2.028993 .605321 .95446

$2.01846 \quad 3.59225 \quad 1.95403$

$2.008013 .57927 \quad 1.95359$

$1.997653 .56638 \quad 1.95315$

$1.987373 .55357 \quad 1.95270$

1.977173 .540851 .95226

$1.96706 \quad 3.52822 \quad 1.95181$

$1.957033 .51567 \quad 1.95135$

$1.947083 .50320 \quad 1.95090$

$1.937203 .49081 \quad 1.95044$

$1.92741 \quad 3.47851 \quad 1.94998$
$T=4900$

2.558034 .241521 .96998

$2.54280 \quad 4.223691 .96967$

2.527744 .206031 .96936

$2.512834 .18852 \quad 1.96904$

$2.49808 \quad 4.171181 .96872$

1100

1110

1120

1130

1140

$2.48348 \quad 4.15398 \quad 1.96839$

2.469034 .136941 .96807

2.454724 .120051 .96774

2.440564 .103301 .96741

$2.42654 \quad 4.08670 \quad 1.96707$

1150

1160

1170

1180

1190

$2.41265 \quad 4.07024 \quad 1.96674$ $2.39891 \quad 4.053921 .96640$

$2.38530 \quad 4.03774 \quad 1.96605$

2.371824 .021691 .96571

$2.35847 \quad 4.00578 \quad 1.96536$

1200

1210

1220

1230

1240

$\begin{array}{llll}2.34525 & 3.98999 & 1.96501\end{array}$

$2.33215 \quad 3.97434 \quad 1.96466$

$2.319183 .95881 \quad 1.96430$

$2.30633 \quad 3.94340 \quad 1.96394$

$2.29360 \quad 3.928121 .96358$

1250

1260

1270

1280

1290

$2.28099 \quad 3.91296 \quad 1.96322$

$2.268493 .89792 \quad 1.96285$

$2.25611 \quad 3.88299 \quad 1.96248$

$2.243843 .86818 \quad 1.96211$

$2.231693 .85348 \quad 1.96173$

1300

1310

1320

1330

1340

$2.21964 \quad 3.83890 \quad 1.96135$ $2.20770 \quad 3.82443 \quad 1.96097$ $2.19586 \quad 3.81006 \quad 1.96059$ $2.18413 \quad 3.79580 \quad 1.96020$ $2.17250 \quad 3.78165 \quad 1.95981$

1350

1360

1370

1380

1390

$2.16098 \quad 3.76761 \quad 1.95942$ $2.149553 .75366 \quad 1.95903$ $2.13822 \quad 3.73982 \quad 1.95863$ $2.12699 \quad 3.72607 \quad 1.95823$ $2.11586 \quad 3.71243 \quad 1.95783$

1400

1410

1420

1430

1440

$2.10482 \quad 3.69888 \quad 1.95742$ 2.093873 .685431 .95701 $2.08302 \quad 3.67207 \quad 1.95660$ $2.072253 .65881 \quad 1.95619$ $2.06158 \quad 3.64564 \quad 1.95577$

1450 1460 1470 1480 1490

$\begin{array}{lllll}2.05099 & 3.63256 & 1.95535 & 1500\end{array}$ $2.04049 \quad 3.61957 \quad 1.95493$ $2.03007 \quad 3.60666 \quad 1.95451$ $2.019743 .59385 \quad 1.95408$ $2.00950 \quad 3.58112 \quad 1.95365$

1510

1520

1530

1540

$\begin{array}{llll}1.99933 & 3.56848 & 1.95322 & 1550\end{array}$

$\begin{array}{llll}1.98925 & 3.55592 & 1.95278 & 1560\end{array}$

$\begin{array}{lllll}1.97925 & 3.54344 & 1.95235 & 1570\end{array}$

$1.96932 \quad 3.53104 \quad 1.95191 \quad 1580$

$\begin{array}{llll}1.95948 & 3.51873 \quad 1.95146 \quad 1590\end{array}$ 
Table II Harmonic Oscillator Contributions to the Thermodynamic

Functions (in units of calories, moles, and ${ }^{\circ} \mathrm{K}$ )

$$
\mathrm{cm}^{-1}-\left(\mathrm{F}^{\circ}-\mathrm{E}_{\mathrm{O}}^{\circ}\right) / \mathrm{T} \quad \mathrm{S}^{\circ}
$$

$T=4700$
$S^{\circ}$

$T=4800$.

$1.917693 .46628 \quad 1.94951$ $1.908053 .45414 \quad 1.94905$ $1.89849 \quad 3.44207 \quad 1.94858$ $1.88900 \quad 3.43008 \quad 1.94811$ $1.87958 \quad 3.41817 \quad 1.94763$

$1.856643 .38909 \quad 1.94645$ $\begin{array}{lllll}1640 \quad 1.84728 & 3.37718 & 1.94595\end{array}$

1650

1660

1670

1680

1690

1700

1710

1720

1730

1740

1750

1760

1770

1780

1790

1800

1810

1820

1830

1840

1850

1860

1870

1880

1890

1900

1910

1920

1930

1940

1950

1960

1970

1980

1990

2000

2010

2020

2030

2040

2050

2060

2070

2080

2090
$1.83798 \quad 3.36535 \quad 1.94545$ $1.82876 \quad 3.35360 \quad 1.94495$ $1.819613 .34192 \quad 1.94445$ $1.810533 .33031 \quad 1.94394$ $1.80152 \quad 3.31878 \quad 1.94343$

$1.792573 .30731 \quad 1.94292$ $1.78370 \quad 3.29592 \quad 1.94241$ $1.774893 .28460 \quad 1.94189$ $1.766143 .27334 \quad 1.94137$ $1.75746 \quad 3.26215 \quad 1.94084$

$1.74885 \quad 3.25103 \quad 1.94032$ $1.74029 \quad 3.23998 \quad 1.93979$ $1.73180 \quad 3.22899 \quad 1.93926$ $1.72338 \quad 3.21806 \quad 1.93872$ $1.715013 .20720 \quad 1.93818$

$1.70670 \quad 3.19641 \quad 1.93764$ 1.698463 .185671 .93710 $1.690273 .17500 \quad 1.93655$ 1.682143 .164391 .93600 $1.674073 .15384 \quad 1.93545$

$1.66606 \quad 3.14336 \quad 1.93490$ $1.65810 \quad 3.13293 \quad 1.93434$ $1.65020 \quad 3.12256 \quad 1.93378$ 1.642353 .112241 .93322 $1.63456 \quad 3.10199 \quad 1.93265$

$1.626823 .09179 \quad 1.93208$ 1.619143 .081651 .93151 $1.611513 .07157 \quad 1.93094$ $1.60393 \quad 3.061541 .93036$ $1.59640 \quad 3.05156 \quad 1.92978$

$1.58893 \quad 3.04164 \quad 1.92920$ $1.58151 \quad 3.03178 \quad 1.92861$ 1.574133 .021961 .92802 $1.566813 .01220 \quad 1.92743$ $1.55953 \quad 3.00249 \quad 1.92684$

$1.55230 \quad 2.99284 \quad 1.92624$ 1.545132 .983231 .92564 $1.53799 \quad 2.97368 \quad 1.92504$ 1.530912 .964171 .92443 1.523872 .954721 .92383

$1.51688 \quad 2.94531 \quad 1.92322$ $1.509942 .93595 \quad 1.92260$ 1.503042 .926641 .92199 $1.496182 .91738 \quad 1.92137$ 1.489372 .908171 .92075
$1.87023 \quad 3.40633 \quad 1.94715$ $1.86096 \quad 3.39457 \quad 1.94667$ $1.85175 \quad 3.382881 .94619$ 1.842623 .371261 .94570 $1.83356 \quad 3.35971 \quad 1.94521$

$1.82456 \quad 3.34824 \quad 1.94472$ 1.815633 .336831 .94423 1.806773 .325501 .94373 $1.79797 \quad 3.31423 \quad 1.94323$ $1.789243 .30303 \quad 1.94273$

$1.78057 \quad 3.29190 \quad 1.94222$ 1.771973 .280841 .94172 $1.76342 \quad 3.26984 \quad 1.94120$ $1.754943 .25890 \quad 1.94069$ $1.746523 .24803 \quad 1.94017$

$1.73817 \quad 3.23722 \quad 1.93966$ $1.729873 .22648 \quad 1.93913$ 1.721633 .215801 .93861 $1.71345 \quad 3.20517 \quad 1.93808$ 1.705333 .194611 .93755

$1.69726 \quad 3.18411 \quad 1.93702$ $1.689253 .17367 \quad 1.93648$ $1.68130 \quad 3.16329 \quad 1.93595$ 1.673403 .152971 .93541 $1.665563 .14270 \quad 1.93486$

$1.65777 \quad 3.13249 \quad 1.93432$ 1.650033 .122341 .93377 $1.642353 .11224 \quad 1.93322$ $1.634723 .10220 \quad 1.93266$ 1.627153 .092221 .93211

$1.619623 .08228 \quad 1.93155$ $1.612143 .07241 \quad 1.93098$ $1.604723 .06258 \quad 1.93042$ $1.59734 \quad 3.05281 \quad 1.92985$ $1.590023 .04309 \quad 1.92928$

$1.58274 \quad 3.03342 \quad 1.92871$ 1.575513 .023801 .92813 $1.56833 \quad 3.01423 \quad 1.92755$ $1.561193 .00471 \quad 1.92697$ $1.554112 .99525 \quad 1.92639$

$1.54706 \quad 2.98583 \quad 1.92580$ $1.540072 .97646 \quad 1.92522$ $1.533122 .96714 \quad 1.92462$ $1.526212 .95786 \quad 1.92403$ $1.51935 \quad 2.94864 \quad 1.92343$
$-\left(F^{\circ}-E_{0}^{\circ}\right) / T$

$S^{\circ}$

$\mathrm{C}_{\mathrm{p}}^{\circ}$

$\mathrm{cm}^{-1}$

$u$
-1

$T=4900$.

$1.949713 .50650 \quad 1.95102$

1.940023 .494341 .95057

$1.93040 \quad 3.48227 \quad 1.95012$

$1.92086 \quad 3.47027 \quad 1.94967$

$1.911393 .45834 \quad 1.94921$

$1.90199 \quad 3.44650 \quad 1.94875$

1.892673 .434721 .94829

1.883413 .423021 .94782

1.874233 .411391 .94736

$1.865113 .39984 \quad 1.94689$

$1.85607 \quad 3.38835 \quad 1.94642$

$1.847093 .37694 \quad 1.94594$

1.838173 .365591 .94546

$1.82932 \quad 3.35432 \quad 1.94498$

1.820543 .343111 .94450

$1.81182 \quad 3.33197 \quad 1.94402$

1.803173 .320891 .94353

$1.79458 \quad 3.309881 .94304$

$1.78605 \quad 3.29894 \quad 1.94254$

1.777583 .288051 .94205

$1.76917 \quad 3.27724 \quad 1.94155$

$1.760823 .26648 \quad 1.94105$

$1.75253 \quad 3.25579 \quad 1.94054$

$1.74430 \quad 3.24516 \quad 1.94004$

1.736133 .234591 .93953

$1.72801 \quad 3.22407 \quad 1.93902$

$1.71995 \quad 3.21362 \quad 1.93850$

$1.71145 \quad 3.203231 .93799$

$1.704013 .19290 \quad 1.93747$

1.696113 .182621 .93694

$1.68827 \quad 3.17240 \quad 1.93642$

$1.680493 .16224 \quad 1.93589$

$1.67276 \quad 3.15213 \quad 1.93536$

$1.66508 \quad 3.14208 \quad 1.93483$

$1.65745 \quad 3.13208 \quad 1.93429$

$1.64988 \quad 3.12213 \quad 1.93376$ 1.642353 .112241 .93322 $1.634883 .10241 \quad 1.93267$ $1.62745 \quad 3.092621 .93213$ $1.62008 \quad 3.082891 .93158$

$1.61275 \quad 3.073211 .93103$ $1.605473 .06358 \quad 1.93048$ $1.59824 \quad 3.05400 \quad 1.92992$ $1.59106 \quad 3.044471 .92936$ 1.583923 .034991 .92880

$1.57683 \quad 3.02556 \quad 1.92824$ $1.56979 \quad 3.01618 \quad 1.92767$ 1.562793 .006851 .92710 $1.55584 \quad 2.99756 \quad 1.92653$ $1.54893 \quad 2.98832 \quad 1.92596$
1600

1610

1620

1630

1640

1650

1660

1670

1680

1690

1700

1710

1720

1730

1740

1750

1760

1770

1780

1790

1800

1810

1820

1830

1840

1850

1860

1870

1880

1890

1900

1910

1920

1930

1940

1950

1960

1970

1980

1990

2000

2010

2020

2030

2040

2050

2060

2070

2080

2090 
Table II Harmonic Oscillator Contributions to the Thermodynamic

Functions (in units of calories, moles, and ${ }^{\circ} \mathrm{K}$ )
$\mathrm{cm}^{-1} \quad-\left(\mathrm{F}^{\circ}-\mathrm{E}_{0}^{\circ}\right) / \mathrm{T} \quad$ So

$\mathrm{C}_{\mathrm{p}}^{\circ} \quad-\left(\mathrm{F}^{\circ}-\mathrm{E}_{\mathrm{O}}^{\mathrm{O}}\right) / \mathrm{T}$

So

$\mathrm{T}=4700$

$T=4800$
$-\left(\mathrm{F}^{\circ}-\mathrm{E}_{\mathrm{O}}^{\mathrm{O}}\right) / \mathrm{T}$

So
2100

2110

2120

2130

2140

2150

2160

2170

2180

2190

2200

2210

2220

2230

2240

2250

2260

2270

2280

2290

2300

2310

2320

2330

2340

2350

2360

2370

2380

2390

2400

2410

2420

2430

2440

2450

2460

2470

2480

2490

2500

2510

2520

2530

2540

2550

2560

2570

2580

2590
$1.48261 \quad 2.89900 \quad 1.92012$ $1.47588 \quad 2.88988 \quad 1.91950$ $\begin{array}{llll}1.46920 \quad 2.88081 & 1.91887\end{array}$ $1.46256 \quad 2.87178 \quad 1.91823$ 1.455972 .862801 .91760

$1.44942 \quad 2.85386 \quad 1.91696$ $1.442912 .84496 \quad 1.91632$ 1.436432 .836111 .91568 $1.43000 \quad 2.82731 \quad 1.91503$ 1.423622 .818541 .91438

$1.41727 \quad 2.80982 \quad 1.91373$ $1.41096 \quad 2.80115 \quad 1.91308$ $1.40468 \quad 2.79251 \quad 1.91242$ 1.398452 .783921 .91176 $1.39226 \quad 2.77537 \quad 1.91110$

$\begin{array}{llll}1.38610 & 2.76685 & 1.91043\end{array}$ $1.37999 \quad 2.75838 \quad 1.90977$ $1.37390 \quad 2.74995 \quad 1.90910$ $1.36786 \quad 2.74156 \quad 1.90842$ $1.36185 \quad 2.73321 \quad 1.90775$

$\begin{array}{llll}1.35588 & 2.72490 & 1.90707\end{array}$ $1.34995 \quad 2.71663 \quad 1.90639$ $1.344052 .70840 \quad 1.90571$ 1.338192 .700201 .90502 $1.33236 \quad 2.692041 .90433$

$1.326572 .68392 \quad 1.90364$ $1.320812 .67584 \quad 1.90295$ $1.31508 \quad 2.66780 \quad 1.90225$ $1.309392 .65979 \quad 1.90155$ $1.303742 .65182 \quad 1.90085$

$1.298112 .64388 \quad 1.90014$ $1.29252 \quad 2.63598 \quad 1.89944$ 1.286962 .628121 .89873 $1.281442 .62029 \quad 1.89801$ $1.275942 .61250 \quad 1.89730$

$1.27048 \quad 2.60474 \quad 1.89658$ $1.265052 .59702 \quad 1.89586$ $1.259652 .58933 \quad 1.89514$ $1.25428 \quad 2.58167 \quad 1.89441$ $1.24895 \quad 2.57405 \quad 1.89368$

$1.243642 .56646 \quad 1.89295$ $1.238362 .55890 \quad 1.89222$ \begin{tabular}{l}
$1.233122 .55138 \quad 1.89148$ \\
\hline
\end{tabular} $\begin{array}{llll}1.22790 & 2.54389 & 1.89075\end{array}$ 1.222722 .536441 .89000

$\begin{array}{lll}1.21756 & 2.52901 & 1.88926\end{array}$ $1.21243 \quad 2.52162 \quad 1.88851$ $\begin{array}{llll}1.20733 & 2.51426 & 1.88777\end{array}$ 1.202262 .506931 .88701 $1.19722 \quad 2.499631 .88626$
$1.512542 .93946 \quad 1.92283$ $1.50576 \quad 2.93032 \quad 1.92223$ 1.499032 .921241 .92163 1.492352 .912191 .92102 $1.48570 \quad 2.90320 \quad 1.92041$

$1.47910 \quad 2.89425 \quad 1.91980$ 1.472542 .885341 .91918 $1.466022 .87648 \quad 1.91856$ $1.459542 .86766 \quad 1.91794$ $1.453102 .85888 \quad 1.91732$

$1.44670 \quad 2.85015 \quad 1.91670$ $1.440342 .84146 \quad 1.91607$ $1.43402 \quad 2.83281 \quad 1.91544$ 1.427742 .824201 .91480 1.421492 .815631 .91417

$1.41529 \quad 2.807111 .91353$ 1.409122 .798621 .91289 1.402992 .790181 .91224 1.396902 .781781 .91160 1.390842 .773411 .91095

1.384832 .765091 .91030 1.378842 .756801 .90964 $1.37290 \quad 2.74855 \quad 1.90899$ 1. $366982.74034 \quad 1.90833$ $1.361112 .73217 \quad 1.90766$

$1.35526 \quad 2.724041 .90700$ $1.34946 \quad 2.71594 \quad 1.90633$ $\begin{array}{llll}1.34368 & 2.70788 & 1.90566\end{array}$ $1.337942 .69986 \quad 1.90499$ $1.332242 .69187 \quad 1.90432$

$1.32657 \quad 2.68392 \quad 1.90364$ 1.320932 .676011 .90296 1.315322 .668131 .90228 1.309752 .660291 .90159 $1.304212 .65248 \quad 1.90091$

$1.29870 \quad 2.64471 \quad 1.90022$ $1.29322 \quad 2.63697 \quad 1.89952$ $1.287772 .62926 \quad 1.89883$ $1.28236 \quad 2.62159 \quad 1.89813$ $1.276972 .61396 \quad 1.89743$

1.271622 .606351 .89673 $\begin{array}{llll}1.26629 & 2.59878 & 1.89603\end{array}$ $1.26100 \quad 2.59125 \quad 1.89532$ $1.255742 .58374 \quad 1.89461$ $1.250502 .57627 \quad 1.89390$

$1.24530 \quad 2.568831 .89318$ 1.240122 .561421 .89246 $1.234972 .55404 \quad 1.89174$ $1.229852 .54670 \quad 1.89102$ 1.224772 .539381 .89030
$1.54206 \quad 2.97913 \quad 1.92538$ $1.53524 \quad 2.96998 \quad 1.92481$ $1.52846 \quad 2.96089 \quad 1.92422$ 1.521732 .951831 .92364 1.515042 .942821 .92305

$\begin{array}{llll}1.50838 & 2.93386 & 1.92247\end{array}$ 1.501772 .924941 .92187 $1.495212 .91606 \quad 1.92128$ $\begin{array}{lll}1.48868 & 2.90723 & 1.92068\end{array}$ $1.482192 .89844 \quad 1.92008$

$\begin{array}{llll}1.47575 & 2.88970 & 1.91948\end{array}$ $1.469342 .88099 \quad 1.91888$ 1.462972 .872331 .91827 $1.456642 .86371 \quad 1.91766$ $1.45035 \quad 2.855131 .91705$

$1.44410 \quad 2.84659 \quad 1.91644$ $1.43788 \quad 2.83810 \quad 1.91582$ $1.431712 .82964 \quad 1.91520$ 1.425572 .821221 .91458 $1.41946 \quad 2.81284 \quad 1.91396$

$1.41340 \quad 2.80451 \quad 1.91333$ $1.407372 .79621 \quad 1.91270$ $1.401372 .78795 \quad 1.91207$ 1.395412 .779721 .91144 $1.389492 .77154 \quad 1.91080$

$\begin{array}{llll}1.38360 & 2.76339 & 1.91016\end{array}$ $1.37775 \quad 2.75528 \quad 1.90952$ $1.371932 .74721 \quad 1.90888$ $1.366142 .73917 \quad 1.90823$ $1.360392 .73117 \quad 1.90758$

$1.354672 .72321 \quad 1.90693$ $1.34898 \quad 2.71528 \quad 1.90628$ 1.343332 .707391 .90562 1.337712 .699531 .90496 $1.33212 \quad 2.69171 \quad 1.90430$

$1.326572 .68392 \quad 1.90364$ $1.321042 .67617 \quad 1.90297$ $1.315552 .66845 \quad 1.90231$ $1.310092 .66077 \quad 1.90164$ 1.304662 .653121 .90096

$1.29926 \quad 2.64550 \quad 1.90029$ $\begin{array}{llll}1.29389 & 2.63791 & 1.89961\end{array}$ $1.288552 .63036 \quad 1.89893$ $1.28324 \quad 2.62284 \quad 1.89825$ $1.27796 \quad 2.61536 \quad 1.89756$

$\begin{array}{llll}1.27271 & 2.60790 \quad 1.89687\end{array}$ $1.267492 .60048 \quad 1.89618$ $1.262292 .59309 \quad 1.89549$ $1.257132 .58573 \quad 1.89480$ $1.251992 .57840 \quad 1.89410$
2100

2110

2120

2130

2140

2150

2160

2170

2180

2190

2200

2210

2220

2230

2240

2250

2260

2270

2280

2290

2300

2310

2320

2330

2340

2350

2360

2370

2380

2390

2400

2410

2420

2430

2440

2450

2460

2470

2480

2490

2500

2510

2520

2530

2540

2550

2560

2570

2580

2590 
Table II Harmonic Oscillator Contributions to the Thermodynamic

Functions (in units of calories, moles, and ${ }^{\circ} \mathrm{K}$ )
$\mathrm{cm}^{-1} \quad-\left(\mathrm{F}^{\circ}-\mathrm{E}_{0}^{\circ}\right) / \mathrm{T}$
So
$\stackrel{\mathrm{C}}{\mathrm{p}}$
$-\left(F^{\circ}-E_{0}^{O}\right) / T \quad S^{\circ}$
$\mathrm{c}_{\mathrm{p}}^{\circ}$
$-\left(F^{\circ}-E_{O}^{\circ}\right) / T$
So

$\nu$

$\mathrm{cm}^{-1}$

$\mathrm{T}=4700$.

2600

2610

2620

2630

2640

2650

2660

2670

2680

2690

2700

2710

2720

2730

2740

2750

2760

2770

2780

2790

2800

2810

2820

2830

2840

2850

2860

2870

2880

2890

2900

2910

2920

2930

2940

2950

2960

2970

2980

2990

3000

3010

3020

3030

3040

3050

3060

3070

3080

3090
$1.192202 .49236 \quad 1.88550$

$1.187212 .48512 \quad 1.88474$

1.182262 .477921 .88398

$1.17732 \quad 2.47074 \quad 1.88322$

$1.17242 \quad 2.46360 \quad 1.88245$

$1.167542 .45648 \quad 1.88168$ $1.162692 .44940 \quad 1.88091$ $1.157872 .44234 \quad 1.88014$ 1.153072 .435311 .87936 $1.14830 \quad 2.42831 \quad 1.87858$

$1.14355 \quad 2.42135 \quad 1.87780$ $1.138832 .41440 \quad 1.87702$ $1.13414 \quad 2.40749 \quad 1.87623$ $1.129472 .40061 \quad 1.87544$ $1.12483 \quad 2.39375 \quad 1.87465$

$1.120212 .38693 \quad 1.87385$ $\begin{array}{llll}1.11561 & 2.38013 & 1.87306\end{array}$ $1.11105 \quad 2.37335 \quad 1.87226$ $1.106502 .36661 \quad 1.87146$ $1.10198 \quad 2.35989 \quad 1.87065$

$1.09748 \quad 2.35320 \quad 1.86984$ $\begin{array}{llll}1.09301 & 2.34653 & 1.86904\end{array}$ $1.088562 .33989 \quad 1.86822$ $\begin{array}{llll}1.08414 & 2.33328 & 1.86741\end{array}$ $1.07973 \quad 2.32670 \quad 1.86659$

$\begin{array}{llll}1.07535 & 2.32014 \quad 1.86577\end{array}$ $1.07100 \quad 2.31360 \quad 1.86495$ 1.066662 .307101 .86413 $1.062352 .30061 \quad 1.86330$ $1.05807 \quad 2.29416 \quad 1.86247$

$1.05380 \quad 2.28772 \quad 1.86164$ $1.049562 .28132 \quad 1.86081$ $1.04533 \quad 2.27494 \quad 1.85997$ $1.04113 \quad 2.26858 \quad 1.85914$ $1.036952 .26224 \quad 1.85829$

$\begin{array}{llll}1.03280 & 2.25594 & 1.85745\end{array}$ $1.02866 \quad 2.249651 .85661$ 1.024552 .243391 .85576 $1.020452 .23716 \quad 1.85491$ $1.016382 .23094 \quad 1.85405$

$1.01233 \quad 2.22475 \quad 1.85320$ $1.00830 \quad 2.21859 \quad 1.85234$ 1.004292 .212451 .85148 $1.00030 \quad 2.20633 \quad 1.85062$ $0.996332 .20023 \quad 1.84975$

$0.99238 \quad 2.19416 \quad 1.84889$ $0.98845 \quad 2.18811 \quad 1.84802$ $0.984542 .18208 \quad 1.84715$ $0.980652 .17607 \quad 1.84627$ $0.97677 \quad 2.17009 \quad 1.84540$

$$
\mathrm{T}=4800 \text {. }
$$

$\begin{array}{llll}1.21970 & 2.53210 \quad 1.88957\end{array}$

$1.21467 \quad 2.52485 \quad 1.88884$

1.209662 .517631 .88811

$1.204692 .51044 \quad 1.88737$

$1.19973 \quad 2.50327 \quad 1.88664$

$1.194812 .49614 \quad 1.88590$ $1.189912 .48904 \quad 1.88516$ $1.18504 \quad 2.48197 \quad 1.88441$ $1.18020 \quad 2.47493 \quad 1.88367$ $\begin{array}{llll}1.17538 & 2.46791 & 1.88292\end{array}$

1.170592 .460931 .88216 $1.16582 \quad 2.45397 \quad 1.88141$ $1.16108 \quad 2.44704 \quad 1.88065$ 1.156372 .440141 .87990 $1.15168 \quad 2.43327 \quad 1.87913$

$1.14701 \quad 2.42642 \quad 1.87837$ $1.142372 .41961 \quad 1.87760$ $1.13776 \quad 2.41282 \quad 1.87684$ $1.13316 \quad 2.40606 \quad 1.87606$ $1.128602 .39932 \quad 1.87529$

$1.12406 \quad 2.39261 \quad 1.87452$ $1.11954 \quad 2.38593 \quad 1.87374$ $1.115042 .37928 \quad 1.87296$ $\begin{array}{llll}1.11057 & 2.37265 & 1.87217\end{array}$ $1.10612 \quad 2.36605 \quad 1.87139$

$1.10170 \quad 2.35947 \quad 1.87060$ $1.097302 .35292 \quad 1.86981$ $1.09292 \quad 2.34639 \quad 1.86902$ $1.088562 .33939 \quad 1.86822$ $1.084232 .33342 \quad 1.86743$

$\begin{array}{llll}1.07992 & 2.32697 & 1.86663\end{array}$ $\begin{array}{llll}1.07563 & 2.32055 & 1.86583\end{array}$ $1.07136 \quad 2.31415 \quad 1.86502$ $1.067112 .30777 \quad 1.86422$ 1.062892 .301421 .86341

$1.058692 .29510 \quad 1.86260$ $\begin{array}{llll}1.05451 & 2.28879 & 1.86178\end{array}$ $1.050352 .28252 \quad 1.86097$ $1.046212 .27626 \quad 1.86015$ $1.042092 .27003 \quad 1.85933$

$\begin{array}{llll}1.03800 & 2.26383 & 1.85850\end{array}$ $1.03392 \quad 2.25764 \quad 1.85768$ $1.02987 \quad 2.25148 \quad 1.85685$ $1.02583 \quad 2.24535 \quad 1.85602$ $1.02182 \quad 2.239231 .85519$

$1.01782 \quad 2.23314 \quad 1.85436$ $1.01385 \quad 2.22707 \quad 1.85352$ $1.009892 .22103 \quad 1.85268$ $1.00596 \quad 2.21500 \quad 1.85184$ 1.002042 .209001 .85100
$\Gamma=4900$.

$1.246892 .57110 \quad 1.89340$

$1.24181 \quad 2.56384 \quad 1.89270$

$1.23676 \quad 2.53660 \quad 1.89199$

1.231732 .549391 .89129

1.226742 .542221 .89058

$\begin{array}{llll}1.22177 & 2.53507 & 1.88987\end{array}$

1.216822 .527951 .88915

1.211912 .520871 .88944

$1.207022 .51391 \quad 1.88772$

$1.20216 \quad 2.50678 \quad 1.88700$

$1.19732 \quad 2.49978 \quad 1.88628$ $\begin{array}{llll}1.19251 & 2.49281 & 1.88555\end{array}$ $1.18772 \quad 2.48586 \quad 1.88482$ $1.18296 \quad 2.47895 \quad 1.88409$ $1.17823 \quad 2.47206 \quad 1.88336$

$1.17352 \quad 2.46520 \quad 1.88262$ 1.168832 .458371 .88189 $1.164172 .45156 \quad 1.88115$ $1.159542 .44478 \quad 1.88041$ $1.15493 \quad 2.43803 \quad 1.87966$

$1.150342 .43131 \quad 1.87892$ $1.14578 \quad 2.42461 \quad 1.87817$ $1.141242 .41794 \quad 1.87742$ 1.136722 .411301 .87666 1.132232 .404681 .87591

$\begin{array}{llll}1.12776 & 2.39809 & 1.87515\end{array}$ $1.12332 \quad 2.391521 .87439$ $\begin{array}{lll}1.11889 & 2.38498 & 1.87363\end{array}$ $\begin{array}{llll}1.11449 & 2.37846 & 1.87286\end{array}$ 1.110122 .371971 .87209

$1.10576 \quad 2.36551 \quad 1.87132$ $1.101432 .35907 \quad 1.87055$ 1.097122 .352651 .86978 $1.092832 .34626 \quad 1.86900$ $1.08856 \quad 2.33989 \quad 1.86822$

$1.08432 \quad 2.33355 \quad 1.86744$ 1.080092 .327231 .86666 $1.075892 .32094 \quad 1.86588$ 1.071712 .314671 .86509 $1.067552 .30842 \quad 1.86430$

$1.06341 \quad 2.30220 \quad 1.86351$ $1.059292 .29600 \quad 1.86271$ 1.055192 .289821 .86191 $1.051112 .28367 \quad 1.86112$ $1.04705 \quad 2.27754 \quad 1.86032$

$1.04302 \quad 2.27143 \quad 1.85951$ $1.03900 \quad 2.26534 \quad 1.85871$ $1.03500 \quad 2.25928 \quad 1.85790$ $1.03102 \quad 2.25324 \quad 1.85709$ $1.02706 \quad 2.24722 \quad 1.85628$
2600

2610

2620

2630

2640

2650

2660

2670

2680

2690

2700

2710

2720

2730

2740

2750

2760

2770

2780

2790

2800

2810

2820

2830

2840

2850

2860

2870

2880

2890

2900

2910

2920

2930

2940

2950

2960

2970

2980

2990

3000

3010

3020

3030

3040

3050

3060

3070

3080

3090 
Table II Harmonic Oscillator Contributions to the Thermodynamic Functions (in units of calories, moles, and ${ }^{\circ} \mathrm{K}$ )
$\nu_{-1}^{-1}-(\mathrm{F} O-E \mathrm{O}) / \mathrm{T} \quad \mathrm{S}$
$\mathrm{co}_{\mathrm{p}}$
$-\left(\mathrm{F}^{\circ}-\mathrm{E}_{\mathrm{O}}\right) / \mathrm{T}$
$S^{\circ}$
$\mathrm{C}_{\mathrm{p}}^{\circ}$
$-\left(F^{\circ}-E_{0}^{0}\right) / T$
So

$T=4700$.

$3100 \quad 0.97292 \quad 2.16413 \quad 1.84452$

$\begin{array}{lllll}3110 & 0.96909 & 2.15819 & 1.84364\end{array}$

$\begin{array}{lllll}3120 & 0.96527 & 2.15227 & 1.84275\end{array}$

$\begin{array}{lllll}3130 & 0.96148 & 2.14638 & 1.84187\end{array}$

$3140 \quad 0.95770 \quad 2.14050 \quad 1.84098$

$\begin{array}{llll}3150 & 0.95395 & 2.13465 & 1.84009\end{array}$

$\begin{array}{lllll}3160 & 0.95021 & 2.12882 & 1.83920\end{array}$

$\begin{array}{lllll}3170 & 0.94649 & 2.12301 & 1.83830\end{array}$

$\begin{array}{lllll}3180 & 0.94278 & 2.11722 & 1.83740\end{array}$

$\begin{array}{llll}3190 & 0.93910 & 2.11145 \quad 1.83650\end{array}$

$\begin{array}{lllll}3200 & 0.93543 & 2.10571 & 1.83560\end{array}$

$\begin{array}{lllll}3210 & 0.93179 & 2.09998 & 1.83470\end{array}$

$\begin{array}{lllll}3220 & 0.92816 & 2.09428 & 1.83379\end{array}$

$\begin{array}{lllll}3230 & 0.92454 & 2.08859 & 1.83288\end{array}$

$\begin{array}{lllll}3240 & 0.92095 & 2.08293 & 1.83197\end{array}$

$\begin{array}{lllll}3250 & 0.91737 & 2.07728 & 1.83106\end{array}$

$\begin{array}{lllll}3260 & 0.91381 & 2.07166 & 1.83014\end{array}$

$\begin{array}{lllll}3270 & 0.91027 & 2.06606 & 1.82922\end{array}$

$\begin{array}{llllll}3280 & 0.90674 & 2.06047 & 1.82830\end{array}$

$\begin{array}{lllll}3290 & 0.90323 & 2.05491 & 1.82738\end{array}$

$\begin{array}{lllll}3300 & 0.89974 & 2.04936 & 1.82646\end{array}$

$\begin{array}{lllll}3310 & 0.89626 & 2.04384 & 1.82553\end{array}$

$\begin{array}{lllll}3320 & 0.89281 & 2.03833 & 1.82460\end{array}$

$\begin{array}{lllll}3330 & 0.88936 & 2.03285 & 1.82367\end{array}$

$\begin{array}{lllll}3340 & 0.88594 & 2.02738 & 1.82274\end{array}$

$\begin{array}{llll}3350 & 0.88253 & 2.02193 \quad 1.82180\end{array}$

$\begin{array}{lllll}3360 & 0.87914 & 2.01650 & 1.82086\end{array}$

$\begin{array}{llll}3370 & 0.87576 & 2.01109 & 1.81992\end{array}$

$\begin{array}{lllll}3380 & 0.87240 & 2.00570 \quad 1.81898\end{array}$

$3390 \quad 0.869052 .00033 \quad 1.81803$

$\begin{array}{llll}3400 \quad 0.86572 & 1.99498 & 1.81709\end{array}$

$\begin{array}{llll}3410 & 0.86241 & 1.98964 & 1.81614\end{array}$

$\begin{array}{lllll}3420 & 0.85911 & 1.98432 & 1.81519\end{array}$

$\begin{array}{lllll}3430 & 0.85583 & 1.97903 & 1.81423\end{array}$

$\begin{array}{llll}3440 & 0.85256 & 1.97375 & 1.81328\end{array}$

$\begin{array}{lllll}3450 & 0.84931 & 1.96848 & 1.81232\end{array}$

$3460 \quad 0.84607 \quad 1.963241 .81136$

$3470 \quad 0.842851 .95801 \quad 1.81040$

$\begin{array}{lllll}3480 & 0.83965 & 1.95280 & 1.80943\end{array}$

$\begin{array}{llllll}3490 & 0.83646 & 1.94761 & 1.80847\end{array}$

$\begin{array}{lllll}3500 & 0.83328 & 1.94244 & 1.80750\end{array}$

$\begin{array}{lllll}3510 & 0.83012 & 1.93729 & 1.80653\end{array}$

$\begin{array}{lllll}3520 & 0.82697 & 1.93215 & 1.80555\end{array}$

$3530 \quad 0.823841 .927031 .80458$

$\begin{array}{llll}3540 & 0.82072 & 1.92192 \quad 1.80360\end{array}$

3550

3560

3570

3580

3590
$0.81762 \quad 1.91684 \quad 1.80262$ 0.814531 .911771 .80164 0.811451 .906711 .80066 0.808391 .901681 .79967 0.805341 .896661 .79868

$T=4800$

$T=4900$.

0.998152 .203021 .85015 0.994272 .197061 .84930 $0.990412 .19113 \quad 1.84845$ 0.986572 .185221 .84760 $0.98275 \quad 2.17932 \quad 1.84675$

$0.97895 \quad 2.17345 \quad 1.84589$ 0.975172 .167601 .84503 $0.97140 \quad 2.16177 \quad 1.84417$ $0.96766 \quad 2.15597 \quad 1.84330$ $0.96393 \quad 2.15018 \quad 1.84244$

$0.96022 \quad 2.14442 \quad 1.84157$ $0.956532 .13867 \quad 1.84070$ $0.952852 .13295 \quad 1.83983$ $0.94920 \quad 2.12724 \quad 1.83895$ $0.94556 \quad 2.12156 \quad 1.83808$

$0.941942 .11590 \quad 1.83720$ $0.938332 .11026 \quad 1.83632$ 0.934752 .104631 .83543 0.931182 .099031 .83455 0.927632 .093451 .83366

$\begin{array}{llll}0.92409 & 2.08788 & 1.83277\end{array}$ $0.920572 .08234 \quad 1.83188$ $0.917072 .07681 \quad 1.83098$ $0.913592 .07131 \quad 1.83008$ 0.910122 .065821 .82919

$0.906672 .06036 \quad 1.82828$ $0.90323 \quad 2.05491 \quad 1.82738$ $\begin{array}{llll}0.89981 & 2.04948 & 1.82648\end{array}$ $0.89641 \quad 2.04407 \quad 1.82557$ 0.893022 .038681 .82466

$0.88965 \quad 2.03330 \quad 1.82375$ 0.886292 .027951 .82283 $0.88295 \quad 2.02261 \quad 1.82192$ 0.879632 .017291 .82100 0.876322 .011991 .82008

$0.87303 \quad 2.00671 \quad 1.81916$ $0.86975 \quad 2.001451 .81823$ $0.86648 \quad 1.99620 \quad 1.81730$ 0.863241 .990971 .81638 $0.86000 \quad 1.98576 \quad 1.81544$

$0.85679 \quad 1.98057 \quad 1.81451$ $0.85358 \quad 1.97539 \quad 1.81358$ $0.850391 .97024 \quad 1.81264$ 0.847221 .965091 .81170 $0.84406 \quad 1.95997 \quad 1.81076$

$0.84091 \quad 1.95486 \quad 1.80982$ $0.83778 \quad 1.94977 \quad 1.80887$ $0.83467 \quad 1.94470 \quad 1.80792$ 0.831571 .939651 .80697 $0.82848 \quad 1.93461 \quad 1.80602$
$1.02313 \quad 2.241231 .85546$ $1.019212 .23525 \quad 1.85465$ $1.015312 .22930 \quad 1.85383$ $1.01142 \quad 2.22337 \quad 1.85301$ $1.00756 \quad 2.21746 \quad 1.85218$

$1.00372 \quad 2.21157 \quad 1.85136$ $0.99989 \quad 2.20570 \quad 1.85053$ $0.996092 .19986 \quad 1.84970$ $0.99230 \quad 2.19403 \quad 1.84887$ $0.98853 \quad 2.18823 \quad 1.84804$

$0.98478 \quad 2.18245 \quad 1.84720$ 0.981042 .176681 .84636 $0.977332 .17094 \quad 1.84552$ 0.973632 .165221 .84468 $0.96995 \quad 2.15952 \quad 1.84393$

$0.966292 .15384 \quad 1.84299$ $0.962642 .14818 \quad 1.84214$ $0.959012 .14254 \quad 1.84129$ $0.95540 \quad 2.13692 \quad 1.84043$ 0.951812 .131321 .83958

$0.948232 .12573 \quad 1.83872$ $0.944672 .12017 \quad 1.83786$ $0.941132 .11463 \quad 1.83700$ $0.93760 \quad 2.10911 \quad 1.83614$ $0.93409 \quad 2.10360 \quad 1.83527$

$\begin{array}{llll}0.93060 \quad 2.09812 & 1.83440\end{array}$ 0.927122 .092651 .83353 $0.92366 \quad 2.08720 \quad 1.83266$ $0.920222 .08177 \quad 1.83178$ $0.916792 .07636 \quad 1.83091$

$0.91338 \quad 2.07097 \quad 1.83003$ $0.90998 \quad 2.06560 \quad 1.82915$ $0.90660 \quad 2.06024 \quad 1.82827$ $0.903232 .05491 \quad 1.82738$ $\begin{array}{llll}0.89988 & 2.04959 & 1.82649\end{array}$

$0.89655 \quad 2.04429 \quad 1.82561$ $0.893232 .03901 \quad 1.82471$ $0.88992 \quad 2.03374 \quad 1.82382$ $0.886642 .02849 \quad 1.82293$ $\begin{array}{llll}0.88336 & 2.02326 & 1.82203\end{array}$

$0.88010 \quad 2.01805 \quad 1.82113$ $0.876862 .01286 \quad 1.82023$ $\begin{array}{llll}0.87363 & 2.00768 & 1.81933\end{array}$ 0.870422 .002521 .81842 $\begin{array}{llll}0.86722 & 1.99738 & 1.81751\end{array}$

0.864031 .992251 .81660 $0.86086 \quad 1.98714 \quad 1.81569$ $0.85770 \quad 1.982051 .81478$ $0.854561 .97698 \quad 1.81386$ $0.851431 .97192 \quad 1.81295$
3100

3110

3120

3130

3140

3150

3160

3170

3180

3190

3200

3210

3220

3230

3240

3250

3260

3270

3280

3290

3300

3310

3320

3330

3340

3350

3360

3370

3380

3390

3400

3410

3420

3430

3440

3450

3460

3470

3480

3490

3500

3510

3520

3530

3540

3550

3560

3570

3580

3590 
Table II Harmonic Oscillator Contributions to the Thermodynamic

Functions (in units of calories, moles, and ${ }^{\circ} \mathrm{K}$ )

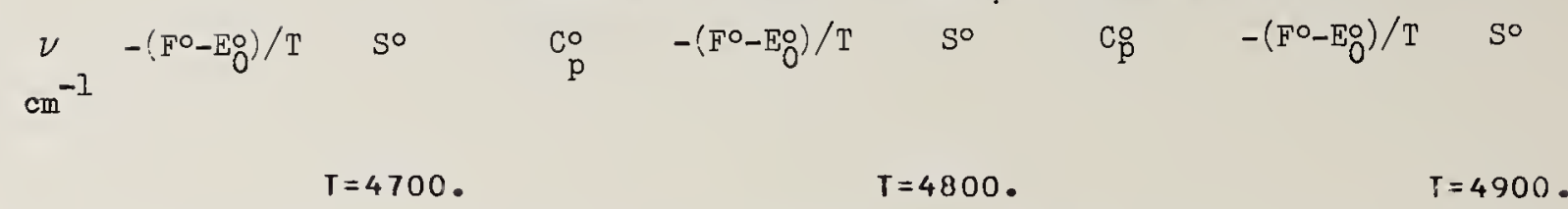

$\begin{array}{lllll}3600 & 0.80231 & 1.89166 & 1.79769\end{array}$

$\begin{array}{lllll}3610 & 0.79929 & 1.88667 & 1.79670\end{array}$

$\begin{array}{lllll}3620 & 0.79629 & 1.88170 & 1.79571\end{array}$

$\begin{array}{lllll}3630 & 0.79330 & 1.87675 & 1.79471\end{array}$

$\begin{array}{llllll}3640 & 0.79032 & 1.87182 & 1.79371\end{array}$

$\begin{array}{lllll}3650 & 0.78735 & 1.86690 & 1.79271\end{array}$

$\begin{array}{lllll}3660 & 0.78440 & 1.86199 & 1.79171\end{array}$

$\begin{array}{llllll}3670 & 0.78147 & 1.85711 & 1.79071\end{array}$

$\begin{array}{lllll}3680 & 0.77854 & 1.85223 & 1.78970\end{array}$

$\begin{array}{lllll}3690 & 0.77563 & 1.84738 & 1.78869\end{array}$

$\begin{array}{lllll}3700 & 0.77273 & 1.84254 & 1.78768\end{array}$

$\begin{array}{lllll}3710 & 0.76985 & 1.83772 & 1.78667\end{array}$

$\begin{array}{lllll}3720 & 0.76698 & 1.83291 & 1.78565\end{array}$

$\begin{array}{lllll}3730 & 0.76412 & 1.82812 & 1.78464\end{array}$

$\begin{array}{lllll}3740 & 0.76127 & 1.82334 & 1.78362\end{array}$

$\begin{array}{llll}3750 & 0.75844 & 1.81858 & 1.78260\end{array}$

$\begin{array}{llll}3760 & 0.75562 & 1.81383 \quad 1.78157\end{array}$

$\begin{array}{lllll}3770 & 0.75281 & 1.80910 & 1.78055\end{array}$

$\begin{array}{lllll}3780 & 0.75001 & 1.80439 & 1.77952\end{array}$

$\begin{array}{lllll}3790 & 0.74723 & 1.79969 & 1.77849\end{array}$

3800

3810

3820

3830

3840

3850

3860

3870

3880

3890

3900

3910

3920

3930

3940

3950

3960

3970

3980

3990

4000

$0.74446 \quad 1.79500 \quad 1.77746$ 0.741701 .790331 .77643 $0.73895 \quad 1.785681 .77540$ $0.736221 .78104 \quad 1.77436$ $0.73350 \quad 1.77641 \quad 1.77332$

$0.730791 .77180 \quad 1.77228$ 0.728091 .767201 .77124 0.725401 .762621 .77019 $0.72273 \quad 1.75806 \quad 1.76914$ 0.720071 .753501 .76810

$0.71742 \quad 1.74896 \quad 1.76704$ $0.71478 \quad 1.74444 \quad 1.76599$ $0.712151 .73993 \quad 1.76494$ $0.70953 \quad 1.73544 \quad 1.76388$ $0.70693 \quad 1.73095 \quad 1.76282$

0.704331 .726491 .76176 $0.70175 \quad 1.72203 \quad 1.76070$ $0.699181 .71760 \quad 1.75964$ 0.696621 .713171 .75857 $0.69407 \quad 1.70876 \quad 1.75750$

$0.69154 \quad 1.70436 \quad 1.75643$ $\begin{array}{llll}0.82540 & 1.92958 & 1.80507\end{array}$ $0.822341 .92458 \quad 1.80411$ $0.81930 \quad 1.91959 \quad 1.80315$ 0.816261 .914621 .80219 $0.813241 .90966 \quad 1.80123$

$0.81024 \quad 1.90472 \quad 1.80027$ $0.80725 \quad 1.89980 \quad 1.79930$

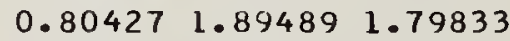
$0.801301 .89000 \quad 1.79736$ 0.798351 .885121 .79639

$\begin{array}{llll}0.79541 & 1.88026 & 1.79542\end{array}$ $0.792491 .87541 \quad 1.79444$ 0.789581 .870591 .79346 $0.78668 \quad 1.86577 \quad 1.79248$ $0.78379 \quad 1.86097 \quad 1.79150$

$0.78092 \quad 1.836191 .79052$ 0.778051 .851421 .78953 $0.77521 \quad 1.84667 \quad 1.78854$ $0.77237 \quad 1.84194 \quad 1.78756$ 0.769551 .837211 .78656 $\begin{array}{llll}0.76674 & 1.83251 & 1.78557\end{array}$

$0.76394 \quad 1.82782 \quad 1.78457$

$0.76115 \quad 1.82314 \quad 1.78358$

0.758381 .818481 .78258

0.755621 .813831 .78157

$\begin{array}{llll}0.75287 & 1.80920 & 1.78057\end{array}$ 0.750131 .804581 .77957 $0.74740 \quad 1.79998 \quad 1.77856$ 0.744691 .795391 .77755 $0.74199 \quad 1.79082 \quad 1.77654$

$0.73930 \quad 1.78626 \quad 1.77552$ $\begin{array}{llll}0.73662 & 1.78171 & 1.77451\end{array}$ $0.73395 \quad 1.77718 \quad 1.77349$ $0.73129 \quad 1.77266 \quad 1.77247$ 0.728651 .768161 .77145

$0.72602 \quad 1.76367 \quad 1.77043$ $0.72340 \quad 1.75920 \quad 1.76941$ $0.720791 .75473 \quad 1.76838$ $0.718191 .75029 \quad 1.76735$ $0.715601 .74585 \quad 1.76632$

$0.71302 \quad 1.741431 .76529$
$0.84832 \quad 1.96688 \quad 1.81203$

0.845221 .961851 .81110

0.842131 .956841 .81018

0.839061 .951851 .80926

$0.836001 .94687 \quad 1.80833$

$0.83296 \quad 1.94191 \quad 1.80740$ $0.829921 .93697 \quad 1.80647$ $0.82691 \quad 1.93204 \quad 1.80553$ 0.823901 .927131 .80460 $0.82091 \quad 1.922231 .80366$

$0.81793 \quad 1.91735 \quad 1.80272$

$0.81497 \quad 1.91249 \quad 1.80178$

$0.812021 .90764 \quad 1.80084$

$0.80908 \quad 1.90281 \quad 1.79989$

$0.806151 .89799 \quad 1.79895$

$0.80324 \quad 1.89319 \quad 1.79800$ $0.800341 .38840 \quad 1.79705$ 0.797451 .883631 .79609 $0.79458 \quad 1.87887 \quad 1.79514$ $0.79171 \quad 1.074131 .79418$

$0.78886 \quad 1.86941 \quad 1.79322$ 0.786031 .864691 .79226 $0.783201 .86000 \quad 1.79130$ $0.780391 .85531 \quad 1.79034$ 0.777591 .850651 .78937

$0.77480 \quad 1.84600 \quad 1.78840$ $0.77202 \quad 1.84136 \quad 1.78743$ $0.76926 \quad 1.83673 \quad 1.78646$ 0.766511 .832121 .78549 $0.76377 \quad 1.82753 \quad 1.78451$

$0.76104 \quad 1.82295 \quad 1.78354$ 0.758321 .818381 .78256 0.755621 .813831 .78157 $0.75292 \quad 1.809291 .78059$ $0.75024 \quad 1.80477 \quad 1.77961$

$0.74757 \quad 1.80026 \quad 1.77862$ $0.74491 \quad 1.79576 \quad 1.77763$ 0.742261 .791281 .77664 0.739631 .786811 .77565 $0.73700 \quad 1.78236 \quad 1.77465$

$0.73439 \quad 1.77792 \quad 1.77366$ $\nu$ $\mathrm{cm}^{-1}$

3600

3610

3620

3630

3640

3650

3660

3670

3680

3690

3700

3710

3720

3730

3740

3750

3760

3770

3780

3790

3800

3810

3820

3830

3840

3850

3860

3870

3880

3890

3900

3910

3920

3930

3940

3950

3960

3970

3980

3990

4000 
Table II Harmonic Oscillator Contributions to the Thermodynamic

Functions (in units of calories, moles, and ${ }^{\circ} \mathrm{K}$ )
$\mathrm{cm}^{-1}-\left(\mathrm{F}^{\circ}-\mathrm{E}_{0}^{\mathrm{o}}\right) / \mathrm{T}$
so
$T=5000$.

$\begin{array}{ccccc}\nu & -\left(F^{\circ}-E_{0}^{\circ}\right) / T & \text { So } & \mathrm{C}_{\mathrm{p}}^{\circ} & \nu \\ \mathrm{cm}^{-1} & & & & \mathrm{~cm}^{-1}\end{array}$

$T=5000$.

600

100

$7.079439 .03814 \quad 1.98703$

110

$6.722818 .67587 \quad 1.98697 \quad 120$

$6.566598 .51683 \quad 1.98694 \quad 130$

$6.422178 .36958 \quad 1.98690 \quad 140$

$\begin{array}{llll}6.28791 & 8.23250 \quad 1.98686 & 150\end{array}$

160

170

$6.044867 .98382 \quad 1.98677$

$5.934117 .87026 \quad 1.98673$

180

190

$\begin{array}{llll}5.73041 & 7.66095 & 1.98662 & 200 \\ 5.63629 & 7.56402 & 1.98657 & 210\end{array}$

$\begin{array}{lllll}5.54667 & 7.47161 & 1.98651 & 220\end{array}$

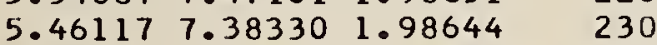

$\begin{array}{llll}5.37942 & 7.29876 & 1.98638 & 240\end{array}$

$\begin{array}{lllll}5.30113 & 7.21768 & 1.98631 & 250\end{array}$

$\begin{array}{lllll}5.22601 & 7.13977 & 1.98624 & 260\end{array}$

$\begin{array}{llll}5.15384 & 7.06481 & 1.98617 & 270\end{array}$

$\begin{array}{llll}5.08439 & 6.99258 & 1.98610 & 280\end{array}$

$\begin{array}{llll}5.01748 & 6.92289 & 1.98602 & 290\end{array}$

$\begin{array}{llll}4.95293 & 6.85556 & 1.98594 & 300\end{array}$

$4.890596 .79044 \quad 1.98585 \quad 310$

$\begin{array}{llll}4.83032 & 6.72740 & 1.98577 & 320\end{array}$

$\begin{array}{llll}4.77198 & 6.66629 & 1.98568 & 330\end{array}$

$\begin{array}{llll}4.71547 & 6.60702 & 1.98559 & 340\end{array}$

330

340

350

360

370

380

$4.66068 \quad 6.54946 \quad 1.98549$

$4.60751 \quad 6.49353 \quad 1.98539$

$\begin{array}{llll}4.55587 & 6.43913 & 1.98529\end{array}$

$4.50569 \quad 6.38619 \quad 1.98519$

$\begin{array}{llll}4.45688 & 6.33462 & 1.98509\end{array}$

$4.40937 \quad 6.28437 \quad 1.98498$

$4.36310 \quad 6.23535 \quad 1.98487$

$4.318026 .18753 \quad 1.98475$

$4.27406 \quad 6.14082 \quad 1.98464$

4.231186 .095201 .98452

$4.18932 \quad 6.05060 \quad 1.98440$

$4.14844 \quad 6.00699 \quad 1.98427$

$4.10850 \quad 5.96432 \quad 1.98414$

$4.06946 \quad 5.92255 \quad 1.98401$

$4.03127 \quad 5.88164 \quad 1.98388$

$3.99392 \quad 5.84156 \quad 1.98375$

500

$3.95736 \quad 5.80228 \quad 1.98361$

$3.92156 \quad 5.76376 \quad 1.98347$

$3.88650 \quad 5.72598 \quad 1.98332$

$3.852145 .68891 \quad 1.98318$

550

$3.81846 \quad 5.65252 \quad 1.98303$

$\begin{array}{llll}3.78544 & 5.61679 & 1.98288\end{array}$

$3.75305 \quad 5.58170 \quad 1.98272$

$3.721275 .54722 \quad 1.98256$

3.690075 .513331 .98240
350

360

370

380

390

400

410

420

430

440

450

460

470

480

490

500

510

520

530

540

550

560

570

580

590

\section{0 \\ 650 \\ 670 \\ 680 \\ 700 \\ 710 \\ 730 \\ 740 \\ 770 \\ 820 \\ 830 \\ 880 \\ 910 \\ 930 \\ 980}

620

630

640

660

690

720

750

760

780

790

800

810

840

850

860

870

890

900

920

940

950

960

970

990

1000

1010

1020

1030

1040

1050

1060

1070

1080

1090
$3.65945 \quad 5.48001 \quad 1.98224$

$3.62938 \quad 5.44725 \quad 1.98208$

$3.59985 \quad 5.41502 \quad 1.98191$

$3.57082 \quad 5.38331 \quad 1.98174$

$3.54230 \quad 5.35210 \quad 1.98156$

$3.51426 \quad 5.32138 \quad 1.98139$

3.486695 .291131 .98121

$3.45958 \quad 5.26134 \quad 1.98103$

$3.43290 \quad 5.23199 \quad 1.98084$

$3.406665 .20307 \quad 1.98065$

$3.38083 \quad 5.17458 \quad 1.98046$

$3.35541 \quad 5.14648 \quad 1.98027$

$3.330375 .11879 \quad 1.98008$

$3.305725 .09148 \quad 1.97988$

$3.28145 \quad 5.06454 \quad 1.97968$

$3.25753 \quad 5.03797 \quad 1.97947$

$3.23396 \quad 5.01175 \quad 1.97927$

$3.210744 .98588 \quad 1.97906$

$3.18785 \quad 4.96035 \quad 1.97885$

$3.165294 .93514 \quad 1.97863$

$3.14304 \quad 4.91025 \quad 1.97842$

$3.121114 .88568 \quad 1.97820$

$3.09947 \quad 4.86140 \quad 1.97798$

3.078134 .837431 .97775

$3.05708 \quad 4.81375 \quad 1.97752$

$3.03630 \quad 4.79034 \quad 1.97729$

$3.01580 \quad 4.76722 \quad 1.97706$

$2.99557 \quad 4.74436 \quad 1.97682$

$2.97560 \quad 4.72177 \quad 1.97658$

$2.95588 \quad 4.69944 \quad 1.97634$

$2.936424 .67736 \quad 1.97610$

$2.917194 .65552 \quad 1.97585$

2.898214 .633931 .97560

$2.87946 \quad 4.61258 \quad 1.97535$

$2.86094 \quad 4.59145 \quad 1.97510$

$2.84264 \quad 4.57055 \quad 1.97484$

$2.82456 \quad 4.54987 \quad 1.97458$

$2.806694 .52941 \quad 1.97432$

$2.78904 \quad 4.50916 \quad 1.97405$

$2.77159 \quad 4.48912 \quad 1.97378$

$2.75434 \quad 4.46929 \quad 1.97351$

$2.73729 \quad 4.44965 \quad 1.97324$

$2.72043 \quad 4.430211 .97296$

$2.70376 \quad 4.41096 \quad 1.97269$

$2.68728 \quad 4.391911 .97240$

$2.67098 \quad 4.37303 \quad 1.97212$

2.638914 .335831 .97154

2.623144 .317491 .97125

2.607534 .299321 .97096
$2.65486 \quad 4.354341 .97183$
600

610

620

630

640

650

660

670

680

690

700

710

720

730

740

750

760

770

780

790

800

810

820

830

840

850

860

870

880

890

900

910

920

930

940

950

960

970

980

990

1000

1010

1020

1030

1040

1050

1060

1070

1080

1090 
Table II Harmonic Oscillator Contributions to the Thermodynamic

Functions (in units of calories, moles, and ${ }^{\circ} \mathrm{K}$ )

$\begin{array}{ccccc}\nu-\left(F^{\circ}-E_{0}^{\circ}\right) / T & \text { So } & c \% & \nu \\ \mathrm{cm}^{-1} & & & \mathrm{~cm}^{-1}\end{array}$

$\mathrm{T}=5000$

$1100 \quad 2.59209 \quad 4.28132 \quad 1.97066$

$1110 \quad 2.576824 .26349 \quad 1.97036$

$1120 \quad 2.56170 \quad 4.24532 \quad 1.97006$

$1130 \quad 2.54674 \quad 4.22831 \quad 1.96975$

$1140 \quad 2.53194 \quad 4.21096 \quad 1.96944$

$1150 \quad 2.51729 \quad 4.19376 \quad 1.96913$

$1160 \quad 2.50278 \quad 4.17671 \quad 1.96882$

$1170 \quad 2.488434 .15981 \quad 1.96850$

$1180 \quad 2.474214 .143061 .96819$

$1190 \quad 2.46014 \quad 4.12645 \quad 1.96787$

$1200 \quad 2.44621 \quad 4.10999 \quad 1.96754$

1210

1220

1230

1240

$2.43241 \quad 4.09366 \quad 1.96721$

$2.41875 \quad 4.07747 \quad 1.96689$

$2.405224 .06141 \quad 1.96655$

2.391824 .045491 .96622

1250

1260

1270

1280

1290

$2.37854 \quad 4.02970 \quad 1.96588$

2.365404 .014041 .96554

$2.35237 \quad 3.998501 .96520$

2.339473 .983091 .96486

$2.326693 .96780 \quad 1.96451$

1300

1310

1320

1330

1340

1350

1360

1370

1380

1390

1400

1410

1420

1430

1440

1450

1460

1470

1480

1490

1500

1510

1520

1530

1540

1550

1560

1570

1580

1590
$2.31403 \quad 3.952631 .96416$

$2.30148 \quad 3.93758 \quad 1.96380$

2.289053 .922651 .96345

$2.27673 \quad 3.90783 \quad 1.96309$

2.264523 .893131 .96273

$2.25242 \quad 3.87854 \quad 1.96237$ 2.240433 .864051 .96200

$2.228543 .84968 \quad 1.96163$

$2.21676 \quad 3.83542 \quad 1.96126$

$2.20508 \quad 3.32126 \quad 1.96089$

$2.19351 \quad 3.80720 \quad 1.96051$

$2.18203 \quad 3.79325 \quad 1.96013$

$2.17065 \quad 3.77940 \quad 1.95975$

2.159373 .765651 .95937

$2.148193 .75199 \quad 1.95898$

$2.13710 \quad 3.73844 \quad 1.95859$

$2.12610 \quad 3.72498 \quad 1.95820$

2.115193 .711611 .95780

$2.10438 \quad 3.69834 \quad 1.95740$

2.093653 .685161 .95700

$2.08302 \quad 3.67207 \quad 1.95660$

$2.072473 .65907 \quad 1.95620$

$2.06200 \quad 3.64616 \quad 1.95579$

$2.051623 .63334 \quad 1.95538$

2.041323 .620601 .95497

$2.031113 .60795 \quad 1.95455$

$2.02098 \quad 3.59538 \quad 1.95413$

$2.01093 \quad 3.58290 \quad 1.95371$

2.000953 .570491 .95329

$1.99106 \quad 3.55817 \quad 1.95286$
1100

1110

1120

1130

1140

1150

1160

1170

1180

1190

1200

1210

1220

1230

1240

1250

1260

1270

1280

1290

1300

1310

1320

1330

1340

1350

1360

1370

1380

1390

1400

1410

1420

1430

1440

1450

1460

1470

1480

1490

1500

1510

1520

1530

1540

1550

1560

1570

1580

1590 $\begin{array}{ccccc}\nu & -\left(F^{\circ}-E_{0}^{\circ}\right) / T & \text { So } & \mathrm{C}_{\mathrm{p}} & \nu \\ \mathrm{cm}^{-1} & & & \mathrm{~cm}^{-1}\end{array}$

$T=3000$

$\begin{array}{lllll}1600 \quad 1.98124 & 3.54593 & 1.95243\end{array}$

$1610 \quad 1.97150 \quad 3.53376 \quad 1.95200$

$\begin{array}{lllll}1620 & 1.96183 & 3.52168 & 1.95157\end{array}$

$\begin{array}{llllll}1630 & 1.95224 & 3.50967 & 1.95113\end{array}$

$\begin{array}{lll}1640 \quad 1.94272 & 3.49774 & 1.95070\end{array}$

$\begin{array}{lllll}1650 & 1.93328 & 3.48588 & 1.95025\end{array}$

$1660 \quad 1.92390 \quad 3.47410 \quad 1.94981$

$\begin{array}{lllll}1670 \quad 1.91460 & 3.46239 & 1.94936\end{array}$

$\begin{array}{lllll}1680 & 1.90537 & 3.45075 & 1.94892\end{array}$

$\begin{array}{lll}1690 \quad 1.89620 & 3.43919 & 1.94846\end{array}$

1700

1710

1720

1730

1740

$1.88711 \quad 3.42769 \quad 1.94801$

$1.87808 \quad 3.41627 \quad 1.94755$

1.869113 .404911 .94710

1.860223 .393631 .94663

$1.851393 .38241 \quad 1.94617$

1750

1760

1770

1780

1790

$1.84262 \quad 3.37126 \quad 1.94570$

$1.833923 .36017 \quad 1.94523$

1.825283 .349151 .94476

$1.81670 \quad 3.33820 \quad 1.94429$

$1.808183 .32731 \quad 1.94381$

1800

1810

1820

1830

1840

$1.79972 \quad 3.31648 \quad 1.94333$

$\begin{array}{llll}1.79133 & 3.30571 & 1.34285\end{array}$

$1.782993 .29501 \quad 1.94236$

$\begin{array}{lllll}1.77471 & 3.28437 & 1.94188\end{array}$

$1.766493 .27379 \quad 1.94139$

1850

1860

1870

1880

1890

$\begin{array}{llll}1.75833 & 3.26327 & 1.94090\end{array}$

$1.750223 .25281 \quad 1.94040$

$1.742173 .24240 \quad 1.93991$

$1.73418 \quad 3.23206 \quad 1.93941$

$1.726243 .22177 \quad 1.93890$

1900

1910

1920

1930

1940

$\begin{array}{llll}1.71835 & 3.21154 & 1.93840\end{array}$

$1.710523 .20137 \quad 1.93789$

$1.70274 \quad 3.19125 \quad 1.93738$

$1.69501 \quad 3.18118 \quad 1.93687$

1950

1960

1970

1980

1990

1.687343 .171181 .93636

2000

2010

2020

2030

2040

$\begin{array}{llll}1.67971 & 3.16122 & 1.93584\end{array}$

$1.67214 \quad 3.15132 \quad 1.93532$

$1.664623 .14147 \quad 1.93480$

$1.657153 .13168 \quad 1.93427$

$1.64973 \quad 3.12194 \quad 1.93375$

2050

2060

2070

2080

2090

1600

1610

1620

1630

$164 \mathrm{C}$

1650

1660

1670

1680

1690

1700

1710

1720

1730

1740

1750

1760

1770

1780

1790

1800

1810

1820

1830

1840

1850

1860

1870

1880

1890

1900

1910

1920

1930

1940

1950

1960

1970

1980

1990

$1.64235 \quad 3.11224 \quad 1.93322$

$1.63503 \quad 3.10260 \quad 1.93268$

$1.627753 .09301 \quad 1.93215$

$1.620523 .08347 \quad 1.93161$

1.613343 .073981 .93107

2000

2010

2020

2030

2040

$1.60620 \quad 3.06454 \quad 1.93053$

2050

$1.599113 .05515,1.92999$

$1.59206 \quad 3.04580 \quad 1.92944$

$1.585063 .03651 \quad 1.92889$

$1.578113 .02726 \quad 1.92834$
2060

2070

2080

2090 
Table II Harmonic Oscillator Contributions to the Thermodynamic

Functions (in units of calories, moles, and ${ }^{\circ} \mathrm{K}$ )
$\nu \quad-\left(F^{\circ}-E_{0}^{\circ}\right) / T \quad S^{\circ}$
$\nu$
$\nu$
$\mathrm{cm}^{-1}$
So
$\mathrm{C}_{\mathrm{p}}^{\circ}$
$\mathrm{cm}^{-1}$

$T=5000$.

$T=5000$

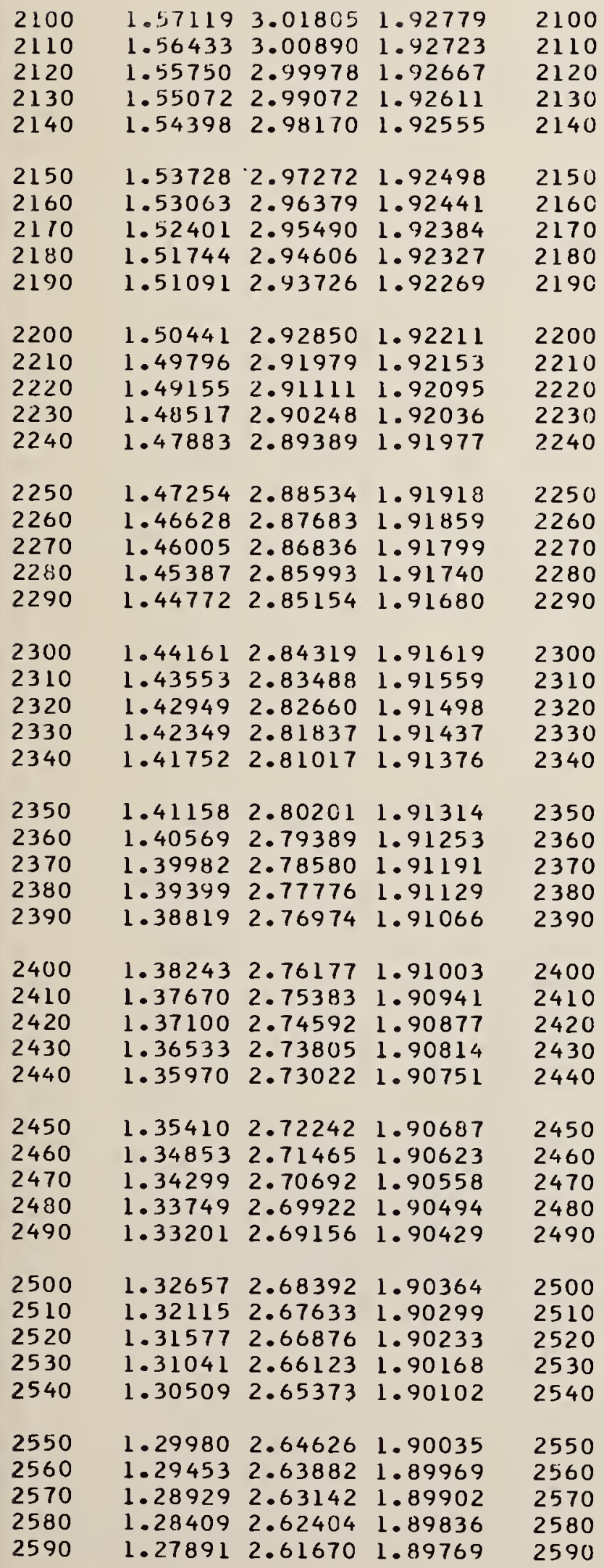

2600

2610

2620

2630

2640

2650

2660

2670

2680

2690

2700

2710

2720

2730

2740

2750

2760

2770

2780

2790

2800

2810

2820

2830

2840

2850

2860

2870

2880

2890

2900

2910

2920

2930

2940

2950

2960

2970

2980

2990

3000

3010

3020

3030

3040

3050

3060

3070

3080

3090
$1.27375 \quad 2.60939 \quad 1.89701$ $1.268632 .60211 \quad 1.89634$ $1.263542 .59486 \quad 1.89566$ 1.258472 .587641 .89498 $1.253432 .58045 \quad 1.89430$

1.248422 .573291 .89361 $1.243432 .56616 \quad 1.89292$ $1.238472 .55906 \quad 1.89223$ $1.233542 .55198 \quad 1.89154$ $1.228632 .54494 \quad 1.89085$

$1.223752 .53792 \quad 1.89015$ $1.21890 \quad 2.53094 \quad 1.88945$ $1.214072 .52398 \quad 1.88875$ $1.209262 .51705 \quad 1.88805$ $1.204492 .51015 \quad 1.88735$

$\begin{array}{llll}1.19973 & 2.50327 & 1.88664\end{array}$ 1.195012 .496431 .88593 $1.19030 \quad 2.48961 \quad 1.88522$ 1.185622 .482821 .88450 1.180972 .476051 .88378

$1.176342 .46931 \quad 1.88307$ $1.171742 .46260 \quad 1.88235$ $1.167152 .45591 \quad 1.88162$ 1.162602 .449251 .88090 1.158062 .442621 .88017

$1.15355 \quad 2.43601 \quad 1.87944$ $1.149062 .42943 \quad 1.87871$ $1.14460 \quad 2.42288 \quad 1.87797$ 1.140152 .416351 .87724 1.135732 .409841 .87650

$1.13134 \quad 2.40336 \quad 1.87576$ $1.126962 .39690 \quad 1.87501$ $\begin{array}{llll}1.12261 & 2.39047 & 1.87427\end{array}$ 1. $11828 \quad 2.38407 \quad 1.87352$ $\begin{array}{llll}1.11397 & 2.37768 & 1.87277\end{array}$

$1.10968 \quad 2.371331 .87202$ $1.105412 .36499 \quad 1.87126$ $1.101172 .35868 \quad 1.87051$ $1.096942 .35240 \quad 1.86975$ 1.092742 .346131 .86899

$1.08856 \quad 2.33989 \quad 1.86822$ $1.08440 \quad 2.33368 \quad 1.86746$ $1.080262 .32749 \quad 1.86669$ 1.076142 .321321 .86592 $1.07204 \quad 2.31517 \quad 1.86515$

$1.06796 \quad 2.30905 \quad 1.86438$ $1.06390 \quad 2.30294 \quad 1.86360$ $1.05986 \quad 2.29687 \quad 1.86282$ 1.055842 .29081
1.086204 $1.051842 .28477 \quad 1.86126$
2600

2610

2620

2630

2640

2650

2660

2670

2680

2690

2700

2710

2720

2730

2740

2750

2760

2770

2780

2790

2800

2810

2820

2830

2840

2850

2860

2870

2880

2890

2900

2910

2920

2930

2940

2950

2960

2970

2980

2990

3000

3010

3020

3030

3040

3050

3060

3070

3080

3090 
Table II Harmonic Oscillator Contributions to the Thermodynamic

Functions (in units of calories, moles, and ${ }^{\circ} \mathrm{K}$ )

$\mathrm{cm}^{-1}-\left(F^{\circ}-\mathrm{E}_{0} \mathrm{O}\right) / \mathrm{T} \quad \mathrm{SO}$

$T=5000$.

$$
\begin{array}{ccccc}
\nu & -\left(F_{-} \mathrm{E}_{\mathrm{O}}\right) / \mathrm{T} & \text { So } & \mathrm{C}_{\mathrm{p}}^{\circ} & \nu \\
\mathrm{cm}^{-1} & & & \mathrm{~cm}^{-1}
\end{array}
$$

$r=5000$.

3100

3110

3120

3130

3140

3150

3160

3170

3180

3190

3200

3210

3220

3230

3240

3250

3260

3270

3280

3290

3300

3310

3320

3330

3340

3350

3360

3370

3380

3390

3400

3410

3420

3430

3440

3450

3460

3470

3480

3490

3500

3510

3520

3530

3540

3550

3560

3570

3580

3590
$1.04786 \quad 2.27876 \quad 1.86048$ $\begin{array}{llll}1.04390 & 2.27277 & 1.85969\end{array}$

$1.03996 \quad 2.26680 \quad 1.85890$

$1.036042 .26085 \mathrm{l} .85811$

1.032132 .254931 .85732

$1.02825 \quad 2.24902 \quad 1.85652$

$1.02438 \quad 2.24314 \quad 1.85572$

$1.020542 .23728 \quad 1.85492$

$1.016712 .23144 \quad 1.85412$

$1.01290 \quad 2.225621 .85332$

$1.00910 \quad 2.21982 \quad 1.85251$

$1.005332 .214 C 4 \quad 1.85171$

$1.001572 .20828 \quad 1.85090$

$0.99783 \quad 2.20254 \quad 1.85008$

$0.994112 .19683 \quad 1.84927$

$0.990412 .19113 \quad 1.84845$

$0.98672 \quad 2.18545 \quad 1.84763$

$0.98306 \quad 2.17979 \quad 1.84681$

$0.97940 \quad 2.17416 \quad 1.84599$

$0.975772 .16854 \quad 1.84517$

$0.97215 \quad 2.16294 \quad 1.84434$

$0.968552 .15736 \quad 1.84351$

$0.964972 .15180 \quad 1.84268$

0.961402 .146261 .84185

$0.957852 .14074 \quad 1.84101$

$0.95432 \quad 2.13524 \quad 1.84018$

$0.95080 \quad 2.12975 \quad 1.83934$

$0.94730 \quad 2.12429 \quad 1.83850$

$0.94382 \quad 2.118841 .83765$

$0.940352 .11341 \quad 1.83681$

$0.93690 \quad 2.10800 \quad 1.83596$

$0.93346 \quad 2.10261 \quad 1.83511$

0.930042 .097241 .83426

0.926642 .091891 .83341

0.923252 .086551 .83255

0.919872 .081231 .83170

0.916512 .075931 .83084

0.913172 .070651 .82998

$0.909842 .06538 \quad 1.82911$

0.906532 .060141 .82825

$0.90323 \quad 2.05491 \quad 1.82738$

0.899952 .049691 .82651

$0.89668 \quad 2.04450 \quad 1.82564$

0.893432 .039321 .82477

0.890192 .034161 .82389

$0.88696 \quad 2.02902 \quad 1.82302$

$0.883752 .02389 \quad 1.82214$

$0.880562 .01878 \quad 1.82126$

0.877382 .013691 .82037

$0.874212 .00861 \quad 1.81949$
3100

3110

3120

3130

3140

3150

3160

3170

3180

3190

3200

3210

3220

3230

3240

3250

3260

3270

3280

3290

3300

3310

3320

3330

3340

3350

3360

3370

3380

3390

3400

3410

3420

3430

3440

3450

3460

3470

3480

3490

3500

3510

3520

3530

3540

3550

3560

3570

3580

3590
3600

3610

3620

3630

3640

3650

3660

3670

3680

3690

3700

3710

3720

3730

3740

3750

3760

3770

3780

3790

3800

3810

3820

3830

3840

3850

3860

3870

3880

3890

3900

3910

3920

3930

3940

3950

3960

3970

3980

3990

4000
$0.87106 \quad 2.00355 \quad 1.81860$

$0.86792 \quad 1.99851 \quad 1.81771$

$0.86479 \quad 1.99348 \quad 1.81682$

$0.861681 .98847 \quad 1.81593$

$0.85859 \quad 1.98348 \quad 1.81503$

$0.85550 \quad 1.77850 \quad 1.81414$

$0.852431 .97353 \quad 1.81324$

$0.84938 \quad 1.96859 \quad 1.81234$

$0.846331 .96366 \quad 1.81144$

$0.843301 .95874 \quad 1.81053$

$0.84029 \quad 1.95384 \quad 1.80963$

$0.83728 \quad 1.94896 \quad 1.80872$

$\begin{array}{llll}0.83429 & 1.944 C 9 & 1.80781\end{array}$

$0.831321 .93924 \quad 1.80690$

$0.828351 .93441 \quad 1.80598$

$0.82540 \quad 1.92958 \quad 1.80507$

$0.82246 \quad 1.92478 \quad 1.80415$

0.819541 .919991 .80323

0.816631 .915211 .80231

0.813731 .910451 .80139

$0.810841 .90571 \quad 1.80046$

$0.80796 \quad 1.90098 \quad 1.79953$

$0.80510 \quad 1.89626 \quad 1.79861$

0.802251 .891561 .79768

$0.799411 .88687 \quad 1.79674$

$0.79659 \quad 1.88220 \quad 1.79581$

$0.79377 \quad 1.87754 \quad 1.79487$

0.790971 .872901 .79393

$0.788181 .86827 \quad 1.79299$

0.785401 .863661 .79205

$0.782641 .85906 \quad 1.79111$

$0.77988 \quad 1.854471 .79016$

$0.77714 \quad 1.84990 \quad 1.78922$

$0.77441 \quad 1.845351 .78827$

$0.771691 .84080 \quad 1.78732$

$\begin{array}{llll}0.76898 & 1.83627 & 1.78636\end{array}$

$0.76629 \quad 1.83176 \quad 1.78541$

$\begin{array}{llll}0.76360 & 1.82726 & 1.78445\end{array}$

$0.760931 .82277 \quad 1.78350$

$0.75827 \quad 1.81829 \quad 1.78254$

$0.75562 \quad 1.813831 .78157$
3600

3610

3620

3630

3640

3650

3660

3670

3680

3690

3700

3710

3720

3730

3740

3750

3760

3770

3780

3790

3800

3810

3820

3830

3840

3850

3860

3870

3880

3890

3900

3910

3920

3930

3940

3950

3960

3970

3980

3990

4000 


\section{THE NATIONAL BUREAU OF STANDARDS}

\section{Functions and Activities}

The functions of the National Bureau of Standards are set forth in the Act of Congress, March 3, 1901, as amended by Congress in Public Law 619, 1950. These include the development and maintenance of the national standards of measurement and the provision of means and methods for making measurements consistent with these standards; the determination of physical constants and properties of materials; the development of methods and instruments for testing materials, devices, and structures; advisory services to govermment agencies on scientific and technical problems; invention and development of devices to serve special needs of the Government; and the development of standard practices, codes, and specifications. The work includes basic and applied research, development, engineering, instrumentation, testing, evaluation, calibration services, and various consultation and information services. Research projects are also performed for other government agencies when the work relates to and supplements the basic program of the Bureau or when the Bureau's unique competence is required. The scope of activities is suggested by the listing of divisions and sections on the next page.

\section{Publications}

The results of the Bureau's research are published either in the Bureau's own series of publications or in the journals of professional and scientific societies. The Bureau itself publishes three periodicals available from the Government Printing Office: The Journal of Research, published in four separate sections, presents complete scientific and technical papers; the Technical News Bulletin presents summary and preliminary reports on work in progress; and Basic Radio Propagation Predictions provides data for determining the best frequencies to use for radio communications throughout the world. There are also five series of nonperiodical publications: Monographs, Applied Mathematics Series, Handbooks, Miscellaneous Publications, and Technical Notes.

A complete listing of the Bureau's publications can be found in National Bureau of Standards Circular 460, Publications of the National Bureau of Standards, 1901 to June 1947 (\$1.25), and the Supplement to National Bureau of Standards Circular 460, July 1947 to June 1957 (\$1.50), and Miscellaneous Publication 240, July 1957 to June 1960 (Includes Titles of Papers Published in Outside Journals 1950 to 1959) (\$2.25); available from the Superintendent of Documents, Government Printing Office, Washington 25, D.C. 


\section{U.S. DEPARTMENT OF COMMERCE \\ Luther H. Hodges, Secretary}

NATIONAL BUREAU OF STANDARDS

A. V. Astin, Director

\section{THE NATIONAL BUREAU OF STANDARDS}

The scope of activities of the National Bureau of Standards at its major laboratories in Washington, D.C., and Boulder Colorado, is suggested in the following listing of the divisions and sections engaged in technical work. In general, each section carries out specialized research, development, and engineering in the field indicated by its title. A brief description of the activities, and of the resultant publications, appears on the preceding page.

\section{WASHINGTON, D.C.}

Electricity. Resistance and Reactance. Electrochemistry. Electrical Instruments. Magnetic Measurements. Dielectrics. High Voltage.

Metrology. Photometry and Colorimetry. Refractometry. Photographic Research. Length. Engineering Metrology. Mass and Scale. Volumetry and Densimetry.

Heat. Temperature Physics. Heat Measurements. Cryogenic Physics. Equation of State. Statistical Physics.

Radiation Physics. X-ray. Radioactivity. Radiation Theory. High Energy Radiation. Radiological Equipment. Nucleonic Instrumentation. Neutron Physics.

Analytical and Inorganic Chemistry. Pure Substances. Spectrochemistry. Solution Chemistry. Standard Reference Materials. Applied Analytical Research.

Mechanics. Sound. Pressure and Vacuum. Fluid Mechanics. Engineering Mechanics. Rheology. Combustion Controls.

Polymers. Macromolecules: Synthesis and Structure. Polymer Chemistry. Polymer Physics. Polymer Characterization. Polymer Evaluation and Testing. Applied Polymer Standards and Research. Dental Research.

Metallurgy. Engineering Metallurgy. Microscopy and Diffraction. Metal Reactions. Metal Physics. Electrolysis and Metal Deposition.

Mineral Products. Engineering Ceramics. Glass. Refractories. Crystal Growth. Physical Properties. Constitution and Microstructure.

Building Research. Structural Engineering. Fire Research. Mechanical Systems. Organic Building Materials. Codes and Safety Standards. Heat Transfer. Inorganic Building Materials. Metallic Building Materials.

Applied Mathematics. Numerical Analysis. Computation. Statistical Engineering. Mathematical Physics. Operations Research.

Data Processing Systems. Components and Techniques. Computer Technology. Measurements Automation. Engineering Applications. Systems Analysis.

Atomic Physics. Spectroscopy. Infrared Spectroscopy. Solid State Physics. Electron Physics. Atomic Physics.

Instrumentation. Engineering Electronics. Electron Devices. Electronic Instrumentation. Mechanical Instruments. Basic Instrumentation.

Physical Chemistry. Thermochemistry. Surface Chemistry. Organic Chemistry. Molecular Spectroscopy. Molecular Kinetics. Mass Spectrometry.

Office of Weights and Measures.

\section{BOULDER, COLO.}

Cryogenic Engineering. Cryogenic Equipment. Cryogenic Processes. Properties of Materials. Cryogenic Technical Services.

Ionosphere Research and Propagation. Low Frequency and Very Low Frequency Research. Ionosphere Research. Prediction Services. Sun-Earth Relationships. Field Engineering. Radio Warning Services. Vertical Soundings Research.

Radio Propagation Engineering. Data Reduction Instrumentation. Radio Noise. Tropospheric Measurements. Tropospheric Analysis. Propagation-Terrain Effects. Radio-Meteorology. Lower Atmosphere Physics.

Radio Standards. High Frequency Electrical Standards. Radio Broadcast Service. Radio and Microwave Materials. Atomic Frequency and Time Interval Standards. Electronic Calibration Center. Millimeter-Wave Research. Microwave Circuit Standards.

Radio Systems. Applied Electromagnetic Theory. High Frequency and Very High Frequency Research. Modulation Research. Antenna Research. Navigation Systems.

Upper Atmosphere and Space Physics. Upper Atmosphere and Plasma Physics. Ionosphere and Exosphere Scatter. Airglow and Aurora. Ionospheric Radio Astronomy.

Radio Physics.

Circuit Standards. 





